Division of Geological \& Geophysical Surveys

GEOPHYSICAL REPORT 1999-17

\title{
PROJECT REPORT OF THE 1999 GEOPHYSICAL SURVEY DATA FOR KETCHIKAN AREA, PARTS OF THE CRAIG, DIXON ENTRANCE, AND KETCHIKAN QUADRANGLES, SOUTHEASTERN ALASKA
}

\author{
by
}

Mark Stephens, Doug McConnell, and Jonathan Rudd

THIS REPORT HAS NOT BEEN REVIEWED FOR

TECHNICAL CONTENT OR FOR CONFORMITY TO THE

EDITORIAL STANDARDS OF DGGS

\author{
Released by
}

STATE OF ALASKA

DEPARTMENT OF NATURAL RESOURCES

Division of Geological \& Geophyscial Surveys

3354 College Rd.

Fairbanks, Alaska 99709-3707 


\section{SUMMARY}

\section{Introduction}

This report describes the logistics and results of a DIGHEMV airborne geophysical survey carried out under contract to WGM Inc., Mining and Geological Consultants, for the State of Alaska, Department of Natural Resources, Division of Geological and Geophysical Surveys. The survey was flown from February 26 to April 7, 1999, over five blocks in the Ketchikan area, southeast Alaska. Three of the areas have been merged with previous DIGHEM surveys for presentation on the maps. Total coverage of the new survey blocks amounts to $2,952.6$ miles $(4,750.8 \mathrm{~km})$. The previous DIGHEM surveys comprise a total of 1,740 miles $(2,801 \mathrm{~km})$.

\section{Purpose}

This airborne geophysical survey is part of a program to acquire such data on Alaska's most promising mineral belts and districts. The information acquired is aimed at catalyzing new private sector exploration, discovery, and ultimate development and production. The purpose of the survey was to map the magnetic and conductive properties of the survey area and to detect conductive mineralization. This purpose was accomplished by using a DIGHEM ${ }^{\mathrm{V}}$ multi-coil, multi-frequency electromagnetic system, supplemented by a high sensitivity cesium magnetometer. A GPS electronic navigation system ensured accurate positioning of the geophysical data with respect to the base maps. Visual flight path recovery techniques were used to confirm the location of the helicopter platform.

\section{Products}

Various maps depicting the survey results are provided at scales of $31,680(1$ " $=$ $1 / 2$ mile) and 1:63,360 ( $1 "=1$ mile). Some of the maps are presented on a topographic base. The data sets are processed and presented using Zones 8 and 9 of the Universal Transverse Mercator projection coordinates using the NAD27 datum. The following geophysical parameters are presented on the maps and/or on the digital archive:

\section{? Total Field Magnetics}

? Apparent Resistivity $-7,200 \mathrm{~Hz}$

? Apparent Resistivity $-56,000 \mathrm{~Hz}$

? Interpreted Discrete Electromagnetic Anomalies

Inverse models of selected total magnetic field responses are provided to aid in the understanding of the geology of the survey area. 


\section{Geology}

The survey area falls in the southeastern geologic region of Alaska which is defined by the 'panhandle'. This region falls within the Cordilleran Orogen. Northwest elongate belts of stratified rock occur throughout the region and show various ages of deposition and degrees of deformation and metamorphism. Intrusive rocks are common and range in age from Cambrian to middle Tertiary. Several phases of deformation and metamorphism have taken place during the history of this region. Three major strike slip fault systems cut the region into many pieces. Thrust, low-angle normal, and dip-slip faults are also common throughout the region.

The rocks of southeastern Alaska have been divided into ten tectonic assemblages, five of which are described as terranes and the other five are described as lithic assemblages. The survey area falls within two of these tectonic assemblages: the first is the Craig subterrane of the Alexander terrane and the second is the Gravina belt. In the vicinity of the survey areas, these two assemblages are divided by the northwest trending Clarence Strait strike slip fault with a dextral displacement of approximately $15 \mathrm{~km}$.

The Alexander terrane has a variety of stratified, plutonic and metamorphic rocks. Volcaniclastics, carbonates and conglomerates are the most common rocks in the terrane. The youngest rock of the terrane, the Bokan Mountain Granite, forms a ring-dyke complex on southern Prince of Wales Island.

The Gravina belt consists of marine argillite and greywacke, andesitic to basaltic volcanic and volcaniclastic rocks, conglomerate, and plutons ranging from quartz diorite to dunite and peridotite. These rocks which are upper Jurassic to mid-Cretaceous in age, form a narrow belt east of the Alexander terrane and define the transition from lower grade metamorphism in the west to the higher grade rocks which flank the Coast Range batholith.

\section{Results and Discussion}

The geophysical results, in general, correlate well with the known geology in the survey area. The results confirm the general trends and serve to extend the mapping of individual geologic units beneath the Paleozoic cover.

The total field magnetic and apparent resistivity data sets have successfully mapped the magnetic and conductive characteristics of the lithologies in the survey area. Numerous faults and contacts have been inferred from the survey results.

The discrete EM anomalies are interpreted to fall within one of four general categories. The first type consists of discrete, well-defined anomalies which are usually 
attributed to conductive sulphides or graphite. The second class of anomalies comprises moderately broad responses which exhibit the characteristics of a half space. Some of these anomalies may reflect conductive rock units or zones of deep weathering. The third class of anomalies consists of negative inphase responses which are indicative of magnetite. The fourth class comprises cultural anomalies.

It is recommended that the survey results be reviewed in detail, in conjunction with all available geophysical, geological and geochemical information. Particular reference should be made to the multi-parameter stacked profiles which clearly define the characteristics of the individual anomalies in the identification of target areas. Image processing of existing geophysical data be considered, in order to extract the maximum amount of information from the survey results. 


\section{CONTENTS}

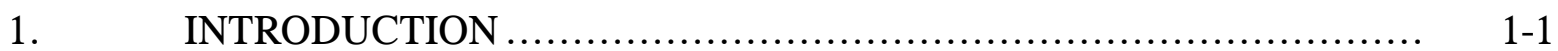

2. SURVEY EQUIPMENT and FIELD PROCEDURES ..................... 2-1

Electromagnetic System ................................................ $2-1$

Mobile Magnetometer ......................................... 2-2

Base Station Magnetometer ......................................... 2-3

Radar Altimeter ...................................................... 2-3

Barometric Pressure and Temperature Sensors .......................... 2-3

Analog Recorder ...................................................... $2-4$

Digital Data Acquisition System .................................... 2-4

Tracking Camera ................................................ $2-4$

Navigation System (RT-DGPS) .................................... $2-5$

Field Processing Software ......................................... $2-6$

3. HISTORICAL DIGHEM SURVEYS .................................... $3-1$

Dolomi Project DIGHEM ${ }^{\mathrm{IV}}$ Survey ....................................... $3-1$

Hetta Inlet and Kasaan Peninsula DIGHEM ${ }^{\mathrm{v}}$ Surveys................... 3- 3-4

4. PRODUCTS and PROCESSING TECHNIQUES ......................... $4-1$

PRODUCTS

Maps.

Other Products................................................. 4-2

PROCESSING TECHNIQUES

Topographic Bases ............................................. $4-2$

Electromagnetic Anomalies ......................................... 4 4

Apparent Resistivity .............................................. 4-5

Total Field Magnetics .................................................. 4-5

Historical Data Processing .......................................... 4 $4-6$

Multi-parameter Stacked Profiles ................................. 4-7

Contour, Colour and Shadow Map Displays.......................... 4 4 4

Total Field Magnetic Data Modeling ............................... 4 4-9

Digital Terrain .................................................... $4-9$

5. SURVEY RESULTS and DISCUSSION .................................. $5-1$

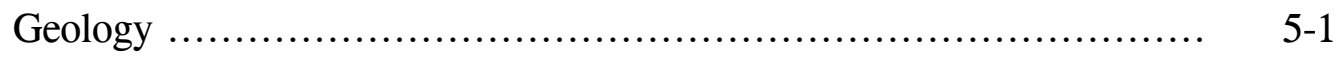

Survey Results .................................................. $5-4$

Salt Chuck and Kasaan Peninsula........................................ $5-15$

Prince of Wales Island, South of Keete Inlet, Hetta Inlet and Dolomi... $\quad$ 5-21

Western Part - Gravina Island ........................................... $\quad 5-34$

Eastern Part - Gravina Island ....................................... $\quad 5-37$

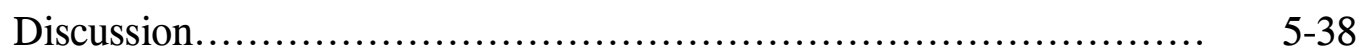


6. CONCLUSIONS and RECOMMENDATIONS _......................... $6-1$

7. REFERENCES .......................................................

APPENDIX A LIST OF PERSONNEL APPENDIX B EM ANOMALY LIST APPENDIX C BACKGROUND INFORMATION APPENDIX D TOTAL FIELD MAGNETIC INVERSE MODELS

\section{LIST OF TABLES}

Table 1-1 Survey Block Descriptions ...................................... 1-2

Table 2-1 DIGHEM System Specifications - Ketchikan Surveys............. 2-1

Table 2-2 The Analog Profiles............................................... 2-6

Table 3-1 Dolomi Project Equipment Specifications......................... 3-2

Table 3-2 Electromagnetic System Specifications - Dolomi Project ......... 3-2

Table 3-3 Hetta Inlet and Kasaan Peninsula Equipment Specifications....... 3-5

Table 3-4 Electromagnetic System Specifications - Hetta Inlet and Kasaan Peninsula Surveys..................................... 3-6

Table 4-1 Multi-parameter Stacked Profiles .................................. 4-8

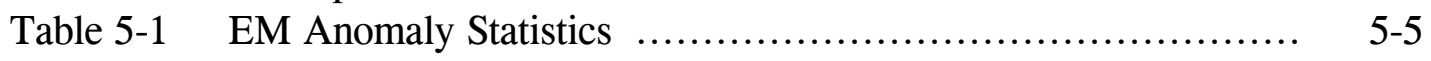

Table B-1 EM Anomaly Grades ...................................... B-2

\section{LIST OF FIGURES}

Figure 1-1 Location of the Ketchikan Survey Areas, Southeast Alaska ....... 1-3

Figure 4-1a Processing Flow Chart - Electromagnetic Data .................. 4-3

Figure 4-1b Processing Flow Chart - Magnetic Data ........................ 4-3

Figure 5-1a Interpretation Sketch of Selected Areas near Ketchikan, Southeast Alaska - Map A - Salt Chuck and Kasaan Peninsula, Prince of Wales Island.

Figure 5-1b Interpretation Sketch of Selected Areas near Ketchikan, Southeast Alaska - Map B - Surveyed Area Immediately North of 55? 15', Prince of Wales Island

Figure 5-1c Interpretation Sketch of Selected Areas near Ketchikan, Southeast Alaska - Map C - Surveyed Area South of 55? 15', Prince of Wales Island.

Figure 5-1d Interpretation Sketch of Selected Areas near Ketchikan, Southeast Alaska - Map D - Western and Eastern Parts, Gravina Island.....

Figure B-1 Typical DIGHEM Anomaly Shapes 


\section{LIST OF MAPS}

GPR 1999-17A Interpretation Map of Selected Areas near Ketchikan, Southeast Alaska - Map A - Salt Chuck and Kasaan Peninsula, Prince of Wales Island .....

GPR 1999-17B Interpretation Map of Selected Areas near Ketchikan, Southeast Alaska - Map B - Surveyed Area Immediately North of 55? 15', Prince of Wales Island

GPR 1999-17C Interpretation Map of Selected Areas near Ketchikan, Southeast Alaska - Map C - Surveyed Area South of 55? 15', Prince of Wales Island

GPR 1999-17D (1) Selected Areas near Ketchikan, Southeas Alaska - Map D - Western and Eastern Parts, Gravina Island ...

map pocket

map pocket

map pocket map pocket 


\section{INTRODUCTION}

A DIGHEMV airborne electromagnetic/resistivity/magnetic survey was flown under contract to WGM Inc., Mining and Geological Consultants, for the State of Alaska, Department of Natural Resources, Division of Geological and Geophysical Surveys (DGGS). The survey was flown from February 26 to April 7, 1999, over five survey blocks located in the Ketchikan area, southeast Alaska. The surveys were carried out in the Craig A-1, A-2, B-1, B-2, B-3, B-4, C-2, Dixon Entrance D-2 and Ketchikan A-6, B5 and B-6 quadrangles.

This airborne geophysical survey is part of a program to acquire such data on Alaska's most promising mineral belts and districts. Major funding for the project comes from the U.S. Bureau of Land Management and the City of Ketchikan. Other contributors include the Sealaska Corporation, the Alaska State Mental Health Division and the City of Thorne Bay. Sealaska Corporation is also contributing data for areas already surveyed (Kasaan Peninsula, Hetta Inlet, and the Dolomi survey area), so that these data can be merged with the new survey data. The Alaska Division of Geological \& Geophysical Surveys is contributing by donating employee time and expertise. The information acquired is aimed at catalyzing new private sector exploration, discovery, and ultimate development and production.

Survey coverage consists of approximately 2,953 miles (4,751 line-km), including 329 miles (529 line-km) of tie lines. A description of the specifications for the individual survey blocks can be found in Table 1-1. The nominal line separation is $1 / 4-$ mile (approximately 400 metres). Tie lines are generally flown perpendicular to the flight line direction with a separation of 3 miles $(5 \mathrm{~km})$.

Historical DIGHEM surveys flown in the area are merged with the current survey for presentation on the final maps and in the archives. A description of these older surveys is included in Table 1-1 and further detail is given in the Historical DIGHEM Surveys section. 
Table 1-1: Survey Block Descriptions

\begin{tabular}{|l|c|c|c|c|}
\hline \multicolumn{1}{|c|}{ Block Name } & $\begin{array}{c}\text { Line Spacing } \\
\text { (mile) }\end{array}$ & $\begin{array}{c}\text { Coverage } \\
\text { (line-miles) }\end{array}$ & $\begin{array}{c}\text { Survey } \\
\text { Speed (mph) }\end{array}$ & $\begin{array}{c}\text { Line Azimuth } \\
\text { (degrees) }\end{array}$ \\
\hline Kasaan Peninsula & $1 / 8$ & 422.0 & 61 & 045 \\
Salt Chuck & $1 / 4$ & 332.7 & 70 & 090 \\
North Prince of Wales & $1 / 4$ & $3,303.5$ & 47 & 000 \\
Hetta Inlet & $1 / 8$ & $1,060.0$ & 63 & 090 \\
Dolomi & $1 / 8$ & 258.0 & 48 & 000 \\
South Prince of Wales & $1 / 4$ & 330.8 & 58 & 000 \\
West Gravina & $1 / 4$ & 398.6 & 41 & 093 \\
East Gravina & $1 / 4$ & 68.4 & 76 & 090 \\
\hline
\end{tabular}

The current survey employs the DIGHEMV electromagnetic system. Ancillary equipment consists of a magnetometer, radar altimeter, video camera, analog and digital recorders and an electronic navigation system.

Section 2 gives a description of the survey equipment and specifications and an outline of the field procedures. Section 3 gives a description of the previous DIGHEM surveys carried out in the Ketchikan area which are provided with the current survey results. Section 4 describes the processing techniques and products. Section 5 describes the results, and the conclusions and recommendations for further work are given in Section 6. 


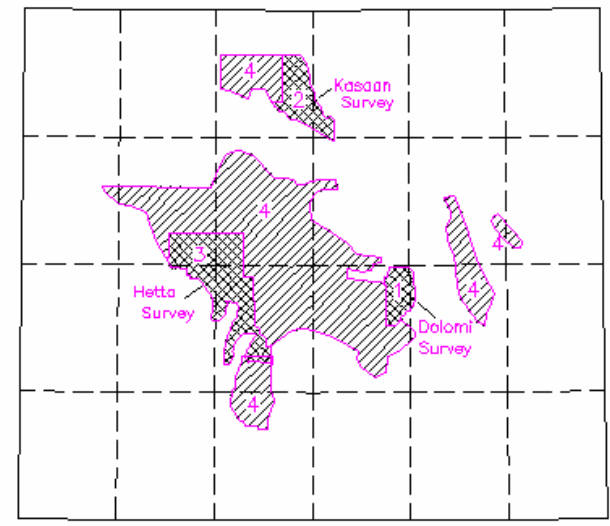

Figure 1-1

Location of the Ketchikan Area Surveys Southeast Alaska 


\section{SURVEY EQUIPMENT AND FIELD PROCEDURES}

The survey instrumentation was installed in an Aerospatiale AS350B2 turbine helicopter (Registration N192-EH) which was owned by Era Aviation Inc. A bird, which houses much of the electromagnetic and magnetic equipment, is suspended approximately 100 feet beneath the helicopter. The helicopter flew at an average air speed of $49 \mathrm{mph}$ (78 $\mathrm{km} / \mathrm{hr}$ ). The EM bird was flown with an approximate terrain clearance of 100 feet (30 metres).

\section{Electromagnetic System}

The survey was flown with a DIGHEM $\mathrm{V}$ system which has a towed bird, symmetric dipole configuration and is operated at a nominal survey altitude of 30 metres. Table 2-1 lists the specifications for the DIGHEM ${ }^{\mathrm{V}}$ system. Five in-phase and five quadrature components are recorded for each of the five coil pairs. The sample rate of 10 per second is equivalent to 1 sample every $7 \mathrm{ft}(2.2 \mathrm{~m})$ at the average survey speed of 49 $\mathrm{mph}(78 \mathrm{~km} / \mathrm{h})$.

\section{Table 2-1: DIGHEM ${ }^{\mathrm{V}}$ System Specifications - Ketchikan Surveys}

\begin{tabular}{|l|c|c|c|c|}
\hline $\begin{array}{c}\text { Coil } \\
\text { Orientation }\end{array}$ & $\begin{array}{c}\text { Nominal } \\
\text { Frequency }(\mathrm{Hz})\end{array}$ & $\begin{array}{c}\text { Actual } \\
\text { Frequency }(\mathrm{Hz})\end{array}$ & $\begin{array}{c}\text { Coil } \\
\text { Separation }(\mathrm{m})\end{array}$ & $\begin{array}{c}\text { Sensitivity } \\
(\mathrm{ppm})\end{array}$ \\
\hline Coaxial & 900 & 1,056 & 8.0 & 0.06 \\
Coplanar & 900 & 883 & 8.0 & 0.06 \\
Coaxial & 5,500 & 5,609 & 8.0 & 0.10 \\
Coplanar & 7,200 & 7,270 & 8.0 & 0.10 \\
Coplanar & 56,000 & 55,736 & 6.3 & 0.30 \\
\hline
\end{tabular}

The electromagnetic system utilizes a multi-coil coaxial/coplanar technique to energize conductors in different directions. The coaxial coils are vertical with their axes in the flight direction. The coplanar coils are horizontal. The secondary fields are sensed simultaneously by means of receiver coils which are maximum coupled to their respective transmitter coils. The system yields an inphase and a quadrature channel from each transmitter-receiver coil-pair.

The DIGHEM calibration procedure involves four stages; primary field bucking, phase calibration, gain calibration, and zero level adjustment. At the beginning of the survey, the primary field at each receiver coil is cancelled, or "bucked out", by precise adjustment of the position of five bucking coils. 
The phase calibration adjusts the phase angle of the receiver to match that of the transmitter. A ferrite bar, which produces a purely in-phase anomaly, is positioned near each receiver coil. The bar is rotated from minimum to maximum field coupling and the responses for the in-phase and quadrature components for each coil pair/frequency are measured. The phase of the response is adjusted at the console to return an in-phase only response for each coil-pair. The EM system was checked for phase calibration at the beginning of each day of operation.

The gain calibration uses external coils designed to produce an equal response on in-phase and quadrature components for each coil pair/frequency. The coil parameters and distances are designed to produce pre-determined responses at the receiver, due to the current induced in the calibration coil by the transmitter when a switch closes the loop at the coil. The gain at the console is adjusted to yield secondary responses of exactly $100 \mathrm{ppm}$. Gain was calibrated at the start and end of the survey flying. Additional gain checks were performed periodically throughout the survey and after any maintenance to the EM system.

The phase and gain calibrations each measure a relative change in the secondary field, rather than an absolute value. This removes any dependency of the calibration procedure on the secondary field due to the ground, except under circumstances of extreme ground conductivity.

During each survey flight, internal (Q-coil) calibration signals are generated to recheck system gain and to establish zero reference levels. These calibrations are carried out at intervals of approximately 20 minutes with the system out of ground effect. At a sensor height of more than $250 \mathrm{~m}$, there is no measurable secondary field from the earth. Any remaining residual is therefore established as the zero level of the system. Linear system drift is automatically removed by interpolating the zero levels between the Q-coil calibrations.

\section{Mobile Magnetometer}

Model: $\quad$ Picodas MEP 710 processor with Geometrics G823A sensor

Type: Optically pumped Cesium vapour

Sensitivity: $\quad 0.01 \mathrm{nT}$

Sample rate: $\quad 10$ per second

The magnetometer sensor is housed in the EM bird $30 \mathrm{~m}$ below the helicopter. 


\section{Base Station Magnetometer}

Model:

Type:

Sensitivity:

Sample rate:

and

Model:

Type:

Sensitivity:

Sample rate:
GEM Systems GSM-19T

Digital recording proton precession

$0.10 \mathrm{nT}$

3 seconds

Picodas MEP710

Digital recording cesium vapor

$0.01 \mathrm{nT}$

1 per second

A digital recorder is operated in conjunction with the base station magnetometers to record the diurnal variations of the earth's magnetic field. The clocks of the base stations are synchronized with that of the airborne system to permit subsequent removal of diurnal drift.

\section{Radar Altimeter}

Manufacturer: Honeywell/Sperry

Type: $\quad$ AA300

Sensitivity: $\quad 0.3 \mathrm{~m}$

The radar altimeter is positioned to measure the vertical distance between the helicopter and the ground.

\section{Barometric Pressure and Temperature Sensors}

Model: $\quad$ DIGHEM D 1300

Type:

Motorola MPX4115AP analog pressure sensor

Sensitivity:

AD592AN high-impedance remote temperature sensors

Sample rate:

Pressure: $\quad 150 \mathrm{mV} / \mathrm{kPa}$

Temperature: $\quad 100 \mathrm{mV} /{ }^{\circ} \mathrm{C}$ or $10 \mathrm{mV} /{ }^{\circ} \mathrm{C}$ (selectable)

Sample rate: $\quad 10$ per second

The D1300 circuit is used in conjunction with one barometric sensor and up to three temperature sensors. Two sensors (baro and temp) are installed in the EM console in the aircraft, to monitor internal operating temperatures. At least one other temperature 
sensor is located in the EM bird to record temperature variations at the receiver coils. The information is recorded by the digital acquisition system, and is displayed on the analog chart records.

\title{
Analog Recorder
}

Manufacturer: $\quad$ RMS Instruments

Type: $\quad$ DGR33 dot-matrix graphics recorder

Resolution: $\quad 4 \times 4$ dots $/ \mathrm{mm}$

Speed: $\quad 1.5 \mathrm{~mm} / \mathrm{sec}$

The analog profiles are recorded on chart paper in the aircraft during the survey. Table 2-1 lists the geophysical data channels and the vertical scale of each profile.

\section{Digital Data Acquisition System}

\author{
Manufacturer: $\quad$ RMS Instruments \\ Model: $\quad$ DGR 33 \\ Recorder: $\quad$ Iomega Zip Plus drive
}

The data are stored on a $100 \mathrm{Mb}$ Zip disc and are downloaded to the field PC workstation at the survey base for verification, backup and preparation of in-field products.

\section{Tracking Camera}

Type: $\quad$ Panasonic VHS colour video camera (NTSC format)

Model: $\quad$ AG 2400/WVCD132

Fiducial numbers are recorded continuously and are displayed on the margin of each image. This procedure ensures accurate correlation of analog and digital data with respect to visible features on the ground. 


\title{
Navigation (Real-Time - Differential Global Positioning System)
}

\author{
$\underline{\text { Airborne Receiver }}$ \\ Model: \\ Ashtech Glonass GG24 \\ Type: \\ SPS (L1 band), 24-channel, C/A code at $1575.42 \mathrm{MHz}$, \\ $\mathrm{S}$ code at $0.5625 \mathrm{MHz}$, Real-time differential. \\ Sensitivity: \\ $-132 \mathrm{dBm}, 0.5$ second update \\ Accuracy: \\ Better than 10 metres real-time \\ $\underline{\text { Base Station }}$ \\ Model: \\ Marconi Allstar OEM, CMT-1200 \\ Type: \\ Code and carrier tracking of L1 band, 12-channel, C/A code \\ Sensitivity: \\ at $1575.42 \mathrm{MHz}$ \\ Accuracy: \\ $-90 \mathrm{dBm}, 1.0$ second update \\ Manufacturer's stated accuracy for differential corrected \\ GPS is 2 metres
}

The Ashtech GG24 is a line of sight, satellite navigation system which utilizes time-coded signals from at least four of forty-eight available satellites. Both Russian GLONASS and American NAVSTAR satellite constellations are used to calculate the position and to provide real time guidance to the helicopter. The Ashtech system is combined with a RACAL GPS receiver which further improves the accuracy of the flying and subsequent flight path recovery to better than 5 metres. The differential corrections, which are obtained from a network of virtual reference stations, are transmitted to the helicopter via a spot-beam satellite.

The Ashtech receiver is coupled with a PNAV navigation system for real-time guidance.

Although the Marconi base station receiver is able to calculate its own latitude and longitude, a higher degree of accuracy can be obtained if the reference unit is established on a known benchmark or triangulation point. For this survey, the GPS station was located at latitude $65 ? 31.81982 \mathrm{~N}$, longitude $148 ? 33.06051$ ' $\mathrm{W}$ at an elevation of 207.56 metres a.m.s.l. The GPS records data relative to the WGS84 ellipsoid, which is the basis of the revised North American Datum (NAD83). The data is differentially post-processed and conversion software is used to transform the WGS84 coordinates to the NAD27 system displayed on the base maps. 
Table 2-2. The Analog Profiles

\begin{tabular}{|c|c|c|c|}
\hline $\begin{array}{l}\text { Channel } \\
\text { Name }\end{array}$ & Parameter & $\begin{array}{c}\text { Scale } \\
\text { Units/mm }\end{array}$ & $\begin{array}{l}\text { Designation on } \\
\text { Digital Profile }\end{array}$ \\
\hline 1X9I & Coaxial inphase $\quad(900 \mathrm{~Hz})$ & $2.5 \mathrm{ppm}$ & CXI900 \\
\hline $1 \mathrm{X9Q}$ & Coaxial quad & $2.5 \mathrm{ppm}$ & CXQ900 \\
\hline 3P9I & Coplanar inphase $(900 \mathrm{~Hz})$ & $2.5 \mathrm{ppm}$ & CPI900 \\
\hline $3 \mathrm{P} 9 \mathrm{Q}$ & Coplanar quad $\quad(900 \mathrm{~Hz})$ & $2.5 \mathrm{ppm}$ & CPQ900 \\
\hline 2P7I & Coplanar inphase $(7,200 \mathrm{~Hz})$ & $5 \mathrm{ppm}$ & CPI7200 \\
\hline $2 \mathrm{P} 7 \mathrm{Q}$ & Coplanar quad $\quad(7,200 \mathrm{~Hz})$ & $5 \mathrm{ppm}$ & CPQ7200 \\
\hline 4X7I & Coaxial inphase $\quad(5,500 \mathrm{~Hz})$ & $5 \mathrm{ppm}$ & CXI5500 \\
\hline 4X7Q & Coaxial quad & $5 \mathrm{ppm}$ & CXQ5500 \\
\hline 5P5I & Coplanar inphase $(56,000 \mathrm{~Hz})$ & $10 \mathrm{ppm}$ & CPI56K \\
\hline $5 \mathrm{P} 5 \mathrm{Q}$ & Coplanar quad $\quad(56,000 \mathrm{~Hz})$ & $10 \mathrm{ppm}$ & CPQ56K \\
\hline ALTR & Altimeter (radar) & $3 \mathrm{~m}$ & ALTR \\
\hline MAGC & Magnetics, coarse & $20 \mathrm{nT}$ & MAG \\
\hline MAGF & Magnetics, fine & $2.0 \mathrm{nT}$ & MAG \\
\hline CXSP & Coaxial spherics monitor & & \\
\hline CPSP & Coplanar spherics monitor & & CPS \\
\hline CXPL & Coaxial powerline monitor & & CXP \\
\hline CPPL & Coplanar powerline monitor & & $\mathrm{CPP}$ \\
\hline 4XSP & Coaxial spherics monitor & & \\
\hline $1 \mathrm{KPA}$ & Altimeter (barometric) & $30 \mathrm{~m}$ & \\
\hline 3TDC & Temperature & $1^{\circ} \mathrm{C}$ & \\
\hline 2TDC & Internal (console) temperature & $1^{\circ} \mathrm{C}$ & \\
\hline
\end{tabular}

\section{Field Workstation Software}

Model:

Manufacturer:

Type:
Geoterrex-Dighem Processing Software

Geoterrex-Dighem

Windows-Based P.C.

A laptop PC-based field workstation is used at the survey base to verify data quality and completeness. Flight data are transferred to a PC to permit the creation of a database. This process allows the field operators to display both the positional (flight path) and geophysical data on a screen or printer. 


\section{HISTORICAL DIGHEM SURVEYS}

\section{Dolomi Project DIGHEM ${ }^{\text {IV }}$ Survey}

A DIGHEM ${ }^{\mathrm{IV}}$ electromagnetic/resistivity/magnetic/VLF survey was flown for American Copper \& Nickel Company, Inc. from March 23 to March 26, 1991, over a survey block located approximately 35 miles west-southwest of Ketchikan, Alaska. The survey area is situated on the southwestern tip of Prince of Wales Island (see Figure 1-1).

Survey coverage consisted of approximately 258 line-miles (415 line-km), including the tie lines. Flight lines were flown in an azimuthal direction of 000 ? with a line separation of $1 / 8$ mile (approximately $200 \mathrm{~m}$ ).

The survey employed the DIGHEM ${ }^{\mathrm{IV}}$ electromagnetic system, a magnetometer, radar altimeter, video camera, analog and digital recorders, a VLF receiver and an electronic navigation system. The instrumentation was installed in an Aerospatiale AS350B turbine helicopter (Registration N157EH) which was provided by ERA Aviation, Inc. The helicopter flew at an average airspeed of $48 \mathrm{mph}(77 \mathrm{~km} / \mathrm{h})$ with a target flying height of approximately $200 \mathrm{ft}(60 \mathrm{~m})^{*}$. Table 3-1 provides a description of the technical specifications for the instrumentation used in the Dolomi Project survey.

Table 3-2 presents the detailed configuration of the electromagnetic system for the Dolomi Project survey.

The DIGHEM ${ }^{\mathrm{IV}}$ electromagnetic system is a towed bird symmetric dipole system which utilizes a multi-coil coaxial/coplanar technique to energize conductors in different directions. The coaxial coils are vertical with their axes in the flight direction. The coplanar coils are horizontal. The secondary fields are sensed simultaneously by means of receiver coils which are maximum coupled to their respective transmitter coils. The system yields an inphase and a quadrature channel from each transmitter-receiver coil-pair.

\footnotetext{
* The actual height of the helicopter was considerably higher than the $60 \mathrm{~m}$ target height due to logistical circumstances, which produced an average additional height of about $17 \mathrm{~m}$ for each of the sensors and the helicopter.
} 


\section{Table 3-1: Dolomi Project Equipment Specifications}

\begin{tabular}{|c|c|c|c|c|c|c|c|}
\hline Equipment & Manufacturer & Model & Type & Accuracy & Sensitivity & Rate & $\begin{array}{c}\text { Nominal } \\
\text { Survey } \\
\text { Altitude }\end{array}$ \\
\hline $\begin{array}{l}\text { Electromagnetic } \\
\text { System }\end{array}$ & Geoterrex-Dighem & DIGHEM $^{\text {IV }}$ & $\begin{array}{l}\text { Towed bird } \\
\text { Symmetric dipole }\end{array}$ & & $\begin{array}{l}0.1 \text { to } \\
0.5 \mathrm{ppm}\end{array}$ & 10 samples/s & $30 \mathrm{~m}^{*}$ \\
\hline $\begin{array}{l}\text { Magnetometer } \\
\text { Sensor }\end{array}$ & Picodas & 3340 & $\begin{array}{l}\text { Optically pumped } \\
\text { Cesium vapour }\end{array}$ & 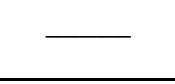 & $0.01 \mathrm{nT}$ & 10 samples/s & $45 \mathrm{~m}^{*}$ \\
\hline $\begin{array}{l}\text { Magnetometer } \\
\text { Base Station }\end{array}$ & Scintrex & MP-3 & $\begin{array}{l}\text { Digital recording } \\
\text { Proton precession }\end{array}$ & 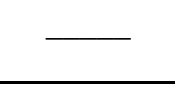 & $0.10 \mathrm{nT}$ & $\begin{array}{l}0.2 \\
\text { samples/s }\end{array}$ & - \\
\hline $\begin{array}{l}\text { VLF-EM } \\
\text { System }\end{array}$ & $\begin{array}{l}\text { Herz Industries } \\
\text { Ltd. }\end{array}$ & Totem-2A & - & + & $0.1 \%$ & 10 samples/s & $50 \mathrm{~m}^{*}$ \\
\hline $\begin{array}{l}\text { Radar } \\
\text { Altimeter }\end{array}$ & $\begin{array}{l}\text { Honeywell/ } \\
\text { Sperry }\end{array}$ & AA220 & $\begin{array}{l}4.3 \mathrm{GHz} \text { short pulse } \\
\text { modulation }\end{array}$ & 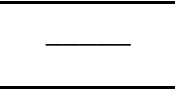 & $0.3 \mathrm{~m}$ & 10 samples/s & \\
\hline $\begin{array}{l}\text { Analog } \\
\text { Recorder }\end{array}$ & $\begin{array}{l}\text { RMS } \\
\text { Instruments }\end{array}$ & DGR33 & $\begin{array}{l}\text { Dot matrix graphics } \\
\text { Recorder }\end{array}$ & $\begin{array}{l}4 \mathrm{x} 4 \\
\text { dots/mm }\end{array}$ & 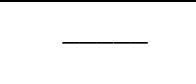 & $1.5 \mathrm{~mm} / \mathrm{s}$ & 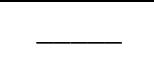 \\
\hline
\end{tabular}

\begin{tabular}{|l|l|l|l|l|l|l|c|}
\hline $\begin{array}{l}\text { Digital Data } \\
\text { Acquisition } \\
\text { System }\end{array}$ & $\begin{array}{l}\text { RMS } \\
\text { Instruments }\end{array}$ & DGR33 & $\begin{array}{l}\text { RMS TCR12 Tape } \\
\text { Deck, 6400 bpi, tape } \\
\text { cartridge recorder }\end{array}$ & - & - & $\begin{array}{l}\text { Up to } \\
9600 \text { bytes/s }\end{array}$ & - \\
\hline Video System & Panasonic & $\begin{array}{l}\text { AG 2400/ } \\
\text { WVCD 132 }\end{array}$ & VHS colour (NTSC) & - & - & Continuous & $60 \mathrm{~m}^{*}$ \\
\hline $\begin{array}{l}\text { Navigation } \\
\text { System }\end{array}$ & Del Norte & 547 & $\begin{array}{l}\text { UHF electronic } \\
\text { Positioning system }\end{array}$ & - & $1 \mathrm{~m}$ & 2 samples/s & - \\
\hline $\begin{array}{l}\text { Field } \\
\text { Workstation }\end{array}$ & Geoterrex-Dighem & FWS & $\begin{array}{l}\text { PC processing } \\
\text { System }\end{array}$ & - & - & - & - \\
\hline
\end{tabular}

Table 3-2: Electromagnetic System Specifications - Dolomi Project

\begin{tabular}{|c|c|c|c|c|c|}
\hline $\begin{array}{c}\text { Coil-pair } \\
\text { Name }\end{array}$ & $\begin{array}{c}\text { Actual } \\
\text { Frequency } \\
\text { (Hz) }\end{array}$ & $\begin{array}{c}\text { Coil } \\
\text { Separation } \\
\text { (m) }\end{array}$ & $\begin{array}{c}\text { Sensitivity } \\
\text { (ppm) }\end{array}$ & $\begin{array}{c}\text { Sample } \\
\text { Rate } \\
\text { (seconds }^{-1} \text { ) }\end{array}$ & $\begin{array}{c}\text { Channels } \\
\text { Recorded }\end{array}$ \\
\hline 900 Hz coaxial & 1,059 & 8.0 & 0.1 & 10 & Inphase/quadrature \\
900 Hz coplanar & 893 & 8.0 & 0.1 & 10 & Inphase/quadrature \\
7,200 Hz coplanar & 7,300 & 8.0 & 0.2 & 10 & Inphase/quadrature \\
56,000 Hz & 55,870 & 6.3 & 0.5 & 10 & Inphase/quadrature \\
coplanar & & & & \\
\hline
\end{tabular}

\footnotetext{
* The actual height of the helicopter was considerably higher than the $60 \mathrm{~m}$ target height due to logistical circumstances, which produced an average additional height of about $17 \mathrm{~m}$ for each of the sensors and the helicopter.
} 


\section{Mobile Magnetometer}

The magnetometer sensor is housed in a separate bird $15 \mathrm{~m}$ below the helicopter.

\section{Base Station Magnetometer}

A digital recorder is operated in conjunction with the base station magnetometer to record the diurnal variations of the earth's magnetic field. The clock of the base station is synchronized with that of the airborne system to permit subsequent removal of diurnal drift.

\section{VLF-EM System}

The VLF receiver measures the total field and vertical quadrature components of the secondary VLF field. Signals from two separate transmitters can be measured simultaneously. The VLF sensor is towed in a bird $10 \mathrm{~m}$ below the helicopter. The signals from Seattle, Washington; NLK, $24.8 \mathrm{kHz}$ and Annapolis, Maryland; NSS, 21.4 $\mathrm{kHz}$ were recorded.

\section{Radar Altimeter}

The radar altimeter is positioned to measure the vertical distance between the helicopter and the ground.

\section{Analog Recorder}

The analog profiles are recorded on chart paper in the aircraft during the survey.

\section{Digital Data Acquisition System}

The data are stored on TCR-12 tape cartridges which are downloaded to the field PC workstation at the survey base for verification, backup and preparation of in-field products.

\section{Tracking Camera}

Fiducial numbers are recorded continuously and are displayed on the margin of each image. This procedure ensures accurate correlation of analog and digital data with respect to visible features on the ground. 


\section{Navigation System}

The navigation system uses ground based transponder stations which transmit distance information back to the helicopter. The ground stations are set up well away from the survey area and are positioned such that the signals cross the survey block at an angle between 30 ? and 150?. The on-board central processing unit takes any two transponder distances and determines the helicopter position relative to these two ground stations in cartesian coordinates.

The cartesian coordinates are transformed to UTM coordinates during data processing. This conversion is accomplished by correlating a number of prominent topographical locations with the navigational data points. The use of numerous visual tie points serves two purposes: to accurately relate the navigation data to the map sheet and to minimize location errors which might result from distortions in uncontrolled photomosaic base maps.

\section{Field Workstation Software}

A PC-based field workstation is used at the survey base to verify data quality and completeness. Flight data are transferred to a PC to permit the creation of a database. This process allows the field operators to display both the positional (flight path) and geophysical data on a screen or printer.

\section{Hetta Inlet and Kasaan Peninsula DIGHEM ${ }^{\mathrm{V}}$ Surveys}

A DIGHEM ${ }^{\mathrm{V}}$ electromagnetic/resistivity/magnetic/VLF survey was flown for American Copper \& Nickel Company, Inc. from May 17 to May 22, 1992, over the Kasaan Peninsula and the Hetta Inlet, southeast Alaska. The survey areas are both situated on Prince of Wales Island (see Figure 1-1).

Survey coverage consisted of approximately 1,060 line-miles (1707 line-km) for the Hetta Inlet survey and 422 line-miles (679 line-km) for the Kasaan Peninsula survey, including the tie lines. Flight lines were flown in an azimuthal direction of 90 ? for the Hetta Inlet survey and 45 ? for the Kasaan Peninsula survey with a line separation of approximately $1 / 8$ mile $(200 \mathrm{~m})$.

The survey employed the DIGHEM ${ }^{\mathrm{V}}$ electromagnetic system, a magnetometer, radar altimeter, video camera, analog and digital recorders, a VLF receiver and an electronic navigation system. The instrumentation was installed in an Aerospatiale AS350B-1 turbine helicopter (Registration N165EH) which was provided by ERA 
Aviation, Inc. The helicopter flew at an average airspeed of $63 \mathrm{mph}(102 \mathrm{~km} / \mathrm{h})$ for the Hetta Inlet survey and $61 \mathrm{mph}(98 \mathrm{~km} / \mathrm{h})$ for the Kasaan Peninsula survey with a target height of approximately $200 \mathrm{ft}(60 \mathrm{~m})^{*}$. The actual flying height was considerably higher due to logistical circumstances. Table 3-3 provides a description of the technical specifications for the instrumentation used in the Hetta Inlet and Kasaan Peninsula surveys.

\section{Table 3-3: Hetta Inlet and Kasaan Peninsula Equipment Specifications}

\begin{tabular}{|c|c|c|c|c|c|c|c|}
\hline Equipment & Manufacturer & Model & Type & Accuracy & Sensitivity & Rate & $\begin{array}{l}\text { Nominal } \\
\text { Survey } \\
\text { Altitude }\end{array}$ \\
\hline $\begin{array}{l}\text { Electromagnetic } \\
\text { System }\end{array}$ & Geoterrex-Dighem & DIGHEM $^{V}$ & $\begin{array}{l}\text { Towed bird } \\
\text { Symmetric dipole }\end{array}$ & & $\begin{array}{l}0.1 \text { to } \\
0.5 \mathrm{ppm}\end{array}$ & 10 samples/s & $30 \mathrm{~m}^{*}$ \\
\hline $\begin{array}{l}\text { Magnetometer } \\
\text { Sensor }\end{array}$ & Picodas & 3340 & $\begin{array}{l}\text { Optically pumped } \\
\text { Cesium vapour }\end{array}$ & & $0.01 \mathrm{nT}$ & 10 samples/s & $40 \mathrm{~m}^{*}$ \\
\hline $\begin{array}{l}\text { Magnetometer } \\
\text { Base Station }\end{array}$ & Scintrex & MP-3 & $\begin{array}{l}\text { Digital recording } \\
\text { Proton precession }\end{array}$ & & $0.10 \mathrm{nT}$ & $\begin{array}{l}0.2 \\
\text { samples/s }\end{array}$ & $\longrightarrow$ \\
\hline $\begin{array}{l}\text { VLF-EM } \\
\text { System }\end{array}$ & $\begin{array}{ll}\text { Herz } & \text { Industries } \\
\text { Ltd. } & \\
\end{array}$ & Totem-2A & & & $0.1 \%$ & 10 samples/s & $50 \mathrm{~m}^{*}$ \\
\hline $\begin{array}{l}\text { Radar } \\
\text { Altimeter }\end{array}$ & $\begin{array}{l}\text { Honeywell/ } \\
\text { Sperry }\end{array}$ & AA220 & $\begin{array}{l}4.3 \mathrm{GHz} \text { short pulse } \\
\text { modulation }\end{array}$ & 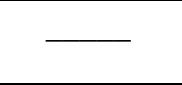 & $0.3 \mathrm{~m}$ & 10 samples/s & \\
\hline $\begin{array}{l}\text { Analog } \\
\text { Recorder }\end{array}$ & $\begin{array}{l}\text { RMS } \\
\text { Instruments }\end{array}$ & DGR33 & $\begin{array}{l}\text { Dot matrix graphics } \\
\text { Recorder }\end{array}$ & $\begin{array}{l}4 \mathrm{x} 4 \\
\text { dots } / \mathrm{mm}\end{array}$ & & $1.5 \mathrm{~mm} / \mathrm{s}$ & \\
\hline $\begin{array}{l}\text { Digital Data } \\
\text { Acquisition } \\
\text { System } \\
\end{array}$ & $\begin{array}{l}\text { RMS } \\
\text { Instruments }\end{array}$ & DGR33 & $\begin{array}{l}\text { RMS TCR12 Tape } \\
\text { Deck, } 6400 \text { bpi, tape } \\
\text { cartridge recorder }\end{array}$ & & & $\begin{array}{l}\text { Up to } \\
9600 \text { bytes/s }\end{array}$ & \\
\hline Video System & Panasonic & $\begin{array}{l}\text { AG 2400/ } \\
\text { WVCD } 132\end{array}$ & VHS colour (NTSC) & & & Continuous & $60 \mathrm{~m}^{*}$ \\
\hline $\begin{array}{l}\text { Navigation } \\
\text { System }\end{array}$ & Del Norte & 547 & $\begin{array}{ll}\text { UHF } & \text { electronic } \\
\text { positioning } & \text { system }\end{array}$ & 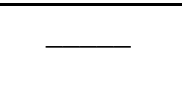 & $1 \mathrm{~m}$ & 2 samples/s & 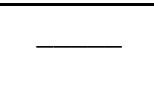 \\
\hline $\begin{array}{l}\text { Field } \\
\text { Workstation }\end{array}$ & Geoterrex-Dighem & FWS & $\begin{array}{l}\text { PC processing } \\
\text { System }\end{array}$ & & & & \\
\hline
\end{tabular}

Table 3-4 presents the detailed configuration of the electromagnetic system for the Hetta Inlet and Kasaan Peninsula surveys.

\footnotetext{
* The actual height of the helicopter was considerably higher than the $60 \mathrm{~m}$ target height due to logistical circumstances, which produced an average additional height of about $17 \mathrm{~m}$ for each of the sensors and the helicopter.
} 
Table 3-4: Electromagnetic System Specifications - Hetta Inlet \& Kasaan Peninsula Surveys

\begin{tabular}{|c|c|c|c|c|c|}
\hline $\begin{array}{c}\text { Coil-pair } \\
\text { Name }\end{array}$ & $\begin{array}{c}\text { Actual } \\
\text { Frequen } \\
\text { cy } \\
\text { (Hz) }\end{array}$ & $\begin{array}{c}\text { Coil } \\
\text { Separation } \\
\text { (m) }\end{array}$ & $\begin{array}{c}\text { Sensitivity } \\
\text { (ppm) }\end{array}$ & $\begin{array}{c}\text { Sample } \\
\text { Rate } \\
\text { (seconds } \mathbf{~} \text { ) }\end{array}$ & $\begin{array}{c}\text { Channels } \\
\text { Recorded }\end{array}$ \\
\hline 900 Hz coaxial & 1,077 & 8.0 & 0.1 & 10 & Inphase/quadrature \\
900 Hz coplanar & 880 & 8.0 & 0.1 & 10 & Inphase/quadrature \\
$7,200 \mathrm{~Hz}$ coaxial & 6,509 & 8.0 & 0.2 & 10 & Inphase/quadrature \\
$7,200 \mathrm{~Hz}$ coplanar & 7,206 & 8.0 & 0.2 & 10 & Inphase/quadrature \\
56,000 Hz coplanar & 54,716 & 6.3 & 0.5 & 10 & Inphase/quadrature \\
\hline
\end{tabular}

The DIGHEM ${ }^{\mathrm{V}}$ electromagnetic system is a towed bird symmetric dipole system which utilizes a multi-coil coaxial/coplanar technique to energize conductors in different directions. The coaxial coils are vertical with their axes in the flight direction. The coplanar coils are horizontal. The secondary fields are sensed simultaneously by means of receiver coils which are maximum coupled to their respective transmitter coils. The system yields an inphase and a quadrature channel from each transmitter-receiver coil-pair.

\section{Mobile Magnetometer}

The magnetometer sensor is housed in a separate bird $20 \mathrm{~m}$ below the helicopter.

\section{Base Station Magnetometer}

A digital recorder is operated in conjunction with the base station magnetometer to record the diurnal variations of the earth's magnetic field. The clock of the base station is synchronized with that of the airborne system to permit subsequent removal of diurnal drift.

\section{VLF-EM System}

The VLF receiver measures the total field and vertical quadrature components of the secondary VLF field. Signals from two separate transmitters can be measured simultaneously. The VLF sensor is housed in the same bird as the magnetometer sensor, and is towed $20 \mathrm{~m}$ below the helicopter. The signal from Annapolis, Maryland; NSS, $21.4 \mathrm{kHz}$ was recorded on all flights for the survey areas. The second signal was chosen 
from Seattle, Washington; NLK, $24.8 \mathrm{kHz}$ and Cutler, Maine; NAA, $24.0 \mathrm{kHz}$ on the basis of availability of adequate signal.

\section{Radar Altimeter}

The radar altimeter is positioned to measure the vertical distance between the helicopter and the ground.

\section{Analog Recorder}

The analog profiles are recorded on chart paper in the aircraft during the survey.

\section{Digital Data Acquisition System}

The data are stored on TCR-12 tape cartridges which are downloaded to the field PC workstation at the survey base for verification, backup and preparation of in-field products.

\section{Tracking Camera}

Fiducial numbers are recorded continuously and are displayed on the margin of each image. This procedure ensures accurate correlation of analog and digital data with respect to visible features on the ground.

\section{Navigation System}

The navigation system uses ground based transponder stations which transmit distance information back to the helicopter. The ground stations are set up well away from the survey area and are positioned such that the signals cross the survey block at an angle between 30 ? and 150? degrees. The on-board central processing unit takes any two transponder distances and determines the helicopter position relative to these two ground stations in cartesian coordinates.

The cartesian coordinates are transformed to UTM coordinates during data processing. This conversion is accomplished by correlating a number of prominent topographical locations with the navigational data points. The use of numerous visual tie points serves two purposes: to accurately relate the navigation data to the map sheet and to minimize location errors which might result from distortions in uncontrolled photomosaic base maps. 


\section{Field Workstation Software}

A PC-based field workstation is used at the survey base to verify data quality and completeness. Flight data are transferred to a PC to permit the creation of a database. This process allows the field operators to display both the positional (flight path) and geophysical data on a screen or printer. 


\section{PRODUCTS AND PROCESSING TECHNIQUES}

This section describes the final delivered products and the techniques employed during data processing, interpretation and presentation. Appendix B provides detailed background information about DIGHEM surveys.

\section{PRODUCTS}

\section{Maps}

Various maps depicting the survey results are provided at scales of 1:31,680 $(1$ ” = $1 / 2$ mile) and 1:63,360 ( $1 "=1$ mile). These maps are available from the State of Alaska, Department of Natural Resources, Division of Geological and Geophysical Surveys. The data sets are processed and presented using Universal Transverse Mercator Zone 8 or 9 projection coordinates using the NAD27 datum. Details of this projection and the conversion from WGS84 are given following:

\section{Projection Description:}

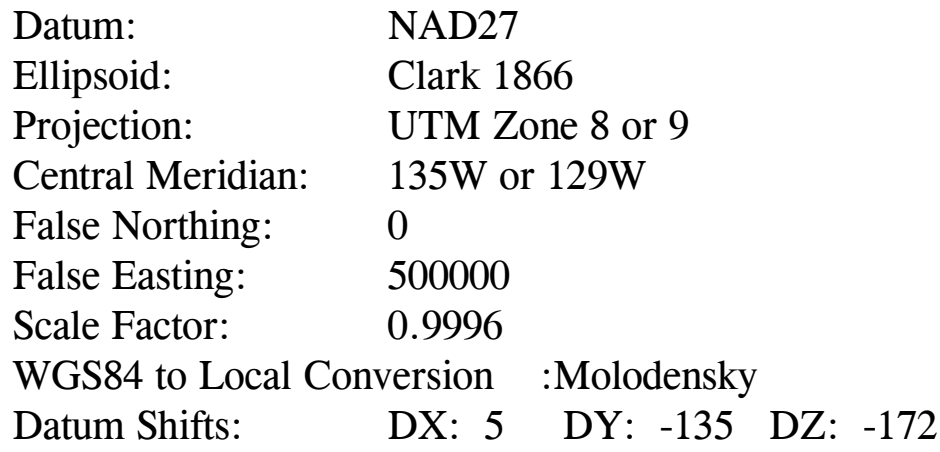

The maps plotted at a scale of 1:63,360 are presented on four map sheets identified as follows:

Map A - Salt Chuck and Kasaan Peninsula, Prince of Wales Island

Map B - Surveyed area immediately north of 55? 15', Prince of Wales Island

Map C - Surveyed area south of 55? 15', Prince of Wales Island

Map D - Western and eastern parts, Gravina Island

Map A contains survey information from a former DIGHEM ${ }^{\mathrm{V}}$ survey on the Kasaan Peninsula and from the current survey (Salt Chuck block). The data sets from these two surveys have been merged for presentation on the maps and the digital grid archive. Map B contains information from the northern part of a former DIGHEM ${ }^{\mathrm{V}}$ 
survey around Hetta Inlet and from the current survey (northern portion, Prince of Wales Island, main block). The data sets from these two surveys have been merged for presentation on the maps and the digital grid archive. Map $\mathrm{C}$ contains information from the southern part of the former DIGHEM ${ }^{\mathrm{V}}$ Hetta Inlet survey, a former DIGHEM ${ }^{\mathrm{V}}$ survey (Dolomi block) and from the current survey (southern portion, Prince of Wales Island, main block and all of the Cordova Bay block). The data sets from these surveys have been merged for presentation on the maps and the digital grid archive. Map D contains information from the western and eastern blocks on Gravina Island from the current survey. Figure 1-1 illustrates the various survey blocks in relation to the map sheets and the local topography.

A map containing an interpretation of the geophysical data can be found in the map pocket at the end of this report. This map presents individual geophysical features, inferred contacts and structural features.

Multi-parameter Stacked Profiles for all survey lines are provided at a scale of 1:63,360. A more detailed description of this product is given later in this section.

\section{Other Products}

The current and historical survey data sets are also provided in digital form as grid and line data archives on CD-ROM. These digital archives are available from the DGGS. The digital data are referenced to the UTM zone 8 or 9 coordinate systems as described above.

\section{PROCESSING TECHNIQUES}

Figure 4-1 depicts the data processing flow for the electromagnetic and magnetic data sets.

\section{Topography Bases}

Topography bases of the survey area have been produced from published topographic maps A-1, A-2, A-3, B-1, B-2, B-3, B-4, C-1 and C-2 of the Craig Quadrangle; A-6, B-5, and B-6 of the Ketchikan Quadrangle; and D-2 of the Dixon Entrance Quadrangle. These bases are photographically reproduced for inclusion on the maps 1999-4 series maps. Scanned images of the topographic bases are presented as gray layers on the 1999-1, 1999-2 and 1999-3 series maps. The remaining maps do not present the topography. 


\section{Figure 4-1. Processing Flow Chart a) Electromagnetic Data}

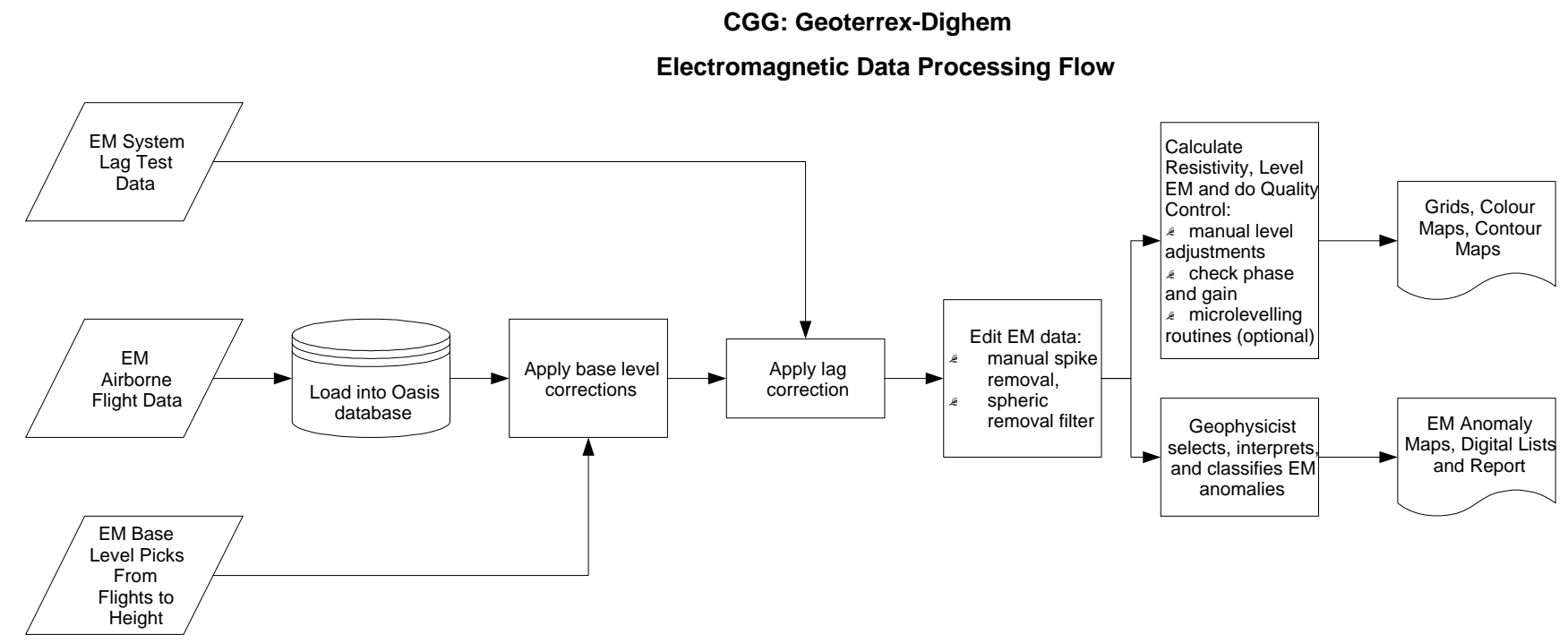

b) Magnetic Data

CGG:Geoterrex-Dighem

Magnetic Data Processing Flow

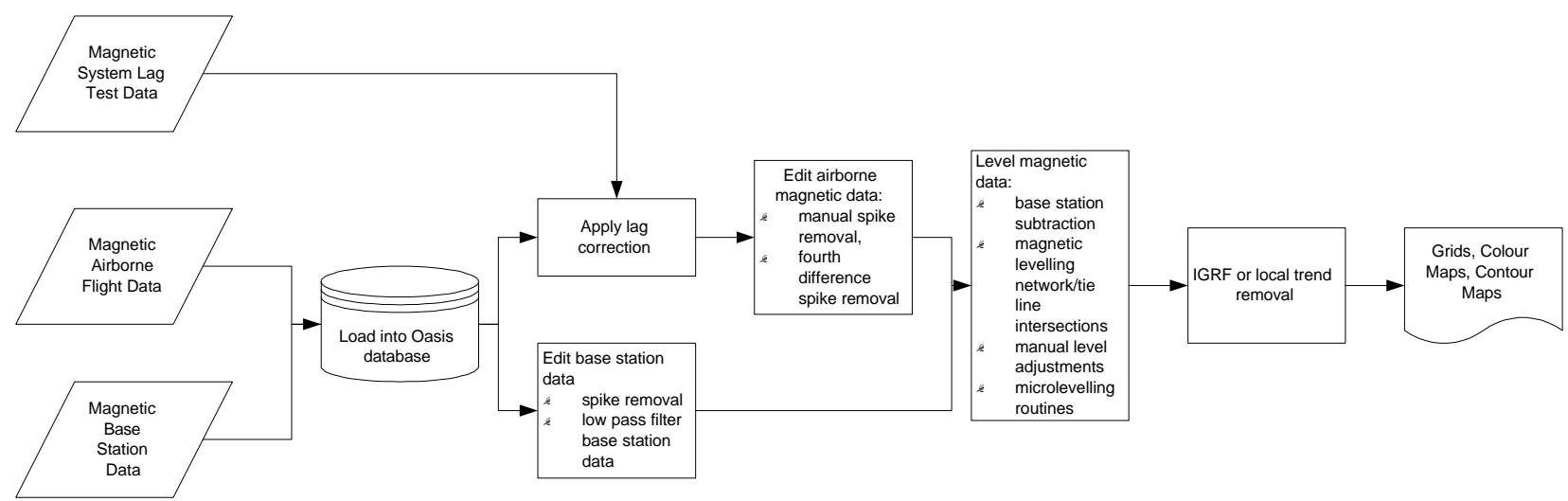




\section{Electromagnetic Anomalies}

The process of interpreting the EM anomalies begins by filtering the EM data with a spike rejection filter. Appropriate median and/or Hanning filters are applied to reduce high frequency noise to acceptable levels. EM test profiles are then created to allow the interpreter to select the most appropriate EM anomaly picking controls for the given survey area. The EM picking parameters depend on several factors but are primarily based on the dynamic range of the resistivity within the survey area, and the types and expected geophysical responses of the geologic target models.

Anomalous electromagnetic responses are selected and analyzed by computer to provide a preliminary electromagnetic anomaly map. The automatic selection algorithm is intentionally oversensitive to assure that no meaningful responses are missed. Using the preliminary map in conjunction with the multi-parameter stacked profiles, the interpreter then reviews and classifies the anomalies according to their source and modifies or eliminates those that are not substantiated by the data, such as those arising from geologic or aerodynamic noise. The final interpreted EM anomaly map includes bedrock, surficial and cultural conductors. A map containing only bedrock conductors can be generated, if desired.

Excellent resolution and discrimination of conductors was accomplished by employing a common frequency on two orthogonal coil-pairs (coaxial and coplanar). The computed "difference channel" parameters often permit differentiation of bedrock and surficial conductors where the computed conductance alone can not.

The anomalies shown on the electromagnetic anomaly maps are based on a nearvertical, half-plane model. This model best reflects "discrete" bedrock conductors. Wide bedrock conductors or flat-lying conductive units, whether from surficial or bedrock sources, may give rise to very broad anomalous responses on the EM profiles. These may not appear on the electromagnetic anomaly map if they have a regional character rather than a locally anomalous character. These broad conductors, which more closely approximate a half space model, will be maximum coupled to the horizontal (coplanar) coil-pair and should be more evident on the resistivity parameter. Resistivity maps, therefore, may be more valuable than the electromagnetic anomaly maps in areas where broad or flat-lying conductors are considered to be of importance (see next - Apparent Resistivity).

Some of the maps available from the DGGS depict the interpreted discrete anomalies as symbols in a vector overlay. Direct magnetic correlation and dip direction are also indicated where they are interpreted. 


\section{Apparent Resistivity}

Apparent resistivity is computed from the in-phase and quadrature EM components for the $900,7,200$ and $56,000 \mathrm{~Hz}$ coplanar data sets using a pseudo-layer half-space model. The resultant apparent resistivity maps portray the variation in apparent resistivity for the given frequency over the entire survey area. This full coverage contrasts with the electromagnetic anomaly map which provides information only over the interpreted discrete conductors. The large dynamic range afforded by the multiple frequencies in the DIGHEM $^{\mathrm{V}}$ system makes the apparent resistivity parameter an excellent mapping tool.

Preliminary apparent resistivity maps and images are carefully inspected to identify lines or line segments which may require base level adjustment. Subtle changes between in-flight calibrations of the system can result in line to line differences which are more readily recognizable in resistive (low signal amplitude) areas. If required, manual level adjustments are carried out to eliminate or minimize resistivity differences which can be attributed in part to changes in operating temperature. These leveling adjustments are usually subtle, and do not result in the degradation of discrete anomalies.

After the leveling process is complete, revised apparent resistivity grids are created. These grids are filtered using a 3 cell by 3 cell smoothing filter prior to the preparation of the final maps. This final filter will not degrade the apparent resistivity given the broad 'footprint' of the parameter and the assumption of a homogeneous half space inherent in the apparent resistivity computation.

The calculated apparent resistivity values are clipped at a maximum value for each of the 900, 7200, and $56000 \mathrm{~Hz}$ data sets. These maxima, 1015, 8360, and $25000 \mathrm{ohm}-$ $\mathrm{m}$, respectively, eliminate the meaningless high apparent resistivity values which would result from very small EM amplitudes.

Contoured resistivity maps, based on the $7,200 \mathrm{~Hz}$ and $56,000 \mathrm{~Hz}$ coplanar data sets are included with this report. The apparent resistivity for all three of the coplanar data sets are included in the digital archives. Values are in ohm-metres on all final products.

\section{Total Field Magnetics}

The aeromagnetic data are corrected for diurnal variation using the magnetic base station data. Manual adjustments are made to any lines that require further leveling as indicated by shadowed images of the gridded magnetic data or tie line/traverse line intercepts. The regional IGRF gradient has been removed from the data. The total field 
magnetic data have been presented as contours on the base maps using a contour interval of $5 \mathrm{nT}$ at a scale of 1:63,360 and 1:31,680.

\section{Historical Data Processing}

Three historical survey blocks are presented with the current survey results. A description of these surveys is given in Section 3. The digital archives from these surveys were recovered from the archive tapes and loaded into databases. These recovered data were verified for completeness through the use of multi-parameter stacked profiles.

A review of the historical geophysical data sets identified errors in the positioning of the Kasaan Peninsula survey. This survey was mispositioned originally by one kilometre due to an error in the annotated UTM location coordinate on the base topographic map used in the tie-down procedure. In addition to the shift, the data set required a rubber sheet correction to move the data sets to its proper position. The procedure for correcting these problems with the positional information was to generate plots of the geophysical data sets for correlation with geophysically distinguishable topographic features. Individual corrections were determined throughout the survey area. Many of these corrections were determined from the response over salt water. The corrections at the individual locations are interpolated to produce a smoothlyvarying correction across the entire survey area. This interpolated correction is then applied to correct the original data. The accuracy of the corrected flight path data is directly related to the nature and frequency of correlation of topographic and cultural features to the geophysical data sets. Good control of the flight path has been achieved given the strong electromagnetic response over salt water which occurs around most of the perimeter of the survey area.

The data sets from these historical surveys have been compared with the results from the current surveys through an examination of the areas where the surveys overlap. Any differences in these regions are characterized to determine the data set to which the error can be attributed. The errors were found to exist predominantly in the historical data sets and can be attributed in large part to the fact that these historical surveys did not have the benefit of recent advancements in instrumentation and acquisition techniques and current state-of-the-art processing and interpretation methods.

The historical apparent resistivity data sets are corrected to match the current survey results by adjusting the amplitudes of the inphase and quadrature components. In like manner, the total magnetic field data sets are adjusted to match the current survey results. The corrected data sets are extracted from the data bases and the resultant grids are merged to produce a seamless final grid for presentation on the maps and in the digital archives. 
The interpreted electromagnetic anomalies are converted from their archived form for presentation and archiving with the anomalies from the current surveys. Magnetite anomalies were not included as an interpretation category for these historical surveys, so no such anomalies appear on the historical survey areas.

An interpretation of the geologic significance of the geophysical survey results from these historical survey blocks has been carried out in conjunction with the interpretation of the current surveys.

\section{Multi-parameter Stacked Profiles}

Distance-based profiles of the survey data sets are generated and plotted. These contain profiles of the recorded data, the calculated parameters and a representation of the interpreted electromagnetic anomalies. A set of preliminary profiles is generated for use throughout the data reduction and interpretation processes. The final profiles are presented on transparent medium, from which prints can be made, at a scale of 1:63,360. Table 4-1 shows the parameters and scales for the multi-parameter stacked profiles.

\section{Contour, Colour and Shadow Map Displays}

The geophysical data are interpolated onto a regular grid using a modified Akima spline technique. The grid cell size is $328 \mathrm{ft}(100 \mathrm{~m})$ for the current surveys and $164 \mathrm{ft}$ $(50 \mathrm{~m})$ for the previous surveys. These cell sizes are approximately $25 \%$ of the nominal line spacing for each survey. The resulting grid is used to generate contours of each geophysical parameter. The contours are labeled, annotated and are presented on the final maps with varying pen weights for ease of viewing.

Colour maps are produced by interpolating the grid to the pixel size. The parameter is then represented with a defined colour for specific amplitude ranges to provide colour "contour" maps. A standard rainbow colour palette is used to define the entire data range. The colours are distributed over the entire data range so that each colour in the palette covers an equal area on the final maps. This equal area distribution is defined over each block independently. In this way the colour distribution is optimized for each map sheet. Since map sheets 2 and 3 depict the same block of data on South Prince of Wales Island, these maps share a common colour distribution. Since the Gravina Island western and eastern parts are displayed on a single map, these blocks also share a common colour distribution. 
Table 4-1. Multi-parameter Stacked Profiles

\begin{tabular}{|c|c|c|}
\hline $\begin{array}{c}\text { Channel } \\
\text { Name (Freq) }\end{array}$ & Observed Parameters & $\begin{array}{c}\text { Scale } \\
\text { Units/mm }\end{array}$ \\
\hline MAG & Magnetics - fine & $5 \mathrm{nT}$ \\
\hline MAG & Magnetics - coarse & $50 \mathrm{nT}$ \\
\hline ALTR & Bird height & $6 \mathrm{~m}$ \\
\hline DTM & Height above mean sea level & $20 \mathrm{~m}$ \\
\hline CXI ( & Vertical coaxial coil-pair inphase & $2 \mathrm{ppm}$ \\
\hline $900 \mathrm{~Hz})$ & Vertical coaxial coil-pair quadrature & $2 \mathrm{ppm}$ \\
\hline CPI $\quad($ & Horizontal coplanar coil-pair inphase & $2 \mathrm{ppm}$ \\
\hline $900 \mathrm{~Hz})$ & Horizontal coplanar coil-pair quadrature & $2 \mathrm{ppm}$ \\
\hline CXI $\quad(5,500 \mathrm{~Hz})$ & Vertical coaxial coil-pair inphase & $4 \mathrm{ppm}$ \\
\hline CXQ $\quad(5,500 \mathrm{~Hz})$ & Vertical coaxial coil-pair quadrature & $4 \mathrm{ppm}$ \\
\hline CPI $\quad(7,200 \mathrm{~Hz})$ & Horizontal coplanar coil-pair inphase & $4 \mathrm{ppm}$ \\
\hline CPQ $\quad(7,200 \mathrm{~Hz})$ & Horizontal coplanar coil-pair quadrature & $4 \mathrm{ppm}$ \\
\hline CPI $\quad(56,000 \mathrm{~Hz})$ & Horizontal coplanar coil-pair inphase & $10 \mathrm{ppm}$ \\
\hline $\mathrm{CPQ} \quad(56,000 \mathrm{~Hz})$ & Horizontal coplanar coil-pair quadrature & $10 \mathrm{ppm}$ \\
\hline 4XS & Coaxial spherics monitor & \\
\hline CXP & Coaxial powerline monitor & \\
\hline CPP & Coplanar powerline monitor & \\
\hline CPS & Coplanar spherics monitor & \\
\hline & Computed Parameters & \\
\hline $900 \mathrm{~Hz})$ & Difference function inphase from CXI and CPI & $2 \mathrm{ppm}$ \\
\hline DFQ $\quad(900 \mathrm{~Hz})$ & Difference function quadrature from $\mathrm{CXQ}$ and $\mathrm{CPQ}$ & $2 \mathrm{ppm}$ \\
\hline RES $\quad(900 \mathrm{~Hz})$ & Log resistivity & .06 decade \\
\hline RES $\quad(7,200 \mathrm{~Hz})$ & Log resistivity & .06 decade \\
\hline RES $\quad(56,000 \mathrm{~Hz})$ & Log resistivity & .06 decade \\
\hline DP $\quad(900 \mathrm{~Hz})$ & Apparent depth & $6 \mathrm{~m}$ \\
\hline$(7,200 \mathrm{~Hz})$ & Apparent depth & $6 \mathrm{~m}$ \\
\hline DP $\quad(56,000 \mathrm{~Hz})$ & Apparent depth & $6 \mathrm{~m}$ \\
\hline CDT & Conductance & 1 grade \\
\hline
\end{tabular}


Shadow maps are generated by employing an artificial sun which casts shadows on a surface defined by the geophysical parameter grids. Shadow maps of the total field magnetic data were combined with the colour magnetic grids to produce colour shadowed total field magnetic maps.

\section{Total Field Magnetic Data Modeling}

Inverse models of 5 selected total field magnetic responses are provided as page size plots. The models were computed from responses on lines 11240 (Map Sheet C), 20100 (Map Sheet A), 30581 (Map Sheet D) and along a diagonal path within Map Sheet $\mathrm{B}$. The model parameters are determined by matching the line data with the theoretical response from one of four simple geometric models. These geometric models range from 2 -dimensional to $23 / 4$ dimensional. The model results assume that the magnetization is induced but remanent magnetization can also be accounted for where it exists. The 5 models are presented in Appendix C.

\section{Digital Terrain}

The radar altimeter values (ALTR - aircraft to ground clearance) were subtracted from the differentially corrected GPS- $Z$ values, which were transformed to the local datum, to produce profiles of the height above mean sea level along the survey lines. These values were gridded to produce contour maps showing approximate elevations within the survey blocks. The resulting digital terrain contours were compared against published topographic maps. The data were manually adjusted to remove differences between the two. The data were then subjected to a microlevelling algorithm to remove any remaining small line-to-line discrepancies.

The accuracy of the elevation calculation is directly dependent on the accuracy of the two input parameters, ALTR and GPS-Z. The ALTR value may be erroneous in areas of heavy tree cover, where the altimeter reflects the distance to the tree canopy rather than the ground. The GPS-Z value is primarily dependent on the number of available satellites. Although post-processing of GPS data will yield $X$ and $Y$ accuracies in the order of 5 metres, the accuracy of the $\mathrm{Z}$ value is usually much less, sometimes in the \pm 20 metre range. Further inaccuracies may be introduced during the interpolation and gridding process.

Because of the inherent inaccuracies of this method, no guarantee is made or implied that the information displayed is a true representation of the height above sea level. Although this product may be of some use as a general reference, $\underline{\text { THIS }}$ PRODUCT MUST NOT BE USED FOR NAVIGATION PURPOSES. 
$-4.10-$

Blank Page 


\section{SURVEY RESULTS AND DISCUSSION}

\section{Geology}

The survey area falls in the southeastern geologic region of Alaska which is defined by the 'panhandle' and is bounded on the south and east by the Canadian border, on the west by the Pacific Ocean and on the north by the northern limit of the 'panhandle'. This region falls within the Cordilleran Orogen and contains rocks which represent a long and complete geologic record beginning in the Proterozoic and ending in the Holocene. Northwest elongate belts of stratified rock occur throughout the region and show various ages of deposition and degrees of deformation and metamorphism. Intrusive rocks are common and range in age from Cambrian to middle Tertiary. The Coast Range batholith (Coast Plutonic Complex) extends along the eastern portion of the region. Several phases of deformation and metamorphism have taken place during the history of this region. Three major strike slip fault systems cut the region into many pieces. Thrust, low-angle normal, and dip-slip faults are also common throughout the region.

The rocks of southeastern Alaska have been divided into ten tectonic assemblages, five of which are described as terranes because they have distinct geologic records and the other five are described as lithic assemblages which contain rocks with depositional, intrusive or unknown contact with the five terranes. The survey area falls within two of these tectonic assemblages: the first is the Craig subterrane of the Alexander terrane and the second is the Gravina belt. In the vicinity of the survey areas, these two assemblages are divided by the northwest trending Clarence Strait strike slip fault, one of the three major regional strike slip fault zones. This fault has a dextral displacement of approximately $15 \mathrm{~km}$.

The Alexander terrane has a variety of stratified, plutonic and metamorphic rocks ranging in age from late Precambrian to middle Jurassic. These rocks underlie much of the Alaska panhandle and extend into British Columbia, the Yukon and the Wrangell Mountains. Volcaniclastics, carbonates and conglomerates are the most common rocks in the terrane. The oldest rocks are arc-type metasedimentary and metavolcanic rocks which form the basement for an arc-type volcanic-plutonic-sedimentary complex which underlies much of the southern portion of the terrane. Related chert and argillite and marine clastic and carbonate rocks in the northern portion of the terrane are thought to have formed in a basin behind the arc. In the southern portion of the terrane, the ensuing Klakas orogeny brought about southwest vergent thrusting, regional metamorphism, uplift and erosion, and felsic intrusion.

Upper Paleozoic erosional remnants in the Alexander terrane include carbonate, clastics, and mafic-intermediate volcanic rocks. Upper Triassic rocks form a narrow belt 
near the eastern margin of the terrane and overlie earlier rocks on a regional unconformity. In the southern portion of the terrane, the general section of these rocks comprises conglomerate, breccia, rhyolite, limestone, argillite, and pillow flows and breccia. These are thought to have formed in a rift environment. The youngest rock of the terrane, the Bokan Mountain Granite, forms a ring-dyke complex on southern Prince of Wales Island.

The Gravina belt consists of marine argillite and greywacke, andesitic to basaltic volcanic and volcaniclastic rocks, conglomerate, and plutons ranging from quartz diorite to dunite and peridotite. These rocks which are upper Jurassic to mid-Cretaceous in age, form a narrow belt east of the Alexander terrane and define the transition from lower grade metamorphism in the west to the higher grade rocks which flank the Coast Range batholith.

Large, isolated, granodiorite plutons of early Cretaceous age intruded the Alexander terrane. Zoned ultramafic complexes formed during the early to midCretaceous occur in both the Alexander and Gravina terranes. Granodioritic, tonalitic and other bodies also from the mid-Cretaceous occur in the Gravina belt. These are thought to have formed in a subduction-related magmatic arc. Oligocene and Miocene stocks of granite, quartz monzonite, granodiorite, diorite and ultra-mafic intrusives intrude the Gravina belt in southern southeast Alaska.

The survey areas represent some of the most prospective lands for hosting economic mineral deposits in southeast Alaska. The survey targets a variety of deposit types that are hosted in several different tectonostratigraphic terranes or rock belts. The survey areas were selected following consultations between the U.S. Bureau of Land Management, Alaska Division of Geological \& Geophysical Surveys, U.S. Geological Survey, and the University of Alaska. Geologic maps are available for the Ketchikan and Prince Rupert quadrangles (Berg and others, 1988). The Craig quadrangle (Eberlein and others, 1983), and the southern Prince of Wales Island - mostly in the Dixon Entrance quadrangle (Gehrels, 1992). A comprehensive review of the mineral deposits in the area was compiled by the Bureau of Mines (Maas and others, 1995).

The targeted rocks on the southern Prince of Wales Island (map sheets B and C) belong to the pre-Ordivician Wales Group, which is part of the Alexander terrane. The Wales Group hosts several known VMS deposits, some with recorded historic production. The Niblack Mine is a VMS type deposit that is currently being evaluated by Abacus Minerals with partner Teck Corporation. The partners have announced a reserve at Niblack of 2.8 million tons grading $0.09 \mathrm{oz} / \mathrm{t}$ gold, $1.17 \mathrm{oz} / \mathrm{t}$ silver, $1.71 \%$ copper, and $3.18 \%$ zinc. The Niblack deposit has recently been evaluated by Cominco, Noranda, Barrick Gold, and LAC Minerals. Additional mines that exploited VMS deposits in the Wales Group include the Khayyam, CopperCity, Corbin, and Big Harbor Mines, each of 
which has reported production. Exploration has also targeted the Ruby Tuesday, and Deer Bay Exhalite deposits and other smaller VMS occurrences in the area.

The Wales Group rocks targeted by the survey also have the potential for vein gold, polymetallic vein, and skarn deposits. Particular interest has been paid to the structure-controlled gold mineralization in the Dolomi area.

The northern portion of the southern Prince of Wales Island survey area is comprised of rocks of the Silurian to Ordovician Descon Formation. It has been included in the survey mainly because of uncertainty in the mapped contact with the Wales Group to the south. Parts of the area have been explored in the recent past for their VMS potential.

The Salt Chuck area targets mainly magmatic segregation deposits such as the past producing Salt Chuck Mine. This mine produced copper, gold, and silver intermittently from 1909 to 1941, but was known particularly for its palladium production. While it was producing, the Salt Chuck Mine was the largest producer of palladium in the U.S.A. This area also has the potential for polymetallic vein and skarn deposits. The area is underlain by the Silurian to Ordovician Descon Formation which contains weakly metamorphosed sedimentary and volcanic rocks that trend generally north-northwest. Major faults in the area also trend to the northwest and in some cases control mineralization.

The Gravina Island western part include early Paleozoic and overlying Triassic rocks of the Alexander terrane. The rocks have been cut by high-angle, northwest and northeast trending faults. Targets in the area include structure-controlled copper mineralization hosted in siliceous breccias and porphyry copper deposits. There is also some indication of massive sulfides hosted in metarhyolites. The area has recently veen of interest to various mineral exploration companies including Phelps-Dodge, Amoco Minerals, and Pacific Northwest Resources.

The Gravina Island eastern part is hosted by Mesozoic, submarine, sedimentary and volcanic rocks of the Gravina Belt overlap sequence, which separates the Alexander terrane on the west from the Taku terrane on the est. Targets in the area are mainly VMS and gold quartz veins. Contacts, metamorphic foliation, an faults generally trend to the northwest. The area was recently examined by Houston Oil and Minerals, particularly for its VMS potential. 


\section{Survey Results}

\section{DISCRETE EM ANOMALY INTERPRETATION}

A total of 3940 discrete anomalous EM responses have been interpreted from the electromagnetic data sets in the current survey areas. Table 5-1 summarizes these responses with respect to conductance grade and interpretation for each of the current survey areas.

The EM anomalies resulting from this survey appear to fall within one of four general categories. The first type consists of discrete, well-defined anomalies which yield marked inflections on the difference channels. These anomalies are usually attributed to conductive sulphides or graphite and are generally given a "B" or "D" interpretive symbol, denoting a bedrock source. 1527 of these types of responses are interpreted in the current survey areas.

The second class of anomalies comprises moderately broad responses which exhibit the characteristics of a half space and do not yield well-defined inflections on the difference channels. Anomalies in this category are usually given an "S" or "H" interpretive symbol. The lack of a difference channel response usually implies a broad or flat-lying conductive source such as overburden. Some of these anomalies may reflect conductive rock units or zones of deep weathering. 858 of these types of responses have been interpreted in the current survey areas.

High-amplitude responses are consistently recorded over salt water because of its strong conductivity. These responses are not considered important from an exploration standpoint and, indeed, are predictable from the topographic maps. For these reasons, anomalies over salt water have not been assigned an interpretation and do not appear on the electromagnetic anomaly layer on the maps.

The effects of conductive overburden are evident over portions of the survey area, particularly in the low-lying areas. Although the difference channels (DFI and DFQ) are extremely valuable in detecting bedrock conductors which are partially masked by conductive overburden, sharp undulations in the bedrock/overburden interface can yield anomalies in the difference channels which may be interpreted as possible bedrock conductors. Such anomalies usually fall into the "S?" or "B?" classification but may also be given an "E" interpretive symbol, denoting a resistivity contrast at the edge of a conductive unit. These types of responses are not distinguished in the anomaly summary. 
TABLE 5-1a

\section{EM ANOMALY STATISTICS}

\section{SURVEY}

\section{SALT CHUCK AREA}

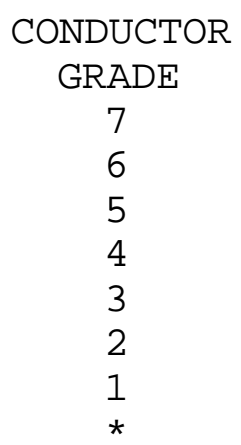

TOTAL

CONDUCTOR

MODEL

D

B

$S$

$\mathrm{E}$

$\mathrm{M}$

TOTAL

\author{
CONDUCTANCE RANGE \\ SIEMENS (MHOS) \\ $>100$ \\ $50-100$ \\ $20-50$ \\ $10-20$ \\ $5-10$ \\ $1-5$ \\ $<1$ \\ INDETERMINATE
}

MOST LIKELY SOURCE

DISCRETE BEDROCK CONDUCTOR

DISCRETE BEDROCK CONDUCTOR CONDUCTIVE COVER

EDGE OF WIDE CONDUCTOR

MAGNETITE
NUMBER OF

RESPONSES

2

1

2

7

9

42

14

116

193

NUMBER OF

RESPONSES

3

8

153

1

28

193 
TABLE 5-1b

EM ANOMALY STATISTICS

1999 SURVEY

\section{PRINCE OF WALES ISLAND AREA}

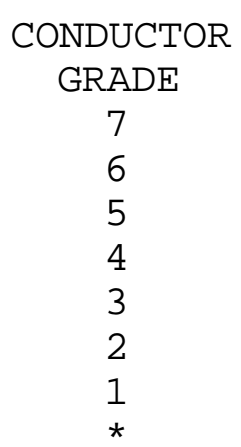

TOTAL

CONDUCTOR
MODEL
D
B
S
H
E
M
L

TOTAL

\author{
CONDUCTANCE RANGE \\ SIEMENS (MHOS)

$$
>100
$$ \\ $50-100$ \\ $20-50$ \\ $10-20$ \\ $5-10$ \\ $1-5$ \\ $<1$
}

INDETERMINATE

MOST LIKELY SOURCE

DISCRETE BEDROCK CONDUCTOR

DISCRETE BEDROCK CONDUCTOR

CONDUCTIVE COVER

ROCK UNIT OR THICK COVER

EDGE OF WIDE CONDUCTOR

MAGNETITE

CULTURE
NUMBER OF
RESPONSES
20
21
118
186
260
627
96
1,917
3,245

NUMBER OF

RESPONSES

232

1,021

562

4

13

1,395

18

3,245 
TABLE 5-1c

EM ANOMALY STATISTICS

\section{SURVEY}

\section{SOUTH OF KEETE INLET AREA}

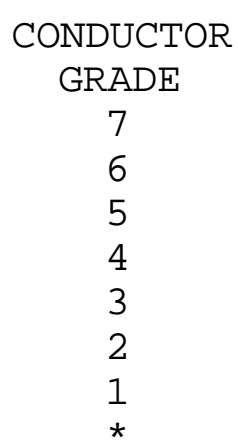

TOTAL

CONDUCTOR
MODEL
D
B
S
M

TOTAL

\author{
CONDUCTANCE RANGE \\ SIEMENS (MHOS)

$$
>100
$$ \\ $50-100$ \\ $20-50$ \\ $10-20$ \\ $5-10$ \\ $1-5$ \\ $<1$ \\ INDETERMINATE
}

MOST LIKELY SOURCE

DISCRETE BEDROCK CONDUCTOR DISCRETE BEDROCK CONDUCTOR CONDUCTIVE COVER

MAGNETITE

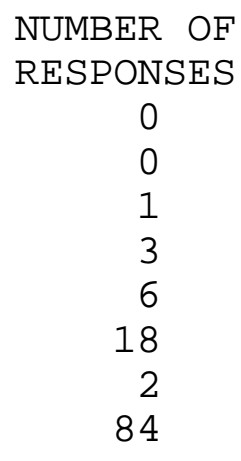

114

NUMBER OF

RESPONSES

14

25

40

35

114 
TABLE 5-1d

\section{EM ANOMALY STATISTICS}

\section{SURVEY}

\section{WESTERN PART - GRAVINA ISLAND}

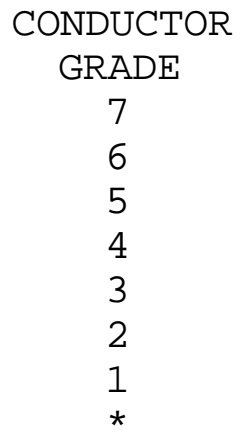

TOTAL

CONDUCTOR
MODEL
D
B
S
H
M

TOTAL

$\begin{array}{cc}\text { CONDUCTANCE RANGE } & \text { NUMBER OF } \\ \text { SIEMENS (MHOS) } & \text { RESPONSES } \\ >100 & 0 \\ 50-100 & 2 \\ 20-50 & 10 \\ 10-20 & 23 \\ 5-10 & 36 \\ 1-5 & 116 \\ \text { INDETERMINATE } & 21 \\ & 138\end{array}$

MOST LIKELY SOURCE

DISCRETE BEDROCK CONDUCTOR DISCRETE BEDROCK CONDUCTOR CONDUCTIVE COVER ROCK UNIT OR THICK COVER MAGNETITE

NUMBER OF RESPONSES

11

211

51

12

61

346 
TABLE 5-1e

EM ANOMALY STATISTICS

1999 SURVEY

\section{EASTERN PART - GRAVINA ISLAND}

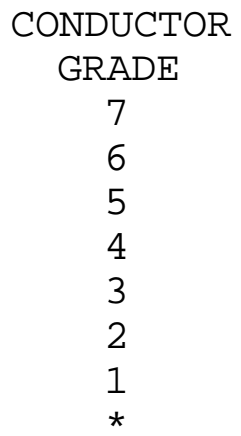

TOTAL

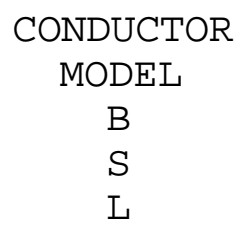

TOTAL

CONDUCTANCE RANGE
SIEMENS (MHOS)
$>100$
$50-100$
$20-50$
$10-20$
$5-10$
$1-5$
INDETERMINATE

MOST LIKELY SOURCE DISCRETE BEDROCK CONDUCTOR CONDUCTIVE COVER CULTURE

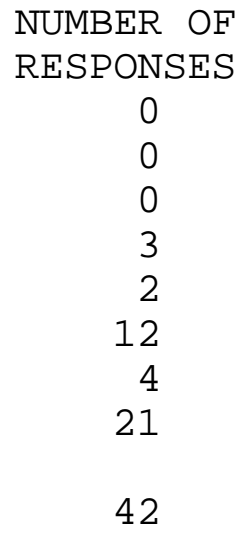

NUMBER OF RESPONSES

2

36

4

42

(SEE EM MAP LEGEND FOR EXPLANATIONS) 
TABLE 5-1f

\section{EM ANOMALY STATISTICS}

\section{HISTORICAL SURVEYS \\ KASAAN AREA}

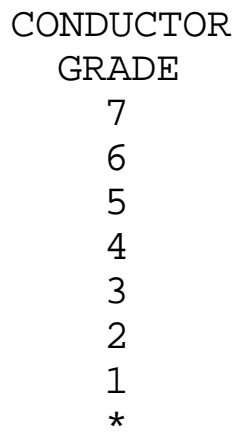

TOTAL

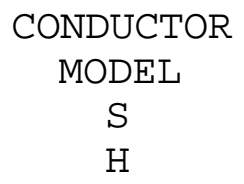

TOTAL

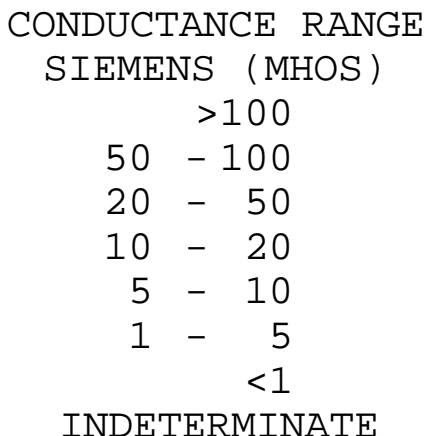

INDETERMINATE

MOST LIKELY SOURCE

CONDUCTIVE COVER

ROCK UNIT OR THICK COVER

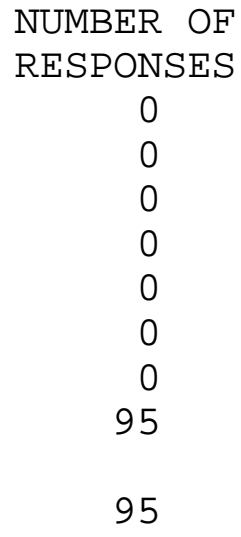

NUMBER OF

RESPONSES

94

1

95

(SEE EM MAP LEGEND FOR EXPLANATIONS) 
$-5.11-$

TABLE 5-1g

EM ANOMALY STATISTICS

HISTORICAL SURVEYS

HETTA INLET (NORTHERN AREA)

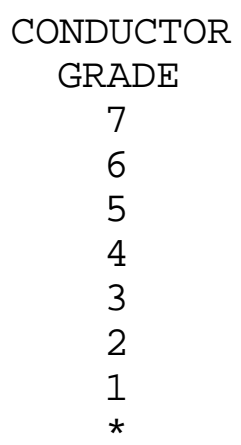

TOTAL

CONDUCTOR

MODEL

$\mathrm{D}$

B

$\mathrm{S}$

$\mathrm{H}$

TOTAL

\author{
CONDUCTANCE RANGE \\ SIEMENS (MHOS)

$$
>100
$$ \\ $50-100$ \\ $20-50$ \\ $10-20$ \\ $5-10$ \\ $1-5$ \\ $<1$
}

INDETERMINATE

MOST LIKELY SOURCE

DISCRETE BEDROCK CONDUCTOR

DISCRETE BEDROCK CONDUCTOR

CONDUCTIVE COVER

ROCK UNIT OR THICK COVER
NUMBER OF

RESPONSES

0

0

0

0

0

0

0

674

674

NUMBER OF

RESPONSES

2

420

221

31

674

(SEE EM MAP LEGEND FOR EXPLANATIONS) 
$-5.12-$

TABLE 5-1h

\section{EM ANOMALY STATISTICS}

\section{HISTORICAL SURVEYS}

\section{HETTA INLET (SOUTHERN AREA)}

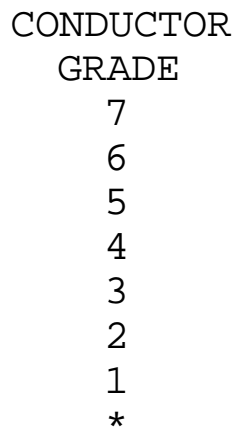

TOTAL

CONDUCTOR
MODEL
B
S
H

TOTAL

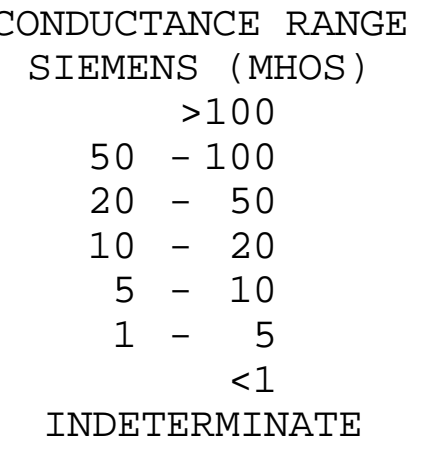

CONDUCTANCE RANGE

$10-20$

$5-10$

$1-5$

INDETERMINATE

MOST LIKELY SOURCE

DISCRETE BEDROCK CONDUCTOR CONDUCTIVE COVER

ROCK UNIT OR THICK COVER

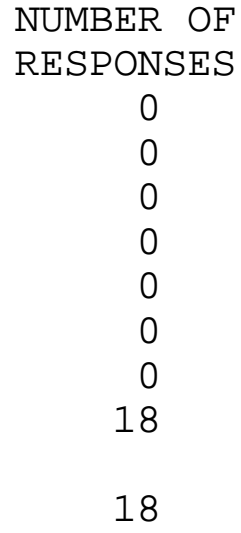

NUMBER OF

RESPONSES

7

8

3

18

(SEE EM MAP LEGEND FOR EXPLANATIONS) 
$-5.13-$

TABLE 5-1i

\section{EM ANOMALY STATISTICS}

\section{HISTORICAL SURVEYS DOLOMI AREA}

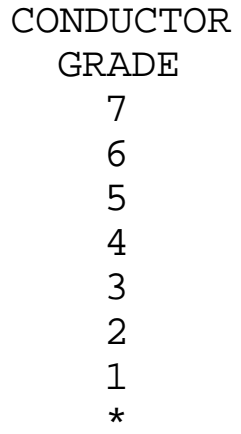

TOTAL

CONDUCTOR

MODEL

$\mathrm{D}$

B

$\mathrm{S}$

$\mathrm{E}$

TOTAL

\author{
CONDUCTANCE RANGE \\ SIEMENS (MHOS)

$$
>100
$$ \\ $50-100$ \\ $20-50$ \\ $10-20$ \\ $5-10$ \\ $1-5$ \\ $<1$ \\ INDETERMINATE
}

MOST LIKELY SOURCE

DISCRETE BEDROCK CONDUCTOR DISCRETE BEDROCK CONDUCTOR CONDUCTIVE COVER

EDGE OF WIDE CONDUCTOR
NUMBER OF RESPONSES

3

2

9

21

32

40

37

146

290

NUMBER OF

RESPONSES

42

95

142

11

290

(SEE EM MAP LEGEND FOR EXPLANATIONS) 
The third class of anomalies consists of negative inphase responses which are indicative of magnetite. These are represented by triangles where anomalies appear on the maps and have been annotated with an " $M$ ". Where a magnetite anomaly has an associated quadrature response, the interpretation of a conductive source will over-ride the magnetite anomaly and a D, B?, or S? interpretation will be assigned based on the strength and shape of the quadrature response. 1519 magnetite anomalies have been interpreted in the survey area.

The fourth class comprises cultural anomalies. 22 responses are attributed to culture and correlate with man-made objects. Any other interpreted conductors which occur in close proximity to these cultural features should be confirmed as bedrock conductors prior to drilling.

The majority of the strong bedrock anomalies have a sufficiently large footprint to produce a strong apparent resistivity low. For this reason, description of these anomalies will be made in the apparent resistivity section. Several other strong responses are evident particularly in the central portion of the survey area, but these are generally of limited strike extent and are reflected on only one survey line. These may be of interest from an exploration standpoint. Details on each of these anomalies are available in the anomaly listing in Appendix C.

In areas where the electromagnetic response is evident primarily on the quadrature component, zones of poor conductivity are indicated. Where these responses are coincident with magnetic anomalies, it is possible that the inphase component amplitudes have been suppressed by the effects of magnetite. Most of these poorly-conductive magnetic features give rise to resistivity anomalies which are only slightly below background. If it is expected that poorly-conductive economic mineralization may be associated with magnetite-rich units, most of these weakly anomalous features will be of interest. In areas where magnetite causes the inphase components to become negative, the apparent conductance and depth of EM anomalies will be unreliable. The conductance values will tend to be understated.

Anomalies which occur near the ends of the survey lines (i.e., outside the survey area), should be viewed with caution. Some of the weaker anomalies could be due to aerodynamic noise, i.e., bird bending, which is created by abnormal stresses to which the bird is subjected during the climb and turn of the aircraft between lines. Such aerodynamic noise is usually manifested by an anomaly on the coaxial inphase channel only, although severe stresses can affect the coplanar inphase channels as well.

In some portions of the survey area, the steep topography forced the pilot to exceed normal terrain clearance for reasons of safety. It is possible that some weak conductors may have escaped detection in areas where the bird height exceeded $120 \mathrm{~m}$. In 
difficult areas where near-vertical climbs were necessary, the forward speed of the helicopter was reduced to a level which permitted excessive bird swinging. This problem, combined with the severe stresses to which the bird was subjected, gave rise to aerodynamic noise levels which are slightly higher than normal. Where warranted, reflights were carried out to minimize these adverse effects.

Interpretation sketches for all the survey areas are shown in Figures 5-1a through $5-1 \mathrm{~d}$. Conductive and magnetic zones have been identified by the letters " $R$ " and " $M$ " respectively.

\section{Salt Chuck and Kasaan Peninsula, Prince of Wales Island}

\section{MAP SHEET A}

The Salt Chuck, Rush \& Brown, Haida, Alarm, It, Poor Man, Uncle Sam, Rich Hill, Mt. Andrews, Stevenstown and Mamie Mines are identified on the USGS topographic maps within the Salt Chuck and Kasaan survey areas. The Mamie, Stevenstown and Mt Andrews mines appear to be associated with discrete, strong magnetic units. Without the flight path videos from the 1992 survey, it is impossible to check if this magnetic activity is the result of man made structures or dumping of mine waste on surface at the mine site. The other mines do not appear to yield discrete EM or magnetic anomalies on the grids. The resolution of the grids from the 200/400 m line spacing, with 50/100 m cell sizes, may be insufficient to resolve the response from such small deposits. Alternatively, the mineralization within the remains of the mined deposits may be too weakly conductive or too weakly magnetic to be detected by the airborne system. Persons with exact knowledge of the location of the mineralization may be able to determine if the flight path intersects one of these deposits, and determine if there are in fact resulting anomalies in the detailed profile data. However, the geological context of the deposits as revealed by the geophysics from this survey may aid in future exploration in this area.

The magnetic domains M1 and M2, which are indicated on the Interpretation Map, outline the inferred boundaries of the gabbro and diorite volcanic intrusives. This information supplements the outcrop information that is presented on the USGS Geologic map ${ }^{1}$. All of the mines mentioned above are located within these two magnetic domains. The Rush \& Brown mine appears to be located very close to a major northeast trending fault which can be inferred from the magnetic data. The Poor man mine is located adjacent to a well-defined north to northeast trending fault or faulted contact labeled F3 on the Interpretation map. These major faults may have

\footnotetext{
1 Open-file Report, 95-215, David Brew (Compiler), Geologic Map of the Craig, Dixon Entrance, and Parts of the Ketchikan and Prince Rupert Quadrangle, Southeastern Alaska.
} 
$-5.16-$

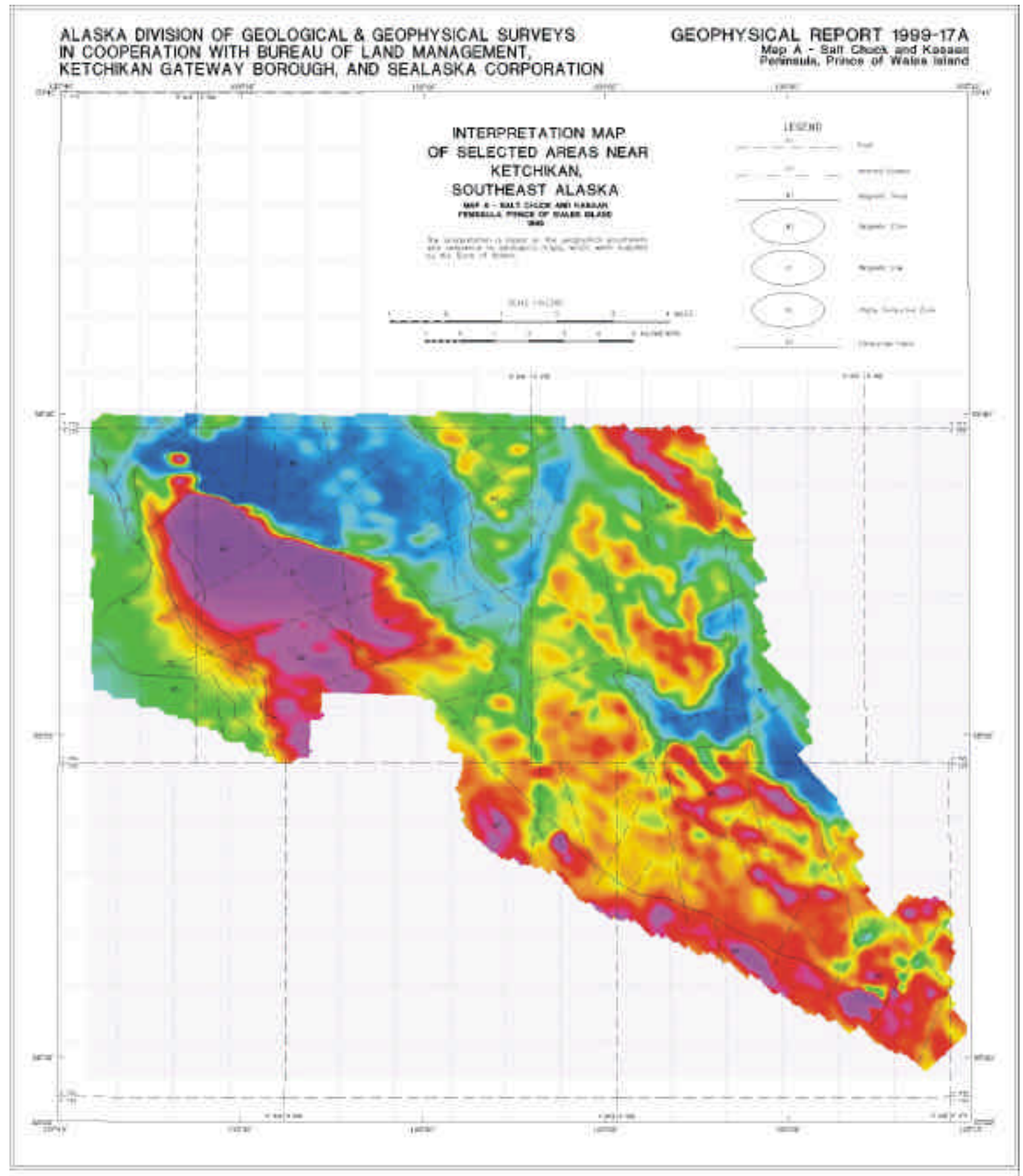

Figure 5-la. Interpretation sketch of selected areas near Ketchikan, Southeast Alaska - Map A - Salt Chuck and Kasam Peninsula, Prince of Wales Island. 
- 5.17 -

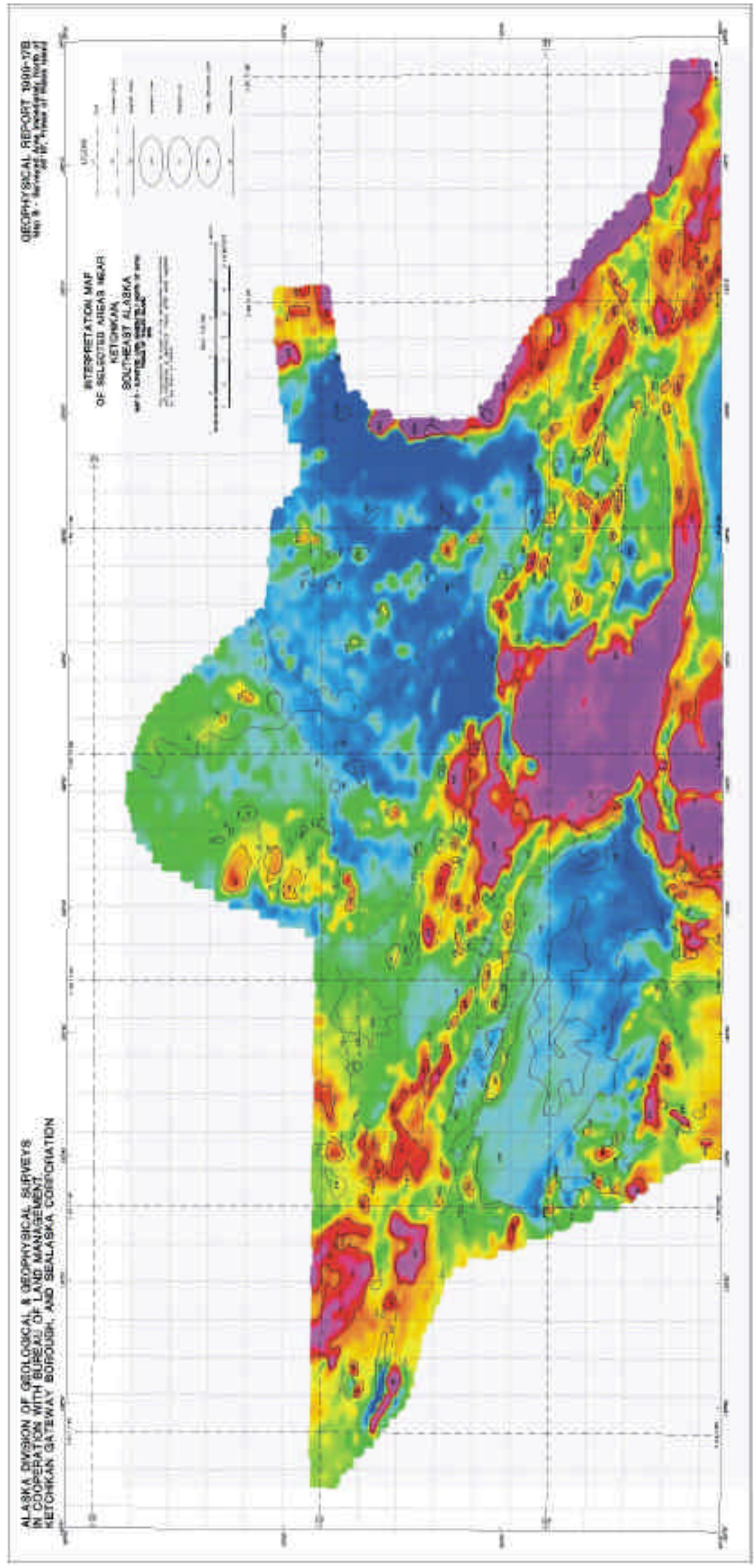

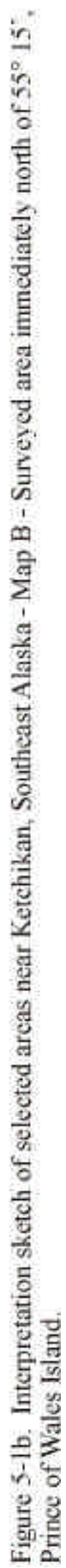


- 5.18 -

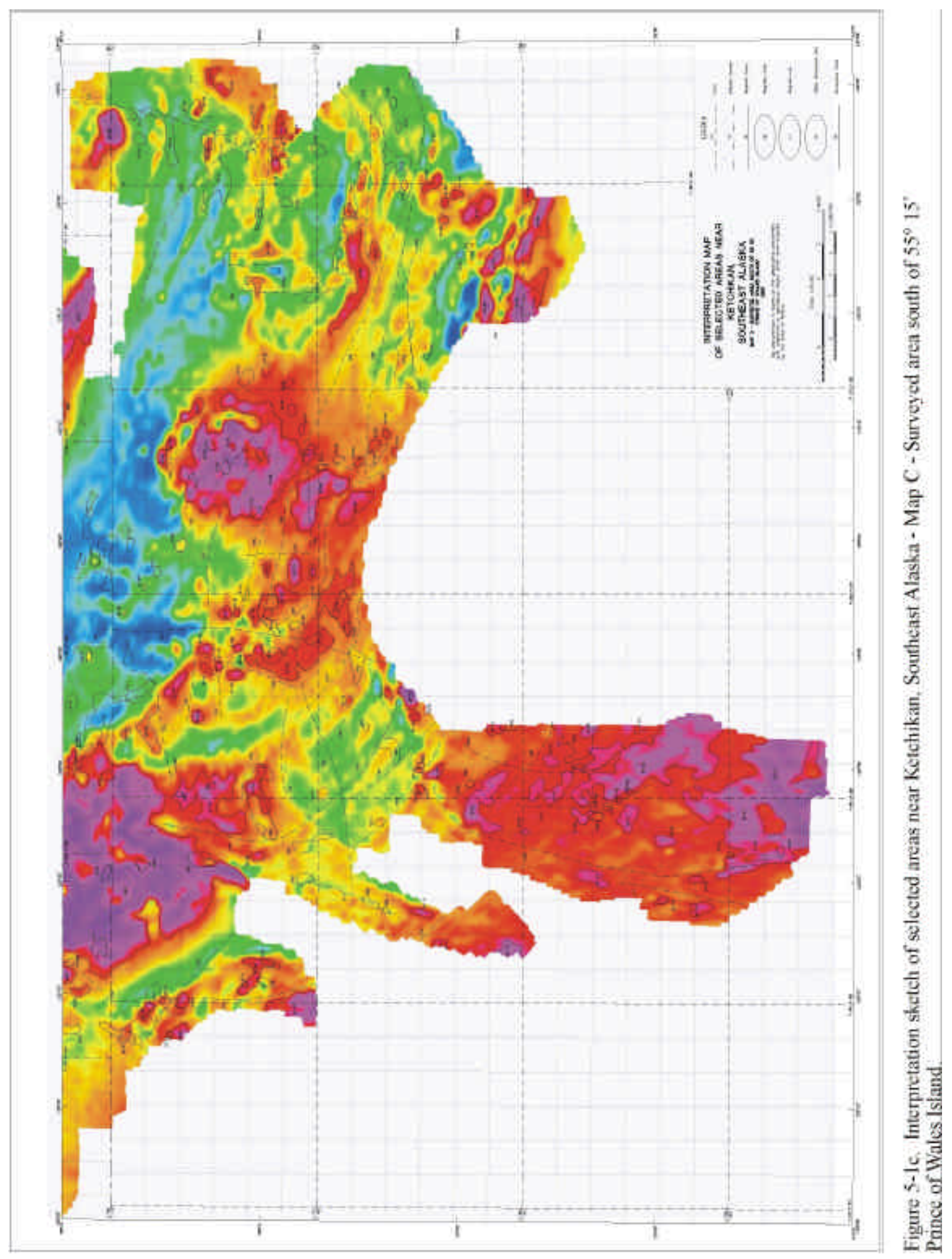




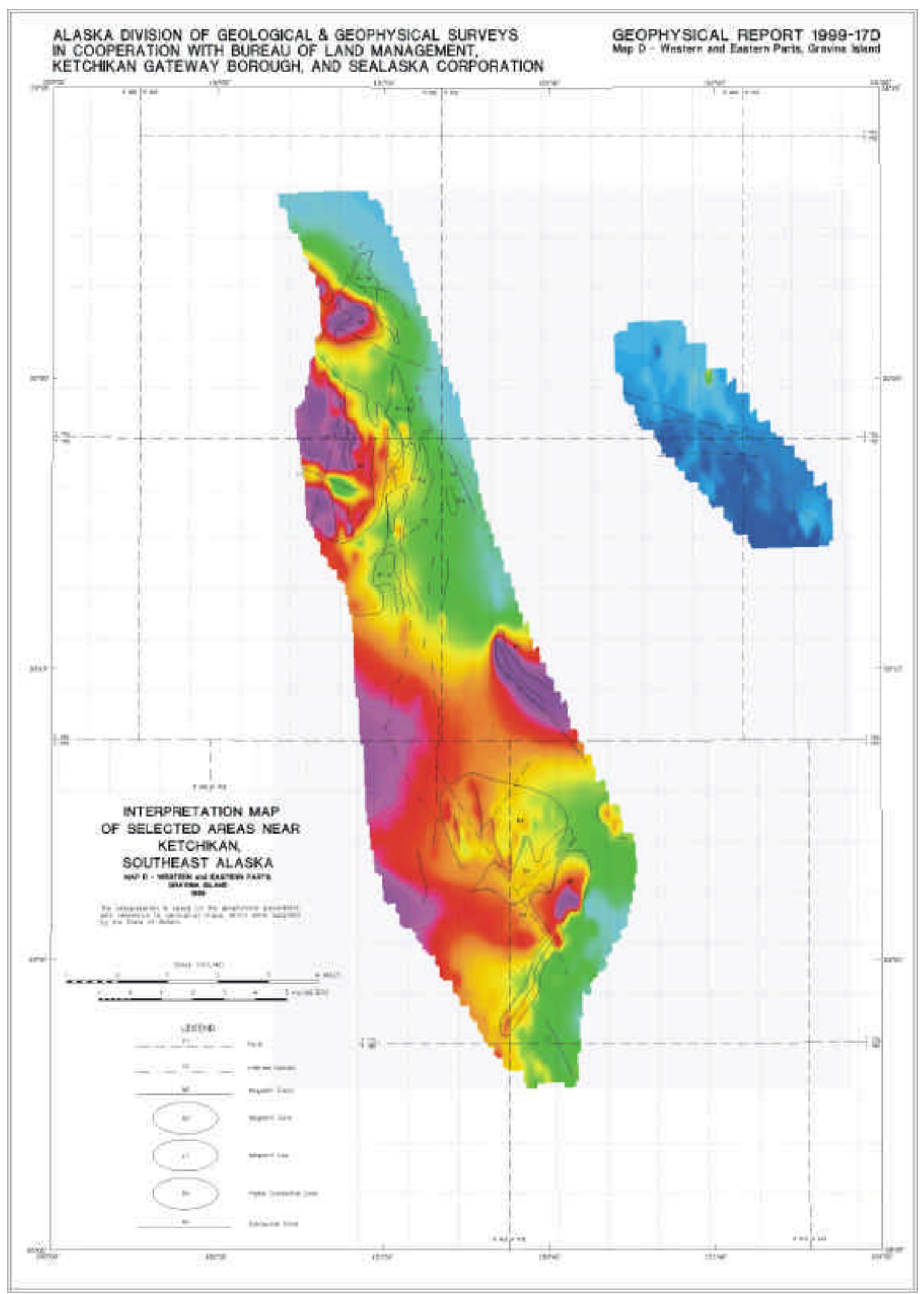

Figure 5-Id. Interpretation sketch of selected areas near Ketchikan, Southeast Alaska - Map D Western and Eastem parts, Gravina Island. 
influenced the deposition of economic mineralization in these locations and elsewhere. The Salt Chuck Mine appears to be located on or very near to the contact of the volcanic intrusives with volcaniclastic sedimentary rocks.

The total field magnetic data for the combined Salt Chuck and Kasaan Peninsula areas ranges from 54,976 to $63,890 \mathrm{nT}$. On average the stratigraphy appears to strike at approximately $\mathrm{N} 45^{\circ} \mathrm{W}$ with many prominent contacts and faulted contacts in this direction. There are also numerous north-northeast and northeast trending faults. Faults and contacts that have been inferred form the magnetic and resistivity data are indicated on the Interpretation Map, which accompanies this report.

The Thorne River and Kasaan faults are obvious as northwest trending lineaments in the magnetic and resistivity data. These have been labeled F1 and F2 respectively on the interpretation Map. The Thorne River Fault bounds the magnetic intrusive units M1 and M2 on the southwest. The Kasaan fault divides the intrusive bodies. An irregular shaped zone of low resistivity R1 appears to be associated with the Thorne River Fault and the low-lying areas to the southeast of the fault. Much of the conductive material here could be attributed to conductive surficial material in lowlying areas and in the fault gouge. However, some of the EM anomalies in R1 have been interpreted as possible bedrock sources based on their anomaly shapes and their calculated depths. Most of the conductivity associated with R1 appears to be situated below surface based on the pseudo layer depths. This suggests bedrock sources such as graphite or sulphides as the cause of the conductivity. However, ancient, buried marine clays are another possible source.

A strong, sharp magnetic low, consisting of several linear segments that probably reflects a major fault has been labeled F3. Fault F3 has been mapped extending north - northeast through Tolstoi Bay to the southern shore of the Kasaan Peninsula. It loosely correlates with the contact between SOdg and SObl on the USGS geology map. Interrupting this fault, at line 20330 fiducial 820 on the Salt Chuck data set (and also on tie line 29030 at fiducial 2660, and at line 10350 fiducial 2810 on the Kasaan survey data) is a strong isolated magnetic anomaly. It is situated at the north end of Poor Man Creek near a small lake. This magnetic anomaly is noteworthy because it is unusual for a major fault to be interrupted by a feature in this manner. There is some conductivity loosely associated with this magnetic anomaly but it may be from lake bottom sediments.

The magnetic domains labeled M1 to M6 on the Interpretation Map represent what appears to be distinct lithologies or groups of rock units with similar geophysical characteristics. 
The areas labeled M1, M2 and M6 loosely correspond to areas with outcropping intrusive volcanic rocks (mostly gabbro, diorite and basalt to andesite; SOpx, SOdi and SOdb on the USGS geology maps). In these domains the magnetic data is characterized by relatively high amplitude responses and complex contour patters, which are typical of volcanic rocks.

The areas labeled M3 appear less strongly magnetized. In most cases there is an obvious contact with the more magnetic rocks of the M1, M2 and M6 volcanic domains or the less magnetically intense M5 sedimentary units. The magnetic domain labeled M3 is situated in an area mapped as andesite breccia of the Luck Creek (SObl).

M4 loosely correlates with an area geologically mapped as hornblende quartz monzodiorite. M5 reflects several areas of lower magnetic intensity. Sedimentary rocks derived from volcanic units (SOdg) are the most prevalent outcrops mapped in this domain.

\section{CONDUCTOR DESCRIPTIONS}

Conductors 20030C, 20040A,B, and 20060A - 20080A

These inferred bedrock conductors ("B", "D" interpretive symbols) or possible bedrock conductors ("B?") are situated within the low-lying areas to the southwest of the Thorne River Fault within R1. The proximity of these conductors to the volcanic intrusive rocks enhances their importance. These conductors may reflect sulphide or graphite mineralization. Sharp bedrock overburden interfaces with buried marine clays are another possible source.

Conductors 20030H, 20040D and 29010A

These conductors are situated at the north edge of the survey area in the vicinity of the Thorne River Fault. They have been interpreted as possible bedrock conductors based on their profile shapes. Graphite or sulphide mineralization are possible sources as well as buried marine clays. Salt water channeling along the Thorne River Fault gouge is another possible source for these conductors.

\section{Prince of Wales Island, South of Keete Inlet, Hetta Inlet and Dolomi (1:63,360 Map Sheets B and C)}

The following discussion describes zones and structural features which have been inferred from the magnetic and resistivity data. 


\section{MAP SHEET B}

Zone R1 is defined on the resistivity maps as a highly conductive zone that contains multiple closely-spaced anomalies indicative of bedrock sources. This conductive zone is situated within geological units described on the geology maps as Silurian to Ordovician basaltic to andesitic volcanic rocks as well as volcaniclastic greywacke, siltstone, mudstone turbidites and minor sedimentary breccia. Low-lying areas consist of Holocene and/or Pleistocene surficial deposits. Although the conductivity of R1 appears to be quite uniform, the magnetic data display varying intensities. The central area of R1 appears to be associated with two small zones of more magnetic rocks labelled M1 and M2. A southwest-trending structural feature, F5, occurs in the southern section of the conductive zone. Several anomalies within R1 are indicative of thin dykelike sources.

Conductive zones R2, R3, R4, R5, R7, R8, R9 and R10 are all situated within a large unit which is mapped as Silurian to Ordovician basaltic to andesitic volcanic rocks. Zones R2 through R4, as well as R7, occur to the east of a magnetic feature that has been fractured into five sections by four possible east-trending faults F1, F2, F3 and F4. The most magnetic portions of this magnetic feature are labelled M3, M4, M5 and M6. M6 consists of a small, strong, discrete magnetic high at its eastern portion just south of structural feature F4. It is located on line 10650, fiducial 5422 and is characterized by a high concentration of magnetite. $\mathrm{R} 4$ is located immediately east of possible structural feature F1. R5 coincides with magnetic high M4 and lies between structural features F1 and F2. R7 is located near the end of three structural features F2, F3 and F4, and approximately half a mile $(600 \mathrm{~m})$ to the north of a southwest-trending possible break F5. Zones R8 through R10 lie in a magnetically inactive area. R8 straddles structural feature F5.

Zone R6 lies to the east of a magnetic feature and in close proximity to possible structural feature F2. It is associated with surficial deposits of Holocene and/or Pleistocene age.

Two conductive zones R11 and R12 are situated near strongly magnetic features M7 through M9. Anomalies R12 and M9 lie on opposite sides of a probable structural break F10 and reflect possible bedrock sources. A number of thin dyke-like sources are associated with these two anomalous features. All are situated within a zone mapped as Andesitic breccia.

Zones R13 and R14 are situated near strong magnetic zones M10, M11 and M12. R13 and R14 are both small, oblate conductive features which are located within a 
Cambrian Greenstone belt. R13 is associated with a thin dyke-like source. R14 rests along the Lake St. Nicholas fault.

R15 rests along a possible fault, F8, and is surrounded by a magnetic high, M11. This conductive zone occurs within a rock unit that is composed of Hornblende quartz monzodiorite with minor granodiorite, quartz diorite, quartz monzonite and monzodiorite.

Conductive zones R16 through R20 fall within a Silurian to Ordovician rock unit that consists of basaltic to andesitic volcanic rocks. R17 represents a conductive portion of a stream that correlates with a circular magnetic high, M14. R18 occurs near the ends of two probable faults, F12 and F13 and lies between four small magnetic highs, M13, M16, M17 and M18. R19 and R20 rest along possible structural features F6 and F7b. Within the same rock unit are magnetic features M19 through M22. All rest in close proximity to faults. Those being F16, F17a and F17b.

Conductive features R21 and F22 are highly conductive features that consist of anomalies that reflect bedrock sources. R21 correlates with a magnetic high. Both features occur close to structural features, namely F14 and F15.

R23 and R24 are situated close to the Trocadero Bay Fault, a portion of which is labelled as F25. R23 straddles the fault and correlates with a magnetic high, M35 and is associated with a number of bedrock and dyke-like sources. It also lies at the end of magnetic feature M36. R24 rests close to a number of magnetic highs, M36 and M40, and at the end of a probable fault, F20.

There are a number of interesting conductive features situated along a major contact, $\mathrm{C} 1$. They consist of R25, R26, R27 and R28. All are relatively conductive and are associated with dyke-like EM anomalies. No magnetic correlation exists for zones R25a through R27. R28, however, lies near magnetic features M27 and M29. Zone R27 lies along a probable structural break, F23, on the other side of which is a magnetic high, M84. All features lie within a Greenstone, Greenshist, black phyllite and quartz-sericite schist unit of Cambrian age.

Two small, circular magnetic features, M23 and M24, lie near contact C1. They occur within a Cambrian unit that consists of Greenstone, Greenschist, black phyllite and quartz-sericite schist. Both magnetic features are associated with bedrock and dyke-like anomalies.

Magnetic zone M30 also lies near contact $\mathrm{C} 1$. Just to the south lies a large conductive zone, R36. A number of dyke-like anomalies occur to the south of this zone which is associated with a Greenstone unit. 
Three small magnetic features, M25, M26 and M28, rest to the northeast of contact $\mathrm{C} 1$. All three lie in close proximity to possible faults, F18 and F25, which run along a portion of the Trocadero Bay Fault. They, too, are associated with a Greenstone unit.

Conductive zones R29 through R33 all lie close to a large magnetite-rich zone, M40, in the lower central portion of map sheet B. The large magnetic zone is broken into various smaller zones by possible faults, such as F19a, F19b, F20, F26 and F27. These faults have produced a number of smaller magnetic zones labelled M32, M33, M34, M35, M36, M37, M38 and M39. Zones R31, R32 and R33 lie along a possible structural break, F19a and are characterized by dyke-like anomalies. The remainder of the conductive zones are associated with bedrock-type and dyke-type anomalies. All conductive zones are located within a large zone of basaltic to andesitic volcanic rocks of Silurian to Ordovician age.

Magnetic zone M31 is a circular magnetic high that coincides with a sinuous conductive feature. A surficial type anomaly, however, is associated with this zone.

Conductive zone R34 is situated near the edge of a highly magnetic zone, M38. A bedrock and dyke-type anomaly are associated with this conductive zone which lies at the edge of a geologic unit consisting of volcanistic greywacke, siltstone, mudstone turbidites, and minor sedimentary breccia, limestone and polymictic conglomerate.

Zone R35 is situated at the westernmost edge of a large magnetic zone, M40, and close to contact $\mathrm{C} 1$. It lies at the corner of a geological zone consisting of marble and minor calcsilicate rocks.

To the south of contact $\mathrm{C} 1$ lies a large conductive zone, R36. It is quite conductive, exhibiting values as low as $10 \mathrm{ohm}-\mathrm{m}$. It is characterized by numerous, closely-spaced bedrock and dyke-type sources, especially at its northern section close to structural feature F23. Generally, this zone shows no direct magnetic correlation as it is situated within a relatively non-magnetic zone. However, it is situated at the western edge of a highly magnetic feature. A possible fault, F23, bounds the northern portion of zone R36. This feature mainly lies within a Greenstone belt.

Just to the south of R36 lies R37. It too is relatively conductive, but unlike R36, it has some weak magnetic correlation. Zone R37 straddles a Greenstone unit as well as a volcanic unit consisting of agglomerate, pillow basalt and aquagene tuff. An oblate resistivity low, within R37, on line 20060, fiducial 2765 lies close to a magnetic high, M86b. 
Surrounding these two large conductive features are a number of small, oblate and circular magnetic highs. These include M83, M84, M85, M86a, M86b, M87, M88, M89 and M90. M84 lies between a possible fault, F23, and a resistivity low, R36. M83 lies near a contact, $\mathrm{C} 1$, and two possible intersecting faults, F22 and F23. Zones M88 and M89 represent two small, circular magnetic highs that occur to the north of a probable fault, F48. Most of the magnetic highs are situated within a large Greenstone zone. M26a and M26b lie on the boundary between a Greenstone zone and one consisting of marble and minor calcsilicate rocks. M90 occurs near a conductive zone, $\mathrm{R} 37$, and is sandwiched between a possible fault, F48, and a contact, C5. It is situated within an agglomerate, pillow basalt and aquagene tuff unit.

An oblate conductive zone, R38, that lies on the boundary of two geologic units consisting of Greenstone and surficial deposits shows some magnetic correlation. It is in close proximity of a strong, circular magnetic high, M88.

Just to the south of a fractured contact, C5, are a number of interesting magnetic and resistivity anomalies. Structural breaks, F47 and F48 split the contact into four pieces. Magnetic zones M91, M92, M93, M94, M95 and M97 are all strong magnetic features that rest immediately to the south of contact C5. Zones M92 through M94 lie along or close to the Cordova Bay Fault and to the east and west of structural breaks F48 and F47 respectively. A resistivity low, R39, is associated with magnetic zone M92. M91 is a strong, circular magnetic high that lies close to a contact, C5, and at the edge of a resistivity low, $\mathrm{R} 37$.

M95 is a magnetic unit that seems to rest at the end of two possible faults, F47 and F48. It is flanked on either side by two circular resistivity lows, R40 and R41. A bedrock anomaly occurs on the northeastern edge of M95. Zone M96 may be of interest as it is a strongly magnetic circular feature that occurs in an area characterized by a moderate magnetic background.

A highly magnetic feature, M97, located close to contact C5 is intersected by a possible fault, F49. Its eastern section is flanked by two resistivity lows, R44 and R45, that reflect bedrock sources

Just to the west of R44 are conductive zones R42 and R43. Zone R42 lies on the eastern end of a structural break, F47.

All the magnetic and conductive zones are situated within a Greenstone unit (east of the Cordova Bay Fault) or within an agglomerate, pillow basalt, tuff and aquagene tuff unit (west of the Cordova Bay Fault). M95 and R39 are the only two zones that are characterized by surficial deposits. 
Two small magnetic zones, M98 and M99, occur near a contact, C5, and near the intersection of two possible faults, F49 and F50. Near the intersection of these two structural breaks lies a resistivity low, R46a. It lies to the west of a large magnetic feature, M40. Along the same possible fault, F49, lies a small, weakly conductive zone, $\mathrm{R} 47$. It is sandwiched between a large magnetic zone, M40, just to the west of a northtrending structural break, F51.

A thin conductive feature, R46b, lies along structural break F50. It is situated on the edge of a magnetic high within M40 and consists of a number of bedrock anomalies.

In the northeastern section of sheet B are a number of magnetic features, namely M46 through M49. All lie within a Greenstone unit. A number of bedrock and magnetite-type anomalies are associated with some of these magnetic zones.

Zones R53 and R54 are conductive zones that occur within a large relatively nonmagnetic zone. A bedrock anomaly occurs on the eastern edge of R53 characterized by a Greenstone unit.

Magnetic zones M41a and M41b represent two magnetic highs in a relatively low magnetic area. To the southwest of these magnetic zones are five conductive features, R48 through R52. They show little or no correlation with the magnetic data. R51 lies at the edge of a lake and coincides with a weakly magnetic circular feature. The abovementioned zones are associated with weakly to moderately strong bedrock conductors as well as a number of dyke-type source conductors. A strong dyke-type anomaly occurs on the southern edge of R52. All features are located within a large Silurian to Ordovician unit consisting of volcaniclastic greywacke, siltstone, mudstone turbidites and minor sedimentary breccia, limestone and polymictic conglomerate.

Magnetic zones M42, M43, M44 and M45 appear to follow a linear southeast trend. All are located within a relative magnetic low area. Surrounding M44 are three conductive zones, R55, R56 and R57. They show no direct magnetic correlation as they too are situated within a relatively non-magnetic zone. A couple of bedrock anomalies occur to the north of M43, whereas zones M44 and M45 are associated with magnetitetype anomalies. Zones R55 and R57 are associated with surficial-type anomalies. All features lie within or near the edges of a volcaniclastic greywacke, siltstone, mudstone turbidites and minor sedimentary breccia, limestone and polymictic conglomerate unit with the exception of M42 and M45, which are situated within a basaltic to andesitic volcanic rock unit and Greenstone unit respectively.

Magnetic zones M50a, M50b, M50c and M50d are situated at the easternmost edge of the survey block. These magnetite-rich zones display little associated conductivity. They exhibit a general correlation with a mapped geologic unit of Early 
Silurian age consisting of diorite, quartz diorite, gabbro, hornblendite, lencogabbro, trondhjemite, pyroxenite and migmatite. Magnetite-rich zones M65 and M66 may be associated with M50. A possible fault, F30, separates M50b and M50c.

Within zone M50b are two conductive features R58 and R60. R58 is associated with a bedrock anomaly and is located along a magnetic contact, C3, whereas R60 rests within the conductive feature M50b.

The southeastern area of sheet B is characterized by numerous magnetic highs and a few resistivity lows surrounded by southeast, east and northeast-trending structural breaks. All anomalous features occur within a Greenstone belt.

R59 and R61 rest to the north of a probable fault, F28. R59 coincides with the magnetic data at its northern point and a dyke-type anomaly that dips toward the north. A dyke-type anomaly is also present at the southern edge of R59 along probable fault F28a. R61 rests within a magnetic low and is characterized by both bedrock and surficial anomalies. A well-defined dyke-type anomaly is evident at the eastern area of R61 on line 11050, fiducial 9870.

Magnetic zones M51 through M63 are all situated close to southeast- or easttrending faults. All, with the exception of M62 and M63, are magnetite-rich, as indicated by the associated strong negative inphase responses. Little conductivity is associated with most of these magnetic zones. Only M57, M59 and M61 correlate with resistivity lows, those being R65, R66 and R67. R66 consists of a strong, well-defined bedrock anomaly. R67 coincides with a portion of magnetic zone M61 and occurs along a possible fault.

Three circular resistivity lows, R62, R63 and R64, form a linear path. R62 occurs within a magnetic low and is associated with two dyke-type anomalies. R63 and R64 both lie on the edge of or correlate with magnetic highs. R64 also lies along a probable fault, F29.

R68 lies along a structural break, F38, within a magnetic low. Two magnetic features, M60 and M61, lie to the northeast.

The remaining magnetic features, M64 through M82, are located near east-, northeast- or southeast-trending possible faults. Most are magnetite-rich and are associated with little conductivity. Zones M68 through M71, along with M76, M78, M79, M80 and M81, occur near intersecting faults and may be of some interest. A resistivity low, R69, characterized by a well-defined dyke-type anomaly rests along a possible fault, F41, and the edge of a strong magnetic high, M76. At the eastern end of the possible fault F41 lies a circular resistivity low, R70, which is characterized by a well-defined dyke-type anomaly. It lies on the edge of a magnetic high marking a 
lithological change between metamorphic rocks of Late and Middle Cambrian to the west and metamorphic rocks of Silurian and/or Ordivician age to the east.

\section{MAP SHEET C}

In the northwestern edge of the survey area on sheet B are two possible intersecting faults, F50 and F52. A number of magnetic highs and resistivity lows occur in the vicinity. They include magnetic zones M100 through M105 and resistivity lows R71 through R73. Zones M101 through M105 are situated near the intersection of possible faults F50 and F52. M101 and M104 coincide with resistivity lows R71 and R73 respectively. R71 is characterized by closely-spaced anomalies that reflect bedrock sources. A possible bedrock source occurs to the right of R73. M103 coincides with a resistivity low, R72, which is in turn flanked to the north and south by magnetic highs, M102 and M105. R72 is associated with a couple of surficial anomalies. M101 and R71 lie within a small Middle Jurassic unit consisting of Gabbro. M104 and R73 lie within a Silurian and Ordivician unit consisting of volcaniclastic greywacke, siltstone, mudstone turbidites with minor sedimentary breccia, limestone and polymictic conglomerate. The remainder lie within a Greenstone unit of Cambrian age.

Just to the east of a south-trending possible fault lie three small conductive features, R74 through R76. All coincide with a strong magnetic high, M106, and occur within a Greenstone unit. Anomalies reflect possible surficial sources.

To the west of structural break F53 lies a highly magnetic feature, M107. It straddles a stratigraphic boundary between volcaniclastic greywacke, siltstone and mudstone turbidites to the west and a Greenstone unit to the east. It is characterized by a combination of possible bedrock and surficial anomalies.

Within a large, highly magnetic unit, M40, at the top of sheet $\mathrm{C}$ are a number of resistivity lows. R77 occurs along a mapped fault within a Greenstone unit and is characterized by closely-spaced bedrock anomalies. Zones R78 and R80 are situated within a zone mapped as hornblende quartz monzodiorite with minor amounts of tonalite, granodiorite, diorite and monzodiorite. Anomalies within R78 reflect a broad source. R80 represents a conductive portion of a lake where anomalies reflect surficial sources. R79 is situated within two geologic units; one consisting of marble and minor calcsilicate rocks, the other consisting of hornblende quartz monzodiorite with minor granodiorite, diorite and monzodiorite. This zone is characterized by possible bedrock anomalies. R81 lies along a semi-circular fault. Anomalies reflect possible bedrock sources which are situated within a Greenstone unit and a unit consisting of marble with minor calcsilicate rocks. 
To the east of magnetic zone M40 lies a known fault, F54. Along it are four resistivity lows, R108, R110, R111 and R112. All reflect bedrock anomalies. R108 and a portion of R110 coincide with magnetic highs. Two dyke-type anomalies occur on the edges of R110. All zones, with the exception of R112, lie within a Greenstone unit and one consisting of marble with minor calcsilicate rocks. R112 lies solely within a Greenstone unit.

A highly conductive, circular feature, M109, consisting of possible surficial and bedrock anomalies, lies to the north of structural break F54. It rests within a marble and calcsilicate rock unit and correlates with a weakly magnetic circular feature.

Magnetic zones M108 through M110 occur in close proximity to possible faults F59 and F60. Two small resistivity lows, R83 and R84, coincide with M108. A bedrock anomaly is associated with R84. Another small resistivity low, R82, lies to the east of M108 along structural break F55 and occurs approximately 100 metres to the west of a dyke-type anomaly. A resistivity low, R85, coincides with M110. A number of bedrock and surficial type anomalies are associated with this feature. A south-dipping dyke-type anomaly occurs on the eastern edge of R85. They are all situated within a large Greenstone unit.

R86 through R89 also occur close to possible faults within a Greenstone unit. R87 lies close to intersecting faults F59 and F61, and comprises of closely-spaced bedrock faults along its eastern edge. R86, R88 and R89 lie along a possible fault, F73. Individual anomalies within these zones reflect surficial sources.

Further to the south are three magnetic features, M111 M112 and M113. M111 and M112 are situated on opposite sides of a possible fault, F74. They are all situated within a Greenstone unit.

M114 and M117 are two moderately magnetic discrete zones that may be of interest. They are situated close to probable faults F59 and F62, and along F66 respectively.

Magnetic zones M115, M116, M118, M119, M120, M121 and M122 represent small, circular or oblate strong magnetic features in a generally moderate magnetic background. All occur close to structural breaks, such as F59, F64, F66, F67b and F69. M115, M116, M118 and M119 are magnetite-rich. M120 and M121 coincide with small resistivity lows, R93 and R94 respectively. M122 lies at the intersection of probable faults F66 and F69.

Three resistivity lows occur close to the above-mentioned magnetic highs. They are R90 through R92. All coincide with magnetic highs and bedrock anomalies. R91 and 
R92 are associated with well-defined dyke-type anomalies. R91 is intersected by a known fault, F91.

The southwestern portion of the survey area on map sheet $\mathrm{C}$ around and to the south of Keete Inlet is highly magnetic and has been mapped as a Greenstone unit. A number of south- and southeast-trending faults occur in the area. Zones M123 through M137 represent anomalous magnetic features in the area. M123 is bounded on all sides by structural breaks F67a (known fault in the area), F68, F69 and F70. A small, circular resistivity low, R95, occurs immediately to the north along F68 and consists of a bedrock anomaly.

A resistivity low, R96, to the east coincides with a magnetic high, M124. A surficial source is indicated in the area.

Zones M126 and M132 are bounded on either side by structural break F72 and a known fault F67a. Along F72, and in close proximity to M132, are a number of resistivity lows, R99, R100, R101, R102 and R103. R99 lies near a magnetic high, M129.

To the east of fault F67a are a number of anomalous features, those being M127, M128, R97, R98 and R104. A resistivity low, R98, coincides with a magnetic high, M128 and M132. It consists of multiple bedrock sources. Two dyke-type anomalies are associated with this feature. A number of bedrock anomalies, including a dyke-type anomaly occur within R104. A small, conductive feature, R97, occurs in close proximity to a known fault, F67a, and a large magnetic high, M132. It consists of a thin dyke-like anomaly.

M130, M133 and M135 are situated near a south-trending possible fault, F71. Both M130 and M135 are rich in magnetite.

A large magnetic feature at the southernmost portion of the survey area, M137, is intersected by a probable fault, F70. Three resistivity lows, R105 through R107, occur near F70. A number of bedrock anomalies, including thin dyke-like anomalies are associated with R106 and R107.

A heavily fractured magnetic unit consisting of zones M138 through M154 occurs in the centre of map sheet C. Structural breaks trend in all directions. A known fault, F92, cuts through the centre of the fractured magnetic unit. M142 to M146 are located near a number of possible faults, F56, F57, F58 and F87. All zones are magnetite-rich. A circular resistivity low occurs along a possible fault, F58 and close to magnetic high M145. It coincides, however, with a lake and may be due to lake-bottom sediments. Other small lakes in the area are not so conductive. 
A resistivity low, R119, that is located along a known fault, F85, may be of interest. It occurs in the vicinity of a number of magnetic highs and is associated with two thin dyke-like anomalies.

R120 is located in the centre of three possible intersecting faults, F58, F62 and F87. It coincides with a magnetic high, M149. A strong circular magnetic high situated on line 11020, fiducial 4300 correlates with the northern end of R120.

R121, R123, R125 and R126 also correlate with magnetic highs. R121 crosses two possible faults, F86 and F87. R125 and R126 are associated with a bedrock and dyke-type anomaly respectively.

R122 and R124 lie along a southeast-trending possible fault, F87. R122 also lies at the eastern end of a structural break, F62. R124 is associated with a dyke-type anomaly. It should be noted that a line of bedrock anomalies stretch from R124 to R126 along the edge of a magnetic feature, M149.

A cluster of resistivity lows, R113 to R117, occur in the top central portion of map sheet C. All are located within a Greenstone unit and show little to no magnetic correlation as they are situated within a relatively non-magnetic zone. R115 is located just to the south of a small magnetic feature, M201. R113 to R116 consist of one or more anomalies that reflect bedrock sources. Two thin dyke-like sources occur on either side of M201 within R115 and on the edge of R113. R117, which is associated with a surficial anomaly, is situated between two small magnetic highs, M199 and M200. These two magnetic highs also line up with two more, M197 and M198. A thin dyke-like anomaly lies on the northern edge of M198.

A number of highly magnetic zones, M155 through M163, in the centre of map sheet $\mathrm{C}$ are separated by five southeast-trending possible faults, F57, F84, F85, F86 and F87. These zones generally correlate with a relatively resistive zone. A few resistivity lows coincide with the magnetic highs and/or faults in the area. R127, R128 and R130 are situated on the edge of M158. R129 and R131 lie along a known mapped fault, F93, that does not show up in the magnetic or resistivity maps. R129 consists of a thin dykelike anomaly, whereas R131 lies immediately to the north of a solitary bedrock anomaly. A possible fault, F57, just south of F93 intersects M158 and R133. R132 lies to the north of a structural break, F85, on the edge of magnetic feature M158. R134 and R135 correlate with moderate magnetic highs.

Another known mapped fault that doesn't show up in the magnetic and resistivity data is F94. It intersects M162 and the southeastern edge of M158. A resistivity low, $\mathrm{R} 136$, also intersected by F94, lies in close proximity to a possible fault, F85 and 
coincides with the southeastern portion of M158. Other resistivity lows and magnetic highs that lie close to possible faults are R137 through R139, and M164 through M167. R139 consists of bedrock type anomalies. All of the above magnetic and conductive features are situated within a number of different geological units consisting of metasedimentary, metavolcanic and sedimentary rocks.

A magnetic contact, C7, is evident in the northeastern section of map sheet $\mathrm{C}$. In the vicinity of the contact are a number of anomalous features. Magnetic feature M168 is located close to the contact and a southwest-trending fault, F75. Three resistivity lows in the area are of interest. They include R140, R141 and R142. R140 and R141 are located near the contact and/or possible fault F75. Both are characterized by dyke-type anomalies. R142 lies on the edge of a narrow, northwest-trending magnetic high and along F75. Closely-spaced anomalies within this feature reflect bedrock anomalies as well as a number of dyke-like sources. M168 crosses three geologic units consisting of quartz diorite, diorite and granodiorite, as well as marble. The resistivity lows occur within a Greenstone unit.

A little to the south are a number of probable intersecting faults which include F75, F76, F77 and F78. In the immediate vicinity are a few magnetic highs and resistivity lows which are located within a Greenstone unit. R143 and R144 are located along or near structural features F75, F76 and F77. Both coincide with small magnetic highs and consist of closely-spaced bedrock features. A number of dyke-like anomalies occur within and to the west of R144.

M171 and M172 represent strong magnetic highs that lie close to structural break F77. M173 is bounded on three sides by possible faults F76, F77 and F78.

Four small resistivity lows, R145 through R148, are located along or close to four probable intersecting faults, F75, F76, F77 and F78. R145, R147 and R148 occur at the edges of lakes. R147 correlates with a small magnetic high, M174, and consists of a bedrock anomaly. R148 rests in between four small magnetic highs, M174 through M178.

R149 lies along structural break F79 within a magnetic low. Anomalies reflect surficial and bedrock anomalies including two dyke-like anomalies along F79. R150 and R151 represent two conductive features that may be of interest. R151 represents a strong resistivity low near a lake that coincides with a small, weak magnetic feature. R150 occurs within a magnetic low, surrounded by magnetic highs.

To the southwest are two semi-circular structural breaks, F79 and F80. A portion of F79 coincides with a known fault in the area. Resistivity lows R152 through R158 rest near these possible faults, as well as F81 and F82. R152 shows no direct magnetic 
correlation and lies along F79. M178 and M179 rest just to the north of R152 and contain moderate amounts of magnetite.

R153 and R154 are intersected by possible faults F80 and F79 respectively and correlate with magnetic highs that also consist of magnetite. R154 lies immediately to the north of a strong magnetic high, M180.

A small resistivity low, R155, occurs near intersecting breaks F79, F81 and F82. It correlates with a small magnetic high, M181, and consists of a couple of bedrock anomalies.

Zones M182 to M185 represent moderate magnetic highs that lie in close proximity to a few east-trending structural breaks.

R156 to R158 are situated between structural breaks F79 and F85. R156 correlates with a magnetic high, M185. Bedrock sources, including a number of dykelike anomalies, are associated with R156. Zones R157 and R158 fall within relative magnetic lows. R157 is associated with a surficial anomaly.

Structural break F89 was inferred from the magnetic data and confirms the existence of a north-dipping fault in the area. However, the magnetic data indicates the fault extends further to the southeast. A number of anomalous features occur in the vicinity of this fault. They include M188, M189, M190, M191, R159 and R160. R159 and R160 are located along and near fault F89 respectively. R159 coincides with the magnetic data. Bedrock sources are associated with this feature, although a number of dyke-like anomalies occur both to the east and west. R160 occurs within a magnetic low and is associated with a surficial anomaly. Magnetic zones M186 to M191 occur near the coast or within Moira Sound. They generally correlate with resistivity highs. M186, M187 and M191 contain moderate amounts of magnetite. All anomalous features have been mapped within a Greenstone unit.

The remaining anomalous features M192 to M196 and R161 to R163 are situated within a Greenstone unit. R161 and R162 are two small resistivity lows that lie along a southeast-trending possible fault, F90. R161 occurs within a magnetic low and reflects a surficial anomaly. R162 occurs on the edge of a magnetic high and reflects a possible bedrock anomaly. R163 correlates with a strong magnetic high, M195, and reflects a bedrock source. Three dyke-like anomalies occur on the edge of the resistivity low. M192 to M196 lie close to a structural break, F91, and represent strong magnetic features that are magnetite-rich. All, with the exception of M195, generally correlate with resistivity highs. M196 consists of a number of bedrock conductors. 


\section{Western Part - Gravina Island}

\section{APPARENT RESISTIVITY}

The West Gravina area has 222 bedrock anomalies, 51 surficial anomalies, 61 magnetite anomalies and no cultural anomalies.

The apparent resistivity data sets range from a low of less that $0.4 \mathrm{ohm}-\mathrm{m}$ over the sea water to over $20,000 \mathrm{ohm}-\mathrm{m}$. The background is 5,000 to $20,000 \mathrm{ohm}-\mathrm{m}$ in the central portion of the survey area. There is only a minor amount of correlation between the trends and individual responses of the apparent resistivity and total field magnetic data sets. This general lack of correlation is attributed only in small part to the presence of surficial material, because of the predominance of outcrop on the island.

Four apparent resistivity lows are identified on the interpretation map and labeled as $\mathrm{R} 1$ through $\mathrm{R} 24$.

$\mathrm{R} 1$ is a long, sinewy conductive feature which extends for 7 miles $(11 \mathrm{~km})$ with a general north-south trend. The feature has been divided into three segments based on interpreted breaks in the continuity of the more conductive portions of the source. Structural features F3 through F5 are interpreted to interrupt the continuity of R1 by creating offsets or breaks. The broken segments are labeled as $\mathrm{a}, \mathrm{b}$ and $\mathrm{c}$ from north to south.

The strongest responses occur on lines 30050, 30070, 30170, and 30200 where the apparent resistivity is computed at $10 \mathrm{ohm}-\mathrm{m}$ or less. The feature pinches and swells from a narrow response to a broad feature several hundred metres in width. In these wider portions, the feature can often be interpreted to comprise two or more closely spaced parallel conductive sources. Where it can be interpreted, the feature displays a consistent easterly dip. In areas such as on lines 30170 and 30180, the source can be seen to extend to great depth beneath the relatively resistive rocks to the east.

R1 has no discrete correlating magnetic response. However, anomalously high magnetic responses on lines 30160 and 30190 indicate that magnetic mineralization is associated with this feature over isolated portions of its extent. R1 occurs within a mapped portion of the Hyd Group containing mafic volcanic and sedimentary rocks. The majority of the feature falls within the Chapin Peak formation and conforms to the general north south trend of the basaltic volcanic and minor sedimentary rocks contained in the formation. R1 is interpreted to reflect graphite or conductive sulphide mineralization with no correlating magnetic mineralization. 
$\mathrm{R} 2$ is a small, isolated, conductive feature which is seen predominantly on line 30220 at approximately fiducial 5172. There is a limited response to the same source on line 30210 but the response is neither strong enough nor broad enough to appear on the $900 \mathrm{~Hz}$ apparent resistivity map. The feature is strongest in the $900 \mathrm{~Hz}$ data set with an apparent resistivity of approximately $12 \mathrm{ohm}-\mathrm{m}$. Like R1, R2 has a clear easterly dip and no discrete correlating magnetic response. The source falls within a mapped unit containing mafic volcanic and sedimentary rocks of the Hyd group. The feature is interpreted to reflect graphite and/or sulphide mineralization with no associated magnetic mineralization.

R3, a small isolated feature similar to R2, occurs on lines 30240 and 30250 near the eastern margin of the survey block. This feature has an apparent resistivity of approximately $30 \mathrm{ohm}-\mathrm{m}$ at depth. R3 dips toward the east and has no discrete correlating magnetic response. The source falls within an area covered with surficial deposits. The mapped rocks immediately to the west of the source consist of sedimentary and minor volcanic rocks of the Gravina Island formation. The quiet magnetic response in this portion of the survey area suggests either a smoothly varying magnetic mineral content or, more probably, a very low magnetic mineral content consistent with felsic volcanic and sedimentary rocks. The source of R3 is interpreted to be graphite and/or conductive sulphides with no significant magnetic mineral content within felsic and/or sedimentary host rocks.

R4 is an odd-shaped conductive feature which occurs in a geophysically and geologically complex portion of the survey area. The feature ranges from weakly conductive to the strongest responses of just under $100 \mathrm{ohm}-\mathrm{m}$ on lines 30540 and 30550. The feature is cut by northwest trending structural feature F6 which is evident in the geophysical data sets and is mapped on the geology map. R4 extends across the survey area with a very general east west trend which conforms to the mapped geologic trends in this portion of the survey block. Portions of this feature correlate well with topographic lows such as valleys and rills and likely are, at least in part, caused by accumulation of surficial sediment. Other portions, however respond strongly in the $900 \mathrm{~Hz}$ apparent resistivity data set and are interpreted to reflect bedrock sources. Although in a generally magnetically active portion of the survey area, R4 has no discrete correlation with the magnetic data set. The strongest portions of the feature are interpreted to reflect conductive mineralization such as graphite and sulphide with no associated magnetic mineralization. The weaker portions may reflect weaker concentrations of this type of mineralization but may also be reflecting accumulations of surficial sediment in the valleys and rills. 


\section{TOTAL MAGNETIC FIELD}

The total magnetic field data amplitudes range from approximately $56,450 \mathrm{nT}$ to $58,100 \mathrm{nT}$ with background ranging from 56,500 to $57,000 \mathrm{nT}$. Individual anomalous features range in amplitude from a few nT to over 1,000 nT. No single trend dominates the magnetic data set. Given the limited spatial extent of the survey area it is difficult to characterize the magnetic responses into discrete domains. Nevertheless, the data set can be separated into two types of magnetic response - active and inactive. The active areas have been defined with boundaries and labeled on the interpretation maps as M1, M2, $\mathrm{M} 3$, etc.

M1 is dominated by a single, large, odd-shaped feature with a maximum amplitude of approximately $400 \mathrm{nT}$ above background. This feature is approximately 1 sq. mi. and may extend to the northwest as a linear feature through lines 30080, 30070, and 30060 at the western limit of the survey area. This northwest extension may be cut by a northeast trending feature, F1. M1 falls on a mapped northwest trending fault boundary between mafic volcanic and sedimentary units to the northeast and felsic intrusives to the southwest. M1 is interpreted to reflect a concentration of magnetic mineralization possibly associated with this structural feature.

M2 is comprised of many strong magnetic highs which range in amplitude up to $500 \mathrm{nT}$. The density and mottled nature of these individual features precludes a confident correlation from line to line. M2 falls within geologic units mapped as felsic to mafic intrusives of the Karheen formation and felsic volcanic rocks of the Hyd group. This zone is interpreted to reflect concentrations of magnetic mineralization which are overprinted on the geologic units possibly through metamorphic processes.

M3 is a linear high which is open to the east of the survey at Bostwick Inlet and extends approximately 2 miles northwest to line 30350. The feature ranges in amplitude up to $1,200 \mathrm{nT}$. It is possible that this feature reflects a body with concentrations of magnetic mineralization which is related to the mapped north northwest trending inferred fault or other mapped thrust faults of the same trend in the vicinity.

M4 is similar to M2 in the mottled texture and presence of many individual highs. The individual responses, which range up to $200 \mathrm{nT}$ in amplitude, however, are of much lower amplitude than those in M2. This zone lies just north of R4 and is cut by structural feature F6 which is manifested by a relative low and break in the occurrence of magnetic highs. This zone falls entirely within a mapped portion of the Chapin Peak formation which contains basaltic volcanic and minor sedimentary rocks. The magnetic response is consistent with the basaltic rocks indicated on the geology map. 
M5 is a high amplitude magnetic feature with a general southwest trend. It is situated just south of conductive feature R4 and has dimensions of approximately $1500 \mathrm{ft}$ $(500 \mathrm{~m})$ by several miles. This magnetic feature is cut by structural feature F6 but may extend southwest of F6 as a narrow magnetic high. M5 falls largely within a mapped unit which contains mafic and felsic plutonic rocks. The main body of this feature is interpreted to reflect a possible mafic intrusive.

The remainder of the survey area is generally inactive in the magnetic data set. However, isolated strong responses of up to several hundred nT in amplitude occur throughout the survey area. These are generally sparse, but do become more common in some areas such as in the southeast portion of the survey area.

\section{STRUCTURE}

Structure is interpreted primarily from the magnetic data set with support from the apparent resistivity data sets as truncation and offset of individual features. A high degree of these breaks are apparent particularly in the northern and southern portions of the survey area. These breaks confirm a high level of structural complexity relative to the flight line spacing which precludes a confident detailed interpretation of structure. However, several prominent structural breaks and lineations are indicated and labeled on the interpretation map as F1, F2, F3, etc.

\section{Eastern Part of Gravina Island}

\section{MAP SHEET D}

The eastern part of Gravina Island comprises primarily Jurassic andesite to basaltic volcanic with minor sedimentary rocks (Jgv on the USGS Geology map), with sedimentary and minor volcanic rocks (Jgs) in one small part at the southwest end of the survey area.

Several possible structural breaks have been indicated on the interpretation map based on the resistivity and magnetic contour patterns. However, the small size of the survey area and relatively wide line spacing makes it difficult to identify lineaments and trends with any certainty.

Most of the conductivity has been interpreted as being due to surficial sources, and most of the resistivity lows are situated in low-lying areas. Judy Hill, at the south end of the survey area, is resistive. This is probably due to a relative lack of conductive overburden. 


\section{Discussion}

The geophysical results, in general, correlate with the known geology in the survey area. The results, in places, confirm the general trends and serve to extend the mapping of individual geologic units beneath the Paleozoic cover.

The resistivity and magnetic contour patterns in the Salt Chuck and Kasaan area appear to supplement the outcrop information on the regional geology maps. The geophysics appears to aid in understanding the lithological and structural relationships and defining regional settings for the deposits in the area. Some of the bedrock conductors in the Salt Chuck area may warrant follow-up if there is supporting geological, geochemical or other geophysical information.

The geology in the present surveys (Prince of Wales Island area and South of Keete Inlet area) and the historical surveys (Hetta Inlet and Dolomi) are geologically complex (see map sheets B and C). A number of large magnetic features dominate the central and southern portions of map sheets B and C. The magnetic data show some correlation with the geology and highlights contacts and known faults in the area. Some strong magnetic features contain moderate amounts of magnetite, most likely representing mafic intrusions. The resistivity data shows little correlation with the geology in the area. A number of large resistivity lows occur in the survey block. They show some agreement with the magnetic trends, suggesting they are related to bedrock features rather than conductive overburden.

The western part, Gravina Island survey area is dominated by a strong apparent resistivity low, R1, which extends with a northern trend through a mapped volcanic/ sedimentary unit. Several large magnetic features and magnetically active areas dominate the magnetic data set. The apparent resistivity data sets largely correlate with the trends and individual units of the geology as mapped. However, conductive surficial sediments toward the eastern margin of the survey area and in the valleys and rills are also evident in the survey results. The magnetic data set does not show this same general correlation with the mapped geology of the survey area. The strong magnetic features and magnetically active zones are interpreted to reflect mafic intrusions in places, but many of the responses can be attributed to concentration of magnetic mineralization through metamorphic processes.

The data covering the eastern part of Gravina Island needs to be further studied against more detailed geological information to identify if the data contains useful information. 


\section{CONCLUSIONS AND RECOMMENDATIONS}

This report describes the equipment, procedures and logistics of the survey and provides a brief description of the survey results.

The total field magnetic and apparent resistivity data sets have successfully mapped the magnetic and conductive characteristics of the geology in the survey areas. Numerous faults and contacts have been inferred from the survey results. There are many discrete electromagnetic anomalies in the survey areas which are typical of massive sulphide or graphite responses. The survey was also successful in locating several larger conductive zones which may also warrant additional work.

It is difficult to assess the relative merits of EM anomalies on the basis of conductance alone. It is recommended that an attempt be made to compile a suite of geophysical "signatures" over areas of interest. Anomaly characteristics and correlation with the other geophysical parameters are perhaps best defined on the Multi-parameter Stacked Profiles.

It is recommended that the survey results be reviewed in detail, in conjunction with all available geophysical, geological and geochemical information. Particular reference should be made to the multi-parameter stacked profiles which clearly define the characteristics of the individual anomalies in the identification of target areas. Image processing of existing geophysical data be considered, in order to extract the maximum amount of information from the survey results.

Respectfully submitted,

\section{GEOTERREX-DIGHEM}

Jonathan Rudd, P.Eng. $\quad$ Mark Stephens, M.Sc. $\quad$ Doug McConnell, P.Eng. Geophysicist

$\mathrm{JR} / \mathrm{MS} / \mathrm{DLM} / \mathrm{sdp}$

R0660JUL.99 


\section{REFERENCES}

Berg, H.C., Elliot, R.L., and Koch, R.D., 1988, Geologic map of the Ketchikan and Prince Rupert quadrangles, southeastern Alaska: Geological Survey Miscellaneous Investigations Map I-1807, 27 p., 1 sheet, scale: 1:250,000.

Eberlein, G.D., Churkin, M., Jr., Carter, C., Berg, H.C., and Ovenshine, A.T., 1983, Geology of the Craig quadrangle: U.S. Geological Survey Open File Report 83-91. 52 p., 7 sheets, scale: $1: 250,000$.

Gehrels, G.E., 1992, Geologic map of the southern Prince of Wales Island, southeastern Alaska: U.S. Geological Survey Miscellaneous Investigations Map I-2169, 23 p., 1 sheet, scale:1:63,360.

Maas, K.M., Bittenbender, P.E., and Still, J.C., 1995, Mineral investigations in the Ketchikan Mining District, southeastern Alaska: U.S. Bureau of Mines Open File Report 11-95, 606 p., 3 sheets.

Pritchard, D.E., 1992, DIGHEM ${ }^{\mathrm{V}}$ Survey for American Copper and Nickel Company, Inc., Kasaan Peninsula, Alaska: Dighem Surveys \& Processing Inc., 40 p.

Pritchard, D.E., 1992, DIGHEM ${ }^{\mathrm{V}}$ Survey for American Copper and Nickel Company, Inc., Hetta Inlet, Alaska: Dighem Surveys \& Processing Inc., 42 p.

Smith, P.A., 1991, DIGHEM ${ }^{\mathrm{V}}$ Survey for American Copper and Nickel Company, Inc., Dolomi Project, Alaska: Dighem Surveys \& Processing Inc., 57 p. 


\section{APPENDIX A}

\section{LIST OF PERSONNEL}

The following personnel were involved in the acquisition, processing, interpretation and presentation of data, relating to a DIGHEM $^{\mathrm{V}}$ airborne geophysical survey carried out under contract to WGM Inc., Mining and Geological Consultants, for the State of Alaska in the Ketchikan area, southeast Alaska.
Chris Nind
Greg Paleolog
Victor Chen
Troy Will
Walter Greaves
Doug McConnell
Stephen Harrison
Mark Stephens
Jonathan Rudd
Lyn Vanderstarren
Susan Pothiah
Albina Tonello

\author{
General Manager, Toronto Office \\ Manager, Helicopter Operations \\ Field Geophysicist \\ Geophysical Operator \\ Pilot (Era Aviation Inc.) \\ Manager, Interpretation and Processing \\ Processing Geophysicist \\ Interpretation Geophysicist \\ Interpretation Geophysicist \\ Drafting Supervisor \\ Word Processing Operator \\ Secretary/Expeditor
}

The survey consisted of 2,952.6 miles $(4,750.8 \mathrm{~km})$ of coverage, flown from February 26 to April 7, 1999.

All personnel are employees of Geoterrex-Dighem, except for the pilot who is an employee of Era Aviation Inc. 
APPENDIX B

BACKGROUND INFORMATION 
- Appendix B-1 -

\section{BACKGROUND INFORMATION}

\section{Electromagnetics}

DIGHEM electromagnetic responses fall into two general classes, discrete and broad. The discrete class consists of sharp, well-defined anomalies from discrete conductors such as sulphide lenses and steeply dipping sheets of graphite and sulfides. The broad class consists of wide anomalies from conductors having a large horizontal surface such as flatly dipping graphite or sulphide sheets, saline water-saturated sedimentary formations, conductive overburden and rock, and geothermal zones. A vertical conductive slab with a width of $200 \mathrm{~m}$ would straddle these two classes.

The vertical sheet (half plane) is the most common model used for the analysis of discrete conductors. All anomalies plotted on the geophysical maps are analyzed according to this model. The following section entitled Discrete Conductor Analysis describes this model in detail, including the effect of using it on anomalies caused by broad conductors such as conductive overburden.

The conductive earth (half space) model is suitable for broad conductors. Resistivity contour maps result from the use of this model. A later section entitled Resistivity Mapping describes the method further, including the effect of using it on anomalies caused by discrete conductors such as sulphide bodies.

\section{Geometric Interpretation}

The geophysical interpreter attempts to determine the geometric shape and dip of the conductor. Figure B-1 shows typical DIGHEM anomaly shapes which are used to guide the geometric interpretation.

\section{Discrete Conductor Analysis}

The EM anomalies appearing on the electromagnetic map are analyzed by computer to give the conductance (i.e., conductivity-thickness product) in siemens (mhos) of a vertical sheet model. This is done regardless of the interpreted geometric shape of the conductor. This is not an unreasonable procedure, because the computed conductance increases as the electrical quality of the conductor increases, regardless of its true shape. DIGHEM anomalies are divided into seven grades of conductance, as shown in Table B1. The conductance in siemens (mhos) is the reciprocal of resistance in ohms. 


\section{- Appendix B-2 -}

The conductance value is a geological parameter because it is a characteristic of the conductor alone. It generally is independent of frequency, flying height or depth of burial, apart from the averaging over a greater portion of the conductor as height increases. Small anomalies from deeply buried strong conductors are not confused with small anomalies from shallow weak conductors because the former will have larger conductance values.

\section{Table B-1. EM Anomaly Grades}

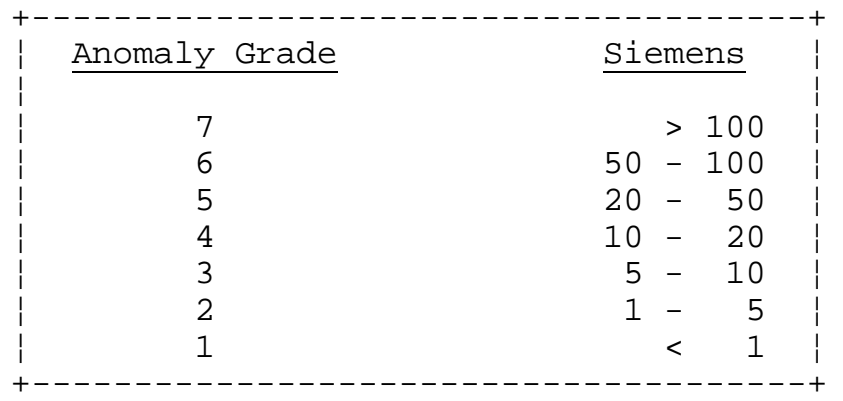

Conductive overburden generally produces broad EM responses which may not be shown as anomalies on the geophysical maps. However, patchy conductive overburden in otherwise resistive areas can yield discrete anomalies with a conductance grade (cf. Table B-1) of 1, 2 or even 3 for conducting clays which have resistivities as low as $50 \mathrm{ohm}-\mathrm{m}$. In areas where ground resistivities are below $10 \mathrm{ohm}-\mathrm{m}$, anomalies caused by weathering variations and similar causes can have any conductance grade. The anomaly shapes from the multiple coils often allow such conductors to be recognized, and these are indicated by the letters $\mathrm{S}, \mathrm{H}$, and sometimes $\mathrm{E}$ on the geophysical maps (see EM legend on maps).

For bedrock conductors, the higher anomaly grades indicate increasingly higher conductances. Examples: DIGHEM's New Insco copper discovery (Noranda, Canada) yielded a grade 5 anomaly, as did the neighbouring copper-zinc Magusi River ore body; Mattabi (copper-zinc, Sturgeon Lake, Canada) and Whistle (nickel, Sudbury, Canada) gave grade 6; and DIGHEM's Montcalm nickel-copper discovery (Timmins, Canada) yielded a grade 7 anomaly. Graphite and sulfides can span all grades but, in any particular survey area, field work may show that the different grades indicate different types of conductors.

Strong conductors (i.e., grades 6 and 7) are characteristic of massive sulfides or graphite. Moderate conductors (grades 4 and 5) typically reflect graphite or sulfides of a less massive character, while weak bedrock conductors (grades 1 to 3 ) can signify poorly connected graphite or heavily disseminated sulfides. Grades 1 and 2 conductors may not respond to ground EM equipment using frequencies less than $2000 \mathrm{~Hz}$. 
The presence of sphalerite or gangue can result in ore deposits having weak to moderate conductances. As an example, the three million ton lead-zinc deposit of Restigouche Mining Corporation near Bathurst, Canada, yielded a well-defined grade 2 conductor. The 10 percent by volume of sphalerite occurs as a coating around the fine grained massive pyrite, thereby inhibiting electrical conduction. Faults, fractures and shear zones may produce anomalies which typically have low conductances (e.g., grades 1 to 3). Conductive rock formations can yield anomalies of any conductance grade. The conductive materials in such rock formations can be salt water, weathered products such as clays, original depositional clays, and carbonaceous material.

For each interpreted electromagnetic anomaly on the geophysical maps, a letter identifier and an interpretive symbol are plotted beside the EM grade symbol. The horizontal rows of dots, under the interpretive symbol, indicate the anomaly amplitude on the flight record. The vertical column of dots, under the anomaly letter, gives the estimated depth. In areas where anomalies are crowded, the letter identifiers, interpretive symbols and dots may be obliterated. The EM grade symbols, however, will always be discernible, and the obliterated information can be obtained from the anomaly listing appended to this report.

The purpose of indicating the anomaly amplitude by dots is to provide an estimate of the reliability of the conductance calculation. Thus, a conductance value obtained from a large ppm anomaly ( 3 or 4 dots) will tend to be accurate whereas one obtained from a small ppm anomaly (no dots) could be quite inaccurate. The absence of amplitude dots indicates that the anomaly from the coaxial coil-pair is $5 \mathrm{ppm}$ or less on both the inphase and quadrature channels. Such small anomalies could reflect a weak conductor at the surface or a stronger conductor at depth. The conductance grade and depth estimate illustrates which of these possibilities fits the recorded data best.

The conductance measurement is considered more reliable than the depth estimate. There are a number of factors which can produce an error in the depth estimate, including the averaging of topographic variations by the altimeter, overlying conductive overburden, and the location and attitude of the conductor relative to the flight line. Conductor location and attitude can provide an erroneous depth estimate because the stronger part of the conductor may be deeper or to one side of the flight line, or because it has a shallow dip. A heavy tree cover can also produce errors in depth estimates. This is because the depth estimate is computed as the distance of bird from conductor, minus the altimeter reading. The altimeter can lock onto the top of a dense forest canopy. This situation yields an erroneously large depth estimate but does not affect the conductance estimate. 
- Appendix B-4 -

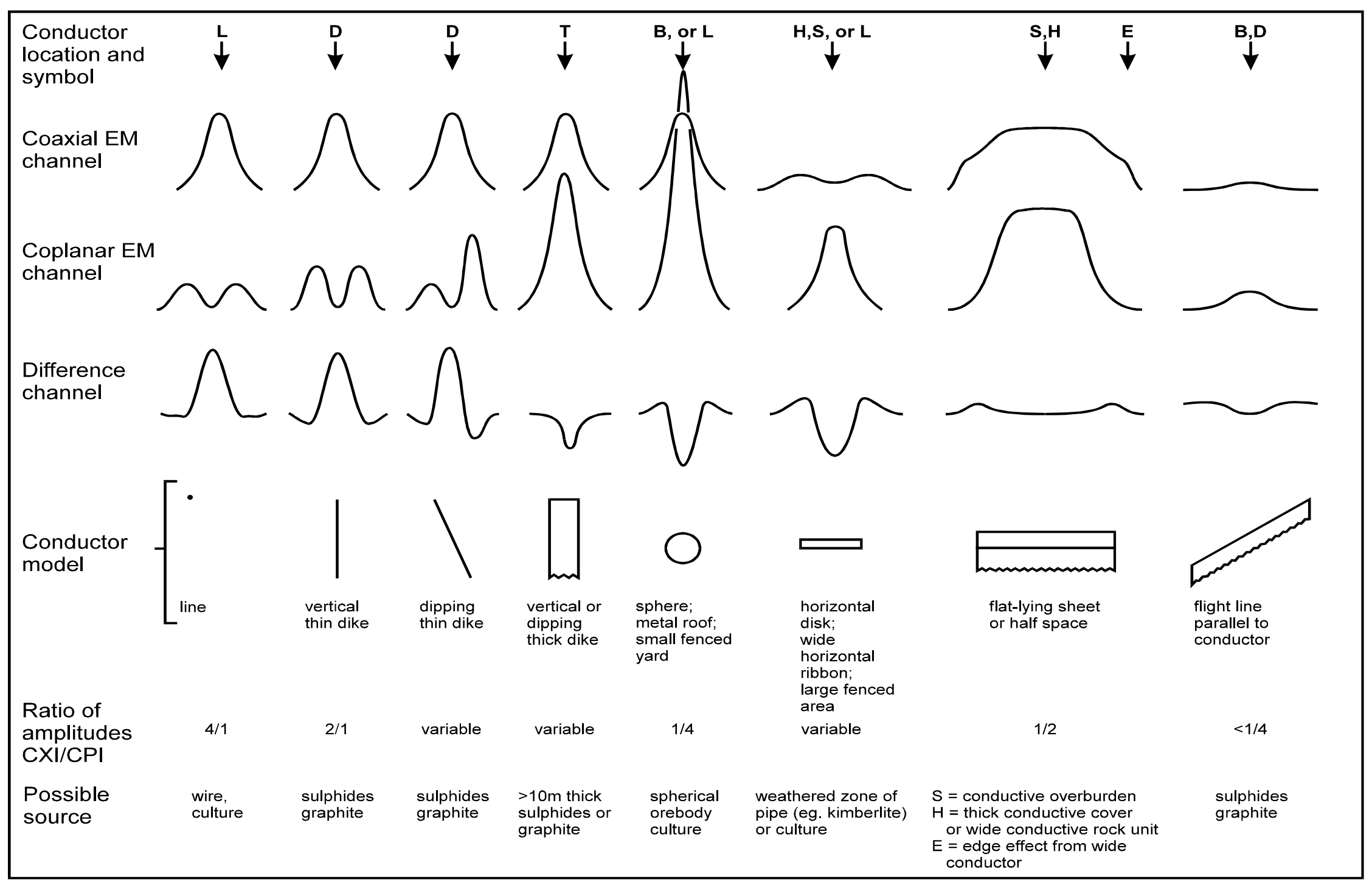

Typical DIGHEM anomaly shapes

Figure B-1 


\section{- Appendix B-5 -}

Dip symbols are used to indicate the direction of dip of conductors. These symbols are used only when the anomaly shapes are unambiguous, which usually requires a fairly resistive environment.

A further interpretation is presented on the EM map by means of the line-to-line correlation of bedrock anomalies, which is based on a comparison of anomaly shapes on adjacent lines. This provides conductor axes which may define the geological structure over portions of the survey area. The absence of conductor axes in an area implies that anomalies could not be correlated from line to line with reasonable confidence.

DIGHEM electromagnetic anomalies are designed to provide a correct impression of conductor quality by means of the conductance grade symbols. The symbols can stand alone with geology when planning a follow-up program. The actual conductance values are printed in the attached anomaly list for those who wish quantitative data. The anomaly ppm and depth are indicated by inconspicuous dots which should not distract from the conductor patterns, while being helpful to those who wish this information. The map provides an interpretation of conductors in terms of length, strike and dip, geometric shape, conductance, depth, and thickness. The accuracy is comparable to an interpretation from a high quality ground EM survey having the same line spacing.

The attached EM anomaly list provides a tabulation of anomalies in ppm, conductance, and depth for the vertical sheet model. The EM anomaly list also shows the conductance and depth for a thin horizontal sheet (whole plane) model, but only the vertical sheet parameters appear on the EM map. The horizontal sheet model is suitable for a flatly dipping thin bedrock conductor such as a sulphide sheet having a thickness less than $10 \mathrm{~m}$. The list also shows the resistivity and depth for a conductive earth (half space) model, which is suitable for thicker slabs such as thick conductive overburden. In the EM anomaly list, a depth value of zero for the conductive earth model, in an area of thick cover, warns that the anomaly may be caused by conductive overburden.

Since discrete bodies normally are the targets of EM surveys, local base (or zero) levels are used to compute local anomaly amplitudes. This contrasts with the use of true zero levels which are used to compute true EM amplitudes. Local anomaly amplitudes are shown in the EM anomaly list and these are used to compute the vertical sheet parameters of conductance and depth. Not shown in the EM anomaly list are the true amplitudes which are used to compute the horizontal sheet and conductive earth parameters.

\section{Questionable Anomalies}

DIGHEM maps may contain EM responses which are displayed as asterisks (*). These responses denote weak anomalies of indeterminate conductance, which may reflect one of the following: a weak conductor near the surface, a strong conductor at depth 


\section{- Appendix B-6 -}

(e.g., 100 to $120 \mathrm{~m}$ below surface) or to one side of the flight line, or aerodynamic noise. Those responses that have the appearance of valid bedrock anomalies on the flight profiles are indicated by appropriate interpretive symbols (see EM legend on maps). The others probably do not warrant further investigation unless their locations are of considerable geological interest.

\section{The Thickness Parameter}

DIGHEM can provide an indication of the thickness of a steeply dipping conductor. The amplitude of the coplanar anomaly (e.g., CPI channel on the digital profile) increases relative to the coaxial anomaly (e.g., CXI) as the apparent thickness increases, i.e., the thickness in the horizontal plane. (The thickness is equal to the conductor width if the conductor dips at 90 degrees and strikes at right angles to the flight line.) This report refers to a conductor as thin when the thickness is likely to be less than $3 \mathrm{~m}$, and thick when in excess of $10 \mathrm{~m}$. Thick conductors are indicated on the EM map by parentheses "( )". For base metal exploration in steeply dipping geology, thick conductors can be high priority targets because many massive sulphide ore bodies are thick, whereas non-economic bedrock conductors are often thin. The system cannot sense the thickness when the strike of the conductor is sub-parallel to the flight line, when the conductor has a shallow dip, when the anomaly amplitudes are small, or when the resistivity of the environment is below $100 \mathrm{ohm}-\mathrm{m}$.

\section{Resistivity Mapping}

Resistivity mapping is useful in areas where broad or flat-lying conductive units are of interest. One example of this is the clay alteration that is associated with Carlintype deposits in the southwest United States. The DIGHEM system was able to identify the clay alteration zone over the Cove deposit. The alteration zone appeared as a strong resistivity low on the $900 \mathrm{~Hz}$ resistivity parameter. The 7,200 Hz and 56,000 Hz resistivities show more of the detail in the covering sediments, and delineate a range front fault. This is typical in many areas of the southwest United States where conductive, nearsurface sediments, which may sometimes be alkalic, attenuate the higher frequencies.

Resistivity mapping has proven successful for locating diatremes in diamond exploration. Weathering products from relatively soft kimberlite pipes produce a resistivity contrast with the unaltered host rock. In many cases weathered kimberlite pipes were associated with thick conductive layers which contrasted with overlying or adjacent relatively thin layers of lake-bottom sediments or overburden.

Areas of widespread conductivity are commonly encountered during surveys. These conductive zones may reflect alteration zones, shallow-dipping sulphide or graphite-rich units or conductive overburden. In such areas, anomalies can be generated 


\section{- Appendix B-7 -}

by decreases of only $5 \mathrm{~m}$ in survey altitude as well as by increases in conductivity. The typical flight record in conductive areas is characterized by inphase and quadrature channels which are continuously active. Local EM peaks reflect either increases in conductivity of the earth or decreases in survey altitude. For such conductive areas, apparent resistivity profiles and contour maps are necessary for the correct interpretation of the airborne data. The advantage of the resistivity parameter is that anomalies caused by altitude changes are virtually eliminated, so the resistivity data reflect only those anomalies caused by conductivity changes. The resistivity analysis also helps the interpreter to differentiate between conductive bedrock and conductive overburden. For example, discrete conductors will generally appear as narrow lows on the contour map and broad conductors (e.g., overburden) will appear as wide lows.

The apparent resistivity is calculated using the pseudo-layer (or buried) half space model defined by Fraser (1978) ${ }^{1}$. This model consists of a resistive layer overlying a conductive half space. The depth channels give the apparent depth below surface of the conductive material. The apparent depth is simply the apparent thickness of the overlying resistive layer. The apparent depth (or thickness) parameter will be positive when the upper layer is more resistive than the underlying material, in which case the apparent depth may be quite close to the true depth.

The apparent depth will be negative when the upper layer is more conductive than the underlying material, and will be zero where a homogeneous half space exists. The apparent depth parameter must be interpreted cautiously because it will contain any error which may exist in the measured altitude of the EM bird (e.g., as caused by a dense tree cover). The inputs to the resistivity algorithm are the inphase and quadrature components of the coplanar coil-pair. The outputs are the apparent resistivity of the conductive half space (the source) and the sensor-source distance. The flying height is not an input variable, and the output resistivity and sensor-source distance are independent of the flying height when the conductivity of the measured material is sufficient to yield significant inphase as well as quadrature responses. The apparent depth, discussed above, is simply the sensor-source distance minus the measured altitude or flying height. Consequently, errors in the measured altitude will affect the apparent depth parameter but not the apparent resistivity parameter.

The apparent depth parameter is a useful indicator of simple layering in areas lacking a heavy tree cover. The DIGHEM system has been flown for purposes of permafrost mapping, where positive apparent depths were used as a measure of

1 Resistivity mapping with an airborne multi-coil electromagnetic system: Geophysics, v. 43, p.144-172 


\section{- Appendix B-8 -}

permafrost thickness. However, little quantitative use has been made of negative apparent depths because the absolute value of the negative depth is not a measure of the thickness of the conductive upper layer and, therefore, is not meaningful physically. Qualitatively, a negative apparent depth estimate usually shows that the EM anomaly is caused by conductive overburden. Consequently, the apparent depth channel can be of significant help in distinguishing between overburden and bedrock conductors.

\section{Interpretation in Conductive Environments}

Environments having low background resistivities (e.g., below $30 \mathrm{ohm}-\mathrm{m}$ for a $900 \mathrm{~Hz}$ system) yield very large responses from the conductive ground. This usually prohibits the recognition of discrete bedrock conductors. However, DIGHEM data processing techniques produce three parameters which contribute significantly to the recognition of bedrock conductors in conductive environments. These are the inphase and quadrature difference channels (DFI and DFQ, which are available only on systems with common frequencies on orthogonal coil pairs), and the resistivity and depth channels (RES and DP) for each coplanar frequency.

The EM difference channels (DFI and DFQ) eliminate most of the responses from conductive ground, leaving responses from bedrock conductors, cultural features (e.g., telephone lines, fences, etc.) and edge effects. Edge effects often occur near the perimeter of broad conductive zones. This can be a source of geologic noise. While edge effects yield anomalies on the EM difference channels, they do not produce resistivity anomalies. Consequently, the resistivity channel aids in eliminating anomalies due to edge effects. On the other hand, resistivity anomalies will coincide with the most highly conductive sections of conductive ground, and this is another source of geologic noise. The recognition of a bedrock conductor in a conductive environment therefore is based on the anomalous responses of the two difference channels (DFI and DFQ) and the resistivity channels (RES). The most favourable situation is where anomalies coincide on all channels.

The DP channels, which give the apparent depth to the conductive material, also help to determine whether a conductive response arises from surficial material or from a conductive zone in the bedrock. When these channels ride above the zero level on the digital profiles (i.e., depth is negative), it implies that the EM and resistivity profiles are responding primarily to a conductive upper layer, i.e., conductive overburden. If the DP channels are below the zero level, it indicates that a resistive upper layer exists, and this usually implies the existence of a bedrock conductor. The case where the low frequency DP channel is below the zero level and the high frequency DP channel is above zero suggests that a bedrock conductor lies beneath conductive cover. 


\section{- Appendix B-9 -}

\section{Reduction of Geologic Noise}

Geologic noise refers to unwanted geophysical responses. For purposes of airborne EM surveying, geologic noise refers to EM responses caused by conductive overburden and magnetic permeability. It was mentioned previously that the EM difference channels (i.e., channel DFI for inphase and DFQ for quadrature) tend to eliminate the response of conductive overburden.

Magnetite produces a form of geological noise on the inphase channels of all EM systems. Rocks containing even less than $1 \%$ magnetite can yield negative inphase anomalies caused by magnetic permeability. When magnetite is widely distributed throughout a survey area, the inphase EM channels may continuously rise and fall, reflecting variations in the magnetite percentage, flying height, and overburden thickness. This can lead to difficulties in recognizing deeply buried bedrock conductors, particularly if conductive overburden also exists. However, the response of broadly distributed magnetite generally vanishes on the inphase difference channel DFI. This feature can be a significant aid in the recognition of conductors which occur in rocks containing accessory magnetite.

\section{EM Magnetite Mapping}

The information content of DIGHEM data consists of a combination of conductive eddy current responses and magnetic permeability responses. The secondary field resulting from conductive eddy current flow is frequency-dependent and consists of both inphase and quadrature components, which are positive in sign. On the other hand, the secondary field resulting from magnetic permeability is independent of frequency and consists of only an inphase component which is negative in sign. When magnetic permeability manifests itself by decreasing the measured amount of positive inphase, its presence may be difficult to recognize. However, when it manifests itself by yielding a negative inphase anomaly (e.g., in the absence of eddy current flow), its presence is assured. In this latter case, the negative component can be used to estimate the percent magnetite content.

A magnetite mapping technique was developed for the coplanar coil-pair of DIGHEM. The method can be complementary to magnetometer mapping in certain cases. Compared to magnetometry, it is far less sensitive but is more able to resolve closely spaced magnetite zones, as well as providing an estimate of the amount of magnetite in the rock. The method is sensitive to $1 / 4 \%$ magnetite by weight when the EM sensor is at a height of $30 \mathrm{~m}$ above a magnetitic half space. It can individually resolve steep dipping narrow magnetite-rich bands which are separated by $60 \mathrm{~m}$. Unlike magnetometry, the EM magnetite method is unaffected by remanent magnetism or magnetic latitude. 
- Appendix B-10 -

The EM magnetite mapping technique provides estimates of magnetite content which are usually correct within a factor of 2 when the magnetite is fairly uniformly distributed. EM magnetite maps can be generated when magnetic permeability is evident as negative inphase responses on the data profiles.

Like magnetometry, the EM magnetite method maps only bedrock features, provided that the overburden is characterized by a general lack of magnetite. This contrasts with resistivity mapping which portrays the combined effect of bedrock and overburden.

\section{Recognition of Culture}

Cultural responses include all EM anomalies caused by man-made metallic objects. Such anomalies may be caused by inductive coupling or current gathering. The concern of the interpreter is to recognize when an EM response is due to culture. Points of consideration used by the interpreter, when coaxial and coplanar coil-pairs are operated at a common frequency, are as follows:

1. Channels CXP and CPP monitor $60 \mathrm{~Hz}$ radiation. An anomaly on these channels shows that the conductor is radiating power. Such an indication is normally a guarantee that the conductor is cultural. However, care must be taken to ensure that the conductor is not a geologic body which strikes across a power line, carrying leakage currents.

2. A flight which crosses a "line" (e.g., fence, telephone line, etc.) yields a centrepeaked coaxial anomaly and an m-shaped coplanar anomaly. ${ }^{2}$ When the flight crosses the cultural line at a high angle of intersection, the amplitude ratio of coaxial/coplanar response is 4 . Such an EM anomaly can only be caused by a line. The geologic body which yields anomalies most closely resembling a line is the vertically dipping thin dike. Such a body, however, yields an amplitude ratio of 2 rather than 4. Consequently, an m-shaped coplanar anomaly with a CXI/CPI amplitude ratio of 4 is virtually a guarantee that the source is a cultural line.

3. A flight which crosses a sphere or horizontal disk yields centre-peaked coaxial and coplanar anomalies with a CXI/CPI amplitude ratio (i.e., coaxial/coplanar) of 1/4. In the absence of geologic bodies of this geometry, the most likely conductor is a

2 See Figure B-1 presented earlier. 
metal roof or small fenced yard. ${ }^{3}$ Anomalies of this type are virtually certain to be cultural if they occur in an area of culture.

4. A flight which crosses a horizontal rectangular body or wide ribbon yields an mshaped coaxial anomaly and a centre-peaked coplanar anomaly. In the absence of geologic bodies of this geometry, the most likely conductor is a large fenced area. ${ }^{5}$ Anomalies of this type are virtually certain to be cultural if they occur in an area of culture.

5. EM anomalies which coincide with culture, as seen on the camera film or video display, are usually caused by culture. However, care is taken with such coincidences because a geologic conductor could occur beneath a fence, for example. In this example, the fence would be expected to yield an m-shaped coplanar anomaly as in case \#2 above. If, instead, a centre-peaked coplanar anomaly occurred, there would be concern that a thick geologic conductor coincided with the cultural line.

6. The above description of anomaly shapes is valid when the culture is not conductively coupled to the environment. In this case, the anomalies arise from inductive coupling to the EM transmitter. However, when the environment is quite conductive (e.g., less than $100 \mathrm{ohm}-\mathrm{m}$ at $900 \mathrm{~Hz}$ ), the cultural conductor may be conductively coupled to the environment. In this latter case, the anomaly shapes tend to be governed by current gathering. Current gathering can completely distort the anomaly shapes, thereby complicating the identification of cultural anomalies. In such circumstances, the interpreter can only rely on the radiation channels and on the camera film or video records.

\section{Magnetics}

Total field magnetics provides information on the magnetic properties of the earth materials in the survey area. The information can be used to locate magnetic bodies of direct interest for exploration, and for structural and lithological mapping.

The total field magnetic response reflects the abundance of magnetic material, in the source. Magnetite is the most common magnetic mineral. Other minerals such as

3 It is a characteristic of EM that geometrically similar anomalies are obtained from: (1) a planar conductor, and (2) a wire which forms a loop having dimensions identical to the perimeter of the equivalent planar conductor. 


\section{- Appendix B-12 -}

ilmenite, pyrrhotite, franklinite, chromite, hematite, arsenopyrite, limonite and pyrite are also magnetic, but to a lesser extent than magnetite on average.

In some geological environments, an EM anomaly with magnetic correlation has a greater likelihood of being produced by sulphides than one that is non-magnetic. However, sulphide ore bodies may be non-magnetic (e.g., the Kidd Creek deposit near Timmins, Canada) as well as magnetic (e.g., the Mattabi deposit near Sturgeon Lake, Canada).

Iron ore deposits will be anomalously magnetic in comparison to surrounding rock due to the concentration of iron minerals such as magnetite, ilmenite and hematite.

Changes in magnetic susceptibility often allow rock units to be differentiated based on the total field magnetic response. Geophysical classifications may differ from geological classifications if various magnetite levels exist within one general geological classification. Geometric considerations of the source such as shape, dip and depth, inclination of the earth's field and remanent magnetization will complicate such an analysis.

In general, mafic lithologies contain more magnetite and are therefore more magnetic than many sediments which tend to be weakly magnetic. Metamorphism and alteration can also increase or decrease the magnetization of a rock unit.

Textural differences on a total field magnetic contour, colour or shadow map due to the frequency of activity of the magnetic parameter resulting from inhomogeneities in the distribution of magnetite within the rock, may define certain lithologies. For example, near surface volcanics may display highly complex contour patterns with little line-to-line correlation.

Rock units may be differentiated based on the plan shapes of their total field magnetic responses. Mafic intrusive plugs can appear as isolated "bulls-eye" anomalies. Granitic intrusives appear as sub-circular zones, and may have contrasting rings due to contact metamorphism. Generally, granitic terrain will lack a pronounced strike direction, although granite gneiss may display strike.

Linear north-south units are theoretically not well-defined on total field magnetic maps in equatorial regions due to the low inclination of the earth's magnetic field. However, most stratigraphic units will have variations in composition along strike which will cause the units to appear as a series of alternating magnetic highs and lows.

Faults and shear zones may be characterized by alteration that causes destruction of magnetite (e.g., weathering) which produces a contrast with surrounding rock. 


\section{- Appendix B-13 -}

Structural breaks may be filled by magnetite-rich, fracture filling material as is the case with diabase dikes, or by non-magnetic felsic material.

Faulting can also be identified by patterns in the magnetic total field contours or colours. Faults and dikes tend to appear as lineaments and often have strike lengths of several kilometres. Offsets in narrow, magnetic, stratigraphic trends also delineate structure. Sharp contrasts in magnetic lithologies may arise due to large displacements along strike-slip or dip-slip faults. 


\section{APPENDIX C}

\section{EM ANOMALY LISTS}


EM Anomaly List

\begin{tabular}{|c|c|c|c|c|c|c|c|c|c|c|c|c|c|}
\hline Label & Fid & Interp & $\underset{\mathrm{m}}{\mathrm{XUTM}}$ & $\begin{array}{c}\text { YUTM } \\
\mathrm{m}\end{array}$ & $\begin{array}{l}\text { CX } \\
\text { Real } \\
\text { ppm }\end{array}$ & $\begin{array}{l}900 \mathrm{HZ} \\
\text { Quad } \\
\text { ppm }\end{array}$ & $\begin{array}{l}\text { CP } \\
\text { Real } \\
\text { ppm }\end{array}$ & $\begin{array}{l}900 \mathrm{HZ} \\
\text { Quad } \\
\text { ppm }\end{array}$ & $\begin{array}{l}\text { CP } \\
\text { Real } \\
\text { ppm }\end{array}$ & $\begin{array}{l}200 \mathrm{HZ} \\
\text { Quad } \\
\text { ppm }\end{array}$ & $\begin{array}{l}\text { Vertica } \\
\text { COND } \\
\text { siemens }\end{array}$ & $\begin{array}{c}\text { al Dike } \\
\text { DEPTH* } \\
\text { m }\end{array}$ & $\begin{array}{c}\text { Mag. Corr } \\
\text { NT }\end{array}$ \\
\hline $\begin{array}{l}\text { LINE } \\
\text { E } \\
\text { D } \\
\text { C } \\
\text { B } \\
\text { A }\end{array}$ & $\begin{array}{l}10010 \\
21441.0 \\
21461.0 \\
21501.0 \\
21541.0 \\
21841.0\end{array}$ & $\begin{array}{r}\text { S } \\
\text { B? } \\
\text { B? } \\
\text { S? } \\
\text { S }\end{array}$ & $\begin{array}{l}684843 \\
684871 \\
684901 \\
684896 \\
685004\end{array}$ & $\begin{array}{l}6121299 \\
6120735 \\
6119635 \\
6118853 \\
6114308\end{array}$ & $\begin{array}{r}10.0 \\
1.0 \\
3.0 \\
1.0 \\
0.0\end{array}$ & $\begin{array}{l}7.0 \\
4.0 \\
4.0 \\
2.0 \\
2.0\end{array}$ & $\begin{array}{r}19.0 \\
1.0 \\
4.0 \\
0.0 \\
1.0\end{array}$ & $\begin{array}{r}23.0 \\
2.0 \\
6.0 \\
1.0 \\
5.0\end{array}$ & $\begin{array}{r}10.0 \\
5.0 \\
17.0 \\
2.0 \\
8.0\end{array}$ & $\begin{array}{r}4.0 \\
4.0 \\
10.0 \\
4.0 \\
3.0\end{array}$ & $\begin{array}{r}10.2 \\
0.9 \\
5.1 \\
--- \\
0.8\end{array}$ & $\begin{array}{r}0 \\
1 \\
27 \\
--- \\
0\end{array}$ & $\begin{array}{r}28 \\
0 \\
0 \\
21 \\
0\end{array}$ \\
\hline $\begin{array}{l}\text { LINE } \\
\text { A } \\
\text { B } \\
\text { C }\end{array}$ & $\begin{array}{l}10021 \\
12676.0 \\
12825.0 \\
12967.0\end{array}$ & $\begin{array}{r}\mathrm{B} ? \\
\mathrm{~S} ? \\
\mathrm{~S}\end{array}$ & $\begin{array}{l}685212 \\
685207 \\
685086\end{array}$ & $\begin{array}{l}6113029 \\
6114570 \\
6116934\end{array}$ & $\begin{array}{l}0.0 \\
1.0 \\
1.0\end{array}$ & $\begin{array}{l}3.0 \\
2.0 \\
2.0\end{array}$ & $\begin{array}{l}0.0 \\
1.0 \\
1.0\end{array}$ & $\begin{array}{l}4.0 \\
7.0 \\
2.0\end{array}$ & $\begin{array}{r}0.0 \\
26.0 \\
2.0\end{array}$ & $\begin{array}{r}7.0 \\
12.0 \\
4.0\end{array}$ & $\begin{array}{l}0.5 \\
1.1 \\
---\end{array}$ & $\begin{array}{r}2 \\
0 \\
---\end{array}$ & $\begin{array}{r}19 \\
0 \\
25\end{array}$ \\
\hline $\begin{array}{l}\text { LINE } \\
\text { A } \\
\text { B } \\
\text { C } \\
\text { D }\end{array}$ & $\begin{array}{l}10022 \\
21182.0 \\
21209.0 \\
21261.0 \\
21293.0\end{array}$ & $\begin{array}{r}\text { S? } \\
\mathrm{B} ? \\
\mathrm{~B} ? \\
\mathrm{E}\end{array}$ & $\begin{array}{l}685060 \\
685031 \\
685014 \\
685014\end{array}$ & $\begin{array}{l}6118931 \\
6119546 \\
6120675 \\
6121342\end{array}$ & $\begin{array}{l}1.0 \\
1.0 \\
0.0 \\
8.0\end{array}$ & $\begin{array}{l}2.0 \\
2.0 \\
2.0 \\
7.0\end{array}$ & $\begin{array}{l}1.0 \\
1.0 \\
0.0 \\
7.0\end{array}$ & $\begin{array}{r}2.0 \\
2.0 \\
2.0 \\
13.0\end{array}$ & $\begin{array}{l}2.0 \\
2.0 \\
2.0 \\
5.0\end{array}$ & $\begin{array}{r}4.0 \\
4.0 \\
4.0 \\
16.0\end{array}$ & $\begin{array}{l}--- \\
--- \\
--- \\
7.4\end{array}$ & $\begin{array}{r}--- \\
---- \\
--- \\
7\end{array}$ & $\begin{array}{l}0 \\
0 \\
0 \\
0\end{array}$ \\
\hline $\begin{array}{l}\text { LINE } \\
\text { E } \\
D \\
\text { C } \\
B \\
A\end{array}$ & $\begin{array}{l}10030 \\
11280.0 \\
11287.0 \\
11359.0 \\
11363.0 \\
11383.0\end{array}$ & $\begin{array}{r}\text { S } \\
\text { E } \\
\text { B? } \\
\text { S? } \\
\text { S }\end{array}$ & $\begin{array}{l}685210 \\
685207 \\
685196 \\
685202 \\
685259\end{array}$ & $\begin{array}{l}6121492 \\
6121327 \\
6119583 \\
6119472 \\
6118938\end{array}$ & $\begin{array}{r}13.0 \\
8.0 \\
1.0 \\
1.0 \\
1.0\end{array}$ & $\begin{array}{l}9.0 \\
9.0 \\
2.0 \\
2.0 \\
2.0\end{array}$ & $\begin{array}{r}14.0 \\
23.0 \\
1.0 \\
1.0 \\
1.0\end{array}$ & $\begin{array}{r}34.0 \\
20.0 \\
2.0 \\
2.0 \\
3.0\end{array}$ & $\begin{array}{r}21.0 \\
12.0 \\
2.0 \\
2.0 \\
5.0\end{array}$ & $\begin{array}{r}12.0 \\
4.0 \\
4.0 \\
4.0 \\
15.0\end{array}$ & $\begin{array}{r}7.8 \\
10.4 \\
--- \\
--- \\
0.2\end{array}$ & $\begin{array}{r}0 \\
6 \\
--- \\
--- \\
0\end{array}$ & $\begin{array}{r}21 \\
22 \\
135 \\
0 \\
0\end{array}$ \\
\hline $\begin{array}{l}\text { LINE } \\
\text { M } \\
\text { L } \\
\text { K } \\
\text { J } \\
\text { I } \\
\text { H } \\
\text { G } \\
\text { F } \\
\text { E } \\
\text { D } \\
\text { C } \\
\text { B } \\
\text { A }\end{array}$ & $\begin{array}{r}10040 \\
2317.0 \\
2322.0 \\
2406.0 \\
2411.0 \\
2444.0 \\
2448.0 \\
2452.0 \\
2509.0 \\
2521.0 \\
2547.0 \\
2570.0 \\
2707.0 \\
2854.0\end{array}$ & $\begin{array}{r}\text { S } \\
\text { E } \\
\text { B? } \\
\text { S? } \\
\text { B? } \\
\text { S? } \\
\text { S? } \\
\text { B? } \\
\text { S? } \\
\text { S } \\
\text { S? } \\
\text { S } \\
\text { B? }\end{array}$ & $\begin{array}{l}685433 \\
685446 \\
685535 \\
685532 \\
685540 \\
685542 \\
685546 \\
685473 \\
685457 \\
685481 \\
685498 \\
685592 \\
685659\end{array}$ & $\begin{array}{l}6121575 \\
6121444 \\
6119600 \\
6119494 \\
6118640 \\
6118530 \\
6118422 \\
6117199 \\
6117005 \\
6116609 \\
6116244 \\
6114324 \\
6113008\end{array}$ & $\begin{array}{r}24.0 \\
35.0 \\
1.0 \\
2.0 \\
2.0 \\
1.0 \\
0.0 \\
0.0 \\
0.0 \\
0.0 \\
0.0 \\
0.0 \\
0.0\end{array}$ & $\begin{array}{r}32.0 \\
32.0 \\
2.0 \\
4.0 \\
9.0 \\
6.0 \\
2.0 \\
1.0 \\
2.0 \\
1.0 \\
2.0 \\
1.0 \\
3.0\end{array}$ & $\begin{array}{r}140.0 \\
104.0 \\
1.0 \\
1.0 \\
1.0 \\
1.0 \\
0.0 \\
0.0 \\
0.0 \\
0.0 \\
0.0 \\
1.0 \\
0.0\end{array}$ & $\begin{array}{r}78.0 \\
84.0 \\
2.0 \\
5.0 \\
10.0 \\
10.0 \\
2.0 \\
1.0 \\
2.0 \\
1.0 \\
2.0 \\
4.0 \\
0.0\end{array}$ & $\begin{array}{r}263.0 \\
15.0 \\
2.0 \\
16.0 \\
52.0 \\
48.0 \\
2.0 \\
0.0 \\
2.0 \\
1.0 \\
0.0 \\
5.0 \\
0.0\end{array}$ & $\begin{array}{r}45.0 \\
21.0 \\
4.0 \\
18.0 \\
81.0 \\
56.0 \\
4.0 \\
4.0 \\
4.0 \\
4.0 \\
4.0 \\
7.0 \\
18.0\end{array}$ & $\begin{array}{r}24.2 \\
20.2 \\
--- \\
1.6 \\
0.8 \\
0.5 \\
--- \\
--- \\
--- \\
--- \\
--- \\
0.5 \\
5.6\end{array}$ & $\begin{array}{r}0 \\
0 \\
--- \\
13 \\
1 \\
0 \\
--- \\
--- \\
--- \\
--- \\
--- \\
0 \\
70\end{array}$ & $\begin{array}{r}28 \\
22 \\
179 \\
0 \\
166 \\
77 \\
176 \\
13 \\
0 \\
22 \\
11 \\
0 \\
160\end{array}$ \\
\hline $\begin{array}{l}\text { LINE } \\
\text { A } \\
B \\
\text { C } \\
\text { D } \\
\text { E }\end{array}$ & $\begin{array}{l}10050 \\
1682.0 \\
1707.0 \\
1825.0 \\
1891.0 \\
1898.0\end{array}$ & $\begin{array}{r}S ? \\
\text { S? } \\
S \\
S ? \\
S\end{array}$ & $\begin{array}{l}685990 \\
686000 \\
685824 \\
685760 \\
685755\end{array}$ & $\begin{array}{l}6112846 \\
6112939 \\
6114421 \\
6115782 \\
6115914\end{array}$ & $\begin{array}{l}0.0 \\
0.0 \\
0.0 \\
0.0 \\
0.0\end{array}$ & $\begin{array}{l}1.0 \\
1.0 \\
3.0 \\
2.0 \\
2.0\end{array}$ & $\begin{array}{l}0.0 \\
0.0 \\
1.0 \\
0.0 \\
0.0\end{array}$ & $\begin{array}{l}1.0 \\
2.0 \\
6.0 \\
2.0 \\
2.0\end{array}$ & $\begin{array}{l}0.0 \\
0.0 \\
3.0 \\
2.0 \\
2.0\end{array}$ & $\begin{array}{l}4.0 \\
4.0 \\
7.0 \\
4.0 \\
4.0\end{array}$ & $\begin{array}{l}--- \\
--- \\
0.7 \\
--- \\
---\end{array}$ & $\begin{array}{r}--- \\
--- \\
0 \\
--- \\
---\end{array}$ & $\begin{array}{r}26 \\
0 \\
0 \\
5 \\
26\end{array}$ \\
\hline
\end{tabular}

$\mathrm{CX}=$ COAXIAL

$\mathrm{CP}=$ COPLANAR

Note: EM values shown above

are local amplitudes

*Estimated Depth may be unreliable because the stronger part of the conductor may be deeper or to one side of the flight line, or because of a shallow dip or magnetite/overburden effects 
EM Anomaly List

\begin{tabular}{|c|c|c|c|c|c|c|c|c|c|c|c|c|c|}
\hline Label & Fid & Interp & $\underset{\mathrm{m}}{\mathrm{XUTM}}$ & $\begin{array}{c}\text { YUTM } \\
\text { m }\end{array}$ & $\begin{array}{l}\text { CX } \\
\text { Real } \\
\text { ppm }\end{array}$ & $\begin{array}{l}900 \mathrm{HZ} \\
\text { Quad } \\
\text { ppm }\end{array}$ & $\begin{array}{l}\text { CP } \\
\text { Real } \\
\text { ppm }\end{array}$ & $\begin{array}{c}900 \mathrm{HZ} \\
\text { Quad } \\
\text { ppm }\end{array}$ & $\begin{array}{l}\text { CP }{ }^{7} \\
\text { Real } \\
\text { ppm }\end{array}$ & $\begin{array}{c}200 \mathrm{HZ} \\
\text { Quad } \\
\text { ppm }\end{array}$ & $\begin{array}{l}\text { Vertica } \\
\text { CoND } \\
\text { siemens }\end{array}$ & $\begin{array}{c}\text { al Dike } \\
\text { DEPTH* } \\
\text { m }\end{array}$ & $\begin{array}{c}\text { Mag. Corr } \\
\text { NT }\end{array}$ \\
\hline $\begin{array}{l}\text { LINE } \\
\text { F } \\
\text { G } \\
\text { H } \\
\text { I } \\
\text { J } \\
\text { K } \\
\text { L } \\
\text { M } \\
\text { N } \\
\text { O }\end{array}$ & $\begin{array}{r}10050 \\
1952.0 \\
2010.0 \\
2018.0 \\
2040.0 \\
2097.0 \\
2119.0 \\
2132.0 \\
2137.0 \\
2173.0 \\
2175.0\end{array}$ & $\begin{array}{r}\text { B? } \\
\text { S } \\
\text { S? } \\
\text { S } \\
\text { D } \\
\text { S? } \\
\text { E } \\
\text { S } \\
\text { E } \\
\text { S }\end{array}$ & $\begin{array}{l}685732 \\
685694 \\
685707 \\
685671 \\
685557 \\
685548 \\
685519 \\
685506 \\
685503 \\
685508\end{array}$ & $\begin{array}{l}6116947 \\
6118616 \\
6118855 \\
6119542 \\
6121424 \\
6122087 \\
6122469 \\
6122623 \\
6123491 \\
6123547\end{array}$ & $\begin{array}{r}0.0 \\
0.0 \\
0.0 \\
1.0 \\
7.0 \\
1.0 \\
16.0 \\
10.0 \\
10.0 \\
11.0\end{array}$ & $\begin{array}{l}2.0 \\
1.0 \\
2.0 \\
2.0 \\
6.0 \\
2.0 \\
5.0 \\
6.0 \\
1.0 \\
2.0\end{array}$ & $\begin{array}{l}1.0 \\
1.0 \\
1.0 \\
1.0 \\
7.0 \\
1.0 \\
6.0 \\
4.0 \\
3.0 \\
3.0\end{array}$ & $\begin{array}{r}2.0 \\
2.0 \\
2.0 \\
2.0 \\
11.0 \\
2.0 \\
13.0 \\
17.0 \\
4.0 \\
4.0\end{array}$ & $\begin{array}{r}2.0 \\
2.0 \\
2.0 \\
2.0 \\
30.0 \\
2.0 \\
4.0 \\
10.0 \\
30.0 \\
30.0\end{array}$ & $\begin{array}{r}4.0 \\
4.0 \\
4.0 \\
4.0 \\
11.0 \\
4.0 \\
8.0 \\
7.0 \\
3.0 \\
3.0\end{array}$ & $\begin{array}{r}--- \\
--- \\
--- \\
--- \\
7.5 \\
---- \\
19.6 \\
6.4 \\
37.1 \\
1.0\end{array}$ & $\begin{array}{r}--- \\
--- \\
--- \\
--- \\
15 \\
--- \\
0 \\
0 \\
0 \\
0\end{array}$ & $\begin{array}{r}26 \\
0 \\
0 \\
0 \\
26 \\
0 \\
0 \\
0 \\
0 \\
0\end{array}$ \\
\hline $\begin{array}{l}\text { LINE } \\
\text { N } \\
\text { M } \\
\text { L } \\
\text { K } \\
\text { J } \\
\text { I } \\
\text { H } \\
\text { G } \\
\text { F } \\
\text { E } \\
\text { D } \\
\text { C } \\
\text { B } \\
\text { A }\end{array}$ & $\begin{array}{c}10060 \\
929.0 \\
935.0 \\
968.0 \\
1023.0 \\
1046.0 \\
1082.0 \\
1090.0 \\
1096.0 \\
1121.0 \\
1176.0 \\
1227.0 \\
1305.0 \\
1330.0 \\
1351.0\end{array}$ & $\begin{array}{r}\text { S } \\
\text { E } \\
\text { S? } \\
\text { B? } \\
\text { B? } \\
\text { B? } \\
\text { D } \\
\text { B? } \\
\text { B? } \\
\text { S } \\
\text { S? } \\
\text { S } \\
\text { S } \\
\text { S? }\end{array}$ & $\begin{array}{l}685712 \\
685714 \\
685722 \\
685872 \\
685904 \\
685996 \\
685995 \\
685982 \\
685935 \\
686008 \\
686018 \\
686059 \\
686119 \\
686156\end{array}$ & $\begin{array}{l}6123471 \\
6123313 \\
6122459 \\
6121156 \\
6120689 \\
6119659 \\
6119427 \\
6119246 \\
6118501 \\
6116907 \\
6115817 \\
6114244 \\
6113818 \\
6113570\end{array}$ & $\begin{array}{l}1.0 \\
1.0 \\
5.0 \\
1.0 \\
1.0 \\
1.0 \\
6.0 \\
4.0 \\
1.0 \\
0.0 \\
0.0 \\
1.0 \\
0.0 \\
0.0\end{array}$ & $\begin{array}{l}0.0 \\
0.0 \\
4.0 \\
2.0 \\
2.0 \\
2.0 \\
9.0 \\
4.0 \\
6.0 \\
1.0 \\
2.0 \\
3.0 \\
1.0 \\
1.0\end{array}$ & $\begin{array}{r}1.0 \\
1.0 \\
18.0 \\
0.0 \\
0.0 \\
1.0 \\
5.0 \\
5.0 \\
0.0 \\
0.0 \\
0.0 \\
1.0 \\
0.0 \\
0.0\end{array}$ & $\begin{array}{r}1.0 \\
1.0 \\
12.0 \\
2.0 \\
2.0 \\
2.0 \\
9.0 \\
10.0 \\
6.0 \\
1.0 \\
2.0 \\
6.0 \\
1.0 \\
1.0\end{array}$ & $\begin{array}{r}2.0 \\
2.0 \\
33.0 \\
2.0 \\
2.0 \\
2.0 \\
17.0 \\
28.0 \\
19.0 \\
0.0 \\
1.0 \\
16.0 \\
0.0 \\
0.0\end{array}$ & $\begin{array}{r}1.0 \\
4.0 \\
8.0 \\
4.0 \\
4.0 \\
4.0 \\
18.0 \\
13.0 \\
25.0 \\
4.0 \\
4.0 \\
19.0 \\
4.0 \\
4.0\end{array}$ & $\begin{array}{r}--- \\
--- \\
13.3 \\
---- \\
--- \\
--- \\
4.7 \\
4.5 \\
0.6 \\
--- \\
--- \\
0.9 \\
--- \\
---\end{array}$ & $\begin{array}{r}--- \\
--- \\
0 \\
--- \\
--- \\
--- \\
16 \\
29 \\
6 \\
--- \\
--- \\
15 \\
--- \\
---\end{array}$ & $\begin{array}{r}0 \\
25 \\
0 \\
0 \\
0 \\
208 \\
0 \\
0 \\
30 \\
22 \\
0 \\
22 \\
63 \\
45\end{array}$ \\
\hline $\begin{array}{l}\text { LINE } \\
\text { A } \\
\text { B } \\
\text { C } \\
\text { D } \\
\text { E } \\
\text { F } \\
\text { G } \\
\text { H } \\
\text { I } \\
\text { J } \\
\text { K }\end{array}$ & $\begin{array}{r}10070 \\
494.0 \\
511.0 \\
571.0 \\
598.0 \\
655.0 \\
669.0 \\
832.0 \\
844.0 \\
850.0 \\
858.0 \\
921.0\end{array}$ & $\begin{array}{r}\text { S } \\
\text { S? } \\
\text { S } \\
\text { S } \\
\text { B? } \\
\text { B? } \\
\text { S? } \\
\text { B? } \\
\text { D } \\
\text { B? } \\
\text { B? }\end{array}$ & $\begin{array}{l}686319 \\
686317 \\
686285 \\
686263 \\
686235 \\
686229 \\
686078 \\
686076 \\
686071 \\
686064 \\
686007\end{array}$ & $\begin{array}{l}6112629 \\
6112827 \\
6113702 \\
6114171 \\
6115148 \\
6115364 \\
6118836 \\
6119219 \\
6119394 \\
6119621 \\
6121488\end{array}$ & $\begin{array}{l}0.0 \\
0.0 \\
0.0 \\
0.0 \\
0.0 \\
0.0 \\
0.0 \\
4.0 \\
8.0 \\
1.0 \\
2.0\end{array}$ & $\begin{array}{r}1.0 \\
1.0 \\
1.0 \\
3.0 \\
2.0 \\
1.0 \\
2.0 \\
6.0 \\
12.0 \\
2.0 \\
3.0\end{array}$ & $\begin{array}{r}0.0 \\
0.0 \\
0.0 \\
1.0 \\
0.0 \\
0.0 \\
1.0 \\
6.0 \\
10.0 \\
1.0 \\
2.0\end{array}$ & $\begin{array}{r}1.0 \\
1.0 \\
2.0 \\
4.0 \\
2.0 \\
2.0 \\
2.0 \\
13.0 \\
17.0 \\
2.0 \\
5.0\end{array}$ & $\begin{array}{r}0.0 \\
0.0 \\
1.0 \\
8.0 \\
0.0 \\
0.0 \\
2.0 \\
2.0 \\
44.0 \\
2.0 \\
2.0\end{array}$ & $\begin{array}{r}4.0 \\
3.0 \\
4.0 \\
8.0 \\
4.0 \\
4.0 \\
4.0 \\
17.0 \\
25.0 \\
4.0 \\
11.0\end{array}$ & $\begin{array}{l}--- \\
0.1 \\
--- \\
0.7 \\
--- \\
--- \\
--- \\
3.4 \\
5.4 \\
--- \\
2.9\end{array}$ & $\begin{array}{r}--- \\
0 \\
--- \\
3 \\
--- \\
--- \\
--- \\
28 \\
13 \\
--- \\
10\end{array}$ & $\begin{array}{r}0 \\
197 \\
72 \\
0 \\
0 \\
0 \\
22 \\
0 \\
22 \\
171 \\
0\end{array}$ \\
\hline $\begin{array}{l}\text { LINE } \\
B \\
A\end{array}$ & $\begin{array}{l}10080 \\
3934.0 \\
3938.0\end{array}$ & $\begin{array}{l}\mathrm{D} \\
\mathrm{D}\end{array}$ & $\begin{array}{l}686098 \\
686117\end{array}$ & $\begin{array}{l}6121487 \\
6121382\end{array}$ & $\begin{array}{r}13.0 \\
4.0\end{array}$ & $\begin{array}{l}12.0 \\
13.0\end{array}$ & $\begin{array}{l}10.0 \\
10.0\end{array}$ & $\begin{array}{l}15.0 \\
15.0\end{array}$ & $\begin{array}{l}52.0 \\
52.0\end{array}$ & $\begin{array}{l}37.0 \\
37.0\end{array}$ & $\begin{array}{l}9.0 \\
3.6\end{array}$ & $\begin{array}{l}3 \\
9\end{array}$ & $\begin{array}{l}0 \\
0\end{array}$ \\
\hline $\begin{array}{l}\text { LINE } \\
\text { F }\end{array}$ & $\begin{array}{r}10081 \\
2532.0\end{array}$ & $\mathrm{~S}$ & 686199 & 6121100 & 3.0 & 3.0 & 1.0 & 3.0 & 12.0 & 2.0 & 1.0 & 0 & 21 \\
\hline
\end{tabular}

$\mathrm{CX}=$ COAXIAL

$\mathrm{CP}=$ COPLANAR

Note: EM values shown above

are local amplitudes

*Estimated Depth may be unreliable because the stronger part of the conductor may be deeper or to one side of the flight line, or because of a shallow dip or magnetite/overburden effects 
EM Anomaly List

\begin{tabular}{|c|c|c|c|c|c|c|c|c|c|c|c|c|c|}
\hline Label & Fid & Interp & $\begin{array}{c}\text { XUTM } \\
\text { m }\end{array}$ & $\begin{array}{c}\text { YUTM } \\
\mathrm{m}\end{array}$ & $\begin{array}{l}\text { CX } \\
\text { Real } \\
\text { ppm }\end{array}$ & $\begin{array}{l}00 \mathrm{HZ} \\
\text { Quad } \\
\text { ppm }\end{array}$ & $\begin{array}{l}\text { CP } \\
\text { Real } \\
\text { ppm }\end{array}$ & $\begin{array}{l}900 \mathrm{HZ} \\
\text { Quad } \\
\text { ppm }\end{array}$ & $\begin{array}{l}\mathrm{CP} \\
\mathrm{Real} \\
\mathrm{ppm}\end{array}$ & $\begin{array}{l}200 \mathrm{HZ} \\
\text { Quad } \\
\text { ppm }\end{array}$ & $\begin{array}{l}\text { Vertica } \\
\text { Cond } \\
\text { siemens }\end{array}$ & $\begin{array}{c}\text { al Dike } \\
\text { DEPTH* } \\
\text { m }\end{array}$ & $\begin{array}{c}\text { Mag. Corr } \\
\text { NT }\end{array}$ \\
\hline $\begin{array}{l}\text { LINE } \\
\text { E } \\
\text { D } \\
\text { C } \\
B \\
\text { A }\end{array}$ & $\begin{array}{r}10081 \\
2555.0 \\
2600.0 \\
2645.0 \\
2700.0 \\
2885.0\end{array}$ & $\begin{array}{r}\text { S } \\
\text { S } \\
\text { B? } \\
\text { S? } \\
\text { S }\end{array}$ & $\begin{array}{l}686227 \\
686283 \\
686255 \\
686357 \\
686487\end{array}$ & $\begin{array}{l}6120642 \\
6119572 \\
6118462 \\
6117150 \\
6114148\end{array}$ & $\begin{array}{l}0.0 \\
1.0 \\
0.0 \\
0.0 \\
0.0\end{array}$ & $\begin{array}{l}1.0 \\
2.0 \\
7.0 \\
2.0 \\
2.0\end{array}$ & $\begin{array}{l}0.0 \\
0.0 \\
0.0 \\
0.0 \\
0.0\end{array}$ & $\begin{array}{r}0.0 \\
2.0 \\
13.0 \\
1.0 \\
2.0\end{array}$ & $\begin{array}{r}0.0 \\
1.0 \\
45.0 \\
2.0 \\
2.0\end{array}$ & $\begin{array}{r}4.0 \\
4.0 \\
93.0 \\
4.0 \\
4.0\end{array}$ & $\begin{array}{l}--- \\
--- \\
0.5 \\
--- \\
---\end{array}$ & $\begin{array}{r}--- \\
--- \\
4 \\
--- \\
---\end{array}$ & $\begin{array}{r}21 \\
0 \\
129 \\
43 \\
0\end{array}$ \\
\hline $\begin{array}{l}\text { LINE } \\
\text { A } \\
\text { B } \\
\text { C } \\
\text { D } \\
\text { E } \\
\text { F } \\
\text { G } \\
\text { H } \\
\text { I } \\
\text { J } \\
\text { K }\end{array}$ & $\begin{array}{r}10090 \\
3127.0 \\
3165.0 \\
3239.0 \\
3302.0 \\
3441.0 \\
3455.0 \\
3463.0 \\
3489.0 \\
3542.0 \\
3657.0 \\
3662.0\end{array}$ & $\begin{array}{r}\text { S? } \\
\text { B? } \\
\text { S } \\
\text { S } \\
\text { S } \\
\text { B? } \\
\text { B? } \\
\text { B? } \\
\text { D } \\
\text { E } \\
\text { E }\end{array}$ & $\begin{array}{l}686569 \\
686583 \\
686622 \\
686640 \\
686551 \\
686549 \\
686549 \\
686550 \\
686445 \\
686293 \\
686287\end{array}$ & $\begin{array}{l}6112368 \\
6112915 \\
6114044 \\
6115086 \\
6118628 \\
6119081 \\
6119312 \\
6119969 \\
6121339 \\
6124369 \\
6124522\end{array}$ & $\begin{array}{r}0.0 \\
0.0 \\
0.0 \\
1.0 \\
0.0 \\
4.0 \\
3.0 \\
0.0 \\
22.0 \\
10.0 \\
57.0\end{array}$ & $\begin{array}{r}1.0 \\
0.0 \\
3.0 \\
1.0 \\
3.0 \\
5.0 \\
5.0 \\
2.0 \\
20.0 \\
39.0 \\
42.0\end{array}$ & $\begin{array}{r}0.0 \\
0.0 \\
0.0 \\
0.0 \\
1.0 \\
4.0 \\
2.0 \\
0.0 \\
12.0 \\
129.0 \\
129.0\end{array}$ & $\begin{array}{r}1.0 \\
1.0 \\
6.0 \\
1.0 \\
4.0 \\
7.0 \\
6.0 \\
2.0 \\
17.0 \\
116.0 \\
117.0\end{array}$ & $\begin{array}{r}0.0 \\
0.0 \\
3.0 \\
1.0 \\
18.0 \\
19.0 \\
17.0 \\
2.0 \\
57.0 \\
248.0 \\
256.0\end{array}$ & $\begin{array}{r}2.0 \\
4.0 \\
7.0 \\
2.0 \\
22.0 \\
13.0 \\
13.0 \\
4.0 \\
62.0 \\
11.0 \\
70.0\end{array}$ & $\begin{array}{c}0.1 \\
--- \\
0.5 \\
--- \\
0.9 \\
4.2 \\
3.0 \\
--- \\
10.7 \\
12.0 \\
23.3\end{array}$ & $\begin{array}{r}0 \\
--- \\
0 \\
--- \\
0 \\
29 \\
26 \\
--- \\
0 \\
0 \\
0\end{array}$ & $\begin{array}{r}287 \\
0 \\
0 \\
29 \\
36 \\
0 \\
25 \\
210 \\
0 \\
29 \\
5\end{array}$ \\
\hline $\begin{array}{l}\text { LINE } \\
\text { H } \\
\text { G } \\
F \\
\text { E } \\
\text { D } \\
\text { C } \\
B \\
\text { A }\end{array}$ & $\begin{array}{r}10100 \\
2548.0 \\
2556.0 \\
2625.0 \\
2634.0 \\
2648.0 \\
2714.0 \\
2854.0 \\
2892.0\end{array}$ & $\begin{array}{r}\text { D } \\
\text { S? } \\
\text { D } \\
\text { B? } \\
\text { S? } \\
\text { S? } \\
\text { S? } \\
\text { S? }\end{array}$ & $\begin{array}{l}686611 \\
686624 \\
686740 \\
686757 \\
686787 \\
686833 \\
686863 \\
686878\end{array}$ & $\begin{array}{l}6121262 \\
6121012 \\
6119228 \\
6118960 \\
6118552 \\
6116862 \\
6114362 \\
6113674\end{array}$ & $\begin{array}{r}11.0 \\
5.0 \\
4.0 \\
1.0 \\
1.0 \\
1.0 \\
0.0 \\
0.0\end{array}$ & $\begin{array}{l}8.0 \\
4.0 \\
4.0 \\
2.0 \\
3.0 \\
1.0 \\
2.0 \\
1.0\end{array}$ & $\begin{array}{l}5.0 \\
5.0 \\
2.0 \\
1.0 \\
1.0 \\
0.0 \\
0.0 \\
0.0\end{array}$ & $\begin{array}{l}6.0 \\
5.0 \\
4.0 \\
2.0 \\
4.0 \\
1.0 \\
2.0 \\
2.0\end{array}$ & $\begin{array}{r}27.0 \\
11.0 \\
16.0 \\
2.0 \\
22.0 \\
0.0 \\
2.0 \\
2.0\end{array}$ & $\begin{array}{r}18.0 \\
7.0 \\
8.0 \\
4.0 \\
30.0 \\
4.0 \\
4.0 \\
4.0\end{array}$ & $\begin{array}{r}10.7 \\
8.4 \\
4.3 \\
--- \\
0.9 \\
--- \\
--- \\
---\end{array}$ & $\begin{array}{r}0 \\
31 \\
30 \\
--- \\
0 \\
--- \\
--- \\
---\end{array}$ & $\begin{array}{r}0 \\
0 \\
7 \\
0 \\
0 \\
26 \\
0 \\
0\end{array}$ \\
\hline $\begin{array}{l}\text { LINE } \\
\text { A } \\
\text { B } \\
\text { C } \\
\text { D } \\
\text { E } \\
\text { F } \\
\text { G } \\
\text { H } \\
\text { I } \\
\text { J } \\
\text { K } \\
\text { L }\end{array}$ & $\begin{array}{r}10110 \\
1662.0 \\
1767.0 \\
1828.0 \\
1855.0 \\
1890.0 \\
1915.0 \\
1995.0 \\
1999.0 \\
2008.0 \\
2061.0 \\
2085.0 \\
2091.0\end{array}$ & $\begin{array}{r}\text { S } \\
\text { S } \\
\text { S? } \\
\text { S? } \\
\text { S } \\
\text { S } \\
\text { D } \\
\text { B? } \\
\text { B? } \\
\text { B? } \\
\text { B? } \\
\text { D }\end{array}$ & $\begin{array}{l}687047 \\
687099 \\
687064 \\
687050 \\
687032 \\
687043 \\
687070 \\
687065 \\
687079 \\
686949 \\
686971 \\
686979\end{array}$ & $\begin{array}{l}6111958 \\
6114217 \\
6115563 \\
6116096 \\
6116786 \\
6117279 \\
6119136 \\
6119219 \\
6119394 \\
6120495 \\
6121137 \\
6121247\end{array}$ & $\begin{array}{r}66.0 \\
0.0 \\
0.0 \\
0.0 \\
0.0 \\
0.0 \\
18.0 \\
1.0 \\
1.0 \\
0.0 \\
1.0 \\
12.0\end{array}$ & $\begin{array}{r}16.0 \\
4.0 \\
2.0 \\
1.0 \\
1.0 \\
1.0 \\
17.0 \\
2.0 \\
2.0 \\
2.0 \\
2.0 \\
9.0\end{array}$ & $\begin{array}{r}176.0 \\
0.0 \\
0.0 \\
0.0 \\
0.0 \\
0.0 \\
12.0 \\
1.0 \\
1.0 \\
1.0 \\
1.0 \\
7.0\end{array}$ & $\begin{array}{r}53.0 \\
6.0 \\
2.0 \\
2.0 \\
1.0 \\
2.0 \\
10.0 \\
2.0 \\
2.0 \\
2.0 \\
2.0 \\
2.0\end{array}$ & $\begin{array}{r}220.0 \\
2.0 \\
0.0 \\
0.0 \\
0.0 \\
0.0 \\
52.0 \\
2.0 \\
2.0 \\
2.0 \\
2.0 \\
57.0\end{array}$ & $\begin{array}{r}23.0 \\
50.0 \\
4.0 \\
4.0 \\
4.0 \\
4.0 \\
34.0 \\
4.0 \\
4.0 \\
4.0 \\
4.0 \\
34.0\end{array}$ & $\begin{array}{r}107.9 \\
0.5 \\
--- \\
--- \\
--- \\
--- \\
12.0 \\
--- \\
--- \\
--- \\
--- \\
16.2\end{array}$ & $\begin{array}{r}0 \\
0 \\
---- \\
---- \\
---- \\
--- \\
5 \\
---- \\
---- \\
--- \\
--- \\
27\end{array}$ & $\begin{array}{r}29 \\
27 \\
0 \\
674 \\
322 \\
249 \\
4 \\
0 \\
124 \\
27 \\
27 \\
27\end{array}$ \\
\hline $\begin{array}{l}\text { LINE } \\
A\end{array}$ & $\begin{array}{l}10120 \\
2017.0\end{array}$ & B & 687110 & 6121231 & 1.0 & 2.0 & 1.0 & 2.0 & 2.0 & 4.0 & --- & --- & 36 \\
\hline
\end{tabular}

$\mathrm{CX}=$ COAXIAL

Note: EM values shown above

are local amplitudes

* Estimated Depth may be unreliable because the stronger part of the conductor may be deeper or to one side of the flight line, or because of a Dolomi 
EM Anomaly List

\begin{tabular}{|c|c|c|c|c|c|c|c|c|c|c|c|c|c|}
\hline Label & Fid & Interp & $\underset{\mathrm{m}}{\mathrm{XUTM}}$ & $\begin{array}{c}\text { YUTM } \\
\mathrm{m}\end{array}$ & $\begin{array}{l}\text { CX } \\
\text { Real } \\
\text { ppm }\end{array}$ & $\begin{array}{l}900 \mathrm{HZ} \\
\text { Quad } \\
\text { ppm }\end{array}$ & $\begin{array}{l}\text { CP } \\
\text { Real } \\
\text { ppm }\end{array}$ & $\begin{array}{c}900 \mathrm{HZ} \\
\text { Quad } \\
\text { ppm }\end{array}$ & $\begin{array}{l}\text { CP } \\
\text { Real } \\
\text { ppm }\end{array}$ & $\begin{array}{c}7200 \mathrm{HZ} \\
\text { Quad } \\
\text { ppm }\end{array}$ & $\begin{array}{l}\text { Vertica } \\
\text { COND } \\
\text { siemens }\end{array}$ & $\begin{array}{c}\text { al Dike } \\
\text { DEPTH* } \\
\text { m }\end{array}$ & $\begin{array}{c}\text { Mag. Corr } \\
\text { NT }\end{array}$ \\
\hline $\begin{array}{l}\text { LINE } \\
\text { G } \\
\text { F } \\
\text { E } \\
\text { D } \\
\text { C } \\
\text { B } \\
\text { A }\end{array}$ & $\begin{array}{r}10121 \\
1005.0 \\
1130.0 \\
1148.0 \\
1181.0 \\
1248.0 \\
1321.0 \\
1433.0\end{array}$ & $\begin{array}{r}\text { B } \\
\text { B? } \\
\text { D } \\
\text { D } \\
\text { S } \\
\text { S? } \\
\text { S? }\end{array}$ & $\begin{array}{l}687156 \\
687137 \\
687153 \\
687210 \\
687213 \\
687239 \\
687333\end{array}$ & $\begin{array}{l}6121199 \\
6119425 \\
6119137 \\
6118614 \\
6117616 \\
6116250 \\
6114222\end{array}$ & $\begin{array}{r}1.0 \\
1.0 \\
14.0 \\
1.0 \\
1.0 \\
0.0 \\
0.0\end{array}$ & $\begin{array}{r}1.0 \\
2.0 \\
16.0 \\
7.0 \\
1.0 \\
2.0 \\
6.0\end{array}$ & $\begin{array}{l}1.0 \\
1.0 \\
4.0 \\
1.0 \\
0.0 \\
0.0 \\
0.0\end{array}$ & $\begin{array}{r}1.0 \\
2.0 \\
14.0 \\
4.0 \\
2.0 \\
2.0 \\
5.0\end{array}$ & $\begin{array}{r}2.0 \\
2.0 \\
36.0 \\
12.0 \\
0.0 \\
0.0 \\
3.0\end{array}$ & $\begin{array}{r}2.0 \\
3.0 \\
16.0 \\
21.0 \\
4.0 \\
4.0 \\
73.0\end{array}$ & $\begin{array}{l}--- \\
--- \\
6.4 \\
1.1 \\
--- \\
--- \\
0.1\end{array}$ & $\begin{array}{r}--- \\
--- \\
1 \\
4 \\
--- \\
--- \\
0\end{array}$ & $\begin{array}{r}0 \\
39 \\
23 \\
6 \\
48 \\
390 \\
0\end{array}$ \\
\hline $\begin{array}{l}\text { LINE } \\
\text { A } \\
\text { B } \\
\text { C } \\
\text { D } \\
\text { E } \\
\text { F } \\
\text { G } \\
\text { H } \\
\text { I } \\
\text { J } \\
\text { K } \\
\text { L } \\
\text { M } \\
\text { N }\end{array}$ & $\begin{array}{r}10130 \\
1092.0 \\
1129.0 \\
1164.0 \\
1238.0 \\
1293.0 \\
1306.0 \\
1307.0 \\
1318.0 \\
1331.0 \\
1338.0 \\
1350.0 \\
1381.0 \\
1438.0 \\
1457.0\end{array}$ & $\begin{array}{r}\text { B? } \\
\text { S? } \\
\text { B? } \\
\text { B? } \\
\text { D } \\
\text { D } \\
\text { D } \\
\text { B } \\
\text { B } \\
\text { D } \\
\text { B? } \\
\text { S } \\
\text { D } \\
\text { S? }\end{array}$ & $\begin{array}{l}687456 \\
687437 \\
687415 \\
687380 \\
687434 \\
687494 \\
687495 \\
687497 \\
687490 \\
687472 \\
687436 \\
687393 \\
687396 \\
687365\end{array}$ & $\begin{array}{l}6114478 \\
6115319 \\
6116091 \\
6117628 \\
6118728 \\
6118969 \\
6118985 \\
6119126 \\
6119259 \\
6119362 \\
6119563 \\
6120113 \\
6121201 \\
6121414\end{array}$ & $\begin{array}{r}1.0 \\
0.0 \\
0.0 \\
0.0 \\
3.0 \\
9.0 \\
12.0 \\
34.0 \\
10.0 \\
6.0 \\
1.0 \\
0.0 \\
14.0 \\
0.0\end{array}$ & $\begin{array}{r}2.0 \\
0.0 \\
3.0 \\
2.0 \\
9.0 \\
21.0 \\
8.0 \\
62.0 \\
7.0 \\
13.0 \\
2.0 \\
1.0 \\
9.0 \\
1.0\end{array}$ & $\begin{array}{r}0.0 \\
0.0 \\
0.0 \\
0.0 \\
3.0 \\
17.0 \\
17.0 \\
2.0 \\
17.0 \\
9.0 \\
0.0 \\
0.0 \\
4.0 \\
0.0\end{array}$ & $\begin{array}{r}2.0 \\
1.0 \\
4.0 \\
2.0 \\
5.0 \\
29.0 \\
29.0 \\
74.0 \\
30.0 \\
8.0 \\
2.0 \\
1.0 \\
24.0 \\
2.0\end{array}$ & $\begin{array}{r}2.0 \\
0.0 \\
0.0 \\
2.0 \\
22.0 \\
89.0 \\
89.0 \\
208.0 \\
79.0 \\
28.0 \\
1.0 \\
1.0 \\
50.0 \\
2.0\end{array}$ & $\begin{array}{r}4.0 \\
4.0 \\
33.0 \\
4.0 \\
35.0 \\
95.0 \\
95.0 \\
240.0 \\
39.0 \\
18.0 \\
4.0 \\
4.0 \\
34.0 \\
3.0\end{array}$ & $\begin{array}{l}--- \\
--- \\
0.1 \\
--- \\
2.3 \\
4.8 \\
9.4 \\
4.6 \\
8.3 \\
4.8 \\
--- \\
--- \\
7.0 \\
---\end{array}$ & $\begin{array}{r}--- \\
--- \\
0 \\
--- \\
6 \\
14 \\
25 \\
0 \\
11 \\
19 \\
--- \\
--- \\
20 \\
---\end{array}$ & $\begin{array}{r}28 \\
0 \\
230 \\
12 \\
38 \\
128 \\
128 \\
0 \\
0 \\
0 \\
137 \\
0 \\
0 \\
0\end{array}$ \\
\hline $\begin{array}{l}\text { LINE } \\
\text { J } \\
\text { I } \\
\text { H } \\
\text { G } \\
\text { F } \\
\text { E } \\
\text { D } \\
\text { C } \\
\text { B } \\
\text { A }\end{array}$ & $\begin{array}{r}10140 \\
3672.0 \\
3865.0 \\
3870.0 \\
3878.0 \\
3933.0 \\
3976.0 \\
3996.0 \\
3999.0 \\
4238.0 \\
4278.0\end{array}$ & $\begin{array}{r}\text { S? } \\
\text { D } \\
\text { B } \\
\text { D } \\
B ? \\
B ? \\
B ? \\
\text { D } \\
\text { S? } \\
\text { B? }\end{array}$ & $\begin{array}{l}687335 \\
687530 \\
687529 \\
687528 \\
687632 \\
687711 \\
687708 \\
687710 \\
687677 \\
687784\end{array}$ & $\begin{array}{l}6125357 \\
6121193 \\
6121109 \\
6120973 \\
6120163 \\
6119563 \\
6119194 \\
6119135 \\
6113716 \\
6112926\end{array}$ & $\begin{array}{r}0.0 \\
14.0 \\
1.0 \\
14.0 \\
1.0 \\
1.0 \\
1.0 \\
13.0 \\
0.0 \\
5.0\end{array}$ & $\begin{array}{r}2.0 \\
7.0 \\
2.0 \\
13.0 \\
2.0 \\
2.0 \\
2.0 \\
15.0 \\
2.0 \\
4.0\end{array}$ & $\begin{array}{r}0.0 \\
8.0 \\
1.0 \\
9.0 \\
0.0 \\
0.0 \\
1.0 \\
10.0 \\
0.0 \\
2.0\end{array}$ & $\begin{array}{r}0.0 \\
15.0 \\
2.0 \\
9.0 \\
3.0 \\
1.0 \\
2.0 \\
28.0 \\
2.0 \\
6.0\end{array}$ & $\begin{array}{r}0.0 \\
42.0 \\
2.0 \\
6.0 \\
7.0 \\
2.0 \\
2.0 \\
22.0 \\
2.0 \\
19.0\end{array}$ & $\begin{array}{r}4.0 \\
23.0 \\
4.0 \\
29.0 \\
28.0 \\
4.0 \\
4.0 \\
39.0 \\
4.0 \\
11.0\end{array}$ & $\begin{array}{r}--- \\
13.1 \\
--- \\
10.2 \\
1.0 \\
--- \\
--- \\
5.9 \\
--- \\
5.3\end{array}$ & $\begin{array}{r}--- \\
0 \\
--- \\
4 \\
29 \\
--- \\
--- \\
8 \\
--- \\
4\end{array}$ & $\begin{array}{r}118 \\
0 \\
0 \\
0 \\
78 \\
164 \\
10 \\
0 \\
25 \\
29\end{array}$ \\
\hline $\begin{array}{l}\text { LINE } \\
\text { A } \\
\text { B } \\
\text { C } \\
\text { D } \\
\text { E } \\
\text { F } \\
\text { G } \\
\text { H }\end{array}$ & $\begin{array}{r}10150 \\
3015.0 \\
3040.0 \\
3064.0 \\
3114.0 \\
3167.0 \\
3202.0 \\
3221.0 \\
3223.0\end{array}$ & $\begin{array}{r}\text { S } \\
\text { S } \\
\text { S } \\
\text { B? } \\
\text { S } \\
\text { B? } \\
\text { B? } \\
\text { B }\end{array}$ & $\begin{array}{l}687840 \\
687872 \\
687872 \\
687829 \\
687778 \\
687788 \\
687788 \\
687789\end{array}$ & $\begin{array}{l}6113881 \\
6114534 \\
6115298 \\
6116429 \\
6117726 \\
6118692 \\
6119136 \\
6119184\end{array}$ & $\begin{array}{r}0.0 \\
0.0 \\
1.0 \\
0.0 \\
1.0 \\
1.0 \\
1.0 \\
11.0\end{array}$ & $\begin{array}{r}1.0 \\
2.0 \\
2.0 \\
5.0 \\
2.0 \\
2.0 \\
0.0 \\
10.0\end{array}$ & $\begin{array}{l}0.0 \\
0.0 \\
0.0 \\
0.0 \\
0.0 \\
0.0 \\
1.0 \\
3.0\end{array}$ & $\begin{array}{r}0.0 \\
0.0 \\
2.0 \\
16.0 \\
2.0 \\
2.0 \\
2.0 \\
13.0\end{array}$ & $\begin{array}{r}2.0 \\
0.0 \\
2.0 \\
42.0 \\
2.0 \\
2.0 \\
2.0 \\
57.0\end{array}$ & $\begin{array}{r}4.0 \\
4.0 \\
4.0 \\
171.0 \\
4.0 \\
4.0 \\
4.0 \\
37.0\end{array}$ & $\begin{array}{l}--- \\
--- \\
--- \\
0.5 \\
--- \\
--- \\
--- \\
6.1\end{array}$ & $\begin{array}{r}--- \\
--- \\
--- \\
4 \\
--- \\
--- \\
--- \\
22\end{array}$ & $\begin{array}{r}31 \\
0 \\
0 \\
334 \\
0 \\
31 \\
0 \\
22\end{array}$ \\
\hline
\end{tabular}

$\mathrm{CX}=$ COAXIAL

$\mathrm{CP}=$ COPLANAR Note:EM values shown above

Dolomi are local amplitudes

*Estimated Depth may be unreliable because the stronger part of the conductor may be deeper or to one side of the flight line, or because of a shallow dip or magnetite/overburden effects 
EM Anomaly List

\begin{tabular}{|c|c|c|c|c|c|c|c|c|c|c|c|c|c|}
\hline Label & Fid & Interp & $\begin{array}{c}\text { XUTM } \\
\text { m }\end{array}$ & $\begin{array}{c}\text { YUTM } \\
\mathrm{m}\end{array}$ & $\begin{array}{l}\text { CX } \\
\text { Real } \\
\text { ppm }\end{array}$ & $\begin{array}{l}900 \mathrm{HZ} \\
\text { Quad } \\
\text { ppm }\end{array}$ & $\begin{array}{l}\text { CP } \\
\text { Real } \\
\text { ppm }\end{array}$ & $\begin{array}{l}900 \mathrm{HZ} \\
\text { Quad } \\
\text { ppm }\end{array}$ & $\begin{array}{l}\text { CP } 7 \\
\text { Real } \\
\text { ppm }\end{array}$ & $\begin{array}{c}7200 \mathrm{HZ} \\
\text { Quad } \\
\text { ppm }\end{array}$ & $\begin{array}{l}\text { Vertica } \\
\text { Cond } \\
\text { siemens }\end{array}$ & $\begin{array}{c}\text { al Dike } \\
\text { DEPTH* } \\
\text { m }\end{array}$ & $\begin{array}{c}\text { Mag. Corr } \\
\text { NT }\end{array}$ \\
\hline $\begin{array}{l}\text { LINE } \\
\text { I } \\
\text { J } \\
\text { K } \\
\text { L } \\
\text { M } \\
\text { N }\end{array}$ & $\begin{array}{r}10150 \\
3239.0 \\
3263.0 \\
3347.0 \\
3509.0 \\
3537.0 \\
3560.0\end{array}$ & $\begin{array}{r}\mathrm{D} \\
\mathrm{D} \\
\mathrm{D} \\
\mathrm{S} \\
\mathrm{S} ? \\
\mathrm{~S}\end{array}$ & $\begin{array}{l}687796 \\
687814 \\
687772 \\
687562 \\
687501 \\
687493\end{array}$ & $\begin{array}{l}6119535 \\
6119853 \\
6121231 \\
6124336 \\
6124895 \\
6125380\end{array}$ & $\begin{array}{r}13.0 \\
1.0 \\
16.0 \\
0.0 \\
0.0 \\
0.0\end{array}$ & $\begin{array}{r}17.0 \\
0.0 \\
16.0 \\
1.0 \\
1.0 \\
1.0\end{array}$ & $\begin{array}{l}2.0 \\
0.0 \\
9.0 \\
0.0 \\
0.0 \\
0.0\end{array}$ & $\begin{array}{r}10.0 \\
1.0 \\
27.0 \\
1.0 \\
0.0 \\
0.0\end{array}$ & $\begin{array}{r}44.0 \\
2.0 \\
96.0 \\
0.0 \\
0.0 \\
0.0\end{array}$ & $\begin{array}{r}44.0 \\
4.0 \\
17.0 \\
2.0 \\
3.0 \\
3.0\end{array}$ & $\begin{array}{l}5.6 \\
--- \\
6.7 \\
--- \\
--- \\
---\end{array}$ & $\begin{array}{r}19 \\
--- \\
0 \\
--- \\
--- \\
---\end{array}$ & $\begin{array}{r}266 \\
6 \\
0 \\
31 \\
0 \\
25\end{array}$ \\
\hline $\begin{array}{l}\text { LINE } \\
\text { M } \\
\text { L } \\
\text { K } \\
\text { J } \\
\text { I } \\
\text { H } \\
\text { G } \\
\text { F } \\
\text { E } \\
\text { D } \\
\text { C } \\
\text { B } \\
\text { A }\end{array}$ & $\begin{array}{r}10160 \\
1960.0 \\
2104.0 \\
2115.0 \\
2151.0 \\
2159.0 \\
2241.0 \\
2246.0 \\
2265.0 \\
2272.0 \\
2291.0 \\
2426.0 \\
2482.0 \\
2525.0\end{array}$ & $\begin{array}{r}\text { S } \\
\text { B? } \\
\text { B? } \\
\text { D } \\
\text { D } \\
\text { B } \\
\text { B? } \\
\text { B } \\
\text { B? } \\
\text { B? } \\
\text { S? } \\
\text { S? } \\
\text { S }\end{array}$ & $\begin{array}{l}687719 \\
687924 \\
687904 \\
687943 \\
687943 \\
688074 \\
688069 \\
688056 \\
688060 \\
688060 \\
688055 \\
688105 \\
688125\end{array}$ & $\begin{array}{l}6124991 \\
6121936 \\
6121777 \\
6121274 \\
6121126 \\
6119568 \\
6119499 \\
6119170 \\
6119038 \\
6118687 \\
6115975 \\
6114821 \\
6114000\end{array}$ & $\begin{array}{r}0.0 \\
0.0 \\
0.0 \\
3.0 \\
21.0 \\
1.0 \\
1.0 \\
7.0 \\
1.0 \\
0.0 \\
0.0 \\
0.0 \\
1.0\end{array}$ & $\begin{array}{r}1.0 \\
2.0 \\
2.0 \\
8.0 \\
27.0 \\
10.0 \\
7.0 \\
7.0 \\
2.0 \\
2.0 \\
1.0 \\
2.0 \\
1.0\end{array}$ & $\begin{array}{r}0.0 \\
0.0 \\
0.0 \\
6.0 \\
12.0 \\
1.0 \\
1.0 \\
2.0 \\
1.0 \\
1.0 \\
0.0 \\
0.0 \\
0.0\end{array}$ & $\begin{array}{r}1.0 \\
0.0 \\
1.0 \\
1.0 \\
28.0 \\
1.0 \\
7.0 \\
5.0 \\
2.0 \\
2.0 \\
2.0 \\
2.0 \\
1.0\end{array}$ & $\begin{array}{r}0.0 \\
0.0 \\
0.0 \\
30.0 \\
89.0 \\
16.0 \\
16.0 \\
29.0 \\
2.0 \\
2.0 \\
0.0 \\
2.0 \\
2.0\end{array}$ & $\begin{array}{r}4.0 \\
4.0 \\
4.0 \\
10.0 \\
97.0 \\
0.0 \\
49.0 \\
12.0 \\
4.0 \\
4.0 \\
4.0 \\
4.0 \\
4.0\end{array}$ & $\begin{array}{l}0.1 \\
--- \\
--- \\
4.7 \\
7.3 \\
0.8 \\
0.6 \\
6.2 \\
--- \\
--- \\
--- \\
--- \\
---\end{array}$ & $\begin{array}{r}0 \\
--- \\
--- \\
22 \\
3 \\
1 \\
0 \\
14 \\
--- \\
--- \\
--- \\
--- \\
---\end{array}$ & $\begin{array}{r}31 \\
249 \\
168 \\
31 \\
31 \\
271 \\
0 \\
0 \\
31 \\
0 \\
541 \\
0 \\
31\end{array}$ \\
\hline $\begin{array}{l}\text { LINE } \\
\text { A } \\
\text { B } \\
\text { C } \\
\text { D } \\
\text { E } \\
\text { F } \\
\text { G }\end{array}$ & $\begin{array}{r}10170 \\
1036.0 \\
1121.0 \\
1191.0 \\
1194.0 \\
1199.0 \\
1215.0 \\
1221.0\end{array}$ & $\begin{array}{r}\text { S? } \\
\text { S } \\
\text { B? } \\
\text { B? } \\
\text { D } \\
\text { D } \\
\text { D }\end{array}$ & $\begin{array}{l}688245 \\
688147 \\
688139 \\
688137 \\
688132 \\
688120 \\
688119\end{array}$ & $\begin{array}{l}6114583 \\
6116933 \\
6118947 \\
6119032 \\
6119158 \\
6119471 \\
6119573\end{array}$ & $\begin{array}{r}0.0 \\
0.0 \\
1.0 \\
4.0 \\
12.0 \\
2.0 \\
2.0\end{array}$ & $\begin{array}{r}2.0 \\
1.0 \\
2.0 \\
9.0 \\
20.0 \\
14.0 \\
11.0\end{array}$ & $\begin{array}{l}1.0 \\
0.0 \\
1.0 \\
8.0 \\
8.0 \\
3.0 \\
2.0\end{array}$ & $\begin{array}{l}2.0 \\
2.0 \\
2.0 \\
8.0 \\
8.0 \\
4.0 \\
7.0\end{array}$ & $\begin{array}{r}2.0 \\
0.0 \\
2.0 \\
47.0 \\
47.0 \\
28.0 \\
24.0\end{array}$ & $\begin{array}{r}4.0 \\
4.0 \\
4.0 \\
42.0 \\
42.0 \\
75.0 \\
71.0\end{array}$ & $\begin{array}{l}--- \\
--- \\
--- \\
4.6 \\
6.2 \\
1.3 \\
0.9\end{array}$ & $\begin{array}{r}--- \\
--- \\
--- \\
23 \\
4 \\
0 \\
4\end{array}$ & $\begin{array}{r}0 \\
155 \\
0 \\
29 \\
67 \\
0 \\
289\end{array}$ \\
\hline $\begin{array}{l}\text { LINE } \\
\text { A } \\
B \\
\text { C } \\
D\end{array}$ & $\begin{array}{r}10171 \\
1607.0 \\
1613.0 \\
1617.0 \\
1783.0\end{array}$ & $\begin{array}{r}\text { D } \\
\text { B? } \\
\text { D } \\
\text { S }\end{array}$ & $\begin{array}{l}688180 \\
688165 \\
688154 \\
687916\end{array}$ & $\begin{array}{l}6121178 \\
6121311 \\
6121399 \\
6124901\end{array}$ & $\begin{array}{r}18.0 \\
1.0 \\
25.0 \\
0.0\end{array}$ & $\begin{array}{r}22.0 \\
2.0 \\
29.0 \\
1.0\end{array}$ & $\begin{array}{r}9.0 \\
1.0 \\
20.0 \\
0.0\end{array}$ & $\begin{array}{r}7.0 \\
2.0 \\
48.0 \\
1.0\end{array}$ & $\begin{array}{r}62.0 \\
2.0 \\
173.0 \\
0.0\end{array}$ & $\begin{array}{r}58.0 \\
4.0 \\
128.0 \\
11.0\end{array}$ & $\begin{array}{l}9.3 \\
--- \\
7.9 \\
0.1\end{array}$ & $\begin{array}{r}8 \\
-- \\
0 \\
0\end{array}$ & $\begin{array}{r}22 \\
0 \\
0 \\
0\end{array}$ \\
\hline $\begin{array}{l}\text { LINE } \\
\text { H } \\
\text { G } \\
F \\
\text { E } \\
\text { D } \\
\text { C } \\
B \\
\text { A }\end{array}$ & $\begin{array}{r}10180 \\
1834.0 \\
1845.0 \\
1853.0 \\
1908.0 \\
1918.0 \\
2079.0 \\
2116.0 \\
2153.0\end{array}$ & $\begin{array}{r}\text { S } \\
\text { D } \\
\text { B? } \\
\text { B? } \\
\text { B? } \\
\text { S } \\
\text { S } \\
\text { B? }\end{array}$ & $\begin{array}{l}688277 \\
688240 \\
688232 \\
688459 \\
688444 \\
688433 \\
688510 \\
688511\end{array}$ & $\begin{array}{l}6121754 \\
6121422 \\
6121157 \\
6119709 \\
6119481 \\
6115038 \\
6114235 \\
6113339\end{array}$ & $\begin{array}{l}0.0 \\
6.0 \\
1.0 \\
1.0 \\
0.0 \\
0.0 \\
0.0 \\
0.0\end{array}$ & $\begin{array}{l}1.0 \\
5.0 \\
2.0 \\
2.0 \\
2.0 \\
1.0 \\
1.0 \\
2.0\end{array}$ & $\begin{array}{l}0.0 \\
6.0 \\
1.0 \\
1.0 \\
0.0 \\
0.0 \\
1.0 \\
0.0\end{array}$ & $\begin{array}{l}2.0 \\
5.0 \\
2.0 \\
2.0 \\
2.0 \\
2.0 \\
2.0 \\
2.0\end{array}$ & $\begin{array}{l}2.0 \\
8.0 \\
2.0 \\
2.0 \\
2.0 \\
2.0 \\
2.0 \\
0.0\end{array}$ & $\begin{array}{l}4.0 \\
9.0 \\
4.0 \\
4.0 \\
4.0 \\
4.0 \\
4.0 \\
4.0\end{array}$ & $\begin{array}{c}--- \\
10.8 \\
--- \\
--- \\
--- \\
--- \\
--- \\
---\end{array}$ & $\begin{array}{l}--- \\
0 \\
--- \\
--- \\
--- \\
--- \\
--- \\
---\end{array}$ & $\begin{array}{r}28 \\
0 \\
28 \\
0 \\
143 \\
28 \\
28 \\
28\end{array}$ \\
\hline
\end{tabular}

$\mathrm{CX}=$ COAXIAL

$\mathrm{CP}=$ COPLANAR

Note: EM values shown above

are local amplitudes

*Estimated Depth may be unreliable because the stronger part of the conductor may be deeper or to one side of the flight line, or because of a shallow dip or magnetite/overburden effects 
EM Anomaly List

\begin{tabular}{|c|c|c|c|c|c|c|c|c|c|c|c|c|c|}
\hline Label & Fid & Interp & $\begin{array}{c}\text { XUTM } \\
\text { m }\end{array}$ & $\begin{array}{c}\text { YUTM } \\
\mathrm{m}\end{array}$ & $\begin{array}{l}\text { CX } \\
\text { Real } \\
\text { ppm }\end{array}$ & $\begin{array}{l}900 \mathrm{HZ} \\
\text { Quad } \\
\text { ppm }\end{array}$ & $\begin{array}{l}\text { CP } \\
\text { Real } \\
\text { ppm }\end{array}$ & $\begin{array}{c}900 \mathrm{HZ} \\
\text { Quad } \\
\text { ppm }\end{array}$ & $\begin{array}{l}\text { CP }{ }^{7} \\
\text { Real } \\
\text { ppm }\end{array}$ & $\begin{array}{c}200 \mathrm{HZ} \\
\text { Quad } \\
\text { ppm }\end{array}$ & $\begin{array}{l}\text { Vertica } \\
\text { COND } \\
\text { siemens }\end{array}$ & $\begin{array}{c}\text { al Dike } \\
\text { DEPTH* } \\
\text { m }\end{array}$ & $\begin{array}{c}\text { Mag. Corr } \\
\text { NT }\end{array}$ \\
\hline $\begin{array}{l}\text { LINE } \\
\text { A } \\
\text { B } \\
\text { C } \\
\text { D } \\
\text { E } \\
\text { F } \\
\text { G } \\
\text { H } \\
\text { I } \\
\text { J } \\
\text { K } \\
\text { L } \\
\text { M } \\
\text { N }\end{array}$ & $\begin{array}{l}10190 \\
881.0 \\
946.0 \\
1047.0 \\
1073.0 \\
1104.0 \\
1118.0 \\
1162.0 \\
1179.0 \\
1256.0 \\
1269.0 \\
1294.0 \\
1354.0 \\
1375.0 \\
1514.0\end{array}$ & $\begin{array}{r}\text { S } \\
\text { S? } \\
\text { B? } \\
\text { S } \\
\text { B } \\
\text { B? } \\
\text { B? } \\
\text { D } \\
\text { D } \\
\text { D } \\
\text { B? } \\
\text { S? } \\
\text { D } \\
\text { S }\end{array}$ & $\begin{array}{l}688692 \\
688626 \\
688557 \\
688550 \\
688559 \\
688551 \\
688568 \\
688613 \\
688590 \\
688577 \\
688528 \\
688510 \\
688477 \\
688311\end{array}$ & $\begin{array}{l}6114106 \\
6115713 \\
6118008 \\
6118285 \\
6118795 \\
6118995 \\
6119457 \\
6119689 \\
6121193 \\
6121395 \\
6121702 \\
6122468 \\
6122774 \\
6125108\end{array}$ & $\begin{array}{r}1.0 \\
1.0 \\
0.0 \\
1.0 \\
6.0 \\
2.0 \\
2.0 \\
3.0 \\
20.0 \\
10.0 \\
0.0 \\
2.0 \\
6.0 \\
0.0\end{array}$ & $\begin{array}{r}1.0 \\
1.0 \\
2.0 \\
1.0 \\
14.0 \\
4.0 \\
12.0 \\
18.0 \\
19.0 \\
8.0 \\
1.0 \\
2.0 \\
10.0 \\
0.0\end{array}$ & $\begin{array}{r}1.0 \\
0.0 \\
0.0 \\
0.0 \\
12.0 \\
2.0 \\
0.0 \\
3.0 \\
10.0 \\
9.0 \\
0.0 \\
1.0 \\
1.0 \\
0.0\end{array}$ & $\begin{array}{r}2.0 \\
1.0 \\
2.0 \\
2.0 \\
30.0 \\
10.0 \\
3.0 \\
25.0 \\
7.0 \\
7.0 \\
2.0 \\
1.0 \\
8.0 \\
1.0\end{array}$ & $\begin{array}{r}2.0 \\
0.0 \\
2.0 \\
2.0 \\
101.0 \\
38.0 \\
30.0 \\
72.0 \\
27.0 \\
31.0 \\
0.0 \\
6.0 \\
24.0 \\
0.0\end{array}$ & $\begin{array}{r}4.0 \\
4.0 \\
4.0 \\
0.0 \\
95.0 \\
40.0 \\
3.0 \\
92.0 \\
46.0 \\
30.0 \\
4.0 \\
13.0 \\
13.0 \\
6.0\end{array}$ & $\begin{array}{c}--- \\
--- \\
---- \\
--- \\
3.5 \\
2.1 \\
0.5 \\
1.2 \\
11.9 \\
12.3 \\
--- \\
3.2 \\
3.2 \\
0.1\end{array}$ & $\begin{array}{r}--- \\
--- \\
--- \\
--- \\
3 \\
16 \\
2 \\
0 \\
10 \\
35 \\
--- \\
58 \\
19 \\
0\end{array}$ & $\begin{array}{r}15 \\
0 \\
33 \\
0 \\
42 \\
0 \\
466 \\
131 \\
0 \\
0 \\
27 \\
0 \\
0 \\
0\end{array}$ \\
\hline $\begin{array}{l}\text { LINE } \\
\text { N } \\
\text { M } \\
\text { L } \\
\text { K } \\
\text { J } \\
\text { I } \\
\text { H } \\
\text { G } \\
\text { F } \\
\text { E } \\
\text { D } \\
\text { C } \\
\text { B } \\
\text { A }\end{array}$ & $\begin{array}{r}10200 \\
3336.0 \\
3382.0 \\
3391.0 \\
3399.0 \\
3455.0 \\
3461.0 \\
3469.0 \\
3499.0 \\
3528.0 \\
3546.0 \\
3550.0 \\
3622.0 \\
3694.0 \\
3704.0\end{array}$ & $\begin{array}{r}\text { B? } \\
\text { S } \\
\text { B? } \\
\text { B } \\
\text { S? } \\
\text { S? } \\
\text { S? } \\
\text { B? } \\
\text { S } \\
\text { D } \\
\text { B? } \\
\text { S } \\
\text { S? } \\
\text { S? }\end{array}$ & $\begin{array}{l}688632 \\
688731 \\
688748 \\
688761 \\
688904 \\
688913 \\
688921 \\
688900 \\
688887 \\
688868 \\
688865 \\
688847 \\
688910 \\
688919\end{array}$ & $\begin{array}{l}6122767 \\
6121626 \\
6121399 \\
6121178 \\
6119854 \\
6119730 \\
6119563 \\
6119009 \\
6118548 \\
6118255 \\
6118191 \\
6116877 \\
6114857 \\
6114582\end{array}$ & $\begin{array}{l}2.0 \\
0.0 \\
1.0 \\
5.0 \\
1.0 \\
0.0 \\
1.0 \\
1.0 \\
0.0 \\
3.0 \\
2.0 \\
0.0 \\
1.0 \\
1.0\end{array}$ & $\begin{array}{r}4.0 \\
1.0 \\
2.0 \\
4.0 \\
10.0 \\
2.0 \\
2.0 \\
2.0 \\
1.0 \\
6.0 \\
7.0 \\
1.0 \\
2.0 \\
2.0\end{array}$ & $\begin{array}{l}0.0 \\
0.0 \\
1.0 \\
3.0 \\
0.0 \\
0.0 \\
0.0 \\
0.0 \\
0.0 \\
4.0 \\
3.0 \\
0.0 \\
1.0 \\
1.0\end{array}$ & $\begin{array}{r}1.0 \\
1.0 \\
1.0 \\
3.0 \\
15.0 \\
2.0 \\
2.0 \\
2.0 \\
2.0 \\
7.0 \\
6.0 \\
2.0 \\
2.0 \\
2.0\end{array}$ & $\begin{array}{r}7.0 \\
1.0 \\
2.0 \\
9.0 \\
38.0 \\
2.0 \\
2.0 \\
2.0 \\
2.0 \\
32.0 \\
12.0 \\
2.0 \\
2.0 \\
2.0\end{array}$ & $\begin{array}{r}6.0 \\
4.0 \\
2.0 \\
8.0 \\
30.0 \\
4.0 \\
4.0 \\
4.0 \\
4.0 \\
4.0 \\
15.0 \\
4.0 \\
4.0 \\
4.0\end{array}$ & $\begin{array}{l}2.1 \\
--- \\
--- \\
8.6 \\
0.5 \\
--- \\
--- \\
--- \\
--- \\
3.6 \\
2.0 \\
--- \\
--- \\
---\end{array}$ & $\begin{array}{r}39 \\
--- \\
--- \\
6 \\
2 \\
--- \\
--- \\
--- \\
--- \\
18 \\
12 \\
--- \\
--- \\
---\end{array}$ & $\begin{array}{r}0 \\
89 \\
0 \\
31 \\
0 \\
31 \\
31 \\
59 \\
0 \\
6 \\
10 \\
31 \\
0 \\
0\end{array}$ \\
\hline $\begin{array}{l}\text { LINE } \\
\text { A } \\
\text { B } \\
\text { C } \\
\text { D } \\
\text { E } \\
\text { F } \\
\text { G } \\
\text { H } \\
\text { I } \\
\text { J } \\
\text { K } \\
\text { L } \\
\text { M }\end{array}$ & $\begin{array}{r}10210 \\
2308.0 \\
2352.0 \\
2385.0 \\
2461.0 \\
2477.0 \\
2517.0 \\
2522.0 \\
2524.0 \\
2575.0 \\
2605.0 \\
2616.0 \\
2709.0 \\
2723.0\end{array}$ & $\begin{array}{r}\text { S } \\
\text { S } \\
\text { S? } \\
\text { S? } \\
\text { B? } \\
\text { D } \\
\text { D } \\
\text { D } \\
\text { S? } \\
\text { S } \\
\text { B? } \\
\text { B? } \\
\text { B }\end{array}$ & $\begin{array}{l}689122 \\
689054 \\
689040 \\
689009 \\
689015 \\
689033 \\
689027 \\
689025 \\
688998 \\
689016 \\
689015 \\
688988 \\
688936\end{array}$ & $\begin{array}{l}6113685 \\
6114775 \\
6115499 \\
6117112 \\
6117399 \\
6118119 \\
6118202 \\
6118236 \\
6119129 \\
6119576 \\
6119751 \\
6121125 \\
6121356\end{array}$ & $\begin{array}{r}48.0 \\
13.0 \\
0.0 \\
0.0 \\
0.0 \\
4.0 \\
5.0 \\
3.0 \\
2.0 \\
1.0 \\
0.0 \\
1.0 \\
11.0\end{array}$ & $\begin{array}{r}20.0 \\
1.0 \\
1.0 \\
1.0 \\
2.0 \\
11.0 \\
11.0 \\
9.0 \\
9.0 \\
2.0 \\
2.0 \\
2.0 \\
10.0\end{array}$ & $\begin{array}{r}148.0 \\
18.0 \\
0.0 \\
0.0 \\
0.0 \\
3.0 \\
7.0 \\
7.0 \\
1.0 \\
0.0 \\
0.0 \\
1.0 \\
3.0\end{array}$ & $\begin{array}{r}61.0 \\
34.0 \\
1.0 \\
3.0 \\
2.0 \\
7.0 \\
8.0 \\
8.0 \\
14.0 \\
4.0 \\
2.0 \\
2.0 \\
18.0\end{array}$ & $\begin{array}{r}172.0 \\
30.0 \\
0.0 \\
0.0 \\
0.0 \\
21.0 \\
46.0 \\
46.0 \\
12.0 \\
15.0 \\
2.0 \\
2.0 \\
54.0\end{array}$ & $\begin{array}{r}31.0 \\
9.0 \\
4.0 \\
16.0 \\
4.0 \\
25.0 \\
25.0 \\
25.0 \\
86.0 \\
32.0 \\
4.0 \\
4.0 \\
39.0\end{array}$ & $\begin{array}{r}56.3 \\
13.1 \\
--- \\
0.1 \\
--- \\
2.4 \\
4.1 \\
3.6 \\
0.8 \\
0.5 \\
--- \\
--- \\
6.1\end{array}$ & $\begin{array}{r}0 \\
4 \\
--- \\
0 \\
--- \\
0 \\
5 \\
7 \\
2 \\
0 \\
--- \\
--- \\
30\end{array}$ & $\begin{array}{r}13 \\
0 \\
368 \\
0 \\
48 \\
0 \\
15 \\
0 \\
42 \\
0 \\
35 \\
0 \\
0\end{array}$ \\
\hline
\end{tabular}

$\mathrm{CX}=$ COAXIAL

Dolomi
Note: EM values shown above

are local amplitudes
* Estimated Depth may be unreliable because the stronger part of the conductor may be deeper or to one side of the flight line, or because of a shallow dip or magnetite/overburden effects 
EM Anomaly List

\begin{tabular}{|c|c|c|c|c|c|c|c|c|c|c|c|c|c|}
\hline Label & Fid & Interp & $\underset{\mathrm{m}}{\mathrm{XUTM}}$ & $\begin{array}{c}\text { YUTM } \\
\mathrm{m}\end{array}$ & $\begin{array}{l}\text { CX } \\
\text { Real } \\
\text { ppm }\end{array}$ & $\begin{array}{l}00 \mathrm{HZ} \\
\text { Quad } \\
\text { ppm }\end{array}$ & $\begin{array}{l}\text { CP } \\
\text { Real } \\
\text { ppm }\end{array}$ & $\begin{array}{l}900 \mathrm{HZ} \\
\text { Quad } \\
\text { ppm }\end{array}$ & $\begin{array}{l}\text { CP } 7 \\
\text { Real } \\
\text { ppm }\end{array}$ & $\begin{array}{c}7200 \mathrm{HZ} \\
\text { Quad } \\
\text { ppm }\end{array}$ & $\begin{array}{l}\text { Vertica } \\
\text { Cond } \\
\text { siemens }\end{array}$ & $\begin{array}{c}\text { al Dike } \\
\text { DEPTH* } \\
\text { m }\end{array}$ & $\begin{array}{c}\text { Mag. Corr } \\
\text { NT }\end{array}$ \\
\hline $\begin{array}{l}\mathrm{LINE} \\
\mathrm{N}\end{array}$ & $\begin{array}{r}10210 \\
2724.0\end{array}$ & B & 688934 & 6121371 & 12.0 & 9.0 & 5.0 & 18.0 & 54.0 & 39.0 & 7.1 & 32 & 0 \\
\hline $\begin{array}{l}\text { LINE } \\
\text { D } \\
\text { C } \\
B \\
\text { A }\end{array}$ & $\begin{array}{r}10221 \\
1855.0 \\
1945.0 \\
2018.0 \\
2099.0\end{array}$ & $\begin{array}{l}\text { S? } \\
\text { B? } \\
\text { B? } \\
\text { S? }\end{array}$ & $\begin{array}{l}688980 \\
689163 \\
689245 \\
689227\end{array}$ & $\begin{array}{l}6124350 \\
6121339 \\
6119094 \\
6117051\end{array}$ & $\begin{array}{l}1.0 \\
4.0 \\
0.0 \\
0.0\end{array}$ & $\begin{array}{l}2.0 \\
2.0 \\
2.0 \\
1.0\end{array}$ & $\begin{array}{l}0.0 \\
4.0 \\
1.0 \\
0.0\end{array}$ & $\begin{array}{l}2.0 \\
5.0 \\
3.0 \\
2.0\end{array}$ & $\begin{array}{l}2.0 \\
2.0 \\
9.0 \\
0.0\end{array}$ & $\begin{array}{r}4.0 \\
5.0 \\
19.0 \\
4.0\end{array}$ & $\begin{array}{r}--- \\
10.7 \\
0.4 \\
---\end{array}$ & $\begin{array}{r}--- \\
2 \\
0 \\
---\end{array}$ & $\begin{array}{r}40 \\
0 \\
80 \\
0\end{array}$ \\
\hline $\begin{array}{l}\text { LINE } \\
\text { A } \\
\text { B } \\
\text { C } \\
\text { D } \\
\text { E } \\
\text { F } \\
\text { G } \\
\text { H } \\
\text { I } \\
\text { J }\end{array}$ & $\begin{array}{r}10230 \\
1128.0 \\
1162.0 \\
1195.0 \\
1224.0 \\
1274.0 \\
1285.0 \\
1291.0 \\
1396.0 \\
1403.0 \\
1486.0\end{array}$ & $\begin{array}{r}\text { S? } \\
\text { B? } \\
\text { S } \\
\text { S? } \\
\text { B } \\
\text { D } \\
\text { D } \\
\text { B? } \\
\text { S? } \\
\text { S? }\end{array}$ & $\begin{array}{l}689260 \\
689232 \\
689247 \\
689308 \\
689388 \\
689414 \\
689425 \\
689285 \\
689275 \\
689164\end{array}$ & $\begin{array}{l}6118245 \\
6119102 \\
6119924 \\
6120377 \\
6121118 \\
6121314 \\
6121419 \\
6122959 \\
6123047 \\
6124253\end{array}$ & $\begin{array}{r}1.0 \\
2.0 \\
0.0 \\
0.0 \\
4.0 \\
13.0 \\
24.0 \\
0.0 \\
0.0 \\
0.0\end{array}$ & $\begin{array}{r}2.0 \\
4.0 \\
2.0 \\
1.0 \\
6.0 \\
21.0 \\
28.0 \\
2.0 \\
2.0 \\
2.0\end{array}$ & $\begin{array}{r}0.0 \\
1.0 \\
0.0 \\
0.0 \\
1.0 \\
2.0 \\
17.0 \\
1.0 \\
0.0 \\
0.0\end{array}$ & $\begin{array}{r}2.0 \\
4.0 \\
1.0 \\
2.0 \\
2.0 \\
5.0 \\
41.0 \\
2.0 \\
2.0 \\
2.0\end{array}$ & $\begin{array}{r}2.0 \\
1.0 \\
2.0 \\
0.0 \\
15.0 \\
15.0 \\
141.0 \\
2.0 \\
1.0 \\
0.0\end{array}$ & $\begin{array}{r}4.0 \\
9.0 \\
4.0 \\
4.0 \\
6.0 \\
53.0 \\
111.0 \\
4.0 \\
4.0 \\
4.0\end{array}$ & $\begin{array}{l}--- \\
0.1 \\
--- \\
--- \\
3.9 \\
5.1 \\
7.7 \\
--- \\
--- \\
---\end{array}$ & $\begin{array}{r}--- \\
0 \\
--- \\
--- \\
7 \\
10 \\
4 \\
--- \\
--- \\
---\end{array}$ & $\begin{array}{r}0 \\
0 \\
35 \\
0 \\
0 \\
35 \\
0 \\
35 \\
0 \\
224\end{array}$ \\
\hline $\begin{array}{l}\text { LINE } \\
\text { D } \\
\text { C } \\
B \\
\text { A }\end{array}$ & $\begin{array}{r}10240 \\
3203.0 \\
3306.0 \\
3313.0 \\
3363.0\end{array}$ & $\begin{array}{r}\text { S } \\
\text { B? } \\
\text { B? } \\
\text { S }\end{array}$ & $\begin{array}{l}689383 \\
689558 \\
689571 \\
689599\end{array}$ & $\begin{array}{l}6124648 \\
6121425 \\
6121158 \\
6119604\end{array}$ & $\begin{array}{l}0.0 \\
1.0 \\
1.0 \\
0.0\end{array}$ & $\begin{array}{l}1.0 \\
2.0 \\
2.0 \\
1.0\end{array}$ & $\begin{array}{l}0.0 \\
1.0 \\
1.0 \\
0.0\end{array}$ & $\begin{array}{l}0.0 \\
2.0 \\
2.0 \\
1.0\end{array}$ & $\begin{array}{l}0.0 \\
2.0 \\
2.0 \\
2.0\end{array}$ & $\begin{array}{l}4.0 \\
4.0 \\
2.0 \\
4.0\end{array}$ & $\begin{array}{l}--- \\
--- \\
--- \\
---\end{array}$ & $\begin{array}{l}--- \\
--- \\
--- \\
---\end{array}$ & $\begin{array}{r}430 \\
0 \\
32 \\
32\end{array}$ \\
\hline $\begin{array}{l}\text { LINE } \\
\text { A } \\
\text { B } \\
\text { C } \\
\text { D } \\
\text { E } \\
\text { F } \\
\text { G } \\
\text { H } \\
\text { I }\end{array}$ & $\begin{array}{l}10250 \\
2457.0 \\
2468.0 \\
2489.0 \\
2497.0 \\
2501.0 \\
2539.0 \\
2545.0 \\
2561.0 \\
2621.0\end{array}$ & $\begin{array}{l}\text { B? } \\
\text { B? } \\
\text { S? } \\
\text { B? } \\
\text { B? } \\
\text { B? } \\
\text { B? } \\
\text { B } \\
\text { D }\end{array}$ & $\begin{array}{l}689766 \\
689782 \\
689862 \\
689875 \\
689872 \\
689804 \\
689804 \\
689777 \\
689582\end{array}$ & $\begin{array}{l}6119626 \\
6119824 \\
6120181 \\
6120328 \\
6120404 \\
6121253 \\
6121415 \\
6121820 \\
6123085\end{array}$ & $\begin{array}{r}1.0 \\
1.0 \\
0.0 \\
1.0 \\
2.0 \\
2.0 \\
2.0 \\
48.0 \\
2.0\end{array}$ & $\begin{array}{r}2.0 \\
2.0 \\
2.0 \\
2.0 \\
7.0 \\
5.0 \\
4.0 \\
74.0 \\
8.0\end{array}$ & $\begin{array}{r}1.0 \\
1.0 \\
0.0 \\
1.0 \\
9.0 \\
1.0 \\
1.0 \\
49.0 \\
0.0\end{array}$ & $\begin{array}{r}2.0 \\
2.0 \\
2.0 \\
2.0 \\
16.0 \\
8.0 \\
8.0 \\
147.0 \\
6.0\end{array}$ & $\begin{array}{r}2.0 \\
2.0 \\
2.0 \\
2.0 \\
47.0 \\
27.0 \\
27.0 \\
537.0 \\
19.0\end{array}$ & $\begin{array}{r}4.0 \\
4.0 \\
4.0 \\
4.0 \\
86.0 \\
20.0 \\
4.0 \\
408.0 \\
38.0\end{array}$ & $\begin{array}{l}--- \\
--- \\
--- \\
--- \\
3.2 \\
1.4 \\
2.0 \\
7.6 \\
0.9\end{array}$ & $\begin{array}{r}--- \\
--- \\
--- \\
--- \\
1 \\
5 \\
10 \\
0 \\
6\end{array}$ & $\begin{array}{r}52 \\
41 \\
280 \\
165 \\
0 \\
0 \\
0 \\
0 \\
0\end{array}$ \\
\hline $\begin{array}{l}\text { LINE } \\
\text { C } \\
\text { B } \\
\text { A }\end{array}$ & $\begin{array}{r}10260 \\
2001.0 \\
2064.0 \\
2074.0\end{array}$ & $\begin{array}{r}\text { B } \\
\text { S? } \\
\text { S? }\end{array}$ & $\begin{array}{l}689934 \\
689973 \\
689984\end{array}$ & $\begin{array}{l}6122414 \\
6120380 \\
6120092\end{array}$ & $\begin{array}{r}13.0 \\
1.0 \\
0.0\end{array}$ & $\begin{array}{r}15.0 \\
3.0 \\
2.0\end{array}$ & $\begin{array}{r}21.0 \\
3.0 \\
0.0\end{array}$ & $\begin{array}{l}7.0 \\
6.0 \\
2.0\end{array}$ & $\begin{array}{r}67.0 \\
5.0 \\
2.0\end{array}$ & $\begin{array}{r}53.0 \\
18.0 \\
4.0\end{array}$ & $\begin{array}{r}15.1 \\
2.3 \\
---\end{array}$ & $\begin{array}{r}9 \\
18 \\
---\end{array}$ & $\begin{array}{r}28 \\
0 \\
242\end{array}$ \\
\hline $\begin{array}{l}\text { LINE } \\
\text { A } \\
B\end{array}$ & $\begin{array}{l}10270 \\
1511.0 \\
1680.0\end{array}$ & $\begin{array}{r}\mathrm{S} ? \\
\mathrm{~S}\end{array}$ & $\begin{array}{l}690336 \\
690050\end{array}$ & $\begin{array}{l}6120086 \\
6123589\end{array}$ & $\begin{array}{l}0.0 \\
0.0\end{array}$ & $\begin{array}{l}1.0 \\
1.0\end{array}$ & $\begin{array}{l}0.0 \\
1.0\end{array}$ & $\begin{array}{l}2.0 \\
2.0\end{array}$ & $\begin{array}{l}2.0 \\
0.0\end{array}$ & $\begin{array}{l}4.0 \\
4.0\end{array}$ & $\begin{array}{l}--- \\
---\end{array}$ & $\begin{array}{l}--- \\
---\end{array}$ & $\begin{array}{r}0 \\
22\end{array}$ \\
\hline
\end{tabular}

$\mathrm{CX}=$ COAXIAL

$\mathrm{CP}=$ COPLANAR

Note: EM values shown above

are local amplitudes

* Estimated Depth may be unreliable because the stronger part of the conductor may be deeper or to one side of the flight line, or because of a shallow dip or magnetite/overburden effects 
EM Anomaly List

\begin{tabular}{|c|c|c|c|c|c|c|c|c|c|c|c|c|c|}
\hline Label & Fid & Interp & $\underset{\mathrm{m}}{\mathrm{XUTM}}$ & $\begin{array}{c}\text { YUTM } \\
\text { m }\end{array}$ & $\begin{array}{l}\text { CX } \\
\text { Real } \\
\text { ppm }\end{array}$ & $\begin{array}{l}00 \mathrm{HZ} \\
\text { Quad } \\
\text { ppm }\end{array}$ & $\begin{array}{l}\text { CP } \\
\text { Real } \\
\text { ppm }\end{array}$ & $\begin{array}{c}900 \mathrm{HZ} \\
\text { Quad } \\
\text { ppm }\end{array}$ & $\begin{array}{l}\text { CP } 7 \\
\text { Real } \\
\text { ppm }\end{array}$ & $\begin{array}{l}200 \mathrm{HZ} \\
\text { Quad } \\
\text { ppm }\end{array}$ & $\begin{array}{l}\text { Vertica } \\
\text { Cond } \\
\text { siemens }\end{array}$ & $\begin{array}{c}\text { al Dike } \\
\text { DEPTH* } \\
\text { m }\end{array}$ & $\begin{array}{c}\text { Mag. Corr } \\
\text { NT }\end{array}$ \\
\hline $\begin{array}{l}\text { LINE } \\
\text { B } \\
\text { A }\end{array}$ & $\begin{array}{c}10281 \\
1231.0 \\
1244.0\end{array}$ & $\begin{array}{l}\text { S? } \\
\text { S? }\end{array}$ & $\begin{array}{l}690497 \\
690493\end{array}$ & $\begin{array}{l}6116768 \\
6116433\end{array}$ & $\begin{array}{l}1.0 \\
7.0\end{array}$ & $\begin{array}{l}2.0 \\
6.0\end{array}$ & $\begin{array}{l}1.0 \\
3.0\end{array}$ & $\begin{array}{l}2.0 \\
4.0\end{array}$ & $\begin{array}{r}2.0 \\
13.0\end{array}$ & $\begin{array}{l}4.0 \\
8.0\end{array}$ & $\begin{array}{l}--- \\
8.8\end{array}$ & $\begin{array}{r}--- \\
0\end{array}$ & $\begin{array}{l}0 \\
0\end{array}$ \\
\hline $\begin{array}{l}\text { LINE } \\
\text { A } \\
B \\
\text { C }\end{array}$ & $\begin{array}{r}10290 \\
1848.0 \\
1992.0 \\
2059.0\end{array}$ & $\begin{array}{l}S \\
S \\
S\end{array}$ & $\begin{array}{l}690595 \\
690551 \\
690525\end{array}$ & $\begin{array}{l}6117015 \\
6120085 \\
6121640\end{array}$ & $\begin{array}{l}0.0 \\
1.0 \\
1.0\end{array}$ & $\begin{array}{l}2.0 \\
2.0 \\
1.0\end{array}$ & $\begin{array}{l}1.0 \\
0.0 \\
0.0\end{array}$ & $\begin{array}{l}2.0 \\
2.0 \\
1.0\end{array}$ & $\begin{array}{l}2.0 \\
2.0 \\
0.0\end{array}$ & $\begin{array}{l}1.0 \\
4.0 \\
4.0\end{array}$ & $\begin{array}{l}--- \\
--- \\
---\end{array}$ & $\begin{array}{l}--- \\
--- \\
---\end{array}$ & $\begin{array}{r}0 \\
28 \\
0\end{array}$ \\
\hline $\begin{array}{l}\text { LINE } \\
A\end{array}$ & $\begin{array}{l}10300 \\
1672.0\end{array}$ & S & 690856 & 6117364 & 0.0 & 2.0 & 1.0 & 2.0 & 2.0 & 4.0 & --- & --- & 0 \\
\hline $\begin{array}{l}\text { LINE } \\
\text { A } \\
B\end{array}$ & $\begin{array}{r}10310 \\
890.0 \\
897.0\end{array}$ & $\begin{array}{l}\text { S? } \\
\text { S? }\end{array}$ & $\begin{array}{l}691211 \\
691179\end{array}$ & $\begin{array}{l}6116738 \\
6116944\end{array}$ & $\begin{array}{l}9.0 \\
6.0\end{array}$ & $\begin{array}{r}8.0 \\
10.0\end{array}$ & $\begin{array}{r}19.0 \\
9.0\end{array}$ & $\begin{array}{r}18.0 \\
8.0\end{array}$ & $\begin{array}{l}31.0 \\
16.0\end{array}$ & $\begin{array}{l}3.0 \\
3.0\end{array}$ & $\begin{array}{r}10.9 \\
6.3\end{array}$ & $\begin{array}{l}0 \\
0\end{array}$ & $\begin{array}{r}0 \\
59\end{array}$ \\
\hline $\begin{array}{l}\text { LINE } \\
\text { A } \\
B\end{array}$ & $\begin{array}{l}10320 \\
3204.0 \\
3217.0\end{array}$ & $\begin{array}{r}E \\
B ?\end{array}$ & $\begin{array}{l}691233 \\
691234\end{array}$ & $\begin{array}{l}6117129 \\
6117418\end{array}$ & $\begin{array}{r}21.0 \\
6.0\end{array}$ & $\begin{array}{l}12.0 \\
10.0\end{array}$ & $\begin{array}{l}50.0 \\
10.0\end{array}$ & $\begin{array}{l}45.0 \\
31.0\end{array}$ & $\begin{array}{l}82.0 \\
95.0\end{array}$ & $\begin{array}{l}13.0 \\
41.0\end{array}$ & $\begin{array}{r}19.3 \\
3.6\end{array}$ & $\begin{array}{l}0 \\
0\end{array}$ & $\begin{array}{l}3 \\
3\end{array}$ \\
\hline $\begin{array}{l}\text { LINE } \\
\mathrm{A}\end{array}$ & $\begin{array}{l}10321 \\
3693.0\end{array}$ & S? & 690941 & 6123272 & 1.0 & 2.0 & 1.0 & 2.0 & 2.0 & 4.0 & --- & --- & 0 \\
\hline $\begin{array}{l}\text { LINE } \\
\text { B } \\
A\end{array}$ & $\begin{array}{r}10330 \\
2829.0 \\
2882.0\end{array}$ & $\begin{array}{r}S \\
\text { S? }\end{array}$ & $\begin{array}{l}691085 \\
691158\end{array}$ & $\begin{array}{l}6125093 \\
6123449\end{array}$ & $\begin{array}{l}1.0 \\
1.0\end{array}$ & $\begin{array}{l}1.0 \\
2.0\end{array}$ & $\begin{array}{l}1.0 \\
0.0\end{array}$ & $\begin{array}{l}4.0 \\
2.0\end{array}$ & $\begin{array}{l}2.0 \\
2.0\end{array}$ & $\begin{array}{l}6.0 \\
4.0\end{array}$ & $\begin{array}{l}0.1 \\
---\end{array}$ & $\begin{array}{r}0 \\
---\end{array}$ & $\begin{array}{r}2 \\
12\end{array}$ \\
\hline $\begin{array}{l}\text { LINE } \\
\text { A } \\
\text { B } \\
\text { C } \\
\text { D }\end{array}$ & $\begin{array}{r}10340 \\
2573.0 \\
2608.0 \\
2641.0 \\
2695.0\end{array}$ & $\begin{array}{l}\text { S } \\
B \\
S \\
\text { S }\end{array}$ & $\begin{array}{l}691415 \\
691384 \\
691355 \\
691307\end{array}$ & $\begin{array}{l}6122406 \\
6123131 \\
6123831 \\
6124977\end{array}$ & $\begin{array}{r}2.0 \\
5.0 \\
1.0 \\
28.0\end{array}$ & $\begin{array}{l}1.0 \\
5.0 \\
2.0 \\
8.0\end{array}$ & $\begin{array}{l}2.0 \\
5.0 \\
1.0 \\
7.0\end{array}$ & $\begin{array}{r}3.0 \\
5.0 \\
5.0 \\
31.0\end{array}$ & $\begin{array}{r}11.0 \\
15.0 \\
8.0 \\
98.0\end{array}$ & $\begin{array}{r}5.0 \\
11.0 \\
4.0 \\
13.0\end{array}$ & $\begin{array}{r}1.0 \\
8.0 \\
0.9 \\
17.1\end{array}$ & $\begin{array}{r}0 \\
25 \\
6 \\
0\end{array}$ & $\begin{array}{l}15 \\
15 \\
15 \\
14\end{array}$ \\
\hline $\begin{array}{l}\text { LINE } \\
\text { C } \\
\text { B } \\
\text { A }\end{array}$ & $\begin{array}{r}10350 \\
1832.0 \\
1858.0 \\
2017.0\end{array}$ & $\begin{array}{r}\text { S? } \\
\text { S } \\
\text { S }\end{array}$ & $\begin{array}{l}691566 \\
691603 \\
691831\end{array}$ & $\begin{array}{l}6123213 \\
6122399 \\
6117275\end{array}$ & $\begin{array}{l}1.0 \\
9.0 \\
3.0\end{array}$ & $\begin{array}{l}2.0 \\
4.0 \\
3.0\end{array}$ & $\begin{array}{r}1.0 \\
6.0 \\
26.0\end{array}$ & $\begin{array}{l}2.0 \\
6.0 \\
9.0\end{array}$ & $\begin{array}{r}2.0 \\
1.0 \\
15.0\end{array}$ & $\begin{array}{r}3.0 \\
2.0 \\
10.0\end{array}$ & $\begin{array}{r}--- \\
17.0 \\
22.2\end{array}$ & $\begin{array}{r}--- \\
0 \\
0\end{array}$ & $\begin{array}{r}11 \\
11 \\
0\end{array}$ \\
\hline $\begin{array}{l}\text { LINE } \\
\text { A } \\
\text { B } \\
\text { C } \\
\text { D } \\
\text { E } \\
\text { F } \\
\text { G }\end{array}$ & $\begin{array}{l}10360 \\
1345.0 \\
1536.0 \\
1541.0 \\
1567.0 \\
1577.0 \\
1588.0 \\
1620.0\end{array}$ & $\begin{array}{r}S \\
E \\
S \\
S \\
E \\
S \\
S ?\end{array}$ & $\begin{array}{l}692028 \\
691860 \\
691856 \\
691833 \\
691824 \\
691815 \\
691786\end{array}$ & $\begin{array}{l}6117457 \\
6121319 \\
6121420 \\
6121946 \\
6122148 \\
6122371 \\
6123018\end{array}$ & $\begin{array}{r}18.0 \\
14.0 \\
10.0 \\
20.0 \\
72.0 \\
6.0 \\
1.0\end{array}$ & $\begin{array}{r}5.0 \\
7.0 \\
4.0 \\
11.0 \\
19.0 \\
10.0 \\
2.0\end{array}$ & $\begin{array}{r}40.0 \\
44.0 \\
44.0 \\
18.0 \\
7.0 \\
2.0 \\
1.0\end{array}$ & $\begin{array}{r}19.0 \\
7.0 \\
1.0 \\
10.0 \\
54.0 \\
11.0 \\
2.0\end{array}$ & $\begin{array}{r}25.0 \\
6.0 \\
17.0 \\
51.0 \\
11.0 \\
49.0 \\
2.0\end{array}$ & $\begin{array}{r}5.0 \\
4.0 \\
9.0 \\
5.0 \\
21.0 \\
25.0 \\
4.0\end{array}$ & $\begin{array}{r}41.6 \\
63.4 \\
174.0 \\
25.8 \\
28.9 \\
3.1 \\
---\end{array}$ & $\begin{array}{r}0 \\
0 \\
0 \\
0 \\
0 \\
0 \\
---\end{array}$ & $\begin{array}{l}0 \\
0 \\
0 \\
0 \\
0 \\
0 \\
0\end{array}$ \\
\hline
\end{tabular}

$\mathrm{CX}=$ COAXIAL

$\mathrm{CP}=$ COPLANAR

Note: EM values shown above

are local amplitudes

*Estimated Depth may be unreliable because the stronger part of the conductor may be deeper or to one side of the flight line, or because of a shallow dip or magnetite/overburden effects 
EM Anomaly List

\begin{tabular}{|c|c|c|c|c|c|c|c|c|c|c|c|c|}
\hline Label & Fid & Interp & $\begin{array}{c}\text { XUTM } \\
\mathrm{m}\end{array}$ & $\begin{array}{c}\text { YUTM } \\
\text { m }\end{array}$ & $\begin{array}{l}\text { CX } \\
\text { Real } \\
\text { ppm }\end{array}$ & $\begin{array}{l}900 \mathrm{HZ} \\
\text { Quad } \\
\text { ppm }\end{array}$ & $\begin{array}{l}\text { CP } \\
\text { Real } \\
\text { ppm }\end{array}$ & $\begin{array}{l}900 \mathrm{~Hz} \\
\text { Quad } \\
\text { ppm }\end{array}$ & $\begin{array}{l}\text { CP } 7 \\
\text { Real } \\
\text { ppm }\end{array}$ & $\begin{array}{l}200 \mathrm{HZ} \\
\text { Quad } \\
\text { ppm }\end{array}$ & \begin{tabular}{cc}
\multicolumn{2}{c}{ Vertical Dike } \\
COND & DEPTH* \\
siemens & $\mathrm{m}$
\end{tabular} & $\begin{array}{l}\text { Mag. Corr } \\
\text { NT }\end{array}$ \\
\hline $\begin{array}{l}\text { LINE } \\
A\end{array}$ & $\begin{array}{l}10380 \\
1027.0\end{array}$ & S & 692387 & 6118525 & 10.0 & 2.0 & 19.0 & 12.0 & 8.0 & 5.0 & 30.9 & 14 \\
\hline $\begin{array}{l}\text { LINE } \\
A\end{array}$ & $\begin{array}{l}19010 \\
22927.0\end{array}$ & $\mathrm{~S}$ & 685457 & 6122441 & 18.0 & 3.0 & 50.0 & 10.0 & 44.0 & 12.0 & 132.2 & 0 \\
\hline
\end{tabular}

$\mathrm{CX}=$ COAXIAL

$\mathrm{CP}=$ COPLANAR

Dolomi
Note: EM values shown above are local amplitudes

*Estimated Depth may be unreliable because the stronger part of the conductor may be deeper or to one side of the flight line, or because of a shallow dip or magnetite/overburden effects 
EM Anomaly List

\begin{tabular}{|c|c|c|c|c|c|c|c|c|c|c|c|c|c|}
\hline Label & Fid & Interp & $\begin{array}{c}\text { XUTM } \\
\text { m }\end{array}$ & $\begin{array}{c}\text { YUTM } \\
\text { m }\end{array}$ & $\begin{array}{l}\text { CX } \\
\text { Real } \\
\text { ppm }\end{array}$ & $\begin{array}{l}00 \mathrm{HZ} \\
\text { Quad } \\
\text { ppm }\end{array}$ & $\begin{array}{l}\text { CP } \\
\text { Real } \\
\text { ppm }\end{array}$ & $\begin{array}{l}900 \mathrm{HZ} \\
\text { Quad } \\
\text { ppm }\end{array}$ & $\begin{array}{l}\text { CP } \\
\text { Real } \\
\text { ppm }\end{array}$ & $\begin{array}{l}200 \mathrm{HZ} \\
\text { Quad } \\
\text { ppm }\end{array}$ & $\begin{array}{l}\text { Vertica } \\
\text { CoND } \\
\text { siemens }\end{array}$ & $\begin{array}{c}\text { al Dike } \\
\text { DEPTH* } \\
\text { m }\end{array}$ & $\begin{array}{c}\text { Mag. Corr } \\
\text { NT }\end{array}$ \\
\hline $\begin{array}{l}\text { LINE } \\
\text { A } \\
B\end{array}$ & $\begin{array}{l}10120 \\
3027.0 \\
3008.0\end{array}$ & $\begin{array}{l}\text { S? } \\
\text { S? }\end{array}$ & $\begin{array}{l}663462 \\
663947\end{array}$ & $\begin{array}{l}6170586 \\
6171061\end{array}$ & $\begin{array}{l}6.8 \\
2.3\end{array}$ & $\begin{array}{l}1.2 \\
0.5\end{array}$ & $\begin{array}{r}28.1 \\
5.0\end{array}$ & $\begin{array}{l}0.7 \\
0.9\end{array}$ & $\begin{array}{r}22.0 \\
4.8\end{array}$ & $\begin{array}{l}1.4 \\
3.5\end{array}$ & $\begin{array}{l}--- \\
---\end{array}$ & $\begin{array}{l}--- \\
---\end{array}$ & $\begin{array}{l}433 \\
102\end{array}$ \\
\hline $\begin{array}{l}\text { LINE } \\
A\end{array}$ & $\begin{array}{c}10130 \\
2894.0\end{array}$ & $\mathrm{H}$ & 662465 & 6169312 & 20.7 & 8.5 & 38.5 & 14.3 & 39.2 & 5.7 & --- & --- & 0 \\
\hline $\begin{array}{l}\text { LINE } \\
\text { A } \\
B \\
\text { C }\end{array}$ & $\begin{array}{r}10140 \\
2675.0 \\
2662.0 \\
2653.0\end{array}$ & $\begin{array}{l}\text { S? } \\
\text { S? } \\
\text { S? }\end{array}$ & $\begin{array}{l}663727 \\
664040 \\
664233\end{array}$ & $\begin{array}{l}6170401 \\
6170700 \\
6170860\end{array}$ & $\begin{array}{r}19.5 \\
15.1 \\
1.7\end{array}$ & $\begin{array}{l}1.1 \\
0.8 \\
0.9\end{array}$ & $\begin{array}{r}2.7 \\
25.4 \\
2.1\end{array}$ & $\begin{array}{l}1.6 \\
0.5 \\
1.7\end{array}$ & $\begin{array}{r}2.1 \\
20.1 \\
1.5\end{array}$ & $\begin{array}{r}5.9 \\
3.2 \\
10.0\end{array}$ & $\begin{array}{l}--- \\
--- \\
---\end{array}$ & $\begin{array}{l}--- \\
--- \\
---\end{array}$ & $\begin{array}{r}158 \\
0 \\
160\end{array}$ \\
\hline $\begin{array}{l}\text { LINE } \\
\text { A } \\
B \\
\text { C }\end{array}$ & $\begin{array}{r}10150 \\
2537.0 \\
2546.0 \\
2552.0\end{array}$ & $\begin{array}{l}\text { S? } \\
\text { S? } \\
\text { S? }\end{array}$ & $\begin{array}{l}663905 \\
664069 \\
664197\end{array}$ & $\begin{array}{l}6170401 \\
6170559 \\
6170679\end{array}$ & $\begin{array}{l}0.0 \\
3.8 \\
0.0\end{array}$ & $\begin{array}{l}0.0 \\
1.8 \\
0.0\end{array}$ & $\begin{array}{r}0.0 \\
14.2 \\
0.0\end{array}$ & $\begin{array}{l}0.0 \\
1.2 \\
0.0\end{array}$ & $\begin{array}{r}0.0 \\
11.2 \\
0.0\end{array}$ & $\begin{array}{l}0.0 \\
4.1 \\
0.0\end{array}$ & $\begin{array}{l}--- \\
--- \\
---\end{array}$ & $\begin{array}{l}--- \\
--- \\
---\end{array}$ & $\begin{array}{r}436 \\
176 \\
0\end{array}$ \\
\hline $\begin{array}{l}\text { LINE } \\
\text { A } \\
B\end{array}$ & $\begin{array}{r}10160 \\
1064.0 \\
1093.0\end{array}$ & $\begin{array}{l}\text { S? } \\
\text { S? }\end{array}$ & $\begin{array}{l}663971 \\
664555\end{array}$ & $\begin{array}{l}6169960 \\
6170554\end{array}$ & $\begin{array}{l}0.8 \\
5.8\end{array}$ & $\begin{array}{l}0.9 \\
1.0\end{array}$ & $\begin{array}{l}0.9 \\
0.4\end{array}$ & $\begin{array}{l}1.4 \\
1.4\end{array}$ & $\begin{array}{l}0.7 \\
1.1\end{array}$ & $\begin{array}{l}2.9 \\
4.3\end{array}$ & $\begin{array}{l}--- \\
---\end{array}$ & $\begin{array}{l}--- \\
---\end{array}$ & $\begin{array}{r}189 \\
84\end{array}$ \\
\hline $\begin{array}{l}\text { LINE } \\
\text { A }\end{array}$ & $\begin{array}{c}10170 \\
734.0\end{array}$ & S? & 664168 & 6169920 & 13.6 & 5.4 & 8.8 & 7.4 & 6.7 & 12.3 & --- & --- & 626 \\
\hline $\begin{array}{l}\text { LINE } \\
\mathrm{A}\end{array}$ & $\begin{array}{c}10180 \\
530.0\end{array}$ & S? & 664538 & 6169908 & 0.0 & 0.0 & 0.0 & 0.0 & 0.0 & 0.0 & --- & --- & 0 \\
\hline $\begin{array}{l}\text { LINE } \\
A\end{array}$ & $\begin{array}{r}10190 \\
2425.0\end{array}$ & S? & 664738 & 6169907 & 6.4 & 0.7 & 13.8 & 0.7 & 10.2 & 8.2 & --- & --- & 0 \\
\hline $\begin{array}{l}\operatorname{LINE} \\
\mathrm{A}\end{array}$ & $\begin{array}{c}10210 \\
194.0\end{array}$ & S? & 665071 & 6169697 & 36.0 & 1.1 & 3.6 & 0.0 & 2.7 & 5.7 & --- & --- & 0 \\
\hline $\begin{array}{l}\text { LINE } \\
\text { A } \\
B\end{array}$ & $\begin{array}{r}10220 \\
465.0 \\
378.0\end{array}$ & $\begin{array}{l}\text { S? } \\
\text { S? }\end{array}$ & $\begin{array}{l}660995 \\
663497\end{array}$ & $\begin{array}{l}6165174 \\
6167823\end{array}$ & $\begin{array}{l}0.6 \\
1.5\end{array}$ & $\begin{array}{l}1.2 \\
0.4\end{array}$ & $\begin{array}{l}0.3 \\
3.1\end{array}$ & $\begin{array}{l}1.5 \\
0.8\end{array}$ & $\begin{array}{l}1.1 \\
2.3\end{array}$ & $\begin{array}{l}9.0 \\
7.4\end{array}$ & $\begin{array}{l}--- \\
---\end{array}$ & $\begin{array}{l}--- \\
---\end{array}$ & $\begin{array}{r}0 \\
36\end{array}$ \\
\hline $\begin{array}{l}\mathrm{LINE} \\
\mathrm{A}\end{array}$ & $\begin{array}{c}10230 \\
721.0\end{array}$ & S? & 665440 & 6169448 & 12.4 & 1.4 & 29.7 & 0.9 & 23.6 & 7.1 & --- & --- & 11 \\
\hline $\begin{array}{l}\text { LINE } \\
\text { A }\end{array}$ & $\begin{array}{l}10250 \\
1185.0\end{array}$ & S? & 663996 & 6167454 & 14.7 & 0.9 & 4.4 & 0.3 & 4.3 & 8.3 & --- & --- & 41 \\
\hline $\begin{array}{l}\mathrm{LINE} \\
\mathrm{A}\end{array}$ & $\begin{array}{c}10260 \\
1436.0\end{array}$ & S? & 664259 & 6167371 & 1.4 & 1.2 & 6.1 & 0.3 & 5.0 & 6.7 & --- & --- & 267 \\
\hline
\end{tabular}

$\mathrm{CX}=$ COAXIAL

$\mathrm{CP}=$ COPLANAR

Note: EM values shown above

are local amplitudes

*Estimated Depth may be unreliable because the stronger part of the conductor may be deeper or to one side of the flight line, or because of a shallow dip or magnetite/overburden effects 
EM Anomaly List

\begin{tabular}{|c|c|c|c|c|c|c|c|c|c|c|c|c|c|}
\hline Label & Fid & Interp & $\begin{array}{c}\text { XUTM } \\
\text { m }\end{array}$ & $\begin{array}{c}\text { YUTM } \\
\text { m }\end{array}$ & $\begin{array}{l}\text { CX } \\
\text { Real } \\
\text { ppm }\end{array}$ & $\begin{array}{l}900 \mathrm{HZ} \\
\text { Quad } \\
\text { ppm }\end{array}$ & $\begin{array}{l}\text { CP } \\
\text { Real } \\
\text { ppm }\end{array}$ & $\begin{array}{l}900 \mathrm{HZ} \\
\text { Quad } \\
\text { ppm }\end{array}$ & $\begin{array}{l}\text { CP } 7 \\
\text { Real } \\
\text { ppm }\end{array}$ & $\begin{array}{l}200 \mathrm{HZ} \\
\text { Quad } \\
\text { ppm }\end{array}$ & $\begin{array}{l}\text { Vertic } \\
\text { CoND } \\
\text { siemens }\end{array}$ & $\begin{array}{c}\text { al Dike } \\
\text { DEPTH* } \\
\text { m }\end{array}$ & $\begin{array}{c}\text { Mag. Corr } \\
\text { NT }\end{array}$ \\
\hline $\begin{array}{ll}\text { LINE } & 1 \\
\text { A } & \\
B\end{array}$ & $\begin{array}{l}10270 \\
1706.0 \\
1880.0\end{array}$ & $\begin{array}{l}\text { S? } \\
\text { S? }\end{array}$ & $\begin{array}{l}660762 \\
664649\end{array}$ & $\begin{array}{l}6163577 \\
6167470\end{array}$ & $\begin{array}{l}0.1 \\
2.0\end{array}$ & $\begin{array}{l}1.4 \\
0.2\end{array}$ & $\begin{array}{l}0.8 \\
2.4\end{array}$ & $\begin{array}{l}2.1 \\
0.8\end{array}$ & $\begin{array}{l}4.8 \\
1.8\end{array}$ & $\begin{array}{l}11.6 \\
11.3\end{array}$ & $\begin{array}{l}--- \\
---\end{array}$ & $\begin{array}{l}--- \\
---\end{array}$ & $\begin{array}{l}73 \\
28\end{array}$ \\
\hline $\begin{array}{ll}\text { LINE } & 1 \\
A\end{array}$ & $\begin{array}{c}10290 \\
540.0\end{array}$ & $S ?$ & 663557 & 6165998 & 4.6 & 1.9 & 4.9 & 1.8 & 3.9 & 18.8 & --- & --- & 70 \\
\hline $\begin{array}{l}\text { LINE } 1 \\
\text { A }\end{array}$ & $\begin{array}{r}10300 \\
838.0\end{array}$ & $S ?$ & 663668 & 6165732 & 0.8 & 0.9 & 4.3 & 1.1 & 3.7 & 8.0 & --- & --- & 120 \\
\hline $\begin{array}{l}\text { LINE } 1 \\
A\end{array}$ & $\begin{array}{l}10310 \\
1493.0\end{array}$ & $S ?$ & 664180 & 6165880 & 5.7 & 0.8 & 16.7 & 0.4 & 13.7 & 4.2 & --- & --- & 41 \\
\hline $\begin{array}{ll}\text { LINE } & 1 \\
A\end{array}$ & $\begin{array}{r}10330 \\
2082.0\end{array}$ & S & 661005 & 6161970 & 0.6 & 4.3 & 2.7 & 4.3 & 10.3 & 22.5 & --- & --- & 0 \\
\hline $\begin{array}{ll}\text { LINE } & 1 \\
A\end{array}$ & $\begin{array}{l}10340 \\
2687.0\end{array}$ & $S ?$ & 659676 & 6160352 & 0.7 & 2.1 & 0.4 & 1.9 & 1.0 & 14.4 & --- & --- & 223 \\
\hline $\begin{array}{ll}\text { LINE } & 1 \\
\text { A } & \\
B\end{array}$ & $\begin{array}{l}10350 \\
2810.0 \\
3025.0\end{array}$ & $\begin{array}{r}S \\
S ?\end{array}$ & $\begin{array}{l}661047 \\
665302\end{array}$ & $\begin{array}{l}6161485 \\
6165907\end{array}$ & $\begin{array}{l}0.8 \\
5.4\end{array}$ & $\begin{array}{l}2.0 \\
0.2\end{array}$ & $\begin{array}{l}1.6 \\
7.1\end{array}$ & $\begin{array}{l}2.8 \\
0.0\end{array}$ & $\begin{array}{l}3.4 \\
4.7\end{array}$ & $\begin{array}{l}8.0 \\
3.5\end{array}$ & $\begin{array}{l}--- \\
---\end{array}$ & $\begin{array}{l}--- \\
---\end{array}$ & $\begin{array}{r}44 \\
244\end{array}$ \\
\hline $\begin{array}{ll}\text { LINE } & 1 \\
\text { A } & \\
\text { B } \\
\text { C }\end{array}$ & $\begin{array}{r}10360 \\
3382.0 \\
3245.0 \\
3224.0\end{array}$ & $\begin{array}{l}\text { S } \\
\text { S? } \\
\text { S? }\end{array}$ & $\begin{array}{l}661030 \\
664872 \\
665336\end{array}$ & $\begin{array}{l}6161168 \\
6165128 \\
6165662\end{array}$ & $\begin{array}{l}3.1 \\
2.0 \\
1.2\end{array}$ & $\begin{array}{l}2.2 \\
1.1 \\
0.4\end{array}$ & $\begin{array}{r}6.6 \\
1.9 \\
90.8\end{array}$ & $\begin{array}{l}3.0 \\
1.4 \\
0.0\end{array}$ & $\begin{array}{l}7.3 \\
1.4 \\
5.6\end{array}$ & $\begin{array}{r}17.5 \\
8.7 \\
1.5\end{array}$ & $\begin{array}{l}--- \\
--- \\
---\end{array}$ & $\begin{array}{l}--- \\
--- \\
---\end{array}$ & $\begin{array}{r}76 \\
147 \\
0\end{array}$ \\
\hline $\begin{array}{ll}\text { LINE } & 1 \\
\text { A } & \\
B \\
\text { C }\end{array}$ & $\begin{array}{l}10370 \\
3706.0 \\
3803.0 \\
3825.0\end{array}$ & $\begin{array}{l}\text { S? } \\
\text { S? } \\
\text { S? }\end{array}$ & $\begin{array}{l}663293 \\
665027 \\
665290\end{array}$ & $\begin{array}{l}6163315 \\
6165067 \\
6165351\end{array}$ & $\begin{array}{l}6.1 \\
0.0 \\
1.1\end{array}$ & $\begin{array}{l}0.4 \\
0.0 \\
0.6\end{array}$ & $\begin{array}{l}3.5 \\
0.0 \\
1.2\end{array}$ & $\begin{array}{l}0.3 \\
0.0 \\
0.2\end{array}$ & $\begin{array}{l}2.6 \\
0.0 \\
1.3\end{array}$ & $\begin{array}{l}7.9 \\
0.0 \\
5.0\end{array}$ & $\begin{array}{l}--- \\
--- \\
---\end{array}$ & $\begin{array}{l}--- \\
--- \\
---\end{array}$ & $\begin{array}{r}458 \\
57 \\
241\end{array}$ \\
\hline $\begin{array}{ll}\text { LINE } & 1 \\
\text { A } & \\
B\end{array}$ & $\begin{array}{r}10380 \\
4032.0 \\
4009.0\end{array}$ & $\begin{array}{l}\text { S? } \\
\text { S? }\end{array}$ & $\begin{array}{l}664854 \\
665318\end{array}$ & $\begin{array}{l}6164561 \\
6165062\end{array}$ & $\begin{array}{r}23.7 \\
2.4\end{array}$ & $\begin{array}{l}0.8 \\
0.6\end{array}$ & $\begin{array}{r}69.2 \\
5.7\end{array}$ & $\begin{array}{l}0.6 \\
0.0\end{array}$ & $\begin{array}{r}53.3 \\
4.7\end{array}$ & $\begin{array}{r}12.9 \\
6.1\end{array}$ & $\begin{array}{l}--- \\
---\end{array}$ & $\begin{array}{l}--- \\
---\end{array}$ & $\begin{array}{l}308 \\
305\end{array}$ \\
\hline $\begin{array}{ll}\text { LINE } & 1 \\
\text { A } & \\
B\end{array}$ & $\begin{array}{l}10390 \\
4364.0 \\
4512.0\end{array}$ & $\begin{array}{l}\text { S? } \\
\text { S? }\end{array}$ & $\begin{array}{l}662871 \\
665766\end{array}$ & $\begin{array}{l}6162241 \\
6165198\end{array}$ & $\begin{array}{r}2.8 \\
36.4\end{array}$ & $\begin{array}{l}1.0 \\
0.4\end{array}$ & $\begin{array}{l}12.1 \\
86.2\end{array}$ & $\begin{array}{l}1.0 \\
0.2\end{array}$ & $\begin{array}{r}8.9 \\
70.0\end{array}$ & $\begin{array}{l}6.0 \\
4.2\end{array}$ & $\begin{array}{l}--- \\
---\end{array}$ & $\begin{array}{l}--- \\
---\end{array}$ & $\begin{array}{r}44 \\
0\end{array}$ \\
\hline $\begin{array}{l}\text { LINE } 1 \\
A\end{array}$ & $\begin{array}{l}10400 \\
4839.0\end{array}$ & S? & 663444 & 6162600 & 2.3 & 1.5 & 1.4 & 2.3 & 0.9 & 15.3 & --- & --- & 59 \\
\hline $\begin{array}{l}\text { LINE } 1 \\
A\end{array}$ & $\begin{array}{l}10410 \\
5214.0\end{array}$ & S? & 663662 & 6162455 & 0.0 & 0.0 & 0.0 & 0.0 & 0.0 & 0.0 & --- & --- & 103 \\
\hline
\end{tabular}

$\mathrm{CX}=$ COAXIAL

$\mathrm{CP}=$ COPLANAR

Note: EM values shown above

are local amplitudes

*Estimated Depth may be unreliable because the stronger part of the conductor may be deeper or to one side of the flight line, or because of a shallow dip or magnetite/overburden effects 
EM Anomaly List

\begin{tabular}{|c|c|c|c|c|c|c|c|c|c|c|c|c|c|}
\hline Label & Fid & Interp & $\begin{array}{c}\text { XUTM } \\
\mathrm{m}\end{array}$ & $\begin{array}{c}\text { YUTM } \\
\mathrm{m}\end{array}$ & $\begin{array}{l}\text { CX } \\
\text { Real } \\
\text { ppm }\end{array}$ & $\begin{array}{l}900 \mathrm{HZ} \\
\text { Quad } \\
\text { ppm }\end{array}$ & $\begin{array}{l}\text { CP } \\
\text { Real } \\
\text { ppm }\end{array}$ & $\begin{array}{l}900 \mathrm{HZ} \\
\text { Quad } \\
\text { ppm }\end{array}$ & $\begin{array}{l}\mathrm{CP} \\
\mathrm{Real} \\
\mathrm{ppm}\end{array}$ & $\begin{array}{l}7200 \mathrm{HZ} \\
\text { Quad } \\
\text { ppm }\end{array}$ & $\begin{array}{l}\text { Vertic } \\
\text { CoND } \\
\text { siemens }\end{array}$ & $\begin{array}{c}\text { al Dike } \\
\text { DEPTH* } \\
\text { m }\end{array}$ & $\begin{array}{c}\text { Mag. Corr } \\
\text { NT }\end{array}$ \\
\hline $\begin{array}{l}\text { LINE } \\
B \\
\text { C }\end{array}$ & $\begin{array}{l}10410 \\
5289.0 \\
5312.0\end{array}$ & $\begin{array}{l}\text { S? } \\
\text { S? }\end{array}$ & $\begin{array}{l}665257 \\
665709\end{array}$ & $\begin{array}{l}6164198 \\
6164651\end{array}$ & $\begin{array}{l}1.0 \\
6.0\end{array}$ & $\begin{array}{l}0.5 \\
0.3\end{array}$ & $\begin{array}{l}0.7 \\
6.6\end{array}$ & $\begin{array}{l}0.4 \\
0.1\end{array}$ & $\begin{array}{l}0.7 \\
5.4\end{array}$ & $\begin{array}{l}4.7 \\
3.9\end{array}$ & $\begin{array}{l}--- \\
---\end{array}$ & $\begin{array}{l}--- \\
---\end{array}$ & $\begin{array}{l}209 \\
357\end{array}$ \\
\hline $\begin{array}{l}\text { LINE } \\
\text { A } \\
B\end{array}$ & $\begin{array}{l}10440 \\
6270.0 \\
6061.0\end{array}$ & $\begin{array}{l}\text { S? } \\
\text { S? }\end{array}$ & $\begin{array}{l}661618 \\
667205\end{array}$ & $\begin{array}{l}6159482 \\
6165127\end{array}$ & $\begin{array}{l}0.9 \\
1.6\end{array}$ & $\begin{array}{l}3.6 \\
0.4\end{array}$ & $\begin{array}{l}1.1 \\
4.3\end{array}$ & $\begin{array}{l}3.8 \\
2.7\end{array}$ & $\begin{array}{l}1.7 \\
4.8\end{array}$ & $\begin{array}{r}11.7 \\
0.0\end{array}$ & $\begin{array}{l}--- \\
---\end{array}$ & $\begin{array}{l}--- \\
---\end{array}$ & $\begin{array}{r}2166 \\
80\end{array}$ \\
\hline $\begin{array}{l}\text { LINE } \\
A\end{array}$ & $\begin{array}{l}10460 \\
6778.0\end{array}$ & S & 663328 & 6160710 & 8.8 & 0.0 & 14.4 & 0.0 & 9.6 & 8.4 & --- & --- & 11 \\
\hline $\begin{array}{l}\text { LINE } \\
\text { A } \\
B\end{array}$ & $\begin{array}{r}10470 \\
7166.0 \\
7238.0\end{array}$ & $\begin{array}{r}\mathrm{S} ? \\
\mathrm{~S}\end{array}$ & $\begin{array}{l}664993 \\
666651\end{array}$ & $\begin{array}{l}6162155 \\
6163857\end{array}$ & $\begin{array}{l}2.5 \\
0.8\end{array}$ & $\begin{array}{l}1.5 \\
2.3\end{array}$ & $\begin{array}{l}4.5 \\
1.6\end{array}$ & $\begin{array}{l}0.9 \\
2.5\end{array}$ & $\begin{array}{l}0.0 \\
7.1\end{array}$ & $\begin{array}{r}8.4 \\
12.6\end{array}$ & $\begin{array}{l}--- \\
---\end{array}$ & $\begin{array}{l}--- \\
---\end{array}$ & $\begin{array}{r}296 \\
47\end{array}$ \\
\hline $\begin{array}{l}\text { LINE } \\
A\end{array}$ & $\begin{array}{l}10490 \\
7684.0\end{array}$ & $\mathrm{~S} ?$ & 664210 & 6160801 & 0.0 & 1.2 & 0.0 & 1.1 & 0.0 & 10.8 & --- & --- & 250 \\
\hline $\begin{array}{l}\operatorname{LINE} \\
\mathrm{A}\end{array}$ & $\begin{array}{l}10500 \\
8036.0\end{array}$ & S? & 664922 & 6161284 & 14.9 & 1.0 & 21.7 & 0.9 & 15.8 & 9.5 & --- & --- & 606 \\
\hline $\begin{array}{l}\text { LINE } \\
\text { A } \\
\text { B } \\
\text { C } \\
\text { D }\end{array}$ & $\begin{array}{r}10510 \\
8300.0 \\
8338.0 \\
8364.0 \\
8457.0\end{array}$ & $\begin{array}{l}\text { S? } \\
\text { S? } \\
\text { S? } \\
\text { S? }\end{array}$ & $\begin{array}{l}664561 \\
665170 \\
665571 \\
667264\end{array}$ & $\begin{array}{l}6160531 \\
6161206 \\
6161627 \\
6163342\end{array}$ & $\begin{array}{l}5.2 \\
0.6 \\
0.0 \\
0.0\end{array}$ & $\begin{array}{l}0.3 \\
1.3 \\
1.3 \\
0.0\end{array}$ & $\begin{array}{l}8.6 \\
6.4 \\
9.4 \\
0.0\end{array}$ & $\begin{array}{l}1.3 \\
1.2 \\
0.3 \\
0.0\end{array}$ & $\begin{array}{l}6.5 \\
3.5 \\
7.5 \\
0.0\end{array}$ & $\begin{array}{r}11.3 \\
10.5 \\
5.3 \\
0.0\end{array}$ & $\begin{array}{l}--- \\
--- \\
--- \\
---\end{array}$ & $\begin{array}{l}--- \\
--- \\
--- \\
---\end{array}$ & $\begin{array}{r}0 \\
386 \\
444 \\
146\end{array}$ \\
\hline $\begin{array}{l}\text { LINE } \\
\mathrm{A}\end{array}$ & $\begin{array}{l}10530 \\
10378.0\end{array}$ & S? & 665228 & 6160639 & 0.0 & 0.0 & 0.0 & 0.0 & 0.0 & 0.0 & --- & --- & 788 \\
\hline $\begin{array}{l}\text { LINE } \\
\text { A } \\
B\end{array}$ & $\begin{array}{l}10540 \\
10834.0 \\
10808.0\end{array}$ & $\begin{array}{r}S ? \\
S\end{array}$ & $\begin{array}{l}664445 \\
664989\end{array}$ & $\begin{array}{l}6159560 \\
6160216\end{array}$ & $\begin{array}{l}0.0 \\
7.7\end{array}$ & $\begin{array}{l}0.0 \\
2.3\end{array}$ & $\begin{array}{l}0.0 \\
7.7\end{array}$ & $\begin{array}{l}0.0 \\
1.8\end{array}$ & $\begin{array}{l}0.0 \\
4.8\end{array}$ & $\begin{array}{r}0.0 \\
10.3\end{array}$ & $\begin{array}{l}--- \\
---\end{array}$ & $\begin{array}{l}--- \\
---\end{array}$ & $\begin{array}{r}0 \\
34\end{array}$ \\
\hline $\begin{array}{l}\text { LINE } \\
A\end{array}$ & $\begin{array}{l}10550 \\
11026.0\end{array}$ & S & 665138 & 6159874 & 7.2 & 1.3 & 13.5 & 0.7 & 11.0 & 3.0 & --- & --- & 46 \\
\hline $\begin{array}{l}\text { LINE } \\
A\end{array}$ & $\begin{array}{l}10570 \\
2476.0\end{array}$ & $\mathrm{~S}$ & 666837 & 6161228 & 18.7 & 0.7 & 33.8 & 0.5 & 25.6 & 6.2 & --- & --- & 0 \\
\hline $\begin{array}{l}\text { LINE } \\
\text { A }\end{array}$ & $\begin{array}{r}10580 \\
2225.0\end{array}$ & S & 664598 & 6158504 & 0.2 & 2.7 & 0.3 & 3.1 & 1.7 & 22.5 & --- & --- & 50 \\
\hline $\begin{array}{l}\text { LINE } \\
\mathrm{A}\end{array}$ & $\begin{array}{l}10600 \\
1345.0\end{array}$ & S? & 667371 & 6161178 & 0.7 & 0.7 & 6.9 & 0.7 & 18.6 & 7.8 & --- & --- & 740 \\
\hline
\end{tabular}

$\mathrm{CX}=$ COAXIAL

$\mathrm{CP}=$ COPLANAR

Note: EM values shown above

are local amplitudes

*Estimated Depth may be unreliable because the stronger part of the conductor may be deeper or to one side of the flight line, or because of a shallow dip or magnetite/overburden effects 
EM Anomaly List

\begin{tabular}{|c|c|c|c|c|c|c|c|c|c|c|c|c|c|}
\hline Label & Fid & Interp & $\begin{array}{l}\text { XUTM } \\
\text { m }\end{array}$ & $\begin{array}{c}\text { YUTM } \\
\mathrm{m}\end{array}$ & $\begin{array}{ll}\text { CX } & 9 \\
\text { Real } & \\
\text { ppm }\end{array}$ & $\begin{array}{l}00 \mathrm{HZ} \\
\text { Quad } \\
\text { ppm }\end{array}$ & $\begin{array}{l}\text { CP } \\
\text { Real } \\
\text { ppm }\end{array}$ & $\begin{array}{l}900 \mathrm{HZ} \\
\text { Quad } \\
\text { ppm }\end{array}$ & $\begin{array}{l}\text { CP }{ }^{7} \\
\text { Real } \\
\text { ppm }\end{array}$ & $\begin{array}{l}200 \mathrm{HZ} \\
\text { Quad } \\
\text { ppm }\end{array}$ & $\begin{array}{l}\text { Vertica } \\
\text { Cond } \\
\text { siemens }\end{array}$ & $\begin{array}{c}\text { al Dike } \\
\text { DEPTH* } \\
\text { m }\end{array}$ & $\begin{array}{c}\text { Mag. Corr } \\
\text { NT }\end{array}$ \\
\hline $\begin{array}{l}\mathrm{LINE} \\
\mathrm{A}\end{array}$ & $\begin{array}{c}10620 \\
868.0\end{array}$ & S? & 665354 & 6158147 & 5.3 & 2.6 & 9.3 & 2.1 & 7.6 & 13.5 & --- & --- & 82 \\
\hline $\begin{array}{l}\text { LINE } \\
\mathrm{A}\end{array}$ & $\begin{array}{c}10630 \\
484.0\end{array}$ & S? & 668286 & 6160847 & 33.6 & 1.1 & 68.2 & 3.6 & 48.4 & 11.0 & --- & --- & 536 \\
\hline $\begin{array}{l}\text { LINE } \\
A\end{array}$ & $\begin{array}{r}10640 \\
3592.0\end{array}$ & S? & 666118 & 6158310 & 0.7 & 2.3 & 1.3 & 1.0 & 1.8 & 5.7 & --- & --- & 58 \\
\hline $\begin{array}{l}\text { LINE } \\
A\end{array}$ & $\begin{array}{l}10650 \\
3156.0\end{array}$ & $\mathrm{~S} ?$ & 666317 & 6158266 & 5.5 & 1.7 & 7.2 & 1.2 & 6.6 & 10.5 & --- & --- & 52 \\
\hline $\begin{array}{l}\text { LINE } \\
\text { A } \\
B\end{array}$ & $\begin{array}{r}10660 \\
2753.0 \\
2704.0\end{array}$ & $\begin{array}{l}\text { S? } \\
\text { S? }\end{array}$ & $\begin{array}{l}667639 \\
668550\end{array}$ & $\begin{array}{l}6159383 \\
6160341\end{array}$ & $\begin{array}{r}19.2 \\
0.3\end{array}$ & $\begin{array}{l}2.5 \\
0.9\end{array}$ & $\begin{array}{r}14.8 \\
4.1\end{array}$ & $\begin{array}{l}2.4 \\
1.5\end{array}$ & $\begin{array}{r}15.1 \\
3.3\end{array}$ & $\begin{array}{l}14.9 \\
10.9\end{array}$ & $\begin{array}{l}--- \\
---\end{array}$ & $\begin{array}{l}--- \\
---\end{array}$ & $\begin{array}{r}53 \\
0\end{array}$ \\
\hline $\begin{array}{l}\text { LINE } \\
\text { A } \\
\text { B }\end{array}$ & $\begin{array}{l}10670 \\
2494.0 \\
2611.0\end{array}$ & $\begin{array}{r}S ? \\
S\end{array}$ & $\begin{array}{l}667192 \\
668948\end{array}$ & $\begin{array}{l}6158758 \\
6160486\end{array}$ & $\begin{array}{l}0.0 \\
9.5\end{array}$ & $\begin{array}{l}0.0 \\
0.8\end{array}$ & $\begin{array}{r}0.0 \\
17.5\end{array}$ & $\begin{array}{l}0.0 \\
0.4\end{array}$ & $\begin{array}{r}0.0 \\
13.4\end{array}$ & $\begin{array}{l}0.0 \\
3.6\end{array}$ & $\begin{array}{l}--- \\
---\end{array}$ & $\begin{array}{l}--- \\
---\end{array}$ & $\begin{array}{l}0 \\
0\end{array}$ \\
\hline $\begin{array}{l}\text { LINE } \\
\text { A }\end{array}$ & $\begin{array}{l}10710 \\
1215.0\end{array}$ & $S ?$ & 668629 & 6158913 & 0.0 & 2.8 & 4.6 & 3.6 & 0.0 & 25.8 & --- & --- & 65 \\
\hline $\begin{array}{l}\text { LINE } \\
\text { A }\end{array}$ & $\begin{array}{c}10720 \\
845.0\end{array}$ & S & 668884 & 6158981 & 1.8 & 2.1 & 4.2 & 1.7 & 4.6 & 13.3 & --- & --- & 52 \\
\hline $\begin{array}{l}\text { LINE } \\
\mathrm{A}\end{array}$ & $\begin{array}{c}10760 \\
5552.0\end{array}$ & S? & 667507 & 6156384 & 0.0 & 0.0 & 0.0 & 0.0 & 0.0 & 0.0 & --- & --- & 0 \\
\hline $\begin{array}{l}\text { LINE } \\
\mathrm{A}\end{array}$ & $\begin{array}{l}10810 \\
4634.0\end{array}$ & S? & 668826 & 6156180 & 0.0 & 2.9 & 1.5 & 3.6 & 1.5 & 24.8 & --- & --- & 234 \\
\hline $\begin{array}{l}\text { LINE } \\
\text { A }\end{array}$ & $\begin{array}{r}10820 \\
4399.0\end{array}$ & S? & 669127 & 6156188 & 6.2 & 2.0 & 10.8 & 3.0 & 11.0 & 18.4 & --- & --- & 0 \\
\hline $\begin{array}{l}\text { LINE } \\
\text { A }\end{array}$ & $\begin{array}{r}10830 \\
4288.0\end{array}$ & S & 669137 & 6155946 & 2.2 & 1.2 & 4.4 & 0.9 & 3.1 & 6.7 & --- & --- & 18 \\
\hline $\begin{array}{l}\text { LINE } \\
\text { A }\end{array}$ & $\begin{array}{l}10880 \\
3286.0\end{array}$ & S? & 670763 & 6156160 & 18.1 & 1.3 & 24.2 & 1.0 & 17.8 & 7.6 & --- & --- & 0 \\
\hline $\begin{array}{l}\text { LINE } \\
\text { A } \\
\text { B } \\
\text { C }\end{array}$ & $\begin{array}{r}10900 \\
2699.0 \\
2743.0 \\
2756.0\end{array}$ & $\begin{array}{l}\text { S? } \\
\text { S? } \\
\text { S? }\end{array}$ & $\begin{array}{l}670205 \\
670393 \\
670517\end{array}$ & $\begin{array}{l}6155110 \\
6155322 \\
6155414\end{array}$ & $\begin{array}{r}205.1 \\
0.0 \\
0.0\end{array}$ & $\begin{array}{l}4.0 \\
0.0 \\
0.0\end{array}$ & $\begin{array}{r}73.3 \\
0.0 \\
0.0\end{array}$ & $\begin{array}{l}3.5 \\
0.0 \\
0.0\end{array}$ & $\begin{array}{r}208.4 \\
0.0 \\
0.0\end{array}$ & $\begin{array}{r}23.5 \\
0.0 \\
0.0\end{array}$ & $\begin{array}{l}--- \\
--- \\
---\end{array}$ & $\begin{array}{l}--- \\
--- \\
---\end{array}$ & $\begin{array}{r}0 \\
0 \\
379\end{array}$ \\
\hline
\end{tabular}

$\mathrm{CX}=$ COAXIAL

$\mathrm{CP}=$ COPLANAR

Note: EM values shown above

are local amplitudes

*Estimated Depth may be unreliable because the stronger part of the conductor may be deeper or to one side of the flight line, or because of a shallow dip or magnetite/overburden effects 
EM Anomaly List

\begin{tabular}{|c|c|c|c|c|c|c|c|c|c|c|c|c|c|}
\hline Label & Fid & Interp & $\begin{array}{l}\text { XUTM } \\
\text { m }\end{array}$ & $\begin{array}{c}\text { YUTM } \\
\text { m }\end{array}$ & $\begin{array}{ll}\text { CX } & 9 \\
\text { Real } & \\
\text { ppm }\end{array}$ & $\begin{array}{l}00 \mathrm{HZ} \\
\text { Quad } \\
\text { ppm }\end{array}$ & $\begin{array}{l}\text { CP } \\
\text { Real } \\
\text { ppm }\end{array}$ & $\begin{array}{c}900 \mathrm{HZ} \\
\text { Quad } \\
\text { ppm }\end{array}$ & $\begin{array}{l}\text { CP } 7 \\
\text { Real } \\
\text { ppm }\end{array}$ & $\begin{array}{l}200 \mathrm{HZ} \\
\text { Quad } \\
\text { ppm }\end{array}$ & $\begin{array}{l}\text { Vertica } \\
\text { CoND } \\
\text { siemens }\end{array}$ & $\begin{array}{c}\text { al Dike } \\
\text { DEPTH* } \\
\text { m }\end{array}$ & $\begin{array}{c}\text { Mag. Corr } \\
\text { NT }\end{array}$ \\
\hline $\begin{array}{l}\text { LINE } \\
A\end{array}$ & $\begin{array}{l}10910 \\
2590.0\end{array}$ & S? & 670558 & 6155244 & 0.0 & 0.0 & 0.0 & 0.0 & 0.0 & 0.0 & --- & --- & 0 \\
\hline $\begin{array}{l}\text { LINE } \\
\text { A } \\
\text { B } \\
\text { C } \\
\text { D }\end{array}$ & $\begin{array}{r}10920 \\
2295.0 \\
2329.0 \\
2364.0 \\
2397.0\end{array}$ & $\begin{array}{r}\text { S? } \\
\text { S? } \\
\text { S? } \\
\text { S }\end{array}$ & $\begin{array}{l}670895 \\
671349 \\
671781 \\
672182\end{array}$ & $\begin{array}{l}6155257 \\
6155690 \\
6156186 \\
6156713\end{array}$ & $\begin{array}{r}0.0 \\
18.9 \\
1.3 \\
1.2\end{array}$ & $\begin{array}{l}0.0 \\
2.9 \\
1.0 \\
1.9\end{array}$ & $\begin{array}{r}0.0 \\
44.5 \\
3.0 \\
1.4\end{array}$ & $\begin{array}{l}0.0 \\
1.3 \\
1.7 \\
2.5\end{array}$ & $\begin{array}{r}0.0 \\
37.9 \\
2.8 \\
1.3\end{array}$ & $\begin{array}{r}0.0 \\
11.9 \\
12.9 \\
13.3\end{array}$ & $\begin{array}{l}--- \\
--- \\
--- \\
---\end{array}$ & $\begin{array}{l}--- \\
--- \\
--- \\
---\end{array}$ & $\begin{array}{r}0 \\
10 \\
120 \\
292\end{array}$ \\
\hline $\begin{array}{l}\text { LINE } \\
\text { A } \\
B\end{array}$ & $\begin{array}{l}10930 \\
2074.0 \\
2063.0\end{array}$ & $\begin{array}{l}\text { S? } \\
\text { S? }\end{array}$ & $\begin{array}{l}671246 \\
671353\end{array}$ & $\begin{array}{l}6155349 \\
6155466\end{array}$ & $\begin{array}{r}681.0 \\
0.0\end{array}$ & $\begin{array}{r}15.5 \\
0.0\end{array}$ & $\begin{array}{r}1326.4 \\
0.0\end{array}$ & $\begin{array}{r}35.1 \\
0.0\end{array}$ & $\begin{array}{r}1066.2 \\
0.0\end{array}$ & $\begin{array}{r}95.9 \\
0.0\end{array}$ & $\begin{array}{l}--- \\
---\end{array}$ & $\begin{array}{l}--- \\
---\end{array}$ & $\begin{array}{r}300 \\
2752\end{array}$ \\
\hline $\begin{array}{l}\text { LINE } \\
\text { A } \\
\text { B } \\
\text { C }\end{array}$ & $\begin{array}{r}10940 \\
1864.0 \\
1924.0 \\
1958.0\end{array}$ & $\begin{array}{r}\text { S } \\
\text { S? } \\
\text { S? }\end{array}$ & $\begin{array}{l}671302 \\
672107 \\
672736\end{array}$ & $\begin{array}{l}6155079 \\
6155998 \\
6156788\end{array}$ & $\begin{array}{r}12.9 \\
3.5 \\
0.0\end{array}$ & $\begin{array}{l}0.8 \\
0.9 \\
0.0\end{array}$ & $\begin{array}{r}32.7 \\
12.6 \\
0.0\end{array}$ & $\begin{array}{l}0.9 \\
1.1 \\
0.0\end{array}$ & $\begin{array}{r}25.9 \\
10.6 \\
0.0\end{array}$ & $\begin{array}{l}4.7 \\
6.5 \\
0.0\end{array}$ & $\begin{array}{l}--- \\
--- \\
---\end{array}$ & $\begin{array}{l}--- \\
--- \\
---\end{array}$ & $\begin{array}{r}80 \\
266 \\
78\end{array}$ \\
\hline $\begin{array}{l}\text { LINE } \\
A\end{array}$ & $\begin{array}{c}11020 \\
979.0\end{array}$ & S? & 672865 & 6154466 & 5.9 & 0.2 & 11.8 & 0.4 & 9.6 & 11.2 & --- & --- & 0 \\
\hline $\begin{array}{l}\text { LINE } \\
A\end{array}$ & $\begin{array}{l}19010 \\
2890.0\end{array}$ & S? & 665809 & 6165519 & 30.3 & 0.0 & 1.4 & 0.0 & 1.0 & 4.6 & --- & --- & 39 \\
\hline $\begin{array}{l}\text { LINE } \\
\text { A }\end{array}$ & $\begin{array}{l}19011 \\
3950.0\end{array}$ & S? & 671553 & 6156194 & 4.2 & 1.3 & 2.4 & 0.9 & 2.2 & 8.3 & --- & --- & 0 \\
\hline $\begin{array}{l}\text { LINE } \\
\text { A } \\
B \\
C\end{array}$ & $\begin{array}{r}19020 \\
3681.0 \\
3749.0 \\
3753.0\end{array}$ & $\begin{array}{l}\text { S } \\
\text { S? } \\
\text { S? }\end{array}$ & $\begin{array}{l}668662 \\
670460 \\
670566\end{array}$ & $\begin{array}{l}6156365 \\
6155341 \\
6155310\end{array}$ & $\begin{array}{l}0.4 \\
0.0 \\
0.0\end{array}$ & $\begin{array}{l}1.0 \\
0.0 \\
0.0\end{array}$ & $\begin{array}{l}0.4 \\
0.0 \\
0.0\end{array}$ & $\begin{array}{l}1.3 \\
0.0 \\
0.0\end{array}$ & $\begin{array}{l}0.0 \\
0.0 \\
0.0\end{array}$ & $\begin{array}{l}5.4 \\
0.0 \\
0.0\end{array}$ & $\begin{array}{l}--- \\
--- \\
---\end{array}$ & $\begin{array}{l}--- \\
--- \\
---\end{array}$ & $\begin{array}{r}106 \\
2474 \\
3782\end{array}$ \\
\hline
\end{tabular}

$\mathrm{CX}=$ COAXIAL

$\mathrm{CP}=$ COPLANAR

Note: EM values shown above

Kasaan are local amplitudes

*Estimated Depth may be unreliable because the stronger part of the conductor may be deeper or to one side of the flight line, or because of shallow dip or magnetite/overburden effects 
EM Anomaly List

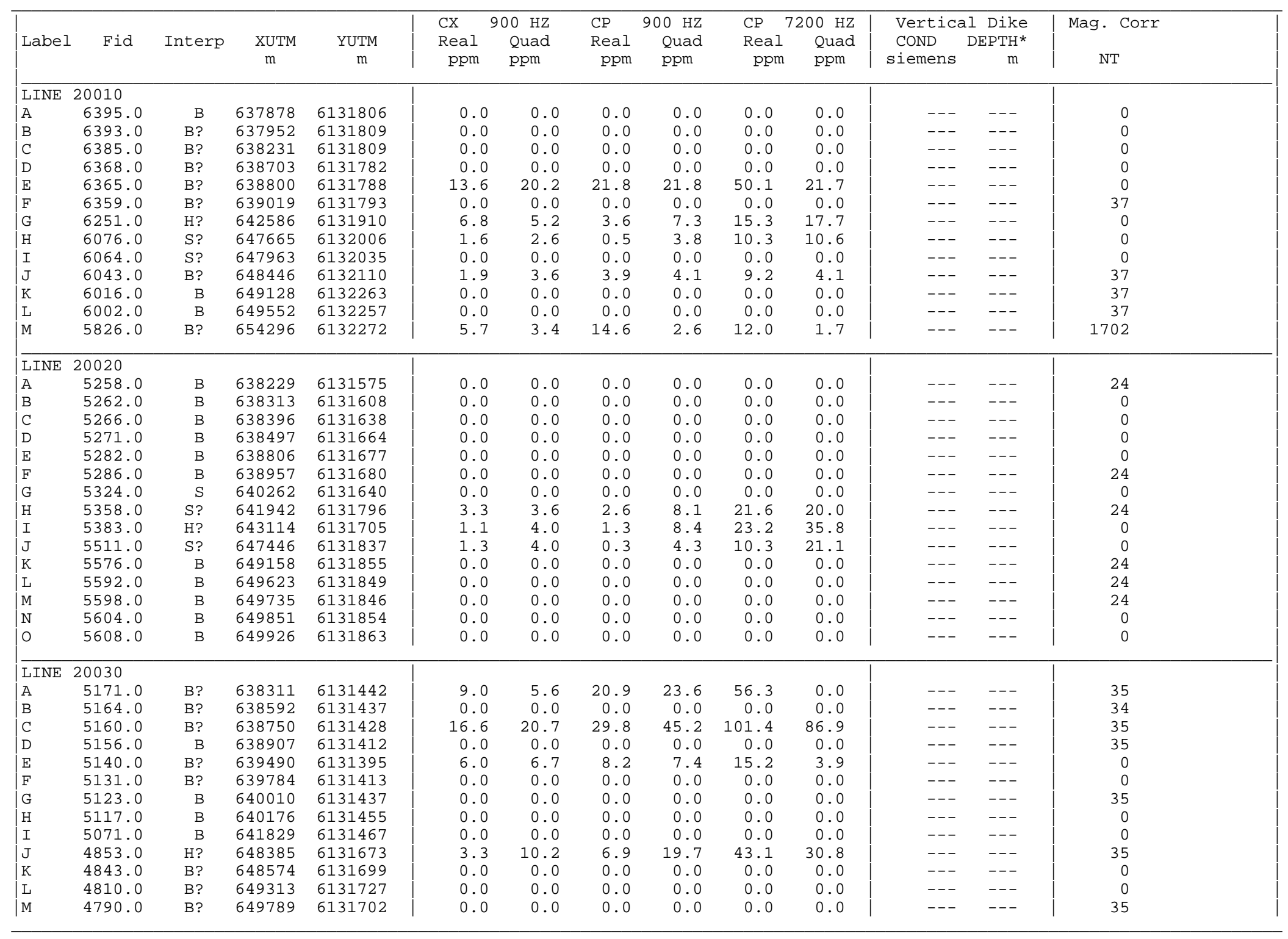

$\mathrm{CX}=$ COAXIAL

$\mathrm{CP}=$ COPLANAR

Note: EM values shown above

are local amplitudes

Estimated Depth may be unreliable because the stronger part of the conductor may be deeper or to one side of the flight line, or because of a shallow dip or magnetite/overburden effects 
EM Anomaly List

\begin{tabular}{|c|c|c|c|c|c|c|c|c|c|c|c|c|c|}
\hline Label & Fid & Interp & $\begin{array}{c}\text { XUTM } \\
\text { m }\end{array}$ & $\begin{array}{c}\text { YUTM } \\
\mathrm{m}\end{array}$ & $\begin{array}{l}\text { CX } \\
\text { Real } \\
\text { ppm }\end{array}$ & $\begin{array}{l}00 \mathrm{HZ} \\
\text { Quad } \\
\text { ppm }\end{array}$ & $\begin{array}{l}\text { CP } \\
\text { Real } \\
\text { ppm }\end{array}$ & $\begin{array}{l}900 \mathrm{HZ} \\
\text { Quad } \\
\text { ppm }\end{array}$ & $\begin{array}{l}\text { CP } 7 \\
\text { Real } \\
\text { ppm }\end{array}$ & $\begin{array}{l}200 \mathrm{HZ} \\
\text { Quad } \\
\text { ppm }\end{array}$ & $\begin{array}{l}\text { Vertica } \\
\text { Cond } \\
\text { siemens }\end{array}$ & $\begin{array}{c}\text { al Dike } \\
\text { DEPTH* } \\
\text { m }\end{array}$ & $\begin{array}{c}\text { Mag. Corr } \\
\text { NT }\end{array}$ \\
\hline $\begin{array}{l}\text { LINE } \\
N \\
O \\
P\end{array}$ & $\begin{array}{r}20030 \\
4788.0 \\
4777.0 \\
4774.0\end{array}$ & $\begin{array}{l}\text { B? } \\
\text { B? } \\
\text { B? }\end{array}$ & $\begin{array}{l}649837 \\
650097 \\
650163\end{array}$ & $\begin{array}{l}6131699 \\
6131686 \\
6131688\end{array}$ & $\begin{array}{r}0.0 \\
11.8 \\
0.0\end{array}$ & $\begin{array}{r}0.0 \\
12.1 \\
0.0\end{array}$ & $\begin{array}{r}0.0 \\
18.9 \\
0.0\end{array}$ & $\begin{array}{r}0.0 \\
20.1 \\
0.0\end{array}$ & $\begin{array}{r}0.0 \\
41.8 \\
0.0\end{array}$ & $\begin{array}{r}0.0 \\
15.3 \\
0.0\end{array}$ & $\begin{array}{l}--- \\
--- \\
---\end{array}$ & $\begin{array}{l}--- \\
--- \\
---\end{array}$ & $\begin{array}{l}0 \\
0 \\
0\end{array}$ \\
\hline $\begin{array}{l}\text { LINE } \\
\text { A } \\
\text { B } \\
\text { C } \\
\text { D } \\
\text { E } \\
\text { F } \\
\text { G } \\
\text { H } \\
\text { I } \\
\text { J } \\
\text { K } \\
\text { L } \\
\text { M } \\
\text { N }\end{array}$ & $\begin{array}{r}20040 \\
4131.0 \\
4153.0 \\
4172.0 \\
4245.0 \\
4251.0 \\
4358.0 \\
4370.0 \\
4400.0 \\
4408.0 \\
4430.0 \\
4437.0 \\
4445.0 \\
4451.0 \\
4454.0\end{array}$ & $\begin{array}{r}\text { B } \\
\text { B } \\
\text { B } \\
\text { B? } \\
\text { H } \\
\text { B? } \\
\text { B? } \\
\text { B } \\
\text { B } \\
\text { B? } \\
\text { B? } \\
\text { B? } \\
\text { B } \\
\text { B }\end{array}$ & $\begin{array}{l}638610 \\
639359 \\
640292 \\
643309 \\
643582 \\
647480 \\
647811 \\
648367 \\
648616 \\
649482 \\
649757 \\
650001 \\
650150 \\
650225\end{array}$ & $\begin{array}{l}6131228 \\
6131240 \\
6131248 \\
6131331 \\
6131348 \\
6131352 \\
6131402 \\
6131492 \\
6131508 \\
6131534 \\
6131538 \\
6131545 \\
6131552 \\
6131555\end{array}$ & $\begin{array}{r}0.0 \\
0.0 \\
0.0 \\
5.2 \\
0.0 \\
0.0 \\
0.0 \\
5.4 \\
0.0 \\
7.9 \\
0.0 \\
18.8 \\
0.0 \\
0.0\end{array}$ & $\begin{array}{r}0.0 \\
0.0 \\
0.0 \\
4.9 \\
0.0 \\
0.0 \\
0.0 \\
2.5 \\
0.0 \\
7.5 \\
0.0 \\
19.3 \\
0.0 \\
0.0\end{array}$ & $\begin{array}{r}0.0 \\
0.0 \\
0.0 \\
2.0 \\
0.0 \\
0.0 \\
0.0 \\
2.9 \\
0.0 \\
3.1 \\
0.0 \\
23.9 \\
0.0 \\
0.0\end{array}$ & $\begin{array}{r}0.0 \\
0.0 \\
0.0 \\
5.4 \\
0.0 \\
0.0 \\
0.0 \\
2.7 \\
0.0 \\
7.9 \\
0.0 \\
25.7 \\
0.0 \\
0.0\end{array}$ & $\begin{array}{r}0.0 \\
0.0 \\
0.0 \\
13.0 \\
0.0 \\
0.0 \\
0.0 \\
5.7 \\
0.0 \\
18.0 \\
0.0 \\
60.4 \\
0.0 \\
0.0\end{array}$ & $\begin{array}{r}0.0 \\
0.0 \\
0.0 \\
6.5 \\
0.0 \\
0.0 \\
0.0 \\
2.8 \\
0.0 \\
24.0 \\
0.0 \\
33.5 \\
0.0 \\
0.0\end{array}$ & $\begin{array}{l}--- \\
--- \\
--- \\
--- \\
--- \\
--- \\
--- \\
--- \\
--- \\
--- \\
--- \\
--- \\
--- \\
---\end{array}$ & $\begin{array}{l}--- \\
--- \\
--- \\
--- \\
--- \\
--- \\
--- \\
--- \\
--- \\
--- \\
--- \\
--- \\
--- \\
---\end{array}$ & $\begin{array}{r}28 \\
28 \\
0 \\
28 \\
0 \\
28 \\
28 \\
27 \\
25 \\
0 \\
28 \\
28 \\
0 \\
0 \\
0\end{array}$ \\
\hline $\begin{array}{l}\text { LINE } \\
\text { A } \\
\text { B } \\
\text { C } \\
\text { D } \\
\text { E } \\
\text { F } \\
\text { G } \\
\text { H }\end{array}$ & $\begin{array}{r}20050 \\
3667.0 \\
3587.0 \\
3577.0 \\
3573.0 \\
3513.0 \\
3503.0 \\
3478.0 \\
3474.0\end{array}$ & $\begin{array}{r}\text { S? } \\
\text { B? } \\
\text { B } \\
\text { B } \\
\text { H? } \\
\text { H? } \\
\text { B? } \\
\text { B? }\end{array}$ & $\begin{array}{l}645434 \\
648076 \\
648377 \\
648486 \\
650248 \\
650635 \\
651566 \\
651699\end{array}$ & $\begin{array}{l}6131303 \\
6131254 \\
6131263 \\
6131268 \\
6131363 \\
6131325 \\
6131400 \\
6131415\end{array}$ & $\begin{array}{l}0.0 \\
0.0 \\
6.6 \\
0.0 \\
0.0 \\
7.9 \\
0.0 \\
4.3\end{array}$ & $\begin{array}{l}0.0 \\
0.0 \\
2.9 \\
0.0 \\
0.0 \\
4.1 \\
0.0 \\
7.2\end{array}$ & $\begin{array}{r}0.0 \\
0.0 \\
19.2 \\
0.0 \\
0.0 \\
5.8 \\
0.0 \\
10.2\end{array}$ & $\begin{array}{r}0.0 \\
0.0 \\
3.6 \\
0.0 \\
0.0 \\
4.4 \\
0.0 \\
14.1\end{array}$ & $\begin{array}{r}0.0 \\
0.0 \\
18.0 \\
0.0 \\
0.0 \\
10.2 \\
0.0 \\
24.4\end{array}$ & $\begin{array}{r}0.0 \\
0.0 \\
15.6 \\
0.0 \\
0.0 \\
2.3 \\
0.0 \\
20.0\end{array}$ & $\begin{array}{l}--- \\
--- \\
--- \\
--- \\
--- \\
--- \\
--- \\
---\end{array}$ & $\begin{array}{l}--- \\
--- \\
--- \\
--- \\
--- \\
--- \\
--- \\
---\end{array}$ & $\begin{array}{r}61 \\
0 \\
57 \\
0 \\
0 \\
0 \\
0 \\
0\end{array}$ \\
\hline $\begin{array}{l}\text { LINE } \\
\text { A } \\
\text { B } \\
\text { C } \\
\text { D } \\
\text { E } \\
\text { F } \\
\text { G } \\
\text { H }\end{array}$ & $\begin{array}{r}20051 \\
4007.0 \\
3849.0 \\
3841.0 \\
3833.0 \\
3829.0 \\
3796.0 \\
3789.0 \\
3782.0\end{array}$ & $\begin{array}{l}\text { S? } \\
\text { B? } \\
\text { B? } \\
\text { B? } \\
\text { B? } \\
\text { B? } \\
\text { B? } \\
\text { B? }\end{array}$ & $\begin{array}{l}637675 \\
643377 \\
643667 \\
643926 \\
644052 \\
644941 \\
645230 \\
645465\end{array}$ & $\begin{array}{l}6130972 \\
6131196 \\
6131222 \\
6131196 \\
6131171 \\
6131148 \\
6131197 \\
6131226\end{array}$ & $\begin{array}{r}0.8 \\
5.8 \\
13.8 \\
0.0 \\
0.0 \\
0.0 \\
0.0 \\
0.0\end{array}$ & $\begin{array}{r}5.0 \\
8.1 \\
19.9 \\
0.0 \\
0.0 \\
0.0 \\
0.0 \\
0.0\end{array}$ & $\begin{array}{r}1.7 \\
12.0 \\
15.5 \\
0.0 \\
0.0 \\
0.0 \\
0.0 \\
0.0\end{array}$ & $\begin{array}{r}4.0 \\
20.5 \\
31.7 \\
0.0 \\
0.0 \\
0.0 \\
0.0 \\
0.0\end{array}$ & $\begin{array}{r}6.8 \\
47.5 \\
69.8 \\
0.0 \\
0.0 \\
0.0 \\
0.0 \\
0.0\end{array}$ & $\begin{array}{r}25.7 \\
40.3 \\
58.7 \\
0.0 \\
0.0 \\
0.0 \\
0.0 \\
0.0\end{array}$ & $\begin{array}{l}--- \\
--- \\
--- \\
--- \\
--- \\
--- \\
--- \\
---\end{array}$ & $\begin{array}{l}--- \\
--- \\
--- \\
--- \\
--- \\
--- \\
--- \\
---\end{array}$ & $\begin{array}{r}5 \\
5 \\
0 \\
5 \\
22 \\
5 \\
0 \\
10\end{array}$ \\
\hline $\begin{array}{l}\text { LINE } \\
\text { A } \\
\text { B } \\
\text { C } \\
\text { D } \\
\text { E }\end{array}$ & $\begin{array}{r}20060 \\
2836.0 \\
2914.0 \\
2938.0 \\
2954.0 \\
2957.0\end{array}$ & $\begin{array}{r}\text { S? } \\
\text { S } \\
\text { B } \\
\mathrm{H} \\
\mathrm{H}\end{array}$ & $\begin{array}{l}639271 \\
642373 \\
643329 \\
643773 \\
643917\end{array}$ & $\begin{array}{l}6130816 \\
6130948 \\
6130926 \\
6130934 \\
6130938\end{array}$ & $\begin{array}{l}0.0 \\
1.9 \\
0.0 \\
0.0 \\
0.0\end{array}$ & $\begin{array}{l}0.0 \\
2.3 \\
0.0 \\
0.0 \\
0.0\end{array}$ & $\begin{array}{l}0.0 \\
1.3 \\
0.0 \\
0.0 \\
0.0\end{array}$ & $\begin{array}{l}0.0 \\
2.8 \\
0.0 \\
0.0 \\
0.0\end{array}$ & $\begin{array}{l}0.0 \\
8.3 \\
0.0 \\
0.0 \\
0.0\end{array}$ & $\begin{array}{l}0.0 \\
5.3 \\
0.0 \\
0.0 \\
0.0\end{array}$ & $\begin{array}{l}--- \\
--- \\
--- \\
--- \\
---\end{array}$ & $\begin{array}{l}--- \\
--- \\
--- \\
--- \\
---\end{array}$ & $\begin{array}{r}0 \\
25 \\
25 \\
0 \\
18\end{array}$ \\
\hline
\end{tabular}

$\mathrm{CX}=$ COAXIAL

Note: EM values shown above

are local amplitudes

*Estimated Depth may be unreliable because the stronger part of the conductor may be deeper or to one side of the flight line, or because of a shallow dip or magnetite/overburden effects 
EM Anomaly List

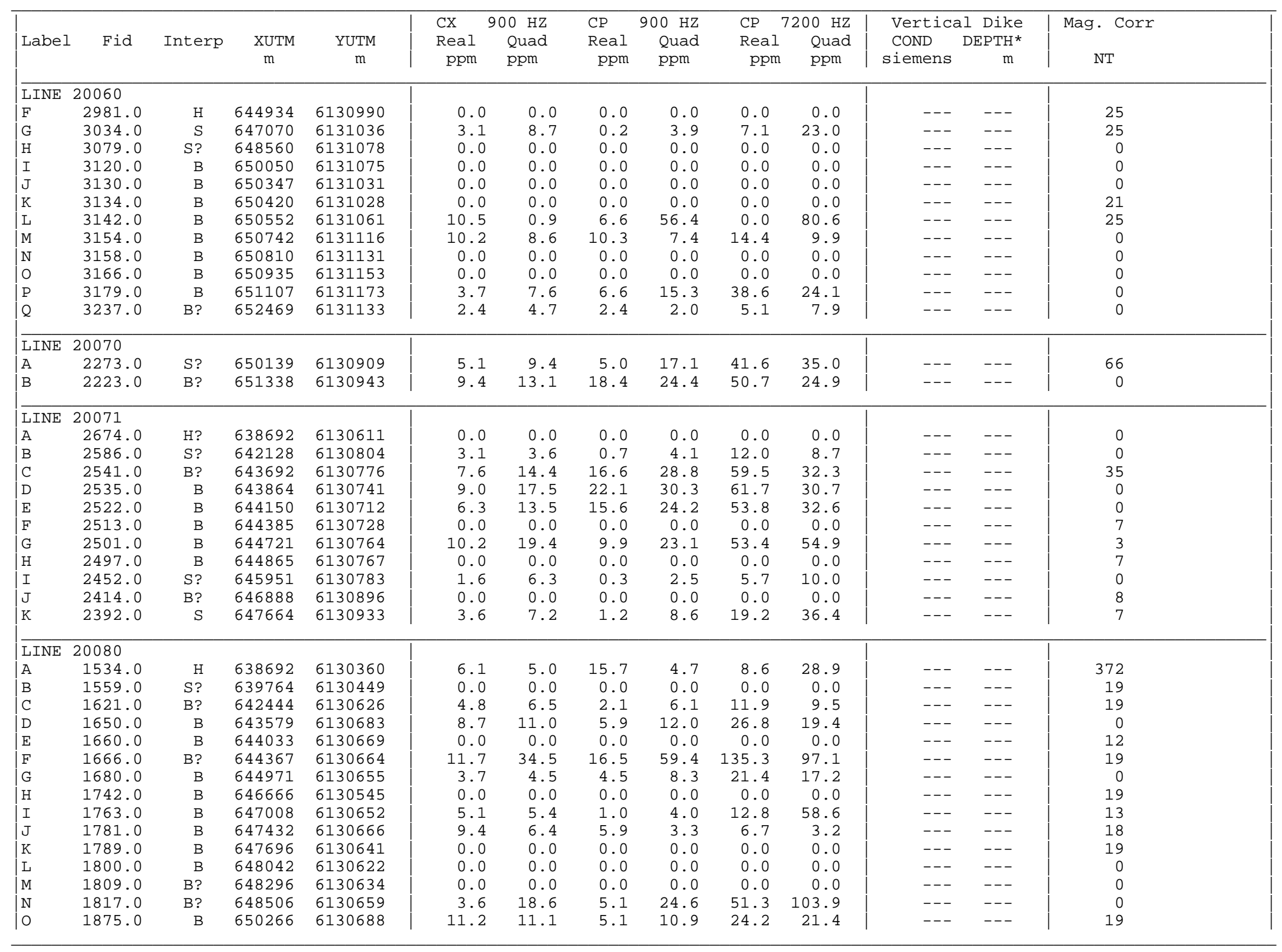

$\mathrm{CX}=$ COAXIAL

Note: EM values shown above

are local amplitudes

Hetta - North/South of 5515
*Estimated Depth may be unreliable because the stronger part of the conductor may be deeper or to one side of the flight line, or because of a shallow dip or magnetite/overburden effects 
EM Anomaly List

\begin{tabular}{|c|c|c|c|c|c|c|c|c|c|c|c|c|c|}
\hline Label & Fid & Interp & $\begin{array}{l}\text { XUTM } \\
\text { m }\end{array}$ & $\begin{array}{c}\text { YUTM } \\
\mathrm{m}\end{array}$ & $\begin{array}{l}\text { CX } \\
\text { Real } \\
\text { ppm }\end{array}$ & $\begin{array}{c}900 \mathrm{HZ} \\
\text { Quad } \\
\text { ppm }\end{array}$ & $\begin{array}{l}\text { CP } \\
\text { Real } \\
\text { ppm }\end{array}$ & $\begin{array}{c}900 \mathrm{HZ} \\
\text { Quad } \\
\text { ppm }\end{array}$ & $\begin{array}{l}\text { CP } \\
\text { Real } \\
\text { ppm }\end{array}$ & $\begin{array}{c}7200 \mathrm{HZ} \\
\text { Quad } \\
\text { ppm }\end{array}$ & $\begin{array}{l}\text { Vertica } \\
\text { COND } \\
\text { siemens }\end{array}$ & $\begin{array}{c}\text { al Dike } \\
\text { DEPTH* } \\
\text { m }\end{array}$ & $\begin{array}{c}\text { Mag. Corr } \\
\text { NT }\end{array}$ \\
\hline $\begin{array}{l}\text { LINE } \\
P \\
Q \\
R\end{array}$ & $\begin{array}{l}20080 \\
1891.0 \\
1993.0 \\
1996.0\end{array}$ & $\begin{array}{l}\text { B } \\
\text { B } \\
\text { B }\end{array}$ & $\begin{array}{l}650601 \\
652239 \\
652331\end{array}$ & $\begin{array}{l}6130603 \\
6130759 \\
6130773\end{array}$ & $\begin{array}{l}9.5 \\
0.0 \\
0.0\end{array}$ & $\begin{array}{r}14.5 \\
0.0 \\
0.0\end{array}$ & $\begin{array}{r}23.9 \\
0.0 \\
0.0\end{array}$ & $\begin{array}{r}19.3 \\
0.0 \\
0.0\end{array}$ & $\begin{array}{r}41.5 \\
0.0 \\
0.0\end{array}$ & $\begin{array}{r}27.1 \\
0.0 \\
0.0\end{array}$ & $\begin{array}{l}--- \\
--- \\
---\end{array}$ & $\begin{array}{l}--- \\
--- \\
---\end{array}$ & $\begin{array}{r}19 \\
104 \\
19\end{array}$ \\
\hline $\begin{array}{l}\text { LINE } \\
\text { A } \\
\text { B } \\
\text { C } \\
\text { D } \\
\text { E } \\
\text { F } \\
\text { G } \\
\text { H } \\
\text { I } \\
\text { J } \\
\text { K } \\
\text { L } \\
\text { M } \\
\text { N } \\
\text { O } \\
\text { P } \\
\text { Q } \\
\text { R } \\
\text { S } \\
\text { T } \\
\text { U } \\
\text { V } \\
\text { W } \\
X \\
\text { Y } \\
\text { Z } \\
\text { AA }\end{array}$ & $\begin{array}{r}20090 \\
1298.0 \\
1295.0 \\
1260.0 \\
1229.0 \\
1190.0 \\
1184.0 \\
1173.0 \\
1169.0 \\
1165.0 \\
1157.0 \\
1151.0 \\
1149.0 \\
1084.0 \\
1081.0 \\
1065.0 \\
1055.0 \\
1046.0 \\
1029.0 \\
1024.0 \\
1019.0 \\
1010.0 \\
996.0 \\
965.0 \\
939.0 \\
867.0 \\
862.0 \\
858.0\end{array}$ & $\begin{array}{r}\text { B } \\
\text { B } \\
\text { S? } \\
\text { B? } \\
\text { B } \\
\text { B } \\
\text { B } \\
\text { B } \\
\text { B } \\
\text { B } \\
\text { B } \\
\text { B } \\
\text { B } \\
\text { B? } \\
\text { B } \\
\text { B } \\
\text { B? } \\
\text { B? } \\
\text { B } \\
\text { B } \\
\text { B } \\
\text { S? } \\
\text { B? } \\
\text { B? } \\
\text { B } \\
\text { B } \\
\text { B }\end{array}$ & $\begin{array}{l}640067 \\
640172 \\
641400 \\
642400 \\
643582 \\
643672 \\
643925 \\
644028 \\
644134 \\
644365 \\
644563 \\
644646 \\
645968 \\
646015 \\
646348 \\
646599 \\
646839 \\
647337 \\
647519 \\
647706 \\
648042 \\
648555 \\
649489 \\
650218 \\
652180 \\
652363 \\
652520\end{array}$ & $\begin{array}{l}6130240 \\
6130239 \\
6130404 \\
6130543 \\
6130520 \\
6130503 \\
6130464 \\
6130452 \\
6130441 \\
6130426 \\
6130421 \\
6130421 \\
6130427 \\
6130427 \\
6130434 \\
6130437 \\
6130407 \\
6130419 \\
6130421 \\
6130422 \\
6130425 \\
6130449 \\
6130491 \\
6130556 \\
6130625 \\
6130651 \\
6130648\end{array}$ & $\begin{array}{r}0.0 \\
25.7 \\
0.0 \\
4.4 \\
0.0 \\
0.0 \\
0.0 \\
0.0 \\
0.0 \\
6.6 \\
0.0 \\
0.0 \\
0.0 \\
5.7 \\
0.0 \\
6.5 \\
0.0 \\
4.6 \\
0.0 \\
0.0 \\
0.0 \\
0.0 \\
2.6 \\
2.2 \\
0.0 \\
0.0 \\
0.0\end{array}$ & $\begin{array}{r}0.0 \\
60.5 \\
0.0 \\
4.9 \\
0.0 \\
0.0 \\
0.0 \\
0.0 \\
0.0 \\
5.1 \\
0.0 \\
0.0 \\
0.0 \\
23.9 \\
0.0 \\
4.5 \\
0.0 \\
7.7 \\
0.0 \\
0.0 \\
0.0 \\
0.0 \\
5.4 \\
5.9 \\
0.0 \\
0.0 \\
0.0\end{array}$ & $\begin{array}{r}0.0 \\
20.8 \\
0.0 \\
4.9 \\
0.0 \\
0.0 \\
0.0 \\
0.0 \\
0.0 \\
12.2 \\
0.0 \\
0.0 \\
0.0 \\
6.6 \\
0.0 \\
7.9 \\
0.0 \\
19.6 \\
0.0 \\
0.0 \\
0.0 \\
0.0 \\
3.5 \\
2.4 \\
0.0 \\
0.0 \\
0.0\end{array}$ & $\begin{array}{r}0.0 \\
75.0 \\
0.0 \\
6.7 \\
0.0 \\
0.0 \\
0.0 \\
0.0 \\
0.0 \\
8.8 \\
0.0 \\
0.0 \\
0.0 \\
30.9 \\
0.0 \\
10.3 \\
0.0 \\
23.6 \\
0.0 \\
0.0 \\
0.0 \\
0.0 \\
6.8 \\
8.5 \\
0.0 \\
0.0 \\
0.0\end{array}$ & $\begin{array}{r}0.0 \\
166.9 \\
0.0 \\
14.1 \\
0.0 \\
0.0 \\
0.0 \\
0.0 \\
0.0 \\
18.1 \\
0.0 \\
0.0 \\
0.0 \\
72.2 \\
0.0 \\
21.6 \\
0.0 \\
52.7 \\
0.0 \\
0.0 \\
0.0 \\
0.0 \\
15.0 \\
23.5 \\
0.0 \\
0.0 \\
0.0\end{array}$ & $\begin{array}{r}0.0 \\
210.1 \\
0.0 \\
8.1 \\
0.0 \\
0.0 \\
0.0 \\
0.0 \\
0.0 \\
11.5 \\
0.0 \\
0.0 \\
0.0 \\
124.2 \\
0.0 \\
8.4 \\
0.0 \\
18.7 \\
0.0 \\
0.0 \\
0.0 \\
0.0 \\
6.1 \\
21.6 \\
0.0 \\
0.0 \\
0.0\end{array}$ & $\begin{array}{l}--- \\
--- \\
--- \\
--- \\
--- \\
--- \\
--- \\
--- \\
--- \\
--- \\
--- \\
--- \\
--- \\
--- \\
--- \\
--- \\
--- \\
--- \\
--- \\
--- \\
--- \\
--- \\
--- \\
--- \\
--- \\
--- \\
---\end{array}$ & $\begin{array}{l}--- \\
--- \\
--- \\
--- \\
--- \\
--- \\
--- \\
--- \\
--- \\
--- \\
--- \\
--- \\
--- \\
--- \\
--- \\
--- \\
--- \\
--- \\
--- \\
--- \\
--- \\
--- \\
--- \\
--- \\
--- \\
--- \\
---\end{array}$ & $\begin{array}{r}16 \\
16 \\
15 \\
16 \\
0 \\
0 \\
0 \\
16 \\
0 \\
0 \\
16 \\
0 \\
16 \\
16 \\
16 \\
0 \\
0 \\
14 \\
0 \\
16 \\
16 \\
0 \\
0 \\
16 \\
392 \\
0 \\
16\end{array}$ \\
\hline $\begin{array}{l}\text { LINE } \\
\text { A } \\
\text { B } \\
\text { C } \\
\text { D } \\
\text { E } \\
\text { F } \\
\text { G } \\
\text { H } \\
\text { I } \\
\text { J } \\
\text { K } \\
\text { L }\end{array}$ & $\begin{array}{r}20100 \\
334.0 \\
349.0 \\
373.0 \\
404.0 \\
410.0 \\
425.0 \\
428.0 \\
437.0 \\
440.0 \\
452.0 \\
470.0 \\
485.0\end{array}$ & $\begin{array}{r}\text { B? } \\
\text { H } \\
\text { B? } \\
\text { B } \\
\text { B } \\
\text { B } \\
\text { B } \\
\text { B } \\
\text { B } \\
\text { B } \\
\text { B } \\
\text { B }\end{array}$ & $\begin{array}{l}640283 \\
641052 \\
642338 \\
643636 \\
643826 \\
644265 \\
644361 \\
644660 \\
644758 \\
645119 \\
645884 \\
646340\end{array}$ & $\begin{array}{l}6130033 \\
6130116 \\
6130190 \\
6130241 \\
6130257 \\
6130281 \\
6130291 \\
6130301 \\
6130296 \\
6130258 \\
6130196 \\
6130217\end{array}$ & $\begin{array}{r}0.0 \\
9.7 \\
5.6 \\
0.0 \\
0.0 \\
0.0 \\
13.6 \\
4.4 \\
0.0 \\
3.9 \\
0.0 \\
0.0\end{array}$ & $\begin{array}{r}0.0 \\
12.7 \\
15.5 \\
0.0 \\
0.0 \\
0.0 \\
11.1 \\
9.3 \\
0.0 \\
1.5 \\
0.0 \\
0.0\end{array}$ & $\begin{array}{r}0.0 \\
14.0 \\
6.1 \\
0.0 \\
0.0 \\
0.0 \\
15.0 \\
13.0 \\
0.0 \\
3.0 \\
0.0 \\
0.0\end{array}$ & $\begin{array}{r}0.0 \\
18.3 \\
15.7 \\
0.0 \\
0.0 \\
0.0 \\
14.6 \\
15.5 \\
0.0 \\
6.0 \\
0.0 \\
0.0\end{array}$ & $\begin{array}{r}0.0 \\
40.1 \\
35.6 \\
0.0 \\
0.0 \\
0.0 \\
26.0 \\
29.1 \\
0.0 \\
13.4 \\
0.0 \\
0.0\end{array}$ & $\begin{array}{r}0.0 \\
29.3 \\
35.9 \\
0.0 \\
0.0 \\
0.0 \\
6.2 \\
19.6 \\
0.0 \\
10.4 \\
0.0 \\
0.0\end{array}$ & $\begin{array}{l}--- \\
--- \\
--- \\
--- \\
--- \\
--- \\
--- \\
--- \\
--- \\
--- \\
--- \\
---\end{array}$ & $\begin{array}{l}--- \\
--- \\
--- \\
--- \\
--- \\
--- \\
--- \\
--- \\
--- \\
--- \\
--- \\
---\end{array}$ & $\begin{array}{r}16 \\
0 \\
0 \\
0 \\
0 \\
16 \\
16 \\
16 \\
0 \\
0 \\
16 \\
0\end{array}$ \\
\hline
\end{tabular}

$\mathrm{CX}=$ COAXIAL

$\mathrm{CP}=$ COPLANAR
*Estimated Depth may be unreliable because the stronger part of the conductor may be deeper or to one side of the flight line, or because of a

shallow dip or magnetite/overburden effects
$-18-$ 
EM Anomaly List

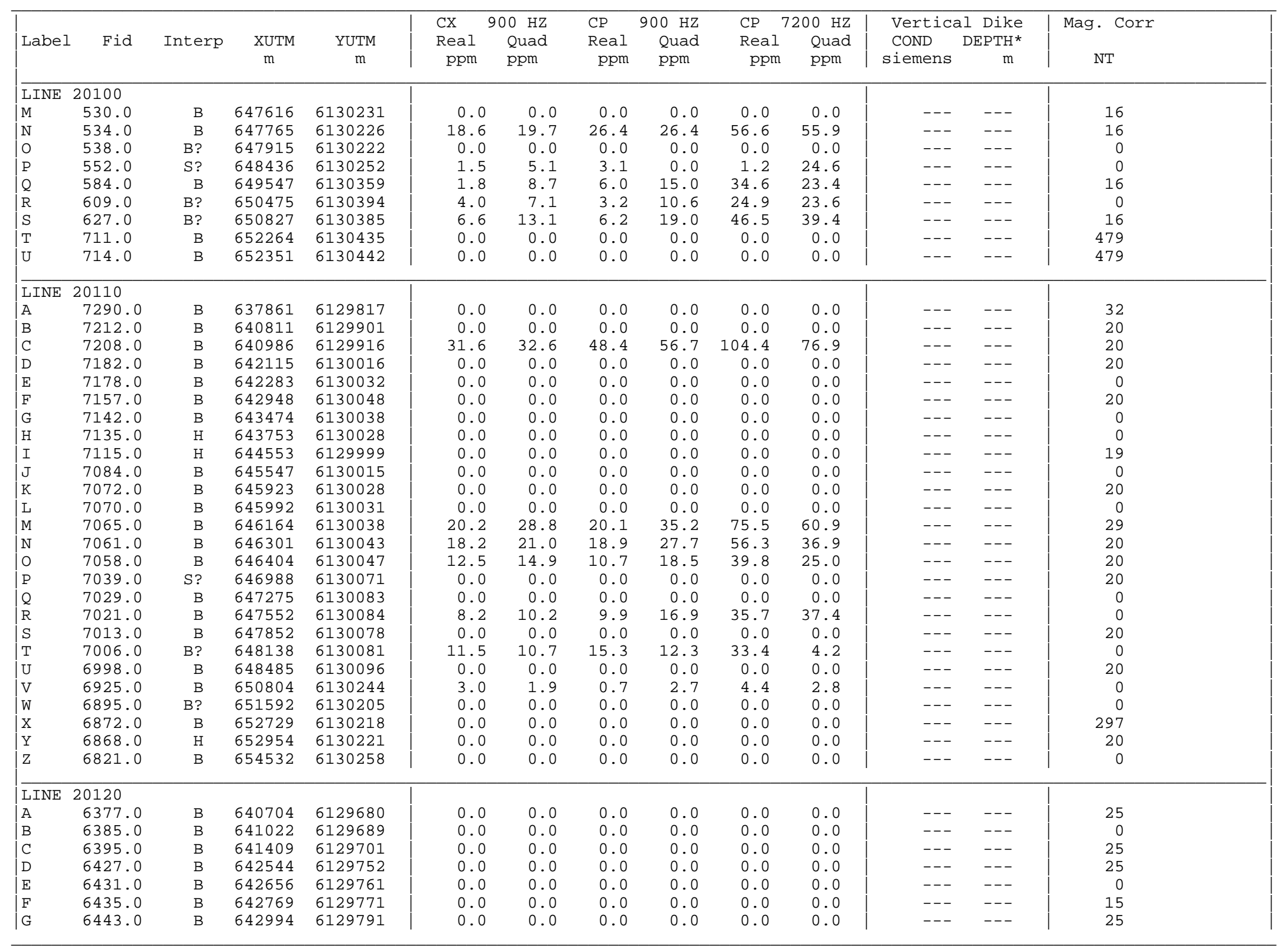

$\mathrm{CX}=$ COAXIAL

Note: EM values shown above

are local amplitudes

Hetta - North/South of 5515
* Estimated Depth may be unreliable because the stronger part of the conductor may be deeper or to one side of the flight line, or because of a shallow dip or magnetite/overburden effects 
EM Anomaly List

\begin{tabular}{|c|c|c|c|c|c|c|c|c|c|c|c|c|c|}
\hline Label & Fid & Interp & $\begin{array}{c}\text { XUTM } \\
\text { m }\end{array}$ & $\begin{array}{c}\text { YUTM } \\
\mathrm{m}\end{array}$ & $\begin{array}{l}\text { CX } \\
\text { Real } \\
\text { ppm }\end{array}$ & $\begin{array}{l}900 \mathrm{HZ} \\
\text { Quad } \\
\text { ppm }\end{array}$ & $\begin{array}{l}\text { CP } \\
\text { Real } \\
\text { ppm }\end{array}$ & $\begin{array}{l}900 \mathrm{HZ} \\
\text { Quad } \\
\text { ppm }\end{array}$ & $\begin{array}{l}\text { CP } 7 \\
\text { Real } \\
\text { ppm }\end{array}$ & $\begin{array}{c}7200 \mathrm{HZ} \\
\text { Quad } \\
\text { ppm }\end{array}$ & $\begin{array}{l}\text { Vertica } \\
\text { Cond } \\
\text { siemens }\end{array}$ & $\begin{array}{c}\text { al Dike } \\
\text { DEPTH* } \\
\text { m }\end{array}$ & $\begin{array}{c}\text { Mag. Corr } \\
\text { NT }\end{array}$ \\
\hline $\begin{array}{l}\text { LINE } \\
\text { H } \\
\text { I } \\
\text { J } \\
\text { K } \\
\text { L } \\
\text { M } \\
\text { N } \\
\text { O } \\
\text { P } \\
\text { Q } \\
\text { R } \\
\text { S } \\
\text { T }\end{array}$ & $\begin{array}{l}20120 \\
6450.0 \\
6457.0 \\
6460.0 \\
6476.0 \\
6488.0 \\
6492.0 \\
6512.0 \\
6516.0 \\
6521.0 \\
6568.0 \\
6602.0 \\
6654.0 \\
6664.0\end{array}$ & $\begin{array}{r}\text { B } \\
\text { B } \\
\text { B } \\
\text { B? } \\
\text { B? } \\
\text { B } \\
\text { B } \\
\text { B } \\
\text { B } \\
\text { B? } \\
\text { H } \\
\text { B } \\
\text { B }\end{array}$ & $\begin{array}{l}643192 \\
643414 \\
643564 \\
644368 \\
644914 \\
645058 \\
645689 \\
645773 \\
645869 \\
646773 \\
648113 \\
649704 \\
650047\end{array}$ & $\begin{array}{l}6129809 \\
6129822 \\
6129822 \\
6129819 \\
6129812 \\
6129814 \\
6129830 \\
6129833 \\
6129835 \\
6129853 \\
6129920 \\
6129998 \\
6129992\end{array}$ & $\begin{array}{r}0.0 \\
0.0 \\
44.4 \\
0.0 \\
0.0 \\
0.0 \\
0.0 \\
0.0 \\
0.0 \\
5.2 \\
0.0 \\
0.0 \\
0.0\end{array}$ & $\begin{array}{r}0.0 \\
0.0 \\
37.2 \\
0.0 \\
0.0 \\
0.0 \\
0.0 \\
0.0 \\
0.0 \\
3.9 \\
0.0 \\
0.0 \\
0.0\end{array}$ & $\begin{array}{r}0.0 \\
0.0 \\
52.5 \\
0.0 \\
0.0 \\
0.0 \\
0.0 \\
0.0 \\
0.0 \\
0.4 \\
0.0 \\
0.0 \\
0.0\end{array}$ & $\begin{array}{r}0.0 \\
0.0 \\
59.4 \\
0.0 \\
0.0 \\
0.0 \\
0.0 \\
0.0 \\
0.0 \\
1.9 \\
0.0 \\
0.0 \\
0.0\end{array}$ & $\begin{array}{r}0.0 \\
0.0 \\
112.5 \\
0.0 \\
0.0 \\
0.0 \\
0.0 \\
0.0 \\
0.0 \\
6.3 \\
0.0 \\
0.0 \\
0.0\end{array}$ & $\begin{array}{r}0.0 \\
0.0 \\
171.0 \\
0.0 \\
0.0 \\
0.0 \\
0.0 \\
0.0 \\
0.0 \\
7.0 \\
0.0 \\
0.0 \\
0.0\end{array}$ & $\begin{array}{l}--- \\
--- \\
--- \\
--- \\
--- \\
--- \\
--- \\
--- \\
--- \\
--- \\
--- \\
--- \\
---\end{array}$ & $\begin{array}{l}--- \\
--- \\
--- \\
--- \\
--- \\
--- \\
--- \\
--- \\
--- \\
--- \\
--- \\
--- \\
---\end{array}$ & $\begin{array}{r}25 \\
0 \\
0 \\
25 \\
25 \\
25 \\
0 \\
25 \\
25 \\
0 \\
0 \\
25 \\
0\end{array}$ \\
\hline $\begin{array}{l}\text { LINE } \\
\text { A } \\
\text { B } \\
\text { C } \\
\text { D } \\
\text { E } \\
\text { F } \\
\text { G } \\
\text { H } \\
\text { I } \\
\text { J } \\
\text { K } \\
\text { L } \\
\text { M } \\
\text { N } \\
\text { O } \\
\text { P } \\
\text { Q } \\
\text { R } \\
\text { S } \\
\text { T } \\
\text { U } \\
\text { V } \\
\text { W } \\
\text { X } \\
\text { Y }\end{array}$ & $\begin{array}{r}20130 \\
6138.0 \\
6136.0 \\
6103.0 \\
6092.0 \\
6085.0 \\
6077.0 \\
6070.0 \\
6064.0 \\
6061.0 \\
6054.0 \\
6044.0 \\
6036.0 \\
6034.0 \\
5974.0 \\
5962.0 \\
5956.0 \\
5950.0 \\
5946.0 \\
5937.0 \\
5929.0 \\
5902.0 \\
5894.0 \\
5825.0 \\
5821.0 \\
5792.0\end{array}$ & $\begin{array}{r}\text { B } \\
\text { B } \\
\text { B? } \\
\text { B } \\
\text { B } \\
\text { B? } \\
\text { B } \\
\text { B } \\
\text { B } \\
\text { B } \\
\text { B } \\
\text { B } \\
\text { B } \\
\text { B? } \\
\text { B } \\
\text { B } \\
\text { B } \\
\text { B } \\
\text { B } \\
\text { B } \\
\text { B? } \\
\text { B } \\
\text { B } \\
\text { B } \\
\text { B }\end{array}$ & $\begin{array}{l}641049 \\
641122 \\
642324 \\
642700 \\
642948 \\
643301 \\
643597 \\
643814 \\
643918 \\
644152 \\
644607 \\
644931 \\
645009 \\
647023 \\
647338 \\
647542 \\
647745 \\
647881 \\
648171 \\
648402 \\
649183 \\
649414 \\
651824 \\
651978 \\
653108\end{array}$ & $\begin{array}{l}6129527 \\
6129529 \\
6129555 \\
6129550 \\
6129551 \\
6129595 \\
6129621 \\
6129618 \\
6129613 \\
6129598 \\
6129606 \\
6129609 \\
6129609 \\
6129714 \\
6129775 \\
6129806 \\
6129837 \\
6129857 \\
6129891 \\
6129902 \\
6129935 \\
6129945 \\
6129870 \\
6129859 \\
6129805\end{array}$ & $\begin{array}{r}0.0 \\
0.0 \\
8.1 \\
0.0 \\
0.0 \\
0.0 \\
0.0 \\
0.0 \\
0.0 \\
10.1 \\
0.8 \\
0.0 \\
0.0 \\
0.0 \\
0.0 \\
0.0 \\
0.0 \\
18.6 \\
10.6 \\
0.0 \\
2.6 \\
0.0 \\
7.9 \\
0.0 \\
0.0\end{array}$ & $\begin{array}{r}0.0 \\
0.0 \\
11.9 \\
0.0 \\
0.0 \\
0.0 \\
0.0 \\
0.0 \\
0.0 \\
15.8 \\
3.2 \\
0.0 \\
0.0 \\
0.0 \\
0.0 \\
0.0 \\
0.0 \\
26.5 \\
11.2 \\
0.0 \\
10.1 \\
0.0 \\
16.2 \\
0.0 \\
0.0\end{array}$ & $\begin{array}{r}0.0 \\
0.0 \\
23.6 \\
0.0 \\
0.0 \\
0.0 \\
0.0 \\
0.0 \\
0.0 \\
12.4 \\
75.6 \\
0.0 \\
0.0 \\
0.0 \\
0.0 \\
0.0 \\
0.0 \\
30.2 \\
13.5 \\
0.0 \\
3.3 \\
0.0 \\
8.2 \\
0.0 \\
0.0\end{array}$ & $\begin{array}{r}0.0 \\
0.0 \\
60.2 \\
0.0 \\
0.0 \\
0.0 \\
0.0 \\
0.0 \\
0.0 \\
20.4 \\
127.6 \\
0.0 \\
0.0 \\
0.0 \\
0.0 \\
0.0 \\
0.0 \\
47.5 \\
20.6 \\
0.0 \\
10.0 \\
0.0 \\
22.0 \\
0.0 \\
0.0\end{array}$ & $\begin{array}{r}0.0 \\
0.0 \\
131.4 \\
0.0 \\
0.0 \\
0.0 \\
0.0 \\
0.0 \\
0.0 \\
50.9 \\
274.0 \\
0.0 \\
0.0 \\
0.0 \\
0.0 \\
0.0 \\
0.0 \\
99.5 \\
47.6 \\
0.0 \\
33.4 \\
0.0 \\
58.3 \\
0.0 \\
0.0\end{array}$ & $\begin{array}{r}0.0 \\
0.0 \\
64.8 \\
0.0 \\
0.0 \\
0.0 \\
0.0 \\
0.0 \\
0.0 \\
51.2 \\
21.7 \\
0.0 \\
0.0 \\
0.0 \\
0.0 \\
0.0 \\
0.0 \\
96.6 \\
32.2 \\
0.0 \\
50.6 \\
0.0 \\
51.8 \\
0.0 \\
0.0\end{array}$ & $\begin{array}{l}--- \\
--- \\
--- \\
--- \\
--- \\
--- \\
--- \\
--- \\
--- \\
--- \\
--- \\
--- \\
--- \\
--- \\
--- \\
--- \\
--- \\
--- \\
--- \\
--- \\
--- \\
--- \\
--- \\
--- \\
--\end{array}$ & $\begin{array}{l}--- \\
--- \\
--- \\
--- \\
--- \\
--- \\
--- \\
--- \\
--- \\
--- \\
--- \\
--- \\
--- \\
--- \\
--- \\
--- \\
--- \\
--- \\
--- \\
--- \\
--- \\
--- \\
--- \\
--- \\
---\end{array}$ & $\begin{array}{r}29 \\
29 \\
29 \\
29 \\
17 \\
29 \\
29 \\
0 \\
0 \\
29 \\
0 \\
0 \\
29 \\
29 \\
0 \\
29 \\
0 \\
0 \\
0 \\
29 \\
29 \\
0 \\
0 \\
0 \\
182\end{array}$ \\
\hline $\begin{array}{l}\text { LINE } \\
\text { A } \\
\text { B } \\
\text { C } \\
\text { D }\end{array}$ & $\begin{array}{l}20140 \\
5187.0 \\
5194.0 \\
5239.0 \\
5264.0\end{array}$ & $\begin{array}{r}\mathrm{B} ? \\
\mathrm{H} \\
\mathrm{S} ? \\
\mathrm{~B}\end{array}$ & $\begin{array}{l}640617 \\
640948 \\
642759 \\
643561\end{array}$ & $\begin{array}{l}6129252 \\
6129263 \\
6129340 \\
6129416\end{array}$ & $\begin{array}{r}0.0 \\
14.7 \\
1.2 \\
13.9\end{array}$ & $\begin{array}{r}0.0 \\
19.6 \\
9.7 \\
7.0\end{array}$ & $\begin{array}{r}0.0 \\
18.6 \\
2.5 \\
15.6\end{array}$ & $\begin{array}{r}0.0 \\
28.2 \\
8.4 \\
8.8\end{array}$ & $\begin{array}{r}0.0 \\
65.7 \\
20.4 \\
18.1\end{array}$ & $\begin{array}{r}0.0 \\
23.6 \\
32.1 \\
0.9\end{array}$ & $\begin{array}{l}--- \\
--- \\
--- \\
---\end{array}$ & $\begin{array}{l}--- \\
--- \\
--- \\
---\end{array}$ & $\begin{array}{r}32 \\
0 \\
0 \\
32\end{array}$ \\
\hline
\end{tabular}

$\mathrm{CX}=$ COAXIAL

Note: EM values shown above

are local amplitudes

Hetta - North/South of 5515
*Estimated Depth may be unreliable because the stronger part of the conductor may be deeper or to one side of the flight line, or because of a shallow dip or magnetite/overburden effects 
EM Anomaly List

\begin{tabular}{|c|c|c|c|c|c|c|c|c|c|c|c|c|c|}
\hline Label & Fid & Interp & $\begin{array}{c}\text { XUTM } \\
\text { m }\end{array}$ & $\begin{array}{c}\text { YUTM } \\
\mathrm{m}\end{array}$ & $\begin{array}{l}\text { CX } \\
\text { Real } \\
\text { ppm }\end{array}$ & $\begin{array}{l}900 \mathrm{HZ} \\
\text { Quad } \\
\text { ppm }\end{array}$ & $\begin{array}{l}\text { CP } \\
\text { Real } \\
\text { ppm }\end{array}$ & $\begin{array}{l}900 \mathrm{HZ} \\
\text { Quad } \\
\text { ppm }\end{array}$ & $\begin{array}{l}\text { CP } 7 \\
\text { Real } \\
\text { ppm }\end{array}$ & $\begin{array}{l}200 \mathrm{HZ} \\
\text { Quad } \\
\text { ppm }\end{array}$ & $\begin{array}{l}\text { Vertica } \\
\text { Cond } \\
\text { siemens }\end{array}$ & $\begin{array}{c}\text { al Dike } \\
\mathrm{DEPTH}^{\star} \\
\mathrm{m}\end{array}$ & $\begin{array}{c}\text { Mag. Corr } \\
\text { NT }\end{array}$ \\
\hline$\overline{\text { LINE }}$ & 20140 & & & & & & & & & & & & \\
\hline$E$ & 5275.0 & B & 643908 & 6129406 & 11.5 & 11.1 & 19.1 & 17.9 & 42.0 & 27.0 & --- & --- & 32 \\
\hline $\mathrm{F}$ & 5286.0 & B & 644253 & 6129393 & 9.1 & 17.8 & 14.2 & 16.1 & 37.0 & 17.2 & --- & --- & 0 \\
\hline G & 5312.0 & B & 645089 & 6129384 & 0.0 & 0.0 & 0.0 & 0.0 & 0.0 & 0.0 & --- & --- & 32 \\
\hline $\mathrm{H}$ & 5315.0 & B & 645186 & 6129394 & 0.0 & 0.0 & 0.0 & 0.0 & 0.0 & 0.0 & --- & --- & 32 \\
\hline I & 5405.0 & S? & 646649 & 6129405 & 2.2 & 10.9 & 1.7 & 10.8 & 27.8 & 54.2 & --- & --- & 0 \\
\hline $\mathrm{J}$ & 5457.0 & B? & 647765 & 6129449 & 0.0 & 0.0 & 0.0 & 0.0 & 0.0 & 0.0 & --- & --- & 0 \\
\hline $\mathrm{K}$ & 5472.0 & B & 648085 & 6129450 & 15.2 & 12.8 & 20.8 & 24.4 & 50.9 & 30.7 & --- & --- & 0 \\
\hline L & 5475.0 & B & 648156 & 6129448 & 26.0 & 26.2 & 32.1 & 39.8 & 86.8 & 51.6 & --- & --- & 0 \\
\hline M & 5481.0 & B & 648330 & 6129447 & 0.0 & 0.0 & 0.0 & 0.0 & 0.0 & 0.0 & --- & --- & 32 \\
\hline $\mathrm{N}$ & 5484.0 & B & 648429 & 6129448 & 0.0 & 0.0 & 0.0 & 0.0 & 0.0 & 0.0 & --- & --- & 0 \\
\hline 0 & 5492.0 & B & 648697 & 6129457 & 45.9 & 51.8 & 80.6 & 75.5 & 78.6 & 38.3 & --- & --- & 32 \\
\hline $\mathrm{P}$ & 5495.0 & B & 648796 & 6129463 & 0.0 & 0.0 & 0.0 & 0.0 & 0.0 & 0.0 & --- & --- & 0 \\
\hline Q & 5515.0 & B & 649366 & 6129524 & 0.0 & 0.0 & 0.0 & 0.0 & 0.0 & 0.0 & --- & --- & 0 \\
\hline $\mathrm{R}$ & 5519.0 & B & 649472 & 6129516 & 0.0 & 0.0 & 0.0 & 0.0 & 0.0 & 0.0 & --- & --- & 32 \\
\hline $\mathrm{S}$ & 5544.0 & $\mathrm{~s}$ & 650354 & 6129541 & 0.0 & 0.0 & 0.0 & 0.0 & 0.0 & 0.0 & --- & --- & 32 \\
\hline $\mathrm{T}$ & 5577.0 & B & 651620 & 6129530 & 0.0 & 0.0 & 0.0 & 0.0 & 0.0 & 0.0 & --- & --- & 0 \\
\hline $\mathrm{U}$ & 5584.0 & B & 651885 & 6129550 & 0.0 & 0.0 & 0.0 & 0.0 & 0.0 & 0.0 & --- & --- & 0 \\
\hline $\mathrm{V}$ & 5597.0 & $B$ ? & 652352 & 6129573 & 0.0 & 0.0 & 0.0 & 0.0 & 0.0 & 0.0 & --- & --- & 0 \\
\hline W & 5605.0 & $\mathrm{~B}$ ? & 652639 & 6129587 & 0.0 & 0.0 & 0.0 & 0.0 & 0.0 & 0.0 & --- & --- & 235 \\
\hline LINE & 20150 & & & & & & & & & & & & \\
\hline A & 5013.0 & $\mathrm{~S}$ & 637540 & 6128972 & 0.0 & 0.0 & 0.0 & 0.0 & 0.0 & 0.0 & --- & --- & 31 \\
\hline B & 4989.0 & $\mathrm{~s}$ & 638480 & 6128991 & 3.7 & 4.5 & 4.8 & 7.1 & 12.8 & 38.6 & --- & --- & 41 \\
\hline $\mathrm{C}$ & 4910.0 & $\mathrm{~s}$ & 641564 & 6129107 & 3.6 & 6.1 & 3.2 & 6.5 & 17.0 & 22.6 & --- & --- & 31 \\
\hline $\mathrm{D}$ & 4856.0 & B & 643888 & 6129242 & 9.7 & 3.6 & 25.9 & 35.1 & 75.1 & 37.9 & --- & --- & 0 \\
\hline $\mathrm{E}$ & 4848.0 & B & 644111 & 6129227 & 0.0 & 0.0 & 0.0 & 0.0 & 0.0 & 0.0 & --- & --- & 27 \\
\hline $\mathrm{F}$ & 4838.0 & B & 644385 & 6129206 & 0.0 & 0.0 & 0.0 & 0.0 & 0.0 & 0.0 & --- & --- & 0 \\
\hline G & 4805.0 & B? & 645224 & 6129208 & 0.0 & 0.0 & 0.0 & 0.0 & 0.0 & 0.0 & --- & --- & 0 \\
\hline $\mathrm{H}$ & 4777.0 & B & 645643 & 6129219 & 0.0 & 0.0 & 0.0 & 0.0 & 0.0 & 0.0 & --- & --- & 31 \\
\hline I & 4767.0 & B & 645760 & 6129216 & 0.0 & 0.0 & 0.0 & 0.0 & 0.0 & 0.0 & --- & --- & 0 \\
\hline $\mathrm{J}$ & 4713.0 & B & 646388 & 6129162 & 2.2 & 7.5 & 0.0 & 12.3 & 21.3 & 52.6 & --- & --- & 0 \\
\hline $\mathrm{K}$ & 4704.0 & B & 646499 & 6129163 & 0.0 & 0.0 & 0.0 & 0.0 & 0.0 & 0.0 & --- & --- & 0 \\
\hline $\mathrm{L}$ & 4662.0 & S? & 647340 & 6129253 & 0.0 & 0.0 & 0.0 & 0.0 & 0.0 & 0.0 & --- & --- & 31 \\
\hline M & 4642.0 & B & 647727 & 6129267 & 0.0 & 0.0 & 0.0 & 0.0 & 0.0 & 0.0 & --- & --- & 0 \\
\hline $\mathrm{N}$ & 4634.0 & B & 647871 & 6129252 & 0.0 & 0.0 & 0.0 & 0.0 & 0.0 & 0.0 & --- & --- & 0 \\
\hline 0 & 4628.0 & B & 647989 & 6129259 & 0.0 & 0.0 & 0.0 & 0.0 & 0.0 & 0.0 & --- & --- & 31 \\
\hline $\mathrm{P}$ & 4623.0 & B & 648120 & 6129264 & 0.0 & 0.0 & 0.0 & 0.0 & 0.0 & 0.0 & --- & --- & 0 \\
\hline Q & 4618.0 & B & 648283 & 6129264 & 0.0 & 0.0 & 0.0 & 0.0 & 0.0 & 0.0 & --- & --- & 24 \\
\hline R & 4614.0 & B & 648414 & 6129262 & 0.0 & 0.0 & 0.0 & 0.0 & 0.0 & 0.0 & --- & --- & 31 \\
\hline S & 4606.0 & B & 648660 & 6129252 & 0.0 & 0.0 & 0.0 & 0.0 & 0.0 & 0.0 & --- & --- & 31 \\
\hline $\mathrm{T}$ & 4598.0 & B & 648890 & 6129250 & 0.0 & 0.0 & 0.0 & 0.0 & 0.0 & 0.0 & --- & --- & 0 \\
\hline $\mathrm{U}$ & 4594.0 & $B$ ? & 649000 & 6129252 & 0.0 & 0.0 & 0.0 & 0.0 & 0.0 & 0.0 & --- & --- & 0 \\
\hline $\mathrm{V}$ & 4586.0 & $B$ ? & 649209 & 6129273 & 3.7 & 4.9 & 1.0 & 1.1 & 2.3 & 4.3 & --- & --- & 0 \\
\hline W & 4577.0 & S? & 649474 & 6129266 & 0.0 & 0.2 & 1.9 & 2.8 & 7.6 & 10.7 & --- & --- & 0 \\
\hline $\mathrm{x}$ & 4557.0 & $B$ ? & 650065 & 6129246 & 0.0 & 0.0 & 0.0 & 0.0 & 0.0 & 0.0 & --- & --- & 0 \\
\hline $\mathrm{Y}$ & 4556.0 & $B$ ? & 650095 & 6129245 & 0.0 & 0.0 & 0.0 & 0.0 & 0.0 & 0.0 & --- & --- & 0 \\
\hline
\end{tabular}

$\mathrm{CX}=$ COAXIAL

Note: EM values shown above

are local amplitudes

Hetta - North/South of 5515
* Estimated Depth may be unreliable because the stronger part of the conductor may be deeper or to one side of the flight line, or because of a shallow dip or magnetite/overburden effects 
EM Anomaly List

\begin{tabular}{|c|c|c|c|c|c|c|c|c|c|c|c|c|c|}
\hline Label & Fid & Interp & $\begin{array}{c}\text { XUTM } \\
\text { m }\end{array}$ & $\begin{array}{c}\text { YUTM } \\
\text { m }\end{array}$ & $\begin{array}{l}\text { CX } \\
\text { Real } \\
\text { ppm }\end{array}$ & $\begin{array}{l}900 \mathrm{HZ} \\
\text { Quad } \\
\text { ppm }\end{array}$ & $\begin{array}{l}\text { CP } \\
\text { Real } \\
\text { ppm }\end{array}$ & $\begin{array}{l}900 \mathrm{HZ} \\
\text { Quad } \\
\text { ppm }\end{array}$ & $\begin{array}{l}\text { CP } 7 \\
\text { Real } \\
\text { ppm }\end{array}$ & $\begin{array}{l}200 \mathrm{HZ} \\
\text { Quad } \\
\text { ppm }\end{array}$ & $\begin{array}{l}\text { Vertica } \\
\text { Cond } \\
\text { siemens }\end{array}$ & $\begin{array}{c}\text { al Dike } \\
\text { DEPTH* } \\
\text { m }\end{array}$ & $\begin{array}{c}\text { Mag. Corr } \\
\text { NT }\end{array}$ \\
\hline $\begin{array}{l}\mathrm{LINE} \\
Z \\
\mathrm{AA}\end{array}$ & $\begin{array}{r}20150 \\
4549.0 \\
4539.0\end{array}$ & $\begin{array}{r}\text { B } \\
\text { B? }\end{array}$ & $\begin{array}{l}650300 \\
650601\end{array}$ & $\begin{array}{l}6129239 \\
6129231\end{array}$ & $\begin{array}{l}0.0 \\
0.0\end{array}$ & $\begin{array}{l}0.0 \\
0.0\end{array}$ & $\begin{array}{l}0.0 \\
0.0\end{array}$ & $\begin{array}{l}0.0 \\
0.0\end{array}$ & $\begin{array}{l}0.0 \\
0.0\end{array}$ & $\begin{array}{l}0.0 \\
0.0\end{array}$ & $\begin{array}{l}--- \\
---\end{array}$ & $\begin{array}{l}--- \\
---\end{array}$ & $\begin{array}{r}31 \\
0\end{array}$ \\
\hline $\begin{array}{l}\text { LINE } \\
\text { A } \\
\text { B } \\
\text { C } \\
\text { D } \\
\text { E } \\
\text { F } \\
\text { G } \\
\text { H } \\
\text { I } \\
\text { J } \\
\text { K } \\
\text { L } \\
\text { M } \\
\text { N } \\
\text { O } \\
\text { P } \\
\text { Q } \\
\text { R } \\
\text { S } \\
\text { T } \\
\text { U } \\
\text { V }\end{array}$ & $\begin{array}{r}20160 \\
3739.0 \\
3753.0 \\
3795.0 \\
3947.0 \\
3988.0 \\
3992.0 \\
3997.0 \\
4076.0 \\
4085.0 \\
4109.0 \\
4135.0 \\
4154.0 \\
4160.0 \\
4165.0 \\
4179.0 \\
4188.0 \\
4191.0 \\
4194.0 \\
4214.0 \\
4238.0 \\
4244.0 \\
4275.0\end{array}$ & $\begin{array}{r}\text { S? } \\
\text { S } \\
\text { S? } \\
\text { S } \\
\text { B? } \\
\text { B? } \\
\text { B? } \\
\text { B? } \\
\text { B? } \\
\text { B? } \\
\text { B? } \\
\text { B } \\
\text { B } \\
\text { B } \\
\text { B } \\
\text { B } \\
\text { B } \\
\text { B } \\
\text { B? } \\
\text { B? } \\
\text { B? } \\
\text { B? }\end{array}$ & $\begin{array}{l}637999 \\
638542 \\
640211 \\
644387 \\
645405 \\
645484 \\
645570 \\
646553 \\
646691 \\
647072 \\
647557 \\
647852 \\
647940 \\
648013 \\
648456 \\
648715 \\
648788 \\
648860 \\
649337 \\
649856 \\
649986 \\
650958\end{array}$ & $\begin{array}{l}6128792 \\
6128812 \\
6128826 \\
6128949 \\
6128948 \\
6128949 \\
6128947 \\
6129007 \\
6128997 \\
6128990 \\
6129036 \\
6129035 \\
6129033 \\
6129031 \\
6129053 \\
6129063 \\
6129062 \\
6129061 \\
6129077 \\
6129102 \\
6129109 \\
6129141\end{array}$ & $\begin{array}{r}3.7 \\
4.7 \\
0.0 \\
0.0 \\
8.1 \\
10.7 \\
10.7 \\
1.4 \\
2.4 \\
3.2 \\
0.0 \\
0.0 \\
9.2 \\
0.0 \\
0.0 \\
0.0 \\
0.0 \\
0.0 \\
0.0 \\
0.0 \\
0.0 \\
1.8\end{array}$ & $\begin{array}{r}6.4 \\
3.1 \\
0.0 \\
0.0 \\
13.0 \\
15.1 \\
12.5 \\
2.7 \\
1.3 \\
11.2 \\
0.0 \\
0.0 \\
25.1 \\
0.0 \\
0.0 \\
0.0 \\
0.0 \\
0.0 \\
0.0 \\
0.0 \\
0.0 \\
0.0\end{array}$ & $\begin{array}{r}3.9 \\
7.1 \\
0.0 \\
0.0 \\
8.5 \\
11.6 \\
15.0 \\
2.8 \\
2.1 \\
2.0 \\
0.0 \\
0.0 \\
13.4 \\
0.0 \\
0.0 \\
0.0 \\
0.0 \\
0.0 \\
0.0 \\
0.0 \\
0.0 \\
0.2\end{array}$ & $\begin{array}{r}8.3 \\
4.9 \\
0.0 \\
0.0 \\
14.8 \\
17.0 \\
20.9 \\
7.9 \\
4.1 \\
9.7 \\
0.0 \\
0.0 \\
33.5 \\
0.0 \\
0.0 \\
0.0 \\
0.0 \\
0.0 \\
0.0 \\
0.0 \\
0.0 \\
42.4\end{array}$ & $\begin{array}{r}4.6 \\
12.1 \\
0.0 \\
0.0 \\
34.1 \\
38.6 \\
43.0 \\
15.3 \\
9.2 \\
26.5 \\
0.0 \\
0.0 \\
73.4 \\
0.0 \\
0.0 \\
0.0 \\
0.0 \\
0.0 \\
0.0 \\
0.0 \\
0.0 \\
0.0\end{array}$ & $\begin{array}{r}52.8 \\
25.4 \\
0.0 \\
0.0 \\
23.6 \\
27.0 \\
27.0 \\
27.1 \\
10.6 \\
36.7 \\
0.0 \\
0.0 \\
44.4 \\
0.0 \\
0.0 \\
0.0 \\
0.0 \\
0.0 \\
0.0 \\
0.0 \\
0.0 \\
2.3\end{array}$ & $\begin{array}{l}--- \\
--- \\
--- \\
--- \\
--- \\
--- \\
--- \\
--- \\
--- \\
--- \\
--- \\
--- \\
--- \\
--- \\
--- \\
--- \\
--- \\
--- \\
--- \\
--- \\
--- \\
---\end{array}$ & $\begin{array}{l}--- \\
--- \\
--- \\
--- \\
--- \\
--- \\
--- \\
--- \\
--- \\
--- \\
--- \\
--- \\
--- \\
--- \\
--- \\
--- \\
--- \\
--- \\
--- \\
--- \\
--- \\
---\end{array}$ & $\begin{array}{r}29 \\
29 \\
29 \\
0 \\
29 \\
29 \\
0 \\
0 \\
34 \\
0 \\
0 \\
27 \\
0 \\
0 \\
29 \\
29 \\
0 \\
0 \\
0 \\
29 \\
0 \\
0\end{array}$ \\
\hline $\begin{array}{l}\text { LINE } \\
\text { A } \\
\text { B } \\
\text { C } \\
\text { D } \\
\text { E } \\
\text { F } \\
\text { G } \\
\text { H } \\
\text { I } \\
\text { J } \\
\text { K }\end{array}$ & $\begin{array}{r}20170 \\
3626.0 \\
3307.0 \\
3300.0 \\
3293.0 \\
3282.0 \\
3253.0 \\
3244.0 \\
3215.0 \\
3197.0 \\
3194.0 \\
3190.0\end{array}$ & $\begin{array}{r}\text { S } \\
\text { B? } \\
\text { B? } \\
\text { B? } \\
\text { B? } \\
\text { B? } \\
\text { H } \\
\text { H } \\
\text { B? } \\
\text { B } \\
\text { B }\end{array}$ & $\begin{array}{l}637898 \\
646973 \\
647047 \\
647122 \\
647236 \\
647656 \\
647819 \\
648605 \\
649262 \\
649372 \\
649519\end{array}$ & $\begin{array}{l}6128547 \\
6128841 \\
6128841 \\
6128842 \\
6128845 \\
6128842 \\
6128839 \\
6128835 \\
6128870 \\
6128876 \\
6128884\end{array}$ & $\begin{array}{r}0.0 \\
3.3 \\
0.0 \\
0.0 \\
0.6 \\
0.0 \\
0.0 \\
0.0 \\
26.5 \\
0.0 \\
0.0\end{array}$ & $\begin{array}{r}0.0 \\
5.9 \\
0.0 \\
0.0 \\
7.3 \\
0.0 \\
0.0 \\
0.0 \\
24.3 \\
0.0 \\
0.0\end{array}$ & $\begin{array}{r}0.0 \\
6.4 \\
0.0 \\
0.0 \\
0.0 \\
0.0 \\
0.0 \\
0.0 \\
36.6 \\
0.0 \\
0.0\end{array}$ & $\begin{array}{r}0.0 \\
18.0 \\
0.0 \\
0.0 \\
8.8 \\
0.0 \\
0.0 \\
0.0 \\
37.7 \\
0.0 \\
0.0\end{array}$ & $\begin{array}{r}0.0 \\
43.7 \\
0.0 \\
0.0 \\
11.4 \\
0.0 \\
0.0 \\
0.0 \\
85.8 \\
0.0 \\
0.0\end{array}$ & $\begin{array}{r}0.0 \\
41.2 \\
0.0 \\
0.0 \\
47.2 \\
0.0 \\
0.0 \\
0.0 \\
57.6 \\
0.0 \\
0.0\end{array}$ & $\begin{array}{l}--- \\
--- \\
--- \\
--- \\
--- \\
--- \\
--- \\
--- \\
--- \\
--- \\
---\end{array}$ & $\begin{array}{l}--- \\
--- \\
--- \\
--- \\
--- \\
--- \\
--- \\
--- \\
--- \\
--- \\
---\end{array}$ & $\begin{array}{r}25 \\
25 \\
25 \\
0 \\
0 \\
25 \\
14 \\
0 \\
25 \\
0 \\
0\end{array}$ \\
\hline $\begin{array}{l}\text { LINE } \\
\text { A } \\
\text { B } \\
\text { C } \\
\text { D } \\
\text { E }\end{array}$ & $\begin{array}{r}20180 \\
2498.0 \\
2836.0 \\
2846.0 \\
2848.0 \\
2863.0\end{array}$ & $\begin{array}{l}\text { S } \\
\text { B } \\
\text { B } \\
\text { B } \\
\text { B }\end{array}$ & $\begin{array}{l}638094 \\
647640 \\
647801 \\
647832 \\
648060\end{array}$ & $\begin{array}{l}6128402 \\
6128640 \\
6128644 \\
6128646 \\
6128655\end{array}$ & $\begin{array}{l}0.0 \\
5.9 \\
0.0 \\
0.0 \\
2.6\end{array}$ & $\begin{array}{l}0.0 \\
4.0 \\
0.0 \\
0.0 \\
6.3\end{array}$ & $\begin{array}{l}0.0 \\
8.0 \\
0.0 \\
0.0 \\
0.9\end{array}$ & $\begin{array}{l}0.0 \\
5.4 \\
0.0 \\
0.0 \\
3.0\end{array}$ & $\begin{array}{r}0.0 \\
10.4 \\
0.0 \\
0.0 \\
6.9\end{array}$ & $\begin{array}{l}0.0 \\
6.3 \\
0.0 \\
0.0 \\
6.7\end{array}$ & $\begin{array}{l}--- \\
--- \\
--- \\
--- \\
---\end{array}$ & $\begin{array}{l}--- \\
--- \\
--- \\
--- \\
---\end{array}$ & $\begin{array}{r}113 \\
0 \\
30 \\
0 \\
11\end{array}$ \\
\hline
\end{tabular}

$\mathrm{CX}=$ COAXIAL

Note: EM values shown above

are local amplitudes

*Estimated Depth may be unreliable because the stronger part of the conductor may be deeper or to one side of the flight line, or because of a

shallow dip or magnetite/overburden effects

Hetta - North/South of 5515 
EM Anomaly List

\begin{tabular}{|c|c|c|c|c|c|c|c|c|c|c|c|c|c|}
\hline Label & Fid & Interp & $\underset{\mathrm{m}}{\mathrm{XUTM}}$ & $\begin{array}{c}\text { YUTM } \\
\mathrm{m}\end{array}$ & $\begin{array}{l}\text { CX } \\
\text { Real } \\
\text { ppm }\end{array}$ & $\begin{array}{l}900 \mathrm{HZ} \\
\text { Quad } \\
\text { ppm }\end{array}$ & $\begin{array}{l}\text { CP } \\
\text { Real } \\
\text { ppm }\end{array}$ & $\begin{array}{c}900 \mathrm{HZ} \\
\text { Quad } \\
\text { ppm }\end{array}$ & $\begin{array}{l}\text { CP }{ }^{7} \\
\text { Real } \\
\text { ppm }\end{array}$ & $\begin{array}{l}200 \mathrm{HZ} \\
\text { Quad } \\
\text { ppm }\end{array}$ & $\begin{array}{l}\text { Vertica } \\
\text { COND } \\
\text { siemens }\end{array}$ & $\begin{array}{c}\text { al Dike } \\
\text { DEPTH* } \\
\text { m }\end{array}$ & $\begin{array}{c}\text { Mag. Corr } \\
\text { NT }\end{array}$ \\
\hline $\begin{array}{l}\text { LINE } \\
\text { F } \\
\text { G } \\
\text { H } \\
\text { I } \\
\text { J } \\
\text { K } \\
\text { L } \\
\text { M }\end{array}$ & $\begin{array}{r}20180 \\
2868.0 \\
2873.0 \\
2881.0 \\
2884.0 \\
2894.0 \\
2899.0 \\
2909.0 \\
3045.0\end{array}$ & $\begin{array}{r}\text { B } \\
\text { B } \\
\text { B? } \\
\text { B } \\
\text { B? } \\
\text { B? } \\
\text { B? } \\
\text { S? }\end{array}$ & $\begin{array}{l}648181 \\
648367 \\
648620 \\
648711 \\
649007 \\
649155 \\
649446 \\
654611\end{array}$ & $\begin{array}{l}6128659 \\
6128665 \\
6128676 \\
6128681 \\
6128696 \\
6128703 \\
6128719 \\
6128771\end{array}$ & $\begin{array}{r}0.0 \\
0.0 \\
0.0 \\
0.0 \\
0.0 \\
19.7 \\
0.0 \\
30.7\end{array}$ & $\begin{array}{r}0.0 \\
0.0 \\
0.0 \\
0.0 \\
0.0 \\
12.9 \\
0.0 \\
14.2\end{array}$ & $\begin{array}{r}0.0 \\
0.0 \\
0.0 \\
0.0 \\
0.0 \\
18.7 \\
0.0 \\
56.0\end{array}$ & $\begin{array}{r}0.0 \\
0.0 \\
0.0 \\
0.0 \\
0.0 \\
18.5 \\
0.0 \\
27.3\end{array}$ & $\begin{array}{r}0.0 \\
0.0 \\
0.0 \\
0.0 \\
0.0 \\
40.0 \\
0.0 \\
64.9\end{array}$ & $\begin{array}{r}0.0 \\
0.0 \\
0.0 \\
0.0 \\
0.0 \\
26.2 \\
0.0 \\
11.1\end{array}$ & $\begin{array}{l}--- \\
--- \\
--- \\
--- \\
--- \\
--- \\
--- \\
---\end{array}$ & $\begin{array}{l}--- \\
--- \\
--- \\
--- \\
--- \\
--- \\
--- \\
---\end{array}$ & $\begin{array}{r}30 \\
30 \\
30 \\
0 \\
0 \\
0 \\
0 \\
30\end{array}$ \\
\hline $\begin{array}{l}\text { LINE } \\
\text { A } \\
B \\
C\end{array}$ & $\begin{array}{r}20190 \\
1916.0 \\
1893.0 \\
1808.0\end{array}$ & $\begin{array}{l}\text { S? } \\
\text { S? } \\
\text { S? }\end{array}$ & $\begin{array}{l}647517 \\
647778 \\
651260\end{array}$ & $\begin{array}{l}6128407 \\
6128430 \\
6128549\end{array}$ & $\begin{array}{l}0.0 \\
9.2 \\
9.2\end{array}$ & $\begin{array}{l}0.0 \\
9.5 \\
6.8\end{array}$ & $\begin{array}{r}0.0 \\
12.0 \\
9.9\end{array}$ & $\begin{array}{r}0.0 \\
11.6 \\
6.9\end{array}$ & $\begin{array}{r}0.0 \\
21.2 \\
14.3\end{array}$ & $\begin{array}{r}0.0 \\
23.1 \\
3.0\end{array}$ & $\begin{array}{l}--- \\
--- \\
---\end{array}$ & $\begin{array}{l}--- \\
--- \\
---\end{array}$ & $\begin{array}{r}0 \\
22 \\
89\end{array}$ \\
\hline $\begin{array}{l}\text { LINE } \\
\text { A } \\
\text { B } \\
\text { C } \\
\text { D }\end{array}$ & $\begin{array}{r}20191 \\
2385.0 \\
2359.0 \\
2283.0 \\
2248.0\end{array}$ & $\begin{array}{l}S \\
S \\
S \\
S\end{array}$ & $\begin{array}{l}637947 \\
639000 \\
641784 \\
643364\end{array}$ & $\begin{array}{l}6128161 \\
6128191 \\
6128280 \\
6128299\end{array}$ & $\begin{array}{l}0.0 \\
4.9 \\
5.9 \\
3.8\end{array}$ & $\begin{array}{l}0.0 \\
2.6 \\
0.9 \\
2.2\end{array}$ & $\begin{array}{l}0.0 \\
3.3 \\
5.6 \\
0.1\end{array}$ & $\begin{array}{l}0.0 \\
2.4 \\
1.2 \\
2.1\end{array}$ & $\begin{array}{l}0.0 \\
3.5 \\
4.6 \\
1.7\end{array}$ & $\begin{array}{r}0.0 \\
9.4 \\
4.1 \\
18.5\end{array}$ & $\begin{array}{l}--- \\
--- \\
--- \\
---\end{array}$ & $\begin{array}{l}--- \\
--- \\
--- \\
---\end{array}$ & $\begin{array}{r}26 \\
0 \\
26 \\
26\end{array}$ \\
\hline $\begin{array}{l}\text { LINE } \\
\text { A } \\
\text { B } \\
\text { C } \\
\text { D }\end{array}$ & $\begin{array}{r}20200 \\
1284.0 \\
1305.0 \\
1387.0 \\
1622.0\end{array}$ & $\begin{array}{l}\text { S? } \\
\text { S? } \\
\text { S? } \\
\text { B? }\end{array}$ & $\begin{array}{l}638134 \\
638948 \\
640927 \\
648852\end{array}$ & $\begin{array}{l}6127996 \\
6128065 \\
6128086 \\
6128270\end{array}$ & $\begin{array}{l}0.0 \\
0.0 \\
0.0 \\
0.0\end{array}$ & $\begin{array}{l}0.0 \\
0.0 \\
0.0 \\
0.0\end{array}$ & $\begin{array}{l}0.0 \\
0.0 \\
0.0 \\
0.0\end{array}$ & $\begin{array}{l}0.0 \\
0.0 \\
0.0 \\
0.0\end{array}$ & $\begin{array}{l}0.0 \\
0.0 \\
0.0 \\
0.0\end{array}$ & $\begin{array}{l}0.0 \\
0.0 \\
0.0 \\
0.0\end{array}$ & $\begin{array}{l}--- \\
--- \\
--- \\
---\end{array}$ & $\begin{array}{l}--- \\
--- \\
--- \\
---\end{array}$ & $\begin{array}{r}29 \\
252 \\
94 \\
29\end{array}$ \\
\hline $\begin{array}{l}\text { LINE } \\
\text { A } \\
\text { B } \\
\text { C } \\
\text { D }\end{array}$ & $\begin{array}{c}20210 \\
1149.0 \\
875.0 \\
865.0 \\
844.0\end{array}$ & $\begin{array}{r}\text { S? } \\
\text { B } \\
\text { B } \\
\text { B }\end{array}$ & $\begin{array}{l}638296 \\
647541 \\
647663 \\
647921\end{array}$ & $\begin{array}{l}6127821 \\
6128064 \\
6128059 \\
6128050\end{array}$ & $\begin{array}{r}68.8 \\
3.3 \\
3.4 \\
3.2\end{array}$ & $\begin{array}{r}38.6 \\
8.8 \\
16.0 \\
10.1\end{array}$ & $\begin{array}{r}118.5 \\
2.6 \\
2.6 \\
2.1\end{array}$ & $\begin{array}{r}58.2 \\
21.2 \\
8.9 \\
5.9\end{array}$ & $\begin{array}{r}124.5 \\
39.7 \\
14.5 \\
13.6\end{array}$ & $\begin{array}{l}18.9 \\
78.9 \\
38.9 \\
31.2\end{array}$ & $\begin{array}{l}--- \\
--- \\
--- \\
---\end{array}$ & $\begin{array}{l}--- \\
--- \\
--- \\
---\end{array}$ & $\begin{array}{r}41 \\
0 \\
0 \\
38\end{array}$ \\
\hline $\begin{array}{l}\text { LINE } \\
\text { A } \\
\text { B } \\
\text { C } \\
\text { D } \\
\text { E } \\
\text { F }\end{array}$ & $\begin{array}{c}20220 \\
98.0 \\
101.0 \\
131.0 \\
264.0 \\
332.0 \\
596.0\end{array}$ & $\begin{array}{r}\text { B? } \\
\text { B? } \\
\text { S? } \\
\text { S } \\
\text { S } \\
\text { B? }\end{array}$ & $\begin{array}{l}636542 \\
636644 \\
637542 \\
640874 \\
643643 \\
652771\end{array}$ & $\begin{array}{l}6127560 \\
6127575 \\
6127600 \\
6127641 \\
6127731 \\
6127983\end{array}$ & $\begin{array}{l}0.0 \\
0.0 \\
0.0 \\
0.0 \\
1.2 \\
0.0\end{array}$ & $\begin{array}{l}0.0 \\
0.0 \\
0.0 \\
0.0 \\
2.0 \\
0.0\end{array}$ & $\begin{array}{l}0.0 \\
0.0 \\
0.0 \\
0.0 \\
1.8 \\
0.0\end{array}$ & $\begin{array}{l}0.0 \\
0.0 \\
0.0 \\
0.0 \\
2.7 \\
0.0\end{array}$ & $\begin{array}{l}0.0 \\
0.0 \\
0.0 \\
0.0 \\
1.1 \\
0.0\end{array}$ & $\begin{array}{r}0.0 \\
0.0 \\
0.0 \\
0.0 \\
18.8 \\
0.0\end{array}$ & $\begin{array}{l}--- \\
--- \\
--- \\
--- \\
--- \\
---\end{array}$ & $\begin{array}{l}--- \\
--- \\
--- \\
--- \\
--- \\
---\end{array}$ & $\begin{array}{l}26 \\
30 \\
32 \\
32 \\
32 \\
32\end{array}$ \\
\hline $\begin{array}{l}\text { LINE } \\
\text { A } \\
\text { B } \\
\text { C } \\
\text { D } \\
\text { E }\end{array}$ & $\begin{array}{l}20230 \\
10582.0 \\
10559.0 \\
10479.0 \\
10300.0 \\
10296.0\end{array}$ & $\begin{array}{l}\text { B? } \\
\text { B? } \\
\text { S? } \\
\text { B? } \\
\text { B? }\end{array}$ & $\begin{array}{l}637332 \\
638118 \\
641332 \\
647506 \\
647602\end{array}$ & $\begin{array}{l}6127320 \\
6127309 \\
6127442 \\
6127642 \\
6127646\end{array}$ & $\begin{array}{l}0.0 \\
0.0 \\
0.0 \\
0.0 \\
0.0\end{array}$ & $\begin{array}{l}0.0 \\
0.0 \\
0.0 \\
0.0 \\
0.0\end{array}$ & $\begin{array}{l}0.0 \\
0.0 \\
0.0 \\
0.0 \\
0.0\end{array}$ & $\begin{array}{l}0.0 \\
0.0 \\
0.0 \\
0.0 \\
0.0\end{array}$ & $\begin{array}{l}0.0 \\
0.0 \\
0.0 \\
0.0 \\
0.0\end{array}$ & $\begin{array}{l}0.0 \\
0.0 \\
0.0 \\
0.0 \\
0.0\end{array}$ & $\begin{array}{l}--- \\
--- \\
--- \\
--- \\
---\end{array}$ & $\begin{array}{l}--- \\
--- \\
--- \\
--- \\
---\end{array}$ & $\begin{array}{r}0 \\
0 \\
29 \\
29 \\
0\end{array}$ \\
\hline
\end{tabular}

$\mathrm{CX}=$ COAXIAL

Note: EM values shown above

are local amplitudes

*Estimated Depth may be unreliable because the stronger part of the conductor may be deeper or to one side of the flight line, or because of a shallow dip or magnetite/overburden effects 
EM Anomaly List

\begin{tabular}{|c|c|c|c|c|c|c|c|c|c|c|c|c|c|}
\hline Label & Fid & Interp & $\underset{\mathrm{m}}{\mathrm{XUTM}}$ & $\begin{array}{c}\text { YUTM } \\
\mathrm{m}\end{array}$ & $\begin{array}{l}\text { CX } \\
\text { Real } \\
\text { ppm }\end{array}$ & $\begin{array}{l}00 \mathrm{HZ} \\
\text { Quad } \\
\text { ppm }\end{array}$ & $\begin{array}{l}\text { CP } \\
\text { Real } \\
\text { ppm }\end{array}$ & $\begin{array}{l}900 \mathrm{HZ} \\
\text { Quad } \\
\text { ppm }\end{array}$ & $\begin{array}{l}\text { CP } 7 \\
\text { Real } \\
\text { ppm }\end{array}$ & $\begin{array}{l}200 \mathrm{HZ} \\
\text { Quad } \\
\text { ppm }\end{array}$ & $\begin{array}{l}\text { Vertica } \\
\text { Cond } \\
\text { siemens }\end{array}$ & $\begin{array}{c}\text { al Dike } \\
\text { DEPTH* } \\
\text { m }\end{array}$ & $\begin{array}{c}\text { Mag. Corr } \\
\text { NT }\end{array}$ \\
\hline $\begin{array}{l}\text { LINE } \\
\text { F } \\
\text { G } \\
\text { H }\end{array}$ & $\begin{array}{l}20230 \\
10284.0 \\
10282.0 \\
10158.0\end{array}$ & $\begin{array}{r}\mathrm{B} ? \\
\mathrm{~B} \\
\mathrm{~S} ?\end{array}$ & $\begin{array}{l}647889 \\
647936 \\
653134\end{array}$ & $\begin{array}{l}6127656 \\
6127658 \\
6127799\end{array}$ & $\begin{array}{l}0.0 \\
0.0 \\
3.6\end{array}$ & $\begin{array}{l}0.0 \\
0.0 \\
2.7\end{array}$ & $\begin{array}{l}0.0 \\
0.0 \\
3.0\end{array}$ & $\begin{array}{l}0.0 \\
0.0 \\
6.2\end{array}$ & $\begin{array}{r}0.0 \\
0.0 \\
10.6\end{array}$ & $\begin{array}{r}0.0 \\
0.0 \\
10.0\end{array}$ & $\begin{array}{l}--- \\
--- \\
---\end{array}$ & $\begin{array}{l}--- \\
--- \\
---\end{array}$ & $\begin{array}{l}29 \\
29 \\
29\end{array}$ \\
\hline $\begin{array}{l}\text { LINE } \\
\text { A } \\
\text { B } \\
\text { C } \\
\text { D } \\
\text { E } \\
\text { F } \\
\text { G } \\
\text { H } \\
\text { I }\end{array}$ & $\begin{array}{l}20240 \\
9589.0 \\
9660.0 \\
9664.0 \\
9693.0 \\
9793.0 \\
9864.0 \\
9876.0 \\
9887.0 \\
9956.0\end{array}$ & $\begin{array}{r}\text { S? } \\
\text { B? } \\
\text { B? } \\
\text { S } \\
\text { S? } \\
\text { B? } \\
\text { B? } \\
\text { B? } \\
\text { S? }\end{array}$ & $\begin{array}{l}638109 \\
640103 \\
640263 \\
641272 \\
645125 \\
647141 \\
647561 \\
647966 \\
650664\end{array}$ & $\begin{array}{l}6127172 \\
6127266 \\
6127270 \\
6127282 \\
6127358 \\
6127437 \\
6127456 \\
6127484 \\
6127526\end{array}$ & $\begin{array}{l}0.0 \\
3.5 \\
0.0 \\
1.5 \\
0.0 \\
4.4 \\
6.9 \\
3.2 \\
7.0\end{array}$ & $\begin{array}{r}0.0 \\
5.5 \\
0.0 \\
3.6 \\
0.0 \\
10.8 \\
14.1 \\
4.8 \\
5.1\end{array}$ & $\begin{array}{l}0.0 \\
2.2 \\
0.0 \\
2.6 \\
0.0 \\
2.5 \\
6.3 \\
1.7 \\
6.5\end{array}$ & $\begin{array}{r}0.0 \\
8.0 \\
0.0 \\
4.5 \\
0.0 \\
8.7 \\
20.6 \\
0.4 \\
5.5\end{array}$ & $\begin{array}{r}0.0 \\
17.9 \\
0.0 \\
2.5 \\
0.0 \\
18.1 \\
42.8 \\
3.6 \\
11.7\end{array}$ & $\begin{array}{r}0.0 \\
20.6 \\
0.0 \\
28.3 \\
0.0 \\
21.3 \\
36.5 \\
9.0 \\
9.9\end{array}$ & $\begin{array}{l}--- \\
--- \\
--- \\
--- \\
--- \\
--- \\
--- \\
--- \\
---\end{array}$ & $\begin{array}{l}--- \\
--- \\
--- \\
--- \\
--- \\
--- \\
--- \\
--- \\
---\end{array}$ & $\begin{array}{r}34 \\
65 \\
34 \\
34 \\
0 \\
34 \\
34 \\
34 \\
491\end{array}$ \\
\hline $\begin{array}{l}\text { LINE } \\
\text { A } \\
B \\
\text { C } \\
\text { D } \\
\text { E } \\
\text { F }\end{array}$ & $\begin{array}{r}20250 \\
9484.0 \\
9468.0 \\
9418.0 \\
9377.0 \\
9291.0 \\
9220.0\end{array}$ & $\begin{array}{r}\text { S? } \\
\text { B? } \\
\text { S } \\
\text { S? } \\
\text { S } \\
\text { S }\end{array}$ & $\begin{array}{l}637257 \\
637918 \\
640058 \\
641786 \\
644991 \\
647311\end{array}$ & $\begin{array}{l}6127008 \\
6126993 \\
6126994 \\
6127068 \\
6127202 \\
6127225\end{array}$ & $\begin{array}{r}24.4 \\
0.0 \\
3.4 \\
0.0 \\
0.0 \\
4.1\end{array}$ & $\begin{array}{l}9.9 \\
0.0 \\
3.4 \\
0.0 \\
0.0 \\
6.3\end{array}$ & $\begin{array}{r}37.0 \\
0.0 \\
3.4 \\
0.0 \\
0.0 \\
1.9\end{array}$ & $\begin{array}{r}17.2 \\
0.0 \\
4.2 \\
0.0 \\
0.0 \\
6.1\end{array}$ & $\begin{array}{r}35.8 \\
0.0 \\
0.1 \\
0.0 \\
0.0 \\
11.5\end{array}$ & $\begin{array}{r}11.6 \\
0.0 \\
33.1 \\
0.0 \\
0.0 \\
18.7\end{array}$ & $\begin{array}{l}--- \\
--- \\
--- \\
--- \\
--- \\
---\end{array}$ & $\begin{array}{l}--- \\
--- \\
--- \\
--- \\
--- \\
---\end{array}$ & $\begin{array}{r}0 \\
41 \\
44 \\
41 \\
0 \\
0\end{array}$ \\
\hline $\begin{array}{l}\text { LINE } \\
\text { A } \\
B \\
\text { C }\end{array}$ & $\begin{array}{r}20260 \\
8414.0 \\
8418.0 \\
8692.0\end{array}$ & $\begin{array}{l}\text { B? } \\
\text { B? } \\
\text { S? }\end{array}$ & $\begin{array}{l}637738 \\
637867 \\
647158\end{array}$ & $\begin{array}{l}6126847 \\
6126834 \\
6127121\end{array}$ & $\begin{array}{l}0.0 \\
0.0 \\
0.9\end{array}$ & $\begin{array}{l}0.0 \\
0.0 \\
5.4\end{array}$ & $\begin{array}{l}0.0 \\
0.0 \\
0.6\end{array}$ & $\begin{array}{l}0.0 \\
0.0 \\
6.0\end{array}$ & $\begin{array}{l}0.0 \\
0.0 \\
0.5\end{array}$ & $\begin{array}{r}0.0 \\
0.0 \\
37.2\end{array}$ & $\begin{array}{l}--- \\
--- \\
---\end{array}$ & $\begin{array}{l}--- \\
--- \\
---\end{array}$ & $\begin{array}{r}47 \\
0 \\
47\end{array}$ \\
\hline $\begin{array}{l}\text { LINE } \\
\text { A } \\
\text { B } \\
\text { C } \\
\text { D } \\
\text { E } \\
\text { F } \\
\text { G } \\
\text { H }\end{array}$ & $\begin{array}{r}20270 \\
8377.0 \\
8340.0 \\
8309.0 \\
8185.0 \\
8135.0 \\
8132.0 \\
8104.0 \\
8029.0\end{array}$ & $\begin{array}{r}\text { S } \\
\text { H? } \\
\text { H? } \\
\text { S } \\
\text { B } \\
\text { B } \\
\text { B? } \\
\text { S }\end{array}$ & $\begin{array}{l}638263 \\
639805 \\
640950 \\
644921 \\
646323 \\
646396 \\
647264 \\
649866\end{array}$ & $\begin{array}{l}6126653 \\
6126594 \\
6126689 \\
6126785 \\
6126816 \\
6126810 \\
6126815 \\
6126918\end{array}$ & $\begin{array}{r}0.0 \\
0.0 \\
0.8 \\
0.0 \\
0.0 \\
17.9 \\
0.0 \\
0.0\end{array}$ & $\begin{array}{r}0.0 \\
0.0 \\
1.3 \\
0.0 \\
0.0 \\
21.0 \\
0.0 \\
0.0\end{array}$ & $\begin{array}{r}0.0 \\
0.0 \\
0.6 \\
0.0 \\
0.0 \\
19.6 \\
0.0 \\
0.0\end{array}$ & $\begin{array}{r}0.0 \\
0.0 \\
3.1 \\
0.0 \\
0.0 \\
26.0 \\
0.0 \\
0.0\end{array}$ & $\begin{array}{r}0.0 \\
0.0 \\
1.2 \\
0.0 \\
0.0 \\
58.4 \\
0.0 \\
0.0\end{array}$ & $\begin{array}{r}0.0 \\
0.0 \\
19.9 \\
0.0 \\
0.0 \\
60.7 \\
0.0 \\
0.0\end{array}$ & $\begin{array}{l}--- \\
--- \\
--- \\
--- \\
--- \\
--- \\
--- \\
---\end{array}$ & $\begin{array}{l}--- \\
--- \\
--- \\
--- \\
--- \\
--- \\
--- \\
---\end{array}$ & $\begin{array}{r}45 \\
45 \\
36 \\
45 \\
45 \\
0 \\
70 \\
0\end{array}$ \\
\hline $\begin{array}{l}\text { LINE } \\
\text { A } \\
B \\
\text { C }\end{array}$ & $\begin{array}{r}20280 \\
7298.0 \\
7405.0 \\
7507.0\end{array}$ & $\begin{array}{r}\mathrm{B} ? \\
\mathrm{~B} \\
\mathrm{~B}\end{array}$ & $\begin{array}{l}639263 \\
643053 \\
646359\end{array}$ & $\begin{array}{l}6126472 \\
6126513 \\
6126634\end{array}$ & $\begin{array}{l}0.0 \\
2.7 \\
0.0\end{array}$ & $\begin{array}{l}0.0 \\
0.6 \\
0.0\end{array}$ & $\begin{array}{l}0.0 \\
0.4 \\
0.0\end{array}$ & $\begin{array}{l}0.0 \\
0.9 \\
0.0\end{array}$ & $\begin{array}{l}0.0 \\
1.1 \\
0.0\end{array}$ & $\begin{array}{l}0.0 \\
4.3 \\
0.0\end{array}$ & $\begin{array}{l}--- \\
--- \\
---\end{array}$ & $\begin{array}{l}--- \\
--- \\
---\end{array}$ & $\begin{array}{r}46 \\
0 \\
46\end{array}$ \\
\hline $\begin{array}{l}\text { LINE } \\
\text { A } \\
B\end{array}$ & $\begin{array}{l}20290 \\
1702.0 \\
1458.0\end{array}$ & $\begin{array}{l}\mathrm{H} \\
\mathrm{B}\end{array}$ & $\begin{array}{l}638861 \\
646383\end{array}$ & $\begin{array}{l}6126190 \\
6126361\end{array}$ & $\begin{array}{l}5.3 \\
0.0\end{array}$ & $\begin{array}{l}4.0 \\
0.0\end{array}$ & $\begin{array}{r}11.1 \\
0.0\end{array}$ & $\begin{array}{l}5.4 \\
0.0\end{array}$ & $\begin{array}{r}12.8 \\
0.0\end{array}$ & $\begin{array}{r}19.8 \\
0.0\end{array}$ & $\begin{array}{l}--- \\
---\end{array}$ & $\begin{array}{l}--- \\
---\end{array}$ & $\begin{array}{l}0 \\
0\end{array}$ \\
\hline
\end{tabular}

$\mathrm{CX}=$ COAXIAL

Note: EM values shown above

are local amplitudes

*Estimated Depth may be unreliable because the stronger part of the conductor may be deeper or to one side of the flight line, or because of a shallow dip or magnetite/overburden effects 
EM Anomaly List

\begin{tabular}{|c|c|c|c|c|c|c|c|c|c|c|c|c|c|}
\hline Label & Fid & Interp & $\begin{array}{c}\text { XUTM } \\
\text { m }\end{array}$ & $\begin{array}{c}\text { YUTM } \\
\mathrm{m}\end{array}$ & $\begin{array}{ll}\text { CX } & 9 \\
\text { Real } & \\
\text { ppm }\end{array}$ & $\begin{array}{l}00 \mathrm{HZ} \\
\text { Quad } \\
\text { ppm }\end{array}$ & $\begin{array}{l}\text { CP } \\
\text { Real } \\
\text { ppm }\end{array}$ & $\begin{array}{l}900 \mathrm{HZ} \\
\text { Quad } \\
\text { ppm }\end{array}$ & $\begin{array}{l}\text { CP } 7 \\
\text { Real } \\
\text { ppm }\end{array}$ & $\begin{array}{l}200 \mathrm{~Hz} \\
\text { Quad } \\
\text { ppm }\end{array}$ & $\begin{array}{l}\text { Vertica } \\
\text { Cond } \\
\text { siemens }\end{array}$ & $\begin{array}{c}\text { al Dike } \\
\text { DEPTH* } \\
\text { m }\end{array}$ & $\begin{array}{c}\text { Mag. Corr } \\
\text { NT }\end{array}$ \\
\hline $\begin{array}{l}\text { LINE } \\
\text { A } \\
B\end{array}$ & $\begin{array}{l}20300 \\
2082.0 \\
2086.0\end{array}$ & $\begin{array}{r}\text { B } \\
\text { B? }\end{array}$ & $\begin{array}{l}646440 \\
646542\end{array}$ & $\begin{array}{l}6126256 \\
6126257\end{array}$ & $\begin{array}{l}0.0 \\
0.0\end{array}$ & $\begin{array}{l}0.0 \\
0.0\end{array}$ & $\begin{array}{l}0.0 \\
0.0\end{array}$ & $\begin{array}{l}0.0 \\
0.0\end{array}$ & $\begin{array}{l}0.0 \\
0.0\end{array}$ & $\begin{array}{l}0.0 \\
0.0\end{array}$ & $\begin{array}{l}--- \\
---\end{array}$ & $\begin{array}{l}--- \\
---\end{array}$ & $\begin{array}{l}49 \\
49\end{array}$ \\
\hline $\begin{array}{l}\text { LINE } \\
A \\
B\end{array}$ & $\begin{array}{r}20310 \\
2845.0 \\
2844.0\end{array}$ & $\begin{array}{l}\mathrm{B} \\
\mathrm{B}\end{array}$ & $\begin{array}{l}646347 \\
646369\end{array}$ & $\begin{array}{l}6125960 \\
6125960\end{array}$ & $\begin{array}{l}0.0 \\
0.0\end{array}$ & $\begin{array}{l}0.0 \\
0.0\end{array}$ & $\begin{array}{l}0.0 \\
0.0\end{array}$ & $\begin{array}{l}0.0 \\
0.0\end{array}$ & $\begin{array}{l}0.0 \\
0.0\end{array}$ & $\begin{array}{l}0.0 \\
0.0\end{array}$ & $\begin{array}{l}--- \\
---\end{array}$ & $\begin{array}{l}--- \\
---\end{array}$ & $\begin{array}{r}54 \\
0\end{array}$ \\
\hline $\begin{array}{l}\text { LINE } \\
A\end{array}$ & $\begin{array}{l}20311 \\
3218.0\end{array}$ & S & 639311 & 6125799 & 3.8 & 1.5 & 0.9 & 2.4 & 3.4 & 6.3 & --- & --- & 0 \\
\hline $\begin{array}{l}\text { LINE } \\
\text { A } \\
\text { B } \\
\text { C } \\
\text { D }\end{array}$ & $\begin{array}{r}20320 \\
3420.0 \\
3458.0 \\
3650.0 \\
3794.0\end{array}$ & $\begin{array}{l}\mathrm{S} \\
\mathrm{S} \\
\mathrm{S} \\
\mathrm{H}\end{array}$ & $\begin{array}{l}639574 \\
640634 \\
645845 \\
649670\end{array}$ & $\begin{array}{l}6125547 \\
6125654 \\
6125820 \\
6125857\end{array}$ & $\begin{array}{l}3.8 \\
8.8 \\
2.1 \\
0.0\end{array}$ & $\begin{array}{l}5.1 \\
1.3 \\
0.4 \\
0.0\end{array}$ & $\begin{array}{l}2.0 \\
9.0 \\
3.2 \\
0.0\end{array}$ & $\begin{array}{l}5.9 \\
2.5 \\
0.2 \\
0.0\end{array}$ & $\begin{array}{r}15.6 \\
7.1 \\
2.1 \\
0.0\end{array}$ & $\begin{array}{r}22.6 \\
15.2 \\
4.3 \\
0.0\end{array}$ & $\begin{array}{l}--- \\
--- \\
--- \\
---\end{array}$ & $\begin{array}{l}--- \\
--- \\
--- \\
---\end{array}$ & $\begin{array}{r}0 \\
78 \\
78 \\
0\end{array}$ \\
\hline $\begin{array}{l}\text { LINE } \\
\text { A } \\
\text { B } \\
\text { C } \\
\text { D }\end{array}$ & $\begin{array}{r}20330 \\
4792.0 \\
4604.0 \\
4418.0 \\
4294.0\end{array}$ & $\begin{array}{r}\text { S? } \\
\text { S } \\
\text { B } \\
\text { B? }\end{array}$ & $\begin{array}{l}638148 \\
642779 \\
646572 \\
649717\end{array}$ & $\begin{array}{l}6125377 \\
6125510 \\
6125591 \\
6125756\end{array}$ & $\begin{array}{l}8.1 \\
1.8 \\
0.0 \\
4.2\end{array}$ & $\begin{array}{l}1.0 \\
1.7 \\
0.0 \\
4.4\end{array}$ & $\begin{array}{l}6.4 \\
0.3 \\
0.0 \\
4.7\end{array}$ & $\begin{array}{l}0.7 \\
2.4 \\
0.0 \\
8.0\end{array}$ & $\begin{array}{r}5.2 \\
0.1 \\
0.0 \\
16.8\end{array}$ & $\begin{array}{r}7.9 \\
16.4 \\
0.0 \\
12.1\end{array}$ & $\begin{array}{l}--- \\
--- \\
--- \\
---\end{array}$ & $\begin{array}{l}--- \\
--- \\
--- \\
---\end{array}$ & $\begin{array}{r}50 \\
0 \\
0 \\
76\end{array}$ \\
\hline $\begin{array}{l}\text { LINE } \\
\text { A } \\
B\end{array}$ & $\begin{array}{r}20340 \\
4850.0 \\
5267.0\end{array}$ & $\begin{array}{l}\mathrm{S} \\
\mathrm{B}\end{array}$ & $\begin{array}{l}638491 \\
649784\end{array}$ & $\begin{array}{l}6125208 \\
6125556\end{array}$ & $\begin{array}{l}5.4 \\
0.0\end{array}$ & $\begin{array}{l}2.1 \\
0.0\end{array}$ & $\begin{array}{l}6.9 \\
0.0\end{array}$ & $\begin{array}{l}1.2 \\
0.0\end{array}$ & $\begin{array}{l}5.5 \\
0.0\end{array}$ & $\begin{array}{l}9.6 \\
0.0\end{array}$ & $\begin{array}{l}--- \\
---\end{array}$ & $\begin{array}{l}--- \\
---\end{array}$ & $\begin{array}{l}43 \\
69\end{array}$ \\
\hline $\begin{array}{l}\text { LINE } \\
\text { A } \\
B\end{array}$ & $\begin{array}{r}20350 \\
6349.0 \\
5971.0\end{array}$ & $\begin{array}{l}\text { S } \\
\text { B }\end{array}$ & $\begin{array}{l}640309 \\
649794\end{array}$ & $\begin{array}{l}6125066 \\
6125341\end{array}$ & $\begin{array}{l}2.1 \\
3.9\end{array}$ & $\begin{array}{l}2.1 \\
5.9\end{array}$ & $\begin{array}{l}0.1 \\
4.6\end{array}$ & $\begin{array}{l}2.7 \\
8.7\end{array}$ & $\begin{array}{r}3.4 \\
20.0\end{array}$ & $\begin{array}{l}18.8 \\
19.5\end{array}$ & $\begin{array}{l}--- \\
---\end{array}$ & $\begin{array}{l}--- \\
---\end{array}$ & $\begin{array}{r}0 \\
47\end{array}$ \\
\hline $\begin{array}{l}\text { LINE } \\
\text { A } \\
\text { B } \\
\text { C } \\
\text { D } \\
\text { E }\end{array}$ & $\begin{array}{l}20360 \\
6622.0 \\
6756.0 \\
6862.0 \\
6925.0 \\
6928.0\end{array}$ & $\begin{array}{l}\text { S } \\
\text { B } \\
\text { B } \\
\text { B } \\
\text { B }\end{array}$ & $\begin{array}{l}643918 \\
646658 \\
649731 \\
650814 \\
650873\end{array}$ & $\begin{array}{l}6124977 \\
6125020 \\
6125147 \\
6125017 \\
6125006\end{array}$ & $\begin{array}{l}0.8 \\
0.0 \\
0.0 \\
0.0 \\
0.0\end{array}$ & $\begin{array}{l}1.8 \\
0.0 \\
0.0 \\
0.0 \\
0.0\end{array}$ & $\begin{array}{l}0.6 \\
0.0 \\
0.0 \\
0.0 \\
0.0\end{array}$ & $\begin{array}{l}2.0 \\
0.0 \\
0.0 \\
0.0 \\
0.0\end{array}$ & $\begin{array}{l}0.2 \\
0.0 \\
0.0 \\
0.0 \\
0.0\end{array}$ & $\begin{array}{r}14.4 \\
0.0 \\
0.0 \\
0.0 \\
0.0\end{array}$ & $\begin{array}{l}--- \\
--- \\
--- \\
--- \\
---\end{array}$ & $\begin{array}{l}--- \\
--- \\
--- \\
--- \\
---\end{array}$ & $\begin{array}{r}33 \\
0 \\
0 \\
0 \\
1956\end{array}$ \\
\hline $\begin{array}{l}\text { LINE } \\
\text { A } \\
\text { B } \\
\text { C } \\
\text { D } \\
\text { E } \\
\text { F } \\
\text { G }\end{array}$ & $\begin{array}{l}20370 \\
7778.0 \\
7765.0 \\
7637.0 \\
7522.0 \\
7432.0 \\
7430.0 \\
7381.0\end{array}$ & $\begin{array}{r}\text { S } \\
\text { S } \\
\text { S } \\
\text { B? } \\
\text { B? } \\
\text { B? } \\
\text { B? }\end{array}$ & $\begin{array}{l}639386 \\
639853 \\
643658 \\
646594 \\
649724 \\
649791 \\
650902\end{array}$ & $\begin{array}{l}6124601 \\
6124643 \\
6124819 \\
6124801 \\
6124954 \\
6124957 \\
6124953\end{array}$ & $\begin{array}{l}3.9 \\
2.4 \\
1.9 \\
0.0 \\
0.0 \\
7.2 \\
0.0\end{array}$ & $\begin{array}{l}3.5 \\
3.6 \\
0.5 \\
0.0 \\
0.0 \\
7.4 \\
0.0\end{array}$ & $\begin{array}{l}1.8 \\
1.2 \\
0.0 \\
0.0 \\
0.0 \\
5.2 \\
0.0\end{array}$ & $\begin{array}{l}4.4 \\
4.5 \\
0.9 \\
0.0 \\
0.0 \\
9.0 \\
0.0\end{array}$ & $\begin{array}{r}8.8 \\
1.5 \\
0.0 \\
0.0 \\
0.0 \\
18.3 \\
0.0\end{array}$ & $\begin{array}{r}22.9 \\
26.4 \\
6.3 \\
0.0 \\
0.0 \\
15.5 \\
0.0\end{array}$ & $\begin{array}{l}--- \\
--- \\
--- \\
--- \\
--- \\
--- \\
---\end{array}$ & $\begin{array}{l}--- \\
--- \\
--- \\
--- \\
--- \\
--- \\
---\end{array}$ & $\begin{array}{r}58 \\
0 \\
0 \\
58 \\
0 \\
0 \\
2391\end{array}$ \\
\hline
\end{tabular}

$\mathrm{CX}=$ COAXIAL

Note: EM values shown above are local amplitudes

*Estimated Depth may be unreliable because the stronger part of the conductor may be deeper or to one side of the flight line, or because of a shallow dip or magnetite/overburden effects 
EM Anomaly List

\begin{tabular}{|c|c|c|c|c|c|c|c|c|c|c|c|c|c|}
\hline Label & Fid & Interp & $\begin{array}{c}\text { XUTM } \\
\text { m }\end{array}$ & $\begin{array}{c}\text { YUTM } \\
\mathrm{m}\end{array}$ & $\begin{array}{l}\text { CX } \\
\text { Real } \\
\text { ppm }\end{array}$ & $\begin{array}{l}00 \mathrm{HZ} \\
\text { Quad } \\
\text { ppm }\end{array}$ & $\begin{array}{l}\text { CP } \\
\text { Real } \\
\text { ppm }\end{array}$ & $\begin{array}{l}900 \mathrm{HZ} \\
\text { Quad } \\
\text { ppm }\end{array}$ & $\begin{array}{l}\text { CP } \\
\text { Real } \\
\text { ppm }\end{array}$ & $\begin{array}{l}200 \mathrm{HZ} \\
\text { Quad } \\
\text { ppm }\end{array}$ & $\begin{array}{l}\text { Vertica } \\
\text { Cond } \\
\text { siemens }\end{array}$ & $\begin{array}{c}\text { al Dike } \\
\text { DEPTH* } \\
\text { m }\end{array}$ & $\begin{array}{c}\text { Mag. Corr } \\
\text { NT }\end{array}$ \\
\hline $\begin{array}{l}\mathrm{LINE} \\
\mathrm{H}\end{array}$ & $\begin{array}{l}20370 \\
7379.0\end{array}$ & $B$ ? & 650939 & 6124950 & 0.0 & 0.0 & 0.0 & 0.0 & 0.0 & 0.0 & --- & --- & 2391 \\
\hline $\begin{array}{l}\text { LINE } \\
\text { A } \\
\text { B } \\
\text { C } \\
\text { D } \\
\text { E }\end{array}$ & $\begin{array}{r}20380 \\
8405.0 \\
8409.0 \\
8526.0 \\
8530.0 \\
8550.0\end{array}$ & $\begin{array}{r}\text { B? } \\
\text { B? } \\
\text { B? } \\
\text { B } \\
\text { S }\end{array}$ & $\begin{array}{l}646423 \\
646547 \\
649796 \\
649935 \\
650694\end{array}$ & $\begin{array}{l}6124599 \\
6124605 \\
6124725 \\
6124743 \\
6124781\end{array}$ & $\begin{array}{l}0.0 \\
7.6 \\
0.0 \\
0.0 \\
0.0\end{array}$ & $\begin{array}{l}0.0 \\
3.5 \\
0.0 \\
0.0 \\
0.0\end{array}$ & $\begin{array}{r}0.0 \\
13.5 \\
0.0 \\
0.0 \\
0.0\end{array}$ & $\begin{array}{l}0.0 \\
6.0 \\
0.0 \\
0.0 \\
0.0\end{array}$ & $\begin{array}{r}0.0 \\
15.2 \\
0.0 \\
0.0 \\
0.0\end{array}$ & $\begin{array}{r}0.0 \\
34.3 \\
0.0 \\
0.0 \\
0.0\end{array}$ & $\begin{array}{l}--- \\
--- \\
--- \\
--- \\
---\end{array}$ & $\begin{array}{l}--- \\
--- \\
--- \\
--- \\
---\end{array}$ & $\begin{array}{r}0 \\
54 \\
0 \\
54 \\
893\end{array}$ \\
\hline $\begin{array}{l}\text { LINE } \\
\text { A } \\
\text { B } \\
\text { C } \\
\text { D }\end{array}$ & $\begin{array}{l}20390 \\
9346.0 \\
9104.0 \\
9099.0 \\
9016.0\end{array}$ & $\begin{array}{r}\mathrm{S} \\
\mathrm{B} \\
\mathrm{B} \\
\mathrm{B} ?\end{array}$ & $\begin{array}{l}639901 \\
646607 \\
646745 \\
649897\end{array}$ & $\begin{array}{l}6124235 \\
6124372 \\
6124385 \\
6124479\end{array}$ & $\begin{array}{l}1.2 \\
0.0 \\
0.0 \\
0.0\end{array}$ & $\begin{array}{l}2.0 \\
0.0 \\
0.0 \\
0.0\end{array}$ & $\begin{array}{l}2.7 \\
0.0 \\
0.0 \\
0.0\end{array}$ & $\begin{array}{l}2.2 \\
0.0 \\
0.0 \\
0.0\end{array}$ & $\begin{array}{l}1.6 \\
0.0 \\
0.0 \\
0.0\end{array}$ & $\begin{array}{r}14.0 \\
0.0 \\
0.0 \\
0.0\end{array}$ & $\begin{array}{l}--- \\
--- \\
--- \\
---\end{array}$ & $\begin{array}{l}--- \\
--- \\
--- \\
---\end{array}$ & $\begin{array}{r}70 \\
0 \\
0 \\
0\end{array}$ \\
\hline $\begin{array}{l}\text { LINE } \\
\text { A } \\
\text { B } \\
\text { C }\end{array}$ & $\begin{array}{l}20400 \\
9436.0 \\
9638.0 \\
9725.0\end{array}$ & $\begin{array}{r}\mathrm{S} \\
\mathrm{H} \\
\mathrm{B} ?\end{array}$ & $\begin{array}{l}639385 \\
646891 \\
649862\end{array}$ & $\begin{array}{l}6123944 \\
6124154 \\
6124280\end{array}$ & $\begin{array}{l}4.2 \\
0.0 \\
0.0\end{array}$ & $\begin{array}{l}3.8 \\
0.0 \\
0.0\end{array}$ & $\begin{array}{l}2.2 \\
0.0 \\
0.0\end{array}$ & $\begin{array}{l}3.6 \\
0.0 \\
0.0\end{array}$ & $\begin{array}{l}7.7 \\
0.0 \\
0.0\end{array}$ & $\begin{array}{r}20.0 \\
0.0 \\
0.0\end{array}$ & $\begin{array}{l}--- \\
--- \\
---\end{array}$ & $\begin{array}{l}--- \\
--- \\
---\end{array}$ & $\begin{array}{r}42 \\
0 \\
42\end{array}$ \\
\hline $\begin{array}{l}\text { LINE } \\
A\end{array}$ & $\begin{array}{l}20410 \\
10037.0\end{array}$ & S & 653223 & 6124177 & 18.0 & 1.0 & 21.1 & 0.1 & 14.3 & 0.7 & --- & --- & 0 \\
\hline $\begin{array}{l}\text { LINE } \\
A\end{array}$ & $\begin{array}{l}20420 \\
10823.0\end{array}$ & S? & 651872 & 6124035 & 0.0 & 0.0 & 0.0 & 0.0 & 0.0 & 0.0 & --- & --- & 0 \\
\hline $\begin{array}{l}\text { LINE } \\
\text { A } \\
\text { B } \\
\text { C } \\
\text { D } \\
\text { E }\end{array}$ & $\begin{array}{l}20430 \\
11485.0 \\
11472.0 \\
11460.0 \\
11457.0 \\
11455.0\end{array}$ & $\begin{array}{l}\text { S? } \\
\text { B? } \\
\text { B? } \\
\text { B? } \\
\text { B }\end{array}$ & $\begin{array}{l}649877 \\
650233 \\
650474 \\
650525 \\
650559\end{array}$ & $\begin{array}{l}6123623 \\
6123654 \\
6123675 \\
6123683 \\
6123688\end{array}$ & $\begin{array}{l}1.0 \\
0.0 \\
4.1 \\
0.0 \\
0.0\end{array}$ & $\begin{array}{r}1.3 \\
0.0 \\
14.1 \\
0.0 \\
0.0\end{array}$ & $\begin{array}{r}1.3 \\
0.0 \\
11.7 \\
0.0 \\
0.0\end{array}$ & $\begin{array}{r}4.6 \\
0.0 \\
36.6 \\
0.0 \\
0.0\end{array}$ & $\begin{array}{r}12.5 \\
0.0 \\
76.7 \\
0.0 \\
0.0\end{array}$ & $\begin{array}{r}8.1 \\
0.0 \\
74.0 \\
0.0 \\
0.0\end{array}$ & $\begin{array}{l}--- \\
--- \\
--- \\
--- \\
---\end{array}$ & $\begin{array}{l}--- \\
--- \\
--- \\
--- \\
---\end{array}$ & $\begin{array}{r}52 \\
0 \\
135 \\
135 \\
0\end{array}$ \\
\hline $\begin{array}{l}\text { LINE } \\
\text { A } \\
\text { B } \\
\text { C } \\
\text { D }\end{array}$ & $\begin{array}{r}20442 \\
479.0 \\
496.0 \\
513.0 \\
520.0\end{array}$ & $\begin{array}{r}\text { B? } \\
\text { B? } \\
\text { B? } \\
\text { B }\end{array}$ & $\begin{array}{l}650103 \\
650370 \\
650602 \\
650728\end{array}$ & $\begin{array}{l}6123481 \\
6123512 \\
6123507 \\
6123512\end{array}$ & $\begin{array}{l}2.5 \\
0.0 \\
5.4 \\
0.0\end{array}$ & $\begin{array}{r}7.8 \\
0.0 \\
18.0 \\
0.0\end{array}$ & $\begin{array}{l}0.1 \\
0.0 \\
6.1 \\
0.0\end{array}$ & $\begin{array}{r}4.3 \\
0.0 \\
23.7 \\
0.0\end{array}$ & $\begin{array}{r}9.9 \\
0.0 \\
52.5 \\
0.0\end{array}$ & $\begin{array}{r}17.9 \\
0.0 \\
75.7 \\
0.0\end{array}$ & $\begin{array}{l}--- \\
--- \\
--- \\
---\end{array}$ & $\begin{array}{l}--- \\
--- \\
--- \\
---\end{array}$ & $\begin{array}{r}71 \\
0 \\
115 \\
74\end{array}$ \\
\hline $\begin{array}{l}\text { LINE } \\
A\end{array}$ & $\begin{array}{r}20443 \\
620.0\end{array}$ & S? & 652197 & 6123541 & 0.0 & 0.0 & 0.0 & 0.0 & 0.0 & 0.0 & --- & --- & 88 \\
\hline $\begin{array}{l}\text { LINE } \\
\text { A } \\
B \\
C\end{array}$ & $\begin{array}{c}20450 \\
1160.0 \\
1026.0 \\
853.0\end{array}$ & $\begin{array}{l}\text { B } \\
\text { S? } \\
\text { H? }\end{array}$ & $\begin{array}{l}646987 \\
650703 \\
653844\end{array}$ & $\begin{array}{l}6123181 \\
6123265 \\
6123385\end{array}$ & $\begin{array}{r}0.0 \\
0.0 \\
2774.6\end{array}$ & $\begin{array}{r}0.0 \\
0.0 \\
108.6\end{array}$ & $\begin{array}{r}0.0 \\
0.0 \\
240.9\end{array}$ & $\begin{array}{l}0.0 \\
0.0 \\
6.7\end{array}$ & $\begin{array}{r}0.0 \\
0.0 \\
159.4\end{array}$ & $\begin{array}{r}0.0 \\
0.0 \\
21.7\end{array}$ & $\begin{array}{l}--- \\
--- \\
---\end{array}$ & $\begin{array}{l}--- \\
--- \\
---\end{array}$ & $\begin{array}{l}0 \\
0 \\
0\end{array}$ \\
\hline
\end{tabular}

$\mathrm{CX}=$ COAXIAL

$\mathrm{CP}=$ COPLANAR

Note: EM values shown above

are local amplitudes

*Estimated Depth may be unreliable because the stronger part of the conductor may be deeper or to one side of the flight line, or because of a shallow dip or magnetite/overburden effects 
EM Anomaly List

\begin{tabular}{|c|c|c|c|c|c|c|c|c|c|c|c|c|c|}
\hline Label & Fid & Interp & $\begin{array}{c}\text { XUTM } \\
\text { m }\end{array}$ & $\begin{array}{c}\text { YUTM } \\
\mathrm{m}\end{array}$ & $\begin{array}{ll}\text { CX } & 9 \\
\text { Real } & \\
\text { ppm }\end{array}$ & $\begin{array}{l}00 \mathrm{HZ} \\
\text { Quad } \\
\text { ppm }\end{array}$ & $\begin{array}{l}\text { CP } \\
\text { Real } \\
\text { ppm }\end{array}$ & $\begin{array}{l}900 \mathrm{HZ} \\
\text { Quad } \\
\text { ppm }\end{array}$ & $\begin{array}{l}\text { CP } 7 \\
\text { Real } \\
\text { ppm }\end{array}$ & $\begin{array}{l}200 \mathrm{HZ} \\
\text { Quad } \\
\text { ppm }\end{array}$ & $\begin{array}{l}\text { Vertica } \\
\text { Cond } \\
\text { siemens }\end{array}$ & $\begin{array}{c}\text { al Dike } \\
\text { DEPTH* } \\
\text { m }\end{array}$ & $\begin{array}{c}\text { Mag. Corr } \\
\text { NT }\end{array}$ \\
\hline $\begin{array}{l}\text { LINE } \\
\text { A } \\
\text { B } \\
\text { C } \\
\text { D }\end{array}$ & $\begin{array}{l}20460 \\
841.0 \\
1002.0 \\
1203.0 \\
1221.0\end{array}$ & $\begin{array}{l}\text { B } \\
\text { H? } \\
\text { H? } \\
\text { B? }\end{array}$ & $\begin{array}{l}646948 \\
650751 \\
653948 \\
654392\end{array}$ & $\begin{array}{l}6122971 \\
6123164 \\
6123182 \\
6123203\end{array}$ & $\begin{array}{l}0.0 \\
3.0 \\
0.0 \\
0.0\end{array}$ & $\begin{array}{l}0.0 \\
6.3 \\
0.0 \\
0.0\end{array}$ & $\begin{array}{l}0.0 \\
5.0 \\
0.0 \\
0.0\end{array}$ & $\begin{array}{l}0.0 \\
7.5 \\
0.0 \\
0.0\end{array}$ & $\begin{array}{r}0.0 \\
16.0 \\
0.0 \\
0.0\end{array}$ & $\begin{array}{r}0.0 \\
14.5 \\
0.0 \\
0.0\end{array}$ & $\begin{array}{l}--- \\
--- \\
--- \\
---\end{array}$ & $\begin{array}{l}--- \\
--- \\
--- \\
---\end{array}$ & $\begin{array}{r}33 \\
49 \\
677 \\
49\end{array}$ \\
\hline $\begin{array}{l}\text { LINE } \\
\text { A } \\
B \\
C\end{array}$ & $\begin{array}{r}20470 \\
1913.0 \\
1840.0 \\
1581.0\end{array}$ & $\begin{array}{r}\text { S } \\
\text { B } \\
\text { S? }\end{array}$ & $\begin{array}{l}644554 \\
646717 \\
652356\end{array}$ & $\begin{array}{l}6122687 \\
6122495 \\
6122937\end{array}$ & $\begin{array}{l}4.5 \\
0.0 \\
0.0\end{array}$ & $\begin{array}{l}0.2 \\
0.0 \\
0.0\end{array}$ & $\begin{array}{l}0.6 \\
0.0 \\
0.0\end{array}$ & $\begin{array}{l}1.2 \\
0.0 \\
0.0\end{array}$ & $\begin{array}{l}1.0 \\
0.0 \\
0.0\end{array}$ & $\begin{array}{l}5.0 \\
0.0 \\
0.0\end{array}$ & $\begin{array}{l}--- \\
--- \\
---\end{array}$ & $\begin{array}{l}--- \\
--- \\
---\end{array}$ & $\begin{array}{l}54 \\
54 \\
54\end{array}$ \\
\hline $\begin{array}{l}\text { LINE } \\
\text { A } \\
\text { B }\end{array}$ & $\begin{array}{r}20480 \\
2204.0 \\
2697.0\end{array}$ & $\begin{array}{r}B \\
\text { S? }\end{array}$ & $\begin{array}{l}646691 \\
657442\end{array}$ & $\begin{array}{l}6122572 \\
6122876\end{array}$ & $\begin{array}{l}0.0 \\
1.7\end{array}$ & $\begin{array}{l}0.0 \\
7.5\end{array}$ & $\begin{array}{l}0.0 \\
2.7\end{array}$ & $\begin{array}{r}0.0 \\
10.9\end{array}$ & $\begin{array}{r}0.0 \\
11.3\end{array}$ & $\begin{array}{r}0.0 \\
65.8\end{array}$ & $\begin{array}{l}--- \\
---\end{array}$ & $\begin{array}{l}--- \\
---\end{array}$ & $\begin{array}{l}87 \\
36\end{array}$ \\
\hline $\begin{array}{l}\text { LINE } \\
\text { A } \\
\text { B } \\
\text { C } \\
\text { D }\end{array}$ & $\begin{array}{r}20490 \\
3231.0 \\
3185.0 \\
2866.0 \\
2722.0\end{array}$ & $\begin{array}{r}\text { S } \\
B \\
S ? \\
S ?\end{array}$ & $\begin{array}{l}645403 \\
646629 \\
654222 \\
657457\end{array}$ & $\begin{array}{l}6122368 \\
6122394 \\
6122597 \\
6122710\end{array}$ & $\begin{array}{l}0.0 \\
0.0 \\
0.0 \\
0.0\end{array}$ & $\begin{array}{l}0.0 \\
0.0 \\
0.0 \\
0.0\end{array}$ & $\begin{array}{l}0.0 \\
0.0 \\
0.0 \\
0.0\end{array}$ & $\begin{array}{l}0.0 \\
0.0 \\
0.0 \\
0.0\end{array}$ & $\begin{array}{l}0.0 \\
0.0 \\
0.0 \\
0.0\end{array}$ & $\begin{array}{l}0.0 \\
0.0 \\
0.0 \\
0.0\end{array}$ & $\begin{array}{l}--- \\
--- \\
--- \\
---\end{array}$ & $\begin{array}{l}--- \\
--- \\
--- \\
---\end{array}$ & $\begin{array}{l}0 \\
0 \\
0 \\
0\end{array}$ \\
\hline $\begin{array}{l}\text { LINE } \\
\text { A } \\
B \\
C\end{array}$ & $\begin{array}{l}20500 \\
3503.0 \\
3506.0 \\
3709.0\end{array}$ & $\begin{array}{l}\text { B } \\
\text { B } \\
\text { S }\end{array}$ & $\begin{array}{l}646560 \\
646628 \\
651212\end{array}$ & $\begin{array}{l}6122194 \\
6122200 \\
6122329\end{array}$ & $\begin{array}{r}0.0 \\
0.0 \\
15.4\end{array}$ & $\begin{array}{l}0.0 \\
0.0 \\
0.5\end{array}$ & $\begin{array}{r}0.0 \\
0.0 \\
24.4\end{array}$ & $\begin{array}{l}0.0 \\
0.0 \\
0.4\end{array}$ & $\begin{array}{r}0.0 \\
0.0 \\
19.2\end{array}$ & $\begin{array}{l}0.0 \\
0.0 \\
4.7\end{array}$ & $\begin{array}{l}--- \\
--- \\
---\end{array}$ & $\begin{array}{l}--- \\
--- \\
---\end{array}$ & $\begin{array}{r}68 \\
0 \\
0\end{array}$ \\
\hline $\begin{array}{l}\text { LINE } \\
\text { A } \\
B \\
C\end{array}$ & $\begin{array}{r}20510 \\
4586.0 \\
4562.0 \\
4381.0\end{array}$ & $\begin{array}{r}\mathrm{B} \\
\mathrm{B} ? \\
\mathrm{~S}\end{array}$ & $\begin{array}{l}646570 \\
646908 \\
651328\end{array}$ & $\begin{array}{l}6122003 \\
6121997 \\
6122086\end{array}$ & $\begin{array}{l}0.0 \\
4.7 \\
0.0\end{array}$ & $\begin{array}{l}0.0 \\
0.3 \\
0.0\end{array}$ & $\begin{array}{l}0.0 \\
5.3 \\
0.0\end{array}$ & $\begin{array}{l}0.0 \\
0.3 \\
0.0\end{array}$ & $\begin{array}{l}0.0 \\
4.3 \\
0.0\end{array}$ & $\begin{array}{l}0.0 \\
7.9 \\
0.0\end{array}$ & $\begin{array}{l}--- \\
--- \\
---\end{array}$ & $\begin{array}{l}--- \\
--- \\
---\end{array}$ & $\begin{array}{r}72 \\
0 \\
0\end{array}$ \\
\hline $\begin{array}{l}\text { LINE } \\
\text { A }\end{array}$ & $\begin{array}{l}20520 \\
4801.0\end{array}$ & B & 646631 & 6121776 & 0.0 & 0.0 & 0.0 & 0.0 & 0.0 & 0.0 & --- & --- & 0 \\
\hline $\begin{array}{l}\text { LINE } \\
\text { A } \\
\text { B } \\
\text { C } \\
\text { D }\end{array}$ & $\begin{array}{r}20530 \\
5770.0 \\
5764.0 \\
5487.0 \\
5452.0\end{array}$ & $\begin{array}{r}\text { B? } \\
\text { B } \\
\text { S } \\
\text { S }\end{array}$ & $\begin{array}{l}646112 \\
646360 \\
653608 \\
654531\end{array}$ & $\begin{array}{l}6121584 \\
6121582 \\
6121755 \\
6121818\end{array}$ & $\begin{array}{l}0.0 \\
0.0 \\
0.0 \\
0.0\end{array}$ & $\begin{array}{l}0.0 \\
0.0 \\
0.0 \\
0.0\end{array}$ & $\begin{array}{l}0.0 \\
0.0 \\
0.0 \\
0.0\end{array}$ & $\begin{array}{l}0.0 \\
0.0 \\
0.0 \\
0.0\end{array}$ & $\begin{array}{l}0.0 \\
0.0 \\
0.0 \\
0.0\end{array}$ & $\begin{array}{l}0.0 \\
0.0 \\
0.0 \\
0.0\end{array}$ & $\begin{array}{l}--- \\
--- \\
--- \\
---\end{array}$ & $\begin{array}{l}--- \\
--- \\
--- \\
---\end{array}$ & $\begin{array}{r}67 \\
0 \\
67 \\
0\end{array}$ \\
\hline $\begin{array}{l}\text { LINE } \\
\text { A } \\
\text { B } \\
\text { C } \\
\text { D }\end{array}$ & $\begin{array}{l}20540 \\
5944.0 \\
5950.0 \\
6254.0 \\
6274.0\end{array}$ & $\begin{array}{l}\text { B } \\
\text { B } \\
\text { S } \\
\text { S }\end{array}$ & $\begin{array}{l}646158 \\
646323 \\
654231 \\
654682\end{array}$ & $\begin{array}{l}6121316 \\
6121323 \\
6121627 \\
6121619\end{array}$ & $\begin{array}{l}6.9 \\
0.0 \\
6.4 \\
8.9\end{array}$ & $\begin{array}{l}5.5 \\
0.0 \\
2.1 \\
2.3\end{array}$ & $\begin{array}{l}2.7 \\
0.0 \\
6.4 \\
6.5\end{array}$ & $\begin{array}{l}2.7 \\
0.0 \\
3.5 \\
2.9\end{array}$ & $\begin{array}{l}4.2 \\
0.0 \\
5.8 \\
7.7\end{array}$ & $\begin{array}{r}3.0 \\
0.0 \\
16.6 \\
15.7\end{array}$ & $\begin{array}{l}--- \\
--- \\
--- \\
---\end{array}$ & $\begin{array}{l}--- \\
--- \\
--- \\
---\end{array}$ & $\begin{array}{r}0 \\
0 \\
65 \\
0\end{array}$ \\
\hline
\end{tabular}

$\mathrm{CX}=$ COAXIAL

$\mathrm{CP}=$ COPLANAR

Note: EM values shown above

are local amplitudes

* Estimated Depth may be unreliable because the stronger part of the conductor may be deeper or to one side of the flight line, or because of a shallow dip or magnetite/overburden effects 
EM Anomaly List

\begin{tabular}{|c|c|c|c|c|c|c|c|c|c|c|c|c|c|}
\hline Label & Fid & Interp & $\begin{array}{l}\text { XUTM } \\
\text { m }\end{array}$ & $\begin{array}{l}\text { YUTM } \\
\text { m }\end{array}$ & $\begin{array}{l}\text { CX } \\
\text { Real } \\
\text { ppm }\end{array}$ & $\begin{array}{l}900 \mathrm{HZ} \\
\text { Quad } \\
\text { ppm }\end{array}$ & $\begin{array}{l}\text { CP } \\
\text { Real } \\
\text { ppm }\end{array}$ & $\begin{array}{l}900 \mathrm{HZ} \\
\text { Quad } \\
\text { ppm }\end{array}$ & $\begin{array}{l}\text { CP } 7 \\
\text { Real } \\
\text { ppm }\end{array}$ & $\begin{array}{l}200 \mathrm{HZ} \\
\text { Quad } \\
\text { ppm }\end{array}$ & $\begin{array}{l}\text { Vertica } \\
\text { Cond } \\
\text { siemens }\end{array}$ & $\begin{array}{c}\text { al Dike } \\
\text { DEPTH* } \\
\text { m }\end{array}$ & $\begin{array}{c}\text { Mag. Corr } \\
\text { NT }\end{array}$ \\
\hline $\begin{array}{l}\text { LINE } \\
\text { A } \\
\text { B } \\
\text { C } \\
\text { D } \\
\text { E }\end{array}$ & $\begin{array}{l}20550 \\
6884.0 \\
6867.0 \\
6866.0 \\
6614.0 \\
6550.0\end{array}$ & $\begin{array}{r}\text { S } \\
\text { B? } \\
\text { B? } \\
\text { S } \\
\text { S }\end{array}$ & $\begin{array}{l}645594 \\
646066 \\
646095 \\
652299 \\
654577\end{array}$ & $\begin{array}{l}6121190 \\
6121212 \\
6121214 \\
6121365 \\
6121367\end{array}$ & $\begin{array}{l}5.5 \\
0.0 \\
6.9 \\
0.7 \\
4.2\end{array}$ & $\begin{array}{l}4.3 \\
0.0 \\
7.3 \\
1.7 \\
2.7\end{array}$ & $\begin{array}{l}1.3 \\
0.0 \\
3.3 \\
2.1 \\
3.9\end{array}$ & $\begin{array}{l}6.1 \\
0.0 \\
8.1 \\
2.8 \\
3.6\end{array}$ & $\begin{array}{r}7.2 \\
0.0 \\
14.9 \\
5.8 \\
6.8\end{array}$ & $\begin{array}{r}35.1 \\
0.0 \\
11.7 \\
10.9 \\
18.8\end{array}$ & $\begin{array}{l}--- \\
--- \\
--- \\
--- \\
---\end{array}$ & $\begin{array}{l}--- \\
--- \\
--- \\
--- \\
---\end{array}$ & $\begin{array}{r}50 \\
50 \\
50 \\
0 \\
0\end{array}$ \\
\hline $\begin{array}{l}\text { LINE } \\
A\end{array}$ & $\begin{array}{c}20571 \\
670.0\end{array}$ & S? & 655104 & 6121008 & 0.0 & 0.0 & 0.0 & 0.0 & 0.0 & 0.0 & --- & --- & 0 \\
\hline $\begin{array}{l}\text { LINE } \\
\text { A }\end{array}$ & $\begin{array}{l}20590 \\
6946.0\end{array}$ & S & 648010 & 6120413 & 6.8 & 1.4 & 3.8 & 1.4 & 3.5 & 10.1 & --- & --- & 14 \\
\hline $\begin{array}{l}\text { LINE } \\
\text { A } \\
B\end{array}$ & $\begin{array}{r}20600 \\
6821.0 \\
6688.0\end{array}$ & $\begin{array}{r}S \\
S ?\end{array}$ & $\begin{array}{l}648150 \\
652052\end{array}$ & $\begin{array}{l}6120193 \\
6120338\end{array}$ & $\begin{array}{l}0.0 \\
0.0\end{array}$ & $\begin{array}{l}0.0 \\
0.0\end{array}$ & $\begin{array}{l}0.0 \\
0.0\end{array}$ & $\begin{array}{l}0.0 \\
0.0\end{array}$ & $\begin{array}{l}0.0 \\
0.0\end{array}$ & $\begin{array}{l}0.0 \\
0.0\end{array}$ & $\begin{array}{l}--- \\
---\end{array}$ & $\begin{array}{l}--- \\
---\end{array}$ & $\begin{array}{r}23 \\
231\end{array}$ \\
\hline $\begin{array}{l}\text { LINE } \\
\text { A }\end{array}$ & $\begin{array}{l}20610 \\
6111.0\end{array}$ & $\mathrm{~B} ?$ & 646742 & 6119924 & 5.9 & 3.9 & 2.7 & 3.3 & 8.3 & 7.3 & --- & --- & 20 \\
\hline $\begin{array}{l}\text { LINE } \\
A\end{array}$ & $\begin{array}{c}20620 \\
5814.0\end{array}$ & $\mathrm{~S} ?$ & 655223 & 6119989 & 0.0 & 0.0 & 0.0 & 0.0 & 0.0 & 0.0 & --- & --- & 2 \\
\hline $\begin{array}{l}\text { LINE } \\
A\end{array}$ & $\begin{array}{l}20640 \\
5014.0\end{array}$ & S & 654591 & 6119460 & 0.0 & 0.0 & 0.0 & 0.0 & 0.0 & 0.0 & --- & --- & 0 \\
\hline $\begin{array}{l}\text { LINE } \\
\text { A } \\
B\end{array}$ & $\begin{array}{l}20650 \\
4440.0 \\
4674.0\end{array}$ & $\begin{array}{r}\mathrm{S} \\
\mathrm{B} ?\end{array}$ & $\begin{array}{l}648390 \\
654807\end{array}$ & $\begin{array}{l}6119279 \\
6119516\end{array}$ & $\begin{array}{l}0.0 \\
0.0\end{array}$ & $\begin{array}{l}0.0 \\
0.0\end{array}$ & $\begin{array}{l}0.0 \\
0.0\end{array}$ & $\begin{array}{l}0.0 \\
0.0\end{array}$ & $\begin{array}{l}0.0 \\
0.0\end{array}$ & $\begin{array}{l}0.0 \\
0.0\end{array}$ & $\begin{array}{l}--- \\
---\end{array}$ & $\begin{array}{l}--- \\
---\end{array}$ & $\begin{array}{r}162 \\
0\end{array}$ \\
\hline $\begin{array}{l}\text { LINE } \\
\text { A } \\
B \\
\text { C }\end{array}$ & $\begin{array}{r}20660 \\
4040.0 \\
4032.0 \\
4028.0\end{array}$ & $\begin{array}{l}\text { B? } \\
\text { B? } \\
\text { B? }\end{array}$ & $\begin{array}{l}654814 \\
654969 \\
655038\end{array}$ & $\begin{array}{l}6119260 \\
6119302 \\
6119323\end{array}$ & $\begin{array}{l}0.0 \\
0.0 \\
0.0\end{array}$ & $\begin{array}{l}0.0 \\
0.0 \\
0.0\end{array}$ & $\begin{array}{l}0.0 \\
0.0 \\
0.0\end{array}$ & $\begin{array}{l}0.0 \\
0.0 \\
0.0\end{array}$ & $\begin{array}{l}0.0 \\
0.0 \\
0.0\end{array}$ & $\begin{array}{l}0.0 \\
0.0 \\
0.0\end{array}$ & $\begin{array}{l}--- \\
--- \\
---\end{array}$ & $\begin{array}{l}--- \\
--- \\
---\end{array}$ & $\begin{array}{r}7 \\
109 \\
112\end{array}$ \\
\hline $\begin{array}{l}\text { LINE } \\
\text { A } \\
\text { B } \\
\text { C } \\
\text { D }\end{array}$ & $\begin{array}{r}20670 \\
3682.0 \\
3724.0 \\
3743.0 \\
3752.0\end{array}$ & $\begin{array}{r}\text { S? } \\
\text { S? } \\
\text { B } \\
B ?\end{array}$ & $\begin{array}{l}653083 \\
654372 \\
654825 \\
655019\end{array}$ & $\begin{array}{l}6118893 \\
6118990 \\
6119033 \\
6119056\end{array}$ & $\begin{array}{l}0.0 \\
2.9 \\
0.0 \\
1.1\end{array}$ & $\begin{array}{l}0.0 \\
1.1 \\
0.0 \\
7.4\end{array}$ & $\begin{array}{r}0.0 \\
19.1 \\
0.0 \\
0.1\end{array}$ & $\begin{array}{r}0.0 \\
1.5 \\
0.0 \\
10.9\end{array}$ & $\begin{array}{r}0.0 \\
14.6 \\
0.0 \\
25.5\end{array}$ & $\begin{array}{r}0.0 \\
5.1 \\
0.0 \\
51.4\end{array}$ & $\begin{array}{l}--- \\
--- \\
--- \\
---\end{array}$ & $\begin{array}{l}--- \\
--- \\
--- \\
---\end{array}$ & $\begin{array}{r}470 \\
689 \\
36 \\
95\end{array}$ \\
\hline $\begin{array}{l}\text { LINE } \\
\text { A } \\
B \\
C\end{array}$ & $\begin{array}{l}20680 \\
3190.0 \\
3181.0 \\
3179.0\end{array}$ & $\begin{array}{r}\mathrm{S} ? \\
\mathrm{~B} \\
\mathrm{~B}\end{array}$ & $\begin{array}{l}654603 \\
654895 \\
654954\end{array}$ & $\begin{array}{l}6118933 \\
6118908 \\
6118899\end{array}$ & $\begin{array}{l}0.0 \\
0.0 \\
0.0\end{array}$ & $\begin{array}{l}0.0 \\
0.0 \\
0.0\end{array}$ & $\begin{array}{l}0.0 \\
0.0 \\
0.0\end{array}$ & $\begin{array}{l}0.0 \\
0.0 \\
0.0\end{array}$ & $\begin{array}{l}0.0 \\
0.0 \\
0.0\end{array}$ & $\begin{array}{l}0.0 \\
0.0 \\
0.0\end{array}$ & $\begin{array}{l}--- \\
--- \\
---\end{array}$ & $\begin{array}{l}--- \\
--- \\
---\end{array}$ & $\begin{array}{l}26 \\
26 \\
14\end{array}$ \\
\hline
\end{tabular}

$\mathrm{CX}=$ COAXIAL

$\mathrm{CP}=$ COPLANAR Note: $\mathrm{EM}$ values shown above are local amplitudes

Hetta - North/South of 5515
*Estimated Depth may be unreliable because the stronger part of the conductor may be deeper or to one side of the flight line, or because of a shallow dip or magnetite/overburden effects 
EM Anomaly List

\begin{tabular}{|c|c|c|c|c|c|c|c|c|c|c|c|c|c|}
\hline Label & Fid & Interp & $\begin{array}{c}\text { XUTM } \\
\text { m }\end{array}$ & $\begin{array}{c}\text { YUTM } \\
\mathrm{m}\end{array}$ & $\begin{array}{l}\text { CX } \\
\text { Real } \\
\text { ppm }\end{array}$ & $\begin{array}{l}00 \mathrm{HZ} \\
\text { Quad } \\
\text { ppm }\end{array}$ & $\begin{array}{l}\text { CP } \\
\text { Real } \\
\text { ppm }\end{array}$ & $\begin{array}{l}900 \mathrm{HZ} \\
\text { Quad } \\
\text { ppm }\end{array}$ & $\begin{array}{l}\text { CP } 7 \\
\text { Real } \\
\text { ppm }\end{array}$ & $\begin{array}{l}200 \mathrm{HZ} \\
\text { Quad } \\
\text { ppm }\end{array}$ & $\begin{array}{l}\text { Vertica } \\
\text { COND } \\
\text { siemens }\end{array}$ & $\begin{array}{c}\text { al Dike } \\
\text { DEPTH* } \\
\text { m }\end{array}$ & $\begin{array}{c}\text { Mag. Corr } \\
\text { NT }\end{array}$ \\
\hline $\begin{array}{l}\text { LINE } \\
\text { A } \\
B\end{array}$ & $\begin{array}{l}20690 \\
2768.0 \\
2940.0\end{array}$ & $\begin{array}{r}S \\
\text { S? }\end{array}$ & $\begin{array}{l}648908 \\
654056\end{array}$ & $\begin{array}{l}6118341 \\
6118658\end{array}$ & $\begin{array}{l}0.0 \\
0.0\end{array}$ & $\begin{array}{l}0.0 \\
0.0\end{array}$ & $\begin{array}{l}0.0 \\
0.0\end{array}$ & $\begin{array}{l}0.0 \\
0.0\end{array}$ & $\begin{array}{l}0.0 \\
0.0\end{array}$ & $\begin{array}{l}0.0 \\
0.0\end{array}$ & $\begin{array}{l}--- \\
---\end{array}$ & $\begin{array}{l}--- \\
---\end{array}$ & $\begin{array}{l}10 \\
87\end{array}$ \\
\hline $\begin{array}{l}\text { LINE } \\
A \\
B\end{array}$ & $\begin{array}{l}20700 \\
2638.0 \\
2477.0\end{array}$ & $\begin{array}{l}\text { S? } \\
\text { S? }\end{array}$ & $\begin{array}{l}649108 \\
654719\end{array}$ & $\begin{array}{l}6118240 \\
6118459\end{array}$ & $\begin{array}{l}0.0 \\
6.6\end{array}$ & $\begin{array}{l}0.0 \\
4.1\end{array}$ & $\begin{array}{l}0.0 \\
6.6\end{array}$ & $\begin{array}{l}0.0 \\
4.1\end{array}$ & $\begin{array}{r}0.0 \\
18.1\end{array}$ & $\begin{array}{r}0.0 \\
23.5\end{array}$ & $\begin{array}{l}--- \\
---\end{array}$ & $\begin{array}{l}--- \\
---\end{array}$ & $\begin{array}{l}19 \\
19\end{array}$ \\
\hline $\begin{array}{l}\text { LINE } \\
\text { A } \\
\text { B } \\
\text { C }\end{array}$ & $\begin{array}{r}20710 \\
2042.0 \\
2207.0 \\
2229.0\end{array}$ & $\begin{array}{l}\mathrm{S} \\
\mathrm{B} \\
\mathrm{B}\end{array}$ & $\begin{array}{l}649186 \\
654594 \\
654989\end{array}$ & $\begin{array}{l}6118168 \\
6118166 \\
6118272\end{array}$ & $\begin{array}{l}0.0 \\
0.0 \\
0.0\end{array}$ & $\begin{array}{l}0.0 \\
0.0 \\
0.0\end{array}$ & $\begin{array}{l}0.0 \\
0.0 \\
0.0\end{array}$ & $\begin{array}{l}0.0 \\
0.0 \\
0.0\end{array}$ & $\begin{array}{l}0.0 \\
0.0 \\
0.0\end{array}$ & $\begin{array}{l}0.0 \\
0.0 \\
0.0\end{array}$ & $\begin{array}{l}--- \\
--- \\
---\end{array}$ & $\begin{array}{l}--- \\
--- \\
---\end{array}$ & $\begin{array}{r}27 \\
0 \\
83\end{array}$ \\
\hline $\begin{array}{l}\text { LINE } \\
\text { A } \\
B\end{array}$ & $\begin{array}{l}20720 \\
1717.0 \\
1604.0\end{array}$ & $\begin{array}{r}S ? \\
S\end{array}$ & $\begin{array}{l}654338 \\
657090\end{array}$ & $\begin{array}{l}6118076 \\
6118096\end{array}$ & $\begin{array}{l}0.0 \\
2.5\end{array}$ & $\begin{array}{l}0.0 \\
3.7\end{array}$ & $\begin{array}{l}0.0 \\
1.2\end{array}$ & $\begin{array}{l}0.0 \\
5.5\end{array}$ & $\begin{array}{r}0.0 \\
15.0\end{array}$ & $\begin{array}{r}0.0 \\
24.9\end{array}$ & $\begin{array}{l}--- \\
---\end{array}$ & $\begin{array}{l}--- \\
---\end{array}$ & $\begin{array}{r}677 \\
0\end{array}$ \\
\hline $\begin{array}{l}\text { LINE } \\
A\end{array}$ & $\begin{array}{l}20721 \\
1930.0\end{array}$ & S? & 649126 & 6117853 & 0.0 & 0.0 & 0.0 & 0.0 & 0.0 & 0.0 & --- & --- & 5 \\
\hline $\begin{array}{l}\text { LINE } \\
\text { A } \\
\text { B } \\
\text { C }\end{array}$ & $\begin{array}{r}20730 \\
1456.0 \\
1463.0 \\
1561.0\end{array}$ & $\begin{array}{r}\mathrm{B} ? \\
\mathrm{~B} ? \\
\mathrm{~S}\end{array}$ & $\begin{array}{l}654635 \\
654830 \\
657325\end{array}$ & $\begin{array}{l}6117833 \\
6117825 \\
6117924\end{array}$ & $\begin{array}{r}34.8 \\
10.6 \\
9.3\end{array}$ & $\begin{array}{l}2.6 \\
6.0 \\
8.3\end{array}$ & $\begin{array}{l}47.3 \\
18.9 \\
18.8\end{array}$ & $\begin{array}{r}4.3 \\
4.0 \\
12.0\end{array}$ & $\begin{array}{l}43.1 \\
21.9 \\
26.8\end{array}$ & $\begin{array}{l}14.8 \\
13.4 \\
14.9\end{array}$ & $\begin{array}{l}--- \\
--- \\
---\end{array}$ & $\begin{array}{l}--- \\
--- \\
---\end{array}$ & $\begin{array}{r}32 \\
0 \\
32\end{array}$ \\
\hline $\begin{array}{l}\text { LINE } \\
\text { A } \\
\text { B } \\
\text { C } \\
\text { D } \\
\text { E } \\
\text { F }\end{array}$ & $\begin{array}{c}20740 \\
1154.0 \\
998.0 \\
994.0 \\
988.0 \\
986.0 \\
874.0\end{array}$ & $\begin{array}{l}\text { S? } \\
\text { B? } \\
\text { B? } \\
\text { B? } \\
\text { B? } \\
\text { S }\end{array}$ & $\begin{array}{l}649680 \\
654273 \\
654348 \\
654446 \\
654478 \\
657128\end{array}$ & $\begin{array}{l}6117484 \\
6117625 \\
6117623 \\
6117618 \\
6117619 \\
6117707\end{array}$ & $\begin{array}{l}0.0 \\
0.0 \\
0.0 \\
0.0 \\
0.0 \\
8.9\end{array}$ & $\begin{array}{l}0.0 \\
0.0 \\
0.0 \\
0.0 \\
0.0 \\
4.5\end{array}$ & $\begin{array}{r}0.0 \\
0.0 \\
0.0 \\
0.0 \\
0.0 \\
10.9\end{array}$ & $\begin{array}{l}0.0 \\
0.0 \\
0.0 \\
0.0 \\
0.0 \\
6.8\end{array}$ & $\begin{array}{r}0.0 \\
0.0 \\
0.0 \\
0.0 \\
0.0 \\
17.4\end{array}$ & $\begin{array}{r}0.0 \\
0.0 \\
0.0 \\
0.0 \\
0.0 \\
15.3\end{array}$ & $\begin{array}{l}--- \\
--- \\
--- \\
--- \\
--- \\
---\end{array}$ & $\begin{array}{l}--- \\
--- \\
--- \\
--- \\
--- \\
---\end{array}$ & $\begin{array}{r}37 \\
0 \\
623 \\
631 \\
304 \\
0\end{array}$ \\
\hline $\begin{array}{l}\text { LINE } \\
\text { A } \\
\text { B } \\
\text { C } \\
\text { D }\end{array}$ & $\begin{array}{r}20750 \\
406.0 \\
623.0 \\
652.0 \\
762.0\end{array}$ & $\begin{array}{r}S \\
S ? \\
S ? \\
S\end{array}$ & $\begin{array}{l}648019 \\
653907 \\
654279 \\
657094\end{array}$ & $\begin{array}{l}6117183 \\
6117420 \\
6117447 \\
6117533\end{array}$ & $\begin{array}{r}0.0 \\
0.0 \\
0.0 \\
10.6\end{array}$ & $\begin{array}{l}0.0 \\
0.0 \\
2.9 \\
6.1\end{array}$ & $\begin{array}{r}0.0 \\
0.0 \\
41.8 \\
18.1\end{array}$ & $\begin{array}{l}0.0 \\
0.0 \\
4.8 \\
8.6\end{array}$ & $\begin{array}{r}0.0 \\
0.0 \\
30.4 \\
20.2\end{array}$ & $\begin{array}{r}0.0 \\
0.0 \\
3.9 \\
20.4\end{array}$ & $\begin{array}{l}--- \\
--- \\
--- \\
---\end{array}$ & $\begin{array}{l}--- \\
--- \\
--- \\
---\end{array}$ & $\begin{array}{r}55 \\
140 \\
0 \\
0\end{array}$ \\
\hline $\begin{array}{l}\text { LINE } \\
\text { A } \\
\text { B } \\
\text { C } \\
\text { D }\end{array}$ & $\begin{array}{l}20760 \\
1793.0 \\
1974.0 \\
2035.0 \\
2060.0\end{array}$ & $\begin{array}{r}\mathrm{S} ? \\
\mathrm{H} ? \\
\mathrm{~S} \\
\mathrm{H}\end{array}$ & $\begin{array}{l}650122 \\
654213 \\
655800 \\
656840\end{array}$ & $\begin{array}{l}6117005 \\
6117245 \\
6117278 \\
6117363\end{array}$ & $\begin{array}{l}2.9 \\
0.0 \\
0.0 \\
0.0\end{array}$ & $\begin{array}{l}1.0 \\
0.0 \\
0.0 \\
0.0\end{array}$ & $\begin{array}{l}5.9 \\
0.0 \\
0.0 \\
0.0\end{array}$ & $\begin{array}{l}1.5 \\
0.0 \\
0.0 \\
0.0\end{array}$ & $\begin{array}{l}5.1 \\
0.0 \\
0.0 \\
0.0\end{array}$ & $\begin{array}{r}10.8 \\
0.0 \\
0.0 \\
0.0\end{array}$ & $\begin{array}{l}--- \\
--- \\
--- \\
---\end{array}$ & $\begin{array}{l}--- \\
--- \\
--- \\
---\end{array}$ & $\begin{array}{r}27 \\
1384 \\
53 \\
0\end{array}$ \\
\hline
\end{tabular}

$\mathrm{CX}=$ COAXIAL

$\mathrm{CP}=$ COPLANAR Note $: \mathrm{EM}$ values shown above are local amplitudes

*Estimated Depth may be unreliable because the stronger part of the conductor may be deeper or to one side of the flight line, or because of a shallow dip or magnetite/overburden effects 
EM Anomaly List

\begin{tabular}{|c|c|c|c|c|c|c|c|c|c|c|c|c|c|}
\hline Label & Fid & Interp & $\begin{array}{c}\text { XUTM } \\
\text { m }\end{array}$ & $\begin{array}{c}\text { YUTM } \\
\mathrm{m}\end{array}$ & $\begin{array}{l}\text { CX } \\
\text { Real } \\
\text { ppm }\end{array}$ & $\begin{array}{l}900 \mathrm{HZ} \\
\text { Quad } \\
\text { ppm }\end{array}$ & $\begin{array}{l}\text { CP } \\
\text { Real } \\
\text { ppm }\end{array}$ & $\begin{array}{l}900 \mathrm{HZ} \\
\text { Quad } \\
\text { ppm }\end{array}$ & $\begin{array}{l}\text { CP } \\
\text { Real } \\
\text { ppm }\end{array}$ & $\begin{array}{l}200 \mathrm{HZ} \\
\text { Quad } \\
\text { ppm }\end{array}$ & $\begin{array}{l}\text { Vertic } \\
\text { Cond } \\
\text { siemens }\end{array}$ & $\begin{array}{c}\text { al Dike } \\
\text { DEPTH* }^{*} \\
\text { m }\end{array}$ & $\begin{array}{c}\text { Mag. Corr } \\
\text { NT }\end{array}$ \\
\hline $\begin{array}{l}\text { LINE } \\
\text { A } \\
B \\
\text { C }\end{array}$ & $\begin{array}{r}20770 \\
2617.0 \\
2569.0 \\
2291.0\end{array}$ & $\begin{array}{r}\mathrm{S} \\
\mathrm{B} ? \\
\mathrm{~S}\end{array}$ & $\begin{array}{l}648078 \\
649912 \\
655823\end{array}$ & $\begin{array}{l}6116793 \\
6116847 \\
6116982\end{array}$ & $\begin{array}{l}0.0 \\
0.0 \\
0.0\end{array}$ & $\begin{array}{l}0.0 \\
0.0 \\
0.0\end{array}$ & $\begin{array}{l}0.0 \\
0.0 \\
0.0\end{array}$ & $\begin{array}{l}0.0 \\
0.0 \\
0.0\end{array}$ & $\begin{array}{l}0.0 \\
0.0 \\
0.0\end{array}$ & $\begin{array}{l}0.0 \\
0.0 \\
0.0\end{array}$ & $\begin{array}{l}--- \\
--- \\
---\end{array}$ & $\begin{array}{l}--- \\
--- \\
---\end{array}$ & $\begin{array}{l}62 \\
98 \\
38\end{array}$ \\
\hline $\begin{array}{l}\text { LINE } \\
\text { A } \\
\text { B } \\
\text { C } \\
\text { D }\end{array}$ & $\begin{array}{r}20780 \\
5396.0 \\
5443.0 \\
5461.0 \\
5518.0\end{array}$ & $\begin{array}{r}S \\
S \\
S \\
B ?\end{array}$ & $\begin{array}{l}654366 \\
655837 \\
656590 \\
658092\end{array}$ & $\begin{array}{l}6116754 \\
6116775 \\
6116794 \\
6116864\end{array}$ & $\begin{array}{r}12.1 \\
0.0 \\
0.0 \\
0.0\end{array}$ & $\begin{array}{l}4.1 \\
0.0 \\
0.0 \\
0.0\end{array}$ & $\begin{array}{r}20.3 \\
0.0 \\
0.0 \\
0.0\end{array}$ & $\begin{array}{l}3.9 \\
0.0 \\
0.0 \\
0.0\end{array}$ & $\begin{array}{r}22.9 \\
0.0 \\
0.0 \\
0.0\end{array}$ & $\begin{array}{r}18.1 \\
0.0 \\
0.0 \\
0.0\end{array}$ & $\begin{array}{l}--- \\
--- \\
--- \\
---\end{array}$ & $\begin{array}{l}--- \\
--- \\
--- \\
---\end{array}$ & $\begin{array}{r}52 \\
52 \\
52 \\
0\end{array}$ \\
\hline $\begin{array}{l}\text { LINE } \\
\text { A } \\
B \\
C\end{array}$ & $\begin{array}{r}20785 \\
2640.0 \\
2670.0 \\
2707.0\end{array}$ & $\begin{array}{r}S \\
S ? \\
S\end{array}$ & $\begin{array}{l}648142 \\
649007 \\
650056\end{array}$ & $\begin{array}{l}6116669 \\
6116675 \\
6116693\end{array}$ & $\begin{array}{r}0.0 \\
12.9 \\
9.0\end{array}$ & $\begin{array}{l}0.0 \\
2.1 \\
1.1\end{array}$ & $\begin{array}{r}0.0 \\
19.6 \\
15.7\end{array}$ & $\begin{array}{l}0.0 \\
2.8 \\
2.1\end{array}$ & $\begin{array}{r}0.0 \\
17.6 \\
13.4\end{array}$ & $\begin{array}{r}0.0 \\
17.3 \\
13.3\end{array}$ & $\begin{array}{l}--- \\
--- \\
---\end{array}$ & $\begin{array}{l}--- \\
--- \\
---\end{array}$ & $\begin{array}{r}34 \\
34 \\
146\end{array}$ \\
\hline $\begin{array}{l}\text { LINE } \\
\text { A } \\
B\end{array}$ & $\begin{array}{l}20790 \\
5736.0 \\
5689.0\end{array}$ & $\begin{array}{l}S \\
S\end{array}$ & $\begin{array}{l}654211 \\
655930\end{array}$ & $\begin{array}{l}6116580 \\
6116586\end{array}$ & $\begin{array}{l}7.4 \\
0.0\end{array}$ & $\begin{array}{l}2.7 \\
0.0\end{array}$ & $\begin{array}{l}8.3 \\
0.0\end{array}$ & $\begin{array}{l}1.8 \\
0.0\end{array}$ & $\begin{array}{l}6.4 \\
0.0\end{array}$ & $\begin{array}{l}5.0 \\
0.0\end{array}$ & $\begin{array}{l}--- \\
---\end{array}$ & $\begin{array}{l}--- \\
---\end{array}$ & $\begin{array}{l}27 \\
27\end{array}$ \\
\hline $\begin{array}{l}\text { LINE } \\
\text { A } \\
B\end{array}$ & $\begin{array}{r}20795 \\
2905.0 \\
2887.0\end{array}$ & $\begin{array}{r}S \\
S ?\end{array}$ & $\begin{array}{l}648235 \\
648964\end{array}$ & $\begin{array}{l}6116336 \\
6116394\end{array}$ & $\begin{array}{l}5.3 \\
0.0\end{array}$ & $\begin{array}{l}7.2 \\
0.0\end{array}$ & $\begin{array}{r}11.3 \\
0.0\end{array}$ & $\begin{array}{l}6.0 \\
0.0\end{array}$ & $\begin{array}{r}17.4 \\
0.0\end{array}$ & $\begin{array}{r}34.8 \\
0.0\end{array}$ & $\begin{array}{l}--- \\
---\end{array}$ & $\begin{array}{l}--- \\
---\end{array}$ & $\begin{array}{r}158 \\
95\end{array}$ \\
\hline $\begin{array}{l}\text { LINE } \\
\text { A } \\
B \\
C\end{array}$ & $\begin{array}{r}20800 \\
5846.0 \\
5861.0 \\
5933.0\end{array}$ & $\begin{array}{l}S \\
S \\
S\end{array}$ & $\begin{array}{l}655677 \\
656313 \\
657505\end{array}$ & $\begin{array}{l}6116466 \\
6116494 \\
6116540\end{array}$ & $\begin{array}{l}0.0 \\
0.0 \\
0.0\end{array}$ & $\begin{array}{l}0.0 \\
0.0 \\
0.0\end{array}$ & $\begin{array}{l}0.0 \\
0.0 \\
0.0\end{array}$ & $\begin{array}{l}0.0 \\
0.0 \\
0.0\end{array}$ & $\begin{array}{l}0.0 \\
0.0 \\
0.0\end{array}$ & $\begin{array}{l}0.0 \\
0.0 \\
0.0\end{array}$ & $\begin{array}{l}--- \\
--- \\
---\end{array}$ & $\begin{array}{l}--- \\
--- \\
---\end{array}$ & $\begin{array}{r}19 \\
41 \\
3\end{array}$ \\
\hline $\begin{array}{l}\text { LINE } \\
\text { A } \\
B\end{array}$ & $\begin{array}{r}20810 \\
6048.0 \\
5977.0\end{array}$ & $\begin{array}{l}S \\
S\end{array}$ & $\begin{array}{l}655727 \\
657508\end{array}$ & $\begin{array}{l}6116285 \\
6116325\end{array}$ & $\begin{array}{r}12.8 \\
0.0\end{array}$ & $\begin{array}{l}9.7 \\
0.0\end{array}$ & $\begin{array}{r}17.4 \\
0.0\end{array}$ & $\begin{array}{r}12.0 \\
0.0\end{array}$ & $\begin{array}{r}23.5 \\
0.0\end{array}$ & $\begin{array}{r}38.6 \\
0.0\end{array}$ & $\begin{array}{l}--- \\
---\end{array}$ & $\begin{array}{l}--- \\
---\end{array}$ & $\begin{array}{r}0 \\
40\end{array}$ \\
\hline $\begin{array}{l}\text { LINE } \\
\text { A } \\
B \\
\text { C }\end{array}$ & $\begin{array}{r}20815 \\
3195.0 \\
3171.0 \\
3155.0\end{array}$ & $\begin{array}{r}S \\
S \\
S ?\end{array}$ & $\begin{array}{l}649076 \\
649926 \\
650365\end{array}$ & $\begin{array}{l}6116098 \\
6116139 \\
6116189\end{array}$ & $\begin{array}{l}1.2 \\
0.0 \\
0.0\end{array}$ & $\begin{array}{l}1.5 \\
0.0 \\
0.0\end{array}$ & $\begin{array}{l}2.1 \\
0.0 \\
0.0\end{array}$ & $\begin{array}{l}2.6 \\
0.0 \\
0.0\end{array}$ & $\begin{array}{l}3.7 \\
0.0 \\
0.0\end{array}$ & $\begin{array}{r}15.6 \\
0.0 \\
0.0\end{array}$ & $\begin{array}{l}--- \\
--- \\
---\end{array}$ & $\begin{array}{l}--- \\
--- \\
---\end{array}$ & $\begin{array}{r}286 \\
0 \\
0\end{array}$ \\
\hline $\begin{array}{l}\text { LINE } \\
\text { A } \\
\text { B } \\
\text { C } \\
\text { D }\end{array}$ & $\begin{array}{r}20820 \\
6131.0 \\
6134.0 \\
6172.0 \\
6249.0\end{array}$ & $\begin{array}{l}\text { B } \\
\text { B } \\
\text { S } \\
\text { S }\end{array}$ & $\begin{array}{l}654383 \\
654483 \\
655575 \\
657444\end{array}$ & $\begin{array}{l}6115997 \\
6116005 \\
6116065 \\
6116172\end{array}$ & $\begin{array}{l}0.0 \\
0.0 \\
0.0 \\
3.8\end{array}$ & $\begin{array}{l}0.0 \\
0.0 \\
0.0 \\
4.5\end{array}$ & $\begin{array}{l}0.0 \\
0.0 \\
0.0 \\
1.7\end{array}$ & $\begin{array}{l}0.0 \\
0.0 \\
0.0 \\
5.7\end{array}$ & $\begin{array}{r}0.0 \\
0.0 \\
0.0 \\
16.3\end{array}$ & $\begin{array}{r}0.0 \\
0.0 \\
0.0 \\
18.2\end{array}$ & $\begin{array}{l}--- \\
--- \\
--- \\
---\end{array}$ & $\begin{array}{l}--- \\
--- \\
--- \\
---\end{array}$ & $\begin{array}{l}31 \\
31 \\
31 \\
20\end{array}$ \\
\hline $\begin{array}{l}\text { LINE } \\
\mathrm{A}\end{array}$ & $\begin{array}{r}20825 \\
3406.0\end{array}$ & S? & 648084 & 6115878 & 0.0 & 0.0 & 0.0 & 0.0 & 0.0 & 0.0 & --- & --- & 3 \\
\hline
\end{tabular}

$\mathrm{CX}=$ COAXIAL

Note: EM values shown above

are local amplitudes

Hetta - North/South of 5515
* Estimated Depth may be unreliable because the stronger part of the conductor may be deeper or to one side of the flight line, or because of a shallow dip or magnetite/overburden effects 
EM Anomaly List

\begin{tabular}{|c|c|c|c|c|c|c|c|c|c|c|c|c|c|}
\hline Label & Fid & Interp & $\begin{array}{l}\text { XUTM } \\
\text { m }\end{array}$ & $\begin{array}{c}\text { YUTM } \\
\mathrm{m}\end{array}$ & $\begin{array}{ll}\text { CX } & 9 \\
\text { Real } & \\
\text { ppm } & \end{array}$ & $\begin{array}{l}00 \mathrm{HZ} \\
\text { Quad } \\
\text { ppm }\end{array}$ & $\begin{array}{l}\text { CP } \\
\text { Real } \\
\text { ppm }\end{array}$ & $\begin{array}{l}900 \mathrm{HZ} \\
\text { Quad } \\
\text { ppm }\end{array}$ & $\begin{array}{l}\text { CP } \\
\text { Real } \\
\text { ppm }\end{array}$ & $\begin{array}{l}200 \mathrm{~Hz} \\
\text { Quad } \\
\text { ppm }\end{array}$ & $\begin{array}{l}\text { Vertica } \\
\text { Cond } \\
\text { siemens }\end{array}$ & $\begin{array}{c}\text { al Dike } \\
\text { DEPTH* } \\
\text { m }\end{array}$ & $\begin{array}{c}\text { Mag. Corr } \\
\text { NT }\end{array}$ \\
\hline $\begin{array}{l}\text { LINE } \\
\text { A } \\
B\end{array}$ & $\begin{array}{l}20835 \\
3665.0 \\
3618.0\end{array}$ & $\begin{array}{r}S \\
S ?\end{array}$ & $\begin{array}{l}648120 \\
650171\end{array}$ & $\begin{array}{l}6115618 \\
6115713\end{array}$ & $\begin{array}{l}0.0 \\
0.0\end{array}$ & $\begin{array}{l}0.0 \\
0.0\end{array}$ & $\begin{array}{l}0.0 \\
0.0\end{array}$ & $\begin{array}{l}0.0 \\
0.0\end{array}$ & $\begin{array}{l}0.0 \\
0.0\end{array}$ & $\begin{array}{l}0.0 \\
0.0\end{array}$ & $\begin{array}{l}--- \\
---\end{array}$ & $\begin{array}{l}--- \\
---\end{array}$ & $\begin{array}{r}51 \\
108\end{array}$ \\
\hline $\begin{array}{l}\text { LINE } \\
A \\
B\end{array}$ & $\begin{array}{l}20840 \\
6480.0 \\
6508.0\end{array}$ & $\begin{array}{l}S \\
S\end{array}$ & $\begin{array}{l}656005 \\
656673\end{array}$ & $\begin{array}{l}6115637 \\
6115696\end{array}$ & $\begin{array}{l}0.0 \\
1.9\end{array}$ & $\begin{array}{l}0.0 \\
3.1\end{array}$ & $\begin{array}{l}0.0 \\
1.6\end{array}$ & $\begin{array}{l}0.0 \\
4.5\end{array}$ & $\begin{array}{r}0.0 \\
12.0\end{array}$ & $\begin{array}{r}0.0 \\
11.2\end{array}$ & $\begin{array}{l}--- \\
---\end{array}$ & $\begin{array}{l}--- \\
---\end{array}$ & $\begin{array}{l}67 \\
24\end{array}$ \\
\hline $\begin{array}{l}\text { LINE } \\
\text { A } \\
\text { B } \\
\text { C }\end{array}$ & $\begin{array}{l}20845 \\
3701.0 \\
3704.0 \\
3763.0\end{array}$ & $\begin{array}{l}\text { B? } \\
\text { B? } \\
\text { S? }\end{array}$ & $\begin{array}{l}648286 \\
648379 \\
650144\end{array}$ & $\begin{array}{l}6115447 \\
6115459 \\
6115485\end{array}$ & $\begin{array}{l}1.3 \\
0.0 \\
0.0\end{array}$ & $\begin{array}{r}12.6 \\
0.0 \\
0.0\end{array}$ & $\begin{array}{l}0.2 \\
0.0 \\
0.0\end{array}$ & $\begin{array}{r}10.4 \\
0.0 \\
0.0\end{array}$ & $\begin{array}{r}22.7 \\
0.0 \\
0.0\end{array}$ & $\begin{array}{r}53.3 \\
0.0 \\
0.0\end{array}$ & $\begin{array}{l}--- \\
--- \\
---\end{array}$ & $\begin{array}{l}--- \\
--- \\
---\end{array}$ & $\begin{array}{r}0 \\
0 \\
214\end{array}$ \\
\hline $\begin{array}{l}\text { LINE } \\
\text { A } \\
\text { B } \\
\text { C }\end{array}$ & $\begin{array}{r}20855 \\
3950.0 \\
3946.0 \\
3925.0\end{array}$ & $\begin{array}{l}\text { B? } \\
\text { B? } \\
\text { S? }\end{array}$ & $\begin{array}{l}648231 \\
648388 \\
649208\end{array}$ & $\begin{array}{l}6115221 \\
6115225 \\
6115249\end{array}$ & $\begin{array}{l}0.0 \\
0.0 \\
6.5\end{array}$ & $\begin{array}{l}0.0 \\
0.0 \\
1.3\end{array}$ & $\begin{array}{r}0.0 \\
0.0 \\
31.5\end{array}$ & $\begin{array}{l}0.0 \\
0.0 \\
2.2\end{array}$ & $\begin{array}{r}0.0 \\
0.0 \\
24.0\end{array}$ & $\begin{array}{l}0.0 \\
0.0 \\
9.9\end{array}$ & $\begin{array}{l}--- \\
--- \\
---\end{array}$ & $\begin{array}{l}--- \\
--- \\
---\end{array}$ & $\begin{array}{l}78 \\
39 \\
18\end{array}$ \\
\hline $\begin{array}{l}\text { LINE } \\
\text { A } \\
B \\
C\end{array}$ & $\begin{array}{r}20865 \\
3986.0 \\
4048.0 \\
4052.0\end{array}$ & $\begin{array}{r}S \\
S ? \\
S\end{array}$ & $\begin{array}{l}648424 \\
650086 \\
650159\end{array}$ & $\begin{array}{l}6115014 \\
6115184 \\
6115164\end{array}$ & $\begin{array}{l}0.0 \\
0.0 \\
0.0\end{array}$ & $\begin{array}{l}0.0 \\
0.0 \\
0.0\end{array}$ & $\begin{array}{l}0.0 \\
0.0 \\
0.0\end{array}$ & $\begin{array}{l}0.0 \\
0.0 \\
0.0\end{array}$ & $\begin{array}{l}0.0 \\
0.0 \\
0.0\end{array}$ & $\begin{array}{l}0.0 \\
0.0 \\
0.0\end{array}$ & $\begin{array}{l}--- \\
--- \\
---\end{array}$ & $\begin{array}{l}--- \\
--- \\
---\end{array}$ & $\begin{array}{r}0 \\
138 \\
53\end{array}$ \\
\hline $\begin{array}{l}\text { LINE } \\
A\end{array}$ & $\begin{array}{r}20875 \\
4214.0\end{array}$ & S & 648275 & 6114776 & 0.0 & 0.0 & 0.0 & 0.0 & 0.0 & 0.0 & --- & --- & 43 \\
\hline $\begin{array}{l}\mathrm{LINE} \\
\mathrm{A}\end{array}$ & $\begin{array}{l}20880 \\
7104.0\end{array}$ & $\mathrm{~S}$ & 655455 & 6114847 & 1.9 & 1.2 & 0.0 & 0.9 & 0.1 & 5.9 & --- & --- & 0 \\
\hline $\begin{array}{l}\text { LINE } \\
A\end{array}$ & $\begin{array}{c}20885 \\
4261.0\end{array}$ & S? & 648722 & 6114572 & 0.0 & 0.0 & 0.0 & 0.0 & 0.0 & 0.0 & --- & --- & 22 \\
\hline $\begin{array}{l}\text { LINE } \\
A\end{array}$ & $\begin{array}{c}20890 \\
7441.0\end{array}$ & S? & 655343 & 6114590 & 0.0 & 0.0 & 0.0 & 0.0 & 0.0 & 0.0 & --- & --- & 97 \\
\hline $\begin{array}{l}\text { LINE } \\
A\end{array}$ & $\begin{array}{r}20895 \\
4424.0\end{array}$ & S? & 649728 & 6114501 & 8.6 & 0.2 & 10.1 & 0.5 & 8.3 & 5.1 & --- & --- & 89 \\
\hline $\begin{array}{l}\text { LINE } \\
\text { A } \\
\text { B } \\
\text { C } \\
\text { D } \\
\text { E }\end{array}$ & $\begin{array}{l}20900 \\
7522.0 \\
7523.0 \\
7546.0 \\
7594.0 \\
7599.0\end{array}$ & $\begin{array}{r}\text { S? } \\
\text { S? } \\
\text { S? } \\
\text { S } \\
\text { B? }\end{array}$ & $\begin{array}{l}653577 \\
653607 \\
654301 \\
655694 \\
655835\end{array}$ & $\begin{array}{l}6114346 \\
6114346 \\
6114387 \\
6114470 \\
6114473\end{array}$ & $\begin{array}{l}7.1 \\
0.0 \\
0.0 \\
6.4 \\
7.8\end{array}$ & $\begin{array}{l}9.5 \\
0.0 \\
0.0 \\
2.1 \\
5.7\end{array}$ & $\begin{array}{r}6.2 \\
0.0 \\
0.0 \\
9.2 \\
10.6\end{array}$ & $\begin{array}{r}11.4 \\
0.0 \\
0.0 \\
2.1 \\
4.6\end{array}$ & $\begin{array}{r}25.6 \\
0.0 \\
0.0 \\
10.1 \\
15.8\end{array}$ & $\begin{array}{r}13.2 \\
0.0 \\
0.0 \\
8.1 \\
20.8\end{array}$ & $\begin{array}{l}--- \\
--- \\
--- \\
--- \\
---\end{array}$ & $\begin{array}{l}--- \\
--- \\
--- \\
--- \\
---\end{array}$ & $\begin{array}{r}5 \\
6 \\
47 \\
16 \\
2\end{array}$ \\
\hline
\end{tabular}

$\mathrm{CX}=$ COAXIAL

$\mathrm{CP}=$ COPLANAR Note $: \mathrm{EM}$ values shown above are local amplitudes

*Estimated Depth may be unreliable because the stronger part of the conductor may be deeper or to one side of the flight line, or because of a shallow dip or magnetite/overburden effects 
EM Anomaly List

\begin{tabular}{|c|c|c|c|c|c|c|c|c|c|c|c|c|c|}
\hline Label & Fid & Interp & $\begin{array}{c}\text { XUTM } \\
\text { m }\end{array}$ & $\begin{array}{c}\text { YUTM } \\
\mathrm{m}\end{array}$ & $\begin{array}{ll}\text { CX } & 9 \\
\text { Real } & \\
\text { ppm } & \end{array}$ & $\begin{array}{l}00 \mathrm{HZ} \\
\text { Quad } \\
\text { ppm }\end{array}$ & $\begin{array}{l}\text { CP } \\
\text { Real } \\
\text { ppm }\end{array}$ & $\begin{array}{l}900 \mathrm{HZ} \\
\text { Quad } \\
\text { ppm }\end{array}$ & $\begin{array}{l}\text { CP } \\
\text { Real } \\
\text { ppm }\end{array}$ & $\begin{array}{l}200 \mathrm{~Hz} \\
\text { Quad } \\
\text { ppm }\end{array}$ & $\begin{array}{l}\text { Vertica } \\
\text { Cond } \\
\text { siemens }\end{array}$ & $\begin{array}{c}\text { al Dike } \\
\text { DEPTH* } \\
\text { m }\end{array}$ & $\begin{array}{c}\text { Mag. Corr } \\
\text { NT }\end{array}$ \\
\hline $\begin{array}{l}\operatorname{LINE} \\
\mathrm{A}\end{array}$ & $\begin{array}{r}20905 \\
4556.0\end{array}$ & S? & 648676 & 6114329 & 0.0 & 0.0 & 0.0 & 0.0 & 0.0 & 0.0 & --- & --- & 42 \\
\hline $\begin{array}{l}\text { LINE } \\
\text { A } \\
B\end{array}$ & $\begin{array}{l}20910 \\
7800.0 \\
7798.0\end{array}$ & $\begin{array}{l}\mathrm{S} ? \\
\mathrm{~B} ?\end{array}$ & $\begin{array}{l}655585 \\
655662\end{array}$ & $\begin{array}{l}6114242 \\
6114242\end{array}$ & $\begin{array}{l}0.0 \\
1.1\end{array}$ & $\begin{array}{l}0.0 \\
6.1\end{array}$ & $\begin{array}{l}0.0 \\
0.1\end{array}$ & $\begin{array}{l}0.0 \\
4.5\end{array}$ & $\begin{array}{l}0.0 \\
7.6\end{array}$ & $\begin{array}{r}0.0 \\
23.6\end{array}$ & $\begin{array}{l}--- \\
---\end{array}$ & $\begin{array}{l}--- \\
---\end{array}$ & $\begin{array}{l}87 \\
87\end{array}$ \\
\hline $\begin{array}{l}\text { LINE } \\
A\end{array}$ & $\begin{array}{r}20920 \\
8185.0\end{array}$ & S? & 655642 & 6114054 & 3.9 & 3.5 & 1.9 & 3.9 & 8.9 & 7.5 & --- & --- & 10 \\
\hline $\begin{array}{l}\text { LINE } \\
\text { A } \\
B \\
\text { C }\end{array}$ & $\begin{array}{r}20930 \\
8323.0 \\
8343.0 \\
8365.0\end{array}$ & $\begin{array}{r}\text { B } \\
\text { S? } \\
\text { S? }\end{array}$ & $\begin{array}{l}654194 \\
654937 \\
655671\end{array}$ & $\begin{array}{l}6113805 \\
6113819 \\
6113845\end{array}$ & $\begin{array}{l}0.0 \\
0.0 \\
2.8\end{array}$ & $\begin{array}{l}0.0 \\
0.0 \\
3.8\end{array}$ & $\begin{array}{l}0.0 \\
0.0 \\
2.5\end{array}$ & $\begin{array}{l}0.0 \\
0.0 \\
5.8\end{array}$ & $\begin{array}{r}0.0 \\
0.0 \\
13.5\end{array}$ & $\begin{array}{r}0.0 \\
0.0 \\
11.3\end{array}$ & $\begin{array}{l}--- \\
--- \\
---\end{array}$ & $\begin{array}{l}--- \\
--- \\
---\end{array}$ & $\begin{array}{l}42 \\
14 \\
39\end{array}$ \\
\hline $\begin{array}{l}\text { LINE } \\
\mathrm{A}\end{array}$ & $\begin{array}{l}20935 \\
4910.0\end{array}$ & $\mathrm{~B}$ ? & 648466 & 6113652 & 4.6 & 4.9 & 1.8 & 3.1 & 5.6 & 4.2 & --- & --- & 230 \\
\hline $\begin{array}{l}\text { LINE } \\
\text { A } \\
\text { B } \\
\text { C }\end{array}$ & $\begin{array}{r}20940 \\
8927.0 \\
8864.0 \\
8860.0\end{array}$ & $\begin{array}{l}\text { S? } \\
\text { B? } \\
\text { B? }\end{array}$ & $\begin{array}{l}653639 \\
655602 \\
655723\end{array}$ & $\begin{array}{l}6113683 \\
6113663 \\
6113672\end{array}$ & $\begin{array}{l}0.0 \\
0.0 \\
3.5\end{array}$ & $\begin{array}{l}0.0 \\
0.0 \\
7.7\end{array}$ & $\begin{array}{l}0.0 \\
0.0 \\
1.9\end{array}$ & $\begin{array}{l}0.0 \\
0.0 \\
5.9\end{array}$ & $\begin{array}{r}0.0 \\
0.0 \\
13.2\end{array}$ & $\begin{array}{r}0.0 \\
0.0 \\
13.3\end{array}$ & $\begin{array}{l}--- \\
--- \\
---\end{array}$ & $\begin{array}{l}--- \\
--- \\
---\end{array}$ & $\begin{array}{r}117 \\
15 \\
0\end{array}$ \\
\hline $\begin{array}{l}\text { LINE } \\
\text { A } \\
B\end{array}$ & $\begin{array}{r}20945 \\
4994.0 \\
5008.0\end{array}$ & $\begin{array}{l}\text { S? } \\
\text { S? }\end{array}$ & $\begin{array}{l}647873 \\
648549\end{array}$ & $\begin{array}{l}6113410 \\
6113477\end{array}$ & $\begin{array}{l}0.0 \\
0.0\end{array}$ & $\begin{array}{l}0.0 \\
0.0\end{array}$ & $\begin{array}{l}0.0 \\
0.0\end{array}$ & $\begin{array}{l}0.0 \\
0.0\end{array}$ & $\begin{array}{l}0.0 \\
0.0\end{array}$ & $\begin{array}{l}0.0 \\
0.0\end{array}$ & $\begin{array}{l}--- \\
---\end{array}$ & $\begin{array}{l}--- \\
---\end{array}$ & $\begin{array}{l}67 \\
36\end{array}$ \\
\hline $\begin{array}{l}\text { LINE } \\
\text { A }\end{array}$ & $\begin{array}{l}20950 \\
9001.0\end{array}$ & S? & 653643 & 6113409 & 0.0 & 0.0 & 0.0 & 0.0 & 0.0 & 0.0 & --- & --- & 168 \\
\hline $\begin{array}{l}\text { LINE } \\
\text { A }\end{array}$ & $\begin{array}{r}20955 \\
5164.0\end{array}$ & S? & 647986 & 6113094 & 1.7 & 3.3 & 0.8 & 0.0 & 0.0 & 5.7 & --- & --- & 0 \\
\hline $\begin{array}{l}\text { LINE } \\
\text { A } \\
\text { B } \\
\text { C } \\
\text { D } \\
\text { E }\end{array}$ & $\begin{array}{l}20960 \\
9340.0 \\
9240.0 \\
9235.0 \\
9191.0 \\
9182.0\end{array}$ & $\begin{array}{r}\text { S? } \\
\text { B? } \\
\text { B } \\
\text { B } \\
\text { B }\end{array}$ & $\begin{array}{l}652891 \\
656050 \\
656140 \\
657095 \\
657404\end{array}$ & $\begin{array}{l}6113115 \\
6113252 \\
6113236 \\
6113285 \\
6113327\end{array}$ & $\begin{array}{l}0.0 \\
0.0 \\
0.0 \\
0.0 \\
0.0\end{array}$ & $\begin{array}{l}0.0 \\
0.0 \\
0.0 \\
0.0 \\
0.0\end{array}$ & $\begin{array}{l}0.0 \\
0.0 \\
0.0 \\
0.0 \\
0.0\end{array}$ & $\begin{array}{l}0.0 \\
0.0 \\
0.0 \\
0.0 \\
0.0\end{array}$ & $\begin{array}{l}0.0 \\
0.0 \\
0.0 \\
0.0 \\
0.0\end{array}$ & $\begin{array}{l}0.0 \\
0.0 \\
0.0 \\
0.0 \\
0.0\end{array}$ & $\begin{array}{l}--- \\
--- \\
--- \\
--- \\
---\end{array}$ & $\begin{array}{l}--- \\
--- \\
--- \\
--- \\
---\end{array}$ & $\begin{array}{r}23 \\
9 \\
0 \\
140 \\
23\end{array}$ \\
\hline $\begin{array}{l}\text { LINE } \\
\text { A } \\
\text { B } \\
\text { C }\end{array}$ & $\begin{array}{l}20970 \\
9400.0 \\
9483.0 \\
9507.0\end{array}$ & $\begin{array}{l}\text { S? } \\
\text { B? } \\
\text { B? }\end{array}$ & $\begin{array}{l}653814 \\
656190 \\
657093\end{array}$ & $\begin{array}{l}6112960 \\
6113129 \\
6113105\end{array}$ & $\begin{array}{l}7.7 \\
0.0 \\
0.0\end{array}$ & $\begin{array}{l}1.4 \\
0.0 \\
0.0\end{array}$ & $\begin{array}{r}12.6 \\
0.0 \\
0.0\end{array}$ & $\begin{array}{l}1.9 \\
0.0 \\
0.0\end{array}$ & $\begin{array}{r}11.6 \\
0.0 \\
0.0\end{array}$ & $\begin{array}{r}12.6 \\
0.0 \\
0.0\end{array}$ & $\begin{array}{l}--- \\
--- \\
---\end{array}$ & $\begin{array}{l}--- \\
--- \\
---\end{array}$ & $\begin{array}{r}14 \\
9 \\
91\end{array}$ \\
\hline
\end{tabular}

$\mathrm{CX}=$ COAXIAL

$\mathrm{CP}=$ COPLANAR Note $: \mathrm{EM}$ values shown above are local amplitudes

*Estimated Depth may be unreliable because the stronger part of the conductor may be deeper or to one side of the flight line, or because of a shallow dip or magnetite/overburden effects 
EM Anomaly List

\begin{tabular}{|c|c|c|c|c|c|c|c|c|c|c|c|c|c|}
\hline Label & Fid & Interp & $\underset{\mathrm{m}}{\mathrm{XUTM}}$ & $\begin{array}{c}\text { YUTM } \\
\text { m }\end{array}$ & $\begin{array}{l}\text { CX } \\
\text { Real } \\
\text { ppm }\end{array}$ & $\begin{array}{l}900 \mathrm{HZ} \\
\text { Quad } \\
\text { ppm }\end{array}$ & $\begin{array}{l}\text { CP } \\
\text { Real } \\
\text { ppm }\end{array}$ & $\begin{array}{c}900 \mathrm{~Hz} \\
\text { Quad } \\
\text { ppm }\end{array}$ & $\begin{array}{l}\text { CP } \\
\text { Real } \\
\text { ppm }\end{array}$ & $\begin{array}{l}200 \mathrm{HZ} \\
\text { Quad } \\
\text { ppm }\end{array}$ & $\begin{array}{l}\text { Vertic } \\
\text { CoND } \\
\text { siemens }\end{array}$ & $\begin{array}{c}\text { al Dike } \\
\text { DEPTH* } \\
\text { m }\end{array}$ & $\begin{array}{c}\text { Mag. Corr } \\
\text { NT }\end{array}$ \\
\hline $\begin{array}{l}\text { LINE } \\
\text { A } \\
\text { B } \\
\text { C }\end{array}$ & $\begin{array}{l}20980 \\
9692.0 \\
9622.0 \\
9594.0\end{array}$ & $\begin{array}{l}\text { S? } \\
\text { S? } \\
\text { B? }\end{array}$ & $\begin{array}{l}652996 \\
655380 \\
656220\end{array}$ & $\begin{array}{l}6112771 \\
6112843 \\
6112930\end{array}$ & $\begin{array}{l}2.1 \\
0.0 \\
3.6\end{array}$ & $\begin{array}{l}2.2 \\
0.0 \\
6.3\end{array}$ & $\begin{array}{l}3.5 \\
0.0 \\
0.5\end{array}$ & $\begin{array}{l}2.8 \\
0.0 \\
3.3\end{array}$ & $\begin{array}{l}3.1 \\
0.0 \\
7.3\end{array}$ & $\begin{array}{r}17.5 \\
0.0 \\
14.4\end{array}$ & $\begin{array}{l}--- \\
--- \\
---\end{array}$ & $\begin{array}{l}--- \\
--- \\
---\end{array}$ & $\begin{array}{r}18 \\
0 \\
0\end{array}$ \\
\hline $\begin{array}{l}\text { LINE } \\
\text { A } \\
B\end{array}$ & $\begin{array}{l}20990 \\
9769.0 \\
9859.0\end{array}$ & $\begin{array}{r}\mathrm{S} ? \\
\mathrm{~B}\end{array}$ & $\begin{array}{l}653504 \\
656198\end{array}$ & $\begin{array}{l}6112556 \\
6112673\end{array}$ & $\begin{array}{l}0.0 \\
0.0\end{array}$ & $\begin{array}{l}0.0 \\
0.0\end{array}$ & $\begin{array}{l}0.0 \\
0.0\end{array}$ & $\begin{array}{l}0.0 \\
0.0\end{array}$ & $\begin{array}{l}0.0 \\
0.0\end{array}$ & $\begin{array}{l}0.0 \\
0.0\end{array}$ & $\begin{array}{l}--- \\
---\end{array}$ & $\begin{array}{l}--- \\
---\end{array}$ & $\begin{array}{l}17 \\
17\end{array}$ \\
\hline $\begin{array}{l}\text { LINE } \\
\text { A } \\
B\end{array}$ & $\begin{array}{l}21000 \\
9960.0 \\
9918.0\end{array}$ & $\begin{array}{r}B ? \\
\mathrm{H}\end{array}$ & $\begin{array}{l}656220 \\
657504\end{array}$ & $\begin{array}{l}6112463 \\
6112533\end{array}$ & $\begin{array}{l}3.4 \\
0.0\end{array}$ & $\begin{array}{l}4.3 \\
0.0\end{array}$ & $\begin{array}{l}0.9 \\
0.0\end{array}$ & $\begin{array}{l}1.8 \\
0.0\end{array}$ & $\begin{array}{l}5.1 \\
0.0\end{array}$ & $\begin{array}{l}5.9 \\
0.0\end{array}$ & $\begin{array}{l}--- \\
---\end{array}$ & $\begin{array}{l}--- \\
---\end{array}$ & $\begin{array}{l}8 \\
0\end{array}$ \\
\hline $\begin{array}{l}\text { LINE } \\
\text { A }\end{array}$ & $\begin{array}{l}21010 \\
10234.0\end{array}$ & S & 656711 & 6112329 & 4.2 & 2.1 & 2.7 & 2.1 & 0.8 & 0.4 & --- & --- & 10 \\
\hline $\begin{array}{l}\text { LINE } \\
\text { A }\end{array}$ & $\begin{array}{l}21020 \\
10294.0\end{array}$ & $\mathrm{~S}$ & 657092 & 6112200 & 0.0 & 0.0 & 0.0 & 0.0 & 0.0 & 0.0 & --- & --- & 12 \\
\hline $\begin{array}{l}\text { LINE } \\
\text { A } \\
B \\
\text { C }\end{array}$ & $\begin{array}{l}21030 \\
266.0 \\
324.0 \\
396.0\end{array}$ & $\begin{array}{r}\text { S? } \\
\text { S? } \\
\text { B }\end{array}$ & $\begin{array}{l}652615 \\
653928 \\
655894\end{array}$ & $\begin{array}{l}6111851 \\
6111833 \\
6111884\end{array}$ & $\begin{array}{r}11.6 \\
3.1 \\
0.0\end{array}$ & $\begin{array}{l}0.8 \\
1.7 \\
0.0\end{array}$ & $\begin{array}{r}10.3 \\
6.1 \\
0.0\end{array}$ & $\begin{array}{l}0.5 \\
2.9 \\
0.0\end{array}$ & $\begin{array}{l}7.9 \\
5.5 \\
0.0\end{array}$ & $\begin{array}{r}6.3 \\
20.6 \\
0.0\end{array}$ & $\begin{array}{l}--- \\
--- \\
---\end{array}$ & $\begin{array}{l}--- \\
--- \\
---\end{array}$ & $\begin{array}{l}65 \\
18 \\
18\end{array}$ \\
\hline $\begin{array}{l}\text { LINE } \\
\text { A } \\
B \\
\text { C }\end{array}$ & $\begin{array}{l}21040 \\
702.0 \\
622.0 \\
587.0\end{array}$ & $\begin{array}{r}\text { S? } \\
\text { B? } \\
\text { H }\end{array}$ & $\begin{array}{l}652769 \\
655226 \\
656098\end{array}$ & $\begin{array}{l}6111615 \\
6111677 \\
6111699\end{array}$ & $\begin{array}{l}9.0 \\
6.4 \\
0.0\end{array}$ & $\begin{array}{l}1.6 \\
8.2 \\
0.0\end{array}$ & $\begin{array}{r}15.2 \\
2.6 \\
0.0\end{array}$ & $\begin{array}{l}1.3 \\
8.2 \\
0.0\end{array}$ & $\begin{array}{r}11.9 \\
22.0 \\
0.0\end{array}$ & $\begin{array}{r}12.3 \\
20.1 \\
0.0\end{array}$ & $\begin{array}{l}--- \\
--- \\
---\end{array}$ & $\begin{array}{l}--- \\
--- \\
---\end{array}$ & $\begin{array}{l}0 \\
0 \\
0\end{array}$ \\
\hline $\begin{array}{l}\text { LINE } \\
\text { A } \\
B \\
\text { C }\end{array}$ & $\begin{array}{l}21050 \\
974.0 \\
981.0 \\
984.0\end{array}$ & $\begin{array}{l}\text { B? } \\
\text { B? } \\
\text { B? }\end{array}$ & $\begin{array}{l}658024 \\
658276 \\
658385\end{array}$ & $\begin{array}{l}6111577 \\
6111586 \\
6111584\end{array}$ & $\begin{array}{l}0.0 \\
0.0 \\
0.0\end{array}$ & $\begin{array}{l}0.0 \\
0.0 \\
0.0\end{array}$ & $\begin{array}{l}0.0 \\
0.0 \\
0.0\end{array}$ & $\begin{array}{l}0.0 \\
0.0 \\
0.0\end{array}$ & $\begin{array}{l}0.0 \\
0.0 \\
0.0\end{array}$ & $\begin{array}{l}0.0 \\
0.0 \\
0.0\end{array}$ & $\begin{array}{l}--- \\
--- \\
---\end{array}$ & $\begin{array}{l}--- \\
--- \\
---\end{array}$ & $\begin{array}{r}8 \\
0 \\
109\end{array}$ \\
\hline $\begin{array}{l}\text { LINE } \\
\text { A }\end{array}$ & $\begin{array}{l}21080 \\
1265.0\end{array}$ & S? & 656844 & 6110859 & 0.0 & 0.0 & 0.0 & 0.0 & 0.0 & 0.0 & --- & --- & 5 \\
\hline $\begin{array}{l}\text { LINE } \\
\text { A }\end{array}$ & $\begin{array}{l}21090 \\
1327.0\end{array}$ & S? & 656029 & 6110640 & 0.0 & 0.0 & 0.0 & 0.0 & 0.0 & 0.0 & --- & --- & 96 \\
\hline $\begin{array}{l}\text { LINE } \\
\text { A } \\
\text { B }\end{array}$ & $\begin{array}{l}21100 \\
1487.0 \\
1458.0\end{array}$ & $\begin{array}{l}\text { B? } \\
\text { S? }\end{array}$ & $\begin{array}{l}655953 \\
657125\end{array}$ & $\begin{array}{l}6110344 \\
6110501\end{array}$ & $\begin{array}{l}0.0 \\
0.0\end{array}$ & $\begin{array}{l}0.0 \\
0.0\end{array}$ & $\begin{array}{l}0.0 \\
0.0\end{array}$ & $\begin{array}{l}0.0 \\
0.0\end{array}$ & $\begin{array}{l}0.0 \\
0.0\end{array}$ & $\begin{array}{l}0.0 \\
0.0\end{array}$ & $\begin{array}{l}--- \\
---\end{array}$ & $\begin{array}{l}--- \\
---\end{array}$ & $\begin{array}{l}19 \\
16\end{array}$ \\
\hline $\begin{array}{l}\text { LINE } \\
\text { A }\end{array}$ & $\begin{array}{l}21110 \\
1531.0\end{array}$ & S? & 655876 & 6110205 & 0.0 & 0.0 & 0.0 & 0.0 & 0.0 & 0.0 & --- & --- & 0 \\
\hline
\end{tabular}

$\mathrm{CX}=$ COAXIAL

Note:EM values shown above

Hetta - North/South of 5515 local amplitudes
* Estimated Depth may be unreliable because the stronger part of the conductor may be deeper or to one side of the flight line, or because of a shallow dip or magnetite/overburden effects 
EM Anomaly List

\begin{tabular}{|c|c|c|c|c|c|c|c|c|c|c|c|c|c|}
\hline Label & Fid & Interp & $\begin{array}{c}\text { XUTM } \\
\text { m }\end{array}$ & $\begin{array}{c}\text { YUTM } \\
\mathrm{m}\end{array}$ & $\begin{array}{ll}\text { CX } & 9 \\
\text { Real } & \\
\text { ppm }\end{array}$ & $\begin{array}{l}00 \mathrm{HZ} \\
\text { Quad } \\
\text { ppm }\end{array}$ & $\begin{array}{l}\text { CP } \\
\text { Real } \\
\text { ppm }\end{array}$ & $\begin{array}{l}900 \mathrm{HZ} \\
\text { Quad } \\
\text { ppm }\end{array}$ & $\begin{array}{l}\text { CP } 7 \\
\text { Real } \\
\text { ppm }\end{array}$ & $\begin{array}{l}200 \mathrm{~Hz} \\
\text { Quad } \\
\text { ppm }\end{array}$ & $\begin{array}{l}\text { Vertica } \\
\text { Cond } \\
\text { siemens }\end{array}$ & $\begin{array}{c}\text { al Dike } \\
\text { DEPTH }^{\star} \\
\text { m }\end{array}$ & $\begin{array}{c}\text { Mag. Corr } \\
\text { NT }\end{array}$ \\
\hline $\begin{array}{l}\mathrm{LINE} \\
\mathrm{B}\end{array}$ & $\begin{array}{r}21110 \\
1532.0\end{array}$ & $B$ ? & 655920 & 6110209 & 0.0 & 0.0 & 0.0 & 0.0 & 0.0 & 0.0 & --- & --- & 0 \\
\hline $\begin{array}{l}\text { LINE } \\
A\end{array}$ & $\begin{array}{l}21120 \\
1707.0\end{array}$ & S? & 655827 & 6110072 & 0.0 & 0.0 & 0.0 & 0.0 & 0.0 & 0.0 & --- & --- & 0 \\
\hline $\begin{array}{l}\text { LINE } \\
\text { A } \\
B\end{array}$ & $\begin{array}{l}21130 \\
1747.0 \\
1749.0\end{array}$ & $\begin{array}{l}\text { S? } \\
\text { S? }\end{array}$ & $\begin{array}{l}656353 \\
656436\end{array}$ & $\begin{array}{l}6109953 \\
6109957\end{array}$ & $\begin{array}{l}0.6 \\
1.1\end{array}$ & $\begin{array}{l}4.8 \\
5.3\end{array}$ & $\begin{array}{l}3.3 \\
3.3\end{array}$ & $\begin{array}{l}5.4 \\
4.9\end{array}$ & $\begin{array}{l}7.2 \\
6.7\end{array}$ & $\begin{array}{l}5.3 \\
6.1\end{array}$ & $\begin{array}{l}--- \\
---\end{array}$ & $\begin{array}{l}--- \\
---\end{array}$ & $\begin{array}{l}51 \\
47\end{array}$ \\
\hline $\begin{array}{l}\text { LINE } \\
\text { A } \\
B\end{array}$ & $\begin{array}{l}21140 \\
1911.0 \\
1884.0\end{array}$ & $\begin{array}{l}S \\
S\end{array}$ & $\begin{array}{l}659651 \\
660716\end{array}$ & $\begin{array}{l}6109848 \\
6109877\end{array}$ & $\begin{array}{l}0.0 \\
0.0\end{array}$ & $\begin{array}{l}0.0 \\
0.0\end{array}$ & $\begin{array}{l}0.0 \\
0.0\end{array}$ & $\begin{array}{l}0.0 \\
0.0\end{array}$ & $\begin{array}{l}0.0 \\
0.0\end{array}$ & $\begin{array}{l}0.0 \\
0.0\end{array}$ & $\begin{array}{l}--- \\
---\end{array}$ & $\begin{array}{l}--- \\
---\end{array}$ & $\begin{array}{l}8 \\
8\end{array}$ \\
\hline $\begin{array}{l}\text { LINE } \\
\text { A } \\
B\end{array}$ & $\begin{array}{r}21180 \\
2415.0 \\
2324.0\end{array}$ & $\begin{array}{r}S ? \\
S\end{array}$ & $\begin{array}{l}658271 \\
660680\end{array}$ & $\begin{array}{l}6108916 \\
6109087\end{array}$ & $\begin{array}{l}0.5 \\
0.9\end{array}$ & $\begin{array}{l}1.7 \\
2.9\end{array}$ & $\begin{array}{l}0.2 \\
1.6\end{array}$ & $\begin{array}{l}4.0 \\
3.7\end{array}$ & $\begin{array}{l}8.8 \\
9.1\end{array}$ & $\begin{array}{l}14.0 \\
19.0\end{array}$ & $\begin{array}{l}--- \\
---\end{array}$ & $\begin{array}{l}--- \\
---\end{array}$ & $\begin{array}{r}31 \\
0\end{array}$ \\
\hline $\begin{array}{l}\text { LINE } \\
\text { A } \\
\text { B } \\
\text { C }\end{array}$ & $\begin{array}{r}21190 \\
2500.0 \\
2542.0 \\
2546.0\end{array}$ & $\begin{array}{l}\text { S? } \\
\text { S? } \\
\text { S? }\end{array}$ & $\begin{array}{l}658346 \\
659420 \\
659503\end{array}$ & $\begin{array}{l}6108766 \\
6108651 \\
6108665\end{array}$ & $\begin{array}{l}0.0 \\
0.0 \\
0.0\end{array}$ & $\begin{array}{l}0.0 \\
0.0 \\
0.0\end{array}$ & $\begin{array}{l}0.0 \\
0.0 \\
0.0\end{array}$ & $\begin{array}{l}0.0 \\
0.0 \\
0.0\end{array}$ & $\begin{array}{l}0.0 \\
0.0 \\
0.0\end{array}$ & $\begin{array}{l}0.0 \\
0.0 \\
0.0\end{array}$ & $\begin{array}{l}--- \\
--- \\
---\end{array}$ & $\begin{array}{l}--- \\
--- \\
---\end{array}$ & $\begin{array}{r}37 \\
375 \\
14\end{array}$ \\
\hline $\begin{array}{l}\text { LINE } \\
\text { A } \\
\text { B } \\
\text { C }\end{array}$ & $\begin{array}{r}21200 \\
2772.0 \\
2729.0 \\
2632.0\end{array}$ & $\begin{array}{l}\text { S? } \\
\text { S? } \\
\text { B? }\end{array}$ & $\begin{array}{l}658355 \\
659450 \\
660701\end{array}$ & $\begin{array}{l}6108598 \\
6108591 \\
6108665\end{array}$ & $\begin{array}{l}3.1 \\
0.0 \\
0.0\end{array}$ & $\begin{array}{l}1.3 \\
0.0 \\
0.0\end{array}$ & $\begin{array}{l}2.8 \\
0.0 \\
0.0\end{array}$ & $\begin{array}{l}2.9 \\
0.0 \\
0.0\end{array}$ & $\begin{array}{l}6.9 \\
0.0 \\
0.0\end{array}$ & $\begin{array}{r}11.3 \\
0.0 \\
0.0\end{array}$ & $\begin{array}{l}--- \\
--- \\
---\end{array}$ & $\begin{array}{l}--- \\
--- \\
---\end{array}$ & $\begin{array}{r}69 \\
0 \\
43\end{array}$ \\
\hline $\begin{array}{l}\text { LINE } \\
\text { A }\end{array}$ & $\begin{array}{l}21220 \\
3114.0\end{array}$ & S? & 659205 & 6108207 & 2.3 & 2.1 & 69.9 & 1.4 & 57.0 & 8.1 & --- & --- & 0 \\
\hline $\begin{array}{l}\text { LINE } \\
A\end{array}$ & $\begin{array}{l}21221 \\
3281.0\end{array}$ & S? & 657399 & 6108180 & 0.0 & 0.0 & 0.0 & 0.0 & 0.0 & 0.0 & --- & --- & 11 \\
\hline $\begin{array}{l}\operatorname{LINE} \\
\mathrm{A} \\
\mathrm{B}\end{array}$ & $\begin{array}{l}21250 \\
3726.0 \\
3822.0\end{array}$ & $\begin{array}{l}S \\
S\end{array}$ & $\begin{array}{l}656787 \\
659378\end{array}$ & $\begin{array}{l}6107563 \\
6107632\end{array}$ & $\begin{array}{l}0.0 \\
0.0\end{array}$ & $\begin{array}{l}0.0 \\
0.0\end{array}$ & $\begin{array}{l}0.0 \\
0.0\end{array}$ & $\begin{array}{l}0.0 \\
0.0\end{array}$ & $\begin{array}{l}0.0 \\
0.0\end{array}$ & $\begin{array}{l}0.0 \\
0.0\end{array}$ & $\begin{array}{l}--- \\
---\end{array}$ & $\begin{array}{l}--- \\
---\end{array}$ & $\begin{array}{l}30 \\
30\end{array}$ \\
\hline $\begin{array}{l}\operatorname{LINE} \\
A\end{array}$ & $\begin{array}{l}21270 \\
4137.0\end{array}$ & S & 657935 & 6107121 & 0.0 & 0.0 & 0.0 & 0.0 & 0.0 & 0.0 & --- & --- & 16 \\
\hline $\begin{array}{l}\text { LINE } \\
\mathrm{A}\end{array}$ & $\begin{array}{r}21280 \\
4349.0\end{array}$ & S? & 659024 & 6106889 & 0.3 & 2.2 & 0.4 & 2.9 & 0.5 & 18.9 & --- & --- & 118 \\
\hline $\begin{array}{l}\text { LINE } \\
\text { A } \\
B\end{array}$ & $\begin{array}{r}21290 \\
4548.0 \\
4579.0\end{array}$ & $\begin{array}{l}\text { B? } \\
\text { S? }\end{array}$ & $\begin{array}{l}658129 \\
659099\end{array}$ & $\begin{array}{l}6106716 \\
6106790\end{array}$ & $\begin{array}{l}0.0 \\
0.0\end{array}$ & $\begin{array}{l}0.0 \\
0.0\end{array}$ & $\begin{array}{l}0.0 \\
0.0\end{array}$ & $\begin{array}{l}0.0 \\
0.0\end{array}$ & $\begin{array}{l}0.0 \\
0.0\end{array}$ & $\begin{array}{l}0.0 \\
0.0\end{array}$ & $\begin{array}{l}--- \\
---\end{array}$ & $\begin{array}{l}--- \\
---\end{array}$ & $\begin{array}{r}26 \\
0\end{array}$ \\
\hline
\end{tabular}

$\mathrm{CX}=$ COAXIAL

$\mathrm{CP}=$ COPLANAR

Note: EM values shown above are local amplitudes

Hetta - North/South of 5515
*Estimated Depth may be unreliable because the stronger part of the conductor may be deeper or to one side of the flight line, or because of a shallow dip or magnetite/overburden effects 
EM Anomaly List

\begin{tabular}{|c|c|c|c|c|c|c|c|c|c|c|c|c|c|}
\hline Label & Fid & Interp & $\begin{array}{l}\text { XUTM } \\
\text { m }\end{array}$ & $\begin{array}{c}\text { YUTM } \\
\mathrm{m}\end{array}$ & $\begin{array}{ll}\text { CX } & 9 \\
\text { Real } & \\
\text { ppm } & \end{array}$ & $\begin{array}{l}00 \mathrm{HZ} \\
\text { Quad } \\
\text { ppm }\end{array}$ & $\begin{array}{l}\text { CP } \\
\text { Real } \\
\text { ppm }\end{array}$ & $\begin{array}{l}900 \mathrm{HZ} \\
\text { Quad } \\
\text { ppm }\end{array}$ & $\begin{array}{l}\text { CP } \\
\text { Real } \\
\text { ppm }\end{array}$ & $\begin{array}{l}200 \mathrm{~Hz} \\
\text { Quad } \\
\text { ppm }\end{array}$ & $\begin{array}{l}\text { Vertica } \\
\text { Cond } \\
\text { siemens }\end{array}$ & $\begin{array}{c}\text { al Dike } \\
\text { DEPTH* } \\
\text { m }\end{array}$ & $\begin{array}{c}\text { Mag. Corr } \\
\text { NT }\end{array}$ \\
\hline $\begin{array}{l}\text { LINE } \\
A\end{array}$ & $\begin{array}{c}21310 \\
630.0\end{array}$ & $B$ ? & 658817 & 6106371 & 0.0 & 0.0 & 0.0 & 0.0 & 0.0 & 0.0 & --- & --- & 20 \\
\hline $\begin{array}{l}\text { LINE } \\
\text { A }\end{array}$ & $\begin{array}{r}21320 \\
1156.0\end{array}$ & S & 658835 & 6106086 & 0.0 & 0.0 & 0.0 & 0.0 & 0.0 & 0.0 & --- & --- & 0 \\
\hline $\begin{array}{l}\text { LINE } \\
A\end{array}$ & $\begin{array}{r}21330 \\
1387.0\end{array}$ & $\mathrm{~S}$ & 657306 & 6105893 & 0.0 & 0.0 & 0.0 & 0.0 & 0.0 & 0.0 & --- & --- & 36 \\
\hline $\begin{array}{l}\text { LINE } \\
A\end{array}$ & $\begin{array}{l}21340 \\
1790.0\end{array}$ & S? & 658126 & 6105690 & 5.1 & 0.8 & 10.0 & 1.4 & 7.8 & 8.4 & --- & --- & 0 \\
\hline $\begin{array}{l}\text { LINE } \\
A\end{array}$ & $\begin{array}{l}21350 \\
2080.0\end{array}$ & S? & 659027 & 6105503 & 0.0 & 0.0 & 0.0 & 0.0 & 0.0 & 0.0 & --- & --- & 15 \\
\hline $\begin{array}{l}\text { LINE } \\
\mathrm{A}\end{array}$ & $\begin{array}{r}21360 \\
2434.0\end{array}$ & S & 658195 & 6105273 & 0.0 & 0.0 & 0.0 & 0.0 & 0.0 & 0.0 & --- & --- & 0 \\
\hline $\begin{array}{l}\text { LINE } \\
\text { A } \\
\text { B } \\
\text { C } \\
\text { D }\end{array}$ & $\begin{array}{r}21390 \\
3136.0 \\
3145.0 \\
3229.0 \\
3336.0\end{array}$ & $\begin{array}{l}\mathrm{S} \\
\mathrm{S} \\
\mathrm{S} \\
\mathrm{S}\end{array}$ & $\begin{array}{l}656142 \\
656396 \\
658432 \\
660466\end{array}$ & $\begin{array}{l}6104589 \\
6104612 \\
6104713 \\
6104806\end{array}$ & $\begin{array}{l}4.0 \\
3.3 \\
0.0 \\
3.0\end{array}$ & $\begin{array}{l}3.0 \\
1.7 \\
0.0 \\
2.3\end{array}$ & $\begin{array}{l}0.2 \\
3.4 \\
0.0 \\
1.3\end{array}$ & $\begin{array}{l}1.6 \\
1.2 \\
0.0 \\
8.7\end{array}$ & $\begin{array}{r}6.5 \\
0.4 \\
0.0 \\
22.0\end{array}$ & $\begin{array}{r}0.2 \\
3.5 \\
0.0 \\
20.3\end{array}$ & $\begin{array}{l}--- \\
--- \\
--- \\
---\end{array}$ & $\begin{array}{l}--- \\
--- \\
--- \\
---\end{array}$ & $\begin{array}{l}15 \\
15 \\
15 \\
15\end{array}$ \\
\hline $\begin{array}{l}\text { LINE } \\
\mathrm{A}\end{array}$ & $\begin{array}{l}29010 \\
269.0\end{array}$ & D & 639791 & 6130858 & 0.0 & 0.0 & 0.0 & 0.0 & 0.0 & 0.0 & --- & --- & 17 \\
\hline $\begin{array}{l}\text { LINE } \\
\text { A } \\
\text { B } \\
\text { C } \\
\text { D } \\
\text { E }\end{array}$ & $\begin{array}{l}29020 \\
627.0 \\
623.0 \\
617.0 \\
549.0 \\
545.0\end{array}$ & $\begin{array}{r}\mathrm{B} \\
\mathrm{B} \\
\mathrm{B} \\
\mathrm{B} ? \\
\mathrm{~B} ?\end{array}$ & $\begin{array}{l}645314 \\
645313 \\
645311 \\
645033 \\
645000\end{array}$ & $\begin{array}{l}6129764 \\
6129902 \\
6130111 \\
6132283 \\
6132382\end{array}$ & $\begin{array}{r}8.0 \\
12.5 \\
10.3 \\
6.6 \\
7.1\end{array}$ & $\begin{array}{l}7.1 \\
7.8 \\
3.7 \\
8.4 \\
8.1\end{array}$ & $\begin{array}{r}12.4 \\
12.0 \\
8.9 \\
2.8 \\
4.4\end{array}$ & $\begin{array}{r}10.9 \\
10.8 \\
6.0 \\
9.1 \\
6.9\end{array}$ & $\begin{array}{l}24.9 \\
24.8 \\
11.0 \\
19.2 \\
13.1\end{array}$ & $\begin{array}{r}11.2 \\
11.7 \\
2.3 \\
24.6 \\
18.4\end{array}$ & $\begin{array}{l}--- \\
--- \\
--- \\
--- \\
---\end{array}$ & $\begin{array}{l}--- \\
--- \\
--- \\
--- \\
---\end{array}$ & $\begin{array}{r}0 \\
7 \\
0 \\
10 \\
10\end{array}$ \\
\hline $\begin{array}{l}\text { LINE } \\
\text { A } \\
B \\
\text { C } \\
\text { D } \\
\text { E }\end{array}$ & $\begin{array}{l}29030 \\
1495.0 \\
1118.0 \\
1115.0 \\
1103.0 \\
1094.0\end{array}$ & $\begin{array}{r}\mathrm{B} ? \\
\mathrm{~B} \\
\mathrm{~B} \\
\mathrm{~B} ? \\
\mathrm{D}\end{array}$ & $\begin{array}{l}654024 \\
651923 \\
651910 \\
651886 \\
651885\end{array}$ & $\begin{array}{l}6119382 \\
6130775 \\
6130901 \\
6131207 \\
6131327\end{array}$ & $\begin{array}{l}6.3 \\
0.0 \\
0.0 \\
0.0 \\
0.0\end{array}$ & $\begin{array}{l}8.8 \\
0.0 \\
0.0 \\
0.0 \\
0.0\end{array}$ & $\begin{array}{l}9.3 \\
0.0 \\
0.0 \\
0.0 \\
0.0\end{array}$ & $\begin{array}{l}6.8 \\
0.0 \\
0.0 \\
0.0 \\
0.0\end{array}$ & $\begin{array}{r}11.4 \\
0.0 \\
0.0 \\
0.0 \\
0.0\end{array}$ & $\begin{array}{r}49.4 \\
0.0 \\
0.0 \\
0.0 \\
0.0\end{array}$ & $\begin{array}{l}--- \\
--- \\
--- \\
--- \\
---\end{array}$ & $\begin{array}{l}--- \\
--- \\
--- \\
--- \\
---\end{array}$ & $\begin{array}{r}48 \\
305 \\
0 \\
49 \\
0\end{array}$ \\
\hline $\begin{array}{l}\text { LINE } \\
\text { A } \\
B\end{array}$ & $\begin{array}{r}29031 \\
1539.0 \\
1524.0\end{array}$ & $\begin{array}{l}\text { S? } \\
\text { S? }\end{array}$ & $\begin{array}{l}654599 \\
654457\end{array}$ & $\begin{array}{l}6118973 \\
6119233\end{array}$ & $\begin{array}{l}0.0 \\
0.0\end{array}$ & $\begin{array}{l}0.0 \\
0.0\end{array}$ & $\begin{array}{l}0.0 \\
0.0\end{array}$ & $\begin{array}{l}0.0 \\
0.0\end{array}$ & $\begin{array}{l}0.0 \\
0.0\end{array}$ & $\begin{array}{l}0.0 \\
0.0\end{array}$ & $\begin{array}{l}--- \\
---\end{array}$ & $\begin{array}{l}--- \\
---\end{array}$ & $\begin{array}{l}0 \\
0\end{array}$ \\
\hline
\end{tabular}

$\mathrm{CX}=$ COAXIAL

$\mathrm{CP}=$ COPLANAR Note $: \mathrm{EM}$ values shown above are local amplitudes

*Estimated Depth may be unreliable because the stronger part of the conductor may be deeper or to one side of the flight line, or because of a shallow dip or magnetite/overburden effects 
EM Anomaly List

\begin{tabular}{|c|c|c|c|c|c|c|c|c|c|c|c|c|c|}
\hline Label & Fid & Interp & $\begin{array}{c}\text { XUTM } \\
\mathrm{m}\end{array}$ & $\begin{array}{c}\text { YUTM } \\
\mathrm{m}\end{array}$ & $\begin{array}{l}\text { CX } \\
\text { Real } \\
\text { ppm }\end{array}$ & $\begin{array}{l}900 \mathrm{HZ} \\
\text { Quad } \\
\text { ppm }\end{array}$ & $\begin{array}{l}\text { CP } \\
\text { Real } \\
\text { ppm }\end{array}$ & $\begin{array}{l}900 \mathrm{HZ} \\
\text { Quad } \\
\text { ppm }\end{array}$ & $\begin{array}{l}\text { CP } 7 \\
\text { Real } \\
\text { ppm }\end{array}$ & $\begin{array}{c}7200 \mathrm{HZ} \\
\text { Quad } \\
\text { ppm }\end{array}$ & $\begin{array}{l}\text { Vertic } \\
\text { CoND } \\
\text { siemens }\end{array}$ & $\begin{array}{c}\text { al Dike } \\
\text { DEPTH* } \\
\text { m }\end{array}$ & $\begin{array}{c}\text { Mag. Corr } \\
\text { NT }\end{array}$ \\
\hline $\begin{array}{l}\text { LINE } 3 \\
\mathrm{~A}\end{array}$ & $\begin{array}{l}30010 \\
1645.0\end{array}$ & $S ?$ & 653991 & 6113346 & 2.3 & 2.1 & 2.2 & 3.2 & 9.9 & 2.4 & --- & --- & 20 \\
\hline $\begin{array}{l}\text { LINE } 3 \\
\text { A }\end{array}$ & $\begin{array}{l}30020 \\
1569.0\end{array}$ & S & 653002 & 6112194 & 0.0 & 0.0 & 0.0 & 0.0 & 0.0 & 0.0 & --- & --- & 19 \\
\hline $\begin{array}{l}\text { LINE } 3 \\
\text { A } \\
B\end{array}$ & $\begin{array}{l}30030 \\
1415.0 \\
1384.0\end{array}$ & $\begin{array}{l}\mathrm{H} ? \\
\mathrm{H} ?\end{array}$ & $\begin{array}{l}652963 \\
653446\end{array}$ & $\begin{array}{l}6111783 \\
6112301\end{array}$ & $\begin{array}{l}0.0 \\
0.0\end{array}$ & $\begin{array}{l}0.0 \\
0.0\end{array}$ & $\begin{array}{l}0.0 \\
0.0\end{array}$ & $\begin{array}{l}0.0 \\
0.0\end{array}$ & $\begin{array}{l}0.0 \\
0.0\end{array}$ & $\begin{array}{l}0.0 \\
0.0\end{array}$ & $\begin{array}{l}--- \\
---\end{array}$ & $\begin{array}{l}--- \\
---\end{array}$ & $\begin{array}{l}82 \\
31\end{array}$ \\
\hline $\begin{array}{l}\text { LINE } 3 \\
A\end{array}$ & $\begin{array}{l}30040 \\
1298.0\end{array}$ & $S ?$ & 653077 & 6111720 & 1.1 & 1.3 & 4.6 & 1.8 & 3.3 & 6.8 & --- & --- & 233 \\
\hline $\begin{array}{l}\text { LINE } 3 \\
\text { A } \\
B\end{array}$ & $\begin{array}{l}30050 \\
1166.0 \\
1145.0\end{array}$ & $\begin{array}{l}\mathrm{B} ? \\
\mathrm{H} \text { ? }\end{array}$ & $\begin{array}{l}651851 \\
652264\end{array}$ & $\begin{array}{l}6110136 \\
6110591\end{array}$ & $\begin{array}{r}29.9 \\
0.0\end{array}$ & $\begin{array}{l}4.4 \\
0.0\end{array}$ & $\begin{array}{r}37.8 \\
0.0\end{array}$ & $\begin{array}{l}6.3 \\
0.0\end{array}$ & $\begin{array}{r}25.2 \\
0.0\end{array}$ & $\begin{array}{r}15.5 \\
0.0\end{array}$ & $\begin{array}{l}--- \\
---\end{array}$ & --- & $\begin{array}{r}0 \\
66\end{array}$ \\
\hline $\begin{array}{l}\text { LINE } 3 \\
A\end{array}$ & $\begin{array}{l}30080 \\
654.0\end{array}$ & S & 653205 & 6110986 & 0.0 & 0.0 & 0.0 & 0.0 & 0.0 & 0.0 & --- & --- & 18 \\
\hline $\begin{array}{l}\text { LINE } 3 \\
A\end{array}$ & $\begin{array}{r}30092 \\
440.0\end{array}$ & S? & 653089 & 6110604 & 0.0 & 0.0 & 0.0 & 0.0 & 0.0 & 0.0 & --- & --- & 13 \\
\hline $\begin{array}{l}\text { LINE } 3 \\
A\end{array}$ & $\begin{array}{c}30100 \\
218.0\end{array}$ & S & 652686 & 6110008 & 8.0 & 1.1 & 0.2 & 2.2 & 0.6 & 19.1 & --- & --- & 13 \\
\hline $\begin{array}{l}\text { LINE } 3 \\
\text { A }\end{array}$ & $\begin{array}{l}30110 \\
2010.0\end{array}$ & S & 652833 & 6109990 & 0.0 & 0.0 & 0.0 & 0.0 & 0.0 & 0.0 & --- & --- & 8 \\
\hline $\begin{array}{l}\text { LINE } 3 \\
\text { A }\end{array}$ & $\begin{array}{l}30130 \\
2384.0\end{array}$ & B & 651554 & 6108115 & 3.2 & 8.7 & 1.4 & 7.2 & 21.6 & 23.7 & --- & --- & 11 \\
\hline $\begin{array}{l}\text { LINE } 3 \\
A\end{array}$ & $\begin{array}{l}30140 \\
2810.0\end{array}$ & B & 651508 & 6107949 & 0.0 & 0.0 & 0.0 & 0.0 & 0.0 & 0.0 & --- & --- & 4 \\
\hline $\begin{array}{l}\text { LINE } 3 \\
A\end{array}$ & $\begin{array}{l}30150 \\
2890.0\end{array}$ & B & 651663 & 6107832 & 0.0 & 0.0 & 0.0 & 0.0 & 0.0 & 0.0 & --- & --- & 0 \\
\hline $\begin{array}{l}\text { LINE } 3 \\
\text { A } \\
B \\
C\end{array}$ & $\begin{array}{r}30210 \\
4216.0 \\
4220.0 \\
4238.0\end{array}$ & $\begin{array}{l}\text { B } \\
\text { B } \\
\text { B }\end{array}$ & $\begin{array}{l}651339 \\
651414 \\
651752\end{array}$ & $\begin{array}{l}6106139 \\
6106227 \\
6106616\end{array}$ & $\begin{array}{l}0.0 \\
0.0 \\
6.2\end{array}$ & $\begin{array}{l}0.0 \\
0.0 \\
3.3\end{array}$ & $\begin{array}{l}0.0 \\
0.0 \\
7.9\end{array}$ & $\begin{array}{l}0.0 \\
0.0 \\
6.2\end{array}$ & $\begin{array}{r}0.0 \\
0.0 \\
13.4\end{array}$ & $\begin{array}{l}0.0 \\
0.0 \\
4.8\end{array}$ & $\begin{array}{l}--- \\
--- \\
---\end{array}$ & $\begin{array}{l}--- \\
--- \\
---\end{array}$ & $\begin{array}{r}3 \\
22 \\
0\end{array}$ \\
\hline $\begin{array}{l}\text { LINE } 3 \\
A\end{array}$ & $\begin{array}{c}30330 \\
6022.0\end{array}$ & S? & 651592 & 6103677 & 0.0 & 0.0 & 0.0 & 0.0 & 0.0 & 0.0 & --- & --- & 0 \\
\hline
\end{tabular}

$\mathrm{CX}=$ COAXIAL

$\mathrm{CP}=$ COPLANAR Note: $\mathrm{EM}$ values shown above

Hetta - South of 55 15, South Portion
* Estimated Depth may be unreliable because the stronger part of the conductor may be deeper or to one side of the flight line, or because of a shallow dip or magnetite/overburden effects 
EM Anomaly List

\begin{tabular}{|c|c|c|c|c|c|c|c|c|c|c|c|c|c|}
\hline Label & Fid & Interp & $\begin{array}{l}\text { XUTM } \\
\mathrm{m}\end{array}$ & $\begin{array}{c}\text { YUTM } \\
\text { m }\end{array}$ & $\begin{array}{ll}\text { CX } & 9 \\
\text { Real } & \\
\text { ppm }\end{array}$ & $\begin{array}{l}00 \mathrm{HZ} \\
\text { Quad } \\
\text { ppm }\end{array}$ & $\begin{array}{l}\text { CP } \\
\text { Real } \\
\text { ppm }\end{array}$ & $\begin{array}{l}900 \mathrm{HZ} \\
\text { Quad } \\
\text { ppm }\end{array}$ & $\begin{array}{l}\text { CP } 7 \\
\text { Real } \\
\text { ppm }\end{array}$ & $\begin{array}{l}200 \mathrm{HZ} \\
\text { Quad } \\
\text { ppm }\end{array}$ & $\begin{array}{l}\text { Vertica } \\
\text { Cond } \\
\text { siemens }\end{array}$ & $\begin{array}{c}\text { al Dike } \\
\text { DEPTH* } \\
\text { m }\end{array}$ & $\begin{array}{c}\text { Mag. Corr } \\
\text { NT }\end{array}$ \\
\hline $\begin{array}{l}\mathrm{LINE} \\
\mathrm{A}\end{array}$ & $\begin{array}{c}10030 \\
404.2\end{array}$ & S & 624057 & 6141089 & 0.1 & 2.1 & 0.3 & 2.4 & 0.3 & 13.7 & 0.5 & 0 & 11 \\
\hline $\begin{array}{l}\text { LINE } \\
\mathrm{A}\end{array}$ & $\begin{array}{c}10040 \\
480.9\end{array}$ & S? & 624480 & 6140700 & 0.4 & 0.8 & 1.2 & 1.8 & 0.9 & 14.8 & 1.7 & 99 & 12 \\
\hline $\begin{array}{l}\text { LINE } \\
A\end{array}$ & $\begin{array}{c}10050 \\
622.2\end{array}$ & S & 624923 & 6140496 & 0.0 & 1.7 & 0.6 & 3.0 & 3.3 & 19.0 & --- & --- & 0 \\
\hline $\begin{array}{l}\text { LINE } \\
\text { A } \\
B \\
C\end{array}$ & $\begin{array}{c}10060 \\
778.0 \\
775.0 \\
714.4\end{array}$ & $\begin{array}{r}\text { B } \\
\text { B? } \\
\text { S? }\end{array}$ & $\begin{array}{l}625386 \\
625395 \\
625304\end{array}$ & $\begin{array}{l}6139148 \\
6139232 \\
6140864\end{array}$ & $\begin{array}{l}5.7 \\
6.0 \\
3.7\end{array}$ & $\begin{array}{l}9.3 \\
5.4 \\
3.3\end{array}$ & $\begin{array}{l}7.4 \\
3.6 \\
0.6\end{array}$ & $\begin{array}{r}12.6 \\
5.4 \\
5.0\end{array}$ & $\begin{array}{r}32.7 \\
16.2 \\
1.2\end{array}$ & $\begin{array}{r}23.5 \\
9.0 \\
33.8\end{array}$ & $\begin{array}{l}3.8 \\
7.1 \\
3.5\end{array}$ & $\begin{array}{r}14 \\
2 \\
53\end{array}$ & $\begin{array}{r}0 \\
0 \\
32\end{array}$ \\
\hline $\begin{array}{l}\text { LINE } \\
\text { A } \\
\text { B } \\
\text { C } \\
\text { D } \\
\text { E }\end{array}$ & $\begin{array}{l}10070 \\
906.9 \\
914.7 \\
933.4 \\
962.4 \\
970.8\end{array}$ & $\begin{array}{r}D \\
S \\
S ? \\
S \\
S ?\end{array}$ & $\begin{array}{l}625735 \\
625721 \\
625741 \\
625722 \\
625682\end{array}$ & $\begin{array}{l}6139082 \\
6139350 \\
6139829 \\
6140837 \\
6141146\end{array}$ & $\begin{array}{l}9.9 \\
1.3 \\
1.0 \\
0.4 \\
1.3\end{array}$ & $\begin{array}{l}5.7 \\
3.0 \\
1.8 \\
3.7 \\
2.8\end{array}$ & $\begin{array}{l}7.1 \\
0.1 \\
0.2 \\
0.5 \\
1.2\end{array}$ & $\begin{array}{l}5.8 \\
2.6 \\
1.7 \\
3.4 \\
4.6\end{array}$ & $\begin{array}{r}16.1 \\
2.0 \\
2.0 \\
7.3 \\
12.4\end{array}$ & $\begin{array}{l}12.2 \\
16.5 \\
12.2 \\
19.6 \\
20.9\end{array}$ & $\begin{array}{r}17.2 \\
1.4 \\
1.7 \\
0.6 \\
1.9\end{array}$ & $\begin{array}{r}16 \\
23 \\
37 \\
0 \\
49\end{array}$ & $\begin{array}{r}0 \\
0 \\
0 \\
0 \\
63\end{array}$ \\
\hline $\begin{array}{l}\text { LINE } \\
\text { A }\end{array}$ & $\begin{array}{l}10080 \\
1034.0\end{array}$ & $\mathrm{H}$ & 626069 & 6140893 & 1.9 & 3.0 & 1.0 & 4.0 & 7.3 & 26.4 & 2.5 & 28 & 0 \\
\hline $\begin{array}{l}\text { LINE } \\
A \\
B\end{array}$ & $\begin{array}{c}10090 \\
1196.5 \\
1224.4\end{array}$ & $\begin{array}{l}\mathrm{B} \text { ? } \\
\mathrm{B} \text { ? }\end{array}$ & $\begin{array}{l}626548 \\
626488\end{array}$ & $\begin{array}{l}6138824 \\
6139709\end{array}$ & $\begin{array}{l}1.5 \\
2.9\end{array}$ & $\begin{array}{l}3.8 \\
3.5\end{array}$ & $\begin{array}{l}5.1 \\
0.5\end{array}$ & $\begin{array}{l}4.5 \\
4.8\end{array}$ & $\begin{array}{r}10.1 \\
8.5\end{array}$ & $\begin{array}{l}20.3 \\
26.5\end{array}$ & $\begin{array}{l}3.4 \\
2.2\end{array}$ & $\begin{array}{l}45 \\
56\end{array}$ & $\begin{array}{r}112 \\
4\end{array}$ \\
\hline $\begin{array}{l}\text { LINE } \\
\text { A } \\
B\end{array}$ & $\begin{array}{r}10100 \\
1458.5 \\
1406.8\end{array}$ & $\begin{array}{l}\text { B? } \\
\text { S? }\end{array}$ & $\begin{array}{l}627007 \\
626976\end{array}$ & $\begin{array}{l}6138744 \\
6140104\end{array}$ & $\begin{array}{l}5.2 \\
0.2\end{array}$ & $\begin{array}{l}6.2 \\
2.2\end{array}$ & $\begin{array}{l}4.8 \\
0.2\end{array}$ & $\begin{array}{l}7.2 \\
4.6\end{array}$ & $\begin{array}{r}15.3 \\
4.3\end{array}$ & $\begin{array}{l}28.6 \\
27.3\end{array}$ & $\begin{array}{l}5.2 \\
---\end{array}$ & $\begin{array}{r}18 \\
---\end{array}$ & $\begin{array}{r}0 \\
79\end{array}$ \\
\hline $\begin{array}{l}\text { LINE } \\
\text { A } \\
\text { B } \\
\text { C } \\
\text { D } \\
\text { E } \\
\text { F } \\
\text { G } \\
\text { H }\end{array}$ & $\begin{array}{r}10110 \\
350.9 \\
357.9 \\
366.8 \\
374.5 \\
379.1 \\
382.7 \\
384.5 \\
386.1\end{array}$ & $\begin{array}{c}\mathrm{B} ? \\
\mathrm{~B} ? \\
\mathrm{M} \\
\mathrm{B} ? \\
\mathrm{M} \\
\mathrm{M} \\
\mathrm{B} \\
\mathrm{B}\end{array}$ & $\begin{array}{l}627394 \\
627386 \\
627387 \\
627387 \\
627374 \\
627364 \\
627361 \\
627359\end{array}$ & $\begin{array}{l}6137945 \\
6138016 \\
6138171 \\
6138375 \\
6138508 \\
6138619 \\
6138676 \\
6138729\end{array}$ & $\begin{array}{r}16.0 \\
4.6 \\
14.7 \\
14.4 \\
0.0 \\
5.1 \\
11.5 \\
9.9\end{array}$ & $\begin{array}{l}1.0 \\
1.2 \\
0.5 \\
7.4 \\
7.4 \\
5.0 \\
8.6 \\
8.5\end{array}$ & $\begin{array}{r}56.1 \\
6.5 \\
21.0 \\
17.2 \\
14.7 \\
3.1 \\
24.2 \\
24.5\end{array}$ & $\begin{array}{r}0.1 \\
1.5 \\
1.1 \\
9.6 \\
9.4 \\
9.1 \\
12.4 \\
12.4\end{array}$ & $\begin{array}{r}46.4 \\
4.9 \\
19.2 \\
28.4 \\
28.4 \\
2.5 \\
22.4 \\
22.4\end{array}$ & $\begin{array}{r}2.1 \\
11.6 \\
3.4 \\
38.7 \\
38.7 \\
21.8 \\
37.7 \\
37.7\end{array}$ & $\begin{array}{r}--- \\
--- \\
--- \\
27.0 \\
--- \\
--- \\
21.4 \\
19.3\end{array}$ & $\begin{array}{r}--- \\
--- \\
--- \\
8 \\
--- \\
--- \\
14 \\
15\end{array}$ & $\begin{array}{r}0 \\
1601 \\
119 \\
130 \\
329 \\
0 \\
130 \\
0\end{array}$ \\
\hline $\begin{array}{l}\text { LINE } \\
\text { A } \\
B \\
C\end{array}$ & $\begin{array}{c}10120 \\
656.9 \\
651.6 \\
648.1\end{array}$ & $\begin{array}{l}\text { S? } \\
B ? \\
M\end{array}$ & $\begin{array}{l}627841 \\
627852 \\
627852\end{array}$ & $\begin{array}{l}6138093 \\
6138262 \\
6138371\end{array}$ & $\begin{array}{r}6.8 \\
10.2 \\
0.0\end{array}$ & $\begin{array}{l}2.1 \\
5.8 \\
5.2\end{array}$ & $\begin{array}{r}11.8 \\
16.9 \\
1.9\end{array}$ & $\begin{array}{l}4.5 \\
8.6 \\
6.8\end{array}$ & $\begin{array}{l}3.1 \\
9.7 \\
3.1\end{array}$ & $\begin{array}{l}32.3 \\
55.0 \\
34.6\end{array}$ & $\begin{array}{r}43.5 \\
24.6 \\
---\end{array}$ & $\begin{array}{r}25 \\
26 \\
---\end{array}$ & $\begin{array}{l}118 \\
499 \\
550\end{array}$ \\
\hline
\end{tabular}

$\mathrm{CX}=$ COAXIAL

Note: EM values shown above are local amplitudes

Ketchikan - North/South of 5515
* Estimated Depth may be unreliable because the stronger part of the conductor may be deeper or to one side of the flight line, or because of a shallow dip or magnetite/overburden effects 
EM Anomaly List

\begin{tabular}{|c|c|c|c|c|c|c|c|c|c|c|c|c|c|}
\hline Label & Fid & Interp & $\begin{array}{c}\text { XUTM } \\
\text { m }\end{array}$ & $\begin{array}{c}\text { YUTM } \\
\mathrm{m}\end{array}$ & $\begin{array}{l}\text { CX } \\
\text { Real } \\
\text { ppm }\end{array}$ & $\begin{array}{l}00 \mathrm{HZ} \\
\text { Quad } \\
\text { ppm }\end{array}$ & $\begin{array}{l}\text { CP } \\
\text { Real } \\
\text { ppm }\end{array}$ & $\begin{array}{l}900 \mathrm{HZ} \\
\text { Quad } \\
\text { ppm }\end{array}$ & $\begin{array}{l}\mathrm{CP} \\
\mathrm{Real} \\
\mathrm{ppm}\end{array}$ & $\begin{array}{l}200 \mathrm{HZ} \\
\text { Quad } \\
\text { ppm }\end{array}$ & $\begin{array}{l}\text { Vertica } \\
\text { Cond } \\
\text { siemens }\end{array}$ & $\begin{array}{c}\text { al Dike } \\
\text { DEPTH* } \\
\text { m }\end{array}$ & $\begin{array}{c}\text { Mag. Corr } \\
\text { NT }\end{array}$ \\
\hline $\begin{array}{l}\text { LINE } \\
\text { D } \\
\text { E } \\
\text { F } \\
\text { G } \\
\text { H } \\
\text { I }\end{array}$ & $\begin{array}{c}10120 \\
646.4 \\
639.5 \\
634.1 \\
613.8 \\
603.4 \\
600.3\end{array}$ & $\begin{array}{c}\mathrm{M} \\
\mathrm{D} \\
\mathrm{B} \\
\mathrm{M} \\
\mathrm{B} ? \\
\mathrm{~B} ?\end{array}$ & $\begin{array}{l}627850 \\
627829 \\
627808 \\
627764 \\
627748 \\
627739\end{array}$ & $\begin{array}{l}6138420 \\
6138621 \\
6138802 \\
6139471 \\
6139824 \\
6139928\end{array}$ & $\begin{array}{r}0.0 \\
33.3 \\
1.7 \\
0.0 \\
0.1 \\
2.6\end{array}$ & $\begin{array}{l}3.8 \\
6.9 \\
3.3 \\
0.5 \\
6.8 \\
6.8\end{array}$ & $\begin{array}{r}18.3 \\
44.9 \\
18.4 \\
0.2 \\
7.4 \\
1.7\end{array}$ & $\begin{array}{r}6.8 \\
12.8 \\
3.7 \\
1.0 \\
5.8 \\
5.6\end{array}$ & $\begin{array}{r}19.6 \\
46.2 \\
6.8 \\
0.2 \\
10.7 \\
14.8\end{array}$ & $\begin{array}{r}31.4 \\
13.8 \\
15.1 \\
5.3 \\
35.9 \\
35.9\end{array}$ & $\begin{array}{r}--- \\
120.7 \\
25.1 \\
--- \\
2.4 \\
2.2\end{array}$ & $\begin{array}{r}--- \\
9 \\
30 \\
--- \\
25 \\
21\end{array}$ & $\begin{array}{r}0 \\
99 \\
94 \\
158 \\
99 \\
0\end{array}$ \\
\hline $\begin{array}{l}\text { LINE } \\
\text { A } \\
\text { B } \\
\text { C } \\
\text { D } \\
\text { E } \\
\text { F }\end{array}$ & $\begin{array}{r}10130 \\
726.3 \\
746.0 \\
771.3 \\
786.7 \\
821.3 \\
833.0\end{array}$ & $\begin{array}{c}\text { S } \\
M \\
D \\
D \\
S ? \\
M\end{array}$ & $\begin{array}{l}628221 \\
628208 \\
628196 \\
628179 \\
628176 \\
628166\end{array}$ & $\begin{array}{l}6137514 \\
6137883 \\
6138490 \\
6138980 \\
6139765 \\
6140142\end{array}$ & $\begin{array}{r}29.1 \\
0.1 \\
22.2 \\
0.8 \\
0.0 \\
6.2\end{array}$ & $\begin{array}{r}26.9 \\
1.3 \\
9.5 \\
2.8 \\
3.1 \\
2.3\end{array}$ & $\begin{array}{r}62.0 \\
0.3 \\
20.7 \\
1.1 \\
0.0 \\
4.4\end{array}$ & $\begin{array}{r}62.2 \\
1.1 \\
16.0 \\
1.4 \\
3.9 \\
3.0\end{array}$ & $\begin{array}{r}121.4 \\
0.0 \\
43.2 \\
1.3 \\
0.5 \\
13.8\end{array}$ & $\begin{array}{r}42.4 \\
5.5 \\
32.8 \\
7.3 \\
26.3 \\
19.6\end{array}$ & $\begin{array}{r}15.6 \\
--- \\
30.0 \\
1.7 \\
--- \\
---\end{array}$ & $\begin{array}{r}0 \\
--- \\
4 \\
26 \\
--- \\
---\end{array}$ & $\begin{array}{r}0 \\
0 \\
81 \\
0 \\
403 \\
5\end{array}$ \\
\hline $\begin{array}{l}\text { LINE } \\
\text { A } \\
B \\
\text { C } \\
\text { D } \\
\text { E }\end{array}$ & $\begin{array}{l}10140 \\
1036.0 \\
1014.8 \\
981.3 \\
954.2 \\
947.7\end{array}$ & $\begin{array}{r}\text { B } \\
\text { D } \\
\text { S? } \\
\text { S? } \\
\text { S? }\end{array}$ & $\begin{array}{l}628596 \\
628555 \\
628555 \\
628517 \\
628499\end{array}$ & $\begin{array}{l}6138681 \\
6139092 \\
6139849 \\
6140756 \\
6140957\end{array}$ & $\begin{array}{r}73.6 \\
0.7 \\
2.0 \\
0.0 \\
2.7\end{array}$ & $\begin{array}{r}24.0 \\
2.1 \\
2.1 \\
1.6 \\
2.3\end{array}$ & $\begin{array}{r}118.8 \\
1.1 \\
0.4 \\
0.3 \\
9.6\end{array}$ & $\begin{array}{r}57.8 \\
1.5 \\
2.9 \\
2.8 \\
3.0\end{array}$ & $\begin{array}{r}152.8 \\
3.6 \\
1.6 \\
2.4 \\
8.8\end{array}$ & $\begin{array}{r}27.0 \\
5.4 \\
17.4 \\
16.1 \\
19.4\end{array}$ & $\begin{array}{r}74.3 \\
1.8 \\
2.3 \\
--- \\
19.4\end{array}$ & $\begin{array}{r}0 \\
46 \\
66 \\
--- \\
37\end{array}$ & $\begin{array}{r}61 \\
53 \\
20 \\
1282 \\
0\end{array}$ \\
\hline $\begin{array}{l}\text { LINE } \\
\text { A } \\
B \\
\text { C } \\
D \\
\text { E }\end{array}$ & $\begin{array}{r}10150 \\
1202.6 \\
1307.2 \\
1312.9 \\
1319.7 \\
1325.3\end{array}$ & $\begin{array}{r}\text { D } \\
\text { S? } \\
\text { S? } \\
\text { S? } \\
\text { M }\end{array}$ & $\begin{array}{l}628976 \\
628951 \\
628931 \\
628915 \\
628901\end{array}$ & $\begin{array}{l}6138689 \\
6140640 \\
6140845 \\
6141093 \\
6141297\end{array}$ & $\begin{array}{r}14.5 \\
0.2 \\
4.0 \\
2.4 \\
5.3\end{array}$ & $\begin{array}{l}5.1 \\
1.6 \\
2.7 \\
2.3 \\
0.2\end{array}$ & $\begin{array}{l}6.1 \\
0.2 \\
5.6 \\
4.3 \\
7.2\end{array}$ & $\begin{array}{l}8.4 \\
2.8 \\
4.6 \\
2.2 \\
1.6\end{array}$ & $\begin{array}{r}15.1 \\
0.5 \\
4.3 \\
4.4 \\
4.4\end{array}$ & $\begin{array}{r}9.1 \\
19.6 \\
30.2 \\
17.4 \\
10.0\end{array}$ & $\begin{array}{r}25.0 \\
--- \\
11.1 \\
9.7 \\
---\end{array}$ & $\begin{array}{r}11 \\
--- \\
41 \\
45 \\
---\end{array}$ & $\begin{array}{r}18 \\
0 \\
0 \\
171 \\
15\end{array}$ \\
\hline $\begin{array}{l}\text { LINE } \\
\text { A } \\
\text { B } \\
\text { C } \\
\text { D }\end{array}$ & $\begin{array}{r}10160 \\
1521.8 \\
1502.1 \\
1380.9 \\
1375.9\end{array}$ & $\begin{array}{r}\text { S } \\
D \\
S ? \\
S ?\end{array}$ & $\begin{array}{l}629456 \\
629396 \\
629293 \\
629268\end{array}$ & $\begin{array}{l}6138194 \\
6138634 \\
6141382 \\
6141502\end{array}$ & $\begin{array}{r}0.0 \\
23.4 \\
4.1 \\
0.2\end{array}$ & $\begin{array}{r}3.8 \\
21.5 \\
2.6 \\
2.5\end{array}$ & $\begin{array}{l}0.0 \\
5.9 \\
1.7 \\
1.2\end{array}$ & $\begin{array}{r}5.2 \\
10.1 \\
2.6 \\
3.4\end{array}$ & $\begin{array}{r}8.8 \\
19.1 \\
1.8 \\
1.2\end{array}$ & $\begin{array}{l}30.3 \\
27.1 \\
16.2 \\
19.4\end{array}$ & $\begin{array}{r}0.5 \\
11.2 \\
9.4 \\
---\end{array}$ & $\begin{array}{r}0 \\
14 \\
36 \\
---\end{array}$ & $\begin{array}{r}0 \\
0 \\
13 \\
55\end{array}$ \\
\hline $\begin{array}{l}\text { LINE } \\
\text { A } \\
B \\
\text { C } \\
D \\
\text { E }\end{array}$ & $\begin{array}{l}10170 \\
1766.0 \\
1782.8 \\
1920.0 \\
1922.8 \\
1925.9\end{array}$ & $\begin{array}{r}\mathrm{S} ? \\
\mathrm{D} \\
\mathrm{M} \\
\mathrm{M} \\
\mathrm{M}\end{array}$ & $\begin{array}{l}629788 \\
629771 \\
629705 \\
629698 \\
629694\end{array}$ & $\begin{array}{l}6138079 \\
6138578 \\
6141098 \\
6141164 \\
6141239\end{array}$ & $\begin{array}{r}0.7 \\
11.6 \\
0.0 \\
2.6 \\
1.1\end{array}$ & $\begin{array}{l}2.9 \\
8.5 \\
0.0 \\
0.0 \\
0.6\end{array}$ & $\begin{array}{l}0.1 \\
1.6 \\
6.7 \\
5.2 \\
6.6\end{array}$ & $\begin{array}{l}3.0 \\
2.5 \\
1.4 \\
0.0 \\
0.4\end{array}$ & $\begin{array}{l}4.6 \\
5.0 \\
6.6 \\
3.9 \\
5.0\end{array}$ & $\begin{array}{r}18.2 \\
0.8 \\
8.1 \\
0.1 \\
2.6\end{array}$ & $\begin{array}{r}--- \\
12.5 \\
--- \\
--- \\
---\end{array}$ & $\begin{array}{r}--- \\
14 \\
--- \\
--- \\
---\end{array}$ & $\begin{array}{r}0 \\
0 \\
211 \\
114 \\
104\end{array}$ \\
\hline $\begin{array}{l}\text { LINE } \\
\text { A } \\
B \\
C\end{array}$ & $\begin{array}{r}10180 \\
2133.8 \\
2110.1 \\
2083.2\end{array}$ & $\begin{array}{l}\text { S } \\
\text { D } \\
\text { B }\end{array}$ & $\begin{array}{l}630237 \\
630208 \\
630198\end{array}$ & $\begin{array}{l}6138006 \\
6138631 \\
6138967\end{array}$ & $\begin{array}{l}2.5 \\
4.0 \\
2.1\end{array}$ & $\begin{array}{l}2.7 \\
4.2 \\
1.0\end{array}$ & $\begin{array}{l}2.0 \\
3.1 \\
0.4\end{array}$ & $\begin{array}{l}3.4 \\
1.5 \\
1.6\end{array}$ & $\begin{array}{l}4.8 \\
3.1 \\
3.4\end{array}$ & $\begin{array}{r}22.5 \\
9.2 \\
7.8\end{array}$ & $\begin{array}{l}4.1 \\
8.0 \\
7.1\end{array}$ & $\begin{array}{l}46 \\
27 \\
77\end{array}$ & $\begin{array}{r}37 \\
0 \\
37\end{array}$ \\
\hline
\end{tabular}

$\mathrm{CX}=$ COAXIAL

Note: EM values shown above

are local amplitudes

Ketchikan - North/South of 5515
* Estimated Depth may be unreliable because the stronger part of the conductor may be deeper or to one side of the flight line, or because of a shallow dip or magnetite/overburden effects 
EM Anomaly List

\begin{tabular}{|c|c|c|c|c|c|c|c|c|c|c|c|c|c|}
\hline Label & Fid & Interp & $\underset{\mathrm{m}}{\mathrm{XUTM}}$ & $\begin{array}{c}\text { YUTM } \\
\mathrm{m}\end{array}$ & $\begin{array}{l}\text { CX } \\
\text { Real } \\
\text { ppm }\end{array}$ & $\begin{array}{l}900 \mathrm{HZ} \\
\text { Quad } \\
\text { ppm }\end{array}$ & $\begin{array}{l}\text { CP } \\
\text { Real } \\
\text { ppm }\end{array}$ & $\begin{array}{l}900 \mathrm{HZ} \\
\text { Quad } \\
\text { ppm }\end{array}$ & $\begin{array}{l}\mathrm{CP} \\
\mathrm{Real} \\
\mathrm{ppm}\end{array}$ & $\begin{array}{l}200 \mathrm{HZ} \\
\text { Quad } \\
\text { ppm }\end{array}$ & $\begin{array}{l}\text { Vertica } \\
\text { ConD } \\
\text { siemens }\end{array}$ & $\begin{array}{c}\text { al Dike } \\
\text { DEPTH* } \\
\text { m }\end{array}$ & $\begin{array}{c}\text { Mag. Corr } \\
\text { NT }\end{array}$ \\
\hline $\begin{array}{l}\text { LINE } \\
\text { D } \\
\text { E } \\
\text { F } \\
\text { G } \\
H \\
\text { I } \\
\text { J }\end{array}$ & $\begin{array}{r}10180 \\
2076.5 \\
2043.9 \\
2014.2 \\
2010.0 \\
2002.7 \\
1988.0 \\
1980.0\end{array}$ & $\begin{array}{c}\mathrm{S} ? \\
\mathrm{M} \\
\mathrm{M} \\
\mathrm{M} \\
\mathrm{M} \\
\mathrm{M} \\
\mathrm{M}\end{array}$ & $\begin{array}{l}630186 \\
630171 \\
630139 \\
630136 \\
630122 \\
630124 \\
630103\end{array}$ & $\begin{array}{l}6139050 \\
6139716 \\
6140447 \\
6140541 \\
6140706 \\
6141078 \\
6141235\end{array}$ & $\begin{array}{l}3.0 \\
2.1 \\
1.5 \\
3.9 \\
0.4 \\
5.8 \\
0.0\end{array}$ & $\begin{array}{l}1.8 \\
0.5 \\
0.3 \\
0.6 \\
0.3 \\
0.4 \\
0.3\end{array}$ & $\begin{array}{r}0.4 \\
0.5 \\
0.2 \\
0.2 \\
1.2 \\
10.6 \\
0.2\end{array}$ & $\begin{array}{l}0.9 \\
0.7 \\
0.9 \\
0.1 \\
0.5 \\
0.6 \\
0.1\end{array}$ & $\begin{array}{l}0.4 \\
0.8 \\
1.4 \\
0.5 \\
1.1 \\
9.3 \\
0.1\end{array}$ & $\begin{array}{l}2.7 \\
3.3 \\
3.3 \\
0.3 \\
5.3 \\
0.9 \\
2.1\end{array}$ & $\begin{array}{l}--- \\
--- \\
--- \\
--- \\
--- \\
--- \\
---\end{array}$ & $\begin{array}{l}--- \\
--- \\
--- \\
--- \\
--- \\
--- \\
---\end{array}$ & $\begin{array}{r}107 \\
17 \\
0 \\
210 \\
0 \\
356 \\
0\end{array}$ \\
\hline $\begin{array}{l}\text { LINE } \\
\text { A } \\
\text { B } \\
\text { C } \\
\text { D } \\
\text { E } \\
\text { F } \\
\text { G } \\
\text { H } \\
\text { I } \\
\text { J }\end{array}$ & $\begin{array}{r}10190 \\
2266.8 \\
2273.9 \\
2287.9 \\
2364.3 \\
2370.0 \\
2378.8 \\
2390.8 \\
2406.0 \\
2412.6 \\
2422.6\end{array}$ & $\begin{array}{l}\mathrm{M} \\
\mathrm{M} \\
\mathrm{D} \\
\mathrm{M} \\
\mathrm{M} \\
\mathrm{M} \\
\mathrm{M} \\
\mathrm{M} \\
\mathrm{M} \\
\mathrm{M}\end{array}$ & $\begin{array}{l}630616 \\
630630 \\
630611 \\
630547 \\
630530 \\
630521 \\
630532 \\
630532 \\
630523 \\
630511\end{array}$ & $\begin{array}{l}6137840 \\
6138117 \\
6138586 \\
6139855 \\
6140044 \\
6140356 \\
6140725 \\
6141005 \\
6141090 \\
6141240\end{array}$ & $\begin{array}{r}0.2 \\
1.3 \\
11.2 \\
3.0 \\
0.8 \\
0.0 \\
4.2 \\
3.5 \\
3.0 \\
13.3\end{array}$ & $\begin{array}{r}2.0 \\
0.9 \\
15.4 \\
1.3 \\
0.4 \\
0.9 \\
1.0 \\
0.2 \\
0.8 \\
0.0\end{array}$ & $\begin{array}{r}1.5 \\
2.6 \\
2.0 \\
3.9 \\
4.1 \\
3.5 \\
5.0 \\
8.5 \\
22.5 \\
9.7\end{array}$ & $\begin{array}{l}2.9 \\
0.6 \\
7.2 \\
1.0 \\
0.7 \\
0.6 \\
0.8 \\
0.2 \\
0.8 \\
0.5\end{array}$ & $\begin{array}{r}1.5 \\
2.0 \\
16.0 \\
4.1 \\
1.0 \\
3.4 \\
4.3 \\
0.8 \\
18.5 \\
5.3\end{array}$ & $\begin{array}{r}16.0 \\
6.1 \\
25.1 \\
6.6 \\
4.7 \\
5.0 \\
5.0 \\
0.2 \\
4.0 \\
2.8\end{array}$ & $\begin{array}{l}--- \\
--- \\
4.6 \\
--- \\
--- \\
--- \\
--- \\
--- \\
--- \\
---\end{array}$ & $\begin{array}{r}--- \\
--- \\
27 \\
--- \\
--- \\
--- \\
--- \\
--- \\
--- \\
---\end{array}$ & $\begin{array}{r}119 \\
29 \\
29 \\
0 \\
123 \\
56 \\
29 \\
0 \\
194 \\
106\end{array}$ \\
\hline $\begin{array}{l}\text { LINE } \\
\text { A } \\
\text { B } \\
\text { C } \\
\text { D } \\
\text { E } \\
\text { F } \\
\text { G }\end{array}$ & $\begin{array}{l}10200 \\
2601.8 \\
2579.4 \\
2567.7 \\
2519.4 \\
2506.8 \\
2472.1 \\
2467.5\end{array}$ & $\begin{array}{r}\text { S? } \\
\text { S? } \\
\text { S? } \\
\text { M } \\
\text { M } \\
\text { S? } \\
\text { S? }\end{array}$ & $\begin{array}{l}631035 \\
631011 \\
630996 \\
630990 \\
630943 \\
630931 \\
630919\end{array}$ & $\begin{array}{l}6137896 \\
6138494 \\
6138749 \\
6139887 \\
6140285 \\
6141205 \\
6141322\end{array}$ & $\begin{array}{l}1.0 \\
8.6 \\
5.8 \\
1.9 \\
1.0 \\
2.6 \\
4.3\end{array}$ & $\begin{array}{l}2.3 \\
2.4 \\
4.1 \\
0.5 \\
0.8 \\
2.4 \\
2.0\end{array}$ & $\begin{array}{r}4.5 \\
16.3 \\
11.5 \\
0.1 \\
0.2 \\
5.8 \\
9.4\end{array}$ & $\begin{array}{l}2.3 \\
2.1 \\
5.4 \\
0.5 \\
1.0 \\
2.8 \\
3.4\end{array}$ & $\begin{array}{r}3.2 \\
10.3 \\
8.3 \\
0.1 \\
0.3 \\
2.6 \\
5.6\end{array}$ & $\begin{array}{r}17.5 \\
14.8 \\
32.6 \\
2.2 \\
5.9 \\
19.5 \\
25.1\end{array}$ & $\begin{array}{r}--- \\
--- \\
18.7 \\
--- \\
--- \\
11.5 \\
---\end{array}$ & $\begin{array}{r}--- \\
--- \\
28 \\
--- \\
--- \\
50 \\
---\end{array}$ & $\begin{array}{r}51 \\
0 \\
0 \\
39 \\
87 \\
0 \\
0\end{array}$ \\
\hline $\begin{array}{l}\text { LINE } \\
\text { A } \\
\text { B } \\
\text { C } \\
\text { D } \\
\text { E } \\
\text { F } \\
\text { G }\end{array}$ & $\begin{array}{r}10210 \\
2746.6 \\
2765.4 \\
2787.5 \\
2807.7 \\
2809.9 \\
2825.7 \\
2840.1\end{array}$ & $\begin{array}{r}\text { S } \\
\text { S? } \\
\text { S? } \\
\text { S } \\
\text { M } \\
\text { S? } \\
\text { S? }\end{array}$ & $\begin{array}{l}631425 \\
631431 \\
631388 \\
631352 \\
631352 \\
631344 \\
631318\end{array}$ & $\begin{array}{l}6137721 \\
6138436 \\
6139193 \\
6139911 \\
6139991 \\
6140498 \\
6140887\end{array}$ & $\begin{array}{l}0.9 \\
0.5 \\
0.2 \\
0.0 \\
1.1 \\
0.0 \\
1.2\end{array}$ & $\begin{array}{l}3.3 \\
3.4 \\
3.7 \\
3.0 \\
0.9 \\
1.9 \\
1.9\end{array}$ & $\begin{array}{l}0.0 \\
0.3 \\
1.0 \\
7.9 \\
0.4 \\
1.2 \\
1.2\end{array}$ & $\begin{array}{l}5.0 \\
5.6 \\
2.9 \\
2.2 \\
2.2 \\
2.9 \\
2.4\end{array}$ & $\begin{array}{l}4.8 \\
1.5 \\
0.6 \\
6.3 \\
0.3 \\
0.7 \\
0.6\end{array}$ & $\begin{array}{l}32.3 \\
35.9 \\
20.5 \\
14.8 \\
15.4 \\
18.9 \\
14.8\end{array}$ & $\begin{array}{l}1.3 \\
--- \\
0.9 \\
5.7 \\
--- \\
--- \\
2.0\end{array}$ & $\begin{array}{r}14 \\
--- \\
15 \\
54 \\
--- \\
--- \\
74\end{array}$ & $\begin{array}{r}200 \\
396 \\
37 \\
64 \\
0 \\
37 \\
92\end{array}$ \\
\hline $\begin{array}{l}\text { LINE } \\
\text { A } \\
\text { B } \\
\text { C } \\
\text { D }\end{array}$ & $\begin{array}{r}10220 \\
3137.3 \\
3063.3 \\
3059.3 \\
3023.0\end{array}$ & $\begin{array}{r}\text { B? } \\
\text { S } \\
B ? \\
\text { S? }\end{array}$ & $\begin{array}{l}631890 \\
631829 \\
631840 \\
631789\end{array}$ & $\begin{array}{l}6136349 \\
6138950 \\
6139081 \\
6140140\end{array}$ & $\begin{array}{r}51.2 \\
1.8 \\
5.2 \\
0.7\end{array}$ & $\begin{array}{r}24.9 \\
3.5 \\
8.7 \\
1.0\end{array}$ & $\begin{array}{r}49.6 \\
4.6 \\
5.1 \\
0.8\end{array}$ & $\begin{array}{r}69.2 \\
5.8 \\
11.6 \\
1.6\end{array}$ & $\begin{array}{r}144.4 \\
29.5 \\
34.9 \\
0.5\end{array}$ & $\begin{array}{l}48.2 \\
41.4 \\
34.3 \\
10.7\end{array}$ & $\begin{array}{r}22.9 \\
--- \\
3.0 \\
---\end{array}$ & $\begin{array}{r}0 \\
--- \\
24 \\
---\end{array}$ & $\begin{array}{r}25 \\
21 \\
0 \\
5\end{array}$ \\
\hline $\begin{array}{l}\text { LINE } \\
\mathrm{A}\end{array}$ & $\begin{array}{r}10230 \\
3297.5\end{array}$ & M & 632249 & 6137435 & 0.1 & 1.2 & 2.9 & 1.0 & 5.1 & 5.5 & --- & --- & 0 \\
\hline
\end{tabular}

$\mathrm{CX}=$ COAXIAL

Note: EM values shown above

are local amplitudes

are 10
of 5515
*Estimated Depth may be unreliable because the stronger part of the conductor may be deeper or to one side of the flight line, or because of a shallow dip or magnetite/overburden effects 
EM Anomaly List

\begin{tabular}{|c|c|c|c|c|c|c|c|c|c|c|c|c|c|}
\hline Label & Fid & Interp & $\begin{array}{c}\text { XUTM } \\
\text { m }\end{array}$ & $\begin{array}{c}\text { YUTM } \\
\mathrm{m}\end{array}$ & $\begin{array}{l}\text { CX } \\
\text { Real } \\
\text { ppm }\end{array}$ & $\begin{array}{l}900 \mathrm{HZ} \\
\text { Quad } \\
\text { ppm }\end{array}$ & $\begin{array}{l}\text { CP } \\
\text { Real } \\
\text { ppm }\end{array}$ & $\begin{array}{c}900 \mathrm{HZ} \\
\text { Quad } \\
\text { ppm }\end{array}$ & $\begin{array}{l}\text { CP } 7 \\
\text { Real } \\
\text { ppm }\end{array}$ & $\begin{array}{l}200 \mathrm{HZ} \\
\text { Quad } \\
\text { ppm }\end{array}$ & $\begin{array}{l}\text { Vertica } \\
\text { CoND } \\
\text { siemens }\end{array}$ & $\begin{array}{c}\text { al Dike } \\
\text { DEPTH* } \\
\text { m }\end{array}$ & $\begin{array}{c}\text { Mag. Corr } \\
\text { NT }\end{array}$ \\
\hline $\begin{array}{l}\text { LINE } \\
\text { B } \\
\text { C } \\
\text { D }\end{array}$ & $\begin{array}{r}10230 \\
3312.7 \\
3342.5 \\
3364.8\end{array}$ & $\begin{array}{c}\mathrm{M} \\
\mathrm{B} ? \\
\mathrm{M}\end{array}$ & $\begin{array}{l}632241 \\
632194 \\
632176\end{array}$ & $\begin{array}{l}6137872 \\
6138886 \\
6139365\end{array}$ & $\begin{array}{l}0.5 \\
5.3 \\
0.2\end{array}$ & $\begin{array}{l}0.3 \\
4.8 \\
0.7\end{array}$ & $\begin{array}{l}0.3 \\
0.5 \\
2.6\end{array}$ & $\begin{array}{l}0.6 \\
4.1 \\
0.7\end{array}$ & $\begin{array}{r}0.3 \\
13.2 \\
2.2\end{array}$ & $\begin{array}{r}2.8 \\
21.3 \\
3.8\end{array}$ & $\begin{array}{l}--- \\
4.8 \\
---\end{array}$ & $\begin{array}{r}--- \\
34 \\
---\end{array}$ & $\begin{array}{r}278 \\
31 \\
0\end{array}$ \\
\hline $\begin{array}{l}\text { LINE } \\
\text { A } \\
\text { B } \\
\text { C } \\
\text { D } \\
\text { E } \\
\text { F } \\
\text { G }\end{array}$ & $\begin{array}{l}10240 \\
3681.0 \\
3650.0 \\
3569.1 \\
3558.1 \\
3543.6 \\
3535.3 \\
3503.3\end{array}$ & $\begin{array}{r}\text { M } \\
\text { S } \\
\text { S? } \\
\text { S? } \\
\text { S? } \\
\text { S } \\
\text { M }\end{array}$ & $\begin{array}{l}632656 \\
632639 \\
632593 \\
632590 \\
632538 \\
632512 \\
632437\end{array}$ & $\begin{array}{l}6137368 \\
6138200 \\
6139981 \\
6140294 \\
6140710 \\
6140945 \\
6141605\end{array}$ & $\begin{array}{r}0.3 \\
1.1 \\
12.6 \\
0.2 \\
9.5 \\
7.1 \\
0.2\end{array}$ & $\begin{array}{l}0.9 \\
1.2 \\
1.2 \\
0.6 \\
1.3 \\
1.0 \\
0.8\end{array}$ & $\begin{array}{r}0.6 \\
1.5 \\
22.9 \\
5.2 \\
24.9 \\
12.4 \\
8.1\end{array}$ & $\begin{array}{l}1.5 \\
1.9 \\
2.3 \\
0.7 \\
2.0 \\
1.9 \\
0.1\end{array}$ & $\begin{array}{r}0.7 \\
1.2 \\
0.1 \\
4.6 \\
0.7 \\
11.2 \\
6.7\end{array}$ & $\begin{array}{r}4.8 \\
12.5 \\
14.1 \\
6.6 \\
14.9 \\
15.4 \\
0.6\end{array}$ & $\begin{array}{r}--- \\
3.8 \\
265.9 \\
28.9 \\
--- \\
122.1 \\
---\end{array}$ & $\begin{array}{r}--- \\
69 \\
34 \\
73 \\
--- \\
43 \\
---\end{array}$ & $\begin{array}{r}227 \\
284 \\
0 \\
50 \\
52 \\
49 \\
87\end{array}$ \\
\hline $\begin{array}{l}\text { LINE } \\
\text { A } \\
\text { B } \\
\text { C } \\
\text { D } \\
\text { E } \\
\text { F } \\
\text { G } \\
\text { H } \\
\text { I } \\
\text { J } \\
\text { K } \\
\text { L }\end{array}$ & $\begin{array}{r}10250 \\
3852.4 \\
3854.8 \\
3929.2 \\
3936.3 \\
3942.0 \\
3946.8 \\
3953.9 \\
3964.2 \\
4018.8 \\
4026.4 \\
4039.9 \\
4058.7\end{array}$ & $\begin{array}{c}\text { B } \\
B \\
M \\
M \\
M \\
M \\
M \\
M \\
S ? \\
M \\
S ? \\
S ?\end{array}$ & $\begin{array}{l}633158 \\
633158 \\
633064 \\
633059 \\
633063 \\
633059 \\
633065 \\
633062 \\
633000 \\
632986 \\
632970 \\
632971\end{array}$ & $\begin{array}{l}6134559 \\
6134644 \\
6137115 \\
6137311 \\
6137481 \\
6137594 \\
6137817 \\
6138153 \\
6139551 \\
6139771 \\
6140175 \\
6140785\end{array}$ & $\begin{array}{r}3.1 \\
3.1 \\
0.2 \\
8.6 \\
2.5 \\
3.5 \\
1.3 \\
15.7 \\
1.4 \\
0.1 \\
1.0 \\
0.5\end{array}$ & $\begin{array}{l}2.0 \\
2.4 \\
0.4 \\
0.7 \\
0.5 \\
0.1 \\
1.5 \\
0.6 \\
1.0 \\
0.5 \\
0.3 \\
2.0\end{array}$ & $\begin{array}{r}2.3 \\
1.6 \\
1.9 \\
13.1 \\
11.1 \\
2.1 \\
6.3 \\
8.9 \\
1.7 \\
3.0 \\
8.5 \\
1.3\end{array}$ & $\begin{array}{l}2.5 \\
3.7 \\
0.5 \\
0.9 \\
0.8 \\
0.5 \\
1.6 \\
0.4 \\
1.1 \\
0.4 \\
0.6 \\
2.3\end{array}$ & $\begin{array}{r}5.8 \\
8.3 \\
1.6 \\
11.1 \\
8.9 \\
1.9 \\
6.9 \\
6.3 \\
3.8 \\
2.4 \\
7.2 \\
2.4\end{array}$ & $\begin{array}{r}14.6 \\
21.3 \\
5.0 \\
5.6 \\
3.8 \\
0.7 \\
12.2 \\
2.9 \\
7.1 \\
3.3 \\
5.4 \\
13.1\end{array}$ & $\begin{array}{r}--- \\
5.3 \\
--- \\
--- \\
--- \\
--- \\
--- \\
--- \\
8.8 \\
--- \\
116.0 \\
---\end{array}$ & $\begin{array}{r}--- \\
40 \\
--- \\
--- \\
--- \\
--- \\
--- \\
--- \\
67 \\
--- \\
75 \\
---\end{array}$ & $\begin{array}{r}45 \\
0 \\
517 \\
33 \\
0 \\
414 \\
338 \\
16 \\
45 \\
0 \\
50 \\
0\end{array}$ \\
\hline $\begin{array}{l}\text { LINE } \\
\text { A } \\
\text { B } \\
\text { C } \\
\text { D } \\
\text { E } \\
\text { F } \\
\text { G } \\
\text { H } \\
\text { I } \\
\text { J } \\
\text { K } \\
\text { L } \\
\text { M } \\
\text { N } \\
\text { O } \\
\text { P }\end{array}$ & $\begin{array}{r}10260 \\
4387.0 \\
4382.1 \\
4376.5 \\
4354.6 \\
4343.3 \\
4287.5 \\
4267.3 \\
4248.7 \\
4235.3 \\
4229.9 \\
4203.6 \\
4191.2 \\
4178.1 \\
4157.3 \\
4138.0 \\
4127.8\end{array}$ & $\begin{array}{r}\text { S } \\
\text { M } \\
\text { S } \\
S \\
S \\
\mathrm{E} \\
\mathrm{M} \\
\mathrm{S} \\
\mathrm{S} \\
\mathrm{S} \\
\mathrm{S} \\
\mathrm{M} \\
\mathrm{S} \\
\mathrm{S} ? \\
\mathrm{M} \\
\mathrm{S}\end{array}$ & $\begin{array}{l}633620 \\
633614 \\
633608 \\
633590 \\
633594 \\
633536 \\
633489 \\
633458 \\
633443 \\
633441 \\
633382 \\
633360 \\
633350 \\
633300 \\
633309 \\
633301\end{array}$ & $\begin{array}{l}6133182 \\
6133364 \\
6133574 \\
6134372 \\
6134711 \\
6136533 \\
6137254 \\
6137857 \\
6138303 \\
6138487 \\
6139453 \\
6139877 \\
6140302 \\
6140896 \\
6141267 \\
6141445\end{array}$ & $\begin{array}{r}3.2 \\
0.2 \\
0.9 \\
0.0 \\
1.8 \\
14.3 \\
0.5 \\
0.3 \\
3.2 \\
1.2 \\
0.3 \\
1.3 \\
0.1 \\
4.4 \\
0.0 \\
3.2\end{array}$ & $\begin{array}{l}1.8 \\
0.4 \\
3.5 \\
0.7 \\
0.7 \\
9.9 \\
0.6 \\
1.4 \\
2.2 \\
1.8 \\
1.1 \\
0.7 \\
1.1 \\
2.0 \\
0.6 \\
0.5\end{array}$ & $\begin{array}{r}5.7 \\
0.5 \\
2.3 \\
2.9 \\
1.3 \\
4.4 \\
7.8 \\
0.3 \\
5.6 \\
3.3 \\
1.9 \\
0.7 \\
0.6 \\
12.0 \\
1.0 \\
1.8\end{array}$ & $\begin{array}{l}3.0 \\
0.3 \\
5.2 \\
1.1 \\
1.7 \\
5.5 \\
1.1 \\
1.9 \\
2.4 \\
1.9 \\
1.7 \\
0.3 \\
1.4 \\
3.1 \\
0.3 \\
0.5\end{array}$ & $\begin{array}{r}5.6 \\
0.3 \\
5.5 \\
2.5 \\
1.0 \\
14.4 \\
6.0 \\
0.5 \\
4.4 \\
5.0 \\
1.7 \\
0.9 \\
0.4 \\
9.3 \\
1.3 \\
1.2\end{array}$ & $\begin{array}{r}22.5 \\
0.0 \\
39.8 \\
6.7 \\
11.5 \\
9.4 \\
4.5 \\
11.2 \\
19.4 \\
13.5 \\
10.4 \\
4.3 \\
9.2 \\
18.4 \\
2.1 \\
5.8\end{array}$ & $\begin{array}{r}--- \\
--- \\
--- \\
5.1 \\
9.3 \\
14.1 \\
--- \\
--- \\
15.8 \\
6.0 \\
--- \\
--- \\
--- \\
--- \\
--- \\
59.6\end{array}$ & $\begin{array}{r}--- \\
--- \\
--- \\
80 \\
70 \\
0 \\
--- \\
--- \\
45 \\
63 \\
--- \\
--- \\
--- \\
--- \\
--- \\
86\end{array}$ & $\begin{array}{r}0 \\
149 \\
39 \\
39 \\
39 \\
0 \\
493 \\
0 \\
0 \\
39 \\
0 \\
146 \\
64 \\
114 \\
370 \\
94\end{array}$ \\
\hline
\end{tabular}

$\mathrm{CX}=$ COAXIAL

Note: EM values shown above

Ketchikan - North/South of 5515
* Estimated Depth may be unreliable because the stronger part of the conductor may be deeper or to one side of the flight line, or because of a shallow dip or magnetite/overburden effects 
EM Anomaly List

\begin{tabular}{|c|c|c|c|c|c|c|c|c|c|c|c|c|c|}
\hline Label & Fid & Interp & $\begin{array}{l}\text { XUTM } \\
\text { m }\end{array}$ & $\begin{array}{c}\text { YUTM } \\
\mathrm{m}\end{array}$ & $\begin{array}{l}\text { CX } \\
\text { Real } \\
\text { ppm }\end{array}$ & $\begin{array}{l}900 \mathrm{HZ} \\
\text { Quad } \\
\text { ppm }\end{array}$ & $\begin{array}{l}\text { CP } \\
\text { Real } \\
\text { ppm }\end{array}$ & $\begin{array}{c}900 \mathrm{HZ} \\
\text { Quad } \\
\text { ppm }\end{array}$ & $\begin{array}{l}\text { CP } 7 \\
\text { Real } \\
\text { ppm }\end{array}$ & $\begin{array}{l}200 \mathrm{HZ} \\
\text { Quad } \\
\text { ppm }\end{array}$ & $\begin{array}{l}\text { Vertica } \\
\text { CoND } \\
\text { siemens }\end{array}$ & $\begin{array}{c}\text { al Dike } \\
\text { DEPTH* } \\
\text { m }\end{array}$ & $\begin{array}{c}\text { Mag. Corr } \\
\text { NT }\end{array}$ \\
\hline $\begin{array}{l}\text { LINE } \\
\text { A } \\
\text { B } \\
\text { C } \\
\text { D } \\
\text { E } \\
\text { F }\end{array}$ & $\begin{array}{r}10270 \\
4477.2 \\
4515.0 \\
4525.0 \\
4545.7 \\
4707.7 \\
4748.8\end{array}$ & $\begin{array}{r}\mathrm{B} \\
\mathrm{M} \\
\mathrm{M} \\
\mathrm{M} \\
\mathrm{M} \\
\mathrm{S} ?\end{array}$ & $\begin{array}{l}634061 \\
634002 \\
633992 \\
634001 \\
633757 \\
633718\end{array}$ & $\begin{array}{l}6132066 \\
6133080 \\
6133403 \\
6134152 \\
6139607 \\
6141090\end{array}$ & $\begin{array}{l}2.9 \\
0.0 \\
0.0 \\
0.1 \\
0.1 \\
0.0\end{array}$ & $\begin{array}{l}4.4 \\
1.2 \\
0.8 \\
0.9 \\
1.2 \\
1.1\end{array}$ & $\begin{array}{l}4.8 \\
1.8 \\
3.9 \\
2.0 \\
1.4 \\
2.2\end{array}$ & $\begin{array}{l}5.2 \\
1.5 \\
0.3 \\
0.7 \\
2.2 \\
1.0\end{array}$ & $\begin{array}{r}16.9 \\
3.7 \\
3.1 \\
2.3 \\
1.2 \\
3.7\end{array}$ & $\begin{array}{r}20.1 \\
8.6 \\
1.8 \\
0.0 \\
12.7 \\
8.7\end{array}$ & $\begin{array}{l}4.4 \\
--- \\
--- \\
--- \\
--- \\
2.4\end{array}$ & $\begin{array}{r}46 \\
--- \\
--- \\
--- \\
--- \\
92\end{array}$ & $\begin{array}{r}31 \\
23 \\
441 \\
470 \\
184 \\
170\end{array}$ \\
\hline $\begin{array}{l}\text { LINE } \\
\text { A } \\
\text { B } \\
\text { C } \\
\text { D } \\
\text { E } \\
\text { F } \\
\text { G } \\
\text { H } \\
\text { I } \\
\text { J } \\
\text { K } \\
\text { L } \\
\text { M } \\
\text { N } \\
\text { O } \\
\text { P } \\
\text { Q } \\
\text { R } \\
\text { S } \\
\text { T }\end{array}$ & $\begin{array}{r}10280 \\
5133.9 \\
5112.6 \\
5083.4 \\
5075.6 \\
5066.3 \\
5034.1 \\
5009.5 \\
5004.0 \\
5000.7 \\
4994.3 \\
4980.5 \\
4971.3 \\
4932.1 \\
4923.3 \\
4908.0 \\
4872.7 \\
4859.7 \\
4851.8 \\
4840.8 \\
4829.9\end{array}$ & $\begin{array}{r}\mathrm{D} \\
\mathrm{S} \\
\mathrm{M} \\
\mathrm{S} \\
\mathrm{S} \\
\mathrm{S} \\
\mathrm{B} \\
\mathrm{B} ? \\
\mathrm{~B} \\
\mathrm{~B} \\
\mathrm{~S} ? \\
\mathrm{~S} ? \\
\mathrm{M} \\
\mathrm{S} ? \\
\mathrm{~S} ? \\
\mathrm{M} \\
\mathrm{M} \\
\mathrm{S} \\
\mathrm{S} ? \\
\mathrm{M}\end{array}$ & $\begin{array}{l}634517 \\
634467 \\
634425 \\
634402 \\
634368 \\
634410 \\
634282 \\
634290 \\
634305 \\
634327 \\
634318 \\
634314 \\
634241 \\
634247 \\
634238 \\
634198 \\
634129 \\
634107 \\
634121 \\
634166\end{array}$ & $\begin{array}{l}6131099 \\
6132023 \\
6133242 \\
6133569 \\
6133951 \\
6135066 \\
6135898 \\
6136086 \\
6136202 \\
6136419 \\
6136849 \\
6137102 \\
6138235 \\
6138521 \\
6138917 \\
6139620 \\
6140008 \\
6140273 \\
6140616 \\
6140940\end{array}$ & $\begin{array}{r}16.2 \\
1.1 \\
1.0 \\
0.2 \\
2.5 \\
2.1 \\
6.8 \\
16.2 \\
16.2 \\
7.5 \\
0.2 \\
0.1 \\
1.3 \\
6.3 \\
1.0 \\
4.1 \\
1.0 \\
3.0 \\
5.8 \\
0.7\end{array}$ & $\begin{array}{r}12.5 \\
1.9 \\
0.9 \\
0.9 \\
2.5 \\
0.9 \\
3.9 \\
15.0 \\
15.0 \\
7.3 \\
2.7 \\
1.5 \\
0.2 \\
3.6 \\
2.8 \\
0.4 \\
0.7 \\
1.9 \\
0.7 \\
0.6\end{array}$ & $\begin{array}{r}9.4 \\
0.4 \\
2.1 \\
1.5 \\
1.0 \\
1.3 \\
6.4 \\
30.0 \\
28.0 \\
5.6 \\
2.8 \\
0.3 \\
11.4 \\
4.6 \\
0.4 \\
0.3 \\
0.7 \\
10.4 \\
3.2 \\
4.3\end{array}$ & $\begin{array}{r}14.1 \\
3.4 \\
1.1 \\
2.5 \\
3.1 \\
1.7 \\
11.3 \\
33.7 \\
34.3 \\
11.4 \\
2.4 \\
1.8 \\
0.5 \\
4.9 \\
3.7 \\
0.1 \\
1.2 \\
3.5 \\
0.8 \\
0.5\end{array}$ & $\begin{array}{r}33.1 \\
5.6 \\
2.4 \\
4.5 \\
3.9 \\
1.6 \\
25.7 \\
60.9 \\
62.0 \\
28.3 \\
7.4 \\
0.3 \\
11.8 \\
8.2 \\
0.8 \\
0.4 \\
0.8 \\
11.1 \\
1.5 \\
3.7\end{array}$ & $\begin{array}{r}11.7 \\
21.6 \\
6.1 \\
17.1 \\
21.1 \\
7.2 \\
7.9 \\
11.5 \\
11.6 \\
6.7 \\
13.9 \\
11.0 \\
3.8 \\
34.9 \\
22.6 \\
2.3 \\
5.6 \\
20.1 \\
6.8 \\
3.0\end{array}$ & $\begin{array}{r}11.5 \\
--- \\
--- \\
--- \\
3.5 \\
--- \\
--- \\
11.6 \\
10.8 \\
--- \\
1.9 \\
--- \\
--- \\
13.2 \\
1.2 \\
--- \\
--- \\
--- \\
--- \\
---\end{array}$ & $\begin{array}{r}0 \\
--- \\
--- \\
--- \\
47 \\
--- \\
--- \\
0 \\
4 \\
--- \\
28 \\
--- \\
--- \\
33 \\
25 \\
--- \\
--- \\
--- \\
--- \\
---\end{array}$ & $\begin{array}{r}0 \\
46 \\
0 \\
0 \\
0 \\
46 \\
0 \\
0 \\
0 \\
0 \\
0 \\
497 \\
0 \\
0 \\
0 \\
172 \\
53 \\
46 \\
0 \\
301\end{array}$ \\
\hline $\begin{array}{l}\text { LINE } \\
\text { A } \\
\text { B } \\
\text { C } \\
\text { D } \\
\text { E } \\
\text { F } \\
\text { G } \\
\text { H } \\
\text { I } \\
\text { J } \\
\text { K } \\
\text { L }\end{array}$ & $\begin{array}{r}10290 \\
5211.6 \\
5222.6 \\
5229.9 \\
5291.6 \\
5293.4 \\
5352.8 \\
5405.4 \\
5415.8 \\
5455.7 \\
5472.6 \\
5492.1 \\
5512.5\end{array}$ & $\begin{array}{c}\mathrm{D} \\
\mathrm{B} \\
\mathrm{D} \\
\mathrm{M} \\
\mathrm{S} \\
\mathrm{D} \\
\mathrm{M} \\
\mathrm{M} \\
\mathrm{M} \\
\mathrm{M} \\
\mathrm{M} \\
\mathrm{B} ?\end{array}$ & $\begin{array}{l}634904 \\
634912 \\
634895 \\
634805 \\
634803 \\
634723 \\
634628 \\
634615 \\
634589 \\
634577 \\
634533 \\
634516\end{array}$ & $\begin{array}{l}6130390 \\
6130788 \\
6131071 \\
6133300 \\
6133369 \\
6135862 \\
6137943 \\
6138312 \\
6139641 \\
6140256 \\
6140837 \\
6141495\end{array}$ & $\begin{array}{r}18.4 \\
1.9 \\
4.1 \\
0.0 \\
4.5 \\
8.6 \\
1.4 \\
0.1 \\
0.8 \\
0.4 \\
0.3 \\
3.5\end{array}$ & $\begin{array}{r}24.4 \\
4.4 \\
7.7 \\
1.2 \\
1.6 \\
9.1 \\
0.7 \\
1.4 \\
0.4 \\
0.9 \\
0.3 \\
3.8\end{array}$ & $\begin{array}{r}26.6 \\
6.7 \\
0.0 \\
2.8 \\
15.2 \\
3.9 \\
0.2 \\
3.6 \\
0.2 \\
1.4 \\
3.7 \\
0.7\end{array}$ & $\begin{array}{r}37.9 \\
8.4 \\
7.0 \\
1.3 \\
1.9 \\
10.2 \\
1.1 \\
1.4 \\
0.4 \\
1.2 \\
0.4 \\
1.4\end{array}$ & $\begin{array}{r}86.8 \\
22.6 \\
23.4 \\
0.4 \\
13.4 \\
32.8 \\
0.0 \\
1.5 \\
0.3 \\
1.5 \\
0.1 \\
2.3\end{array}$ & $\begin{array}{r}52.2 \\
36.9 \\
43.3 \\
11.0 \\
11.1 \\
25.1 \\
3.8 \\
7.3 \\
2.2 \\
5.8 \\
1.9 \\
7.8\end{array}$ & $\begin{array}{l}7.6 \\
3.2 \\
2.4 \\
--- \\
--- \\
5.4 \\
--- \\
--- \\
--- \\
--- \\
--- \\
4.7\end{array}$ & $\begin{array}{r}0 \\
27 \\
6 \\
--- \\
--- \\
0 \\
---- \\
--- \\
--- \\
--- \\
--- \\
47\end{array}$ & $\begin{array}{r}30 \\
10 \\
0 \\
226 \\
30 \\
30 \\
0 \\
241 \\
30 \\
105 \\
109 \\
0\end{array}$ \\
\hline $\begin{array}{l}\text { LINE } \\
\mathrm{A}\end{array}$ & $\begin{array}{l}10300 \\
5883.1\end{array}$ & $\mathrm{H}$ & 635375 & 6129126 & 2.1 & 2.2 & 1.6 & 2.5 & 3.9 & 16.7 & 4.2 & 46 & 26 \\
\hline
\end{tabular}

$\mathrm{CX}=$ COAXIAL

Note: EM values shown above

Ketchikan - North/South of 5515
* Estimated Depth may be unreliable because the stronger part of the conductor may be deeper or the side of the flight line, or because of 
EM Anomaly List

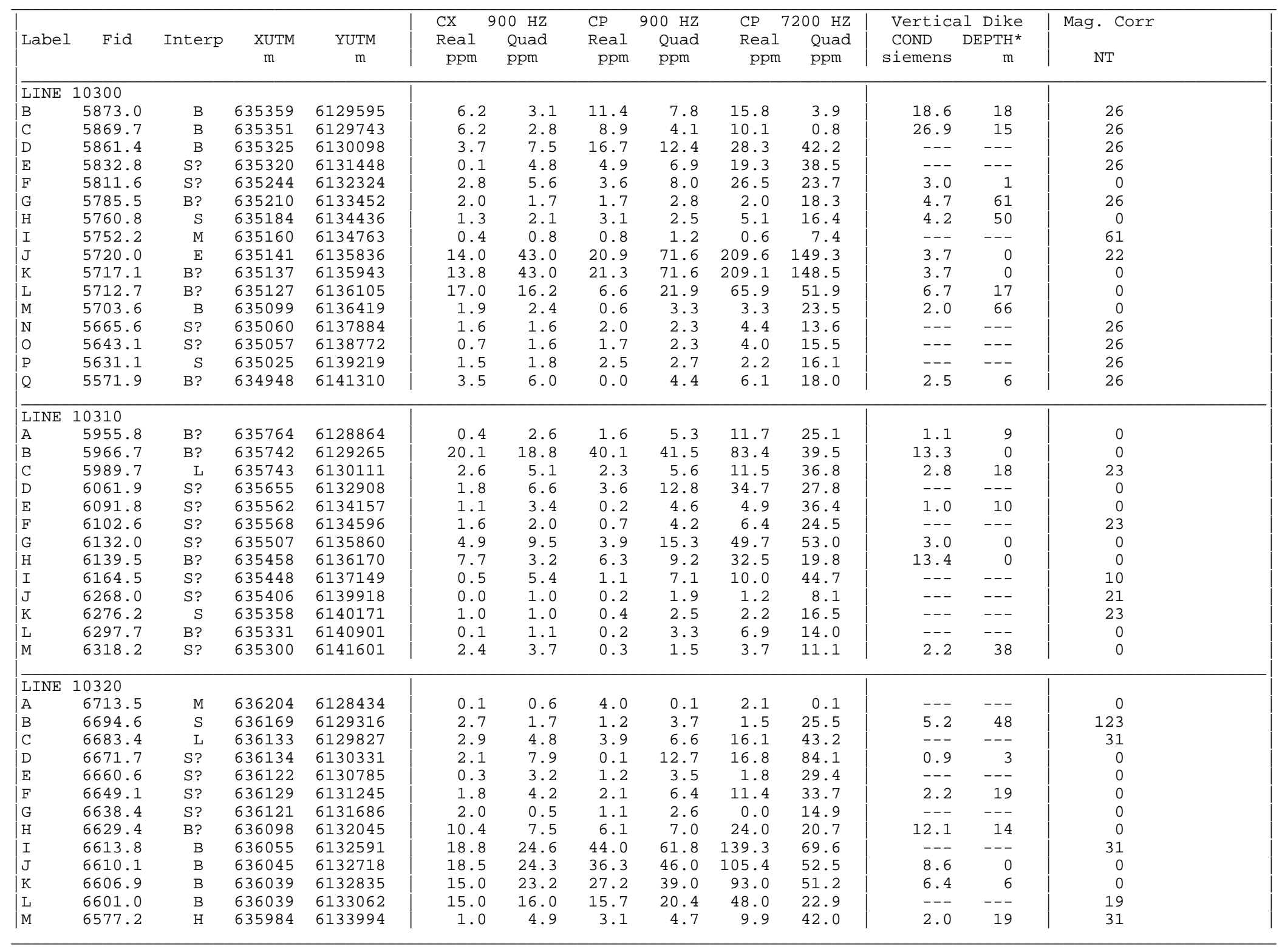

$\mathrm{CX}=$ COAXIAL

Note: EM values shown above

are local amplitudes

are lo
5515
*Estimated Depth may be unreliable because the stronger part of the conductor may be deeper or to one side of the flight line, or because of a shallow dip or magnetite/overburden effects 
EM Anomaly List

\begin{tabular}{|c|c|c|c|c|c|c|c|c|c|c|c|c|c|}
\hline Label & Fid & Interp & $\begin{array}{c}\text { XUTM } \\
\text { m }\end{array}$ & $\begin{array}{c}\text { YUTM } \\
\mathrm{m}\end{array}$ & $\begin{array}{l}\text { CX } \\
\text { Real } \\
\text { ppm }\end{array}$ & $\begin{array}{l}900 \mathrm{HZ} \\
\text { Quad } \\
\text { ppm }\end{array}$ & $\begin{array}{l}\text { CP } \\
\text { Real } \\
\text { ppm }\end{array}$ & $\begin{array}{c}900 \mathrm{HZ} \\
\text { Quad } \\
\text { ppm }\end{array}$ & $\begin{array}{l}\text { CP } \\
\text { Real } \\
\text { ppm }\end{array}$ & $\begin{array}{c}7200 \mathrm{HZ} \\
\text { Quad } \\
\text { ppm }\end{array}$ & $\begin{array}{l}\text { Vertica } \\
\text { CoND } \\
\text { siemens }\end{array}$ & $\begin{array}{c}\text { al Dike } \\
\text { DEPTH* } \\
\text { m }\end{array}$ & $\begin{array}{c}\text { Mag. Corr } \\
\text { NT }\end{array}$ \\
\hline $\begin{array}{l}\text { LINE } \\
\text { N } \\
O \\
P \\
Q \\
R \\
\text { S } \\
\text { T } \\
\text { U }\end{array}$ & $\begin{array}{r}10320 \\
6529.3 \\
6523.4 \\
6519.4 \\
6512.9 \\
6504.7 \\
6440.2 \\
6435.9 \\
6381.9\end{array}$ & $\begin{array}{r}\text { B? } \\
\text { B? } \\
\text { D } \\
\text { S? } \\
\text { S? } \\
\text { M } \\
\text { S? } \\
\text { B }\end{array}$ & $\begin{array}{l}635956 \\
635945 \\
635937 \\
635910 \\
635896 \\
635815 \\
635798 \\
635779\end{array}$ & $\begin{array}{l}6135879 \\
6136126 \\
6136293 \\
6136562 \\
6136896 \\
6139290 \\
6139387 \\
6140690\end{array}$ & $\begin{array}{r}7.5 \\
3.0 \\
13.8 \\
1.8 \\
2.8 \\
1.7 \\
2.7 \\
37.2\end{array}$ & $\begin{array}{r}17.1 \\
17.9 \\
14.2 \\
1.8 \\
4.0 \\
0.4 \\
1.1 \\
54.0\end{array}$ & $\begin{array}{r}7.6 \\
2.4 \\
3.8 \\
1.6 \\
1.0 \\
2.8 \\
0.1 \\
50.0\end{array}$ & $\begin{array}{r}36.1 \\
27.9 \\
9.6 \\
3.7 \\
6.8 \\
0.9 \\
1.5 \\
92.1\end{array}$ & $\begin{array}{r}109.9 \\
73.4 \\
24.9 \\
3.2 \\
12.3 \\
1.0 \\
0.2 \\
216.1\end{array}$ & $\begin{array}{r}96.0 \\
119.4 \\
43.2 \\
20.3 \\
38.4 \\
6.5 \\
10.1 \\
151.0\end{array}$ & $\begin{array}{l}3.0 \\
1.0 \\
7.2 \\
3.3 \\
2.6 \\
--- \\
9.1 \\
7.4\end{array}$ & $\begin{array}{r}3 \\
0 \\
10 \\
54 \\
21 \\
--- \\
66 \\
0\end{array}$ & $\begin{array}{r}0 \\
64 \\
31 \\
22 \\
0 \\
23 \\
70 \\
31\end{array}$ \\
\hline $\begin{array}{l}\text { LINE } \\
\text { A } \\
\text { B } \\
\text { C } \\
\text { D } \\
\text { E } \\
\text { F } \\
\text { G } \\
\text { H } \\
\text { I } \\
\text { J } \\
\text { K } \\
\text { L } \\
\text { M } \\
\text { N } \\
\text { O } \\
\text { P } \\
\text { Q } \\
\text { R } \\
\text { S }\end{array}$ & $\begin{array}{r}10330 \\
6887.3 \\
6898.6 \\
6934.2 \\
6957.1 \\
6983.0 \\
6997.3 \\
7006.2 \\
7009.3 \\
7015.0 \\
7034.6 \\
7036.5 \\
7055.2 \\
7061.7 \\
7064.2 \\
7087.5 \\
7097.7 \\
7133.3 \\
7175.5 \\
7221.5\end{array}$ & $\begin{array}{r}\mathrm{M} \\
\mathrm{M} \\
\mathrm{M} \\
\mathrm{H} \\
\mathrm{M} \\
\mathrm{B} \\
\mathrm{D} \\
\mathrm{B} \\
\mathrm{B} \\
\mathrm{B} \\
\mathrm{B} \\
\mathrm{L} \\
\mathrm{B} ? \\
\mathrm{~B} ? \\
\mathrm{~B} ? \\
\mathrm{D} \\
\mathrm{S} ? \\
\mathrm{~B} \\
\mathrm{~B}\end{array}$ & $\begin{array}{l}636607 \\
636594 \\
636576 \\
636500 \\
636477 \\
636466 \\
636477 \\
636478 \\
636465 \\
636369 \\
636364 \\
636407 \\
636419 \\
636414 \\
636312 \\
636327 \\
636281 \\
636245 \\
636145\end{array}$ & $\begin{array}{l}6128085 \\
6128455 \\
6129649 \\
6130522 \\
6131591 \\
6132004 \\
6132272 \\
6132389 \\
6132614 \\
6133477 \\
6133563 \\
6134329 \\
6134614 \\
6134726 \\
6135750 \\
6136198 \\
6137742 \\
6139424 \\
6140972\end{array}$ & $\begin{array}{r}0.3 \\
0.3 \\
0.9 \\
0.2 \\
1.8 \\
13.1 \\
13.5 \\
8.7 \\
11.8 \\
9.4 \\
9.0 \\
2.4 \\
7.1 \\
7.1 \\
1.2 \\
12.1 \\
0.6 \\
5.5 \\
9.2\end{array}$ & $\begin{array}{r}2.2 \\
0.8 \\
0.8 \\
1.2 \\
0.0 \\
11.1 \\
24.4 \\
9.0 \\
12.3 \\
14.9 \\
14.9 \\
3.9 \\
5.6 \\
5.6 \\
6.6 \\
9.6 \\
1.1 \\
3.6 \\
7.7\end{array}$ & $\begin{array}{r}3.1 \\
0.3 \\
7.8 \\
0.2 \\
3.4 \\
4.6 \\
13.8 \\
10.8 \\
8.5 \\
8.5 \\
14.0 \\
0.2 \\
5.9 \\
5.9 \\
2.2 \\
1.2 \\
1.7 \\
4.2 \\
6.8\end{array}$ & $\begin{array}{r}4.9 \\
2.1 \\
1.7 \\
1.1 \\
0.3 \\
10.8 \\
27.9 \\
19.5 \\
19.5 \\
28.4 \\
33.9 \\
3.0 \\
11.4 \\
11.4 \\
9.8 \\
4.7 \\
2.3 \\
4.0 \\
11.4\end{array}$ & $\begin{array}{r}11.9 \\
0.4 \\
8.5 \\
3.6 \\
6.3 \\
34.6 \\
74.5 \\
45.0 \\
45.2 \\
69.3 \\
77.9 \\
7.0 \\
28.2 \\
28.2 \\
23.9 \\
8.6 \\
3.9 \\
11.1 \\
33.6\end{array}$ & $\begin{array}{r}11.8 \\
10.2 \\
11.4 \\
7.6 \\
1.8 \\
24.0 \\
66.3 \\
37.5 \\
37.5 \\
46.6 \\
46.6 \\
28.9 \\
19.6 \\
17.6 \\
43.9 \\
7.2 \\
13.7 \\
6.4 \\
16.7\end{array}$ & $\begin{array}{l}--- \\
--- \\
--- \\
--- \\
--- \\
8.5 \\
4.0 \\
6.0 \\
6.0 \\
4.2 \\
3.4 \\
2.4 \\
6.9 \\
6.9 \\
--- \\
9.6 \\
--- \\
--- \\
8.1\end{array}$ & $\begin{array}{r}--- \\
--- \\
--- \\
--- \\
--- \\
14 \\
4 \\
10 \\
13 \\
0 \\
12 \\
19 \\
19 \\
19 \\
--- \\
12 \\
--- \\
--- \\
15\end{array}$ & $\begin{array}{r}146 \\
89 \\
634 \\
0 \\
283 \\
0 \\
0 \\
32 \\
34 \\
0 \\
0 \\
34 \\
34 \\
0 \\
34 \\
0 \\
24 \\
0 \\
0\end{array}$ \\
\hline $\begin{array}{l}\text { LINE } \\
\text { A } \\
\text { B } \\
\text { C } \\
\text { D } \\
\text { E } \\
\text { F } \\
\text { G } \\
\text { H } \\
\text { I } \\
\text { J } \\
\text { K } \\
\text { L } \\
\text { M } \\
\text { N } \\
\text { O }\end{array}$ & $\begin{array}{r}10340 \\
7684.7 \\
7680.3 \\
7673.2 \\
7654.6 \\
7643.5 \\
7586.1 \\
7546.5 \\
7522.9 \\
7502.1 \\
7484.8 \\
7461.9 \\
7453.3 \\
7415.6 \\
7409.1 \\
7369.3\end{array}$ & $\begin{array}{r}\text { B } \\
\text { B? } \\
\text { B? } \\
\text { B? } \\
\text { S? } \\
\text { S? } \\
\text { B } \\
\text { D } \\
\text { B } \\
\text { B } \\
\text { B } \\
\text { B? } \\
\text { D } \\
\text { S } \\
\text { S }\end{array}$ & $\begin{array}{l}637003 \\
637009 \\
637023 \\
636994 \\
636983 \\
636913 \\
636873 \\
636838 \\
636805 \\
636796 \\
636779 \\
636790 \\
636724 \\
636708 \\
636647\end{array}$ & $\begin{array}{l}6127494 \\
6127675 \\
6127979 \\
6128766 \\
6129182 \\
6131078 \\
6132275 \\
6132703 \\
6133303 \\
6133941 \\
6134588 \\
6134901 \\
6136231 \\
6136492 \\
6138057\end{array}$ & $\begin{array}{r}3.5 \\
5.1 \\
0.1 \\
1.3 \\
1.7 \\
1.8 \\
12.9 \\
28.1 \\
5.1 \\
9.6 \\
0.8 \\
3.0 \\
7.6 \\
0.9 \\
1.6\end{array}$ & $\begin{array}{r}4.6 \\
6.2 \\
3.3 \\
1.2 \\
3.4 \\
4.4 \\
14.8 \\
42.8 \\
8.2 \\
11.7 \\
6.0 \\
5.0 \\
9.1 \\
2.3 \\
2.1\end{array}$ & $\begin{array}{r}0.8 \\
5.5 \\
1.7 \\
2.5 \\
1.1 \\
0.1 \\
19.4 \\
30.6 \\
2.2 \\
15.4 \\
1.6 \\
4.3 \\
1.3 \\
3.6 \\
1.1\end{array}$ & $\begin{array}{r}8.7 \\
8.9 \\
5.4 \\
1.7 \\
5.0 \\
7.1 \\
32.9 \\
67.2 \\
7.0 \\
25.0 \\
6.0 \\
7.5 \\
6.3 \\
3.3 \\
3.0\end{array}$ & $\begin{array}{r}24.3 \\
24.6 \\
8.3 \\
3.2 \\
2.8 \\
7.9 \\
72.1 \\
161.3 \\
20.2 \\
56.3 \\
13.8 \\
18.1 \\
11.5 \\
4.5 \\
3.4\end{array}$ & $\begin{array}{r}33.3 \\
30.5 \\
31.7 \\
9.9 \\
27.9 \\
44.6 \\
37.7 \\
121.7 \\
25.6 \\
21.6 \\
26.4 \\
25.3 \\
16.8 \\
18.8 \\
17.4\end{array}$ & $\begin{array}{l}--- \\
4.8 \\
--- \\
--- \\
2.0 \\
1.2 \\
6.6 \\
5.8 \\
2.6 \\
6.0 \\
1.0 \\
3.0 \\
4.3 \\
--- \\
---\end{array}$ & $\begin{array}{r}--- \\
19 \\
--- \\
--- \\
21 \\
8 \\
0 \\
3 \\
26 \\
13 \\
0 \\
33 \\
16 \\
--- \\
---\end{array}$ & $\begin{array}{r}0 \\
28 \\
69 \\
0 \\
67 \\
18 \\
0 \\
0 \\
0 \\
0 \\
0 \\
28 \\
0 \\
0 \\
0\end{array}$ \\
\hline
\end{tabular}

$\mathrm{CX}=$ COAXIAL

Note: EM values shown above

are local amplitudes

Ketchikan - North/South of 5515
* Estimated Depth may be unreliable because the stronger part of the conductor may be deeper or to one side of the flight line, or because of a shallow dip or magnetite/overburden effects 
EM Anomaly List

\begin{tabular}{|c|c|c|c|c|c|c|c|c|c|c|c|c|c|}
\hline Label & Fid & Interp & $\underset{\mathrm{m}}{\mathrm{XUTM}}$ & $\begin{array}{c}\text { YUTM } \\
\text { m }\end{array}$ & $\begin{array}{ll}\text { CX } & 9 \\
\text { Real } & \\
\text { ppm }\end{array}$ & $\begin{array}{l}00 \mathrm{HZ} \\
\text { Quad } \\
\text { ppm }\end{array}$ & $\begin{array}{l}\text { CP } \\
\text { Real } \\
\text { ppm }\end{array}$ & $\begin{array}{l}900 \mathrm{HZ} \\
\text { Quad } \\
\text { ppm }\end{array}$ & $\begin{array}{l}\text { CP } 7 \\
\text { Real } \\
\text { ppm }\end{array}$ & $\begin{array}{l}7200 \mathrm{HZ} \\
\text { Quad } \\
\text { ppm }\end{array}$ & $\begin{array}{l}\text { Vertica } \\
\text { CoND } \\
\text { siemens }\end{array}$ & $\begin{array}{c}\text { al Dike } \\
\text { DEPTH* } \\
\text { m }\end{array}$ & $\begin{array}{c}\text { Mag. Corr } \\
\text { NT }\end{array}$ \\
\hline $\begin{array}{ll}\text { LINE } & 1 \\
P & \\
\text { Q } & \\
\text { R } & \end{array}$ & $\begin{array}{r}10340 \\
7310.6 \\
7287.9 \\
7283.3\end{array}$ & $\begin{array}{r}\text { S? } \\
\text { D } \\
\text { B }\end{array}$ & $\begin{array}{l}636541 \\
636572 \\
636553\end{array}$ & $\begin{array}{l}6140302 \\
6141167 \\
6141323\end{array}$ & $\begin{array}{r}0.7 \\
16.5 \\
9.0\end{array}$ & $\begin{array}{r}2.3 \\
17.7 \\
7.8\end{array}$ & $\begin{array}{r}0.3 \\
14.5 \\
7.0\end{array}$ & $\begin{array}{r}2.2 \\
30.7 \\
12.8\end{array}$ & $\begin{array}{r}5.2 \\
77.1 \\
34.1\end{array}$ & $\begin{array}{l}11.3 \\
40.5 \\
21.6\end{array}$ & $\begin{array}{l}1.1 \\
6.7 \\
---\end{array}$ & $\begin{array}{r}10 \\
10 \\
---\end{array}$ & $\begin{array}{r}28 \\
0 \\
2\end{array}$ \\
\hline $\begin{array}{l}\text { LINE } 1 \\
\text { A } \\
\text { B } \\
\text { C } \\
\text { D } \\
\text { E } \\
\text { F } \\
\text { G } \\
\text { H } \\
\text { I } \\
\text { J } \\
\text { K } \\
\text { L } \\
\text { M } \\
\text { N } \\
\text { O } \\
\text { P } \\
\text { Q } \\
\text { R } \\
\text { S } \\
\text { T } \\
\text { U } \\
\text { V } \\
\text { W } \\
\text { X } \\
\text { Y }\end{array}$ & $\begin{array}{r}10350 \\
7835.1 \\
7851.8 \\
7855.8 \\
7881.0 \\
7902.2 \\
7965.2 \\
7987.7 \\
7996.0 \\
8015.0 \\
8023.7 \\
8033.5 \\
8040.1 \\
8076.5 \\
8103.3 \\
8114.0 \\
8136.8 \\
8155.0 \\
8171.5 \\
8187.2 \\
8193.3 \\
8197.3 \\
8213.0 \\
8224.0 \\
8235.0 \\
8251.4\end{array}$ & $\begin{array}{r}\text { D } \\
\text { S? } \\
\text { M } \\
\text { S? } \\
\text { S? } \\
\text { B } \\
\text { B } \\
\text { B } \\
\text { B } \\
\text { B } \\
\text { B } \\
\text { B? } \\
\text { S? } \\
\text { B? } \\
\text { B } \\
\text { S? } \\
\text { S? } \\
\text { S } \\
\text { S? } \\
\text { D } \\
\text { B } \\
\text { B? } \\
\text { S? } \\
\text { S } \\
\text { B? }\end{array}$ & $\begin{array}{l}637445 \\
637396 \\
637388 \\
637381 \\
637367 \\
637287 \\
637295 \\
637309 \\
637257 \\
637239 \\
637223 \\
637214 \\
637163 \\
637137 \\
637105 \\
637122 \\
637055 \\
637026 \\
637044 \\
637027 \\
637009 \\
636961 \\
636970 \\
636988 \\
636906\end{array}$ & $\begin{array}{l}6127574 \\
6128105 \\
6128242 \\
6129130 \\
6129663 \\
6131900 \\
6132310 \\
6132567 \\
6133187 \\
6133467 \\
6133794 \\
6133950 \\
6135272 \\
6136303 \\
6136700 \\
6137521 \\
6138261 \\
6138795 \\
6139318 \\
6139492 \\
6139589 \\
6140095 \\
6140474 \\
6140914 \\
6141606\end{array}$ & $\begin{array}{r}4.3 \\
0.0 \\
1.9 \\
1.1 \\
0.9 \\
16.2 \\
6.3 \\
28.2 \\
3.4 \\
3.5 \\
5.8 \\
7.4 \\
1.0 \\
1.3 \\
1.3 \\
0.7 \\
0.1 \\
1.9 \\
0.9 \\
11.3 \\
3.7 \\
0.2 \\
0.1 \\
1.9 \\
7.6\end{array}$ & $\begin{array}{r}6.4 \\
0.8 \\
1.1 \\
4.2 \\
1.6 \\
20.1 \\
9.7 \\
30.4 \\
9.6 \\
2.1 \\
5.3 \\
3.2 \\
1.6 \\
4.0 \\
5.6 \\
3.7 \\
2.5 \\
2.0 \\
2.3 \\
4.8 \\
1.4 \\
6.6 \\
2.6 \\
3.8 \\
14.6\end{array}$ & $\begin{array}{r}1.4 \\
2.8 \\
1.2 \\
0.0 \\
1.6 \\
19.8 \\
14.7 \\
40.2 \\
8.9 \\
0.3 \\
0.8 \\
0.0 \\
0.3 \\
0.1 \\
0.3 \\
0.4 \\
5.0 \\
0.1 \\
1.0 \\
8.9 \\
6.9 \\
1.2 \\
2.0 \\
2.6 \\
2.4\end{array}$ & $\begin{array}{r}5.1 \\
1.5 \\
1.5 \\
5.0 \\
2.5 \\
28.4 \\
20.0 \\
55.3 \\
6.6 \\
1.3 \\
3.0 \\
6.6 \\
1.7 \\
4.9 \\
7.3 \\
5.6 \\
3.2 \\
2.7 \\
2.7 \\
2.1 \\
5.4 \\
9.2 \\
2.5 \\
5.3 \\
11.0\end{array}$ & $\begin{array}{r}12.5 \\
1.8 \\
1.0 \\
8.2 \\
2.8 \\
70.6 \\
47.8 \\
129.3 \\
18.8 \\
3.3 \\
3.9 \\
18.6 \\
1.4 \\
9.4 \\
12.0 \\
10.0 \\
3.2 \\
1.3 \\
4.6 \\
12.8 \\
31.1 \\
19.3 \\
1.1 \\
6.7 \\
32.4\end{array}$ & $\begin{array}{r}12.6 \\
9.5 \\
7.8 \\
30.5 \\
16.5 \\
40.0 \\
34.1 \\
67.5 \\
58.6 \\
14.2 \\
22.1 \\
24.0 \\
11.3 \\
23.2 \\
42.9 \\
36.3 \\
19.7 \\
13.6 \\
18.8 \\
8.5 \\
19.1 \\
50.6 \\
14.0 \\
35.5 \\
50.6\end{array}$ & $\begin{array}{r}2.8 \\
3.6 \\
--- \\
0.9 \\
2.0 \\
7.5 \\
5.3 \\
10.6 \\
3.6 \\
8.7 \\
6.0 \\
---- \\
--- \\
1.1 \\
0.8 \\
--- \\
--- \\
--- \\
2.2 \\
40.7 \\
16.5 \\
0.5 \\
1.3 \\
--- \\
3.5\end{array}$ & $\begin{array}{r}37 \\
84 \\
--- \\
6 \\
73 \\
8 \\
13 \\
5 \\
28 \\
56 \\
29 \\
--- \\
--- \\
0 \\
4 \\
--- \\
--- \\
--- \\
42 \\
16 \\
14 \\
0 \\
27 \\
--- \\
0\end{array}$ & $\begin{array}{r}21 \\
0 \\
0 \\
63 \\
0 \\
0 \\
0 \\
21 \\
0 \\
21 \\
37 \\
21 \\
51 \\
0 \\
0 \\
122 \\
269 \\
21 \\
0 \\
0 \\
0 \\
21 \\
21 \\
0 \\
0\end{array}$ \\
\hline $\begin{array}{ll}\text { LINE } & 1 \\
\text { A } & \\
\text { B } \\
\text { C } \\
\text { D } \\
\text { E } \\
\text { F } \\
\text { G } \\
\text { H } \\
\text { I } \\
\text { J } \\
\text { K } \\
\text { L } \\
\text { M } \\
\text { N }\end{array}$ & $\begin{array}{r}10360 \\
8782.6 \\
8773.5 \\
8706.4 \\
8683.6 \\
8660.6 \\
8658.4 \\
8657.3 \\
8634.6 \\
8617.4 \\
8607.7 \\
8605.5 \\
8596.7 \\
8592.1 \\
8577.6\end{array}$ & $\begin{array}{r}\text { B? } \\
\text { S? } \\
\text { S } \\
\text { S? } \\
\text { B } \\
\text { B } \\
\text { B } \\
\text { B } \\
\text { D } \\
\text { B } \\
\text { B } \\
\text { D } \\
\text { L } \\
\text { D }\end{array}$ & $\begin{array}{l}637797 \\
637818 \\
637771 \\
637719 \\
637757 \\
637744 \\
637735 \\
637696 \\
637598 \\
637583 \\
637584 \\
637589 \\
637594 \\
637585\end{array}$ & $\begin{array}{l}6128133 \\
6128486 \\
6130130 \\
6130841 \\
6131703 \\
6131785 \\
6131823 \\
6132532 \\
6133130 \\
6133451 \\
6133521 \\
6133820 \\
6133963 \\
6134349\end{array}$ & $\begin{array}{r}7.2 \\
3.4 \\
1.2 \\
0.1 \\
27.0 \\
27.0 \\
14.6 \\
31.8 \\
5.0 \\
2.6 \\
1.8 \\
1.6 \\
4.2 \\
0.4\end{array}$ & $\begin{array}{r}25.5 \\
5.8 \\
1.6 \\
2.2 \\
21.0 \\
21.0 \\
16.3 \\
43.2 \\
1.9 \\
6.6 \\
6.3 \\
4.3 \\
6.3 \\
3.9\end{array}$ & $\begin{array}{r}4.7 \\
2.7 \\
2.1 \\
0.5 \\
39.4 \\
39.4 \\
32.6 \\
48.6 \\
3.5 \\
3.1 \\
2.9 \\
2.0 \\
2.6 \\
1.7\end{array}$ & $\begin{array}{r}33.0 \\
7.7 \\
1.7 \\
2.2 \\
38.1 \\
38.1 \\
32.1 \\
87.9 \\
3.9 \\
9.5 \\
9.7 \\
6.5 \\
5.1 \\
7.6\end{array}$ & $\begin{array}{r}106.2 \\
23.9 \\
2.4 \\
5.0 \\
89.6 \\
89.6 \\
75.8 \\
203.8 \\
12.6 \\
27.2 \\
27.0 \\
16.9 \\
14.7 \\
21.6\end{array}$ & $\begin{array}{r}151.1 \\
37.4 \\
14.6 \\
14.5 \\
19.4 \\
19.4 \\
24.0 \\
116.0 \\
20.4 \\
35.4 \\
32.5 \\
28.8 \\
15.8 \\
28.8\end{array}$ & $\begin{array}{r}2.1 \\
3.1 \\
--- \\
0.5 \\
17.1 \\
17.1 \\
11.1 \\
7.5 \\
16.4 \\
2.3 \\
1.8 \\
1.9 \\
3.3 \\
0.8\end{array}$ & $\begin{array}{r}0 \\
5 \\
--- \\
0 \\
0 \\
0 \\
4 \\
0 \\
27 \\
6 \\
7 \\
8 \\
19 \\
3\end{array}$ & $\begin{array}{r}27 \\
27 \\
12 \\
0 \\
27 \\
0 \\
0 \\
27 \\
0 \\
0 \\
0 \\
27 \\
27 \\
0\end{array}$ \\
\hline
\end{tabular}

$\mathrm{CX}=$ COAXIAL

Note: EM values shown above

are local amplitudes

Ketchikan - North/South of 5515
* Estimated Depth may be unreliable because the stronger part of the conductor may be deeper or to one side of the flight line, or because of a shallow dip or magnetite/overburden effects 
EM Anomaly List

\begin{tabular}{|c|c|c|c|c|c|c|c|c|c|c|c|c|c|}
\hline Label & Fid & Interp & $\begin{array}{c}\text { XUTM } \\
\text { m }\end{array}$ & $\begin{array}{c}\text { YUTM } \\
\mathrm{m}\end{array}$ & $\begin{array}{l}\text { CX } \\
\text { Real } \\
\text { ppm }\end{array}$ & $\begin{array}{l}900 \mathrm{HZ} \\
\text { Quad } \\
\text { ppm }\end{array}$ & $\begin{array}{l}\text { CP } \\
\text { Real } \\
\text { ppm }\end{array}$ & $\begin{array}{l}900 \mathrm{HZ} \\
\text { Quad } \\
\text { ppm }\end{array}$ & $\begin{array}{l}\text { CP } 7 \\
\text { Real } \\
\text { ppm }\end{array}$ & $\begin{array}{c}7200 \mathrm{HZ} \\
\text { Quad } \\
\text { ppm }\end{array}$ & $\begin{array}{l}\text { Vertica } \\
\text { Cond } \\
\text { siemens }\end{array}$ & $\begin{array}{c}\text { al Dike } \\
\text { DEPTH* } \\
\text { m }\end{array}$ & $\begin{array}{c}\text { Mag. Corr } \\
\text { NT }\end{array}$ \\
\hline $\begin{array}{l}\text { LINE } \\
O \\
P \\
Q \\
\text { R } \\
\text { S } \\
\text { T } \\
\text { U } \\
\text { V }\end{array}$ & $\begin{array}{r}10360 \\
8572.2 \\
8568.9 \\
8539.7 \\
8534.1 \\
8430.2 \\
8397.2 \\
8394.5 \\
8376.6\end{array}$ & $\begin{array}{r}\text { D } \\
\text { B } \\
\text { S? } \\
\text { S? } \\
\text { S? } \\
\text { B } \\
\text { B } \\
\text { B? }\end{array}$ & $\begin{array}{l}637577 \\
637570 \\
637536 \\
637541 \\
637340 \\
637367 \\
637355 \\
637288\end{array}$ & $\begin{array}{l}6134549 \\
6134674 \\
6135602 \\
6135828 \\
6139671 \\
6140837 \\
6140941 \\
6141608\end{array}$ & $\begin{array}{r}7.0 \\
3.1 \\
1.2 \\
1.0 \\
0.2 \\
10.1 \\
8.7 \\
0.1\end{array}$ & $\begin{array}{r}19.1 \\
11.8 \\
5.1 \\
5.1 \\
0.5 \\
15.3 \\
15.3 \\
2.9\end{array}$ & $\begin{array}{r}11.1 \\
4.0 \\
2.7 \\
1.4 \\
1.9 \\
15.5 \\
15.5 \\
0.5\end{array}$ & $\begin{array}{r}32.8 \\
13.3 \\
6.5 \\
6.1 \\
0.9 \\
26.3 \\
25.9 \\
2.8\end{array}$ & $\begin{array}{r}87.2 \\
39.5 \\
12.3 \\
10.6 \\
0.7 \\
58.5 \\
58.0 \\
6.4\end{array}$ & $\begin{array}{r}46.9 \\
25.6 \\
34.8 \\
40.0 \\
3.8 \\
66.1 \\
66.1 \\
28.0\end{array}$ & $\begin{array}{l}3.3 \\
--- \\
--- \\
--- \\
5.3 \\
4.9 \\
4.3 \\
0.5\end{array}$ & $\begin{array}{r}0 \\
--- \\
---- \\
--- \\
89 \\
18 \\
11 \\
0\end{array}$ & $\begin{array}{r}27 \\
27 \\
0 \\
27 \\
245 \\
0 \\
157 \\
27\end{array}$ \\
\hline $\begin{array}{l}\text { LINE } \\
\text { A } \\
\text { B } \\
\text { C } \\
\text { D } \\
\text { E } \\
\text { F } \\
\text { G } \\
\text { H } \\
\text { I } \\
\text { J } \\
\text { K } \\
\text { L } \\
\text { M } \\
\text { N } \\
\text { O } \\
\text { P } \\
\text { Q } \\
\text { R } \\
\text { S } \\
\text { T } \\
\text { U } \\
\text { V } \\
\text { W } \\
\text { X }\end{array}$ & $\begin{array}{r}10370 \\
9030.6 \\
9037.4 \\
9050.2 \\
9066.0 \\
9075.2 \\
9079.0 \\
9082.9 \\
9085.0 \\
9101.6 \\
9110.3 \\
9114.0 \\
9122.2 \\
9128.6 \\
9134.6 \\
9171.5 \\
9178.8 \\
9185.4 \\
9206.0 \\
9291.1 \\
9297.9 \\
9311.3 \\
9319.2 \\
9327.9 \\
9331.5\end{array}$ & $\begin{array}{r}\text { B } \\
\text { B? } \\
\text { B } \\
\text { B } \\
\text { B } \\
\text { B } \\
\text { B } \\
\text { B } \\
\text { B } \\
\text { L } \\
\text { B } \\
\text { B } \\
\text { D } \\
\text { B? } \\
\text { B? } \\
\text { D } \\
\text { B? } \\
\text { S? } \\
\text { S? } \\
\text { M } \\
\text { S? } \\
\text { B? } \\
\text { B } \\
\text { B }\end{array}$ & $\begin{array}{l}638085 \\
638054 \\
638066 \\
638114 \\
638115 \\
638086 \\
638056 \\
638042 \\
637999 \\
637984 \\
637977 \\
637969 \\
637973 \\
637983 \\
637958 \\
637950 \\
637940 \\
637916 \\
637870 \\
637857 \\
637786 \\
637754 \\
637788 \\
637816\end{array}$ & $\begin{array}{l}6131557 \\
6131696 \\
6131888 \\
6132192 \\
6132464 \\
6132583 \\
6132714 \\
6132785 \\
6133305 \\
6133581 \\
6133710 \\
6134018 \\
6134272 \\
6134524 \\
6135354 \\
6135559 \\
6135776 \\
6136420 \\
6139494 \\
6139795 \\
6140397 \\
6140729 \\
6141077 \\
6141223\end{array}$ & $\begin{array}{r}29.2 \\
4.6 \\
2.1 \\
8.6 \\
11.2 \\
5.0 \\
22.2 \\
22.2 \\
5.9 \\
0.2 \\
0.7 \\
3.1 \\
6.3 \\
0.9 \\
2.7 \\
5.6 \\
2.2 \\
0.0 \\
3.1 \\
1.5 \\
0.2 \\
6.3 \\
4.8 \\
4.5\end{array}$ & $\begin{array}{r}26.3 \\
7.1 \\
1.5 \\
7.6 \\
25.8 \\
15.3 \\
31.0 \\
31.0 \\
26.4 \\
7.3 \\
4.2 \\
4.7 \\
7.5 \\
7.6 \\
9.6 \\
3.7 \\
13.8 \\
1.6 \\
1.8 \\
0.4 \\
1.4 \\
4.3 \\
2.9 \\
8.0\end{array}$ & $\begin{array}{r}39.5 \\
22.5 \\
3.7 \\
9.1 \\
16.6 \\
5.6 \\
20.6 \\
20.1 \\
2.8 \\
0.7 \\
0.0 \\
0.8 \\
2.7 \\
2.0 \\
3.4 \\
0.2 \\
6.2 \\
0.4 \\
1.5 \\
1.7 \\
1.4 \\
4.6 \\
5.7 \\
7.8\end{array}$ & $\begin{array}{r}41.8 \\
23.6 \\
6.0 \\
12.3 \\
35.6 \\
38.6 \\
47.1 \\
45.7 \\
28.8 \\
7.4 \\
7.2 \\
0.4 \\
5.4 \\
8.1 \\
16.3 \\
0.2 \\
25.9 \\
1.6 \\
2.7 \\
0.2 \\
2.4 \\
7.1 \\
8.2 \\
14.2\end{array}$ & $\begin{array}{r}101.6 \\
50.1 \\
15.1 \\
28.7 \\
87.4 \\
103.3 \\
121.2 \\
117.4 \\
81.9 \\
16.4 \\
18.5 \\
3.2 \\
18.0 \\
14.5 \\
48.1 \\
1.0 \\
78.2 \\
1.5 \\
1.4 \\
0.9 \\
5.7 \\
17.3 \\
15.9 \\
29.2\end{array}$ & $\begin{array}{r}54.3 \\
22.9 \\
6.3 \\
8.3 \\
100.0 \\
118.5 \\
83.9 \\
77.2 \\
132.0 \\
38.0 \\
16.2 \\
0.6 \\
23.4 \\
29.5 \\
70.4 \\
2.7 \\
87.6 \\
8.9 \\
19.7 \\
0.4 \\
12.7 \\
8.7 \\
17.3 \\
51.9\end{array}$ & $\begin{array}{r}14.6 \\
7.4 \\
4.9 \\
8.4 \\
4.6 \\
--- \\
5.6 \\
5.6 \\
1.6 \\
0.5 \\
0.5 \\
--- \\
4.9 \\
1.0 \\
1.8 \\
12.3 \\
1.6 \\
--- \\
7.7 \\
--- \\
--- \\
8.8 \\
8.4 \\
3.2\end{array}$ & $\begin{array}{r}2 \\
9 \\
40 \\
13 \\
0 \\
--- \\
0 \\
0 \\
0 \\
0 \\
0 \\
--- \\
20 \\
0 \\
0 \\
25 \\
0 \\
--- \\
48 \\
--- \\
--- \\
0 \\
18 \\
17\end{array}$ & $\begin{array}{r}28 \\
0 \\
0 \\
0 \\
0 \\
28 \\
0 \\
0 \\
16 \\
28 \\
0 \\
22 \\
0 \\
0 \\
0 \\
0 \\
0 \\
0 \\
28 \\
380 \\
28 \\
0 \\
0 \\
0\end{array}$ \\
\hline $\begin{array}{l}\text { LINE } \\
\text { A } \\
\text { B } \\
\text { C } \\
\text { D } \\
\text { E } \\
\text { F } \\
\text { G } \\
\text { H } \\
\text { I } \\
\text { J }\end{array}$ & $\begin{array}{c}10380 \\
528.4 \\
514.4 \\
504.7 \\
496.7 \\
482.6 \\
479.0 \\
474.6 \\
463.3 \\
451.1 \\
427.6\end{array}$ & $\begin{array}{l}\text { B } \\
\text { B } \\
\text { B } \\
\text { B } \\
\text { B } \\
\text { B } \\
\text { B } \\
\text { D } \\
\text { D } \\
\text { B }\end{array}$ & $\begin{array}{l}638539 \\
638478 \\
638469 \\
638473 \\
638436 \\
638424 \\
638419 \\
638443 \\
638447 \\
638404\end{array}$ & $\begin{array}{l}6131846 \\
6132112 \\
6132395 \\
6132704 \\
6133255 \\
6133392 \\
6133567 \\
6134017 \\
6134436 \\
6135022\end{array}$ & $\begin{array}{r}10.5 \\
9.4 \\
10.0 \\
20.4 \\
14.8 \\
8.3 \\
2.3 \\
4.7 \\
3.7 \\
5.2\end{array}$ & $\begin{array}{r}9.2 \\
8.9 \\
14.2 \\
20.8 \\
8.5 \\
12.8 \\
8.6 \\
9.7 \\
6.5 \\
13.3\end{array}$ & $\begin{array}{r}11.4 \\
8.8 \\
9.4 \\
36.3 \\
14.3 \\
8.1 \\
4.3 \\
1.6 \\
2.5 \\
6.0\end{array}$ & $\begin{array}{r}20.9 \\
16.0 \\
19.5 \\
40.8 \\
15.3 \\
14.4 \\
9.5 \\
4.3 \\
5.3 \\
23.5\end{array}$ & $\begin{array}{r}47.7 \\
39.4 \\
49.3 \\
84.4 \\
38.5 \\
43.5 \\
23.5 \\
9.2 \\
11.5 \\
65.1\end{array}$ & $\begin{array}{l}29.2 \\
34.5 \\
41.8 \\
40.7 \\
13.2 \\
44.0 \\
36.8 \\
30.0 \\
14.3 \\
76.6\end{array}$ & $\begin{array}{r}7.2 \\
6.8 \\
4.5 \\
11.6 \\
16.6 \\
4.3 \\
2.2 \\
3.1 \\
2.6 \\
2.7\end{array}$ & $\begin{array}{r}10 \\
3 \\
6 \\
0 \\
0 \\
10 \\
1 \\
12 \\
22 \\
0\end{array}$ & $\begin{array}{r}21 \\
25 \\
25 \\
0 \\
25 \\
0 \\
25 \\
0 \\
0 \\
0\end{array}$ \\
\hline
\end{tabular}

$\mathrm{CX}=$ COAXIAL

Note: EM values shown above

are local amplitudes
*Estimated Depth may be unreliable because the stronger part of the conductor may be deeper or to one side of the flight line, or because of a shallow dip or magnetite/overburden effects 
EM Anomaly List

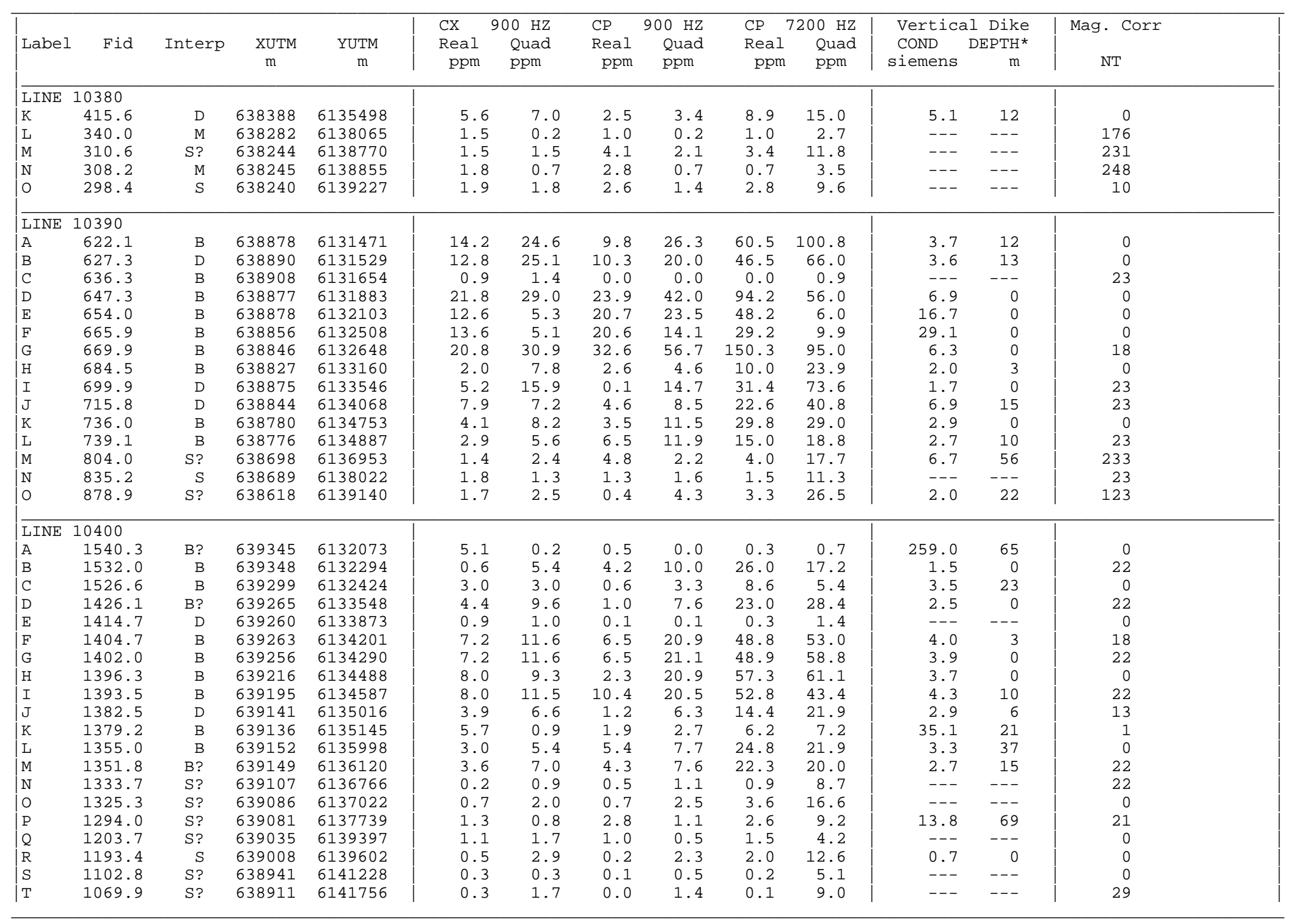

$\mathrm{CX}=$ COAXIAL

Note: EM values shown above

Ketchikan - North/South of 5515
* Estimated Depth may be unreliable because the stronger part of the conductor may be deeper or to one side of the flight line, or because of a shallow dip or magnetite/overburden effects 
EM Anomaly List

\begin{tabular}{|c|c|c|c|c|c|c|c|c|c|c|c|c|c|}
\hline Label & Fid & Interp & $\begin{array}{c}\text { XUTM } \\
\text { m }\end{array}$ & $\begin{array}{c}\text { YUTM } \\
\mathrm{m}\end{array}$ & $\begin{array}{ll}\text { CX } & 9 \\
\text { Real } & \\
\text { ppm }\end{array}$ & $\begin{array}{l}900 \mathrm{HZ} \\
\text { Quad } \\
\text { ppm }\end{array}$ & $\begin{array}{l}\text { CP } \\
\text { Real } \\
\text { ppm }\end{array}$ & $\begin{array}{l}900 \mathrm{HZ} \\
\text { Quad } \\
\text { ppm }\end{array}$ & \multicolumn{2}{|c|}{$\begin{array}{ll}\text { CP } & 7200 \mathrm{HZ} \\
\text { Real } & \text { Quad } \\
\text { ppm } & \text { ppm }\end{array}$} & \multicolumn{2}{|c|}{\begin{tabular}{lc}
\multicolumn{2}{c}{ Vertical Dike } \\
COND & DEPTH* \\
siemens & $\mathrm{m}$
\end{tabular}} & $\begin{array}{l}\text { Mag. Corr } \\
\text { NT }\end{array}$ \\
\hline LINE & 10410 & & & & & & & & & & & & \\
\hline A & 1601.8 & $B$ ? & 639713 & 6131458 & 2.0 & 3.6 & 2.5 & 9.1 & 26.0 & 30.8 & 2.3 & 3 & 31 \\
\hline B & 1627.3 & B? & 639691 & 6132055 & 2.6 & 4.0 & 1.3 & 1.8 & 4.9 & 7.4 & --- & --- & 25 \\
\hline $\mathrm{C}$ & 1639.2 & B & 639685 & 6132401 & 12.8 & 17.3 & 11.8 & 24.3 & 56.3 & 34.2 & 5.1 & 1 & 0 \\
\hline $\mathrm{D}$ & 1645.9 & B & 639676 & 6132575 & 6.1 & 8.2 & 12.6 & 23.6 & 49.1 & 27.7 & --- & --- & 0 \\
\hline $\mathrm{E}$ & 1669.5 & D & 639664 & 6133150 & 2.9 & 2.3 & 0.3 & 0.3 & 1.2 & 1.9 & --- & --- & 0 \\
\hline $\mathrm{F}$ & 1684.9 & S? & 639614 & 6133491 & 2.5 & 11.7 & 0.2 & 11.6 & 23.3 & 65.6 & 1.0 & 0 & 20 \\
\hline G & 1699.5 & B & 639620 & 6133864 & 1.4 & 9.7 & 0.9 & 9.6 & 17.7 & 50.6 & 0.8 & 0 & 25 \\
\hline $\mathrm{H}$ & 1702.3 & B & 639624 & 6133950 & 2.4 & 11.3 & 1.4 & 15.5 & 40.1 & 78.0 & 1.1 & 0 & 0 \\
\hline I & 1710.3 & $\mathrm{~B}$ & 639619 & 6134199 & 2.7 & 6.2 & 2.1 & 5.9 & 14.1 & 24.5 & 2.5 & 15 & 0 \\
\hline $\mathrm{J}$ & 1723.2 & $B$ ? & 639606 & 6134542 & 1.8 & 0.1 & 0.9 & 0.7 & 1.2 & 3.5 & --- & --- & 0 \\
\hline $\mathrm{K}$ & 1733.3 & D & 639580 & 6134868 & 4.6 & 4.2 & 1.9 & 2.9 & 9.2 & 9.3 & 6.6 & 26 & 0 \\
\hline L & 1764.7 & B & 639541 & 6135743 & 5.2 & 5.1 & 0.2 & 0.2 & 0.3 & 3.2 & 6.9 & 31 & 25 \\
\hline M & 1771.8 & $\mathrm{D}$ & 639555 & 6135954 & 9.2 & 11.8 & 3.0 & 12.2 & 33.5 & 35.9 & 3.9 & 9 & 25 \\
\hline $\mathrm{N}$ & 1790.0 & B? & 639520 & 6136300 & 0.2 & 0.9 & 2.4 & 0.8 & 2.8 & 6.8 & 4.4 & 93 & 0 \\
\hline 0 & 1843.0 & M & 639528 & 6136637 & 0.2 & 0.2 & 0.2 & 0.0 & 0.3 & 0.8 & -- & --- & 107 \\
\hline $\mathrm{P}$ & 1869.0 & S? & 639513 & 6137285 & 3.1 & 1.7 & 7.9 & 1.7 & 7.5 & 11.2 & --- & --- & 25 \\
\hline Q & 1871.9 & M & 639515 & 6137369 & 0.3 & 1.8 & 7.9 & 2.0 & 8.0 & 11.5 & --- & --- & 0 \\
\hline R & 1882.6 & $\mathrm{M}$ & 639496 & 6137686 & 1.7 & 1.4 & 5.2 & 0.9 & 4.5 & 5.5 & --- & --- & 0 \\
\hline $\mathrm{S}$ & 1898.3 & $\mathrm{~S}$ & 639497 & 6138171 & 0.1 & 0.8 & 3.1 & 1.1 & 3.1 & 7.4 & 5.7 & 80 & 0 \\
\hline $\mathrm{T}$ & 1922.8 & $\mathrm{M}$ & 639476 & 6138782 & 2.1 & 0.5 & 2.9 & 0.4 & 2.7 & 2.4 & -- & --- & 63 \\
\hline U & 1947.9 & S? & 639427 & 6139419 & 2.5 & 2.1 & 0.0 & 2.4 & 3.6 & 14.0 & 3.6 & 57 & 8 \\
\hline LINE & 10420 & & & & & & & & & & & & \\
\hline A & 2763.2 & $B$ ? & 640123 & 6131610 & 1.2 & 5.4 & 2.1 & 11.4 & 37.9 & 44.4 & 1.2 & 0 & 0 \\
\hline B & 2751.2 & B & 640112 & 6131975 & 1.6 & 9.4 & 1.6 & 15.1 & 46.5 & 62.1 & 0.9 & 0 & 28 \\
\hline $\mathrm{C}$ & 2729.3 & B & 640081 & 6132519 & 9.1 & 7.4 & 8.3 & 11.9 & 27.3 & 18.6 & 8.9 & 10 & 0 \\
\hline D & 2682.4 & B? & 640055 & 6133517 & 6.4 & 7.8 & 3.7 & 7.7 & 23.6 & 23.1 & 4.6 & 11 & 28 \\
\hline $\mathrm{E}$ & 2672.4 & B & 640033 & 6133829 & 7.3 & 20.7 & 12.9 & 35.2 & 91.2 & 79.2 & 3.4 & 0 & 0 \\
\hline $\mathrm{F}$ & 2645.3 & M & 640044 & 6134614 & 3.1 & 0.7 & 5.1 & 0.4 & 3.3 & 4.7 & -- & --- & 374 \\
\hline G & 2628.8 & $\mathrm{~S}$ & 639992 & 6135190 & 0.0 & 4.2 & 1.6 & 4.7 & 7.6 & 26.8 & 0.6 & 0 & 28 \\
\hline $\mathrm{H}$ & 2611.5 & B? & 639960 & 6135745 & 0.9 & 3.0 & 3.7 & 1.6 & 13.1 & 7.2 & 3.3 & 47 & 0 \\
\hline I & 2603.1 & B? & 639988 & 6135935 & 9.4 & 14.2 & 1.5 & 10.2 & 29.6 & 41.9 & 3.1 & 12 & 0 \\
\hline $\mathrm{J}$ & 2550.5 & M & 639940 & 6136930 & 0.1 & 0.7 & 2.5 & 0.9 & 2.4 & 4.4 & -- & --- & 0 \\
\hline K & 2529.8 & $\mathrm{~S}$ & 639919 & 6137531 & 3.3 & 1.6 & 7.7 & 1.9 & 7.7 & 11.6 & 33.0 & 24 & 0 \\
\hline L & 2523.0 & $\mathrm{M}$ & 639911 & 6137730 & 0.2 & 1.3 & 0.6 & 1.9 & 0.5 & 12.8 & -- & --- & 85 \\
\hline M & 2410.3 & S? & 639828 & 6138992 & 2.4 & 0.9 & 1.9 & 2.3 & 1.1 & 9.1 & --- & --- & 135 \\
\hline $\mathrm{N}$ & 2354.0 & B? & 639808 & 6140055 & 0.8 & 0.6 & 0.0 & 0.4 & 0.7 & 2.5 & --- & --- & 0 \\
\hline 0 & 2317.0 & $\dot{M}$ & 639795 & 6140724 & 0.2 & 0.3 & 0.5 & 0.5 & 0.4 & 4.5 & --- & --- & 93 \\
\hline $\mathrm{P}$ & 2312.7 & M & 639794 & 6140813 & 4.4 & 0.4 & 11.6 & 0.7 & 9.6 & 3.5 & --- & --- & 0 \\
\hline 0 & 2307.8 & $\mathrm{M}$ & 639789 & 6140901 & 0.4 & 0.4 & 6.0 & 0.8 & 5.2 & 1.7 & --- & --- & 0 \\
\hline R & 2300.9 & M & 639780 & 6140979 & 0.3 & 0.0 & 0.2 & 0.0 & 0.2 & 0.7 & --- & --- & 0 \\
\hline $\mathrm{S}$ & 2276.3 & $\mathrm{~B}$ ? & 639760 & 6141255 & 1.4 & 0.5 & 13.1 & 0.2 & 11.1 & 3.6 & --- & --- & 103 \\
\hline$\overline{\text { LINE }}$ & 10430 & & & & & & & & & & & & \\
\hline A & 2803.7 & B & 640549 & 6131504 & 0.4 & 3.7 & 1.3 & 5.3 & 10.9 & 15.2 & 0.7 & 0 & 0 \\
\hline
\end{tabular}

$\mathrm{CX}=$ COAXIAL

Note: EM values shown above

Ketchikan - North/South of 5515
* Estimated Depth may be unreliable because the stronger part of the conductor may be deeper or of side of the flight line, or because of 
EM Anomaly List

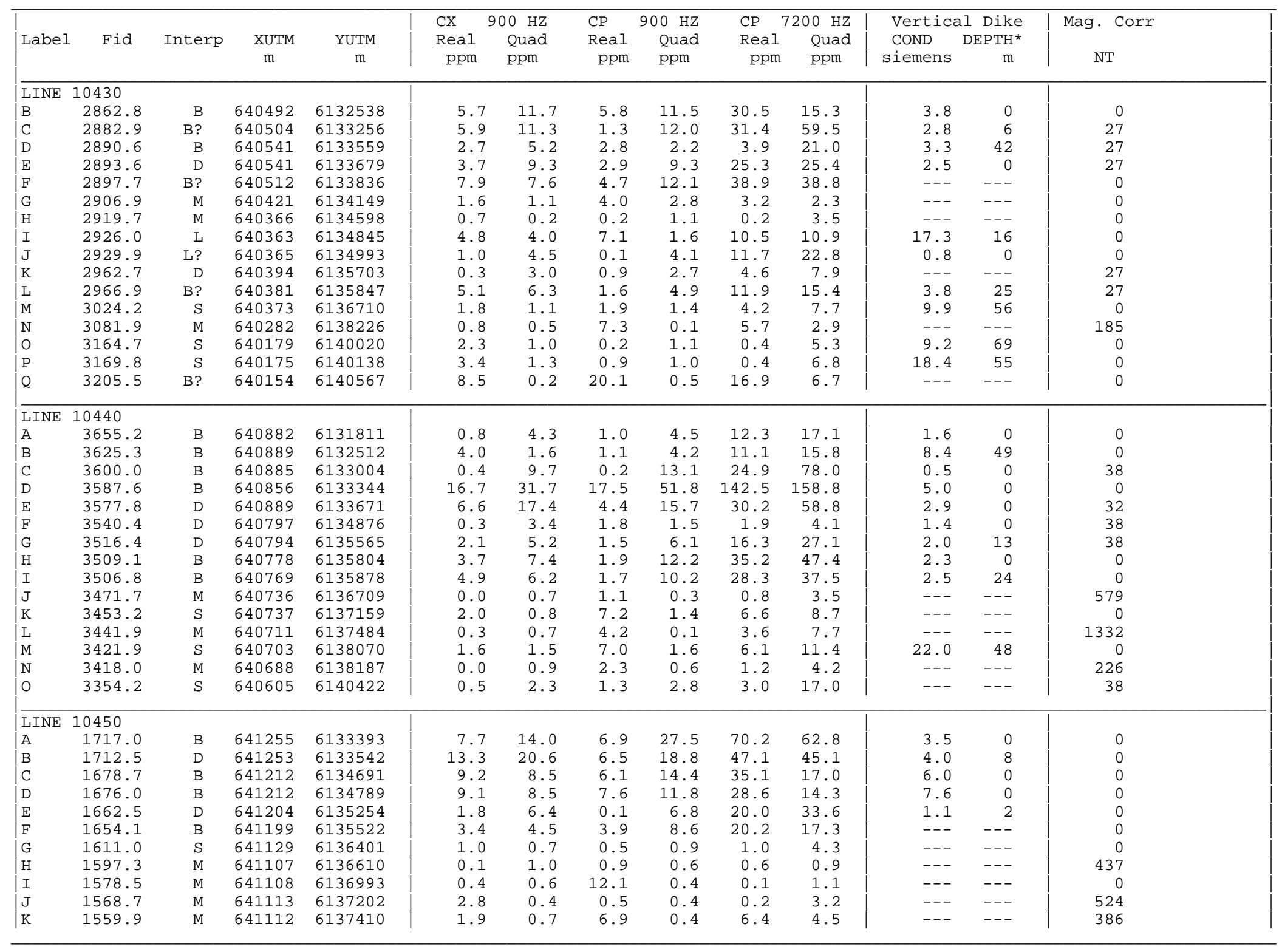

$\mathrm{CX}=$ COAXIAL

Note: EM values shown above

are local amplitudes
*Estimated Depth may be unreliable because the stronger part of the conductor may be deeper or to one side of the flight line, or because of a shallow dip or magnetite/overburden effects 
EM Anomaly List

\begin{tabular}{|c|c|c|c|c|c|c|c|c|c|c|c|c|c|}
\hline Label & Fid & Interp & $\begin{array}{l}\text { XUTM } \\
\text { m }\end{array}$ & $\begin{array}{c}\text { YUTM } \\
\mathrm{m}\end{array}$ & $\begin{array}{l}\text { CX } \\
\text { Real } \\
\text { ppm }\end{array}$ & $\begin{array}{c}900 \mathrm{HZ} \\
\text { Quad } \\
\text { ppm }\end{array}$ & $\begin{array}{l}\text { CP } \\
\text { Real } \\
\text { ppm }\end{array}$ & $\begin{array}{c}900 \mathrm{HZ} \\
\text { Quad } \\
\text { ppm }\end{array}$ & $\begin{array}{l}\text { CP } \\
\text { Real } \\
\text { ppm }\end{array}$ & $\begin{array}{c}7200 \mathrm{HZ} \\
\text { Quad } \\
\text { ppm }\end{array}$ & $\begin{array}{l}\text { Vertica } \\
\text { CoND } \\
\text { siemens }\end{array}$ & $\begin{array}{c}\text { al Dike } \\
\text { DEPTH* } \\
\text { m }\end{array}$ & $\begin{array}{c}\text { Mag. Corr } \\
\text { NT }\end{array}$ \\
\hline $\begin{array}{l}\text { LINE } \\
\text { L } \\
M \\
N \\
O \\
P\end{array}$ & $\begin{array}{l}10450 \\
1538.1 \\
1517.9 \\
1497.8 \\
1459.4 \\
1388.1\end{array}$ & $\begin{array}{c}\mathrm{S} \\
\mathrm{M} \\
\mathrm{M} \\
\mathrm{B} ? \\
\mathrm{M}\end{array}$ & $\begin{array}{l}641084 \\
641071 \\
641051 \\
641005 \\
640945\end{array}$ & $\begin{array}{l}6137999 \\
6138394 \\
6138793 \\
6140000 \\
6141700\end{array}$ & $\begin{array}{l}2.0 \\
0.4 \\
0.6 \\
0.7 \\
1.2\end{array}$ & $\begin{array}{l}1.9 \\
0.5 \\
1.0 \\
3.6 \\
0.3\end{array}$ & $\begin{array}{l}0.3 \\
2.4 \\
1.0 \\
0.6 \\
2.6\end{array}$ & $\begin{array}{l}2.3 \\
0.5 \\
0.5 \\
2.5 \\
0.1\end{array}$ & $\begin{array}{l}3.9 \\
0.1 \\
0.2 \\
4.1 \\
1.9\end{array}$ & $\begin{array}{r}18.2 \\
0.1 \\
2.8 \\
16.6 \\
3.8\end{array}$ & $\begin{array}{l}3.0 \\
--- \\
--- \\
0.9 \\
---\end{array}$ & $\begin{array}{r}73 \\
--- \\
--- \\
17 \\
---\end{array}$ & $\begin{array}{r}32 \\
0 \\
147 \\
22 \\
32\end{array}$ \\
\hline $\begin{array}{l}\text { LINE } \\
\text { A } \\
\text { B } \\
\text { C } \\
\text { D } \\
\text { E } \\
\text { F }\end{array}$ & $\begin{array}{r}10460 \\
725.2 \\
688.0 \\
684.9 \\
661.2 \\
621.8 \\
562.6\end{array}$ & $\begin{array}{l}\mathrm{M} \\
\mathrm{M} \\
\mathrm{M} \\
\mathrm{M} \\
\mathrm{S} \\
\mathrm{S}\end{array}$ & $\begin{array}{l}641578 \\
641512 \\
641502 \\
641539 \\
641470 \\
641359\end{array}$ & $\begin{array}{l}6135489 \\
6136412 \\
6136519 \\
6137294 \\
6138481 \\
6140935\end{array}$ & $\begin{array}{l}1.0 \\
2.0 \\
0.0 \\
0.0 \\
1.6 \\
0.7\end{array}$ & $\begin{array}{l}0.6 \\
0.8 \\
0.6 \\
0.4 \\
2.9 \\
1.3\end{array}$ & $\begin{array}{l}2.2 \\
1.1 \\
4.4 \\
5.1 \\
1.5 \\
0.9\end{array}$ & $\begin{array}{l}0.8 \\
0.3 \\
0.8 \\
0.5 \\
5.8 \\
2.1\end{array}$ & $\begin{array}{l}3.6 \\
0.6 \\
3.5 \\
4.7 \\
4.2 \\
3.1\end{array}$ & $\begin{array}{r}3.2 \\
3.2 \\
3.0 \\
7.7 \\
37.0 \\
12.2\end{array}$ & $\begin{array}{l}--- \\
--- \\
--- \\
--- \\
2.1 \\
---\end{array}$ & $\begin{array}{r}--- \\
--- \\
--- \\
--- \\
24 \\
---\end{array}$ & $\begin{array}{r}0 \\
531 \\
9 \\
850 \\
33 \\
33\end{array}$ \\
\hline $\begin{array}{l}\text { LINE } \\
\text { A } \\
\text { B } \\
\text { C } \\
\text { D } \\
\text { E } \\
\text { F } \\
\text { G } \\
\text { H } \\
\text { I } \\
\text { J } \\
\text { K } \\
\text { L }\end{array}$ & $\begin{array}{c}10461 \\
912.4 \\
923.7 \\
929.9 \\
934.4 \\
936.2 \\
965.7 \\
991.6 \\
997.0 \\
1051.8 \\
1061.7 \\
1068.7 \\
1090.8\end{array}$ & $\begin{array}{l}\text { B } \\
B \\
D \\
B \\
D \\
B \\
D \\
D \\
M \\
M \\
M \\
M\end{array}$ & $\begin{array}{l}641696 \\
641693 \\
641679 \\
641668 \\
641664 \\
641638 \\
641603 \\
641578 \\
641556 \\
641569 \\
641559 \\
641513\end{array}$ & $\begin{array}{l}6132784 \\
6133112 \\
6133309 \\
6133461 \\
6133524 \\
6134504 \\
6134983 \\
6135143 \\
6136122 \\
6136367 \\
6136582 \\
6137262\end{array}$ & $\begin{array}{r}0.1 \\
2.9 \\
14.0 \\
8.4 \\
25.6 \\
14.8 \\
3.7 \\
1.3 \\
1.6 \\
0.9 \\
2.4 \\
3.2\end{array}$ & $\begin{array}{r}1.3 \\
4.9 \\
23.9 \\
22.9 \\
39.1 \\
17.9 \\
7.5 \\
4.2 \\
0.8 \\
0.1 \\
1.0 \\
0.5\end{array}$ & $\begin{array}{r}0.1 \\
1.5 \\
4.5 \\
13.3 \\
13.7 \\
12.5 \\
1.8 \\
0.9 \\
1.8 \\
8.4 \\
6.1 \\
8.9\end{array}$ & $\begin{array}{r}2.3 \\
4.4 \\
22.6 \\
37.1 \\
43.1 \\
27.6 \\
4.3 \\
3.7 \\
0.4 \\
0.2 \\
0.7 \\
0.6\end{array}$ & $\begin{array}{r}5.4 \\
9.3 \\
65.6 \\
84.4 \\
106.9 \\
71.9 \\
8.0 \\
6.4 \\
1.8 \\
7.0 \\
5.4 \\
7.0\end{array}$ & $\begin{array}{r}12.6 \\
16.7 \\
72.1 \\
87.5 \\
111.2 \\
56.8 \\
15.5 \\
17.3 \\
0.8 \\
0.3 \\
2.8 \\
5.7\end{array}$ & $\begin{array}{l}0.5 \\
--- \\
4.5 \\
3.6 \\
4.8 \\
5.7 \\
3.0 \\
1.5 \\
--- \\
--- \\
--- \\
---\end{array}$ & $\begin{array}{r}0 \\
--- \\
0 \\
0 \\
5 \\
4 \\
6 \\
20 \\
--- \\
--- \\
--- \\
---\end{array}$ & $\begin{array}{r}0 \\
45 \\
0 \\
0 \\
45 \\
0 \\
45 \\
45 \\
0 \\
717 \\
81 \\
471\end{array}$ \\
\hline $\begin{array}{l}\text { LINE } \\
\text { A } \\
\text { B } \\
\text { C } \\
\text { D } \\
\text { E } \\
\text { F } \\
\text { G } \\
\text { H } \\
\text { I } \\
\text { J } \\
\text { K } \\
\text { L } \\
\text { M } \\
\text { N } \\
\text { O } \\
\text { P } \\
\text { Q }\end{array}$ & $\begin{array}{r}10470 \\
224.8 \\
237.3 \\
245.8 \\
251.0 \\
256.9 \\
273.1 \\
284.2 \\
288.7 \\
306.0 \\
327.8 \\
332.0 \\
340.3 \\
343.9 \\
345.8 \\
352.4 \\
377.9 \\
446.7\end{array}$ & $\begin{array}{r}\text { B } \\
\text { B? } \\
\text { B } \\
\text { B } \\
\text { D } \\
\text { B? } \\
\text { D } \\
\text { B? } \\
\text { D } \\
\text { M } \\
\text { B? } \\
\text { M } \\
\text { S } \\
\text { M } \\
\text { M } \\
\text { M } \\
\text { B? }\end{array}$ & $\begin{array}{l}642086 \\
642113 \\
642067 \\
642023 \\
641990 \\
641952 \\
641959 \\
641977 \\
641977 \\
641929 \\
641918 \\
641892 \\
641883 \\
641882 \\
641895 \\
641947 \\
641852\end{array}$ & $\begin{array}{l}6132498 \\
6132868 \\
6133171 \\
6133367 \\
6133588 \\
6134145 \\
6134471 \\
6134592 \\
6135137 \\
6135759 \\
6135861 \\
6136046 \\
6136139 \\
6136192 \\
6136386 \\
6137195 \\
6139516\end{array}$ & $\begin{array}{r}2.2 \\
1.4 \\
12.1 \\
2.2 \\
14.1 \\
4.2 \\
8.2 \\
2.8 \\
7.6 \\
0.0 \\
2.2 \\
0.0 \\
8.0 \\
9.8 \\
0.2 \\
1.9 \\
3.1\end{array}$ & $\begin{array}{r}3.5 \\
9.5 \\
16.4 \\
7.0 \\
29.0 \\
4.9 \\
3.1 \\
3.1 \\
12.6 \\
0.7 \\
0.3 \\
0.8 \\
1.4 \\
0.5 \\
0.7 \\
0.6 \\
5.5\end{array}$ & $\begin{array}{r}0.7 \\
0.6 \\
3.5 \\
4.6 \\
5.3 \\
3.4 \\
7.6 \\
8.2 \\
1.4 \\
4.3 \\
3.8 \\
3.3 \\
20.5 \\
22.2 \\
5.5 \\
1.2 \\
0.9\end{array}$ & $\begin{array}{r}1.0 \\
8.5 \\
12.5 \\
13.7 \\
32.0 \\
4.9 \\
8.0 \\
8.6 \\
3.3 \\
0.9 \\
1.1 \\
0.6 \\
1.7 \\
1.7 \\
0.6 \\
0.4 \\
5.0\end{array}$ & $\begin{array}{r}2.4 \\
15.3 \\
37.1 \\
33.4 \\
79.8 \\
15.3 \\
19.2 \\
20.2 \\
7.4 \\
3.2 \\
3.5 \\
1.7 \\
17.6 \\
19.2 \\
5.3 \\
4.0 \\
11.2\end{array}$ & $\begin{array}{r}6.7 \\
41.0 \\
40.9 \\
47.1 \\
86.6 \\
27.2 \\
10.3 \\
11.2 \\
14.5 \\
7.4 \\
7.7 \\
6.5 \\
10.8 \\
10.8 \\
5.3 \\
0.4 \\
34.8\end{array}$ & $\begin{array}{r}---- \\
0.7 \\
4.4 \\
2.2 \\
3.8 \\
5.0 \\
18.5 \\
6.5 \\
3.4 \\
--- \\
--- \\
--- \\
176.7 \\
--- \\
--- \\
--- \\
2.6\end{array}$ & $\begin{array}{r}--- \\
0 \\
14 \\
9 \\
6 \\
30 \\
14 \\
34 \\
11 \\
--- \\
--- \\
--- \\
43 \\
--- \\
--- \\
--- \\
15\end{array}$ & $\begin{array}{r}27 \\
0 \\
26 \\
27 \\
0 \\
0 \\
27 \\
0 \\
0 \\
23 \\
27 \\
23 \\
27 \\
0 \\
748 \\
96 \\
0\end{array}$ \\
\hline
\end{tabular}

$\mathrm{CX}=$ COAXIAL

Note: EM values shown above

are local amplitudes

Ketchikan - North/South of 5515
* Estimated Depth may be unreliable because the stronger part of the conductor may be deeper or to one side of the flight line, or because of a shallow dip or magnetite/overburden effects 
EM Anomaly List

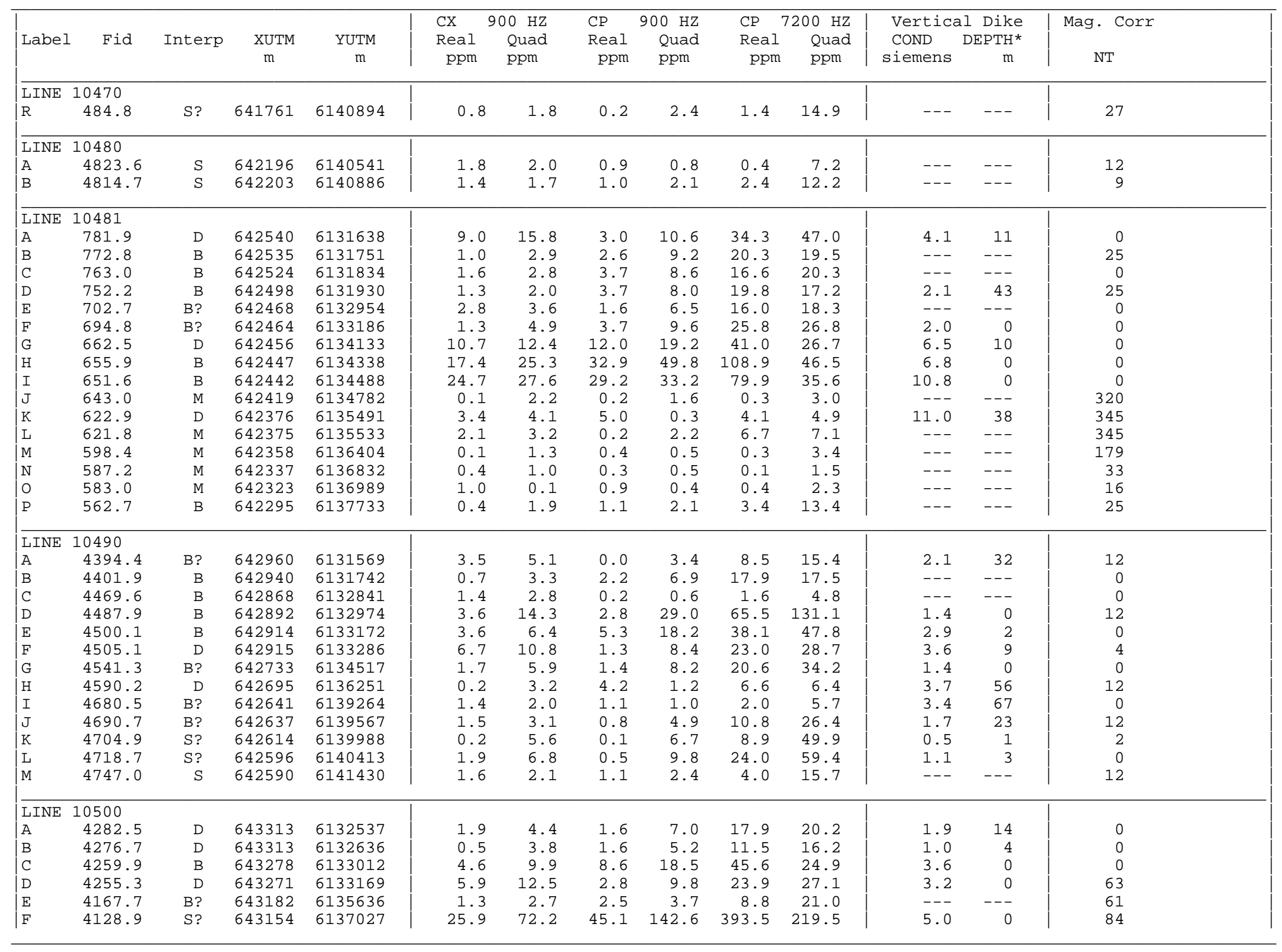

$\mathrm{CX}=$ COAXIAL

Note: EM values shown above

are local amplitudes

Ketchikan - North/South of 5515
* Estimated Depth may be unreliable because the stronger part of the conductor may be deeper or to one side of the flight line, or because of a shallow dip or magnetite/overburden effects 
EM Anomaly List

\begin{tabular}{|c|c|c|c|c|c|c|c|c|c|c|c|c|c|}
\hline Label & Fid & Interp & $\underset{\mathrm{m}}{\mathrm{XUTM}}$ & $\begin{array}{c}\text { YUTM } \\
\text { m }\end{array}$ & $\begin{array}{l}\text { CX } \\
\text { Real } \\
\text { ppm }\end{array}$ & $\begin{array}{c}900 \mathrm{HZ} \\
\text { Quad } \\
\text { ppm }\end{array}$ & $\begin{array}{l}\text { CP } \\
\text { Real } \\
\text { ppm }\end{array}$ & $\begin{array}{c}900 \mathrm{HZ} \\
\text { Quad } \\
\text { ppm }\end{array}$ & $\begin{array}{l}\text { CP } \\
\text { Real } \\
\text { ppm }\end{array}$ & $\begin{array}{c}7200 \mathrm{HZ} \\
\text { Quad } \\
\text { ppm }\end{array}$ & $\begin{array}{l}\text { Vertica } \\
\text { COND } \\
\text { siemens }\end{array}$ & $\begin{array}{c}\text { al Dike } \\
\text { DEPTH* } \\
\text { m }\end{array}$ & $\begin{array}{c}\text { Mag. Corr } \\
\text { NT }\end{array}$ \\
\hline $\begin{array}{l}\text { LINE } \\
\text { G } \\
\text { H } \\
\text { I } \\
\text { J } \\
\text { K } \\
\text { L } \\
\text { M } \\
\text { N }\end{array}$ & $\begin{array}{r}10500 \\
4124.9 \\
4113.4 \\
4078.3 \\
4068.4 \\
4054.4 \\
4038.4 \\
4023.9 \\
4012.8\end{array}$ & $\begin{array}{r}\text { S? } \\
\text { S? } \\
\text { B? } \\
\text { D } \\
\text { B } \\
\text { S? } \\
\text { S? } \\
\text { S? }\end{array}$ & $\begin{array}{l}643147 \\
643125 \\
643055 \\
643045 \\
643051 \\
643039 \\
643002 \\
642968\end{array}$ & $\begin{array}{l}6137193 \\
6137666 \\
6139085 \\
6139473 \\
6140012 \\
6140596 \\
6141100 \\
6141480\end{array}$ & $\begin{array}{r}19.3 \\
8.4 \\
2.2 \\
2.0 \\
0.0 \\
1.4 \\
2.6 \\
4.1\end{array}$ & $\begin{array}{r}24.9 \\
27.7 \\
2.5 \\
5.7 \\
6.9 \\
6.1 \\
3.4 \\
2.6\end{array}$ & $\begin{array}{r}16.1 \\
6.1 \\
0.9 \\
2.0 \\
0.7 \\
0.9 \\
0.3 \\
4.2\end{array}$ & $\begin{array}{r}39.2 \\
46.2 \\
2.8 \\
4.0 \\
9.8 \\
9.0 \\
5.0 \\
2.1\end{array}$ & $\begin{array}{r}56.1 \\
132.2 \\
10.6 \\
10.4 \\
26.8 \\
25.1 \\
6.5 \\
4.7\end{array}$ & $\begin{array}{r}41.3 \\
173.5 \\
5.0 \\
6.2 \\
54.9 \\
44.2 \\
32.9 \\
14.7\end{array}$ & $\begin{array}{r}5.6 \\
2.2 \\
--- \\
2.2 \\
0.5 \\
1.0 \\
2.5 \\
15.0\end{array}$ & $\begin{array}{r}0 \\
0 \\
--- \\
15 \\
2 \\
0 \\
21 \\
35\end{array}$ & $\begin{array}{r}0 \\
17 \\
0 \\
17 \\
0 \\
46 \\
0 \\
0\end{array}$ \\
\hline $\begin{array}{l}\text { LINE } \\
\text { A } \\
\text { B } \\
\text { C } \\
\text { D } \\
\text { E } \\
\text { F }\end{array}$ & $\begin{array}{r}10511 \\
3435.8 \\
3361.3 \\
3346.5 \\
3336.0 \\
3326.2 \\
3318.5\end{array}$ & $\begin{array}{l}\text { S? } \\
\text { S? } \\
\text { B? } \\
\text { B? } \\
\text { B } \\
\text { D }\end{array}$ & $\begin{array}{l}643720 \\
643755 \\
643766 \\
643743 \\
643683 \\
643681\end{array}$ & $\begin{array}{l}6131766 \\
6132344 \\
6132476 \\
6132673 \\
6132872 \\
6132983\end{array}$ & $\begin{array}{l}0.3 \\
1.6 \\
0.7 \\
1.6 \\
1.1 \\
4.2\end{array}$ & $\begin{array}{r}1.6 \\
1.2 \\
2.4 \\
4.0 \\
11.0 \\
3.4\end{array}$ & $\begin{array}{l}0.8 \\
0.1 \\
0.0 \\
1.5 \\
2.5 \\
1.6\end{array}$ & $\begin{array}{r}1.8 \\
2.1 \\
1.1 \\
2.6 \\
13.3 \\
3.6\end{array}$ & $\begin{array}{r}3.1 \\
4.8 \\
3.4 \\
3.7 \\
34.3 \\
6.8\end{array}$ & $\begin{array}{r}12.5 \\
14.5 \\
5.7 \\
7.5 \\
53.5 \\
18.6\end{array}$ & $\begin{array}{l}--- \\
--- \\
1.1 \\
2.2 \\
0.9 \\
---\end{array}$ & $\begin{array}{r}--- \\
--- \\
10 \\
17 \\
0 \\
---\end{array}$ & $\begin{array}{r}0 \\
6 \\
6 \\
29 \\
0 \\
7\end{array}$ \\
\hline $\begin{array}{l}\text { LINE } \\
\text { A } \\
\text { B } \\
\text { C } \\
\text { D } \\
\text { E } \\
\text { F } \\
\text { G } \\
\text { H } \\
\text { I } \\
\text { J } \\
\text { K } \\
\text { L } \\
\text { M } \\
\text { N }\end{array}$ & $\begin{array}{r}10512 \\
3682.3 \\
3690.9 \\
3694.3 \\
3700.7 \\
3703.4 \\
3735.0 \\
3745.5 \\
3749.4 \\
3751.1 \\
3795.4 \\
3809.8 \\
3815.1 \\
3886.7 \\
3919.2\end{array}$ & $\begin{array}{r}\text { B } \\
\text { B? } \\
\text { B } \\
\text { B } \\
\text { D } \\
\text { B } \\
\text { B? } \\
\text { B } \\
\text { M } \\
\text { B? } \\
\text { S? } \\
\text { S? } \\
\text { B? } \\
\text { B? }\end{array}$ & $\begin{array}{l}643692 \\
643676 \\
643667 \\
643657 \\
643656 \\
643658 \\
643635 \\
643619 \\
643612 \\
643594 \\
643569 \\
643572 \\
643464 \\
643417\end{array}$ & $\begin{array}{l}6132631 \\
6132858 \\
6132946 \\
6133097 \\
6133170 \\
6133915 \\
6134124 \\
6134207 \\
6134245 \\
6135623 \\
6136095 \\
6136277 \\
6138956 \\
6140053\end{array}$ & $\begin{array}{r}4.2 \\
4.5 \\
4.5 \\
3.5 \\
11.4 \\
1.2 \\
7.4 \\
6.5 \\
3.1 \\
2.2 \\
3.8 \\
3.6 \\
3.7 \\
1.0\end{array}$ & $\begin{array}{r}6.0 \\
14.5 \\
12.0 \\
9.4 \\
29.7 \\
2.9 \\
9.9 \\
9.6 \\
9.6 \\
5.5 \\
19.9 \\
35.4 \\
11.7 \\
2.6\end{array}$ & $\begin{array}{l}1.1 \\
2.5 \\
0.4 \\
0.8 \\
2.8 \\
0.2 \\
8.9 \\
7.2 \\
4.0 \\
0.4 \\
5.5 \\
4.9 \\
3.0 \\
0.1\end{array}$ & $\begin{array}{r}3.7 \\
14.8 \\
16.5 \\
9.9 \\
17.3 \\
2.9 \\
10.5 \\
10.8 \\
10.5 \\
8.4 \\
26.0 \\
75.8 \\
16.5 \\
2.0\end{array}$ & $\begin{array}{r}7.4 \\
38.7 \\
44.8 \\
25.5 \\
56.2 \\
3.8 \\
36.8 \\
36.8 \\
32.3 \\
19.5 \\
76.2 \\
245.5 \\
47.9 \\
2.8\end{array}$ & $\begin{array}{r}6.0 \\
62.4 \\
75.4 \\
22.4 \\
76.1 \\
18.6 \\
37.3 \\
38.6 \\
38.0 \\
40.7 \\
132.5 \\
297.9 \\
36.9 \\
21.4\end{array}$ & $\begin{array}{l}3.0 \\
2.0 \\
1.7 \\
--- \\
3.2 \\
1.4 \\
6.0 \\
4.6 \\
--- \\
1.5 \\
1.7 \\
0.9 \\
1.9 \\
1.2\end{array}$ & $\begin{array}{r}25 \\
0 \\
0 \\
--- \\
0 \\
34 \\
21 \\
33 \\
--- \\
9 \\
0 \\
0 \\
0 \\
22\end{array}$ & $\begin{array}{r}0 \\
0 \\
32 \\
0 \\
0 \\
0 \\
0 \\
0 \\
251 \\
0 \\
17 \\
107 \\
1 \\
0\end{array}$ \\
\hline $\begin{array}{l}\text { LINE } \\
\text { A } \\
\text { B } \\
\text { C } \\
\text { D } \\
\text { E } \\
\text { F }\end{array}$ & $\begin{array}{r}10520 \\
3069.2 \\
3061.2 \\
3056.4 \\
3004.9 \\
2996.0 \\
2962.6\end{array}$ & $\begin{array}{r}\text { B? } \\
\text { S? } \\
\text { D } \\
\text { D } \\
\text { B? } \\
\text { S? }\end{array}$ & $\begin{array}{l}644086 \\
644093 \\
644093 \\
644072 \\
644061 \\
644043\end{array}$ & $\begin{array}{l}6132719 \\
6132970 \\
6133108 \\
6134007 \\
6134161 \\
6135187\end{array}$ & $\begin{array}{l}5.5 \\
3.4 \\
5.9 \\
2.1 \\
2.9 \\
1.5\end{array}$ & $\begin{array}{r}8.1 \\
8.0 \\
12.2 \\
4.6 \\
4.7 \\
4.0\end{array}$ & $\begin{array}{l}1.0 \\
2.9 \\
1.1 \\
0.2 \\
2.1 \\
1.9\end{array}$ & $\begin{array}{r}5.5 \\
13.2 \\
6.8 \\
4.3 \\
9.8 \\
5.9\end{array}$ & $\begin{array}{l}16.5 \\
39.2 \\
18.2 \\
14.2 \\
26.3 \\
12.5\end{array}$ & $\begin{array}{l}20.0 \\
30.2 \\
23.7 \\
19.2 \\
25.4 \\
36.6\end{array}$ & $\begin{array}{l}2.9 \\
2.3 \\
3.0 \\
1.8 \\
--- \\
---\end{array}$ & $\begin{array}{r}9 \\
0 \\
8 \\
18 \\
--- \\
---\end{array}$ & $\begin{array}{r}0 \\
0 \\
3 \\
20 \\
0 \\
19\end{array}$ \\
\hline $\begin{array}{l}\text { LINE } \\
\text { A } \\
\text { B } \\
\text { C } \\
\text { D }\end{array}$ & $\begin{array}{r}10530 \\
2487.3 \\
2492.2 \\
2503.9 \\
2544.8\end{array}$ & $\begin{array}{r}\text { S? } \\
\text { B } \\
\text { B? } \\
\text { S? }\end{array}$ & $\begin{array}{l}644457 \\
644454 \\
644484 \\
644422\end{array}$ & $\begin{array}{l}6133659 \\
6133755 \\
6133964 \\
6134949\end{array}$ & $\begin{array}{l}0.6 \\
3.2 \\
2.0 \\
1.3\end{array}$ & $\begin{array}{l}2.7 \\
1.5 \\
7.0 \\
2.5\end{array}$ & $\begin{array}{l}0.5 \\
0.6 \\
2.0 \\
1.5\end{array}$ & $\begin{array}{l}3.0 \\
1.0 \\
8.4 \\
3.5\end{array}$ & $\begin{array}{r}3.9 \\
5.8 \\
20.5 \\
4.3\end{array}$ & $\begin{array}{r}17.7 \\
4.2 \\
31.0 \\
22.6\end{array}$ & $\begin{array}{l}--- \\
--- \\
1.6 \\
2.4\end{array}$ & $\begin{array}{r}--- \\
-- \\
1 \\
30\end{array}$ & $\begin{array}{l}0 \\
0 \\
0 \\
0\end{array}$ \\
\hline
\end{tabular}

$\mathrm{CX}=$ COAXIAL

Note: EM values shown above

are local amplitudes

Ketchikan - North/South of 5515
* Estimated Depth may be unreliable because the stronger part of the conductor may be deeper or to one side of the flight line, or because of a shallow dip or magnetite/overburden effects 
EM Anomaly List

\begin{tabular}{|c|c|c|c|c|c|c|c|c|c|c|c|c|c|}
\hline Label & Fid & Interp & $\begin{array}{c}\text { XUTM } \\
\text { m }\end{array}$ & $\begin{array}{c}\text { YUTM } \\
\mathrm{m}\end{array}$ & $\begin{array}{l}\text { CX } \\
\text { Real } \\
\text { ppm }\end{array}$ & $\begin{array}{l}00 \mathrm{HZ} \\
\text { Quad } \\
\text { ppm }\end{array}$ & $\begin{array}{l}\text { CP } \\
\text { Real } \\
\text { ppm }\end{array}$ & $\begin{array}{l}900 \mathrm{HZ} \\
\text { Quad } \\
\text { ppm }\end{array}$ & $\begin{array}{l}\mathrm{CP} \\
\mathrm{Real} \\
\mathrm{ppm}\end{array}$ & $\begin{array}{c}7200 \mathrm{HZ} \\
\text { Quad } \\
\text { ppm }\end{array}$ & $\begin{array}{l}\text { Vertica } \\
\text { Cond } \\
\text { siemens }\end{array}$ & $\begin{array}{c}\text { al Dike } \\
\text { DEPTH* } \\
\text { m }\end{array}$ & $\begin{array}{c}\text { Mag. Corr } \\
\text { NT }\end{array}$ \\
\hline $\begin{array}{l}\text { LINE } \\
\text { E } \\
F \\
G \\
H \\
I\end{array}$ & $\begin{array}{l}10530 \\
2550.1 \\
2559.8 \\
2562.3 \\
2613.1 \\
2714.4\end{array}$ & $\begin{array}{c}\mathrm{L} \\
\mathrm{S} ? \\
\mathrm{M} \\
\mathrm{B} ? \\
\mathrm{~B} ?\end{array}$ & $\begin{array}{l}644418 \\
644406 \\
644406 \\
644385 \\
644219\end{array}$ & $\begin{array}{l}6135117 \\
6135401 \\
6135469 \\
6136861 \\
6140511\end{array}$ & $\begin{array}{r}0.8 \\
15.6 \\
5.4 \\
1.3 \\
0.3\end{array}$ & $\begin{array}{l}1.5 \\
2.4 \\
2.5 \\
3.1 \\
3.4\end{array}$ & $\begin{array}{r}13.8 \\
1.0 \\
4.6 \\
0.2 \\
0.5\end{array}$ & $\begin{array}{l}2.8 \\
2.4 \\
2.4 \\
1.9 \\
5.4\end{array}$ & $\begin{array}{r}4.9 \\
2.3 \\
3.7 \\
0.2 \\
11.2\end{array}$ & $\begin{array}{l}16.3 \\
23.9 \\
24.1 \\
14.2 \\
28.0\end{array}$ & $\begin{array}{c}31.8 \\
--- \\
--- \\
1.6 \\
0.7\end{array}$ & $\begin{array}{r}41 \\
--- \\
--- \\
28 \\
6\end{array}$ & $\begin{array}{r}18 \\
0 \\
572 \\
0 \\
34\end{array}$ \\
\hline $\begin{array}{l}\text { LINE } \\
\text { A } \\
\text { B } \\
\text { C } \\
\text { D } \\
\text { E } \\
\text { F }\end{array}$ & $\begin{array}{r}10540 \\
1988.4 \\
1992.2 \\
2002.4 \\
2078.1 \\
2251.4 \\
2300.9\end{array}$ & $\begin{array}{r}\mathrm{D} \\
\mathrm{B} \\
\mathrm{B} \\
\mathrm{B} ? \\
\mathrm{D} \\
\mathrm{B} ?\end{array}$ & $\begin{array}{l}644920 \\
644916 \\
644898 \\
644888 \\
644661 \\
644589\end{array}$ & $\begin{array}{l}6132338 \\
6132440 \\
6132710 \\
6134019 \\
6138443 \\
6140410\end{array}$ & $\begin{array}{r}6.1 \\
3.2 \\
3.4 \\
5.2 \\
31.9 \\
0.8\end{array}$ & $\begin{array}{r}9.4 \\
5.9 \\
8.0 \\
6.0 \\
16.3 \\
5.7\end{array}$ & $\begin{array}{r}1.8 \\
5.4 \\
2.1 \\
0.5 \\
32.0 \\
3.0\end{array}$ & $\begin{array}{r}9.2 \\
15.5 \\
12.0 \\
4.0 \\
22.6 \\
9.1\end{array}$ & $\begin{array}{r}25.2 \\
41.0 \\
34.4 \\
8.5 \\
52.3 \\
21.6\end{array}$ & $\begin{array}{l}26.9 \\
34.2 \\
35.2 \\
11.9 \\
25.5 \\
39.9\end{array}$ & $\begin{array}{r}2.6 \\
--- \\
2.1 \\
3.8 \\
30.6 \\
1.3\end{array}$ & $\begin{array}{r}21 \\
--- \\
0 \\
22 \\
0 \\
3\end{array}$ & $\begin{array}{r}0 \\
12 \\
0 \\
12 \\
169 \\
49\end{array}$ \\
\hline $\begin{array}{l}\text { LINE } \\
\text { A } \\
\text { B } \\
\text { C } \\
\text { D } \\
\text { E } \\
\text { F } \\
\text { G } \\
\text { H } \\
\text { I } \\
\text { J } \\
\text { K } \\
\text { L }\end{array}$ & $\begin{array}{r}10550 \\
2817.5 \\
2807.2 \\
2791.1 \\
2682.3 \\
2662.1 \\
2634.1 \\
2627.5 \\
2578.1 \\
2522.1 \\
2520.0 \\
2499.5 \\
2484.8\end{array}$ & $\begin{array}{r}\text { B? } \\
\text { B? } \\
\text { B? } \\
\text { B } \\
\text { M } \\
\text { B } \\
\text { B } \\
\text { S? } \\
\text { B? } \\
\text { D } \\
\text { B? } \\
\text { B? }\end{array}$ & $\begin{array}{l}645305 \\
645302 \\
645291 \\
645293 \\
645255 \\
645237 \\
645220 \\
645169 \\
645121 \\
645117 \\
645115 \\
645113\end{array}$ & $\begin{array}{l}6132162 \\
6132397 \\
6132704 \\
6133957 \\
6134411 \\
6135221 \\
6135329 \\
6136353 \\
6138158 \\
6138219 \\
6138646 \\
6139019\end{array}$ & $\begin{array}{r}2.6 \\
6.1 \\
0.8 \\
10.2 \\
0.5 \\
1.6 \\
1.5 \\
1.3 \\
14.7 \\
14.7 \\
4.2 \\
1.6\end{array}$ & $\begin{array}{r}5.6 \\
8.2 \\
2.9 \\
15.0 \\
0.1 \\
3.0 \\
1.8 \\
1.6 \\
6.5 \\
6.5 \\
3.3 \\
5.5\end{array}$ & $\begin{array}{r}3.7 \\
2.2 \\
1.7 \\
12.5 \\
0.2 \\
1.3 \\
0.2 \\
0.5 \\
22.3 \\
22.3 \\
0.4 \\
0.5\end{array}$ & $\begin{array}{r}9.6 \\
4.0 \\
2.9 \\
30.2 \\
0.0 \\
3.5 \\
2.3 \\
2.5 \\
17.1 \\
17.1 \\
7.1 \\
6.2\end{array}$ & $\begin{array}{r}19.3 \\
11.8 \\
9.5 \\
75.3 \\
0.1 \\
4.7 \\
3.0 \\
2.9 \\
38.5 \\
38.5 \\
15.6 \\
17.7\end{array}$ & $\begin{array}{r}14.1 \\
24.5 \\
8.3 \\
47.7 \\
0.2 \\
21.2 \\
11.2 \\
15.6 \\
12.0 \\
12.0 \\
47.3 \\
39.1\end{array}$ & $\begin{array}{r}2.7 \\
4.4 \\
1.8 \\
3.9 \\
--- \\
2.3 \\
1.8 \\
1.7 \\
24.4 \\
24.4 \\
3.3 \\
1.1\end{array}$ & $\begin{array}{r}8 \\
24 \\
16 \\
19 \\
--- \\
32 \\
83 \\
78 \\
3 \\
5 \\
44 \\
8\end{array}$ & $\begin{array}{r}0 \\
0 \\
11 \\
0 \\
179 \\
11 \\
0 \\
19 \\
11 \\
0 \\
11 \\
23\end{array}$ \\
\hline $\begin{array}{l}\text { LINE } \\
\text { A } \\
\text { B } \\
\text { C } \\
\text { D } \\
\text { E } \\
\text { F } \\
\text { G } \\
\text { H } \\
\text { I } \\
\text { J }\end{array}$ & $\begin{array}{r}10560 \\
2911.3 \\
2933.0 \\
2943.5 \\
3010.5 \\
3160.4 \\
3167.8 \\
3173.0 \\
3181.6 \\
3200.7 \\
3229.9\end{array}$ & $\begin{array}{r}\text { B? } \\
\text { B } \\
\text { B? } \\
\text { B? } \\
\text { S? } \\
\text { M } \\
\text { S? } \\
\text { B } \\
\text { B? } \\
\text { B? }\end{array}$ & $\begin{array}{l}645710 \\
645702 \\
645658 \\
645648 \\
645553 \\
645517 \\
645495 \\
645483 \\
645481 \\
645464\end{array}$ & $\begin{array}{l}6132026 \\
6132537 \\
6132824 \\
6133869 \\
6137421 \\
6137631 \\
6137787 \\
6138046 \\
6138571 \\
6139509\end{array}$ & $\begin{array}{r}1.7 \\
3.2 \\
0.8 \\
2.3 \\
2.7 \\
1.7 \\
0.6 \\
101.2 \\
0.4 \\
0.2\end{array}$ & $\begin{array}{r}1.8 \\
4.1 \\
2.8 \\
4.8 \\
3.0 \\
0.2 \\
2.1 \\
56.9 \\
8.4 \\
2.2\end{array}$ & $\begin{array}{r}0.9 \\
1.1 \\
1.1 \\
2.8 \\
2.0 \\
5.1 \\
7.5 \\
102.2 \\
0.3 \\
0.1\end{array}$ & $\begin{array}{r}1.4 \\
4.1 \\
3.8 \\
3.9 \\
4.4 \\
0.6 \\
3.3 \\
119.8 \\
5.1 \\
1.6\end{array}$ & $\begin{array}{r}1.5 \\
11.3 \\
10.8 \\
13.3 \\
7.5 \\
1.2 \\
6.2 \\
256.7 \\
14.0 \\
2.9\end{array}$ & $\begin{array}{r}6.4 \\
13.5 \\
9.2 \\
13.3 \\
31.8 \\
4.2 \\
21.5 \\
120.2 \\
29.9 \\
10.7\end{array}$ & $\begin{array}{r}--- \\
--- \\
1.3 \\
2.4 \\
3.6 \\
---- \\
7.5 \\
29.8 \\
0.5 \\
---\end{array}$ & $\begin{array}{r}--- \\
--- \\
6 \\
24 \\
48 \\
--- \\
54 \\
2 \\
0 \\
----\end{array}$ & $\begin{array}{r}10 \\
15 \\
0 \\
0 \\
0 \\
85 \\
18 \\
0 \\
0 \\
74\end{array}$ \\
\hline $\begin{array}{l}\text { LINE } \\
\text { A } \\
B \\
\text { C } \\
\text { D } \\
\text { E }\end{array}$ & $\begin{array}{r}10570 \\
3750.6 \\
3746.6 \\
3742.0 \\
3739.2 \\
3731.5\end{array}$ & $\begin{array}{l}\text { B } \\
\text { B } \\
\text { B } \\
\text { B } \\
\text { B }\end{array}$ & $\begin{array}{l}646155 \\
646149 \\
646148 \\
646145 \\
646130\end{array}$ & $\begin{array}{l}6132414 \\
6132569 \\
6132745 \\
6132846 \\
6133100\end{array}$ & $\begin{array}{r}8.5 \\
9.3 \\
9.8 \\
7.0 \\
14.6\end{array}$ & $\begin{array}{r}10.9 \\
17.3 \\
14.2 \\
9.2 \\
16.3\end{array}$ & $\begin{array}{r}9.0 \\
14.3 \\
9.7 \\
0.6 \\
14.9\end{array}$ & $\begin{array}{r}16.5 \\
25.3 \\
22.0 \\
1.0 \\
20.3\end{array}$ & $\begin{array}{r}28.5 \\
81.3 \\
58.9 \\
6.6 \\
47.6\end{array}$ & $\begin{array}{l}22.9 \\
55.1 \\
52.3 \\
12.8 \\
29.8\end{array}$ & $\begin{array}{l}5.0 \\
--- \\
4.1 \\
4.8 \\
8.1\end{array}$ & $\begin{array}{r}19 \\
-- \\
16 \\
34 \\
6\end{array}$ & $\begin{array}{l}0 \\
0 \\
0 \\
9 \\
0\end{array}$ \\
\hline
\end{tabular}

$\mathrm{CX}=$ COAXIAL

Note: EM values shown above

are local amplitudes

Ketchikan - North/South of 5515
* Estimated Depth may be unreliable because the stronger part of the conductor may be deeper or to one side of the flight line, or because of a shallow dip or magnetite/overburden effects 
EM Anomaly List

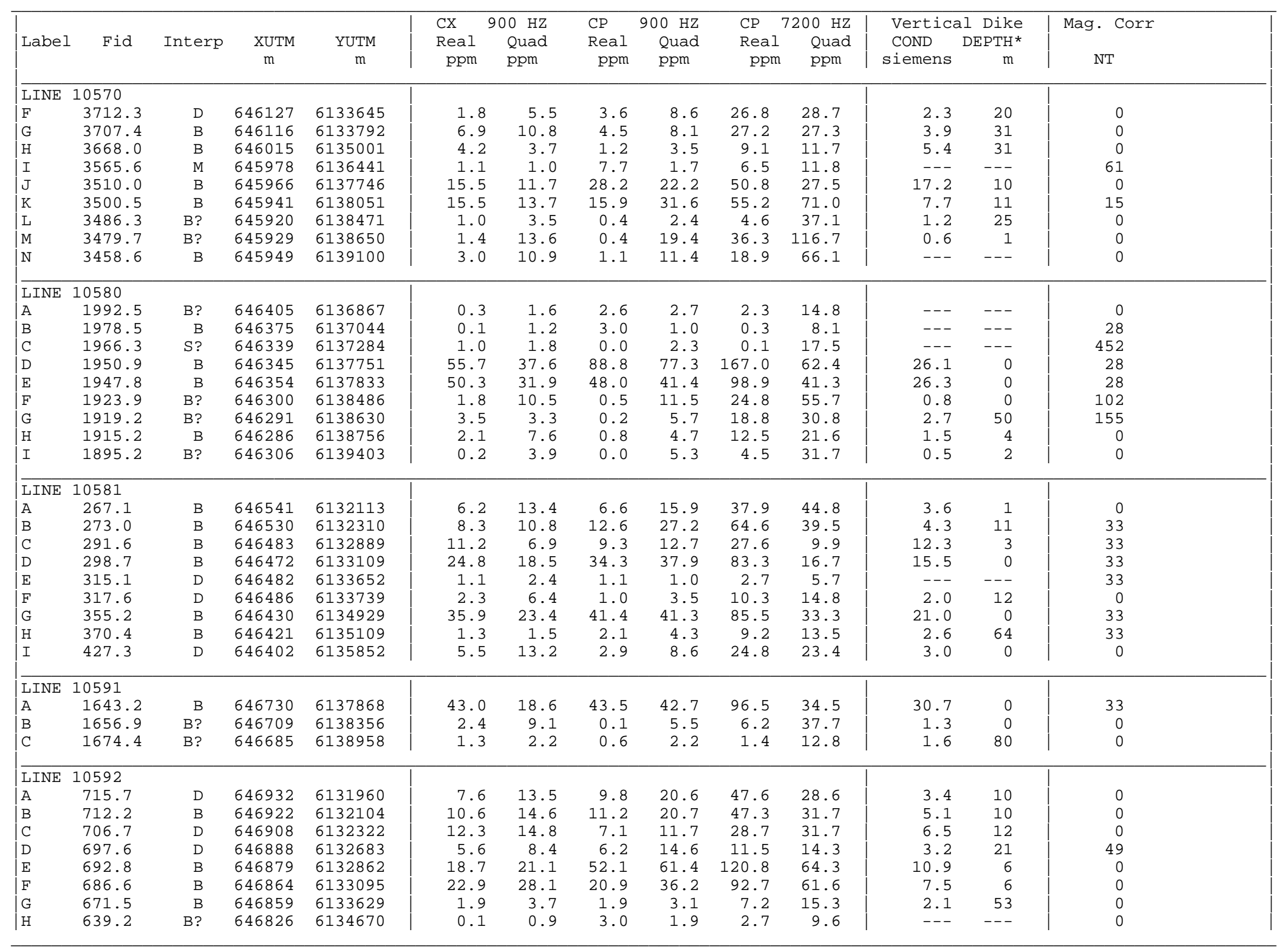

$\mathrm{CX}=$ COAXIAL

Note: EM values shown above

are local amplitudes

Ketchikan - North/South of 5515
*Estimated Depth may be unreliable because the stronger part of the conductor may be deeper or to one side of the flight line, or because of a shallow dip or magnetite/overburden effects 
EM Anomaly List

\begin{tabular}{|c|c|c|c|c|c|c|c|c|c|c|c|c|c|}
\hline Label & Fid & Interp & $\underset{\mathrm{m}}{\mathrm{XUTM}}$ & $\begin{array}{c}\text { YUTM } \\
\text { m }\end{array}$ & $\begin{array}{l}\text { CX } \\
\text { Real } \\
\text { ppm }\end{array}$ & $\begin{array}{l}900 \mathrm{HZ} \\
\text { Quad } \\
\text { ppm }\end{array}$ & $\begin{array}{l}\text { CP } \\
\text { Real } \\
\text { ppm }\end{array}$ & $\begin{array}{c}900 \mathrm{~Hz} \\
\text { Quad } \\
\text { ppm }\end{array}$ & $\begin{array}{l}\text { CP } \\
\text { Real } \\
\text { ppm }\end{array}$ & $\begin{array}{c}7200 \mathrm{HZ} \\
\text { Quad } \\
\text { ppm }\end{array}$ & $\begin{array}{l}\text { Vertica } \\
\text { CoND } \\
\text { siemens }\end{array}$ & $\begin{array}{c}\text { al Dike } \\
\text { DEPTH* } \\
\text { m }\end{array}$ & $\begin{array}{c}\text { Mag. Corr } \\
\text { NT }\end{array}$ \\
\hline $\begin{array}{l}\text { LINE } \\
\text { I } \\
\text { J } \\
\text { K } \\
\text { L } \\
\text { M } \\
\text { N } \\
\text { O } \\
\text { P }\end{array}$ & $\begin{array}{r}10592 \\
583.2 \\
576.6 \\
562.0 \\
548.5 \\
540.0 \\
530.0 \\
528.0 \\
522.1\end{array}$ & $\begin{array}{c}\mathrm{B} \\
\mathrm{D} \\
\mathrm{M} \\
\mathrm{D} \\
\mathrm{M} \\
\mathrm{S} ? \\
\mathrm{M} \\
\mathrm{M}\end{array}$ & $\begin{array}{l}646801 \\
646793 \\
646784 \\
646761 \\
646734 \\
646724 \\
646724 \\
646720\end{array}$ & $\begin{array}{l}6135779 \\
6135934 \\
6136364 \\
6136750 \\
6136995 \\
6137244 \\
6137297 \\
6137450\end{array}$ & $\begin{array}{r}46.6 \\
54.8 \\
2.7 \\
6.6 \\
1.2 \\
3.9 \\
0.1 \\
0.0\end{array}$ & $\begin{array}{r}37.2 \\
42.0 \\
0.7 \\
1.9 \\
0.9 \\
1.9 \\
2.2 \\
0.6\end{array}$ & $\begin{array}{r}62.2 \\
84.0 \\
0.5 \\
13.4 \\
1.7 \\
10.8 \\
9.8 \\
14.6\end{array}$ & $\begin{array}{r}80.2 \\
75.3 \\
0.9 \\
2.5 \\
0.8 \\
3.1 \\
3.1 \\
0.7\end{array}$ & $\begin{array}{r}165.5 \\
163.6 \\
0.6 \\
12.4 \\
1.2 \\
8.2 \\
6.4 \\
10.7\end{array}$ & $\begin{array}{r}68.5 \\
87.7 \\
7.5 \\
16.2 \\
4.3 \\
19.0 \\
19.1 \\
5.6\end{array}$ & $\begin{array}{r}16.3 \\
23.1 \\
--- \\
77.9 \\
--- \\
--- \\
--- \\
---\end{array}$ & $\begin{array}{r}0 \\
0 \\
--- \\
28 \\
--- \\
--- \\
--- \\
---\end{array}$ & $\begin{array}{r}0 \\
0 \\
98 \\
234 \\
2 \\
445 \\
451 \\
372\end{array}$ \\
\hline $\begin{array}{l}\text { LINE } \\
\text { A } \\
\text { B } \\
\text { C } \\
\text { D } \\
\text { E } \\
\text { F } \\
\text { G } \\
\text { H } \\
\text { I } \\
\text { J }\end{array}$ & $\begin{array}{r}10600 \\
1862.6 \\
1851.4 \\
1834.2 \\
1824.0 \\
1809.6 \\
1801.1 \\
1794.4 \\
1758.1 \\
1736.2 \\
1669.1\end{array}$ & $\begin{array}{r}\mathrm{M} \\
\mathrm{M} \\
\mathrm{M} \\
\mathrm{M} \\
\mathrm{D} \\
\mathrm{B} ? \\
\mathrm{D} \\
\mathrm{M} \\
\mathrm{M} \\
\mathrm{M}\end{array}$ & $\begin{array}{l}647183 \\
647164 \\
647131 \\
647114 \\
647085 \\
647087 \\
647089 \\
647056 \\
647060 \\
646991\end{array}$ & $\begin{array}{l}6136299 \\
6136629 \\
6137224 \\
6137549 \\
6138069 \\
6138366 \\
6138607 \\
6139726 \\
6140170 \\
6141836\end{array}$ & $\begin{array}{r}0.1 \\
0.8 \\
3.5 \\
1.2 \\
16.5 \\
2.5 \\
1.4 \\
0.2 \\
0.2 \\
0.4\end{array}$ & $\begin{array}{r}1.2 \\
0.9 \\
0.8 \\
0.6 \\
13.5 \\
12.9 \\
7.2 \\
0.7 \\
0.5 \\
0.0\end{array}$ & $\begin{array}{l}0.1 \\
0.6 \\
9.4 \\
6.5 \\
3.0 \\
1.1 \\
0.9 \\
0.4 \\
0.1 \\
1.0\end{array}$ & $\begin{array}{r}2.6 \\
1.4 \\
1.4 \\
0.6 \\
9.0 \\
12.2 \\
7.5 \\
0.9 \\
0.9 \\
0.3\end{array}$ & $\begin{array}{r}0.5 \\
0.2 \\
7.9 \\
5.4 \\
20.1 \\
24.0 \\
18.6 \\
0.1 \\
0.2 \\
0.5\end{array}$ & $\begin{array}{r}6.6 \\
11.4 \\
8.0 \\
4.6 \\
15.1 \\
100.6 \\
33.1 \\
5.5 \\
4.2 \\
2.1\end{array}$ & $\begin{array}{l}--- \\
--- \\
--- \\
--- \\
9.9 \\
1.1 \\
--- \\
--- \\
--- \\
---\end{array}$ & $\begin{array}{r}--- \\
--- \\
--- \\
--- \\
8 \\
0 \\
--- \\
--- \\
--- \\
---\end{array}$ & $\begin{array}{r}0 \\
90 \\
194 \\
46 \\
46 \\
134 \\
0 \\
0 \\
81 \\
55\end{array}$ \\
\hline $\begin{array}{l}\text { LINE } \\
\text { A } \\
\text { B } \\
\text { C } \\
\text { D } \\
\text { E } \\
\text { F } \\
\text { G } \\
\text { H } \\
\text { I } \\
\text { J } \\
\text { K }\end{array}$ & $\begin{array}{l}10601 \\
773.6 \\
790.4 \\
798.6 \\
804.1 \\
826.1 \\
850.4 \\
984.7 \\
992.5 \\
997.1 \\
1026.8 \\
1033.8\end{array}$ & $\begin{array}{r}\text { B } \\
\text { D } \\
\text { B } \\
\text { B } \\
\text { B } \\
\text { D } \\
\text { M } \\
\text { S? } \\
\text { D } \\
\text { M } \\
\text { M }\end{array}$ & $\begin{array}{l}647341 \\
647354 \\
647347 \\
647336 \\
647316 \\
647289 \\
647210 \\
647211 \\
647204 \\
647162 \\
647164\end{array}$ & $\begin{array}{l}6131727 \\
6131891 \\
6132028 \\
6132158 \\
6132766 \\
6133474 \\
6135646 \\
6135877 \\
6136035 \\
6137135 \\
6137405\end{array}$ & $\begin{array}{l}1.0 \\
5.7 \\
2.0 \\
3.7 \\
4.4 \\
4.6 \\
0.6 \\
5.4 \\
5.6 \\
1.6 \\
0.6\end{array}$ & $\begin{array}{r}3.3 \\
15.3 \\
2.5 \\
11.4 \\
5.8 \\
6.9 \\
0.4 \\
2.4 \\
4.8 \\
0.2 \\
0.6\end{array}$ & $\begin{array}{r}0.4 \\
1.2 \\
4.6 \\
8.3 \\
1.9 \\
3.0 \\
0.4 \\
16.6 \\
3.8 \\
5.0 \\
4.1\end{array}$ & $\begin{array}{r}1.5 \\
7.2 \\
8.2 \\
18.8 \\
7.3 \\
5.0 \\
0.4 \\
4.6 \\
2.7 \\
0.8 \\
0.6\end{array}$ & $\begin{array}{r}3.9 \\
15.4 \\
16.1 \\
40.7 \\
18.0 \\
14.7 \\
0.3 \\
17.9 \\
6.9 \\
5.9 \\
3.9\end{array}$ & $\begin{array}{r}8.0 \\
38.9 \\
15.3 \\
25.4 \\
31.2 \\
15.5 \\
1.2 \\
24.5 \\
10.2 \\
2.6 \\
4.7\end{array}$ & $\begin{array}{r}1.3 \\
2.5 \\
3.3 \\
3.0 \\
2.8 \\
3.7 \\
--- \\
--- \\
10.0 \\
--- \\
---\end{array}$ & $\begin{array}{r}30 \\
5 \\
21 \\
0 \\
39 \\
27 \\
--- \\
--- \\
23 \\
--- \\
---\end{array}$ & $\begin{array}{r}48 \\
0 \\
0 \\
0 \\
43 \\
0 \\
772 \\
48 \\
0 \\
0 \\
103\end{array}$ \\
\hline $\begin{array}{l}\text { LINE } \\
\text { A } \\
\text { B } \\
\text { C } \\
\text { D } \\
\text { E } \\
\text { F } \\
\text { G } \\
\text { H } \\
\text { I } \\
\text { J } \\
\text { K }\end{array}$ & $\begin{array}{l}10610 \\
967.9 \\
972.1 \\
1029.3 \\
1062.0 \\
1068.4 \\
1073.3 \\
1096.7 \\
1116.4 \\
1138.2 \\
1143.6 \\
1165.1\end{array}$ & $\begin{array}{l}B \\
B \\
B \\
D \\
M \\
M \\
M \\
M \\
M \\
M \\
D\end{array}$ & $\begin{array}{l}647767 \\
647786 \\
647741 \\
647719 \\
647731 \\
647725 \\
647671 \\
647653 \\
647650 \\
647643 \\
647616\end{array}$ & $\begin{array}{l}6131951 \\
6132069 \\
6132967 \\
6133535 \\
6133738 \\
6133899 \\
6134658 \\
6134936 \\
6135248 \\
6135380 \\
6136012\end{array}$ & $\begin{array}{r}7.2 \\
16.5 \\
42.8 \\
7.1 \\
1.2 \\
1.9 \\
1.1 \\
0.1 \\
3.1 \\
2.8 \\
5.5\end{array}$ & $\begin{array}{r}24.7 \\
28.0 \\
38.4 \\
7.2 \\
1.0 \\
0.6 \\
0.3 \\
0.2 \\
0.6 \\
0.7 \\
9.3\end{array}$ & $\begin{array}{r}4.3 \\
10.7 \\
50.7 \\
2.3 \\
4.2 \\
1.7 \\
0.4 \\
4.2 \\
1.9 \\
2.4 \\
3.0\end{array}$ & $\begin{array}{r}24.2 \\
36.6 \\
42.4 \\
1.2 \\
3.8 \\
2.4 \\
0.6 \\
0.8 \\
0.7 \\
0.4 \\
5.0\end{array}$ & $\begin{array}{r}48.2 \\
94.9 \\
91.0 \\
8.4 \\
14.3 \\
1.8 \\
3.4 \\
4.3 \\
1.8 \\
1.9 \\
8.1\end{array}$ & $\begin{array}{r}118.8 \\
172.3 \\
17.8 \\
8.8 \\
10.4 \\
10.4 \\
3.2 \\
2.8 \\
4.5 \\
1.6 \\
21.3\end{array}$ & $\begin{array}{r}2.3 \\
5.0 \\
18.8 \\
8.3 \\
--- \\
--- \\
--- \\
--- \\
--- \\
--- \\
3.3\end{array}$ & $\begin{array}{r}0 \\
0 \\
0 \\
12 \\
--- \\
--- \\
--- \\
--- \\
--- \\
--- \\
30\end{array}$ & $\begin{array}{r}34 \\
0 \\
0 \\
0 \\
130 \\
192 \\
287 \\
0 \\
263 \\
0 \\
36\end{array}$ \\
\hline
\end{tabular}

$\mathrm{CX}=$ COAXIAL

Note: EM values shown above

are local amplitudes

Ketchikan - North/South of 5515
* Estimated Depth may be unreliable because the stronger part of the conductor may be deeper or to one side of the flight line, or because of a shallow dip or magnetite/overburden effects 
EM Anomaly List

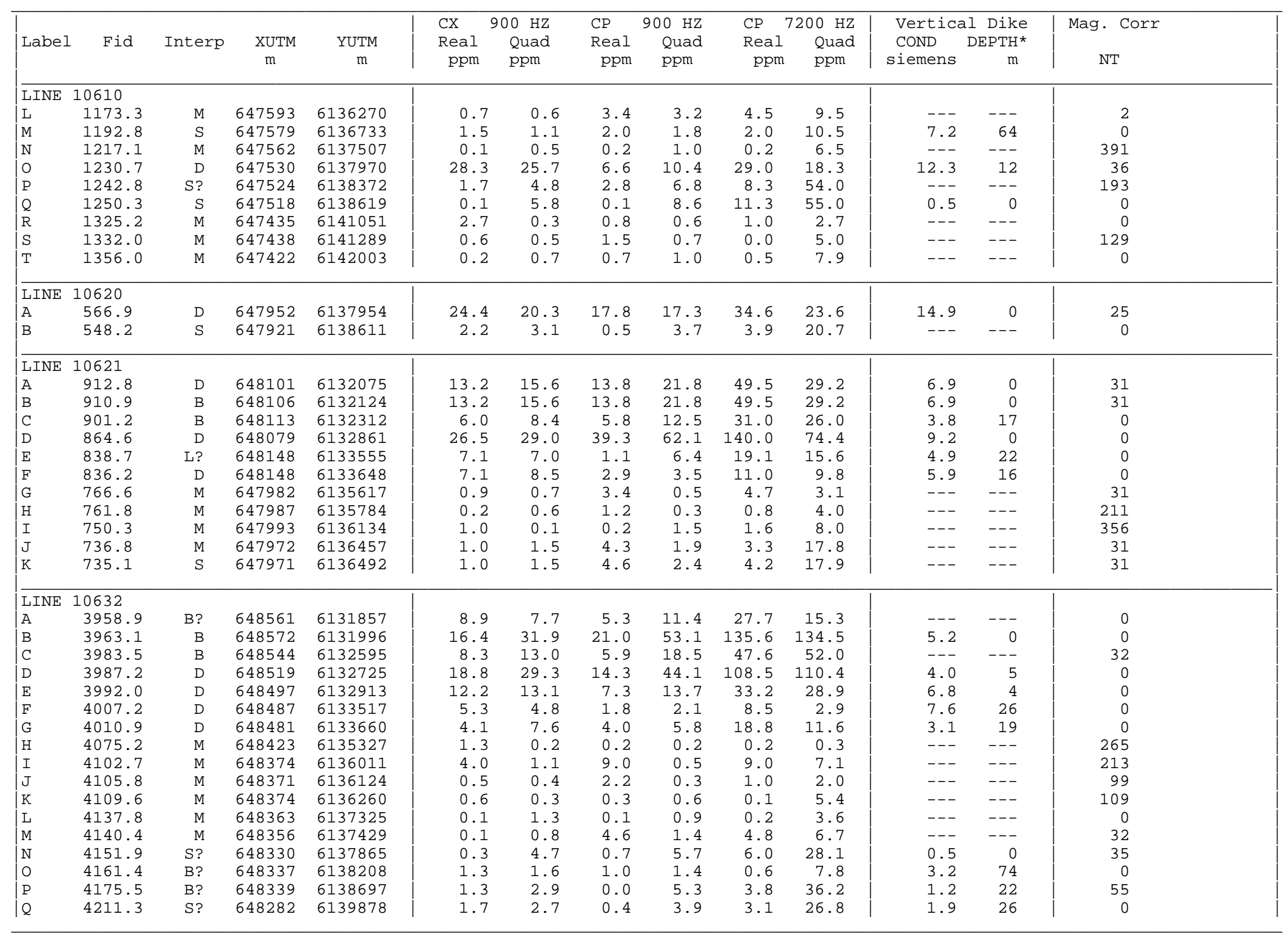

$\mathrm{CX}=$ COAXIAL

Note: EM values shown above

Ketchikan - North/South of 5515
*Estimated Depth may be unreliable because the stronger part of the conductor may be deeper or to one side of the flight line, or because of a shallow dip or magnetite/overburden effects 
EM Anomaly List

\begin{tabular}{|c|c|c|c|c|c|c|c|c|c|c|c|c|c|}
\hline Label & Fid & Interp & $\begin{array}{c}\text { XUTM } \\
\text { m }\end{array}$ & $\begin{array}{c}\text { YUTM } \\
\text { m }\end{array}$ & $\begin{array}{l}\text { CX } \\
\text { Real } \\
\text { ppm }\end{array}$ & $\begin{array}{l}900 \mathrm{HZ} \\
\text { Quad } \\
\text { ppm }\end{array}$ & $\begin{array}{l}\text { CP } \\
\text { Real } \\
\text { ppm }\end{array}$ & $\begin{array}{l}900 \mathrm{HZ} \\
\text { Quad } \\
\text { ppm }\end{array}$ & \multicolumn{2}{|c|}{$\begin{array}{ll}\text { CP } & 7200 \mathrm{HZ} \\
\text { Real } & \text { Quad } \\
\text { ppm } & \text { ppm }\end{array}$} & \multicolumn{2}{|c|}{\begin{tabular}{lc}
\multicolumn{2}{c}{ Vertical Dike } \\
COND & DEPTH* \\
siemens & $m$
\end{tabular}} & $\begin{array}{c}\text { Mag. Corr } \\
\text { NT }\end{array}$ \\
\hline LINE 1 & 10640 & & & & & & & & & & & & \\
\hline A & 5073.7 & B & 648970 & 6131878 & 18.0 & 27.5 & 20.8 & 40.1 & 101.0 & 93.3 & 5.4 & 2 & 45 \\
\hline B & 5064.3 & B & 648947 & 6132136 & 42.2 & 64.2 & 42.3 & 75.1 & 185.1 & 181.4 & 7.5 & 0 & 44 \\
\hline $\mathrm{C}$ & 5053.7 & B & 648939 & 6132410 & 8.0 & 14.5 & 20.1 & 25.8 & 68.1 & 36.0 & 5.3 & 0 & 0 \\
\hline D & 5050.7 & B & 648936 & 6132494 & 13.0 & 14.5 & 20.6 & 27.8 & 74.1 & 39.0 & 8.1 & 0 & 44 \\
\hline $\mathrm{E}$ & 5046.6 & B & 648936 & 6132621 & 10.4 & 11.7 & 9.3 & 18.2 & 44.0 & 24.6 & --- & --- & 0 \\
\hline $\mathrm{F}$ & 5024.4 & D & 648938 & 6133353 & 1.9 & 2.9 & 2.1 & 2.8 & 21.4 & 6.9 & 3.1 & 50 & 0 \\
\hline G & 5018.8 & D & 648928 & 6133528 & 5.2 & 9.3 & 2.6 & 2.6 & 6.9 & 12.1 & 3.4 & 29 & 0 \\
\hline $\mathrm{H}$ & 4993.8 & $\mathrm{M}$ & 648879 & 6134226 & 0.4 & 0.9 & 0.2 & 1.0 & 0.3 & 4.4 & -- & --- & 252 \\
\hline I & 4968.8 & $\mathrm{M}$ & 648866 & 6134680 & 0.7 & 0.1 & 0.0 & 0.3 & 0.1 & 1.3 & --- & --- & 456 \\
\hline $\mathrm{J}$ & 4942.2 & $\mathrm{M}$ & 648850 & 6135179 & 0.8 & 0.6 & 10.9 & 0.2 & 8.2 & 2.0 & --- & --- & 370 \\
\hline K & 4912.9 & $\mathrm{M}$ & 648801 & 6135685 & 3.2 & 0.5 & 0.5 & 0.4 & 0.4 & 2.0 & --- & --- & 32 \\
\hline $\mathrm{L}$ & 4897.8 & M & 648775 & 6136147 & 2.1 & 0.2 & 0.5 & 0.1 & 0.9 & 3.2 & --- & --- & 0 \\
\hline M & 4875.9 & $\mathrm{M}$ & 648789 & 6136841 & 0.4 & 0.7 & 1.7 & 0.3 & 10.0 & 1.5 & --- & --- & 407 \\
\hline $\mathrm{N}$ & 4867.9 & M & 648795 & 6137099 & 0.4 & 0.6 & 1.0 & 0.8 & 1.4 & 6.7 & --- & --- & 194 \\
\hline 0 & 4839.9 & D & 648783 & 6138021 & 4.5 & 8.8 & 0.1 & 1.3 & 8.0 & 14.1 & 3.0 & 24 & 0 \\
\hline $\mathrm{P}$ & 4790.9 & $\mathrm{~s}$ & 648689 & 6139761 & 0.3 & 1.4 & 0.6 & 1.4 & 1.6 & 10.3 & --- & --- & 44 \\
\hline$Q$ & 4755.5 & $\mathrm{~s}$ & 648640 & 6140791 & 1.1 & 1.5 & 1.7 & 1.6 & 0.0 & 11.0 & --- & --- & 0 \\
\hline R & 4709.3 & $\mathrm{M}$ & 648586 & 6141986 & 0.6 & 0.8 & 0.1 & 0.8 & 0.2 & 3.7 & --- & --- & 156 \\
\hline $\mathrm{S}$ & 4699.2 & $\mathrm{M}$ & 648570 & 6142277 & 0.2 & 0.4 & 0.8 & 0.8 & 0.7 & 3.2 & --- & --- & 158 \\
\hline $\mathrm{T}$ & 4573.5 & B & 648545 & 6145081 & 0.3 & 2.1 & 0.5 & 1.7 & 1.8 & 10.5 & --- & --- & 0 \\
\hline U & 4527.7 & B & 648469 & 6146467 & 9.2 & 18.3 & 8.9 & 26.4 & 73.5 & 81.2 & 4.0 & 0 & 0 \\
\hline V & 4524.2 & $\mathrm{~B}$ ? & 648467 & 6146572 & 6.7 & 13.9 & 4.2 & 14.7 & 39.9 & 57.6 & --- & --- & 0 \\
\hline$\overline{\text { LINE } 1}$ & 10650 & & & & & & & & & & & & \\
\hline A & 5110.2 & B & 649357 & 6131897 & 13.3 & 17.4 & 25.0 & 30.7 & 71.5 & 35.9 & --- & --- & 45 \\
\hline B & 5115.4 & B & 649321 & 6132017 & 7.9 & 20.6 & 22.1 & 38.6 & 100.8 & 74.6 & 4.8 & 0 & 0 \\
\hline $\mathrm{C}$ & 5118.3 & B & 649308 & 6132082 & 7.9 & 20.6 & 24.1 & 40.1 & 103.3 & 74.6 & 5.0 & 0 & 0 \\
\hline D & 5124.5 & B & 649300 & 6132210 & 3.3 & 8.4 & 13.0 & 19.0 & 44.5 & 37.5 & 3.3 & 20 & 0 \\
\hline $\mathrm{E}$ & 5131.0 & $\mathrm{~B}$ & 649314 & 6132348 & 13.1 & 7.5 & 11.9 & 19.8 & 50.5 & 32.0 & 11.5 & 2 & 0 \\
\hline $\mathrm{F}$ & 5207.3 & $\mathrm{M}$ & 649243 & 6134589 & 0.5 & 0.3 & 5.3 & 0.6 & 4.3 & 2.3 & -- & --- & 672 \\
\hline G & 5237.2 & M & 649270 & 6134917 & 0.2 & 0.5 & 0.1 & 0.4 & 0.4 & 2.3 & --- & --- & 457 \\
\hline $\mathrm{H}$ & 5284.1 & $\mathrm{M}$ & 649257 & 6135781 & 0.0 & 0.2 & 2.3 & 0.4 & 1.0 & 2.9 & --- & --- & 134 \\
\hline I & 5318.7 & M & 649178 & 6136683 & 1.9 & 0.6 & 4.0 & 0.5 & 2.7 & 1.4 & --- & --- & 318 \\
\hline $\mathrm{J}$ & 5328.5 & M & 649167 & 6137065 & 3.2 & 0.3 & 0.5 & 0.3 & 0.5 & 0.9 & --- & --- & 164 \\
\hline K & 5340.2 & $\mathrm{M}$ & 649156 & 6137533 & 3.3 & 0.8 & 1.0 & 0.6 & 0.1 & 2.6 & --- & --- & 35 \\
\hline L & 5349.0 & D & 649135 & 6137908 & 5.2 & 3.4 & 3.5 & 1.9 & 5.7 & 2.5 & 13.9 & 12 & 0 \\
\hline M & 5374.9 & B? & 649114 & 6139000 & 0.6 & 2.9 & 0.1 & 4.6 & 1.8 & 33.3 & 0.6 & 4 & 108 \\
\hline $\mathrm{N}$ & 5408.3 & S? & 649087 & 6140264 & 1.8 & 1.5 & 2.1 & 1.5 & 1.9 & 8.7 & --- & --- & 0 \\
\hline 0 & 5429.2 & M & 649064 & 6140822 & 0.7 & 0.8 & 0.2 & 0.6 & 0.2 & 5.3 & --- & --- & 777 \\
\hline $\mathrm{P}$ & 5472.6 & $\mathrm{M}$ & 648976 & 6142394 & 0.2 & 0.9 & 0.3 & 0.8 & 0.3 & 6.3 & --- & --- & 43 \\
\hline$Q$ & 5479.0 & $\mathrm{M}$ & 648950 & 6142644 & 3.7 & 0.6 & 3.0 & 0.5 & 1.4 & 1.1 & --- & --- & 0 \\
\hline $\begin{array}{l}\text { LINE } 1 \\
\end{array}$ & 10660 & & & & & & & & & & & & \\
\hline A & 3757.8 & B & 649779 & 6131775 & 4.8 & 7.0 & 17.2 & 5.4 & 19.9 & 24.7 & 14.4 & 2 & 0 \\
\hline $\mathrm{B}$ & 3763.3 & B & 649797 & 6131902 & 9.8 & 7.5 & 1.1 & 9.9 & 26.7 & 35.2 & 6.3 & 18 & 0 \\
\hline
\end{tabular}

$\mathrm{CX}=$ COAXIAL

Note: EM values shown above

Ketchikan - North/South of 5515
* Estimated Depth may be unreliable because the stronger part of the conductor may be deeper or shale side of the flight line, or because 
EM Anomaly List

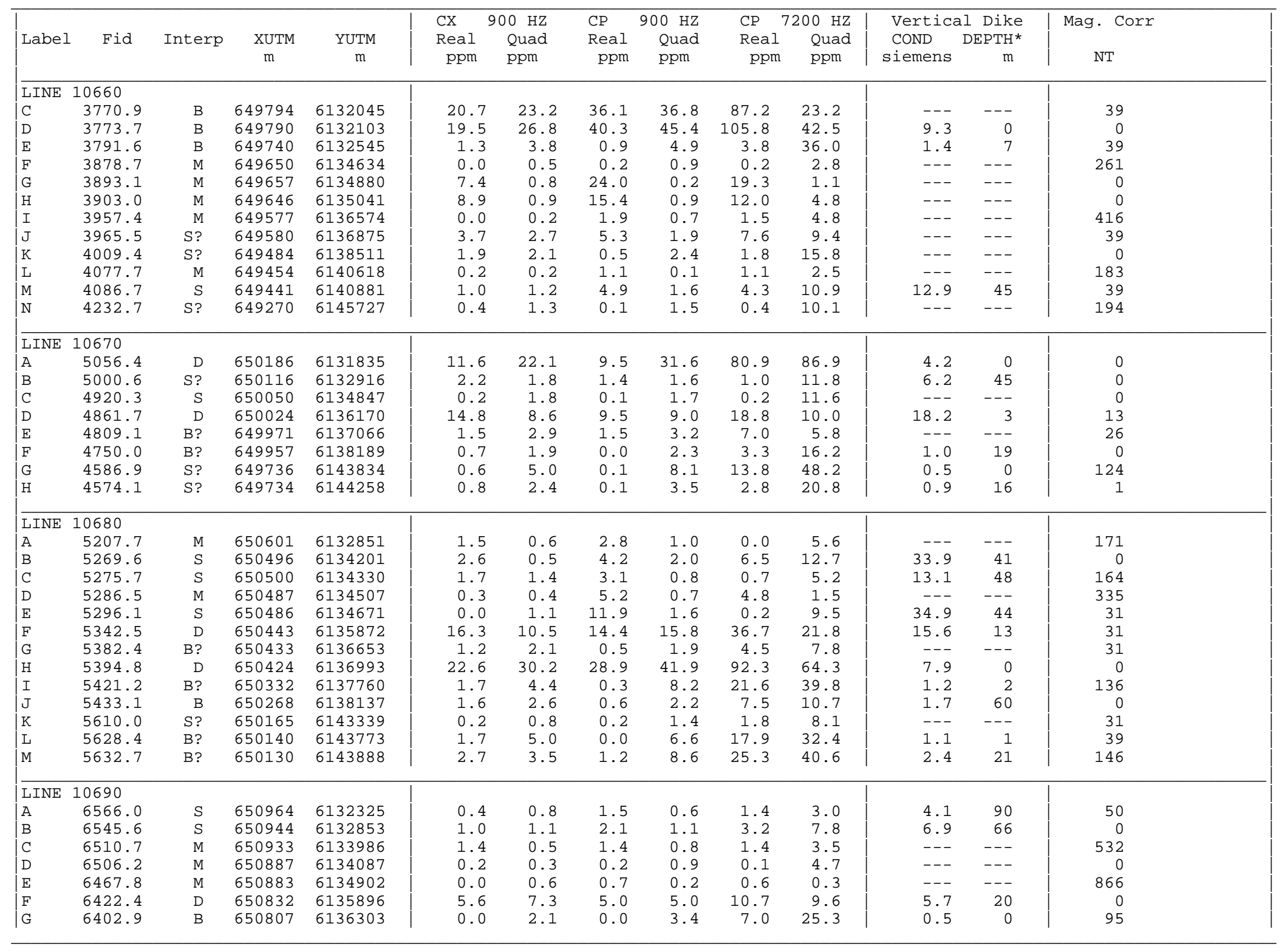

$\mathrm{CX}=$ COAXIAL

Note: EM values shown above

are local amplitudes

Ketchikan - North/South of 5515
* Estimated Depth may be unreliable because the stronger part of the conductor may be deeper or to one side of the flight line, or because of a shallow dip or magnetite/overburden effects 
EM Anomaly List

\begin{tabular}{|c|c|c|c|c|c|c|c|c|c|c|c|c|c|}
\hline Label & Fid & Interp & $\begin{array}{c}\text { XUTM } \\
\text { m }\end{array}$ & $\begin{array}{c}\text { YUTM } \\
\mathrm{m}\end{array}$ & $\begin{array}{l}\text { CX } \\
\text { Real } \\
\text { ppm }\end{array}$ & $\begin{array}{l}900 \mathrm{HZ} \\
\text { Quad } \\
\text { ppm }\end{array}$ & $\begin{array}{l}\text { CP } \\
\text { Real } \\
\text { ppm }\end{array}$ & $\begin{array}{l}900 \mathrm{HZ} \\
\text { Quad } \\
\text { ppm }\end{array}$ & $\begin{array}{l}\text { CP } 7 \\
\text { Real } \\
\text { ppm }\end{array}$ & $\begin{array}{l}200 \mathrm{HZ} \\
\text { Quad } \\
\text { ppm }\end{array}$ & $\begin{array}{l}\text { Vertica } \\
\text { Cond } \\
\text { siemens }\end{array}$ & $\begin{array}{c}\text { al Dike } \\
\text { DEPTH* } \\
\text { m }\end{array}$ & $\begin{array}{c}\text { Mag. Corr } \\
\text { NT }\end{array}$ \\
\hline $\begin{array}{l}\text { LINE } \\
H \\
I \\
\text { J } \\
\text { K } \\
\text { L } \\
M \\
N \\
O \\
P \\
Q \\
R\end{array}$ & $\begin{array}{r}10690 \\
6389.0 \\
6380.2 \\
6354.3 \\
6336.3 \\
6321.1 \\
6280.5 \\
6161.4 \\
6137.7 \\
6002.6 \\
6000.1 \\
5888.2\end{array}$ & $\begin{array}{r}\text { B } \\
\text { B } \\
\text { B } \\
\text { B } \\
\text { B } \\
\text { B? } \\
\text { M } \\
\text { S } \\
\text { S? } \\
\text { S? } \\
\text { M }\end{array}$ & $\begin{array}{l}650805 \\
650812 \\
650798 \\
650818 \\
650829 \\
650768 \\
650627 \\
650616 \\
650468 \\
650467 \\
650361\end{array}$ & $\begin{array}{l}6136541 \\
6136629 \\
6136973 \\
6137093 \\
6137199 \\
6138385 \\
6141662 \\
6142347 \\
6146151 \\
6146228 \\
6148884\end{array}$ & $\begin{array}{l}6.7 \\
3.4 \\
3.9 \\
3.7 \\
6.7 \\
1.6 \\
0.2 \\
0.1 \\
0.8 \\
1.7 \\
0.3\end{array}$ & $\begin{array}{l}6.6 \\
4.1 \\
1.1 \\
1.2 \\
5.0 \\
2.1 \\
0.3 \\
1.4 \\
4.8 \\
4.8 \\
0.3\end{array}$ & $\begin{array}{r}6.2 \\
15.9 \\
6.6 \\
8.7 \\
7.8 \\
2.0 \\
0.6 \\
0.3 \\
0.9 \\
0.8 \\
1.0\end{array}$ & $\begin{array}{r}8.3 \\
14.8 \\
6.7 \\
6.0 \\
7.7 \\
3.5 \\
0.7 \\
2.4 \\
14.9 \\
13.4 \\
0.3\end{array}$ & $\begin{array}{r}20.0 \\
29.7 \\
10.5 \\
13.3 \\
20.4 \\
2.6 \\
0.3 \\
0.0 \\
42.8 \\
38.1 \\
0.0\end{array}$ & $\begin{array}{r}10.8 \\
9.3 \\
5.0 \\
6.1 \\
14.1 \\
23.2 \\
4.2 \\
17.3 \\
81.9 \\
75.4 \\
3.2\end{array}$ & $\begin{array}{r}7.1 \\
8.4 \\
14.9 \\
19.5 \\
--- \\
--- \\
--- \\
--- \\
--- \\
1.0 \\
---\end{array}$ & $\begin{array}{r}23 \\
16 \\
38 \\
27 \\
--- \\
--- \\
--- \\
--- \\
--- \\
0 \\
---\end{array}$ & $\begin{array}{r}32 \\
0 \\
0 \\
0 \\
0 \\
0 \\
232 \\
0 \\
32 \\
0 \\
0\end{array}$ \\
\hline $\begin{array}{l}\text { LINE } \\
\text { A } \\
\text { B } \\
\text { C } \\
\text { D } \\
\text { E } \\
\text { F } \\
\text { G } \\
\text { H } \\
\text { I } \\
\text { J } \\
\text { K } \\
\text { L } \\
\text { M } \\
\text { N } \\
\text { O } \\
P \\
\text { Q } \\
R \\
\text { S } \\
\text { T } \\
\text { U } \\
\text { V }\end{array}$ & $\begin{array}{r}10700 \\
6955.4 \\
6963.2 \\
6965.8 \\
6982.7 \\
6986.7 \\
7019.3 \\
7037.9 \\
7048.3 \\
7067.2 \\
7121.6 \\
7128.0 \\
7134.8 \\
7146.1 \\
7175.6 \\
7182.7 \\
7207.3 \\
7382.4 \\
7413.5 \\
7536.4 \\
7645.3 \\
7652.9 \\
7680.9\end{array}$ & $\begin{array}{r}\mathrm{M} \\
\mathrm{M} \\
\mathrm{M} \\
\mathrm{M} \\
\mathrm{S} \\
\mathrm{M} \\
\mathrm{M} \\
\mathrm{M} \\
\mathrm{M} \\
\mathrm{B} \\
\mathrm{B} \\
\mathrm{B} \\
\mathrm{B} ? \\
\mathrm{D} \\
\mathrm{B} \\
\mathrm{S} \\
\mathrm{S} \\
\mathrm{S} \\
\mathrm{S} ? \\
\mathrm{M} \\
\mathrm{M} \\
\mathrm{B} ?\end{array}$ & $\begin{array}{l}651370 \\
651379 \\
651372 \\
651308 \\
651294 \\
651293 \\
651272 \\
651258 \\
651251 \\
651251 \\
651239 \\
651236 \\
651217 \\
651206 \\
651164 \\
651167 \\
651041 \\
651004 \\
650880 \\
650821 \\
650817 \\
650810\end{array}$ & $\begin{array}{l}6133476 \\
6133643 \\
6133710 \\
6134186 \\
6134279 \\
6134749 \\
6135040 \\
6135209 \\
6135463 \\
6135896 \\
6135983 \\
6136104 \\
6136307 \\
6136961 \\
6137129 \\
6137798 \\
6141732 \\
6142677 \\
6145831 \\
6148248 \\
6148360 \\
6148780\end{array}$ & $\begin{array}{r}3.2 \\
0.0 \\
0.2 \\
0.0 \\
3.5 \\
7.0 \\
0.0 \\
1.8 \\
14.2 \\
25.5 \\
26.9 \\
12.7 \\
4.8 \\
12.9 \\
4.3 \\
1.3 \\
0.1 \\
1.5 \\
1.4 \\
4.0 \\
3.0 \\
0.3\end{array}$ & $\begin{array}{r}0.4 \\
0.5 \\
0.2 \\
0.6 \\
0.9 \\
0.4 \\
0.7 \\
0.4 \\
0.8 \\
17.2 \\
18.1 \\
3.1 \\
0.6 \\
18.1 \\
12.2 \\
1.4 \\
2.6 \\
5.7 \\
4.2 \\
0.3 \\
0.0 \\
1.4\end{array}$ & $\begin{array}{r}3.2 \\
13.8 \\
6.5 \\
6.5 \\
5.3 \\
1.0 \\
5.1 \\
10.9 \\
9.0 \\
21.0 \\
76.6 \\
31.3 \\
6.9 \\
3.7 \\
8.9 \\
0.2 \\
1.8 \\
1.5 \\
0.6 \\
5.7 \\
1.5 \\
1.0\end{array}$ & $\begin{array}{r}0.4 \\
0.6 \\
0.8 \\
1.1 \\
1.7 \\
0.8 \\
0.8 \\
0.6 \\
0.1 \\
21.7 \\
41.0 \\
6.3 \\
0.0 \\
17.2 \\
17.2 \\
1.7 \\
4.2 \\
10.2 \\
7.3 \\
0.4 \\
0.1 \\
1.8\end{array}$ & $\begin{array}{r}11.1 \\
10.8 \\
5.7 \\
8.8 \\
4.0 \\
0.6 \\
3.5 \\
9.4 \\
9.4 \\
51.3 \\
103.6 \\
30.5 \\
5.9 \\
40.4 \\
37.9 \\
1.1 \\
5.8 \\
14.2 \\
21.0 \\
5.5 \\
1.0 \\
0.6\end{array}$ & $\begin{array}{r}1.8 \\
3.5 \\
5.9 \\
6.4 \\
11.8 \\
6.8 \\
2.4 \\
1.0 \\
1.6 \\
32.0 \\
34.9 \\
0.0 \\
1.6 \\
55.0 \\
46.8 \\
8.7 \\
26.9 \\
66.8 \\
40.9 \\
2.6 \\
1.0 \\
9.5\end{array}$ & $\begin{array}{r}--- \\
--- \\
--- \\
--- \\
43.8 \\
--- \\
--- \\
--- \\
--- \\
18.0 \\
32.7 \\
109.5 \\
319.6 \\
3.9 \\
3.4 \\
1.9 \\
--- \\
1.2 \\
1.1 \\
--- \\
--- \\
---\end{array}$ & $\begin{array}{r}--- \\
--- \\
--- \\
--- \\
45 \\
--- \\
--- \\
--- \\
--- \\
7 \\
0 \\
0 \\
29 \\
23 \\
0 \\
73 \\
--- \\
0 \\
0 \\
--- \\
--- \\
---\end{array}$ & $\begin{array}{r}175 \\
0 \\
40 \\
217 \\
0 \\
0 \\
234 \\
321 \\
1292 \\
0 \\
0 \\
0 \\
0 \\
20 \\
0 \\
40 \\
0 \\
0 \\
0 \\
133 \\
0 \\
40\end{array}$ \\
\hline $\begin{array}{l}\text { LINE } \\
\text { A } \\
\text { B } \\
\text { C } \\
\text { D } \\
\text { E } \\
\text { F } \\
\text { G } \\
\text { H } \\
\text { I }\end{array}$ & $\begin{array}{l}10710 \\
1283.4 \\
1258.9 \\
1239.9 \\
1237.0 \\
1230.6 \\
1168.1 \\
1028.1 \\
960.0 \\
819.5\end{array}$ & $\begin{array}{c}\text { M } \\
\text { D } \\
\text { B } \\
\text { B } \\
\text { D } \\
\text { S } \\
\text { M } \\
\text { M } \\
\text { S? }\end{array}$ & $\begin{array}{l}651647 \\
651628 \\
651641 \\
651637 \\
651634 \\
651608 \\
651535 \\
651477 \\
651413\end{array}$ & $\begin{array}{l}6135708 \\
6135800 \\
6135999 \\
6136051 \\
6136141 \\
6136592 \\
6139336 \\
6140352 \\
6142103\end{array}$ & $\begin{array}{r}46.2 \\
10.5 \\
16.9 \\
16.9 \\
12.9 \\
2.1 \\
3.0 \\
1.3 \\
0.1\end{array}$ & $\begin{array}{r}0.8 \\
16.5 \\
9.5 \\
9.5 \\
9.9 \\
2.3 \\
3.5 \\
0.9 \\
4.6\end{array}$ & $\begin{array}{r}142.2 \\
4.8 \\
45.1 \\
45.1 \\
24.0 \\
1.2 \\
0.5 \\
2.0 \\
0.7\end{array}$ & $\begin{array}{r}0.9 \\
4.7 \\
20.7 \\
20.7 \\
21.9 \\
1.2 \\
0.1 \\
0.3 \\
7.9\end{array}$ & $\begin{array}{r}115.2 \\
18.3 \\
55.0 \\
55.0 \\
45.0 \\
0.4 \\
2.9 \\
1.6 \\
16.6\end{array}$ & $\begin{array}{r}1.5 \\
23.0 \\
10.3 \\
10.3 \\
21.3 \\
10.3 \\
1.8 \\
2.2 \\
48.4\end{array}$ & $\begin{array}{r}--- \\
5.1 \\
35.2 \\
35.2 \\
14.3 \\
4.6 \\
--- \\
--- \\
---\end{array}$ & $\begin{array}{r}--- \\
29 \\
0 \\
0 \\
9 \\
56 \\
--- \\
--- \\
---\end{array}$ & $\begin{array}{r}52 \\
0 \\
22 \\
0 \\
0 \\
0 \\
0 \\
55 \\
0\end{array}$ \\
\hline
\end{tabular}

$\mathrm{CX}=$ COAXIAL

Note: EM values shown above

are local amplitudes

Ketchikan - North/South of 5515
* Estimated Depth may be unreliable because the stronger part of the conductor may be deeper or to one side of the flight line, or because of a shallow dip or magnetite/overburden effects 
EM Anomaly List

\begin{tabular}{|c|c|c|c|c|c|c|c|c|c|c|c|c|c|}
\hline Label & Fid & Interp & $\begin{array}{c}\text { XUTM } \\
\mathrm{m}\end{array}$ & $\begin{array}{c}\text { YUTM } \\
\mathrm{m}\end{array}$ & $\begin{array}{ll}\text { CX } & 9 \\
\text { Real } \\
\text { ppm }\end{array}$ & $\begin{array}{l}00 \mathrm{HZ} \\
\text { Quad } \\
\text { ppm }\end{array}$ & $\begin{array}{l}\text { CP } \\
\text { Real } \\
\text { ppm }\end{array}$ & $\begin{array}{c}900 \mathrm{HZ} \\
\text { Quad } \\
\text { ppm }\end{array}$ & $\begin{array}{l}\text { CP }{ }^{7} \\
\text { Real } \\
\text { ppm }\end{array}$ & $\begin{array}{c}200 \mathrm{HZ} \\
\text { Quad } \\
\text { ppm }\end{array}$ & $\begin{array}{l}\text { Vertica } \\
\text { COND } \\
\text { siemens }\end{array}$ & $\begin{array}{c}\text { al Dike } \\
\text { DEPTH* } \\
\text { m }\end{array}$ & $\begin{array}{c}\text { Mag. Corr } \\
\text { NT }\end{array}$ \\
\hline $\begin{array}{l}\text { LINE } \\
\text { J } \\
K \\
\text { L }\end{array}$ & $\begin{array}{l}10710 \\
692.9 \\
640.0 \\
534.0\end{array}$ & $\begin{array}{r}\text { S } \\
\text { S? } \\
\text { S? }\end{array}$ & $\begin{array}{l}651248 \\
651232 \\
651177\end{array}$ & $\begin{array}{l}6145810 \\
6146984 \\
6148629\end{array}$ & $\begin{array}{l}1.6 \\
1.1 \\
0.2\end{array}$ & $\begin{array}{l}2.7 \\
0.7 \\
0.5\end{array}$ & $\begin{array}{l}0.5 \\
0.6 \\
0.4\end{array}$ & $\begin{array}{l}2.5 \\
0.9 \\
0.8\end{array}$ & $\begin{array}{l}1.1 \\
0.8 \\
0.1\end{array}$ & $\begin{array}{r}16.2 \\
3.4 \\
5.2\end{array}$ & $\begin{array}{l}--- \\
6.0 \\
---\end{array}$ & $\begin{array}{r}--- \\
93 \\
---\end{array}$ & $\begin{array}{l}0 \\
0 \\
0\end{array}$ \\
\hline $\begin{array}{l}\text { LINE } \\
\text { A } \\
\text { B } \\
\text { C } \\
\text { D } \\
\text { E } \\
\text { F } \\
\text { G }\end{array}$ & $\begin{array}{l}10711 \\
1650.2 \\
1635.0 \\
1552.5 \\
1506.0 \\
1487.9 \\
1480.9 \\
1438.1\end{array}$ & $\begin{array}{l}\mathrm{M} \\
\mathrm{M} \\
\mathrm{S} \\
\mathrm{M} \\
\mathrm{M} \\
\mathrm{M} \\
\mathrm{M}\end{array}$ & $\begin{array}{l}651773 \\
651755 \\
651721 \\
651654 \\
651647 \\
651640 \\
651675\end{array}$ & $\begin{array}{l}6132123 \\
6132517 \\
6133917 \\
6134950 \\
6135167 \\
6135248 \\
6135597\end{array}$ & $\begin{array}{r}0.7 \\
0.6 \\
0.5 \\
0.2 \\
2.2 \\
18.6 \\
4.0\end{array}$ & $\begin{array}{l}0.2 \\
0.6 \\
2.2 \\
0.5 \\
0.4 \\
0.7 \\
0.3\end{array}$ & $\begin{array}{r}0.8 \\
0.1 \\
0.9 \\
2.1 \\
34.5 \\
43.1 \\
5.4\end{array}$ & $\begin{array}{l}0.8 \\
0.7 \\
2.8 \\
0.2 \\
0.4 \\
0.4 \\
0.2\end{array}$ & $\begin{array}{r}0.0 \\
0.7 \\
2.8 \\
0.8 \\
28.5 \\
33.7 \\
5.3\end{array}$ & $\begin{array}{r}3.1 \\
2.0 \\
18.4 \\
3.6 \\
7.1 \\
1.6 \\
1.8\end{array}$ & $\begin{array}{l}--- \\
--- \\
--- \\
--- \\
--- \\
--- \\
---\end{array}$ & $\begin{array}{l}--- \\
--- \\
--- \\
--- \\
--- \\
--- \\
---\end{array}$ & $\begin{array}{r}250 \\
0 \\
0 \\
0 \\
707 \\
157 \\
156\end{array}$ \\
\hline $\begin{array}{l}\text { LINE } \\
\text { A } \\
\text { B } \\
\text { C } \\
\text { D } \\
\text { E } \\
\text { F } \\
\text { G } \\
\text { H } \\
\text { I } \\
\text { J } \\
\text { K } \\
\text { L } \\
\text { M } \\
\text { N } \\
\text { O } \\
\text { P } \\
\text { Q } \\
\text { R } \\
\text { S } \\
\text { T } \\
\text { U }\end{array}$ & $\begin{array}{r}10720 \\
1791.7 \\
1801.7 \\
1833.6 \\
1862.0 \\
1897.0 \\
1911.2 \\
1916.0 \\
1926.8 \\
1962.1 \\
1993.3 \\
2014.3 \\
2029.3 \\
2061.7 \\
2076.3 \\
2131.6 \\
2146.8 \\
2155.8 \\
2169.8 \\
2184.2 \\
2214.4 \\
2226.9\end{array}$ & $\begin{array}{c}\mathrm{M} \\
\mathrm{M} \\
\mathrm{M} \\
\mathrm{M} \\
\mathrm{M} \\
\mathrm{M} \\
\mathrm{M} \\
\mathrm{M} \\
\mathrm{M} \\
\mathrm{M} \\
\mathrm{M} \\
\mathrm{M} \\
\mathrm{M} \\
\mathrm{M} \\
\mathrm{S} ? \\
\mathrm{D} \\
\mathrm{B} \\
\mathrm{D} \\
\mathrm{D} \\
\mathrm{B} \\
\mathrm{B}\end{array}$ & $\begin{array}{l}652177 \\
652182 \\
652181 \\
652174 \\
652148 \\
652109 \\
652084 \\
652041 \\
652048 \\
652067 \\
652059 \\
652059 \\
652060 \\
652055 \\
652029 \\
652024 \\
652022 \\
652025 \\
652009 \\
651997 \\
651956\end{array}$ & $\begin{array}{l}6132710 \\
6132811 \\
6133110 \\
6133237 \\
6133716 \\
6134103 \\
6134232 \\
6134513 \\
6135079 \\
6135246 \\
6135378 \\
6135491 \\
6135653 \\
6135709 \\
6135888 \\
6136033 \\
6136126 \\
6136351 \\
6136661 \\
6137311 \\
6137454\end{array}$ & $\begin{array}{r}1.1 \\
0.1 \\
0.7 \\
2.6 \\
0.0 \\
0.3 \\
2.6 \\
0.0 \\
0.1 \\
0.3 \\
0.1 \\
0.0 \\
0.6 \\
24.3 \\
2.1 \\
22.0 \\
3.3 \\
9.9 \\
0.4 \\
2.0 \\
0.9\end{array}$ & $\begin{array}{r}0.2 \\
0.4 \\
0.1 \\
0.6 \\
0.9 \\
0.4 \\
0.5 \\
0.3 \\
0.2 \\
0.4 \\
0.5 \\
0.1 \\
0.8 \\
0.7 \\
3.8 \\
24.1 \\
5.7 \\
10.3 \\
2.7 \\
2.8 \\
0.7\end{array}$ & $\begin{array}{r}1.6 \\
0.2 \\
2.0 \\
12.3 \\
2.5 \\
0.3 \\
9.7 \\
9.8 \\
0.8 \\
5.5 \\
9.1 \\
16.5 \\
29.8 \\
57.8 \\
0.7 \\
1.7 \\
16.2 \\
4.1 \\
1.7 \\
0.2 \\
0.1\end{array}$ & $\begin{array}{r}0.7 \\
0.2 \\
0.6 \\
0.1 \\
0.6 \\
0.2 \\
0.1 \\
0.6 \\
0.7 \\
0.5 \\
0.3 \\
0.2 \\
0.0 \\
0.8 \\
2.6 \\
7.0 \\
12.2 \\
4.0 \\
2.0 \\
3.0 \\
3.6\end{array}$ & $\begin{array}{r}1.0 \\
0.0 \\
0.2 \\
9.4 \\
2.6 \\
0.3 \\
8.3 \\
7.9 \\
1.0 \\
4.4 \\
7.8 \\
14.1 \\
25.4 \\
46.5 \\
5.6 \\
13.0 \\
23.0 \\
9.5 \\
3.5 \\
2.3 \\
1.5\end{array}$ & $\begin{array}{r}1.3 \\
0.6 \\
2.5 \\
3.7 \\
3.3 \\
1.3 \\
0.6 \\
4.5 \\
6.6 \\
3.7 \\
1.2 \\
1.0 \\
2.7 \\
5.2 \\
10.9 \\
49.3 \\
9.7 \\
8.7 \\
8.9 \\
18.2 \\
18.0\end{array}$ & $\begin{array}{l}--- \\
--- \\
--- \\
--- \\
--- \\
--- \\
--- \\
--- \\
--- \\
--- \\
--- \\
--- \\
--- \\
--- \\
2.4 \\
8.1 \\
--- \\
8.3 \\
2.0 \\
1.7 \\
---\end{array}$ & $\begin{array}{r}--- \\
--- \\
--- \\
--- \\
--- \\
--- \\
--- \\
--- \\
--- \\
--- \\
--- \\
--- \\
--- \\
--- \\
16 \\
21 \\
--- \\
0 \\
17 \\
66 \\
---\end{array}$ & $\begin{array}{r}0 \\
0 \\
0 \\
0 \\
0 \\
430 \\
72 \\
0 \\
0 \\
82 \\
255 \\
409 \\
0 \\
0 \\
0 \\
0 \\
72 \\
72 \\
0 \\
72 \\
0\end{array}$ \\
\hline $\begin{array}{l}\text { LINE } \\
\text { A } \\
\text { B } \\
\text { C } \\
\text { D } \\
\text { E } \\
\text { F } \\
\text { G } \\
\text { H }\end{array}$ & $\begin{array}{r}10721 \\
2438.1 \\
2447.3 \\
2523.8 \\
2562.1 \\
2578.7 \\
2590.5 \\
2611.9 \\
2722.6\end{array}$ & $\begin{array}{r}\text { B? } \\
\text { B } \\
\text { B? } \\
\text { B? } \\
\text { B } \\
\text { B } \\
\text { B } \\
\text { S }\end{array}$ & $\begin{array}{l}651922 \\
651892 \\
651754 \\
651725 \\
651730 \\
651725 \\
651709 \\
651588\end{array}$ & $\begin{array}{l}6141443 \\
6141643 \\
6143459 \\
6144484 \\
6144812 \\
6144931 \\
6145165 \\
6147568\end{array}$ & $\begin{array}{l}4.5 \\
3.6 \\
0.6 \\
1.7 \\
2.2 \\
1.2 \\
1.6 \\
0.2\end{array}$ & $\begin{array}{l}3.6 \\
2.5 \\
7.5 \\
2.7 \\
7.5 \\
2.9 \\
3.7 \\
3.0\end{array}$ & $\begin{array}{l}2.9 \\
1.9 \\
1.6 \\
1.3 \\
0.4 \\
2.0 \\
1.5 \\
0.8\end{array}$ & $\begin{array}{r}2.9 \\
3.1 \\
14.3 \\
4.9 \\
6.3 \\
4.8 \\
7.7 \\
2.8\end{array}$ & $\begin{array}{r}4.6 \\
4.1 \\
40.8 \\
14.5 \\
18.9 \\
13.2 \\
18.8 \\
1.8\end{array}$ & $\begin{array}{r}11.7 \\
6.5 \\
55.1 \\
20.2 \\
28.0 \\
16.4 \\
16.8 \\
15.2\end{array}$ & $\begin{array}{l}--- \\
7.3 \\
0.6 \\
2.3 \\
1.4 \\
--- \\
1.7 \\
0.6\end{array}$ & $\begin{array}{r}--- \\
55 \\
0 \\
24 \\
4 \\
--- \\
16 \\
0\end{array}$ & $\begin{array}{r}0 \\
14 \\
0 \\
0 \\
0 \\
15 \\
0 \\
15\end{array}$ \\
\hline
\end{tabular}

$\mathrm{CX}=$ COAXIAL

Note: EM values shown above

Ketchikan - North/South of 5515
*Estimated Depth may be unreliable because the stronger part of the conductor may be deeper or to one side of the flight line, or because of shallow dip or magnetite/overburden effects 
EM Anomaly List

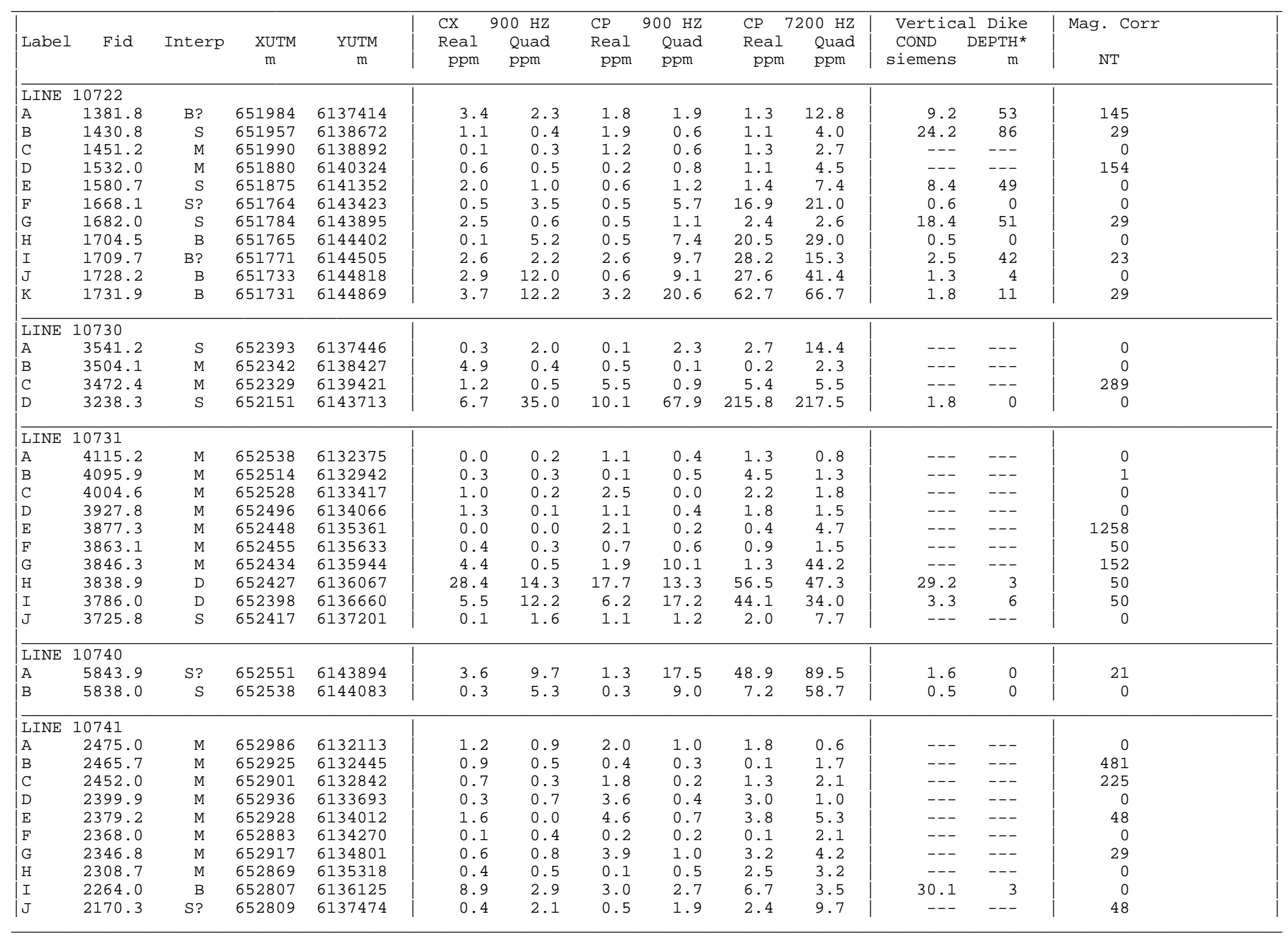

$\mathrm{CX}=$ COAXIAL

Note: EM values shown above

Ketchikan - North/South of 5515
* Estimated Depth may be unreliable because the stronger part of the conductor may be deeper or to one side of the flight line, or because of a shallow dip or magnetite/overburden effects 
EM Anomaly List

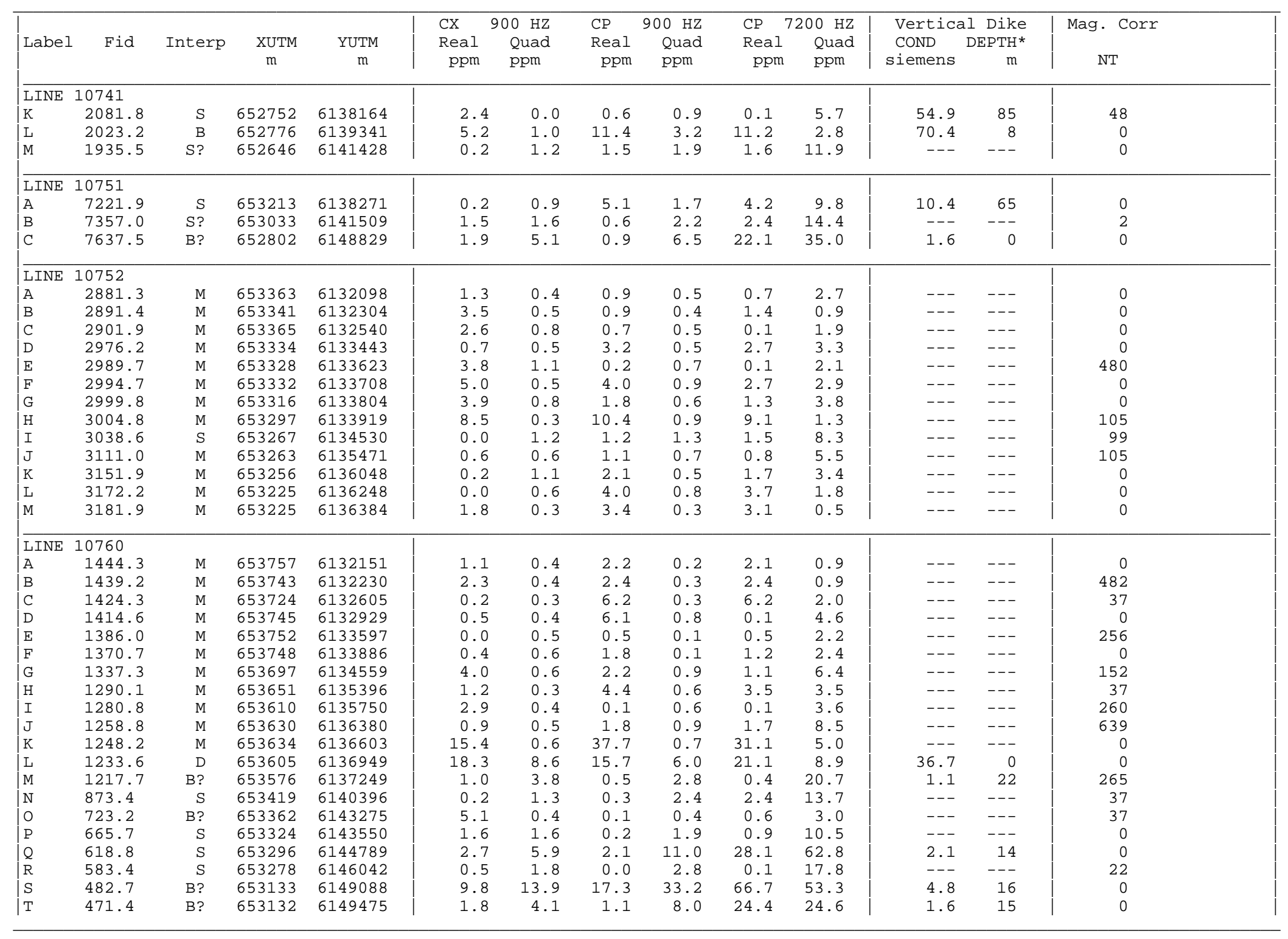

$\mathrm{CX}=$ COAXIAL

Note: EM values shown above

Ketchikan - North/South of 5515
* Estimated Depth may be unreliable because the stronger part of the conductor may be deeper or to one side of the flight line, or because of a shallow dip or magnetite/overburden effects 
EM Anomaly List

\begin{tabular}{|c|c|c|c|c|c|c|c|c|c|c|c|c|c|}
\hline Label & Fid & Interp & $\underset{\mathrm{m}}{\mathrm{XUTM}}$ & $\begin{array}{c}\text { YUTM } \\
\mathrm{m}\end{array}$ & $\begin{array}{l}\text { CX } \\
\text { Real } \\
\text { ppm }\end{array}$ & $\begin{array}{l}900 \mathrm{HZ} \\
\text { Quad } \\
\text { ppm }\end{array}$ & $\begin{array}{l}\text { CP } \\
\text { Real } \\
\text { ppm }\end{array}$ & $\begin{array}{c}900 \mathrm{HZ} \\
\text { Quad } \\
\text { ppm }\end{array}$ & $\begin{array}{l}\text { CP } \\
\text { Real } \\
\text { ppm }\end{array}$ & $\begin{array}{c}200 \mathrm{~Hz} \\
\text { Quad } \\
\text { ppm }\end{array}$ & $\begin{array}{l}\text { Vertica } \\
\text { CoND } \\
\text { siemens }\end{array}$ & $\begin{array}{c}\text { al Dike } \\
\text { DEPTH* } \\
\text { m }\end{array}$ & $\begin{array}{c}\text { Mag. Corr } \\
\text { NT }\end{array}$ \\
\hline LINE & 10770 & & & & & & & & & & & & \\
\hline A & 2363.3 & M & 654340 & 6128961 & 1.1 & 0.2 & 4.5 & 0.9 & 4.6 & 3.0 & --- & --- & 0 \\
\hline B & 2412.0 & M & 654266 & 6129278 & 3.7 & 0.0 & 13.8 & 0.2 & 10.7 & 2.1 & --- & --- & 0 \\
\hline C & 2431.2 & M & 654281 & 6129377 & 0.6 & 0.1 & 43.3 & 0.6 & 35.1 & 1.9 & --- & --- & 0 \\
\hline D & 2450.1 & M & 654299 & 6129483 & 0.0 & 0.2 & 31.1 & 0.1 & 21.4 & 3.1 & --- & --- & 0 \\
\hline $\mathrm{E}$ & 2471.9 & M & 654323 & 6129847 & 9.3 & 0.1 & 8.2 & 0.2 & 5.5 & 1.3 & --- & --- & 234 \\
\hline $\mathrm{F}$ & 2479.3 & M & 654316 & 6130070 & 1.2 & 0.1 & 14.0 & 0.5 & 10.1 & 4.7 & --- & --- & 0 \\
\hline G & 2493.2 & S? & 654243 & 6130514 & 2.4 & 1.3 & 12.2 & 1.7 & 10.5 & 11.2 & 58.5 & 31 & 0 \\
\hline $\mathrm{H}$ & 2501.3 & S? & 654228 & 6130791 & 24.6 & 3.2 & 40.0 & 3.4 & 40.1 & 19.9 & 279.5 & 11 & 0 \\
\hline I & 2511.0 & M & 654226 & 6131064 & 1.3 & 0.1 & 0.6 & 0.9 & 0.7 & 6.5 & --- & --- & 592 \\
\hline $\mathrm{J}$ & 2527.8 & M & 654217 & 6131198 & 1.0 & 0.5 & 4.8 & 0.5 & 4.1 & 1.0 & --- & --- & 148 \\
\hline K & 2539.9 & M & 654216 & 6131241 & 6.5 & 0.7 & 0.4 & 0.4 & 0.1 & 1.1 & --- & --- & 36 \\
\hline $\mathrm{L}$ & 2583.8 & M & 654239 & 6131457 & 1.5 & 0.5 & 0.6 & 0.1 & 0.4 & 1.3 & --- & --- & 0 \\
\hline M & 2591.3 & M & 654230 & 6131548 & 4.7 & 0.3 & 11.3 & 0.5 & 0.1 & 1.0 & --- & --- & 52 \\
\hline $\mathrm{N}$ & 2623.3 & M & 654244 & 6131933 & 16.8 & 0.0 & 25.6 & 0.2 & 19.4 & 0.7 & --- & --- & 0 \\
\hline 0 & 2625.7 & M & 654243 & 6131977 & 9.8 & 0.8 & 42.2 & 0.6 & 35.2 & 1.0 & --- & --- & 0 \\
\hline $\mathrm{P}$ & 2646.8 & M & 654210 & 6132299 & 0.0 & 0.2 & 1.2 & 0.3 & 0.4 & 1.3 & --- & --- & 0 \\
\hline Q & 2669.2 & M & 654205 & 6132641 & 0.5 & 0.5 & 2.3 & 0.2 & 2.4 & 1.0 & --- & --- & 0 \\
\hline $\mathrm{R}$ & 2712.6 & M & 654181 & 6133779 & 7.2 & 0.6 & 2.0 & 0.3 & 2.3 & 1.9 & --- & --- & 0 \\
\hline $\mathrm{S}$ & 2721.2 & M & 654157 & 6133994 & 0.0 & 0.2 & 5.1 & 0.4 & 3.7 & 3.3 & --- & --- & 0 \\
\hline $\mathrm{T}$ & 2732.0 & $\mathrm{M}$ & 654125 & 6134270 & 11.8 & 0.5 & 18.1 & 0.6 & 15.1 & 3.7 & --- & --- & 0 \\
\hline LINE & 10771 & & & & & & & & & & & & \\
\hline A & 3456.1 & B & 653667 & 6147290 & 2.2 & 3.8 & 0.6 & 5.0 & 11.5 & 32.7 & 2.1 & 26 & 0 \\
\hline B & 3510.5 & B & 653574 & 6148954 & 10.1 & 20.8 & 11.4 & 33.2 & 103.3 & 71.3 & 4.1 & 0 & 5 \\
\hline C & 3513.6 & B & 653570 & 6149064 & 10.1 & 20.8 & 11.1 & 33.2 & 103.3 & 71.0 & 4.1 & 0 & 4 \\
\hline LINE & 10772 & & & & & & & & & & & & \\
\hline A & 4419.4 & M & 654111 & 6134633 & 0.0 & 0.9 & 7.8 & 0.8 & 5.9 & 5.2 & --- & --- & 86 \\
\hline B & 4398.6 & M & 654084 & 6134943 & 0.9 & 0.3 & 30.1 & 0.5 & 24.3 & 4.4 & --- & --- & 0 \\
\hline C & 4388.0 & M & 654087 & 6135042 & 0.0 & 0.2 & 0.0 & 0.4 & 11.3 & 5.4 & --- & --- & 0 \\
\hline D & 4338.4 & S? & 654032 & 6136304 & 0.4 & 1.5 & 0.7 & 1.7 & 1.2 & 11.7 & --- & --- & 615 \\
\hline $\mathrm{E}$ & 4317.8 & B & 654000 & 6136866 & 23.4 & 18.8 & 28.3 & 30.8 & 73.3 & 33.2 & 14.6 & 0 & 36 \\
\hline $\mathrm{F}$ & 4274.1 & $\mathrm{~S}$ & 654000 & 6137614 & 0.6 & 2.8 & 0.4 & 3.9 & 8.8 & 24.4 & --- & --- & 36 \\
\hline G & 4182.2 & M & 653966 & 6138363 & 0.7 & 0.5 & 1.7 & 0.2 & 1.4 & 2.4 & --- & --- & 0 \\
\hline $\mathrm{H}$ & 4173.0 & M & 653960 & 6138438 & 0.2 & 0.5 & 1.5 & 0.4 & 1.5 & 1.3 & --- & --- & 0 \\
\hline I & 4038.0 & $S$ & 653869 & 6141020 & 1.4 & 1.6 & 0.4 & 2.8 & 3.1 & 18.4 & --- & --- & 0 \\
\hline LINE & 10773 & & & & & & & & & & & & \\
\hline A & 5016.7 & M & 654481 & 6123652 & 0.2 & 0.6 & 1.7 & 0.3 & 1.4 & 2.0 & --- & --- & 264 \\
\hline B & 5001.8 & M & 654473 & 6123821 & 0.2 & 0.7 & 0.9 & 0.1 & 0.1 & 0.6 & --- & --- & 0 \\
\hline C & 4986.8 & M & 654495 & 6123909 & 0.1 & 0.5 & 0.4 & 0.4 & 0.2 & 0.7 & --- & --- & 0 \\
\hline D & 4896.4 & M & 654445 & 6125261 & 15.9 & 0.5 & 16.2 & 0.3 & 13.0 & 1.8 & --- & --- & 0 \\
\hline $\mathrm{E}$ & 4876.3 & M & 654424 & 6125331 & 20.3 & 0.1 & 41.9 & 0.3 & 35.1 & 1.1 & --- & --- & 0 \\
\hline $\mathrm{F}$ & 4865.6 & M & 654417 & 6125379 & 29.6 & 0.4 & 20.7 & 0.6 & 15.2 & 2.0 & --- & --- & 0 \\
\hline G & 4843.8 & M & 654425 & 6125485 & 19.1 & 0.5 & 33.9 & 0.6 & 26.4 & 2.0 & --- & --- & 0 \\
\hline
\end{tabular}

$\mathrm{CX}=$ COAXIAL

Note: EM values shown above

Ketchikan - North/South of 5515
* Estimated Depth may be unreliable because the stronger part of the conductor may be deeper or to one side of the flight line, or because of a shallow dip or magnetite/overburden effects 
EM Anomaly List

\begin{tabular}{|c|c|c|c|c|c|c|c|c|c|c|c|c|c|}
\hline Label & Fid & Interp & $\begin{array}{c}\text { XUTM } \\
\text { m }\end{array}$ & $\begin{array}{c}\text { YUTM } \\
\mathrm{m}\end{array}$ & $\begin{array}{l}\text { CX } \\
\text { Real } \\
\text { ppm }\end{array}$ & $\begin{array}{l}00 \mathrm{HZ} \\
\text { Quad } \\
\text { ppm }\end{array}$ & $\begin{array}{l}\text { CP } \\
\text { Real } \\
\text { ppm }\end{array}$ & $\begin{array}{l}900 \mathrm{HZ} \\
\text { Quad } \\
\text { ppm }\end{array}$ & $\begin{array}{l}\text { CP } 7 \\
\text { Real } \\
\text { ppm }\end{array}$ & $\begin{array}{l}200 \mathrm{~Hz} \\
\text { Quad } \\
\text { ppm }\end{array}$ & $\begin{array}{l}\text { Vertica } \\
\text { ConD } \\
\text { siemens }\end{array}$ & $\begin{array}{c}\text { al Dike } \\
\text { DEPTH* } \\
\text { m }\end{array}$ & $\begin{array}{c}\text { Mag. Corr } \\
\text { NT }\end{array}$ \\
\hline $\begin{array}{l}\text { LINE } \\
\text { H } \\
\text { I } \\
\text { J } \\
\text { K } \\
\text { L } \\
\text { M } \\
\text { N } \\
\text { O }\end{array}$ & $\begin{array}{r}10773 \\
4830.9 \\
4820.1 \\
4787.8 \\
4780.3 \\
4772.7 \\
4754.3 \\
4740.3 \\
4664.3\end{array}$ & $\begin{array}{l}\mathrm{M} \\
\mathrm{M} \\
\mathrm{M} \\
\mathrm{M} \\
\mathrm{M} \\
\mathrm{M} \\
\mathrm{M} \\
\mathrm{M}\end{array}$ & $\begin{array}{l}654437 \\
654425 \\
654357 \\
654380 \\
654401 \\
654384 \\
654367 \\
654323\end{array}$ & $\begin{array}{l}6125574 \\
6125730 \\
6126259 \\
6126471 \\
6126653 \\
6126977 \\
6127133 \\
6127784\end{array}$ & $\begin{array}{l}5.0 \\
0.2 \\
7.2 \\
1.0 \\
6.4 \\
2.7 \\
1.2 \\
1.1\end{array}$ & $\begin{array}{l}0.4 \\
0.5 \\
0.1 \\
0.7 \\
0.2 \\
0.3 \\
0.1 \\
0.4\end{array}$ & $\begin{array}{r}0.8 \\
0.2 \\
21.8 \\
0.4 \\
12.0 \\
2.7 \\
0.3 \\
5.9\end{array}$ & $\begin{array}{l}0.0 \\
0.2 \\
0.5 \\
0.2 \\
0.1 \\
0.2 \\
0.1 \\
0.2\end{array}$ & $\begin{array}{r}0.9 \\
0.4 \\
17.9 \\
0.4 \\
10.3 \\
2.4 \\
0.1 \\
4.2\end{array}$ & $\begin{array}{l}0.7 \\
2.2 \\
0.9 \\
0.8 \\
0.5 \\
2.8 \\
2.2 \\
1.9\end{array}$ & $\begin{array}{l}--- \\
--- \\
--- \\
--- \\
--- \\
--- \\
--- \\
---\end{array}$ & $\begin{array}{l}--- \\
--- \\
--- \\
--- \\
--- \\
--- \\
--- \\
---\end{array}$ & $\begin{array}{r}0 \\
0 \\
0 \\
1441 \\
0 \\
0 \\
0 \\
0\end{array}$ \\
\hline $\begin{array}{l}\text { LINE } \\
\text { A } \\
\text { B } \\
\text { C } \\
\text { D } \\
\text { E } \\
\text { F } \\
\text { G } \\
\text { H }\end{array}$ & $\begin{array}{r}10781 \\
7413.8 \\
7440.0 \\
7466.9 \\
7487.9 \\
7526.0 \\
7544.2 \\
7563.5 \\
7600.0\end{array}$ & $\begin{array}{l}\mathrm{M} \\
\mathrm{M} \\
\mathrm{M} \\
\mathrm{M} \\
\mathrm{M} \\
\mathrm{M} \\
\mathrm{M} \\
\mathrm{M}\end{array}$ & $\begin{array}{l}654915 \\
654912 \\
654919 \\
654883 \\
654888 \\
654890 \\
654901 \\
654865\end{array}$ & $\begin{array}{l}6122345 \\
6122666 \\
6122990 \\
6123298 \\
6123518 \\
6123647 \\
6123923 \\
6124089\end{array}$ & $\begin{array}{l}1.9 \\
6.9 \\
0.0 \\
0.2 \\
0.5 \\
1.4 \\
1.6 \\
0.1\end{array}$ & $\begin{array}{l}0.3 \\
0.9 \\
0.9 \\
0.4 \\
0.3 \\
0.3 \\
0.5 \\
0.4\end{array}$ & $\begin{array}{r}0.6 \\
15.3 \\
6.2 \\
1.2 \\
6.5 \\
0.3 \\
0.0 \\
0.1\end{array}$ & $\begin{array}{l}0.2 \\
0.5 \\
0.5 \\
0.1 \\
0.2 \\
0.0 \\
0.5 \\
0.1\end{array}$ & $\begin{array}{r}0.7 \\
13.1 \\
0.0 \\
2.3 \\
5.7 \\
0.0 \\
0.1 \\
3.3\end{array}$ & $\begin{array}{l}0.7 \\
2.3 \\
3.1 \\
0.8 \\
1.4 \\
0.7 \\
0.6 \\
1.0\end{array}$ & $\begin{array}{l}--- \\
--- \\
--- \\
--- \\
--- \\
--- \\
--- \\
---\end{array}$ & $\begin{array}{l}--- \\
--- \\
--- \\
--- \\
--- \\
--- \\
--- \\
---\end{array}$ & $\begin{array}{r}0 \\
0 \\
111 \\
0 \\
0 \\
0 \\
0 \\
0\end{array}$ \\
\hline $\begin{array}{l}\text { LINE } \\
\text { A } \\
\text { B } \\
\text { C } \\
\text { D } \\
\text { E }\end{array}$ & $\begin{array}{r}10782 \\
7736.0 \\
7728.3 \\
7702.0 \\
7683.3 \\
7677.0\end{array}$ & $\begin{array}{l}\mathrm{M} \\
\mathrm{M} \\
\mathrm{M} \\
\mathrm{M} \\
\mathrm{M}\end{array}$ & $\begin{array}{l}654867 \\
654867 \\
654869 \\
654884 \\
654881\end{array}$ & $\begin{array}{l}6124056 \\
6124119 \\
6124260 \\
6124372 \\
6124475\end{array}$ & $\begin{array}{l}3.8 \\
0.4 \\
3.4 \\
0.0 \\
3.6\end{array}$ & $\begin{array}{l}0.1 \\
0.4 \\
0.3 \\
0.4 \\
1.4\end{array}$ & $\begin{array}{r}10.9 \\
2.3 \\
1.5 \\
0.2 \\
15.9\end{array}$ & $\begin{array}{l}0.2 \\
0.3 \\
0.0 \\
0.2 \\
1.7\end{array}$ & $\begin{array}{r}8.8 \\
1.8 \\
1.3 \\
0.2 \\
13.5\end{array}$ & $\begin{array}{l}0.4 \\
0.4 \\
1.1 \\
0.8 \\
8.9\end{array}$ & $\begin{array}{l}--- \\
--- \\
--- \\
--- \\
---\end{array}$ & $\begin{array}{l}--- \\
--- \\
--- \\
--- \\
---\end{array}$ & $\begin{array}{r}0 \\
30 \\
0 \\
11 \\
0\end{array}$ \\
\hline $\begin{array}{l}\text { LINE } \\
\text { A } \\
\text { B } \\
\text { C } \\
\text { D } \\
\text { E } \\
\text { F } \\
\text { G } \\
\text { H } \\
\text { I } \\
\text { J } \\
\text { K }\end{array}$ & $\begin{array}{r}10783 \\
7842.4 \\
7863.9 \\
7887.8 \\
7898.2 \\
7907.8 \\
7917.3 \\
7964.7 \\
7990.2 \\
8008.1 \\
8019.7 \\
8036.1\end{array}$ & $\begin{array}{c}\mathrm{M} \\
\mathrm{M} \\
\mathrm{M} \\
\mathrm{M} \\
\mathrm{M} \\
\mathrm{M} \\
\mathrm{M} \\
\mathrm{M} \\
\mathrm{B} \\
\mathrm{S} ? \\
\mathrm{~S}\end{array}$ & $\begin{array}{l}654824 \\
654828 \\
654805 \\
654787 \\
654760 \\
654753 \\
654780 \\
654796 \\
654717 \\
654690 \\
654731\end{array}$ & $\begin{array}{l}6124992 \\
6125248 \\
6125667 \\
6125929 \\
6126156 \\
6126373 \\
6127058 \\
6127338 \\
6127581 \\
6127716 \\
6127886\end{array}$ & $\begin{array}{l}3.6 \\
0.0 \\
2.0 \\
6.0 \\
0.6 \\
7.5 \\
1.2 \\
0.5 \\
2.4 \\
0.8 \\
0.0\end{array}$ & $\begin{array}{l}0.5 \\
1.0 \\
0.4 \\
0.2 \\
0.4 \\
0.4 \\
0.2 \\
0.8 \\
2.8 \\
2.7 \\
1.9\end{array}$ & $\begin{array}{r}5.7 \\
11.7 \\
4.9 \\
16.3 \\
6.3 \\
0.7 \\
1.4 \\
0.7 \\
3.1 \\
0.2 \\
0.5\end{array}$ & $\begin{array}{l}0.6 \\
0.0 \\
0.4 \\
0.2 \\
0.2 \\
0.3 \\
0.2 \\
0.2 \\
4.7 \\
0.9 \\
0.8\end{array}$ & $\begin{array}{r}5.1 \\
10.5 \\
3.8 \\
12.6 \\
4.9 \\
0.6 \\
0.8 \\
0.2 \\
5.2 \\
6.4 \\
2.0\end{array}$ & $\begin{array}{r}8.7 \\
1.2 \\
1.7 \\
0.7 \\
0.9 \\
0.8 \\
0.6 \\
2.1 \\
28.0 \\
0.2 \\
5.8\end{array}$ & $\begin{array}{l}--- \\
--- \\
--- \\
--- \\
--- \\
--- \\
--- \\
--- \\
--- \\
1.2 \\
---\end{array}$ & $\begin{array}{l}--- \\
--- \\
--- \\
--- \\
--- \\
--- \\
--- \\
--- \\
--- \\
26 \\
---\end{array}$ & $\begin{array}{r}86 \\
0 \\
0 \\
0 \\
731 \\
1020 \\
1051 \\
0 \\
146 \\
0 \\
146\end{array}$ \\
\hline $\begin{array}{l}\text { LINE } \\
\text { A } \\
\text { B } \\
\text { C } \\
\text { D } \\
\text { E } \\
\text { F }\end{array}$ & $\begin{array}{r}10784 \\
6830.0 \\
6793.9 \\
6784.8 \\
6775.4 \\
6761.2 \\
6740.1\end{array}$ & $\begin{array}{l}\mathrm{M} \\
\mathrm{M} \\
\mathrm{M} \\
\mathrm{M} \\
\mathrm{M} \\
\mathrm{M}\end{array}$ & $\begin{array}{l}654678 \\
654670 \\
654658 \\
654673 \\
654676 \\
654682\end{array}$ & $\begin{array}{l}6128984 \\
6129426 \\
6129593 \\
6129895 \\
6130407 \\
6131039\end{array}$ & $\begin{array}{l}0.6 \\
0.4 \\
2.8 \\
0.0 \\
0.6 \\
0.1\end{array}$ & $\begin{array}{l}0.7 \\
0.3 \\
0.3 \\
0.5 \\
0.4 \\
0.3\end{array}$ & $\begin{array}{r}6.8 \\
10.3 \\
0.2 \\
10.8 \\
0.5 \\
0.6\end{array}$ & $\begin{array}{l}0.7 \\
0.0 \\
0.1 \\
0.7 \\
0.3 \\
0.4\end{array}$ & $\begin{array}{l}4.4 \\
8.3 \\
0.3 \\
9.4 \\
0.1 \\
1.3\end{array}$ & $\begin{array}{l}3.0 \\
1.6 \\
1.2 \\
2.9 \\
3.3 \\
4.5\end{array}$ & $\begin{array}{l}--- \\
--- \\
--- \\
--- \\
--- \\
---\end{array}$ & $\begin{array}{l}--- \\
--- \\
--- \\
--- \\
--- \\
---\end{array}$ & $\begin{array}{r}0 \\
0 \\
77 \\
0 \\
396 \\
0\end{array}$ \\
\hline
\end{tabular}

$\mathrm{CX}=$ COAXIAL

Note: EM values shown above

are local amplitudes

Ketchikan - North/South of 5515
*Estimated Depth may be unreliable because the

stronger part of the conductor may be deeper or hallow dip or magnetite/ov 
EM Anomaly List

\begin{tabular}{|c|c|c|c|c|c|c|c|c|c|c|c|c|c|}
\hline Label & Fid & Interp & $\begin{array}{c}\text { XUTM } \\
\text { m }\end{array}$ & $\begin{array}{c}\text { YUTM } \\
\mathrm{m}\end{array}$ & $\begin{array}{l}\text { CX } \\
\text { Real } \\
\text { ppm }\end{array}$ & $\begin{array}{l}900 \mathrm{HZ} \\
\text { Quad } \\
\text { ppm }\end{array}$ & $\begin{array}{l}\text { CP } \\
\text { Real } \\
\text { ppm }\end{array}$ & $\begin{array}{l}900 \mathrm{HZ} \\
\text { Quad } \\
\text { ppm }\end{array}$ & $\begin{array}{l}\text { CP } 7 \\
\text { Real } \\
\text { ppm }\end{array}$ & $\begin{array}{l}200 \mathrm{~Hz} \\
\text { Quad } \\
\text { ppm }\end{array}$ & $\begin{array}{l}\text { Vertica } \\
\text { Cond } \\
\text { siemens }\end{array}$ & $\begin{array}{c}\text { al Dike } \\
\text { DEPTH* } \\
\text { m }\end{array}$ & $\begin{array}{c}\text { Mag. Corr } \\
\text { NT }\end{array}$ \\
\hline LINE & 10784 & & & & & & & & & & & & \\
\hline G & 6726.2 & M & 654592 & 6131423 & 4.5 & 0.6 & 4.4 & 1.0 & 4.5 & 5.6 & --- & --- & 0 \\
\hline $\mathrm{H}$ & 6720.0 & M & 654586 & 6131532 & 0.4 & 0.7 & 12.4 & 0.7 & 10.0 & 3.8 & --- & --- & 0 \\
\hline I & 6698.0 & $\mathrm{M}$ & 654614 & 6131778 & 1.5 & 0.7 & 0.7 & 0.1 & 0.1 & 1.0 & --- & --- & 0 \\
\hline $\mathrm{J}$ & 6693.7 & $\mathrm{M}$ & 654608 & 6131870 & 1.3 & 0.4 & 0.8 & 0.2 & 0.8 & 1.6 & --- & --- & 77 \\
\hline $\mathrm{K}$ & 6679.8 & $\mathrm{M}$ & 654592 & 6132252 & 0.1 & 0.6 & 0.5 & 0.6 & 0.3 & 2.2 & --- & --- & 0 \\
\hline L & 6649.9 & $\mathrm{M}$ & 654567 & 6132730 & 0.1 & 0.6 & 6.5 & 0.3 & 0.0 & 2.2 & --- & --- & 0 \\
\hline M & 6631.7 & M & 654534 & 6132898 & 0.5 & 0.3 & 0.0 & 0.3 & 0.6 & 1.4 & --- & --- & 0 \\
\hline $\mathrm{N}$ & 6612.6 & M & 654547 & 6133287 & 0.3 & 0.2 & 0.4 & 0.1 & 0.1 & 1.7 & --- & --- & 0 \\
\hline 0 & 6593.0 & M & 654519 & 6133968 & 0.5 & 0.5 & 1.6 & 0.8 & 1.3 & 3.9 & --- & --- & 0 \\
\hline P & 6573.9 & M & 654474 & 6134618 & 6.9 & 0.8 & 13.7 & 0.4 & 11.1 & 1.7 & --- & --- & 77 \\
\hline Q & 6565.8 & M & 654494 & 6134845 & 0.1 & 0.6 & 0.3 & 0.1 & 0.3 & 5.5 & --- & --- & 576 \\
\hline $\mathrm{R}$ & 6531.3 & $\mathrm{M}$ & 654452 & 6135776 & 0.6 & 0.4 & 4.8 & 0.2 & 4.8 & 1.4 & --- & --- & 77 \\
\hline $\mathrm{S}$ & 6524.6 & $\mathrm{M}$ & 654454 & 6136000 & 1.5 & 0.3 & 1.1 & 0.3 & 1.3 & 0.3 & --- & --- & 359 \\
\hline $\mathrm{T}$ & 6520.8 & $\mathrm{M}$ & 654449 & 6136113 & 7.0 & 0.4 & 9.5 & 0.3 & 7.7 & 0.5 & --- & --- & 0 \\
\hline $\mathrm{U}$ & 6514.0 & M & 654437 & 6136308 & 0.2 & 0.5 & 1.6 & 0.3 & 1.5 & 1.1 & --- & --- & 150 \\
\hline $\mathrm{V}$ & 6489.4 & B & 654446 & 6136948 & 19.8 & 21.7 & 19.2 & 25.7 & 70.3 & 55.0 & 9.2 & 0 & 77 \\
\hline W & 6276.8 & $\mathrm{~S}$ & 654234 & 6141309 & 0.3 & 2.2 & 0.8 & 2.5 & 0.8 & 16.5 & --- & --- & 0 \\
\hline $\mathrm{x}$ & 6134.8 & $\mathrm{~S}$ & 654148 & 6145302 & 0.1 & 2.6 & 0.6 & 3.1 & 2.6 & 19.0 & 0.5 & 0 & 0 \\
\hline $\mathrm{Y}$ & 6073.9 & $B$ ? & 654046 & 6147490 & 3.1 & 2.9 & 0.7 & 4.2 & 11.2 & 22.2 & 3.2 & 45 & 0 \\
\hline $\mathrm{z}$ & 6027.3 & B & 653972 & 6149087 & 12.5 & 18.1 & 13.5 & 28.4 & 67.1 & 35.7 & 4.7 & 13 & 65 \\
\hline $\mathrm{AA}$ & 6009.2 & B & 653961 & 6149582 & 3.2 & 7.9 & 2.9 & 15.4 & 45.8 & 50.8 & 2.1 & 5 & 0 \\
\hline$A B$ & 6004.8 & B & 653957 & 6149686 & 2.4 & 7.4 & 0.9 & 7.1 & 18.5 & 31.9 & 1.6 & 14 & 0 \\
\hline LINE & 10790 & & & & & & & & & & & & \\
\hline A & 7236.7 & $\mathrm{M}$ & 655289 & 6122933 & 1.8 & 0.4 & 0.1 & 0.0 & 0.2 & 2.6 & --- & --- & 0 \\
\hline B & 7193.5 & M & 655302 & 6123510 & 0.9 & 0.0 & 0.6 & 0.1 & 0.4 & 1.2 & --- & --- & 0 \\
\hline $\mathrm{C}$ & 7186.8 & M & 655283 & 6123638 & 2.3 & 0.8 & 3.0 & 0.3 & 2.4 & 1.3 & --- & --- & 0 \\
\hline $\mathrm{D}$ & 7177.9 & M & 655266 & 6123825 & 3.3 & 0.5 & 4.8 & 0.2 & 3.7 & 0.5 & --- & --- & 0 \\
\hline $\mathrm{E}$ & 7168.5 & $\mathrm{M}$ & 655263 & 6123992 & 0.0 & 0.5 & 0.1 & 0.2 & 4.1 & 0.7 & --- & --- & 0 \\
\hline $\mathrm{F}$ & 7142.6 & $\mathrm{M}$ & 655275 & 6124300 & 0.9 & 0.0 & 0.1 & 0.1 & 1.2 & 0.9 & --- & --- & 6 \\
\hline G & 7099.3 & M & 655198 & 6125107 & 3.2 & 0.1 & 0.7 & 0.0 & 0.4 & 1.1 & --- & --- & 0 \\
\hline $\mathrm{H}$ & 7057.9 & $\mathrm{M}$ & 655233 & 6125493 & 12.7 & 0.0 & 13.2 & 0.1 & 12.5 & 4.1 & --- & --- & 0 \\
\hline I & 7027.8 & M & 655212 & 6125727 & 0.0 & 0.4 & 6.8 & 0.2 & 0.0 & 2.0 & --- & --- & 0 \\
\hline $\mathrm{J}$ & 6996.3 & M & 655203 & 6126435 & 0.6 & 0.5 & 0.6 & 0.3 & 0.5 & 1.0 & --- & --- & 0 \\
\hline $\mathrm{K}$ & 6984.3 & $\mathrm{M}$ & 655195 & 6126596 & 5.0 & 0.6 & 0.1 & 0.5 & 0.3 & 1.2 & --- & --- & 0 \\
\hline L & 6973.0 & $\mathrm{~S}$ & 655156 & 6126738 & 0.2 & 1.1 & 0.1 & 1.1 & 0.1 & 4.8 & --- & --- & 0 \\
\hline M & 6930.8 & $\mathrm{M}$ & 655120 & 6127301 & 0.0 & 0.2 & 7.9 & 1.1 & 7.2 & 3.7 & --- & --- & 0 \\
\hline $\mathrm{N}$ & 6781.4 & $\mathrm{M}$ & 655101 & 6128964 & 0.1 & 0.3 & 0.3 & 0.4 & 0.5 & 2.3 & --- & --- & 0 \\
\hline 0 & 6757.3 & M & 655074 & 6129224 & 3.5 & 0.3 & 0.0 & 0.4 & 0.2 & 0.5 & --- & --- & 0 \\
\hline $\mathrm{P}$ & 6743.6 & M & 655098 & 6129361 & 6.1 & 0.1 & 25.7 & 0.1 & 20.9 & 1.0 & --- & --- & 0 \\
\hline Q & 6730.3 & M & 655111 & 6129697 & 0.2 & 0.9 & 0.8 & 1.0 & 0.7 & 7.7 & --- & --- & 0 \\
\hline R & 6726.3 & M & 655091 & 6129815 & 0.0 & 1.1 & 31.7 & 0.8 & 26.1 & 6.5 & --- & --- & 0 \\
\hline $\mathrm{S}$ & 6703.8 & M & 655055 & 6130410 & 0.2 & 0.7 & 0.3 & 0.5 & 0.5 & 3.5 & --- & --- & 353 \\
\hline $\mathrm{T}$ & 6672.9 & M & 655019 & 6130860 & 0.0 & 0.6 & 47.4 & 0.2 & 38.7 & 2.9 & --- & --- & 0 \\
\hline U & 6663.3 & $\mathrm{M}$ & 655010 & 6130939 & 8.2 & 0.4 & 5.3 & 0.6 & 5.0 & 1.1 & --- & --- & 94 \\
\hline V & 6659.1 & M & 655011 & 6131007 & 0.0 & 0.1 & 3.2 & 0.4 & 2.5 & 2.5 & --- & --- & 94 \\
\hline
\end{tabular}

$\mathrm{CX}=$ COAXIAL

Note: EM values shown above

are local amplitudes

Ketchikan - North/South of 5515
* Estimated Depth may be unreliable because the stronger part of the conductor may be deeper or to one side of the flight line, or because of a shallow dip or magnetite/overburden effects 
EM Anomaly List

\begin{tabular}{|c|c|c|c|c|c|c|c|c|c|c|c|c|c|}
\hline Label & Fid & Interp & $\begin{array}{l}\text { XUTM } \\
\text { m }\end{array}$ & $\begin{array}{l}\text { YUTM } \\
\text { m }\end{array}$ & $\begin{array}{l}\text { CX } \\
\text { Real } \\
\text { ppm }\end{array}$ & $\begin{array}{l}900 \mathrm{HZ} \\
\text { Quad } \\
\text { ppm }\end{array}$ & $\begin{array}{l}\text { CP } \\
\text { Real } \\
\text { ppm }\end{array}$ & $\begin{array}{l}900 \mathrm{HZ} \\
\text { Quad } \\
\text { ppm }\end{array}$ & \multicolumn{2}{|c|}{$\begin{array}{ll}\text { CP } & 7200 \mathrm{HZ} \\
\text { Real } & \text { Quad } \\
\text { ppm } & \text { ppm }\end{array}$} & \multicolumn{2}{|c|}{$\begin{array}{l}\text { Vertical Dike } \\
\text { COND DEPTH* } \\
\text { siemens }\end{array}$} & $\begin{array}{c}\text { Mag. Corr } \\
\text { NT }\end{array}$ \\
\hline LINE & 10790 & & & & & & & & & & & & \\
\hline W & 6656.4 & M & 655017 & 6131064 & 25.0 & 0.5 & 54.8 & 0.4 & 45.5 & 1.3 & --- & --- & 18 \\
\hline $\mathrm{x}$ & 6644.6 & M & 655022 & 6131383 & 5.2 & 0.5 & 9.4 & 0.6 & 8.9 & 4.9 & --- & --- & 157 \\
\hline $\mathrm{Y}$ & 6623.6 & M & 654992 & 6131808 & 0.0 & 0.4 & 2.0 & 0.5 & 1.5 & 2.1 & --- & --- & 58 \\
\hline z & 6562.1 & M & 654960 & 6132498 & 4.3 & 0.5 & 4.3 & 0.2 & 2.7 & 0.5 & --- & --- & 1504 \\
\hline $\mathrm{AA}$ & 6539.9 & M & 654962 & 6132774 & 0.0 & 0.5 & 2.8 & 0.7 & 2.3 & 1.9 & --- & --- & 0 \\
\hline$A B$ & 6473.9 & M & 654932 & 6133517 & 2.0 & 0.2 & 1.3 & 0.4 & 0.4 & 2.3 & --- & --- & 0 \\
\hline $\mathrm{AC}$ & 6466.7 & M & 654940 & 6133664 & 0.1 & 0.2 & 0.2 & 0.4 & 0.6 & 2.4 & --- & --- & 0 \\
\hline $\mathrm{AD}$ & 6419.9 & M & 654880 & 6134743 & 0.2 & 0.5 & 2.1 & 1.0 & 2.4 & 7.1 & --- & --- & 0 \\
\hline $\mathrm{AE}$ & 6402.5 & M & 654852 & 6135185 & 0.3 & 0.9 & 1.0 & 1.0 & 0.6 & 8.6 & --- & --- & 0 \\
\hline $\mathrm{AF}$ & 6384.1 & M & 654862 & 6135875 & 1.9 & 0.3 & 0.2 & 0.2 & 4.1 & 1.4 & --- & --- & 94 \\
\hline AG & 6380.7 & M & 654865 & 6136017 & 0.0 & 0.1 & 0.4 & 0.4 & 0.4 & 1.3 & --- & --- & 224 \\
\hline $\mathrm{AH}$ & 6373.0 & M & 654857 & 6136329 & 1.7 & 0.7 & 3.4 & 0.6 & 3.5 & 3.0 & --- & --- & 0 \\
\hline AI & 6363.1 & M & 654839 & 6136725 & 0.4 & 0.6 & 0.4 & 0.5 & 0.4 & 2.3 & --- & --- & 0 \\
\hline AJ & 6346.9 & $\mathrm{D}$ & 654798 & 6137263 & 3.4 & 8.1 & 2.8 & 9.4 & 21.2 & 32.9 & --- & --- & 0 \\
\hline $\mathrm{AK}$ & 6111.7 & S? & 654644 & 6141389 & 1.3 & 2.6 & 2.0 & 3.6 & 0.0 & 25.0 & --- & --- & 0 \\
\hline $\mathrm{AL}$ & 6043.1 & $\mathrm{~S}$ & 654593 & 6143236 & 0.6 & 1.7 & 0.4 & 2.6 & 2.5 & 16.9 & 1.6 & 28 & 85 \\
\hline $\mathrm{AM}$ & 5921.5 & $\mathrm{~S}$ & 654443 & 6146348 & 1.4 & 3.0 & 0.3 & 4.8 & 5.8 & 35.0 & 1.4 & 12 & 0 \\
\hline AN & 5885.8 & $B$ ? & 654454 & 6147589 & 0.3 & 3.3 & 1.8 & 6.2 & 18.3 & 13.3 & --- & --- & 0 \\
\hline $\mathrm{AO}$ & 5873.5 & $B$ ? & 654420 & 6148024 & 1.9 & 5.4 & 4.4 & 7.6 & 13.2 & 21.6 & 2.7 & 19 & 0 \\
\hline $\mathrm{AP}$ & 5860.2 & B & 654363 & 6148502 & 0.6 & 2.0 & 0.2 & 5.1 & 14.8 & 28.0 & 0.8 & 3 & 0 \\
\hline $\mathrm{AQ}$ & 5831.0 & $B$ ? & 654352 & 6149331 & 4.8 & 20.4 & 6.0 & 32.4 & 92.9 & 77.7 & 1.9 & 0 & 0 \\
\hline $\mathrm{AR}$ & 5825.8 & B & 654353 & 6149465 & 11.4 & 9.2 & 21.3 & 27.3 & 83.6 & 34.6 & --- & --- & 0 \\
\hline AS & 5816.8 & B & 654334 & 6149714 & 72.4 & 57.6 & 159.4 & 128.2 & 249.2 & 63.5 & 28.0 & 0 & 0 \\
\hline LINE & 10800 & & & & & & & & & & & & \\
\hline A & 4145.3 & M & 655741 & 6122533 & 5.7 & 0.8 & 7.2 & 0.2 & 5.7 & 1.3 & --- & --- & 106 \\
\hline B & 4155.9 & $\mathrm{M}$ & 655742 & 6122630 & 7.6 & 0.9 & 10.6 & 0.3 & 8.3 & 1.1 & --- & --- & 0 \\
\hline $\mathrm{C}$ & 4194.8 & M & 655736 & 6123231 & 3.6 & 0.6 & 2.5 & 0.6 & 2.0 & 1.9 & --- & --- & 0 \\
\hline D & 4205.8 & M & 655673 & 6123390 & 8.7 & 0.9 & 15.4 & 0.7 & 12.9 & 1.3 & --- & --- & 440 \\
\hline $\mathrm{E}$ & 4217.8 & S? & 655660 & 6123617 & 8.5 & 2.7 & 17.5 & 5.1 & 20.5 & 32.9 & 55.4 & 22 & 0 \\
\hline $\mathrm{F}$ & 4224.6 & M & 655664 & 6123771 & 0.5 & 0.1 & 0.5 & 2.1 & 0.7 & 15.6 & --- & --- & 205 \\
\hline G & 4237.6 & M & 655659 & 6123978 & 0.1 & 0.5 & 0.9 & 0.3 & 0.5 & 1.6 & --- & --- & 39 \\
\hline $\mathrm{H}$ & 4247.6 & M & 655662 & 6124117 & 0.6 & 0.5 & 0.6 & 0.3 & 1.1 & 1.5 & --- & --- & 21 \\
\hline I & 4261.8 & M & 655658 & 6124307 & 3.9 & 1.3 & 6.2 & 0.7 & 5.4 & 5.4 & --- & --- & 0 \\
\hline J & 4276.4 & M & 655661 & 6124430 & 2.7 & 0.2 & 0.3 & 0.6 & 0.1 & 1.0 & --- & --- & 457 \\
\hline $\mathrm{K}$ & 4303.1 & M & 655643 & 6124732 & 40.8 & 0.0 & 59.4 & 0.4 & 45.9 & 2.8 & --- & --- & 495 \\
\hline $\mathrm{L}$ & 4322.4 & M & 655653 & 6124806 & 5.0 & 0.6 & 6.6 & 0.1 & 5.4 & 6.6 & --- & --- & 0 \\
\hline M & 4360.7 & M & 655659 & 6125068 & 0.0 & 0.8 & 11.6 & 0.2 & 8.8 & 2.9 & --- & --- & 0 \\
\hline $\mathrm{N}$ & 4382.2 & M & 655629 & 6125350 & 6.8 & 0.5 & 19.7 & 0.7 & 16.5 & 3.3 & --- & --- & 0 \\
\hline 0 & 4388.5 & M & 655626 & 6125459 & 4.2 & 0.5 & 9.7 & 0.2 & 8.1 & 2.3 & --- & --- & 0 \\
\hline $\mathrm{P}$ & 4409.6 & M & 655623 & 6125762 & 0.3 & 0.0 & 1.0 & 0.3 & 0.8 & 1.5 & --- & --- & 228 \\
\hline Q & 4424.1 & M & 655614 & 6125861 & 3.3 & 0.4 & 0.9 & 0.1 & 0.6 & 0.8 & --- & --- & 0 \\
\hline $\mathrm{R}$ & 4463.6 & $\mathrm{~S}$ & 655570 & 6126395 & 2.2 & 3.3 & 5.5 & 5.3 & 11.9 & 35.4 & 4.8 & 33 & 106 \\
\hline $\mathrm{s}$ & 4485.9 & M & 655588 & 6126851 & 0.1 & 0.6 & 0.5 & 0.4 & 0.5 & 0.5 & --- & --- & 178 \\
\hline $\mathrm{T}$ & 4561.5 & B & 655568 & 6127513 & 0.9 & 1.7 & 0.1 & 0.5 & 1.6 & 4.1 & --- & --- & 106 \\
\hline U & 4651.5 & M & 655491 & 6129021 & 0.0 & 0.2 & 13.3 & 0.2 & 11.1 & 3.7 & --- & --- & 0 \\
\hline
\end{tabular}

$\mathrm{CX}=$ COAXIAL

Note: EM values shown above

are local amplitudes

Ketchikan - North/South of 5515
* Estimated Depth may be unreliable because the stronger part of the conductor may be deeper or to one side of the flight line, or because of a shallow dip or magnetite/overburden effects 
EM Anomaly List

\begin{tabular}{|c|c|c|c|c|c|c|c|c|c|c|c|c|c|}
\hline Label & Fid & Interp & $\begin{array}{c}\text { XUTM } \\
\text { m }\end{array}$ & $\begin{array}{c}\text { YUTM } \\
\text { m }\end{array}$ & $\begin{array}{l}\text { CX } \\
\text { Real } \\
\text { ppm }\end{array}$ & $\begin{array}{l}900 \mathrm{HZ} \\
\text { Quad } \\
\text { ppm }\end{array}$ & $\begin{array}{l}\text { CP } \\
\text { Real } \\
\text { ppm }\end{array}$ & $\begin{array}{l}900 \mathrm{HZ} \\
\text { Quad } \\
\text { ppm }\end{array}$ & \multicolumn{2}{|c|}{$\begin{array}{ll}\text { CP } & 7200 \mathrm{~Hz} \\
\text { Real } & \text { Quad } \\
\text { ppm } & \text { ppm }\end{array}$} & \multicolumn{2}{|c|}{$\begin{array}{cc}\text { Vertical Dike } \\
\text { COND } & \text { DEPTH* } \\
\text { siemens } & m\end{array}$} & $\begin{array}{l}\text { Mag. Corr } \\
\text { NT }\end{array}$ \\
\hline LINE & 10800 & & & & & & & & & & & & \\
\hline V & 4664.1 & M & 655492 & 6129106 & 1.4 & 0.0 & 1.7 & 0.3 & 1.2 & 1.8 & --- & --- & 75 \\
\hline W & 4715.2 & M & 655489 & 6129848 & 0.0 & 0.4 & 0.2 & 0.3 & 0.1 & 2.3 & --- & --- & 0 \\
\hline $\mathrm{X}$ & 4742.6 & M & 655438 & 6130301 & 1.2 & 1.3 & 4.1 & 1.2 & 3.4 & 9.1 & --- & --- & 765 \\
\hline $\mathrm{Y}$ & 4758.1 & M & 655436 & 6130710 & 1.6 & 0.2 & 2.4 & 0.5 & 2.4 & 2.3 & --- & --- & 0 \\
\hline z & 4783.5 & $\mathrm{~S}$ & 655422 & 6131508 & 1.0 & 7.9 & 4.3 & 14.7 & 44.7 & 72.9 & 1.3 & 0 & 0 \\
\hline $\mathrm{AA}$ & 4798.1 & M & 655404 & 6131959 & 2.6 & 0.3 & 2.0 & 0.6 & 2.2 & 1.7 & --- & --- & 23 \\
\hline$A B$ & 4824.4 & M & 655388 & 6132474 & 0.7 & 0.2 & 3.0 & 0.1 & 3.5 & 0.9 & --- & --- & 26 \\
\hline $\mathrm{AC}$ & 4854.0 & M & 655349 & 6132955 & 1.3 & 0.4 & 1.2 & 0.2 & 1.4 & 0.6 & --- & --- & 1195 \\
\hline $\mathrm{AD}$ & 4864.7 & M & 655348 & 6133124 & 0.4 & 0.1 & 0.9 & 0.1 & 0.3 & 0.7 & --- & --- & 0 \\
\hline $\mathrm{AE}$ & 4875.9 & M & 655361 & 6133334 & 3.6 & 0.5 & 0.2 & 0.1 & 0.1 & 0.4 & --- & --- & 32 \\
\hline $\mathrm{AF}$ & 4900.6 & M & 655341 & 6133668 & 0.4 & 0.6 & 0.1 & 0.7 & 0.1 & 1.3 & --- & --- & 28 \\
\hline$A G$ & 4916.0 & M & 655324 & 6133882 & 0.7 & 0.6 & 0.4 & 0.3 & 0.4 & 4.1 & --- & --- & 435 \\
\hline $\mathrm{AH}$ & 4932.9 & $S$ & 655283 & 6134310 & 4.8 & 1.2 & 8.6 & 1.8 & 7.3 & 12.2 & --- & --- & 106 \\
\hline AI & 4943.6 & M & 655314 & 6134620 & 0.1 & 1.0 & 6.5 & 0.9 & 5.7 & 3.5 & --- & --- & 0 \\
\hline AJ & 4964.7 & M & 655298 & 6134997 & 10.5 & 1.4 & 28.5 & 0.7 & 24.3 & 13.3 & --- & --- & 670 \\
\hline $\mathrm{AK}$ & 4973.6 & M & 655287 & 6135135 & 10.0 & 0.2 & 1.1 & 0.1 & 0.6 & 0.4 & --- & --- & 639 \\
\hline $\mathrm{AL}$ & 4989.6 & M & 655289 & 6135560 & 3.7 & 0.2 & 5.9 & 0.6 & 4.9 & 2.7 & --- & --- & 0 \\
\hline $\mathrm{AM}$ & 4999.9 & M & 655274 & 6135845 & 1.9 & 0.3 & 1.4 & 0.2 & 0.7 & 2.4 & --- & --- & 407 \\
\hline AN & 5008.0 & M & 655255 & 6136087 & 0.6 & 0.5 & 2.4 & 1.0 & 2.6 & 7.4 & --- & --- & 54 \\
\hline $\mathrm{AO}$ & 5024.0 & M & 655248 & 6136603 & 2.3 & 0.5 & 0.8 & 0.2 & 0.7 & 2.8 & --- & --- & 532 \\
\hline $\mathrm{AP}$ & 5048.7 & D & 655193 & 6137137 & 2.8 & 5.5 & 1.4 & 2.3 & 5.0 & 12.9 & 2.2 & 46 & 106 \\
\hline $\mathrm{AQ}$ & 5290.3 & S & 655033 & 6141507 & 0.0 & 1.4 & 0.1 & 1.6 & 1.1 & 9.4 & --- & --- & 0 \\
\hline$A R$ & 5329.0 & $B$ ? & 655005 & 6142708 & 2.2 & 3.3 & 0.5 & 4.3 & 12.5 & 22.2 & 2.3 & 37 & 0 \\
\hline AS & 5338.6 & B & 655028 & 6142850 & 0.8 & 2.4 & 0.3 & 1.0 & 4.1 & 1.5 & --- & --- & 106 \\
\hline $\mathrm{AT}$ & 5468.5 & $B$ ? & 654884 & 6145716 & 3.0 & 8.3 & 1.6 & 12.6 & 36.7 & 50.9 & 1.7 & 0 & 0 \\
\hline $\mathrm{AU}$ & 5489.0 & $B$ ? & 654854 & 6146436 & 0.7 & 4.8 & 1.2 & 11.6 & 38.7 & 52.6 & 0.6 & 0 & 0 \\
\hline AV & 5510.2 & B? & 654833 & 6147204 & 6.9 & 10.3 & 5.2 & 16.8 & 47.0 & 34.7 & 2.9 & 18 & 0 \\
\hline AW & 5521.1 & B & 654848 & 6147570 & 0.5 & 3.0 & 5.8 & 7.9 & 16.3 & 9.4 & -- & --- & 0 \\
\hline$A X$ & 5533.1 & B & 654835 & 6147996 & 3.4 & 4.1 & 7.3 & 4.0 & 5.1 & 3.0 & --- & --- & 106 \\
\hline$A Y$ & 5540.3 & B & 654811 & 6148253 & 7.8 & 10.2 & 0.2 & 3.8 & 11.6 & 12.0 & 4.0 & 8 & 0 \\
\hline AZ & 5557.1 & B & 654775 & 6148882 & 0.1 & 4.4 & 9.9 & 21.8 & 54.0 & 19.8 & 2.3 & 0 & 0 \\
\hline $\mathrm{BA}$ & 5570.6 & B & 654746 & 6149310 & 59.8 & 53.3 & 101.5 & 121.3 & 250.0 & 101.3 & 17.4 & 0 & 0 \\
\hline BB & 5572.9 & B & 654741 & 6149378 & 57.7 & 48.4 & 168.1 & 134.3 & 264.0 & 16.2 & 26.6 & 0 & 106 \\
\hline $\mathrm{BC}$ & 5576.1 & B & 654734 & 6149471 & 32.4 & 14.5 & 48.4 & 34.8 & 57.2 & 17.5 & 33.6 & 0 & 0 \\
\hline $\mathrm{BD}$ & 5579.9 & B & 654728 & 6149594 & 28.3 & 26.9 & 55.6 & 55.6 & 114.9 & 44.9 & --- & --- & 0 \\
\hline LINE & 10810 & & & & & & & & & & & & \\
\hline A & 4031.4 & M & 656141 & 6122618 & 15.7 & 0.4 & 67.6 & 0.0 & 54.0 & 1.1 & --- & --- & 0 \\
\hline B & 4010.4 & M & 656146 & 6122702 & 13.6 & 0.2 & 4.3 & 0.1 & 4.0 & 1.5 & --- & --- & 0 \\
\hline $\mathrm{C}$ & 3989.6 & M & 656138 & 6122826 & 0.0 & 0.4 & 24.6 & 0.0 & 20.7 & 1.5 & --- & --- & 0 \\
\hline D & 3971.8 & M & 656123 & 6123067 & 0.4 & 0.5 & 0.2 & 0.2 & 0.1 & 1.0 & --- & --- & 0 \\
\hline $\mathrm{E}$ & 3918.3 & M & 656079 & 6124203 & 9.4 & 0.0 & 19.7 & 0.4 & 15.2 & 2.0 & --- & --- & 369 \\
\hline $\mathrm{F}$ & 3911.6 & M & 656079 & 6124314 & 4.0 & 0.5 & 2.9 & 0.5 & 2.0 & 2.7 & --- & --- & 0 \\
\hline G & 3880.3 & M & 656076 & 6124697 & 21.6 & 0.4 & 19.8 & 0.3 & 15.3 & 2.0 & --- & --- & 0 \\
\hline $\mathrm{H}$ & 3847.8 & M & 656058 & 6124897 & 69.1 & 0.2 & 107.2 & 1.2 & 91.1 & 0.6 & --- & --- & 0 \\
\hline I & 3781.8 & M & 656022 & 6125826 & 3.6 & 0.7 & 1.9 & 0.2 & 2.0 & 1.1 & --- & --- & 0 \\
\hline
\end{tabular}

$\mathrm{CX}=$ COAXIAL

Note: EM values shown above

are local amplitudes

Ketchikan - North/South of 5515
* Estimated Depth may be unreliable because the stronger part of the conductor may be deeper or to one side of the flight line, or because of a shallow dip or magnetite/overburden effects 
EM Anomaly List

\begin{tabular}{|c|c|c|c|c|c|c|c|c|c|c|c|c|c|}
\hline Label & Fid & Interp & $\begin{array}{c}\text { XUTM } \\
\text { m }\end{array}$ & $\begin{array}{c}\text { YUTM } \\
\mathrm{m}\end{array}$ & $\begin{array}{l}\text { CX } \\
\text { Real } \\
\text { ppm }\end{array}$ & $\begin{array}{l}900 \mathrm{HZ} \\
\text { Quad } \\
\text { ppm }\end{array}$ & $\begin{array}{l}\text { CP } \\
\text { Real } \\
\text { ppm }\end{array}$ & $\begin{array}{l}900 \mathrm{HZ} \\
\text { Quad } \\
\text { ppm }\end{array}$ & $\begin{array}{l}\text { CP } 7 \\
\text { Real } \\
\text { ppm }\end{array}$ & $\begin{array}{c}7200 \mathrm{HZ} \\
\text { Quad } \\
\text { ppm }\end{array}$ & $\begin{array}{l}\text { Vertica } \\
\text { ConD } \\
\text { siemens }\end{array}$ & $\begin{array}{c}\text { al Dike } \\
\text { DEPTH* } \\
\text { m }\end{array}$ & $\begin{array}{c}\text { Mag. Corr } \\
\text { NT }\end{array}$ \\
\hline LINE & 10810 & & & & & & & & & & & & \\
\hline $\mathrm{J}$ & 3761.8 & M & 656015 & 6125922 & 1.2 & 0.1 & 5.0 & 0.1 & 4.2 & 1.8 & --- & --- & 0 \\
\hline $\mathrm{K}$ & 3749.0 & M & 656018 & 6125994 & 0.1 & 0.2 & 3.9 & 0.2 & 3.3 & 0.7 & --- & --- & 0 \\
\hline L & 3734.0 & $\mathrm{M}$ & 656007 & 6126041 & 2.4 & 0.6 & 6.2 & 0.4 & 5.2 & 0.1 & --- & --- & 0 \\
\hline M & 3726.5 & $\mathrm{M}$ & 656005 & 6126077 & 1.9 & 0.1 & 3.7 & 0.3 & 2.8 & 0.5 & --- & --- & 107 \\
\hline $\mathrm{N}$ & 3600.4 & B & 655937 & 6128051 & 3.1 & 4.4 & 6.1 & 12.0 & 29.3 & 14.1 & 3.2 & 31 & 0 \\
\hline 0 & 3595.1 & S? & 655946 & 6128197 & 7.4 & 7.3 & 6.4 & 12.4 & 29.7 & 12.5 & 5.9 & 0 & 0 \\
\hline $\mathrm{P}$ & 3570.8 & M & 655903 & 6128871 & 0.5 & 0.6 & 0.1 & 1.1 & 0.0 & 6.1 & --- & --- & 107 \\
\hline Q & 3525.0 & M & 655889 & 6129492 & 4.3 & 0.4 & 5.1 & 0.1 & 4.9 & 0.3 & --- & --- & 1439 \\
\hline R & 3480.0 & M & 655838 & 6130071 & 0.3 & 0.3 & 0.7 & 0.8 & 0.6 & 0.6 & --- & --- & 0 \\
\hline $\mathrm{S}$ & 3460.7 & M & 655867 & 6130460 & 0.3 & 0.1 & 6.5 & 0.4 & 6.3 & 2.4 & --- & --- & 107 \\
\hline $\mathrm{T}$ & 3407.1 & $\mathrm{~S}$ & 655769 & 6131786 & 0.1 & 3.9 & 0.7 & 5.8 & 3.6 & 37.7 & --- & --- & 73 \\
\hline $\mathrm{U}$ & 3383.1 & M & 655715 & 6132621 & 0.7 & 0.7 & 4.7 & 0.5 & 4.7 & 4.1 & --- & --- & 0 \\
\hline $\mathrm{V}$ & 3369.7 & $\mathrm{M}$ & 655716 & 6133031 & 0.1 & 0.4 & 0.2 & 0.4 & 0.2 & 2.2 & --- & --- & 929 \\
\hline W & 3348.0 & $\mathrm{M}$ & 655735 & 6133540 & 1.1 & 0.3 & 0.2 & 0.2 & 0.1 & 1.5 & --- & --- & 0 \\
\hline $\mathrm{x}$ & 3331.0 & M & 655743 & 6133807 & 16.4 & 0.0 & 33.2 & 0.3 & 26.7 & 3.0 & --- & --- & 0 \\
\hline $\mathrm{Y}$ & 3324.4 & M & 655737 & 6133961 & 0.0 & 0.4 & 26.9 & 0.1 & 22.8 & 4.1 & --- & --- & 595 \\
\hline $\mathrm{z}$ & 3297.3 & M & 655693 & 6134806 & 0.0 & 0.9 & 0.9 & 0.7 & 0.3 & 3.9 & --- & --- & 889 \\
\hline $\mathrm{AA}$ & 3276.0 & M & 655717 & 6135332 & 0.0 & 0.5 & 0.5 & 0.3 & 0.2 & 4.7 & --- & --- & 0 \\
\hline$A B$ & 3260.8 & S? & 655700 & 6135746 & 0.2 & 1.2 & 4.8 & 1.4 & 4.0 & 10.4 & 8.1 & 58 & 597 \\
\hline $\mathrm{AC}$ & 3217.9 & $\mathrm{~S}$ & 655670 & 6136887 & 2.4 & 0.6 & 7.7 & 1.6 & 7.2 & 10.8 & -- & --- & 107 \\
\hline $\mathrm{AD}$ & 2928.1 & $\mathrm{~s}$ & 655410 & 6142561 & 0.9 & 2.7 & 0.4 & 2.9 & 2.1 & 20.9 & 1.2 & 21 & 0 \\
\hline $\mathrm{AE}$ & 2908.0 & $\mathrm{~S}$ & 655369 & 6143045 & 1.9 & 0.8 & 0.0 & 1.1 & 0.3 & 6.2 & 8.3 & 71 & 107 \\
\hline $\mathrm{AF}$ & 2860.0 & B? & 655354 & 6144202 & 0.3 & 1.7 & 2.1 & 7.1 & 18.5 & 21.6 & 1.2 & 8 & 0 \\
\hline AG & 2787.4 & S & 655292 & 6146203 & 0.4 & 2.3 & 0.9 & 2.3 & 2.3 & 13.7 & 1.1 & 9 & 0 \\
\hline $\mathrm{AH}$ & 2776.7 & B & 655272 & 6146619 & 3.2 & 21.0 & 15.4 & 52.4 & 127.3 & 68.4 & 2.2 & 0 & 0 \\
\hline $\mathrm{AI}$ & 2766.6 & B & 655260 & 6147013 & 10.2 & 21.2 & 20.8 & 47.9 & 122.2 & 98.2 & --- & --- & 0 \\
\hline AJ & 2743.1 & B & 655223 & 6147878 & 56.2 & 55.5 & 101.7 & 113.4 & 269.7 & 128.9 & 16.8 & 0 & 0 \\
\hline AK & 2733.6 & B & 655211 & 6148162 & 10.3 & 11.6 & 48.8 & 60.8 & 136.0 & 53.3 & 9.4 & 5 & 0 \\
\hline $\mathrm{AL}$ & 2727.1 & B & 655204 & 6148301 & 16.4 & 26.2 & 12.8 & 27.6 & 70.5 & 60.5 & 4.7 & 10 & 107 \\
\hline $\mathrm{AM}$ & 2718.1 & B & 655198 & 6148477 & 9.8 & 3.3 & 26.1 & 7.3 & 17.3 & 0.1 & 62.0 & 3 & 0 \\
\hline AN & 2710.7 & B & 655198 & 6148706 & 26.7 & 17.0 & 66.4 & 32.8 & 72.1 & 18.0 & --- & --- & 0 \\
\hline $\mathrm{AO}$ & 2706.8 & B & 655201 & 6148833 & 29.1 & 17.0 & 69.1 & 49.3 & 101.0 & 16.9 & 28.0 & 0 & 107 \\
\hline $\mathrm{AP}$ & 2699.9 & B & 655192 & 6149076 & 7.7 & 9.5 & 7.5 & 4.8 & 18.1 & 15.9 & 8.1 & 15 & 0 \\
\hline $\mathrm{AQ}$ & 2685.1 & B & 655170 & 6149581 & 35.2 & 19.3 & 68.3 & 33.5 & 93.2 & 19.4 & 40.3 & 0 & 0 \\
\hline $\begin{array}{l}\text { LINE } \\
A\end{array}$ & $\begin{array}{c}10820 \\
369.2\end{array}$ & $\mathrm{M}$ & 656539 & 6122474 & 2.2 & 0.1 & 104.6 & 0.1 & 86.6 & 2.5 & --- & --- & 45 \\
\hline $\begin{array}{l}\text { LINE } \\
\mathrm{A}\end{array}$ & $\begin{array}{c}10821 \\
533.0\end{array}$ & M & 656492 & 6124285 & 0.1 & 1.0 & 7.4 & 0.5 & 6.3 & 2.4 & --- & --- & 0 \\
\hline$\overline{\mathrm{LINE}}$ & 10822 & & & & & & & & & & & & \\
\hline A & 864.9 & M & 656458 & 6124719 & 7.1 & 0.9 & 17.6 & 0.4 & 36.0 & 1.7 & --- & --- & 0 \\
\hline B & 719.3 & $\mathrm{~S}$ & 656462 & 6125233 & 2.2 & 0.6 & 0.5 & 1.4 & 0.4 & 7.5 & --- & --- & 0 \\
\hline
\end{tabular}

$\mathrm{CX}=$ COAXIAL
$\mathrm{CP}=$ COPLANAR

Note: EM values shown above

Ketchikan - North/South of 5515
*Estimated Depth may be unreliable because the stronger part of the conductor may be deeper or to one side of the flight line, or because of a shallow dip or magnetite/overburden effects 
EM Anomaly List

\begin{tabular}{|c|c|c|c|c|c|c|c|c|c|c|c|c|c|}
\hline Label & Fid & Interp & $\begin{array}{l}\text { XUTM } \\
\text { m }\end{array}$ & $\begin{array}{c}\text { YUTM } \\
\text { m }\end{array}$ & $\begin{array}{ll}\text { CX } & 9 \\
\text { Real } & \\
\text { ppm }\end{array}$ & $\begin{array}{l}00 \mathrm{HZ} \\
\text { Quad } \\
\text { ppm }\end{array}$ & $\begin{array}{l}\text { CP } \\
\text { Real } \\
\text { ppm }\end{array}$ & $\begin{array}{l}900 \mathrm{HZ} \\
\text { Quad } \\
\text { ppm }\end{array}$ & $\begin{array}{l}\text { CP } \\
\text { Real } \\
\text { ppm }\end{array}$ & $\begin{array}{l}7200 \mathrm{HZ} \\
\text { Quad } \\
\text { ppm }\end{array}$ & $\begin{array}{l}\text { Vertica } \\
\text { Cond } \\
\text { siemens }\end{array}$ & $\begin{array}{c}\text { al Dike } \\
\text { DEPTH* } \\
\text { m }\end{array}$ & $\begin{array}{c}\text { Mag. Corr } \\
\text { NT }\end{array}$ \\
\hline $\begin{array}{ll}\text { LINE } & 1 \\
A\end{array}$ & $\begin{array}{c}10823 \\
1058.1\end{array}$ & $B$ ? & 656390 & 6125223 & 2.6 & 2.1 & 1.2 & 2.7 & 3.1 & 20.4 & --- & --- & 0 \\
\hline $\begin{array}{ll}\text { LINE } & 1 \\
\text { A } & \\
\text { B } \\
\text { C }\end{array}$ & $\begin{array}{r}10824 \\
1445.8 \\
1434.0 \\
1346.0\end{array}$ & $\begin{array}{r}\mathrm{B} \\
\mathrm{B} ? \\
\mathrm{~B}\end{array}$ & $\begin{array}{l}656418 \\
656426 \\
656402\end{array}$ & $\begin{array}{l}6126226 \\
6126381 \\
6127199\end{array}$ & $\begin{array}{l}0.0 \\
0.2 \\
3.6\end{array}$ & $\begin{array}{l}4.7 \\
0.6 \\
2.2\end{array}$ & $\begin{array}{l}0.4 \\
3.4 \\
1.8\end{array}$ & $\begin{array}{l}4.2 \\
3.9 \\
2.9\end{array}$ & $\begin{array}{r}13.6 \\
9.4 \\
9.8\end{array}$ & $\begin{array}{r}25.8 \\
6.4 \\
7.8\end{array}$ & $\begin{array}{l}0.5 \\
3.2 \\
---\end{array}$ & $\begin{array}{r}0 \\
61 \\
---\end{array}$ & $\begin{array}{l}0 \\
0 \\
0\end{array}$ \\
\hline $\begin{array}{l}\text { LINE } 1 \\
\end{array}$ & 10825 & & & & & & & & & & & & \\
\hline A & 1660.2 & M & 656331 & 6128701 & 0.0 & 0.0 & 7.7 & 0.4 & 5.7 & 4.1 & --- & --- & 78 \\
\hline B & 1672.1 & S? & 656277 & 6128935 & 13.0 & 2.8 & 19.8 & 4.3 & 21.4 & 27.9 & 104.6 & 15 & 82 \\
\hline $\mathrm{C}$ & 1676.2 & M & 656267 & 6129014 & 0.0 & 2.9 & 10.4 & 4.4 & 13.0 & 28.7 & -- & --- & 0 \\
\hline D & 1695.3 & $\mathrm{M}$ & 656279 & 6129194 & 0.1 & 0.5 & 7.6 & 0.3 & 6.3 & 3.4 & --- & --- & 0 \\
\hline $\mathrm{E}$ & 1806.2 & $\mathrm{M}$ & 656280 & 6129917 & 0.0 & 0.3 & 0.9 & 0.1 & 0.7 & 1.0 & --- & --- & 0 \\
\hline $\mathrm{F}$ & 1816.6 & M & 656280 & 6130078 & 1.0 & 0.5 & 1.7 & 0.1 & 1.8 & 1.9 & --- & --- & 158 \\
\hline G & 1840.6 & $\mathrm{~S} ?$ & 656256 & 6130592 & 0.1 & 1.1 & 0.1 & 1.4 & 0.2 & 9.5 & --- & --- & 0 \\
\hline $\mathrm{H}$ & 1844.5 & M & 656254 & 6130681 & 1.6 & 0.5 & 2.7 & 0.7 & 1.9 & 9.9 & --- & --- & 0 \\
\hline I & 1851.2 & M & 656239 & 6130825 & 4.6 & 1.1 & 9.5 & 1.5 & 8.5 & 8.8 & --- & --- & 0 \\
\hline $\mathrm{J}$ & 1855.4 & $\mathrm{M}$ & 656226 & 6130911 & 2.5 & 0.2 & 7.7 & 0.3 & 6.3 & 5.7 & --- & --- & 341 \\
\hline $\mathrm{K}$ & 1864.2 & M & 656207 & 6131078 & 0.0 & 0.5 & 0.4 & 0.4 & 0.1 & 1.2 & --- & --- & 0 \\
\hline L & 1884.7 & M & 656227 & 6131465 & 0.0 & 0.7 & 1.0 & 0.1 & 0.6 & 2.9 & --- & --- & 110 \\
\hline M & 1919.7 & M & 656145 & 6132574 & 0.5 & 0.0 & 0.3 & 0.1 & 0.5 & 4.5 & --- & --- & 933 \\
\hline N & 1934.0 & M & 656145 & 6132847 & 1.6 & 0.3 & 4.2 & 0.2 & 3.2 & 1.1 & --- & --- & 1322 \\
\hline 0 & 1943.9 & $\mathrm{M}$ & 656145 & 6133076 & 8.8 & 0.3 & 10.9 & 0.4 & 9.2 & 0.6 & --- & --- & 0 \\
\hline $\mathrm{P}$ & 1962.8 & M & 656139 & 6133651 & 2.8 & 0.6 & 13.3 & 0.5 & 13.7 & 5.6 & --- & --- & 941 \\
\hline Q & 2023.3 & M & 656069 & 6135698 & 0.0 & 0.4 & 13.0 & 0.4 & 11.9 & 4.3 & --- & --- & 217 \\
\hline $\begin{array}{l}2 \\
\mathrm{R}\end{array}$ & 2026.5 & M & 656059 & 6135798 & 1.4 & 0.6 & 3.8 & 0.5 & 3.4 & 4.5 & --- & --- & 2 \\
\hline S & 2053.0 & $\mathrm{~S}$ & 656008 & 6136488 & 1.7 & 1.3 & 0.7 & 1.2 & 0.9 & 6.5 & --- & --- & 0 \\
\hline $\mathrm{T}$ & 2221.6 & $B$ ? & 655918 & 6141081 & 0.3 & 0.8 & 0.7 & 3.2 & 5.9 & 19.4 & --- & --- & 0 \\
\hline $\mathrm{U}$ & 2227.2 & $B$ ? & 655912 & 6141227 & 0.3 & 0.3 & 0.1 & 2.8 & 2.1 & 12.9 & --- & --- & 78 \\
\hline $\mathrm{V}$ & 2252.5 & B? & 655869 & 6141849 & 2.4 & 6.0 & 0.9 & 3.3 & 9.4 & 15.3 & 2.1 & 0 & 0 \\
\hline W & 2278.8 & $\mathrm{~S}$ & 655830 & 6142686 & 1.9 & 1.5 & 1.7 & 0.8 & 0.4 & 7.5 & -- & --- & 0 \\
\hline $\mathrm{x}$ & 2297.9 & M & 655811 & 6143266 & 0.0 & 0.8 & 4.2 & 1.6 & 1.7 & 7.0 & --- & --- & 0 \\
\hline $\mathrm{Y}$ & 2319.3 & D & 655798 & 6143641 & 18.1 & 12.6 & 4.5 & 11.6 & 31.3 & 21.7 & 11.9 & 15 & 0 \\
\hline $\mathrm{Z}$ & 2377.5 & B & 655778 & 6144299 & 2.6 & 10.7 & 4.5 & 27.9 & 80.9 & 71.9 & 1.5 & 0 & 0 \\
\hline $\mathrm{AA}$ & 2390.9 & B & 655767 & 6144634 & 2.6 & 5.4 & 10.7 & 17.9 & 39.6 & 23.9 & 3.1 & 24 & 82 \\
\hline$A B$ & 2406.7 & B & 655746 & 6145006 & 6.5 & 14.8 & 2.4 & 14.0 & 35.6 & 56.3 & 2.8 & 0 & 0 \\
\hline $\mathrm{AC}$ & 2427.6 & B & 655739 & 6145565 & 17.9 & 24.4 & 40.6 & 41.7 & 84.2 & 47.0 & 10.0 & 0 & 0 \\
\hline $\mathrm{AD}$ & 2430.0 & B & 655732 & 6145637 & 22.0 & 24.7 & 46.4 & 34.6 & $\begin{array}{l}80.5 \\
80.5\end{array}$ & 35.9 & 15.0 & 2 & 0 \\
\hline $\mathrm{AE}$ & 2440.3 & B & 655685 & 6145948 & 1.6 & 4.2 & 2.5 & 15.6 & 44.6 & 55.4 & 1.6 & 7 & 0 \\
\hline $\mathrm{AF}$ & 2448.2 & B & 655684 & 6146198 & 18.3 & 32.8 & 20.2 & 56.2 & 131.3 & 124.3 & 5.4 & 3 & 0 \\
\hline $\mathrm{AG}$ & 2451.8 & B & 655690 & 6146317 & 35.5 & 54.7 & 41.6 & 137.4 & 350.1 & 169.7 & 6.7 & 0 & 172 \\
\hline $\mathrm{AH}$ & 2454.7 & B & 655693 & 6146415 & 30.2 & 43.5 & 43.9 & 73.2 & 209.8 & 80.3 & 7.5 & 8 & 0 \\
\hline $\mathrm{AI}$ & 2457.8 & B & 655693 & 6146522 & 26.6 & 39.7 & 29.2 & 61.2 & 146.6 & 93.6 & 5.9 & 10 & 0 \\
\hline $\mathrm{AJ}$ & 2462.0 & B & 655689 & 6146672 & 6.5 & 9.4 & 8.3 & 21.9 & 163.5 & 60.4 & -- & --- & 0 \\
\hline AK & 2465.4 & B & 655682 & 6146795 & 22.6 & 50.6 & 42.4 & 92.0 & 215.7 & 209.0 & 6.1 & 0 & 0 \\
\hline
\end{tabular}

$\mathrm{CX}=$ COAXIAL

Note: EM values shown above

Ketchikan - North/South of 5515
*Estimated Depth may be unreliable because the stronger part of the conductor may be deeper or to one side of the flight line, or because of shallow dip or magnetite/overburden effects 
EM Anomaly List

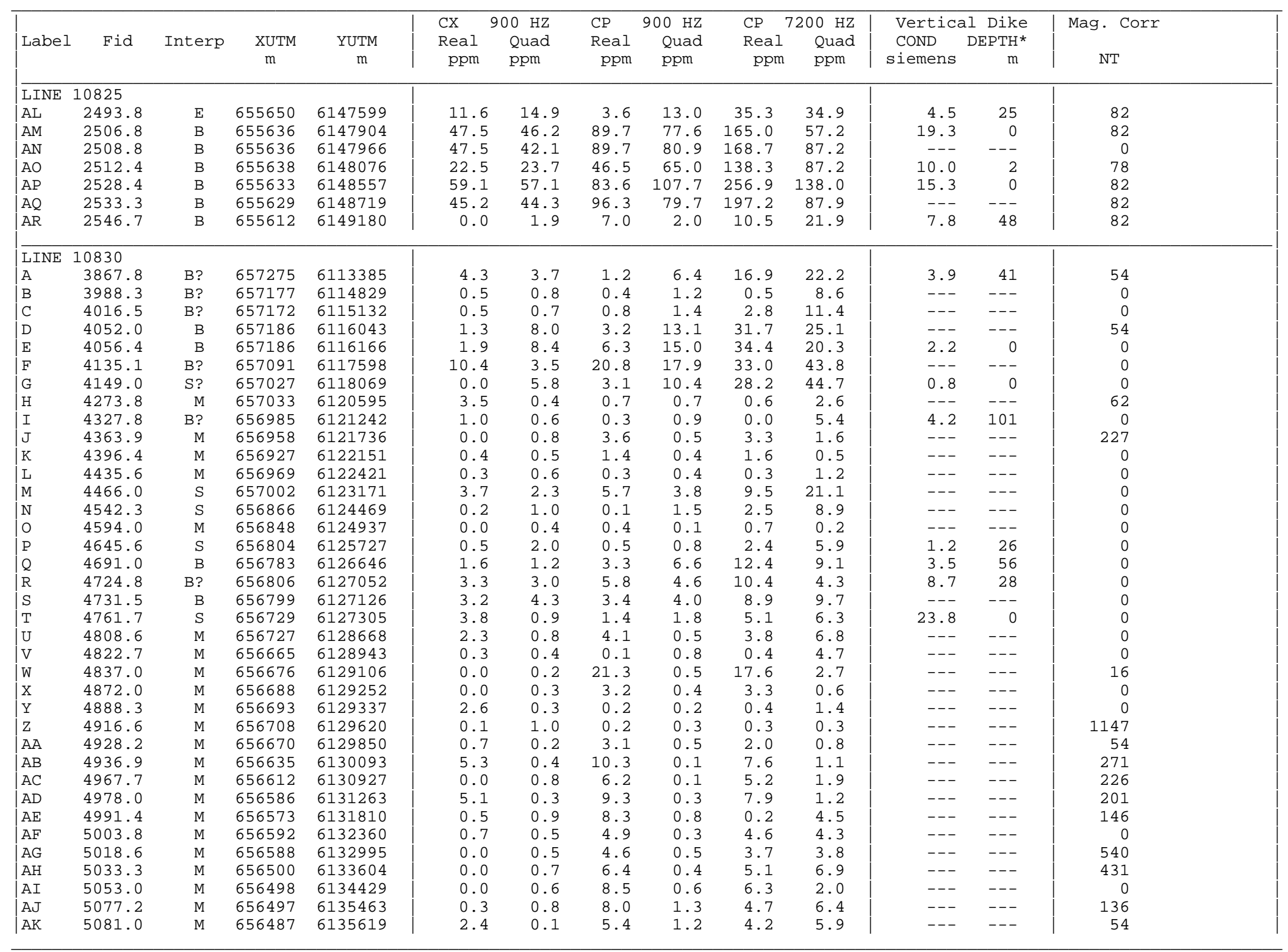

$\mathrm{CX}=$ COAXIAL

Note: EM values shown above

are local amplitudes
*Estimated Depth may be unreliable because the stronger part of the conductor may be deeper or to one side of the flight line, or because of a shallow dip or magnetite/overburden effects 
EM Anomaly List

\begin{tabular}{|c|c|c|c|c|c|c|c|c|c|c|c|c|c|}
\hline Label & Fid & Interp & $\begin{array}{c}\text { XUTM } \\
\mathrm{m}\end{array}$ & $\begin{array}{c}\text { YUTM } \\
\mathrm{m}\end{array}$ & $\begin{array}{l}\text { CX } \\
\text { Real } \\
\text { ppm }\end{array}$ & $\begin{array}{l}900 \mathrm{HZ} \\
\text { Quad } \\
\text { ppm }\end{array}$ & $\begin{array}{l}\text { CP } \\
\text { Real } \\
\text { ppm }\end{array}$ & $\begin{array}{l}900 \mathrm{HZ} \\
\text { Quad } \\
\text { ppm }\end{array}$ & \multicolumn{2}{|c|}{$\begin{array}{ll}\text { CP } & 7200 \mathrm{HZ} \\
\text { Real } & \text { Quad } \\
\text { ppm } & \text { ppm }\end{array}$} & $\begin{array}{l}\text { Vertica } \\
\text { COND } \\
\text { siemens }\end{array}$ & $\begin{array}{c}\text { al Dike } \\
\text { DEPTH* } \\
\text { m }\end{array}$ & $\begin{array}{c}\text { Mag. Corr } \\
\text { NT }\end{array}$ \\
\hline LINE & 10830 & & & & & & & & & & & & \\
\hline $\mathrm{AL}$ & 5084.6 & M & 656473 & 6135757 & 1.9 & 0.3 & 4.1 & 1.0 & 2.3 & 5.9 & --- & --- & 118 \\
\hline $\mathrm{AM}$ & 5127.7 & M & 656417 & 6137361 & 0.6 & 0.7 & 1.2 & 0.2 & 0.2 & 2.7 & --- & --- & 407 \\
\hline AN & 5274.1 & $\mathrm{~B}$ ? & 656266 & 6141450 & 1.9 & 1.4 & 0.3 & 0.8 & 2.2 & 10.4 & 6.0 & 67 & 0 \\
\hline $\mathrm{AO}$ & 5328.0 & B & 656245 & 6141787 & 5.5 & 8.1 & 3.3 & 6.9 & 17.7 & 21.5 & 3.6 & 20 & 42 \\
\hline $\mathrm{AP}$ & 5336.3 & B? & 656244 & 6141881 & 5.2 & 16.5 & 4.7 & 19.7 & 45.6 & 65.4 & 2.4 & 0 & 0 \\
\hline $\mathrm{AQ}$ & 5378.2 & B & 656228 & 6142271 & 1.1 & 3.8 & 0.6 & 5.2 & 12.6 & 22.6 & -- & --- & 0 \\
\hline$A R$ & 5406.9 & S? & 656192 & 6142927 & 1.5 & 4.2 & 0.9 & 5.5 & 6.2 & 33.3 & 1.5 & 11 & 0 \\
\hline AS & 5417.9 & B & 656176 & 6143329 & 4.2 & 13.1 & 5.5 & 27.4 & 65.7 & 64.9 & 2.1 & 0 & 0 \\
\hline $\mathrm{AT}$ & 5420.5 & B & 656177 & 6143408 & 7.9 & 14.3 & 7.0 & 28.7 & 69.1 & 66.1 & 3.5 & 0 & 43 \\
\hline $\mathrm{AU}$ & 5447.3 & B & 656179 & 6143812 & 1.8 & 2.5 & 0.2 & 0.1 & 0.5 & 2.4 & 3.2 & 81 & 54 \\
\hline $\mathrm{AV}$ & 5493.6 & B? & 656149 & 6144395 & 0.9 & 2.5 & 1.5 & 6.7 & 18.8 & 28.3 & 1.4 & 11 & 0 \\
\hline AW & 5512.3 & B & 656127 & 6144854 & 5.3 & 9.6 & 4.5 & 10.1 & 24.0 & 13.3 & 2.8 & 18 & 0 \\
\hline$A X$ & 5522.9 & D & 656150 & 6145014 & 16.2 & 21.8 & 1.2 & 3.8 & 15.1 & 23.7 & 5.8 & 14 & 0 \\
\hline$A Y$ & 5534.5 & B & 656151 & 6145234 & 15.1 & 24.0 & 22.8 & 35.3 & 86.1 & 64.6 & 5.8 & 4 & 0 \\
\hline $\mathrm{AZ}$ & 5540.2 & B & 656144 & 6145373 & 25.4 & 28.7 & 43.2 & 54.9 & 111.1 & 61.8 & 10.5 & 7 & 0 \\
\hline $\mathrm{BA}$ & 5543.1 & B & 656137 & 6145452 & 25.4 & 28.7 & 42.1 & 54.3 & 108.0 & 52.4 & 10.4 & 0 & 54 \\
\hline $\mathrm{BB}$ & 5571.3 & B & 656073 & 6146435 & 7.0 & 19.2 & 16.9 & 36.6 & 100.6 & 70.3 & 4.0 & 0 & 0 \\
\hline $\mathrm{BC}$ & 5581.4 & B & 656087 & 6146813 & 18.8 & 7.3 & 26.2 & 8.3 & 5.3 & 2.3 & 53.7 & 12 & 0 \\
\hline $\mathrm{BD}$ & 5586.9 & D & 656063 & 6147021 & 10.6 & 9.6 & 36.2 & 23.2 & 63.9 & 28.4 & 18.0 & 14 & 52 \\
\hline $\mathrm{BE}$ & 5595.1 & B & 656047 & 6147337 & 35.0 & 24.6 & 47.0 & 45.3 & 88.2 & 34.8 & 20.1 & 0 & 0 \\
\hline $\mathrm{BF}$ & 5596.3 & B & 656044 & 6147385 & 35.7 & 40.6 & 85.6 & 90.6 & 185.2 & 57.9 & 14.2 & 0 & 54 \\
\hline BG & 5598.2 & B & 656043 & 6147459 & 33.9 & 39.0 & 86.5 & 94.3 & 190.3 & 66.4 & 13.7 & 4 & 54 \\
\hline $\mathrm{BH}$ & 5613.2 & B & 656054 & 6148015 & 9.9 & 13.4 & 12.8 & 24.7 & 50.5 & 31.0 & 4.8 & 13 & 47 \\
\hline $\mathrm{BI}$ & 5616.2 & B & 656053 & 6148136 & 4.6 & 14.9 & 18.3 & 38.6 & 78.5 & 38.9 & 3.8 & 0 & 0 \\
\hline $\mathrm{BJ}$ & 5628.7 & B & 656027 & 6148625 & 22.3 & 27.3 & 38.8 & 62.0 & 129.5 & 61.6 & 8.0 & 0 & 0 \\
\hline BK & 5638.9 & B & 655988 & 6149005 & 31.7 & 28.3 & 81.4 & 75.9 & 159.0 & 61.4 & 18.0 & 0 & 0 \\
\hline LINE & 10840 & & & & & & & & & & & & \\
\hline A & 3658.4 & B & 657720 & 6112994 & 0.8 & 2.1 & 0.5 & 4.5 & 15.5 & 16.1 & 1.1 & 25 & 0 \\
\hline B & 3618.1 & S? & 657647 & 6113673 & 1.0 & 1.9 & 0.1 & 2.9 & 4.2 & 20.4 & 1.4 & 25 & 0 \\
\hline $\mathrm{C}$ & 3521.7 & S? & 657584 & 6115637 & 1.2 & 1.0 & 0.1 & 2.6 & 0.6 & 11.0 & -- & --- & 35 \\
\hline D & 3462.1 & B & 657560 & 6116481 & 1.4 & 7.3 & 1.4 & 12.5 & 38.5 & 52.1 & 1.0 & 0 & 0 \\
\hline $\mathrm{E}$ & 3451.4 & B & 657542 & 6116691 & 2.5 & 4.4 & 0.7 & 5.3 & 15.3 & 23.0 & 2.2 & 14 & 0 \\
\hline $\mathrm{F}$ & 3352.5 & $\mathrm{~S}$ & 657527 & 6117991 & 1.5 & 3.6 & 0.7 & 6.6 & 11.5 & 39.8 & 1.4 & 2 & 0 \\
\hline G & 3203.4 & $B$ ? & 657471 & 6121464 & 2.9 & 2.2 & 1.0 & 2.6 & 3.5 & 15.1 & -- & --- & 57 \\
\hline $\mathrm{H}$ & 3195.5 & S? & 657422 & 6121680 & 1.2 & 3.5 & 0.5 & 6.0 & 8.8 & 38.3 & 1.1 & 4 & 0 \\
\hline I & 3132.3 & $\mathrm{~S}$ & 657316 & 6123216 & 7.1 & 4.6 & 12.5 & 6.9 & 14.9 & 45.5 & 18.8 & 27 & 0 \\
\hline $\mathrm{J}$ & 3120.0 & M & 657269 & 6123472 & 8.8 & 0.3 & 17.3 & 0.4 & 14.2 & 0.3 & -- & -- & 517 \\
\hline $\mathrm{K}$ & 3112.4 & M & 657269 & 6123616 & 6.2 & 0.7 & 11.5 & 0.0 & 9.8 & 2.2 & --- & --- & 440 \\
\hline L & 2923.2 & S? & 657199 & 6126189 & 0.9 & 1.7 & 2.1 & 1.5 & 3.2 & 6.9 & 3.4 & 69 & 0 \\
\hline M & 2899.8 & B & 657175 & 6126623 & 6.9 & 6.9 & 14.2 & 9.7 & 23.2 & 7.8 & 11.8 & 1 & 0 \\
\hline $\mathrm{N}$ & 2883.0 & B? & 657179 & 6126893 & 6.7 & 8.7 & 4.7 & 11.9 & 28.5 & 23.3 & 3.9 & 29 & 21 \\
\hline 0 & 2860.7 & B & 657152 & 6127105 & 12.1 & 13.1 & 12.6 & 18.4 & 37.2 & 11.8 & 7.5 & 8 & 57 \\
\hline $\mathrm{P}$ & 2829.3 & B & 657119 & 6127590 & 9.9 & 9.6 & 22.4 & 21.4 & 45.0 & 11.4 & 11.0 & 6 & 57 \\
\hline$Q$ & 2800.6 & $\mathrm{M}$ & 657140 & 6128454 & 0.2 & 0.8 & 1.9 & 1.0 & 0.8 & 5.5 & -- & --- & 0 \\
\hline $\mathrm{R}$ & 2787.3 & M & 657108 & 6128668 & 0.6 & 0.8 & 0.1 & 0.7 & 0.3 & 3.4 & --- & --- & 0 \\
\hline
\end{tabular}

$\mathrm{CX}=$ COAXIAL

Note: EM values shown above

are local amplitudes
*Estimated Depth may be unreliable because the stronger part of the conductor may be deeper or to one side of the flight line, or because of a shallow dip or magnetite/overburden effects 
EM Anomaly List

\begin{tabular}{|c|c|c|c|c|c|c|c|c|c|c|c|c|c|}
\hline Label & Fid & Interp & $\begin{array}{c}\text { XUTM } \\
\text { m }\end{array}$ & $\begin{array}{c}\text { YUTM } \\
\mathrm{m}\end{array}$ & $\begin{array}{l}\text { CX } \\
\text { Real } \\
\text { ppm }\end{array}$ & $\begin{array}{l}900 \mathrm{HZ} \\
\text { Quad } \\
\text { ppm }\end{array}$ & $\begin{array}{l}\text { CP } \\
\text { Real } \\
\text { ppm }\end{array}$ & $\begin{array}{l}900 \mathrm{HZ} \\
\text { Quad } \\
\text { ppm }\end{array}$ & $\begin{array}{l}\text { CP } \\
\text { Real } \\
\text { ppm }\end{array}$ & $\begin{array}{l}7200 \mathrm{HZ} \\
\text { Quad } \\
\text { ppm }\end{array}$ & $\begin{array}{l}\text { Vertica } \\
\text { CoND } \\
\text { siemens }\end{array}$ & $\begin{array}{c}\text { al Dike } \\
\text { DEPTH* } \\
\text { m }\end{array}$ & $\begin{array}{c}\text { Mag. Corr } \\
\text { NT }\end{array}$ \\
\hline LINE 1 & 10840 & & & & & & & & & & & & \\
\hline $\mathrm{S}$ & 2733.3 & M & 657085 & 6129372 & 0.1 & 0.4 & 0.0 & 0.5 & 0.1 & 1.6 & --- & --- & 0 \\
\hline $\mathrm{T}$ & 2713.8 & M & 657093 & 6129521 & 2.1 & 0.2 & 0.3 & 0.4 & 0.5 & 0.7 & --- & --- & 0 \\
\hline $\mathrm{U}$ & 2696.0 & $\mathrm{M}$ & 657096 & 6129599 & 3.5 & 0.2 & 3.1 & 0.2 & 3.2 & 1.1 & --- & --- & 0 \\
\hline $\mathrm{V}$ & 2673.5 & $\mathrm{M}$ & 657098 & 6129702 & 5.3 & 0.2 & 0.7 & 0.0 & 0.8 & 0.9 & --- & --- & 0 \\
\hline W & 2657.2 & $\mathrm{M}$ & 657103 & 6129788 & 1.3 & 0.3 & 10.3 & 0.1 & 9.3 & 1.1 & --- & --- & 0 \\
\hline $\mathrm{x}$ & 2636.0 & $\mathrm{M}$ & 657109 & 6129888 & 85.6 & 1.0 & 180.8 & 0.1 & 148.0 & 0.0 & --- & --- & 74 \\
\hline $\mathrm{Y}$ & 2621.8 & M & 657107 & 6129931 & 111.1 & 1.1 & 160.8 & 0.1 & 135.0 & 2.9 & --- & --- & 0 \\
\hline $\mathrm{Z}$ & 2609.6 & M & 657105 & 6129961 & 63.5 & 0.9 & 145.3 & 0.1 & 119.3 & 1.8 & --- & --- & 0 \\
\hline $\mathrm{AA}$ & 2550.3 & M & 657062 & 6131236 & 0.1 & 0.3 & 2.3 & 0.6 & 1.6 & 2.4 & --- & --- & 332 \\
\hline$A B$ & 2543.0 & M & 657046 & 6131443 & 0.2 & 0.5 & 3.5 & 0.6 & 3.2 & 3.6 & --- & --- & 216 \\
\hline $\mathrm{AC}$ & 2531.8 & M & 657027 & 6131712 & 5.5 & 0.2 & 5.0 & 0.6 & 3.7 & 7.9 & --- & --- & 0 \\
\hline $\mathrm{AD}$ & 2527.9 & $\mathrm{M}$ & 657014 & 6131832 & 6.4 & 0.5 & 14.0 & 0.3 & 0.4 & 3.4 & --- & --- & 348 \\
\hline $\mathrm{AE}$ & 2512.0 & $\mathrm{M}$ & 656984 & 6132402 & 0.3 & 0.9 & 0.8 & 0.8 & 0.6 & 8.7 & --- & --- & 0 \\
\hline $\mathrm{AF}$ & 2499.3 & $\mathrm{M}$ & 656960 & 6132864 & 0.5 & 0.6 & 1.9 & 0.4 & 1.7 & 10.5 & --- & --- & 413 \\
\hline AG & 2474.8 & M & 656960 & 6133814 & 0.0 & 0.4 & 0.2 & 0.1 & 0.1 & 6.2 & --- & --- & 0 \\
\hline $\mathrm{AH}$ & 2459.4 & M & 656932 & 6134369 & 3.6 & 0.8 & 3.1 & 0.7 & 2.9 & 2.8 & --- & --- & 0 \\
\hline $\mathrm{AI}$ & 2454.2 & M & 656920 & 6134550 & 1.9 & 0.0 & 0.4 & 0.0 & 0.2 & 0.8 & --- & --- & 0 \\
\hline AJ & 2440.4 & M & 656887 & 6135066 & 1.4 & 1.0 & 2.1 & 2.5 & 6.2 & 10.8 & --- & --- & 331 \\
\hline $\mathrm{AK}$ & 2428.1 & S? & 656854 & 6135580 & 0.0 & 3.1 & 2.5 & 4.7 & 8.9 & 27.4 & --- & --- & 0 \\
\hline $\mathrm{AL}$ & 2316.6 & S? & 656681 & 6139915 & 2.1 & 3.7 & 1.3 & 4.8 & 11.3 & 17.7 & 2.4 & 8 & 0 \\
\hline $\mathrm{AM}$ & 2282.9 & D & 656661 & 6141079 & 7.7 & 7.8 & 14.5 & 17.0 & 30.5 & 29.5 & 8.3 & 9 & 0 \\
\hline AN & 2191.6 & D & 656619 & 6142220 & 4.2 & 4.4 & 0.1 & 2.8 & 5.9 & 4.7 & --- & --- & 57 \\
\hline $\mathrm{AO}$ & 2139.5 & D & 656647 & 6142427 & 5.3 & 10.2 & 3.8 & 5.6 & 11.5 & 8.0 & 3.0 & 35 & 0 \\
\hline $\mathrm{AP}$ & 2122.4 & $\mathrm{D}$ & 656608 & 6142709 & 3.3 & 7.8 & 1.0 & 8.4 & 19.6 & 29.8 & 2.1 & 1 & 0 \\
\hline $\mathrm{AQ}$ & 2098.4 & $B$ ? & 656622 & 6143441 & 4.9 & 8.8 & 3.2 & 14.1 & 39.1 & 35.8 & 3.0 & 0 & 0 \\
\hline$A R$ & 2090.8 & $B$ ? & 656608 & 6143604 & 1.9 & 4.6 & 2.0 & 3.6 & 6.7 & 10.3 & --- & --- & 0 \\
\hline AS & 2060.0 & $B$ ? & 656583 & 6144082 & 0.5 & 1.9 & 0.3 & 1.8 & 0.1 & 11.5 & --- & --- & 0 \\
\hline $\mathrm{AT}$ & 2020.5 & B & 656535 & 6144913 & 26.4 & 26.8 & 56.1 & 71.4 & 144.8 & 69.0 & 11.7 & 0 & 42 \\
\hline $\mathrm{AU}$ & 2015.3 & B & 656518 & 6145006 & 30.1 & 33.1 & 36.6 & 52.0 & 98.8 & 30.1 & 10.3 & 0 & 0 \\
\hline $\mathrm{AV}$ & 2001.9 & B & 656541 & 6145184 & 8.7 & 14.5 & 11.8 & 11.9 & 23.5 & 12.1 & 5.6 & 17 & 0 \\
\hline AW & 1992.0 & B & 656562 & 6145280 & 21.3 & 59.4 & 44.7 & 147.5 & 369.2 & 368.2 & 4.7 & 0 & 0 \\
\hline$A X$ & 1977.6 & B & 656552 & 6145452 & 168.3 & 262.7 & 164.2 & 294.5 & 636.5 & 476.5 & 11.5 & 1 & 57 \\
\hline$A Y$ & 1966.5 & B? & 656519 & 6145724 & 13.9 & 12.0 & 2.7 & 14.9 & 36.2 & 25.8 & 6.6 & 21 & 57 \\
\hline $\mathrm{AZ}$ & 1956.2 & $\mathrm{~B}$ & 656514 & 6146087 & 16.7 & 14.5 & 20.1 & 33.8 & 83.0 & 69.0 & 9.0 & 17 & 0 \\
\hline $\mathrm{BA}$ & 1953.2 & B & 656514 & 6146201 & 16.7 & 15.3 & 20.1 & 29.1 & 70.4 & 68.2 & 9.7 & 12 & 57 \\
\hline $\mathrm{BB}$ & 1942.6 & B & 656485 & 6146616 & 54.3 & 55.1 & 140.9 & 135.6 & 284.5 & 145.2 & 19.4 & 0 & 57 \\
\hline $\mathrm{BC}$ & 1940.5 & B & 656479 & 6146698 & 43.7 & 42.5 & 98.8 & 98.2 & 203.4 & 117.7 & 17.6 & 1 & 57 \\
\hline $\mathrm{BD}$ & 1934.0 & B & 656469 & 6146944 & 4.0 & 5.2 & 4.3 & 10.0 & 18.2 & 14.1 & 3.3 & 40 & 0 \\
\hline $\mathrm{BE}$ & 1917.6 & B & 656414 & 6147566 & 43.1 & 57.8 & 94.0 & 121.3 & 259.8 & 162.0 & 11.4 & 0 & 57 \\
\hline $\mathrm{BF}$ & 1910.7 & B & 656394 & 6147828 & 43.5 & 67.0 & 121.5 & 184.0 & 377.4 & 231.7 & 9.5 & 0 & 56 \\
\hline BG & 1907.3 & B & 656408 & 6147954 & 21.1 & 25.4 & 54.2 & 58.3 & 135.4 & 111.9 & 11.5 & 6 & 57 \\
\hline $\mathrm{BH}$ & 1899.8 & B & 656462 & 6148214 & 2.3 & 1.5 & 0.4 & 2.1 & 7.2 & 12.3 & 5.6 & 57 & 57 \\
\hline BI & 1893.1 & B & 656510 & 6148454 & 32.0 & 40.1 & 89.7 & 103.1 & 214.1 & 87.2 & 12.4 & 0 & 0 \\
\hline $\mathrm{BJ}$ & 1883.6 & B & 656432 & 6148769 & 19.9 & 11.2 & 38.4 & 17.0 & 43.9 & 8.0 & 34.8 & 0 & 0 \\
\hline
\end{tabular}

$\mathrm{CX}=$ COAXIAL

Note: EM values shown above

Ketchikan - North/South of 5515
*Estimated Depth may be unreliable because the stronger part of the conductor may be deeper or chal side of the flight line, or because 
EM Anomaly List

\begin{tabular}{|c|c|c|c|c|c|c|c|c|c|c|c|c|c|}
\hline Label & Fid & Interp & $\begin{array}{c}\text { XUTM } \\
\text { m }\end{array}$ & $\begin{array}{c}\text { YUTM } \\
\mathrm{m}\end{array}$ & $\begin{array}{l}\text { CX } \\
\text { Real } \\
\text { ppm }\end{array}$ & $\begin{array}{c}900 \mathrm{HZ} \\
\text { Quad } \\
\text { ppm }\end{array}$ & $\begin{array}{l}\text { CP } \\
\text { Real } \\
\text { ppm }\end{array}$ & $\begin{array}{c}900 \mathrm{HZ} \\
\text { Quad } \\
\text { ppm }\end{array}$ & $\begin{array}{l}\text { CP }{ }^{7} \\
\text { Real } \\
\text { ppm }\end{array}$ & $\begin{array}{c}7200 \mathrm{~Hz} \\
\text { Quad } \\
\text { ppm }\end{array}$ & $\begin{array}{l}\text { Vertica } \\
\text { CoND } \\
\text { siemens }\end{array}$ & $\begin{array}{c}\text { al Dike } \\
\text { DEPTH* } \\
\text { m }\end{array}$ & $\begin{array}{c}\text { Mag. Corr } \\
\text { NT }\end{array}$ \\
\hline $\begin{array}{l}\text { LINE } \\
\text { A } \\
\text { B } \\
\text { C } \\
\text { D } \\
\text { E } \\
\text { F } \\
\text { G } \\
\text { H } \\
\text { I } \\
\text { J } \\
\text { K } \\
\text { L } \\
\text { M } \\
\text { N }\end{array}$ & $\begin{array}{l}10850 \\
10110.0 \\
10122.3 \\
10148.4 \\
10153.8 \\
10353.8 \\
10405.6 \\
10456.5 \\
10630.4 \\
10639.2 \\
10645.7 \\
10650.2 \\
10677.1 \\
10683.4 \\
10689.7\end{array}$ & $\begin{array}{r}\text { S? } \\
\text { S } \\
\text { S? } \\
\text { B? } \\
\text { S } \\
\text { S? } \\
\text { S } \\
\text { B } \\
\text { B } \\
\text { B } \\
\text { B } \\
\text { B } \\
\text { D } \\
\text { B }\end{array}$ & $\begin{array}{l}658018 \\
658050 \\
657970 \\
657950 \\
657843 \\
657773 \\
657742 \\
657661 \\
657641 \\
657620 \\
657625 \\
657627 \\
657648 \\
657688\end{array}$ & $\begin{array}{l}6115369 \\
6115587 \\
6116359 \\
6116528 \\
6119894 \\
6121653 \\
6122982 \\
6125978 \\
6126134 \\
6126232 \\
6126315 \\
6126933 \\
6127055 \\
6127205\end{array}$ & $\begin{array}{r}0.7 \\
0.2 \\
2.3 \\
4.1 \\
0.2 \\
0.9 \\
6.0 \\
5.1 \\
7.5 \\
9.6 \\
10.6 \\
13.6 \\
16.0 \\
15.9\end{array}$ & $\begin{array}{r}2.2 \\
1.8 \\
9.8 \\
9.6 \\
1.5 \\
12.2 \\
5.2 \\
11.0 \\
3.5 \\
7.6 \\
12.8 \\
9.0 \\
8.3 \\
4.2\end{array}$ & $\begin{array}{r}0.1 \\
0.9 \\
5.3 \\
2.9 \\
0.1 \\
1.8 \\
11.0 \\
9.0 \\
0.6 \\
12.6 \\
10.4 \\
15.2 \\
13.6 \\
22.1\end{array}$ & $\begin{array}{r}2.2 \\
3.1 \\
13.9 \\
14.2 \\
2.2 \\
22.2 \\
7.7 \\
17.6 \\
0.4 \\
19.5 \\
19.5 \\
12.0 \\
13.3 \\
9.5\end{array}$ & $\begin{array}{r}3.2 \\
6.2 \\
39.8 \\
40.5 \\
7.7 \\
31.6 \\
13.5 \\
46.8 \\
2.8 \\
42.6 \\
42.6 \\
26.8 \\
26.8 \\
21.5\end{array}$ & $\begin{array}{r}13.8 \\
15.4 \\
32.0 \\
51.5 \\
14.8 \\
138.3 \\
50.6 \\
26.3 \\
6.6 \\
26.7 \\
27.1 \\
11.5 \\
10.6 \\
1.7\end{array}$ & $\begin{array}{r}--- \\
--- \\
2.1 \\
2.4 \\
0.5 \\
0.6 \\
--- \\
3.9 \\
22.2 \\
8.3 \\
5.6 \\
17.5 \\
19.8 \\
57.5\end{array}$ & $\begin{array}{r}--- \\
--- \\
0 \\
1 \\
0 \\
3 \\
--- \\
0 \\
29 \\
6 \\
3 \\
16 \\
0 \\
5\end{array}$ & $\begin{array}{r}0 \\
40 \\
40 \\
237 \\
76 \\
10 \\
40 \\
9 \\
0 \\
0 \\
0 \\
0 \\
40 \\
0\end{array}$ \\
\hline $\begin{array}{l}\text { LINE } \\
\text { A } \\
\text { B } \\
\text { C } \\
\text { D } \\
\text { E } \\
\text { F } \\
\text { G }\end{array}$ & $\begin{array}{r}10851 \\
185.1 \\
228.5 \\
241.8 \\
312.0 \\
444.2 \\
453.2 \\
487.3\end{array}$ & $\begin{array}{l}\mathrm{B} \\
\mathrm{M} \\
\mathrm{M} \\
\mathrm{M} \\
\mathrm{M} \\
\mathrm{M} \\
\mathrm{M}\end{array}$ & $\begin{array}{l}657622 \\
657529 \\
657533 \\
657524 \\
657506 \\
657497 \\
657497\end{array}$ & $\begin{array}{l}6127246 \\
6128329 \\
6128445 \\
6128799 \\
6129297 \\
6129341 \\
6129450\end{array}$ & $\begin{array}{r}27.5 \\
0.0 \\
24.0 \\
0.2 \\
20.4 \\
0.0 \\
0.0\end{array}$ & $\begin{array}{r}11.6 \\
0.1 \\
0.1 \\
0.4 \\
0.7 \\
0.2 \\
0.6\end{array}$ & $\begin{array}{r}28.6 \\
15.2 \\
58.6 \\
2.2 \\
39.8 \\
20.5 \\
1.3\end{array}$ & $\begin{array}{r}16.8 \\
0.2 \\
0.0 \\
0.3 \\
0.6 \\
0.1 \\
0.0\end{array}$ & $\begin{array}{r}36.2 \\
12.4 \\
47.1 \\
0.6 \\
32.0 \\
16.4 \\
0.7\end{array}$ & $\begin{array}{l}8.0 \\
6.2 \\
5.7 \\
3.6 \\
0.8 \\
1.7 \\
2.1\end{array}$ & $\begin{array}{c}38.5 \\
--- \\
--- \\
--- \\
--- \\
--- \\
---\end{array}$ & $\begin{array}{c}0 \\
--- \\
--- \\
--- \\
--- \\
--- \\
---\end{array}$ & $\begin{array}{l}0 \\
0 \\
0 \\
0 \\
0 \\
0 \\
0\end{array}$ \\
\hline $\begin{array}{l}\text { LINE } \\
\text { A } \\
\text { B } \\
\text { C } \\
\text { D } \\
\text { E } \\
\text { F } \\
\text { G } \\
\text { H } \\
\text { I }\end{array}$ & $\begin{array}{r}10852 \\
818.3 \\
805.1 \\
788.0 \\
770.0 \\
713.1 \\
664.0 \\
640.0 \\
634.8 \\
613.9\end{array}$ & $\begin{array}{l}\mathrm{M} \\
\mathrm{M} \\
\mathrm{M} \\
\mathrm{M} \\
\mathrm{M} \\
\mathrm{M} \\
\mathrm{M} \\
\mathrm{M} \\
\mathrm{M}\end{array}$ & $\begin{array}{l}657495 \\
657493 \\
657496 \\
657492 \\
657468 \\
657461 \\
657479 \\
657483 \\
657424\end{array}$ & $\begin{array}{l}6129571 \\
6129598 \\
6129631 \\
6129686 \\
6129888 \\
6130073 \\
6130297 \\
6130388 \\
6130949\end{array}$ & $\begin{array}{r}3.2 \\
5.9 \\
5.0 \\
12.4 \\
4.3 \\
1.4 \\
0.0 \\
11.7 \\
0.1\end{array}$ & $\begin{array}{l}0.4 \\
0.4 \\
0.2 \\
0.1 \\
0.2 \\
0.1 \\
0.4 \\
0.6 \\
0.5\end{array}$ & $\begin{array}{r}43.3 \\
5.9 \\
28.4 \\
2.4 \\
40.8 \\
6.4 \\
16.0 \\
22.8 \\
8.5\end{array}$ & $\begin{array}{l}0.5 \\
0.4 \\
0.0 \\
0.0 \\
0.9 \\
0.5 \\
0.8 \\
1.0 \\
0.9\end{array}$ & $\begin{array}{r}34.0 \\
5.5 \\
22.4 \\
2.6 \\
33.4 \\
5.2 \\
13.3 \\
18.7 \\
6.4\end{array}$ & $\begin{array}{l}0.6 \\
1.0 \\
1.5 \\
1.3 \\
1.4 \\
2.5 \\
4.1 \\
1.6 \\
5.7\end{array}$ & $\begin{array}{l}--- \\
--- \\
--- \\
--- \\
--- \\
--- \\
--- \\
--- \\
---\end{array}$ & $\begin{array}{l}--- \\
--- \\
--- \\
--- \\
--- \\
--- \\
--- \\
--- \\
---\end{array}$ & $\begin{array}{r}0 \\
0 \\
0 \\
75 \\
168 \\
16 \\
0 \\
1021 \\
0\end{array}$ \\
\hline $\begin{array}{l}\text { LINE } \\
\text { A } \\
\text { B } \\
\text { C } \\
\text { D } \\
\text { E } \\
\text { F } \\
\text { G } \\
\text { H } \\
\text { I }\end{array}$ & $\begin{array}{l}10853 \\
950.9 \\
958.6 \\
964.6 \\
987.7 \\
998.8 \\
1019.4 \\
1049.4 \\
1057.3 \\
1220.7\end{array}$ & $\begin{array}{r}S ? \\
M \\
M \\
M \\
M \\
M \\
M \\
D \\
S ?\end{array}$ & $\begin{array}{l}657411 \\
657416 \\
657421 \\
657420 \\
657391 \\
657335 \\
657315 \\
657316 \\
657095\end{array}$ & $\begin{array}{l}6131203 \\
6131383 \\
6131544 \\
6132280 \\
6132682 \\
6133535 \\
6134741 \\
6135071 \\
6140635\end{array}$ & $\begin{array}{r}25.3 \\
14.8 \\
39.0 \\
23.0 \\
0.1 \\
6.0 \\
0.0 \\
7.8 \\
1.1\end{array}$ & $\begin{array}{l}2.6 \\
0.3 \\
0.0 \\
0.9 \\
0.3 \\
0.6 \\
0.5 \\
4.5 \\
1.7\end{array}$ & $\begin{array}{r}39.6 \\
29.0 \\
2.3 \\
6.3 \\
0.5 \\
1.5 \\
0.3 \\
6.8 \\
0.5\end{array}$ & $\begin{array}{l}0.9 \\
0.8 \\
1.3 \\
1.4 \\
0.8 \\
1.4 \\
0.7 \\
2.7 \\
4.2\end{array}$ & $\begin{array}{r}31.3 \\
23.1 \\
2.3 \\
6.3 \\
0.4 \\
0.9 \\
1.1 \\
13.4 \\
5.1\end{array}$ & $\begin{array}{r}15.1 \\
3.9 \\
9.0 \\
10.7 \\
4.9 \\
10.6 \\
2.7 \\
10.6 \\
25.5\end{array}$ & $\begin{array}{r}--- \\
--- \\
--- \\
--- \\
--- \\
--- \\
--- \\
22.3 \\
1.7\end{array}$ & $\begin{array}{r}--- \\
--- \\
--- \\
--- \\
--- \\
--- \\
--- \\
22 \\
36\end{array}$ & $\begin{array}{r}1173 \\
0 \\
283 \\
936 \\
0 \\
607 \\
0 \\
96 \\
0\end{array}$ \\
\hline
\end{tabular}

$\mathrm{CX}=$ COAXIAL

Note: EM values shown above

Ketchikan - North/South of 5515
*Estimated Depth may be unreliable because the stronger part of the conductor may be deeper or to one side of the flight line, or because of shallow dip or magnetite/overburden effects 
EM Anomaly List

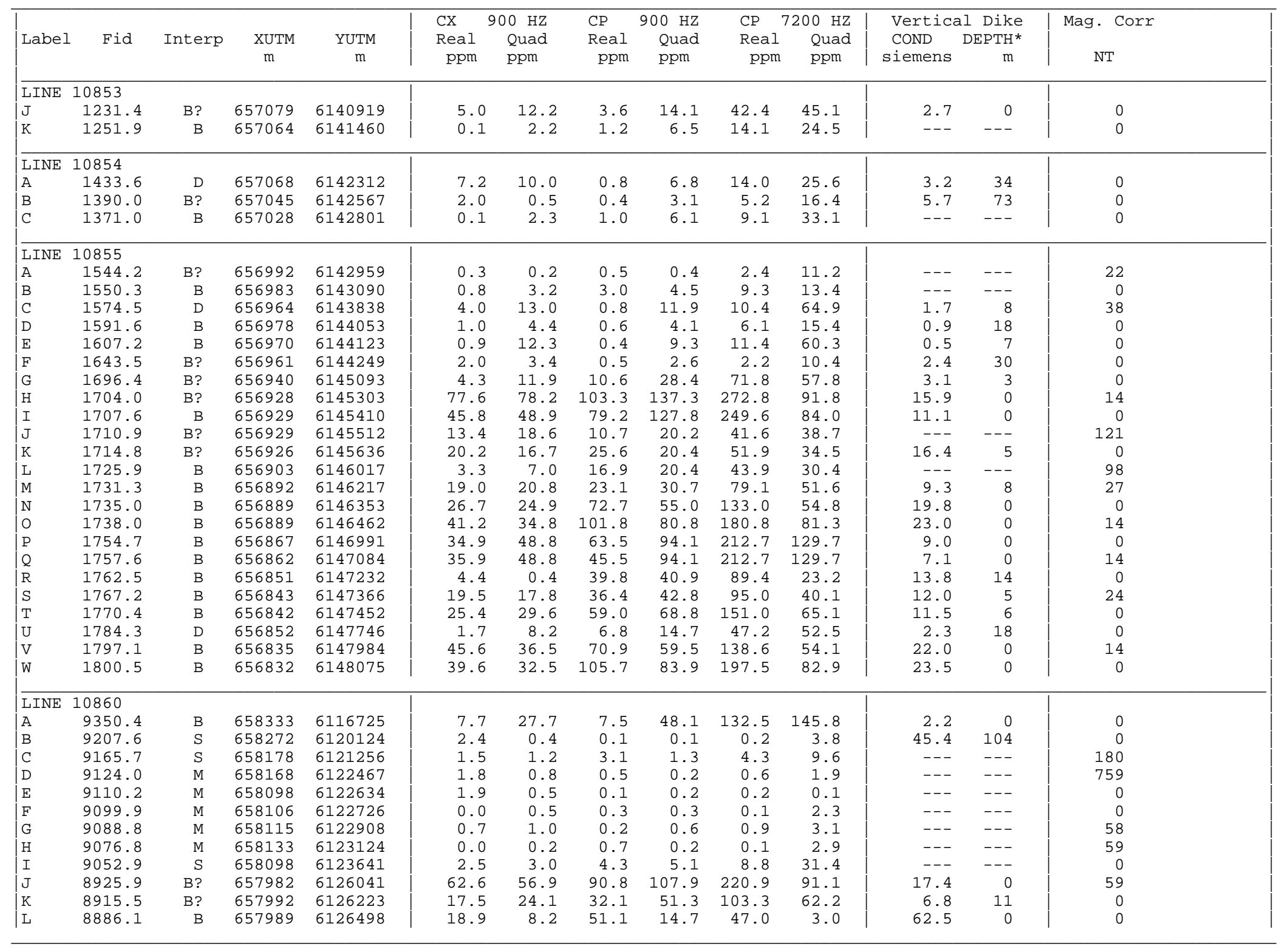

$\mathrm{CX}=$ COAXIAL

Note: EM values shown above

are local amplitudes are 10
15
*Estimated Depth may be unreliable because the stronger part of the conductor may be deeper or to one side of the flight line, or because of a shallow dip or magnetite/overburden effects 
EM Anomaly List

\begin{tabular}{|c|c|c|c|c|c|c|c|c|c|c|c|c|c|}
\hline Label & Fid & Interp & $\begin{array}{c}\text { XUTM } \\
\text { m }\end{array}$ & $\begin{array}{c}\text { YUTM } \\
\text { m }\end{array}$ & $\begin{array}{l}\text { CX } \\
\text { Real } \\
\text { ppm }\end{array}$ & $\begin{array}{l}900 \mathrm{HZ} \\
\text { Quad } \\
\text { ppm }\end{array}$ & $\begin{array}{l}\text { CP } \\
\text { Real } \\
\text { ppm }\end{array}$ & $\begin{array}{l}900 \mathrm{HZ} \\
\text { Quad } \\
\text { ppm }\end{array}$ & $\begin{array}{l}\text { CP } \\
\text { Real } \\
\text { ppm }\end{array}$ & $\begin{array}{l}7200 \mathrm{HZ} \\
\text { Quad } \\
\text { ppm }\end{array}$ & $\begin{array}{l}\text { Vertica } \\
\text { CoND } \\
\text { siemens }\end{array}$ & $\begin{array}{c}\text { al Dike } \\
\text { DEPTH* } \\
\text { m }\end{array}$ & $\begin{array}{c}\text { Mag. Corr } \\
\text { NT }\end{array}$ \\
\hline LINE & 10860 & & & & & & & & & & & & \\
\hline M & 8872.0 & B & 657998 & 6126623 & 10.7 & 10.2 & 2.4 & 11.5 & 21.8 & 16.0 & 5.6 & 20 & 59 \\
\hline $\mathrm{N}$ & 8838.0 & B & 657961 & 6127505 & 62.1 & 32.9 & 72.5 & 66.9 & 159.8 & 69.6 & 31.0 & 0 & 59 \\
\hline 0 & 8813.3 & M & 657927 & 6128121 & 0.6 & 0.6 & 11.6 & 0.7 & 9.0 & 4.8 & --- & --- & 0 \\
\hline P & 8805.0 & M & 657926 & 6128227 & 8.9 & 0.7 & 3.7 & 1.1 & 3.4 & 4.6 & --- & --- & 98 \\
\hline$Q$ & 8764.1 & M & 657877 & 6128889 & 2.0 & 0.5 & 1.4 & 0.4 & 1.1 & 1.9 & --- & --- & 0 \\
\hline $\mathrm{R}$ & 8693.8 & M & 657873 & 6129841 & 0.0 & 0.1 & 26.3 & 0.0 & 22.3 & 1.4 & --- & --- & 59 \\
\hline $\mathrm{S}$ & 8681.8 & M & 657875 & 6129882 & 3.0 & 0.3 & 3.1 & 0.1 & 2.5 & 1.9 & --- & --- & 59 \\
\hline $\mathrm{T}$ & 8661.5 & M & 657871 & 6130057 & 6.0 & 0.3 & 4.2 & 0.0 & 4.1 & 2.0 & --- & --- & 59 \\
\hline $\mathrm{U}$ & 8641.8 & M & 657882 & 6130377 & 0.2 & 0.3 & 1.0 & 1.2 & 1.1 & 3.9 & --- & --- & 96 \\
\hline $\mathrm{V}$ & 8630.0 & M & 657872 & 6130837 & 0.0 & 0.8 & 8.8 & 0.6 & 5.1 & 3.7 & --- & --- & 435 \\
\hline W & 8609.9 & M & 657830 & 6131467 & 0.3 & 1.5 & 0.9 & 0.8 & 0.8 & 5.5 & --- & --- & 0 \\
\hline $\mathrm{x}$ & 8595.4 & B? & 657807 & 6131869 & 8.5 & 10.2 & 5.1 & 8.9 & 7.9 & 27.0 & --- & --- & 1452 \\
\hline $\mathrm{Y}$ & 8547.6 & M & 657726 & 6133169 & 1.1 & 1.3 & 0.6 & 1.0 & 0.8 & 7.0 & --- & --- & 0 \\
\hline z & 8538.7 & M & 657729 & 6133519 & 11.8 & 0.4 & 6.8 & 0.5 & 6.2 & 0.4 & --- & --- & 84 \\
\hline $\mathrm{AA}$ & 8500.5 & D & 657706 & 6135084 & 9.5 & 11.5 & 6.0 & 5.2 & 12.4 & 12.2 & 7.4 & 19 & 0 \\
\hline$A B$ & 8356.1 & $\mathrm{~S}$ & 657519 & 6140663 & 0.6 & 1.6 & 0.0 & 1.5 & 0.1 & 11.7 & --- & --- & 0 \\
\hline $\mathrm{AC}$ & 8279.3 & $B$ ? & 657418 & 6142644 & 0.1 & 1.1 & 0.3 & 2.2 & 4.9 & 12.3 & --- & --- & 0 \\
\hline $\mathrm{AD}$ & 8257.2 & $\mathrm{~S}$ & 657409 & 6142842 & 1.2 & 3.3 & 0.1 & 0.1 & 0.1 & 1.1 & --- & --- & 0 \\
\hline $\mathrm{AE}$ & 8227.0 & S? & 657404 & 6143623 & 4.9 & 18.8 & 3.4 & 25.4 & 62.9 & 111.4 & 1.7 & 0 & 59 \\
\hline $\mathrm{AF}$ & 8208.2 & $B$ ? & 657335 & 6144221 & 2.3 & 2.8 & 1.2 & 6.5 & 11.4 & 33.3 & 2.6 & 22 & 0 \\
\hline$A G$ & 8181.8 & B & 657328 & 6144888 & 44.8 & 65.9 & 98.3 & 115.2 & 241.3 & 130.7 & 11.5 & 0 & 59 \\
\hline $\mathrm{AH}$ & 8178.0 & B & 657323 & 6145013 & 36.9 & 19.8 & 55.1 & 36.3 & 47.9 & 13.3 & 32.9 & 0 & 0 \\
\hline AI & 8171.8 & B? & 657313 & 6145200 & 17.9 & 12.9 & 34.5 & 28.5 & 42.5 & 5.4 & 18.1 & 5 & 0 \\
\hline AJ & 8165.4 & B & 657315 & 6145390 & 0.7 & 10.8 & 29.5 & 15.1 & 21.7 & 32.5 & --- & --- & 0 \\
\hline $\mathrm{AK}$ & 8159.7 & B & 657316 & 6145574 & 22.6 & 7.2 & 28.6 & 3.7 & 4.8 & 31.3 & 100.2 & 9 & 3 \\
\hline $\mathrm{AL}$ & 8154.6 & B & 657312 & 6145743 & 73.6 & 49.5 & 161.6 & 117.4 & 246.0 & 44.3 & 34.1 & 0 & 0 \\
\hline $\mathrm{AM}$ & 8131.1 & B & 657281 & 6146590 & 18.8 & 3.1 & 30.2 & 9.3 & 33.7 & 13.4 & -- & --- & 0 \\
\hline AN & 8128.0 & B & 657275 & 6146702 & 6.5 & 2.6 & 6.6 & 3.5 & 2.4 & 8.2 & 26.1 & 22 & 59 \\
\hline $\mathrm{AO}$ & 8114.6 & B & 657252 & 6147146 & 102.7 & 84.9 & 182.4 & 165.0 & 365.0 & 145.2 & 27.2 & 0 & 0 \\
\hline $\mathrm{AP}$ & 8094.6 & B & 657222 & 6147825 & 11.1 & 19.3 & 24.0 & 45.0 & 92.4 & 46.7 & 4.4 & 12 & 59 \\
\hline $\mathrm{AQ}$ & 8091.8 & B & 657214 & 6147907 & 12.4 & 19.3 & 23.1 & 45.0 & 92.4 & 46.7 & 4.8 & 2 & 0 \\
\hline LINE & 10870 & & & & & & & & & & & & \\
\hline A & 6240.1 & B & 658918 & 6113083 & 0.5 & 1.6 & 0.2 & 1.0 & 2.6 & 7.2 & --- & --- & 0 \\
\hline B & 6257.4 & $B$ ? & 658894 & 6113269 & 0.2 & 0.7 & 0.1 & 1.0 & 0.2 & 5.1 & --- & --- & 77 \\
\hline C & 6269.8 & $B$ ? & 658889 & 6113379 & 0.3 & 0.8 & 0.6 & 0.9 & 1.1 & 5.0 & --- & --- & 0 \\
\hline D & 6401.7 & $B$ ? & 658777 & 6115521 & 4.9 & 21.1 & 15.1 & 59.0 & 160.5 & 117.6 & 2.5 & 0 & 52 \\
\hline $\mathrm{E}$ & 6431.2 & D & 658812 & 6116346 & 6.0 & 17.4 & 3.0 & 14.3 & 42.1 & 43.7 & 2.5 & 0 & 0 \\
\hline $\mathrm{F}$ & 6475.2 & $\mathrm{~S}$ & 658750 & 6117611 & 4.1 & 1.6 & 3.7 & 3.5 & 6.6 & 23.4 & 15.8 & 21 & 0 \\
\hline G & 6674.8 & $B$ ? & 658540 & 6122905 & 5.5 & 1.4 & 4.5 & 1.6 & 3.6 & 8.1 & 46.2 & 43 & 0 \\
\hline $\mathrm{H}$ & 6680.0 & $B$ ? & 658548 & 6123003 & 2.8 & 2.2 & 2.9 & 4.3 & 1.1 & 27.8 & 5.9 & 58 & 0 \\
\hline I & 6688.5 & $B$ ? & 658573 & 6123208 & 6.0 & 2.1 & 5.5 & 3.2 & 6.6 & 22.4 & 27.8 & 41 & 0 \\
\hline $\mathrm{J}$ & 6774.0 & B & 658454 & 6124974 & 2.2 & 0.4 & 1.0 & 0.5 & 1.4 & 2.4 & 43.0 & 67 & 0 \\
\hline K & 6804.9 & B & 658436 & 6125173 & 14.8 & 30.1 & 1.1 & 11.4 & 30.7 & 52.9 & 4.1 & 9 & 0 \\
\hline L & 6814.7 & B & 658439 & 6125203 & 0.0 & 4.4 & 7.2 & 19.6 & 45.6 & 23.6 & 1.7 & 21 & 0 \\
\hline M & 6864.0 & $B$ ? & 658420 & 6125872 & 2.4 & 2.0 & 0.0 & 1.4 & 1.4 & 1.6 & 4.4 & 35 & 52 \\
\hline
\end{tabular}

$\mathrm{CX}=$ COAXIAL

Note: EM values shown above

are local amplitudes

Ketchikan - North/South of 5515
* Estimated Depth may be unreliable because the stronger part of the conductor may be deeper or to one side of the flight line, or because of a shallow dip or magnetite/overburden effects 
EM Anomaly List

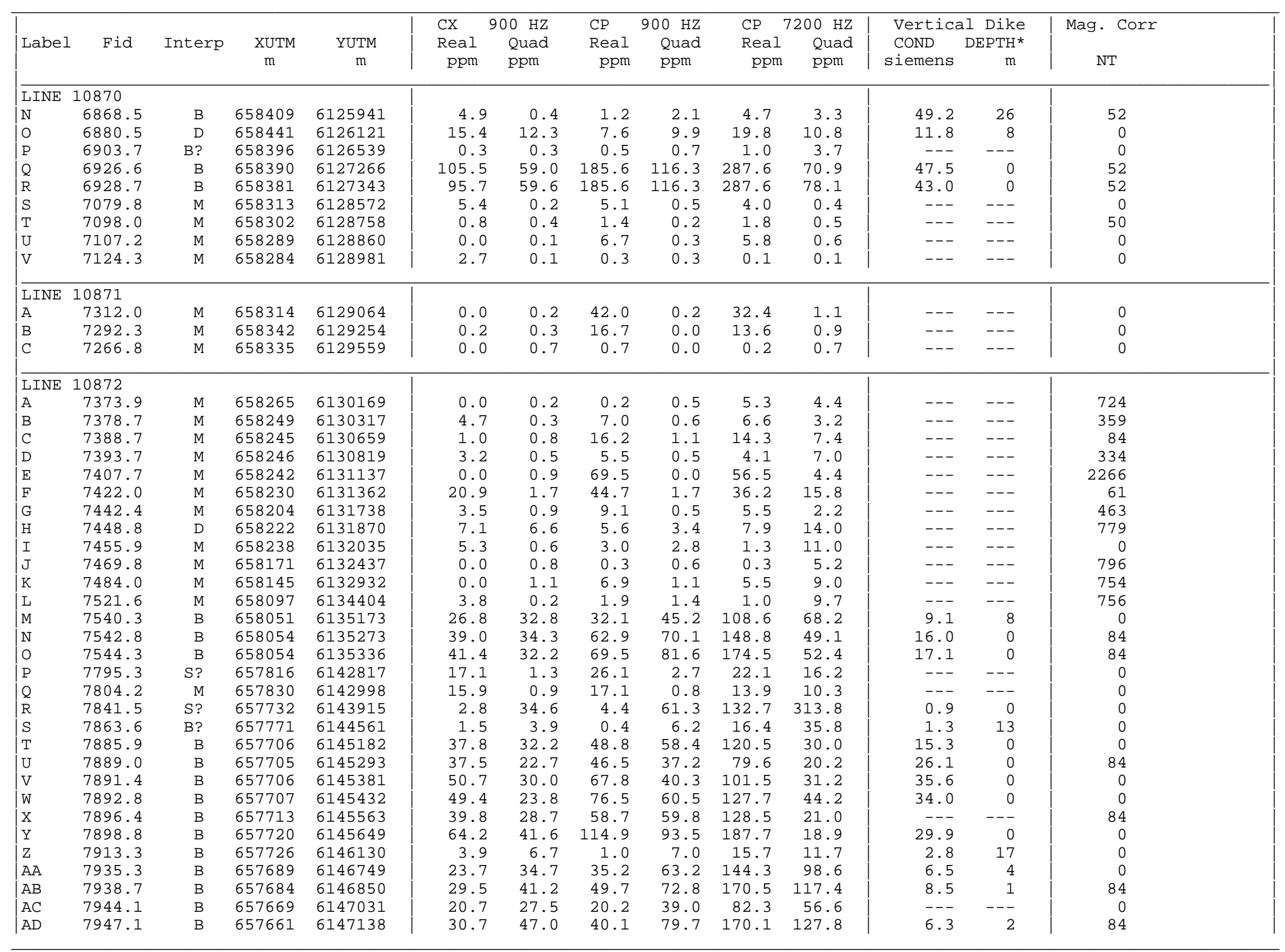

$\mathrm{CX}=$ COAXIAL

Note: EM values shown above

are local amplitudes
* Estimated Depth may be unreliable because the stronger part of the conductor may be deeper or to one side of the flight line, or because of a shallow dip or magnetite/overburden effects 
EM Anomaly List

\begin{tabular}{|c|c|c|c|c|c|c|c|c|c|c|c|c|c|}
\hline Label & Fid & Interp & $\begin{array}{c}\text { XUTM } \\
\text { m }\end{array}$ & $\begin{array}{c}\text { YUTM } \\
\mathrm{m}\end{array}$ & $\begin{array}{l}\text { CX } \\
\text { Real } \\
\text { ppm }\end{array}$ & $\begin{array}{l}900 \mathrm{HZ} \\
\text { Quad } \\
\text { ppm }\end{array}$ & $\begin{array}{l}\text { CP } \\
\text { Real } \\
\text { ppm }\end{array}$ & $\begin{array}{l}900 \mathrm{HZ} \\
\text { Quad } \\
\text { ppm }\end{array}$ & $\begin{array}{l}\text { CP } \\
\text { Real } \\
\text { ppm }\end{array}$ & $\begin{array}{l}7200 \mathrm{HZ} \\
\text { Quad } \\
\text { ppm }\end{array}$ & $\begin{array}{l}\text { Vertica } \\
\text { Cond } \\
\text { siemens }\end{array}$ & $\begin{array}{c}\text { al Dike } \\
\text { DEPTH* } \\
\text { m }\end{array}$ & $\begin{array}{c}\text { Mag. Corr } \\
\text { NT }\end{array}$ \\
\hline$\overline{\text { LINE } 1}$ & 10880 & & & & & & & & & & & & \\
\hline A & 5700.8 & $\mathrm{~S}$ & 659202 & 6114882 & 1.7 & 6.1 & 2.4 & 11.5 & 19.2 & 66.1 & 1.5 & 2 & 0 \\
\hline B & 5666.2 & $B$ ? & 659213 & 6115882 & 2.4 & 9.4 & 6.3 & 19.2 & 47.7 & 54.2 & -- & --- & 0 \\
\hline $\mathrm{C}$ & 5656.0 & $B$ ? & 659191 & 6116153 & 0.9 & 6.1 & 0.3 & 11.4 & 30.0 & 65.1 & --- & --- & 0 \\
\hline D & 5609.3 & M & 659157 & 6117143 & 4.1 & 0.1 & 1.2 & 0.0 & 1.1 & 2.1 & --- & --- & 0 \\
\hline $\mathrm{E}$ & 5597.2 & $\mathrm{M}$ & 659156 & 6117210 & 1.8 & 0.2 & 10.1 & 0.0 & 8.9 & 3.1 & --- & --- & 0 \\
\hline $\mathrm{F}$ & 5550.5 & $\mathrm{~s}$ & 659134 & 6117746 & 0.1 & 1.2 & 0.3 & 1.7 & 2.0 & 8.2 & --- & --- & 0 \\
\hline G & 5435.9 & $\mathrm{D}$ & 659067 & 6120241 & 1.6 & 4.6 & 0.8 & 1.7 & 1.7 & 12.4 & 1.8 & 29 & 0 \\
\hline $\mathrm{H}$ & 5370.2 & M & 659000 & 6122012 & 0.0 & 0.4 & 1.1 & 0.4 & 1.0 & 3.0 & -- & --- & 281 \\
\hline I & 5353.2 & M & 658931 & 6122423 & 4.1 & 0.4 & 1.2 & 0.3 & 0.9 & 3.4 & --- & --- & 190 \\
\hline $\mathrm{J}$ & 5251.0 & M & 658907 & 6123617 & 1.3 & 0.5 & 0.5 & 0.1 & 0.4 & 1.5 & --- & --- & 42 \\
\hline $\mathrm{K}$ & 5238.8 & M & 658904 & 6123694 & 0.4 & 0.1 & 5.7 & 0.4 & 4.6 & 1.7 & --- & --- & 119 \\
\hline L & 5218.0 & $\mathrm{M}$ & 658895 & 6123811 & 0.1 & 0.1 & 0.2 & 0.2 & 0.6 & 1.3 & --- & --- & 0 \\
\hline M & 5179.8 & B & 658880 & 6124546 & 14.9 & 10.3 & 19.3 & 18.9 & 40.4 & 12.9 & 15.0 & 7 & 0 \\
\hline $\mathrm{N}$ & 5091.1 & D & 658851 & 6125365 & 8.9 & 10.7 & 0.4 & 0.8 & 3.0 & 4.3 & 5.9 & 20 & 0 \\
\hline 0 & 5024.9 & B & 658835 & 6126067 & 2.5 & 6.2 & 7.8 & 16.5 & 40.1 & 31.4 & 3.2 & 15 & 0 \\
\hline $\mathrm{P}$ & 4997.9 & B & 658824 & 6126185 & 8.4 & 7.0 & 19.5 & 18.3 & 38.7 & 24.1 & 11.9 & 26 & 0 \\
\hline Q & 4967.4 & B & 658805 & 6126331 & 8.4 & 5.2 & 23.7 & 12.8 & 35.5 & 9.5 & 23.4 & 7 & 0 \\
\hline R & 4954.9 & B & 658799 & 6126413 & 68.7 & 43.2 & 111.0 & 69.0 & 158.7 & 38.4 & 37.4 & 0 & 72 \\
\hline $\mathrm{S}$ & 4937.2 & B & 658799 & 6126943 & 44.2 & 22.0 & 93.7 & 39.0 & 99.2 & 18.1 & 53.6 & 0 & 0 \\
\hline $\mathrm{T}$ & 4928.3 & B & 658784 & 6127230 & 19.7 & 24.0 & 19.8 & 36.8 & 100.7 & 52.1 & 6.9 & 0 & 0 \\
\hline U & 4852.2 & M & 658715 & 6128438 & 0.3 & 0.1 & 1.1 & 0.2 & 0.8 & 1.9 & --- & --- & 0 \\
\hline $\mathrm{V}$ & 4826.0 & M & 658722 & 6128674 & 2.6 & 0.2 & 2.6 & 0.1 & 1.5 & 2.5 & --- & --- & 10 \\
\hline W & 4808.3 & $\mathrm{M}$ & 658716 & 6128760 & 12.6 & 0.3 & 135.5 & 2.3 & 114.5 & 3.4 & --- & --- & 2591 \\
\hline $\mathrm{x}$ & 4770.8 & M & 658713 & 6129061 & 0.2 & 0.5 & 1.4 & 0.0 & 1.0 & 3.7 & --- & --- & 72 \\
\hline $\mathrm{Y}$ & 4752.0 & M & 658714 & 6129392 & 35.8 & 0.3 & 89.5 & 0.1 & 76.6 & 1.1 & --- & --- & 0 \\
\hline $\mathrm{z}$ & 4740.3 & M & 658714 & 6129442 & 19.9 & 0.0 & 13.3 & 0.2 & 11.3 & 6.2 & --- & --- & 0 \\
\hline $\mathrm{AA}$ & 4687.2 & $\mathrm{~S}$ & 658738 & 6130119 & 3.8 & 3.5 & 6.6 & 4.3 & 6.8 & 25.3 & 10.2 & 26 & 72 \\
\hline$A B$ & 4644.2 & M & 658629 & 6131267 & 0.0 & 1.2 & 0.5 & 0.1 & 0.7 & 3.3 & -- & -- & 1196 \\
\hline $\mathrm{AC}$ & 4630.0 & $\mathrm{M}$ & 658642 & 6131517 & 3.1 & 0.6 & 5.5 & 0.6 & 3.7 & 3.4 & --- & --- & 34 \\
\hline $\mathrm{AD}$ & 4610.3 & $\mathrm{M}$ & 658630 & 6131751 & 1.7 & 0.0 & 0.1 & 0.2 & 0.1 & 1.5 & --- & --- & 747 \\
\hline $\mathrm{AE}$ & 4588.8 & M & 658627 & 6131883 & 0.5 & 0.5 & 16.0 & 0.0 & 13.0 & 2.5 & --- & --- & 0 \\
\hline $\mathrm{AF}$ & 4567.8 & $\mathrm{M}$ & 658639 & 6132000 & 0.7 & 0.5 & 3.6 & 0.0 & 3.9 & 2.6 & --- & --- & 0 \\
\hline AG & 4538.2 & M & 658575 & 6132636 & 0.0 & 0.4 & 2.2 & 0.1 & 2.1 & 2.0 & --- & --- & 1248 \\
\hline $\mathrm{AH}$ & 4501.2 & M & 658585 & 6133740 & 1.0 & 0.5 & 2.3 & 0.1 & 1.7 & 7.6 & --- & --- & 0 \\
\hline $\mathrm{AI}$ & 4472.9 & M & 658550 & 6134723 & 0.8 & 0.9 & 12.6 & 1.5 & 11.6 & 7.1 & --- & --- & 0 \\
\hline $\mathrm{AJ}$ & 4454.9 & $\mathrm{D}$ & 658577 & 6135465 & 4.1 & 7.1 & 3.8 & 6.4 & 15.1 & 16.6 & 3.0 & 26 & 72 \\
\hline AK & 4435.9 & B? & 658464 & 6136203 & 23.0 & 51.8 & 33.3 & 113.0 & 291.2 & 150.1 & 5.0 & 0 & 72 \\
\hline $\mathrm{AL}$ & 4269.6 & S? & 658224 & 6142383 & 0.8 & 3.6 & 0.4 & 3.7 & 5.6 & 18.8 & 0.9 & 0 & 0 \\
\hline $\mathrm{AM}$ & 4247.3 & $\mathrm{~S}$ & 658218 & 6142944 & 0.4 & 1.0 & 0.6 & 1.9 & 1.2 & 16.3 & --- & --- & 72 \\
\hline AN & 4235.3 & $\mathrm{~s}$ & 658243 & 6143176 & 0.1 & 1.1 & 1.2 & 1.7 & 1.3 & 13.8 & --- & --- & 0 \\
\hline AO & 4220.2 & S? & 658250 & 6143393 & 12.8 & 2.6 & 6.7 & 5.3 & 26.4 & 36.3 & 50.2 & 29 & 72 \\
\hline AP & 4208.0 & $\mathrm{M}$ & 658217 & 6143683 & 0.2 & 0.3 & 0.8 & 0.0 & 0.8 & 2.1 & -- & --- & 362 \\
\hline $\mathrm{AQ}$ & 4188.8 & S? & 658187 & 6144263 & 1.5 & 5.2 & 1.8 & 7.6 & 18.5 & 35.3 & 1.5 & 0 & 0 \\
\hline $\mathrm{AR}$ & 4170.9 & S? & 658175 & 6144892 & 4.7 & 7.1 & 0.7 & 9.9 & 31.6 & 36.7 & 2.8 & 5 & 0 \\
\hline AS & 4137.7 & B & 658116 & 6145914 & 10.3 & 24.0 & 16.5 & 45.8 & 119.0 & 77.1 & 4.1 & 0 & 0 \\
\hline
\end{tabular}

$\mathrm{CX}=$ COAXIAL

Note: EM values shown above

Ketchikan - North/South of 5515
*Estimated Depth may be unreliable because the stronger part of the conductor may be deeper or to one side of the flight line, or because of a shallow dip or magnetite/overburden effects 
EM Anomaly List

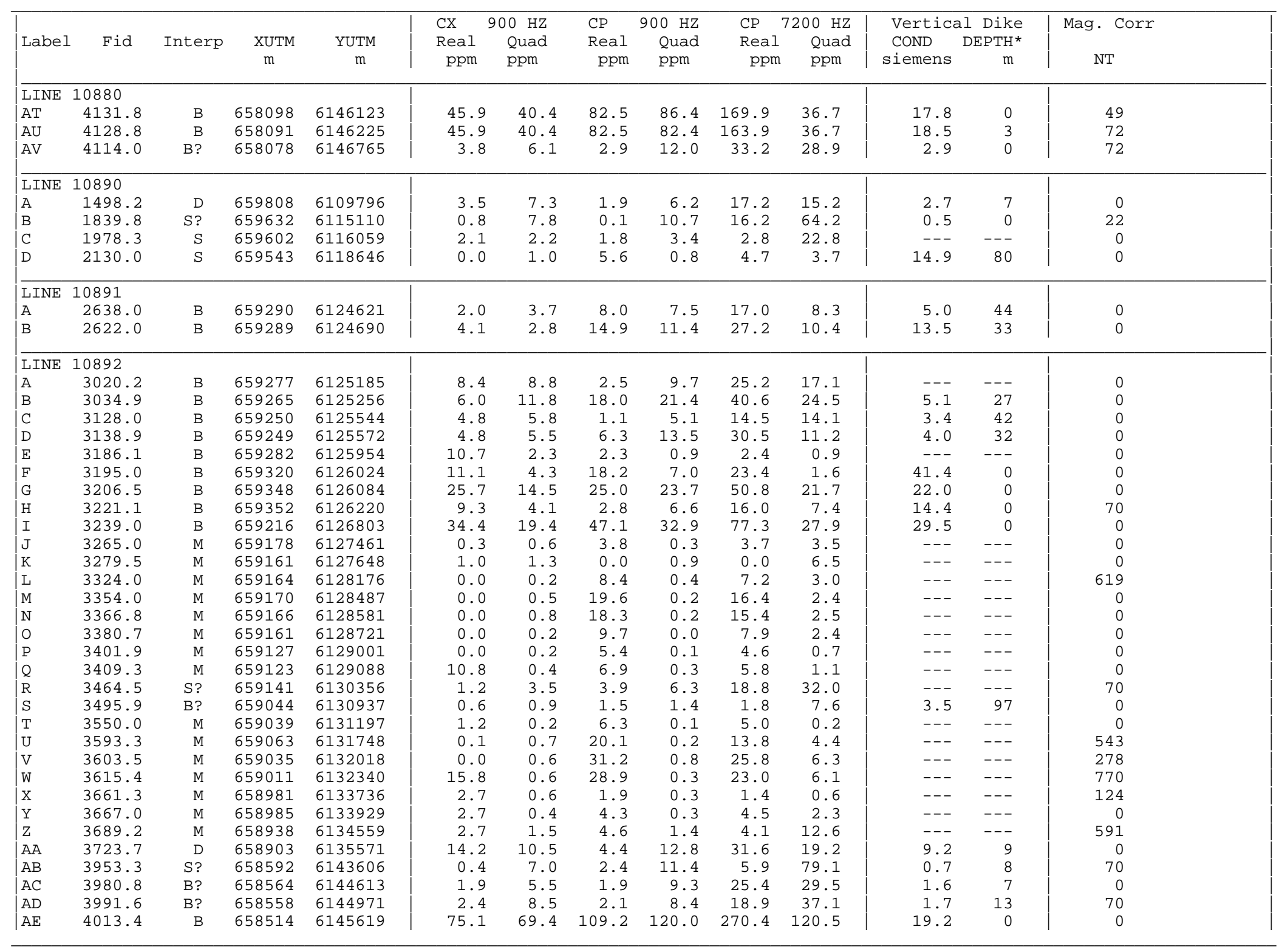

$\mathrm{CX}=$ COAXIAL

Note: EM values shown above

are local amplitudes

Ketchikan - North/South of 5515
* Estimated Depth may be unreliable because the stronger part of the conductor may be deeper or to one side of the flight line, or because of a shallow dip or magnetite/overburden effects 
EM Anomaly List

\begin{tabular}{|c|c|c|c|c|c|c|c|c|c|c|c|c|c|}
\hline Label & Fid & Interp & $\underset{\mathrm{m}}{\mathrm{XUTM}}$ & $\begin{array}{c}\text { YUTM } \\
\text { m }\end{array}$ & $\begin{array}{ll}\text { CX } & 9 \\
\text { Real } & \\
\text { ppm }\end{array}$ & $\begin{array}{l}00 \mathrm{HZ} \\
\text { Quad } \\
\text { ppm }\end{array}$ & $\begin{array}{l}\text { CP } \\
\text { Real } \\
\text { ppm }\end{array}$ & $\begin{array}{l}900 \mathrm{HZ} \\
\text { Quad } \\
\text { ppm }\end{array}$ & $\begin{array}{l}\text { CP }{ }^{7} \\
\text { Real } \\
\text { ppm }\end{array}$ & $\begin{array}{l}200 \mathrm{HZ} \\
\text { Quad } \\
\text { ppm }\end{array}$ & $\begin{array}{l}\text { Vertica } \\
\text { Cond } \\
\text { siemens }\end{array}$ & $\begin{array}{c}\text { al Dike } \\
\text { DEPTH* } \\
\text { m }\end{array}$ & $\begin{array}{c}\text { Mag. Corr } \\
\text { NT }\end{array}$ \\
\hline $\begin{array}{l}\text { LINE } \\
\text { A } \\
\text { B } \\
\text { C } \\
\text { D } \\
\text { E } \\
\text { F }\end{array}$ & $\begin{array}{r}10900 \\
359.6 \\
330.0 \\
322.0 \\
304.9 \\
288.5 \\
249.0\end{array}$ & $\begin{array}{l}\mathrm{D} \\
\mathrm{M} \\
\mathrm{M} \\
\mathrm{M} \\
\mathrm{M} \\
\mathrm{M}\end{array}$ & $\begin{array}{l}659703 \\
659713 \\
659728 \\
659750 \\
659752 \\
659742\end{array}$ & $\begin{array}{l}6123292 \\
6123546 \\
6123579 \\
6123638 \\
6123676 \\
6123896\end{array}$ & $\begin{array}{r}5.5 \\
1.2 \\
10.3 \\
6.0 \\
0.0 \\
0.0\end{array}$ & $\begin{array}{l}6.6 \\
0.1 \\
0.7 \\
0.6 \\
0.1 \\
0.0\end{array}$ & $\begin{array}{r}3.8 \\
1.4 \\
21.0 \\
23.4 \\
4.9 \\
8.6\end{array}$ & $\begin{array}{l}2.2 \\
0.3 \\
0.5 \\
0.2 \\
0.2 \\
0.5\end{array}$ & $\begin{array}{r}8.8 \\
0.9 \\
16.0 \\
16.7 \\
3.1 \\
7.6\end{array}$ & $\begin{array}{l}7.9 \\
6.2 \\
2.6 \\
1.2 \\
0.3 \\
2.2\end{array}$ & $\begin{array}{l}7.0 \\
--- \\
--- \\
--- \\
--- \\
---\end{array}$ & $\begin{array}{c}11 \\
--- \\
--- \\
--- \\
--- \\
---\end{array}$ & $\begin{array}{r}0 \\
4 \\
0 \\
26 \\
0 \\
0\end{array}$ \\
\hline $\begin{array}{l}\text { LINE } \\
\text { A } \\
B \\
\text { C } \\
\text { D } \\
\text { E }\end{array}$ & $\begin{array}{l}10901 \\
1258.2 \\
1253.9 \\
1201.5 \\
968.5 \\
917.8\end{array}$ & $\begin{array}{c}\text { S? } \\
\text { M } \\
\text { S } \\
\text { D } \\
\text { S }\end{array}$ & $\begin{array}{l}660270 \\
660272 \\
660222 \\
660036 \\
659978\end{array}$ & $\begin{array}{l}6108690 \\
6108721 \\
6109793 \\
6114914 \\
6116152\end{array}$ & $\begin{array}{l}4.3 \\
0.6 \\
2.1 \\
1.9 \\
0.5\end{array}$ & $\begin{array}{l}0.7 \\
0.7 \\
5.8 \\
2.8 \\
1.7\end{array}$ & $\begin{array}{r}36.9 \\
0.5 \\
0.8 \\
1.0 \\
2.0\end{array}$ & $\begin{array}{l}1.4 \\
0.6 \\
6.0 \\
2.4 \\
3.0\end{array}$ & $\begin{array}{r}29.1 \\
0.2 \\
16.2 \\
7.4 \\
4.5\end{array}$ & $\begin{array}{r}6.8 \\
7.1 \\
17.5 \\
14.2 \\
18.1\end{array}$ & $\begin{array}{r}504.9 \\
--- \\
1.6 \\
--- \\
---\end{array}$ & $\begin{array}{r}44 \\
--- \\
12 \\
--- \\
---\end{array}$ & $\begin{array}{r}24 \\
117 \\
0 \\
4 \\
0\end{array}$ \\
\hline $\begin{array}{l}\text { LINE } \\
\text { A } \\
\text { B } \\
\text { C } \\
\text { D } \\
\text { E } \\
\text { F } \\
\text { G } \\
\text { H } \\
\text { I } \\
\text { J } \\
\text { K } \\
\text { L } \\
\text { M } \\
\text { N } \\
\text { O } \\
\text { P } \\
\text { Q }\end{array}$ & $\begin{array}{r}10902 \\
3496.6 \\
3484.6 \\
3419.5 \\
3391.0 \\
3320.3 \\
3274.7 \\
3250.3 \\
3222.0 \\
3207.2 \\
3190.0 \\
2897.5 \\
2882.8 \\
2876.6 \\
2784.7 \\
2692.5 \\
2477.9 \\
2476.4\end{array}$ & $\begin{array}{r}\text { B } \\
\mathrm{B} \\
\mathrm{D} \\
\mathrm{D} \\
\mathrm{B} \\
\mathrm{B} \\
\mathrm{B} \\
\mathrm{B} ? \\
\mathrm{D} \\
\mathrm{M} \\
\mathrm{M} \\
\mathrm{M} \\
\mathrm{M} \\
\mathrm{S} \\
\mathrm{S} \\
\mathrm{B} ? \\
\mathrm{~B}\end{array}$ & $\begin{array}{l}659739 \\
659688 \\
659682 \\
659613 \\
659655 \\
659663 \\
659675 \\
659655 \\
659608 \\
659623 \\
659454 \\
659468 \\
659468 \\
659333 \\
659250 \\
658964 \\
658961\end{array}$ & $\begin{array}{l}6124158 \\
6124381 \\
6125288 \\
6125505 \\
6125855 \\
6125929 \\
6126033 \\
6126255 \\
6126786 \\
6127258 \\
6131230 \\
6131435 \\
6131598 \\
6134413 \\
6137339 \\
6144932 \\
6144986\end{array}$ & $\begin{array}{r}7.3 \\
15.0 \\
4.8 \\
11.4 \\
15.5 \\
75.5 \\
9.5 \\
6.4 \\
6.8 \\
0.7 \\
1.0 \\
3.4 \\
1.1 \\
0.2 \\
0.2 \\
1.6 \\
1.6\end{array}$ & $\begin{array}{r}2.8 \\
15.1 \\
5.0 \\
8.8 \\
7.6 \\
41.1 \\
10.0 \\
3.0 \\
7.3 \\
0.6 \\
0.4 \\
0.8 \\
0.1 \\
2.4 \\
1.4 \\
4.1 \\
3.9\end{array}$ & $\begin{array}{r}17.0 \\
28.9 \\
0.8 \\
1.4 \\
1.8 \\
95.8 \\
23.5 \\
9.6 \\
8.7 \\
0.9 \\
0.3 \\
6.1 \\
2.1 \\
0.2 \\
1.1 \\
0.3 \\
0.9\end{array}$ & $\begin{array}{r}9.2 \\
31.2 \\
2.0 \\
4.6 \\
0.1 \\
82.4 \\
18.3 \\
6.9 \\
4.9 \\
1.2 \\
0.2 \\
0.8 \\
0.4 \\
2.2 \\
1.9 \\
4.5 \\
4.5\end{array}$ & $\begin{array}{r}22.5 \\
63.5 \\
6.2 \\
11.0 \\
2.2 \\
181.2 \\
36.9 \\
12.9 \\
13.9 \\
0.5 \\
0.3 \\
4.4 \\
2.5 \\
0.2 \\
1.7 \\
7.1 \\
6.9\end{array}$ & $\begin{array}{r}5.5 \\
33.1 \\
8.0 \\
8.9 \\
2.5 \\
62.7 \\
12.9 \\
3.4 \\
9.4 \\
5.7 \\
2.3 \\
6.6 \\
2.0 \\
15.8 \\
14.6 \\
27.8 \\
27.7\end{array}$ & $\begin{array}{r}28.4 \\
11.0 \\
5.2 \\
--- \\
29.4 \\
34.0 \\
12.2 \\
18.8 \\
10.2 \\
--- \\
--- \\
--- \\
--- \\
--- \\
--- \\
1.4 \\
1.7\end{array}$ & $\begin{array}{r}9 \\
9 \\
16 \\
--- \\
0 \\
0 \\
22 \\
0 \\
6 \\
--- \\
--- \\
--- \\
--- \\
--- \\
--- \\
9 \\
13\end{array}$ & $\begin{array}{r}0 \\
0 \\
0 \\
50 \\
52 \\
0 \\
48 \\
52 \\
0 \\
160 \\
0 \\
0 \\
529 \\
0 \\
0 \\
4 \\
4\end{array}$ \\
\hline $\begin{array}{l}\text { LINE } \\
A\end{array}$ & $\begin{array}{l}10903 \\
3761.2\end{array}$ & $\mathrm{M}$ & 659759 & 6122975 & 0.4 & 0.6 & 2.1 & 0.3 & 0.1 & 0.8 & --- & --- & 0 \\
\hline $\begin{array}{l}\text { LINE } \\
\text { A } \\
\text { B } \\
\text { C } \\
\text { D } \\
\text { E } \\
\text { F } \\
\text { G } \\
\text { H }\end{array}$ & $\begin{array}{r}10910 \\
9480.3 \\
9473.5 \\
9095.0 \\
8890.1 \\
8876.7 \\
8866.3 \\
8801.0 \\
8735.5\end{array}$ & $\begin{array}{r}\mathrm{D} \\
\mathrm{S} \\
\mathrm{B} ? \\
\mathrm{~S} \\
\mathrm{~B} ? \\
\mathrm{~B} \\
\mathrm{~B} \\
\mathrm{~B}\end{array}$ & $\begin{array}{l}660570 \\
660589 \\
660346 \\
660091 \\
660114 \\
660133 \\
660107 \\
660053\end{array}$ & $\begin{array}{l}6110527 \\
6110750 \\
6117657 \\
6123127 \\
6123444 \\
6123707 \\
6124634 \\
6125735\end{array}$ & $\begin{array}{r}7.1 \\
0.9 \\
1.7 \\
0.4 \\
15.2 \\
32.6 \\
3.5 \\
5.0\end{array}$ & $\begin{array}{r}13.7 \\
3.8 \\
2.2 \\
2.3 \\
7.4 \\
20.8 \\
3.2 \\
4.4\end{array}$ & $\begin{array}{r}0.2 \\
0.1 \\
0.9 \\
1.7 \\
34.0 \\
42.9 \\
4.5 \\
9.1\end{array}$ & $\begin{array}{r}6.2 \\
6.7 \\
3.1 \\
2.6 \\
30.3 \\
34.6 \\
7.1 \\
13.3\end{array}$ & $\begin{array}{r}18.7 \\
6.5 \\
9.7 \\
1.5 \\
68.8 \\
78.8 \\
16.5 \\
27.5\end{array}$ & $\begin{array}{l}26.5 \\
44.8 \\
14.1 \\
17.3 \\
19.0 \\
22.1 \\
11.4 \\
16.1\end{array}$ & $\begin{array}{r}3.2 \\
0.7 \\
--- \\
--- \\
20.2 \\
--- \\
5.4 \\
6.6\end{array}$ & $\begin{array}{r}10 \\
0 \\
--- \\
--- \\
0 \\
--- \\
35 \\
0\end{array}$ & $\begin{array}{r}38 \\
38 \\
56 \\
38 \\
0 \\
0 \\
0 \\
0\end{array}$ \\
\hline
\end{tabular}

$\mathrm{CX}=$ COAXIAL

Note: EM values shown above

Ketchikan - North/South of 5515
* Estimated Depth may be unreliable because the stronger part of the conductor may be deeper or to one side of the flight line, or because of a shallow dip or magnetite/overburden effects 
EM Anomaly List

\begin{tabular}{|c|c|c|c|c|c|c|c|c|c|c|c|c|c|}
\hline Label & Fid & Interp & $\begin{array}{l}\text { XUTM } \\
\text { m }\end{array}$ & $\begin{array}{c}\text { YUTM } \\
\mathrm{m}\end{array}$ & $\begin{array}{l}\text { CX } \\
\text { Real } \\
\text { ppm }\end{array}$ & $\begin{array}{l}900 \mathrm{HZ} \\
\text { Quad } \\
\text { ppm }\end{array}$ & $\begin{array}{l}\text { CP } \\
\text { Real } \\
\text { ppm }\end{array}$ & $\begin{array}{c}900 \mathrm{HZ} \\
\text { Quad } \\
\text { ppm }\end{array}$ & $\begin{array}{l}\text { CP } 7 \\
\text { Real } \\
\text { ppm }\end{array}$ & $\begin{array}{l}200 \mathrm{HZ} \\
\text { Quad } \\
\text { ppm }\end{array}$ & $\begin{array}{l}\text { Vertica } \\
\text { CoND } \\
\text { siemens }\end{array}$ & $\begin{array}{c}\text { al Dike } \\
\text { DEPTH* } \\
\text { m }\end{array}$ & $\begin{array}{c}\text { Mag. Corr } \\
\text { NT }\end{array}$ \\
\hline $\begin{array}{l}\text { LINE } \\
\text { I } \\
\text { J } \\
\text { K } \\
\text { L } \\
\text { M } \\
N \\
O \\
P \\
Q \\
R \\
\text { S } \\
\text { T } \\
\text { U }\end{array}$ & $\begin{array}{r}10910 \\
8721.7 \\
8713.1 \\
8688.1 \\
8679.0 \\
8654.8 \\
8647.2 \\
8627.2 \\
8598.0 \\
8487.9 \\
8460.2 \\
8392.8 \\
8293.2 \\
8069.1\end{array}$ & $\begin{array}{c}\mathrm{B} \\
\mathrm{B} \\
\mathrm{D} \\
\mathrm{M} \\
\mathrm{M} \\
\mathrm{M} \\
\mathrm{M} \\
\mathrm{M} \\
\mathrm{M} \\
\mathrm{M} \\
\mathrm{M} \\
\mathrm{M} \\
\mathrm{B}\end{array}$ & $\begin{array}{l}660051 \\
660071 \\
660010 \\
660008 \\
659983 \\
659984 \\
660014 \\
659944 \\
659844 \\
659846 \\
659776 \\
659667 \\
659348\end{array}$ & $\begin{array}{l}6126008 \\
6126259 \\
6127009 \\
6127236 \\
6127688 \\
6127868 \\
6128424 \\
6128922 \\
6130738 \\
6131191 \\
6133303 \\
6136788 \\
6145062\end{array}$ & $\begin{array}{r}8.8 \\
17.3 \\
9.2 \\
2.5 \\
1.0 \\
0.2 \\
9.6 \\
0.1 \\
0.9 \\
0.0 \\
0.4 \\
0.7 \\
2.5\end{array}$ & $\begin{array}{l}8.3 \\
6.8 \\
6.4 \\
1.4 \\
1.1 \\
0.8 \\
0.7 \\
0.7 \\
0.6 \\
0.6 \\
0.7 \\
0.1 \\
7.0\end{array}$ & $\begin{array}{r}7.4 \\
4.9 \\
11.5 \\
0.2 \\
0.2 \\
0.8 \\
10.4 \\
0.8 \\
0.3 \\
4.2 \\
7.9 \\
0.4 \\
1.0\end{array}$ & $\begin{array}{r}10.0 \\
8.9 \\
4.4 \\
0.9 \\
0.5 \\
0.5 \\
0.8 \\
0.0 \\
0.4 \\
0.8 \\
0.5 \\
0.8 \\
5.3\end{array}$ & $\begin{array}{r}21.8 \\
18.7 \\
17.3 \\
0.6 \\
0.3 \\
0.6 \\
6.2 \\
0.6 \\
0.4 \\
3.8 \\
7.2 \\
0.1 \\
11.9\end{array}$ & $\begin{array}{r}20.9 \\
8.1 \\
16.8 \\
7.8 \\
1.8 \\
2.1 \\
3.3 \\
2.3 \\
1.8 \\
3.2 \\
5.1 \\
16.7 \\
25.7\end{array}$ & $\begin{array}{r}8.1 \\
23.3 \\
20.8 \\
--- \\
--- \\
--- \\
--- \\
--- \\
--- \\
--- \\
--- \\
--- \\
1.8\end{array}$ & $\begin{array}{r}8 \\
4 \\
5 \\
--- \\
--- \\
--- \\
--- \\
--- \\
--- \\
--- \\
--- \\
--- \\
15\end{array}$ & $\begin{array}{r}0 \\
0 \\
0 \\
0 \\
0 \\
717 \\
96 \\
39 \\
0 \\
578 \\
38 \\
293 \\
31\end{array}$ \\
\hline $\begin{array}{l}\text { LINE } \\
\text { A } \\
\text { B } \\
\text { C } \\
\text { D } \\
\text { E }\end{array}$ & $\begin{array}{l}10920 \\
517.4 \\
514.8 \\
509.9 \\
505.3 \\
422.6\end{array}$ & $\begin{array}{l}\text { S? } \\
\text { S? } \\
\text { S? } \\
\text { S? } \\
\text { B? }\end{array}$ & $\begin{array}{l}659903 \\
659906 \\
659909 \\
659902 \\
659766\end{array}$ & $\begin{array}{l}6141217 \\
6141321 \\
6141520 \\
6141703 \\
6145114\end{array}$ & $\begin{array}{r}40.5 \\
39.1 \\
18.6 \\
22.1 \\
4.3\end{array}$ & $\begin{array}{r}24.0 \\
23.6 \\
11.9 \\
6.3 \\
6.1\end{array}$ & $\begin{array}{r}76.6 \\
52.3 \\
14.7 \\
1.4 \\
4.9\end{array}$ & $\begin{array}{r}56.6 \\
39.9 \\
16.8 \\
6.4 \\
9.4\end{array}$ & $\begin{array}{r}102.0 \\
78.3 \\
21.1 \\
3.5 \\
22.2\end{array}$ & $\begin{array}{r}15.1 \\
13.5 \\
7.5 \\
1.9 \\
18.4\end{array}$ & $\begin{array}{r}29.5 \\
27.5 \\
16.0 \\
38.8 \\
3.6\end{array}$ & $\begin{array}{r}0 \\
0 \\
0 \\
0 \\
27\end{array}$ & $\begin{array}{r}17 \\
0 \\
17 \\
17 \\
11\end{array}$ \\
\hline $\begin{array}{l}\text { LINE } \\
\text { A } \\
\text { B } \\
\text { C } \\
\text { D } \\
\text { E } \\
\text { F } \\
\text { G } \\
\text { H } \\
\text { I } \\
\text { J } \\
\text { K } \\
\text { L } \\
\text { M }\end{array}$ & $\begin{array}{r}10921 \\
6180.8 \\
6269.8 \\
6875.9 \\
6888.8 \\
6921.6 \\
7008.2 \\
7017.8 \\
7045.5 \\
7111.2 \\
7114.7 \\
7139.5 \\
7171.4 \\
7193.3\end{array}$ & $\begin{array}{r}\text { S? } \\
\text { B } \\
\text { M } \\
\text { M } \\
\text { B? } \\
\text { B } \\
\text { B } \\
B ? \\
\text { S } \\
\text { M } \\
\text { M } \\
\text { M } \\
\text { M }\end{array}$ & $\begin{array}{l}661093 \\
661011 \\
660565 \\
660558 \\
660546 \\
660468 \\
660431 \\
660482 \\
660451 \\
660457 \\
660433 \\
660400 \\
660339\end{array}$ & $\begin{array}{l}6107712 \\
6110428 \\
6122403 \\
6122627 \\
6123336 \\
6124580 \\
6124720 \\
6125531 \\
6126910 \\
6126976 \\
6127200 \\
6127493 \\
6127799\end{array}$ & $\begin{array}{r}0.8 \\
2.6 \\
0.5 \\
0.3 \\
16.8 \\
2.3 \\
5.1 \\
10.4 \\
2.0 \\
2.6 \\
0.0 \\
1.1 \\
0.2\end{array}$ & $\begin{array}{r}0.9 \\
3.2 \\
0.9 \\
0.8 \\
17.6 \\
3.4 \\
3.4 \\
10.1 \\
2.2 \\
2.3 \\
0.6 \\
0.7 \\
0.1\end{array}$ & $\begin{array}{r}1.7 \\
0.7 \\
4.3 \\
0.7 \\
22.8 \\
2.2 \\
2.9 \\
5.3 \\
3.9 \\
0.2 \\
2.6 \\
5.3 \\
1.1\end{array}$ & $\begin{array}{r}1.6 \\
2.5 \\
0.3 \\
0.5 \\
46.6 \\
4.7 \\
4.6 \\
13.7 \\
0.9 \\
2.0 \\
0.8 \\
0.1 \\
0.3\end{array}$ & $\begin{array}{r}3.6 \\
5.1 \\
3.4 \\
0.9 \\
108.1 \\
12.1 \\
11.0 \\
32.4 \\
5.8 \\
4.2 \\
2.8 \\
0.1 \\
0.6\end{array}$ & $\begin{array}{r}11.4 \\
10.1 \\
1.1 \\
1.8 \\
67.5 \\
10.6 \\
7.4 \\
20.1 \\
4.1 \\
10.7 \\
4.5 \\
1.2 \\
0.4\end{array}$ & $\begin{array}{l}4.5 \\
3.0 \\
--- \\
--- \\
6.7 \\
--- \\
--- \\
6.1 \\
--- \\
--- \\
--- \\
--- \\
---\end{array}$ & $\begin{array}{r}75 \\
58 \\
--- \\
--- \\
2 \\
--- \\
--- \\
0 \\
--- \\
--- \\
--- \\
--- \\
---\end{array}$ & $\begin{array}{r}87 \\
0 \\
234 \\
18 \\
77 \\
0 \\
0 \\
25 \\
25 \\
30 \\
0 \\
180 \\
0\end{array}$ \\
\hline $\begin{array}{l}\text { LINE } \\
\text { A } \\
\text { B } \\
\text { C } \\
\text { D }\end{array}$ & $\begin{array}{r}10922 \\
4418.2 \\
4442.6 \\
4471.4 \\
4499.0\end{array}$ & $\begin{array}{l}\mathrm{M} \\
\mathrm{M} \\
\mathrm{M} \\
\mathrm{M}\end{array}$ & $\begin{array}{l}660409 \\
660428 \\
660429 \\
660412\end{array}$ & $\begin{array}{l}6126979 \\
6127259 \\
6127445 \\
6127710\end{array}$ & $\begin{array}{l}0.0 \\
0.0 \\
0.1 \\
0.3\end{array}$ & $\begin{array}{l}1.9 \\
0.1 \\
0.8 \\
1.1\end{array}$ & $\begin{array}{l}4.2 \\
9.8 \\
5.3 \\
1.3\end{array}$ & $\begin{array}{l}1.7 \\
0.2 \\
0.3 \\
0.2\end{array}$ & $\begin{array}{l}5.7 \\
0.1 \\
4.7 \\
1.4\end{array}$ & $\begin{array}{r}11.2 \\
3.8 \\
1.6 \\
0.6\end{array}$ & $\begin{array}{l}--- \\
--- \\
--- \\
---\end{array}$ & $\begin{array}{l}--- \\
--- \\
--- \\
---\end{array}$ & $\begin{array}{r}0 \\
0 \\
0 \\
643\end{array}$ \\
\hline $\begin{array}{l}\text { LINE } \\
\text { A }\end{array}$ & $\begin{array}{r}10923 \\
4788.8\end{array}$ & S? & 660331 & 6129515 & 1.8 & 0.1 & 1.4 & 0.3 & 0.9 & 4.2 & 99.1 & 103 & 0 \\
\hline
\end{tabular}

$\mathrm{CX}=$ COAXIAL

Note: EM values shown above

Ketchikan - North/South of 5515
* Estimated Depth may be unreliable because the stronger part of the conductor may be deeper or to one side of the flight line, or because of a shallow dip or magnetite/overburden effects 
EM Anomaly List

\begin{tabular}{|c|c|c|c|c|c|c|c|c|c|c|c|c|c|}
\hline Label & Fid & Interp & $\begin{array}{l}\text { XUTM } \\
\text { m }\end{array}$ & $\begin{array}{c}\text { YUTM } \\
\mathrm{m}\end{array}$ & $\begin{array}{l}\text { CX } \\
\text { Real } \\
\text { ppm }\end{array}$ & $\begin{array}{l}00 \mathrm{HZ} \\
\text { Quad } \\
\text { ppm }\end{array}$ & $\begin{array}{l}\text { CP } \\
\text { Real } \\
\text { ppm }\end{array}$ & $\begin{array}{c}900 \mathrm{HZ} \\
\text { Quad } \\
\text { ppm }\end{array}$ & $\begin{array}{l}\text { CP } 7 \\
\text { Real } \\
\text { ppm }\end{array}$ & $\begin{array}{l}7200 \mathrm{HZ} \\
\text { Quad } \\
\text { ppm }\end{array}$ & $\begin{array}{l}\text { Vertica } \\
\text { COND } \\
\text { siemens }\end{array}$ & $\begin{array}{c}\text { al Dike } \\
\text { DEPTH* } \\
\text { m }\end{array}$ & $\begin{array}{c}\text { Mag. Corr } \\
\text { NT }\end{array}$ \\
\hline $\begin{array}{l}\text { LINE } \\
\mathrm{A}\end{array}$ & $\begin{array}{c}10924 \\
5136.5\end{array}$ & S? & 660263 & 6131465 & 2.4 & 0.8 & 0.1 & 0.7 & 0.7 & 6.3 & 13.9 & 59 & 47 \\
\hline $\begin{array}{l}\text { LINE } \\
\text { A } \\
\text { B } \\
\text { C } \\
\text { D } \\
\text { E } \\
\text { F } \\
\text { G } \\
\text { H } \\
\text { I } \\
\text { J } \\
\text { K } \\
\text { L } \\
\text { M } \\
\text { N } \\
\text { O } \\
\text { P }\end{array}$ & $\begin{array}{r}10930 \\
6328.7 \\
6348.2 \\
6866.8 \\
6871.2 \\
6896.4 \\
6940.4 \\
7007.9 \\
7076.0 \\
7093.9 \\
7107.0 \\
7206.5 \\
7216.0 \\
7227.5 \\
7421.9 \\
7671.8 \\
7676.4\end{array}$ & $\begin{array}{r}\text { S } \\
\text { B? } \\
\text { B? } \\
\text { B? } \\
\text { B? } \\
\text { S? } \\
\text { B? } \\
\text { M } \\
\text { M } \\
\text { M } \\
\text { M } \\
\text { M } \\
\text { M } \\
\text { M } \\
\text { B? } \\
\text { B? }\end{array}$ & $\begin{array}{l}661406 \\
661362 \\
660966 \\
660962 \\
660954 \\
660874 \\
660865 \\
660790 \\
660801 \\
660815 \\
660787 \\
660775 \\
660762 \\
660693 \\
660320 \\
660318\end{array}$ & $\begin{array}{l}6110668 \\
6111161 \\
6123358 \\
6123429 \\
6123816 \\
6124457 \\
6125564 \\
6126979 \\
6127094 \\
6127180 \\
6128076 \\
6128148 \\
6128210 \\
6131017 \\
6139929 \\
6140092\end{array}$ & $\begin{array}{r}0.2 \\
2.3 \\
16.5 \\
8.6 \\
23.0 \\
4.8 \\
20.2 \\
0.4 \\
3.3 \\
0.1 \\
0.2 \\
8.9 \\
2.2 \\
0.0 \\
38.5 \\
45.3\end{array}$ & $\begin{array}{r}3.0 \\
3.5 \\
20.0 \\
8.7 \\
56.7 \\
4.0 \\
29.5 \\
0.8 \\
0.3 \\
1.7 \\
0.5 \\
0.1 \\
1.0 \\
0.7 \\
31.6 \\
23.2\end{array}$ & $\begin{array}{r}1.0 \\
0.8 \\
20.6 \\
9.7 \\
25.8 \\
2.1 \\
51.0 \\
6.8 \\
6.2 \\
17.2 \\
0.4 \\
28.4 \\
1.6 \\
4.9 \\
41.8 \\
85.4\end{array}$ & $\begin{array}{r}2.1 \\
4.8 \\
30.3 \\
13.9 \\
95.1 \\
6.6 \\
76.7 \\
0.9 \\
0.3 \\
0.6 \\
0.4 \\
0.7 \\
0.1 \\
0.8 \\
43.8 \\
71.9\end{array}$ & $\begin{array}{r}1.1 \\
10.0 \\
75.0 \\
33.3 \\
249.8 \\
13.7 \\
152.6 \\
0.1 \\
5.8 \\
13.6 \\
0.3 \\
23.4 \\
2.2 \\
3.6 \\
98.6 \\
130.0\end{array}$ & $\begin{array}{r}15.0 \\
34.6 \\
52.8 \\
19.5 \\
225.5 \\
10.2 \\
62.5 \\
6.4 \\
3.4 \\
1.9 \\
2.0 \\
1.7 \\
2.3 \\
6.4 \\
34.9 \\
24.0\end{array}$ & $\begin{array}{c}3.4 \\
2.4 \\
7.6 \\
7.2 \\
4.6 \\
--- \\
7.6 \\
---- \\
--- \\
--- \\
--- \\
--- \\
--- \\
--- \\
17.2 \\
30.0\end{array}$ & $\begin{array}{r}43 \\
38 \\
5 \\
28 \\
0 \\
--- \\
0 \\
--- \\
--- \\
--- \\
--- \\
--- \\
--- \\
--- \\
0 \\
0\end{array}$ & $\begin{array}{r}28 \\
0 \\
0 \\
0 \\
0 \\
0 \\
28 \\
12 \\
0 \\
107 \\
0 \\
0 \\
12 \\
340 \\
28 \\
0\end{array}$ \\
\hline 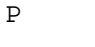 & 7676.4 & $\mathrm{~B} ?$ & 660318 & 6140092 & 45.3 & 23.2 & 85.4 & 71.9 & 130.0 & 24.0 & 30.0 & & 0 \\
\hline $\begin{array}{l}\text { LINE } \\
\text { A } \\
\text { B } \\
\text { C } \\
\text { D } \\
\text { E } \\
\text { F } \\
\text { G } \\
\text { H } \\
\text { I } \\
\text { J } \\
\text { K } \\
\text { L } \\
\text { M } \\
\text { N } \\
\text { O } \\
\text { P } \\
\text { Q }\end{array}$ & $\begin{array}{r}10940 \\
6116.1 \\
5973.5 \\
5658.7 \\
5648.2 \\
5452.1 \\
5421.4 \\
5301.8 \\
5156.8 \\
5130.7 \\
5117.9 \\
5107.9 \\
5096.0 \\
5086.0 \\
4976.7 \\
4957.2 \\
4947.8 \\
4929.1\end{array}$ & $\begin{array}{r}M \\
S ? \\
S ? \\
M \\
B \\
B ? \\
B ? \\
M \\
M \\
M \\
M \\
M \\
M \\
S ? \\
M \\
M \\
S ?\end{array}$ & $\begin{array}{l}661864 \\
661768 \\
661428 \\
661430 \\
661377 \\
661293 \\
661309 \\
661195 \\
661160 \\
661153 \\
661152 \\
661185 \\
661197 \\
661093 \\
661084 \\
661077 \\
661076\end{array}$ & $\begin{array}{l}6108837 \\
6111566 \\
6119703 \\
6120081 \\
6123160 \\
6124156 \\
6125466 \\
6127515 \\
6127873 \\
6128064 \\
6128224 \\
6128369 \\
6128497 \\
6130764 \\
6131422 \\
6131694 \\
6132344\end{array}$ & $\begin{array}{l}0.0 \\
0.3 \\
1.1 \\
2.4 \\
5.7 \\
3.1 \\
4.1 \\
1.1 \\
0.0 \\
0.7 \\
0.0 \\
0.6 \\
1.9 \\
1.7 \\
1.1 \\
1.1 \\
0.2\end{array}$ & $\begin{array}{l}0.3 \\
1.0 \\
0.3 \\
0.8 \\
4.4 \\
2.0 \\
2.5 \\
0.9 \\
0.4 \\
0.6 \\
0.9 \\
0.8 \\
0.0 \\
1.2 \\
0.6 \\
0.6 \\
3.1\end{array}$ & $\begin{array}{r}0.4 \\
0.1 \\
0.6 \\
0.8 \\
9.8 \\
1.2 \\
6.8 \\
0.8 \\
2.0 \\
5.2 \\
13.1 \\
5.9 \\
1.3 \\
0.6 \\
1.0 \\
0.3 \\
1.2\end{array}$ & $\begin{array}{l}0.5 \\
2.0 \\
0.9 \\
1.0 \\
9.8 \\
4.2 \\
7.4 \\
0.4 \\
1.3 \\
0.1 \\
0.1 \\
0.3 \\
0.3 \\
1.6 \\
0.2 \\
0.1 \\
6.1\end{array}$ & $\begin{array}{r}0.5 \\
2.0 \\
0.0 \\
0.3 \\
17.8 \\
10.3 \\
15.1 \\
0.7 \\
1.7 \\
4.4 \\
9.7 \\
4.4 \\
0.9 \\
0.5 \\
1.4 \\
0.2 \\
18.7\end{array}$ & $\begin{array}{r}2.6 \\
4.6 \\
4.7 \\
4.7 \\
5.2 \\
7.0 \\
7.0 \\
1.1 \\
3.8 \\
2.7 \\
4.1 \\
4.3 \\
3.3 \\
11.1 \\
2.1 \\
3.6 \\
29.1\end{array}$ & $\begin{array}{r}--- \\
--- \\
9.9 \\
--- \\
10.1 \\
5.0 \\
--- \\
--- \\
--- \\
--- \\
--- \\
--- \\
--- \\
4.6 \\
--- \\
--- \\
---\end{array}$ & $\begin{array}{r}--- \\
--- \\
77 \\
--- \\
24 \\
33 \\
--- \\
--- \\
--- \\
--- \\
--- \\
--- \\
--- \\
68 \\
--- \\
--- \\
---\end{array}$ & $\begin{array}{r}0 \\
0 \\
2 \\
0 \\
0 \\
0 \\
0 \\
311 \\
0 \\
0 \\
0 \\
0 \\
0 \\
0 \\
116 \\
0 \\
0\end{array}$ \\
\hline $\begin{array}{l}\text { LINE } \\
\text { A } \\
B \\
\text { C } \\
\text { D } \\
\text { E }\end{array}$ & $\begin{array}{r}10950 \\
2543.7 \\
2621.2 \\
2634.3 \\
2657.3 \\
2734.1\end{array}$ & $\begin{array}{r}\text { S? } \\
\text { S? } \\
\text { B? } \\
\text { M } \\
\text { S? }\end{array}$ & $\begin{array}{l}662250 \\
662155 \\
662152 \\
662146 \\
662107\end{array}$ & $\begin{array}{l}6109779 \\
6111357 \\
6111619 \\
6111949 \\
6113572\end{array}$ & $\begin{array}{r}0.4 \\
0.7 \\
1.2 \\
0.0 \\
89.6\end{array}$ & $\begin{array}{r}1.2 \\
1.1 \\
2.6 \\
0.8 \\
61.3\end{array}$ & $\begin{array}{r}1.0 \\
1.0 \\
1.4 \\
0.8 \\
72.4\end{array}$ & $\begin{array}{r}1.4 \\
0.7 \\
2.1 \\
0.1 \\
110.7\end{array}$ & $\begin{array}{r}1.0 \\
1.2 \\
1.1 \\
1.2 \\
230.3\end{array}$ & $\begin{array}{r}8.7 \\
5.7 \\
11.6 \\
2.3 \\
82.2\end{array}$ & $\begin{array}{r}--- \\
3.1 \\
1.9 \\
--- \\
21.2\end{array}$ & $\begin{array}{r}--- \\
90 \\
80 \\
--- \\
0\end{array}$ & $\begin{array}{r}25 \\
0 \\
25 \\
126 \\
68\end{array}$ \\
\hline
\end{tabular}

$\mathrm{CX}=$ COAXIAL

Note: EM values shown above

Ketchikan - North/South of 5515
* Estimated Depth may be unreliable because the stronger part of the conductor may be deeper or to one side of the flight line, or because of a shallow dip or magnetite/overburden effects 
EM Anomaly List

\begin{tabular}{|c|c|c|c|c|c|c|c|c|c|c|c|c|c|}
\hline Label & Fid & Interp & $\begin{array}{c}\text { XUTM } \\
\text { m }\end{array}$ & $\begin{array}{c}\text { YUTM } \\
\mathrm{m}\end{array}$ & $\begin{array}{l}\text { CX } \\
\text { Real } \\
\text { ppm }\end{array}$ & $\begin{array}{l}900 \mathrm{HZ} \\
\text { Quad } \\
\text { ppm }\end{array}$ & $\begin{array}{l}\text { CP } \\
\text { Real } \\
\text { ppm }\end{array}$ & $\begin{array}{l}900 \mathrm{HZ} \\
\text { Quad } \\
\text { ppm }\end{array}$ & $\begin{array}{l}\text { CP } 7 \\
\text { Real } \\
\text { ppm }\end{array}$ & $\begin{array}{c}7200 \mathrm{HZ} \\
\text { Quad } \\
\text { ppm }\end{array}$ & $\begin{array}{l}\text { Vertica } \\
\text { ConD } \\
\text { siemens }\end{array}$ & $\begin{array}{c}\text { al Dike } \\
\text { DEPTH* } \\
\text { m }\end{array}$ & $\begin{array}{c}\text { Mag. Corr } \\
\text { NT }\end{array}$ \\
\hline $\begin{array}{l}\text { LINE } \\
\text { F } \\
\text { G } \\
\text { H }\end{array}$ & $\begin{array}{r}10950 \\
2741.2 \\
2769.6 \\
2796.8\end{array}$ & $\begin{array}{r}S ? \\
S \\
S\end{array}$ & $\begin{array}{l}662073 \\
662025 \\
661990\end{array}$ & $\begin{array}{l}6113809 \\
6114839 \\
6115880\end{array}$ & $\begin{array}{r}40.8 \\
0.4 \\
3.9\end{array}$ & $\begin{array}{r}39.0 \\
6.5 \\
1.6\end{array}$ & $\begin{array}{r}36.0 \\
1.4 \\
0.5\end{array}$ & $\begin{array}{r}62.6 \\
11.1 \\
2.3\end{array}$ & $\begin{array}{r}114.2 \\
33.8 \\
2.4\end{array}$ & $\begin{array}{r}136.7 \\
55.5 \\
14.5\end{array}$ & $\begin{array}{r}11.4 \\
0.5 \\
11.4\end{array}$ & $\begin{array}{r}0 \\
0 \\
37\end{array}$ & $\begin{array}{l}25 \\
20 \\
25\end{array}$ \\
\hline $\begin{array}{l}\text { LINE } \\
\text { A } \\
B\end{array}$ & $\begin{array}{l}10951 \\
3226.7 \\
3179.8\end{array}$ & $\begin{array}{l}\mathrm{S} \\
\mathrm{B}\end{array}$ & $\begin{array}{l}661805 \\
661762\end{array}$ & $\begin{array}{l}6121980 \\
6123052\end{array}$ & $\begin{array}{l}2.3 \\
2.4\end{array}$ & $\begin{array}{l}0.8 \\
9.3\end{array}$ & $\begin{array}{l}0.0 \\
3.1\end{array}$ & $\begin{array}{r}0.4 \\
15.6\end{array}$ & $\begin{array}{r}0.1 \\
46.5\end{array}$ & $\begin{array}{r}7.0 \\
47.8\end{array}$ & $\begin{array}{r}16.7 \\
1.6\end{array}$ & $\begin{array}{r}66 \\
5\end{array}$ & $\begin{array}{r}37 \\
0\end{array}$ \\
\hline $\begin{array}{l}\text { LINE } \\
\text { A } \\
\text { B } \\
\text { C } \\
\text { D } \\
\text { E } \\
\text { F } \\
\text { G } \\
\text { H } \\
\text { I } \\
\text { J } \\
\text { K } \\
\text { L } \\
\text { M } \\
\text { N } \\
\text { O } \\
\text { P } \\
\text { Q } \\
\text { R }\end{array}$ & $\begin{array}{r}10952 \\
3558.3 \\
3574.4 \\
3606.2 \\
3619.1 \\
3698.8 \\
3745.6 \\
3791.2 \\
3798.4 \\
3809.7 \\
3855.0 \\
3873.7 \\
3976.2 \\
4035.3 \\
4040.1 \\
4107.9 \\
4140.7 \\
4163.2 \\
4296.9\end{array}$ & $\begin{array}{r}\text { B } \\
\text { B? } \\
\text { B? } \\
\text { B? } \\
\text { S? } \\
\text { S } \\
\text { M } \\
\text { M } \\
\text { M } \\
\text { M } \\
\text { M } \\
\text { M } \\
\text { M } \\
\text { M } \\
\text { S } \\
\text { M } \\
\text { M } \\
\text { S? }\end{array}$ & $\begin{array}{l}661745 \\
661740 \\
661672 \\
661667 \\
661711 \\
661693 \\
661642 \\
661633 \\
661631 \\
661617 \\
661612 \\
661517 \\
661447 \\
661452 \\
661387 \\
661372 \\
661313 \\
661210\end{array}$ & $\begin{array}{l}6123002 \\
6123224 \\
6123731 \\
6123984 \\
6124974 \\
6125773 \\
6126917 \\
6126999 \\
6127069 \\
6127396 \\
6127472 \\
6130017 \\
6131488 \\
6131615 \\
6133796 \\
6134479 \\
6135072 \\
6138538\end{array}$ & $\begin{array}{r}6.8 \\
3.3 \\
3.8 \\
4.9 \\
55.1 \\
2.2 \\
0.5 \\
5.0 \\
1.5 \\
0.0 \\
0.8 \\
0.4 \\
3.3 \\
1.4 \\
1.2 \\
1.1 \\
0.4 \\
2.1\end{array}$ & $\begin{array}{r}5.9 \\
3.6 \\
6.2 \\
9.0 \\
50.0 \\
1.8 \\
0.4 \\
0.4 \\
1.1 \\
0.6 \\
0.3 \\
0.4 \\
0.1 \\
0.8 \\
0.9 \\
1.0 \\
0.6 \\
3.1\end{array}$ & $\begin{array}{r}3.3 \\
0.6 \\
3.0 \\
0.4 \\
110.7 \\
0.3 \\
9.5 \\
13.5 \\
5.0 \\
3.5 \\
2.5 \\
4.9 \\
2.2 \\
2.7 \\
1.2 \\
3.1 \\
0.8 \\
2.9\end{array}$ & $\begin{array}{r}11.2 \\
2.4 \\
12.0 \\
10.8 \\
92.5 \\
0.9 \\
0.7 \\
0.9 \\
0.1 \\
0.3 \\
0.2 \\
0.2 \\
0.1 \\
0.2 \\
1.0 \\
0.7 \\
0.9 \\
4.4\end{array}$ & $\begin{array}{r}33.1 \\
3.9 \\
29.2 \\
19.9 \\
170.6 \\
0.8 \\
8.9 \\
11.0 \\
4.5 \\
3.3 \\
1.9 \\
4.5 \\
1.7 \\
2.1 \\
1.1 \\
10.1 \\
0.2 \\
3.6\end{array}$ & $\begin{array}{r}28.1 \\
9.8 \\
26.9 \\
62.0 \\
60.3 \\
2.5 \\
1.0 \\
0.9 \\
0.9 \\
3.1 \\
2.6 \\
2.2 \\
0.1 \\
1.8 \\
6.8 \\
4.0 \\
3.9 \\
28.7\end{array}$ & $\begin{array}{r}4.9 \\
--- \\
2.9 \\
2.5 \\
22.2 \\
5.2 \\
--- \\
--- \\
--- \\
--- \\
--- \\
--- \\
--- \\
--- \\
6.4 \\
--- \\
--- \\
---\end{array}$ & $\begin{array}{r}20 \\
--- \\
11 \\
16 \\
0 \\
31 \\
---- \\
--- \\
--- \\
--- \\
--- \\
--- \\
--- \\
--- \\
63 \\
--- \\
--- \\
---\end{array}$ & $\begin{array}{r}0 \\
0 \\
0 \\
0 \\
30 \\
30 \\
184 \\
30 \\
0 \\
30 \\
0 \\
305 \\
364 \\
0 \\
30 \\
532 \\
191 \\
228\end{array}$ \\
\hline $\begin{array}{l}\text { LINE } \\
\text { A } \\
\text { B } \\
\text { C } \\
\text { D } \\
\text { E } \\
\text { F } \\
\text { G } \\
\text { H } \\
\text { I } \\
\text { J } \\
\text { K } \\
\text { L } \\
\text { M } \\
\text { N } \\
\text { O } \\
\text { P } \\
\text { Q }\end{array}$ & $\begin{array}{l}10960 \\
1312.6 \\
1301.7 \\
1263.9 \\
1260.5 \\
1240.7 \\
1225.9 \\
1214.0 \\
1185.4 \\
948.1 \\
929.2 \\
844.3 \\
703.9 \\
471.4 \\
457.1 \\
451.3 \\
446.3 \\
442.7\end{array}$ & $\begin{array}{r}\text { B } \\
\mathrm{B} \\
\mathrm{B} \\
\mathrm{B} \\
\mathrm{B} ? \\
\mathrm{~B} \\
\mathrm{~S} ? \\
\mathrm{~B} \\
\mathrm{M} \\
\mathrm{M} \\
\mathrm{M} \\
\mathrm{M} \\
\mathrm{B} \\
\mathrm{B} \\
\mathrm{D} \\
\mathrm{B} ? \\
\mathrm{~B}\end{array}$ & $\begin{array}{l}662213 \\
662203 \\
662140 \\
662135 \\
662153 \\
662166 \\
662126 \\
662062 \\
661973 \\
661991 \\
661871 \\
661738 \\
661507 \\
661461 \\
661449 \\
661450 \\
661446\end{array}$ & $\begin{array}{l}6121882 \\
6122214 \\
6123167 \\
6123274 \\
6123755 \\
6123924 \\
6124251 \\
6125031 \\
6127501 \\
6128015 \\
6130647 \\
6134306 \\
6141739 \\
6142104 \\
6142281 \\
6142471 \\
6142620\end{array}$ & $\begin{array}{r}7.2 \\
6.4 \\
7.4 \\
5.4 \\
2.3 \\
10.0 \\
4.4 \\
6.8 \\
0.2 \\
0.0 \\
0.5 \\
1.5 \\
5.2 \\
5.4 \\
1.1 \\
10.2 \\
10.1\end{array}$ & $\begin{array}{r}6.4 \\
8.4 \\
9.9 \\
9.3 \\
4.8 \\
14.2 \\
2.3 \\
14.0 \\
0.3 \\
0.6 \\
0.7 \\
1.2 \\
2.1 \\
9.7 \\
3.6 \\
8.1 \\
8.2\end{array}$ & $\begin{array}{r}3.1 \\
5.3 \\
13.0 \\
11.1 \\
1.9 \\
4.3 \\
0.6 \\
8.4 \\
0.3 \\
7.6 \\
0.3 \\
3.0 \\
0.7 \\
3.3 \\
1.0 \\
12.3 \\
7.7\end{array}$ & $\begin{array}{r}10.0 \\
13.5 \\
24.8 \\
22.2 \\
8.4 \\
24.0 \\
1.6 \\
34.5 \\
0.1 \\
0.9 \\
0.6 \\
1.0 \\
5.1 \\
11.3 \\
0.9 \\
22.7 \\
11.7\end{array}$ & $\begin{array}{r}26.3 \\
28.8 \\
51.8 \\
47.9 \\
20.2 \\
67.7 \\
1.1 \\
92.9 \\
0.0 \\
5.8 \\
0.2 \\
1.9 \\
10.1 \\
29.8 \\
2.3 \\
43.0 \\
23.5\end{array}$ & $\begin{array}{r}24.8 \\
23.0 \\
27.9 \\
28.0 \\
41.4 \\
59.5 \\
7.1 \\
69.0 \\
5.7 \\
6.1 \\
4.2 \\
8.5 \\
9.3 \\
28.1 \\
0.5 \\
23.6 \\
12.2\end{array}$ & $\begin{array}{r}5.4 \\
3.7 \\
4.5 \\
3.3 \\
2.1 \\
4.0 \\
11.6 \\
3.1 \\
--- \\
--- \\
--- \\
--- \\
--- \\
3.4 \\
1.9 \\
7.4 \\
9.0\end{array}$ & $\begin{array}{r}28 \\
17 \\
20 \\
25 \\
18 \\
0 \\
24 \\
0 \\
--- \\
--- \\
--- \\
--- \\
--- \\
14 \\
26 \\
1 \\
0\end{array}$ & $\begin{array}{r}0 \\
34 \\
0 \\
0 \\
0 \\
34 \\
34 \\
0 \\
0 \\
0 \\
166 \\
188 \\
34 \\
0 \\
34 \\
34 \\
0\end{array}$ \\
\hline
\end{tabular}

$\mathrm{CX}=$ COAXIAL

Note: EM values shown above

are local amplitudes

Ketchikan - North/South of 5515
* Estimated Depth may be unreliable because the stronger part of the conductor may be deeper or to one side of the flight line, or because of a shallow dip or magnetite/overburden effects 
EM Anomaly List

\begin{tabular}{|c|c|c|c|c|c|c|c|c|c|c|c|c|c|}
\hline Label & Fid & Interp & $\begin{array}{c}\text { XUTM } \\
\text { m }\end{array}$ & $\begin{array}{c}\text { YUTM } \\
\text { m }\end{array}$ & $\begin{array}{l}\text { CX } \\
\text { Real } \\
\text { ppm }\end{array}$ & $\begin{array}{l}00 \mathrm{HZ} \\
\text { Quad } \\
\text { ppm }\end{array}$ & $\begin{array}{l}\text { CP } \\
\text { Real } \\
\text { ppm }\end{array}$ & $\begin{array}{l}900 \mathrm{HZ} \\
\text { Quad } \\
\text { ppm }\end{array}$ & $\begin{array}{l}\text { CP } 7 \\
\text { Real } \\
\text { ppm }\end{array}$ & $\begin{array}{l}200 \mathrm{HZ} \\
\text { Quad } \\
\text { ppm }\end{array}$ & $\begin{array}{l}\text { Vertica } \\
\text { COND } \\
\text { siemens }\end{array}$ & $\begin{array}{c}\text { al Dike } \\
\text { DEPTH* } \\
\text { m }\end{array}$ & $\begin{array}{c}\text { Mag. Corr } \\
\text { NT }\end{array}$ \\
\hline $\begin{array}{l}\mathrm{LINE} \\
\mathrm{R}\end{array}$ & $\begin{array}{c}10960 \\
423.4\end{array}$ & $B$ ? & 661429 & 6143365 & 0.1 & 2.1 & 0.3 & 2.8 & 9.6 & 22.8 & 0.5 & 0 & 0 \\
\hline $\begin{array}{l}\text { LINE } \\
\text { A } \\
\text { B } \\
\text { C } \\
\text { D } \\
\text { E } \\
\text { F } \\
\text { G } \\
\text { H }\end{array}$ & $\begin{array}{r}10962 \\
2366.0 \\
2365.0 \\
2314.8 \\
2269.0 \\
2263.5 \\
2244.9 \\
2196.6 \\
2064.4\end{array}$ & $\begin{array}{l}\mathrm{M} \\
\mathrm{M} \\
\mathrm{M} \\
\mathrm{M} \\
\mathrm{M} \\
\mathrm{M} \\
\mathrm{S} \\
\mathrm{S}\end{array}$ & $\begin{array}{l}662643 \\
662642 \\
662643 \\
662599 \\
662586 \\
662551 \\
662616 \\
662451\end{array}$ & $\begin{array}{l}6109302 \\
6109318 \\
6109496 \\
6109895 \\
6110094 \\
6110668 \\
6111399 \\
6114566\end{array}$ & $\begin{array}{l}9.2 \\
9.0 \\
9.3 \\
1.3 \\
0.5 \\
0.4 \\
0.6 \\
2.1\end{array}$ & $\begin{array}{l}0.8 \\
0.8 \\
0.8 \\
0.2 \\
0.9 \\
0.8 \\
4.0 \\
2.7\end{array}$ & $\begin{array}{r}7.8 \\
2.4 \\
48.3 \\
5.9 \\
1.2 \\
0.0 \\
0.3 \\
1.2\end{array}$ & $\begin{array}{l}0.3 \\
0.2 \\
0.1 \\
0.3 \\
0.6 \\
0.5 \\
6.1 \\
6.0\end{array}$ & $\begin{array}{r}6.8 \\
2.2 \\
40.5 \\
5.6 \\
0.7 \\
1.7 \\
5.6 \\
19.3\end{array}$ & $\begin{array}{r}1.2 \\
1.2 \\
3.5 \\
0.1 \\
2.6 \\
4.7 \\
40.3 \\
24.7\end{array}$ & $\begin{array}{l}--- \\
--- \\
--- \\
--- \\
--- \\
--- \\
0.6 \\
---\end{array}$ & $\begin{array}{l}--- \\
--- \\
--- \\
--- \\
--- \\
--- \\
0 \\
---\end{array}$ & $\begin{array}{r}341 \\
0 \\
37 \\
354 \\
0 \\
95 \\
0 \\
0\end{array}$ \\
\hline $\begin{array}{l}\text { LINE } \\
\text { A } \\
\text { B } \\
\text { C } \\
\text { D } \\
\text { E } \\
\text { F } \\
\text { G } \\
\text { H } \\
\text { I } \\
\text { J } \\
\text { K } \\
\text { L } \\
\text { M } \\
\text { N } \\
\text { O }\end{array}$ & $\begin{array}{r}10970 \\
2469.4 \\
2538.3 \\
3114.1 \\
3166.9 \\
3345.8 \\
3500.0 \\
3707.7 \\
3731.8 \\
3845.6 \\
3866.6 \\
3964.7 \\
3980.6 \\
4004.6 \\
4028.9 \\
4037.3\end{array}$ & $\begin{array}{r}\text { M } \\
\text { M } \\
\text { B } \\
\text { B? } \\
\text { S? } \\
\text { M } \\
\text { D } \\
\text { D } \\
\text { S? } \\
\text { S? } \\
\text { S? } \\
\text { S? } \\
\text { B } \\
\text { D } \\
\text { B }\end{array}$ & $\begin{array}{l}663048 \\
663052 \\
662493 \\
662514 \\
662432 \\
662315 \\
662113 \\
662132 \\
662060 \\
661991 \\
661873 \\
661927 \\
661856 \\
661843 \\
661851\end{array}$ & $\begin{array}{l}6109463 \\
6110260 \\
6122174 \\
6123501 \\
6127152 \\
6129794 \\
6134184 \\
6134766 \\
6137423 \\
6137971 \\
6140673 \\
6141198 \\
6141961 \\
6142786 \\
6143067\end{array}$ & $\begin{array}{r}0.0 \\
0.4 \\
6.1 \\
7.1 \\
1.4 \\
1.1 \\
5.6 \\
19.6 \\
0.8 \\
0.6 \\
0.6 \\
0.1 \\
0.2 \\
4.0 \\
1.4\end{array}$ & $\begin{array}{r}0.4 \\
0.3 \\
5.0 \\
13.1 \\
0.6 \\
0.4 \\
5.1 \\
27.6 \\
3.8 \\
3.6 \\
1.8 \\
1.4 \\
6.6 \\
7.2 \\
4.2\end{array}$ & $\begin{array}{l}0.1 \\
6.2 \\
9.7 \\
8.2 \\
0.6 \\
1.6 \\
0.1 \\
4.9 \\
0.9 \\
0.5 \\
0.3 \\
0.0 \\
2.5 \\
1.5 \\
4.8\end{array}$ & $\begin{array}{r}0.2 \\
0.1 \\
10.0 \\
22.5 \\
0.8 \\
0.4 \\
0.1 \\
13.0 \\
2.1 \\
5.4 \\
1.1 \\
1.6 \\
14.5 \\
8.6 \\
13.5\end{array}$ & $\begin{array}{r}0.5 \\
4.8 \\
23.8 \\
49.4 \\
0.1 \\
1.2 \\
0.2 \\
31.4 \\
1.7 \\
7.5 \\
1.6 \\
0.8 \\
41.8 \\
21.2 \\
30.1\end{array}$ & $\begin{array}{r}0.5 \\
1.7 \\
12.4 \\
45.7 \\
7.6 \\
2.5 \\
3.7 \\
33.7 \\
14.9 \\
34.3 \\
5.7 \\
9.5 \\
36.2 \\
36.5 \\
30.9\end{array}$ & $\begin{array}{r}--- \\
--- \\
9.6 \\
3.9 \\
10.4 \\
--- \\
7.8 \\
5.6 \\
1.2 \\
0.6 \\
--- \\
--- \\
0.6 \\
2.7 \\
---\end{array}$ & $\begin{array}{r}--- \\
--- \\
11 \\
6 \\
81 \\
--- \\
24 \\
11 \\
8 \\
0 \\
--- \\
--- \\
0 \\
13 \\
---\end{array}$ & $\begin{array}{r}574 \\
526 \\
0 \\
0 \\
1 \\
227 \\
0 \\
0 \\
0 \\
33 \\
0 \\
0 \\
0 \\
0 \\
0\end{array}$ \\
\hline $\begin{array}{l}\text { LINE } \\
\text { A } \\
\text { B } \\
\text { C } \\
\text { D } \\
\text { E }\end{array}$ & $\begin{array}{r}10975 \\
250.3 \\
243.4 \\
222.8 \\
218.3 \\
161.5\end{array}$ & $\begin{array}{l}\text { B } \\
\text { B } \\
\text { B } \\
\text { B } \\
\text { B }\end{array}$ & $\begin{array}{l}662593 \\
662594 \\
662592 \\
662578 \\
662527\end{array}$ & $\begin{array}{l}6121411 \\
6121520 \\
6121934 \\
6122050 \\
6123441\end{array}$ & $\begin{array}{r}8.1 \\
6.0 \\
19.6 \\
23.4 \\
0.6\end{array}$ & $\begin{array}{r}8.7 \\
11.3 \\
14.9 \\
13.3 \\
5.7\end{array}$ & $\begin{array}{r}6.3 \\
12.8 \\
46.3 \\
25.8 \\
5.8\end{array}$ & $\begin{array}{l}16.2 \\
19.3 \\
36.3 \\
22.3 \\
11.6\end{array}$ & $\begin{array}{l}37.7 \\
39.0 \\
71.7 \\
43.6 \\
21.8\end{array}$ & $\begin{array}{l}27.7 \\
23.2 \\
24.7 \\
24.7 \\
13.6\end{array}$ & $\begin{array}{r}4.7 \\
4.0 \\
19.3 \\
22.2 \\
1.9\end{array}$ & $\begin{array}{l}4 \\
7 \\
0 \\
0 \\
0\end{array}$ & $\begin{array}{r}0 \\
0 \\
5 \\
10 \\
6\end{array}$ \\
\hline $\begin{array}{l}\text { LINE } \\
\text { A } \\
\text { B } \\
\text { C } \\
\text { D } \\
\text { E } \\
\text { F } \\
\text { G } \\
\text { H }\end{array}$ & $\begin{array}{c}10980 \\
978.8 \\
705.4 \\
666.6 \\
656.9 \\
652.1 \\
268.4 \\
237.2 \\
232.6\end{array}$ & $\begin{array}{l}\text { M } \\
\text { M } \\
\text { S? } \\
\text { S? } \\
\text { S? } \\
\text { B? } \\
\text { B? } \\
\text { B? }\end{array}$ & $\begin{array}{l}662687 \\
662585 \\
662518 \\
662553 \\
662580 \\
662255 \\
662227 \\
662221\end{array}$ & $\begin{array}{l}6130232 \\
6133648 \\
6134255 \\
6134460 \\
6134565 \\
6142834 \\
6143727 \\
6143882\end{array}$ & $\begin{array}{r}0.1 \\
2.7 \\
12.8 \\
0.2 \\
0.5 \\
4.1 \\
1.5 \\
2.0\end{array}$ & $\begin{array}{r}0.2 \\
0.8 \\
5.7 \\
2.5 \\
2.5 \\
11.0 \\
1.7 \\
2.8\end{array}$ & $\begin{array}{l}0.1 \\
1.2 \\
6.1 \\
1.6 \\
0.4 \\
1.9 \\
0.5 \\
0.8\end{array}$ & $\begin{array}{r}0.1 \\
0.7 \\
0.9 \\
3.3 \\
3.9 \\
16.6 \\
1.9 \\
6.3\end{array}$ & $\begin{array}{r}0.1 \\
1.3 \\
13.8 \\
4.1 \\
7.5 \\
41.1 \\
5.1 \\
19.9\end{array}$ & $\begin{array}{r}1.6 \\
2.0 \\
5.8 \\
23.6 \\
23.7 \\
58.2 \\
4.2 \\
21.4\end{array}$ & $\begin{array}{r}--- \\
--- \\
37.5 \\
--- \\
0.7 \\
1.9 \\
2.5 \\
2.1\end{array}$ & $\begin{array}{r}--- \\
--- \\
0 \\
--- \\
0 \\
0 \\
65 \\
16\end{array}$ & $\begin{array}{r}39 \\
431 \\
0 \\
0 \\
0 \\
0 \\
0 \\
0\end{array}$ \\
\hline
\end{tabular}

$\mathrm{CX}=$ COAXIAL

Note: EM values shown above

Ketchikan - North/South of 5515
*Estimated Depth may be unreliable because the stronger part of the conductor may be deeper or to one side of the flight line, or because of shallow dip or magnetite/overburden effects 
EM Anomaly List

\begin{tabular}{|c|c|c|c|c|c|c|c|c|c|c|c|c|c|}
\hline Label & Fid & Interp & $\begin{array}{c}\text { XUTM } \\
\text { m }\end{array}$ & $\begin{array}{c}\text { YUTM } \\
\mathrm{m}\end{array}$ & $\begin{array}{l}\text { CX } \\
\text { Real } \\
\text { ppm }\end{array}$ & $\begin{array}{c}900 \mathrm{HZ} \\
\text { Quad } \\
\text { ppm }\end{array}$ & $\begin{array}{l}\text { CP } \\
\text { Real } \\
\text { ppm }\end{array}$ & $\begin{array}{l}900 \mathrm{HZ} \\
\text { Quad } \\
\text { ppm }\end{array}$ & $\begin{array}{l}\text { CP } \\
\text { Real } \\
\text { ppm }\end{array}$ & $\begin{array}{l}200 \mathrm{HZ} \\
\text { Quad } \\
\text { ppm }\end{array}$ & $\begin{array}{l}\text { Vertica } \\
\text { COND } \\
\text { siemens }\end{array}$ & $\begin{array}{c}\text { al Dike } \\
\text { DEPTH* } \\
\text { m }\end{array}$ & $\begin{array}{c}\text { Mag. Corr } \\
\text { NT }\end{array}$ \\
\hline $\begin{array}{l}\text { LINE } \\
\text { A } \\
B \\
C\end{array}$ & $\begin{array}{r}10981 \\
2141.9 \\
2083.2 \\
2054.1\end{array}$ & $\begin{array}{r}\mathrm{M} \\
\mathrm{B} ? \\
\mathrm{~S}\end{array}$ & $\begin{array}{l}663396 \\
663361 \\
663349\end{array}$ & $\begin{array}{l}6110492 \\
6111768 \\
6112393\end{array}$ & $\begin{array}{l}2.0 \\
0.8 \\
1.2\end{array}$ & $\begin{array}{l}0.5 \\
2.4 \\
0.9\end{array}$ & $\begin{array}{l}1.3 \\
0.3 \\
2.3\end{array}$ & $\begin{array}{l}0.6 \\
2.8 \\
1.9\end{array}$ & $\begin{array}{l}0.8 \\
8.0 \\
1.5\end{array}$ & $\begin{array}{r}4.3 \\
16.1 \\
11.0\end{array}$ & $\begin{array}{l}--- \\
1.1 \\
7.4\end{array}$ & $\begin{array}{r}--- \\
12 \\
50\end{array}$ & $\begin{array}{r}0 \\
0 \\
22\end{array}$ \\
\hline $\begin{array}{l}\text { LINE } \\
\text { A } \\
\text { B } \\
\text { C } \\
\text { D }\end{array}$ & $\begin{array}{r}10982 \\
853.5 \\
869.8 \\
880.5 \\
922.3\end{array}$ & $\begin{array}{r}\mathrm{D} \\
\mathrm{B} \\
\mathrm{B} \\
\mathrm{S} ?\end{array}$ & $\begin{array}{l}662995 \\
662997 \\
663018 \\
662961\end{array}$ & $\begin{array}{l}6121028 \\
6121173 \\
6121401 \\
6122434\end{array}$ & $\begin{array}{l}4.9 \\
1.4 \\
9.2 \\
4.8\end{array}$ & $\begin{array}{r}6.5 \\
4.4 \\
16.9 \\
1.5\end{array}$ & $\begin{array}{l}1.6 \\
4.6 \\
1.6 \\
0.8\end{array}$ & $\begin{array}{r}6.5 \\
6.0 \\
14.9 \\
2.5\end{array}$ & $\begin{array}{r}19.9 \\
11.6 \\
36.7 \\
5.2\end{array}$ & $\begin{array}{l}16.9 \\
11.8 \\
64.6 \\
15.4\end{array}$ & $\begin{array}{r}3.0 \\
2.9 \\
3.4 \\
16.6\end{array}$ & $\begin{array}{r}28 \\
6 \\
0 \\
33\end{array}$ & $\begin{array}{r}0 \\
0 \\
21 \\
0\end{array}$ \\
\hline $\begin{array}{l}\text { LINE } \\
\text { A } \\
\text { B } \\
\text { C } \\
\text { D } \\
\text { E } \\
\text { F } \\
\text { G } \\
\text { H } \\
\text { I } \\
\text { J } \\
\text { K } \\
\text { L } \\
\text { M } \\
\text { N } \\
\text { O } \\
\text { P } \\
\text { Q } \\
\text { R } \\
\text { S } \\
\text { T } \\
\text { U } \\
\text { V }\end{array}$ & $\begin{array}{l}10990 \\
10558.8 \\
10520.7 \\
10486.2 \\
10277.0 \\
10263.5 \\
10249.2 \\
10225.1 \\
10195.3 \\
10178.6 \\
10133.5 \\
9754.9 \\
9678.7 \\
9540.0 \\
9503.0 \\
9418.6 \\
9380.0 \\
9330.0 \\
9238.4 \\
9214.6 \\
9200.8 \\
9174.9 \\
9160.7\end{array}$ & $\begin{array}{r}\mathrm{M} \\
\mathrm{D} \\
\mathrm{B} ? \\
\mathrm{M} \\
\mathrm{M} \\
\mathrm{M} \\
\mathrm{M} \\
\mathrm{M} \\
\mathrm{M} \\
\mathrm{B} \\
\mathrm{B} ? \\
\mathrm{M} \\
\mathrm{M} \\
\mathrm{M} \\
\mathrm{M} \\
\mathrm{S} \\
\mathrm{M} \\
\mathrm{B} ? \\
\mathrm{~B} \\
\mathrm{~B} ? \\
\mathrm{~B} ? \\
\mathrm{D}\end{array}$ & $\begin{array}{l}663813 \\
663759 \\
663734 \\
663575 \\
663523 \\
663531 \\
663518 \\
663457 \\
663461 \\
663393 \\
663159 \\
663105 \\
663007 \\
662930 \\
662926 \\
662888 \\
662850 \\
662764 \\
662686 \\
662700 \\
662626 \\
662644\end{array}$ & $\begin{array}{l}6110472 \\
6111740 \\
6112436 \\
6117621 \\
6117982 \\
6118432 \\
6119077 \\
6120050 \\
6120467 \\
6121554 \\
6129054 \\
6130619 \\
6132809 \\
6133539 \\
6135630 \\
6136482 \\
6137607 \\
6140652 \\
6141533 \\
6142009 \\
6142778 \\
6143282\end{array}$ & $\begin{array}{l}0.4 \\
2.1 \\
1.4 \\
1.2 \\
0.5 \\
0.0 \\
1.3 \\
0.5 \\
0.5 \\
1.7 \\
0.5 \\
0.0 \\
1.0 \\
0.0 \\
1.2 \\
1.0 \\
0.2 \\
8.2 \\
1.8 \\
2.6 \\
0.5 \\
2.3\end{array}$ & $\begin{array}{r}1.0 \\
3.3 \\
1.0 \\
0.3 \\
0.6 \\
0.5 \\
0.3 \\
0.4 \\
0.4 \\
2.5 \\
0.3 \\
0.9 \\
0.6 \\
0.4 \\
0.4 \\
4.1 \\
0.5 \\
20.6 \\
2.3 \\
2.6 \\
1.3 \\
5.7\end{array}$ & $\begin{array}{r}0.5 \\
2.0 \\
0.2 \\
2.6 \\
1.8 \\
1.4 \\
0.4 \\
0.9 \\
1.7 \\
1.2 \\
0.3 \\
0.1 \\
3.8 \\
0.2 \\
0.7 \\
1.3 \\
0.3 \\
11.1 \\
0.3 \\
1.3 \\
0.8 \\
0.3\end{array}$ & $\begin{array}{r}1.6 \\
1.3 \\
1.0 \\
0.2 \\
0.8 \\
0.9 \\
0.7 \\
0.6 \\
0.9 \\
3.8 \\
1.4 \\
0.4 \\
0.5 \\
0.6 \\
0.6 \\
6.5 \\
0.6 \\
33.7 \\
2.5 \\
6.3 \\
5.2 \\
7.6\end{array}$ & $\begin{array}{r}0.3 \\
3.4 \\
0.1 \\
1.9 \\
1.6 \\
1.1 \\
1.2 \\
0.7 \\
2.2 \\
8.3 \\
0.6 \\
0.2 \\
2.5 \\
0.1 \\
0.8 \\
8.2 \\
0.3 \\
77.6 \\
8.3 \\
15.4 \\
7.6 \\
29.0\end{array}$ & $\begin{array}{r}8.5 \\
4.9 \\
6.3 \\
0.9 \\
3.6 \\
3.5 \\
2.7 \\
3.0 \\
3.5 \\
9.0 \\
9.7 \\
0.6 \\
3.3 \\
4.7 \\
3.2 \\
40.6 \\
2.0 \\
79.6 \\
16.1 \\
14.7 \\
22.8 \\
25.9\end{array}$ & $\begin{array}{l}--- \\
4.0 \\
4.2 \\
--- \\
--- \\
--- \\
--- \\
--- \\
--- \\
--- \\
--- \\
--- \\
--- \\
--- \\
--- \\
--- \\
--- \\
3.5 \\
--- \\
--- \\
1.1 \\
1.5\end{array}$ & $\begin{array}{r}--- \\
58 \\
92 \\
--- \\
--- \\
--- \\
--- \\
--- \\
--- \\
--- \\
--- \\
--- \\
--- \\
--- \\
--- \\
--- \\
--- \\
0 \\
--- \\
--- \\
25 \\
12\end{array}$ & $\begin{array}{r}290 \\
41 \\
0 \\
39 \\
0 \\
225 \\
0 \\
251 \\
0 \\
38 \\
0 \\
210 \\
231 \\
0 \\
19 \\
0 \\
503 \\
0 \\
0 \\
0 \\
41 \\
111\end{array}$ \\
\hline $\begin{array}{l}\text { LINE } \\
\text { A } \\
\text { B } \\
\text { C } \\
\text { D } \\
\text { E } \\
\text { F } \\
\text { G } \\
\text { H } \\
\text { I } \\
\text { J }\end{array}$ & $\begin{array}{l}11000 \\
7574.7 \\
7585.8 \\
7638.9 \\
7911.9 \\
7928.1 \\
7942.2 \\
7970.1 \\
7982.8 \\
8012.3 \\
8027.3\end{array}$ & $\begin{array}{r}\text { B } \\
\text { B? } \\
\text { B } \\
\text { M } \\
\text { M } \\
\text { M } \\
\text { M } \\
\text { M } \\
\text { M } \\
\text { M }\end{array}$ & $\begin{array}{l}664191 \\
664173 \\
664166 \\
663925 \\
663933 \\
663934 \\
663955 \\
663953 \\
663907 \\
663860\end{array}$ & $\begin{array}{l}6111512 \\
6111795 \\
6112511 \\
6117770 \\
6117945 \\
6118053 \\
6118365 \\
6118591 \\
6119304 \\
6119681\end{array}$ & $\begin{array}{l}3.0 \\
2.0 \\
1.8 \\
0.2 \\
0.0 \\
0.0 \\
0.2 \\
0.1 \\
1.2 \\
0.5\end{array}$ & $\begin{array}{l}7.2 \\
6.6 \\
1.5 \\
0.7 \\
0.8 \\
0.8 \\
0.2 \\
0.8 \\
0.4 \\
0.7\end{array}$ & $\begin{array}{r}3.6 \\
5.1 \\
0.8 \\
4.0 \\
9.3 \\
24.6 \\
26.2 \\
0.5 \\
2.2 \\
3.9\end{array}$ & $\begin{array}{r}9.9 \\
19.0 \\
1.6 \\
1.3 \\
1.2 \\
0.8 \\
0.5 \\
1.2 \\
0.6 \\
0.5\end{array}$ & $\begin{array}{r}29.3 \\
54.8 \\
2.4 \\
4.5 \\
7.7 \\
21.8 \\
21.0 \\
0.7 \\
1.6 \\
0.2\end{array}$ & $\begin{array}{r}25.4 \\
32.4 \\
9.4 \\
5.4 \\
6.4 \\
3.9 \\
2.9 \\
6.1 \\
2.7 \\
2.1\end{array}$ & $\begin{array}{l}2.6 \\
2.0 \\
4.6 \\
--- \\
--- \\
--- \\
--- \\
--- \\
--- \\
---\end{array}$ & $\begin{array}{r}20 \\
10 \\
61 \\
--- \\
--- \\
--- \\
--- \\
--- \\
--- \\
---\end{array}$ & $\begin{array}{r}35 \\
0 \\
0 \\
79 \\
0 \\
71 \\
0 \\
35 \\
0 \\
222\end{array}$ \\
\hline
\end{tabular}

$\mathrm{CX}=$ COAXIAL

Note: EM values shown above

Ketchikan - North/South of 5515
*Estimated Depth may be unreliable because the stronger part of the conductor may be deeper or to one side of the flight line, or because of 
EM Anomaly List

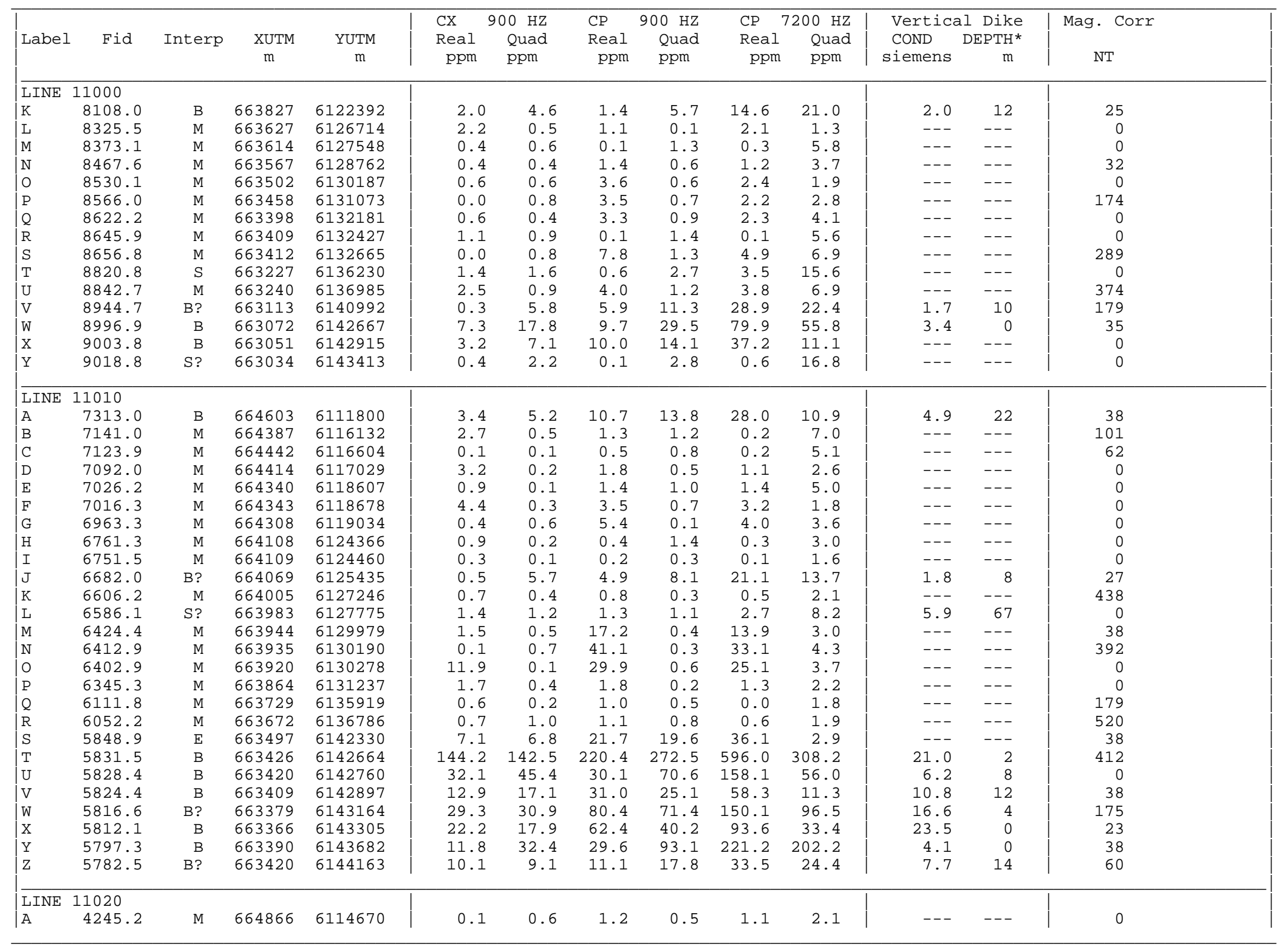

$\mathrm{CX}=$ COAXIAL

Note: EM values shown above

are local amplitudes

are loca
5515
*Estimated Depth may be unreliable because the stronger part of the conductor may be deeper or to one side of the flight line, or because of a shallow dip or magnetite/overburden effects 
EM Anomaly List

\begin{tabular}{|c|c|c|c|c|c|c|c|c|c|c|c|c|c|}
\hline Label & Fid & Interp & $\begin{array}{l}\text { XUTM } \\
\text { m }\end{array}$ & $\begin{array}{c}\text { YUTM } \\
\mathrm{m}\end{array}$ & $\begin{array}{l}\text { CX } \\
\text { Real } \\
\text { ppm }\end{array}$ & $\begin{array}{l}900 \mathrm{HZ} \\
\text { Quad } \\
\text { ppm }\end{array}$ & $\begin{array}{l}\text { CP } \\
\text { Real } \\
\text { ppm }\end{array}$ & $\begin{array}{c}900 \mathrm{HZ} \\
\text { Quad } \\
\text { ppm }\end{array}$ & $\begin{array}{l}\text { CP } \\
\text { Real } \\
\text { ppm }\end{array}$ & $\begin{array}{c}7200 \mathrm{~Hz} \\
\text { Quad } \\
\text { ppm }\end{array}$ & $\begin{array}{l}\text { Vertica } \\
\text { COND } \\
\text { siemens }\end{array}$ & $\begin{array}{c}\text { al Dike } \\
\text { DEPTH* } \\
\text { m }\end{array}$ & $\begin{array}{c}\text { Mag. Corr } \\
\text { NT }\end{array}$ \\
\hline LINE & 11020 & & & & & & & & & & & & \\
\hline B & 4298.0 & M & 664807 & 6116217 & 0.5 & 0.5 & 5.4 & 0.3 & 5.6 & 6.0 & --- & --- & 0 \\
\hline C & 4339.5 & M & 664777 & 6117297 & 0.0 & 0.4 & 2.5 & 0.3 & 2.1 & 1.9 & --- & --- & 514 \\
\hline D & 4391.8 & M & 664787 & 6118665 & 0.0 & 0.2 & 14.1 & 0.2 & 12.3 & 2.4 & --- & --- & 522 \\
\hline $\mathrm{E}$ & 4396.2 & M & 664786 & 6118786 & 4.1 & 0.3 & 12.8 & 0.2 & 9.9 & 2.1 & --- & --- & 213 \\
\hline $\mathrm{F}$ & 4403.4 & M & 664769 & 6118991 & 5.2 & 0.6 & 9.4 & 0.2 & 7.8 & 1.4 & --- & --- & 70 \\
\hline G & 4829.0 & M & 664442 & 6127041 & 0.7 & 1.0 & 3.4 & 0.7 & 1.8 & 4.9 & --- & --- & 41 \\
\hline $\mathrm{H}$ & 4853.5 & M & 664452 & 6127305 & 0.5 & 0.3 & 5.8 & 0.5 & 4.4 & 3.2 & --- & --- & 454 \\
\hline I & 5065.0 & M & 664327 & 6130787 & 2.8 & 0.3 & 35.1 & 0.5 & 28.5 & 1.5 & --- & --- & 0 \\
\hline $\mathrm{J}$ & 5116.2 & M & 664279 & 6131472 & 0.5 & 0.3 & 0.8 & 0.6 & 1.3 & 1.1 & --- & --- & 7 \\
\hline K & 5163.2 & $\mathrm{M}$ & 664231 & 6132312 & 0.0 & 0.9 & 2.8 & 0.8 & 2.4 & 5.3 & --- & --- & 0 \\
\hline $\mathrm{L}$ & 5223.3 & S? & 664207 & 6133410 & 1.2 & 1.6 & 1.6 & 2.5 & 2.9 & 12.2 & 2.8 & 61 & 0 \\
\hline M & 5244.2 & M & 664179 & 6133754 & 0.0 & 0.6 & 3.7 & 0.7 & 4.7 & 4.1 & --- & --- & 124 \\
\hline $\mathrm{N}$ & 5310.7 & M & 664103 & 6135521 & 0.3 & 0.9 & 0.4 & 1.0 & 0.1 & 5.0 & --- & --- & 0 \\
\hline 0 & 5439.1 & $\mathrm{~s}$ & 664074 & 6137822 & 1.0 & 1.6 & 0.5 & 1.7 & 2.7 & 11.6 & --- & --- & 0 \\
\hline $\mathrm{P}$ & 5620.6 & B & 663860 & 6142846 & 36.4 & 30.2 & 34.2 & 50.0 & 93.3 & 30.2 & 13.6 & 0 & 41 \\
\hline Q & 5629.0 & D & 663847 & 6143100 & 2.9 & 4.5 & 6.9 & 10.0 & 17.0 & 29.1 & 3.9 & 38 & 359 \\
\hline $\mathrm{R}$ & 5646.2 & B & 663823 & 6143517 & 5.8 & 27.7 & 12.3 & 54.0 & 122.6 & 98.5 & 2.2 & 0 & 0 \\
\hline$S$ & 5648.7 & B & 663822 & 6143593 & 9.2 & 27.7 & 12.7 & 60.4 & 143.3 & 102.6 & 2.8 & 0 & 41 \\
\hline $\mathrm{T}$ & 5656.8 & B & 663833 & 6143832 & 52.3 & 48.2 & 61.8 & 89.7 & 185.2 & 91.8 & 14.0 & 1 & 209 \\
\hline U & 5659.1 & B & 663836 & 6143901 & 68.6 & 81.8 & 206.4 & 168.1 & 352.6 & 116.1 & 22.2 & 0 & 0 \\
\hline V & 5664.6 & B & 663826 & 6144077 & 1.3 & 3.5 & 13.3 & 11.1 & 42.9 & 72.0 & 6.4 & 30 & 0 \\
\hline LINE & 11030 & & & & & & & & & & & & \\
\hline A & 3887.9 & S? & 665310 & 6113543 & 0.0 & 1.3 & 0.2 & 2.1 & 0.2 & 13.1 & --- & --- & 145 \\
\hline B & 3606.9 & M & 665162 & 6117876 & 8.4 & 0.4 & 10.7 & 0.2 & 8.7 & 1.2 & --- & --- & 0 \\
\hline $\mathrm{C}$ & 3553.7 & M & 665089 & 6118473 & 0.6 & 0.2 & 0.7 & 0.2 & 0.3 & 1.2 & --- & --- & 197 \\
\hline D & 3522.3 & $\mathrm{M}$ & 665094 & 6119272 & 1.3 & 0.5 & 0.3 & 0.4 & 0.3 & 1.7 & --- & --- & 0 \\
\hline $\mathrm{E}$ & 3253.0 & B & 664901 & 6125095 & 4.2 & 1.8 & 0.2 & 3.2 & 6.7 & 18.2 & --- & --- & 0 \\
\hline $\mathrm{F}$ & 3057.8 & B & 664759 & 6128897 & 1.3 & 1.0 & 1.6 & 1.2 & 1.4 & 7.9 & 7.5 & 93 & 0 \\
\hline G & 2924.5 & M & 664799 & 6129836 & 2.0 & 0.5 & 5.4 & 0.5 & 5.5 & 0.6 & --- & --- & 0 \\
\hline $\mathrm{H}$ & 2915.7 & M & 664751 & 6129954 & 0.8 & 0.6 & 2.6 & 0.5 & 2.2 & 1.7 & --- & --- & 0 \\
\hline I & 2890.0 & M & 664754 & 6130367 & 1.8 & 0.6 & 0.3 & 1.2 & 0.1 & 3.0 & --- & --- & 0 \\
\hline $\mathrm{J}$ & 2880.0 & M & 664749 & 6130423 & 1.5 & 1.1 & 1.8 & 0.5 & 1.6 & 1.2 & --- & --- & 33 \\
\hline K & 2863.9 & M & 664731 & 6130495 & 0.1 & 0.3 & 1.7 & 0.3 & 0.9 & 1.3 & --- & --- & 38 \\
\hline L & 2781.0 & M & 664664 & 6131095 & 0.5 & 0.4 & 2.5 & 0.2 & 2.0 & 1.5 & --- & --- & 119 \\
\hline M & 2769.9 & M & 664658 & 6131136 & 0.1 & 0.1 & 0.1 & 0.2 & 0.3 & 0.8 & --- & --- & 0 \\
\hline $\mathrm{N}$ & 2759.5 & M & 664667 & 6131196 & 1.4 & 0.2 & 3.1 & 0.1 & 2.2 & 0.6 & --- & --- & 0 \\
\hline 0 & 2746.2 & M & 664680 & 6131282 & 0.4 & 0.0 & 6.2 & 0.1 & 4.1 & 1.3 & --- & --- & 31 \\
\hline P & 2660.0 & M & 664622 & 6132778 & 0.7 & 0.5 & 4.1 & 1.3 & 4.3 & 6.1 & --- & --- & 408 \\
\hline$Q$ & 2654.1 & M & 664618 & 6132931 & 0.8 & 0.4 & 0.0 & 0.0 & 0.3 & 0.3 & --- & --- & 507 \\
\hline $\mathrm{R}$ & 2620.1 & B & 664607 & 6133661 & 1.2 & 2.8 & 5.9 & 10.1 & 21.0 & 7.2 & 2.4 & 36 & 41 \\
\hline $\mathrm{S}$ & 2403.2 & M & 664406 & 6138470 & 1.7 & 0.8 & 2.3 & 1.6 & 2.2 & 7.7 & --- & --- & 39 \\
\hline $\mathrm{T}$ & 2337.6 & B & 664341 & 6140293 & 4.6 & 7.0 & 3.8 & 13.0 & 39.6 & 44.7 & 3.4 & 7 & 0 \\
\hline $\mathrm{U}$ & 2198.5 & B & 664224 & 6143242 & 10.4 & 36.5 & 17.7 & 74.1 & 241.6 & 300.1 & 3.0 & 0 & 204 \\
\hline $\mathrm{V}$ & 2196.3 & $B$ ? & 664228 & 6143310 & 7.2 & 40.9 & 19.2 & 91.9 & 233.6 & 288.5 & 2.2 & 0 & 231 \\
\hline W & 2179.4 & B & 664226 & 6143830 & 8.9 & 22.0 & 19.5 & 40.7 & 96.5 & 76.3 & 4.5 & 0 & 128 \\
\hline
\end{tabular}

$\mathrm{CX}=$ COAXIAL

Note: EM values shown above

are local amplitudes

are 10
55
15
*Estimated Depth may be unreliable because the stronger part of the conductor may be deeper or to one side of the flight line, or because of a shallow dip or magnetite/overburden effects 
EM Anomaly List

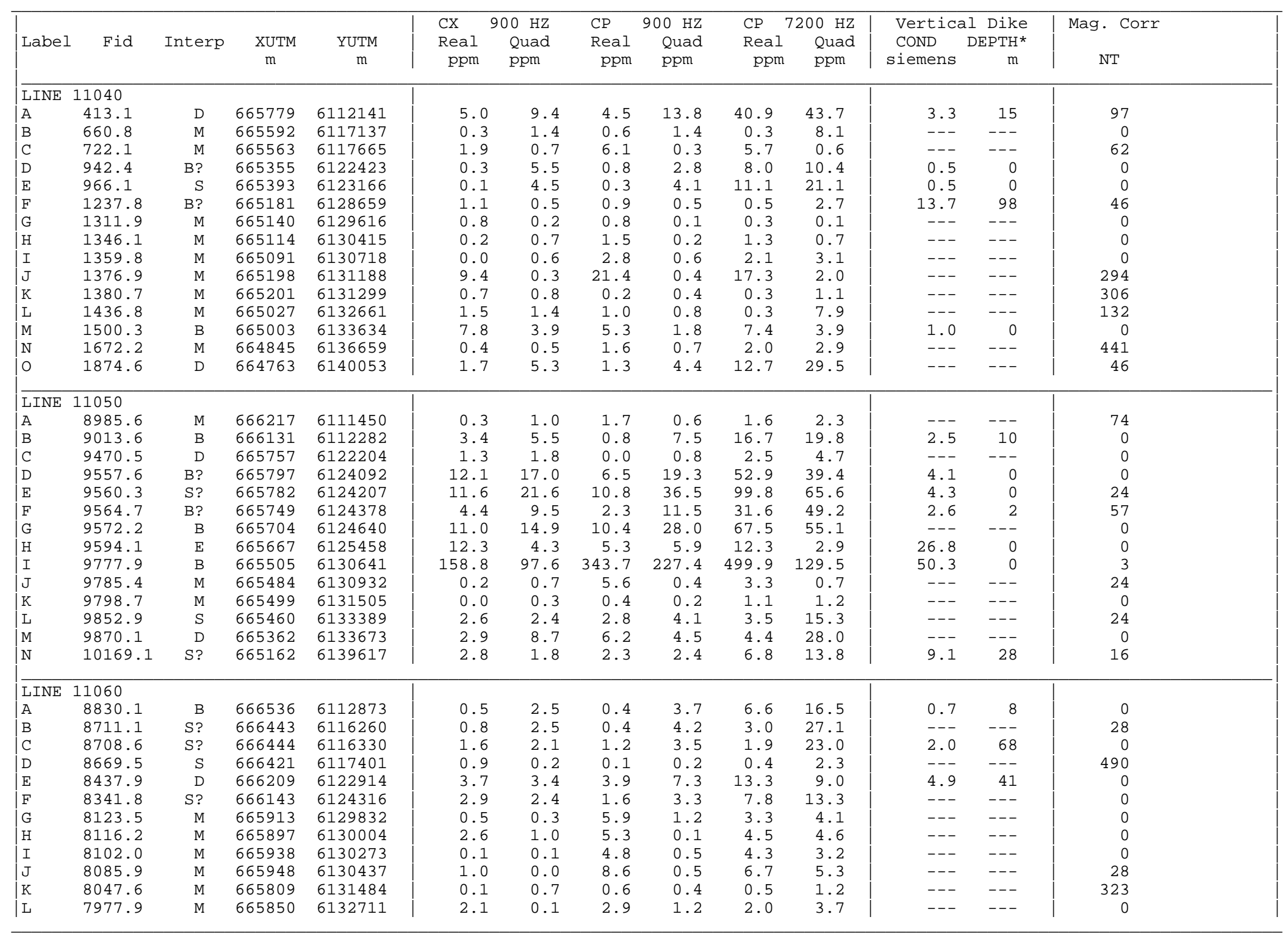

$\mathrm{CX}=$ COAXIAL

Note: EM values shown above

Ketchikan - North/South of 5515
*Estimated Depth may be unreliable because the stronger part of the conductor may be deeper or shallow dip or magnetite/overburden effects 
EM Anomaly List

\begin{tabular}{|c|c|c|c|c|c|c|c|c|c|c|c|c|c|}
\hline Label & Fid & Interp & $\begin{array}{c}\text { XUTM } \\
\text { m }\end{array}$ & $\begin{array}{c}\text { YUTM } \\
\text { m }\end{array}$ & $\begin{array}{l}\text { CX } \\
\text { Real } \\
\text { ppm }\end{array}$ & $\begin{array}{l}00 \mathrm{HZ} \\
\text { Quad } \\
\text { ppm }\end{array}$ & $\begin{array}{l}\text { CP } \\
\text { Real } \\
\text { ppm }\end{array}$ & $\begin{array}{l}900 \mathrm{HZ} \\
\text { Quad } \\
\text { ppm }\end{array}$ & $\begin{array}{l}\text { CP } 7 \\
\text { Real } \\
\text { ppm }\end{array}$ & $\begin{array}{l}200 \mathrm{HZ} \\
\text { Quad } \\
\text { ppm }\end{array}$ & $\begin{array}{l}\text { Vertica } \\
\text { Cond } \\
\text { siemens }\end{array}$ & $\begin{array}{c}\text { al Dike } \\
\text { DEPTH* } \\
\text { m }\end{array}$ & $\begin{array}{c}\text { Mag. Corr } \\
\text { NT }\end{array}$ \\
\hline $\begin{array}{l}\text { LINE } \\
M\end{array}$ & $\begin{array}{c}11060 \\
7855.1\end{array}$ & M & 665787 & 6135260 & 0.4 & 0.7 & 0.1 & 0.6 & 0.2 & 2.7 & --- & --- & 89 \\
\hline $\begin{array}{l}\text { LINE } \\
\text { A } \\
\text { B } \\
\text { C } \\
\text { D } \\
\text { E }\end{array}$ & $\begin{array}{r}11070 \\
6023.6 \\
6669.5 \\
6815.3 \\
6890.7 \\
6932.8\end{array}$ & $\begin{array}{r}\mathrm{D} \\
\mathrm{B} ? \\
\mathrm{M} \\
\mathrm{M} \\
\mathrm{D}\end{array}$ & $\begin{array}{l}666942 \\
666427 \\
666259 \\
666219 \\
666198\end{array}$ & $\begin{array}{l}6113288 \\
6127506 \\
6130949 \\
6132722 \\
6133525\end{array}$ & $\begin{array}{l}2.0 \\
0.2 \\
0.2 \\
1.4 \\
2.6\end{array}$ & $\begin{array}{l}2.8 \\
1.6 \\
0.5 \\
0.3 \\
3.7\end{array}$ & $\begin{array}{l}1.0 \\
0.0 \\
0.8 \\
0.4 \\
0.8\end{array}$ & $\begin{array}{l}4.1 \\
1.1 \\
0.2 \\
0.7 \\
2.8\end{array}$ & $\begin{array}{l}9.5 \\
2.1 \\
0.4 \\
0.3 \\
6.1\end{array}$ & $\begin{array}{r}11.6 \\
7.2 \\
2.4 \\
5.4 \\
5.1\end{array}$ & $\begin{array}{l}--- \\
1.6 \\
--- \\
--- \\
2.5\end{array}$ & $\begin{array}{r}--- \\
94 \\
--- \\
--- \\
41\end{array}$ & $\begin{array}{r}23 \\
0 \\
0 \\
243 \\
0\end{array}$ \\
\hline $\begin{array}{l}\text { LINE } \\
\text { A } \\
\text { B } \\
\text { C } \\
\text { D } \\
\text { E } \\
\text { F } \\
\text { G } \\
\text { H } \\
\text { I } \\
\text { J } \\
\text { K } \\
\text { L } \\
\text { M } \\
\text { N } \\
\text { O }\end{array}$ & $\begin{array}{r}11080 \\
5803.5 \\
5745.3 \\
5688.4 \\
5588.4 \\
5545.3 \\
5356.4 \\
5342.3 \\
5221.4 \\
4942.8 \\
4929.2 \\
4753.1 \\
4648.8 \\
4629.8 \\
4617.8 \\
4406.5\end{array}$ & $\begin{array}{r}\text { B } \\
\text { D } \\
\text { S } \\
\text { D } \\
\text { M } \\
\text { B? } \\
\text { B? } \\
\text { B } \\
\text { M } \\
\text { M } \\
\text { D } \\
\text { S? } \\
\text { M } \\
\text { M } \\
\text { B }\end{array}$ & $\begin{array}{l}667329 \\
667327 \\
667289 \\
667250 \\
667133 \\
667042 \\
667034 \\
666938 \\
666749 \\
666741 \\
666614 \\
666589 \\
666594 \\
666602 \\
666367\end{array}$ & $\begin{array}{l}6112521 \\
6113738 \\
6114692 \\
6116945 \\
6117929 \\
6121463 \\
6121611 \\
6123262 \\
6129768 \\
6129951 \\
6133438 \\
6133901 \\
6134111 \\
6134404 \\
6140064\end{array}$ & $\begin{array}{l}0.4 \\
0.3 \\
0.5 \\
1.7 \\
7.4 \\
0.5 \\
0.4 \\
6.1 \\
0.0 \\
0.7 \\
8.2 \\
1.3 \\
2.4 \\
1.2 \\
2.7\end{array}$ & $\begin{array}{r}2.7 \\
2.1 \\
3.4 \\
3.9 \\
0.8 \\
0.7 \\
1.4 \\
4.9 \\
0.7 \\
0.8 \\
12.1 \\
0.7 \\
0.2 \\
0.7 \\
2.7\end{array}$ & $\begin{array}{l}0.5 \\
0.5 \\
0.8 \\
0.2 \\
0.1 \\
0.1 \\
2.0 \\
2.2 \\
1.5 \\
3.0 \\
2.6 \\
0.8 \\
0.9 \\
0.9 \\
3.0\end{array}$ & $\begin{array}{l}3.7 \\
0.4 \\
5.2 \\
3.4 \\
0.8 \\
1.2 \\
1.3 \\
7.6 \\
0.1 \\
0.9 \\
2.1 \\
1.1 \\
0.3 \\
0.4 \\
2.7\end{array}$ & $\begin{array}{r}8.3 \\
1.3 \\
3.9 \\
8.2 \\
0.5 \\
0.1 \\
2.2 \\
18.8 \\
1.3 \\
3.6 \\
8.2 \\
0.7 \\
0.8 \\
0.6 \\
7.0\end{array}$ & $\begin{array}{r}19.7 \\
2.5 \\
32.5 \\
16.3 \\
3.5 \\
5.3 \\
8.5 \\
11.0 \\
2.8 \\
5.7 \\
2.7 \\
5.3 \\
2.6 \\
1.1 \\
4.5\end{array}$ & $\begin{array}{l}--- \\
--- \\
--- \\
--- \\
--- \\
--- \\
--- \\
5.6 \\
--- \\
--- \\
5.0 \\
6.5 \\
--- \\
--- \\
---\end{array}$ & $\begin{array}{r}--- \\
--- \\
--- \\
--- \\
--- \\
--- \\
--- \\
7 \\
--- \\
--- \\
32 \\
84 \\
--- \\
--- \\
---\end{array}$ & $\begin{array}{r}0 \\
26 \\
0 \\
0 \\
237 \\
0 \\
26 \\
0 \\
204 \\
0 \\
0 \\
0 \\
0 \\
26 \\
0\end{array}$ \\
\hline $\begin{array}{l}\text { LINE } \\
\text { A } \\
\text { B } \\
\text { C } \\
\text { D } \\
\text { E } \\
\text { F } \\
\text { G } \\
\text { H } \\
\text { I } \\
\text { J } \\
\text { K } \\
\text { L }\end{array}$ & $\begin{array}{r}11090 \\
2714.4 \\
2779.8 \\
2787.9 \\
2838.1 \\
2945.2 \\
3014.8 \\
3347.9 \\
3753.4 \\
3766.8 \\
3774.3 \\
3785.5 \\
3836.6\end{array}$ & $\begin{array}{c}\mathrm{S} \\
\mathrm{M} \\
\mathrm{M} \\
\mathrm{D} \\
\mathrm{M} \\
\mathrm{B} ? \\
\mathrm{~B} ? \\
\mathrm{M} \\
\mathrm{M} \\
\mathrm{M} \\
\mathrm{M} \\
\mathrm{M}\end{array}$ & $\begin{array}{l}667685 \\
667680 \\
667676 \\
667648 \\
667570 \\
667528 \\
667400 \\
667120 \\
667118 \\
667109 \\
667086 \\
667056\end{array}$ & $\begin{array}{l}6114640 \\
6115463 \\
6115529 \\
6116783 \\
6119289 \\
6120583 \\
6123381 \\
6130094 \\
6130470 \\
6130700 \\
6131053 \\
6132763\end{array}$ & $\begin{array}{l}1.0 \\
0.0 \\
1.9 \\
0.3 \\
0.0 \\
0.7 \\
3.2 \\
1.5 \\
0.1 \\
0.6 \\
0.4 \\
0.3\end{array}$ & $\begin{array}{l}2.0 \\
0.4 \\
0.5 \\
2.7 \\
0.8 \\
2.5 \\
3.9 \\
0.4 \\
1.0 \\
1.5 \\
0.3 \\
0.3\end{array}$ & $\begin{array}{l}0.6 \\
1.1 \\
2.6 \\
0.2 \\
0.0 \\
2.6 \\
3.5 \\
2.0 \\
0.4 \\
2.0 \\
0.4 \\
0.7\end{array}$ & $\begin{array}{l}2.2 \\
0.5 \\
0.3 \\
0.9 \\
0.5 \\
3.3 \\
5.6 \\
0.4 \\
0.6 \\
0.9 \\
0.6 \\
1.5\end{array}$ & $\begin{array}{r}1.7 \\
0.8 \\
2.4 \\
1.1 \\
2.8 \\
0.4 \\
14.2 \\
2.3 \\
3.8 \\
2.5 \\
0.0 \\
0.5\end{array}$ & $\begin{array}{r}11.8 \\
3.6 \\
2.1 \\
2.4 \\
1.8 \\
21.4 \\
4.9 \\
1.5 \\
4.3 \\
5.0 \\
3.4 \\
7.7\end{array}$ & $\begin{array}{l}1.8 \\
--- \\
--- \\
--- \\
--- \\
--- \\
4.1 \\
--- \\
--- \\
--- \\
--- \\
---\end{array}$ & $\begin{array}{r}7 \\
--- \\
--- \\
--- \\
--- \\
--- \\
8 \\
--- \\
--- \\
--- \\
--- \\
---\end{array}$ & $\begin{array}{r}0 \\
0 \\
0 \\
0 \\
289 \\
0 \\
0 \\
171 \\
99 \\
0 \\
418 \\
229\end{array}$ \\
\hline $\begin{array}{l}\text { LINE } \\
\text { A } \\
\text { B } \\
\text { C } \\
\text { D } \\
\text { E }\end{array}$ & $\begin{array}{r}11100 \\
2068.1 \\
1846.6 \\
1803.1 \\
1713.5 \\
1509.9\end{array}$ & $\begin{array}{r}\text { S? } \\
\text { B? } \\
\text { B } \\
\text { D } \\
\text { M }\end{array}$ & $\begin{array}{l}667948 \\
667786 \\
667774 \\
667687 \\
667562\end{array}$ & $\begin{array}{l}6119785 \\
6122644 \\
6123357 \\
6125530 \\
6129809\end{array}$ & $\begin{array}{l}1.8 \\
1.3 \\
8.8 \\
2.7 \\
1.1\end{array}$ & $\begin{array}{l}0.3 \\
0.3 \\
6.8 \\
4.3 \\
0.7\end{array}$ & $\begin{array}{l}2.8 \\
1.6 \\
5.2 \\
1.4 \\
0.3\end{array}$ & $\begin{array}{l}0.6 \\
0.7 \\
8.7 \\
4.0 \\
0.5\end{array}$ & $\begin{array}{r}1.1 \\
1.4 \\
21.9 \\
8.8 \\
0.2\end{array}$ & $\begin{array}{r}3.8 \\
5.5 \\
14.2 \\
8.4 \\
3.6\end{array}$ & $\begin{array}{r}50.5 \\
27.7 \\
8.7 \\
--- \\
---\end{array}$ & $\begin{array}{r}95 \\
84 \\
10 \\
--- \\
---\end{array}$ & $\begin{array}{r}0 \\
29 \\
15 \\
0 \\
310\end{array}$ \\
\hline
\end{tabular}

$\mathrm{CX}=$ COAXIAL

Note: EM values shown above

are local amplitudes

Ketchikan - North/South of 5515
*Estimated Depth may be unreliable because the stronger part of the conductor may be deeper or to one side of the flight line, or because of a shallow dip or magnetite/overburden effects 
EM Anomaly List

\begin{tabular}{|c|c|c|c|c|c|c|c|c|c|c|c|c|c|}
\hline Label & Fid & Interp & $\begin{array}{c}\text { XUTM } \\
\text { m }\end{array}$ & $\begin{array}{c}\text { YUTM } \\
\mathrm{m}\end{array}$ & $\begin{array}{l}\text { CX } \\
\text { Real } \\
\text { ppm }\end{array}$ & $\begin{array}{l}900 \mathrm{HZ} \\
\text { Quad } \\
\text { ppm }\end{array}$ & $\begin{array}{l}\text { CP } \\
\text { Real } \\
\text { ppm }\end{array}$ & $\begin{array}{c}900 \mathrm{HZ} \\
\text { Quad } \\
\text { ppm }\end{array}$ & $\begin{array}{l}\text { CP } 7 \\
\text { Real } \\
\text { ppm }\end{array}$ & $\begin{array}{l}200 \mathrm{HZ} \\
\text { Quad } \\
\text { ppm }\end{array}$ & $\begin{array}{l}\text { Vertica } \\
\text { COND } \\
\text { siemens }\end{array}$ & $\begin{array}{c}\text { al Dike } \\
\text { DEPTH* }^{\star} \\
\text { m }\end{array}$ & $\begin{array}{c}\text { Mag. Corr } \\
\text { NT }\end{array}$ \\
\hline $\begin{array}{l}\text { LINE } \\
\text { F } \\
\text { G } \\
\text { H } \\
\text { I } \\
\text { J } \\
\text { K } \\
\text { L } \\
\text { M }\end{array}$ & $\begin{array}{r}11100 \\
1499.8 \\
1487.3 \\
1452.1 \\
1417.9 \\
1358.0 \\
1187.2 \\
1151.0 \\
1040.4\end{array}$ & $\begin{array}{l}\mathrm{M} \\
\mathrm{M} \\
\mathrm{M} \\
\mathrm{M} \\
\mathrm{M} \\
\mathrm{M} \\
\mathrm{B} \\
\mathrm{B}\end{array}$ & $\begin{array}{l}667536 \\
667528 \\
667536 \\
667485 \\
667415 \\
667249 \\
667185 \\
667118\end{array}$ & $\begin{array}{l}6130077 \\
6130263 \\
6131015 \\
6131653 \\
6133321 \\
6138863 \\
6139535 \\
6142970\end{array}$ & $\begin{array}{r}0.5 \\
0.3 \\
0.3 \\
0.2 \\
0.7 \\
4.1 \\
42.6 \\
8.4\end{array}$ & $\begin{array}{l}0.6 \\
0.1 \\
0.4 \\
1.0 \\
0.4 \\
1.0 \\
39.8 \\
11.3\end{array}$ & $\begin{array}{r}1.1 \\
0.8 \\
1.2 \\
0.7 \\
2.2 \\
0.6 \\
68.4 \\
42.3\end{array}$ & $\begin{array}{r}0.3 \\
0.6 \\
0.2 \\
1.1 \\
0.7 \\
0.9 \\
81.0 \\
32.3\end{array}$ & $\begin{array}{r}1.1 \\
0.2 \\
1.1 \\
0.5 \\
2.4 \\
0.5 \\
175.8 \\
62.2\end{array}$ & $\begin{array}{r}3.0 \\
1.8 \\
2.0 \\
7.3 \\
5.5 \\
2.6 \\
80.4 \\
26.6\end{array}$ & $\begin{array}{c}--- \\
--- \\
--- \\
--- \\
--- \\
--- \\
15.1 \\
13.0\end{array}$ & $\begin{array}{r}--- \\
--- \\
--- \\
--- \\
--- \\
--- \\
0 \\
0\end{array}$ & $\begin{array}{r}0 \\
0 \\
293 \\
251 \\
29 \\
140 \\
0 \\
0\end{array}$ \\
\hline $\begin{array}{l}\text { LINE } \\
\text { A } \\
\text { B } \\
\text { C } \\
\text { D } \\
\text { E } \\
\text { F } \\
\text { G } \\
\text { H } \\
\text { I } \\
\text { J } \\
\text { K } \\
\text { L } \\
\text { M }\end{array}$ & $\begin{array}{r}11110 \\
2977.4 \\
2894.7 \\
2820.7 \\
2746.5 \\
2714.2 \\
2699.9 \\
2644.2 \\
2621.3 \\
2517.4 \\
2470.8 \\
2440.9 \\
2426.0 \\
2341.3\end{array}$ & $\begin{array}{r}\text { B? } \\
\text { S } \\
\text { S? } \\
\text { B } \\
\text { B } \\
\text { B } \\
\text { B } \\
\text { B } \\
\text { S } \\
\text { M } \\
\text { M } \\
\text { M } \\
\text { M }\end{array}$ & $\begin{array}{l}668435 \\
668401 \\
668275 \\
668177 \\
668134 \\
668130 \\
668092 \\
668096 \\
667966 \\
667934 \\
667905 \\
667905 \\
667850\end{array}$ & $\begin{array}{l}6116688 \\
6119390 \\
6121180 \\
6123264 \\
6123877 \\
6124214 \\
6125526 \\
6125723 \\
6128174 \\
6129684 \\
6130492 \\
6130916 \\
6132947\end{array}$ & $\begin{array}{r}29.6 \\
1.8 \\
0.2 \\
1.9 \\
3.0 \\
11.0 \\
0.8 \\
3.1 \\
1.7 \\
0.7 \\
0.6 \\
0.0 \\
0.1\end{array}$ & $\begin{array}{r}25.2 \\
0.6 \\
1.9 \\
4.9 \\
6.2 \\
12.4 \\
1.6 \\
1.6 \\
3.2 \\
0.2 \\
0.1 \\
0.5 \\
0.0\end{array}$ & $\begin{array}{r}71.7 \\
1.0 \\
0.8 \\
5.1 \\
2.9 \\
9.5 \\
1.0 \\
2.4 \\
1.9 \\
0.3 \\
0.8 \\
0.5 \\
1.1\end{array}$ & $\begin{array}{r}65.0 \\
0.9 \\
3.6 \\
12.4 \\
8.5 \\
21.6 \\
4.0 \\
6.4 \\
4.5 \\
0.7 \\
0.2 \\
0.7 \\
0.7\end{array}$ & $\begin{array}{r}139.0 \\
1.0 \\
11.5 \\
28.5 \\
22.2 \\
51.0 \\
10.6 \\
16.6 \\
4.1 \\
0.5 \\
0.3 \\
0.6 \\
1.1\end{array}$ & $\begin{array}{r}69.2 \\
6.0 \\
18.4 \\
20.3 \\
18.6 \\
33.8 \\
6.5 \\
20.3 \\
31.4 \\
2.7 \\
2.1 \\
3.7 \\
2.5\end{array}$ & $\begin{array}{r}18.4 \\
15.3 \\
--- \\
2.6 \\
2.7 \\
5.4 \\
1.6 \\
5.3 \\
--- \\
--- \\
--- \\
--- \\
---\end{array}$ & $\begin{array}{r}0 \\
70 \\
--- \\
0 \\
5 \\
11 \\
34 \\
42 \\
--- \\
--- \\
--- \\
--- \\
---\end{array}$ & $\begin{array}{r}0 \\
0 \\
0 \\
0 \\
27 \\
0 \\
0 \\
0 \\
0 \\
200 \\
29 \\
210 \\
156\end{array}$ \\
\hline $\begin{array}{l}\text { LINE } \\
\text { A } \\
\text { B } \\
\text { C } \\
\text { D } \\
\text { E } \\
\text { F } \\
\text { G } \\
\text { H } \\
\text { I } \\
\text { J } \\
\text { K } \\
\text { L } \\
\text { M } \\
\text { N } \\
\text { O } \\
\text { P } \\
\text { Q } \\
\text { R } \\
\text { S } \\
\text { T } \\
\text { U }\end{array}$ & $\begin{array}{l}11120 \\
561.4 \\
694.6 \\
971.9 \\
997.2 \\
1027.9 \\
1052.1 \\
1114.7 \\
1121.4 \\
1192.0 \\
1321.7 \\
1361.0 \\
1391.0 \\
1636.0 \\
1650.0 \\
1670.2 \\
1685.8 \\
1700.3 \\
1736.0 \\
1759.7 \\
1773.2 \\
1788.7\end{array}$ & $\begin{array}{r}\text { B? } \\
\text { S } \\
\text { L? } \\
\text { L? } \\
\text { D } \\
\text { D } \\
\text { D } \\
\text { B? } \\
\text { M } \\
\text { M } \\
\text { M } \\
\text { M } \\
\text { B? } \\
\text { M } \\
\text { M } \\
\text { M } \\
\text { M } \\
\text { M } \\
\text { M } \\
\text { M } \\
\text { M }\end{array}$ & $\begin{array}{l}668848 \\
668696 \\
668609 \\
668577 \\
668524 \\
668525 \\
668508 \\
668503 \\
668393 \\
668348 \\
668286 \\
668297 \\
668035 \\
668050 \\
668038 \\
668041 \\
668053 \\
667969 \\
667972 \\
667991 \\
667989\end{array}$ & $\begin{array}{l}6116000 \\
6120270 \\
6123313 \\
6123585 \\
6123970 \\
6124454 \\
6125523 \\
6125619 \\
6127176 \\
6129556 \\
6130742 \\
6131313 \\
6137900 \\
6138241 \\
6138556 \\
6138767 \\
6139023 \\
6139753 \\
6139986 \\
6140200 \\
6140529\end{array}$ & $\begin{array}{l}0.6 \\
0.0 \\
1.1 \\
4.8 \\
6.8 \\
4.6 \\
3.8 \\
2.7 \\
0.1 \\
0.0 \\
0.9 \\
0.9 \\
0.4 \\
0.2 \\
0.2 \\
0.1 \\
2.5 \\
1.5 \\
0.0 \\
4.1 \\
0.2\end{array}$ & $\begin{array}{l}2.4 \\
0.9 \\
1.8 \\
7.2 \\
7.3 \\
6.5 \\
8.6 \\
1.0 \\
0.2 \\
0.3 \\
0.5 \\
0.1 \\
2.1 \\
0.6 \\
0.5 \\
0.4 \\
0.4 \\
0.5 \\
0.7 \\
0.4 \\
0.1\end{array}$ & $\begin{array}{l}1.7 \\
0.1 \\
0.9 \\
0.2 \\
3.4 \\
2.7 \\
3.2 \\
1.9 \\
3.3 \\
1.6 \\
5.0 \\
0.0 \\
1.3 \\
2.7 \\
7.8 \\
1.3 \\
7.4 \\
1.4 \\
1.4 \\
0.1 \\
7.3\end{array}$ & $\begin{array}{l}2.8 \\
1.1 \\
6.6 \\
2.0 \\
7.5 \\
2.9 \\
9.2 \\
2.5 \\
0.3 \\
0.1 \\
0.6 \\
0.1 \\
6.8 \\
0.9 \\
0.1 \\
0.2 \\
0.5 \\
1.2 \\
0.4 \\
0.1 \\
0.4\end{array}$ & $\begin{array}{r}4.3 \\
0.9 \\
16.0 \\
4.0 \\
18.3 \\
10.9 \\
25.1 \\
4.4 \\
3.1 \\
1.3 \\
4.5 \\
0.1 \\
21.4 \\
1.3 \\
6.1 \\
1.5 \\
6.2 \\
1.4 \\
1.7 \\
0.1 \\
5.8\end{array}$ & $\begin{array}{r}8.0 \\
7.1 \\
30.1 \\
5.7 \\
15.9 \\
9.7 \\
12.3 \\
3.1 \\
4.0 \\
1.2 \\
3.5 \\
0.0 \\
27.0 \\
3.2 \\
2.1 \\
0.6 \\
1.3 \\
5.3 \\
1.5 \\
0.7 \\
2.6\end{array}$ & $\begin{array}{l}1.7 \\
--- \\
1.5 \\
3.1 \\
5.3 \\
4.5 \\
2.8 \\
--- \\
--- \\
--- \\
--- \\
--- \\
--- \\
--- \\
--- \\
--- \\
--- \\
--- \\
--- \\
--- \\
---\end{array}$ & $\begin{array}{r}26 \\
--- \\
24 \\
34 \\
23 \\
28 \\
3 \\
--- \\
--- \\
--- \\
--- \\
--- \\
--- \\
--- \\
--- \\
--- \\
--- \\
--- \\
--- \\
--- \\
---\end{array}$ & $\begin{array}{r}122 \\
40 \\
0 \\
0 \\
0 \\
0 \\
0 \\
0 \\
90 \\
165 \\
339 \\
189 \\
0 \\
0 \\
38 \\
0 \\
0 \\
40 \\
110 \\
0 \\
0\end{array}$ \\
\hline
\end{tabular}

$\mathrm{CX}=$ COAXIAL

Note: EM values shown above

are local amplitudes

Ketchikan - North/South of 5515
* Estimated Depth may be unreliable because the stronger part of the conductor may be deeper or to one side of the flight line, or because of a shallow dip or magnetite/overburden effects 
EM Anomaly List

\begin{tabular}{|c|c|c|c|c|c|c|c|c|c|c|c|c|c|}
\hline Label & Fid & Interp & $\begin{array}{c}\text { XUTM } \\
\text { m }\end{array}$ & $\begin{array}{c}\text { YUTM } \\
\mathrm{m}\end{array}$ & $\begin{array}{l}\text { CX } \\
\text { Real } \\
\text { ppm }\end{array}$ & $\begin{array}{l}00 \mathrm{HZ} \\
\text { Quad } \\
\text { ppm }\end{array}$ & $\begin{array}{l}\text { CP } \\
\text { Real } \\
\text { ppm }\end{array}$ & $\begin{array}{l}900 \mathrm{HZ} \\
\text { Quad } \\
\text { ppm }\end{array}$ & $\begin{array}{l}\mathrm{CP} \\
\mathrm{Real} \\
\mathrm{ppm}\end{array}$ & $\begin{array}{l}200 \mathrm{HZ} \\
\text { Quad } \\
\text { ppm }\end{array}$ & $\begin{array}{l}\text { Vertica } \\
\text { Cond } \\
\text { siemens }\end{array}$ & $\begin{array}{c}\text { al Dike } \\
\text { DEPTH* } \\
\text { m }\end{array}$ & $\begin{array}{c}\text { Mag. Corr } \\
\text { NT }\end{array}$ \\
\hline $\begin{array}{l}\text { LINE } \\
\mathrm{V}\end{array}$ & $\begin{array}{c}11120 \\
1800.7\end{array}$ & M & 667984 & 6140797 & 1.6 & 0.8 & 5.6 & 0.9 & 7.1 & 6.7 & --- & --- & 146 \\
\hline $\begin{array}{l}\text { LINE } \\
\text { A } \\
\text { B } \\
\text { C } \\
\text { D } \\
\text { E } \\
\text { F } \\
\text { G } \\
\text { H } \\
\text { I } \\
\text { J } \\
\text { K } \\
\text { L } \\
\text { M } \\
\text { N } \\
\text { O } \\
\text { P } \\
\text { Q }\end{array}$ & $\begin{array}{l}11130 \\
10418.7 \\
10301.8 \\
10260.6 \\
10150.0 \\
10109.1 \\
10107.7 \\
10072.6 \\
9819.8 \\
9722.7 \\
9695.9 \\
9691.3 \\
9684.9 \\
9672.1 \\
9660.4 \\
9627.9 \\
9608.2 \\
9597.1\end{array}$ & $\begin{array}{r}\mathrm{B} \\
\mathrm{B} \\
\mathrm{B} ? \\
\mathrm{M} \\
\mathrm{M} \\
\mathrm{S} \\
\mathrm{S} ? \\
\mathrm{~S} ? \\
\mathrm{M} \\
\mathrm{M} \\
\mathrm{M} \\
\mathrm{M} \\
\mathrm{M} \\
\mathrm{M} \\
\mathrm{M} \\
\mathrm{M} \\
\mathrm{M}\end{array}$ & $\begin{array}{l}669047 \\
668892 \\
668980 \\
668851 \\
668800 \\
668794 \\
668765 \\
668555 \\
668439 \\
668473 \\
668481 \\
668480 \\
668438 \\
668398 \\
668418 \\
668344 \\
668350\end{array}$ & $\begin{array}{l}6120690 \\
6123770 \\
6124708 \\
6126990 \\
6127873 \\
6127922 \\
6129070 \\
6134612 \\
6136953 \\
6137864 \\
6138044 \\
6138299 \\
6138779 \\
6139154 \\
6140381 \\
6140725 \\
6140860\end{array}$ & $\begin{array}{r}0.7 \\
3.2 \\
3.9 \\
0.2 \\
0.3 \\
0.6 \\
0.6 \\
2.7 \\
0.3 \\
10.6 \\
34.7 \\
0.8 \\
0.3 \\
2.3 \\
0.0 \\
3.0 \\
0.1\end{array}$ & $\begin{array}{l}1.0 \\
3.2 \\
1.7 \\
0.5 \\
2.4 \\
2.5 \\
0.9 \\
1.4 \\
0.4 \\
0.4 \\
0.7 \\
0.1 \\
0.0 \\
0.2 \\
0.1 \\
0.1 \\
0.1\end{array}$ & $\begin{array}{r}0.5 \\
3.2 \\
5.0 \\
7.2 \\
0.3 \\
4.4 \\
0.4 \\
1.3 \\
0.3 \\
10.5 \\
64.5 \\
5.5 \\
2.1 \\
2.9 \\
23.1 \\
0.0 \\
0.4\end{array}$ & $\begin{array}{l}2.5 \\
3.7 \\
5.8 \\
0.9 \\
3.4 \\
3.4 \\
1.5 \\
2.2 \\
0.6 \\
0.7 \\
0.4 \\
0.0 \\
0.3 \\
0.4 \\
0.4 \\
0.3 \\
0.8\end{array}$ & $\begin{array}{r}5.2 \\
11.5 \\
12.5 \\
5.8 \\
0.2 \\
0.1 \\
0.4 \\
2.2 \\
0.2 \\
9.6 \\
53.0 \\
4.8 \\
2.0 \\
3.3 \\
0.3 \\
0.1 \\
0.3\end{array}$ & $\begin{array}{r}12.5 \\
6.6 \\
5.1 \\
4.6 \\
21.9 \\
21.9 \\
8.5 \\
9.6 \\
2.5 \\
1.9 \\
1.2 \\
1.7 \\
5.7 \\
2.3 \\
2.7 \\
1.2 \\
4.5\end{array}$ & $\begin{array}{r}--- \\
5.9 \\
11.7 \\
--- \\
--- \\
2.9 \\
--- \\
8.8 \\
--- \\
--- \\
--- \\
--- \\
--- \\
--- \\
--- \\
--- \\
---\end{array}$ & $\begin{array}{r}--- \\
0 \\
22 \\
--- \\
--- \\
60 \\
--- \\
41 \\
--- \\
--- \\
--- \\
--- \\
--- \\
--- \\
--- \\
--- \\
---\end{array}$ & $\begin{array}{r}0 \\
52 \\
0 \\
296 \\
245 \\
0 \\
0 \\
0 \\
176 \\
269 \\
172 \\
761 \\
52 \\
0 \\
1288 \\
31 \\
0\end{array}$ \\
\hline $\begin{array}{l}\text { LINE } \\
\text { A } \\
\text { B } \\
\text { C } \\
\text { D } \\
\text { E } \\
\text { F } \\
\text { G } \\
\text { H } \\
\text { I } \\
\text { J } \\
\text { K } \\
\text { L }\end{array}$ & $\begin{array}{r}11140 \\
8163.9 \\
8207.5 \\
8225.3 \\
8471.3 \\
8490.2 \\
8504.0 \\
8580.8 \\
8740.3 \\
8773.8 \\
8829.1 \\
8880.0 \\
8940.0\end{array}$ & $\begin{array}{r}\text { B } \\
\text { B? } \\
\text { B } \\
\text { B? } \\
\text { B } \\
\text { B } \\
\text { B? } \\
\text { S? } \\
\text { S? } \\
\text { S } \\
\text { M } \\
\text { M }\end{array}$ & $\begin{array}{l}669715 \\
669640 \\
669676 \\
669440 \\
669391 \\
669380 \\
669330 \\
669154 \\
669150 \\
669187 \\
669089 \\
669045\end{array}$ & $\begin{array}{l}6114785 \\
6115961 \\
6116522 \\
6122548 \\
6123109 \\
6123520 \\
6124879 \\
6129002 \\
6129397 \\
6130411 \\
6130910 \\
6132365\end{array}$ & $\begin{array}{r}2.4 \\
15.3 \\
12.7 \\
2.6 \\
1.5 \\
6.9 \\
2.1 \\
0.3 \\
0.4 \\
1.0 \\
0.3 \\
0.2\end{array}$ & $\begin{array}{r}6.9 \\
33.9 \\
10.1 \\
5.1 \\
4.5 \\
5.6 \\
5.7 \\
1.9 \\
0.8 \\
0.7 \\
0.1 \\
0.5\end{array}$ & $\begin{array}{r}6.4 \\
18.3 \\
5.5 \\
0.8 \\
0.4 \\
15.8 \\
3.1 \\
4.6 \\
0.5 \\
0.8 \\
2.7 \\
2.8\end{array}$ & $\begin{array}{r}12.7 \\
56.9 \\
12.2 \\
8.3 \\
3.1 \\
13.3 \\
10.0 \\
1.7 \\
0.8 \\
1.4 \\
0.3 \\
0.3\end{array}$ & $\begin{array}{r}30.1 \\
141.5 \\
29.9 \\
25.8 \\
6.5 \\
28.0 \\
26.7 \\
0.8 \\
0.3 \\
0.9 \\
3.6 \\
2.1\end{array}$ & $\begin{array}{r}24.7 \\
73.6 \\
12.7 \\
30.7 \\
8.9 \\
7.2 \\
21.5 \\
11.9 \\
6.1 \\
2.5 \\
1.1 \\
0.4\end{array}$ & $\begin{array}{r}2.9 \\
4.5 \\
8.8 \\
1.9 \\
1.4 \\
12.4 \\
2.1 \\
--- \\
--- \\
--- \\
--- \\
---\end{array}$ & $\begin{array}{r}0 \\
0 \\
0 \\
0 \\
16 \\
1 \\
10 \\
--- \\
--- \\
--- \\
--- \\
---\end{array}$ & $\begin{array}{r}21 \\
0 \\
0 \\
0 \\
21 \\
0 \\
21 \\
0 \\
0 \\
0 \\
0 \\
126\end{array}$ \\
\hline $\begin{array}{l}\text { LINE } \\
\text { A } \\
\text { B } \\
\text { C } \\
\text { D } \\
\text { E }\end{array}$ & $\begin{array}{l}11141 \\
9238.2 \\
9243.9 \\
9251.8 \\
9268.0 \\
9282.9\end{array}$ & $\begin{array}{l}\mathrm{M} \\
\mathrm{M} \\
\mathrm{M} \\
\mathrm{M} \\
\mathrm{M}\end{array}$ & $\begin{array}{l}668807 \\
668786 \\
668781 \\
668796 \\
668771\end{array}$ & $\begin{array}{l}6139742 \\
6139914 \\
6140140 \\
6140513 \\
6140735\end{array}$ & $\begin{array}{r}9.0 \\
1.9 \\
5.8 \\
39.4 \\
6.6\end{array}$ & $\begin{array}{l}0.6 \\
0.2 \\
0.5 \\
0.3 \\
0.1\end{array}$ & $\begin{array}{r}20.5 \\
4.9 \\
0.6 \\
49.7 \\
6.6\end{array}$ & $\begin{array}{l}0.5 \\
0.4 \\
0.2 \\
0.3 \\
0.1\end{array}$ & $\begin{array}{r}17.2 \\
3.5 \\
0.3 \\
39.6 \\
5.0\end{array}$ & $\begin{array}{l}2.1 \\
1.7 \\
0.8 \\
0.3 \\
1.3\end{array}$ & $\begin{array}{l}--- \\
--- \\
--- \\
--- \\
---\end{array}$ & $\begin{array}{l}--- \\
--- \\
--- \\
--- \\
---\end{array}$ & $\begin{array}{r}460 \\
404 \\
936 \\
0 \\
49\end{array}$ \\
\hline $\begin{array}{l}\text { LINE } \\
\text { A } \\
B \\
\text { C }\end{array}$ & $\begin{array}{r}11150 \\
7520.0 \\
7513.7 \\
7504.4\end{array}$ & $\begin{array}{r}\mathrm{D} \\
\mathrm{D} \\
\mathrm{L} ?\end{array}$ & $\begin{array}{l}669988 \\
669982 \\
669954\end{array}$ & $\begin{array}{l}6118446 \\
6118606 \\
6118868\end{array}$ & $\begin{array}{l}0.9 \\
2.7 \\
4.4\end{array}$ & $\begin{array}{l}0.9 \\
1.2 \\
2.5\end{array}$ & $\begin{array}{l}0.8 \\
0.3 \\
3.4\end{array}$ & $\begin{array}{l}0.4 \\
0.2 \\
1.5\end{array}$ & $\begin{array}{l}1.5 \\
0.1 \\
2.5\end{array}$ & $\begin{array}{l}1.3 \\
1.6 \\
4.8\end{array}$ & $\begin{array}{r}5.1 \\
17.5 \\
17.8\end{array}$ & $\begin{array}{l}75 \\
68 \\
41\end{array}$ & $\begin{array}{r}0 \\
19 \\
12\end{array}$ \\
\hline
\end{tabular}

$\mathrm{CX}=$ COAXIAL

Note: EM values shown above

are local amplitudes

Ketchikan - North/South of 5515
*Estimated Depth may be unreliable because the stronger part of the conductor may be deeper or to one side of the flight line, or because of a shallow dip or magnetite/overburden effects 
EM Anomaly List

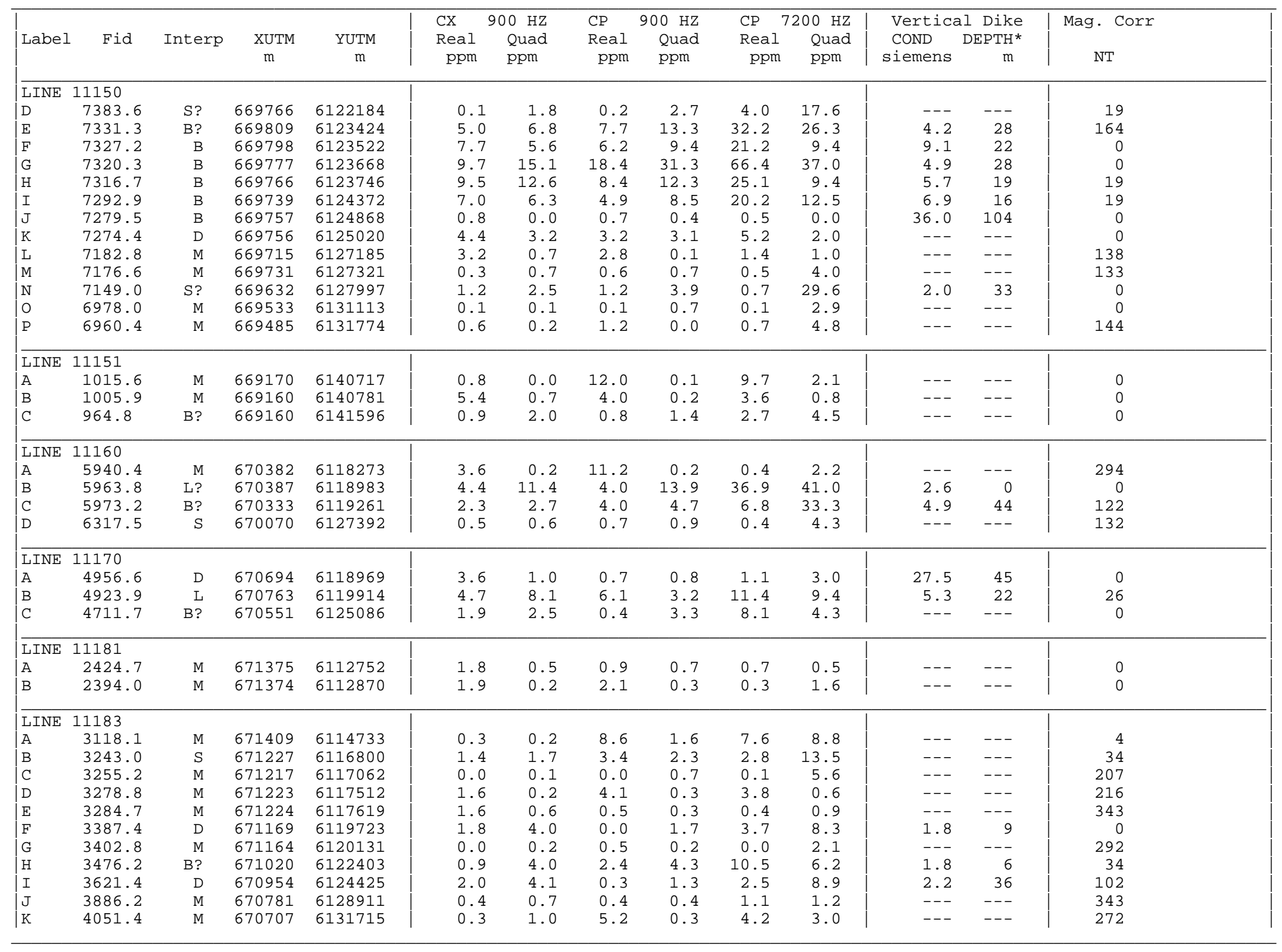

$\mathrm{CX}=$ COAXIAL

Note: EM values shown above

are local amplitudes

Ketchikan - North/South of 5515
* Estimated Depth may be unreliable because the stronger part of the conductor may be deeper or to one side of the flight line, or because of a shallow dip or magnetite/overburden effects 
EM Anomaly List

\begin{tabular}{|c|c|c|c|c|c|c|c|c|c|c|c|c|c|}
\hline Label & Fid & Interp & $\underset{\mathrm{m}}{\mathrm{XUTM}}$ & $\begin{array}{c}\text { YUTM } \\
\text { m }\end{array}$ & $\begin{array}{l}\text { CX } \\
\text { Real } \\
\text { ppm }\end{array}$ & $\begin{array}{l}00 \mathrm{HZ} \\
\text { Quad } \\
\text { ppm }\end{array}$ & $\begin{array}{l}\text { CP } \\
\text { Real } \\
\text { ppm }\end{array}$ & $\begin{array}{l}900 \mathrm{HZ} \\
\text { Quad } \\
\text { ppm }\end{array}$ & $\begin{array}{l}\text { CP } \\
\text { Real } \\
\text { ppm }\end{array}$ & $\begin{array}{l}7200 \mathrm{HZ} \\
\text { Quad } \\
\text { ppm }\end{array}$ & $\begin{array}{l}\text { Vertica } \\
\text { CoND } \\
\text { siemens }\end{array}$ & $\begin{array}{c}\text { al Dike } \\
\text { DEPTH* } \\
\text { m }\end{array}$ & $\begin{array}{c}\text { Mag. Corr } \\
\text { NT }\end{array}$ \\
\hline $\begin{array}{l}\text { LINE } \\
\text { L } \\
M\end{array}$ & $\begin{array}{c}11183 \\
4121.7 \\
4140.4\end{array}$ & $\begin{array}{l}\mathrm{M} \\
\mathrm{S}\end{array}$ & $\begin{array}{l}670635 \\
670611\end{array}$ & $\begin{array}{l}6133641 \\
6134304\end{array}$ & $\begin{array}{l}0.6 \\
0.5\end{array}$ & $\begin{array}{l}0.4 \\
2.5\end{array}$ & $\begin{array}{l}0.3 \\
0.9\end{array}$ & $\begin{array}{l}0.6 \\
3.9\end{array}$ & $\begin{array}{l}0.2 \\
1.9\end{array}$ & $\begin{array}{r}3.4 \\
22.4\end{array}$ & $\begin{array}{l}--- \\
---\end{array}$ & $\begin{array}{l}--- \\
---\end{array}$ & $\begin{array}{r}145 \\
0\end{array}$ \\
\hline $\begin{array}{l}\text { LINE } \\
A\end{array}$ & $\begin{array}{c}11184 \\
7274.4\end{array}$ & B & 670307 & 6143118 & 1.7 & 3.0 & 2.8 & 7.0 & 17.6 & 11.6 & 2.6 & 16 & 0 \\
\hline $\begin{array}{l}\text { LINE } \\
\text { A } \\
B\end{array}$ & $\begin{array}{l}11190 \\
7367.2 \\
7364.9\end{array}$ & $\begin{array}{l}\mathrm{B} \\
\mathrm{B}\end{array}$ & $\begin{array}{l}670745 \\
670732\end{array}$ & $\begin{array}{l}6143296 \\
6143388\end{array}$ & $\begin{array}{l}3.3 \\
3.9\end{array}$ & $\begin{array}{l}5.1 \\
5.3\end{array}$ & $\begin{array}{r}11.6 \\
5.2\end{array}$ & $\begin{array}{r}12.2 \\
5.1\end{array}$ & $\begin{array}{l}24.4 \\
10.0\end{array}$ & $\begin{array}{r}14.6 \\
5.5\end{array}$ & $\begin{array}{l}5.8 \\
5.4\end{array}$ & $\begin{array}{l}7 \\
7\end{array}$ & $\begin{array}{r}14 \\
0\end{array}$ \\
\hline $\begin{array}{l}\text { LINE } \\
\text { A } \\
\text { B } \\
\text { C } \\
\text { D } \\
\text { E } \\
\text { F } \\
\text { G } \\
\text { H } \\
\text { I } \\
\text { J } \\
\text { K } \\
\text { L }\end{array}$ & $\begin{array}{r}11191 \\
2672.9 \\
2406.0 \\
2311.5 \\
2185.8 \\
1745.1 \\
1720.2 \\
1714.3 \\
1418.2 \\
1347.0 \\
1322.8 \\
1313.4 \\
1291.9\end{array}$ & $\begin{array}{r}\mathrm{M} \\
\mathrm{M} \\
\mathrm{M} \\
\mathrm{B} ? \\
\mathrm{~B} \\
\mathrm{~B} ? \\
\mathrm{~B} ? \\
\mathrm{~B} ? \\
\mathrm{M} \\
\mathrm{M} \\
\mathrm{M} \\
\mathrm{M}\end{array}$ & $\begin{array}{l}671762 \\
671701 \\
671690 \\
671606 \\
671341 \\
671354 \\
671334 \\
671127 \\
671071 \\
671051 \\
671048 \\
671018\end{array}$ & $\begin{array}{l}6112640 \\
6114562 \\
6115181 \\
6117129 \\
6124423 \\
6125096 \\
6125275 \\
6130903 \\
6132382 \\
6132951 \\
6133248 \\
6133878\end{array}$ & $\begin{array}{l}8.9 \\
1.3 \\
0.9 \\
0.1 \\
2.4 \\
4.6 \\
1.3 \\
1.7 \\
0.4 \\
0.2 \\
0.2 \\
0.1\end{array}$ & $\begin{array}{l}0.0 \\
0.3 \\
0.3 \\
0.8 \\
3.7 \\
4.9 \\
2.9 \\
0.6 \\
0.6 \\
0.3 \\
0.5 \\
0.6\end{array}$ & $\begin{array}{l}5.7 \\
0.0 \\
0.2 \\
0.3 \\
1.0 \\
4.1 \\
6.7 \\
2.3 \\
1.3 \\
1.1 \\
3.6 \\
0.8\end{array}$ & $\begin{array}{l}0.0 \\
0.1 \\
0.7 \\
1.7 \\
2.5 \\
8.3 \\
6.8 \\
3.9 \\
0.4 \\
0.1 \\
0.6 \\
0.7\end{array}$ & $\begin{array}{r}6.0 \\
0.0 \\
0.4 \\
0.9 \\
6.3 \\
18.4 \\
14.4 \\
6.7 \\
1.6 \\
0.8 \\
3.3 \\
0.8\end{array}$ & $\begin{array}{r}1.9 \\
2.8 \\
0.7 \\
7.5 \\
12.4 \\
9.3 \\
7.2 \\
12.7 \\
3.3 \\
1.6 \\
3.2 \\
2.6\end{array}$ & $\begin{array}{l}--- \\
--- \\
--- \\
--- \\
2.6 \\
4.6 \\
--- \\
6.2 \\
--- \\
--- \\
--- \\
---\end{array}$ & $\begin{array}{r}--- \\
--- \\
--- \\
--- \\
52 \\
13 \\
--- \\
65 \\
--- \\
--- \\
--- \\
---\end{array}$ & $\begin{array}{r}0 \\
0 \\
0 \\
73 \\
0 \\
0 \\
28 \\
162 \\
122 \\
0 \\
156 \\
94\end{array}$ \\
\hline $\begin{array}{l}\text { LINE } \\
\text { A } \\
\text { B } \\
\text { C }\end{array}$ & $\begin{array}{r}11202 \\
3638.0 \\
3612.2 \\
3420.0\end{array}$ & $\begin{array}{l}\mathrm{M} \\
\mathrm{M} \\
\mathrm{M}\end{array}$ & $\begin{array}{l}672137 \\
672125 \\
672120\end{array}$ & $\begin{array}{l}6114567 \\
6114602 \\
6115107\end{array}$ & $\begin{array}{l}4.9 \\
5.9 \\
0.4\end{array}$ & $\begin{array}{l}0.3 \\
0.6 \\
0.7\end{array}$ & $\begin{array}{r}16.3 \\
14.6 \\
0.4\end{array}$ & $\begin{array}{l}0.2 \\
0.0 \\
0.0\end{array}$ & $\begin{array}{r}14.0 \\
12.3 \\
0.4\end{array}$ & $\begin{array}{l}0.0 \\
1.3 \\
1.7\end{array}$ & $\begin{array}{l}--- \\
--- \\
---\end{array}$ & $\begin{array}{l}--- \\
--- \\
---\end{array}$ & $\begin{array}{l}0 \\
0 \\
0\end{array}$ \\
\hline $\begin{array}{l}\text { LINE } \\
\text { A } \\
B \\
C \\
D \\
\text { E }\end{array}$ & $\begin{array}{l}11203 \\
402.0 \\
535.9 \\
542.1 \\
813.3 \\
833.9\end{array}$ & $\begin{array}{r}\mathrm{M} \\
\mathrm{S} \\
\mathrm{S} ? \\
\mathrm{M} \\
\mathrm{M}\end{array}$ & $\begin{array}{l}671972 \\
671801 \\
671819 \\
671577 \\
671567\end{array}$ & $\begin{array}{l}6119928 \\
6123951 \\
6124186 \\
6130017 \\
6130301\end{array}$ & $\begin{array}{r}1.0 \\
44.4 \\
47.3 \\
0.8 \\
0.3\end{array}$ & $\begin{array}{r}0.7 \\
51.6 \\
58.1 \\
0.1 \\
0.2\end{array}$ & $\begin{array}{r}0.0 \\
58.0 \\
64.3 \\
1.7 \\
0.0\end{array}$ & $\begin{array}{r}1.0 \\
97.3 \\
103.0 \\
0.1 \\
0.1\end{array}$ & $\begin{array}{r}0.1 \\
223.7 \\
216.0 \\
1.4 \\
1.8\end{array}$ & $\begin{array}{r}2.9 \\
96.5 \\
140.2 \\
0.5 \\
0.8\end{array}$ & $\begin{array}{r}--- \\
10.0 \\
10.1 \\
--- \\
---\end{array}$ & $\begin{array}{r}--- \\
0 \\
0 \\
--- \\
---\end{array}$ & $\begin{array}{r}0 \\
40 \\
4 \\
249 \\
201\end{array}$ \\
\hline $\begin{array}{l}\text { LINE } \\
\text { A } \\
\text { B } \\
\text { C } \\
\text { D } \\
\text { E } \\
\text { F } \\
\text { G } \\
\text { H } \\
\text { I }\end{array}$ & $\begin{array}{r}11211 \\
2535.9 \\
2474.0 \\
2462.1 \\
2449.9 \\
2442.2 \\
2436.4 \\
1915.3 \\
1806.8 \\
1676.0\end{array}$ & $\begin{array}{r}\mathrm{M} \\
\mathrm{M} \\
\mathrm{M} \\
\mathrm{M} \\
\mathrm{M} \\
\mathrm{M} \\
\mathrm{S} \\
\mathrm{B} ? \\
\mathrm{~B} ?\end{array}$ & $\begin{array}{l}672686 \\
672594 \\
672593 \\
672576 \\
672574 \\
672581 \\
672403 \\
672357 \\
672267\end{array}$ & $\begin{array}{l}6111597 \\
6112528 \\
6112632 \\
6112736 \\
6112834 \\
6112926 \\
6118293 \\
6119583 \\
6122221\end{array}$ & $\begin{array}{l}0.5 \\
0.2 \\
1.9 \\
1.4 \\
2.0 \\
0.0 \\
0.5 \\
0.2 \\
4.1\end{array}$ & $\begin{array}{l}0.8 \\
0.5 \\
0.1 \\
0.3 \\
0.9 \\
0.6 \\
0.8 \\
0.2 \\
5.4\end{array}$ & $\begin{array}{l}1.0 \\
1.7 \\
0.9 \\
3.3 \\
2.6 \\
0.7 \\
0.2 \\
5.8 \\
0.6\end{array}$ & $\begin{array}{l}1.0 \\
0.2 \\
0.6 \\
0.3 \\
0.1 \\
0.4 \\
1.1 \\
0.7 \\
5.9\end{array}$ & $\begin{array}{r}0.7 \\
1.2 \\
0.8 \\
0.5 \\
2.3 \\
0.4 \\
0.5 \\
5.0 \\
13.2\end{array}$ & $\begin{array}{r}3.0 \\
1.8 \\
1.2 \\
0.5 \\
1.3 \\
1.6 \\
8.4 \\
5.9 \\
10.1\end{array}$ & $\begin{array}{r}--- \\
--- \\
--- \\
--- \\
--- \\
--- \\
--- \\
64.6 \\
2.4\end{array}$ & $\begin{array}{r}--- \\
--- \\
--- \\
--- \\
--- \\
--- \\
--- \\
93 \\
2\end{array}$ & $\begin{array}{r}0 \\
129 \\
0 \\
0 \\
212 \\
116 \\
0 \\
29 \\
0\end{array}$ \\
\hline
\end{tabular}

$\mathrm{CX}=$ COAXIAL

Note: EM values shown above

are local amplitudes

Ketchikan - North/South of 5515
* Estimated Depth may be unreliable because the stronger part of the conductor may be deeper or to one side of the flight line, or because of a shallow dip or magnetite/overburden effects 
EM Anomaly List

\begin{tabular}{|c|c|c|c|c|c|c|c|c|c|c|c|c|c|}
\hline Label & Fid & Interp & $\begin{array}{c}\text { XUTM } \\
\text { m }\end{array}$ & $\begin{array}{c}\text { YUTM } \\
\text { m }\end{array}$ & $\begin{array}{l}\text { CX } \\
\text { Real } \\
\text { ppm }\end{array}$ & $\begin{array}{l}00 \mathrm{HZ} \\
\text { Quad } \\
\text { ppm }\end{array}$ & $\begin{array}{l}\text { CP } \\
\text { Real } \\
\text { ppm }\end{array}$ & $\begin{array}{l}900 \mathrm{HZ} \\
\text { Quad } \\
\text { ppm }\end{array}$ & $\begin{array}{l}\text { CP } \\
\text { Real } \\
\text { ppm }\end{array}$ & $\begin{array}{c}7200 \mathrm{HZ} \\
\text { Quad } \\
\text { ppm }\end{array}$ & $\begin{array}{l}\text { Vertica } \\
\text { Cond } \\
\text { siemens }\end{array}$ & $\begin{array}{c}\text { al Dike } \\
\text { DEPTH* } \\
\text { m }\end{array}$ & $\begin{array}{c}\text { Mag. Corr } \\
\text { NT }\end{array}$ \\
\hline $\begin{array}{l}\text { LINE } \\
\text { J } \\
\text { K } \\
L \\
M \\
N\end{array}$ & $\begin{array}{r}11211 \\
1608.4 \\
1596.2 \\
1324.2 \\
1309.4 \\
1288.8\end{array}$ & $\begin{array}{c}\text { S? } \\
\text { S? } \\
\text { M } \\
\text { M } \\
\text { S? }\end{array}$ & $\begin{array}{l}672168 \\
672165 \\
671921 \\
671916 \\
671916\end{array}$ & $\begin{array}{l}6124613 \\
6124966 \\
6130998 \\
6131363 \\
6131786\end{array}$ & $\begin{array}{r}27.4 \\
18.6 \\
1.9 \\
2.4 \\
2.8\end{array}$ & $\begin{array}{r}25.6 \\
8.8 \\
0.4 \\
0.3 \\
3.8\end{array}$ & $\begin{array}{r}20.4 \\
19.9 \\
1.1 \\
1.1 \\
0.2\end{array}$ & $\begin{array}{r}39.6 \\
18.6 \\
0.7 \\
0.3 \\
6.1\end{array}$ & $\begin{array}{r}102.1 \\
33.8 \\
0.5 \\
0.9 \\
6.3\end{array}$ & $\begin{array}{r}59.3 \\
18.4 \\
4.3 \\
1.1 \\
39.9\end{array}$ & $\begin{array}{r}--- \\
22.4 \\
--- \\
--- \\
2.4\end{array}$ & $\begin{array}{r}--- \\
0 \\
--- \\
--- \\
23\end{array}$ & $\begin{array}{r}42 \\
0 \\
33 \\
495 \\
0\end{array}$ \\
\hline $\begin{array}{l}\text { LINE } \\
\text { A } \\
\text { B } \\
\text { C } \\
\text { D } \\
\text { E } \\
\text { F } \\
\text { G }\end{array}$ & $\begin{array}{l}11220 \\
1255.7 \\
1223.8 \\
1214.2 \\
966.0 \\
594.0 \\
503.9 \\
458.8\end{array}$ & $\begin{array}{l}\mathrm{M} \\
\mathrm{M} \\
\mathrm{M} \\
\mathrm{B} \\
\mathrm{M} \\
\mathrm{M} \\
\mathrm{M}\end{array}$ & $\begin{array}{l}672767 \\
672818 \\
672811 \\
672689 \\
672378 \\
672326 \\
672282\end{array}$ & $\begin{array}{l}6118999 \\
6119320 \\
6119373 \\
6121307 \\
6130359 \\
6131475 \\
6132430\end{array}$ & $\begin{array}{l}0.0 \\
3.5 \\
4.2 \\
1.4 \\
8.4 \\
0.2 \\
0.1\end{array}$ & $\begin{array}{l}0.6 \\
0.2 \\
0.4 \\
4.1 \\
0.6 \\
0.3 \\
1.3\end{array}$ & $\begin{array}{r}2.3 \\
1.5 \\
2.0 \\
2.6 \\
12.5 \\
1.9 \\
1.1\end{array}$ & $\begin{array}{r}0.5 \\
0.7 \\
0.3 \\
12.0 \\
0.5 \\
0.3 \\
0.5\end{array}$ & $\begin{array}{r}1.1 \\
1.3 \\
2.1 \\
21.5 \\
9.2 \\
1.2 \\
1.2\end{array}$ & $\begin{array}{r}1.7 \\
4.1 \\
4.6 \\
34.8 \\
1.1 \\
3.1 \\
3.8\end{array}$ & $\begin{array}{l}--- \\
--- \\
--- \\
1.6 \\
--- \\
--- \\
---\end{array}$ & $\begin{array}{r}--- \\
--- \\
--- \\
12 \\
--- \\
--- \\
---\end{array}$ & $\begin{array}{r}350 \\
0 \\
0 \\
0 \\
13 \\
93 \\
86\end{array}$ \\
\hline $\begin{array}{l}\text { LINE } \\
A\end{array}$ & $\begin{array}{r}11221 \\
7695.5\end{array}$ & B & 671858 & 6143987 & 38.4 & 25.1 & 58.9 & 59.5 & 117.8 & 30.7 & 21.0 & 0 & 0 \\
\hline $\begin{array}{l}\text { LINE } \\
\text { A } \\
\text { B } \\
\text { C } \\
\text { D } \\
\text { E } \\
\text { F } \\
\text { G } \\
\text { H } \\
\text { I } \\
\text { J } \\
\text { K } \\
\text { L } \\
\text { M } \\
\text { N } \\
\text { O } \\
\text { P } \\
\text { Q } \\
\text { R }\end{array}$ & $\begin{array}{r}11230 \\
4528.7 \\
4601.2 \\
4618.7 \\
4637.2 \\
4748.5 \\
4798.7 \\
5217.5 \\
5375.0 \\
5430.0 \\
5445.6 \\
5490.5 \\
5494.6 \\
5512.3 \\
5529.5 \\
5538.9 \\
5560.0 \\
5572.0 \\
5647.2\end{array}$ & $\begin{array}{r}\text { S } \\
\text { B } \\
\text { B? } \\
\text { B? } \\
\text { M } \\
\text { D } \\
\text { B? } \\
\text { S? } \\
\text { M } \\
\text { D } \\
\text { S } \\
\text { M } \\
\text { M } \\
\text { M } \\
\text { B } \\
\text { B } \\
\text { M } \\
\text { M }\end{array}$ & $\begin{array}{l}673400 \\
673330 \\
673290 \\
673279 \\
673211 \\
673195 \\
673022 \\
672902 \\
672815 \\
672821 \\
672795 \\
672780 \\
672766 \\
672742 \\
672727 \\
672747 \\
672748 \\
672676\end{array}$ & $\begin{array}{l}6113697 \\
6115455 \\
6115616 \\
6115705 \\
6117760 \\
6118156 \\
6122165 \\
6127165 \\
6128264 \\
6128562 \\
6129340 \\
6129448 \\
6129917 \\
6130238 \\
6130403 \\
6130746 \\
6130995 \\
6132799\end{array}$ & $\begin{array}{r}1.1 \\
1.2 \\
1.9 \\
0.5 \\
0.0 \\
3.6 \\
2.0 \\
10.6 \\
0.3 \\
12.1 \\
0.7 \\
0.2 \\
8.7 \\
0.3 \\
2.7 \\
3.4 \\
0.9 \\
0.6\end{array}$ & $\begin{array}{r}2.2 \\
3.4 \\
8.6 \\
1.3 \\
0.5 \\
5.1 \\
3.5 \\
81.7 \\
0.7 \\
18.0 \\
1.9 \\
1.7 \\
1.0 \\
0.3 \\
1.4 \\
0.5 \\
0.6 \\
0.1\end{array}$ & $\begin{array}{r}1.8 \\
0.3 \\
2.6 \\
0.3 \\
3.5 \\
1.0 \\
0.1 \\
18.5 \\
1.2 \\
14.8 \\
6.6 \\
2.9 \\
18.5 \\
18.3 \\
9.4 \\
5.7 \\
6.5 \\
3.2\end{array}$ & $\begin{array}{r}1.0 \\
3.1 \\
7.4 \\
1.2 \\
0.2 \\
2.2 \\
1.6 \\
164.7 \\
1.0 \\
13.0 \\
2.4 \\
2.5 \\
0.6 \\
0.0 \\
2.0 \\
1.1 \\
0.8 \\
0.1\end{array}$ & $\begin{array}{r}1.8 \\
5.2 \\
2.1 \\
1.2 \\
2.2 \\
5.0 \\
4.1 \\
538.3 \\
1.0 \\
32.6 \\
4.4 \\
2.3 \\
14.4 \\
0.0 \\
6.8 \\
4.6 \\
5.7 \\
2.6\end{array}$ & $\begin{array}{r}9.2 \\
19.0 \\
52.7 \\
13.0 \\
3.0 \\
1.3 \\
11.4 \\
548.4 \\
3.7 \\
21.7 \\
15.3 \\
17.4 \\
4.5 \\
1.3 \\
12.3 \\
6.4 \\
3.7 \\
0.9\end{array}$ & $\begin{array}{c}3.0 \\
1.3 \\
1.7 \\
--- \\
--- \\
3.3 \\
2.3 \\
1.6 \\
--- \\
7.3 \\
8.8 \\
--- \\
--- \\
--- \\
--- \\
76.4 \\
--- \\
---\end{array}$ & $\begin{array}{r}66 \\
23 \\
29 \\
--- \\
--- \\
20 \\
27 \\
0 \\
--- \\
11 \\
47 \\
--- \\
--- \\
--- \\
--- \\
74 \\
--- \\
---\end{array}$ & $\begin{array}{r}0 \\
0 \\
21 \\
0 \\
0 \\
0 \\
38 \\
35 \\
0 \\
38 \\
0 \\
148 \\
0 \\
72 \\
38 \\
0 \\
101 \\
0\end{array}$ \\
\hline $\begin{array}{l}\text { LINE } \\
A \\
B\end{array}$ & $\begin{array}{r}11231 \\
7758.6 \\
7755.4\end{array}$ & $\begin{array}{r}\text { B } \\
\text { B? }\end{array}$ & $\begin{array}{l}672268 \\
672261\end{array}$ & $\begin{array}{l}6144192 \\
6144320\end{array}$ & $\begin{array}{l}21.6 \\
21.3\end{array}$ & $\begin{array}{l}14.1 \\
11.6\end{array}$ & $\begin{array}{l}77.3 \\
18.8\end{array}$ & $\begin{array}{l}40.3 \\
19.8\end{array}$ & $\begin{array}{l}94.0 \\
49.3\end{array}$ & $\begin{array}{r}9.7 \\
16.0\end{array}$ & $\begin{array}{l}33.7 \\
20.0\end{array}$ & $\begin{array}{l}0 \\
0\end{array}$ & $\begin{array}{r}31 \\
0\end{array}$ \\
\hline $\begin{array}{l}\text { LINE } \\
\text { A } \\
B \\
C\end{array}$ & $\begin{array}{r}11240 \\
4175.6 \\
4156.4 \\
4015.2\end{array}$ & $\begin{array}{r}\mathrm{B} ? \\
\mathrm{~B} \\
\mathrm{~B} ?\end{array}$ & $\begin{array}{l}673838 \\
673787 \\
673688\end{array}$ & $\begin{array}{l}6111306 \\
6111710 \\
6115480\end{array}$ & $\begin{array}{l}0.9 \\
2.0 \\
2.1\end{array}$ & $\begin{array}{l}2.0 \\
1.9 \\
4.7\end{array}$ & $\begin{array}{l}0.0 \\
1.5 \\
1.0\end{array}$ & $\begin{array}{l}1.8 \\
1.9 \\
4.6\end{array}$ & $\begin{array}{r}1.6 \\
5.6 \\
13.4\end{array}$ & $\begin{array}{r}8.9 \\
7.4 \\
21.2\end{array}$ & $\begin{array}{l}--- \\
--- \\
2.0\end{array}$ & $\begin{array}{r}--- \\
--- \\
8\end{array}$ & $\begin{array}{r}41 \\
0 \\
101\end{array}$ \\
\hline
\end{tabular}

$\mathrm{CX}=$ COAXIAL

Note: EM values shown above

are local amplitudes

Ketchikan - North/South of 5515
*Estimated Depth may be unreliable because the stronger part of the conductor may be deeper or to one side of the flight line, or because of a shallow dip or magnetite/overburden effects 
EM Anomaly List

\begin{tabular}{|c|c|c|c|c|c|c|c|c|c|c|c|c|c|}
\hline Label & Fid & Interp & $\begin{array}{c}\text { XUTM } \\
\text { m }\end{array}$ & $\begin{array}{c}\text { YUTM } \\
\mathrm{m}\end{array}$ & $\begin{array}{l}\text { CX } \\
\text { Real } \\
\text { ppm }\end{array}$ & $\begin{array}{l}900 \mathrm{HZ} \\
\text { Quad } \\
\text { ppm }\end{array}$ & $\begin{array}{l}\text { CP } \\
\text { Real } \\
\text { ppm }\end{array}$ & $\begin{array}{c}900 \mathrm{HZ} \\
\text { Quad } \\
\text { ppm }\end{array}$ & $\begin{array}{l}\text { CP }{ }^{7} \\
\text { Real } \\
\text { ppm }\end{array}$ & $\begin{array}{c}7200 \mathrm{~Hz} \\
\text { Quad } \\
\text { ppm }\end{array}$ & $\begin{array}{l}\text { Vertica } \\
\text { CoND } \\
\text { siemens }\end{array}$ & $\begin{array}{c}\text { al Dike } \\
\text { DEPTH* } \\
\text { m }\end{array}$ & $\begin{array}{c}\text { Mag. Corr } \\
\text { NT }\end{array}$ \\
\hline LINE & 11240 & & & & & & & & & & & & \\
\hline D & 3790.5 & M & 673644 & 6117945 & 1.7 & 0.4 & 0.7 & 0.8 & 0.9 & 3.9 & --- & --- & 0 \\
\hline $\mathrm{E}$ & 3773.0 & $\mathrm{~S}$ & 673635 & 6118172 & 0.9 & 1.4 & 5.2 & 2.0 & 5.7 & 11.0 & --- & --- & 0 \\
\hline $\mathrm{F}$ & 3695.5 & M & 673567 & 6120051 & 1.3 & 0.4 & 1.8 & 0.2 & 1.5 & 4.2 & --- & --- & 41 \\
\hline G & 3674.8 & M & 673554 & 6120315 & 0.1 & 0.4 & 0.9 & 0.3 & 0.8 & 2.4 & --- & --- & 0 \\
\hline $\mathrm{H}$ & 3665.9 & M & 673550 & 6120431 & 0.0 & 0.3 & 0.2 & 0.2 & 0.6 & 2.2 & --- & --- & 0 \\
\hline I & 3622.9 & M & 673453 & 6120937 & 0.8 & 0.9 & 1.1 & 0.6 & 0.3 & 5.9 & --- & --- & 41 \\
\hline $\mathrm{J}$ & 3572.0 & B? & 673485 & 6121813 & 7.9 & 19.6 & 14.8 & 33.8 & 88.6 & 70.1 & 4.0 & 0 & 41 \\
\hline $\mathrm{K}$ & 3567.5 & $B$ ? & 673468 & 6121947 & 23.8 & 35.6 & 42.2 & 75.1 & 161.1 & 60.4 & 6.5 & 0 & 0 \\
\hline L & 3532.1 & L? & 673444 & 6123021 & 2.6 & 3.6 & 1.2 & 1.1 & 0.2 & 11.0 & 3.8 & 51 & 50 \\
\hline M & 3503.0 & D & 673420 & 6123840 & 18.0 & 20.5 & 19.7 & 26.9 & 61.3 & 38.1 & 8.6 & 0 & 0 \\
\hline $\mathrm{N}$ & 3498.1 & $B$ ? & 673428 & 6124008 & 3.4 & 5.7 & 5.7 & 5.4 & 13.5 & 8.0 & 4.4 & 23 & 41 \\
\hline 0 & 3491.2 & S? & 673427 & 6124239 & 2.6 & 2.0 & 0.9 & 2.3 & 4.9 & 14.9 & 5.4 & 49 & 0 \\
\hline P & 3372.4 & M & 673253 & 6127605 & 2.5 & 0.6 & 0.7 & 0.6 & 0.1 & 0.2 & --- & --- & 317 \\
\hline Q & 3334.0 & M & 673241 & 6128344 & 0.5 & 0.3 & 7.3 & 0.7 & 5.1 & 2.8 & --- & --- & 0 \\
\hline $\mathrm{R}$ & 3315.3 & S? & 673251 & 6128735 & 1.2 & 0.9 & 7.5 & 1.8 & 8.1 & 10.0 & --- & --- & 0 \\
\hline $\mathrm{S}$ & 3303.7 & M & 673220 & 6128904 & 0.6 & 0.5 & 0.2 & 0.6 & 0.1 & 0.3 & --- & --- & 0 \\
\hline $\mathrm{T}$ & 3279.9 & $\mathrm{~s}$ & 673202 & 6129395 & 10.5 & 4.2 & 27.7 & 6.6 & 27.0 & 43.3 & --- & --- & 0 \\
\hline U & 3175.6 & S? & 673101 & 6132168 & 0.1 & 2.4 & 1.0 & 2.9 & 2.3 & 19.2 & --- & --- & 0 \\
\hline V & 3144.5 & $\mathrm{M}$ & 673090 & 6132879 & 0.9 & 0.4 & 0.8 & 0.3 & 1.1 & 1.5 & --- & --- & 0 \\
\hline LINE & 11242 & & & & & & & & & & & & \\
\hline A & 427.7 & M & 672678 & 6143262 & 0.4 & 0.8 & 1.4 & 0.9 & 1.5 & 6.0 & --- & --- & 86 \\
\hline B & 442.8 & $\mathrm{~S}$ & 672667 & 6143808 & 2.1 & 1.3 & 1.9 & 1.1 & 1.9 & 10.2 & --- & --- & 19 \\
\hline LINE & 11250 & & & & & & & & & & & & \\
\hline A & 1976.2 & $B$ ? & 674268 & 6110912 & 0.2 & 2.5 & 1.4 & 1.6 & 0.6 & 11.8 & 1.2 & 44 & 0 \\
\hline B & 2165.8 & $B$ ? & 674095 & 6115398 & 3.3 & 4.4 & 2.3 & 7.3 & 22.4 & 25.7 & --- & --- & 0 \\
\hline C & 2351.9 & $B$ ? & 674058 & 6116869 & 3.9 & 0.1 & 0.5 & 0.1 & 0.5 & 1.0 & 889.4 & 49 & 40 \\
\hline D & 2382.4 & M & 674031 & 6118061 & 0.0 & 1.0 & 1.8 & 0.9 & 1.1 & 5.3 & --- & --- & 159 \\
\hline $\mathrm{E}$ & 2410.9 & M & 673999 & 6119164 & 0.2 & 0.0 & 31.1 & 0.4 & 26.4 & 2.4 & --- & --- & 630 \\
\hline $\mathrm{F}$ & 2464.1 & $\mathrm{~S}$ & 673889 & 6120640 & 0.2 & 1.2 & 0.4 & 2.1 & 0.8 & 13.2 & --- & --- & 40 \\
\hline G & 2526.4 & S? & 673850 & 6122506 & 1.1 & 1.7 & 1.0 & 2.8 & 0.3 & 21.8 & --- & --- & 40 \\
\hline $\mathrm{H}$ & 2537.0 & L & 673772 & 6122829 & 1.6 & 3.4 & 0.2 & 3.2 & 3.2 & 19.1 & 1.6 & 16 & 0 \\
\hline I & 2575.0 & B & 673805 & 6123627 & 31.8 & 38.2 & 9.2 & 35.7 & 84.0 & 91.1 & 6.6 & 12 & 40 \\
\hline $\mathrm{J}$ & 2580.2 & B & 673792 & 6123764 & 24.1 & 32.2 & 48.3 & 78.8 & 166.0 & 122.7 & 7.7 & 0 & 0 \\
\hline $\mathrm{K}$ & 2597.6 & D & 673803 & 6124281 & 7.2 & 7.3 & 1.9 & 4.7 & 8.4 & 19.4 & 5.9 & 28 & 0 \\
\hline $\mathrm{L}$ & 2774.1 & M & 673667 & 6127332 & 0.0 & 0.9 & 0.3 & 0.8 & 0.2 & 4.7 & --- & --- & 118 \\
\hline M & 2788.5 & $\mathrm{~s}$ & 673664 & 6127625 & 0.9 & 0.9 & 1.0 & 1.8 & 1.6 & 10.9 & --- & --- & 40 \\
\hline $\mathrm{N}$ & 2809.9 & M & 673640 & 6128011 & 1.9 & 0.4 & 3.7 & 0.1 & 3.8 & 0.8 & --- & --- & 125 \\
\hline 0 & 2816.8 & M & 673661 & 6128156 & 1.3 & 0.9 & 5.2 & 0.4 & 4.5 & 1.4 & --- & --- & 23 \\
\hline $\mathrm{P}$ & 2826.3 & M & 673670 & 6128389 & 0.0 & 0.7 & 8.2 & 1.2 & 7.3 & 5.0 & --- & --- & 0 \\
\hline Q & 2838.7 & M & 673647 & 6128611 & 1.9 & 1.0 & 8.2 & 0.6 & 8.9 & 2.8 & --- & --- & 64 \\
\hline $\mathrm{R}$ & 2858.7 & S? & 673603 & 6129095 & 0.4 & 2.1 & 0.5 & 2.6 & 1.4 & 18.8 & --- & --- & 38 \\
\hline $\mathrm{S}$ & 2883.6 & S? & 673539 & 6129875 & 1.1 & 0.9 & 0.7 & 1.7 & 1.6 & 8.8 & --- & --- & 0 \\
\hline $\mathrm{T}$ & 2894.4 & M & 673520 & 6130193 & 3.4 & 0.4 & 0.8 & 1.1 & 1.4 & 5.6 & --- & --- & 85 \\
\hline U & 2906.8 & M & 673500 & 6130376 & 2.6 & 0.7 & 5.0 & 1.4 & 1.3 & 4.2 & --- & --- & 338 \\
\hline
\end{tabular}

$\mathrm{CX}=$ COAXIAL

Note: EM values shown above

are local amplitudes

Ketchikan - North/South of 5515
* Estimated Depth may be unreliable because the stronger part of the conductor may be deeper or to one side of the flight line, or because of a shallow dip or magnetite/overburden effects 
EM Anomaly List

\begin{tabular}{|c|c|c|c|c|c|c|c|c|c|c|c|c|c|}
\hline Label & Fid & Interp & $\begin{array}{c}\text { XUTM } \\
\text { m }\end{array}$ & $\begin{array}{c}\text { YUTM } \\
\text { m }\end{array}$ & $\begin{array}{l}\text { CX } \\
\text { Real } \\
\text { ppm }\end{array}$ & $\begin{array}{l}00 \mathrm{HZ} \\
\text { Quad } \\
\text { ppm }\end{array}$ & $\begin{array}{l}\text { CP } \\
\text { Real } \\
\text { ppm }\end{array}$ & $\begin{array}{l}900 \mathrm{HZ} \\
\text { Quad } \\
\text { ppm }\end{array}$ & $\begin{array}{l}\text { CP } 7 \\
\text { Real } \\
\text { ppm }\end{array}$ & $\begin{array}{l}200 \mathrm{HZ} \\
\text { Quad } \\
\text { ppm }\end{array}$ & $\begin{array}{l}\text { Vertica } \\
\text { Cond } \\
\text { siemens }\end{array}$ & $\begin{array}{c}\text { al Dike } \\
\text { DEPTH* } \\
\text { m }\end{array}$ & $\begin{array}{c}\text { Mag. Corr } \\
\text { NT }\end{array}$ \\
\hline $\begin{array}{l}\mathrm{LINE} \\
\mathrm{V}\end{array}$ & $\begin{array}{r}11250 \\
2936.6\end{array}$ & M & 673534 & 6131206 & 0.1 & 0.9 & 1.4 & 1.3 & 3.1 & 10.0 & --- & --- & 89 \\
\hline $\begin{array}{l}\text { LINE } \\
\text { A } \\
B\end{array}$ & $\begin{array}{r}11251 \\
8072.2 \\
8056.4\end{array}$ & $\begin{array}{l}\mathrm{M} \\
\mathrm{M}\end{array}$ & $\begin{array}{l}673130 \\
673121\end{array}$ & $\begin{array}{l}6142775 \\
6143340\end{array}$ & $\begin{array}{l}2.6 \\
0.1\end{array}$ & $\begin{array}{l}0.4 \\
0.1\end{array}$ & $\begin{array}{l}3.2 \\
0.5\end{array}$ & $\begin{array}{l}0.3 \\
0.4\end{array}$ & $\begin{array}{l}2.5 \\
0.3\end{array}$ & $\begin{array}{l}0.0 \\
1.3\end{array}$ & $\begin{array}{l}--- \\
---\end{array}$ & $\begin{array}{l}--- \\
---\end{array}$ & $\begin{array}{r}0 \\
65\end{array}$ \\
\hline $\begin{array}{l}\text { LINE } \\
\text { A } \\
\text { B } \\
\text { C } \\
\text { D } \\
\text { E } \\
\text { F } \\
\text { G } \\
\text { H } \\
\text { I } \\
\text { J } \\
\text { K } \\
\text { L } \\
\text { M } \\
\text { N } \\
\text { O } \\
\text { P } \\
\text { Q } \\
\text { R } \\
\text { S } \\
\text { T } \\
\text { U } \\
\text { V } \\
\text { W } \\
X \\
Y \\
\text { Z }\end{array}$ & $\begin{array}{c}11260 \\
1369.5 \\
1366.0 \\
1340.1 \\
1337.2 \\
1290.4 \\
1063.9 \\
1054.8 \\
1050.0 \\
1041.2 \\
955.9 \\
927.3 \\
907.4 \\
887.9 \\
874.0 \\
857.8 \\
753.3 \\
722.9 \\
683.0 \\
671.5 \\
607.6 \\
580.2 \\
578.8 \\
565.3 \\
529.2 \\
495.7 \\
477.9\end{array}$ & 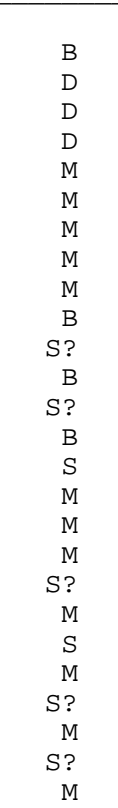 & $\begin{array}{l}674533 \\
674538 \\
674477 \\
674477 \\
674459 \\
674327 \\
674300 \\
674295 \\
674309 \\
674217 \\
674181 \\
674219 \\
674184 \\
674167 \\
674182 \\
674060 \\
674046 \\
674029 \\
674031 \\
674001 \\
673930 \\
673927 \\
673902 \\
673944 \\
673869 \\
673871\end{array}$ & $\begin{array}{l}6114981 \\
6115072 \\
6115489 \\
6115536 \\
6116804 \\
6118830 \\
6119114 \\
6119268 \\
6119555 \\
6122366 \\
6123176 \\
6123609 \\
6124135 \\
6124379 \\
6124734 \\
6127570 \\
6128182 \\
6128598 \\
6128781 \\
6129972 \\
6130788 \\
6130825 \\
6131125 \\
6131841 \\
6132450 \\
6132779\end{array}$ & $\begin{array}{l}4.2 \\
3.2 \\
2.9 \\
1.7 \\
1.4 \\
0.4 \\
0.6 \\
9.3 \\
0.1 \\
1.2 \\
0.1 \\
9.8 \\
7.8 \\
1.6 \\
0.2 \\
1.0 \\
1.8 \\
5.5 \\
3.1 \\
1.9 \\
2.6 \\
1.8 \\
0.3 \\
0.2 \\
1.3 \\
3.1\end{array}$ & $\begin{array}{l}4.8 \\
5.2 \\
5.5 \\
5.7 \\
0.4 \\
0.2 \\
0.5 \\
0.5 \\
0.3 \\
3.4 \\
4.7 \\
8.4 \\
6.0 \\
1.6 \\
1.6 \\
0.6 \\
0.4 \\
0.5 \\
0.9 \\
0.3 \\
1.4 \\
0.8 \\
1.0 \\
0.8 \\
2.2 \\
0.4\end{array}$ & $\begin{array}{r}11.4 \\
3.0 \\
0.2 \\
0.4 \\
1.6 \\
1.6 \\
0.7 \\
16.4 \\
0.2 \\
0.7 \\
0.3 \\
1.9 \\
0.2 \\
3.1 \\
0.3 \\
1.8 \\
0.1 \\
0.2 \\
2.8 \\
1.5 \\
3.9 \\
0.4 \\
0.6 \\
0.5 \\
0.3 \\
3.0\end{array}$ & $\begin{array}{r}11.0 \\
5.0 \\
5.6 \\
3.5 \\
1.1 \\
2.2 \\
1.2 \\
0.5 \\
0.7 \\
1.6 \\
3.6 \\
6.3 \\
2.7 \\
2.7 \\
3.2 \\
0.7 \\
2.2 \\
0.8 \\
0.6 \\
0.2 \\
0.5 \\
1.1 \\
1.4 \\
0.7 \\
3.5 \\
0.3\end{array}$ & $\begin{array}{r}26.0 \\
12.1 \\
4.5 \\
2.2 \\
1.0 \\
1.0 \\
0.3 \\
13.1 \\
6.4 \\
1.7 \\
0.3 \\
14.5 \\
7.0 \\
4.8 \\
0.6 \\
1.4 \\
0.6 \\
0.8 \\
1.9 \\
1.3 \\
2.9 \\
0.2 \\
0.9 \\
0.3 \\
6.0 \\
4.7\end{array}$ & $\begin{array}{r}17.7 \\
13.8 \\
34.6 \\
22.9 \\
3.0 \\
12.9 \\
7.3 \\
2.6 \\
5.3 \\
11.8 \\
20.1 \\
12.9 \\
10.6 \\
14.8 \\
17.0 \\
4.5 \\
12.4 \\
4.7 \\
5.7 \\
0.0 \\
8.1 \\
8.1 \\
7.3 \\
4.0 \\
15.7 \\
1.0\end{array}$ & $\begin{array}{c}7.7 \\
3.1 \\
2.1 \\
1.4 \\
--- \\
--- \\
--- \\
---- \\
--- \\
--- \\
0.5 \\
7.5 \\
0.5 \\
---- \\
--- \\
--- \\
--- \\
--- \\
43.5 \\
--- \\
--- \\
--- \\
--- \\
--- \\
--- \\
---\end{array}$ & $\begin{array}{r}17 \\
33 \\
28 \\
23 \\
--- \\
--- \\
--- \\
--- \\
--- \\
--- \\
5 \\
19 \\
0 \\
--- \\
--- \\
--- \\
--- \\
--- \\
60 \\
--- \\
--- \\
--- \\
--- \\
--- \\
--- \\
---\end{array}$ & $\begin{array}{r}17 \\
0 \\
0 \\
39 \\
506 \\
39 \\
752 \\
0 \\
0 \\
0 \\
39 \\
0 \\
0 \\
0 \\
0 \\
27 \\
32 \\
0 \\
0 \\
307 \\
39 \\
44 \\
0 \\
140 \\
0 \\
347\end{array}$ \\
\hline $\begin{array}{l}\text { LINE } \\
\text { A } \\
B\end{array}$ & $\begin{array}{r}11261 \\
8139.9 \\
8150.8\end{array}$ & $\begin{array}{l}\mathrm{B} ? \\
\mathrm{M}\end{array}$ & $\begin{array}{l}673564 \\
673520\end{array}$ & $\begin{array}{l}6143307 \\
6143756\end{array}$ & $\begin{array}{r}21.0 \\
1.4\end{array}$ & $\begin{array}{r}16.5 \\
0.3\end{array}$ & $\begin{array}{r}72.8 \\
2.6\end{array}$ & $\begin{array}{r}44.6 \\
0.4\end{array}$ & $\begin{array}{r}97.8 \\
1.6\end{array}$ & $\begin{array}{r}16.5 \\
1.9\end{array}$ & $\begin{array}{r}25.8 \\
---\end{array}$ & $\begin{array}{r}0 \\
---\end{array}$ & $\begin{array}{l}57 \\
52\end{array}$ \\
\hline $\begin{array}{l}\text { LINE } \\
A\end{array}$ & $\begin{array}{r}11271 \\
8522.2\end{array}$ & B & 674990 & 6114153 & 3.3 & 9.8 & 2.9 & 14.9 & 53.0 & 50.7 & --- & --- & 0 \\
\hline $\begin{array}{l}\text { LINE } \\
\text { A } \\
B \\
C\end{array}$ & $\begin{array}{r}11272 \\
8863.0 \\
8884.0 \\
8895.8\end{array}$ & $\begin{array}{r}\mathrm{B} ? \\
\mathrm{D} \\
\mathrm{D}\end{array}$ & $\begin{array}{l}674941 \\
674935 \\
674921\end{array}$ & $\begin{array}{l}6114098 \\
6114740 \\
6115008\end{array}$ & $\begin{array}{l}3.3 \\
9.6 \\
3.5\end{array}$ & $\begin{array}{r}6.4 \\
12.6 \\
13.6\end{array}$ & $\begin{array}{r}1.1 \\
10.2 \\
1.6\end{array}$ & $\begin{array}{r}9.4 \\
17.0 \\
13.6\end{array}$ & $\begin{array}{l}31.4 \\
42.3 \\
31.9\end{array}$ & $\begin{array}{l}37.2 \\
26.7 \\
60.2\end{array}$ & $\begin{array}{l}2.2 \\
5.4 \\
1.5\end{array}$ & $\begin{array}{r}3 \\
5 \\
12\end{array}$ & $\begin{array}{r}0 \\
0 \\
54\end{array}$ \\
\hline
\end{tabular}

$\mathrm{CX}=$ COAXIAL

Note: EM values shown above

Ketchikan - North/South of 5515
* Estimated Depth may be unreliable because the stronger part of the conductor may be deeper or to one side of the flight line, or because of a shallow dip or magnetite/overburden effects 
EM Anomaly List

\begin{tabular}{|c|c|c|c|c|c|c|c|c|c|c|c|c|c|}
\hline Label & Fid & Interp & $\begin{array}{l}\text { XUTM } \\
\text { m }\end{array}$ & $\begin{array}{c}\text { YUTM } \\
\mathrm{m}\end{array}$ & $\begin{array}{l}\text { CX } \\
\text { Real } \\
\text { ppm }\end{array}$ & $\begin{array}{c}900 \mathrm{HZ} \\
\text { Quad } \\
\text { ppm }\end{array}$ & $\begin{array}{l}\text { CP } \\
\text { Real } \\
\text { ppm }\end{array}$ & $\begin{array}{c}900 \mathrm{HZ} \\
\text { Quad } \\
\text { ppm }\end{array}$ & $\begin{array}{l}\text { CP }{ }^{7} \\
\text { Real } \\
\text { ppm }\end{array}$ & $\begin{array}{l}200 \mathrm{HZ} \\
\text { Quad } \\
\text { ppm }\end{array}$ & $\begin{array}{l}\text { Vertica } \\
\text { COND } \\
\text { siemens }\end{array}$ & $\begin{array}{c}\text { al Dike } \\
\text { DEPTH* } \\
\text { m }\end{array}$ & $\begin{array}{c}\text { Mag. Corr } \\
\text { NT }\end{array}$ \\
\hline $\begin{array}{l}\text { LINE } \\
\text { A } \\
B \\
C\end{array}$ & $\begin{array}{r}11273 \\
9253.9 \\
9179.9 \\
9171.9\end{array}$ & $\begin{array}{l}\mathrm{B} ? \\
\mathrm{M} \\
\mathrm{M}\end{array}$ & $\begin{array}{l}674824 \\
674775 \\
674777\end{array}$ & $\begin{array}{l}6117955 \\
6118940 \\
6119077\end{array}$ & $\begin{array}{l}1.8 \\
1.4 \\
0.7\end{array}$ & $\begin{array}{l}0.1 \\
0.4 \\
0.3\end{array}$ & $\begin{array}{l}0.9 \\
0.6 \\
0.0\end{array}$ & $\begin{array}{l}0.6 \\
0.8 \\
0.2\end{array}$ & $\begin{array}{l}0.8 \\
0.3 \\
0.2\end{array}$ & $\begin{array}{l}7.6 \\
3.8 \\
1.1\end{array}$ & $\begin{array}{r}50.5 \\
--- \\
---\end{array}$ & $\begin{array}{l}107 \\
--- \\
---\end{array}$ & $\begin{array}{r}8 \\
0 \\
41\end{array}$ \\
\hline $\begin{array}{l}\text { LINE } \\
\text { A } \\
\text { B } \\
\text { C } \\
\text { D } \\
\text { E } \\
\text { F } \\
\text { G } \\
\text { H } \\
\text { I } \\
\text { J } \\
\text { K } \\
\text { L } \\
\text { M } \\
\text { N } \\
\text { O } \\
\text { P } \\
\text { Q } \\
\text { R }\end{array}$ & $\begin{array}{r}11274 \\
9486.6 \\
9509.4 \\
9615.5 \\
9623.2 \\
9647.2 \\
9703.9 \\
9709.0 \\
9719.2 \\
9734.1 \\
9746.3 \\
9756.8 \\
9773.1 \\
9776.8 \\
9785.9 \\
9795.5 \\
9804.5 \\
9812.5 \\
9821.6\end{array}$ & $\begin{array}{r}\mathrm{L} \\
\mathrm{B} ? \\
\mathrm{M} \\
\mathrm{M} \\
\mathrm{M} \\
\mathrm{S} ? \\
\mathrm{M} \\
\mathrm{M} \\
\mathrm{M} \\
\mathrm{M} \\
\mathrm{M} \\
\mathrm{M} \\
\mathrm{M} \\
\mathrm{M} \\
\mathrm{M} \\
\mathrm{M} \\
\mathrm{S} ? \\
\mathrm{M}\end{array}$ & $\begin{array}{l}674579 \\
674582 \\
674407 \\
674402 \\
674447 \\
674402 \\
674405 \\
674424 \\
674385 \\
674350 \\
674349 \\
674355 \\
674351 \\
674338 \\
674309 \\
674287 \\
674261 \\
674247\end{array}$ & $\begin{array}{l}6123930 \\
6124673 \\
6127856 \\
6127970 \\
6128514 \\
6129724 \\
6129850 \\
6130111 \\
6130579 \\
6130962 \\
6131281 \\
6131684 \\
6131783 \\
6132050 \\
6132256 \\
6132391 \\
6132501 \\
6132619\end{array}$ & $\begin{array}{r}4.8 \\
2.4 \\
0.1 \\
0.6 \\
1.4 \\
0.1 \\
1.5 \\
0.4 \\
2.0 \\
4.1 \\
1.9 \\
0.3 \\
2.9 \\
2.2 \\
1.1 \\
0.0 \\
32.0 \\
5.8\end{array}$ & $\begin{array}{r}10.6 \\
3.0 \\
0.3 \\
0.1 \\
0.5 \\
3.0 \\
0.9 \\
0.6 \\
0.3 \\
0.5 \\
0.0 \\
0.3 \\
0.8 \\
0.6 \\
0.1 \\
0.3 \\
3.1 \\
0.2\end{array}$ & $\begin{array}{r}2.8 \\
1.7 \\
3.0 \\
0.8 \\
1.0 \\
7.8 \\
3.3 \\
0.9 \\
3.5 \\
5.5 \\
11.1 \\
6.4 \\
2.9 \\
8.1 \\
1.1 \\
13.7 \\
19.7 \\
2.4\end{array}$ & $\begin{array}{l}8.2 \\
1.9 \\
0.3 \\
0.4 \\
0.4 \\
3.3 \\
3.1 \\
0.4 \\
2.1 \\
0.6 \\
0.2 \\
0.3 \\
0.2 \\
0.8 \\
0.3 \\
0.0 \\
4.7 \\
0.0\end{array}$ & $\begin{array}{r}21.7 \\
2.3 \\
2.6 \\
0.2 \\
0.1 \\
7.7 \\
5.5 \\
0.5 \\
2.5 \\
4.4 \\
8.3 \\
5.4 \\
1.9 \\
6.3 \\
0.7 \\
11.3 \\
18.6 \\
2.3\end{array}$ & $\begin{array}{r}24.9 \\
10.8 \\
1.3 \\
3.0 \\
2.3 \\
24.5 \\
21.5 \\
1.1 \\
14.4 \\
4.8 \\
0.0 \\
1.3 \\
0.7 \\
5.2 \\
1.0 \\
2.5 \\
27.8 \\
6.1\end{array}$ & $\begin{array}{r}3.0 \\
4.3 \\
--- \\
--- \\
--- \\
4.9 \\
--- \\
--- \\
--- \\
--- \\
--- \\
--- \\
--- \\
--- \\
--- \\
--- \\
217.0 \\
---\end{array}$ & $\begin{array}{r}0 \\
49 \\
--- \\
--- \\
--- \\
48 \\
--- \\
--- \\
--- \\
--- \\
--- \\
--- \\
--- \\
--- \\
--- \\
--- \\
17 \\
---\end{array}$ & $\begin{array}{r}45 \\
0 \\
115 \\
0 \\
0 \\
35 \\
214 \\
160 \\
19 \\
178 \\
55 \\
112 \\
0 \\
0 \\
566 \\
0 \\
43 \\
76\end{array}$ \\
\hline $\begin{array}{l}\text { LINE } \\
\text { A }\end{array}$ & $\begin{array}{r}11275 \\
8254.9\end{array}$ & $\mathrm{M}$ & 673947 & 6142446 & 0.0 & 0.9 & 1.4 & 1.4 & 1.1 & 1.3 & --- & --- & 23 \\
\hline $\begin{array}{l}\text { LINE } \\
\text { A } \\
\text { B } \\
\text { C } \\
\text { D } \\
\text { E } \\
\text { F } \\
\text { G } \\
\text { H } \\
\text { I } \\
\text { J } \\
\text { K } \\
\text { L }\end{array}$ & $\begin{array}{r}11280 \\
7916.0 \\
7901.2 \\
7599.7 \\
7596.5 \\
7454.1 \\
7441.3 \\
7424.0 \\
7402.1 \\
7219.2 \\
7007.3 \\
6938.7 \\
6933.9\end{array}$ & $\begin{array}{c}\mathrm{M} \\
\mathrm{M} \\
\mathrm{S} \\
\mathrm{B} ? \\
\mathrm{M} \\
\mathrm{M} \\
\mathrm{M} \\
\mathrm{M} \\
\mathrm{B} ? \\
\mathrm{M} \\
\mathrm{M} \\
\mathrm{M}\end{array}$ & $\begin{array}{l}675446 \\
675432 \\
675332 \\
675326 \\
675215 \\
675218 \\
675222 \\
675217 \\
675000 \\
674772 \\
674709 \\
674676\end{array}$ & $\begin{array}{l}6110546 \\
6110798 \\
6114453 \\
6114560 \\
6117891 \\
6118062 \\
6118251 \\
6118418 \\
6124261 \\
6130387 \\
6132136 \\
6132239\end{array}$ & $\begin{array}{r}3.1 \\
0.0 \\
35.4 \\
25.0 \\
0.8 \\
4.5 \\
0.9 \\
1.0 \\
0.7 \\
0.3 \\
1.5 \\
6.4\end{array}$ & $\begin{array}{r}0.9 \\
0.7 \\
24.1 \\
24.1 \\
0.2 \\
0.5 \\
0.1 \\
0.5 \\
1.7 \\
0.2 \\
0.6 \\
0.0\end{array}$ & $\begin{array}{r}2.4 \\
1.5 \\
69.6 \\
35.0 \\
0.4 \\
8.2 \\
0.5 \\
1.0 \\
2.2 \\
0.9 \\
1.1 \\
0.4\end{array}$ & $\begin{array}{r}0.6 \\
0.8 \\
52.1 \\
48.4 \\
0.4 \\
0.3 \\
0.2 \\
0.3 \\
1.2 \\
0.2 \\
0.5 \\
0.4\end{array}$ & $\begin{array}{r}2.3 \\
1.2 \\
99.0 \\
98.2 \\
0.2 \\
6.4 \\
1.0 \\
0.2 \\
4.1 \\
0.8 \\
0.6 \\
9.4\end{array}$ & $\begin{array}{r}3.9 \\
2.2 \\
59.4 \\
59.5 \\
0.8 \\
1.2 \\
1.5 \\
0.4 \\
4.5 \\
3.6 \\
2.2 \\
1.5\end{array}$ & $\begin{array}{c}--- \\
--- \\
--- \\
11.0 \\
--- \\
--- \\
--- \\
--- \\
3.0 \\
--- \\
--- \\
---\end{array}$ & $\begin{array}{r}--- \\
--- \\
--- \\
0 \\
--- \\
--- \\
--- \\
--- \\
64 \\
--- \\
--- \\
---\end{array}$ & $\begin{array}{r}0 \\
153 \\
0 \\
0 \\
637 \\
0 \\
222 \\
0 \\
40 \\
604 \\
420 \\
0\end{array}$ \\
\hline $\begin{array}{l}\text { LINE } \\
\text { A } \\
B \\
C\end{array}$ & $\begin{array}{r}11290 \\
5708.8 \\
6166.5 \\
6182.8\end{array}$ & $\begin{array}{r}\text { S } \\
B \\
S ?\end{array}$ & $\begin{array}{l}675939 \\
675762 \\
675720\end{array}$ & $\begin{array}{l}6109389 \\
6114230 \\
6114642\end{array}$ & $\begin{array}{r}0.2 \\
33.2 \\
28.0\end{array}$ & $\begin{array}{r}1.1 \\
22.4 \\
15.4\end{array}$ & $\begin{array}{r}0.6 \\
87.0 \\
63.6\end{array}$ & $\begin{array}{r}1.4 \\
59.9 \\
36.5\end{array}$ & $\begin{array}{r}0.2 \\
114.1 \\
82.8\end{array}$ & $\begin{array}{l}11.2 \\
45.3 \\
32.8\end{array}$ & $\begin{array}{r}--- \\
28.0 \\
---\end{array}$ & $\begin{array}{r}--- \\
0 \\
---\end{array}$ & $\begin{array}{r}33 \\
0 \\
0\end{array}$ \\
\hline
\end{tabular}

$\mathrm{CX}=$ COAXIAL

Note: EM values shown above

Ketchikan - North/South of 5515
* Estimated Depth may be unreliable because the stronger part of the conductor may be deeper or to one side of the flight line, or because of shallow dip or magnetite/overburden effects 
EM Anomaly List

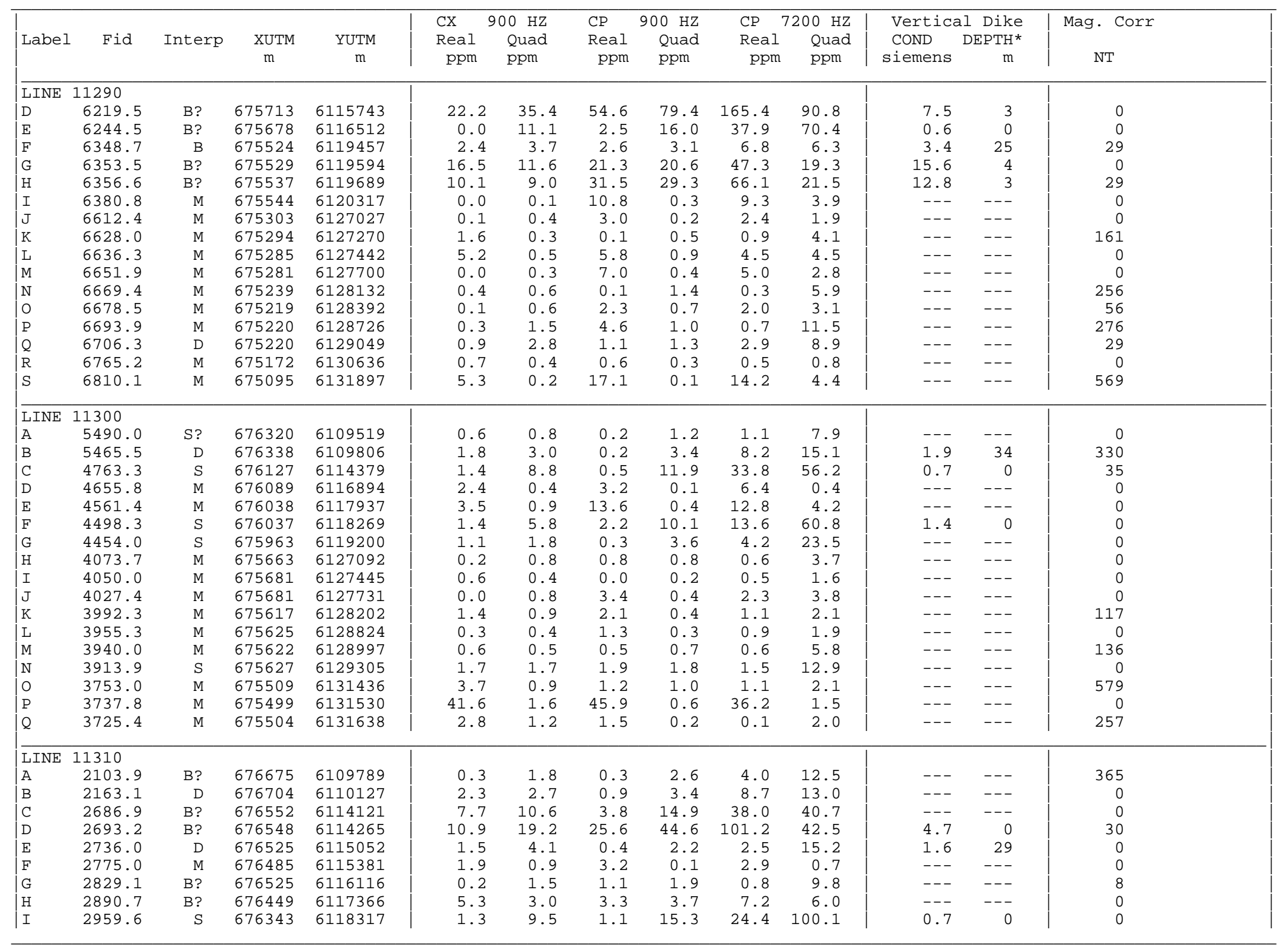

$\mathrm{CX}=$ COAXIAL

Note: EM values shown above

are local amplitudes

Ketchikan - North/South of 5515
* Estimated Depth may be unreliable because the stronger part of the conductor may be deeper or to one side of the flight line, or because of a shallow dip or magnetite/overburden effects 
EM Anomaly List

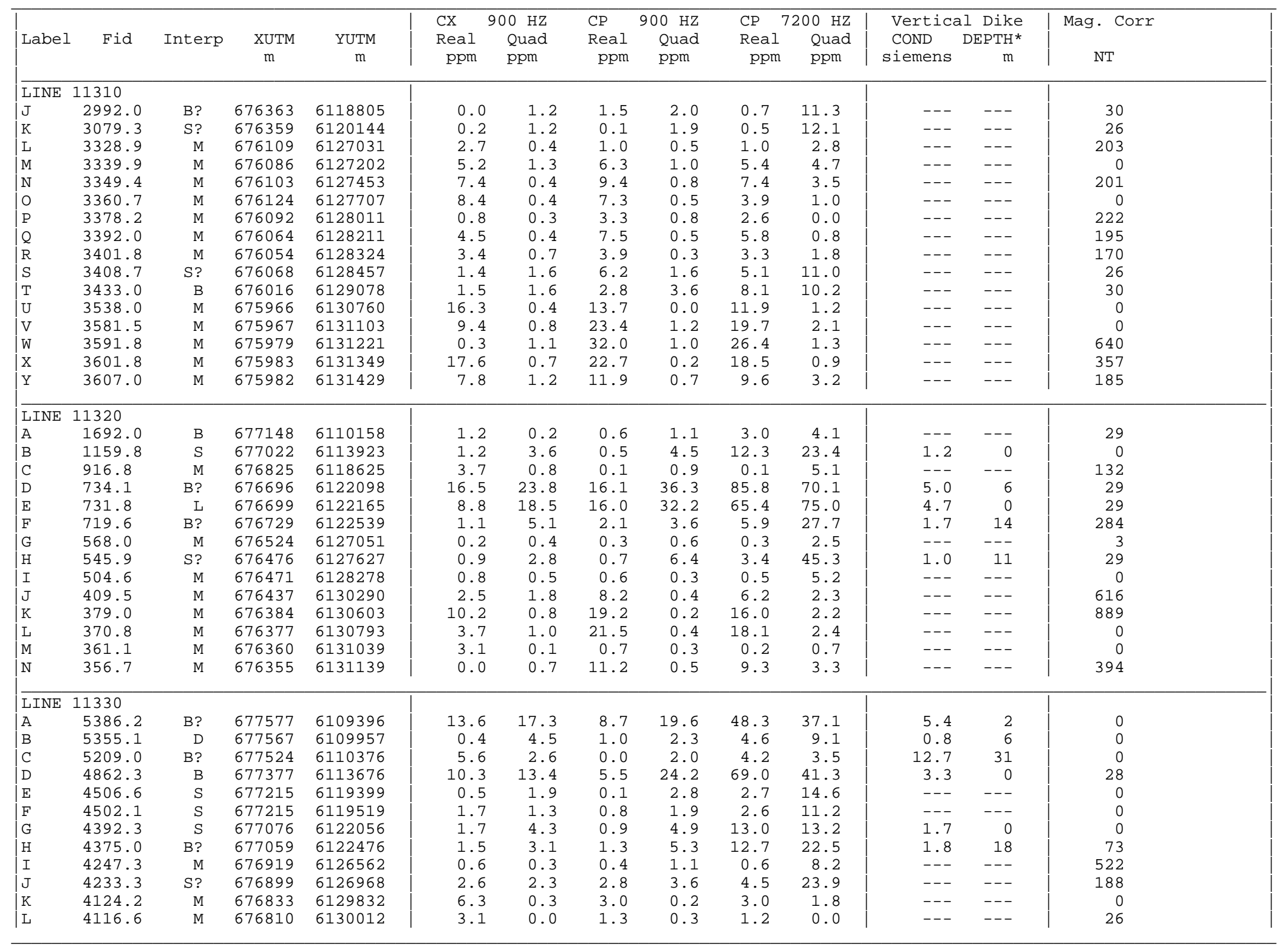

$\mathrm{CX}=$ COAXIAL

Note: EM values shown above

are local amplitudes

Ketchikan - North/South of 5515
* Estimated Depth may be unreliable because the stronger part of the conductor may be deeper or to one side of the flight line, or because of a shallow dip or magnetite/overburden effects 
EM Anomaly List

\begin{tabular}{|c|c|c|c|c|c|c|c|c|c|c|c|c|c|}
\hline Label & Fid & Interp & $\begin{array}{l}\text { XUTM } \\
\text { m }\end{array}$ & $\begin{array}{c}\text { YUTM } \\
\mathrm{m}\end{array}$ & $\begin{array}{l}\text { CX } \\
\text { Real } \\
\text { ppm }\end{array}$ & $\begin{array}{l}900 \mathrm{HZ} \\
\text { Quad } \\
\text { ppm }\end{array}$ & $\begin{array}{l}\text { CP } \\
\text { Real } \\
\text { ppm }\end{array}$ & $\begin{array}{l}900 \mathrm{HZ} \\
\text { Quad } \\
\text { ppm }\end{array}$ & $\begin{array}{l}\text { CP }{ }^{7} \\
\text { Real } \\
\text { ppm }\end{array}$ & $\begin{array}{c}200 \mathrm{HZ} \\
\text { Quad } \\
\text { ppm }\end{array}$ & $\begin{array}{l}\text { Vertica } \\
\text { CoND } \\
\text { siemens }\end{array}$ & $\begin{array}{c}\text { al Dike } \\
\text { DEPTH* } \\
\text { m }\end{array}$ & $\begin{array}{c}\text { Mag. Corr } \\
\text { NT }\end{array}$ \\
\hline $\begin{array}{l}\text { LINE } \\
\text { M } \\
N \\
O\end{array}$ & $\begin{array}{r}11330 \\
4102.0 \\
4085.3 \\
4069.4\end{array}$ & $\begin{array}{l}\mathrm{M} \\
\mathrm{M} \\
\mathrm{M}\end{array}$ & $\begin{array}{l}676761 \\
676735 \\
676755\end{array}$ & $\begin{array}{l}6130376 \\
6130765 \\
6131049\end{array}$ & $\begin{array}{l}0.5 \\
0.0 \\
0.0\end{array}$ & $\begin{array}{l}0.2 \\
0.4 \\
0.6\end{array}$ & $\begin{array}{r}1.1 \\
6.1 \\
21.0\end{array}$ & $\begin{array}{l}0.2 \\
0.5 \\
0.2\end{array}$ & $\begin{array}{r}1.0 \\
5.3 \\
17.5\end{array}$ & $\begin{array}{l}0.0 \\
2.3 \\
1.7\end{array}$ & $\begin{array}{l}--- \\
--- \\
---\end{array}$ & $\begin{array}{l}--- \\
--- \\
---\end{array}$ & $\begin{array}{r}589 \\
0 \\
247\end{array}$ \\
\hline $\begin{array}{l}\text { LINE } \\
\text { A } \\
B \\
C\end{array}$ & $\begin{array}{r}11341 \\
2472.0 \\
2354.4 \\
2333.3\end{array}$ & $\begin{array}{l}\mathrm{B} \\
\mathrm{B} \\
\mathrm{B}\end{array}$ & $\begin{array}{l}677920 \\
677916 \\
677919\end{array}$ & $\begin{array}{l}6109654 \\
6110027 \\
6110100\end{array}$ & $\begin{array}{l}8.9 \\
1.7 \\
7.4\end{array}$ & $\begin{array}{r}14.3 \\
4.7 \\
6.6\end{array}$ & $\begin{array}{r}11.4 \\
2.4 \\
6.6\end{array}$ & $\begin{array}{r}21.3 \\
9.9 \\
6.5\end{array}$ & $\begin{array}{l}52.7 \\
25.2 \\
17.7\end{array}$ & $\begin{array}{l}28.9 \\
29.8 \\
20.1\end{array}$ & $\begin{array}{l}4.2 \\
1.8 \\
9.4\end{array}$ & $\begin{array}{r}9 \\
7 \\
22\end{array}$ & $\begin{array}{r}0 \\
22 \\
0\end{array}$ \\
\hline $\begin{array}{l}\text { LINE } \\
\text { A } \\
\text { B } \\
\text { C }\end{array}$ & $\begin{array}{l}11342 \\
3017.3 \\
3612.7 \\
3643.7\end{array}$ & $\begin{array}{c}\text { B? } \\
\text { S? } \\
\text { M }\end{array}$ & $\begin{array}{l}677799 \\
677506 \\
677505\end{array}$ & $\begin{array}{l}6113410 \\
6121000 \\
6121863\end{array}$ & $\begin{array}{r}20.7 \\
0.3 \\
3.3\end{array}$ & $\begin{array}{r}21.5 \\
0.7 \\
0.2\end{array}$ & $\begin{array}{r}44.2 \\
0.0 \\
7.3\end{array}$ & $\begin{array}{r}43.6 \\
1.5 \\
0.9\end{array}$ & $\begin{array}{r}80.5 \\
0.2 \\
6.0\end{array}$ & $\begin{array}{r}35.0 \\
10.9 \\
3.8\end{array}$ & $\begin{array}{r}13.0 \\
--- \\
---\end{array}$ & $\begin{array}{r}0 \\
--- \\
---\end{array}$ & $\begin{array}{r}0 \\
17 \\
0\end{array}$ \\
\hline $\begin{array}{l}\text { LINE } \\
\text { A } \\
\text { B } \\
\text { C } \\
\text { D } \\
\text { E } \\
\text { F } \\
\text { G } \\
\text { H } \\
\text { I } \\
\text { J } \\
\text { K } \\
\text { L } \\
\text { M } \\
\text { N }\end{array}$ & $\begin{array}{r}11343 \\
3791.3 \\
3804.1 \\
3818.2 \\
3827.9 \\
3846.8 \\
3907.6 \\
3910.5 \\
3934.5 \\
3945.5 \\
3953.3 \\
3964.7 \\
3971.0 \\
3984.6 \\
3995.5\end{array}$ & $\begin{array}{r}\mathrm{M} \\
\mathrm{M} \\
\mathrm{M} \\
\mathrm{M} \\
\mathrm{M} \\
\mathrm{B} ? \\
\mathrm{D} \\
\mathrm{M} \\
\mathrm{M} \\
\mathrm{M} \\
\mathrm{M} \\
\mathrm{S} \\
\mathrm{M} \\
\mathrm{M}\end{array}$ & $\begin{array}{l}677366 \\
677347 \\
677338 \\
677327 \\
677295 \\
677241 \\
677239 \\
677230 \\
677225 \\
677210 \\
677200 \\
677207 \\
677209 \\
677186\end{array}$ & $\begin{array}{l}6126354 \\
6126575 \\
6126883 \\
6127075 \\
6127449 \\
6129118 \\
6129193 \\
6129587 \\
6129773 \\
6129899 \\
6130088 \\
6130240 \\
6130448 \\
6130600\end{array}$ & $\begin{array}{r}1.8 \\
0.6 \\
0.2 \\
0.4 \\
0.0 \\
4.4 \\
7.7 \\
1.7 \\
0.0 \\
8.0 \\
0.0 \\
65.0 \\
0.0 \\
34.9\end{array}$ & $\begin{array}{l}0.2 \\
0.2 \\
0.1 \\
0.4 \\
0.8 \\
3.6 \\
3.9 \\
0.4 \\
0.5 \\
0.6 \\
1.4 \\
3.4 \\
0.1 \\
0.9\end{array}$ & $\begin{array}{r}0.6 \\
6.2 \\
0.3 \\
1.2 \\
1.8 \\
1.2 \\
6.4 \\
21.9 \\
39.9 \\
41.9 \\
12.7 \\
137.6 \\
35.0 \\
88.8\end{array}$ & $\begin{array}{l}0.2 \\
0.7 \\
0.1 \\
0.5 \\
0.6 \\
7.2 \\
3.6 \\
0.3 \\
0.2 \\
0.4 \\
0.3 \\
2.6 \\
0.1 \\
0.5\end{array}$ & $\begin{array}{r}1.0 \\
5.3 \\
0.4 \\
0.9 \\
1.3 \\
17.2 \\
9.7 \\
18.2 \\
32.8 \\
34.3 \\
10.4 \\
111.3 \\
27.7 \\
71.9\end{array}$ & $\begin{array}{r}2.3 \\
3.4 \\
2.4 \\
1.9 \\
3.1 \\
16.9 \\
9.0 \\
1.2 \\
0.8 \\
0.2 \\
3.2 \\
11.7 \\
0.8 \\
3.5\end{array}$ & $\begin{array}{c}--- \\
--- \\
--- \\
--- \\
--- \\
3.8 \\
21.3 \\
--- \\
--- \\
--- \\
--- \\
--- \\
--- \\
---\end{array}$ & $\begin{array}{l}--- \\
--- \\
--- \\
--- \\
--- \\
41 \\
30 \\
--- \\
--- \\
--- \\
--- \\
--- \\
--- \\
---\end{array}$ & $\begin{array}{r}0 \\
0 \\
134 \\
235 \\
0 \\
0 \\
0 \\
644 \\
607 \\
0 \\
613 \\
0 \\
652 \\
0\end{array}$ \\
\hline $\begin{array}{l}\text { LINE } \\
\text { A } \\
\text { B } \\
\text { C } \\
\text { D } \\
\text { E } \\
\text { F } \\
\text { G } \\
\text { H } \\
\text { I } \\
\text { J } \\
\text { K } \\
\text { L } \\
\text { M } \\
\text { N } \\
\text { O }\end{array}$ & $\begin{array}{r}11350 \\
587.4 \\
543.9 \\
534.2 \\
527.0 \\
517.0 \\
507.1 \\
440.4 \\
432.5 \\
427.3 \\
419.7 \\
411.3 \\
400.0 \\
396.3 \\
385.6 \\
368.2\end{array}$ & $\begin{array}{l}\mathrm{M} \\
\mathrm{M} \\
\mathrm{M} \\
\mathrm{M} \\
\mathrm{M} \\
\mathrm{M} \\
\mathrm{M} \\
\mathrm{M} \\
\mathrm{M} \\
\mathrm{M} \\
\mathrm{M} \\
\mathrm{M} \\
\mathrm{M} \\
\mathrm{M} \\
\mathrm{M}\end{array}$ & $\begin{array}{l}677709 \\
677720 \\
677705 \\
677694 \\
677698 \\
677702 \\
677640 \\
677643 \\
677648 \\
677647 \\
677648 \\
677647 \\
677643 \\
677622 \\
677619\end{array}$ & $\begin{array}{l}6126150 \\
6126965 \\
6127262 \\
6127483 \\
6127788 \\
6128053 \\
6129247 \\
6129334 \\
6129412 \\
6129557 \\
6129699 \\
6129919 \\
6129977 \\
6130095 \\
6130304\end{array}$ & $\begin{array}{r}1.0 \\
0.8 \\
1.7 \\
0.0 \\
1.6 \\
0.0 \\
3.8 \\
6.9 \\
45.9 \\
20.7 \\
20.3 \\
3.5 \\
0.0 \\
0.0 \\
0.8\end{array}$ & $\begin{array}{l}0.5 \\
0.1 \\
0.1 \\
0.5 \\
0.8 \\
0.3 \\
0.4 \\
0.6 \\
1.6 \\
0.3 \\
0.6 \\
0.7 \\
0.3 \\
0.1 \\
0.4\end{array}$ & $\begin{array}{r}0.2 \\
0.3 \\
4.1 \\
0.5 \\
1.8 \\
0.4 \\
4.6 \\
10.1 \\
125.6 \\
7.7 \\
49.3 \\
2.4 \\
50.5 \\
10.5 \\
2.7\end{array}$ & $\begin{array}{l}2.0 \\
0.5 \\
0.3 \\
0.1 \\
0.7 \\
0.4 \\
0.4 \\
0.4 \\
0.9 \\
0.1 \\
0.4 \\
0.2 \\
0.5 \\
0.2 \\
0.8\end{array}$ & $\begin{array}{r}1.6 \\
0.5 \\
3.1 \\
0.5 \\
3.6 \\
0.2 \\
4.8 \\
7.3 \\
101.8 \\
6.9 \\
42.9 \\
2.0 \\
42.2 \\
8.9 \\
1.1\end{array}$ & $\begin{array}{l}2.9 \\
2.7 \\
0.9 \\
1.6 \\
3.4 \\
1.0 \\
0.0 \\
1.8 \\
4.0 \\
0.7 \\
0.8 \\
0.2 \\
1.1 \\
0.8 \\
1.8\end{array}$ & $\begin{array}{l}--- \\
--- \\
--- \\
--- \\
--- \\
--- \\
--- \\
--- \\
--- \\
--- \\
--- \\
--- \\
--- \\
--- \\
---\end{array}$ & $\begin{array}{l}--- \\
--- \\
--- \\
--- \\
--- \\
--- \\
--- \\
--- \\
--- \\
--- \\
--- \\
--- \\
--- \\
--- \\
---\end{array}$ & $\begin{array}{r}286 \\
0 \\
298 \\
0 \\
84 \\
0 \\
0 \\
84 \\
0 \\
670 \\
0 \\
1 \\
0 \\
84 \\
102\end{array}$ \\
\hline
\end{tabular}

$\mathrm{CX}=$ COAXIAL

Note: EM values shown above

are local amplitudes

Ketchikan - North/South of 5515
* Estimated Depth may be unreliable because the stronger part of the conductor may be deeper or to one side of the flight line, or because of a shallow dip or magnetite/overburden effects 
EM Anomaly List

\begin{tabular}{|c|c|c|c|c|c|c|c|c|c|c|c|c|c|}
\hline Label & Fid & Interp & $\underset{\mathrm{m}}{\mathrm{XUTM}}$ & $\begin{array}{c}\text { YUTM } \\
\mathrm{m}\end{array}$ & $\begin{array}{l}\text { CX } \\
\text { Real } \\
\text { ppm }\end{array}$ & $\begin{array}{c}900 \mathrm{HZ} \\
\text { Quad } \\
\text { ppm }\end{array}$ & $\begin{array}{l}\text { CP } \\
\text { Real } \\
\text { ppm }\end{array}$ & $\begin{array}{l}900 \mathrm{HZ} \\
\text { Quad } \\
\text { ppm }\end{array}$ & $\begin{array}{l}\text { CP }{ }^{7} \\
\text { Real } \\
\text { ppm }\end{array}$ & $\begin{array}{c}7200 \mathrm{HZ} \\
\text { Quad } \\
\text { ppm }\end{array}$ & $\begin{array}{l}\text { Vertica } \\
\text { COND } \\
\text { siemens }\end{array}$ & $\begin{array}{c}\text { al Dike } \\
\text { DEPTH* } \\
\text { m }\end{array}$ & $\begin{array}{c}\text { Mag. Corr } \\
\text { NT }\end{array}$ \\
\hline $\begin{array}{l}\text { LINE } \\
\text { A } \\
\text { B } \\
\text { C } \\
\text { D } \\
\text { E }\end{array}$ & $\begin{array}{l}11351 \\
1259.4 \\
1109.9 \\
833.0 \\
774.1 \\
709.4\end{array}$ & $\begin{array}{r}\text { B? } \\
\text { M } \\
\text { S? } \\
\text { S } \\
\text { B? }\end{array}$ & $\begin{array}{l}678159 \\
678088 \\
678014 \\
677964 \\
677867\end{array}$ & $\begin{array}{l}6114028 \\
6116193 \\
6119959 \\
6120557 \\
6122201\end{array}$ & $\begin{array}{l}1.1 \\
1.0 \\
0.4 \\
0.6 \\
6.3\end{array}$ & $\begin{array}{l}1.7 \\
0.1 \\
0.6 \\
2.1 \\
9.3\end{array}$ & $\begin{array}{l}3.6 \\
0.7 \\
0.3 \\
0.3 \\
8.1\end{array}$ & $\begin{array}{l}1.3 \\
0.6 \\
1.0 \\
3.4 \\
9.4\end{array}$ & $\begin{array}{r}1.7 \\
0.5 \\
0.6 \\
3.6 \\
25.6\end{array}$ & $\begin{array}{r}7.7 \\
1.7 \\
6.7 \\
19.7 \\
29.5\end{array}$ & $\begin{array}{l}7.4 \\
--- \\
--- \\
--- \\
5.3\end{array}$ & $\begin{array}{r}73 \\
--- \\
--- \\
--- \\
12\end{array}$ & $\begin{array}{r}0 \\
139 \\
0 \\
0 \\
0\end{array}$ \\
\hline $\begin{array}{l}\text { LINE } \\
\text { A } \\
\text { B } \\
\text { C } \\
\text { D } \\
\text { E } \\
\text { F }\end{array}$ & $\begin{array}{r}11353 \\
1895.9 \\
1883.8 \\
1853.0 \\
1852.3 \\
1838.6 \\
1596.9\end{array}$ & $\begin{array}{r}\text { B } \\
\text { B } \\
\text { B } \\
\text { B } \\
B ? \\
\text { S? }\end{array}$ & $\begin{array}{l}678348 \\
678339 \\
678322 \\
678323 \\
678348 \\
678160\end{array}$ & $\begin{array}{l}6109608 \\
6109689 \\
6109984 \\
6109995 \\
6110276 \\
6113291\end{array}$ & $\begin{array}{r}7.3 \\
6.5 \\
14.0 \\
13.8 \\
0.6 \\
3.0\end{array}$ & $\begin{array}{r}30.1 \\
15.8 \\
10.4 \\
11.2 \\
4.8 \\
9.1\end{array}$ & $\begin{array}{r}1.7 \\
13.2 \\
35.7 \\
35.7 \\
2.9 \\
5.9\end{array}$ & $\begin{array}{r}20.8 \\
30.6 \\
26.3 \\
26.3 \\
6.8 \\
26.8\end{array}$ & $\begin{array}{l}58.8 \\
74.6 \\
59.8 \\
59.8 \\
15.7 \\
76.4\end{array}$ & $\begin{array}{r}100.7 \\
73.3 \\
18.5 \\
17.4 \\
17.9 \\
57.3\end{array}$ & $\begin{array}{r}1.8 \\
3.9 \\
18.7 \\
--- \\
1.4 \\
---\end{array}$ & $\begin{array}{r}0 \\
0 \\
1 \\
--- \\
10 \\
---\end{array}$ & $\begin{array}{r}0 \\
15 \\
0 \\
0 \\
0 \\
0\end{array}$ \\
\hline $\begin{array}{l}\text { LINE } \\
\text { A } \\
\text { B } \\
\text { C } \\
\text { D } \\
\text { E } \\
\text { F } \\
\text { G } \\
\text { H } \\
\text { I } \\
\text { J } \\
\text { K } \\
\text { L }\end{array}$ & $\begin{array}{r}11360 \\
3890.1 \\
3820.9 \\
3812.8 \\
3806.9 \\
3787.0 \\
3768.8 \\
3707.3 \\
3697.0 \\
3650.0 \\
3629.8 \\
3625.9 \\
3622.8\end{array}$ & $\begin{array}{l}\mathrm{M} \\
\mathrm{M} \\
\mathrm{M} \\
\mathrm{M} \\
\mathrm{M} \\
\mathrm{M} \\
\mathrm{M} \\
\mathrm{M} \\
\mathrm{M} \\
\mathrm{M} \\
\mathrm{M} \\
\mathrm{M}\end{array}$ & $\begin{array}{l}678149 \\
678110 \\
678090 \\
678081 \\
678131 \\
678111 \\
678040 \\
678041 \\
678025 \\
678041 \\
678060 \\
678064\end{array}$ & $\begin{array}{l}6125881 \\
6127051 \\
6127235 \\
6127357 \\
6127764 \\
6128149 \\
6129076 \\
6129152 \\
6129543 \\
6129648 \\
6129681 \\
6129715\end{array}$ & $\begin{array}{r}0.0 \\
6.7 \\
5.3 \\
11.4 \\
0.1 \\
0.6 \\
1.1 \\
8.3 \\
2.3 \\
22.9 \\
1.5 \\
36.3\end{array}$ & $\begin{array}{l}0.4 \\
0.4 \\
0.5 \\
0.4 \\
0.3 \\
0.5 \\
0.3 \\
0.4 \\
0.3 \\
0.7 \\
0.0 \\
0.3\end{array}$ & $\begin{array}{r}0.9 \\
2.7 \\
8.3 \\
17.5 \\
1.4 \\
0.1 \\
5.7 \\
11.4 \\
0.3 \\
31.0 \\
11.2 \\
61.4\end{array}$ & $\begin{array}{l}0.5 \\
0.5 \\
0.3 \\
0.2 \\
0.7 \\
0.7 \\
0.8 \\
0.2 \\
0.3 \\
1.0 \\
0.1 \\
0.2\end{array}$ & $\begin{array}{r}0.8 \\
2.5 \\
7.6 \\
14.5 \\
1.6 \\
0.1 \\
4.8 \\
9.1 \\
0.5 \\
24.4 \\
11.1 \\
49.0\end{array}$ & $\begin{array}{l}2.4 \\
5.4 \\
3.8 \\
1.1 \\
2.3 \\
4.4 \\
2.1 \\
0.9 \\
1.2 \\
2.0 \\
0.2 \\
1.5\end{array}$ & $\begin{array}{l}--- \\
--- \\
--- \\
--- \\
--- \\
--- \\
--- \\
--- \\
--- \\
--- \\
--- \\
---\end{array}$ & $\begin{array}{l}--- \\
--- \\
--- \\
--- \\
--- \\
--- \\
--- \\
--- \\
--- \\
--- \\
--- \\
---\end{array}$ & $\begin{array}{r}32 \\
0 \\
0 \\
0 \\
182 \\
0 \\
0 \\
413 \\
76 \\
49 \\
76 \\
14\end{array}$ \\
\hline $\begin{array}{l}\text { LINE } \\
\text { A } \\
B\end{array}$ & $\begin{array}{r}11361 \\
4416.7 \\
4055.8\end{array}$ & $\begin{array}{r}\text { M } \\
\text { B? }\end{array}$ & $\begin{array}{l}678526 \\
678325\end{array}$ & $\begin{array}{l}6115786 \\
6122044\end{array}$ & $\begin{array}{l}0.1 \\
2.1\end{array}$ & $\begin{array}{l}0.5 \\
5.8\end{array}$ & $\begin{array}{l}1.8 \\
6.2\end{array}$ & $\begin{array}{l}0.7 \\
9.6\end{array}$ & $\begin{array}{r}1.4 \\
28.2\end{array}$ & $\begin{array}{r}2.6 \\
40.5\end{array}$ & $\begin{array}{l}--- \\
3.2\end{array}$ & $\begin{array}{r}--- \\
13\end{array}$ & $\begin{array}{r}67 \\
0\end{array}$ \\
\hline $\begin{array}{l}\text { LINE } \\
\text { A } \\
\text { B } \\
\text { C } \\
\text { D }\end{array}$ & $\begin{array}{r}11362 \\
3363.8 \\
3415.3 \\
3440.7 \\
3630.5\end{array}$ & $\begin{array}{r}\mathrm{B} ? \\
\mathrm{~B} \\
\mathrm{~B} ? \\
\mathrm{M}\end{array}$ & $\begin{array}{l}678777 \\
678733 \\
678708 \\
678730\end{array}$ & $\begin{array}{l}6109280 \\
6109718 \\
6110534 \\
6113600\end{array}$ & $\begin{array}{r}0.8 \\
24.3 \\
0.7 \\
1.1\end{array}$ & $\begin{array}{r}0.8 \\
29.5 \\
1.0 \\
0.1\end{array}$ & $\begin{array}{r}0.2 \\
32.9 \\
0.6 \\
0.0\end{array}$ & $\begin{array}{r}1.0 \\
58.8 \\
1.2 \\
0.3\end{array}$ & $\begin{array}{r}0.3 \\
126.9 \\
0.6 \\
0.1\end{array}$ & $\begin{array}{r}6.0 \\
71.8 \\
6.5 \\
0.8\end{array}$ & $\begin{array}{l}--- \\
7.6 \\
--- \\
---\end{array}$ & $\begin{array}{r}--- \\
0 \\
--- \\
---\end{array}$ & $\begin{array}{r}0 \\
22 \\
176 \\
114\end{array}$ \\
\hline $\begin{array}{l}\text { LINE } \\
\text { A } \\
\text { B } \\
\text { C } \\
\text { D } \\
\text { E } \\
\text { F }\end{array}$ & $\begin{array}{r}11370 \\
554.2 \\
518.0 \\
489.1 \\
485.7 \\
465.8 \\
437.2\end{array}$ & $\begin{array}{c}\mathrm{B} ? \\
\mathrm{M} \\
\mathrm{M} \\
\mathrm{M} \\
\mathrm{M} \\
\mathrm{M}\end{array}$ & $\begin{array}{l}678613 \\
678555 \\
678513 \\
678507 \\
678515 \\
678512\end{array}$ & $\begin{array}{l}6125188 \\
6125681 \\
6126061 \\
6126139 \\
6126736 \\
6127273\end{array}$ & $\begin{array}{l}8.0 \\
0.1 \\
3.1 \\
5.1 \\
0.9 \\
0.6\end{array}$ & $\begin{array}{l}8.7 \\
0.2 \\
0.3 \\
0.3 \\
1.2 \\
0.6\end{array}$ & $\begin{array}{r}25.1 \\
0.2 \\
6.1 \\
13.3 \\
0.9 \\
0.3\end{array}$ & $\begin{array}{r}22.3 \\
0.8 \\
0.2 \\
0.3 \\
0.4 \\
0.6\end{array}$ & $\begin{array}{r}44.0 \\
2.1 \\
4.8 \\
11.5 \\
1.0 \\
0.2\end{array}$ & $\begin{array}{l}9.9 \\
2.8 \\
2.0 \\
3.1 \\
4.2 \\
2.8\end{array}$ & $\begin{array}{c}11.0 \\
--- \\
--- \\
--- \\
--- \\
---\end{array}$ & $\begin{array}{l}0 \\
--- \\
--- \\
--- \\
--- \\
---\end{array}$ & $\begin{array}{r}0 \\
5 \\
193 \\
42 \\
198 \\
0\end{array}$ \\
\hline
\end{tabular}

$\mathrm{CX}=$ COAXIAL

Note: EM values shown above

Ketchikan - North/South of 5515
* Estimated Depth may be unreliable because the stronger part of the conductor may be deeper or to one side of the flight line, or because of a shallow dip or magnetite/overburden effects 
EM Anomaly List

\begin{tabular}{|c|c|c|c|c|c|c|c|c|c|c|c|c|c|}
\hline Label & Fid & Interp & $\begin{array}{c}\text { XUTM } \\
\text { m }\end{array}$ & $\begin{array}{c}\text { YUTM } \\
\mathrm{m}\end{array}$ & $\begin{array}{l}\text { CX } \\
\text { Real } \\
\text { ppm }\end{array}$ & $\begin{array}{l}00 \mathrm{HZ} \\
\text { Quad } \\
\text { ppm }\end{array}$ & $\begin{array}{l}\text { CP } \\
\text { Real } \\
\text { ppm }\end{array}$ & $\begin{array}{l}900 \mathrm{HZ} \\
\text { Quad } \\
\text { ppm }\end{array}$ & $\begin{array}{l}\text { CP } 7 \\
\text { Real } \\
\text { ppm }\end{array}$ & $\begin{array}{l}200 \mathrm{HZ} \\
\text { Quad } \\
\text { ppm }\end{array}$ & $\begin{array}{l}\text { Vertica } \\
\text { COND } \\
\text { siemens }\end{array}$ & $\begin{array}{c}\text { al Dike } \\
\text { DEPTH* } \\
\text { m }\end{array}$ & $\begin{array}{c}\text { Mag. Corr } \\
\text { NT }\end{array}$ \\
\hline $\begin{array}{l}\text { LINE } \\
\text { G } \\
\text { H } \\
\text { I }\end{array}$ & $\begin{array}{c}11370 \\
384.6 \\
336.1 \\
317.0\end{array}$ & $\begin{array}{l}\mathrm{M} \\
\mathrm{M} \\
\mathrm{M}\end{array}$ & $\begin{array}{l}678518 \\
678386 \\
678421\end{array}$ & $\begin{array}{l}6128042 \\
6129167 \\
6129399\end{array}$ & $\begin{array}{r}0.1 \\
0.8 \\
17.4\end{array}$ & $\begin{array}{l}0.6 \\
0.8 \\
0.8\end{array}$ & $\begin{array}{r}0.9 \\
4.7 \\
53.1\end{array}$ & $\begin{array}{l}0.9 \\
0.8 \\
0.7\end{array}$ & $\begin{array}{r}1.0 \\
4.5 \\
44.0\end{array}$ & $\begin{array}{l}2.8 \\
2.3 \\
1.3\end{array}$ & $\begin{array}{l}--- \\
--- \\
---\end{array}$ & $\begin{array}{l}--- \\
--- \\
---\end{array}$ & $\begin{array}{r}175 \\
0 \\
0\end{array}$ \\
\hline $\begin{array}{l}\text { LINE } \\
\text { A } \\
\text { B } \\
\text { C } \\
\text { D } \\
\text { E } \\
\text { F }\end{array}$ & $\begin{array}{c}11371 \\
1252.1 \\
1187.6 \\
886.6 \\
871.5 \\
801.9 \\
725.0\end{array}$ & $\begin{array}{r}\mathrm{M} \\
\mathrm{S} \\
\mathrm{D} \\
\mathrm{S} \\
\mathrm{B} ? \\
\mathrm{M}\end{array}$ & $\begin{array}{l}679020 \\
678957 \\
678809 \\
678771 \\
678753 \\
678726\end{array}$ & $\begin{array}{l}6113508 \\
6114270 \\
6119183 \\
6119422 \\
6120362 \\
6121003\end{array}$ & $\begin{array}{l}0.0 \\
0.0 \\
5.9 \\
1.2 \\
3.0 \\
2.8\end{array}$ & $\begin{array}{l}0.5 \\
3.4 \\
7.8 \\
4.6 \\
0.6 \\
0.3\end{array}$ & $\begin{array}{l}1.1 \\
1.1 \\
1.8 \\
0.8 \\
0.7 \\
1.1\end{array}$ & $\begin{array}{l}0.1 \\
7.3 \\
9.0 \\
8.4 \\
1.6 \\
0.2\end{array}$ & $\begin{array}{r}0.4 \\
13.2 \\
21.6 \\
6.1 \\
0.9 \\
0.2\end{array}$ & $\begin{array}{r}2.1 \\
43.1 \\
31.2 \\
52.6 \\
10.3 \\
1.9\end{array}$ & $\begin{array}{r}--- \\
0.5 \\
3.0 \\
1.0 \\
19.9 \\
---\end{array}$ & $\begin{array}{r}--- \\
0 \\
42 \\
14 \\
64 \\
---\end{array}$ & $\begin{array}{r}28 \\
0 \\
0 \\
0 \\
0 \\
0\end{array}$ \\
\hline $\begin{array}{l}\text { LINE } \\
\text { A } \\
\text { B } \\
\text { C } \\
\text { D } \\
\text { E }\end{array}$ & $\begin{array}{r}11372 \\
4003.2 \\
3994.4 \\
3869.2 \\
3790.6 \\
3783.3\end{array}$ & $\begin{array}{r}\text { B? } \\
\text { B? } \\
\text { D } \\
\text { M } \\
\text { M }\end{array}$ & $\begin{array}{l}679150 \\
679150 \\
679071 \\
678991 \\
678999\end{array}$ & $\begin{array}{l}6109662 \\
6109850 \\
6111964 \\
6113380 \\
6113500\end{array}$ & $\begin{array}{l}5.0 \\
7.1 \\
8.2 \\
0.1 \\
3.4\end{array}$ & $\begin{array}{r}9.2 \\
11.1 \\
8.6 \\
0.4 \\
0.4\end{array}$ & $\begin{array}{r}9.8 \\
11.7 \\
1.3 \\
1.3 \\
2.4\end{array}$ & $\begin{array}{r}18.5 \\
18.6 \\
6.1 \\
0.3 \\
0.5\end{array}$ & $\begin{array}{r}41.8 \\
42.1 \\
16.2 \\
1.0 \\
0.0\end{array}$ & $\begin{array}{r}28.0 \\
29.1 \\
12.8 \\
2.3 \\
1.9\end{array}$ & $\begin{array}{l}3.2 \\
4.5 \\
5.3 \\
--- \\
---\end{array}$ & $\begin{array}{r}6 \\
5 \\
0 \\
--- \\
---\end{array}$ & $\begin{array}{r}0 \\
0 \\
8 \\
163 \\
92\end{array}$ \\
\hline $\begin{array}{l}\text { LINE } \\
\text { A } \\
\text { B } \\
\text { C } \\
\text { D } \\
\text { E } \\
\text { F } \\
\text { G } \\
\text { H } \\
\text { I } \\
\text { J } \\
\text { K }\end{array}$ & $\begin{array}{r}11380 \\
5916.7 \\
6039.1 \\
6081.9 \\
6179.3 \\
6184.1 \\
6524.2 \\
6664.7 \\
6673.2 \\
6678.6 \\
6691.1 \\
6699.4\end{array}$ & $\begin{array}{r}\text { S } \\
\text { B? } \\
\text { D } \\
\text { B } \\
\text { B? } \\
\text { S } \\
\text { B? } \\
\text { S? } \\
\text { B } \\
\text { B? } \\
\text { B? }\end{array}$ & $\begin{array}{l}679596 \\
679478 \\
679544 \\
679394 \\
679397 \\
679264 \\
679136 \\
679126 \\
679126 \\
679114 \\
679111\end{array}$ & $\begin{array}{l}6108031 \\
6109586 \\
6109971 \\
6111784 \\
6111879 \\
6118140 \\
6120834 \\
6120975 \\
6121072 \\
6121323 \\
6121476\end{array}$ & $\begin{array}{r}1.4 \\
1.3 \\
19.6 \\
3.2 \\
9.7 \\
2.3 \\
1.5 \\
1.4 \\
1.4 \\
1.5 \\
0.8\end{array}$ & $\begin{array}{r}1.5 \\
4.2 \\
18.3 \\
3.7 \\
6.4 \\
6.7 \\
5.3 \\
2.1 \\
5.8 \\
6.4 \\
4.4\end{array}$ & $\begin{array}{l}0.8 \\
1.5 \\
0.1 \\
2.0 \\
4.7 \\
0.7 \\
0.9 \\
0.7 \\
0.9 \\
0.3 \\
1.2\end{array}$ & $\begin{array}{r}3.3 \\
7.8 \\
15.3 \\
5.6 \\
7.7 \\
13.3 \\
8.2 \\
1.1 \\
6.6 \\
5.8 \\
2.7\end{array}$ & $\begin{array}{r}1.5 \\
19.6 \\
33.2 \\
14.5 \\
16.3 \\
23.9 \\
14.6 \\
12.1 \\
15.3 \\
8.2 \\
7.2\end{array}$ & $\begin{array}{r}19.9 \\
27.4 \\
27.9 \\
14.3 \\
10.8 \\
83.1 \\
49.4 \\
2.0 \\
17.6 \\
40.0 \\
18.8\end{array}$ & $\begin{array}{r}1.9 \\
1.4 \\
6.7 \\
--- \\
10.9 \\
1.3 \\
1.1 \\
--- \\
1.1 \\
0.9 \\
1.2\end{array}$ & $\begin{array}{r}63 \\
3 \\
16 \\
--- \\
0 \\
0 \\
2 \\
--- \\
7 \\
13 \\
17\end{array}$ & $\begin{array}{r}0 \\
0 \\
0 \\
0 \\
41 \\
21 \\
0 \\
0 \\
21 \\
0 \\
0\end{array}$ \\
\hline $\begin{array}{l}\text { LINE } \\
\text { A } \\
\text { B } \\
\text { C } \\
\text { D } \\
\text { E } \\
\text { F } \\
\text { G }\end{array}$ & $\begin{array}{r}11381 \\
6883.1 \\
6891.1 \\
6896.3 \\
6942.3 \\
6971.2 \\
6981.9 \\
6997.2\end{array}$ & $\begin{array}{l}\mathrm{S} \\
\mathrm{M} \\
\mathrm{M} \\
\mathrm{S} \\
\mathrm{M} \\
\mathrm{M} \\
\mathrm{M}\end{array}$ & $\begin{array}{l}678940 \\
678906 \\
678878 \\
678817 \\
678816 \\
678836 \\
678859\end{array}$ & $\begin{array}{l}6126239 \\
6126490 \\
6126653 \\
6127989 \\
6128343 \\
6128425 \\
6128653\end{array}$ & $\begin{array}{l}3.5 \\
0.0 \\
2.8 \\
0.6 \\
1.2 \\
1.9 \\
1.0\end{array}$ & $\begin{array}{l}1.9 \\
1.1 \\
0.2 \\
1.8 \\
0.6 \\
0.5 \\
0.4\end{array}$ & $\begin{array}{l}9.2 \\
4.6 \\
1.1 \\
0.8 \\
3.6 \\
0.5 \\
3.9\end{array}$ & $\begin{array}{l}2.9 \\
0.6 \\
0.5 \\
1.5 \\
0.4 \\
0.8 \\
0.4\end{array}$ & $\begin{array}{l}9.0 \\
3.7 \\
2.2 \\
0.1 \\
2.3 \\
0.3 \\
0.1\end{array}$ & $\begin{array}{r}16.2 \\
3.3 \\
3.7 \\
8.7 \\
1.0 \\
1.4 \\
1.2\end{array}$ & $\begin{array}{l}--- \\
--- \\
--- \\
1.6 \\
--- \\
--- \\
---\end{array}$ & $\begin{array}{l}--- \\
--- \\
--- \\
28 \\
--- \\
--- \\
---\end{array}$ & $\begin{array}{r}0 \\
0 \\
0 \\
66 \\
0 \\
461 \\
110\end{array}$ \\
\hline $\begin{array}{l}\text { LINE } \\
\text { A } \\
\text { B } \\
\text { C } \\
\text { D }\end{array}$ & $\begin{array}{r}11390 \\
7326.1 \\
7296.6 \\
7270.0 \\
7246.4\end{array}$ & $\begin{array}{c}\text { S } \\
\text { S? } \\
\text { M } \\
\text { S }\end{array}$ & $\begin{array}{l}679370 \\
679364 \\
679282 \\
679289\end{array}$ & $\begin{array}{l}6125717 \\
6126087 \\
6126599 \\
6127192\end{array}$ & $\begin{array}{l}1.2 \\
2.9 \\
1.4 \\
1.3\end{array}$ & $\begin{array}{l}0.7 \\
3.8 \\
1.2 \\
1.7\end{array}$ & $\begin{array}{r}3.1 \\
10.8 \\
2.8 \\
3.6\end{array}$ & $\begin{array}{l}1.5 \\
6.4 \\
0.9 \\
3.2\end{array}$ & $\begin{array}{r}3.6 \\
11.7 \\
2.0 \\
5.0\end{array}$ & $\begin{array}{r}9.6 \\
37.1 \\
2.9 \\
20.4\end{array}$ & $\begin{array}{r}13.1 \\
9.8 \\
--- \\
---\end{array}$ & $\begin{array}{r}68 \\
37 \\
--- \\
---\end{array}$ & $\begin{array}{r}93 \\
0 \\
94 \\
93\end{array}$ \\
\hline
\end{tabular}

$\mathrm{CX}=$ COAXIAL

$\mathrm{CP}=$ COPLANAR Note:EM values shown above

are local amplitudes

are $10 c a 1$
55
15
*Estimated Depth may be unreliable because the stronger part of the conductor may be deeper or to one side of the flight line, or because of a shallow dip or magnetite/overburden effects 
EM Anomaly List

\begin{tabular}{|c|c|c|c|c|c|c|c|c|c|c|c|c|c|}
\hline Label & Fid & Interp & $\underset{\mathrm{m}}{\mathrm{XUTM}}$ & $\begin{array}{c}\text { YUTM } \\
\mathrm{m}\end{array}$ & $\begin{array}{l}\text { CX } \\
\text { Real } \\
\text { ppm }\end{array}$ & $\begin{array}{c}900 \mathrm{HZ} \\
\text { Quad } \\
\text { ppm }\end{array}$ & $\begin{array}{l}\text { CP } \\
\text { Real } \\
\text { ppm }\end{array}$ & $\begin{array}{l}900 \mathrm{HZ} \\
\text { Quad } \\
\text { ppm }\end{array}$ & $\begin{array}{l}\text { CP }{ }^{7} \\
\text { Real } \\
\text { ppm }\end{array}$ & $\begin{array}{c}7200 \mathrm{HZ} \\
\text { Quad } \\
\text { ppm }\end{array}$ & $\begin{array}{l}\text { Vertica } \\
\text { CoND } \\
\text { siemens }\end{array}$ & $\begin{array}{c}\text { al Dike } \\
\text { DEPTH* } \\
\text { m }\end{array}$ & $\begin{array}{c}\text { Mag. Corr } \\
\text { NT }\end{array}$ \\
\hline $\begin{array}{l}\text { LINE } \\
\text { E } \\
F \\
\text { G } \\
H \\
I\end{array}$ & $\begin{array}{r}11390 \\
7222.9 \\
7184.1 \\
7146.9 \\
7135.2 \\
7128.3\end{array}$ & $\begin{array}{l}\mathrm{D} \\
\mathrm{M} \\
\mathrm{M} \\
\mathrm{M} \\
\mathrm{M}\end{array}$ & $\begin{array}{l}679308 \\
679242 \\
679228 \\
679229 \\
679230\end{array}$ & $\begin{array}{l}6127706 \\
6128214 \\
6128367 \\
6128432 \\
6128481\end{array}$ & $\begin{array}{r}4.3 \\
2.6 \\
73.1 \\
1.2 \\
83.4\end{array}$ & $\begin{array}{l}2.0 \\
0.8 \\
2.5 \\
2.9 \\
2.5\end{array}$ & $\begin{array}{r}1.7 \\
8.0 \\
95.4 \\
14.6 \\
70.9\end{array}$ & $\begin{array}{l}1.3 \\
0.4 \\
1.0 \\
0.3 \\
0.5\end{array}$ & $\begin{array}{r}1.3 \\
7.1 \\
65.8 \\
15.2 \\
54.5\end{array}$ & $\begin{array}{l}7.7 \\
1.4 \\
5.3 \\
3.3 \\
2.5\end{array}$ & $\begin{array}{c}17.5 \\
--- \\
--- \\
--- \\
---\end{array}$ & $\begin{array}{l}39 \\
--- \\
--- \\
--- \\
---\end{array}$ & $\begin{array}{r}93 \\
0 \\
0 \\
0 \\
908\end{array}$ \\
\hline $\begin{array}{l}\text { LINE } \\
\text { A } \\
\text { B } \\
\text { C } \\
\text { D } \\
\text { E } \\
\text { F } \\
\text { G } \\
\text { H } \\
\text { I } \\
\text { J } \\
\text { K } \\
\text { L } \\
\text { M } \\
\text { N }\end{array}$ & $\begin{array}{r}11391 \\
6887.0 \\
6878.7 \\
6868.0 \\
6857.3 \\
6779.2 \\
6742.2 \\
6689.3 \\
6676.3 \\
6654.8 \\
6640.3 \\
6533.1 \\
6530.0 \\
6361.5 \\
6275.5\end{array}$ & $\begin{array}{r}\mathrm{M} \\
\mathrm{M} \\
\mathrm{M} \\
\mathrm{M} \\
\mathrm{S} ? \\
\mathrm{~S} ? \\
\mathrm{~B} ? \\
\mathrm{~B} \\
\mathrm{~B} ? \\
\mathrm{~S} ? \\
\mathrm{~B} ? \\
\mathrm{~B} ? \\
\mathrm{~B} ? \\
\mathrm{~B} ?\end{array}$ & $\begin{array}{l}679995 \\
680016 \\
680023 \\
680022 \\
680036 \\
679989 \\
679954 \\
679944 \\
679948 \\
679969 \\
679847 \\
679849 \\
679698 \\
679644\end{array}$ & $\begin{array}{l}6107173 \\
6107307 \\
6107416 \\
6107493 \\
6108101 \\
6108675 \\
6109521 \\
6109646 \\
6109852 \\
6110288 \\
6112311 \\
6112376 \\
6115952 \\
6118287\end{array}$ & $\begin{array}{r}3.4 \\
4.8 \\
2.8 \\
1.4 \\
0.5 \\
0.4 \\
2.1 \\
6.5 \\
3.0 \\
0.0 \\
23.4 \\
12.5 \\
73.7 \\
0.3\end{array}$ & $\begin{array}{r}0.6 \\
0.3 \\
0.1 \\
0.1 \\
4.8 \\
0.7 \\
3.0 \\
10.0 \\
3.3 \\
1.2 \\
27.1 \\
16.9 \\
92.6 \\
1.0\end{array}$ & $\begin{array}{r}5.2 \\
13.4 \\
4.3 \\
3.4 \\
1.2 \\
0.1 \\
1.8 \\
0.5 \\
0.0 \\
0.4 \\
39.4 \\
6.8 \\
141.9 \\
0.5\end{array}$ & $\begin{array}{r}0.6 \\
1.2 \\
0.3 \\
0.3 \\
6.6 \\
0.8 \\
3.6 \\
2.0 \\
1.5 \\
1.8 \\
57.5 \\
14.7 \\
221.0 \\
1.1\end{array}$ & $\begin{array}{r}5.1 \\
8.3 \\
3.8 \\
2.9 \\
6.1 \\
0.5 \\
3.3 \\
7.6 \\
3.3 \\
1.7 \\
105.4 \\
30.1 \\
427.4 \\
0.8\end{array}$ & $\begin{array}{r}2.8 \\
0.8 \\
2.5 \\
1.7 \\
42.2 \\
6.1 \\
7.9 \\
15.5 \\
6.8 \\
12.1 \\
33.3 \\
21.1 \\
151.8 \\
7.6\end{array}$ & $\begin{array}{r}--- \\
--- \\
--- \\
--- \\
0.6 \\
--- \\
2.8 \\
3.5 \\
3.5 \\
--- \\
9.1 \\
5.1 \\
12.1 \\
---\end{array}$ & $\begin{array}{r}--- \\
--- \\
--- \\
--- \\
5 \\
--- \\
61 \\
26 \\
43 \\
--- \\
0 \\
0 \\
0 \\
---\end{array}$ & $\begin{array}{r}56 \\
0 \\
22 \\
0 \\
0 \\
0 \\
0 \\
0 \\
0 \\
137 \\
0 \\
0 \\
13 \\
22\end{array}$ \\
\hline $\begin{array}{l}\text { LINE } \\
\text { A } \\
\text { B } \\
\text { C } \\
\text { D } \\
\text { E } \\
\text { F } \\
\text { G } \\
\text { H } \\
\text { I } \\
\text { J } \\
\text { K } \\
\text { L }\end{array}$ & $\begin{array}{r}11400 \\
7500.2 \\
7528.3 \\
7533.2 \\
7541.4 \\
7550.9 \\
7567.9 \\
7581.7 \\
7590.1 \\
7602.0 \\
7644.3 \\
7654.0 \\
7665.2\end{array}$ & $\begin{array}{l}\mathrm{M} \\
\mathrm{S} \\
\mathrm{M} \\
\mathrm{M} \\
\mathrm{M} \\
\mathrm{M} \\
\mathrm{M} \\
\mathrm{M} \\
\mathrm{M} \\
\mathrm{M} \\
\mathrm{M} \\
\mathrm{M}\end{array}$ & $\begin{array}{l}679738 \\
679730 \\
679723 \\
679713 \\
679697 \\
679708 \\
679707 \\
679707 \\
679726 \\
679642 \\
679643 \\
679618\end{array}$ & $\begin{array}{l}6125424 \\
6125877 \\
6125996 \\
6126172 \\
6126356 \\
6126620 \\
6126805 \\
6126955 \\
6127212 \\
6128106 \\
6128220 \\
6128393\end{array}$ & $\begin{array}{r}2.0 \\
12.3 \\
7.9 \\
8.0 \\
1.3 \\
1.9 \\
0.0 \\
6.2 \\
3.3 \\
1.3 \\
1.0 \\
0.1\end{array}$ & $\begin{array}{l}0.5 \\
1.9 \\
0.8 \\
0.3 \\
1.3 \\
0.4 \\
1.0 \\
0.9 \\
1.0 \\
0.9 \\
0.2 \\
0.8\end{array}$ & $\begin{array}{r}1.4 \\
32.2 \\
3.2 \\
11.5 \\
2.9 \\
3.8 \\
1.3 \\
5.8 \\
0.2 \\
3.2 \\
0.6 \\
2.3\end{array}$ & $\begin{array}{l}0.9 \\
2.9 \\
2.6 \\
0.1 \\
0.6 \\
0.5 \\
1.0 \\
0.4 \\
1.1 \\
0.5 \\
0.1 \\
0.6\end{array}$ & $\begin{array}{r}0.8 \\
27.2 \\
3.4 \\
9.5 \\
1.8 \\
4.0 \\
1.1 \\
3.0 \\
0.1 \\
3.0 \\
0.2 \\
1.8\end{array}$ & $\begin{array}{r}2.0 \\
18.1 \\
15.8 \\
0.1 \\
4.6 \\
1.7 \\
6.5 \\
6.2 \\
3.6 \\
0.9 \\
0.1 \\
2.1\end{array}$ & $\begin{array}{r}--- \\
211.1 \\
--- \\
--- \\
--- \\
--- \\
--- \\
--- \\
--- \\
--- \\
--- \\
---\end{array}$ & $\begin{array}{r}--- \\
25 \\
--- \\
--- \\
--- \\
--- \\
--- \\
--- \\
--- \\
--- \\
--- \\
---\end{array}$ & $\begin{array}{r}193 \\
0 \\
67 \\
545 \\
0 \\
0 \\
0 \\
0 \\
65 \\
228 \\
103 \\
57\end{array}$ \\
\hline $\begin{array}{l}\text { LINE } \\
\text { A } \\
B\end{array}$ & $\begin{array}{r}11401 \\
7029.5 \\
7048.0\end{array}$ & $\begin{array}{l}\text { B? } \\
\text { B? }\end{array}$ & $\begin{array}{l}680505 \\
680489\end{array}$ & $\begin{array}{l}6105100 \\
6105231\end{array}$ & $\begin{array}{l}2.0 \\
0.4\end{array}$ & $\begin{array}{l}0.4 \\
0.8\end{array}$ & $\begin{array}{l}0.2 \\
0.5\end{array}$ & $\begin{array}{l}1.2 \\
0.7\end{array}$ & $\begin{array}{l}1.1 \\
0.8\end{array}$ & $\begin{array}{l}5.5 \\
4.0\end{array}$ & $\begin{array}{r}14.0 \\
---\end{array}$ & $\begin{array}{r}84 \\
---\end{array}$ & $\begin{array}{l}0 \\
0\end{array}$ \\
\hline $\begin{array}{l}\text { LINE } \\
\text { A } \\
\text { B } \\
\text { C } \\
\text { D } \\
\text { E }\end{array}$ & $\begin{array}{r}11402 \\
7381.8 \\
7348.8 \\
7309.3 \\
7290.4 \\
7279.8\end{array}$ & $\begin{array}{l}\mathrm{B} \\
\mathrm{B} \\
\mathrm{M} \\
\mathrm{M} \\
\mathrm{M}\end{array}$ & $\begin{array}{l}680483 \\
680468 \\
680455 \\
680438 \\
680434\end{array}$ & $\begin{array}{l}6106230 \\
6106392 \\
6106774 \\
6106971 \\
6107238\end{array}$ & $\begin{array}{r}10.5 \\
2.4 \\
11.9 \\
0.8 \\
0.0\end{array}$ & $\begin{array}{l}0.8 \\
2.9 \\
0.1 \\
0.1 \\
0.0\end{array}$ & $\begin{array}{r}19.1 \\
0.7 \\
0.3 \\
3.0 \\
8.0\end{array}$ & $\begin{array}{l}1.3 \\
0.9 \\
0.8 \\
0.6 \\
0.2\end{array}$ & $\begin{array}{r}18.5 \\
2.0 \\
0.2 \\
2.8 \\
5.7\end{array}$ & $\begin{array}{l}4.8 \\
0.8 \\
4.3 \\
3.5 \\
2.4\end{array}$ & $\begin{array}{l}--- \\
--- \\
--- \\
--- \\
---\end{array}$ & $\begin{array}{l}--- \\
--- \\
--- \\
--- \\
---\end{array}$ & $\begin{array}{r}0 \\
0 \\
319 \\
57 \\
0\end{array}$ \\
\hline
\end{tabular}

$\mathrm{CX}=$ COAXIAL

Note: EM values shown above

are local amplitudes

Ketchikan - North/South of 5515
* Estimated Depth may be unreliable because the stronger part of the conductor may be deeper or to one side of the flight line, or because of a shallow dip or magnetite/overburden effects 
EM Anomaly List

\begin{tabular}{|c|c|c|c|c|c|c|c|c|c|c|c|c|c|}
\hline Label & Fid & Interp & $\begin{array}{l}\text { XUTM } \\
\text { m }\end{array}$ & $\begin{array}{c}\text { YUTM } \\
\mathrm{m}\end{array}$ & $\begin{array}{l}\text { CX } \\
\text { Real } \\
\text { ppm }\end{array}$ & $\begin{array}{c}900 \mathrm{HZ} \\
\text { Quad } \\
\text { ppm }\end{array}$ & $\begin{array}{l}\text { CP } \\
\text { Real } \\
\text { ppm }\end{array}$ & $\begin{array}{c}900 \mathrm{HZ} \\
\text { Quad } \\
\text { ppm }\end{array}$ & $\begin{array}{l}\text { CP } \\
\text { Real } \\
\text { ppm }\end{array}$ & $\begin{array}{c}7200 \mathrm{~Hz} \\
\text { Quad } \\
\text { ppm }\end{array}$ & $\begin{array}{l}\text { Vertica } \\
\text { COND } \\
\text { siemens }\end{array}$ & $\begin{array}{c}\text { al Dike } \\
\text { DEPTH* } \\
\text { m }\end{array}$ & $\begin{array}{c}\text { Mag. Corr } \\
\text { NT }\end{array}$ \\
\hline $\begin{array}{l}\text { LINE } \\
\text { A } \\
\text { B } \\
\text { C } \\
\text { D } \\
\text { E } \\
\text { F } \\
\text { G } \\
\text { H } \\
\text { I } \\
\text { J }\end{array}$ & $\begin{array}{r}11403 \\
7506.0 \\
7662.8 \\
7687.6 \\
7728.5 \\
7900.7 \\
8038.6 \\
8076.8 \\
8108.5 \\
8195.8 \\
8206.6\end{array}$ & $\begin{array}{r}\mathrm{M} \\
\mathrm{B} ? \\
\mathrm{D} \\
\mathrm{M} \\
\mathrm{M} \\
\mathrm{B} ? \\
\mathrm{~S} \\
\mathrm{~S} \\
\mathrm{~B} ? \\
\mathrm{~B}\end{array}$ & $\begin{array}{l}680460 \\
680381 \\
680351 \\
680358 \\
680226 \\
680055 \\
680002 \\
680031 \\
679937 \\
679904\end{array}$ & $\begin{array}{l}6107181 \\
6109319 \\
6109737 \\
6110622 \\
6114350 \\
6117658 \\
6118464 \\
6119530 \\
6122113 \\
6122497\end{array}$ & $\begin{array}{r}1.5 \\
0.6 \\
8.1 \\
0.3 \\
2.1 \\
0.3 \\
2.6 \\
2.8 \\
98.6 \\
1.0\end{array}$ & $\begin{array}{r}0.1 \\
1.4 \\
9.0 \\
0.3 \\
1.0 \\
0.6 \\
0.8 \\
5.1 \\
73.5 \\
7.5\end{array}$ & $\begin{array}{r}0.8 \\
0.6 \\
5.9 \\
1.6 \\
0.8 \\
0.2 \\
0.5 \\
2.0 \\
149.4 \\
4.7\end{array}$ & $\begin{array}{r}0.4 \\
3.2 \\
10.7 \\
1.2 \\
1.7 \\
0.9 \\
1.9 \\
8.2 \\
126.2 \\
11.4\end{array}$ & $\begin{array}{r}0.4 \\
5.6 \\
21.8 \\
1.5 \\
1.3 \\
0.2 \\
1.7 \\
18.2 \\
282.0 \\
32.0\end{array}$ & $\begin{array}{r}1.5 \\
16.3 \\
15.4 \\
7.7 \\
9.6 \\
4.1 \\
10.0 \\
42.5 \\
104.9 \\
22.5\end{array}$ & $\begin{array}{r}--- \\
1.2 \\
5.8 \\
--- \\
--- \\
--- \\
--- \\
2.5 \\
29.9 \\
1.6\end{array}$ & $\begin{array}{r}--- \\
32 \\
7 \\
--- \\
--- \\
--- \\
--- \\
8 \\
0 \\
0\end{array}$ & $\begin{array}{r}0 \\
50 \\
0 \\
4 \\
75 \\
0 \\
0 \\
25 \\
0 \\
0\end{array}$ \\
\hline $\begin{array}{l}\text { LINE } \\
\text { A } \\
\text { B } \\
\text { C } \\
\text { D } \\
\text { E } \\
\text { F } \\
\text { G } \\
\text { H } \\
\text { I } \\
\text { J } \\
\text { K } \\
\text { L }\end{array}$ & $\begin{array}{r}11410 \\
7933.3 \\
7905.3 \\
7894.1 \\
7883.2 \\
7875.0 \\
7843.4 \\
7829.4 \\
7823.2 \\
7766.8 \\
7750.9 \\
7742.0 \\
7733.0\end{array}$ & $\begin{array}{r}\text { B? } \\
\text { M } \\
\text { M } \\
\text { M } \\
\text { M } \\
\text { S? } \\
\text { D } \\
\text { D } \\
\text { S } \\
\text { M } \\
\text { M } \\
\text { B? }\end{array}$ & $\begin{array}{l}680187 \\
680225 \\
680224 \\
680179 \\
680155 \\
680107 \\
680119 \\
680115 \\
680121 \\
680096 \\
680074 \\
680046\end{array}$ & $\begin{array}{l}6125162 \\
6125587 \\
6125839 \\
6126064 \\
6126232 \\
6126774 \\
6127050 \\
6127174 \\
6127938 \\
6128080 \\
6128160 \\
6128258\end{array}$ & $\begin{array}{r}9.9 \\
0.6 \\
0.3 \\
0.1 \\
5.3 \\
0.0 \\
1.3 \\
4.7 \\
0.9 \\
4.5 \\
14.7 \\
10.4\end{array}$ & $\begin{array}{l}1.0 \\
0.2 \\
1.2 \\
0.5 \\
1.1 \\
1.3 \\
2.6 \\
4.2 \\
1.1 \\
0.6 \\
1.0 \\
1.4\end{array}$ & $\begin{array}{r}4.1 \\
0.2 \\
1.9 \\
1.1 \\
4.4 \\
4.5 \\
8.1 \\
0.7 \\
0.1 \\
12.0 \\
0.5 \\
3.4\end{array}$ & $\begin{array}{l}0.8 \\
0.3 \\
0.9 \\
0.3 \\
0.2 \\
1.2 \\
9.8 \\
3.6 \\
1.5 \\
0.7 \\
0.7 \\
1.1\end{array}$ & $\begin{array}{r}2.8 \\
0.2 \\
1.7 \\
0.7 \\
11.3 \\
2.3 \\
24.2 \\
6.6 \\
0.1 \\
11.8 \\
0.3 \\
2.9\end{array}$ & $\begin{array}{r}3.6 \\
0.7 \\
3.9 \\
3.0 \\
1.9 \\
4.4 \\
27.0 \\
8.1 \\
9.5 \\
3.3 \\
5.8 \\
9.0\end{array}$ & $\begin{array}{r}--- \\
--- \\
--- \\
--- \\
--- \\
--- \\
--- \\
5.1 \\
--- \\
--- \\
--- \\
103.2\end{array}$ & $\begin{array}{r}--- \\
--- \\
--- \\
--- \\
---- \\
--- \\
--- \\
47 \\
---- \\
--- \\
--- \\
58\end{array}$ & $\begin{array}{r}52 \\
0 \\
619 \\
165 \\
54 \\
98 \\
0 \\
0 \\
0 \\
0 \\
0 \\
0\end{array}$ \\
\hline $\begin{array}{l}\text { LINE } \\
\text { A } \\
\text { B } \\
\text { C } \\
\text { D } \\
\text { E } \\
\text { F } \\
\text { G } \\
\text { H } \\
\text { I } \\
\text { J } \\
\text { K } \\
\text { L } \\
\text { M } \\
\text { N } \\
\text { O } \\
\text { P } \\
\text { Q } \\
\text { R }\end{array}$ & $\begin{array}{r}11411 \\
9178.8 \\
9166.3 \\
9131.3 \\
9118.1 \\
9052.6 \\
9040.5 \\
9027.1 \\
8982.7 \\
8848.4 \\
8646.1 \\
8559.5 \\
8546.7 \\
8499.3 \\
8482.1 \\
8445.3 \\
8439.2 \\
8397.1 \\
8361.2\end{array}$ & $\begin{array}{r}\mathrm{D} \\
\mathrm{M} \\
\mathrm{M} \\
\mathrm{M} \\
\mathrm{M} \\
\mathrm{M} \\
\mathrm{M} \\
\mathrm{M} \\
\mathrm{D} \\
\mathrm{S} \\
\mathrm{M} \\
\mathrm{B} ? \\
\mathrm{D} \\
\mathrm{S} ? \\
\mathrm{~S} \\
\mathrm{~S} \\
\mathrm{~B} \\
\mathrm{E}\end{array}$ & $\begin{array}{l}680988 \\
680925 \\
680913 \\
680903 \\
680813 \\
680836 \\
680850 \\
680806 \\
680739 \\
680550 \\
680557 \\
680551 \\
680488 \\
680456 \\
680411 \\
680398 \\
680361 \\
680289\end{array}$ & $\begin{array}{l}6104610 \\
6104855 \\
6105240 \\
6105391 \\
6106542 \\
6106736 \\
6106882 \\
6107594 \\
6109738 \\
6114171 \\
6116015 \\
6116478 \\
6118145 \\
6118690 \\
6119974 \\
6120202 \\
6121721 \\
6122589\end{array}$ & $\begin{array}{r}5.9 \\
115.4 \\
0.0 \\
0.1 \\
17.7 \\
0.1 \\
2.9 \\
0.3 \\
2.6 \\
0.0 \\
1.2 \\
14.6 \\
3.8 \\
1.7 \\
0.7 \\
2.1 \\
36.7 \\
16.8\end{array}$ & $\begin{array}{r}9.0 \\
0.0 \\
0.3 \\
0.3 \\
0.9 \\
0.0 \\
0.2 \\
0.6 \\
2.0 \\
1.5 \\
0.9 \\
14.6 \\
1.6 \\
8.5 \\
5.0 \\
4.9 \\
23.0 \\
4.3\end{array}$ & $\begin{array}{r}5.8 \\
31.9 \\
0.9 \\
10.1 \\
35.1 \\
13.7 \\
5.6 \\
0.1 \\
1.5 \\
0.9 \\
0.3 \\
45.4 \\
4.9 \\
1.8 \\
1.2 \\
0.9 \\
70.3 \\
25.7\end{array}$ & $\begin{array}{r}8.6 \\
0.5 \\
0.1 \\
0.7 \\
0.3 \\
0.3 \\
0.2 \\
0.5 \\
1.9 \\
3.0 \\
1.4 \\
44.4 \\
3.8 \\
15.9 \\
6.4 \\
7.1 \\
44.9 \\
10.6\end{array}$ & $\begin{array}{r}21.3 \\
26.4 \\
0.5 \\
9.2 \\
29.5 \\
12.1 \\
5.5 \\
0.4 \\
5.3 \\
0.0 \\
0.5 \\
80.5 \\
10.4 \\
20.5 \\
7.7 \\
7.1 \\
105.5 \\
30.7\end{array}$ & $\begin{array}{r}25.8 \\
2.5 \\
3.8 \\
5.2 \\
3.5 \\
3.9 \\
2.2 \\
4.2 \\
7.0 \\
18.2 \\
7.5 \\
18.6 \\
18.7 \\
103.3 \\
39.2 \\
44.3 \\
23.7 \\
6.1\end{array}$ & $\begin{array}{r}4.2 \\
--- \\
--- \\
--- \\
--- \\
--- \\
--- \\
--- \\
7.0 \\
--- \\
--- \\
12.9 \\
--- \\
1.0 \\
--- \\
1.7 \\
30.6 \\
61.6\end{array}$ & $\begin{array}{r}34 \\
--- \\
--- \\
--- \\
--- \\
--- \\
--- \\
--- \\
42 \\
--- \\
--- \\
0 \\
--- \\
0 \\
--- \\
19 \\
0 \\
0\end{array}$ & $\begin{array}{r}83 \\
155 \\
0 \\
44 \\
0 \\
0 \\
0 \\
0 \\
0 \\
44 \\
87 \\
0 \\
0 \\
44 \\
0 \\
44 \\
0 \\
44\end{array}$ \\
\hline
\end{tabular}

$\mathrm{CX}=$ COAXIAL

Note: EM values shown above

Ketchikan - North/South of 5515
* Estimated Depth may be unreliable because the stronger part of the conductor may be deeper or to one side of the flight line, or because 
EM Anomaly List

\begin{tabular}{|c|c|c|c|c|c|c|c|c|c|c|c|c|c|}
\hline Label & Fid & Interp & $\begin{array}{c}\text { XUTM } \\
\text { m }\end{array}$ & $\begin{array}{c}\text { YUTM } \\
\mathrm{m}\end{array}$ & $\begin{array}{l}\text { CX } \\
\text { Real } \\
\text { ppm }\end{array}$ & $\begin{array}{l}00 \mathrm{HZ} \\
\text { Quad } \\
\text { ppm }\end{array}$ & $\begin{array}{l}\text { CP } \\
\text { Real } \\
\text { ppm }\end{array}$ & $\begin{array}{l}900 \mathrm{HZ} \\
\text { Quad } \\
\text { ppm }\end{array}$ & $\begin{array}{l}\text { CP } \\
\text { Real } \\
\text { ppm }\end{array}$ & $\begin{array}{l}200 \mathrm{HZ} \\
\text { Quad } \\
\text { ppm }\end{array}$ & $\begin{array}{l}\text { Vertica } \\
\text { Cond } \\
\text { siemens }\end{array}$ & $\begin{array}{c}\text { al Dike } \\
\text { DEPTH* } \\
\text { m }\end{array}$ & $\begin{array}{c}\text { Mag. Corr } \\
\text { NT }\end{array}$ \\
\hline $\begin{array}{l}\text { LINE } \\
\text { A } \\
\text { B } \\
\text { C } \\
\text { D } \\
\text { E } \\
\text { F } \\
\text { G }\end{array}$ & $\begin{array}{l}11420 \\
8079.1 \\
8088.0 \\
8101.9 \\
8113.4 \\
8153.6 \\
8218.9 \\
8223.0\end{array}$ & $\begin{array}{c}\mathrm{M} \\
\mathrm{M} \\
\mathrm{B} ? \\
\mathrm{M} \\
\mathrm{D} \\
\mathrm{M} \\
\mathrm{M}\end{array}$ & $\begin{array}{l}680550 \\
680549 \\
680539 \\
680532 \\
680522 \\
680430 \\
680446\end{array}$ & $\begin{array}{l}6125298 \\
6125462 \\
6125670 \\
6125881 \\
6126738 \\
6128257 \\
6128374\end{array}$ & $\begin{array}{r}1.2 \\
1.8 \\
116.1 \\
121.7 \\
11.0 \\
22.9 \\
2.3\end{array}$ & $\begin{array}{l}0.7 \\
0.6 \\
4.1 \\
1.2 \\
8.6 \\
0.9 \\
1.0\end{array}$ & $\begin{array}{r}2.7 \\
10.2 \\
157.4 \\
293.0 \\
1.1 \\
46.4 \\
7.5\end{array}$ & $\begin{array}{l}0.5 \\
0.3 \\
1.8 \\
1.0 \\
3.1 \\
0.7 \\
0.4\end{array}$ & $\begin{array}{r}2.5 \\
5.5 \\
130.2 \\
234.4 \\
9.0 \\
39.1 \\
5.5\end{array}$ & $\begin{array}{r}2.2 \\
0.7 \\
12.2 \\
5.7 \\
6.9 \\
3.7 \\
0.4\end{array}$ & $\begin{array}{r}--- \\
--- \\
999.0 \\
--- \\
10.4 \\
--- \\
---\end{array}$ & $\begin{array}{r}--- \\
--- \\
18 \\
--- \\
22 \\
--- \\
---\end{array}$ & $\begin{array}{r}9 \\
401 \\
486 \\
0 \\
0 \\
0 \\
107\end{array}$ \\
\hline $\begin{array}{l}\text { LINE } \\
\text { A } \\
\text { B } \\
\text { C } \\
\text { D } \\
\text { E } \\
\text { F } \\
\text { G } \\
\text { H } \\
\text { I } \\
\text { J } \\
\text { K } \\
\text { L } \\
\text { M } \\
\text { N } \\
\text { O } \\
\text { P } \\
\text { Q }\end{array}$ & $\begin{array}{l}11421 \\
9396.8 \\
9416.5 \\
9416.6 \\
9466.8 \\
9485.8 \\
9486.4 \\
9524.0 \\
9583.9 \\
9661.9 \\
9672.0 \\
9686.8 \\
9723.7 \\
9971.2 \\
9972.8 \\
9981.6 \\
10022.2 \\
10106.5\end{array}$ & $\begin{array}{r}\mathrm{D} \\
\mathrm{M} \\
\mathrm{M} \\
\mathrm{M} \\
\mathrm{M} \\
\mathrm{B} ? \\
\mathrm{M} \\
\mathrm{M} \\
\mathrm{S} \\
\mathrm{B} ? \\
\mathrm{~B} ? \\
\mathrm{D} \\
\mathrm{B} ? \\
\mathrm{~B} ? \\
\mathrm{E} \\
\mathrm{M} \\
\mathrm{B}\end{array}$ & $\begin{array}{l}681351 \\
681339 \\
681339 \\
681256 \\
681252 \\
681253 \\
681309 \\
681212 \\
681177 \\
681183 \\
681166 \\
681097 \\
680968 \\
680959 \\
680899 \\
680849 \\
680692\end{array}$ & $\begin{array}{l}6104405 \\
6104676 \\
6104676 \\
6105176 \\
6105530 \\
6105541 \\
6106008 \\
6106617 \\
6108600 \\
6108905 \\
6109105 \\
6109723 \\
6116274 \\
6116341 \\
6116693 \\
6118315 \\
6121521\end{array}$ & $\begin{array}{r}12.2 \\
0.0 \\
0.0 \\
12.3 \\
5.1 \\
0.4 \\
0.0 \\
26.3 \\
0.6 \\
2.5 \\
0.9 \\
0.4 \\
1.3 \\
0.9 \\
6.0 \\
3.2 \\
28.0\end{array}$ & $\begin{array}{r}11.0 \\
0.5 \\
0.5 \\
0.1 \\
0.3 \\
0.4 \\
0.1 \\
0.1 \\
2.9 \\
2.2 \\
2.0 \\
1.8 \\
3.7 \\
4.7 \\
10.2 \\
0.7 \\
23.1\end{array}$ & $\begin{array}{r}12.8 \\
0.5 \\
0.8 \\
20.8 \\
5.0 \\
4.8 \\
20.3 \\
1.1 \\
1.4 \\
2.0 \\
1.3 \\
0.5 \\
2.2 \\
2.6 \\
4.2 \\
1.2 \\
58.5\end{array}$ & $\begin{array}{r}13.1 \\
0.4 \\
0.4 \\
0.7 \\
2.0 \\
2.4 \\
0.5 \\
0.3 \\
3.6 \\
4.8 \\
5.1 \\
2.4 \\
8.7 \\
10.1 \\
7.9 \\
0.8 \\
50.7\end{array}$ & $\begin{array}{r}30.1 \\
19.4 \\
0.1 \\
16.3 \\
5.6 \\
3.2 \\
17.3 \\
0.9 \\
4.7 \\
9.3 \\
15.7 \\
0.7 \\
25.4 \\
29.1 \\
16.8 \\
1.1 \\
105.6\end{array}$ & $\begin{array}{r}16.8 \\
3.8 \\
3.8 \\
3.4 \\
9.8 \\
12.0 \\
3.4 \\
1.1 \\
23.3 \\
13.2 \\
21.9 \\
12.9 \\
28.8 \\
32.2 \\
6.5 \\
3.2 \\
31.0\end{array}$ & $\begin{array}{r}10.9 \\
--- \\
--- \\
--- \\
--- \\
11.8 \\
--- \\
--- \\
1.2 \\
--- \\
1.6 \\
--- \\
1.7 \\
1.3 \\
3.3 \\
--- \\
18.6\end{array}$ & $\begin{array}{r}11 \\
--- \\
--- \\
--- \\
--- \\
77 \\
--- \\
--- \\
11 \\
--- \\
30 \\
--- \\
0 \\
0 \\
21 \\
--- \\
0\end{array}$ & $\begin{array}{r}0 \\
0 \\
0 \\
141 \\
0 \\
37 \\
437 \\
84 \\
0 \\
42 \\
0 \\
37 \\
37 \\
37 \\
0 \\
102 \\
37\end{array}$ \\
\hline $\begin{array}{l}\text { LINE } \\
\text { A } \\
\text { B } \\
\text { C } \\
\text { D } \\
\text { E } \\
\text { F } \\
\text { G } \\
\text { H } \\
\text { I } \\
\text { J }\end{array}$ & $\begin{array}{r}11430 \\
8577.4 \\
8533.0 \\
8508.2 \\
8467.8 \\
8452.8 \\
8437.2 \\
8423.2 \\
8413.2 \\
8406.1 \\
8392.0\end{array}$ & $\begin{array}{r}\text { S? } \\
\text { B? } \\
\text { S } \\
\text { M } \\
\text { M } \\
\text { M } \\
\text { M } \\
\text { B? } \\
\text { M } \\
\text { M }\end{array}$ & $\begin{array}{l}680956 \\
680927 \\
680926 \\
680897 \\
680891 \\
680903 \\
680904 \\
680908 \\
680910 \\
680894\end{array}$ & $\begin{array}{l}6125421 \\
6126374 \\
6126921 \\
6127500 \\
6127669 \\
6127945 \\
6128075 \\
6128205 \\
6128287 \\
6128425\end{array}$ & $\begin{array}{l}0.2 \\
1.0 \\
0.2 \\
0.5 \\
0.7 \\
0.1 \\
7.0 \\
8.1 \\
2.1 \\
8.5\end{array}$ & $\begin{array}{l}1.2 \\
2.6 \\
2.3 \\
0.9 \\
1.4 \\
0.6 \\
1.5 \\
4.7 \\
1.0 \\
1.3\end{array}$ & $\begin{array}{l}1.0 \\
0.1 \\
0.4 \\
3.8 \\
0.5 \\
0.6 \\
4.8 \\
0.4 \\
1.0 \\
13.0\end{array}$ & $\begin{array}{l}1.1 \\
4.8 \\
2.5 \\
1.1 \\
0.7 \\
0.1 \\
0.6 \\
6.3 \\
0.3 \\
1.8\end{array}$ & $\begin{array}{r}0.5 \\
9.0 \\
1.1 \\
3.6 \\
1.7 \\
0.5 \\
4.5 \\
0.4 \\
1.0 \\
11.7\end{array}$ & $\begin{array}{r}6.6 \\
20.3 \\
15.5 \\
4.0 \\
3.1 \\
2.3 \\
5.5 \\
44.4 \\
3.1 \\
10.0\end{array}$ & $\begin{array}{l}--- \\
0.9 \\
0.7 \\
--- \\
--- \\
--- \\
--- \\
8.3 \\
--- \\
---\end{array}$ & $\begin{array}{r}--- \\
12 \\
4 \\
--- \\
--- \\
--- \\
--- \\
39 \\
--- \\
---\end{array}$ & $\begin{array}{r}302 \\
0 \\
0 \\
0 \\
71 \\
0 \\
0 \\
69 \\
16 \\
69\end{array}$ \\
\hline $\begin{array}{l}\text { LINE } \\
\text { A } \\
B \\
\text { C } \\
\text { D } \\
\text { E }\end{array}$ & $\begin{array}{r}11431 \\
1418.0 \\
1392.0 \\
1239.1 \\
1152.1 \\
1129.0\end{array}$ & $\begin{array}{l}\mathrm{B} \\
\mathrm{M} \\
\mathrm{M} \\
\mathrm{M} \\
\mathrm{M}\end{array}$ & $\begin{array}{l}681821 \\
681724 \\
681739 \\
681677 \\
681683\end{array}$ & $\begin{array}{l}6104350 \\
6104766 \\
6105215 \\
6106244 \\
6106391\end{array}$ & $\begin{array}{l}2.5 \\
2.2 \\
0.9 \\
0.3 \\
8.7\end{array}$ & $\begin{array}{l}2.0 \\
0.1 \\
0.7 \\
0.6 \\
0.2\end{array}$ & $\begin{array}{l}4.6 \\
0.7 \\
0.7 \\
0.4 \\
0.6\end{array}$ & $\begin{array}{l}6.4 \\
0.1 \\
0.1 \\
0.3 \\
0.2\end{array}$ & $\begin{array}{r}15.9 \\
0.6 \\
0.4 \\
0.6 \\
0.5\end{array}$ & $\begin{array}{r}13.7 \\
0.7 \\
1.8 \\
0.9 \\
1.6\end{array}$ & $\begin{array}{l}5.6 \\
--- \\
--- \\
--- \\
---\end{array}$ & $\begin{array}{l}26 \\
--- \\
--- \\
--- \\
---\end{array}$ & $\begin{array}{r}0 \\
591 \\
0 \\
68 \\
0\end{array}$ \\
\hline
\end{tabular}

$\mathrm{CX}=$ COAXIAL

Note: EM values shown above

Ketchikan - North/South of 5515
* Estimated Depth may be unreliable because the stronger part of the conductor may be deeper or to one side of the flight line, or because of shallow dip or magnetite/overburden effects 
EM Anomaly List

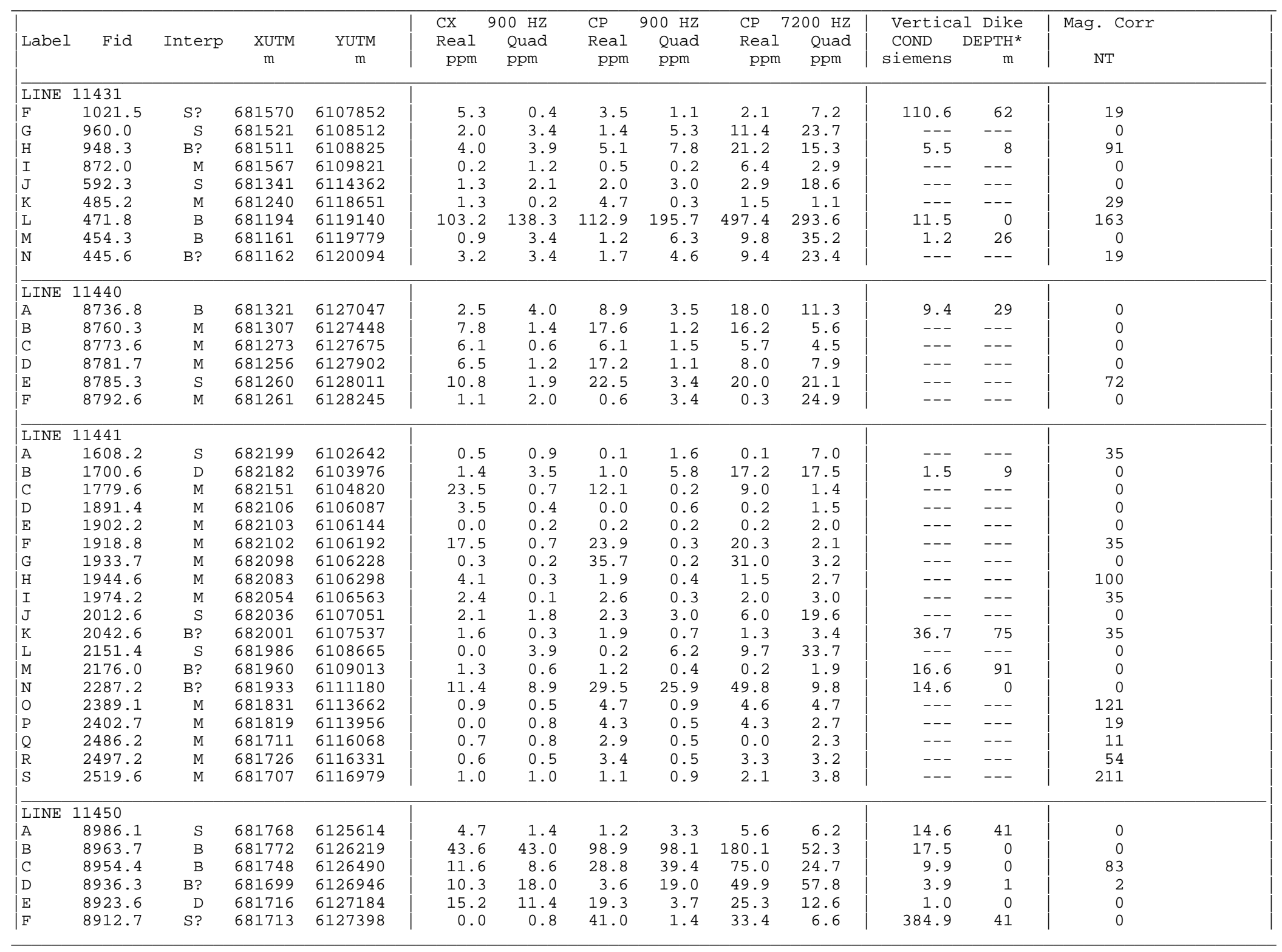

$\mathrm{CX}=$ COAXIAL

Note: EM values shown above

are local amplitudes

Ketchikan - North/South of 5515
*Estimated Depth may be unreliable because the stronger part of the conductor may be deeper or to one side of the flight line, or because of a shallow dip or magnetite/overburden effects 
EM Anomaly List

\begin{tabular}{|c|c|c|c|c|c|c|c|c|c|c|c|c|c|}
\hline Label & Fid & Interp & $\begin{array}{c}\text { XUTM } \\
\text { m }\end{array}$ & $\begin{array}{c}\text { YUTM } \\
\text { m }\end{array}$ & $\begin{array}{l}\text { CX } \\
\text { Real } \\
\text { ppm }\end{array}$ & $\begin{array}{l}900 \mathrm{~Hz} \\
\text { Quad } \\
\text { ppm }\end{array}$ & $\begin{array}{l}\text { CP } \\
\text { Real } \\
\text { ppm }\end{array}$ & $\begin{array}{l}900 \mathrm{~Hz} \\
\text { Quad } \\
\text { ppm }\end{array}$ & $\begin{array}{l}\text { CP } 7 \\
\text { Real } \\
\text { ppm }\end{array}$ & $\begin{array}{l}200 \mathrm{HZ} \\
\text { Quad } \\
\text { ppm }\end{array}$ & $\begin{array}{l}\text { Vertica } \\
\text { COND } \\
\text { siemens }\end{array}$ & $\begin{array}{c}\text { al Dike } \\
\text { DEPTH* } \\
\text { m }\end{array}$ & $\begin{array}{c}\text { Mag. Corr } \\
\text { NT }\end{array}$ \\
\hline $\begin{array}{l}\text { LINE } \\
\text { G } \\
\text { H } \\
\text { I } \\
\text { J } \\
\text { K } \\
\text { L }\end{array}$ & $\begin{array}{r}11450 \\
8904.7 \\
8895.3 \\
8872.0 \\
8854.7 \\
8849.3 \\
8843.2\end{array}$ & $\begin{array}{c}\mathrm{M} \\
\mathrm{M} \\
\mathrm{B} ? \\
\mathrm{M} \\
\mathrm{M} \\
\mathrm{M}\end{array}$ & $\begin{array}{l}681692 \\
681678 \\
681618 \\
681642 \\
681667 \\
681671\end{array}$ & $\begin{array}{l}6127520 \\
6127639 \\
6127892 \\
6128114 \\
6128231 \\
6128354\end{array}$ & $\begin{array}{r}12.3 \\
0.0 \\
0.0 \\
16.1 \\
13.0 \\
0.3\end{array}$ & $\begin{array}{l}0.7 \\
0.7 \\
2.0 \\
0.7 \\
0.9 \\
1.3\end{array}$ & $\begin{array}{r}30.2 \\
4.5 \\
3.0 \\
38.0 \\
2.6 \\
18.8\end{array}$ & $\begin{array}{l}0.9 \\
0.4 \\
1.9 \\
1.1 \\
0.7 \\
1.4\end{array}$ & $\begin{array}{r}22.6 \\
4.0 \\
2.6 \\
31.2 \\
2.4 \\
13.6\end{array}$ & $\begin{array}{l}3.9 \\
2.9 \\
9.9 \\
4.9 \\
3.8 \\
4.1\end{array}$ & $\begin{array}{l}--- \\
--- \\
1.6 \\
--- \\
--- \\
---\end{array}$ & $\begin{array}{r}--- \\
--- \\
90 \\
--- \\
--- \\
---\end{array}$ & $\begin{array}{r}63 \\
0 \\
83 \\
0 \\
363 \\
568\end{array}$ \\
\hline $\begin{array}{l}\text { LINE } \\
\text { A } \\
\text { B } \\
\text { C } \\
\text { D } \\
\text { E } \\
\text { F } \\
\text { G } \\
\text { H } \\
\text { I } \\
\text { J } \\
\text { K } \\
\text { L } \\
\text { M }\end{array}$ & $\begin{array}{r}11451 \\
3702.0 \\
3651.6 \\
3605.0 \\
3394.1 \\
3352.7 \\
3337.0 \\
3075.7 \\
3064.4 \\
3057.6 \\
3044.1 \\
3033.9 \\
3018.0 \\
2942.1\end{array}$ & $\begin{array}{c}\text { S? } \\
M \\
M \\
S \\
S \\
S \\
M \\
M \\
M \\
M \\
M \\
M \\
M\end{array}$ & $\begin{array}{l}682638 \\
682534 \\
682523 \\
682443 \\
682413 \\
682363 \\
682193 \\
682171 \\
682158 \\
682139 \\
682149 \\
682166 \\
682094\end{array}$ & $\begin{array}{l}6103001 \\
6104117 \\
6104968 \\
6106971 \\
6108201 \\
6108643 \\
6113759 \\
6114162 \\
6114421 \\
6114859 \\
6115094 \\
6115277 \\
6117098\end{array}$ & $\begin{array}{l}0.4 \\
1.2 \\
3.1 \\
3.1 \\
1.5 \\
0.8 \\
3.3 \\
1.4 \\
0.3 \\
0.3 \\
0.2 \\
0.5 \\
0.3\end{array}$ & $\begin{array}{l}0.5 \\
0.5 \\
0.3 \\
3.9 \\
5.8 \\
2.4 \\
0.3 \\
0.9 \\
0.7 \\
0.2 \\
0.1 \\
0.2 \\
0.2\end{array}$ & $\begin{array}{r}1.1 \\
3.5 \\
10.1 \\
3.6 \\
2.1 \\
0.6 \\
2.8 \\
0.2 \\
0.4 \\
0.6 \\
1.3 \\
0.9 \\
1.4\end{array}$ & $\begin{array}{l}2.2 \\
0.8 \\
0.2 \\
7.3 \\
9.6 \\
1.9 \\
0.0 \\
1.2 \\
0.5 \\
0.3 \\
0.8 \\
1.3 \\
0.2\end{array}$ & $\begin{array}{r}2.1 \\
2.0 \\
8.1 \\
11.5 \\
11.6 \\
3.2 \\
2.1 \\
0.3 \\
0.3 \\
0.8 \\
1.3 \\
0.2 \\
0.9\end{array}$ & $\begin{array}{r}11.6 \\
1.6 \\
1.6 \\
40.2 \\
57.7 \\
10.7 \\
2.8 \\
6.6 \\
2.1 \\
0.7 \\
5.7 \\
0.7 \\
2.0\end{array}$ & $\begin{array}{l}--- \\
--- \\
--- \\
--- \\
--- \\
--- \\
--- \\
--- \\
--- \\
--- \\
--- \\
--- \\
---\end{array}$ & $\begin{array}{l}--- \\
--- \\
--- \\
--- \\
--- \\
--- \\
--- \\
--- \\
--- \\
--- \\
--- \\
--- \\
---\end{array}$ & $\begin{array}{r}31 \\
209 \\
0 \\
0 \\
31 \\
0 \\
86 \\
44 \\
126 \\
68 \\
31 \\
0 \\
0\end{array}$ \\
\hline $\begin{array}{l}\text { LINE } \\
\text { A } \\
B\end{array}$ & $\begin{array}{l}11460 \\
954.8 \\
1000.0\end{array}$ & $\begin{array}{l}\mathrm{B} \\
\mathrm{M}\end{array}$ & $\begin{array}{l}682115 \\
682138\end{array}$ & $\begin{array}{l}6126983 \\
6128295\end{array}$ & $\begin{array}{r}2.5 \\
25.7\end{array}$ & $\begin{array}{l}3.0 \\
0.0\end{array}$ & $\begin{array}{r}0.1 \\
59.5\end{array}$ & $\begin{array}{l}1.6 \\
0.4\end{array}$ & $\begin{array}{r}4.9 \\
56.6\end{array}$ & $\begin{array}{l}5.4 \\
7.0\end{array}$ & $\begin{array}{l}--- \\
---\end{array}$ & $\begin{array}{l}--- \\
---\end{array}$ & $\begin{array}{r}0 \\
385\end{array}$ \\
\hline $\begin{array}{l}\text { LINE } \\
\text { A } \\
\text { B } \\
\text { C } \\
\text { D } \\
\text { E } \\
\text { F } \\
\text { G } \\
\text { H } \\
\text { I } \\
\text { J } \\
\text { K } \\
\text { L } \\
\text { M } \\
\text { N } \\
\text { O } \\
\text { P } \\
\text { Q } \\
\text { R }\end{array}$ & $\begin{array}{r}11461 \\
3928.1 \\
3963.9 \\
3988.8 \\
3998.5 \\
4024.5 \\
4064.0 \\
4117.0 \\
4122.1 \\
4217.5 \\
4422.2 \\
4454.1 \\
4465.9 \\
4494.0 \\
4512.7 \\
4535.9 \\
4734.2 \\
4773.3 \\
4786.8\end{array}$ & $\begin{array}{c}\text { M } \\
\text { M } \\
\text { S } \\
\text { B? } \\
\text { B? } \\
\text { M } \\
\text { M } \\
\text { M } \\
\text { S } \\
\text { M } \\
\text { M } \\
\text { M } \\
\text { M } \\
\text { M } \\
\text { M } \\
\text { M } \\
\text { B? } \\
\text { B? }\end{array}$ & $\begin{array}{l}682977 \\
682989 \\
682984 \\
682971 \\
682969 \\
682970 \\
682933 \\
682934 \\
682823 \\
682670 \\
682672 \\
682670 \\
682586 \\
682608 \\
682600 \\
682393 \\
682362 \\
682429\end{array}$ & $\begin{array}{l}6103398 \\
6103770 \\
6104124 \\
6104304 \\
6104722 \\
6105248 \\
6106131 \\
6106238 \\
6108308 \\
6112270 \\
6113088 \\
6113436 \\
6114143 \\
6114527 \\
6114864 \\
6119673 \\
6120664 \\
6121063\end{array}$ & $\begin{array}{r}0.7 \\
0.5 \\
0.9 \\
3.3 \\
1.9 \\
2.1 \\
5.1 \\
17.0 \\
0.2 \\
2.2 \\
0.0 \\
0.7 \\
1.3 \\
0.9 \\
0.2 \\
0.7 \\
23.8 \\
25.7\end{array}$ & $\begin{array}{r}0.5 \\
0.1 \\
1.3 \\
0.6 \\
2.4 \\
0.9 \\
0.3 \\
0.2 \\
1.3 \\
0.1 \\
0.4 \\
0.6 \\
0.3 \\
0.5 \\
0.3 \\
0.8 \\
7.4 \\
14.4\end{array}$ & $\begin{array}{r}4.8 \\
0.7 \\
0.1 \\
0.7 \\
2.9 \\
0.8 \\
19.4 \\
13.6 \\
1.4 \\
3.8 \\
3.7 \\
6.8 \\
3.4 \\
0.3 \\
0.1 \\
0.1 \\
30.4 \\
19.8\end{array}$ & $\begin{array}{r}1.1 \\
0.2 \\
2.2 \\
1.3 \\
1.9 \\
0.9 \\
2.1 \\
0.4 \\
2.2 \\
0.3 \\
0.6 \\
0.5 \\
1.1 \\
0.4 \\
0.6 \\
1.4 \\
18.4 \\
31.0\end{array}$ & $\begin{array}{r}4.3 \\
0.4 \\
1.1 \\
0.3 \\
3.8 \\
1.0 \\
16.4 \\
10.9 \\
7.9 \\
2.6 \\
3.3 \\
5.3 \\
2.2 \\
0.3 \\
0.2 \\
0.4 \\
38.6 \\
61.7\end{array}$ & $\begin{array}{r}5.0 \\
3.4 \\
12.7 \\
4.9 \\
12.8 \\
3.0 \\
6.0 \\
2.0 \\
14.5 \\
2.3 \\
4.4 \\
3.1 \\
5.1 \\
1.7 \\
3.3 \\
8.3 \\
10.4 \\
23.5\end{array}$ & $\begin{array}{r}--- \\
--- \\
1.8 \\
26.4 \\
5.8 \\
--- \\
--- \\
--- \\
--- \\
--- \\
--- \\
--- \\
--- \\
--- \\
--- \\
--- \\
44.4 \\
16.1\end{array}$ & $\begin{array}{r}--- \\
--- \\
43 \\
69 \\
58 \\
--- \\
--- \\
--- \\
--- \\
--- \\
--- \\
--- \\
--- \\
--- \\
--- \\
--- \\
0 \\
0\end{array}$ & $\begin{array}{r}0 \\
109 \\
0 \\
0 \\
0 \\
0 \\
33 \\
0 \\
0 \\
65 \\
33 \\
100 \\
101 \\
0 \\
22 \\
86 \\
33 \\
81\end{array}$ \\
\hline
\end{tabular}

$\mathrm{CX}=$ COAXIAL

Note: EM values shown above

Ketchikan - North/South of 5515
*Estimated Depth may be unreliable because the stronger part of the conductor may be deeper or to one side of the flight line, or because of shallow dip or magnetite/overburden effects 
EM Anomaly List

\begin{tabular}{|c|c|c|c|c|c|c|c|c|c|c|c|c|c|}
\hline Label & Fid & Interp & $\underset{\mathrm{m}}{\mathrm{XUTM}}$ & $\begin{array}{c}\text { YUTM } \\
\mathrm{m}\end{array}$ & $\begin{array}{l}\text { CX } \\
\text { Real } \\
\text { ppm }\end{array}$ & $\begin{array}{c}900 \mathrm{HZ} \\
\text { Quad } \\
\text { ppm }\end{array}$ & $\begin{array}{l}\text { CP } \\
\text { Real } \\
\text { ppm }\end{array}$ & $\begin{array}{l}900 \mathrm{HZ} \\
\text { Quad } \\
\text { ppm }\end{array}$ & $\begin{array}{l}\text { CP } \\
\text { Real } \\
\text { ppm }\end{array}$ & $\begin{array}{l}200 \mathrm{HZ} \\
\text { Quad } \\
\text { ppm }\end{array}$ & $\begin{array}{l}\text { Vertica } \\
\text { COND } \\
\text { siemens }\end{array}$ & $\begin{array}{c}\text { al Dike } \\
\text { DEPTH* } \\
\text { m }\end{array}$ & $\begin{array}{c}\text { Mag. Corr } \\
\text { NT }\end{array}$ \\
\hline $\begin{array}{l}\text { LINE } \\
\text { A } \\
\text { B } \\
\text { C } \\
\text { D }\end{array}$ & $\begin{array}{c}11470 \\
1117.1 \\
1112.2 \\
1042.2 \\
1027.0\end{array}$ & $\begin{array}{l}\mathrm{D} \\
\mathrm{D} \\
\mathrm{M} \\
\mathrm{M}\end{array}$ & $\begin{array}{l}682540 \\
682543 \\
682444 \\
682403\end{array}$ & $\begin{array}{l}6127168 \\
6127271 \\
6128168 \\
6128303\end{array}$ & $\begin{array}{r}1.8 \\
5.8 \\
17.0 \\
0.0\end{array}$ & $\begin{array}{l}2.9 \\
5.8 \\
0.5 \\
0.0\end{array}$ & $\begin{array}{l}3.5 \\
8.1 \\
1.4 \\
1.5\end{array}$ & $\begin{array}{l}4.8 \\
2.7 \\
0.7 \\
0.0\end{array}$ & $\begin{array}{r}24.2 \\
17.4 \\
1.9 \\
1.2\end{array}$ & $\begin{array}{r}21.0 \\
7.2 \\
2.5 \\
8.8\end{array}$ & $\begin{array}{r}--- \\
13.0 \\
--- \\
---\end{array}$ & $\begin{array}{r}--- \\
32 \\
--- \\
---\end{array}$ & $\begin{array}{r}0 \\
0 \\
704 \\
105\end{array}$ \\
\hline $\begin{array}{l}\text { LINE } \\
\text { A } \\
\text { B } \\
\text { C } \\
\text { D } \\
\text { E } \\
\text { F } \\
\text { G } \\
\text { H } \\
\text { I }\end{array}$ & $\begin{array}{r}11471 \\
5639.3 \\
5632.8 \\
5619.4 \\
5573.4 \\
5527.4 \\
5341.0 \\
5319.3 \\
5312.8 \\
5300.3\end{array}$ & $\begin{array}{r}\text { M } \\
\text { M } \\
\text { M } \\
\text { B? } \\
\text { B? } \\
\text { M } \\
\text { S } \\
\text { M } \\
\text { S }\end{array}$ & $\begin{array}{l}683257 \\
683237 \\
683271 \\
683128 \\
683176 \\
683058 \\
683019 \\
682984 \\
683015\end{array}$ & $\begin{array}{l}6106199 \\
6106413 \\
6106780 \\
6108267 \\
6108891 \\
6112650 \\
6112984 \\
6113141 \\
6113340\end{array}$ & $\begin{array}{l}0.4 \\
0.9 \\
1.1 \\
0.5 \\
1.0 \\
0.2 \\
1.3 \\
0.1 \\
0.6\end{array}$ & $\begin{array}{l}0.2 \\
0.6 \\
0.7 \\
1.9 \\
1.0 \\
0.5 \\
0.5 \\
0.8 \\
1.9\end{array}$ & $\begin{array}{r}3.2 \\
5.3 \\
7.1 \\
1.0 \\
1.6 \\
2.0 \\
11.5 \\
0.7 \\
6.6\end{array}$ & $\begin{array}{l}0.5 \\
0.3 \\
0.8 \\
2.8 \\
1.1 \\
1.2 \\
1.6 \\
0.5 \\
1.4\end{array}$ & $\begin{array}{r}3.0 \\
3.1 \\
13.2 \\
5.7 \\
0.1 \\
1.7 \\
10.9 \\
0.9 \\
6.5\end{array}$ & $\begin{array}{l}1.8 \\
4.9 \\
3.2 \\
3.2 \\
7.0 \\
5.1 \\
7.9 \\
1.6 \\
8.0\end{array}$ & $\begin{array}{l}--- \\
--- \\
--- \\
--- \\
--- \\
--- \\
--- \\
--- \\
---\end{array}$ & $\begin{array}{l}--- \\
--- \\
--- \\
--- \\
--- \\
--- \\
--- \\
--- \\
---\end{array}$ & $\begin{array}{r}707 \\
0 \\
32 \\
0 \\
0 \\
221 \\
0 \\
297 \\
0\end{array}$ \\
\hline $\begin{array}{l}\text { LINE } \\
\text { A } \\
\text { B } \\
\text { C } \\
\text { D } \\
\text { E }\end{array}$ & $\begin{array}{r}11480 \\
1235.4 \\
1244.4 \\
1256.5 \\
1265.2 \\
1277.5\end{array}$ & $\begin{array}{l}\mathrm{M} \\
\mathrm{M} \\
\mathrm{M} \\
\mathrm{M} \\
\mathrm{M}\end{array}$ & $\begin{array}{l}682933 \\
682936 \\
682919 \\
682907 \\
682899\end{array}$ & $\begin{array}{l}6127127 \\
6127319 \\
6127674 \\
6127912 \\
6128173\end{array}$ & $\begin{array}{l}0.6 \\
7.1 \\
7.1 \\
0.0 \\
2.9\end{array}$ & $\begin{array}{l}0.8 \\
0.5 \\
0.8 \\
0.3 \\
0.3\end{array}$ & $\begin{array}{r}5.1 \\
12.4 \\
2.8 \\
10.8 \\
47.6\end{array}$ & $\begin{array}{l}0.1 \\
0.8 \\
0.4 \\
0.2 \\
0.4\end{array}$ & $\begin{array}{r}6.0 \\
12.2 \\
3.4 \\
10.4 \\
44.2\end{array}$ & $\begin{array}{l}3.8 \\
4.2 \\
1.1 \\
1.3 \\
4.2\end{array}$ & $\begin{array}{l}--- \\
--- \\
--- \\
--- \\
---\end{array}$ & $\begin{array}{l}--- \\
--- \\
--- \\
--- \\
---\end{array}$ & $\begin{array}{r}586 \\
181 \\
0 \\
25 \\
755\end{array}$ \\
\hline $\begin{array}{l}\text { LINE } \\
\text { A } \\
\text { B } \\
\text { C } \\
\text { D } \\
\text { E } \\
\text { F } \\
\text { G } \\
\text { H } \\
\text { I }\end{array}$ & $\begin{array}{r}11481 \\
5985.1 \\
6133.7 \\
6181.2 \\
6192.8 \\
6234.7 \\
6532.1 \\
6532.3 \\
6842.8 \\
6853.4\end{array}$ & $\begin{array}{r}\text { S? } \\
M \\
M \\
M \\
S \\
S \\
S ? \\
M \\
M\end{array}$ & $\begin{array}{l}683815 \\
683712 \\
683705 \\
683701 \\
683644 \\
683347 \\
683346 \\
683368 \\
683359\end{array}$ & $\begin{array}{l}6102990 \\
6105017 \\
6105938 \\
6106252 \\
6107475 \\
6113850 \\
6113852 \\
6115977 \\
6116288\end{array}$ & $\begin{array}{l}1.2 \\
2.3 \\
0.0 \\
4.5 \\
1.5 \\
8.8 \\
8.8 \\
0.1 \\
0.0\end{array}$ & $\begin{array}{l}1.8 \\
0.4 \\
0.2 \\
0.4 \\
3.7 \\
0.3 \\
0.3 \\
0.8 \\
0.5\end{array}$ & $\begin{array}{r}2.1 \\
14.6 \\
13.2 \\
28.1 \\
7.7 \\
2.9 \\
2.6 \\
3.0 \\
7.2\end{array}$ & $\begin{array}{l}2.4 \\
0.6 \\
3.8 \\
0.8 \\
8.6 \\
0.1 \\
0.1 \\
0.6 \\
0.6\end{array}$ & $\begin{array}{r}4.3 \\
9.9 \\
17.3 \\
21.7 \\
20.4 \\
0.1 \\
0.0 \\
2.7 \\
1.0\end{array}$ & $\begin{array}{r}15.5 \\
1.2 \\
8.3 \\
3.5 \\
20.7 \\
0.8 \\
0.9 \\
3.6 \\
3.7\end{array}$ & $\begin{array}{r}--- \\
--- \\
--- \\
--- \\
--- \\
999.0 \\
--- \\
--- \\
---\end{array}$ & $\begin{array}{c}--- \\
--- \\
--- \\
--- \\
--- \\
33 \\
--- \\
--- \\
---\end{array}$ & $\begin{array}{r}0 \\
0 \\
776 \\
36 \\
39 \\
0 \\
0 \\
65 \\
54\end{array}$ \\
\hline $\begin{array}{l}\text { LINE } \\
\text { A } \\
B\end{array}$ & $\begin{array}{l}11490 \\
1362.3 \\
1330.8\end{array}$ & $\begin{array}{l}\mathrm{M} \\
\mathrm{M}\end{array}$ & $\begin{array}{l}683373 \\
683260\end{array}$ & $\begin{array}{l}6127022 \\
6127888\end{array}$ & $\begin{array}{l}0.0 \\
5.4\end{array}$ & $\begin{array}{l}0.5 \\
0.5\end{array}$ & $\begin{array}{r}1.6 \\
11.1\end{array}$ & $\begin{array}{l}0.5 \\
1.5\end{array}$ & $\begin{array}{l}2.6 \\
9.9\end{array}$ & $\begin{array}{l}0.6 \\
5.7\end{array}$ & $\begin{array}{l}--- \\
---\end{array}$ & $\begin{array}{l}--- \\
---\end{array}$ & $\begin{array}{l}0 \\
1\end{array}$ \\
\hline $\begin{array}{l}\text { LINE } \\
\text { A } \\
\text { B } \\
\text { C } \\
\text { D } \\
\text { E } \\
\text { F }\end{array}$ & $\begin{array}{r}11491 \\
996.9 \\
889.5 \\
842.2 \\
818.2 \\
638.3 \\
592.0\end{array}$ & $\begin{array}{r}\mathrm{D} \\
\mathrm{B} ? \\
\mathrm{M} \\
\mathrm{M} \\
\mathrm{M} \\
\mathrm{M}\end{array}$ & $\begin{array}{l}684217 \\
684068 \\
684009 \\
684041 \\
683893 \\
683869\end{array}$ & $\begin{array}{l}6104012 \\
6107098 \\
6107842 \\
6108128 \\
6111815 \\
6112461\end{array}$ & $\begin{array}{l}8.6 \\
3.4 \\
0.1 \\
3.1 \\
0.5 \\
0.7\end{array}$ & $\begin{array}{l}4.8 \\
4.0 \\
0.3 \\
0.2 \\
0.3 \\
0.3\end{array}$ & $\begin{array}{l}1.4 \\
5.5 \\
0.7 \\
5.7 \\
3.0 \\
0.4\end{array}$ & $\begin{array}{l}2.7 \\
6.2 \\
0.1 \\
0.1 \\
0.1 \\
0.7\end{array}$ & $\begin{array}{r}5.8 \\
17.8 \\
0.8 \\
5.4 \\
1.9 \\
0.2\end{array}$ & $\begin{array}{r}10.2 \\
38.1 \\
0.8 \\
2.7 \\
1.8 \\
1.4\end{array}$ & $\begin{array}{r}14.5 \\
5.6 \\
--- \\
--- \\
--- \\
---\end{array}$ & $\begin{array}{r}37 \\
33 \\
--- \\
--- \\
--- \\
---\end{array}$ & $\begin{array}{r}10 \\
19 \\
0 \\
38 \\
0 \\
0\end{array}$ \\
\hline
\end{tabular}

$\mathrm{CX}=$ COAXIAL

Note: EM values shown above

Ketchikan - North/South of 5515
* Estimated Depth may be unreliable because the stronger part of the conductor may be deeper or to one side of the flight line, or because of a shallow dip or magnetite/overburden effects 
EM Anomaly List

\begin{tabular}{|c|c|c|c|c|c|c|c|c|c|c|c|c|c|}
\hline Label & Fid & Interp & $\begin{array}{l}\text { XUTM } \\
\text { m }\end{array}$ & $\begin{array}{c}\text { YUTM } \\
\mathrm{m}\end{array}$ & $\begin{array}{l}\text { CX } \\
\text { Real } \\
\text { ppm }\end{array}$ & $\begin{array}{c}900 \mathrm{HZ} \\
\text { Quad } \\
\text { ppm }\end{array}$ & $\begin{array}{l}\text { CP } \\
\text { Real } \\
\text { ppm }\end{array}$ & $\begin{array}{c}900 \mathrm{HZ} \\
\text { Quad } \\
\text { ppm }\end{array}$ & $\begin{array}{l}\text { CP } \\
\text { Real } \\
\text { ppm }\end{array}$ & $\begin{array}{c}200 \mathrm{HZ} \\
\text { Quad } \\
\text { ppm }\end{array}$ & $\begin{array}{l}\text { Vertica } \\
\text { COND } \\
\text { siemens }\end{array}$ & $\begin{array}{c}\text { al Dike } \\
\text { DEPTH* } \\
\text { m }\end{array}$ & $\begin{array}{c}\text { Mag. Corr } \\
\text { NT }\end{array}$ \\
\hline $\begin{array}{l}\text { LINE } \\
\text { G } \\
\text { H } \\
\text { I } \\
\text { J } \\
\text { K } \\
\text { L }\end{array}$ & $\begin{array}{r}11491 \\
564.3 \\
352.7 \\
327.8 \\
309.3 \\
250.5 \\
220.7\end{array}$ & $\begin{array}{r}M \\
M \\
S ? \\
M \\
B \\
B ?\end{array}$ & $\begin{array}{l}683878 \\
683707 \\
683684 \\
683693 \\
683612 \\
683620\end{array}$ & $\begin{array}{l}6112901 \\
6116524 \\
6117003 \\
6117321 \\
6118929 \\
6119709\end{array}$ & $\begin{array}{r}3.1 \\
10.4 \\
0.0 \\
0.5 \\
65.8 \\
5.1\end{array}$ & $\begin{array}{r}0.4 \\
0.1 \\
0.3 \\
0.3 \\
48.0 \\
1.1\end{array}$ & $\begin{array}{r}1.1 \\
0.8 \\
0.0 \\
0.2 \\
177.5 \\
4.7\end{array}$ & $\begin{array}{r}0.1 \\
0.3 \\
1.4 \\
0.4 \\
127.3 \\
3.0\end{array}$ & $\begin{array}{r}0.8 \\
0.9 \\
0.1 \\
0.3 \\
232.5 \\
7.5\end{array}$ & $\begin{array}{r}1.7 \\
6.1 \\
16.0 \\
5.0 \\
57.7 \\
4.0\end{array}$ & $\begin{array}{c}--- \\
--- \\
0.1 \\
--- \\
32.6 \\
35.3\end{array}$ & $\begin{array}{r}--- \\
--- \\
0 \\
--- \\
0 \\
19\end{array}$ & $\begin{array}{r}38 \\
231 \\
0 \\
0 \\
38 \\
0\end{array}$ \\
\hline $\begin{array}{l}\text { LINE } \\
\text { A } \\
\text { B } \\
\text { C } \\
\text { D } \\
\text { E }\end{array}$ & $\begin{array}{r}11500 \\
1446.2 \\
1457.8 \\
1468.7 \\
1474.0 \\
1478.3\end{array}$ & $\begin{array}{l}\mathrm{D} \\
\mathrm{M} \\
\mathrm{M} \\
\mathrm{M} \\
\mathrm{M}\end{array}$ & $\begin{array}{l}683744 \\
683732 \\
683728 \\
683714 \\
683707\end{array}$ & $\begin{array}{l}6127211 \\
6127548 \\
6127800 \\
6127922 \\
6128034\end{array}$ & $\begin{array}{r}11.3 \\
4.0 \\
6.0 \\
6.1 \\
2.4\end{array}$ & $\begin{array}{l}8.6 \\
0.2 \\
0.4 \\
0.4 \\
0.6\end{array}$ & $\begin{array}{r}15.8 \\
5.8 \\
10.9 \\
30.7 \\
28.5\end{array}$ & $\begin{array}{r}19.1 \\
0.3 \\
0.1 \\
0.7 \\
1.0\end{array}$ & $\begin{array}{r}43.4 \\
8.4 \\
10.2 \\
28.1 \\
26.4\end{array}$ & $\begin{array}{l}7.7 \\
1.4 \\
1.9 \\
5.8 \\
6.4\end{array}$ & $\begin{array}{c}11.1 \\
--- \\
--- \\
--- \\
---\end{array}$ & $\begin{array}{r}3 \\
--- \\
--- \\
--- \\
---\end{array}$ & $\begin{array}{r}0 \\
0 \\
208 \\
25 \\
85\end{array}$ \\
\hline $\begin{array}{l}\text { LINE } \\
\text { A } \\
\text { B } \\
\text { C } \\
\text { D } \\
\text { E } \\
\text { F } \\
\text { G } \\
\text { H } \\
\text { I } \\
\text { J } \\
\text { K } \\
\text { L } \\
\text { M } \\
\text { N } \\
\text { O } \\
\text { P } \\
\text { Q }\end{array}$ & $\begin{array}{r}11501 \\
1276.8 \\
1348.7 \\
1387.0 \\
1408.7 \\
1466.8 \\
1603.4 \\
1638.9 \\
1707.7 \\
1735.8 \\
1764.7 \\
1777.0 \\
1789.6 \\
2028.7 \\
2033.5 \\
2043.3 \\
2113.6 \\
2142.0\end{array}$ & $\begin{array}{r}\text { S } \\
\text { B } \\
\text { D } \\
\text { S } \\
\text { B } \\
M \\
\text { B? } \\
M \\
M \\
M \\
M \\
M \\
M \\
M \\
M \\
D \\
D\end{array}$ & $\begin{array}{l}684651 \\
684617 \\
684602 \\
684576 \\
684540 \\
684409 \\
684356 \\
684301 \\
684293 \\
684271 \\
684252 \\
684261 \\
684131 \\
684119 \\
684104 \\
684032 \\
684011\end{array}$ & $\begin{array}{l}6102490 \\
6103438 \\
6103937 \\
6104507 \\
6106218 \\
6108715 \\
6109499 \\
6111631 \\
6112255 \\
6112572 \\
6112652 \\
6112749 \\
6116724 \\
6116818 \\
6117056 \\
6119055 \\
6119887\end{array}$ & $\begin{array}{r}0.0 \\
0.6 \\
3.9 \\
5.6 \\
1.2 \\
5.1 \\
0.5 \\
0.2 \\
1.3 \\
1.7 \\
0.7 \\
3.8 \\
6.1 \\
1.9 \\
4.6 \\
4.7 \\
25.9\end{array}$ & $\begin{array}{r}0.9 \\
0.9 \\
4.0 \\
1.1 \\
2.3 \\
0.6 \\
3.8 \\
0.8 \\
0.4 \\
0.3 \\
0.5 \\
0.5 \\
0.4 \\
0.6 \\
0.3 \\
4.5 \\
23.3\end{array}$ & $\begin{array}{l}1.7 \\
0.5 \\
0.2 \\
0.9 \\
2.2 \\
0.4 \\
1.7 \\
2.0 \\
1.0 \\
3.2 \\
0.1 \\
1.2 \\
7.5 \\
1.4 \\
8.6 \\
1.6 \\
27.6\end{array}$ & $\begin{array}{r}2.2 \\
1.6 \\
1.6 \\
1.6 \\
3.9 \\
0.5 \\
5.3 \\
0.7 \\
0.6 \\
1.0 \\
0.8 \\
0.3 \\
0.8 \\
1.1 \\
0.8 \\
4.2 \\
45.1\end{array}$ & $\begin{array}{r}1.9 \\
3.6 \\
4.8 \\
3.2 \\
6.0 \\
0.4 \\
12.6 \\
2.1 \\
1.7 \\
3.0 \\
0.4 \\
1.0 \\
6.8 \\
1.1 \\
2.4 \\
10.5 \\
98.0\end{array}$ & $\begin{array}{r}10.0 \\
10.1 \\
6.0 \\
3.7 \\
5.0 \\
2.6 \\
15.6 \\
3.3 \\
3.1 \\
2.8 \\
4.2 \\
1.0 \\
4.2 \\
8.8 \\
5.3 \\
11.8 \\
55.7\end{array}$ & $\begin{array}{r}--- \\
--- \\
4.5 \\
37.6 \\
--- \\
--- \\
--- \\
--- \\
--- \\
--- \\
--- \\
--- \\
--- \\
--- \\
--- \\
--- \\
10.5\end{array}$ & $\begin{array}{r}--- \\
--- \\
37 \\
10 \\
--- \\
--- \\
--- \\
--- \\
--- \\
--- \\
--- \\
--- \\
--- \\
--- \\
--- \\
--- \\
0\end{array}$ & $\begin{array}{r}0 \\
0 \\
33 \\
363 \\
0 \\
54 \\
0 \\
0 \\
243 \\
33 \\
12 \\
60 \\
69 \\
33 \\
0 \\
59 \\
33\end{array}$ \\
\hline $\begin{array}{l}\text { LINE } \\
\text { A } \\
B \\
C\end{array}$ & $\begin{array}{r}11510 \\
1527.2 \\
1522.2 \\
1513.5\end{array}$ & $\begin{array}{l}\mathrm{M} \\
\mathrm{M} \\
\mathrm{M}\end{array}$ & $\begin{array}{l}684074 \\
684059 \\
684024\end{array}$ & $\begin{array}{l}6127943 \\
6128035 \\
6128200\end{array}$ & $\begin{array}{r}0.8 \\
27.3 \\
0.0\end{array}$ & $\begin{array}{l}0.1 \\
0.5 \\
0.5\end{array}$ & $\begin{array}{r}10.1 \\
68.1 \\
0.0\end{array}$ & $\begin{array}{l}0.7 \\
1.4 \\
0.1\end{array}$ & $\begin{array}{r}9.8 \\
62.8 \\
0.0\end{array}$ & $\begin{array}{l}3.5 \\
6.0 \\
3.1\end{array}$ & $\begin{array}{l}--- \\
--- \\
---\end{array}$ & $\begin{array}{l}--- \\
--- \\
---\end{array}$ & $\begin{array}{l}91 \\
91 \\
82\end{array}$ \\
\hline $\begin{array}{l}\text { LINE } \\
\text { A } \\
\text { B } \\
\text { C } \\
\text { D } \\
\text { E } \\
\text { F } \\
\text { G }\end{array}$ & $\begin{array}{r}11511 \\
3941.2 \\
4259.9 \\
4300.0 \\
4318.6 \\
4349.8 \\
4432.8 \\
4437.7\end{array}$ & $\begin{array}{c}\mathrm{B} ? \\
\mathrm{M} \\
\mathrm{M} \\
\mathrm{M} \\
\mathrm{M} \\
\mathrm{M} \\
\mathrm{S}\end{array}$ & $\begin{array}{l}684968 \\
684688 \\
684671 \\
684643 \\
684656 \\
684600 \\
684560\end{array}$ & $\begin{array}{l}6105999 \\
6112232 \\
6112662 \\
6113024 \\
6113506 \\
6114303 \\
6114448\end{array}$ & $\begin{array}{l}8.3 \\
0.2 \\
0.1 \\
0.3 \\
0.6 \\
0.8 \\
1.2\end{array}$ & $\begin{array}{r}11.0 \\
0.9 \\
0.3 \\
1.3 \\
0.4 \\
6.5 \\
2.5\end{array}$ & $\begin{array}{l}5.3 \\
0.1 \\
0.0 \\
2.0 \\
2.4 \\
1.2 \\
0.0\end{array}$ & $\begin{array}{r}17.7 \\
0.5 \\
0.4 \\
0.8 \\
0.4 \\
10.1 \\
8.4\end{array}$ & $\begin{array}{r}48.8 \\
0.2 \\
0.5 \\
1.7 \\
2.1 \\
21.8 \\
16.5\end{array}$ & $\begin{array}{r}33.1 \\
2.3 \\
1.0 \\
4.0 \\
1.8 \\
59.6 \\
48.5\end{array}$ & $\begin{array}{l}3.5 \\
--- \\
--- \\
--- \\
--- \\
--- \\
---\end{array}$ & $\begin{array}{l}0 \\
--- \\
--- \\
--- \\
--- \\
--- \\
---\end{array}$ & $\begin{array}{r}29 \\
199 \\
16 \\
492 \\
0 \\
29 \\
29\end{array}$ \\
\hline
\end{tabular}

$\mathrm{CX}=$ COAXIAL

Note: EM values shown above

are local amplitudes

Ketchikan - North/South of 5515
*Estimated Depth may be unreliable because the stronger part of the conductor may be deeper or to one side of the flight line, or because of a shallow dip or magnetite/overburden effects 
EM Anomaly List

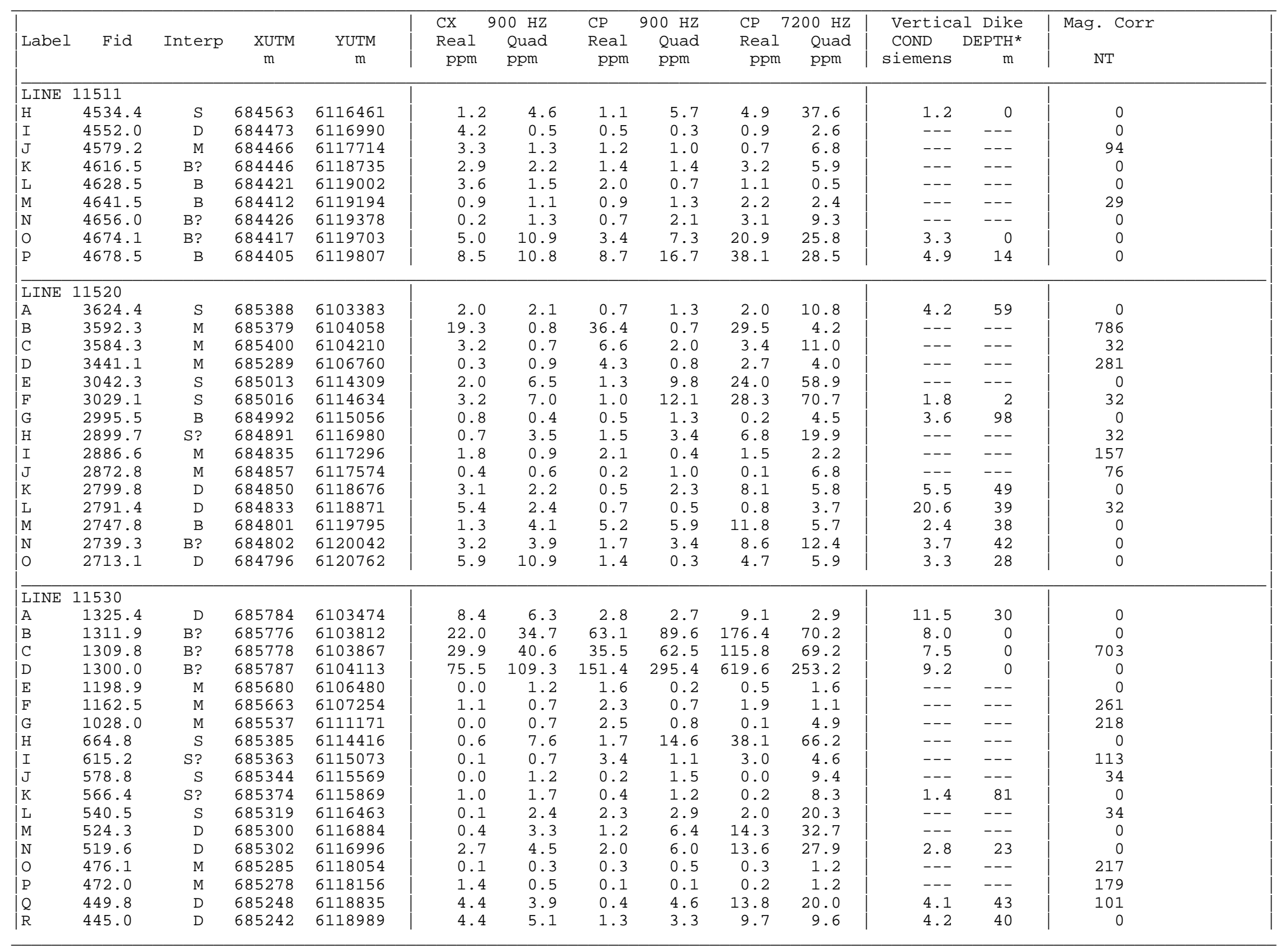

$\mathrm{CX}=$ COAXIAL

Note: EM values shown above

are local amplitudes

Ketchikan - North/South of 5515
*Estimated Depth may be unreliable because the stronger part of the conductor may be deeper or to one side of the flight line, or because of a shallow dip or magnetite/overburden effects 
EM Anomaly List

\begin{tabular}{|c|c|c|c|c|c|c|c|c|c|c|c|c|c|}
\hline Label & Fid & Interp & $\underset{\mathrm{m}}{\mathrm{XUTM}}$ & $\begin{array}{c}\text { YUTM } \\
\text { m }\end{array}$ & $\begin{array}{l}\text { CX } \\
\text { Real } \\
\text { ppm }\end{array}$ & $\begin{array}{l}00 \mathrm{HZ} \\
\text { Quad } \\
\text { ppm }\end{array}$ & $\begin{array}{l}\text { CP } \\
\text { Real } \\
\text { ppm }\end{array}$ & $\begin{array}{l}900 \mathrm{HZ} \\
\text { Quad } \\
\text { ppm }\end{array}$ & $\begin{array}{l}\text { CP } \\
\text { Real } \\
\text { ppm }\end{array}$ & $\begin{array}{c}200 \mathrm{HZ} \\
\text { Quad } \\
\text { ppm }\end{array}$ & $\begin{array}{l}\text { Vertica } \\
\text { CoND } \\
\text { siemens }\end{array}$ & $\begin{array}{c}\text { al Dike } \\
\text { DEPTH* } \\
\text { m }\end{array}$ & $\begin{array}{c}\text { Mag. Corr } \\
\text { NT }\end{array}$ \\
\hline $\begin{array}{l}\text { LINE } \\
\text { S } \\
\text { T } \\
\text { U } \\
\text { V } \\
\text { W } \\
X \\
Y \\
\text { Z }\end{array}$ & $\begin{array}{r}11530 \\
431.6 \\
428.0 \\
424.9 \\
419.2 \\
361.7 \\
356.9 \\
351.1 \\
346.2\end{array}$ & $\begin{array}{r}\mathrm{D} \\
\mathrm{D} \\
\mathrm{B} \\
\mathrm{B} ? \\
\mathrm{E} \\
\mathrm{B} ? \\
\mathrm{~B} \\
\mathrm{~B} ?\end{array}$ & $\begin{array}{l}685244 \\
685244 \\
685240 \\
685231 \\
685174 \\
685175 \\
685163 \\
685144\end{array}$ & $\begin{array}{l}6119461 \\
6119582 \\
6119685 \\
6119868 \\
6121330 \\
6121480 \\
6121660 \\
6121806\end{array}$ & $\begin{array}{r}8.5 \\
7.9 \\
4.4 \\
2.2 \\
13.5 \\
44.6 \\
34.3 \\
10.7\end{array}$ & $\begin{array}{r}6.1 \\
11.4 \\
4.5 \\
2.8 \\
14.9 \\
13.6 \\
10.9 \\
8.9\end{array}$ & $\begin{array}{r}1.2 \\
4.7 \\
4.4 \\
1.5 \\
28.3 \\
72.5 \\
42.7 \\
6.6\end{array}$ & $\begin{array}{r}11.2 \\
8.5 \\
7.2 \\
2.0 \\
28.5 \\
52.8 \\
27.8 \\
6.8\end{array}$ & $\begin{array}{r}27.3 \\
17.8 \\
14.2 \\
2.3 \\
60.8 \\
101.5 \\
55.0 \\
12.7\end{array}$ & $\begin{array}{r}24.6 \\
24.1 \\
18.9 \\
3.5 \\
29.1 \\
22.8 \\
3.6 \\
4.5\end{array}$ & $\begin{array}{r}--- \\
4.4 \\
5.1 \\
3.7 \\
10.6 \\
45.6 \\
47.0 \\
11.1\end{array}$ & $\begin{array}{r}--- \\
28 \\
39 \\
54 \\
8 \\
0 \\
0 \\
0\end{array}$ & $\begin{array}{r}0 \\
0 \\
214 \\
157 \\
0 \\
0 \\
0 \\
0\end{array}$ \\
\hline $\begin{array}{l}\text { LINE } \\
\text { A } \\
B\end{array}$ & $\begin{array}{r}11540 \\
1658.6 \\
1688.0\end{array}$ & $\begin{array}{l}\mathrm{B} \\
\mathrm{M}\end{array}$ & $\begin{array}{l}685932 \\
685890\end{array}$ & $\begin{array}{l}6110414 \\
6111067\end{array}$ & $\begin{array}{l}2.2 \\
3.4\end{array}$ & $\begin{array}{l}3.7 \\
0.6\end{array}$ & $\begin{array}{l}3.6 \\
2.1\end{array}$ & $\begin{array}{l}4.3 \\
0.8\end{array}$ & $\begin{array}{l}9.6 \\
0.1\end{array}$ & $\begin{array}{l}4.8 \\
5.7\end{array}$ & $\begin{array}{l}3.4 \\
---\end{array}$ & $\begin{array}{r}9 \\
---\end{array}$ & $\begin{array}{r}0 \\
161\end{array}$ \\
\hline $\begin{array}{l}\text { LINE } \\
\text { A } \\
B\end{array}$ & $\begin{array}{l}11541 \\
1877.9 \\
1998.0\end{array}$ & $\begin{array}{l}\mathrm{M} \\
\mathrm{M}\end{array}$ & $\begin{array}{l}685887 \\
685885\end{array}$ & $\begin{array}{l}6112210 \\
6112843\end{array}$ & $\begin{array}{l}0.9 \\
1.5\end{array}$ & $\begin{array}{l}0.4 \\
0.1\end{array}$ & $\begin{array}{l}2.7 \\
0.0\end{array}$ & $\begin{array}{l}0.5 \\
0.4\end{array}$ & $\begin{array}{l}4.8 \\
0.2\end{array}$ & $\begin{array}{l}2.4 \\
1.2\end{array}$ & $\begin{array}{l}--- \\
---\end{array}$ & $\begin{array}{l}--- \\
---\end{array}$ & $\begin{array}{l}0 \\
0\end{array}$ \\
\hline $\begin{array}{l}\text { LINE } \\
\text { A } \\
\text { B } \\
\text { C } \\
\text { D } \\
\text { E } \\
\text { F } \\
\text { G } \\
\text { H } \\
\text { I } \\
\text { J }\end{array}$ & $\begin{array}{r}11542 \\
2240.3 \\
2318.2 \\
2350.0 \\
2395.8 \\
2400.6 \\
2403.8 \\
2408.4 \\
2424.3 \\
2479.1 \\
2492.9\end{array}$ & $\begin{array}{r}\text { S } \\
\text { S? } \\
\text { B? } \\
\text { M } \\
\text { S? } \\
\text { B? } \\
\text { B? } \\
\text { B? } \\
\text { D } \\
\text { B? }\end{array}$ & $\begin{array}{l}685801 \\
685766 \\
685717 \\
685664 \\
685636 \\
685629 \\
685625 \\
685637 \\
685517 \\
685583\end{array}$ & $\begin{array}{l}6114457 \\
6115774 \\
6116875 \\
6118330 \\
6118504 \\
6118625 \\
6118798 \\
6119414 \\
6121383 \\
6121866\end{array}$ & $\begin{array}{r}3.0 \\
0.5 \\
3.4 \\
2.0 \\
7.3 \\
4.8 \\
4.5 \\
4.9 \\
18.7 \\
3.4\end{array}$ & $\begin{array}{r}11.0 \\
3.4 \\
5.2 \\
1.2 \\
10.0 \\
8.9 \\
8.2 \\
5.0 \\
21.2 \\
1.2\end{array}$ & $\begin{array}{r}1.8 \\
1.5 \\
4.1 \\
0.6 \\
9.8 \\
8.6 \\
1.2 \\
0.2 \\
17.6 \\
1.5\end{array}$ & $\begin{array}{r}17.2 \\
5.9 \\
3.6 \\
2.2 \\
12.7 \\
10.7 \\
7.8 \\
5.5 \\
38.1 \\
0.6\end{array}$ & $\begin{array}{r}42.1 \\
3.8 \\
6.6 \\
5.6 \\
40.5 \\
32.2 \\
17.4 \\
15.9 \\
95.4 \\
1.2\end{array}$ & $\begin{array}{r}88.0 \\
39.2 \\
24.1 \\
10.7 \\
61.8 \\
64.6 \\
30.6 \\
17.4 \\
53.4 \\
5.5\end{array}$ & $\begin{array}{l}1.4 \\
--- \\
--- \\
--- \\
5.7 \\
4.0 \\
2.8 \\
3.4 \\
6.5 \\
---\end{array}$ & $\begin{array}{r}0 \\
--- \\
--- \\
--- \\
14 \\
23 \\
8 \\
37 \\
0 \\
---\end{array}$ & $\begin{array}{r}0 \\
0 \\
0 \\
0 \\
54 \\
95 \\
0 \\
0 \\
23 \\
23\end{array}$ \\
\hline $\begin{array}{l}\text { LINE } \\
\text { A } \\
\text { B } \\
\text { C } \\
\text { D } \\
\text { E } \\
\text { F } \\
\text { G }\end{array}$ & $\begin{array}{c}11551 \\
737.3 \\
710.0 \\
661.8 \\
567.3 \\
554.0 \\
543.0 \\
534.0\end{array}$ & $\begin{array}{c}B ? \\
M \\
M \\
M \\
M \\
M \\
M\end{array}$ & $\begin{array}{l}686350 \\
686330 \\
686331 \\
686287 \\
686279 \\
686290 \\
686281\end{array}$ & $\begin{array}{l}6110700 \\
6111019 \\
6111312 \\
6112898 \\
6112969 \\
6113023 \\
6113082\end{array}$ & $\begin{array}{l}0.7 \\
0.2 \\
1.0 \\
0.1 \\
1.3 \\
0.0 \\
3.2\end{array}$ & $\begin{array}{l}2.3 \\
0.1 \\
0.1 \\
0.3 \\
0.4 \\
0.4 \\
0.5\end{array}$ & $\begin{array}{l}0.2 \\
0.8 \\
2.2 \\
3.4 \\
1.1 \\
0.3 \\
5.5\end{array}$ & $\begin{array}{l}0.1 \\
0.6 \\
0.3 \\
0.0 \\
0.0 \\
0.1 \\
0.4\end{array}$ & $\begin{array}{l}0.3 \\
0.9 \\
1.2 \\
2.8 \\
0.7 \\
3.5 \\
4.7\end{array}$ & $\begin{array}{l}3.1 \\
3.4 \\
0.4 \\
0.9 \\
1.9 \\
2.5 \\
3.9\end{array}$ & $\begin{array}{l}--- \\
--- \\
--- \\
--- \\
--- \\
--- \\
---\end{array}$ & $\begin{array}{l}--- \\
--- \\
--- \\
--- \\
--- \\
--- \\
---\end{array}$ & $\begin{array}{r}46 \\
0 \\
0 \\
285 \\
0 \\
0 \\
0\end{array}$ \\
\hline $\begin{array}{l}\text { LINE } \\
\text { A } \\
\text { B } \\
\text { C } \\
\text { D } \\
\text { E } \\
\text { F } \\
\text { G }\end{array}$ & $\begin{array}{r}11560 \\
1192.8 \\
1202.0 \\
1214.0 \\
1302.1 \\
1317.2 \\
1394.0 \\
1418.0\end{array}$ & $\begin{array}{r}\text { M } \\
\text { B? } \\
\text { B? } \\
\text { M } \\
\text { M } \\
\text { M } \\
\text { M }\end{array}$ & $\begin{array}{l}686754 \\
686754 \\
686744 \\
686696 \\
686698 \\
686641 \\
686693\end{array}$ & $\begin{array}{l}6110225 \\
6110510 \\
6110879 \\
6112229 \\
6112278 \\
6112522 \\
6112873\end{array}$ & $\begin{array}{r}3.0 \\
2.7 \\
1.7 \\
4.6 \\
11.8 \\
1.3 \\
0.0\end{array}$ & $\begin{array}{l}0.1 \\
3.2 \\
3.4 \\
0.3 \\
0.3 \\
0.4 \\
1.0\end{array}$ & $\begin{array}{r}2.7 \\
6.1 \\
0.2 \\
1.2 \\
10.9 \\
5.9 \\
0.6\end{array}$ & $\begin{array}{l}1.0 \\
1.9 \\
2.1 \\
1.3 \\
0.2 \\
0.1 \\
1.0\end{array}$ & $\begin{array}{l}2.0 \\
6.7 \\
0.2 \\
0.9 \\
8.4 \\
5.6 \\
0.7\end{array}$ & $\begin{array}{r}5.4 \\
11.1 \\
16.3 \\
2.8 \\
0.5 \\
0.6 \\
4.9\end{array}$ & $\begin{array}{r}--- \\
10.7 \\
1.9 \\
--- \\
--- \\
--- \\
---\end{array}$ & $\begin{array}{r}--- \\
38 \\
29 \\
--- \\
--- \\
--- \\
---\end{array}$ & $\begin{array}{r}292 \\
0 \\
67 \\
0 \\
0 \\
0 \\
247\end{array}$ \\
\hline
\end{tabular}

$\mathrm{CX}=$ COAXIAL

Note: EM values shown above

are local amplitudes

Ketchikan - North/South of 5515
*Estimated Depth may be unreliable because the stronger part of the conductor may be deeper or to one side of the flight line, or because of a shallow dip or magnetite/overburden effects 
EM Anomaly List

\begin{tabular}{|c|c|c|c|c|c|c|c|c|c|c|c|c|c|}
\hline Label & Fid & Interp & $\underset{\mathrm{m}}{\mathrm{XUTM}}$ & $\begin{array}{c}\text { YUTM } \\
\mathrm{m}\end{array}$ & $\begin{array}{l}\text { CX } \\
\text { Real } \\
\text { ppm }\end{array}$ & $\begin{array}{l}00 \mathrm{HZ} \\
\text { Quad } \\
\text { ppm }\end{array}$ & $\begin{array}{l}\text { CP } \\
\text { Real } \\
\text { ppm }\end{array}$ & $\begin{array}{l}900 \mathrm{HZ} \\
\text { Quad } \\
\text { ppm }\end{array}$ & $\begin{array}{l}\text { CP } 7 \\
\text { Real } \\
\text { ppm }\end{array}$ & $\begin{array}{l}200 \mathrm{HZ} \\
\text { Quad } \\
\text { ppm }\end{array}$ & $\begin{array}{l}\text { Vertica } \\
\text { COND } \\
\text { siemens }\end{array}$ & $\begin{array}{c}\text { al Dike } \\
\text { DEPTH* } \\
\text { m }\end{array}$ & $\begin{array}{c}\text { Mag. Corr } \\
\text { NT }\end{array}$ \\
\hline $\begin{array}{l}\text { LINE } \\
\mathrm{H}\end{array}$ & $\begin{array}{c}11560 \\
1436.8\end{array}$ & $\mathrm{M}$ & 686674 & 6113136 & 0.2 & 0.6 & 0.5 & 0.8 & 1.0 & 2.9 & --- & --- & 0 \\
\hline $\begin{array}{l}\text { LINE } \\
\text { A } \\
\text { B } \\
\text { C } \\
\text { D } \\
\text { E } \\
\text { F } \\
\text { G } \\
\text { H }\end{array}$ & $\begin{array}{r}11570 \\
1775.2 \\
1756.2 \\
1742.5 \\
1727.3 \\
1693.4 \\
1678.0 \\
1667.4 \\
1525.0\end{array}$ & $\begin{array}{c}\mathrm{M} \\
\mathrm{M} \\
\mathrm{M} \\
\mathrm{M} \\
\mathrm{D} \\
\mathrm{B} ? \\
\mathrm{M} \\
\mathrm{M}\end{array}$ & $\begin{array}{l}687236 \\
687225 \\
687206 \\
687181 \\
687170 \\
687157 \\
687148 \\
687093\end{array}$ & $\begin{array}{l}6108570 \\
6108928 \\
6109182 \\
6109545 \\
6110376 \\
6110794 \\
6110987 \\
6112437\end{array}$ & $\begin{array}{l}0.8 \\
0.7 \\
0.5 \\
1.0 \\
3.6 \\
3.7 \\
0.2 \\
0.2\end{array}$ & $\begin{array}{l}0.6 \\
0.3 \\
0.3 \\
0.1 \\
3.7 \\
1.5 \\
0.7 \\
0.1\end{array}$ & $\begin{array}{r}1.1 \\
1.0 \\
0.2 \\
0.8 \\
3.1 \\
10.5 \\
2.7 \\
0.5\end{array}$ & $\begin{array}{l}1.0 \\
0.2 \\
0.8 \\
0.3 \\
2.6 \\
0.8 \\
0.9 \\
0.2\end{array}$ & $\begin{array}{l}1.5 \\
0.5 \\
0.1 \\
0.8 \\
5.4 \\
9.2 \\
2.3 \\
0.4\end{array}$ & $\begin{array}{r}3.5 \\
1.7 \\
3.0 \\
1.2 \\
19.3 \\
3.8 \\
5.1 \\
4.4\end{array}$ & $\begin{array}{l}--- \\
--- \\
--- \\
--- \\
6.9 \\
--- \\
--- \\
---\end{array}$ & $\begin{array}{r}--- \\
--- \\
--- \\
--- \\
36 \\
--- \\
--- \\
---\end{array}$ & $\begin{array}{r}0 \\
0 \\
0 \\
0 \\
0 \\
59 \\
0 \\
0\end{array}$ \\
\hline $\begin{array}{l}\text { LINE } \\
\text { A } \\
\text { B } \\
\text { C } \\
\text { D } \\
\text { E } \\
\text { F }\end{array}$ & $\begin{array}{r}11580 \\
1938.7 \\
1982.5 \\
1986.8 \\
1997.4 \\
2010.3 \\
2044.2\end{array}$ & $\begin{array}{r}\mathrm{M} \\
\mathrm{D} \\
\mathrm{B} ? \\
\mathrm{~B} \\
\mathrm{~B} ? \\
\mathrm{~S} ?\end{array}$ & $\begin{array}{l}687580 \\
687578 \\
687561 \\
687529 \\
687517 \\
687554\end{array}$ & $\begin{array}{l}6108795 \\
6110136 \\
6110214 \\
6110378 \\
6110658 \\
6111147\end{array}$ & $\begin{array}{l}0.3 \\
1.3 \\
0.2 \\
0.1 \\
2.6 \\
1.0\end{array}$ & $\begin{array}{l}0.2 \\
5.4 \\
4.4 \\
4.1 \\
3.8 \\
1.0\end{array}$ & $\begin{array}{l}3.1 \\
0.0 \\
0.4 \\
0.1 \\
1.4 \\
1.3\end{array}$ & $\begin{array}{l}0.5 \\
7.0 \\
5.1 \\
4.9 \\
3.5 \\
1.2\end{array}$ & $\begin{array}{r}3.3 \\
20.5 \\
11.1 \\
6.8 \\
2.3 \\
2.0\end{array}$ & $\begin{array}{r}3.8 \\
31.8 \\
34.6 \\
30.0 \\
25.4 \\
8.6\end{array}$ & $\begin{array}{l}--- \\
0.8 \\
--- \\
--- \\
2.6 \\
4.7\end{array}$ & $\begin{array}{r}--- \\
6 \\
--- \\
--- \\
51 \\
72\end{array}$ & $\begin{array}{r}231 \\
0 \\
294 \\
0 \\
69 \\
0\end{array}$ \\
\hline $\begin{array}{l}\text { LINE } \\
\text { A } \\
\text { B } \\
\text { C } \\
\text { D }\end{array}$ & $\begin{array}{r}11590 \\
2314.6 \\
2306.0 \\
2263.0 \\
2232.4\end{array}$ & $\begin{array}{c}\text { B? } \\
\text { M } \\
\text { B? } \\
\text { B }\end{array}$ & $\begin{array}{l}688017 \\
688028 \\
687958 \\
687944\end{array}$ & $\begin{array}{l}6108770 \\
6109043 \\
6110360 \\
6111135\end{array}$ & $\begin{array}{l}0.3 \\
1.8 \\
1.7 \\
1.2\end{array}$ & $\begin{array}{l}2.0 \\
0.5 \\
3.3 \\
5.4\end{array}$ & $\begin{array}{l}1.8 \\
0.6 \\
0.6 \\
0.7\end{array}$ & $\begin{array}{l}1.6 \\
0.4 \\
4.0 \\
5.7\end{array}$ & $\begin{array}{r}3.2 \\
0.3 \\
8.1 \\
16.9\end{array}$ & $\begin{array}{r}8.4 \\
1.1 \\
20.7 \\
30.5\end{array}$ & $\begin{array}{l}1.7 \\
--- \\
1.9 \\
1.0\end{array}$ & $\begin{array}{r}40 \\
--- \\
30 \\
0\end{array}$ & $\begin{array}{r}30 \\
236 \\
29 \\
29\end{array}$ \\
\hline $\begin{array}{l}\text { LINE } \\
\text { A } \\
\text { B } \\
\text { C } \\
\text { D } \\
\text { E } \\
\text { F }\end{array}$ & $\begin{array}{r}11600 \\
2522.3 \\
2548.1 \\
2552.0 \\
2589.9 \\
2596.1 \\
2602.0\end{array}$ & $\begin{array}{r}\mathrm{M} \\
\mathrm{M} \\
\mathrm{M} \\
\mathrm{B} ? \\
\mathrm{~B} ? \\
\mathrm{D}\end{array}$ & $\begin{array}{l}688385 \\
688365 \\
688344 \\
688379 \\
688372 \\
688360\end{array}$ & $\begin{array}{l}6109079 \\
6109959 \\
6110096 \\
6110950 \\
6111063 \\
6111164\end{array}$ & $\begin{array}{l}1.1 \\
3.9 \\
3.3 \\
0.8 \\
3.2 \\
2.2\end{array}$ & $\begin{array}{l}0.6 \\
0.8 \\
1.5 \\
1.6 \\
1.0 \\
2.4\end{array}$ & $\begin{array}{l}0.2 \\
3.3 \\
3.5 \\
0.6 \\
0.8 \\
2.0\end{array}$ & $\begin{array}{l}0.6 \\
1.6 \\
2.9 \\
2.3 \\
2.5 \\
2.1\end{array}$ & $\begin{array}{l}0.1 \\
1.4 \\
4.5 \\
3.4 \\
3.0 \\
3.2\end{array}$ & $\begin{array}{r}0.9 \\
7.3 \\
12.3 \\
11.7 \\
10.2 \\
9.6\end{array}$ & $\begin{array}{r}--- \\
--- \\
--- \\
--- \\
11.3 \\
5.2\end{array}$ & $\begin{array}{r}--- \\
--- \\
--- \\
--- \\
60 \\
52\end{array}$ & $\begin{array}{r}377 \\
33 \\
2 \\
32 \\
41 \\
33\end{array}$ \\
\hline $\begin{array}{l}\text { LINE } \\
\text { A } \\
\text { B } \\
\text { C } \\
\text { D } \\
\text { E }\end{array}$ & $\begin{array}{r}11610 \\
2923.7 \\
2902.1 \\
2884.5 \\
2845.2 \\
2729.2\end{array}$ & $\begin{array}{c}\mathrm{M} \\
\mathrm{M} \\
\mathrm{B} ? \\
\mathrm{M} \\
\mathrm{S}\end{array}$ & $\begin{array}{l}688816 \\
688843 \\
688854 \\
688763 \\
688692\end{array}$ & $\begin{array}{l}6108429 \\
6109038 \\
6109601 \\
6110510 \\
6113300\end{array}$ & $\begin{array}{l}0.4 \\
0.8 \\
2.3 \\
4.6 \\
0.4\end{array}$ & $\begin{array}{l}0.4 \\
0.3 \\
2.4 \\
0.3 \\
1.4\end{array}$ & $\begin{array}{l}1.7 \\
0.3 \\
2.8 \\
6.1 \\
1.5\end{array}$ & $\begin{array}{l}1.5 \\
0.1 \\
1.3 \\
0.9 \\
2.1\end{array}$ & $\begin{array}{l}2.8 \\
0.3 \\
1.3 \\
4.9 \\
1.2\end{array}$ & $\begin{array}{r}2.7 \\
1.1 \\
7.1 \\
4.6 \\
12.7\end{array}$ & $\begin{array}{l}--- \\
--- \\
--- \\
--- \\
---\end{array}$ & $\begin{array}{l}--- \\
--- \\
--- \\
--- \\
---\end{array}$ & $\begin{array}{r}66 \\
0 \\
6 \\
0 \\
37\end{array}$ \\
\hline $\begin{array}{l}\text { LINE } \\
A\end{array}$ & $\begin{array}{c}11620 \\
3135.2\end{array}$ & S? & 689219 & 6110703 & 0.0 & 2.0 & 0.4 & 1.8 & 1.2 & 9.0 & --- & --- & 0 \\
\hline $\begin{array}{l}\text { LINE } \\
\mathrm{A}\end{array}$ & $\begin{array}{c}11630 \\
3514.4\end{array}$ & $\mathrm{~S}$ & 689581 & 6109710 & 1.5 & 1.1 & 0.1 & 1.5 & 1.0 & 9.3 & --- & --- & 19 \\
\hline
\end{tabular}

$\mathrm{CX}=$ COAXIAL

$\mathrm{CP}=$ COPLANAR Note: $\mathrm{EM}$ values shown above

Ketchikan - North/South of 5515 local amplitudes
*Estimated Depth may be unreliable because the stronger part of the conductor may be deeper or to one side of the flight line, or because of a shallow dip or magnetite/overburden effects 
EM Anomaly List

\begin{tabular}{|c|c|c|c|c|c|c|c|c|c|c|c|c|c|}
\hline Label & Fid & Interp & $\begin{array}{l}\text { XUTM } \\
\text { m }\end{array}$ & $\begin{array}{c}\text { YUTM } \\
\text { m }\end{array}$ & $\begin{array}{ll}\text { CX } & 9 \\
\text { Real } & \\
\text { ppm }\end{array}$ & $\begin{array}{l}00 \mathrm{HZ} \\
\text { Quad } \\
\text { ppm }\end{array}$ & $\begin{array}{l}\text { CP } \\
\text { Real } \\
\text { ppm }\end{array}$ & $\begin{array}{l}900 \mathrm{HZ} \\
\text { Quad } \\
\text { ppm }\end{array}$ & $\begin{array}{l}\text { CP } 7 \\
\text { Real } \\
\text { ppm }\end{array}$ & $\begin{array}{l}200 \mathrm{HZ} \\
\text { Quad } \\
\text { ppm }\end{array}$ & $\begin{array}{l}\text { Vertica } \\
\text { CoND } \\
\text { siemens }\end{array}$ & $\begin{array}{c}\text { al Dike } \\
\text { DEPTH* } \\
\text { m }\end{array}$ & $\begin{array}{c}\text { Mag. Corr } \\
\text { NT }\end{array}$ \\
\hline $\begin{array}{l}\text { LINE } \\
B\end{array}$ & $\begin{array}{c}11630 \\
3457.5\end{array}$ & S & 689513 & 6110684 & 0.1 & 2.7 & 0.0 & 2.6 & 3.1 & 15.3 & 0.5 & 0 & 0 \\
\hline $\begin{array}{l}\text { LINE } \\
\text { A } \\
B\end{array}$ & $\begin{array}{l}11640 \\
3753.1 \\
3788.9\end{array}$ & $\begin{array}{l}\mathrm{S} \\
\mathrm{M}\end{array}$ & $\begin{array}{l}689975 \\
689926\end{array}$ & $\begin{array}{l}6110344 \\
6111178\end{array}$ & $\begin{array}{l}0.1 \\
0.2\end{array}$ & $\begin{array}{l}2.1 \\
1.9\end{array}$ & $\begin{array}{l}0.2 \\
1.7\end{array}$ & $\begin{array}{l}2.2 \\
2.0\end{array}$ & $\begin{array}{l}2.0 \\
1.3\end{array}$ & $\begin{array}{l}12.9 \\
12.4\end{array}$ & $\begin{array}{l}--- \\
---\end{array}$ & $\begin{array}{l}--- \\
---\end{array}$ & $\begin{array}{r}21 \\
106\end{array}$ \\
\hline $\begin{array}{l}\text { LINE } \\
\text { A } \\
B\end{array}$ & $\begin{array}{r}11650 \\
4003.0 \\
3982.1\end{array}$ & $\begin{array}{l}S \\
S\end{array}$ & $\begin{array}{l}690365 \\
690353\end{array}$ & $\begin{array}{l}6111493 \\
6111932\end{array}$ & $\begin{array}{l}2.4 \\
0.1\end{array}$ & $\begin{array}{l}1.1 \\
2.6\end{array}$ & $\begin{array}{l}1.0 \\
2.5\end{array}$ & $\begin{array}{l}1.1 \\
1.6\end{array}$ & $\begin{array}{l}2.4 \\
3.1\end{array}$ & $\begin{array}{l}7.5 \\
9.8\end{array}$ & $\begin{array}{l}--- \\
1.7\end{array}$ & $\begin{array}{r}--- \\
19\end{array}$ & $\begin{array}{r}0 \\
13\end{array}$ \\
\hline $\begin{array}{l}\text { LINE } \\
A\end{array}$ & $\begin{array}{l}11660 \\
4180.2\end{array}$ & $\mathrm{M}$ & 690749 & 6111410 & 3.5 & 0.5 & 1.5 & 0.3 & 1.0 & 0.5 & --- & --- & 54 \\
\hline $\begin{array}{l}\text { LINE } \\
A\end{array}$ & $\begin{array}{l}11680 \\
4522.1\end{array}$ & $\mathrm{E}$ & 691540 & 6111747 & 12.2 & 9.9 & 3.9 & 6.0 & 16.7 & 11.9 & --- & --- & 9 \\
\hline $\begin{array}{l}\text { LINE } \\
\text { A } \\
\text { B } \\
\text { C } \\
\text { D } \\
\text { E } \\
\text { F } \\
\text { G } \\
\text { H } \\
\text { I } \\
\text { J } \\
\text { K } \\
\text { L } \\
\text { M } \\
\text { N } \\
\text { O } \\
\text { P }\end{array}$ & $\begin{array}{r}19010 \\
8514.8 \\
8632.4 \\
8664.2 \\
8672.6 \\
8676.3 \\
8682.2 \\
8687.8 \\
8692.2 \\
8696.5 \\
8707.5 \\
8709.9 \\
8729.4 \\
8734.4 \\
8737.3 \\
8740.4 \\
8743.1\end{array}$ & $\begin{array}{r}\text { S } \\
\text { S } \\
\text { E } \\
\text { B } \\
\text { B } \\
\text { B } \\
\text { B } \\
\text { B } \\
\text { B } \\
\text { B } \\
\text { B } \\
\text { B } \\
\text { B? } \\
\text { B } \\
\text { B } \\
\text { B }\end{array}$ & $\begin{array}{l}650636 \\
653813 \\
654964 \\
655289 \\
655431 \\
655652 \\
655855 \\
656010 \\
656170 \\
656592 \\
656682 \\
657329 \\
657522 \\
657638 \\
657762 \\
657867\end{array}$ & $\begin{array}{l}6146683 \\
6146810 \\
6146922 \\
6146916 \\
6146911 \\
6146909 \\
6146918 \\
6146930 \\
6146937 \\
6146934 \\
6146927 \\
6146921 \\
6146943 \\
6146958 \\
6146973 \\
6146988\end{array}$ & $\begin{array}{r}0.5 \\
0.5 \\
4.2 \\
24.9 \\
35.5 \\
4.8 \\
14.5 \\
0.4 \\
0.9 \\
20.7 \\
22.9 \\
22.8 \\
28.5 \\
24.2 \\
50.2 \\
50.2\end{array}$ & $\begin{array}{r}0.9 \\
1.3 \\
10.7 \\
21.6 \\
27.1 \\
1.3 \\
12.0 \\
6.0 \\
5.7 \\
23.9 \\
23.9 \\
15.5 \\
33.8 \\
31.5 \\
42.6 \\
42.6\end{array}$ & $\begin{array}{r}0.7 \\
0.1 \\
1.4 \\
12.7 \\
58.6 \\
45.3 \\
1.3 \\
14.2 \\
0.4 \\
31.4 \\
33.4 \\
31.5 \\
14.5 \\
43.4 \\
76.0 \\
76.5\end{array}$ & $\begin{array}{r}1.7 \\
1.6 \\
14.2 \\
27.1 \\
52.3 \\
29.7 \\
7.2 \\
0.6 \\
2.2 \\
36.4 \\
37.6 \\
24.0 \\
50.9 \\
61.5 \\
82.6 \\
82.6\end{array}$ & $\begin{array}{r}0.0 \\
1.8 \\
31.3 \\
72.8 \\
110.9 \\
26.3 \\
17.8 \\
7.0 \\
9.5 \\
81.8 \\
83.9 \\
58.0 \\
104.5 \\
112.8 \\
176.2 \\
176.2\end{array}$ & $\begin{array}{r}8.9 \\
13.1 \\
78.2 \\
31.5 \\
31.7 \\
41.2 \\
16.3 \\
16.6 \\
16.6 \\
44.9 \\
45.8 \\
27.3 \\
66.6 \\
74.0 \\
68.3 \\
68.3\end{array}$ & $\begin{array}{r}--- \\
1.3 \\
2.0 \\
10.1 \\
--- \\
24.9 \\
9.0 \\
9.7 \\
0.8 \\
10.1 \\
11.5 \\
20.9 \\
5.9 \\
8.6 \\
18.2 \\
18.3\end{array}$ & $\begin{array}{r}--- \\
16 \\
0 \\
1 \\
--- \\
0 \\
10 \\
20 \\
0 \\
0 \\
0 \\
0 \\
0 \\
0 \\
0 \\
0\end{array}$ & $\begin{array}{r}0 \\
0 \\
0 \\
24 \\
10 \\
44 \\
0 \\
1 \\
0 \\
10 \\
0 \\
10 \\
78 \\
10 \\
0 \\
0\end{array}$ \\
\hline $\begin{array}{l}\text { LINE } \\
\text { A } \\
B \\
C\end{array}$ & $\begin{array}{r}19020 \\
1537.6 \\
1497.7 \\
1477.3\end{array}$ & $\begin{array}{c}\mathrm{S} ? \\
\mathrm{M} \\
\mathrm{M}\end{array}$ & $\begin{array}{l}671896 \\
672990 \\
673300\end{array}$ & $\begin{array}{l}6143938 \\
6143955 \\
6143969\end{array}$ & $\begin{array}{l}3.0 \\
0.1 \\
0.6\end{array}$ & $\begin{array}{l}7.5 \\
0.8 \\
0.2\end{array}$ & $\begin{array}{l}7.6 \\
0.6 \\
1.8\end{array}$ & $\begin{array}{r}18.3 \\
0.5 \\
0.1\end{array}$ & $\begin{array}{r}49.9 \\
0.7 \\
1.3\end{array}$ & $\begin{array}{r}50.7 \\
2.3 \\
0.6\end{array}$ & $\begin{array}{l}3.0 \\
--- \\
---\end{array}$ & $\begin{array}{r}0 \\
--- \\
---\end{array}$ & $\begin{array}{r}43 \\
0 \\
0\end{array}$ \\
\hline $\begin{array}{l}\text { LINE } \\
\text { A } \\
\text { B } \\
\text { C } \\
\text { D } \\
\text { E } \\
\text { F }\end{array}$ & $\begin{array}{r}19030 \\
7353.2 \\
7301.7 \\
7200.2 \\
7194.0 \\
7189.3 \\
7186.5\end{array}$ & $\begin{array}{l}S \\
S \\
S \\
M \\
M \\
S\end{array}$ & $\begin{array}{l}624528 \\
625664 \\
628473 \\
628709 \\
628875 \\
628968\end{array}$ & $\begin{array}{l}6140958 \\
6140992 \\
6141076 \\
6141085 \\
6141099 \\
6141104\end{array}$ & $\begin{array}{l}1.3 \\
1.4 \\
3.9 \\
2.5 \\
0.3 \\
1.7\end{array}$ & $\begin{array}{l}1.0 \\
2.7 \\
1.4 \\
0.5 \\
0.3 \\
1.8\end{array}$ & $\begin{array}{l}0.2 \\
0.7 \\
9.9 \\
0.1 \\
0.4 \\
4.8\end{array}$ & $\begin{array}{l}1.4 \\
4.7 \\
2.6 \\
0.3 \\
2.3 \\
2.3\end{array}$ & $\begin{array}{r}2.8 \\
13.4 \\
9.4 \\
0.3 \\
0.3 \\
5.3\end{array}$ & $\begin{array}{r}10.2 \\
20.5 \\
16.9 \\
1.1 \\
16.9 \\
16.9\end{array}$ & $\begin{array}{l}3.9 \\
--- \\
--- \\
--- \\
--- \\
---\end{array}$ & $\begin{array}{r}73 \\
--- \\
--- \\
--- \\
--- \\
---\end{array}$ & $\begin{array}{r}0 \\
0 \\
0 \\
586 \\
459 \\
24\end{array}$ \\
\hline
\end{tabular}

$\mathrm{CX}=$ COAXIAL

$\mathrm{CP}=$ COPLANAR Note:EM values shown above

Ketchikan - North/South of 5515 local amplitudes
*Estimated Depth may be unreliable because the stronger part of the conductor may be deeper or to one side of the flight line, or because of a shallow dip or magnetite/overburden effects 
EM Anomaly List

\begin{tabular}{|c|c|c|c|c|c|c|c|c|c|c|c|c|c|}
\hline Label & Fid & Interp & $\begin{array}{c}\text { XUTM } \\
\text { m }\end{array}$ & $\begin{array}{c}\text { YUTM } \\
\mathrm{m}\end{array}$ & $\begin{array}{l}\text { CX } \\
\text { Real } \\
\text { ppm }\end{array}$ & $\begin{array}{l}900 \mathrm{HZ} \\
\text { Quad } \\
\text { ppm }\end{array}$ & $\begin{array}{l}\text { CP } \\
\text { Real } \\
\text { ppm }\end{array}$ & $\begin{array}{l}900 \mathrm{HZ} \\
\text { Quad } \\
\text { ppm }\end{array}$ & $\begin{array}{l}\text { CP } \\
\text { Real } \\
\text { ppm }\end{array}$ & $\begin{array}{l}200 \mathrm{HZ} \\
\text { Quad } \\
\text { ppm }\end{array}$ & $\begin{array}{l}\text { Vertica } \\
\text { COND } \\
\text { siemens }\end{array}$ & $\begin{array}{c}\text { al Dike } \\
\text { DEPTH* } \\
\text { m }\end{array}$ & $\begin{array}{c}\text { Mag. Corr } \\
\text { NT }\end{array}$ \\
\hline LINE 1 & 19030 & & & & & & & & & & & & \\
\hline G & 7163.5 & M & 629751 & 6141139 & 3.9 & 0.1 & 3.0 & 0.5 & 3.5 & 0.4 & --- & --- & 0 \\
\hline $\mathrm{H}$ & 7158.6 & M & 629891 & 6141147 & 0.4 & 0.7 & 0.0 & 0.2 & 0.5 & 1.0 & --- & --- & 276 \\
\hline I & 7148.6 & $\mathrm{M}$ & 630122 & 6141148 & 0.4 & 0.6 & 3.0 & 0.5 & 2.6 & 4.2 & --- & --- & 0 \\
\hline $\mathrm{J}$ & 7122.1 & $\mathrm{M}$ & 630685 & 6141174 & 0.1 & 0.2 & 4.0 & 0.1 & 1.0 & 6.9 & --- & --- & 12 \\
\hline $\mathrm{K}$ & 7111.8 & $\mathrm{M}$ & 630998 & 6141185 & 0.2 & 1.6 & 0.4 & 2.4 & 2.8 & 16.1 & --- & --- & 42 \\
\hline L & 7035.3 & $\mathrm{M}$ & 632986 & 6141250 & 8.4 & 0.3 & 1.3 & 0.5 & 1.3 & 0.7 & --- & --- & 145 \\
\hline M & 7025.4 & M & 633156 & 6141266 & 1.1 & 0.3 & 7.1 & 1.2 & 5.3 & 7.3 & --- & --- & 174 \\
\hline $\mathrm{N}$ & 6957.7 & $\mathrm{~B}$ & 634917 & 6141329 & 3.4 & 3.0 & 0.5 & 0.8 & 2.1 & 12.7 & 6.7 & 37 & 23 \\
\hline 0 & 6891.0 & B & 636645 & 6141357 & 3.8 & 7.1 & 3.5 & 15.4 & 40.8 & 32.3 & 2.7 & 5 & 23 \\
\hline P & 6882.3 & B & 636814 & 6141377 & 8.5 & 8.8 & 1.0 & 9.2 & 29.3 & 24.9 & 4.4 & 25 & 23 \\
\hline$Q$ & 6861.0 & $\mathrm{~s}$ & 637451 & 6141419 & 0.2 & 2.7 & 3.9 & 4.8 & 7.9 & 9.7 & 2.0 & 19 & 0 \\
\hline $\mathrm{R}$ & 6776.1 & M & 639482 & 6141497 & 0.2 & 0.6 & 4.6 & 0.6 & 4.5 & 1.4 & -- & --- & 92 \\
\hline $\mathrm{S}$ & 6748.0 & $\mathrm{M}$ & 640091 & 6141502 & 3.4 & 0.3 & 12.0 & 0.1 & 1.5 & 3.5 & --- & --- & 23 \\
\hline $\mathrm{T}$ & 6736.1 & M & 640393 & 6141519 & 0.4 & 0.6 & 2.0 & 0.2 & 0.4 & 0.8 & --- & --- & 12 \\
\hline $\mathrm{U}$ & 6682.1 & S? & 641569 & 6141554 & 0.7 & 1.3 & 0.1 & 1.7 & 2.2 & 11.4 & --- & --- & 0 \\
\hline $\mathrm{V}$ & 6639.0 & S & 642848 & 6141660 & 2.6 & 3.4 & 0.6 & 6.4 & 3.5 & 37.0 & 2.4 & 27 & 0 \\
\hline W & 6605.2 & S? & 643823 & 6141595 & 1.6 & 1.8 & 0.4 & 2.6 & 1.4 & 16.4 & --- & --- & 0 \\
\hline $\mathrm{x}$ & 6399.9 & $\dot{M}$ & 648852 & 6141840 & 0.7 & 0.1 & 0.4 & 0.0 & 0.6 & 0.7 & --- & --- & 179 \\
\hline $\mathrm{Y}$ & 6288.1 & S? & 651320 & 6141956 & 1.6 & 2.5 & 1.2 & 4.7 & 10.9 & 24.6 & 2.3 & 1 & 27 \\
\hline $\mathrm{z}$ & 6284.2 & S? & 651412 & 6141915 & 2.7 & 3.0 & 0.2 & 3.9 & 6.9 & 18.7 & --- & --- & 0 \\
\hline $\mathrm{AA}$ & 6241.8 & $\dot{S}$ & 651975 & 6141904 & 0.5 & 0.3 & 0.1 & 0.7 & 0.3 & 2.8 & --- & --- & 0 \\
\hline$A B$ & 6230.7 & S? & 652142 & 6141939 & 0.1 & 0.8 & 0.1 & 1.1 & 0.1 & 4.5 & --- & --- & 0 \\
\hline $\mathrm{AC}$ & 6152.3 & S? & 654068 & 6142010 & 1.7 & 0.6 & 0.6 & 0.6 & 0.3 & 6.3 & 15.3 & 78 & 23 \\
\hline$A D$ & 6138.5 & S? & 654206 & 6142010 & 1.7 & 0.7 & 0.1 & 0.5 & 0.1 & 5.0 & 10.8 & 83 & 0 \\
\hline $\mathrm{AE}$ & 6082.9 & S & 655121 & 6142043 & 0.4 & 1.0 & 0.9 & 1.4 & 2.4 & 9.4 & -- & --- & 23 \\
\hline $\mathrm{AF}$ & 6055.2 & $\mathrm{~S}$ & 656062 & 6142082 & 0.0 & 3.9 & 0.1 & 2.7 & 6.6 & 12.6 & --- & --- & 23 \\
\hline$A G$ & 6038.9 & D & 656440 & 6142102 & 5.4 & 12.2 & 6.2 & 26.6 & 65.5 & 58.8 & --- & --- & 0 \\
\hline $\mathrm{AH}$ & 6034.4 & B & 656498 & 6142108 & 3.3 & 10.5 & 6.3 & 25.4 & 62.1 & 62.0 & 2.2 & 0 & 0 \\
\hline $\mathrm{AI}$ & 6023.8 & B & 656591 & 6142087 & 5.8 & 8.8 & 2.2 & 5.1 & 5.1 & 14.3 & 3.4 & 29 & 0 \\
\hline $\mathrm{AJ}$ & 5993.5 & B? & 657043 & 6142122 & 0.2 & 1.8 & 5.5 & 5.1 & 10.1 & 8.2 & --- & --- & 0 \\
\hline AK & 5972.8 & B? & 657396 & 6142129 & 1.1 & 1.8 & 0.8 & 3.9 & 6.5 & 21.0 & --- & --- & 12 \\
\hline $\mathrm{AL}$ & 5928.0 & $\mathrm{~S}$ & 657915 & 6142184 & 3.0 & 0.4 & 0.1 & 1.8 & 3.0 & 10.1 & 20.3 & 49 & 0 \\
\hline$A M$ & 5803.1 & B? & 661637 & 6142254 & 1.7 & 4.1 & 1.3 & 5.7 & 15.0 & 21.7 & 1.8 & 3 & 23 \\
\hline AN & 5758.6 & D & 662824 & 6142329 & 6.2 & 7.5 & 1.7 & 6.2 & 15.8 & 12.0 & 4.0 & 29 & 171 \\
\hline AO & 5736.1 & $\mathrm{E}$ & 663327 & 6142337 & 4.0 & 4.8 & 8.7 & 11.8 & 26.1 & 12.9 & 5.3 & 17 & 0 \\
\hline $\mathrm{AP}$ & 5730.1 & B & 663498 & 6142366 & 5.7 & 4.7 & 13.4 & 14.2 & 27.9 & 5.8 & --- & --- & 0 \\
\hline $\mathrm{AQ}$ & 5716.3 & D & 663907 & 6142392 & 1.0 & 3.5 & 1.4 & 2.9 & 6.6 & 9.2 & 1.7 & 24 & 23 \\
\hline$A R$ & 5691.8 & B? & 664689 & 6142391 & 7.5 & 7.0 & 1.1 & 10.0 & 33.4 & 21.9 & 4.2 & 0 & 59 \\
\hline AS & 5599.0 & D & 667573 & 6142517 & 0.3 & 2.4 & 0.2 & 1.4 & 2.1 & 7.9 & 0.7 & 3 & 0 \\
\hline $\mathrm{AT}$ & 5483.3 & $B$ ? & 671120 & 6142638 & 5.3 & 3.6 & 2.4 & 1.2 & 2.6 & 2.3 & 13.8 & 22 & 0 \\
\hline $\mathrm{AU}$ & 5474.1 & M & 671377 & 6142639 & 0.6 & 0.6 & 3.6 & 0.8 & 2.4 & 2.9 & -- & --- & 0 \\
\hline $\mathrm{AV}$ & 5463.0 & M & 671679 & 6142640 & 0.7 & 0.0 & 1.4 & 0.6 & 0.9 & 2.1 & --- & --- & 400 \\
\hline AW & 5425.8 & M & 672684 & 6142696 & 0.7 & 0.5 & 0.5 & 0.3 & 0.5 & 2.1 & --- & --- & 23 \\
\hline$A X$ & 5404.1 & M & 673111 & 6142679 & 2.7 & 1.3 & 4.3 & 1.2 & 3.1 & 6.7 & --- & --- & 0 \\
\hline $\mathrm{AY}$ & 5399.2 & M & 673244 & 6142687 & 3.5 & 0.7 & 0.5 & 0.6 & 0.2 & 2.5 & --- & --- & 159 \\
\hline $\mathrm{AZ}$ & 5389.9 & $\mathrm{M}$ & 673415 & 6142738 & 5.8 & 0.2 & 0.2 & 0.6 & 0.1 & 3.2 & --- & --- & 0 \\
\hline
\end{tabular}

$\mathrm{CX}=$ COAXIAL

Note: EM values shown above

are local amplitudes

*Estimated Depth may be unreliable because the stronger part of the conductor may be deeper or to one side of the flight line, or because of a shallow dip or magnetite/overburden effects 
EM Anomaly List

\begin{tabular}{|c|c|c|c|c|c|c|c|c|c|c|c|c|c|}
\hline Label & Fid & Interp & $\begin{array}{c}\text { XUTM } \\
\text { m }\end{array}$ & $\begin{array}{c}\text { YUTM } \\
\mathrm{m}\end{array}$ & $\begin{array}{l}\text { CX } \\
\text { Real } \\
\text { ppm }\end{array}$ & $\begin{array}{l}900 \mathrm{HZ} \\
\text { Quad } \\
\text { ppm }\end{array}$ & $\begin{array}{l}\text { CP } \\
\text { Real } \\
\text { ppm }\end{array}$ & $\begin{array}{c}900 \mathrm{HZ} \\
\text { Quad } \\
\text { ppm }\end{array}$ & $\begin{array}{l}\text { CP } 7 \\
\text { Real } \\
\text { ppm }\end{array}$ & $\begin{array}{c}7200 \mathrm{HZ} \\
\text { Quad } \\
\text { ppm }\end{array}$ & $\begin{array}{l}\text { Vertica } \\
\text { COND } \\
\text { siemens }\end{array}$ & $\begin{array}{c}\text { al Dike } \\
\text { DEPTH* }^{\star} \\
\text { m }\end{array}$ & $\begin{array}{c}\text { Mag. Corr } \\
\text { NT }\end{array}$ \\
\hline $\begin{array}{l}\text { LINE } \\
\text { BA } \\
\text { BB } \\
B C\end{array}$ & $\begin{array}{r}19030 \\
5384.7 \\
5375.3 \\
5365.8\end{array}$ & $\begin{array}{l}\mathrm{M} \\
\mathrm{M} \\
\mathrm{M}\end{array}$ & $\begin{array}{l}673535 \\
673784 \\
674018\end{array}$ & $\begin{array}{l}6142748 \\
6142715 \\
6142696\end{array}$ & $\begin{array}{l}0.0 \\
0.1 \\
1.7\end{array}$ & $\begin{array}{l}0.8 \\
0.7 \\
0.3\end{array}$ & $\begin{array}{r}16.5 \\
9.5 \\
1.3\end{array}$ & $\begin{array}{l}0.7 \\
0.4 \\
0.0\end{array}$ & $\begin{array}{l}2.1 \\
8.0 \\
0.9\end{array}$ & $\begin{array}{l}3.3 \\
2.4 \\
0.3\end{array}$ & $\begin{array}{l}--- \\
--- \\
---\end{array}$ & $\begin{array}{l}--- \\
--- \\
---\end{array}$ & $\begin{array}{r}0 \\
0 \\
58\end{array}$ \\
\hline $\begin{array}{l}\text { LINE } \\
\text { A } \\
\text { B } \\
\text { C } \\
\text { D } \\
\text { E } \\
\text { F } \\
\text { G } \\
\text { H } \\
\text { I } \\
\text { J } \\
\text { K } \\
\text { L } \\
\text { M } \\
\text { N } \\
\text { O } \\
\text { P } \\
\text { Q } \\
\text { R } \\
\text { S } \\
\text { T } \\
\text { U } \\
\text { V } \\
\text { W } \\
X \\
\text { Y } \\
\text { Z } \\
\text { AA }\end{array}$ & $\begin{array}{r}19040 \\
3732.0 \\
3752.8 \\
3777.1 \\
3886.7 \\
3889.6 \\
3914.0 \\
3924.8 \\
3955.4 \\
3960.8 \\
3976.0 \\
3978.7 \\
4085.7 \\
4101.6 \\
4134.6 \\
4146.0 \\
4154.0 \\
4163.7 \\
4240.1 \\
4447.6 \\
4488.0 \\
4647.4 \\
4694.2 \\
4726.7 \\
4796.0 \\
5010.3 \\
5047.9 \\
5059.4\end{array}$ & $\begin{array}{r}\mathrm{S} \\
\mathrm{S} \\
\mathrm{S} \\
\mathrm{M} \\
\mathrm{M} \\
\mathrm{M} \\
\mathrm{M} \\
\mathrm{M} \\
\mathrm{B} ? \\
\mathrm{~S} \\
\mathrm{~S} \\
\mathrm{M} \\
\mathrm{M} \\
\mathrm{M} \\
\mathrm{M} \\
\mathrm{M} \\
\mathrm{M} \\
\mathrm{B} \\
\mathrm{B} ? \\
\mathrm{M} \\
\mathrm{S} \\
\mathrm{S} ? \\
\mathrm{M} \\
\mathrm{S} \\
\mathrm{B} \\
\mathrm{M} \\
\mathrm{M}\end{array}$ & $\begin{array}{l}635047 \\
635888 \\
636883 \\
640695 \\
640788 \\
641460 \\
641711 \\
642653 \\
642869 \\
643454 \\
643563 \\
646468 \\
646976 \\
648226 \\
648665 \\
648988 \\
649350 \\
651012 \\
654819 \\
656130 \\
660680 \\
662002 \\
663014 \\
664238 \\
667996 \\
668338 \\
668646\end{array}$ & $\begin{array}{l}6136544 \\
6136547 \\
6136599 \\
6136681 \\
6136678 \\
6136728 \\
6136787 \\
6136742 \\
6136746 \\
6136874 \\
6136891 \\
6136939 \\
6136965 \\
6137025 \\
6137017 \\
6137025 \\
6137036 \\
6137084 \\
6137225 \\
6137346 \\
6137448 \\
6137507 \\
6137476 \\
6137490 \\
6137679 \\
6137692 \\
6137660\end{array}$ & $\begin{array}{r}0.0 \\
1.1 \\
2.0 \\
5.0 \\
0.5 \\
0.4 \\
3.2 \\
0.1 \\
5.9 \\
62.4 \\
89.9 \\
0.3 \\
0.1 \\
0.1 \\
0.7 \\
2.5 \\
2.5 \\
9.2 \\
0.8 \\
0.8 \\
0.3 \\
0.9 \\
1.9 \\
2.1 \\
1.0 \\
0.6 \\
0.7\end{array}$ & $\begin{array}{r}2.5 \\
3.8 \\
2.2 \\
0.3 \\
0.5 \\
1.2 \\
2.0 \\
1.0 \\
2.2 \\
117.6 \\
172.0 \\
1.1 \\
1.2 \\
0.3 \\
0.1 \\
0.8 \\
0.6 \\
12.6 \\
3.1 \\
1.1 \\
1.0 \\
1.6 \\
0.6 \\
2.4 \\
0.9 \\
0.3 \\
0.3\end{array}$ & $\begin{array}{r}1.5 \\
2.0 \\
0.0 \\
11.4 \\
0.8 \\
0.9 \\
0.9 \\
0.2 \\
1.4 \\
84.3 \\
118.7 \\
0.1 \\
0.2 \\
0.4 \\
1.2 \\
0.2 \\
0.8 \\
13.0 \\
0.2 \\
0.1 \\
0.2 \\
0.4 \\
0.2 \\
1.5 \\
1.7 \\
0.1 \\
0.6\end{array}$ & $\begin{array}{r}4.0 \\
5.5 \\
4.1 \\
0.6 \\
0.4 \\
0.6 \\
1.7 \\
1.3 \\
1.0 \\
188.3 \\
314.7 \\
1.1 \\
1.7 \\
0.3 \\
0.2 \\
0.7 \\
0.6 \\
20.0 \\
3.6 \\
0.5 \\
1.0 \\
1.6 \\
0.7 \\
3.1 \\
2.0 \\
0.1 \\
0.3\end{array}$ & $\begin{array}{r}10.3 \\
12.5 \\
8.0 \\
9.7 \\
0.9 \\
0.6 \\
0.5 \\
0.9 \\
2.1 \\
436.9 \\
816.3 \\
0.3 \\
0.6 \\
0.6 \\
0.9 \\
0.1 \\
0.9 \\
46.5 \\
7.0 \\
1.3 \\
0.3 \\
1.8 \\
0.2 \\
9.6 \\
5.5 \\
0.1 \\
0.8\end{array}$ & $\begin{array}{r}21.4 \\
25.7 \\
24.6 \\
3.3 \\
3.8 \\
5.2 \\
11.1 \\
3.2 \\
0.8 \\
174.5 \\
477.2 \\
7.5 \\
11.3 \\
1.6 \\
3.6 \\
5.7 \\
2.0 \\
51.8 \\
13.5 \\
3.7 \\
7.5 \\
12.4 \\
1.9 \\
13.1 \\
7.0 \\
1.2 \\
3.4\end{array}$ & $\begin{array}{c}0.8 \\
--- \\
--- \\
--- \\
--- \\
--- \\
--- \\
--- \\
25.9 \\
8.7 \\
9.2 \\
--- \\
--- \\
--- \\
--- \\
--- \\
--- \\
5.5 \\
--- \\
--- \\
--- \\
--- \\
--- \\
3.4 \\
4.6 \\
--- \\
---\end{array}$ & $\begin{array}{r}0 \\
--- \\
--- \\
--- \\
--- \\
--- \\
--- \\
--- \\
17 \\
0 \\
0 \\
---- \\
--- \\
--- \\
--- \\
--- \\
--- \\
7 \\
--- \\
--- \\
--- \\
--- \\
--- \\
37 \\
70 \\
--- \\
---\end{array}$ & $\begin{array}{r}25 \\
0 \\
0 \\
475 \\
0 \\
56 \\
8 \\
49 \\
25 \\
25 \\
0 \\
91 \\
57 \\
20 \\
134 \\
25 \\
219 \\
25 \\
25 \\
25 \\
25 \\
40 \\
0 \\
24 \\
0 \\
950 \\
0\end{array}$ \\
\hline $\begin{array}{l}\text { LINE } \\
\text { A } \\
\text { B } \\
\text { C } \\
\text { D } \\
\text { E } \\
\text { F } \\
\text { G } \\
\text { H } \\
\text { I } \\
\text { J } \\
\text { K } \\
\text { L }\end{array}$ & $\begin{array}{r}19050 \\
2959.5 \\
2934.6 \\
2919.4 \\
2913.8 \\
2843.0 \\
2834.3 \\
2821.0 \\
2818.0 \\
2798.7 \\
2776.3 \\
2764.8 \\
2757.6\end{array}$ & $\begin{array}{c}\text { S? } \\
\text { S? } \\
\text { M } \\
\text { S? } \\
\text { B } \\
\text { B } \\
\text { B } \\
\text { B } \\
\text { B } \\
\text { B } \\
\text { B } \\
\text { E }\end{array}$ & $\begin{array}{l}634329 \\
634959 \\
635481 \\
635669 \\
637960 \\
638126 \\
638337 \\
638380 \\
638533 \\
638683 \\
638993 \\
639213\end{array}$ & $\begin{array}{l}6131706 \\
6131672 \\
6131687 \\
6131685 \\
6131813 \\
6131790 \\
6131782 \\
6131786 \\
6131802 \\
6131819 \\
6131796 \\
6131809\end{array}$ & $\begin{array}{r}1.1 \\
0.6 \\
1.2 \\
0.5 \\
4.5 \\
5.7 \\
4.6 \\
1.6 \\
3.0 \\
7.4 \\
13.1 \\
2.0\end{array}$ & $\begin{array}{r}4.9 \\
6.4 \\
0.8 \\
2.6 \\
5.8 \\
6.1 \\
7.1 \\
5.7 \\
4.5 \\
11.0 \\
12.7 \\
6.6\end{array}$ & $\begin{array}{r}0.0 \\
2.8 \\
2.3 \\
4.1 \\
4.6 \\
4.8 \\
7.7 \\
6.8 \\
2.9 \\
10.0 \\
18.1 \\
2.8\end{array}$ & $\begin{array}{r}6.5 \\
7.9 \\
1.5 \\
2.7 \\
7.5 \\
3.8 \\
15.0 \\
11.2 \\
7.1 \\
20.5 \\
30.8 \\
4.8\end{array}$ & $\begin{array}{r}10.2 \\
16.6 \\
1.8 \\
8.6 \\
22.8 \\
8.5 \\
33.9 \\
24.1 \\
16.2 \\
48.6 \\
74.7 \\
8.8\end{array}$ & $\begin{array}{r}41.9 \\
35.1 \\
11.4 \\
22.1 \\
20.8 \\
4.9 \\
27.4 \\
17.6 \\
9.2 \\
46.7 \\
41.8 \\
13.0\end{array}$ & $\begin{array}{l}0.7 \\
1.1 \\
--- \\
--- \\
4.4 \\
7.8 \\
3.4 \\
2.9 \\
2.6 \\
4.0 \\
7.5 \\
---\end{array}$ & $\begin{array}{r}0 \\
0 \\
--- \\
--- \\
29 \\
28 \\
18 \\
13 \\
44 \\
23 \\
0 \\
---\end{array}$ & $\begin{array}{r}0 \\
0 \\
0 \\
52 \\
48 \\
0 \\
0 \\
0 \\
52 \\
0 \\
0 \\
0\end{array}$ \\
\hline
\end{tabular}

$\mathrm{CX}=$ COAXIAL

Note: EM values shown above

are local amplitudes

Ketchikan - North/South of 5515
* Estimated Depth may be unreliable because the stronger part of the conductor may be deeper or to one side of the flight line, or because of a shallow dip or magnetite/overburden effects 
EM Anomaly List

\begin{tabular}{|c|c|c|c|c|c|c|c|c|c|c|c|c|c|}
\hline Label & Fid & Interp & $\begin{array}{c}\text { XUTM } \\
\text { m }\end{array}$ & $\begin{array}{c}\text { YUTM } \\
\mathrm{m}\end{array}$ & $\begin{array}{l}\text { CX } \\
\text { Real } \\
\text { ppm }\end{array}$ & $\begin{array}{l}900 \mathrm{HZ} \\
\text { Quad } \\
\text { ppm }\end{array}$ & $\begin{array}{l}\text { CP } \\
\text { Real } \\
\text { ppm }\end{array}$ & $\begin{array}{l}900 \mathrm{HZ} \\
\text { Quad } \\
\text { ppm }\end{array}$ & $\begin{array}{l}\text { CP } \\
\text { Real } \\
\text { ppm }\end{array}$ & $\begin{array}{c}200 \mathrm{HZ} \\
\text { Quad } \\
\text { ppm }\end{array}$ & $\begin{array}{l}\text { Vertica } \\
\text { ConD } \\
\text { siemens }\end{array}$ & $\begin{array}{c}\text { al Dike } \\
\text { DEPTH* } \\
\text { m }\end{array}$ & $\begin{array}{c}\text { Mag. Corr } \\
\text { NT }\end{array}$ \\
\hline $\begin{array}{l}\text { LINE } 1 \\
\end{array}$ & 19050 & & & & & & & & & & & & \\
\hline M & 2710.9 & $B$ ? & 640183 & 6131870 & 0.6 & 2.2 & 0.5 & 1.1 & 2.1 & 7.4 & --- & --- & 0 \\
\hline $\mathrm{N}$ & 2683.0 & B & 640716 & 6131837 & 2.3 & 3.8 & 1.5 & 8.1 & 20.0 & 14.6 & --- & --- & 0 \\
\hline 0 & 2669.2 & D & 641114 & 6131899 & 1.6 & 1.5 & 1.2 & 4.5 & 14.9 & 16.5 & --- & --- & 34 \\
\hline $\mathrm{P}$ & 2654.1 & B & 641527 & 6131918 & 1.3 & 4.4 & 1.5 & 6.3 & 16.7 & 22.0 & 1.5 & 4 & 0 \\
\hline Q & 2609.4 & B & 642460 & 6131926 & 2.0 & 6.0 & 4.3 & 9.9 & 24.5 & 27.3 & 2.4 & 2 & 52 \\
\hline R & 2429.8 & B? & 645852 & 6132021 & 1.6 & 1.1 & 0.3 & 2.5 & 2.3 & 15.9 & 2.8 & 74 & 52 \\
\hline $\mathrm{S}$ & 2389.7 & B & 646797 & 6132132 & 3.2 & 5.2 & 11.0 & 18.0 & 41.0 & 16.8 & 3.7 & 3 & 52 \\
\hline $\mathrm{T}$ & 2380.1 & B & 647043 & 6132108 & 2.9 & 4.7 & 5.1 & 5.9 & 12.1 & 12.6 & 4.0 & 16 & 0 \\
\hline U & 2374.4 & B & 647166 & 6132091 & 4.0 & 3.8 & 5.2 & 5.6 & 13.4 & 6.8 & 7.1 & 14 & 0 \\
\hline $\mathrm{V}$ & 2370.0 & B & 647260 & 6132083 & 2.7 & 7.2 & 2.3 & 12.0 & 27.2 & 40.8 & 1.9 & 0 & 0 \\
\hline W & 2364.2 & B & 647384 & 6132084 & 3.3 & 6.2 & 8.4 & 11.5 & 25.5 & 4.3 & 3.7 & 15 & 52 \\
\hline $\mathrm{x}$ & 2358.7 & B & 647498 & 6132096 & 3.4 & 4.9 & 7.0 & 15.8 & 32.1 & 19.6 & 2.8 & 4 & 0 \\
\hline $\mathrm{Y}$ & 2353.2 & B & 647605 & 6132113 & 3.3 & 11.7 & 4.7 & 14.2 & 35.7 & 19.6 & 2.2 & 0 & 0 \\
\hline $\mathrm{Z}$ & 2347.0 & B? & 647703 & 6132139 & 3.5 & 4.5 & 0.2 & 10.4 & 30.0 & 30.8 & -- & --- & 0 \\
\hline AA & 2338.4 & B & 647814 & 6132172 & 6.3 & 8.1 & 4.4 & 1.0 & 7.5 & 0.1 & 7.6 & 15 & 0 \\
\hline$A B$ & 2299.2 & B & 648237 & 6132140 & 6.6 & 7.3 & 0.4 & 0.4 & 0.9 & 1.7 & 6.3 & 28 & 44 \\
\hline $\mathrm{AC}$ & 2292.2 & B? & 648295 & 6132149 & 0.1 & 3.3 & 4.6 & 13.2 & 32.9 & 29.0 & 1.4 & 2 & 0 \\
\hline $\mathrm{AD}$ & 2282.0 & B & 648382 & 6132173 & 2.8 & 8.0 & 5.2 & 14.7 & 36.5 & 32.3 & 2.5 & 0 & 52 \\
\hline $\mathrm{AE}$ & 2251.7 & B? & 648538 & 6132172 & 0.2 & 0.5 & 6.6 & 22.5 & 54.5 & 71.7 & --- & --- & 52 \\
\hline $\mathrm{AF}$ & 2246.7 & B & 648584 & 6132166 & 3.0 & 1.9 & 0.2 & 0.2 & 0.3 & 0.9 & 9.8 & 53 & 51 \\
\hline$A G$ & 2235.5 & B & 648721 & 6132167 & 0.8 & 2.2 & 0.0 & 1.0 & 1.8 & 3.8 & 2.0 & 69 & 0 \\
\hline $\mathrm{AH}$ & 2215.8 & B & 648956 & 6132199 & 21.6 & 26.5 & 34.0 & 58.9 & 132.6 & 93.9 & 7.4 & 4 & 51 \\
\hline $\mathrm{AI}$ & 2210.7 & B & 649058 & 6132206 & 20.2 & 19.3 & 21.3 & 30.1 & 70.6 & 43.9 & 10.1 & 0 & 0 \\
\hline AJ & 2204.0 & B & 649223 & 6132198 & 10.5 & 11.4 & 18.3 & 17.2 & 40.2 & 57.6 & 10.0 & 0 & 0 \\
\hline $\mathrm{AK}$ & 2193.8 & B & 649395 & 6132193 & 6.5 & 10.3 & 10.1 & 24.8 & 64.7 & 32.8 & 3.2 & 17 & 0 \\
\hline $\mathrm{AL}$ & 2187.8 & B & 649493 & 6132185 & 4.2 & 6.4 & 23.0 & 13.7 & 32.9 & 22.3 & 12.2 & 11 & 52 \\
\hline $\mathrm{AM}$ & 2183.1 & B? & 649601 & 6132175 & 1.2 & 10.3 & 20.0 & 29.7 & 82.7 & 54.7 & 3.8 & 0 & 0 \\
\hline AN & 2177.7 & B & 649707 & 6132172 & 22.3 & 34.2 & 10.4 & 22.4 & 61.1 & 60.6 & 5.4 & 2 & 52 \\
\hline $\mathrm{AO}$ & 2158.5 & $\mathrm{E}$ & 649951 & 6132168 & 2.7 & 3.6 & 2.3 & 1.5 & 3.7 & 3.6 & 4.9 & 41 & 0 \\
\hline $\mathrm{AP}$ & 2113.0 & $\mathrm{M}$ & 650755 & 6132240 & 0.1 & 0.6 & 0.2 & 0.4 & 3.9 & 2.8 & --- & --- & 51 \\
\hline$A Q$ & 2080.9 & M & 651380 & 6132277 & 0.3 & 0.9 & 0.3 & 0.9 & 0.3 & 5.1 & --- & --- & 189 \\
\hline$A R$ & 2011.8 & $\mathrm{M}$ & 652483 & 6132323 & 0.9 & 0.3 & 2.1 & 0.3 & 1.4 & 0.8 & --- & --- & 405 \\
\hline AS & 1909.9 & M & 653091 & 6132326 & 2.4 & 0.5 & 5.9 & 0.1 & 5.1 & 1.5 & --- & --- & 1 \\
\hline $\mathrm{AT}$ & 1881.7 & M & 653641 & 6132348 & 0.2 & 0.2 & 8.6 & 0.6 & 6.5 & 4.1 & --- & --- & 0 \\
\hline $\mathrm{AU}$ & 1864.9 & M & 654009 & 6132356 & 0.0 & 0.6 & 2.4 & 0.0 & 1.6 & 1.8 & --- & --- & 55 \\
\hline $\mathrm{AV}$ & 1838.8 & M & 654409 & 6132407 & 5.1 & 0.5 & 4.4 & 0.1 & 0.0 & 0.4 & --- & --- & 1736 \\
\hline AW & 1820.8 & M & 654748 & 6132386 & 5.8 & 0.1 & 2.6 & 0.3 & 2.2 & 1.5 & --- & --- & 52 \\
\hline $\mathrm{AX}$ & 1808.4 & M & 654965 & 6132397 & 0.0 & 0.3 & 22.9 & 0.2 & 17.4 & 1.3 & --- & --- & 1493 \\
\hline$A Y$ & 1798.1 & M & 655076 & 6132406 & 0.0 & 0.5 & 13.5 & 0.5 & 11.2 & 1.3 & --- & --- & 0 \\
\hline $\mathrm{AZ}$ & 1787.4 & $\mathrm{M}$ & 655245 & 6132408 & 7.1 & 0.6 & 5.4 & 0.2 & $\begin{array}{r}1.2 \\
5.0\end{array}$ & 1.2 & --- & --- & 292 \\
\hline $\mathrm{BA}$ & 1743.5 & M & 656250 & 6132473 & 0.0 & 0.5 & 0.5 & 0.6 & 0.4 & 5.9 & --- & --- & 0 \\
\hline BB & 1724.8 & M & 656833 & 6132437 & 4.6 & 0.3 & 3.8 & 0.5 & 4.5 & 3.3 & --- & --- & 1181 \\
\hline $\mathrm{BC}$ & 1711.0 & M & 657318 & 6132453 & 7.3 & 1.4 & 9.3 & 0.6 & 6.8 & 2.8 & --- & --- & 556 \\
\hline $\mathrm{BD}$ & 1697.0 & M & 657710 & 6132481 & 1.7 & 0.3 & 0.2 & 0.3 & 0.4 & 3.7 & --- & --- & 0 \\
\hline $\mathrm{BE}$ & 1690.7 & M & 657862 & 6132493 & 5.3 & 0.6 & 11.2 & 0.1 & 9.3 & 3.4 & --- & --- & 178 \\
\hline $\mathrm{BF}$ & 1683.8 & $\mathrm{M}$ & 658029 & 6132499 & 5.8 & 0.7 & 6.5 & 0.8 & 5.8 & 4.1 & --- & --- & 52 \\
\hline
\end{tabular}

$\mathrm{CX}=$ COAXIAL

Note: EM values shown above

are local amplitudes

$\begin{array}{rl}\text { are local } & \text { loc } \\ 55 & 15\end{array}$
*Estimated Depth may be unreliable because the stronger part of the conductor may be deeper or to one side of the flight line, or because of a shallow dip or magnetite/overburden effects 
EM Anomaly List

\begin{tabular}{|c|c|c|c|c|c|c|c|c|c|c|c|c|c|}
\hline Label & Fid & Interp & $\begin{array}{c}\text { XUTM } \\
\text { m }\end{array}$ & $\begin{array}{c}\text { YUTM } \\
\mathrm{m}\end{array}$ & $\begin{array}{l}\text { CX } \\
\text { Real } \\
\text { ppm }\end{array}$ & $\begin{array}{l}900 \mathrm{HZ} \\
\text { Quad } \\
\text { ppm }\end{array}$ & $\begin{array}{l}\text { CP } \\
\text { Real } \\
\text { ppm }\end{array}$ & $\begin{array}{l}900 \mathrm{HZ} \\
\text { Quad } \\
\text { ppm }\end{array}$ & $\begin{array}{l}\text { CP } 7 \\
\text { Real } \\
\text { ppm }\end{array}$ & $\begin{array}{l}200 \mathrm{HZ} \\
\text { Quad } \\
\text { ppm }\end{array}$ & $\begin{array}{l}\text { Vertica } \\
\text { CoND } \\
\text { siemens }\end{array}$ & $\begin{array}{c}\text { al Dike } \\
\text { DEPTH* } \\
\text { m }\end{array}$ & $\begin{array}{c}\text { Mag. Corr } \\
\text { NT }\end{array}$ \\
\hline $\begin{array}{l}\text { LINE } \\
\text { BG } \\
B H \\
B I \\
B J \\
B K \\
B L \\
B M \\
B N \\
B O \\
B P \\
B Q \\
B R \\
B S\end{array}$ & $\begin{array}{l}19050 \\
1664.5 \\
1638.2 \\
1540.0 \\
1490.1 \\
1345.0 \\
1336.5 \\
1321.3 \\
1288.8 \\
1279.3 \\
1170.0 \\
955.8 \\
894.2 \\
821.0\end{array}$ & $\begin{array}{c}\mathrm{M} \\
\mathrm{M} \\
\mathrm{M} \\
\mathrm{B} ? \\
\mathrm{M} \\
\mathrm{M} \\
\mathrm{M} \\
\mathrm{M} \\
\mathrm{M} \\
\mathrm{M} \\
\mathrm{M} \\
\mathrm{M} \\
\mathrm{M}\end{array}$ & $\begin{array}{l}658484 \\
658988 \\
659641 \\
660890 \\
664676 \\
664893 \\
665159 \\
665747 \\
666019 \\
667183 \\
671625 \\
672812 \\
673754\end{array}$ & $\begin{array}{l}6132526 \\
6132532 \\
6132544 \\
6132589 \\
6132756 \\
6132753 \\
6132758 \\
6132768 \\
6132786 \\
6132863 \\
6132982 \\
6133017 \\
6133072\end{array}$ & $\begin{array}{r}0.0 \\
0.0 \\
1.2 \\
12.1 \\
0.6 \\
0.0 \\
0.2 \\
1.0 \\
0.0 \\
2.2 \\
0.2 \\
0.0 \\
5.2\end{array}$ & $\begin{array}{r}1.0 \\
1.4 \\
0.2 \\
32.9 \\
0.2 \\
0.5 \\
0.3 \\
0.8 \\
0.4 \\
0.7 \\
0.4 \\
0.5 \\
0.5\end{array}$ & $\begin{array}{r}0.2 \\
4.0 \\
2.2 \\
26.9 \\
4.8 \\
1.1 \\
2.7 \\
0.0 \\
0.1 \\
2.7 \\
0.1 \\
1.5 \\
14.5\end{array}$ & $\begin{array}{r}0.7 \\
0.9 \\
0.3 \\
65.1 \\
1.0 \\
0.7 \\
0.5 \\
0.3 \\
0.6 \\
0.1 \\
0.4 \\
0.2 \\
0.2\end{array}$ & $\begin{array}{r}1.8 \\
3.5 \\
1.6 \\
159.8 \\
2.7 \\
0.5 \\
2.4 \\
0.0 \\
0.1 \\
2.2 \\
0.9 \\
0.2 \\
11.1\end{array}$ & $\begin{array}{r}3.7 \\
9.0 \\
2.6 \\
92.1 \\
3.8 \\
3.0 \\
1.6 \\
1.6 \\
3.8 \\
4.0 \\
2.0 \\
2.1 \\
4.4\end{array}$ & $\begin{array}{l}--- \\
--- \\
--- \\
4.5 \\
--- \\
--- \\
--- \\
--- \\
--- \\
--- \\
--- \\
--- \\
---\end{array}$ & $\begin{array}{l}--- \\
--- \\
--- \\
0 \\
--- \\
--- \\
--- \\
--- \\
--- \\
--- \\
--- \\
--- \\
---\end{array}$ & $\begin{array}{r}0 \\
0 \\
0 \\
52 \\
287 \\
0 \\
0 \\
0 \\
0 \\
0 \\
193 \\
35 \\
0\end{array}$ \\
\hline$\overline{\text { LINE }}$ & 19060 & & & & & & & & & & & & \\
\hline A & 179.8 & $\mathrm{~S}$ & 655423 & 6127464 & 0.3 & 0.9 & 0.2 & 1.6 & 4.2 & 12.9 & --- & --- & 0 \\
\hline B & 253.8 & B & 657288 & 6127665 & 9.7 & 6.7 & 22.1 & 10.9 & 23.7 & 6.8 & 22.8 & 0 & 33 \\
\hline $\mathrm{C}$ & 259.1 & B & 657506 & 6127700 & 16.6 & 8.7 & 35.3 & 19.5 & 46.9 & 6.0 & 30.4 & 0 & 0 \\
\hline D & 271.3 & M & 657996 & 6127686 & 0.2 & 0.6 & 1.1 & 3.1 & 1.1 & 8.6 & --- & --- & 318 \\
\hline $\mathrm{E}$ & 281.3 & $\mathrm{M}$ & 658280 & 6127669 & 4.0 & 0.7 & 8.0 & 1.0 & 7.0 & 6.4 & --- & --- & 0 \\
\hline $\mathrm{F}$ & 318.5 & M & 658590 & 6127657 & 3.9 & 0.5 & 5.4 & 0.3 & 0.1 & 0.6 & --- & --- & 0 \\
\hline G & 336.5 & M & 658658 & 6127663 & 0.9 & 0.2 & 0.2 & 0.2 & 2.1 & 1.1 & --- & --- & 107 \\
\hline $\mathrm{H}$ & 348.3 & $\mathrm{M}$ & 658743 & 6127661 & 1.0 & 0.3 & 6.0 & 0.4 & 5.1 & 0.9 & --- & --- & 0 \\
\hline I & 360.7 & M & 658865 & 6127685 & 1.9 & 0.5 & 0.7 & 0.3 & 0.6 & 1.1 & --- & --- & 246 \\
\hline $\mathrm{J}$ & 412.0 & M & 659678 & 6127711 & 0.4 & 0.4 & 4.5 & 1.0 & 3.3 & 3.4 & --- & --- & 0 \\
\hline $\mathrm{K}$ & 430.1 & M & 659769 & 6127722 & 0.0 & 0.3 & 9.3 & 0.6 & 7.0 & 3.3 & --- & --- & 122 \\
\hline L & 440.2 & $\mathrm{M}$ & 659891 & 6127729 & 0.5 & 0.9 & 3.8 & 0.5 & 0.1 & 3.2 & --- & --- & 0 \\
\hline M & 454.7 & M & 660177 & 6127730 & 0.5 & 0.2 & 0.6 & 0.0 & 0.2 & 1.7 & --- & --- & 264 \\
\hline $\mathrm{N}$ & 485.2 & $\mathrm{M}$ & 660916 & 6127784 & 0.3 & 0.2 & 0.4 & 0.6 & 0.7 & 3.3 & --- & --- & 155 \\
\hline 0 & 506.1 & $\mathrm{M}$ & 661302 & 6127802 & 0.2 & 0.2 & 0.5 & 0.1 & 1.1 & 2.3 & --- & --- & 0 \\
\hline $\mathrm{P}$ & 518.0 & M & 661451 & 6127816 & 1.2 & 0.4 & 0.8 & 0.6 & 1.2 & 4.1 & --- & --- & 0 \\
\hline$Q$ & 532.3 & $\mathrm{M}$ & 661680 & 6127820 & 0.4 & 0.5 & 0.8 & 0.8 & 0.5 & 4.5 & --- & --- & 0 \\
\hline $\mathrm{R}$ & 544.5 & M & 662002 & 6127823 & 0.1 & 0.9 & 0.4 & 0.4 & 0.5 & 3.0 & --- & --- & 0 \\
\hline $\mathrm{S}$ & 854.9 & $\mathrm{~S} ?$ & 667600 & 6128036 & 1.3 & 1.2 & 0.6 & 1.4 & 1.3 & 8.8 & --- & --- & 0 \\
\hline $\mathrm{T}$ & 895.9 & M & 669294 & 6128068 & 0.6 & 0.5 & 3.1 & 0.5 & 1.8 & 3.3 & --- & --- & 113 \\
\hline U & 918.8 & S? & 670245 & 6128117 & 0.7 & 1.8 & 0.1 & 2.5 & 0.9 & 18.4 & --- & --- & 0 \\
\hline $\mathrm{V}$ & 967.1 & $\mathrm{~S}$ & 671774 & 6128149 & 1.3 & 2.1 & 1.5 & 3.2 & 2.0 & 20.5 & --- & --- & 0 \\
\hline W & 1030.2 & M & 673024 & 6128228 & 2.4 & 0.4 & 2.5 & 0.5 & 0.7 & 2.5 & --- & --- & 0 \\
\hline $\mathrm{x}$ & 1057.3 & M & 673562 & 6128244 & 0.3 & 0.3 & 0.1 & 1.2 & 0.6 & 4.0 & --- & --- & 48 \\
\hline $\mathrm{Y}$ & 1066.8 & M & 673819 & 6128230 & 2.0 & 0.7 & 5.9 & 1.0 & 3.6 & 5.0 & --- & --- & 0 \\
\hline $\mathrm{z}$ & 1078.3 & M & 674111 & 6128216 & 4.1 & 0.8 & 0.4 & 0.7 & 0.4 & 6.7 & --- & --- & 157 \\
\hline $\mathrm{AA}$ & 1111.1 & $\mathrm{~B}$ ? & 674845 & 6128315 & 0.9 & 2.2 & 3.4 & 3.1 & 3.7 & 23.3 & --- & --- & 48 \\
\hline$A B$ & 1117.3 & $B$ ? & 675065 & 6128314 & 3.4 & 3.1 & 4.3 & 5.3 & 6.1 & 36.8 & --- & --- & 0 \\
\hline $\mathrm{AC}$ & 1148.7 & M & 675664 & 6128294 & 0.4 & 0.5 & 0.4 & 0.3 & 0.1 & 3.0 & --- & --- & 0 \\
\hline $\mathrm{AD}$ & 1160.3 & $\mathrm{M}$ & 675810 & 6128327 & 4.8 & 0.9 & 0.4 & 0.4 & 0.3 & 3.0 & --- & --- & 0 \\
\hline $\mathrm{AE}$ & 1168.0 & M & 675986 & 6128339 & 4.4 & 0.5 & 5.6 & 0.7 & 3.8 & 1.5 & --- & --- & 0 \\
\hline
\end{tabular}

$\mathrm{CX}=$ COAXIAL

Note: EM values shown above

are local amplitudes

are 10
15
* Estimated Depth may be unreliable because the stronger part of the conductor may be deeper or to one side of the flight line, or because of a shallow dip or magnetite/overburden effects 
EM Anomaly List

\begin{tabular}{|c|c|c|c|c|c|c|c|c|c|c|c|c|c|}
\hline Label & Fid & Interp & $\underset{\mathrm{m}}{\mathrm{XUTM}}$ & $\begin{array}{c}\text { YUTM } \\
\mathrm{m}\end{array}$ & $\begin{array}{l}\text { CX } \\
\text { Real } \\
\text { ppm }\end{array}$ & $\begin{array}{c}900 \mathrm{HZ} \\
\text { Quad } \\
\text { ppm }\end{array}$ & $\begin{array}{l}\text { CP } \\
\text { Real } \\
\text { ppm }\end{array}$ & $\begin{array}{l}900 \mathrm{HZ} \\
\text { Quad } \\
\text { ppm }\end{array}$ & $\begin{array}{l}\text { CP }{ }^{7} \\
\text { Real } \\
\text { ppm }\end{array}$ & $\begin{array}{l}200 \mathrm{HZ} \\
\text { Quad } \\
\text { ppm }\end{array}$ & $\begin{array}{l}\text { Vertica } \\
\text { COND } \\
\text { siemens }\end{array}$ & $\begin{array}{c}\text { al Dike } \\
\text { DEPTH* } \\
\text { m }\end{array}$ & $\begin{array}{c}\text { Mag. Corr } \\
\text { NT }\end{array}$ \\
\hline $\begin{array}{l}\text { LINE } \\
\text { AF } \\
\text { AG } \\
\text { AH } \\
\text { AI } \\
\text { AJ } \\
\text { AK } \\
\text { AL } \\
\text { AM }\end{array}$ & $\begin{array}{r}19060 \\
1187.4 \\
1208.7 \\
1227.1 \\
1268.0 \\
1283.0 \\
1299.2 \\
1316.4 \\
1326.7\end{array}$ & $\begin{array}{l}\mathrm{M} \\
\mathrm{M} \\
\mathrm{M} \\
\mathrm{M} \\
\mathrm{M} \\
\mathrm{M} \\
\mathrm{M} \\
\mathrm{M}\end{array}$ & $\begin{array}{l}676529 \\
677310 \\
677613 \\
678720 \\
678955 \\
679250 \\
679523 \\
679739\end{array}$ & $\begin{array}{l}6128307 \\
6128402 \\
6128433 \\
6128456 \\
6128455 \\
6128412 \\
6128454 \\
6128477\end{array}$ & $\begin{array}{r}0.9 \\
0.1 \\
3.1 \\
0.8 \\
1.3 \\
0.3 \\
15.3 \\
269.7\end{array}$ & $\begin{array}{l}0.8 \\
1.3 \\
0.7 \\
0.5 \\
0.4 \\
0.6 \\
0.5 \\
0.5\end{array}$ & $\begin{array}{r}0.3 \\
0.5 \\
4.2 \\
8.7 \\
0.7 \\
7.9 \\
0.6 \\
755.8\end{array}$ & $\begin{array}{l}0.8 \\
1.0 \\
1.1 \\
0.3 \\
0.1 \\
0.2 \\
0.5 \\
4.5\end{array}$ & $\begin{array}{r}0.2 \\
0.3 \\
2.7 \\
7.0 \\
0.5 \\
6.3 \\
0.3 \\
623.4\end{array}$ & $\begin{array}{r}2.6 \\
8.5 \\
4.7 \\
2.6 \\
2.9 \\
1.4 \\
4.0 \\
23.7\end{array}$ & $\begin{array}{l}--- \\
--- \\
--- \\
--- \\
--- \\
--- \\
--- \\
---\end{array}$ & $\begin{array}{l}--- \\
--- \\
--- \\
--- \\
--- \\
--- \\
--- \\
---\end{array}$ & $\begin{array}{r}48 \\
78 \\
48 \\
130 \\
0 \\
0 \\
0 \\
323\end{array}$ \\
\hline $\begin{array}{l}\text { LINE } \\
\text { A } \\
\text { B } \\
\text { C } \\
\text { D } \\
\text { E } \\
\text { F } \\
\text { G } \\
\text { H }\end{array}$ & $\begin{array}{r}19061 \\
461.9 \\
392.8 \\
374.6 \\
356.2 \\
309.0 \\
283.3 \\
271.4 \\
262.0\end{array}$ & $\begin{array}{l}\mathrm{S} \\
\mathrm{M} \\
\mathrm{M} \\
\mathrm{M} \\
\mathrm{M} \\
\mathrm{M} \\
\mathrm{M} \\
\mathrm{M}\end{array}$ & $\begin{array}{l}677180 \\
678626 \\
678849 \\
679085 \\
679342 \\
679626 \\
679838 \\
679974\end{array}$ & $\begin{array}{l}6128391 \\
6128448 \\
6128462 \\
6128467 \\
6128471 \\
6128423 \\
6128463 \\
6128486\end{array}$ & $\begin{array}{r}0.5 \\
3.7 \\
2.0 \\
0.0 \\
0.0 \\
12.4 \\
15.2 \\
0.0\end{array}$ & $\begin{array}{l}2.2 \\
0.5 \\
0.8 \\
0.3 \\
0.1 \\
0.6 \\
0.0 \\
0.3\end{array}$ & $\begin{array}{r}0.3 \\
0.8 \\
5.1 \\
7.4 \\
21.4 \\
44.2 \\
29.5 \\
21.9\end{array}$ & $\begin{array}{l}2.5 \\
0.8 \\
0.3 \\
0.2 \\
0.9 \\
1.4 \\
0.7 \\
0.2\end{array}$ & $\begin{array}{r}0.5 \\
0.3 \\
6.7 \\
6.4 \\
18.1 \\
26.4 \\
23.8 \\
2.5\end{array}$ & $\begin{array}{r}17.8 \\
1.2 \\
1.0 \\
1.3 \\
4.4 \\
3.5 \\
2.7 \\
2.4\end{array}$ & $\begin{array}{l}0.8 \\
--- \\
--- \\
--- \\
--- \\
--- \\
--- \\
---\end{array}$ & $\begin{array}{r}6 \\
--- \\
--- \\
--- \\
--- \\
--- \\
--- \\
---\end{array}$ & $\begin{array}{r}71 \\
83 \\
36 \\
0 \\
64 \\
64 \\
0 \\
0\end{array}$ \\
\hline $\begin{array}{l}\text { LINE } \\
\mathrm{A}\end{array}$ & $\begin{array}{c}19062 \\
3271.0\end{array}$ & B & 637869 & 6126956 & 33.3 & 34.1 & 58.4 & 75.8 & 128.8 & 35.4 & 12.2 & 0 & 0 \\
\hline $\begin{array}{l}\text { LINE } \\
\text { A } \\
\text { B } \\
\text { C } \\
\text { D } \\
\text { E } \\
\text { F } \\
\text { G } \\
\text { H } \\
\text { I } \\
\text { J }\end{array}$ & $\begin{array}{r}19070 \\
385.6 \\
377.9 \\
368.2 \\
352.8 \\
343.9 \\
337.9 \\
334.2 \\
302.2 \\
290.4 \\
279.2\end{array}$ & $\begin{array}{l}\mathrm{M} \\
\mathrm{M} \\
\mathrm{M} \\
\mathrm{M} \\
\mathrm{M} \\
\mathrm{B} \\
\mathrm{B} \\
\mathrm{M} \\
\mathrm{M} \\
\mathrm{M}\end{array}$ & $\begin{array}{l}680960 \\
681084 \\
681186 \\
681509 \\
681803 \\
682004 \\
682128 \\
683054 \\
683316 \\
683630\end{array}$ & $\begin{array}{l}6127419 \\
6127409 \\
6127401 \\
6127387 \\
6127413 \\
6127434 \\
6127440 \\
6127469 \\
6127471 \\
6127469\end{array}$ & $\begin{array}{l}0.1 \\
5.2 \\
0.4 \\
3.5 \\
3.1 \\
2.7 \\
6.3 \\
0.2 \\
2.1 \\
0.0\end{array}$ & $\begin{array}{l}0.5 \\
0.3 \\
0.5 \\
0.3 \\
0.3 \\
5.4 \\
8.9 \\
0.5 \\
0.4 \\
0.4\end{array}$ & $\begin{array}{r}2.3 \\
11.1 \\
8.3 \\
0.1 \\
1.0 \\
17.1 \\
9.0 \\
10.2 \\
23.9 \\
9.5\end{array}$ & $\begin{array}{r}0.4 \\
0.7 \\
0.6 \\
0.7 \\
0.1 \\
22.8 \\
10.4 \\
0.8 \\
1.0 \\
0.3\end{array}$ & $\begin{array}{r}2.5 \\
9.1 \\
6.8 \\
0.0 \\
2.1 \\
62.4 \\
33.3 \\
7.2 \\
18.7 \\
8.6\end{array}$ & $\begin{array}{r}2.7 \\
0.4 \\
1.8 \\
4.4 \\
2.5 \\
36.6 \\
30.2 \\
5.6 \\
8.3 \\
4.1\end{array}$ & $\begin{array}{l}--- \\
--- \\
--- \\
--- \\
--- \\
4.8 \\
5.6 \\
--- \\
--- \\
---\end{array}$ & $\begin{array}{r}--- \\
--- \\
--- \\
--- \\
--- \\
23 \\
24 \\
--- \\
--- \\
---\end{array}$ & $\begin{array}{r}53 \\
0 \\
0 \\
0 \\
409 \\
0 \\
66 \\
56 \\
66 \\
0\end{array}$ \\
\hline $\begin{array}{l}\text { LINE } \\
\text { A } \\
\text { B } \\
\text { C } \\
\text { D } \\
\text { E } \\
\text { F }\end{array}$ & $\begin{array}{r}19080 \\
826.8 \\
838.0 \\
848.3 \\
854.0 \\
862.0 \\
884.6\end{array}$ & $\begin{array}{l}\mathrm{M} \\
\mathrm{M} \\
\mathrm{M} \\
\mathrm{M} \\
\mathrm{M} \\
\mathrm{M}\end{array}$ & $\begin{array}{l}679888 \\
680205 \\
680460 \\
680607 \\
680823 \\
681567\end{array}$ & $\begin{array}{l}6125468 \\
6125481 \\
6125489 \\
6125486 \\
6125499 \\
6125536\end{array}$ & $\begin{array}{l}0.0 \\
0.3 \\
5.4 \\
3.9 \\
0.7 \\
0.1\end{array}$ & $\begin{array}{l}1.1 \\
0.4 \\
0.7 \\
0.6 \\
0.9 \\
0.7\end{array}$ & $\begin{array}{l}0.3 \\
0.4 \\
0.2 \\
5.7 \\
0.9 \\
0.9\end{array}$ & $\begin{array}{l}0.7 \\
0.3 \\
0.4 \\
0.1 \\
0.6 \\
0.7\end{array}$ & $\begin{array}{l}0.2 \\
0.3 \\
5.9 \\
4.1 \\
0.6 \\
0.6\end{array}$ & $\begin{array}{l}2.4 \\
2.3 \\
0.7 \\
0.2 \\
3.1 \\
2.6\end{array}$ & $\begin{array}{l}--- \\
--- \\
--- \\
--- \\
--- \\
---\end{array}$ & $\begin{array}{l}--- \\
--- \\
--- \\
--- \\
--- \\
---\end{array}$ & $\begin{array}{r}0 \\
22 \\
0 \\
225 \\
376 \\
144\end{array}$ \\
\hline $\begin{array}{l}\text { LINE } \\
\text { A } \\
B \\
\text { C }\end{array}$ & $\begin{array}{r}19090 \\
8111.0 \\
8090.0 \\
8080.5\end{array}$ & $\begin{array}{l}\mathrm{M} \\
\mathrm{M} \\
\mathrm{M}\end{array}$ & $\begin{array}{l}654345 \\
654666 \\
654949\end{array}$ & $\begin{array}{l}6122682 \\
6122675 \\
6122698\end{array}$ & $\begin{array}{l}0.4 \\
1.6 \\
0.0\end{array}$ & $\begin{array}{l}0.6 \\
0.1 \\
0.2\end{array}$ & $\begin{array}{l}0.9 \\
5.6 \\
2.8\end{array}$ & $\begin{array}{l}0.3 \\
0.2 \\
0.5\end{array}$ & $\begin{array}{l}0.8 \\
4.6 \\
2.7\end{array}$ & $\begin{array}{l}0.8 \\
0.9 \\
1.4\end{array}$ & $\begin{array}{l}--- \\
--- \\
---\end{array}$ & $\begin{array}{l}--- \\
--- \\
---\end{array}$ & $\begin{array}{r}0 \\
34 \\
0\end{array}$ \\
\hline
\end{tabular}

$\mathrm{CX}=$ COAXIAL

Note: EM values shown above

are local amplitudes

Ketchikan - North/South of 5515
*Estimated Depth may be unreliable because the stronger part of the conductor may be deeper or to one side of the flight line, or because of a shallow dip or magnetite/overburden effects 
EM Anomaly List

\begin{tabular}{|c|c|c|c|c|c|c|c|c|c|c|c|c|c|}
\hline Label & Fid & Interp & $\begin{array}{c}\text { XUTM } \\
\text { m }\end{array}$ & $\begin{array}{c}\text { YUTM } \\
\mathrm{m}\end{array}$ & $\begin{array}{l}\text { CX } \\
\text { Real } \\
\text { ppm }\end{array}$ & $\begin{array}{l}900 \mathrm{HZ} \\
\text { Quad } \\
\text { ppm }\end{array}$ & $\begin{array}{l}\text { CP } \\
\text { Real } \\
\text { ppm }\end{array}$ & $\begin{array}{l}900 \mathrm{HZ} \\
\text { Quad } \\
\text { ppm }\end{array}$ & $\begin{array}{l}\text { CP } \\
\text { Real } \\
\text { ppm }\end{array}$ & $\begin{array}{l}200 \mathrm{HZ} \\
\text { Quad } \\
\text { ppm }\end{array}$ & $\begin{array}{l}\text { Vertica } \\
\text { CoND } \\
\text { siemens }\end{array}$ & $\begin{array}{c}\text { al Dike } \\
\text { DEPTH* } \\
\text { m }\end{array}$ & $\begin{array}{c}\text { Mag. Corr } \\
\text { NT }\end{array}$ \\
\hline LINE & 19090 & & & & & & & & & & & & \\
\hline D & 8037.0 & M & 655737 & 6122744 & 0.0 & 0.3 & 4.2 & 0.2 & 3.4 & 1.6 & --- & --- & 28 \\
\hline $\mathrm{E}$ & 8009.0 & M & 655899 & 6122756 & 0.0 & 0.7 & 1.2 & 0.5 & 3.4 & 0.4 & --- & --- & 0 \\
\hline $\mathrm{F}$ & 7986.0 & M & 656157 & 6122751 & 0.0 & 0.1 & 9.2 & 0.1 & 8.2 & 1.0 & --- & --- & 0 \\
\hline G & 7970.8 & $\mathrm{M}$ & 656348 & 6122771 & 0.5 & 0.0 & 4.1 & 0.5 & 3.4 & 1.5 & --- & --- & 0 \\
\hline $\mathrm{H}$ & 7949.1 & M & 656585 & 6122758 & 0.6 & 0.3 & 4.4 & 0.1 & 3.9 & 1.6 & --- & --- & 0 \\
\hline I & 7919.9 & $\mathrm{~S}$ & 657408 & 6122820 & 0.8 & 2.4 & 2.2 & 4.6 & 5.6 & 27.3 & --- & --- & 34 \\
\hline $\mathrm{J}$ & 7902.6 & M & 657853 & 6122798 & 1.3 & 0.5 & 4.8 & 0.5 & 3.5 & 1.1 & --- & --- & 0 \\
\hline $\mathrm{K}$ & 7888.0 & $\mathrm{M}$ & 658034 & 6122827 & 10.4 & 0.2 & 18.6 & 0.1 & 15.9 & 1.0 & --- & --- & 163 \\
\hline $\mathrm{L}$ & 7876.7 & M & 658164 & 6122843 & 3.5 & 0.4 & 5.6 & 0.1 & 4.7 & 1.1 & --- & --- & 269 \\
\hline M & 7859.4 & M & 658350 & 6122836 & 30.7 & 0.3 & 30.6 & 0.9 & 24.9 & 2.5 & --- & --- & 136 \\
\hline $\mathrm{N}$ & 7798.8 & M & 659217 & 6122861 & 1.0 & 0.6 & 4.4 & 0.4 & 2.0 & 2.7 & --- & --- & 0 \\
\hline 0 & 7659.3 & $\mathrm{~S}$ & 661229 & 6122960 & 1.4 & 1.1 & 1.1 & 2.1 & 7.3 & 3.5 & --- & --- & 0 \\
\hline P & 7636.8 & $B$ ? & 661710 & 6122947 & 0.0 & 3.5 & 2.5 & 4.4 & 10.9 & 3.0 & 1.1 & 0 & 34 \\
\hline$Q$ & 7626.7 & $B$ ? & 661845 & 6122961 & 5.4 & 8.8 & 1.4 & 9.8 & 25.4 & 36.2 & 3.1 & 7 & 34 \\
\hline $\mathrm{R}$ & 7590.4 & D & 662140 & 6123014 & 2.9 & 3.3 & 0.7 & 2.9 & 6.1 & 8.5 & 3.2 & 49 & 0 \\
\hline $\mathrm{S}$ & 7224.2 & B & 666866 & 6123182 & 2.1 & 4.1 & 3.3 & 10.6 & 28.5 & 20.2 & 2.4 & 17 & 0 \\
\hline $\mathrm{T}$ & 7210.2 & B & 667039 & 6123197 & 5.4 & 7.1 & 5.8 & 12.3 & 29.3 & 15.8 & 3.9 & 20 & 0 \\
\hline U & 7166.9 & $B$ ? & 667996 & 6123200 & 5.4 & 1.7 & 0.1 & 0.1 & 0.1 & 0.2 & --- & --- & 0 \\
\hline $\mathrm{V}$ & 7123.8 & B & 668440 & 6123216 & 1.9 & 0.0 & 0.5 & 0.8 & 1.1 & 5.1 & 51.9 & 75 & 0 \\
\hline W & 7093.9 & S? & 668626 & 6123221 & 0.2 & 1.3 & 0.1 & 1.2 & 1.6 & 4.3 & --- & --- & 0 \\
\hline LINE & 19100 & & & & & & & & & & & & \\
\hline$A$ & 4715.4 & B & 657190 & 6117964 & 5.0 & 6.0 & 16.3 & 10.8 & 18.4 & 5.9 & 11.1 & 9 & 0 \\
\hline B & 4821.6 & $B$ ? & 658235 & 6118023 & 4.8 & 0.9 & 0.5 & 0.2 & 0.5 & 1.1 & --- & --- & 31 \\
\hline C & 4947.8 & $B$ ? & 660359 & 6118151 & 3.0 & 1.3 & 0.7 & 2.0 & 4.9 & 9.4 & --- & --- & 0 \\
\hline D & 5140.5 & M & 663507 & 6118206 & 0.3 & 0.4 & 0.0 & 0.4 & 0.1 & 3.0 & --- & --- & 0 \\
\hline $\mathrm{E}$ & 5160.0 & M & 663672 & 6118219 & 0.2 & 0.5 & 0.1 & 0.2 & 0.1 & 0.9 & --- & --- & 154 \\
\hline $\mathrm{F}$ & 5176.5 & M & 663743 & 6118226 & 1.1 & 0.4 & 0.6 & 0.2 & 0.2 & 4.0 & --- & --- & 0 \\
\hline G & 5191.3 & M & 663789 & 6118226 & 6.6 & 0.3 & 4.7 & 0.3 & 3.9 & 2.1 & --- & --- & 0 \\
\hline $\mathrm{H}$ & 5206.1 & M & 663824 & 6118236 & 9.9 & 0.3 & 3.6 & 0.2 & 3.0 & 0.9 & --- & --- & 31 \\
\hline I & 5215.7 & M & 663850 & 6118223 & 4.6 & 0.5 & 7.0 & 0.6 & 5.7 & 2.0 & --- & --- & 0 \\
\hline $\mathrm{J}$ & 5228.6 & M & 663908 & 6118216 & 3.9 & 0.5 & 1.1 & 0.1 & 1.3 & 1.7 & --- & --- & 280 \\
\hline $\mathrm{K}$ & 5457.4 & M & 666315 & 6118309 & 5.6 & 0.4 & 2.1 & 0.2 & 2.0 & 1.1 & --- & --- & 157 \\
\hline L & 5680.2 & M & 670982 & 6118493 & 0.0 & 0.5 & 2.7 & 0.8 & 0.0 & 5.1 & --- & --- & 112 \\
\hline M & 5761.1 & M & 672029 & 6118513 & 3.3 & 0.5 & 0.6 & 0.2 & 0.5 & 2.4 & --- & --- & 123 \\
\hline $\mathrm{N}$ & 5817.0 & M & 672550 & 6118482 & 23.8 & 0.8 & 7.1 & 0.2 & 6.7 & 1.5 & --- & --- & 0 \\
\hline 0 & 5844.0 & M & 672609 & 6118480 & 19.9 & 0.2 & 14.8 & 0.9 & 11.3 & 3.2 & --- & --- & 31 \\
\hline $\mathrm{P}$ & 6004.7 & B & 675684 & 6118674 & 42.9 & 22.5 & 113.4 & 56.6 & 135.0 & 23.8 & 46.8 & 0 & 0 \\
\hline Q & 6170.9 & S? & 678314 & 6118719 & 0.6 & 2.7 & 1.8 & 3.1 & 3.3 & 20.4 & 1.7 & 29 & 0 \\
\hline $\mathrm{R}$ & 6257.9 & $\mathrm{~S}$ & 680113 & 6118754 & 0.7 & 3.8 & 1.1 & 5.5 & 6.0 & 35.7 & --- & --- & 0 \\
\hline $\mathrm{S}$ & 6267.9 & $\mathrm{~s}$ & 680456 & 6118799 & 0.1 & 3.3 & 1.1 & 3.5 & 4.1 & 21.9 & --- & --- & 0 \\
\hline $\mathrm{T}$ & 6289.4 & M & 681129 & 6118859 & 0.0 & 1.3 & 0.5 & 1.0 & 1.4 & 6.7 & --- & --- & 8 \\
\hline U & 6298.2 & M & 681408 & 6118866 & 2.7 & 0.6 & 0.3 & 0.0 & 0.3 & 0.1 & --- & --- & 223 \\
\hline $\mathrm{V}$ & 6387.3 & D & 684352 & 6118949 & 6.9 & 4.4 & 5.3 & 9.2 & 17.1 & 6.8 & 8.8 & 27 & 0 \\
\hline W & 6400.0 & $B$ ? & 684617 & 6118940 & 2.2 & 4.9 & 3.8 & 14.2 & 39.0 & 30.5 & 2.2 & 12 & 230 \\
\hline $\mathrm{x}$ & 6418.0 & B & 685184 & 6119001 & 0.2 & 2.7 & 1.1 & 5.7 & 14.3 & 12.1 & 0.7 & 0 & 31 \\
\hline
\end{tabular}

$\mathrm{CX}=$ COAXIAL

Note: EM values shown above

are local amplitudes

Ketchikan - North/South of 5515
* Estimated Depth may be unreliable because the stronger part of the conductor may be deeper or to one side of the flight line, or because of a shallow dip or magnetite/overburden effects 
EM Anomaly List

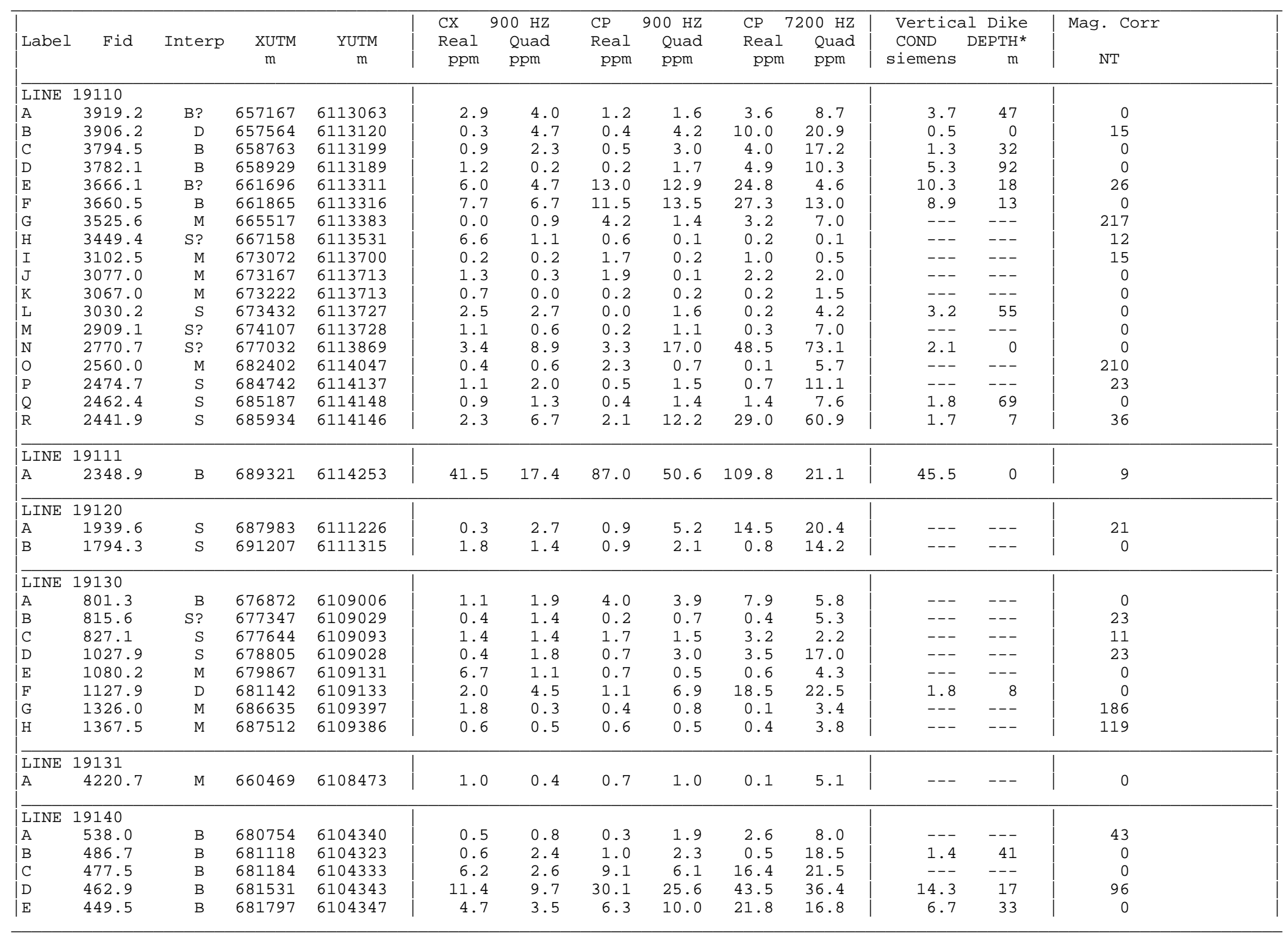

$\mathrm{CX}=$ COAXIAL

Note: EM values shown above

Ketchikan - North/South of 5515
* Estimated Depth may be unreliable because the stronger part of the conductor may be deeper or to one side of the flight line, or because of a shallow dip or magnetite/overburden effects 
EM Anomaly List

\begin{tabular}{|c|c|c|c|c|c|c|c|c|c|c|c|c|c|}
\hline Label & Fid & Interp & $\underset{\mathrm{m}}{\mathrm{XUTM}}$ & $\begin{array}{c}\text { YUTM } \\
\mathrm{m}\end{array}$ & $\begin{array}{l}\text { CX } \\
\text { Real } \\
\text { ppm }\end{array}$ & $\begin{array}{c}900 \mathrm{HZ} \\
\text { Quad } \\
\text { ppm }\end{array}$ & $\begin{array}{l}\text { CP } \\
\text { Real } \\
\text { ppm }\end{array}$ & $\begin{array}{c}900 \mathrm{HZ} \\
\text { Quad } \\
\text { ppm }\end{array}$ & $\begin{array}{l}\mathrm{CP} \\
\mathrm{Real} \\
\mathrm{ppm}\end{array}$ & $\begin{array}{c}200 \mathrm{HZ} \\
\text { Quad } \\
\text { ppm }\end{array}$ & $\begin{array}{l}\text { Vertica } \\
\text { COND } \\
\text { siemens }\end{array}$ & $\begin{array}{c}\text { al Dike } \\
\text { DEPTH* } \\
\text { m }\end{array}$ & $\begin{array}{c}\text { Mag. Corr } \\
\text { NT }\end{array}$ \\
\hline $\begin{array}{l}\text { LINE } \\
\mathrm{F} \\
\mathrm{G} \\
\mathrm{H}\end{array}$ & $\begin{array}{r}19140 \\
357.8 \\
345.9 \\
326.1\end{array}$ & $\begin{array}{l}\mathrm{M} \\
\mathrm{M} \\
\mathrm{M}\end{array}$ & $\begin{array}{l}683633 \\
683923 \\
684380\end{array}$ & $\begin{array}{l}6104415 \\
6104424 \\
6104426\end{array}$ & $\begin{array}{l}0.4 \\
0.6 \\
0.1\end{array}$ & $\begin{array}{l}1.0 \\
0.4 \\
0.6\end{array}$ & $\begin{array}{l}2.4 \\
0.2 \\
3.9\end{array}$ & $\begin{array}{l}1.2 \\
0.3 \\
1.2\end{array}$ & $\begin{array}{l}2.2 \\
0.0 \\
2.9\end{array}$ & $\begin{array}{l}7.3 \\
3.0 \\
3.4\end{array}$ & $\begin{array}{l}--- \\
--- \\
---\end{array}$ & $\begin{array}{l}--- \\
--- \\
---\end{array}$ & $\begin{array}{r}83 \\
1 \\
483\end{array}$ \\
\hline $\begin{array}{l}\text { LINE } \\
\text { A } \\
\text { B } \\
\text { C } \\
\text { D } \\
\text { E } \\
\text { F } \\
\text { G } \\
\text { H }\end{array}$ & $\begin{array}{r}19150 \\
7782.2 \\
7818.0 \\
7827.2 \\
7836.4 \\
7839.6 \\
7845.0 \\
7847.7 \\
7855.4\end{array}$ & $\begin{array}{l}\mathrm{B} \\
\mathrm{M} \\
\mathrm{M} \\
\mathrm{M} \\
\mathrm{M} \\
\mathrm{M} \\
\mathrm{S} \\
\mathrm{M}\end{array}$ & $\begin{array}{l}626218 \\
627134 \\
627387 \\
627627 \\
627707 \\
627858 \\
627941 \\
628187\end{array}$ & $\begin{array}{l}6139236 \\
6138726 \\
6138592 \\
6138463 \\
6138418 \\
6138331 \\
6138289 \\
6138163\end{array}$ & $\begin{array}{l}2.6 \\
4.3 \\
1.5 \\
0.5 \\
0.4 \\
1.8 \\
1.0 \\
0.8\end{array}$ & $\begin{array}{l}3.0 \\
3.3 \\
1.5 \\
2.1 \\
0.2 \\
2.1 \\
2.7 \\
1.7\end{array}$ & $\begin{array}{l}2.2 \\
1.5 \\
0.3 \\
0.3 \\
0.6 \\
3.2 \\
2.4 \\
1.4\end{array}$ & $\begin{array}{l}4.2 \\
4.5 \\
3.8 \\
3.5 \\
0.4 \\
3.4 \\
3.8 \\
2.6\end{array}$ & $\begin{array}{r}11.0 \\
7.1 \\
0.1 \\
14.7 \\
4.6 \\
2.7 \\
4.5 \\
0.8\end{array}$ & $\begin{array}{r}16.0 \\
8.0 \\
10.6 \\
3.3 \\
0.7 \\
19.7 \\
23.3 \\
14.7\end{array}$ & $\begin{array}{l}3.7 \\
--- \\
--- \\
--- \\
--- \\
--- \\
--- \\
---\end{array}$ & $\begin{array}{l}41 \\
--- \\
--- \\
--- \\
--- \\
--- \\
--- \\
---\end{array}$ & $\begin{array}{r}0 \\
67 \\
266 \\
255 \\
268 \\
244 \\
288 \\
47\end{array}$ \\
\hline
\end{tabular}

$\mathrm{CX}=$ COAXIAL

$\mathrm{CP}=$ COPLANAR

Note:EM values shown above are local amplitudes

Ketchikan - North/South of 5515
* Estimated Depth may be unreliable because the stronger part of the conductor may be deeper or shallow dip or magnetite/overburden effects 
EM Anomaly List

\begin{tabular}{|c|c|c|c|c|c|c|c|c|c|c|c|c|c|}
\hline Label & Fid & Interp & $\underset{\mathrm{m}}{\mathrm{XUTM}}$ & $\begin{array}{c}\text { YUTM } \\
\text { m }\end{array}$ & $\begin{array}{ll}\text { CX } & 9 \\
\text { Real } \\
\text { ppm }\end{array}$ & $\begin{array}{l}00 \mathrm{HZ} \\
\text { Quad } \\
\text { ppm }\end{array}$ & $\begin{array}{l}\text { CP } \\
\text { Real } \\
\text { ppm }\end{array}$ & $\begin{array}{c}900 \mathrm{HZ} \\
\text { Quad } \\
\text { ppm }\end{array}$ & $\begin{array}{l}\text { CP } \\
\text { Real } \\
\text { ppm }\end{array}$ & $\begin{array}{c}7200 \mathrm{HZ} \\
\text { Quad } \\
\text { ppm }\end{array}$ & $\begin{array}{l}\text { Vertica } \\
\text { ConD } \\
\text { siemens }\end{array}$ & $\begin{array}{c}\text { al Dike } \\
\text { DEPTH* } \\
\text { m }\end{array}$ & $\begin{array}{c}\text { Mag. Corr } \\
\text { NT }\end{array}$ \\
\hline $\begin{array}{l}\text { LINE } \\
\text { A } \\
\text { B } \\
\text { C } \\
\text { D } \\
\text { E }\end{array}$ & $\begin{array}{l}20010 \\
1778.9 \\
1735.9 \\
1720.0 \\
1482.0 \\
1476.0\end{array}$ & $\begin{array}{r}\text { S } \\
\text { S } \\
\text { S } \\
S ? \\
S ?\end{array}$ & $\begin{array}{l}648127 \\
648032 \\
648034 \\
647851 \\
647932\end{array}$ & $\begin{array}{l}6163532 \\
6165026 \\
6165606 \\
6170863 \\
6170972\end{array}$ & $\begin{array}{l}1.1 \\
1.6 \\
0.3 \\
2.8 \\
5.7\end{array}$ & $\begin{array}{l}2.4 \\
2.9 \\
1.4 \\
6.3 \\
5.9\end{array}$ & $\begin{array}{l}0.3 \\
0.7 \\
0.1 \\
1.1 \\
0.4\end{array}$ & $\begin{array}{r}4.1 \\
4.2 \\
1.9 \\
10.4 \\
9.7\end{array}$ & $\begin{array}{r}1.7 \\
7.5 \\
1.8 \\
37.4 \\
31.2\end{array}$ & $\begin{array}{l}31.3 \\
21.4 \\
12.7 \\
40.1 \\
45.9\end{array}$ & $\begin{array}{l}1.3 \\
1.9 \\
0.8 \\
1.8 \\
2.8\end{array}$ & $\begin{array}{r}23 \\
21 \\
15 \\
0 \\
24\end{array}$ & $\begin{array}{r}0 \\
16 \\
93 \\
0 \\
0\end{array}$ \\
\hline $\begin{array}{l}\text { LINE } \\
\text { A } \\
\text { B } \\
\text { C } \\
\text { D } \\
\text { E }\end{array}$ & $\begin{array}{l}20020 \\
1224.5 \\
1237.3 \\
1263.3 \\
1396.8 \\
1435.8\end{array}$ & $\begin{array}{l}S \\
S \\
S \\
S \\
S\end{array}$ & $\begin{array}{l}648421 \\
648447 \\
648417 \\
648293 \\
648254\end{array}$ & $\begin{array}{l}6164694 \\
6165079 \\
6165508 \\
6169540 \\
6170796\end{array}$ & $\begin{array}{l}2.1 \\
2.2 \\
0.7 \\
0.2 \\
1.1\end{array}$ & $\begin{array}{l}3.6 \\
2.2 \\
2.8 \\
1.9 \\
6.8\end{array}$ & $\begin{array}{l}2.1 \\
0.3 \\
1.0 \\
1.5 \\
1.7\end{array}$ & $\begin{array}{r}3.3 \\
4.2 \\
2.7 \\
4.6 \\
11.2\end{array}$ & $\begin{array}{r}8.5 \\
14.3 \\
10.0 \\
5.8 \\
30.8\end{array}$ & $\begin{array}{l}24.2 \\
18.5 \\
14.3 \\
29.4 \\
40.0\end{array}$ & $\begin{array}{l}--- \\
--- \\
1.3 \\
--- \\
0.9\end{array}$ & $\begin{array}{r}--- \\
--- \\
32 \\
--- \\
0\end{array}$ & $\begin{array}{r}0 \\
12 \\
0 \\
0 \\
0\end{array}$ \\
\hline $\begin{array}{l}\text { LINE } \\
\text { A } \\
\text { B } \\
\text { C } \\
\text { D } \\
\text { E } \\
\text { F } \\
\text { G } \\
\text { H }\end{array}$ & $\begin{array}{l}20030 \\
1079.8 \\
1054.1 \\
1046.2 \\
1037.3 \\
917.8 \\
906.2 \\
894.1 \\
878.7\end{array}$ & $\begin{array}{r}\text { S } \\
\text { S } \\
\text { D } \\
\text { S } \\
\text { S } \\
\text { S } \\
\text { S } \\
\text { B? }\end{array}$ & $\begin{array}{l}648867 \\
648824 \\
648799 \\
648775 \\
648688 \\
648683 \\
648673 \\
648664\end{array}$ & $\begin{array}{l}6164585 \\
6165514 \\
6165795 \\
6166098 \\
6169659 \\
6170042 \\
6170477 \\
6170995\end{array}$ & $\begin{array}{l}0.6 \\
3.8 \\
1.8 \\
0.3 \\
1.1 \\
1.5 \\
3.5 \\
3.9\end{array}$ & $\begin{array}{r}4.2 \\
10.1 \\
5.6 \\
2.6 \\
6.0 \\
4.9 \\
25.4 \\
6.6\end{array}$ & $\begin{array}{l}1.6 \\
2.6 \\
0.7 \\
0.8 \\
1.2 \\
1.3 \\
5.2 \\
5.3\end{array}$ & $\begin{array}{r}6.5 \\
16.2 \\
4.0 \\
3.4 \\
7.9 \\
6.8 \\
41.6 \\
10.5\end{array}$ & $\begin{array}{r}17.3 \\
50.5 \\
7.6 \\
2.6 \\
17.1 \\
15.3 \\
132.5 \\
22.0\end{array}$ & $\begin{array}{r}21.4 \\
63.6 \\
20.4 \\
22.3 \\
55.6 \\
39.8 \\
170.1 \\
48.0\end{array}$ & $\begin{array}{l}--- \\
2.0 \\
1.5 \\
--- \\
0.9 \\
1.4 \\
1.2 \\
---\end{array}$ & $\begin{array}{r}--- \\
0 \\
15 \\
--- \\
0 \\
15 \\
0 \\
---\end{array}$ & $\begin{array}{r}12 \\
12 \\
12 \\
0 \\
12 \\
0 \\
0 \\
0\end{array}$ \\
\hline $\begin{array}{l}\text { LINE } \\
\text { A } \\
\text { B } \\
\text { C } \\
\text { D }\end{array}$ & $\begin{array}{r}20040 \\
694.0 \\
703.3 \\
706.2 \\
835.8\end{array}$ & $\begin{array}{r}\mathrm{D} \\
\mathrm{B} ? \\
\mathrm{D} \\
\mathrm{B} ?\end{array}$ & $\begin{array}{l}649232 \\
649216 \\
649210 \\
649079\end{array}$ & $\begin{array}{l}6165754 \\
6166090 \\
6166195 \\
6170791\end{array}$ & $\begin{array}{l}0.2 \\
0.9 \\
0.8 \\
7.7\end{array}$ & $\begin{array}{l}3.2 \\
4.3 \\
6.0 \\
7.4\end{array}$ & $\begin{array}{r}0.7 \\
0.3 \\
0.3 \\
14.1\end{array}$ & $\begin{array}{r}2.5 \\
6.7 \\
9.5 \\
12.6\end{array}$ & $\begin{array}{r}2.5 \\
20.4 \\
25.1 \\
25.0\end{array}$ & $\begin{array}{l}12.6 \\
42.4 \\
63.2 \\
13.6\end{array}$ & $\begin{array}{r}0.6 \\
0.6 \\
0.5 \\
10.4\end{array}$ & $\begin{array}{r}0 \\
7 \\
1 \\
10\end{array}$ & $\begin{array}{r}0 \\
20 \\
0 \\
0\end{array}$ \\
\hline $\begin{array}{l}\text { LINE } \\
\text { A } \\
\text { B } \\
\text { C } \\
\text { D } \\
\text { E } \\
\text { F } \\
\text { G } \\
\text { H } \\
\text { I }\end{array}$ & $\begin{array}{r}20050 \\
398.6 \\
351.5 \\
313.6 \\
295.3 \\
263.2 \\
251.7 \\
246.3 \\
180.3 \\
161.3\end{array}$ & $\begin{array}{r}\text { S } \\
\text { S } \\
\text { S } \\
\text { S } \\
\text { S? } \\
\text { M } \\
\text { S? } \\
\text { S } \\
\text { S }\end{array}$ & $\begin{array}{l}649679 \\
649629 \\
649570 \\
649590 \\
649576 \\
649536 \\
649522 \\
649457 \\
649452\end{array}$ & $\begin{array}{l}6164278 \\
6165722 \\
6166316 \\
6166842 \\
6167813 \\
6168130 \\
6168274 \\
6170367 \\
6171021\end{array}$ & $\begin{array}{r}2.3 \\
2.9 \\
0.9 \\
0.6 \\
6.4 \\
1.9 \\
54.1 \\
1.5 \\
2.0\end{array}$ & $\begin{array}{r}3.2 \\
10.3 \\
1.9 \\
1.7 \\
3.3 \\
0.0 \\
5.9 \\
6.2 \\
2.8\end{array}$ & $\begin{array}{r}1.0 \\
2.1 \\
0.5 \\
0.7 \\
16.9 \\
10.1 \\
104.4 \\
0.9 \\
2.1\end{array}$ & $\begin{array}{r}4.0 \\
11.2 \\
3.1 \\
4.0 \\
5.6 \\
11.4 \\
11.0 \\
9.4 \\
4.2\end{array}$ & $\begin{array}{r}11.8 \\
34.0 \\
7.6 \\
6.4 \\
18.5 \\
9.7 \\
87.8 \\
6.5 \\
17.2\end{array}$ & $\begin{array}{l}17.9 \\
58.8 \\
18.1 \\
27.1 \\
30.5 \\
84.6 \\
70.8 \\
68.3 \\
20.5\end{array}$ & $\begin{array}{l}2.1 \\
1.7 \\
--- \\
--- \\
--- \\
--- \\
--- \\
1.0 \\
---\end{array}$ & $\begin{array}{r}56 \\
0 \\
--- \\
--- \\
--- \\
--- \\
--- \\
3 \\
---\end{array}$ & $\begin{array}{r}3 \\
21 \\
21 \\
0 \\
0 \\
51 \\
0 \\
0 \\
48\end{array}$ \\
\hline $\begin{array}{l}\text { LINE } \\
\text { A } \\
\text { B } \\
\text { C } \\
\text { D }\end{array}$ & $\begin{array}{l}20060 \\
9811.1 \\
9828.1 \\
9856.2 \\
9870.1\end{array}$ & $\begin{array}{l}\text { B } \\
\text { S } \\
\text { S } \\
\text { S }\end{array}$ & $\begin{array}{l}650083 \\
650052 \\
650064 \\
650045\end{array}$ & $\begin{array}{l}6163984 \\
6164442 \\
6165359 \\
6165795\end{array}$ & $\begin{array}{l}1.1 \\
0.0 \\
0.7 \\
1.2\end{array}$ & $\begin{array}{l}6.3 \\
1.5 \\
2.2 \\
5.4\end{array}$ & $\begin{array}{l}0.5 \\
0.7 \\
0.2 \\
1.2\end{array}$ & $\begin{array}{l}6.6 \\
3.3 \\
2.1 \\
6.7\end{array}$ & $\begin{array}{r}18.7 \\
12.9 \\
2.9 \\
17.1\end{array}$ & $\begin{array}{r}35.8 \\
6.7 \\
14.7 \\
35.1\end{array}$ & $\begin{array}{l}0.7 \\
--- \\
--- \\
1.1\end{array}$ & $\begin{array}{r}0 \\
--- \\
--- \\
0\end{array}$ & $\begin{array}{r}0 \\
0 \\
0 \\
45\end{array}$ \\
\hline
\end{tabular}

$\mathrm{CX}=$ COAXIAL

Note: EM values shown above

are local amplitudes

*Estimated Depth may be unreliable because the stronger part of the conductor may be deeper or to one side of the flight line, or because of a shallow dip or magnetite/overburden effects 
EM Anomaly List

\begin{tabular}{|c|c|c|c|c|c|c|c|c|c|c|c|c|c|}
\hline Label & Fid & Interp & $\underset{\mathrm{m}}{\mathrm{XUTM}}$ & $\begin{array}{c}\text { YUTM } \\
\mathrm{m}\end{array}$ & $\begin{array}{l}\text { CX } \\
\text { Real } \\
\text { ppm }\end{array}$ & $\begin{array}{c}900 \mathrm{HZ} \\
\text { Quad } \\
\text { ppm }\end{array}$ & $\begin{array}{l}\text { CP } \\
\text { Real } \\
\text { ppm }\end{array}$ & $\begin{array}{c}900 \mathrm{HZ} \\
\text { Quad } \\
\text { ppm }\end{array}$ & $\begin{array}{l}\text { CP }{ }^{7} \\
\text { Real } \\
\text { ppm }\end{array}$ & $\begin{array}{l}200 \mathrm{HZ} \\
\text { Quad } \\
\text { ppm }\end{array}$ & $\begin{array}{l}\text { Vertica } \\
\text { COND } \\
\text { siemens }\end{array}$ & $\begin{array}{c}\text { al Dike } \\
\text { DEPTH* } \\
\text { m }\end{array}$ & $\begin{array}{c}\text { Mag. Corr } \\
\text { NT }\end{array}$ \\
\hline $\begin{array}{l}\text { LINE } \\
\text { E } \\
F \\
\text { G } \\
H \\
I\end{array}$ & $\begin{array}{l}20060 \\
9899.8 \\
9912.7 \\
9933.8 \\
9974.0 \\
10010.1\end{array}$ & $\begin{array}{l}\mathrm{S} \\
\mathrm{S} \\
\mathrm{S} \\
\mathrm{M} \\
\mathrm{S}\end{array}$ & $\begin{array}{l}650000 \\
650010 \\
649961 \\
649937 \\
649908\end{array}$ & $\begin{array}{l}6166746 \\
6167160 \\
6167883 \\
6169381 \\
6170718\end{array}$ & $\begin{array}{l}0.2 \\
0.9 \\
9.9 \\
0.0 \\
0.8\end{array}$ & $\begin{array}{l}3.2 \\
3.4 \\
4.9 \\
0.5 \\
2.6\end{array}$ & $\begin{array}{r}0.8 \\
2.0 \\
16.6 \\
6.8 \\
1.7\end{array}$ & $\begin{array}{l}5.9 \\
4.9 \\
8.0 \\
0.7 \\
2.4\end{array}$ & $\begin{array}{r}9.9 \\
13.2 \\
15.6 \\
6.5 \\
3.8\end{array}$ & $\begin{array}{r}30.4 \\
39.0 \\
57.0 \\
5.0 \\
18.0\end{array}$ & $\begin{array}{l}--- \\
1.7 \\
--- \\
--- \\
---\end{array}$ & $\begin{array}{r}--- \\
23 \\
--- \\
--- \\
---\end{array}$ & $\begin{array}{r}45 \\
0 \\
0 \\
272 \\
41\end{array}$ \\
\hline $\begin{array}{l}\text { LINE } \\
\text { A } \\
\text { B } \\
\text { C } \\
\text { D } \\
\text { E } \\
\text { F } \\
\text { G } \\
\text { H } \\
\text { I } \\
\text { J } \\
\text { K } \\
\text { L }\end{array}$ & $\begin{array}{r}20070 \\
9654.5 \\
9637.0 \\
9634.2 \\
9590.3 \\
9569.0 \\
9545.6 \\
9531.0 \\
9516.0 \\
9488.0 \\
9454.0 \\
9440.4 \\
9428.2\end{array}$ & $\begin{array}{r}\text { S } \\
\text { B } \\
\text { S? } \\
\text { S? } \\
\text { S } \\
\text { S } \\
\text { S } \\
\text { S } \\
\text { S } \\
\text { M } \\
\text { S } \\
\text { S }\end{array}$ & $\begin{array}{l}650538 \\
650503 \\
650500 \\
650429 \\
650434 \\
650372 \\
650353 \\
650367 \\
650349 \\
650304 \\
650275 \\
650287\end{array}$ & $\begin{array}{l}6163401 \\
6164023 \\
6164129 \\
6165616 \\
6166378 \\
6167188 \\
6167711 \\
6168189 \\
6168911 \\
6170015 \\
6170479 \\
6170876\end{array}$ & $\begin{array}{r}0.4 \\
5.3 \\
3.8 \\
0.6 \\
1.9 \\
1.6 \\
3.5 \\
0.5 \\
0.1 \\
3.4 \\
19.0 \\
0.7\end{array}$ & $\begin{array}{l}0.6 \\
7.0 \\
6.0 \\
6.0 \\
6.1 \\
5.1 \\
1.8 \\
1.0 \\
1.5 \\
1.2 \\
1.2 \\
4.9\end{array}$ & $\begin{array}{r}0.6 \\
2.1 \\
2.1 \\
1.4 \\
0.4 \\
0.4 \\
28.0 \\
0.7 \\
12.5 \\
1.0 \\
29.8 \\
1.3\end{array}$ & $\begin{array}{r}1.4 \\
7.9 \\
7.9 \\
11.2 \\
8.7 \\
7.4 \\
2.2 \\
1.7 \\
1.8 \\
1.3 \\
1.4 \\
8.1\end{array}$ & $\begin{array}{r}0.2 \\
24.6 \\
24.6 \\
35.6 \\
16.2 \\
2.8 \\
8.0 \\
0.8 \\
12.0 \\
0.7 \\
30.2 \\
20.4\end{array}$ & $\begin{array}{r}10.6 \\
25.1 \\
22.7 \\
64.6 \\
59.5 \\
56.9 \\
16.0 \\
12.6 \\
13.0 \\
8.5 \\
10.7 \\
53.6\end{array}$ & $\begin{array}{l}--- \\
--- \\
--- \\
0.6 \\
1.2 \\
1.4 \\
--- \\
--- \\
--- \\
--- \\
--- \\
---\end{array}$ & $\begin{array}{r}--- \\
--- \\
--- \\
0 \\
5 \\
4 \\
--- \\
--- \\
--- \\
--- \\
--- \\
---\end{array}$ & $\begin{array}{r}0 \\
0 \\
0 \\
0 \\
0 \\
88 \\
137 \\
0 \\
137 \\
2618 \\
153 \\
0\end{array}$ \\
\hline $\begin{array}{l}\text { LINE } \\
\text { A } \\
\text { B } \\
\text { C } \\
\text { D } \\
\text { E } \\
\text { F } \\
\text { G } \\
\text { H } \\
\text { I } \\
\text { J } \\
\text { K } \\
\text { L }\end{array}$ & $\begin{array}{r}20080 \\
9150.5 \\
9185.0 \\
9193.9 \\
9213.3 \\
9220.4 \\
9286.0 \\
9297.6 \\
9304.0 \\
9332.0 \\
9344.0 \\
9344.2 \\
9375.4\end{array}$ & $\begin{array}{r}B ? \\
E \\
S ? \\
S \\
S \\
\text { M } \\
\text { S } \\
\text { M } \\
\text { S? } \\
\text { S? } \\
\text { S? } \\
\text { S }\end{array}$ & $\begin{array}{l}650913 \\
650847 \\
650830 \\
650833 \\
650837 \\
650752 \\
650742 \\
650746 \\
650731 \\
650693 \\
650692 \\
650746\end{array}$ & $\begin{array}{l}6163831 \\
6164848 \\
6165143 \\
6165853 \\
6166083 \\
6168089 \\
6168456 \\
6168669 \\
6169151 \\
6169440 \\
6169444 \\
6170327\end{array}$ & $\begin{array}{r}0.9 \\
2.9 \\
2.0 \\
1.4 \\
0.1 \\
1.4 \\
30.6 \\
2.4 \\
0.1 \\
3.8 \\
4.2 \\
0.1\end{array}$ & $\begin{array}{l}2.7 \\
5.0 \\
4.1 \\
4.4 \\
3.3 \\
0.8 \\
1.4 \\
0.8 \\
1.5 \\
1.4 \\
1.4 \\
1.0\end{array}$ & $\begin{array}{r}1.0 \\
0.5 \\
0.7 \\
1.5 \\
0.5 \\
8.9 \\
70.4 \\
8.6 \\
0.8 \\
1.8 \\
3.0 \\
0.4\end{array}$ & $\begin{array}{l}2.4 \\
7.0 \\
7.7 \\
6.1 \\
6.8 \\
0.2 \\
2.1 \\
0.9 \\
1.5 \\
1.2 \\
1.2 \\
1.1\end{array}$ & $\begin{array}{r}4.7 \\
19.9 \\
29.5 \\
6.8 \\
11.2 \\
8.8 \\
67.4 \\
8.1 \\
1.0 \\
1.0 \\
2.2 \\
0.5\end{array}$ & $\begin{array}{r}12.3 \\
40.9 \\
44.7 \\
47.3 \\
50.7 \\
3.9 \\
18.7 \\
11.0 \\
12.5 \\
10.7 \\
10.7 \\
7.7\end{array}$ & $\begin{array}{l}--- \\
--- \\
1.6 \\
1.6 \\
--- \\
--- \\
--- \\
--- \\
--- \\
--- \\
--- \\
---\end{array}$ & $\begin{array}{r}--- \\
--- \\
10 \\
22 \\
---- \\
--- \\
--- \\
--- \\
--- \\
--- \\
--- \\
---\end{array}$ & $\begin{array}{r}0 \\
0 \\
0 \\
0 \\
0 \\
2230 \\
0 \\
1745 \\
0 \\
1193 \\
1193 \\
0\end{array}$ \\
\hline $\begin{array}{l}\text { LINE } \\
\text { A } \\
\text { B } \\
\text { C } \\
\text { D } \\
\text { E } \\
\text { F } \\
\text { G } \\
\text { H }\end{array}$ & $\begin{array}{l}20090 \\
8871.1 \\
8817.0 \\
8802.6 \\
8783.0 \\
8760.2 \\
8713.0 \\
8703.4 \\
8677.9\end{array}$ & $\begin{array}{r}\text { S? } \\
\text { S } \\
\text { B? } \\
\text { M } \\
\text { M } \\
\text { M } \\
\text { S } \\
\text { S }\end{array}$ & $\begin{array}{l}651318 \\
651274 \\
651245 \\
651230 \\
651214 \\
651165 \\
651145 \\
651123\end{array}$ & $\begin{array}{l}6164720 \\
6166391 \\
6166760 \\
6167130 \\
6167825 \\
6168958 \\
6169296 \\
6170186\end{array}$ & $\begin{array}{r}1.8 \\
5.9 \\
4.2 \\
5.3 \\
0.0 \\
0.1 \\
26.3 \\
0.0\end{array}$ & $\begin{array}{l}3.4 \\
3.0 \\
4.6 \\
0.3 \\
1.2 \\
1.8 \\
5.2 \\
1.2\end{array}$ & $\begin{array}{r}0.6 \\
9.2 \\
28.6 \\
7.9 \\
18.1 \\
0.7 \\
41.1 \\
0.5\end{array}$ & $\begin{array}{l}2.3 \\
3.5 \\
4.5 \\
0.1 \\
1.1 \\
4.2 \\
9.1 \\
1.6\end{array}$ & $\begin{array}{r}6.5 \\
12.3 \\
27.6 \\
7.3 \\
16.8 \\
0.4 \\
61.3 \\
0.1\end{array}$ & $\begin{array}{r}12.9 \\
31.1 \\
36.9 \\
5.6 \\
8.9 \\
25.8 \\
61.2 \\
10.5\end{array}$ & $\begin{array}{l}--- \\
--- \\
--- \\
--- \\
--- \\
--- \\
--- \\
---\end{array}$ & $\begin{array}{l}--- \\
--- \\
--- \\
--- \\
--- \\
--- \\
--- \\
---\end{array}$ & $\begin{array}{r}197 \\
0 \\
1787 \\
0 \\
0 \\
0 \\
197 \\
197\end{array}$ \\
\hline $\begin{array}{l}\text { LINE } \\
\mathrm{A}\end{array}$ & $\begin{array}{l}20100 \\
8372.6\end{array}$ & $S$ & 651665 & 6164238 & 1.1 & 1.3 & 0.6 & 1.9 & 6.5 & 8.7 & --- & --- & 148 \\
\hline
\end{tabular}

$\mathrm{CX}=$ COAXIAL

Note: EM values shown above

Ketchikan - Salt Chuck Block
$121-$
* Estimated Depth may be unreliable because the stronger part of the conductor may be deeper or to one side of the flight line, or because of a shallow dip or magnetite/overburden effects 
EM Anomaly List

\begin{tabular}{|c|c|c|c|c|c|c|c|c|c|c|c|c|c|}
\hline Label & Fid & Interp & $\begin{array}{c}\text { XUTM } \\
\text { m }\end{array}$ & $\begin{array}{c}\text { YUTM } \\
\mathrm{m}\end{array}$ & $\begin{array}{l}\text { CX } \\
\text { Real } \\
\text { ppm }\end{array}$ & $\begin{array}{l}00 \mathrm{HZ} \\
\text { Quad } \\
\text { ppm }\end{array}$ & $\begin{array}{l}\text { CP } \\
\text { Real } \\
\text { ppm }\end{array}$ & $\begin{array}{l}900 \mathrm{HZ} \\
\text { Quad } \\
\text { ppm }\end{array}$ & $\begin{array}{l}\text { CP } 7 \\
\text { Real } \\
\text { ppm }\end{array}$ & $\begin{array}{l}200 \mathrm{HZ} \\
\text { Quad } \\
\text { ppm }\end{array}$ & $\begin{array}{l}\text { Vertica } \\
\text { ConD } \\
\text { siemens }\end{array}$ & $\begin{array}{c}\text { al Dike } \\
\text { DEPTH* } \\
\text { m }\end{array}$ & $\begin{array}{c}\text { Mag. Corr } \\
\text { NT }\end{array}$ \\
\hline $\begin{array}{l}\text { LINE } \\
\text { B } \\
\text { C } \\
\text { D } \\
\text { E } \\
\text { F } \\
\text { G } \\
\text { H } \\
\text { I }\end{array}$ & $\begin{array}{r}0100 \\
8397.5 \\
8425.8 \\
8444.6 \\
8466.5 \\
8479.4 \\
8526.1 \\
8535.6 \\
8542.4\end{array}$ & $\begin{array}{l}\mathrm{S} \\
\mathrm{S} \\
\mathrm{S} \\
\mathrm{S} \\
\mathrm{M} \\
\mathrm{M} \\
\mathrm{S} \\
\mathrm{S}\end{array}$ & $\begin{array}{l}651702 \\
651619 \\
651608 \\
651617 \\
651602 \\
651577 \\
651581 \\
651568\end{array}$ & $\begin{array}{l}6164990 \\
6165752 \\
6166262 \\
6166907 \\
6167336 \\
6168585 \\
6168833 \\
6169018\end{array}$ & $\begin{array}{r}0.9 \\
0.1 \\
3.4 \\
3.2 \\
21.2 \\
8.2 \\
9.4 \\
15.2\end{array}$ & $\begin{array}{l}5.7 \\
2.9 \\
2.3 \\
1.9 \\
0.9 \\
0.6 \\
5.5 \\
3.5\end{array}$ & $\begin{array}{r}0.9 \\
0.1 \\
2.2 \\
7.6 \\
54.2 \\
15.0 \\
15.7 \\
26.5\end{array}$ & $\begin{array}{l}8.9 \\
2.1 \\
3.2 \\
1.8 \\
0.7 \\
0.7 \\
8.7 \\
3.9\end{array}$ & $\begin{array}{r}25.4 \\
1.5 \\
3.9 \\
6.8 \\
52.9 \\
14.6 \\
23.4 \\
15.3\end{array}$ & $\begin{array}{r}41.3 \\
17.3 \\
26.7 \\
12.8 \\
7.3 \\
4.2 \\
62.2 \\
30.1\end{array}$ & $\begin{array}{r}0.7 \\
--- \\
7.6 \\
--- \\
--- \\
--- \\
--- \\
105.5\end{array}$ & $\begin{array}{r}0 \\
--- \\
52 \\
--- \\
--- \\
--- \\
--- \\
9\end{array}$ & $\begin{array}{r}0 \\
52 \\
0 \\
137 \\
3202 \\
0 \\
0 \\
0\end{array}$ \\
\hline $\begin{array}{l}\text { LINE } \\
\text { A } \\
\text { B } \\
\text { C } \\
\text { D }\end{array}$ & $\begin{array}{r}20110 \\
8163.0 \\
8077.2 \\
8034.0 \\
8028.0\end{array}$ & $\begin{array}{l}\mathrm{S} \\
\mathrm{S} \\
\mathrm{M} \\
\mathrm{S}\end{array}$ & $\begin{array}{l}652058 \\
651967 \\
651929 \\
651908\end{array}$ & $\begin{array}{l}6164841 \\
6167414 \\
6168670 \\
6168865\end{array}$ & $\begin{array}{r}1.0 \\
10.7 \\
1.3 \\
35.7\end{array}$ & $\begin{array}{l}2.7 \\
1.5 \\
1.8 \\
8.1\end{array}$ & $\begin{array}{r}0.5 \\
24.5 \\
1.8 \\
65.0\end{array}$ & $\begin{array}{r}3.3 \\
1.9 \\
7.1 \\
11.7\end{array}$ & $\begin{array}{r}7.7 \\
25.5 \\
1.4 \\
61.7\end{array}$ & $\begin{array}{l}17.8 \\
13.5 \\
49.7 \\
87.2\end{array}$ & $\begin{array}{r}1.3 \\
--- \\
--- \\
162.9\end{array}$ & $\begin{array}{r}6 \\
--- \\
--- \\
1\end{array}$ & $\begin{array}{l}0 \\
0 \\
0 \\
0\end{array}$ \\
\hline $\begin{array}{l}\text { LINE } \\
\text { A } \\
B \\
\text { C } \\
D \\
\text { E }\end{array}$ & $\begin{array}{l}20120 \\
7674.0 \\
7732.1 \\
7804.7 \\
7826.0 \\
7850.0\end{array}$ & $\begin{array}{r}S \\
S \\
S \\
S ? \\
S\end{array}$ & $\begin{array}{l}652584 \\
652495 \\
652402 \\
652406 \\
652356\end{array}$ & $\begin{array}{l}6162883 \\
6164864 \\
6167133 \\
6167826 \\
6168522\end{array}$ & $\begin{array}{r}1.0 \\
1.0 \\
13.1 \\
13.3 \\
0.7\end{array}$ & $\begin{array}{l}5.8 \\
1.7 \\
2.6 \\
5.2 \\
8.7\end{array}$ & $\begin{array}{r}0.7 \\
0.4 \\
24.4 \\
21.6 \\
8.2\end{array}$ & $\begin{array}{r}7.8 \\
2.4 \\
3.9 \\
8.5 \\
14.4\end{array}$ & $\begin{array}{r}21.0 \\
0.5 \\
25.3 \\
18.9 \\
42.9\end{array}$ & $\begin{array}{l}43.7 \\
19.6 \\
26.7 \\
54.3 \\
82.9\end{array}$ & $\begin{array}{r}--- \\
--- \\
--- \\
43.2 \\
2.1\end{array}$ & $\begin{array}{r}--- \\
--- \\
--- \\
8 \\
0\end{array}$ & $\begin{array}{r}0 \\
85 \\
0 \\
1066 \\
85\end{array}$ \\
\hline $\begin{array}{l}\text { LINE } \\
\text { A } \\
\text { B } \\
\text { C } \\
\text { D } \\
\text { E } \\
\text { F }\end{array}$ & $\begin{array}{r}20130 \\
7518.3 \\
7502.9 \\
7474.0 \\
7457.4 \\
7328.9 \\
7257.5\end{array}$ & $\begin{array}{r}\text { S } \\
\text { S } \\
\text { S? } \\
\text { S? } \\
\text { S } \\
\text { S }\end{array}$ & $\begin{array}{l}652981 \\
652965 \\
652936 \\
652924 \\
652791 \\
652726\end{array}$ & $\begin{array}{l}6162278 \\
6162777 \\
6163492 \\
6163989 \\
6168076 \\
6170539\end{array}$ & $\begin{array}{l}5.5 \\
8.8 \\
0.4 \\
0.1 \\
0.4 \\
1.0\end{array}$ & $\begin{array}{r}12.0 \\
14.6 \\
5.6 \\
6.8 \\
1.7 \\
2.1\end{array}$ & $\begin{array}{l}4.3 \\
7.7 \\
1.5 \\
0.7 \\
2.5 \\
0.3\end{array}$ & $\begin{array}{r}17.9 \\
19.9 \\
9.2 \\
12.0 \\
2.6 \\
2.8\end{array}$ & $\begin{array}{r}62.0 \\
65.0 \\
8.7 \\
16.8 \\
3.1 \\
4.1\end{array}$ & $\begin{array}{r}54.6 \\
46.6 \\
67.6 \\
88.3 \\
8.3 \\
19.4\end{array}$ & $\begin{array}{l}2.9 \\
3.3 \\
0.6 \\
0.5 \\
--- \\
1.4\end{array}$ & $\begin{array}{r}0 \\
0 \\
0 \\
0 \\
--- \\
11\end{array}$ & $\begin{array}{r}0 \\
0 \\
73 \\
73 \\
0 \\
0\end{array}$ \\
\hline $\begin{array}{l}\text { LINE } \\
\text { A } \\
\text { B } \\
\text { C } \\
\text { D }\end{array}$ & $\begin{array}{r}20140 \\
6988.5 \\
7038.9 \\
7063.6 \\
7134.0\end{array}$ & $\begin{array}{l}\mathrm{S} \\
\mathrm{S} \\
\mathrm{M} \\
\mathrm{S}\end{array}$ & $\begin{array}{l}653247 \\
653217 \\
653134 \\
653150\end{array}$ & $\begin{array}{l}6165443 \\
6166941 \\
6167788 \\
6170034\end{array}$ & $\begin{array}{r}2.5 \\
16.8 \\
6.9 \\
1.9\end{array}$ & $\begin{array}{r}1.5 \\
14.9 \\
1.2 \\
1.9\end{array}$ & $\begin{array}{r}2.1 \\
31.9 \\
10.1 \\
1.1\end{array}$ & $\begin{array}{r}2.9 \\
34.0 \\
1.2 \\
3.1\end{array}$ & $\begin{array}{r}4.2 \\
72.3 \\
10.2 \\
2.2\end{array}$ & $\begin{array}{l}21.2 \\
18.4 \\
11.3 \\
23.1\end{array}$ & $\begin{array}{r}7.6 \\
12.6 \\
--- \\
3.1\end{array}$ & $\begin{array}{r}32 \\
0 \\
-- \\
52\end{array}$ & $\begin{array}{l}0 \\
0 \\
0 \\
0\end{array}$ \\
\hline $\begin{array}{l}\text { LINE } \\
\text { A } \\
\text { B } \\
\text { C } \\
\text { D } \\
\text { E }\end{array}$ & $\begin{array}{l}20150 \\
6797.5 \\
6790.7 \\
6704.2 \\
6668.2 \\
6654.0\end{array}$ & $\begin{array}{l}S \\
S \\
S \\
S \\
M\end{array}$ & $\begin{array}{l}653790 \\
653780 \\
653668 \\
653617 \\
653606\end{array}$ & $\begin{array}{l}6162410 \\
6162684 \\
6165772 \\
6166955 \\
6167497\end{array}$ & $\begin{array}{r}22.6 \\
7.4 \\
1.6 \\
10.5 \\
0.7\end{array}$ & $\begin{array}{r}14.0 \\
8.4 \\
2.4 \\
10.0 \\
0.5\end{array}$ & $\begin{array}{r}19.6 \\
6.0 \\
4.0 \\
10.3 \\
2.7\end{array}$ & $\begin{array}{r}27.9 \\
7.3 \\
2.0 \\
21.4 \\
1.2\end{array}$ & $\begin{array}{r}56.3 \\
23.1 \\
5.2 \\
60.0 \\
3.4\end{array}$ & $\begin{array}{r}21.7 \\
21.2 \\
14.1 \\
33.1 \\
4.2\end{array}$ & $\begin{array}{r}15.0 \\
6.7 \\
--- \\
6.3 \\
---\end{array}$ & $\begin{array}{r}0 \\
0 \\
--- \\
0 \\
---\end{array}$ & $\begin{array}{r}0 \\
0 \\
0 \\
0 \\
4035\end{array}$ \\
\hline $\begin{array}{l}\text { LINE } \\
\text { A } \\
B\end{array}$ & $\begin{array}{l}20160 \\
6251.2 \\
6315.0\end{array}$ & $\begin{array}{l}\mathrm{S} \\
\mathrm{M}\end{array}$ & $\begin{array}{l}654101 \\
653989\end{array}$ & $\begin{array}{l}6164737 \\
6167042\end{array}$ & $\begin{array}{l}3.3 \\
0.0\end{array}$ & $\begin{array}{l}2.8 \\
1.2\end{array}$ & $\begin{array}{l}10.9 \\
10.0\end{array}$ & $\begin{array}{r}3.8 \\
10.7\end{array}$ & $\begin{array}{l}16.4 \\
35.5\end{array}$ & $\begin{array}{l}20.4 \\
35.3\end{array}$ & $\begin{array}{r}19.2 \\
---\end{array}$ & $\begin{array}{r}21 \\
---\end{array}$ & $\begin{array}{r}98 \\
0\end{array}$ \\
\hline
\end{tabular}

$\mathrm{CX}=$ COAXIAL

Note: EM values shown above

are local amplitudes

* Estimated Depth may be unreliable because the stronger part of the conductor may be deeper or to one side of the flight line, or because of a shallow dip or magnetite/overburden effects 
EM Anomaly List

\begin{tabular}{|c|c|c|c|c|c|c|c|c|c|c|c|c|c|}
\hline Label & Fid & Interp & $\begin{array}{c}\text { XUTM } \\
\text { m }\end{array}$ & $\begin{array}{c}\text { YUTM } \\
\mathrm{m}\end{array}$ & $\begin{array}{l}\text { CX } \\
\text { Real } \\
\text { ppm }\end{array}$ & $\begin{array}{l}00 \mathrm{HZ} \\
\text { Quad } \\
\text { ppm }\end{array}$ & $\begin{array}{l}\text { CP } \\
\text { Real } \\
\text { ppm }\end{array}$ & $\begin{array}{l}900 \mathrm{HZ} \\
\text { Quad } \\
\text { ppm }\end{array}$ & $\begin{array}{l}\text { CP } \\
\text { Real } \\
\text { ppm }\end{array}$ & $\begin{array}{c}7200 \mathrm{HZ} \\
\text { Quad } \\
\text { ppm }\end{array}$ & $\begin{array}{l}\text { Vertica } \\
\text { ConD } \\
\text { siemens }\end{array}$ & $\begin{array}{c}\text { al Dike } \\
\text { DEPTH* } \\
\text { m }\end{array}$ & $\begin{array}{c}\text { Mag. Corr } \\
\text { NT }\end{array}$ \\
\hline $\begin{array}{l}\text { LINE } \\
\text { A } \\
B \\
\text { C } \\
\text { D } \\
\text { E }\end{array}$ & $\begin{array}{l}20170 \\
5888.0 \\
5878.4 \\
5868.0 \\
5838.6 \\
5727.0\end{array}$ & $\begin{array}{l}\mathrm{S} \\
\mathrm{M} \\
\mathrm{M} \\
\mathrm{M} \\
\mathrm{S}\end{array}$ & $\begin{array}{l}654429 \\
654425 \\
654423 \\
654389 \\
654299\end{array}$ & $\begin{array}{l}6166611 \\
6166896 \\
6167149 \\
6167539 \\
6170881\end{array}$ & $\begin{array}{r}49.8 \\
10.0 \\
2.8 \\
10.1 \\
0.5\end{array}$ & $\begin{array}{r}51.7 \\
3.9 \\
1.3 \\
5.4 \\
0.7\end{array}$ & $\begin{array}{r}71.2 \\
8.2 \\
11.6 \\
26.3 \\
0.9\end{array}$ & $\begin{array}{r}101.1 \\
6.7 \\
1.3 \\
8.0 \\
2.1\end{array}$ & $\begin{array}{r}257.3 \\
18.8 \\
10.9 \\
26.4 \\
4.1\end{array}$ & $\begin{array}{l}83.5 \\
42.1 \\
10.4 \\
62.0 \\
13.0\end{array}$ & $\begin{array}{c}12.8 \\
--- \\
--- \\
--- \\
---\end{array}$ & $\begin{array}{r}0 \\
--- \\
--- \\
--- \\
---\end{array}$ & $\begin{array}{r}0 \\
394 \\
0 \\
0 \\
0\end{array}$ \\
\hline $\begin{array}{l}\text { LINE } \\
\text { A } \\
B \\
C\end{array}$ & $\begin{array}{r}20180 \\
5465.0 \\
5494.0 \\
5600.0\end{array}$ & $\begin{array}{l}\mathrm{S} \\
\mathrm{M} \\
\mathrm{M}\end{array}$ & $\begin{array}{l}654858 \\
654832 \\
654685\end{array}$ & $\begin{array}{l}6166188 \\
6167182 \\
6170490\end{array}$ & $\begin{array}{l}3.6 \\
0.4 \\
1.9\end{array}$ & $\begin{array}{l}1.7 \\
1.6 \\
0.6\end{array}$ & $\begin{array}{l}4.7 \\
6.5 \\
5.2\end{array}$ & $\begin{array}{l}3.8 \\
1.3 \\
0.9\end{array}$ & $\begin{array}{r}11.9 \\
5.6 \\
4.8\end{array}$ & $\begin{array}{l}6.6 \\
9.1 \\
7.7\end{array}$ & $\begin{array}{l}--- \\
--- \\
---\end{array}$ & $\begin{array}{l}--- \\
--- \\
---\end{array}$ & $\begin{array}{l}0 \\
0 \\
0\end{array}$ \\
\hline $\begin{array}{l}\text { LINE } \\
\text { A } \\
B\end{array}$ & $\begin{array}{r}20190 \\
5245.9 \\
5160.0\end{array}$ & $\begin{array}{l}\mathrm{M} \\
\mathrm{S}\end{array}$ & $\begin{array}{l}655233 \\
655163\end{array}$ & $\begin{array}{l}6166930 \\
6169643\end{array}$ & $\begin{array}{l}3.5 \\
1.6\end{array}$ & $\begin{array}{l}0.2 \\
1.3\end{array}$ & $\begin{array}{l}8.6 \\
1.8\end{array}$ & $\begin{array}{l}0.1 \\
1.1\end{array}$ & $\begin{array}{l}8.4 \\
1.5\end{array}$ & $\begin{array}{r}5.5 \\
11.4\end{array}$ & $\begin{array}{l}--- \\
8.3\end{array}$ & $\begin{array}{r}--- \\
46\end{array}$ & $\begin{array}{r}639 \\
0\end{array}$ \\
\hline $\begin{array}{l}\text { LINE } \\
\text { A } \\
B \\
C\end{array}$ & $\begin{array}{r}20200 \\
4906.2 \\
4925.0 \\
5000.2\end{array}$ & $\begin{array}{l}\mathrm{M} \\
\mathrm{M} \\
\mathrm{S}\end{array}$ & $\begin{array}{l}655594 \\
655602 \\
655514\end{array}$ & $\begin{array}{l}6167669 \\
6168139 \\
6170364\end{array}$ & $\begin{array}{l}5.6 \\
3.5 \\
2.2\end{array}$ & $\begin{array}{l}0.4 \\
0.5 \\
1.5\end{array}$ & $\begin{array}{r}24.0 \\
12.2 \\
1.7\end{array}$ & $\begin{array}{l}0.1 \\
0.2 \\
2.2\end{array}$ & $\begin{array}{r}23.3 \\
12.0 \\
3.3\end{array}$ & $\begin{array}{r}2.5 \\
2.3 \\
11.9\end{array}$ & $\begin{array}{l}--- \\
--- \\
---\end{array}$ & $\begin{array}{l}--- \\
--- \\
---\end{array}$ & $\begin{array}{r}220 \\
82 \\
67\end{array}$ \\
\hline $\begin{array}{l}\text { LINE } \\
\text { A } \\
\text { B } \\
\text { C } \\
\text { D }\end{array}$ & $\begin{array}{r}20210 \\
4556.0 \\
4516.3 \\
4449.5 \\
4415.6\end{array}$ & $\begin{array}{l}\mathrm{S} \\
\mathrm{M} \\
\mathrm{S} \\
\mathrm{S}\end{array}$ & $\begin{array}{l}655997 \\
656012 \\
655948 \\
655958\end{array}$ & $\begin{array}{l}6166506 \\
6167314 \\
6168909 \\
6170081\end{array}$ & $\begin{array}{l}1.6 \\
2.1 \\
0.3 \\
1.6\end{array}$ & $\begin{array}{l}1.1 \\
0.5 \\
2.1 \\
8.6\end{array}$ & $\begin{array}{l}1.6 \\
5.6 \\
5.8 \\
0.8\end{array}$ & $\begin{array}{r}0.9 \\
0.2 \\
2.7 \\
13.8\end{array}$ & $\begin{array}{r}1.8 \\
6.4 \\
8.5 \\
33.8\end{array}$ & $\begin{array}{r}7.7 \\
2.4 \\
18.6 \\
88.5\end{array}$ & $\begin{array}{l}--- \\
--- \\
--- \\
0.8\end{array}$ & $\begin{array}{r}--- \\
--- \\
-- \\
0\end{array}$ & $\begin{array}{l}0 \\
0 \\
0 \\
0\end{array}$ \\
\hline $\begin{array}{l}\text { LINE } \\
\text { A } \\
B\end{array}$ & $\begin{array}{l}20220 \\
4275.2 \\
4279.7\end{array}$ & $\begin{array}{l}S \\
S\end{array}$ & $\begin{array}{l}656375 \\
656355\end{array}$ & $\begin{array}{l}6169252 \\
6169421\end{array}$ & $\begin{array}{l}2.1 \\
2.7\end{array}$ & $\begin{array}{r}8.6 \\
13.7\end{array}$ & $\begin{array}{l}1.1 \\
3.9\end{array}$ & $\begin{array}{l}14.0 \\
23.7\end{array}$ & $\begin{array}{l}18.8 \\
53.0\end{array}$ & $\begin{array}{l}102.3 \\
149.9\end{array}$ & $\begin{array}{l}--- \\
1.4\end{array}$ & $\begin{array}{r}--- \\
1\end{array}$ & $\begin{array}{l}45 \\
45\end{array}$ \\
\hline $\begin{array}{l}\text { LINE } \\
\text { A } \\
\text { B } \\
\text { C } \\
\text { D }\end{array}$ & $\begin{array}{r}20230 \\
4044.0 \\
3996.6 \\
3919.8 \\
3870.7\end{array}$ & $\begin{array}{r}\text { S? } \\
\text { S } \\
\text { S } \\
\text { S }\end{array}$ & $\begin{array}{l}656886 \\
656878 \\
656772 \\
656691\end{array}$ & $\begin{array}{l}6164716 \\
6166238 \\
6169167 \\
6170924\end{array}$ & $\begin{array}{l}2.4 \\
3.0 \\
2.9 \\
1.7\end{array}$ & $\begin{array}{l}1.7 \\
0.5 \\
3.8 \\
3.2\end{array}$ & $\begin{array}{r}2.0 \\
20.1 \\
0.7 \\
1.7\end{array}$ & $\begin{array}{l}1.9 \\
1.0 \\
5.4 \\
5.1\end{array}$ & $\begin{array}{r}5.6 \\
18.6 \\
11.8 \\
15.6\end{array}$ & $\begin{array}{r}7.8 \\
9.3 \\
38.5 \\
23.3\end{array}$ & $\begin{array}{l}8.3 \\
--- \\
2.8 \\
2.3\end{array}$ & $\begin{array}{r}41 \\
--- \\
25 \\
12\end{array}$ & $\begin{array}{l}0 \\
0 \\
0 \\
0\end{array}$ \\
\hline $\begin{array}{l}\text { LINE } \\
\text { A } \\
\text { B } \\
\text { C } \\
\text { D } \\
\text { E } \\
\text { F }\end{array}$ & $\begin{array}{l}20240 \\
3682.5 \\
3701.9 \\
3707.3 \\
3732.2 \\
3743.4 \\
3798.4\end{array}$ & $\begin{array}{l}S \\
S \\
S \\
S \\
S \\
S\end{array}$ & $\begin{array}{l}657243 \\
657209 \\
657216 \\
657240 \\
657231 \\
657123\end{array}$ & $\begin{array}{l}6166475 \\
6167189 \\
6167407 \\
6168242 \\
6168591 \\
6170287\end{array}$ & $\begin{array}{l}1.6 \\
0.2 \\
3.6 \\
0.9 \\
2.7 \\
2.3\end{array}$ & $\begin{array}{l}0.7 \\
4.7 \\
3.4 \\
1.1 \\
0.7 \\
2.7\end{array}$ & $\begin{array}{l}0.7 \\
1.4 \\
0.9 \\
1.3 \\
1.3 \\
0.8\end{array}$ & $\begin{array}{l}0.6 \\
7.9 \\
5.4 \\
0.9 \\
0.1 \\
5.6\end{array}$ & $\begin{array}{r}0.5 \\
13.6 \\
9.1 \\
2.3 \\
0.8 \\
6.6\end{array}$ & $\begin{array}{r}4.9 \\
54.6 \\
40.8 \\
8.2 \\
1.1 \\
43.5\end{array}$ & $\begin{array}{l}--- \\
--- \\
3.3 \\
--- \\
--- \\
2.6\end{array}$ & $\begin{array}{r}--- \\
--- \\
39 \\
--- \\
--- \\
29\end{array}$ & $\begin{array}{r}27 \\
27 \\
0 \\
27 \\
0 \\
0\end{array}$ \\
\hline
\end{tabular}

$\mathrm{CX}=$ COAXIAL

$\mathrm{CP}=$ COPLANAR Note $: \mathrm{EM}$ values shown above

Ketchikan - Salt Chuck Block
*Estimated Depth may be unreliable because the stronger part of the conductor may be deeper or to one side of the flight line, or because 
EM Anomaly List

\begin{tabular}{|c|c|c|c|c|c|c|c|c|c|c|c|c|c|}
\hline Label & Fid & Interp & $\begin{array}{c}\text { XUTM } \\
\text { m }\end{array}$ & $\begin{array}{c}\text { YUTM } \\
\mathrm{m}\end{array}$ & $\begin{array}{l}\text { CX } \\
\text { Real } \\
\text { ppm }\end{array}$ & $\begin{array}{l}00 \mathrm{HZ} \\
\text { Quad } \\
\text { ppm }\end{array}$ & $\begin{array}{l}\text { CP } \\
\text { Real } \\
\text { ppm }\end{array}$ & $\begin{array}{l}900 \mathrm{HZ} \\
\text { Quad } \\
\text { ppm }\end{array}$ & $\begin{array}{l}\text { CP } 7 \\
\text { Real } \\
\text { ppm }\end{array}$ & $\begin{array}{c}7200 \mathrm{HZ} \\
\text { Quad } \\
\text { ppm }\end{array}$ & $\begin{array}{l}\text { Vertica } \\
\text { ConD } \\
\text { siemens }\end{array}$ & $\begin{array}{c}\text { al Dike } \\
\text { DEPTH* } \\
\text { m }\end{array}$ & $\begin{array}{c}\text { Mag. Corr } \\
\text { NT }\end{array}$ \\
\hline $\begin{array}{l}\text { LINE } \\
\text { A } \\
\text { B } \\
\text { C } \\
\text { D } \\
\text { E } \\
\text { F }\end{array}$ & $\begin{array}{r}20250 \\
3533.7 \\
3476.6 \\
3456.0 \\
3422.0 \\
3414.3 \\
3387.7\end{array}$ & $\begin{array}{l}S \\
S \\
S \\
S \\
S \\
S\end{array}$ & $\begin{array}{l}657822 \\
657688 \\
657661 \\
657668 \\
657645 \\
657576\end{array}$ & $\begin{array}{l}6163220 \\
6165466 \\
6166216 \\
6167431 \\
6167715 \\
6168627\end{array}$ & $\begin{array}{l}3.0 \\
0.6 \\
0.6 \\
0.3 \\
0.5 \\
0.0\end{array}$ & $\begin{array}{l}6.7 \\
1.7 \\
3.1 \\
1.6 \\
1.7 \\
1.3\end{array}$ & $\begin{array}{l}4.1 \\
2.3 \\
1.9 \\
0.8 \\
1.5 \\
1.5\end{array}$ & $\begin{array}{r}12.6 \\
1.8 \\
5.7 \\
2.7 \\
2.6 \\
1.6\end{array}$ & $\begin{array}{r}34.1 \\
3.1 \\
9.1 \\
4.2 \\
9.0 \\
1.2\end{array}$ & $\begin{array}{r}16.0 \\
12.1 \\
44.4 \\
14.8 \\
12.8 \\
9.6\end{array}$ & $\begin{array}{l}2.6 \\
--- \\
--- \\
--- \\
1.7 \\
---\end{array}$ & $\begin{array}{r}0 \\
--- \\
--- \\
-- \\
33 \\
---\end{array}$ & $\begin{array}{r}0 \\
25 \\
25 \\
25 \\
25 \\
0\end{array}$ \\
\hline $\begin{array}{l}\text { LINE } \\
\text { A } \\
\text { B } \\
\text { C } \\
\text { D }\end{array}$ & $\begin{array}{c}20260 \\
3004.2 \\
3021.4 \\
3067.2 \\
3146.0\end{array}$ & $\begin{array}{l}\mathrm{S} \\
\mathrm{S} \\
\mathrm{S} \\
\mathrm{S}\end{array}$ & $\begin{array}{l}658205 \\
658185 \\
658132 \\
658068\end{array}$ & $\begin{array}{l}6162747 \\
6163381 \\
6164987 \\
6167391\end{array}$ & $\begin{array}{r}25.4 \\
0.8 \\
0.0 \\
0.5\end{array}$ & $\begin{array}{r}61.9 \\
4.1 \\
1.3 \\
1.1\end{array}$ & $\begin{array}{r}29.5 \\
2.6 \\
2.1 \\
0.4\end{array}$ & $\begin{array}{r}104.4 \\
8.2 \\
1.2 \\
1.6\end{array}$ & $\begin{array}{r}324.1 \\
32.6 \\
2.6 \\
1.3\end{array}$ & $\begin{array}{r}173.2 \\
27.9 \\
6.3 \\
6.4\end{array}$ & $\begin{array}{l}4.8 \\
--- \\
--- \\
---\end{array}$ & $\begin{array}{r}0 \\
--- \\
--- \\
---\end{array}$ & $\begin{array}{r}27 \\
3 \\
550 \\
0\end{array}$ \\
\hline $\begin{array}{l}\text { LINE } \\
\text { A } \\
\text { B } \\
\text { C } \\
\text { D }\end{array}$ & $\begin{array}{r}20270 \\
2826.5 \\
2768.9 \\
2666.9 \\
2636.9\end{array}$ & $\begin{array}{l}\mathrm{S} \\
\mathrm{S} \\
\mathrm{S} \\
\mathrm{S}\end{array}$ & $\begin{array}{l}658595 \\
658516 \\
658374 \\
658411\end{array}$ & $\begin{array}{l}6162834 \\
6164910 \\
6168548 \\
6169629\end{array}$ & $\begin{array}{l}3.0 \\
1.8 \\
0.5 \\
8.0\end{array}$ & $\begin{array}{r}7.8 \\
1.0 \\
9.5 \\
15.8\end{array}$ & $\begin{array}{r}3.8 \\
2.5 \\
0.3 \\
14.4\end{array}$ & $\begin{array}{r}15.3 \\
2.1 \\
18.0 \\
32.8\end{array}$ & $\begin{array}{r}53.1 \\
4.7 \\
45.9 \\
88.1\end{array}$ & $\begin{array}{r}41.8 \\
14.7 \\
122.0 \\
65.8\end{array}$ & $\begin{array}{l}2.2 \\
--- \\
0.5 \\
4.4\end{array}$ & $\begin{array}{r}1 \\
--- \\
0 \\
0\end{array}$ & $\begin{array}{r}0 \\
0 \\
23 \\
0\end{array}$ \\
\hline $\begin{array}{l}\text { LINE } \\
\text { A } \\
\text { B } \\
\text { C }\end{array}$ & $\begin{array}{r}20280 \\
2266.9 \\
2308.5 \\
2449.9\end{array}$ & $\begin{array}{l}\mathrm{S} \\
\mathrm{S} \\
\mathrm{S}\end{array}$ & $\begin{array}{l}659065 \\
658932 \\
658843\end{array}$ & $\begin{array}{l}6160946 \\
6162509 \\
6167687\end{array}$ & $\begin{array}{r}28.2 \\
1.4 \\
0.7\end{array}$ & $\begin{array}{r}29.9 \\
2.0 \\
9.4\end{array}$ & $\begin{array}{r}47.6 \\
1.2 \\
1.6\end{array}$ & $\begin{array}{r}60.5 \\
1.8 \\
18.2\end{array}$ & $\begin{array}{r}153.5 \\
2.9 \\
58.0\end{array}$ & $\begin{array}{r}81.7 \\
13.0 \\
110.4\end{array}$ & $\begin{array}{r}11.4 \\
--- \\
0.6\end{array}$ & $\begin{array}{r}0 \\
--- \\
0\end{array}$ & $\begin{array}{r}0 \\
13 \\
0\end{array}$ \\
\hline $\begin{array}{l}\text { LINE } \\
\text { A } \\
\text { B } \\
\text { C } \\
\text { D }\end{array}$ & $\begin{array}{r}20290 \\
2228.5 \\
2220.5 \\
2205.6 \\
2097.0\end{array}$ & $\begin{array}{l}M \\
S \\
S \\
S\end{array}$ & $\begin{array}{l}659458 \\
659445 \\
659424 \\
659272\end{array}$ & $\begin{array}{l}6161063 \\
6161401 \\
6161994 \\
6166077\end{array}$ & $\begin{array}{l}0.0 \\
5.7 \\
1.2 \\
3.2\end{array}$ & $\begin{array}{l}0.0 \\
2.6 \\
2.4 \\
2.0\end{array}$ & $\begin{array}{l}6.7 \\
5.0 \\
0.3 \\
1.1\end{array}$ & $\begin{array}{l}5.3 \\
3.7 \\
1.9 \\
1.1\end{array}$ & $\begin{array}{r}7.5 \\
12.3 \\
2.9 \\
1.1\end{array}$ & $\begin{array}{l}50.0 \\
28.5 \\
12.0 \\
12.0\end{array}$ & $\begin{array}{l}--- \\
--- \\
--- \\
9.9\end{array}$ & $\begin{array}{r}--- \\
--- \\
--- \\
49\end{array}$ & $\begin{array}{r}0 \\
0 \\
0 \\
37\end{array}$ \\
\hline $\begin{array}{l}\text { LINE } \\
A \\
B\end{array}$ & $\begin{array}{l}20300 \\
1707.2 \\
1774.1\end{array}$ & $\begin{array}{l}S \\
S\end{array}$ & $\begin{array}{l}659757 \\
659664\end{array}$ & $\begin{array}{l}6163813 \\
6166604\end{array}$ & $\begin{array}{l}0.9 \\
1.7\end{array}$ & $\begin{array}{l}1.8 \\
1.5\end{array}$ & $\begin{array}{l}1.5 \\
2.0\end{array}$ & $\begin{array}{l}3.6 \\
1.7\end{array}$ & $\begin{array}{l}7.0 \\
5.3\end{array}$ & $\begin{array}{l}25.1 \\
11.0\end{array}$ & $\begin{array}{l}--- \\
---\end{array}$ & $\begin{array}{l}--- \\
---\end{array}$ & $\begin{array}{r}44 \\
0\end{array}$ \\
\hline $\begin{array}{l}\text { LINE } \\
\text { A } \\
\text { B } \\
\text { C } \\
\text { D } \\
\text { E }\end{array}$ & $\begin{array}{l}20310 \\
1516.4 \\
1433.1 \\
1414.2 \\
1367.1 \\
1212.3\end{array}$ & $\begin{array}{l}\text { S } \\
\text { S } \\
\text { S } \\
\text { S } \\
\text { S }\end{array}$ & $\begin{array}{l}660230 \\
660154 \\
660153 \\
660151 \\
659892\end{array}$ & $\begin{array}{l}6161108 \\
6163929 \\
6164606 \\
6166265 \\
6171390\end{array}$ & $\begin{array}{r}1.9 \\
0.8 \\
0.3 \\
3.1 \\
76.8\end{array}$ & $\begin{array}{r}0.6 \\
1.2 \\
1.5 \\
7.9 \\
25.3\end{array}$ & $\begin{array}{r}2.2 \\
0.9 \\
0.2 \\
9.4 \\
154.0\end{array}$ & $\begin{array}{r}1.0 \\
2.2 \\
1.3 \\
19.0 \\
59.9\end{array}$ & $\begin{array}{r}1.9 \\
3.0 \\
1.2 \\
44.5 \\
180.4\end{array}$ & $\begin{array}{r}7.1 \\
14.4 \\
9.9 \\
17.7 \\
17.5\end{array}$ & $\begin{array}{r}--- \\
--- \\
--- \\
3.4 \\
90.8\end{array}$ & $\begin{array}{r}--- \\
--- \\
--- \\
0 \\
0\end{array}$ & $\begin{array}{r}36 \\
0 \\
50 \\
0 \\
55\end{array}$ \\
\hline $\begin{array}{l}\text { LINE } \\
A\end{array}$ & $\begin{array}{c}20320 \\
1169.0\end{array}$ & $\mathrm{~S}$ & 660331 & 6171054 & 0.5 & 3.3 & 1.0 & 3.8 & 10.9 & 21.2 & --- & --- & 39 \\
\hline
\end{tabular}

$\mathrm{CX}=$ COAXIAL
$\mathrm{CP}=$ COPLANAR

Note: EM values shown above

Ketchikan - Salt Chuck Block
*Estimated Depth may be unreliable because the stronger part of the conductor may be deeper or to one side of the flight line, or because 
EM Anomaly List

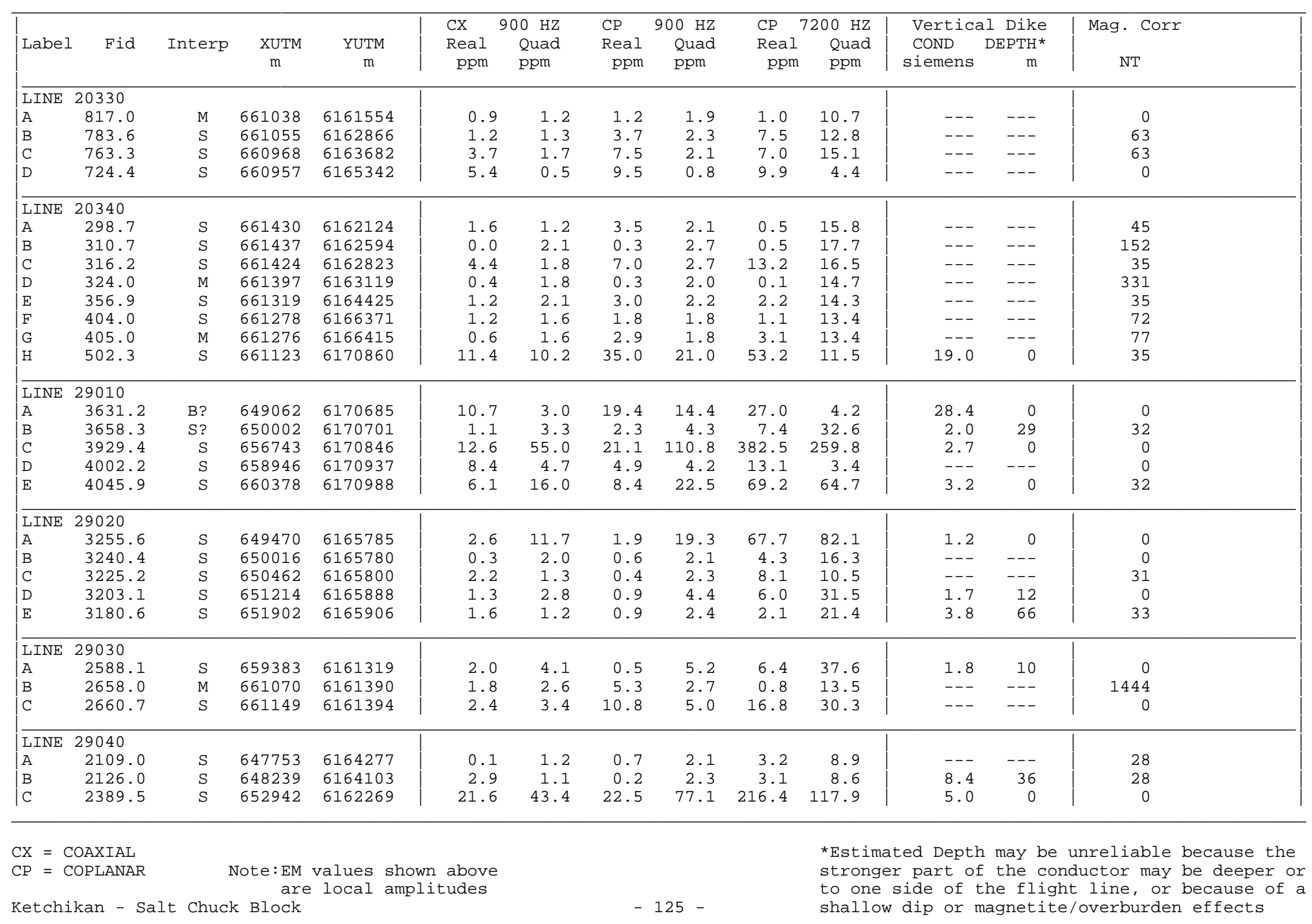


EM Anomaly List

\begin{tabular}{|c|c|c|c|c|c|c|c|c|c|c|c|c|c|}
\hline Label & Fid & Interp & $\begin{array}{c}\text { XUTM } \\
\text { m }\end{array}$ & $\begin{array}{c}\text { YUTM } \\
\text { m }\end{array}$ & $\begin{array}{l}\text { CX } \\
\text { Real } \\
\text { ppm }\end{array}$ & $\begin{array}{c}900 \mathrm{HZ} \\
\text { Quad } \\
\text { ppm }\end{array}$ & $\begin{array}{l}\text { CP } \\
\text { Real } \\
\text { ppm }\end{array}$ & $\begin{array}{l}900 \mathrm{HZ} \\
\text { Quad } \\
\text { ppm }\end{array}$ & $\begin{array}{l}\mathrm{CP} \\
\mathrm{Real} \\
\mathrm{ppm}\end{array}$ & $\begin{array}{c}7200 \mathrm{~Hz} \\
\text { Quad } \\
\text { ppm }\end{array}$ & $\begin{array}{l}\text { Vertica } \\
\text { CoND } \\
\text { siemens }\end{array}$ & $\begin{array}{c}\text { al Dike } \\
\text { DEPTH* } \\
\text { m }\end{array}$ & $\begin{array}{c}\text { Mag. Corr } \\
\text { NT }\end{array}$ \\
\hline $\begin{array}{l}\text { LINE } \\
\text { A } \\
B\end{array}$ & $\begin{array}{c}30020 \\
506.2 \\
539.1\end{array}$ & $\begin{array}{r}\mathrm{B} ? \\
\mathrm{~B}\end{array}$ & $\begin{array}{l}318231 \\
319309\end{array}$ & $\begin{array}{l}6140557 \\
6140465\end{array}$ & $\begin{array}{r}4.6 \\
15.7\end{array}$ & $\begin{array}{r}5.1 \\
14.5\end{array}$ & $\begin{array}{r}0.5 \\
24.7\end{array}$ & $\begin{array}{r}4.5 \\
25.5\end{array}$ & $\begin{array}{l}14.6 \\
53.8\end{array}$ & $\begin{array}{l}15.5 \\
12.9\end{array}$ & $\begin{array}{r}3.4 \\
12.0\end{array}$ & $\begin{array}{r}34 \\
4\end{array}$ & $\begin{array}{l}0 \\
0\end{array}$ \\
\hline $\begin{array}{l}\text { LINE } \\
\text { A } \\
B \\
\text { C } \\
\text { D }\end{array}$ & $\begin{array}{c}30030 \\
649.2 \\
643.0 \\
611.0 \\
603.2\end{array}$ & $\begin{array}{l}\text { B? } \\
\text { B? } \\
\text { B? } \\
\text { B? }\end{array}$ & $\begin{array}{l}318384 \\
318581 \\
319603 \\
319851\end{array}$ & $\begin{array}{l}6140149 \\
6140125 \\
6140078 \\
6140054\end{array}$ & $\begin{array}{r}2.5 \\
2.2 \\
38.2 \\
13.1\end{array}$ & $\begin{array}{r}3.2 \\
3.8 \\
26.4 \\
21.2\end{array}$ & $\begin{array}{r}0.8 \\
0.4 \\
82.7 \\
5.5\end{array}$ & $\begin{array}{r}6.2 \\
3.7 \\
56.1 \\
30.6\end{array}$ & $\begin{array}{r}15.0 \\
8.2 \\
118.6 \\
93.4\end{array}$ & $\begin{array}{r}39.8 \\
25.8 \\
6.2 \\
59.2\end{array}$ & $\begin{array}{r}2.5 \\
--- \\
28.2 \\
4.2\end{array}$ & $\begin{array}{r}26 \\
--- \\
0 \\
0\end{array}$ & $\begin{array}{l}2 \\
0 \\
0 \\
0\end{array}$ \\
\hline $\begin{array}{l}\text { LINE } \\
\text { A } \\
B \\
\text { C } \\
\text { D } \\
\text { E } \\
\text { F }\end{array}$ & $\begin{array}{r}30040 \\
742.0 \\
753.3 \\
758.0 \\
765.9 \\
782.4 \\
789.0\end{array}$ & $\begin{array}{r}\text { B } \\
\text { B } \\
\text { B } \\
\text { D } \\
\text { S } \\
\text { B? }\end{array}$ & $\begin{array}{l}318844 \\
319203 \\
319351 \\
319605 \\
320147 \\
320383\end{array}$ & $\begin{array}{l}6139738 \\
6139728 \\
6139738 \\
6139729 \\
6139680 \\
6139672\end{array}$ & $\begin{array}{l}2.5 \\
3.7 \\
6.7 \\
4.2 \\
4.1 \\
1.1\end{array}$ & $\begin{array}{r}10.1 \\
16.8 \\
7.4 \\
10.1 \\
9.9 \\
3.1\end{array}$ & $\begin{array}{l}4.6 \\
1.3 \\
3.3 \\
2.4 \\
3.8 \\
3.5\end{array}$ & $\begin{array}{r}15.1 \\
18.4 \\
19.1 \\
7.8 \\
19.0 \\
11.9\end{array}$ & $\begin{array}{l}59.1 \\
61.0 \\
69.5 \\
18.2 \\
67.9 \\
37.6\end{array}$ & $\begin{array}{r}51.1 \\
106.9 \\
81.2 \\
46.8 \\
36.7 \\
25.9\end{array}$ & $\begin{array}{l}2.0 \\
1.3 \\
3.8 \\
2.7 \\
2.3 \\
1.8\end{array}$ & $\begin{array}{l}0 \\
0 \\
2 \\
9 \\
0 \\
0\end{array}$ & $\begin{array}{l}1 \\
1 \\
0 \\
3 \\
0 \\
0\end{array}$ \\
\hline $\begin{array}{l}\text { LINE } \\
\text { A } \\
B \\
C \\
D \\
E\end{array}$ & $\begin{array}{l}30050 \\
891.1 \\
885.8 \\
875.8 \\
867.0 \\
853.6\end{array}$ & $\begin{array}{r}\mathrm{B} \\
\mathrm{B} ? \\
\mathrm{D} \\
\mathrm{B} ? \\
\mathrm{~B}\end{array}$ & $\begin{array}{l}318449 \\
318648 \\
319014 \\
319330 \\
319790\end{array}$ & $\begin{array}{l}6139312 \\
6139289 \\
6139273 \\
6139274 \\
6139281\end{array}$ & $\begin{array}{r}14.9 \\
1.5 \\
14.0 \\
0.7 \\
38.3\end{array}$ & $\begin{array}{r}11.8 \\
1.6 \\
24.7 \\
2.8 \\
28.5\end{array}$ & $\begin{array}{r}10.5 \\
1.6 \\
9.0 \\
1.0 \\
72.4\end{array}$ & $\begin{array}{r}20.8 \\
2.3 \\
28.1 \\
5.3 \\
61.5\end{array}$ & $\begin{array}{r}53.3 \\
10.9 \\
91.1 \\
11.0 \\
127.3\end{array}$ & $\begin{array}{l}20.9 \\
10.5 \\
69.2 \\
51.7 \\
55.7\end{array}$ & $\begin{array}{r}8.9 \\
4.0 \\
4.8 \\
1.0 \\
22.2\end{array}$ & $\begin{array}{r}0 \\
54 \\
0 \\
10 \\
0\end{array}$ & $\begin{array}{l}0 \\
3 \\
0 \\
0 \\
0\end{array}$ \\
\hline $\begin{array}{l}\text { LINE } \\
\text { A } \\
B \\
\text { C } \\
\text { D }\end{array}$ & $\begin{array}{c}30060 \\
964.1 \\
976.1 \\
979.2 \\
982.9\end{array}$ & $\begin{array}{r}\mathrm{B} \\
\mathrm{B} \\
\mathrm{B} \\
\mathrm{B} ?\end{array}$ & $\begin{array}{l}319055 \\
319468 \\
319580 \\
319701\end{array}$ & $\begin{array}{l}6138960 \\
6138915 \\
6138905 \\
6138896\end{array}$ & $\begin{array}{r}5.7 \\
15.0 \\
26.6 \\
15.3\end{array}$ & $\begin{array}{l}10.3 \\
19.9 \\
33.5 \\
33.1\end{array}$ & $\begin{array}{r}0.8 \\
9.4 \\
39.4 \\
40.4\end{array}$ & $\begin{array}{r}8.3 \\
28.5 \\
65.1 \\
66.9\end{array}$ & $\begin{array}{r}33.1 \\
85.1 \\
152.0 \\
157.3\end{array}$ & $\begin{array}{l}46.9 \\
78.2 \\
79.7 \\
74.1\end{array}$ & $\begin{array}{l}3.0 \\
4.5 \\
8.0 \\
---\end{array}$ & $\begin{array}{r}0 \\
3 \\
0 \\
---\end{array}$ & $\begin{array}{l}0 \\
0 \\
0 \\
0\end{array}$ \\
\hline $\begin{array}{l}\text { LINE } 3 \\
\text { A } \\
\text { B } \\
\text { C }\end{array}$ & $\begin{array}{l}30070 \\
1107.8 \\
1100.5 \\
1097.8\end{array}$ & $\begin{array}{r}\mathrm{B} ? \\
\mathrm{~B} \\
\mathrm{~B}\end{array}$ & $\begin{array}{l}319154 \\
319421 \\
319520\end{array}$ & $\begin{array}{l}6138483 \\
6138478 \\
6138479\end{array}$ & $\begin{array}{r}5.9 \\
50.8 \\
57.7\end{array}$ & $\begin{array}{l}14.4 \\
45.9 \\
45.9\end{array}$ & $\begin{array}{r}6.7 \\
105.7 \\
105.6\end{array}$ & $\begin{array}{l}27.1 \\
92.1 \\
80.6\end{array}$ & $\begin{array}{r}91.1 \\
193.5 \\
176.4\end{array}$ & $\begin{array}{l}69.4 \\
87.5 \\
90.9\end{array}$ & $\begin{array}{r}2.8 \\
21.2 \\
26.1\end{array}$ & $\begin{array}{l}0 \\
0 \\
0\end{array}$ & $\begin{array}{l}0 \\
0 \\
0\end{array}$ \\
\hline $\begin{array}{l}\text { LINE } 3 \\
\text { A } \\
\text { B } \\
\text { C }\end{array}$ & $\begin{array}{l}30080 \\
1199.8 \\
1208.5 \\
1211.8\end{array}$ & $\begin{array}{r}\mathrm{B} ? \\
\mathrm{~B} \\
\mathrm{~B}\end{array}$ & $\begin{array}{l}319566 \\
319840 \\
319944\end{array}$ & $\begin{array}{l}6138122 \\
6138084 \\
6138076\end{array}$ & $\begin{array}{r}4.0 \\
12.6 \\
15.1\end{array}$ & $\begin{array}{r}7.4 \\
7.4 \\
15.8\end{array}$ & $\begin{array}{r}3.1 \\
10.4 \\
33.4\end{array}$ & $\begin{array}{r}8.1 \\
15.5 \\
41.6\end{array}$ & $\begin{array}{l}23.3 \\
39.4 \\
98.0\end{array}$ & $\begin{array}{l}35.7 \\
24.6 \\
39.3\end{array}$ & $\begin{array}{r}3.2 \\
12.6 \\
9.8\end{array}$ & $\begin{array}{l}1 \\
5 \\
0\end{array}$ & $\begin{array}{r}12 \\
0 \\
0\end{array}$ \\
\hline $\begin{array}{l}\text { LINE } 3 \\
\mathrm{~A}\end{array}$ & $\begin{array}{l}30090 \\
1347.9\end{array}$ & B & 320011 & 6137678 & 1.6 & 5.8 & 2.1 & 4.8 & 13.9 & 14.3 & 1.8 & 0 & 0 \\
\hline $\begin{array}{l}\text { LINE } 3 \\
\mathrm{~A}\end{array}$ & $\begin{array}{l}30100 \\
1492.0\end{array}$ & B & 320075 & 6137253 & 8.6 & 10.2 & 8.6 & 16.9 & 42.7 & 20.1 & 5.2 & 16 & 0 \\
\hline
\end{tabular}

$\mathrm{CX}=$ COAXIAL

Note: EM values shown above

are local amplitudes

Ketchikan - Gravina Island West
* Estimated Depth may be unreliable because the stronger part of the conductor may be deeper or to one side of the flight line, or because of a shallow dip or magnetite/overburden effects 
EM Anomaly List

\begin{tabular}{|c|c|c|c|c|c|c|c|c|c|c|c|c|c|}
\hline Label & Fid & Interp & $\begin{array}{c}\text { XUTM } \\
\text { m }\end{array}$ & $\begin{array}{c}\text { YUTM } \\
\mathrm{m}\end{array}$ & $\begin{array}{l}\text { CX } \\
\text { Real } \\
\text { ppm }\end{array}$ & $\begin{array}{l}900 \mathrm{HZ} \\
\text { Quad } \\
\text { ppm }\end{array}$ & $\begin{array}{l}\text { CP } \\
\text { Real } \\
\text { ppm }\end{array}$ & $\begin{array}{l}900 \mathrm{HZ} \\
\text { Quad } \\
\text { ppm }\end{array}$ & $\begin{array}{l}\mathrm{CP} \\
\mathrm{Real} \\
\mathrm{ppm}\end{array}$ & $\begin{array}{l}200 \mathrm{HZ} \\
\text { Quad } \\
\text { ppm }\end{array}$ & $\begin{array}{l}\text { Vertica } \\
\text { CoND } \\
\text { siemens }\end{array}$ & $\begin{array}{c}\text { al Dike } \\
\text { DEPTH* } \\
\text { m }\end{array}$ & $\begin{array}{c}\text { Mag. Corr } \\
\text { NT }\end{array}$ \\
\hline $\begin{array}{l}\text { LINE } \\
\text { B } \\
\text { C } \\
\text { D } \\
\text { E }\end{array}$ & $\begin{array}{l}30100 \\
1499.0 \\
1503.0 \\
1508.6 \\
1579.7\end{array}$ & $\begin{array}{r}\mathrm{B} \\
\mathrm{B} \\
\mathrm{B} \\
\mathrm{B} ?\end{array}$ & $\begin{array}{l}320228 \\
320298 \\
320377 \\
321217\end{array}$ & $\begin{array}{l}6137253 \\
6137253 \\
6137247 \\
6137199\end{array}$ & $\begin{array}{l}2.7 \\
3.9 \\
3.9 \\
3.5\end{array}$ & $\begin{array}{r}2.8 \\
2.3 \\
4.1 \\
13.0\end{array}$ & $\begin{array}{l}4.7 \\
6.3 \\
7.1 \\
1.2\end{array}$ & $\begin{array}{r}3.1 \\
10.1 \\
11.9 \\
15.3\end{array}$ & $\begin{array}{r}6.1 \\
24.5 \\
30.1 \\
52.8\end{array}$ & $\begin{array}{r}3.2 \\
4.8 \\
13.5 \\
65.8\end{array}$ & $\begin{array}{l}8.2 \\
6.9 \\
--- \\
1.4\end{array}$ & $\begin{array}{r}32 \\
23 \\
--- \\
0\end{array}$ & $\begin{array}{r}0 \\
0 \\
0 \\
20\end{array}$ \\
\hline $\begin{array}{l}\text { LINE } \\
\text { A } \\
B \\
C\end{array}$ & $\begin{array}{r}30110 \\
1827.4 \\
1819.0 \\
1762.3\end{array}$ & $\begin{array}{l}\text { B? } \\
\text { B? } \\
\text { B? }\end{array}$ & $\begin{array}{l}320457 \\
320576 \\
321405\end{array}$ & $\begin{array}{l}6136844 \\
6136834 \\
6136781\end{array}$ & $\begin{array}{l}1.9 \\
4.8 \\
0.2\end{array}$ & $\begin{array}{l}5.1 \\
3.6 \\
3.3\end{array}$ & $\begin{array}{l}2.8 \\
0.7 \\
0.5\end{array}$ & $\begin{array}{l}3.3 \\
6.1 \\
3.3\end{array}$ & $\begin{array}{r}6.9 \\
17.0 \\
11.3\end{array}$ & $\begin{array}{r}5.6 \\
6.5 \\
20.0\end{array}$ & $\begin{array}{l}2.7 \\
4.5 \\
---\end{array}$ & $\begin{array}{r}4 \\
17 \\
---\end{array}$ & $\begin{array}{l}0 \\
0 \\
0\end{array}$ \\
\hline $\begin{array}{l}\text { LINE } \\
\text { A } \\
B \\
C\end{array}$ & $\begin{array}{r}30120 \\
2048.8 \\
2057.3 \\
2113.5\end{array}$ & $\begin{array}{l}\text { B? } \\
\text { B? } \\
\text { B? }\end{array}$ & $\begin{array}{l}320528 \\
320602 \\
321524\end{array}$ & $\begin{array}{l}6136434 \\
6136437 \\
6136352\end{array}$ & $\begin{array}{r}7.5 \\
11.8 \\
1.2\end{array}$ & $\begin{array}{r}3.7 \\
11.9 \\
2.9\end{array}$ & $\begin{array}{l}0.2 \\
0.3 \\
0.5\end{array}$ & $\begin{array}{l}2.4 \\
5.8 \\
4.2\end{array}$ & $\begin{array}{r}7.0 \\
19.8 \\
10.6\end{array}$ & $\begin{array}{l}11.0 \\
30.7 \\
21.6\end{array}$ & $\begin{array}{l}0.5 \\
6.3 \\
---\end{array}$ & $\begin{array}{r}0 \\
19 \\
---\end{array}$ & $\begin{array}{l}7 \\
0 \\
0\end{array}$ \\
\hline $\begin{array}{l}\text { LINE } \\
\text { A } \\
B\end{array}$ & $\begin{array}{l}30130 \\
2250.8 \\
2180.3\end{array}$ & $\begin{array}{l}B \\
S\end{array}$ & $\begin{array}{l}320602 \\
321827\end{array}$ & $\begin{array}{l}6136012 \\
6135949\end{array}$ & $\begin{array}{l}4.3 \\
2.5\end{array}$ & $\begin{array}{l}5.4 \\
4.8\end{array}$ & $\begin{array}{l}0.2 \\
0.0\end{array}$ & $\begin{array}{l}4.5 \\
5.3\end{array}$ & $\begin{array}{l}16.1 \\
21.8\end{array}$ & $\begin{array}{l}17.0 \\
30.0\end{array}$ & $\begin{array}{l}2.7 \\
1.9\end{array}$ & $\begin{array}{r}29 \\
0\end{array}$ & $\begin{array}{l}0 \\
0\end{array}$ \\
\hline $\begin{array}{l}\text { LINE } \\
\text { A } \\
B \\
\text { C }\end{array}$ & $\begin{array}{r}30140 \\
2618.2 \\
2632.0 \\
2676.8\end{array}$ & $\begin{array}{r}\text { B? } \\
\text { B } \\
\text { S }\end{array}$ & $\begin{array}{l}320439 \\
320644 \\
321738\end{array}$ & $\begin{array}{l}6135600 \\
6135617 \\
6135576\end{array}$ & $\begin{array}{l}4.1 \\
0.0 \\
1.9\end{array}$ & $\begin{array}{l}5.5 \\
1.7 \\
6.8\end{array}$ & $\begin{array}{l}0.4 \\
3.6 \\
0.3\end{array}$ & $\begin{array}{l}3.5 \\
3.7 \\
9.9\end{array}$ & $\begin{array}{l}14.5 \\
11.4 \\
28.6\end{array}$ & $\begin{array}{r}15.3 \\
2.2 \\
52.1\end{array}$ & $\begin{array}{l}--- \\
1.7 \\
1.0\end{array}$ & $\begin{array}{r}--- \\
64 \\
0\end{array}$ & $\begin{array}{l}0 \\
0 \\
0\end{array}$ \\
\hline $\begin{array}{l}\text { LINE } \\
\text { A } \\
\text { B } \\
\text { C }\end{array}$ & $\begin{array}{r}30150 \\
2929.6 \\
2781.6 \\
2776.5\end{array}$ & $\begin{array}{r}\mathrm{M} \\
\mathrm{B} \\
\mathrm{B} ?\end{array}$ & $\begin{array}{l}318546 \\
320465 \\
320567\end{array}$ & $\begin{array}{l}6135262 \\
6135188 \\
6135187\end{array}$ & $\begin{array}{r}2.1 \\
15.6 \\
5.9\end{array}$ & $\begin{array}{r}0.9 \\
14.8 \\
11.0\end{array}$ & $\begin{array}{r}0.2 \\
14.5 \\
14.9\end{array}$ & $\begin{array}{r}0.8 \\
28.8 \\
24.8\end{array}$ & $\begin{array}{r}1.8 \\
71.6 \\
52.9\end{array}$ & $\begin{array}{r}0.2 \\
26.0 \\
22.0\end{array}$ & $\begin{array}{l}--- \\
7.5 \\
3.9\end{array}$ & $\begin{array}{r}--- \\
7 \\
17\end{array}$ & $\begin{array}{r}159 \\
0 \\
7\end{array}$ \\
\hline $\begin{array}{l}\text { LINE } \\
\text { A } \\
B \\
C\end{array}$ & $\begin{array}{r}30160 \\
3136.0 \\
3145.0 \\
3185.4\end{array}$ & $\begin{array}{r}\mathrm{B} \\
\mathrm{B} \\
\mathrm{B} ?\end{array}$ & $\begin{array}{l}320528 \\
320717 \\
321495\end{array}$ & $\begin{array}{l}6134802 \\
6134796 \\
6134764\end{array}$ & $\begin{array}{l}6.7 \\
2.0 \\
1.8\end{array}$ & $\begin{array}{r}12.3 \\
2.0 \\
6.7\end{array}$ & $\begin{array}{l}7.8 \\
7.8 \\
0.1\end{array}$ & $\begin{array}{r}16.8 \\
10.2 \\
8.1\end{array}$ & $\begin{array}{l}44.6 \\
21.6 \\
23.8\end{array}$ & $\begin{array}{r}32.8 \\
1.0 \\
46.5\end{array}$ & $\begin{array}{l}3.1 \\
5.2 \\
1.0\end{array}$ & $\begin{array}{r}22 \\
24 \\
0\end{array}$ & $\begin{array}{l}0 \\
0 \\
0\end{array}$ \\
\hline $\begin{array}{l}\text { LINE } \\
\text { A } \\
\text { B } \\
\text { C } \\
\text { D } \\
\text { E } \\
\text { F }\end{array}$ & $\begin{array}{r}30170 \\
3624.0 \\
3608.7 \\
3596.2 \\
3493.7 \\
3483.2 \\
3470.0\end{array}$ & $\begin{array}{r}\mathrm{M} \\
\mathrm{M} \\
\mathrm{M} \\
\mathrm{B} \\
\mathrm{B} ? \\
\mathrm{~B}\end{array}$ & $\begin{array}{l}318461 \\
318806 \\
319034 \\
320362 \\
320523 \\
320668\end{array}$ & $\begin{array}{l}6134492 \\
6134487 \\
6134477 \\
6134400 \\
6134383 \\
6134376\end{array}$ & $\begin{array}{r}0.0 \\
0.1 \\
0.8 \\
14.0 \\
7.9 \\
4.5\end{array}$ & $\begin{array}{r}0.0 \\
2.3 \\
0.5 \\
14.8 \\
11.7 \\
4.6\end{array}$ & $\begin{array}{r}0.3 \\
0.2 \\
0.1 \\
16.9 \\
31.0 \\
9.2\end{array}$ & $\begin{array}{r}3.0 \\
3.9 \\
0.7 \\
33.6 \\
29.6 \\
7.6\end{array}$ & $\begin{array}{r}0.5 \\
7.9 \\
0.4 \\
92.2 \\
67.4 \\
17.2\end{array}$ & $\begin{array}{r}29.2 \\
29.8 \\
2.9 \\
35.2 \\
37.1 \\
6.6\end{array}$ & $\begin{array}{l}--- \\
--- \\
--- \\
6.4 \\
9.0 \\
8.9\end{array}$ & $\begin{array}{r}--- \\
--- \\
-- \\
0 \\
12 \\
37\end{array}$ & $\begin{array}{r}0 \\
51 \\
190 \\
0 \\
0 \\
18\end{array}$ \\
\hline $\begin{array}{l}\text { LINE } \\
\text { A } \\
\text { B } \\
\text { C }\end{array}$ & $\begin{array}{r}30180 \\
3716.0 \\
3820.2 \\
3826.4\end{array}$ & $\begin{array}{l}\mathrm{M} \\
\mathrm{B} \\
\mathrm{B}\end{array}$ & $\begin{array}{l}318929 \\
320293 \\
320402\end{array}$ & $\begin{array}{l}6134096 \\
6134005 \\
6133992\end{array}$ & $\begin{array}{r}0.1 \\
8.0 \\
13.3\end{array}$ & $\begin{array}{r}0.1 \\
9.1 \\
23.6\end{array}$ & $\begin{array}{r}0.1 \\
3.7 \\
25.9\end{array}$ & $\begin{array}{r}0.4 \\
9.1 \\
43.4\end{array}$ & $\begin{array}{r}0.0 \\
24.3 \\
123.9\end{array}$ & $\begin{array}{r}3.7 \\
22.2 \\
83.5\end{array}$ & $\begin{array}{l}--- \\
--- \\
5.0\end{array}$ & $\begin{array}{r}--- \\
--- \\
5\end{array}$ & $\begin{array}{l}0 \\
0 \\
0\end{array}$ \\
\hline
\end{tabular}

$\mathrm{CX}=$ COAXIAL

Note: EM values shown above

are local amplitudes

Ketchikan - Gravina Island West
$-127-$
*Estimated Depth may be unreliable because the stronger part of the conductor may be deeper or to one side of the flight line, or because of a shallow dip or magnetite/overburden effects 
EM Anomaly List

\begin{tabular}{|c|c|c|c|c|c|c|c|c|c|c|c|c|c|}
\hline Label & Fid & Interp & $\begin{array}{c}\text { XUTM } \\
\text { m }\end{array}$ & $\begin{array}{c}\text { YUTM } \\
\mathrm{m}\end{array}$ & $\begin{array}{l}\text { CX } \\
\text { Real } \\
\text { ppm }\end{array}$ & $\begin{array}{c}900 \mathrm{HZ} \\
\text { Quad } \\
\text { ppm }\end{array}$ & $\begin{array}{l}\text { CP } \\
\text { Real } \\
\text { ppm }\end{array}$ & $\begin{array}{c}900 \mathrm{HZ} \\
\text { Quad } \\
\text { ppm }\end{array}$ & $\begin{array}{l}\text { CP } \\
\text { Real } \\
\text { ppm }\end{array}$ & $\begin{array}{c}200 \mathrm{HZ} \\
\text { Quad } \\
\text { ppm }\end{array}$ & $\begin{array}{l}\text { Vertica } \\
\text { COND } \\
\text { siemens }\end{array}$ & $\begin{array}{c}\text { al Dike } \\
\text { DEPTH* } \\
\text { m }\end{array}$ & $\begin{array}{c}\text { Mag. Corr } \\
\text { NT }\end{array}$ \\
\hline $\begin{array}{l}\text { LINE } \\
\text { D } \\
\text { E } \\
\text { F } \\
G\end{array}$ & $\begin{array}{r}30180 \\
3833.9 \\
3836.4 \\
3842.0 \\
3903.3\end{array}$ & $\begin{array}{l}\text { B } \\
\text { B } \\
\text { B } \\
\text { S }\end{array}$ & $\begin{array}{l}320557 \\
320612 \\
320734 \\
321982\end{array}$ & $\begin{array}{l}6133959 \\
6133953 \\
6133960 \\
6133898\end{array}$ & $\begin{array}{r}24.2 \\
24.2 \\
14.2 \\
0.1\end{array}$ & $\begin{array}{r}31.4 \\
31.4 \\
14.8 \\
9.3\end{array}$ & $\begin{array}{r}46.3 \\
46.0 \\
31.9 \\
5.5\end{array}$ & $\begin{array}{l}48.3 \\
67.3 \\
34.3 \\
24.5\end{array}$ & $\begin{array}{r}115.5 \\
165.7 \\
91.6 \\
63.4\end{array}$ & $\begin{array}{l}54.6 \\
77.1 \\
40.8 \\
78.8\end{array}$ & $\begin{array}{r}10.8 \\
8.5 \\
10.8 \\
0.9\end{array}$ & $\begin{array}{l}0 \\
0 \\
0 \\
0\end{array}$ & $\begin{array}{l}0 \\
0 \\
0 \\
0\end{array}$ \\
\hline $\begin{array}{l}\text { LINE } \\
\text { A } \\
\text { B } \\
\text { C } \\
\text { D } \\
\text { E } \\
\text { F } \\
\text { G } \\
\text { H } \\
\text { I } \\
\text { J } \\
\text { K } \\
\text { L } \\
\text { M }\end{array}$ & $\begin{array}{r}30190 \\
4224.7 \\
4214.0 \\
4204.5 \\
4197.0 \\
4192.7 \\
4186.4 \\
4174.5 \\
4166.0 \\
4062.0 \\
4042.7 \\
4038.0 \\
4033.0 \\
3967.0\end{array}$ & $\begin{array}{l}\text { M } \\
M \\
M \\
M \\
M \\
M \\
M \\
M \\
M \\
B \\
B \\
B \\
S\end{array}$ & $\begin{array}{l}318359 \\
318598 \\
318786 \\
318922 \\
318999 \\
319112 \\
319301 \\
319409 \\
320395 \\
320652 \\
320731 \\
320833 \\
322108\end{array}$ & $\begin{array}{l}6133644 \\
6133616 \\
6133624 \\
6133636 \\
6133637 \\
6133623 \\
6133603 \\
6133591 \\
6133556 \\
6133556 \\
6133550 \\
6133546 \\
6133515\end{array}$ & $\begin{array}{r}1.0 \\
1.7 \\
2.2 \\
4.0 \\
4.1 \\
2.3 \\
0.0 \\
1.7 \\
0.1 \\
12.6 \\
5.3 \\
7.0 \\
1.6\end{array}$ & $\begin{array}{r}0.0 \\
1.1 \\
0.7 \\
0.2 \\
0.4 \\
0.9 \\
0.6 \\
0.5 \\
0.4 \\
10.6 \\
9.4 \\
6.6 \\
4.3\end{array}$ & $\begin{array}{r}1.1 \\
0.2 \\
3.1 \\
12.1 \\
0.8 \\
0.1 \\
3.2 \\
0.9 \\
0.2 \\
20.5 \\
20.6 \\
9.5 \\
2.3\end{array}$ & $\begin{array}{r}0.5 \\
0.8 \\
0.7 \\
0.2 \\
0.7 \\
0.9 \\
0.8 \\
0.4 \\
0.7 \\
28.1 \\
27.2 \\
9.8 \\
6.8\end{array}$ & $\begin{array}{r}1.1 \\
0.3 \\
3.3 \\
10.7 \\
1.2 \\
0.4 \\
3.6 \\
0.8 \\
2.0 \\
71.9 \\
67.6 \\
24.4 \\
18.2\end{array}$ & $\begin{array}{r}6.2 \\
8.6 \\
2.6 \\
0.6 \\
7.2 \\
8.7 \\
5.2 \\
1.9 \\
1.7 \\
32.9 \\
35.5 \\
4.9 \\
21.6\end{array}$ & $\begin{array}{l}--- \\
--- \\
--- \\
--- \\
--- \\
--- \\
--- \\
--- \\
--- \\
9.7 \\
5.3 \\
8.9 \\
2.0\end{array}$ & $\begin{array}{r}--- \\
--- \\
--- \\
--- \\
--- \\
--- \\
--- \\
--- \\
--- \\
18 \\
13 \\
4 \\
3\end{array}$ & $\begin{array}{r}0 \\
5 \\
0 \\
0 \\
19 \\
2 \\
180 \\
0 \\
0 \\
0 \\
0 \\
0 \\
0\end{array}$ \\
\hline $\begin{array}{l}\text { LINE } \\
\text { A } \\
\text { B } \\
\text { C } \\
\text { D } \\
\text { E } \\
\text { F }\end{array}$ & $\begin{array}{r}30200 \\
4301.4 \\
4307.0 \\
4504.0 \\
4515.9 \\
4555.2 \\
4577.0\end{array}$ & $\begin{array}{r}\mathrm{M} \\
\mathrm{M} \\
\mathrm{B} \\
\mathrm{B} ? \\
\mathrm{~B} ? \\
\mathrm{~S}\end{array}$ & $\begin{array}{l}318327 \\
318487 \\
320835 \\
321002 \\
321924 \\
322545\end{array}$ & $\begin{array}{l}6133305 \\
6133298 \\
6133193 \\
6133203 \\
6133149 \\
6133120\end{array}$ & $\begin{array}{r}2.9 \\
1.8 \\
11.7 \\
11.2 \\
0.1 \\
0.9\end{array}$ & $\begin{array}{r}0.6 \\
0.3 \\
13.7 \\
8.7 \\
4.9 \\
5.8\end{array}$ & $\begin{array}{r}5.5 \\
6.6 \\
29.9 \\
8.3 \\
0.3 \\
4.0\end{array}$ & $\begin{array}{r}0.6 \\
0.2 \\
20.2 \\
13.8 \\
5.0 \\
12.5\end{array}$ & $\begin{array}{r}2.3 \\
5.8 \\
44.3 \\
37.7 \\
11.2 \\
34.5\end{array}$ & $\begin{array}{r}7.3 \\
2.1 \\
1.9 \\
10.1 \\
34.9 \\
42.2\end{array}$ & $\begin{array}{r}--- \\
--- \\
13.4 \\
9.2 \\
--- \\
1.5\end{array}$ & $\begin{array}{r}--- \\
--- \\
11 \\
9 \\
--- \\
0\end{array}$ & $\begin{array}{r}53 \\
92 \\
0 \\
0 \\
0 \\
21\end{array}$ \\
\hline $\begin{array}{l}\text { LINE } \\
\text { A } \\
\text { B } \\
\text { C } \\
\text { D } \\
\text { E } \\
\text { F } \\
\text { G }\end{array}$ & $\begin{array}{r}30210 \\
4915.4 \\
4896.0 \\
4886.0 \\
4766.0 \\
4760.5 \\
4674.2 \\
4626.9\end{array}$ & $\begin{array}{r}\mathrm{M} \\
\mathrm{M} \\
\mathrm{M} \\
\mathrm{M} \\
\mathrm{D} \\
\mathrm{B} \\
\mathrm{B} ?\end{array}$ & $\begin{array}{l}318354 \\
318762 \\
318899 \\
319896 \\
319972 \\
320986 \\
322023\end{array}$ & $\begin{array}{l}6132868 \\
6132851 \\
6132835 \\
6132799 \\
6132801 \\
6132730 \\
6132756\end{array}$ & $\begin{array}{l}1.0 \\
1.3 \\
0.2 \\
0.0 \\
1.0 \\
7.8 \\
0.1\end{array}$ & $\begin{array}{l}0.4 \\
0.2 \\
0.6 \\
0.6 \\
2.5 \\
5.3 \\
4.3\end{array}$ & $\begin{array}{l}1.1 \\
2.1 \\
0.2 \\
0.1 \\
4.5 \\
6.0 \\
0.2\end{array}$ & $\begin{array}{l}0.1 \\
0.4 \\
0.5 \\
1.6 \\
2.0 \\
7.8 \\
4.6\end{array}$ & $\begin{array}{r}1.1 \\
0.0 \\
0.1 \\
0.3 \\
9.2 \\
19.3 \\
17.4\end{array}$ & $\begin{array}{r}5.0 \\
0.4 \\
5.2 \\
9.5 \\
8.4 \\
13.9 \\
28.1\end{array}$ & $\begin{array}{r}--- \\
--- \\
--- \\
--- \\
5.2 \\
10.5 \\
0.5\end{array}$ & $\begin{array}{r}--- \\
--- \\
--- \\
--- \\
55 \\
14 \\
0\end{array}$ & $\begin{array}{r}44 \\
0 \\
8 \\
0 \\
0 \\
17 \\
0\end{array}$ \\
\hline $\begin{array}{l}\text { LINE } \\
\text { A } \\
\text { B } \\
\text { C } \\
\text { D } \\
\text { E } \\
\text { F } \\
\text { G }\end{array}$ & $\begin{array}{r}30220 \\
4992.0 \\
5143.0 \\
5171.7 \\
5188.0 \\
5224.0 \\
5235.9 \\
5297.9\end{array}$ & $\begin{array}{r}\mathrm{M} \\
\mathrm{M} \\
\mathrm{D} \\
\mathrm{B} \\
\mathrm{B} \\
\mathrm{B} \\
\mathrm{B} ?\end{array}$ & $\begin{array}{l}318483 \\
319688 \\
319992 \\
320236 \\
320672 \\
320840 \\
321996\end{array}$ & $\begin{array}{l}6132486 \\
6132446 \\
6132444 \\
6132424 \\
6132399 \\
6132387 \\
6132350\end{array}$ & $\begin{array}{r}0.3 \\
0.2 \\
20.6 \\
4.3 \\
9.4 \\
13.3 \\
0.5\end{array}$ & $\begin{array}{r}0.5 \\
0.4 \\
19.5 \\
6.6 \\
11.7 \\
18.7 \\
3.5\end{array}$ & $\begin{array}{r}3.2 \\
1.0 \\
7.9 \\
2.5 \\
7.5 \\
13.1 \\
0.3\end{array}$ & $\begin{array}{r}0.9 \\
0.2 \\
12.8 \\
0.2 \\
16.2 \\
17.2 \\
4.0\end{array}$ & $\begin{array}{r}4.0 \\
0.2 \\
35.6 \\
6.6 \\
45.0 \\
41.5 \\
12.7\end{array}$ & $\begin{array}{r}1.4 \\
2.6 \\
28.1 \\
7.3 \\
19.0 \\
33.2 \\
18.3\end{array}$ & $\begin{array}{r}--- \\
--- \\
10.2 \\
5.1 \\
4.9 \\
6.4 \\
---\end{array}$ & $\begin{array}{r}--- \\
--- \\
13 \\
36 \\
11 \\
21 \\
---\end{array}$ & $\begin{array}{r}288 \\
0 \\
0 \\
0 \\
0 \\
0 \\
0\end{array}$ \\
\hline
\end{tabular}

$\mathrm{CX}=$ COAXIAL

Note: EM values shown above

are local amplitudes

*Estimated Depth may be unreliable because the stronger part of the conductor may be deeper or to one side of the flight line, or because of a shallow dip or magnetite/overburden effects

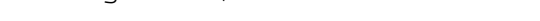


EM Anomaly List

\begin{tabular}{|c|c|c|c|c|c|c|c|c|c|c|c|c|c|}
\hline Label & Fid & Interp & $\begin{array}{c}\text { XUTM } \\
\text { m }\end{array}$ & $\begin{array}{c}\text { YUTM } \\
\mathrm{m}\end{array}$ & $\begin{array}{l}\text { CX } \\
\text { Real } \\
\text { ppm }\end{array}$ & $\begin{array}{l}00 \mathrm{HZ} \\
\text { Quad } \\
\text { ppm }\end{array}$ & $\begin{array}{l}\text { CP } \\
\text { Real } \\
\text { ppm }\end{array}$ & $\begin{array}{l}900 \mathrm{HZ} \\
\text { Quad } \\
\text { ppm }\end{array}$ & $\begin{array}{l}\text { CP } 7 \\
\text { Real } \\
\text { ppm }\end{array}$ & $\begin{array}{l}200 \mathrm{HZ} \\
\text { Quad } \\
\text { ppm }\end{array}$ & $\begin{array}{l}\text { Vertica } \\
\text { COND } \\
\text { siemens }\end{array}$ & $\begin{array}{c}\text { al Dike } \\
\text { DEPTH* } \\
\text { m }\end{array}$ & $\begin{array}{c}\text { Mag. Corr } \\
\text { NT }\end{array}$ \\
\hline $\begin{array}{l}\text { LINE } \\
\text { A } \\
\text { B } \\
\text { C } \\
\text { D } \\
\text { E } \\
\text { F }\end{array}$ & $\begin{array}{r}30230 \\
5613.5 \\
5607.2 \\
5600.7 \\
5595.0 \\
5486.4 \\
5463.9\end{array}$ & $\begin{array}{r}\text { B? } \\
\text { B } \\
\text { B } \\
\text { B } \\
\text { S } \\
\text { S }\end{array}$ & $\begin{array}{l}320228 \\
320363 \\
320535 \\
320702 \\
322256 \\
322720\end{array}$ & $\begin{array}{l}6132011 \\
6132000 \\
6131989 \\
6131977 \\
6131878 \\
6131924\end{array}$ & $\begin{array}{l}1.6 \\
2.4 \\
0.4 \\
8.9 \\
1.2 \\
0.2\end{array}$ & $\begin{array}{l}3.7 \\
3.2 \\
3.9 \\
8.4 \\
6.7 \\
4.2\end{array}$ & $\begin{array}{l}0.5 \\
2.2 \\
6.2 \\
4.2 \\
2.5 \\
0.5\end{array}$ & $\begin{array}{r}2.0 \\
5.4 \\
8.5 \\
11.6 \\
12.8 \\
6.9\end{array}$ & $\begin{array}{r}8.6 \\
21.0 \\
27.8 \\
32.8 \\
33.3 \\
19.8\end{array}$ & $\begin{array}{r}17.2 \\
7.1 \\
39.7 \\
23.4 \\
44.5 \\
37.2\end{array}$ & $\begin{array}{l}1.9 \\
2.6 \\
2.6 \\
5.7 \\
1.1 \\
0.5\end{array}$ & $\begin{array}{r}18 \\
29 \\
11 \\
6 \\
0 \\
0\end{array}$ & $\begin{array}{r}0 \\
10 \\
0 \\
0 \\
0 \\
0\end{array}$ \\
\hline $\begin{array}{l}\text { LINE } \\
\text { A } \\
\text { B } \\
\text { C } \\
\text { D } \\
\text { E }\end{array}$ & $\begin{array}{l}30240 \\
5934.0 \\
5963.5 \\
5973.4 \\
6070.9 \\
6078.6\end{array}$ & $\begin{array}{r}\text { B? } \\
\text { B? } \\
\text { B? } \\
\text { B } \\
\text { B }\end{array}$ & $\begin{array}{l}320187 \\
320393 \\
320526 \\
322166 \\
322391\end{array}$ & $\begin{array}{l}6131598 \\
6131591 \\
6131586 \\
6131496 \\
6131448\end{array}$ & $\begin{array}{r}4.8 \\
1.4 \\
3.6 \\
11.8 \\
4.7\end{array}$ & $\begin{array}{l}6.0 \\
2.4 \\
5.7 \\
9.0 \\
7.0\end{array}$ & $\begin{array}{r}3.5 \\
4.8 \\
1.7 \\
4.2 \\
14.5\end{array}$ & $\begin{array}{r}5.7 \\
4.9 \\
6.7 \\
12.8 \\
14.5\end{array}$ & $\begin{array}{l}17.6 \\
10.8 \\
18.4 \\
39.7 \\
26.8\end{array}$ & $\begin{array}{r}4.8 \\
9.5 \\
13.3 \\
23.8 \\
25.9\end{array}$ & $\begin{array}{l}4.5 \\
--- \\
--- \\
7.8 \\
---\end{array}$ & $\begin{array}{r}39 \\
--- \\
--- \\
0 \\
---\end{array}$ & $\begin{array}{r}0 \\
0 \\
10 \\
0 \\
8\end{array}$ \\
\hline $\begin{array}{l}\text { LINE } \\
\text { A } \\
\text { B } \\
\text { C }\end{array}$ & $\begin{array}{r}30250 \\
6307.0 \\
6294.3 \\
6159.8\end{array}$ & $\begin{array}{r}\text { B? } \\
\text { B? } \\
\text { B }\end{array}$ & $\begin{array}{l}320272 \\
320427 \\
322172\end{array}$ & $\begin{array}{l}6131189 \\
6131157 \\
6131111\end{array}$ & $\begin{array}{r}3.2 \\
3.7 \\
11.5\end{array}$ & $\begin{array}{r}2.6 \\
6.9 \\
16.6\end{array}$ & $\begin{array}{r}2.4 \\
2.5 \\
15.9\end{array}$ & $\begin{array}{r}11.1 \\
3.5 \\
33.1\end{array}$ & $\begin{array}{r}31.1 \\
8.3 \\
80.1\end{array}$ & $\begin{array}{l}16.7 \\
13.7 \\
50.2\end{array}$ & $\begin{array}{l}2.5 \\
2.8 \\
4.6\end{array}$ & $\begin{array}{l}27 \\
25 \\
17\end{array}$ & $\begin{array}{l}0 \\
0 \\
0\end{array}$ \\
\hline $\begin{array}{l}\text { LINE } \\
\text { A } \\
\text { B } \\
\text { C }\end{array}$ & $\begin{array}{r}30260 \\
6594.7 \\
6599.0 \\
6731.4\end{array}$ & $\begin{array}{l}\mathrm{B} \\
\mathrm{B} \\
\mathrm{H}\end{array}$ & $\begin{array}{l}320265 \\
320339 \\
322750\end{array}$ & $\begin{array}{l}6130820 \\
6130820 \\
6130712\end{array}$ & $\begin{array}{l}5.6 \\
4.7 \\
1.6\end{array}$ & $\begin{array}{l}7.2 \\
3.6 \\
3.0\end{array}$ & $\begin{array}{l}3.8 \\
8.0 \\
0.8\end{array}$ & $\begin{array}{l}6.4 \\
9.9 \\
4.4\end{array}$ & $\begin{array}{l}18.6 \\
25.6 \\
10.0\end{array}$ & $\begin{array}{r}6.9 \\
9.7 \\
33.4\end{array}$ & $\begin{array}{l}4.6 \\
7.9 \\
2.0\end{array}$ & $\begin{array}{l}11 \\
22 \\
27\end{array}$ & $\begin{array}{r}0 \\
10 \\
0\end{array}$ \\
\hline $\begin{array}{l}\text { LINE } \\
\text { A } \\
\text { B } \\
\text { C } \\
\text { D } \\
\text { E }\end{array}$ & $\begin{array}{l}30270 \\
553.6 \\
546.4 \\
537.0 \\
435.2 \\
237.4\end{array}$ & $\begin{array}{r}\mathrm{S} \\
\mathrm{M} \\
\mathrm{M} \\
\mathrm{B} \\
\mathrm{B} ?\end{array}$ & $\begin{array}{l}318859 \\
318972 \\
319103 \\
320339 \\
322779\end{array}$ & $\begin{array}{l}6130433 \\
6130415 \\
6130413 \\
6130404 \\
6130272\end{array}$ & $\begin{array}{l}7.0 \\
0.0 \\
1.2 \\
2.9 \\
1.8\end{array}$ & $\begin{array}{l}2.4 \\
1.1 \\
1.0 \\
3.4 \\
7.6\end{array}$ & $\begin{array}{r}11.5 \\
0.3 \\
2.6 \\
3.0 \\
0.9\end{array}$ & $\begin{array}{r}1.9 \\
1.7 \\
0.6 \\
7.4 \\
11.7\end{array}$ & $\begin{array}{r}10.7 \\
0.3 \\
2.2 \\
20.3 \\
22.6\end{array}$ & $\begin{array}{r}16.4 \\
11.9 \\
5.7 \\
10.8 \\
60.6\end{array}$ & $\begin{array}{l}--- \\
--- \\
--- \\
--- \\
1.0\end{array}$ & $\begin{array}{l}--- \\
--- \\
--- \\
--- \\
0\end{array}$ & $\begin{array}{r}0 \\
0 \\
269 \\
15 \\
0\end{array}$ \\
\hline $\begin{array}{l}\mathrm{LINE} \\
\mathrm{A} \\
\mathrm{B}\end{array}$ & $\begin{array}{l}30280 \\
877.3 \\
959.0\end{array}$ & $\begin{array}{r}\mathrm{S} ? \\
\mathrm{H}\end{array}$ & $\begin{array}{l}321122 \\
322920\end{array}$ & $\begin{array}{l}6129944 \\
6129877\end{array}$ & $\begin{array}{l}0.8 \\
0.5\end{array}$ & $\begin{array}{l}1.8 \\
4.2\end{array}$ & $\begin{array}{l}0.3 \\
1.5\end{array}$ & $\begin{array}{l}3.0 \\
6.3\end{array}$ & $\begin{array}{l}0.1 \\
4.6\end{array}$ & $\begin{array}{l}23.5 \\
41.7\end{array}$ & $\begin{array}{l}--- \\
0.8\end{array}$ & $\begin{array}{r}--- \\
1\end{array}$ & $\begin{array}{l}6 \\
0\end{array}$ \\
\hline $\begin{array}{l}\text { LINE } \\
\text { A } \\
B \\
C\end{array}$ & $\begin{array}{r}30290 \\
1251.2 \\
1216.0 \\
1054.6\end{array}$ & $\begin{array}{r}\text { B } \\
\text { B? } \\
\text { D }\end{array}$ & $\begin{array}{l}319976 \\
320360 \\
322031\end{array}$ & $\begin{array}{l}6129607 \\
6129602 \\
6129507\end{array}$ & $\begin{array}{l}5.6 \\
0.8 \\
3.9\end{array}$ & $\begin{array}{l}5.5 \\
1.2 \\
7.4\end{array}$ & $\begin{array}{l}2.0 \\
1.0 \\
1.9\end{array}$ & $\begin{array}{l}4.0 \\
2.9 \\
9.4\end{array}$ & $\begin{array}{r}11.7 \\
6.2 \\
29.5\end{array}$ & $\begin{array}{r}6.3 \\
10.1 \\
40.2\end{array}$ & $\begin{array}{l}5.9 \\
1.5 \\
2.7\end{array}$ & $\begin{array}{r}25 \\
70 \\
4\end{array}$ & $\begin{array}{l}0 \\
0 \\
5\end{array}$ \\
\hline $\begin{array}{l}\text { LINE } \\
\text { A } \\
\text { B } \\
\text { C } \\
\text { D }\end{array}$ & $\begin{array}{l}30300 \\
1440.5 \\
1445.5 \\
1449.9 \\
1558.9\end{array}$ & $\begin{array}{l}\text { B } \\
\text { B } \\
\text { B } \\
\text { D }\end{array}$ & $\begin{array}{l}319750 \\
319818 \\
319873 \\
320394\end{array}$ & $\begin{array}{l}6129226 \\
6129225 \\
6129222 \\
6129234\end{array}$ & $\begin{array}{r}18.8 \\
18.7 \\
12.9 \\
5.6\end{array}$ & $\begin{array}{r}13.9 \\
9.3 \\
11.4 \\
5.5\end{array}$ & $\begin{array}{r}10.7 \\
15.3 \\
20.5 \\
1.6\end{array}$ & $\begin{array}{r}17.3 \\
19.3 \\
24.5 \\
4.8\end{array}$ & $\begin{array}{l}43.7 \\
43.2 \\
52.4 \\
12.5\end{array}$ & $\begin{array}{l}21.9 \\
13.7 \\
15.0 \\
12.4\end{array}$ & $\begin{array}{r}12.1 \\
18.2 \\
10.5 \\
5.1\end{array}$ & $\begin{array}{r}13 \\
2 \\
4 \\
27\end{array}$ & $\begin{array}{l}0 \\
0 \\
0 \\
2\end{array}$ \\
\hline
\end{tabular}

$\mathrm{CX}=$ COAXIAL

Note: EM values shown above

are local amplitudes

Ketchikan - Gravina Island West
* Estimated Depth may be unreliable because the stronger part of the conductor may be deeper or to one side of the flight line, or because of a shallow dip or magnetite/overburden effects 
EM Anomaly List

\begin{tabular}{|c|c|c|c|c|c|c|c|c|c|c|c|c|c|}
\hline Label & Fid & Interp & $\underset{\mathrm{m}}{\mathrm{XUTM}}$ & $\begin{array}{c}\text { YUTM } \\
\mathrm{m}\end{array}$ & $\begin{array}{l}\text { CX } \\
\text { Real } \\
\text { ppm }\end{array}$ & $\begin{array}{l}900 \mathrm{HZ} \\
\text { Quad } \\
\text { ppm }\end{array}$ & $\begin{array}{l}\text { CP } \\
\text { Real } \\
\text { ppm }\end{array}$ & $\begin{array}{l}900 \mathrm{HZ} \\
\text { Quad } \\
\text { ppm }\end{array}$ & $\begin{array}{l}\text { CP } \\
\text { Real } \\
\text { ppm }\end{array}$ & $\begin{array}{l}200 \mathrm{HZ} \\
\text { Quad } \\
\text { ppm }\end{array}$ & $\begin{array}{l}\text { Vertica } \\
\text { CoND } \\
\text { siemens }\end{array}$ & $\begin{array}{c}\text { al Dike } \\
\text { DEPTH* } \\
\text { m }\end{array}$ & $\begin{array}{c}\text { Mag. Corr } \\
\text { NT }\end{array}$ \\
\hline $\begin{array}{l}\text { LINE } \\
\text { E } \\
F \\
G \\
H\end{array}$ & $\begin{array}{l}30300 \\
1711.6 \\
1720.4 \\
1727.7 \\
1763.0\end{array}$ & $\begin{array}{r}\mathrm{B} ? \\
\mathrm{D} \\
\mathrm{B} ? \\
\mathrm{~B} ?\end{array}$ & $\begin{array}{l}321643 \\
321825 \\
321997 \\
322948\end{array}$ & $\begin{array}{l}6129141 \\
6129127 \\
6129114 \\
6129074\end{array}$ & $\begin{array}{l}3.1 \\
4.6 \\
1.1 \\
3.1\end{array}$ & $\begin{array}{r}4.4 \\
11.2 \\
4.4 \\
7.8\end{array}$ & $\begin{array}{l}0.8 \\
1.2 \\
0.1 \\
0.1\end{array}$ & $\begin{array}{l}2.2 \\
5.5 \\
4.5 \\
9.6\end{array}$ & $\begin{array}{r}8.2 \\
16.1 \\
10.4 \\
19.4\end{array}$ & $\begin{array}{l}13.3 \\
24.0 \\
31.6 \\
62.1\end{array}$ & $\begin{array}{l}2.9 \\
2.5 \\
2.1 \\
1.6\end{array}$ & $\begin{array}{r}56 \\
6 \\
20 \\
4\end{array}$ & $\begin{array}{l}0 \\
0 \\
3 \\
0\end{array}$ \\
\hline $\begin{array}{l}\text { LINE } \\
\text { A } \\
\text { B } \\
\text { C } \\
\text { D } \\
\text { E } \\
\text { F } \\
\text { G } \\
\text { H } \\
\text { I } \\
\text { J } \\
\text { K } \\
\text { L }\end{array}$ & $\begin{array}{r}30310 \\
2088.0 \\
2081.7 \\
2076.6 \\
2064.5 \\
2057.2 \\
2043.2 \\
1912.1 \\
1904.6 \\
1891.9 \\
1850.0 \\
1844.9 \\
1838.5\end{array}$ & $\begin{array}{r}\text { B? } \\
\text { B } \\
\text { B } \\
\text { B } \\
\text { B } \\
\text { B } \\
\text { B } \\
\text { B? } \\
\text { S } \\
\text { B? } \\
\text { B? } \\
\text { B? }\end{array}$ & $\begin{array}{l}319852 \\
319931 \\
319991 \\
320142 \\
320227 \\
320366 \\
321794 \\
321969 \\
322271 \\
323265 \\
323398 \\
323565\end{array}$ & $\begin{array}{l}6128793 \\
6128794 \\
6128789 \\
6128774 \\
6128759 \\
6128776 \\
6128696 \\
6128692 \\
6128677 \\
6128603 \\
6128602 \\
6128600\end{array}$ & $\begin{array}{r}17.0 \\
34.3 \\
15.9 \\
1.0 \\
2.0 \\
4.5 \\
1.3 \\
3.1 \\
0.4 \\
0.6 \\
0.8 \\
1.8\end{array}$ & $\begin{array}{r}12.3 \\
39.0 \\
15.2 \\
4.2 \\
3.7 \\
5.8 \\
6.9 \\
4.5 \\
7.4 \\
9.0 \\
6.7 \\
14.4\end{array}$ & $\begin{array}{r}27.2 \\
38.7 \\
14.0 \\
0.5 \\
2.1 \\
1.0 \\
1.3 \\
1.2 \\
0.3 \\
2.5 \\
4.6 \\
4.4\end{array}$ & $\begin{array}{r}38.9 \\
61.2 \\
25.3 \\
3.6 \\
4.5 \\
9.0 \\
6.2 \\
2.1 \\
8.1 \\
12.9 \\
15.1 \\
23.2\end{array}$ & $\begin{array}{r}95.0 \\
138.9 \\
69.2 \\
6.5 \\
16.8 \\
23.2 \\
15.9 \\
5.7 \\
9.7 \\
33.5 \\
47.1 \\
52.1\end{array}$ & $\begin{array}{r}49.6 \\
63.0 \\
42.2 \\
17.3 \\
15.5 \\
22.8 \\
25.3 \\
8.8 \\
73.6 \\
60.8 \\
69.7 \\
92.1\end{array}$ & $\begin{array}{r}11.2 \\
9.7 \\
--- \\
1.0 \\
2.0 \\
3.1 \\
1.1 \\
--- \\
0.5 \\
0.7 \\
1.4 \\
1.2\end{array}$ & $\begin{array}{r}0 \\
10 \\
--- \\
15 \\
49 \\
11 \\
0 \\
--- \\
0 \\
0 \\
4 \\
0\end{array}$ & $\begin{array}{l}0 \\
2 \\
2 \\
0 \\
0 \\
2 \\
0 \\
4 \\
0 \\
2 \\
0 \\
2\end{array}$ \\
\hline $\begin{array}{l}\text { LINE } \\
\text { A } \\
\text { B } \\
\text { C } \\
\text { D } \\
\text { E }\end{array}$ & $\begin{array}{r}30320 \\
2215.5 \\
2228.5 \\
2242.0 \\
2332.5 \\
2350.1\end{array}$ & $\begin{array}{r}\mathrm{B} ? \\
\mathrm{~B} ? \\
\mathrm{~B} ? \\
\mathrm{~B} \\
\mathrm{~B} ?\end{array}$ & $\begin{array}{l}319924 \\
320226 \\
320478 \\
321443 \\
321746\end{array}$ & $\begin{array}{l}6128398 \\
6128375 \\
6128357 \\
6128346 \\
6128333\end{array}$ & $\begin{array}{l}2.9 \\
1.9 \\
2.6 \\
1.3 \\
0.5\end{array}$ & $\begin{array}{l}3.3 \\
3.0 \\
2.4 \\
4.5 \\
2.5\end{array}$ & $\begin{array}{l}0.6 \\
1.5 \\
7.4 \\
0.2 \\
1.2\end{array}$ & $\begin{array}{r}1.0 \\
2.5 \\
12.9 \\
2.2 \\
5.2\end{array}$ & $\begin{array}{r}5.1 \\
10.1 \\
34.3 \\
2.2 \\
16.4\end{array}$ & $\begin{array}{r}5.9 \\
7.3 \\
16.3 \\
17.7 \\
14.0\end{array}$ & $\begin{array}{l}--- \\
2.6 \\
4.3 \\
1.2 \\
---\end{array}$ & $\begin{array}{r}--- \\
54 \\
29 \\
16 \\
---\end{array}$ & $\begin{array}{l}0 \\
2 \\
2 \\
2 \\
2\end{array}$ \\
\hline $\begin{array}{l}\text { LINE } \\
\text { A } \\
\text { B } \\
\text { C } \\
\text { D } \\
\text { E }\end{array}$ & $\begin{array}{l}30330 \\
2773.0 \\
2764.9 \\
2748.9 \\
2679.6 \\
2584.7\end{array}$ & $\begin{array}{r}\mathrm{B} \\
\mathrm{B} \\
\mathrm{B} ? \\
\mathrm{~B} \\
\mathrm{~B}\end{array}$ & $\begin{array}{l}320362 \\
320509 \\
320723 \\
321657 \\
323689\end{array}$ & $\begin{array}{l}6127988 \\
6127983 \\
6127978 \\
6127951 \\
6127806\end{array}$ & $\begin{array}{l}5.3 \\
5.7 \\
3.9 \\
3.0 \\
2.1\end{array}$ & $\begin{array}{l}3.2 \\
5.2 \\
3.8 \\
4.4 \\
5.8\end{array}$ & $\begin{array}{l}2.9 \\
4.7 \\
1.5 \\
1.3 \\
3.1\end{array}$ & $\begin{array}{l}3.7 \\
6.5 \\
3.4 \\
3.8 \\
6.7\end{array}$ & $\begin{array}{r}8.2 \\
17.3 \\
11.3 \\
9.7 \\
19.6\end{array}$ & $\begin{array}{r}5.8 \\
10.2 \\
11.2 \\
11.1 \\
25.9\end{array}$ & $\begin{array}{r}10.9 \\
7.1 \\
5.0 \\
2.6 \\
2.4\end{array}$ & $\begin{array}{l}23 \\
10 \\
45 \\
35 \\
12\end{array}$ & $\begin{array}{l}4 \\
0 \\
0 \\
4 \\
4\end{array}$ \\
\hline $\begin{array}{l}\text { LINE } \\
\text { A } \\
\text { B } \\
\text { C } \\
\text { D } \\
\text { E } \\
\text { F }\end{array}$ & $\begin{array}{r}30340 \\
2904.0 \\
2924.5 \\
3025.0 \\
3148.2 \\
3158.8 \\
3170.3\end{array}$ & $\begin{array}{r}\text { B } \\
\text { B } \\
\text { B? } \\
\text { B? } \\
\text { B? } \\
\text { B? }\end{array}$ & $\begin{array}{l}320392 \\
320631 \\
321568 \\
323476 \\
323817 \\
324186\end{array}$ & $\begin{array}{l}6127560 \\
6127548 \\
6127500 \\
6127437 \\
6127407 \\
6127399\end{array}$ & $\begin{array}{l}5.8 \\
3.2 \\
1.0 \\
1.0 \\
2.0 \\
4.1\end{array}$ & $\begin{array}{r}1.4 \\
4.1 \\
1.0 \\
7.2 \\
6.9 \\
12.5\end{array}$ & $\begin{array}{l}1.4 \\
1.4 \\
0.6 \\
0.8 \\
3.6 \\
5.3\end{array}$ & $\begin{array}{r}1.6 \\
2.4 \\
3.5 \\
9.8 \\
9.5 \\
21.2\end{array}$ & $\begin{array}{r}5.5 \\
7.2 \\
10.7 \\
25.3 \\
21.4 \\
74.2\end{array}$ & $\begin{array}{r}3.1 \\
5.2 \\
12.6 \\
55.5 \\
24.7 \\
61.0\end{array}$ & $\begin{array}{r}34.0 \\
3.8 \\
2.0 \\
0.7 \\
2.0 \\
2.3\end{array}$ & $\begin{array}{r}33 \\
39 \\
42 \\
0 \\
4 \\
0\end{array}$ & $\begin{array}{l}9 \\
0 \\
0 \\
0 \\
7 \\
0\end{array}$ \\
\hline $\begin{array}{l}\text { LINE } \\
\text { A } \\
\text { B } \\
\text { C } \\
\text { D }\end{array}$ & $\begin{array}{r}30350 \\
3494.8 \\
3476.5 \\
3468.8 \\
3421.6\end{array}$ & $\begin{array}{r}\mathrm{B} ? \\
\mathrm{~B} \\
\mathrm{~B} \\
\mathrm{D}\end{array}$ & $\begin{array}{l}320111 \\
320463 \\
320589 \\
321273\end{array}$ & $\begin{array}{l}6127216 \\
6127164 \\
6127177 \\
6127172\end{array}$ & $\begin{array}{l}0.8 \\
6.8 \\
8.7 \\
0.4\end{array}$ & $\begin{array}{l}3.8 \\
3.2 \\
8.4 \\
2.3\end{array}$ & $\begin{array}{l}1.9 \\
0.4 \\
1.9 \\
0.4\end{array}$ & $\begin{array}{l}4.3 \\
2.7 \\
7.4 \\
1.0\end{array}$ & $\begin{array}{r}11.2 \\
8.2 \\
21.4 \\
2.1\end{array}$ & $\begin{array}{r}23.8 \\
11.5 \\
23.8 \\
8.3\end{array}$ & $\begin{array}{l}--- \\
0.6 \\
--- \\
1.0\end{array}$ & $\begin{array}{r}--- \\
0 \\
--- \\
18\end{array}$ & $\begin{array}{l}2 \\
0 \\
0 \\
0\end{array}$ \\
\hline
\end{tabular}

$\mathrm{CX}=$ COAXIAL

Note: EM values shown above

are local amplitudes

*Estimated Depth may be unreliable because the stronger part of the conductor may be deeper or to one side of the flight line, or because of a

shallow dip or magnetite/overburden effects 
EM Anomaly List

\begin{tabular}{|c|c|c|c|c|c|c|c|c|c|c|c|c|c|}
\hline Label & Fid & Interp & $\begin{array}{l}\text { XUTM } \\
\text { m }\end{array}$ & $\begin{array}{c}\text { YUTM } \\
\text { m }\end{array}$ & $\begin{array}{l}\text { CX } \\
\text { Real } \\
\text { ppm }\end{array}$ & $\begin{array}{l}00 \mathrm{HZ} \\
\text { Quad } \\
\text { ppm }\end{array}$ & $\begin{array}{l}\text { CP } \\
\text { Real } \\
\text { ppm }\end{array}$ & $\begin{array}{l}900 \mathrm{HZ} \\
\text { Quad } \\
\text { ppm }\end{array}$ & $\begin{array}{l}\text { CP } \\
\text { Real } \\
\text { ppm }\end{array}$ & $\begin{array}{c}7200 \mathrm{HZ} \\
\text { Quad } \\
\text { ppm }\end{array}$ & $\begin{array}{l}\text { Vertica } \\
\text { CoND } \\
\text { siemens }\end{array}$ & $\begin{array}{c}\text { al Dike } \\
\text { DEPTH* } \\
\text { m }\end{array}$ & $\begin{array}{c}\text { Mag. Corr } \\
\text { NT }\end{array}$ \\
\hline $\begin{array}{l}\text { LINE } \\
\mathrm{E}\end{array}$ & $\begin{array}{r}30350 \\
3272.6\end{array}$ & S & 322422 & 6127014 & 0.7 & 4.7 & 0.3 & 7.1 & 3.9 & 52.9 & 0.5 & 6 & 0 \\
\hline $\begin{array}{l}\text { LINE } \\
\text { A } \\
\text { B } \\
\text { C }\end{array}$ & $\begin{array}{r}30360 \\
3595.4 \\
3603.5 \\
3772.0\end{array}$ & $\begin{array}{r}\mathrm{B} ? \\
\mathrm{~B} ? \\
\mathrm{~S}\end{array}$ & $\begin{array}{l}320250 \\
320486 \\
323195\end{array}$ & $\begin{array}{l}6126768 \\
6126744 \\
6126671\end{array}$ & $\begin{array}{l}5.2 \\
2.8 \\
1.9\end{array}$ & $\begin{array}{l}3.2 \\
5.9 \\
6.5\end{array}$ & $\begin{array}{l}3.5 \\
3.9 \\
0.8\end{array}$ & $\begin{array}{l}2.5 \\
8.2 \\
8.1\end{array}$ & $\begin{array}{r}4.2 \\
21.6 \\
11.9\end{array}$ & $\begin{array}{r}0.4 \\
16.8 \\
58.6\end{array}$ & $\begin{array}{l}--- \\
--- \\
1.3\end{array}$ & $\begin{array}{r}--- \\
--- \\
12\end{array}$ & $\begin{array}{r}0 \\
0 \\
16\end{array}$ \\
\hline $\begin{array}{l}\text { LINE } \\
\text { A } \\
\text { B } \\
\text { C } \\
\text { D } \\
\text { E } \\
\text { F } \\
\text { G } \\
\text { H } \\
\text { I }\end{array}$ & $\begin{array}{r}30370 \\
4094.8 \\
4091.2 \\
4086.0 \\
3989.6 \\
3966.6 \\
3892.8 \\
3880.0 \\
3868.8 \\
3863.5\end{array}$ & $\begin{array}{r}\text { B } \\
\text { B } \\
\text { B } \\
\text { D } \\
\text { S } \\
\text { B? } \\
\text { B? } \\
\text { S } \\
\text { M }\end{array}$ & $\begin{array}{l}320155 \\
320225 \\
320324 \\
321567 \\
321883 \\
322927 \\
323211 \\
323535 \\
323706\end{array}$ & $\begin{array}{l}6126356 \\
6126364 \\
6126365 \\
6126338 \\
6126290 \\
6126188 \\
6126241 \\
6126279 \\
6126284\end{array}$ & $\begin{array}{l}5.8 \\
5.8 \\
8.5 \\
3.2 \\
0.0 \\
0.9 \\
1.4 \\
3.9 \\
0.0\end{array}$ & $\begin{array}{r}10.7 \\
10.7 \\
13.3 \\
10.9 \\
2.0 \\
1.7 \\
2.3 \\
5.0 \\
0.4\end{array}$ & $\begin{array}{r}14.0 \\
12.9 \\
8.5 \\
0.0 \\
0.3 \\
0.9 \\
0.1 \\
6.0 \\
0.0\end{array}$ & $\begin{array}{r}23.1 \\
22.1 \\
23.2 \\
8.5 \\
2.9 \\
0.5 \\
1.4 \\
6.3 \\
0.3\end{array}$ & $\begin{array}{r}54.2 \\
54.2 \\
65.5 \\
19.1 \\
1.3 \\
0.1 \\
3.5 \\
8.3 \\
0.1\end{array}$ & $\begin{array}{r}35.8 \\
35.8 \\
51.6 \\
53.0 \\
26.2 \\
6.4 \\
5.1 \\
51.2 \\
4.2\end{array}$ & $\begin{array}{l}3.9 \\
3.8 \\
3.3 \\
1.4 \\
--- \\
2.5 \\
--- \\
--- \\
---\end{array}$ & $\begin{array}{r}21 \\
19 \\
13 \\
5 \\
--- \\
87 \\
--- \\
--- \\
---\end{array}$ & $\begin{array}{r}0 \\
0 \\
28 \\
0 \\
0 \\
28 \\
0 \\
0 \\
976\end{array}$ \\
\hline $\begin{array}{l}\text { LINE } \\
\text { A } \\
\text { B } \\
\text { C } \\
\text { D }\end{array}$ & $\begin{array}{r}30380 \\
4213.0 \\
4399.4 \\
4459.4 \\
4512.4\end{array}$ & $\begin{array}{r}B \\
B \\
S ? \\
M\end{array}$ & $\begin{array}{l}319768 \\
321518 \\
322483 \\
323884\end{array}$ & $\begin{array}{l}6126027 \\
6125914 \\
6125875 \\
6125844\end{array}$ & $\begin{array}{l}6.8 \\
2.0 \\
0.4 \\
0.0\end{array}$ & $\begin{array}{l}3.9 \\
3.9 \\
2.8 \\
1.1\end{array}$ & $\begin{array}{l}9.2 \\
0.8 \\
0.3 \\
1.4\end{array}$ & $\begin{array}{l}6.6 \\
1.6 \\
3.2 \\
2.1\end{array}$ & $\begin{array}{r}15.1 \\
0.5 \\
2.0 \\
1.6\end{array}$ & $\begin{array}{r}10.0 \\
12.8 \\
26.2 \\
4.4\end{array}$ & $\begin{array}{l}--- \\
1.9 \\
0.6 \\
---\end{array}$ & $\begin{array}{r}--- \\
59 \\
0 \\
---\end{array}$ & $\begin{array}{r}28 \\
0 \\
0 \\
1121\end{array}$ \\
\hline $\begin{array}{l}\text { LINE } \\
A \\
B\end{array}$ & $\begin{array}{r}30390 \\
4909.7 \\
4778.5\end{array}$ & $\begin{array}{r}B ? \\
S\end{array}$ & $\begin{array}{l}319842 \\
322531\end{array}$ & $\begin{array}{l}6125556 \\
6125473\end{array}$ & $\begin{array}{l}1.1 \\
0.4\end{array}$ & $\begin{array}{l}4.1 \\
1.2\end{array}$ & $\begin{array}{l}1.9 \\
0.2\end{array}$ & $\begin{array}{l}4.9 \\
1.0\end{array}$ & $\begin{array}{r}12.8 \\
0.1\end{array}$ & $\begin{array}{r}14.5 \\
8.7\end{array}$ & $\begin{array}{l}--- \\
---\end{array}$ & $\begin{array}{l}--- \\
---\end{array}$ & $\begin{array}{l}0 \\
0\end{array}$ \\
\hline $\begin{array}{l}\text { LINE } \\
A\end{array}$ & $\begin{array}{l}30400 \\
5034.9\end{array}$ & B? & 321161 & 6125115 & 1.3 & 3.3 & 1.1 & 7.9 & 23.1 & 48.4 & 1.4 & 0 & 0 \\
\hline $\begin{array}{l}\text { LINE } \\
\text { A } \\
B \\
C \\
D \\
\text { E }\end{array}$ & $\begin{array}{l}30410 \\
5573.8 \\
5566.7 \\
5554.2 \\
5394.0 \\
5268.7\end{array}$ & $\begin{array}{r}\text { B? } \\
\text { S } \\
\text { B } \\
\text { B } \\
\text { S }\end{array}$ & $\begin{array}{l}319799 \\
319914 \\
320046 \\
321748 \\
323629\end{array}$ & $\begin{array}{l}6124759 \\
6124759 \\
6124772 \\
6124694 \\
6124600\end{array}$ & $\begin{array}{l}0.3 \\
0.9 \\
0.4 \\
1.2 \\
0.8\end{array}$ & $\begin{array}{l}0.6 \\
2.2 \\
3.6 \\
1.6 \\
1.4\end{array}$ & $\begin{array}{l}0.7 \\
0.8 \\
0.2 \\
0.1 \\
0.6\end{array}$ & $\begin{array}{l}2.3 \\
1.2 \\
3.6 \\
1.4 \\
1.8\end{array}$ & $\begin{array}{l}3.3 \\
3.0 \\
2.2 \\
4.0 \\
1.0\end{array}$ & $\begin{array}{r}24.9 \\
10.1 \\
25.6 \\
6.2 \\
15.0\end{array}$ & $\begin{array}{l}--- \\
--- \\
0.5 \\
1.7 \\
---\end{array}$ & $\begin{array}{r}--- \\
--- \\
4 \\
68 \\
---\end{array}$ & $\begin{array}{l}0 \\
2 \\
6 \\
0 \\
0\end{array}$ \\
\hline $\begin{array}{l}\text { LINE } \\
\text { A } \\
B\end{array}$ & $\begin{array}{l}30420 \\
5643.6 \\
5739.0\end{array}$ & $\begin{array}{r}\mathrm{B} ? \\
\mathrm{D}\end{array}$ & $\begin{array}{l}319805 \\
321587\end{array}$ & $\begin{array}{l}6124390 \\
6124294\end{array}$ & $\begin{array}{l}2.2 \\
1.4\end{array}$ & $\begin{array}{l}2.0 \\
1.9\end{array}$ & $\begin{array}{l}2.2 \\
0.5\end{array}$ & $\begin{array}{l}0.9 \\
0.7\end{array}$ & $\begin{array}{l}2.7 \\
1.6\end{array}$ & $\begin{array}{l}6.5 \\
4.1\end{array}$ & $\begin{array}{l}--- \\
2.9\end{array}$ & $\begin{array}{r}--- \\
58\end{array}$ & $\begin{array}{l}1 \\
0\end{array}$ \\
\hline $\begin{array}{l}\text { LINE } \\
\text { A } \\
\text { B } \\
\text { C }\end{array}$ & $\begin{array}{r}30430 \\
6543.5 \\
6351.8 \\
6308.6\end{array}$ & $\begin{array}{l}\text { B? } \\
\text { B? } \\
\text { B? }\end{array}$ & $\begin{array}{l}319685 \\
321250 \\
321741\end{array}$ & $\begin{array}{l}6124002 \\
6123927 \\
6123953\end{array}$ & $\begin{array}{l}7.9 \\
0.9 \\
2.3\end{array}$ & $\begin{array}{r}10.7 \\
8.5 \\
4.8\end{array}$ & $\begin{array}{l}9.6 \\
0.4 \\
0.1\end{array}$ & $\begin{array}{r}14.3 \\
13.7 \\
0.3\end{array}$ & $\begin{array}{r}39.7 \\
19.6 \\
0.6\end{array}$ & $\begin{array}{r}36.6 \\
101.8 \\
5.7\end{array}$ & $\begin{array}{l}5.4 \\
0.5 \\
---\end{array}$ & $\begin{array}{r}25 \\
0 \\
---\end{array}$ & $\begin{array}{l}1 \\
0 \\
0\end{array}$ \\
\hline
\end{tabular}

$\mathrm{CX}=$ COAXIAL

$\mathrm{CP}=$ COPLANAR

Note: EM values shown above

are local amplitudes

Ketchikan - Gravina Island West
$-131-$
*Estimated Depth may be unreliable because the stronger part of the conductor may be deeper or to one side of the flight line, or because of a shallow dip or magnetite/overburden effects 
EM Anomaly List

\begin{tabular}{|c|c|c|c|c|c|c|c|c|c|c|c|c|c|}
\hline Label & Fid & Interp & $\begin{array}{l}\text { XUTM } \\
\text { m }\end{array}$ & $\begin{array}{c}\text { YUTM } \\
\text { m }\end{array}$ & $\begin{array}{l}\text { CX } \\
\text { Real } \\
\text { ppm }\end{array}$ & $\begin{array}{l}900 \mathrm{HZ} \\
\text { Quad } \\
\text { ppm }\end{array}$ & $\begin{array}{l}\text { CP } \\
\text { Real } \\
\text { ppm }\end{array}$ & $\begin{array}{c}900 \mathrm{HZ} \\
\text { Quad } \\
\text { ppm }\end{array}$ & $\begin{array}{l}\text { CP } 7 \\
\text { Real } \\
\text { ppm }\end{array}$ & $\begin{array}{l}200 \mathrm{HZ} \\
\text { Quad } \\
\text { ppm }\end{array}$ & $\begin{array}{l}\text { Vertica } \\
\text { ConD } \\
\text { siemens }\end{array}$ & $\begin{array}{c}\text { al Dike } \\
\text { DEPTH* } \\
\text { m }\end{array}$ & $\begin{array}{c}\text { Mag. Corr } \\
\text { NT }\end{array}$ \\
\hline $\begin{array}{l}\text { LINE } \\
\text { D }\end{array}$ & $\begin{array}{l}30430 \\
6097.4\end{array}$ & S & 324921 & 6123743 & 1.3 & 3.1 & 0.5 & 4.2 & 6.3 & 34.1 & 1.5 & 14 & 0 \\
\hline $\begin{array}{l}\text { LINE } \\
\text { A } \\
B\end{array}$ & $\begin{array}{l}30440 \\
6846.6 \\
6963.8\end{array}$ & $\begin{array}{r}\text { S } \\
\text { B? }\end{array}$ & $\begin{array}{l}321419 \\
322429\end{array}$ & $\begin{array}{l}6123506 \\
6123488\end{array}$ & $\begin{array}{l}0.0 \\
0.4\end{array}$ & $\begin{array}{l}2.1 \\
2.1\end{array}$ & $\begin{array}{l}0.1 \\
0.2\end{array}$ & $\begin{array}{l}2.6 \\
3.0\end{array}$ & $\begin{array}{l}1.0 \\
7.5\end{array}$ & $\begin{array}{l}16.3 \\
20.2\end{array}$ & $\begin{array}{l}--- \\
---\end{array}$ & $\begin{array}{l}--- \\
---\end{array}$ & $\begin{array}{l}1 \\
0\end{array}$ \\
\hline $\begin{array}{l}\text { LINE } \\
\text { A } \\
B\end{array}$ & $\begin{array}{l}30450 \\
7641.9 \\
7410.0\end{array}$ & $\begin{array}{r}B ? \\
S\end{array}$ & $\begin{array}{l}320076 \\
322325\end{array}$ & $\begin{array}{l}6123133 \\
6123058\end{array}$ & $\begin{array}{l}2.3 \\
0.5\end{array}$ & $\begin{array}{l}2.4 \\
0.7\end{array}$ & $\begin{array}{l}1.3 \\
0.5\end{array}$ & $\begin{array}{l}2.2 \\
0.5\end{array}$ & $\begin{array}{l}2.1 \\
0.7\end{array}$ & $\begin{array}{l}6.9 \\
7.3\end{array}$ & $\begin{array}{l}4.5 \\
---\end{array}$ & $\begin{array}{r}62 \\
---\end{array}$ & $\begin{array}{l}0 \\
1\end{array}$ \\
\hline $\begin{array}{l}\text { LINE } \\
\text { A } \\
\text { B } \\
\text { C } \\
\text { D }\end{array}$ & $\begin{array}{r}30460 \\
7847.3 \\
8086.5 \\
8179.2 \\
8247.5\end{array}$ & $\begin{array}{r}\text { B? } \\
\text { S } \\
\text { S } \\
\text { S }\end{array}$ & $\begin{array}{l}320094 \\
321806 \\
323154 \\
324211\end{array}$ & $\begin{array}{l}6122785 \\
6122721 \\
6122656 \\
6122615\end{array}$ & $\begin{array}{l}0.4 \\
0.6 \\
0.4 \\
0.1\end{array}$ & $\begin{array}{l}4.4 \\
0.7 \\
1.4 \\
2.2\end{array}$ & $\begin{array}{l}1.1 \\
0.7 \\
0.1 \\
0.3\end{array}$ & $\begin{array}{l}4.9 \\
1.5 \\
1.6 \\
0.6\end{array}$ & $\begin{array}{r}12.4 \\
1.6 \\
4.6 \\
5.8\end{array}$ & $\begin{array}{r}20.2 \\
10.1 \\
15.5 \\
5.1\end{array}$ & $\begin{array}{l}--- \\
--- \\
--- \\
0.5\end{array}$ & $\begin{array}{r}--- \\
--- \\
--- \\
10\end{array}$ & $\begin{array}{l}0 \\
1 \\
0 \\
0\end{array}$ \\
\hline $\begin{array}{l}\text { LINE } \\
\text { A } \\
\text { B } \\
\text { C } \\
\text { D } \\
\text { E }\end{array}$ & $\begin{array}{r}30470 \\
8886.5 \\
8619.2 \\
8547.6 \\
8518.9 \\
8399.5\end{array}$ & $\begin{array}{l}\text { B } \\
\text { S } \\
\text { S } \\
\text { S } \\
\text { S }\end{array}$ & $\begin{array}{l}320113 \\
322206 \\
322973 \\
323297 \\
325383\end{array}$ & $\begin{array}{l}6122275 \\
6122245 \\
6122186 \\
6122186 \\
6122111\end{array}$ & $\begin{array}{l}7.4 \\
1.6 \\
1.0 \\
0.4 \\
9.3\end{array}$ & $\begin{array}{r}10.5 \\
3.2 \\
2.2 \\
1.6 \\
16.0\end{array}$ & $\begin{array}{r}5.9 \\
0.3 \\
0.3 \\
0.0 \\
15.2\end{array}$ & $\begin{array}{r}13.9 \\
3.9 \\
0.6 \\
2.0 \\
33.9\end{array}$ & $\begin{array}{r}29.6 \\
4.0 \\
2.2 \\
1.0 \\
90.3\end{array}$ & $\begin{array}{r}36.8 \\
29.0 \\
4.1 \\
16.0 \\
38.3\end{array}$ & $\begin{array}{l}--- \\
--- \\
--- \\
--- \\
3.6\end{array}$ & $\begin{array}{l}--- \\
--- \\
--- \\
--- \\
0\end{array}$ & $\begin{array}{l}0 \\
0 \\
0 \\
0 \\
0\end{array}$ \\
\hline $\begin{array}{l}\text { LINE } \\
\text { A } \\
B\end{array}$ & $\begin{array}{l}30480 \\
9013.3 \\
9454.3\end{array}$ & $\begin{array}{r}\mathrm{S} \\
\mathrm{B} ?\end{array}$ & $\begin{array}{l}320695 \\
325691\end{array}$ & $\begin{array}{l}6121920 \\
6121679\end{array}$ & $\begin{array}{l}0.9 \\
4.1\end{array}$ & $\begin{array}{l}1.9 \\
8.0\end{array}$ & $\begin{array}{l}0.5 \\
1.6\end{array}$ & $\begin{array}{l}2.3 \\
9.4\end{array}$ & $\begin{array}{r}6.2 \\
35.7\end{array}$ & $\begin{array}{r}7.6 \\
44.0\end{array}$ & $\begin{array}{l}--- \\
2.6\end{array}$ & $\begin{array}{r}--- \\
3\end{array}$ & $\begin{array}{l}0 \\
0\end{array}$ \\
\hline $\begin{array}{l}\text { LINE } \\
\text { A } \\
B \\
C\end{array}$ & $\begin{array}{l}30490 \\
855.2 \\
694.0 \\
574.6\end{array}$ & $\begin{array}{l}\mathrm{H} \\
\mathrm{M} \\
\mathrm{M}\end{array}$ & $\begin{array}{l}320693 \\
321793 \\
322837\end{array}$ & $\begin{array}{l}6121538 \\
6121416 \\
6121400\end{array}$ & $\begin{array}{l}2.4 \\
0.8 \\
0.1\end{array}$ & $\begin{array}{l}4.5 \\
0.6 \\
0.7\end{array}$ & $\begin{array}{l}1.9 \\
6.3 \\
1.0\end{array}$ & $\begin{array}{l}6.1 \\
0.6 \\
0.4\end{array}$ & $\begin{array}{r}18.3 \\
6.0 \\
0.1\end{array}$ & $\begin{array}{r}24.7 \\
2.6 \\
1.8\end{array}$ & $\begin{array}{l}2.6 \\
--- \\
---\end{array}$ & $\begin{array}{r}17 \\
--- \\
---\end{array}$ & $\begin{array}{l}0 \\
0 \\
0\end{array}$ \\
\hline $\begin{array}{l}\text { LINE } \\
\text { A } \\
B\end{array}$ & $\begin{array}{l}30500 \\
957.0 \\
1230.0\end{array}$ & $\begin{array}{r}\mathrm{B} ? \\
\mathrm{M}\end{array}$ & $\begin{array}{l}320495 \\
321948\end{array}$ & $\begin{array}{l}6121122 \\
6121121\end{array}$ & $\begin{array}{l}0.5 \\
2.7\end{array}$ & $\begin{array}{l}3.5 \\
0.4\end{array}$ & $\begin{array}{l}1.8 \\
7.3\end{array}$ & $\begin{array}{l}7.2 \\
0.1\end{array}$ & $\begin{array}{r}21.1 \\
7.3\end{array}$ & $\begin{array}{r}29.8 \\
1.7\end{array}$ & $\begin{array}{l}1.0 \\
---\end{array}$ & $\begin{array}{r}0 \\
---\end{array}$ & $\begin{array}{r}10 \\
0\end{array}$ \\
\hline $\begin{array}{l}\text { LINE } \\
\text { A } \\
\text { B } \\
\text { C } \\
\text { D } \\
\text { E } \\
\text { F } \\
\text { G } \\
\text { H }\end{array}$ & $\begin{array}{r}30510 \\
2553.7 \\
2546.4 \\
2400.0 \\
2369.0 \\
2245.0 \\
2136.0 \\
2068.0 \\
1853.0\end{array}$ & $\begin{array}{c}\text { B? } \\
\text { B? } \\
\text { M } \\
\text { M } \\
\text { S } \\
\text { M } \\
\text { M } \\
\text { H }\end{array}$ & $\begin{array}{l}320731 \\
320888 \\
322079 \\
322193 \\
322752 \\
323620 \\
323983 \\
325710\end{array}$ & $\begin{array}{l}6120760 \\
6120761 \\
6120699 \\
6120707 \\
6120629 \\
6120603 \\
6120572 \\
6120497\end{array}$ & $\begin{array}{l}1.4 \\
1.2 \\
4.7 \\
0.5 \\
0.7 \\
4.4 \\
1.4 \\
3.3\end{array}$ & $\begin{array}{l}2.4 \\
2.9 \\
0.3 \\
0.4 \\
2.9 \\
0.3 \\
0.2 \\
3.7\end{array}$ & $\begin{array}{r}2.5 \\
0.8 \\
6.3 \\
5.0 \\
1.1 \\
14.3 \\
0.7 \\
1.0\end{array}$ & $\begin{array}{l}7.7 \\
1.6 \\
0.3 \\
0.5 \\
3.4 \\
0.1 \\
0.4 \\
3.9\end{array}$ & $\begin{array}{r}26.3 \\
2.8 \\
5.7 \\
4.7 \\
3.4 \\
13.6 \\
0.5 \\
11.5\end{array}$ & $\begin{array}{r}29.5 \\
9.8 \\
0.6 \\
1.6 \\
24.3 \\
0.1 \\
3.7 \\
20.8\end{array}$ & $\begin{array}{l}2.2 \\
--- \\
--- \\
--- \\
--- \\
--- \\
--- \\
3.3\end{array}$ & $\begin{array}{c}16 \\
--- \\
--- \\
--- \\
--- \\
--- \\
--- \\
41\end{array}$ & $\begin{array}{r}0 \\
0 \\
0 \\
0 \\
0 \\
15 \\
73 \\
18\end{array}$ \\
\hline
\end{tabular}

$\mathrm{CX}=$ COAXIAL

Note: EM values shown above

are local amplitudes

Ketchikan - Gravina Island West
* Estimated Depth may be unreliable because the stronger part of the conductor may be deeper or to one side of the flight line, or because of a shallow dip or magnetite/overburden effects 
EM Anomaly List

\begin{tabular}{|c|c|c|c|c|c|c|c|c|c|c|c|c|c|}
\hline Label & Fid & Interp & $\begin{array}{c}\text { XUTM } \\
\text { m }\end{array}$ & $\begin{array}{c}\text { YUTM } \\
\mathrm{m}\end{array}$ & $\begin{array}{l}\text { CX } \\
\text { Real } \\
\text { ppm }\end{array}$ & $\begin{array}{l}900 \mathrm{HZ} \\
\text { Quad } \\
\text { ppm }\end{array}$ & $\begin{array}{l}\text { CP } \\
\text { Real } \\
\text { ppm }\end{array}$ & $\begin{array}{c}900 \mathrm{HZ} \\
\text { Quad } \\
\text { ppm }\end{array}$ & $\begin{array}{l}\text { CP } 7 \\
\text { Real } \\
\text { ppm }\end{array}$ & $\begin{array}{l}7200 \mathrm{HZ} \\
\text { Quad } \\
\text { ppm }\end{array}$ & $\begin{array}{l}\text { Vertica } \\
\text { COND } \\
\text { siemens }\end{array}$ & $\begin{array}{c}\text { al Dike } \\
\text { DEPTH* }^{\star} \\
\text { m }\end{array}$ & $\begin{array}{c}\text { Mag. Corr } \\
\text { NT }\end{array}$ \\
\hline $\begin{array}{l}\text { LINE } \\
\text { A } \\
\text { B } \\
\text { C } \\
\text { D } \\
\text { E } \\
\text { F } \\
\text { G } \\
\text { H } \\
\text { I } \\
\text { J }\end{array}$ & $\begin{array}{r}30520 \\
2666.0 \\
2690.0 \\
2880.0 \\
3081.5 \\
3100.0 \\
3104.0 \\
3192.5 \\
3305.3 \\
3345.0 \\
3364.0\end{array}$ & $\begin{array}{r}\mathrm{H} \\
\mathrm{B} ? \\
\mathrm{M} \\
\mathrm{M} \\
\mathrm{H} \\
\mathrm{M} \\
\mathrm{B} ? \\
\mathrm{~S} \\
\mathrm{~B} ? \\
\mathrm{~B} ?\end{array}$ & $\begin{array}{l}320876 \\
321432 \\
322313 \\
323542 \\
323659 \\
323669 \\
324361 \\
325456 \\
326101 \\
326572\end{array}$ & $\begin{array}{l}6120323 \\
6120248 \\
6120237 \\
6120215 \\
6120211 \\
6120208 \\
6119967 \\
6120101 \\
6120067 \\
6120027\end{array}$ & $\begin{array}{l}2.1 \\
1.5 \\
1.2 \\
0.5 \\
0.4 \\
2.0 \\
0.9 \\
2.2 \\
1.1 \\
4.8\end{array}$ & $\begin{array}{l}1.9 \\
1.9 \\
0.1 \\
0.4 \\
0.3 \\
0.5 \\
1.8 \\
4.6 \\
3.7 \\
8.8\end{array}$ & $\begin{array}{l}2.5 \\
0.1 \\
2.0 \\
1.0 \\
4.9 \\
3.8 \\
0.4 \\
0.9 \\
1.0 \\
6.4\end{array}$ & $\begin{array}{r}3.7 \\
1.3 \\
0.2 \\
0.2 \\
0.5 \\
0.1 \\
1.4 \\
7.8 \\
4.5 \\
12.0\end{array}$ & $\begin{array}{r}7.7 \\
1.2 \\
2.1 \\
0.8 \\
4.0 \\
3.7 \\
4.0 \\
30.4 \\
10.8 \\
32.3\end{array}$ & $\begin{array}{r}9.4 \\
6.6 \\
2.2 \\
3.1 \\
1.1 \\
0.9 \\
4.5 \\
29.8 \\
26.7 \\
28.0\end{array}$ & $\begin{array}{c}4.8 \\
2.1 \\
--- \\
--- \\
52.0 \\
---- \\
--- \\
1.8 \\
--- \\
3.1\end{array}$ & $\begin{array}{r}32 \\
61 \\
--- \\
--- \\
78 \\
--- \\
--- \\
11 \\
--- \\
36\end{array}$ & $\begin{array}{l}0 \\
0 \\
0 \\
0 \\
0 \\
0 \\
9 \\
0 \\
0 \\
0\end{array}$ \\
\hline $\begin{array}{l}\text { LINE } \\
\text { A } \\
\text { B } \\
\text { C } \\
\text { D } \\
\text { E } \\
\text { F } \\
\text { G } \\
\text { H } \\
\text { I } \\
\text { J } \\
\text { K } \\
\text { L } \\
\text { M }\end{array}$ & $\begin{array}{r}30530 \\
4288.3 \\
4270.3 \\
4261.3 \\
4253.5 \\
4010.0 \\
4006.0 \\
4000.0 \\
3986.0 \\
3956.0 \\
3715.1 \\
3575.9 \\
3574.0 \\
3545.0\end{array}$ & $\begin{array}{r}\text { S } \\
\text { B } \\
\text { B? } \\
\text { B? } \\
\text { M } \\
\text { M } \\
\text { M } \\
\text { M } \\
\text { M } \\
\text { B } \\
\text { B? } \\
\text { B? } \\
\text { B }\end{array}$ & $\begin{array}{l}320267 \\
320748 \\
320957 \\
321127 \\
323374 \\
323424 \\
323516 \\
323691 \\
323824 \\
325182 \\
326366 \\
326422 \\
327201\end{array}$ & $\begin{array}{l}6119907 \\
6119894 \\
6119894 \\
6119872 \\
6119811 \\
6119820 \\
6119817 \\
6119785 \\
6119748 \\
6119710 \\
6119674 \\
6119674 \\
6119602\end{array}$ & $\begin{array}{r}26.8 \\
0.7 \\
10.6 \\
6.0 \\
0.4 \\
0.9 \\
1.0 \\
1.4 \\
1.0 \\
3.6 \\
1.6 \\
0.6 \\
1.7\end{array}$ & $\begin{array}{r}24.6 \\
4.6 \\
14.0 \\
8.0 \\
0.4 \\
0.6 \\
0.4 \\
0.6 \\
0.2 \\
4.6 \\
3.6 \\
2.2 \\
2.1\end{array}$ & $\begin{array}{r}63.5 \\
3.2 \\
6.9 \\
6.5 \\
3.6 \\
5.2 \\
0.9 \\
0.2 \\
1.0 \\
0.9 \\
0.7 \\
1.4 \\
4.6\end{array}$ & $\begin{array}{r}64.2 \\
11.3 \\
22.1 \\
12.4 \\
0.1 \\
0.3 \\
0.2 \\
0.1 \\
0.1 \\
3.5 \\
3.9 \\
3.7 \\
4.9\end{array}$ & $\begin{array}{r}121.5 \\
34.5 \\
81.3 \\
37.6 \\
3.4 \\
5.4 \\
1.0 \\
0.7 \\
1.6 \\
14.1 \\
9.0 \\
8.5 \\
17.4\end{array}$ & $\begin{array}{r}14.9 \\
41.9 \\
68.8 \\
37.0 \\
0.2 \\
2.9 \\
3.2 \\
0.6 \\
1.2 \\
13.1 \\
19.7 \\
15.4 \\
0.5\end{array}$ & $\begin{array}{r}15.4 \\
1.3 \\
3.9 \\
--- \\
--- \\
--- \\
--- \\
--- \\
--- \\
--- \\
1.8 \\
1.4 \\
---\end{array}$ & $\begin{array}{r}0 \\
0 \\
12 \\
--- \\
--- \\
--- \\
--- \\
--- \\
--- \\
--- \\
17 \\
21 \\
---\end{array}$ & $\begin{array}{r}0 \\
0 \\
0 \\
0 \\
45 \\
0 \\
29 \\
0 \\
0 \\
7 \\
0 \\
0 \\
0\end{array}$ \\
\hline $\begin{array}{l}\text { LINE } \\
\text { A } \\
\text { B } \\
\text { C } \\
\text { D } \\
\text { E } \\
\text { F } \\
\text { G } \\
\text { H } \\
\text { I } \\
\text { J }\end{array}$ & $\begin{array}{r}30540 \\
4359.2 \\
4375.8 \\
4390.4 \\
4570.5 \\
4690.0 \\
4702.9 \\
4716.1 \\
4734.1 \\
4852.1 \\
4867.6\end{array}$ & $\begin{array}{r}\text { B } \\
\text { B? } \\
\mathrm{H} \\
\mathrm{H} \\
\mathrm{B} \\
\mathrm{B} \\
\mathrm{B} ? \\
\mathrm{~B} \\
\mathrm{~B} ? \\
\mathrm{~S}\end{array}$ & $\begin{array}{l}320458 \\
320980 \\
321433 \\
323294 \\
324517 \\
324638 \\
324775 \\
324965 \\
326409 \\
326852\end{array}$ & $\begin{array}{l}6119541 \\
6119522 \\
6119480 \\
6119382 \\
6119325 \\
6119315 \\
6119301 \\
6119295 \\
6119237 \\
6119234\end{array}$ & $\begin{array}{r}1.6 \\
12.1 \\
1.8 \\
3.3 \\
1.7 \\
1.6 \\
2.6 \\
6.7 \\
8.7 \\
45.0\end{array}$ & $\begin{array}{r}4.2 \\
16.3 \\
0.0 \\
6.5 \\
2.5 \\
4.5 \\
4.6 \\
6.1 \\
15.8 \\
36.9\end{array}$ & $\begin{array}{r}0.5 \\
17.1 \\
3.5 \\
1.1 \\
0.3 \\
0.8 \\
1.5 \\
0.4 \\
12.7 \\
90.1\end{array}$ & $\begin{array}{r}2.1 \\
32.1 \\
1.2 \\
5.4 \\
0.7 \\
1.0 \\
2.1 \\
2.4 \\
31.7 \\
93.6\end{array}$ & $\begin{array}{r}3.6 \\
93.6 \\
4.0 \\
17.6 \\
2.5 \\
9.5 \\
8.1 \\
9.9 \\
91.3 \\
184.1\end{array}$ & $\begin{array}{r}15.9 \\
52.4 \\
6.2 \\
18.1 \\
3.0 \\
0.1 \\
26.4 \\
6.2 \\
49.0 \\
38.1\end{array}$ & $\begin{array}{r}1.7 \\
5.3 \\
--- \\
2.5 \\
--- \\
2.0 \\
--- \\
6.7 \\
4.4 \\
18.9\end{array}$ & $\begin{array}{r}12 \\
11 \\
--- \\
7 \\
--- \\
28 \\
--- \\
19 \\
0 \\
0\end{array}$ & $\begin{array}{l}0 \\
0 \\
0 \\
0 \\
0 \\
5 \\
0 \\
0 \\
0 \\
0\end{array}$ \\
\hline $\begin{array}{l}\text { LINE } \\
\text { A } \\
\text { B } \\
\text { C } \\
\text { D } \\
\text { E } \\
\text { F }\end{array}$ & $\begin{array}{r}30550 \\
5612.6 \\
5607.4 \\
5601.4 \\
5512.1 \\
5492.3 \\
5349.5\end{array}$ & $\begin{array}{r}\mathrm{B} ? \\
\mathrm{~B} \\
\mathrm{~B} \\
\mathrm{~B} ? \\
\mathrm{~B} ? \\
\mathrm{~B} ?\end{array}$ & $\begin{array}{l}320862 \\
321001 \\
321165 \\
322484 \\
322595 \\
323386\end{array}$ & $\begin{array}{l}6119072 \\
6119077 \\
6119081 \\
6119032 \\
6119045 \\
6119000\end{array}$ & $\begin{array}{l}1.7 \\
4.6 \\
1.3 \\
3.8 \\
2.1 \\
3.2\end{array}$ & $\begin{array}{r}11.1 \\
13.1 \\
7.1 \\
5.0 \\
3.3 \\
0.5\end{array}$ & $\begin{array}{l}0.3 \\
3.1 \\
2.4 \\
6.0 \\
1.8 \\
4.8\end{array}$ & $\begin{array}{r}13.2 \\
17.9 \\
6.3 \\
11.8 \\
1.6 \\
3.8\end{array}$ & $\begin{array}{r}24.3 \\
77.7 \\
20.6 \\
31.5 \\
4.3 \\
7.2\end{array}$ & $\begin{array}{r}95.4 \\
111.3 \\
39.4 \\
22.6 \\
2.6 \\
5.2\end{array}$ & $\begin{array}{l}0.6 \\
2.1 \\
1.4 \\
3.6 \\
--- \\
---\end{array}$ & $\begin{array}{r}0 \\
2 \\
5 \\
26 \\
--- \\
---\end{array}$ & $\begin{array}{l}7 \\
1 \\
7 \\
0 \\
0 \\
0\end{array}$ \\
\hline
\end{tabular}

$\mathrm{CX}=$ COAXIAL

Note: EM values shown above

are local amplitudes

*Estimated Depth may be unreliable because the stronger part of the conductor may be deeper or to one side of the flight line, or because of a shallow dip or magnetite/overburden effects 
EM Anomaly List

\begin{tabular}{|c|c|c|c|c|c|c|c|c|c|c|c|c|c|}
\hline Label & Fid & Interp & $\begin{array}{c}\text { XUTM } \\
\text { m }\end{array}$ & $\begin{array}{c}\text { YUTM } \\
\mathrm{m}\end{array}$ & $\begin{array}{l}\text { CX } \\
\text { Real } \\
\text { ppm }\end{array}$ & $\begin{array}{l}900 \mathrm{HZ} \\
\text { Quad } \\
\text { ppm }\end{array}$ & $\begin{array}{l}\text { CP } \\
\text { Real } \\
\text { ppm }\end{array}$ & $\begin{array}{l}900 \mathrm{HZ} \\
\text { Quad } \\
\text { ppm }\end{array}$ & $\begin{array}{l}\mathrm{CP} \\
\mathrm{Real} \\
\mathrm{ppm}\end{array}$ & $\begin{array}{c}7200 \mathrm{HZ} \\
\text { Quad } \\
\text { ppm }\end{array}$ & $\begin{array}{l}\text { Vertica } \\
\text { CoND } \\
\text { siemens }\end{array}$ & $\begin{array}{c}\text { al Dike } \\
\text { DEPTH* } \\
\text { m }\end{array}$ & $\begin{array}{c}\text { Mag. Corr } \\
\text { NT }\end{array}$ \\
\hline $\begin{array}{l}\text { LINE } \\
\text { G } \\
\text { H } \\
\text { I }\end{array}$ & $\begin{array}{r}30550 \\
5241.9 \\
5061.0 \\
5052.0\end{array}$ & $\begin{array}{l}\mathrm{B} \\
\mathrm{M} \\
\mathrm{B}\end{array}$ & $\begin{array}{l}324892 \\
325952 \\
326120\end{array}$ & $\begin{array}{l}6118964 \\
6118880 \\
6118861\end{array}$ & $\begin{array}{l}1.1 \\
1.0 \\
1.7\end{array}$ & $\begin{array}{l}5.8 \\
0.2 \\
2.1\end{array}$ & $\begin{array}{l}0.5 \\
0.0 \\
2.7\end{array}$ & $\begin{array}{l}5.0 \\
0.3 \\
1.7\end{array}$ & $\begin{array}{l}54.2 \\
43.8 \\
39.2\end{array}$ & $\begin{array}{r}26.5 \\
4.3 \\
9.6\end{array}$ & $\begin{array}{l}0.8 \\
--- \\
5.9\end{array}$ & $\begin{array}{r}14 \\
--- \\
52\end{array}$ & $\begin{array}{l}0 \\
0 \\
0\end{array}$ \\
\hline $\begin{array}{l}\text { LINE } \\
\text { A } \\
B \\
C\end{array}$ & $\begin{array}{r}30560 \\
5739.5 \\
5904.9 \\
6095.0\end{array}$ & $\begin{array}{r}\mathrm{B} ? \\
\mathrm{~B} \\
\mathrm{M}\end{array}$ & $\begin{array}{l}322035 \\
323445 \\
325765\end{array}$ & $\begin{array}{l}6118634 \\
6118603 \\
6118533\end{array}$ & $\begin{array}{l}3.2 \\
1.1 \\
0.7\end{array}$ & $\begin{array}{l}1.3 \\
2.0 \\
0.4\end{array}$ & $\begin{array}{l}1.1 \\
0.8 \\
1.6\end{array}$ & $\begin{array}{l}3.7 \\
0.2 \\
0.9\end{array}$ & $\begin{array}{r}10.6 \\
1.4 \\
0.3\end{array}$ & $\begin{array}{r}11.1 \\
8.6 \\
4.3\end{array}$ & $\begin{array}{l}7.3 \\
--- \\
---\end{array}$ & $\begin{array}{r}27 \\
--- \\
---\end{array}$ & $\begin{array}{l}0 \\
0 \\
0\end{array}$ \\
\hline $\begin{array}{l}\text { LINE } \\
\text { A } \\
B\end{array}$ & $\begin{array}{r}30571 \\
6635.3 \\
6631.5\end{array}$ & $\begin{array}{l}\text { B? } \\
\text { B? }\end{array}$ & $\begin{array}{l}321672 \\
321745\end{array}$ & $\begin{array}{l}6118272 \\
6118269\end{array}$ & $\begin{array}{l}4.6 \\
4.0\end{array}$ & $\begin{array}{l}17.2 \\
13.1\end{array}$ & $\begin{array}{l}6.1 \\
1.6\end{array}$ & $\begin{array}{l}35.3 \\
13.3\end{array}$ & $\begin{array}{r}132.1 \\
48.0\end{array}$ & $\begin{array}{r}113.2 \\
59.0\end{array}$ & $\begin{array}{l}1.9 \\
---\end{array}$ & $\begin{array}{r}0 \\
---\end{array}$ & $\begin{array}{l}8 \\
0\end{array}$ \\
\hline $\begin{array}{l}\text { LINE } \\
\text { A } \\
B\end{array}$ & $\begin{array}{l}30581 \\
6765.2 \\
7122.5\end{array}$ & $\begin{array}{l}\mathrm{B} \\
\mathrm{M}\end{array}$ & $\begin{array}{l}321850 \\
326227\end{array}$ & $\begin{array}{l}6117851 \\
6117668\end{array}$ & $\begin{array}{l}0.9 \\
1.3\end{array}$ & $\begin{array}{l}3.2 \\
0.5\end{array}$ & $\begin{array}{l}0.8 \\
1.4\end{array}$ & $\begin{array}{l}3.3 \\
0.4\end{array}$ & $\begin{array}{l}9.5 \\
1.7\end{array}$ & $\begin{array}{r}15.2 \\
1.8\end{array}$ & $\begin{array}{l}1.2 \\
---\end{array}$ & $\begin{array}{r}10 \\
---\end{array}$ & $\begin{array}{r}0 \\
105\end{array}$ \\
\hline $\begin{array}{l}\text { LINE } \\
\text { A } \\
B \\
\text { C }\end{array}$ & $\begin{array}{r}30591 \\
6347.8 \\
6343.3 \\
6332.8\end{array}$ & $\begin{array}{l}\text { B? } \\
\text { B? } \\
\text { B? }\end{array}$ & $\begin{array}{l}321536 \\
321686 \\
322008\end{array}$ & $\begin{array}{l}6117438 \\
6117439 \\
6117471\end{array}$ & $\begin{array}{l}2.7 \\
0.2 \\
4.2\end{array}$ & $\begin{array}{l}6.1 \\
7.1 \\
2.8\end{array}$ & $\begin{array}{l}2.6 \\
0.4 \\
2.0\end{array}$ & $\begin{array}{l}9.6 \\
7.3 \\
3.7\end{array}$ & $\begin{array}{r}22.4 \\
15.0 \\
5.5\end{array}$ & $\begin{array}{l}40.9 \\
41.9 \\
14.1\end{array}$ & $\begin{array}{l}2.3 \\
--- \\
---\end{array}$ & $\begin{array}{r}11 \\
--- \\
---\end{array}$ & $\begin{array}{l}0 \\
0 \\
1\end{array}$ \\
\hline $\begin{array}{l}\text { LINE } \\
\text { A }\end{array}$ & $\begin{array}{l}30602 \\
6072.3\end{array}$ & $\mathrm{~B}$ ? & 326114 & 6116866 & 1.5 & 2.2 & 1.3 & 2.5 & 3.1 & 19.4 & 2.4 & 51 & 8 \\
\hline $\begin{array}{l}\text { LINE } \\
\mathrm{A}\end{array}$ & $\begin{array}{l}30612 \\
5790.4\end{array}$ & B? & 324705 & 6116516 & 3.8 & 1.0 & 0.0 & 0.5 & 1.2 & 3.4 & --- & --- & 5 \\
\hline $\begin{array}{l}\text { LINE } \\
\text { A } \\
B\end{array}$ & $\begin{array}{l}30621 \\
5601.5 \\
5655.3\end{array}$ & $\begin{array}{l}\text { B? } \\
\text { B? }\end{array}$ & $\begin{array}{l}322573 \\
324362\end{array}$ & $\begin{array}{l}6116150 \\
6116135\end{array}$ & $\begin{array}{l}6.4 \\
2.1\end{array}$ & $\begin{array}{l}2.2 \\
2.3\end{array}$ & $\begin{array}{r}16.2 \\
1.6\end{array}$ & $\begin{array}{r}16.1 \\
3.3\end{array}$ & $\begin{array}{r}38.8 \\
6.4\end{array}$ & $\begin{array}{l}17.3 \\
21.2\end{array}$ & $\begin{array}{r}15.2 \\
3.5\end{array}$ & $\begin{array}{r}0 \\
47\end{array}$ & $\begin{array}{l}7 \\
0\end{array}$ \\
\hline $\begin{array}{l}\text { LINE } \\
\text { A } \\
B \\
\text { C }\end{array}$ & $\begin{array}{r}30631 \\
5432.6 \\
5403.2 \\
5393.0\end{array}$ & $\begin{array}{r}\text { S } \\
\text { B? } \\
\text { B? }\end{array}$ & $\begin{array}{l}322604 \\
323836 \\
324245\end{array}$ & $\begin{array}{l}6115777 \\
6115740 \\
6115720\end{array}$ & $\begin{array}{r}99.2 \\
2.7 \\
0.1\end{array}$ & $\begin{array}{r}87.8 \\
2.8 \\
2.6\end{array}$ & $\begin{array}{r}166.3 \\
1.6 \\
0.5\end{array}$ & $\begin{array}{r}194.9 \\
2.7 \\
2.9\end{array}$ & $\begin{array}{r}342.7 \\
2.8 \\
3.5\end{array}$ & $\begin{array}{l}62.0 \\
15.8 \\
20.1\end{array}$ & $\begin{array}{r}21.0 \\
4.4 \\
---\end{array}$ & $\begin{array}{r}0 \\
42 \\
---\end{array}$ & $\begin{array}{l}0 \\
0 \\
0\end{array}$ \\
\hline $\begin{array}{l}\text { LINE } \\
\text { A } \\
B \\
\text { C }\end{array}$ & $\begin{array}{r}30641 \\
5231.4 \\
5253.2 \\
5268.9\end{array}$ & $\begin{array}{r}\mathrm{S} \\
\mathrm{B} ? \\
\mathrm{~B}\end{array}$ & $\begin{array}{l}322622 \\
323329 \\
323875\end{array}$ & $\begin{array}{l}6115405 \\
6115339 \\
6115376\end{array}$ & $\begin{array}{r}39.6 \\
1.0 \\
4.3\end{array}$ & $\begin{array}{r}44.9 \\
2.0 \\
0.4\end{array}$ & $\begin{array}{r}70.2 \\
0.5 \\
0.5\end{array}$ & $\begin{array}{r}98.5 \\
2.3 \\
0.0\end{array}$ & $\begin{array}{r}188.5 \\
3.6 \\
0.6\end{array}$ & $\begin{array}{r}49.2 \\
15.0 \\
0.6\end{array}$ & $\begin{array}{r}11.3 \\
--- \\
---\end{array}$ & $\begin{array}{r}0 \\
--- \\
---\end{array}$ & $\begin{array}{l}7 \\
7 \\
0\end{array}$ \\
\hline $\begin{array}{l}\text { LINE } \\
\text { A } \\
B \\
C\end{array}$ & $\begin{array}{r}30651 \\
5171.2 \\
5142.9 \\
5136.6\end{array}$ & $\begin{array}{r}\mathrm{B} ? \\
\mathrm{~S} \\
\mathrm{~S}\end{array}$ & $\begin{array}{l}323207 \\
324410 \\
324679\end{array}$ & $\begin{array}{l}6114997 \\
6114868 \\
6114854\end{array}$ & $\begin{array}{r}2.5 \\
24.6 \\
7.7\end{array}$ & $\begin{array}{r}2.1 \\
23.9 \\
14.1\end{array}$ & $\begin{array}{r}1.0 \\
77.7 \\
1.7\end{array}$ & $\begin{array}{r}2.1 \\
62.7 \\
15.9\end{array}$ & $\begin{array}{r}4.3 \\
116.5 \\
50.4\end{array}$ & $\begin{array}{r}9.8 \\
21.4 \\
45.5\end{array}$ & $\begin{array}{r}5.1 \\
18.5 \\
3.1\end{array}$ & $\begin{array}{r}42 \\
0 \\
0\end{array}$ & $\begin{array}{l}0 \\
0 \\
8\end{array}$ \\
\hline
\end{tabular}

$\mathrm{CX}=$ COAXIAL

$\mathrm{CP}=$ COPLANAR

Note: EM values shown above

are local amplitudes

Ketchikan - Gravina Island West
$-134-$
*Estimated Depth may be unreliable because the stronger part of the conductor may be deeper or to one side of the flight line, or because of a shallow dip or magnetite/overburden effects 
EM Anomaly List

\begin{tabular}{|c|c|c|c|c|c|c|c|c|c|c|c|c|c|}
\hline Label & Fid & Interp & $\begin{array}{c}\text { XUTM } \\
\text { m }\end{array}$ & $\begin{array}{c}\text { YUTM } \\
\mathrm{m}\end{array}$ & $\begin{array}{ll}\text { CX } & 9 \\
\text { Real } & \\
\text { ppm }\end{array}$ & $\begin{array}{l}00 \mathrm{HZ} \\
\text { Quad } \\
\text { ppm }\end{array}$ & $\begin{array}{l}\text { CP } \\
\text { Real } \\
\text { ppm }\end{array}$ & $\begin{array}{l}900 \mathrm{HZ} \\
\text { Quad } \\
\text { ppm }\end{array}$ & $\begin{array}{l}\text { CP } 7 \\
\text { Real } \\
\text { ppm }\end{array}$ & $\begin{array}{l}200 \mathrm{HZ} \\
\text { Quad } \\
\text { ppm }\end{array}$ & $\begin{array}{l}\text { Vertica } \\
\text { Cond } \\
\text { siemens }\end{array}$ & $\begin{array}{c}\text { al Dike } \\
\text { DEPTH* } \\
\text { m }\end{array}$ & $\begin{array}{c}\text { Mag. Corr } \\
\text { NT }\end{array}$ \\
\hline $\begin{array}{l}\mathrm{LINE} \\
\mathrm{D}\end{array}$ & $\begin{array}{c}30651 \\
5130.2\end{array}$ & $S$ & 324944 & 6114852 & 28.9 & 32.8 & 53.7 & 64.1 & 113.8 & 33.4 & 11.6 & 0 & 4 \\
\hline $\begin{array}{l}\text { LINE } \\
\text { A } \\
\text { B } \\
\text { C }\end{array}$ & $\begin{array}{r}30661 \\
5010.2 \\
5028.8 \\
5059.5\end{array}$ & $\begin{array}{l}\text { M } \\
\text { B? } \\
\text { B? }\end{array}$ & $\begin{array}{l}323342 \\
323926 \\
324918\end{array}$ & $\begin{array}{l}6114568 \\
6114517 \\
6114465\end{array}$ & $\begin{array}{l}0.0 \\
1.2 \\
1.6\end{array}$ & $\begin{array}{l}0.3 \\
2.1 \\
4.2\end{array}$ & $\begin{array}{l}3.1 \\
0.2 \\
1.1\end{array}$ & $\begin{array}{l}0.3 \\
3.0 \\
4.7\end{array}$ & $\begin{array}{r}4.3 \\
5.4 \\
11.6\end{array}$ & $\begin{array}{r}4.2 \\
19.0 \\
23.6\end{array}$ & $\begin{array}{l}--- \\
1.6 \\
1.7\end{array}$ & $\begin{array}{r}--- \\
20 \\
17\end{array}$ & $\begin{array}{r}221 \\
12 \\
0\end{array}$ \\
\hline $\begin{array}{l}\text { LINE } \\
A\end{array}$ & $\begin{array}{l}30670 \\
1293.8\end{array}$ & $B$ ? & 325044 & 6114072 & 1.6 & 2.5 & 1.5 & 1.8 & 1.9 & 9.5 & 2.9 & 48 & 0 \\
\hline $\begin{array}{l}\text { LINE } \\
A\end{array}$ & $\begin{array}{l}30680 \\
1126.2\end{array}$ & B? & 324404 & 6113684 & 0.3 & 1.1 & 0.5 & 0.9 & 0.3 & 6.8 & --- & --- & 52 \\
\hline $\begin{array}{l}\text { LINE } \\
\text { A } \\
B\end{array}$ & $\begin{array}{c}30700 \\
936.8 \\
931.2\end{array}$ & $\begin{array}{l}S \\
S\end{array}$ & $\begin{array}{l}324044 \\
324246\end{array}$ & $\begin{array}{l}6112901 \\
6112930\end{array}$ & $\begin{array}{l}21.7 \\
34.8\end{array}$ & $\begin{array}{l}16.5 \\
22.7\end{array}$ & $\begin{array}{l}27.9 \\
60.4\end{array}$ & $\begin{array}{l}11.7 \\
55.4\end{array}$ & $\begin{array}{r}35.3 \\
106.5\end{array}$ & $\begin{array}{l}18.7 \\
20.4\end{array}$ & $\begin{array}{l}24.5 \\
22.0\end{array}$ & $\begin{array}{l}0 \\
0\end{array}$ & $\begin{array}{r}0 \\
10\end{array}$ \\
\hline $\begin{array}{l}\text { LINE } \\
\text { A } \\
\text { B } \\
\text { C } \\
\text { D } \\
\text { E } \\
\text { F } \\
\text { G } \\
\text { H } \\
\text { I } \\
\text { J }\end{array}$ & $\begin{array}{r}39010 \\
5396.0 \\
5324.3 \\
5303.6 \\
5272.3 \\
5205.8 \\
5203.2 \\
5198.8 \\
5184.0 \\
5164.3 \\
5148.6\end{array}$ & $\begin{array}{l}\mathrm{M} \\
\mathrm{M} \\
\mathrm{M} \\
\mathrm{S} \\
\mathrm{S} \\
\mathrm{M} \\
\mathrm{M} \\
\mathrm{S} \\
\mathrm{S} \\
\mathrm{B}\end{array}$ & $\begin{array}{l}318763 \\
318866 \\
318868 \\
318921 \\
319013 \\
319011 \\
319007 \\
319041 \\
319102 \\
319102\end{array}$ & $\begin{array}{l}6130502 \\
6133042 \\
6133673 \\
6134725 \\
6136846 \\
6136939 \\
6137098 \\
6137636 \\
6138388 \\
6138950\end{array}$ & $\begin{array}{r}0.9 \\
0.2 \\
5.1 \\
1.0 \\
8.1 \\
6.5 \\
0.2 \\
93.5 \\
1.2 \\
2.9\end{array}$ & $\begin{array}{r}1.0 \\
0.1 \\
0.5 \\
4.7 \\
1.4 \\
0.4 \\
0.2 \\
41.9 \\
6.7 \\
8.8\end{array}$ & $\begin{array}{r}0.8 \\
0.1 \\
8.7 \\
1.3 \\
18.6 \\
14.3 \\
0.2 \\
196.0 \\
0.6 \\
2.5\end{array}$ & $\begin{array}{l}0.7 \\
0.2 \\
0.2 \\
7.5 \\
1.8 \\
0.4 \\
0.1 \\
85.2 \\
10.8 \\
15.3\end{array}$ & $\begin{array}{r}0.8 \\
2.0 \\
8.0 \\
16.5 \\
18.9 \\
13.9 \\
0.3 \\
228.3 \\
38.3 \\
56.8\end{array}$ & $\begin{array}{r}3.9 \\
0.4 \\
5.0 \\
56.5 \\
12.0 \\
2.2 \\
6.6 \\
5.7 \\
66.5 \\
53.8\end{array}$ & $\begin{array}{c}--- \\
--- \\
--- \\
1.0 \\
--- \\
--- \\
--- \\
72.0 \\
0.7 \\
1.7\end{array}$ & $\begin{array}{r}--- \\
--- \\
--- \\
0 \\
--- \\
--- \\
--- \\
0 \\
0 \\
0\end{array}$ & $\begin{array}{r}29 \\
100 \\
29 \\
0 \\
0 \\
225 \\
300 \\
223 \\
29 \\
0\end{array}$ \\
\hline$\overline{\mathrm{LINE}}$ & 39015 & & & & & & & & & & & & \\
\hline A & 1576.4 & S & 320218 & 6119997 & 20.0 & 38.2 & 34.5 & 79.6 & 182.6 & 72.0 & 6.0 & 0 & 0 \\
\hline B & 1634.0 & $B$ ? & 319971 & 6122274 & 3.6 & 4.6 & 0.1 & 2.6 & 7.4 & 12.6 & --- & --- & 15 \\
\hline $\mathrm{C}$ & 1638.6 & B? & 319954 & 6122461 & 5.1 & 6.5 & 8.3 & 11.5 & 26.6 & 16.8 & --- & --- & 0 \\
\hline D & 1661.1 & $\mathrm{H}$ & 319885 & 6123356 & 5.5 & 9.9 & 9.7 & 20.4 & 52.7 & 46.0 & 3.1 & 12 & 0 \\
\hline $\mathrm{E}$ & 1755.0 & B? & 319598 & 6126788 & 12.4 & 11.5 & 17.2 & 24.3 & 60.6 & 31.1 & --- & --- & 15 \\
\hline $\mathrm{F}$ & 1780.7 & D & 319512 & 6127762 & 30.6 & 21.8 & 27.0 & 31.7 & 65.0 & 33.9 & 16.8 & 2 & 0 \\
\hline G & 1979.8 & $\mathrm{M}$ & 319170 & 6133413 & 1.3 & 0.3 & 1.9 & 0.6 & 1.2 & 2.3 & -- & --- & 174 \\
\hline $\mathrm{H}$ & 1990.2 & $\mathrm{M}$ & 319133 & 6133780 & 0.1 & 0.5 & 0.9 & 1.0 & 0.3 & 5.9 & --- & --- & 0 \\
\hline I & 1996.6 & M & 319099 & 6134007 & 3.2 & 0.7 & 2.9 & 0.7 & 2.6 & 7.3 & --- & --- & 155 \\
\hline $\mathrm{J}$ & 2014.0 & $\mathrm{~S}$ & 319040 & 6134670 & 4.2 & 7.5 & 6.7 & 12.9 & 21.3 & 86.4 & 3.0 & 31 & 0 \\
\hline K & 2028.0 & M & 318999 & 6135188 & 1.4 & 0.9 & 0.1 & 1.5 & 1.2 & 10.3 & --- & --- & 15 \\
\hline L & 2052.0 & M & 318959 & 6135793 & 1.3 & 0.6 & 2.5 & 1.5 & 2.1 & 5.8 & --- & --- & 43 \\
\hline M & 2084.6 & M & 318852 & 6136900 & 0.2 & 1.2 & 0.3 & 1.2 & 0.3 & 6.8 & --- & --- & 145 \\
\hline $\mathrm{N}$ & 2089.0 & M & 318843 & 6137075 & 1.4 & 0.9 & 3.4 & 0.9 & 4.2 & 2.7 & --- & --- & 262 \\
\hline 0 & 2114.7 & $\mathrm{~s}$ & 318767 & 6138051 & 14.2 & 12.1 & 32.7 & 31.9 & 67.0 & 21.9 & 13.4 & 0 & 0 \\
\hline $\mathrm{P}$ & 2148.8 & $\mathrm{~B}$ ? & 318690 & 6139307 & 5.4 & 12.7 & 2.1 & 14.0 & 45.7 & 50.7 & 2.5 & 0 & 0 \\
\hline
\end{tabular}

$\mathrm{CX}=$ COAXIAL

Note: EM values shown above

are local amplitudes

*Estimated Depth may be unreliable because the stronger part of the conductor may be deeper or to one side of the flight line, or because of a

shallow dip or magnetite/overburden effects 
EM Anomaly List

\begin{tabular}{|c|c|c|c|c|c|c|c|c|c|c|c|c|c|}
\hline Label & Fid & Interp & $\underset{\mathrm{m}}{\mathrm{XUTM}}$ & $\begin{array}{c}\text { YUTM } \\
\mathrm{m}\end{array}$ & $\begin{array}{l}\text { CX } \\
\text { Real } \\
\text { ppm }\end{array}$ & $\begin{array}{l}00 \mathrm{HZ} \\
\text { Quad } \\
\text { ppm }\end{array}$ & $\begin{array}{l}\text { CP } \\
\text { Real } \\
\text { ppm }\end{array}$ & $\begin{array}{l}900 \mathrm{HZ} \\
\text { Quad } \\
\text { ppm }\end{array}$ & $\begin{array}{l}\text { CP } \\
\text { Real } \\
\text { ppm }\end{array}$ & $\begin{array}{l}200 \mathrm{HZ} \\
\text { Quad } \\
\text { ppm }\end{array}$ & $\begin{array}{l}\text { Vertica } \\
\text { COND } \\
\text { siemens }\end{array}$ & $\begin{array}{c}\text { al Dike } \\
\text { DEPTH* } \\
\text { m }\end{array}$ & $\begin{array}{c}\text { Mag. Corr } \\
\text { NT }\end{array}$ \\
\hline $\begin{array}{l}\text { LINE } \\
Q\end{array}$ & $\begin{array}{r}39015 \\
2158.3\end{array}$ & B? & 318662 & 6139684 & 7.2 & 13.1 & 6.8 & 23.3 & 80.2 & 41.6 & 3.7 & 0 & 0 \\
\hline $\begin{array}{l}\text { LINE } \\
\text { A } \\
\text { B } \\
\text { C } \\
\text { D } \\
\text { E } \\
\text { F }\end{array}$ & $\begin{array}{r}39020 \\
6601.7 \\
6271.7 \\
6036.0 \\
6026.0 \\
5959.3 \\
5905.1\end{array}$ & $\begin{array}{l}\mathrm{H} \\
\mathrm{S} \\
\mathrm{B} \\
\mathrm{B} \\
\mathrm{S} \\
\mathrm{H}\end{array}$ & $\begin{array}{l}320747 \\
320923 \\
321217 \\
321208 \\
321315 \\
321393\end{array}$ & $\begin{array}{l}6121382 \\
6126521 \\
6132791 \\
6133097 \\
6135316 \\
6137350\end{array}$ & $\begin{array}{l}1.2 \\
0.2 \\
0.8 \\
4.2 \\
1.1 \\
2.2\end{array}$ & $\begin{array}{l}2.7 \\
0.4 \\
0.3 \\
0.8 \\
3.7 \\
5.0\end{array}$ & $\begin{array}{l}0.8 \\
0.4 \\
2.6 \\
3.0 \\
0.5 \\
3.9\end{array}$ & $\begin{array}{l}4.0 \\
1.0 \\
1.4 \\
1.8 \\
6.6 \\
9.0\end{array}$ & $\begin{array}{r}13.4 \\
0.9 \\
3.8 \\
4.6 \\
16.8 \\
24.9\end{array}$ & $\begin{array}{r}17.5 \\
5.9 \\
0.6 \\
3.5 \\
43.0 \\
35.9\end{array}$ & $\begin{array}{r}--- \\
--- \\
13.9 \\
42.2 \\
1.0 \\
---\end{array}$ & $\begin{array}{r}--- \\
--- \\
64 \\
29 \\
0 \\
---\end{array}$ & $\begin{array}{r}0 \\
0 \\
0 \\
16 \\
0 \\
0\end{array}$ \\
\hline $\begin{array}{l}\text { LINE } \\
\text { A } \\
\text { B } \\
\text { C } \\
\text { D } \\
\text { E } \\
\text { F } \\
\text { G } \\
\text { H } \\
\text { I } \\
\text { J } \\
\text { K } \\
\text { L } \\
\text { M }\end{array}$ & $\begin{array}{l}39025 \\
1247.2 \\
1206.4 \\
1146.7 \\
1138.9 \\
949.0 \\
818.1 \\
785.6 \\
774.4 \\
770.7 \\
616.4 \\
596.0 \\
479.6 \\
461.8\end{array}$ & $\begin{array}{r}\mathrm{M} \\
\mathrm{M} \\
\mathrm{B} \\
\mathrm{B} \\
\mathrm{M} \\
\mathrm{B} ? \\
\mathrm{~B} \\
\mathrm{~B} \\
\mathrm{~S} \\
\mathrm{~B} \\
\mathrm{~B} \\
\mathrm{~B} \\
\mathrm{~B} ?\end{array}$ & $\begin{array}{l}326417 \\
326076 \\
325656 \\
325604 \\
323976 \\
322709 \\
322363 \\
322254 \\
322216 \\
320783 \\
320672 \\
319910 \\
319761\end{array}$ & $\begin{array}{l}6117807 \\
6118984 \\
6120282 \\
6120471 \\
6125956 \\
6130264 \\
6131359 \\
6131738 \\
6131857 \\
6136418 \\
6136877 \\
6139413 \\
6139979\end{array}$ & $\begin{array}{r}0.0 \\
1.7 \\
4.4 \\
2.9 \\
0.2 \\
0.3 \\
8.6 \\
6.4 \\
3.8 \\
1.4 \\
3.8 \\
30.3 \\
9.8\end{array}$ & $\begin{array}{r}0.7 \\
0.1 \\
9.2 \\
11.1 \\
5.1 \\
4.3 \\
1.6 \\
2.1 \\
9.9 \\
1.7 \\
0.9 \\
24.1 \\
24.0\end{array}$ & $\begin{array}{r}1.3 \\
0.3 \\
1.6 \\
3.4 \\
0.1 \\
1.1 \\
21.5 \\
10.4 \\
3.4 \\
5.1 \\
4.1 \\
37.7 \\
16.9\end{array}$ & $\begin{array}{r}0.1 \\
1.8 \\
9.6 \\
13.5 \\
7.8 \\
6.2 \\
4.1 \\
14.8 \\
14.8 \\
3.3 \\
2.4 \\
40.2 \\
44.0\end{array}$ & $\begin{array}{r}1.1 \\
0.1 \\
27.3 \\
32.5 \\
18.1 \\
10.7 \\
14.7 \\
29.0 \\
29.6 \\
7.9 \\
7.0 \\
87.7 \\
112.3\end{array}$ & $\begin{array}{r}1.3 \\
14.2 \\
26.8 \\
44.3 \\
38.6 \\
40.8 \\
0.4 \\
66.0 \\
67.3 \\
6.9 \\
0.2 \\
42.8 \\
55.3\end{array}$ & $\begin{array}{r}--- \\
--- \\
2.6 \\
1.8 \\
--- \\
--- \\
--- \\
11.3 \\
2.3 \\
7.9 \\
31.2 \\
16.2 \\
4.1\end{array}$ & $\begin{array}{r}--- \\
--- \\
12 \\
0 \\
--- \\
--- \\
--- \\
24 \\
5 \\
47 \\
38 \\
0 \\
0\end{array}$ & $\begin{array}{r}86 \\
0 \\
0 \\
8 \\
0 \\
8 \\
0 \\
6 \\
0 \\
0 \\
7 \\
0 \\
7\end{array}$ \\
\hline $\begin{array}{l}\text { LINE } \\
A\end{array}$ & $\begin{array}{l}39030 \\
3937.8\end{array}$ & $\mathrm{H}$ & 323031 & 6119143 & 3.5 & 3.3 & 2.3 & 8.7 & 28.2 & 25.3 & --- & --- & 0 \\
\hline $\begin{array}{l}\text { LINE } \\
\text { A } \\
\text { B } \\
\text { C } \\
\text { D }\end{array}$ & $\begin{array}{r}39040 \\
3423.2 \\
3310.8 \\
3139.0 \\
3128.1\end{array}$ & $\begin{array}{c}\mathrm{B} ? \\
\mathrm{M} \\
\mathrm{B} \\
\mathrm{B}\end{array}$ & $\begin{array}{l}325115 \\
325217 \\
325414 \\
325432\end{array}$ & $\begin{array}{l}6113506 \\
6117504 \\
6120160 \\
6120488\end{array}$ & $\begin{array}{l}1.5 \\
0.4 \\
6.0 \\
0.1\end{array}$ & $\begin{array}{l}3.2 \\
0.2 \\
6.7 \\
2.9\end{array}$ & $\begin{array}{l}1.9 \\
0.0 \\
2.5 \\
1.0\end{array}$ & $\begin{array}{l}3.4 \\
0.2 \\
6.6 \\
3.6\end{array}$ & $\begin{array}{r}12.5 \\
0.2 \\
19.0 \\
9.1\end{array}$ & $\begin{array}{r}9.7 \\
0.4 \\
14.5 \\
9.0\end{array}$ & $\begin{array}{l}1.9 \\
--- \\
4.6 \\
---\end{array}$ & $\begin{array}{r}43 \\
--- \\
18 \\
---\end{array}$ & $\begin{array}{r}0 \\
251 \\
0 \\
0\end{array}$ \\
\hline
\end{tabular}

\section{$\mathrm{CX}=$ COAXIAL}

COPIANAR

Note: EM values shown above are local amplitudes

Ketchikan - Gravina Island West
$-136-$
*Estimated Depth may be unreliable because the stronger part of the conductor may be deeper or to one side of the flight line, or because of shallow dip or magnetite/overburden effects 
EM Anomaly List

\begin{tabular}{|c|c|c|c|c|c|c|c|c|c|c|c|c|c|}
\hline Label & Fid & Interp & $\begin{array}{c}\text { XUTM } \\
\text { m }\end{array}$ & $\begin{array}{c}\text { YUTM } \\
\mathrm{m}\end{array}$ & $\begin{array}{ll}\text { CX } & 9 \\
\text { Real } & \\
\text { ppm } & \end{array}$ & $\begin{array}{l}00 \mathrm{HZ} \\
\text { Quad } \\
\text { ppm }\end{array}$ & $\begin{array}{l}\mathrm{CP} \\
\text { Real } \\
\text { ppm }\end{array}$ & $\begin{array}{l}900 \mathrm{HZ} \\
\text { Quad } \\
\text { ppm }\end{array}$ & $\begin{array}{l}\text { CP } 7 \\
\text { Real } \\
\text { ppm }\end{array}$ & $\begin{array}{l}200 \mathrm{~Hz} \\
\text { Quad } \\
\text { ppm }\end{array}$ & $\begin{array}{l}\text { Vertica } \\
\text { Cond } \\
\text { siemens }\end{array}$ & $\begin{array}{c}\text { al Dike } \\
\text { DEPTH* } \\
\text { m }\end{array}$ & $\begin{array}{c}\text { Mag. Corr } \\
\text { NT }\end{array}$ \\
\hline $\begin{array}{l}\text { LINE } \\
\mathrm{A}\end{array}$ & $\begin{array}{c}40010 \\
263.0\end{array}$ & $\mathrm{~L}$ & 329039 & 6136423 & 7.1 & 9.2 & 1.8 & 6.3 & 14.0 & 24.9 & 4.0 & 9 & 9 \\
\hline $\begin{array}{l}\text { LINE } \\
\text { A } \\
B\end{array}$ & $\begin{array}{l}40020 \\
319.3 \\
308.7\end{array}$ & $\begin{array}{l}S \\
S\end{array}$ & $\begin{array}{l}328940 \\
329259\end{array}$ & $\begin{array}{l}6135988 \\
6135968\end{array}$ & $\begin{array}{l}0.1 \\
0.5\end{array}$ & $\begin{array}{l}3.2 \\
4.0\end{array}$ & $\begin{array}{l}0.4 \\
1.3\end{array}$ & $\begin{array}{l}5.8 \\
5.2\end{array}$ & $\begin{array}{l}12.6 \\
11.6\end{array}$ & $\begin{array}{l}30.3 \\
28.1\end{array}$ & $\begin{array}{l}--- \\
---\end{array}$ & $\begin{array}{l}--- \\
---\end{array}$ & $\begin{array}{l}1 \\
0\end{array}$ \\
\hline $\begin{array}{l}\text { LINE } \\
\mathrm{A}\end{array}$ & $\begin{array}{c}40030 \\
429.7\end{array}$ & $\mathrm{~s}$ & 328979 & 6135679 & 1.7 & 4.1 & 1.9 & 5.8 & 14.2 & 33.0 & --- & --- & 6 \\
\hline $\begin{array}{l}\text { LINE } \\
A\end{array}$ & $\begin{array}{c}40040 \\
529.3\end{array}$ & S & 328923 & 6135251 & 0.5 & 4.2 & 0.5 & 5.5 & 13.3 & 40.4 & 0.5 & 0 & 6 \\
\hline $\begin{array}{l}\text { LINE } \\
\text { A } \\
B \\
\text { C }\end{array}$ & $\begin{array}{l}40050 \\
631.7 \\
656.5 \\
669.0\end{array}$ & $\begin{array}{l}S \\
S \\
S\end{array}$ & $\begin{array}{l}328665 \\
329539 \\
329997\end{array}$ & $\begin{array}{l}6134851 \\
6134816 \\
6134793\end{array}$ & $\begin{array}{l}2.6 \\
1.0 \\
0.0\end{array}$ & $\begin{array}{l}2.7 \\
1.7 \\
1.1\end{array}$ & $\begin{array}{l}2.3 \\
3.1 \\
1.4\end{array}$ & $\begin{array}{l}3.1 \\
2.8 \\
0.8\end{array}$ & $\begin{array}{l}7.1 \\
6.6 \\
2.4\end{array}$ & $\begin{array}{r}21.4 \\
16.1 \\
6.8\end{array}$ & $\begin{array}{l}4.8 \\
--- \\
---\end{array}$ & $\begin{array}{r}48 \\
--- \\
---\end{array}$ & $\begin{array}{r}0 \\
0 \\
23\end{array}$ \\
\hline $\begin{array}{l}\text { LINE } \\
\text { A } \\
B\end{array}$ & $\begin{array}{l}40060 \\
793.0 \\
763.9\end{array}$ & $\begin{array}{l}S \\
S\end{array}$ & $\begin{array}{l}328695 \\
329701\end{array}$ & $\begin{array}{l}6134452 \\
6134384\end{array}$ & $\begin{array}{l}1.7 \\
1.1\end{array}$ & $\begin{array}{l}2.3 \\
1.8\end{array}$ & $\begin{array}{l}1.4 \\
2.9\end{array}$ & $\begin{array}{l}2.3 \\
3.3\end{array}$ & $\begin{array}{l}5.4 \\
7.7\end{array}$ & $\begin{array}{l}20.1 \\
15.5\end{array}$ & $\begin{array}{l}3.1 \\
---\end{array}$ & $\begin{array}{r}50 \\
---\end{array}$ & $\begin{array}{r}12 \\
3\end{array}$ \\
\hline $\begin{array}{l}\text { LINE } \\
\text { A } \\
B\end{array}$ & $\begin{array}{l}40070 \\
892.1 \\
921.6\end{array}$ & $\begin{array}{l}S \\
L\end{array}$ & $\begin{array}{l}329530 \\
330718\end{array}$ & $\begin{array}{l}6134008 \\
6133946\end{array}$ & $\begin{array}{l}0.8 \\
2.0\end{array}$ & $\begin{array}{l}2.1 \\
2.9\end{array}$ & $\begin{array}{l}0.5 \\
1.1\end{array}$ & $\begin{array}{l}1.8 \\
0.4\end{array}$ & $\begin{array}{l}2.4 \\
3.3\end{array}$ & $\begin{array}{r}10.9 \\
0.9\end{array}$ & $\begin{array}{l}--- \\
---\end{array}$ & $\begin{array}{l}--- \\
---\end{array}$ & $\begin{array}{l}9 \\
0\end{array}$ \\
\hline $\begin{array}{l}\text { LINE } \\
\text { A } \\
B\end{array}$ & $\begin{array}{l}40080 \\
1043.0 \\
992.3\end{array}$ & $\begin{array}{l}S \\
L\end{array}$ & $\begin{array}{l}329271 \\
331081\end{array}$ & $\begin{array}{l}6133584 \\
6133566\end{array}$ & $\begin{array}{l}0.1 \\
3.8\end{array}$ & $\begin{array}{l}0.6 \\
5.5\end{array}$ & $\begin{array}{l}0.9 \\
2.8\end{array}$ & $\begin{array}{l}0.7 \\
4.1\end{array}$ & $\begin{array}{r}1.6 \\
13.0\end{array}$ & $\begin{array}{r}5.8 \\
12.0\end{array}$ & $\begin{array}{l}--- \\
3.9\end{array}$ & $\begin{array}{r}--- \\
21\end{array}$ & $\begin{array}{l}0 \\
3\end{array}$ \\
\hline $\begin{array}{l}\text { LINE } \\
A\end{array}$ & $\begin{array}{l}40090 \\
1235.9\end{array}$ & S & 329330 & 6133240 & 0.6 & 1.5 & 0.7 & 1.7 & 1.9 & 11.2 & --- & --- & 0 \\
\hline $\begin{array}{l}\text { LINE } \\
\text { A } \\
B\end{array}$ & $\begin{array}{l}40100 \\
1418.9 \\
1403.3\end{array}$ & $\begin{array}{r}S ? \\
S\end{array}$ & $\begin{array}{l}329860 \\
330421\end{array}$ & $\begin{array}{l}6132779 \\
6132756\end{array}$ & $\begin{array}{l}3.5 \\
0.0\end{array}$ & $\begin{array}{l}1.5 \\
1.6\end{array}$ & $\begin{array}{l}2.1 \\
1.2\end{array}$ & $\begin{array}{l}2.7 \\
2.8\end{array}$ & $\begin{array}{l}3.9 \\
2.9\end{array}$ & $\begin{array}{l}17.5 \\
20.2\end{array}$ & $\begin{array}{l}--- \\
0.9\end{array}$ & $\begin{array}{r}--- \\
12\end{array}$ & $\begin{array}{l}2 \\
2\end{array}$ \\
\hline $\begin{array}{l}\text { LINE } \\
\text { A } \\
B\end{array}$ & $\begin{array}{l}40110 \\
1490.6 \\
1510.7\end{array}$ & $\begin{array}{r}S \\
S ?\end{array}$ & $\begin{array}{l}329077 \\
329716\end{array}$ & $\begin{array}{l}6132415 \\
6132400\end{array}$ & $\begin{array}{l}4.0 \\
1.9\end{array}$ & $\begin{array}{l}8.4 \\
3.9\end{array}$ & $\begin{array}{l}6.2 \\
0.7\end{array}$ & $\begin{array}{r}24.0 \\
2.8\end{array}$ & $\begin{array}{r}67.8 \\
4.1\end{array}$ & $\begin{array}{l}25.3 \\
18.4\end{array}$ & $\begin{array}{l}2.7 \\
2.2\end{array}$ & $\begin{array}{r}0 \\
21\end{array}$ & $\begin{array}{r}9 \\
14\end{array}$ \\
\hline $\begin{array}{l}\text { LINE } \\
\text { A } \\
B\end{array}$ & $\begin{array}{l}40120 \\
1717.9 \\
1698.0\end{array}$ & $\begin{array}{l}S \\
S\end{array}$ & $\begin{array}{l}329743 \\
330458\end{array}$ & $\begin{array}{l}6131997 \\
6131968\end{array}$ & $\begin{array}{l}2.0 \\
0.3\end{array}$ & $\begin{array}{l}2.4 \\
1.4\end{array}$ & $\begin{array}{l}5.1 \\
0.4\end{array}$ & $\begin{array}{l}2.5 \\
1.4\end{array}$ & $\begin{array}{l}8.9 \\
1.0\end{array}$ & $\begin{array}{l}5.3 \\
8.4\end{array}$ & $\begin{array}{l}--- \\
---\end{array}$ & $\begin{array}{l}--- \\
---\end{array}$ & $\begin{array}{l}0 \\
0\end{array}$ \\
\hline
\end{tabular}

$\mathrm{CX}=$ COAXIAL

$\mathrm{CP}=$ COPLANAR Note: $\mathrm{EM}$ values shown above

are local amplitudes

Ketchikan - Gravina Island East
* Estimated Depth may be unreliable because the stronger part of the conductor may be deeper or to one side of the flight line, or because 
EM Anomaly List

\begin{tabular}{|c|c|c|c|c|c|c|c|c|c|c|c|c|c|}
\hline Label & Fid & Interp & $\begin{array}{c}\text { XUTM } \\
\text { m }\end{array}$ & $\begin{array}{c}\text { YUTM } \\
\mathrm{m}\end{array}$ & $\begin{array}{l}\text { CX } \\
\text { Real } \\
\text { ppm }\end{array}$ & $\begin{array}{l}00 \mathrm{HZ} \\
\text { Quad } \\
\text { ppm }\end{array}$ & $\begin{array}{l}\text { CP } \\
\text { Real } \\
\text { ppm }\end{array}$ & $\begin{array}{c}900 \mathrm{HZ} \\
\text { Quad } \\
\text { ppm }\end{array}$ & $\begin{array}{l}\text { CP } 7 \\
\text { Real } \\
\text { ppm }\end{array}$ & $\begin{array}{l}200 \mathrm{HZ} \\
\text { Quad } \\
\text { ppm }\end{array}$ & $\begin{array}{l}\text { Vertica } \\
\text { COND } \\
\text { siemens }\end{array}$ & $\begin{array}{c}\text { al Dike } \\
\text { DEPTH* } \\
\text { m }\end{array}$ & $\begin{array}{c}\text { Mag. Corr } \\
\text { NT }\end{array}$ \\
\hline $\begin{array}{l}\text { LINE } 4 \\
A\end{array}$ & $\begin{array}{l}40130 \\
1779.6\end{array}$ & $\mathrm{~S}$ & 330399 & 6131588 & 13.5 & 11.9 & 18.9 & 22.5 & 53.6 & 17.4 & 10.6 & 0 & 3 \\
\hline $\begin{array}{l}\text { LINE } 4 \\
\text { A }\end{array}$ & $\begin{array}{l}40140 \\
2007.6\end{array}$ & S? & 331036 & 6131169 & 2.7 & 2.5 & 2.9 & 2.3 & 7.3 & 7.1 & --- & --- & 0 \\
\hline $\begin{array}{l}\text { LINE } 4 \\
\text { A }\end{array}$ & $\begin{array}{l}40150 \\
2303.7\end{array}$ & $B$ ? & 333085 & 6130593 & 0.9 & 3.4 & 0.1 & 3.0 & 6.2 & 20.1 & 0.9 & 12 & 0 \\
\hline $\begin{array}{l}\text { LINE } 4 \\
\text { A } \\
B \\
\text { C }\end{array}$ & $\begin{array}{r}40160 \\
2447.3 \\
2396.2 \\
2387.3\end{array}$ & $\begin{array}{r}\mathrm{S} \\
\mathrm{S} \\
\mathrm{B} ?\end{array}$ & $\begin{array}{l}331765 \\
333288 \\
333580\end{array}$ & $\begin{array}{l}6130339 \\
6130250 \\
6130240\end{array}$ & $\begin{array}{l}4.3 \\
2.8 \\
2.3\end{array}$ & $\begin{array}{r}10.0 \\
3.3 \\
3.3\end{array}$ & $\begin{array}{l}3.0 \\
3.2 \\
1.7\end{array}$ & $\begin{array}{r}17.3 \\
7.6 \\
4.9\end{array}$ & $\begin{array}{l}58.8 \\
23.2 \\
12.2\end{array}$ & $\begin{array}{l}59.7 \\
11.7 \\
15.0\end{array}$ & $\begin{array}{l}2.3 \\
--- \\
2.3\end{array}$ & $\begin{array}{r}6 \\
--- \\
45\end{array}$ & $\begin{array}{r}9 \\
30 \\
0\end{array}$ \\
\hline $\begin{array}{l}\text { LINE } 4 \\
\text { A } \\
\text { B } \\
\text { C } \\
\text { D }\end{array}$ & $\begin{array}{l}40170 \\
2530.2 \\
2558.3 \\
2570.0 \\
2579.9\end{array}$ & $\begin{array}{l}S \\
S \\
S \\
S\end{array}$ & $\begin{array}{l}332024 \\
332977 \\
333418 \\
333781\end{array}$ & $\begin{array}{l}6129887 \\
6129850 \\
6129813 \\
6129758\end{array}$ & $\begin{array}{r}18.9 \\
0.7 \\
5.9 \\
6.0\end{array}$ & $\begin{array}{r}15.2 \\
4.0 \\
6.0 \\
12.8\end{array}$ & $\begin{array}{r}25.7 \\
0.4 \\
6.0 \\
10.8\end{array}$ & $\begin{array}{r}28.9 \\
7.1 \\
10.3 \\
16.0\end{array}$ & $\begin{array}{l}69.5 \\
20.0 \\
35.4 \\
43.1\end{array}$ & $\begin{array}{l}35.3 \\
39.0 \\
41.9 \\
22.8\end{array}$ & $\begin{array}{r}13.2 \\
0.6 \\
5.6 \\
3.5\end{array}$ & $\begin{array}{r}1 \\
1 \\
24 \\
0\end{array}$ & $\begin{array}{r}0 \\
3 \\
10 \\
2\end{array}$ \\
\hline $\begin{array}{l}\text { LINE } \\
\text { A } \\
\text { B } \\
\text { C } \\
\text { D } \\
\text { E } \\
\text { F } \\
\text { G }\end{array}$ & $\begin{array}{l}4910 \\
7238.5 \\
7274.3 \\
7316.4 \\
7366.9 \\
7384.7 \\
7395.3 \\
7472.8\end{array}$ & $\begin{array}{l}\mathrm{S} \\
\mathrm{S} \\
\mathrm{S} \\
\mathrm{S} \\
\mathrm{S} \\
\mathrm{S} \\
\mathrm{S}\end{array}$ & $\begin{array}{l}332800 \\
332002 \\
331066 \\
329934 \\
329580 \\
329327 \\
327493\end{array}$ & $\begin{array}{l}6128850 \\
6129862 \\
6131096 \\
6132392 \\
6132923 \\
6133222 \\
6135503\end{array}$ & $\begin{array}{r}18.0 \\
11.6 \\
1.3 \\
1.4 \\
0.2 \\
0.0 \\
0.2\end{array}$ & $\begin{array}{r}0.5 \\
10.1 \\
3.7 \\
2.3 \\
1.4 \\
2.6 \\
1.9\end{array}$ & $\begin{array}{r}47.0 \\
19.3 \\
0.3 \\
0.3 \\
0.4 \\
0.8 \\
0.8\end{array}$ & $\begin{array}{r}3.1 \\
23.0 \\
4.3 \\
2.1 \\
1.3 \\
4.3 \\
2.3\end{array}$ & $\begin{array}{r}37.2 \\
57.8 \\
10.6 \\
6.6 \\
1.4 \\
4.3 \\
2.7\end{array}$ & $\begin{array}{r}1.2 \\
22.7 \\
30.0 \\
13.6 \\
8.2 \\
31.6 \\
17.5\end{array}$ & $\begin{array}{r}--- \\
10.3 \\
1.2 \\
--- \\
--- \\
--- \\
---\end{array}$ & $\begin{array}{r}--- \\
0 \\
8 \\
--- \\
--- \\
--- \\
---\end{array}$ & $\begin{array}{l}0 \\
0 \\
0 \\
0 \\
0 \\
5 \\
0\end{array}$ \\
\hline $\begin{array}{l}\text { LINE } 4 \\
\text { A } \\
\text { B } \\
\text { C } \\
\text { D }\end{array}$ & $\begin{array}{l}49020 \\
7744.7 \\
7721.5 \\
7666.3 \\
7662.1\end{array}$ & $\begin{array}{l}\mathrm{L} \\
\mathrm{S} \\
\mathrm{S} \\
\mathrm{S}\end{array}$ & $\begin{array}{l}331256 \\
330689 \\
329186 \\
329086\end{array}$ & $\begin{array}{l}6133689 \\
6134488 \\
6136243 \\
6136381\end{array}$ & $\begin{array}{r}12.2 \\
3.6 \\
1.6 \\
1.1\end{array}$ & $\begin{array}{l}8.7 \\
4.9 \\
2.7 \\
3.5\end{array}$ & $\begin{array}{l}5.0 \\
7.1 \\
0.7 \\
0.3\end{array}$ & $\begin{array}{r}26.8 \\
9.7 \\
3.8 \\
2.0\end{array}$ & $\begin{array}{r}84.6 \\
33.3 \\
10.0 \\
4.4\end{array}$ & $\begin{array}{r}0.5 \\
21.6 \\
15.3 \\
17.0\end{array}$ & $\begin{array}{l}5.1 \\
4.6 \\
--- \\
1.3\end{array}$ & $\begin{array}{r}5 \\
3 \\
--- \\
0\end{array}$ & $\begin{array}{l}0 \\
8 \\
0 \\
3\end{array}$ \\
\hline
\end{tabular}

\section{$\mathrm{CX}=$ COAXIAL}

Note: EM values shown above are local amplitudes

Ketchikan - Gravina Island East
$-138-$
*Estimated Depth may be unreliable because the stronger part of the conductor may be deeper or to one side of the flight line, or because of a shallow dip or magnetite/overburden effects 
EM Anomaly List

\begin{tabular}{|c|c|c|c|c|c|c|c|c|c|c|c|c|c|}
\hline Label & Fid & Interp & $\begin{array}{c}\text { XUTM } \\
\text { m }\end{array}$ & $\begin{array}{c}\text { YUTM } \\
\text { m }\end{array}$ & $\begin{array}{l}\text { CX } \\
\text { Real } \\
\text { ppm }\end{array}$ & $\begin{array}{l}00 \mathrm{HZ} \\
\text { Quad } \\
\text { ppm }\end{array}$ & $\begin{array}{l}\text { CP } \\
\text { Real } \\
\text { ppm }\end{array}$ & $\begin{array}{l}900 \mathrm{HZ} \\
\text { Quad } \\
\text { ppm }\end{array}$ & $\begin{array}{l}\text { CP } 7 \\
\text { Real } \\
\text { ppm }\end{array}$ & $\begin{array}{l}200 \mathrm{HZ} \\
\text { Quad } \\
\text { ppm }\end{array}$ & $\begin{array}{l}\text { Vertica } \\
\text { Cond } \\
\text { siemens }\end{array}$ & $\begin{array}{c}\text { al Dike } \\
\text { DEPTH* } \\
\text { m }\end{array}$ & $\begin{array}{c}\text { Mag. Corr } \\
\text { NT }\end{array}$ \\
\hline $\begin{array}{l}\mathrm{LINE} \\
\mathrm{A}\end{array}$ & $\begin{array}{c}50010 \\
559.0\end{array}$ & $\mathrm{~S}$ & 658618 & 6105267 & 4.4 & 1.1 & 1.4 & 1.3 & 0.8 & 8.1 & --- & --- & 0 \\
\hline $\begin{array}{l}\text { LINE } \\
\text { A } \\
B\end{array}$ & $\begin{array}{l}50020 \\
860.0 \\
1067.0\end{array}$ & $\begin{array}{l}S \\
S\end{array}$ & $\begin{array}{l}658651 \\
661366\end{array}$ & $\begin{array}{l}6104860 \\
6104877\end{array}$ & $\begin{array}{l}0.3 \\
0.6\end{array}$ & $\begin{array}{l}1.2 \\
4.0\end{array}$ & $\begin{array}{l}0.2 \\
2.1\end{array}$ & $\begin{array}{l}1.6 \\
6.5\end{array}$ & $\begin{array}{r}0.5 \\
20.7\end{array}$ & $\begin{array}{l}11.8 \\
22.4\end{array}$ & $\begin{array}{l}--- \\
1.1\end{array}$ & $\begin{array}{r}--- \\
17\end{array}$ & $\begin{array}{l}18 \\
74\end{array}$ \\
\hline $\begin{array}{l}\text { LINE } \\
\text { A } \\
\text { B } \\
\text { C }\end{array}$ & $\begin{array}{l}50090 \\
2794.9 \\
2767.3 \\
2746.0\end{array}$ & $\begin{array}{l}\text { S? } \\
\text { S? } \\
\text { S? }\end{array}$ & $\begin{array}{l}659752 \\
660513 \\
661044\end{array}$ & $\begin{array}{l}6102075 \\
6102101 \\
6102152\end{array}$ & $\begin{array}{l}0.7 \\
2.3 \\
3.0\end{array}$ & $\begin{array}{l}2.9 \\
2.7 \\
2.8\end{array}$ & $\begin{array}{l}0.8 \\
0.5 \\
0.3\end{array}$ & $\begin{array}{l}7.1 \\
4.3 \\
2.4\end{array}$ & $\begin{array}{r}11.6 \\
8.2 \\
7.0\end{array}$ & $\begin{array}{l}46.4 \\
23.8 \\
15.4\end{array}$ & $\begin{array}{l}--- \\
--- \\
---\end{array}$ & $\begin{array}{l}--- \\
--- \\
---\end{array}$ & $\begin{array}{r}0 \\
21 \\
0\end{array}$ \\
\hline $\begin{array}{l}\text { LINE } \\
\text { A } \\
\text { B } \\
\text { C } \\
\text { D }\end{array}$ & $\begin{array}{l}50100 \\
3175.9 \\
3204.7 \\
3343.0 \\
3378.8\end{array}$ & $\begin{array}{l}\text { S } \\
\text { S } \\
\text { D } \\
\text { B }\end{array}$ & $\begin{array}{l}656363 \\
656885 \\
660018 \\
661010\end{array}$ & $\begin{array}{l}6101513 \\
6101547 \\
6101687 \\
6101643\end{array}$ & $\begin{array}{l}0.4 \\
0.9 \\
1.7 \\
1.7\end{array}$ & $\begin{array}{l}1.0 \\
1.8 \\
9.8 \\
6.2\end{array}$ & $\begin{array}{l}0.8 \\
1.6 \\
2.5 \\
2.5\end{array}$ & $\begin{array}{r}2.5 \\
1.1 \\
19.9 \\
6.1\end{array}$ & $\begin{array}{r}0.9 \\
0.3 \\
57.1 \\
17.5\end{array}$ & $\begin{array}{r}16.5 \\
5.9 \\
54.9 \\
14.7\end{array}$ & $\begin{array}{l}--- \\
--- \\
--- \\
1.9\end{array}$ & $\begin{array}{r}--- \\
--- \\
--- \\
0\end{array}$ & $\begin{array}{r}16 \\
0 \\
16 \\
16\end{array}$ \\
\hline $\begin{array}{l}\text { LINE } \\
\text { A } \\
\text { B } \\
\text { C } \\
\text { D } \\
\text { E } \\
\text { F } \\
\text { G } \\
\text { H } \\
\text { I }\end{array}$ & $\begin{array}{l}50110 \\
3684.0 \\
3669.0 \\
3604.5 \\
3595.1 \\
3576.2 \\
3560.0 \\
3552.4 \\
3533.7 \\
3527.8\end{array}$ & $\begin{array}{r}\mathrm{M} \\
\mathrm{M} \\
\mathrm{D} \\
\mathrm{D} \\
\mathrm{B} ? \\
\mathrm{~B} \\
\mathrm{~B} \\
\mathrm{~B} ? \\
\mathrm{~B} ?\end{array}$ & $\begin{array}{l}657644 \\
657854 \\
659565 \\
659784 \\
660318 \\
660763 \\
660918 \\
661288 \\
661429\end{array}$ & $\begin{array}{l}6101218 \\
6101266 \\
6101323 \\
6101345 \\
6101382 \\
6101354 \\
6101325 \\
6101245 \\
6101222\end{array}$ & $\begin{array}{l}0.1 \\
0.0 \\
0.4 \\
1.5 \\
4.2 \\
5.2 \\
3.1 \\
1.2 \\
2.7\end{array}$ & $\begin{array}{r}0.6 \\
0.8 \\
5.9 \\
4.7 \\
9.1 \\
11.7 \\
4.1 \\
3.4 \\
3.9\end{array}$ & $\begin{array}{l}5.0 \\
6.7 \\
1.4 \\
0.2 \\
0.5 \\
4.5 \\
1.4 \\
0.4 \\
0.0\end{array}$ & $\begin{array}{r}0.7 \\
0.6 \\
9.0 \\
6.7 \\
1.0 \\
20.0 \\
1.4 \\
3.9 \\
4.0\end{array}$ & $\begin{array}{r}3.7 \\
5.7 \\
19.8 \\
17.6 \\
8.1 \\
60.5 \\
4.1 \\
12.2 \\
11.8\end{array}$ & $\begin{array}{r}3.6 \\
6.1 \\
55.4 \\
44.1 \\
8.3 \\
47.1 \\
7.3 \\
18.2 \\
14.6\end{array}$ & $\begin{array}{l}--- \\
--- \\
--- \\
--- \\
2.9 \\
2.7 \\
--- \\
--- \\
2.4\end{array}$ & $\begin{array}{r}--- \\
--- \\
--- \\
--- \\
14 \\
0 \\
--- \\
--- \\
22\end{array}$ & $\begin{array}{r}340 \\
22 \\
0 \\
0 \\
0 \\
22 \\
22 \\
5 \\
0\end{array}$ \\
\hline $\begin{array}{l}\text { LINE } \\
\text { A } \\
\text { B } \\
\text { C } \\
\text { D } \\
\text { E } \\
\text { F } \\
\text { G } \\
\text { H }\end{array}$ & $\begin{array}{r}0120 \\
3937.0 \\
4039.5 \\
4114.4 \\
4131.1 \\
4133.9 \\
4137.1 \\
4144.6 \\
4184.2\end{array}$ & $\begin{array}{l}\text { M } \\
\text { S } \\
\text { D } \\
\text { D } \\
\text { B } \\
\text { B } \\
\text { D } \\
\text { B }\end{array}$ & $\begin{array}{l}654855 \\
657629 \\
659766 \\
660380 \\
660475 \\
660577 \\
660832 \\
661374\end{array}$ & $\begin{array}{l}6100714 \\
6100776 \\
6100822 \\
6100853 \\
6100859 \\
6100872 \\
6100890 \\
6100915\end{array}$ & $\begin{array}{l}0.0 \\
0.5 \\
1.2 \\
5.1 \\
5.4 \\
4.5 \\
2.0 \\
3.5\end{array}$ & $\begin{array}{r}1.4 \\
1.6 \\
7.1 \\
11.9 \\
11.6 \\
4.6 \\
4.3 \\
0.2\end{array}$ & $\begin{array}{l}0.9 \\
1.6 \\
1.3 \\
1.8 \\
2.1 \\
2.8 \\
2.3 \\
0.0\end{array}$ & $\begin{array}{r}1.1 \\
2.0 \\
8.2 \\
12.9 \\
19.3 \\
22.8 \\
6.4 \\
0.0\end{array}$ & $\begin{array}{r}0.7 \\
1.7 \\
24.5 \\
41.0 \\
55.1 \\
68.4 \\
18.7 \\
0.0\end{array}$ & $\begin{array}{r}7.3 \\
9.0 \\
37.6 \\
53.0 \\
82.5 \\
86.0 \\
24.3 \\
2.6\end{array}$ & $\begin{array}{l}--- \\
--- \\
0.9 \\
2.4 \\
2.3 \\
2.6 \\
--- \\
---\end{array}$ & $\begin{array}{r}--- \\
-- \\
0 \\
0 \\
0 \\
0 \\
--- \\
---\end{array}$ & $\begin{array}{r}154 \\
0 \\
33 \\
0 \\
0 \\
0 \\
30 \\
40\end{array}$ \\
\hline $\begin{array}{l}\text { LINE } \\
\text { A } \\
\text { B } \\
\text { C } \\
\text { D } \\
\text { E } \\
\text { F }\end{array}$ & $\begin{array}{r}50130 \\
4415.9 \\
4328.5 \\
4281.8 \\
4274.5 \\
4260.1 \\
4249.6\end{array}$ & $\begin{array}{r}\mathrm{S} ? \\
\mathrm{~S} \\
\mathrm{~B} ? \\
\mathrm{~B} \\
\mathrm{~B} \\
\mathrm{~B}\end{array}$ & $\begin{array}{l}657444 \\
659359 \\
660499 \\
660736 \\
661119 \\
661314\end{array}$ & $\begin{array}{l}6100414 \\
6100469 \\
6100521 \\
6100523 \\
6100540 \\
6100557\end{array}$ & $\begin{array}{l}2.0 \\
0.8 \\
1.1 \\
1.8 \\
3.2 \\
2.5\end{array}$ & $\begin{array}{l}1.6 \\
1.6 \\
2.5 \\
4.0 \\
8.8 \\
3.3\end{array}$ & $\begin{array}{l}2.3 \\
1.3 \\
0.5 \\
0.6 \\
7.0 \\
0.9\end{array}$ & $\begin{array}{r}2.5 \\
1.9 \\
2.2 \\
5.3 \\
19.5 \\
6.1\end{array}$ & $\begin{array}{r}5.0 \\
1.4 \\
10.3 \\
12.2 \\
50.2 \\
11.1\end{array}$ & $\begin{array}{r}15.8 \\
13.9 \\
7.2 \\
31.6 \\
37.4 \\
21.8\end{array}$ & $\begin{array}{l}--- \\
--- \\
1.6 \\
1.7 \\
2.7 \\
---\end{array}$ & $\begin{array}{r}--- \\
--- \\
32 \\
7 \\
7 \\
---\end{array}$ & $\begin{array}{r}26 \\
66 \\
0 \\
0 \\
0 \\
0\end{array}$ \\
\hline
\end{tabular}

$\mathrm{CX}=$ COAXIAL

Note: EM values shown above

are local amplitudes

Ketchikan - South of Keete Inlet
*Estimated Depth may be unreliable because the stronger part of the conductor may be deeper or to one side of the flight line, or because of a shallow dip or magnetite/overburden effects 
EM Anomaly List

\begin{tabular}{|c|c|c|c|c|c|c|c|c|c|c|c|c|c|}
\hline Label & Fid & Interp & $\begin{array}{c}\text { XUTM } \\
\text { m }\end{array}$ & $\begin{array}{c}\text { YUTM } \\
\mathrm{m}\end{array}$ & $\begin{array}{l}\text { CX } \\
\text { Real } \\
\text { ppm }\end{array}$ & $\begin{array}{l}900 \mathrm{HZ} \\
\text { Quad } \\
\text { ppm }\end{array}$ & $\begin{array}{l}\text { CP } \\
\text { Real } \\
\text { ppm }\end{array}$ & $\begin{array}{c}900 \mathrm{HZ} \\
\text { Quad } \\
\text { ppm }\end{array}$ & $\begin{array}{l}\mathrm{CP} \\
\mathrm{Real} \\
\mathrm{ppm}\end{array}$ & $\begin{array}{l}200 \mathrm{HZ} \\
\text { Quad } \\
\text { ppm }\end{array}$ & $\begin{array}{l}\text { Vertica } \\
\text { CoND } \\
\text { siemens }\end{array}$ & $\begin{array}{c}\text { al Dike } \\
\text { DEPTH* } \\
\text { m }\end{array}$ & $\begin{array}{c}\text { Mag. Corr } \\
\text { NT }\end{array}$ \\
\hline $\begin{array}{l}\text { LINE } \\
\text { A } \\
B \\
\text { C }\end{array}$ & $\begin{array}{r}50140 \\
4650.0 \\
4914.0 \\
4949.2\end{array}$ & $\begin{array}{r}M \\
S ? \\
S\end{array}$ & $\begin{array}{l}654306 \\
660089 \\
660725\end{array}$ & $\begin{array}{l}6099886 \\
6100072 \\
6100082\end{array}$ & $\begin{array}{l}0.0 \\
2.8 \\
0.9\end{array}$ & $\begin{array}{l}0.7 \\
1.3 \\
0.9\end{array}$ & $\begin{array}{l}1.2 \\
0.3 \\
0.4\end{array}$ & $\begin{array}{l}0.7 \\
1.7 \\
0.8\end{array}$ & $\begin{array}{l}1.4 \\
2.7 \\
1.5\end{array}$ & $\begin{array}{l}5.9 \\
5.6 \\
6.2\end{array}$ & $\begin{array}{l}--- \\
8.6 \\
4.1\end{array}$ & $\begin{array}{r}--- \\
57 \\
82\end{array}$ & $\begin{array}{r}288 \\
0 \\
4\end{array}$ \\
\hline $\begin{array}{l}\text { LINE } \\
\text { A } \\
\text { B } \\
\text { C } \\
\text { D } \\
\text { E }\end{array}$ & $\begin{array}{l}50150 \\
5211.7 \\
5184.0 \\
5128.9 \\
5078.6 \\
5055.0\end{array}$ & $\begin{array}{r}\text { S } \\
\text { M } \\
\text { M } \\
\text { S? } \\
\text { S? }\end{array}$ & $\begin{array}{l}657970 \\
658453 \\
659414 \\
660344 \\
661076\end{array}$ & $\begin{array}{l}6099655 \\
6099597 \\
6099648 \\
6099612 \\
6099698\end{array}$ & $\begin{array}{l}0.6 \\
8.1 \\
0.0 \\
0.8 \\
2.2\end{array}$ & $\begin{array}{l}2.2 \\
1.3 \\
0.4 \\
0.9 \\
0.8\end{array}$ & $\begin{array}{l}4.1 \\
0.4 \\
4.5 \\
0.1 \\
0.8\end{array}$ & $\begin{array}{l}2.3 \\
1.8 \\
0.3 \\
1.4 \\
1.7\end{array}$ & $\begin{array}{l}3.8 \\
3.8 \\
4.2 \\
1.5 \\
2.4\end{array}$ & $\begin{array}{r}18.9 \\
9.8 \\
3.7 \\
7.1 \\
5.8\end{array}$ & $\begin{array}{c}--- \\
--- \\
--- \\
--- \\
10.3\end{array}$ & $\begin{array}{l}--- \\
--- \\
--- \\
--- \\
51\end{array}$ & $\begin{array}{r}0 \\
290 \\
0 \\
0 \\
0\end{array}$ \\
\hline $\begin{array}{l}\text { LINE } \\
\text { A } \\
B \\
C\end{array}$ & $\begin{array}{r}50160 \\
5636.5 \\
5761.4 \\
5774.0\end{array}$ & $\begin{array}{r}\text { S } \\
\text { B? } \\
\text { S? }\end{array}$ & $\begin{array}{l}658191 \\
660645 \\
660991\end{array}$ & $\begin{array}{l}6099185 \\
6099241 \\
6099293\end{array}$ & $\begin{array}{l}2.3 \\
1.5 \\
1.2\end{array}$ & $\begin{array}{l}1.5 \\
1.8 \\
1.3\end{array}$ & $\begin{array}{l}1.7 \\
1.1 \\
0.6\end{array}$ & $\begin{array}{l}1.7 \\
2.5 \\
1.9\end{array}$ & $\begin{array}{l}2.1 \\
7.6 \\
1.7\end{array}$ & $\begin{array}{r}13.4 \\
10.1 \\
9.5\end{array}$ & $\begin{array}{l}--- \\
--- \\
---\end{array}$ & $\begin{array}{l}--- \\
--- \\
---\end{array}$ & $\begin{array}{r}0 \\
0 \\
78\end{array}$ \\
\hline $\begin{array}{l}\text { LINE } \\
\text { A } \\
B \\
C\end{array}$ & $\begin{array}{r}50170 \\
6016.5 \\
5912.8 \\
5884.1\end{array}$ & $\begin{array}{l}S \\
S \\
D\end{array}$ & $\begin{array}{l}658778 \\
660761 \\
661404\end{array}$ & $\begin{array}{l}6098802 \\
6098894 \\
6098927\end{array}$ & $\begin{array}{l}1.0 \\
2.3 \\
1.9\end{array}$ & $\begin{array}{l}2.0 \\
3.9 \\
1.9\end{array}$ & $\begin{array}{l}3.0 \\
1.2 \\
0.7\end{array}$ & $\begin{array}{l}2.1 \\
3.6 \\
1.2\end{array}$ & $\begin{array}{l}3.6 \\
5.7 \\
3.2\end{array}$ & $\begin{array}{r}14.8 \\
24.6 \\
4.7\end{array}$ & $\begin{array}{l}--- \\
2.0 \\
---\end{array}$ & $\begin{array}{r}--- \\
32 \\
---\end{array}$ & $\begin{array}{r}0 \\
28 \\
0\end{array}$ \\
\hline $\begin{array}{l}\text { LINE } \\
\text { A } \\
\text { B } \\
\text { C } \\
\text { D }\end{array}$ & $\begin{array}{r}50180 \\
6429.6 \\
6451.1 \\
6529.1 \\
6538.1\end{array}$ & $\begin{array}{l}\text { S } \\
\text { S } \\
\text { D } \\
\text { B }\end{array}$ & $\begin{array}{l}658755 \\
659498 \\
661435 \\
661532\end{array}$ & $\begin{array}{l}6098418 \\
6098433 \\
6098517 \\
6098524\end{array}$ & $\begin{array}{l}2.6 \\
0.0 \\
1.5 \\
6.6\end{array}$ & $\begin{array}{l}0.5 \\
1.4 \\
5.6 \\
5.4\end{array}$ & $\begin{array}{l}1.2 \\
0.5 \\
2.2 \\
0.3\end{array}$ & $\begin{array}{l}1.5 \\
1.1 \\
8.7 \\
1.8\end{array}$ & $\begin{array}{r}3.0 \\
0.7 \\
20.9 \\
7.7\end{array}$ & $\begin{array}{r}7.0 \\
5.9 \\
25.9 \\
5.2\end{array}$ & $\begin{array}{l}--- \\
--- \\
1.5 \\
---\end{array}$ & $\begin{array}{r}--- \\
--- \\
8 \\
---\end{array}$ & $\begin{array}{r}20 \\
20 \\
0 \\
0\end{array}$ \\
\hline $\begin{array}{l}\text { LINE } \\
\text { A } \\
B \\
C\end{array}$ & $\begin{array}{r}50190 \\
6800.6 \\
6743.0 \\
6579.6\end{array}$ & $\begin{array}{l}\text { S } \\
\text { S } \\
\text { B }\end{array}$ & $\begin{array}{l}655824 \\
657339 \\
661660\end{array}$ & $\begin{array}{l}6097887 \\
6097908 \\
6098143\end{array}$ & $\begin{array}{r}3.4 \\
0.1 \\
10.8\end{array}$ & $\begin{array}{r}0.6 \\
0.8 \\
12.3\end{array}$ & $\begin{array}{l}3.4 \\
4.0 \\
7.7\end{array}$ & $\begin{array}{l}0.8 \\
1.5 \\
1.7\end{array}$ & $\begin{array}{l}0.2 \\
0.0 \\
8.5\end{array}$ & $\begin{array}{r}4.1 \\
11.6 \\
15.6\end{array}$ & $\begin{array}{l}--- \\
7.4 \\
---\end{array}$ & $\begin{array}{r}--- \\
69 \\
---\end{array}$ & $\begin{array}{l}0 \\
7 \\
0\end{array}$ \\
\hline $\begin{array}{l}\text { LINE } \\
\text { A } \\
B\end{array}$ & $\begin{array}{l}50200 \\
7027.6 \\
7131.3\end{array}$ & $\begin{array}{l}S \\
S\end{array}$ & $\begin{array}{l}655779 \\
658863\end{array}$ & $\begin{array}{l}6097488 \\
6097656\end{array}$ & $\begin{array}{l}0.3 \\
1.1\end{array}$ & $\begin{array}{l}1.7 \\
1.7\end{array}$ & $\begin{array}{l}1.8 \\
1.4\end{array}$ & $\begin{array}{l}1.8 \\
2.6\end{array}$ & $\begin{array}{l}2.5 \\
3.4\end{array}$ & $\begin{array}{l}11.6 \\
14.9\end{array}$ & $\begin{array}{l}--- \\
---\end{array}$ & $\begin{array}{l}--- \\
---\end{array}$ & $\begin{array}{r}0 \\
23\end{array}$ \\
\hline $\begin{array}{l}\text { LINE } \\
A\end{array}$ & $\begin{array}{l}50210 \\
7401.8\end{array}$ & $S$ & 658337 & 6097235 & 0.5 & 1.8 & 1.3 & 2.5 & 3.7 & 13.7 & --- & --- & 0 \\
\hline $\begin{array}{l}\text { LINE } \\
\text { A } \\
B\end{array}$ & $\begin{array}{l}50220 \\
7797.2 \\
7810.7\end{array}$ & $\begin{array}{l}S \\
S\end{array}$ & $\begin{array}{l}657431 \\
657942\end{array}$ & $\begin{array}{l}6096788 \\
6096807\end{array}$ & $\begin{array}{l}2.3 \\
0.3\end{array}$ & $\begin{array}{l}1.7 \\
2.2\end{array}$ & $\begin{array}{l}1.0 \\
2.0\end{array}$ & $\begin{array}{l}2.1 \\
3.2\end{array}$ & $\begin{array}{l}3.1 \\
5.1\end{array}$ & $\begin{array}{l}13.8 \\
21.7\end{array}$ & $\begin{array}{l}--- \\
---\end{array}$ & $\begin{array}{l}--- \\
---\end{array}$ & $\begin{array}{r}57 \\
0\end{array}$ \\
\hline $\begin{array}{l}\text { LINE } \\
A\end{array}$ & $\begin{array}{c}50250 \\
535.0\end{array}$ & M & 653520 & 6095376 & 2.2 & 0.1 & 0.6 & 0.2 & 0.1 & 3.4 & --- & --- & 427 \\
\hline
\end{tabular}

$\mathrm{CX}=$ COAXIAL

Note: EM values shown above

are local amplitudes

Ketchikan - South of Keete Inlet
* Estimated Depth may be unreliable because the stronger part of the conductor may be deeper or to one side of the flight line, or because of a shallow dip or magnetite/overburden effects 
EM Anomaly List

\begin{tabular}{|c|c|c|c|c|c|c|c|c|c|c|c|c|c|}
\hline Label & Fid & Interp & $\begin{array}{c}\text { XUTM } \\
\text { m }\end{array}$ & $\begin{array}{c}\text { YUTM } \\
\mathrm{m}\end{array}$ & $\begin{array}{ll}\text { CX } & 9 \\
\text { Real } & \\
\text { ppm }\end{array}$ & $\begin{array}{l}00 \mathrm{HZ} \\
\text { Quad } \\
\text { ppm }\end{array}$ & $\begin{array}{l}\text { CP } \\
\text { Real } \\
\text { ppm }\end{array}$ & $\begin{array}{l}900 \mathrm{HZ} \\
\text { Quad } \\
\text { ppm }\end{array}$ & $\begin{array}{l}\text { CP } 7 \\
\text { Real } \\
\text { ppm }\end{array}$ & $\begin{array}{l}200 \mathrm{HZ} \\
\text { Quad } \\
\text { ppm }\end{array}$ & $\begin{array}{l}\text { Vertica } \\
\text { Cond } \\
\text { siemens }\end{array}$ & $\begin{array}{c}\text { al Dike } \\
\text { DEPTH* } \\
\text { m }\end{array}$ & $\begin{array}{c}\text { Mag. Corr } \\
\text { NT }\end{array}$ \\
\hline $\begin{array}{l}\text { LINE } \\
B \\
\text { C }\end{array}$ & $\begin{array}{l}50250 \\
681.3 \\
684.0\end{array}$ & $\begin{array}{l}\mathrm{S} \\
\mathrm{M}\end{array}$ & $\begin{array}{l}658214 \\
658291\end{array}$ & $\begin{array}{l}6095549 \\
6095558\end{array}$ & $\begin{array}{l}2.6 \\
2.6\end{array}$ & $\begin{array}{l}1.0 \\
0.8\end{array}$ & $\begin{array}{l}9.2 \\
9.2\end{array}$ & $\begin{array}{l}1.5 \\
1.2\end{array}$ & $\begin{array}{l}7.3 \\
7.3\end{array}$ & $\begin{array}{l}10.4 \\
10.0\end{array}$ & $\begin{array}{l}--- \\
---\end{array}$ & $\begin{array}{l}--- \\
---\end{array}$ & $\begin{array}{r}41 \\
315\end{array}$ \\
\hline $\begin{array}{l}\mathrm{LINE} \\
\mathrm{A} \\
\mathrm{B}\end{array}$ & $\begin{array}{l}50260 \\
978.0 \\
956.0\end{array}$ & $\begin{array}{l}M \\
M\end{array}$ & $\begin{array}{l}657938 \\
658825\end{array}$ & $\begin{array}{l}6095110 \\
6095169\end{array}$ & $\begin{array}{l}0.4 \\
2.1\end{array}$ & $\begin{array}{l}0.0 \\
0.4\end{array}$ & $\begin{array}{l}0.4 \\
1.4\end{array}$ & $\begin{array}{l}0.7 \\
0.1\end{array}$ & $\begin{array}{l}0.5 \\
1.0\end{array}$ & $\begin{array}{l}4.4 \\
3.8\end{array}$ & $\begin{array}{l}--- \\
---\end{array}$ & $\begin{array}{l}--- \\
---\end{array}$ & $\begin{array}{r}131 \\
26\end{array}$ \\
\hline $\begin{array}{l}\text { LINE } \\
\text { A } \\
\text { B } \\
\text { C }\end{array}$ & $\begin{array}{r}50270 \\
1412.0 \\
1492.0 \\
1508.7\end{array}$ & $\begin{array}{r}\mathrm{M} \\
\mathrm{M} \\
\mathrm{B} ?\end{array}$ & $\begin{array}{l}656982 \\
658747 \\
659116\end{array}$ & $\begin{array}{l}6094712 \\
6094767 \\
6094798\end{array}$ & $\begin{array}{l}0.1 \\
3.4 \\
2.1\end{array}$ & $\begin{array}{l}0.8 \\
0.2 \\
2.3\end{array}$ & $\begin{array}{l}8.9 \\
9.2 \\
3.3\end{array}$ & $\begin{array}{l}0.6 \\
0.4 \\
2.7\end{array}$ & $\begin{array}{l}7.6 \\
8.1 \\
8.2\end{array}$ & $\begin{array}{r}4.2 \\
1.5 \\
15.7\end{array}$ & $\begin{array}{l}--- \\
--- \\
---\end{array}$ & $\begin{array}{l}--- \\
--- \\
---\end{array}$ & $\begin{array}{r}108 \\
135 \\
0\end{array}$ \\
\hline $\begin{array}{l}\text { LINE } \\
\text { A } \\
\text { B } \\
\text { C } \\
\text { D } \\
\text { E }\end{array}$ & $\begin{array}{l}50280 \\
1958.7 \\
1860.3 \\
1847.9 \\
1836.6 \\
1822.0\end{array}$ & $\begin{array}{l}\mathrm{M} \\
\mathrm{M} \\
\mathrm{M} \\
\mathrm{M} \\
\mathrm{M}\end{array}$ & $\begin{array}{l}655066 \\
656799 \\
657134 \\
657348 \\
657632\end{array}$ & $\begin{array}{l}6094223 \\
6094301 \\
6094329 \\
6094320 \\
6094320\end{array}$ & $\begin{array}{l}5.2 \\
0.2 \\
0.1 \\
1.5 \\
1.6\end{array}$ & $\begin{array}{l}0.2 \\
0.8 \\
1.0 \\
0.9 \\
0.9\end{array}$ & $\begin{array}{r}14.0 \\
1.0 \\
0.0 \\
3.1 \\
3.8\end{array}$ & $\begin{array}{l}0.8 \\
1.0 \\
1.2 \\
1.6 \\
0.3\end{array}$ & $\begin{array}{r}11.9 \\
0.9 \\
0.8 \\
0.2 \\
2.8\end{array}$ & $\begin{array}{r}3.1 \\
6.5 \\
6.8 \\
13.1 \\
2.7\end{array}$ & $\begin{array}{l}--- \\
--- \\
--- \\
--- \\
---\end{array}$ & $\begin{array}{l}--- \\
--- \\
--- \\
--- \\
---\end{array}$ & $\begin{array}{r}0 \\
0 \\
9 \\
11 \\
0\end{array}$ \\
\hline $\begin{array}{l}\text { LINE } \\
\text { A } \\
\text { B } \\
\text { C } \\
\text { D } \\
\text { E }\end{array}$ & $\begin{array}{r}50290 \\
2296.0 \\
2317.9 \\
2383.3 \\
2413.7 \\
2448.0\end{array}$ & $\begin{array}{c}\mathrm{M} \\
\mathrm{M} \\
\mathrm{S} ? \\
\mathrm{M} \\
\mathrm{S}\end{array}$ & $\begin{array}{l}655336 \\
655761 \\
657214 \\
657801 \\
658349\end{array}$ & $\begin{array}{l}6093828 \\
6093844 \\
6093901 \\
6093899 \\
6093942\end{array}$ & $\begin{array}{l}0.1 \\
2.5 \\
5.9 \\
0.0 \\
0.8\end{array}$ & $\begin{array}{l}0.2 \\
0.3 \\
2.7 \\
0.5 \\
2.0\end{array}$ & $\begin{array}{r}6.7 \\
8.1 \\
18.4 \\
5.6 \\
12.2\end{array}$ & $\begin{array}{l}0.1 \\
1.2 \\
2.6 \\
0.1 \\
2.3\end{array}$ & $\begin{array}{r}6.0 \\
6.4 \\
16.1 \\
4.5 \\
4.2\end{array}$ & $\begin{array}{r}1.7 \\
7.0 \\
19.8 \\
2.2 \\
17.8\end{array}$ & $\begin{array}{l}--- \\
--- \\
--- \\
--- \\
---\end{array}$ & $\begin{array}{l}--- \\
--- \\
--- \\
--- \\
---\end{array}$ & $\begin{array}{r}145 \\
0 \\
7 \\
1111 \\
48\end{array}$ \\
\hline $\begin{array}{l}\text { LINE } \\
\mathrm{A}\end{array}$ & $\begin{array}{l}50300 \\
2809.0\end{array}$ & M & 656841 & 6093416 & 0.1 & 0.7 & 1.0 & 0.3 & 0.7 & 4.8 & --- & --- & 64 \\
\hline $\begin{array}{l}\text { LINE } \\
\text { A } \\
\text { B } \\
\text { C } \\
\text { D }\end{array}$ & $\begin{array}{l}50310 \\
310.2 \\
443.2 \\
506.0 \\
610.0\end{array}$ & $\begin{array}{r}\mathrm{S} ? \\
\mathrm{D} \\
\mathrm{M} \\
\mathrm{M}\end{array}$ & $\begin{array}{l}654278 \\
655797 \\
656964 \\
659416\end{array}$ & $\begin{array}{l}6093043 \\
6093059 \\
6093105 \\
6093205\end{array}$ & $\begin{array}{l}0.7 \\
1.8 \\
0.0 \\
0.0\end{array}$ & $\begin{array}{l}2.1 \\
5.1 \\
0.4 \\
0.0\end{array}$ & $\begin{array}{l}0.7 \\
5.2 \\
0.6 \\
0.0\end{array}$ & $\begin{array}{l}3.2 \\
5.3 \\
0.4 \\
7.0\end{array}$ & $\begin{array}{r}8.4 \\
16.3 \\
0.6 \\
15.4\end{array}$ & $\begin{array}{r}8.1 \\
11.7 \\
7.1 \\
6.0\end{array}$ & $\begin{array}{l}--- \\
--- \\
--- \\
---\end{array}$ & $\begin{array}{l}--- \\
--- \\
--- \\
---\end{array}$ & $\begin{array}{r}0 \\
47 \\
29 \\
450\end{array}$ \\
\hline $\begin{array}{l}\text { LINE } \\
\text { A } \\
B\end{array}$ & $\begin{array}{l}50320 \\
972.5 \\
952.0\end{array}$ & $\begin{array}{l}\mathrm{D} \\
\mathrm{M}\end{array}$ & $\begin{array}{l}655887 \\
656253\end{array}$ & $\begin{array}{l}6092619 \\
6092664\end{array}$ & $\begin{array}{l}2.6 \\
1.4\end{array}$ & $\begin{array}{l}6.7 \\
1.0\end{array}$ & $\begin{array}{l}1.2 \\
2.0\end{array}$ & $\begin{array}{l}5.6 \\
0.5\end{array}$ & $\begin{array}{r}13.6 \\
1.5\end{array}$ & $\begin{array}{r}15.8 \\
5.6\end{array}$ & $\begin{array}{l}2.0 \\
---\end{array}$ & $\begin{array}{r}22 \\
---\end{array}$ & $\begin{array}{r}39 \\
0\end{array}$ \\
\hline $\begin{array}{l}\mathrm{LINE} \\
\mathrm{A} \\
\mathrm{B}\end{array}$ & $\begin{array}{l}50330 \\
1233.9 \\
1247.0\end{array}$ & $\begin{array}{l}\mathrm{D} \\
\mathrm{M}\end{array}$ & $\begin{array}{l}655868 \\
656035\end{array}$ & $\begin{array}{l}6092274 \\
6092275\end{array}$ & $\begin{array}{l}1.8 \\
2.6\end{array}$ & $\begin{array}{r}10.6 \\
0.7\end{array}$ & $\begin{array}{l}1.5 \\
4.0\end{array}$ & $\begin{array}{l}3.2 \\
0.7\end{array}$ & $\begin{array}{l}9.3 \\
6.3\end{array}$ & $\begin{array}{r}20.0 \\
2.2\end{array}$ & $\begin{array}{l}1.3 \\
---\end{array}$ & $\begin{array}{r}10 \\
---\end{array}$ & $\begin{array}{r}0 \\
300\end{array}$ \\
\hline $\begin{array}{l}\text { LINE } \\
\text { A } \\
B\end{array}$ & $\begin{array}{l}50340 \\
1704.0 \\
1681.6\end{array}$ & $\begin{array}{l}\mathrm{S} \\
\mathrm{D}\end{array}$ & $\begin{array}{l}655484 \\
655919\end{array}$ & $\begin{array}{l}6091824 \\
6091854\end{array}$ & $\begin{array}{l}0.1 \\
4.7\end{array}$ & $\begin{array}{l}2.2 \\
5.0\end{array}$ & $\begin{array}{l}0.3 \\
3.8\end{array}$ & $\begin{array}{l}2.0 \\
1.7\end{array}$ & $\begin{array}{l}1.3 \\
2.8\end{array}$ & $\begin{array}{r}13.9 \\
4.9\end{array}$ & $\begin{array}{l}--- \\
8.4\end{array}$ & --- & $\begin{array}{r}0 \\
416\end{array}$ \\
\hline
\end{tabular}

$\mathrm{CX}=$ COAXIAL

Note: EM values shown above

are local amplitudes

Ketchikan - South of Keete Inlet
*Estimated Depth may be unreliable because the stronger part of the conductor may be deeper or to one side of the flight line, or because of a shallow dip or magnetite/overburden effects 
EM Anomaly List

\begin{tabular}{|c|c|c|c|c|c|c|c|c|c|c|c|c|c|}
\hline Label & Fid & Interp & $\begin{array}{l}\text { XUTM } \\
\text { m }\end{array}$ & $\begin{array}{c}\text { YUTM } \\
\text { m }\end{array}$ & $\begin{array}{l}\text { CX } \\
\text { Real } \\
\text { ppm }\end{array}$ & $\begin{array}{l}00 \mathrm{HZ} \\
\text { Quad } \\
\text { ppm }\end{array}$ & $\begin{array}{l}\text { CP } \\
\text { Real } \\
\text { ppm }\end{array}$ & $\begin{array}{l}900 \mathrm{HZ} \\
\text { Quad } \\
\text { ppm }\end{array}$ & $\begin{array}{l}\text { CP } 7 \\
\text { Real } \\
\text { ppm }\end{array}$ & $\begin{array}{l}200 \mathrm{HZ} \\
\text { Quad } \\
\text { ppm }\end{array}$ & $\begin{array}{l}\text { Vertica } \\
\text { CoND } \\
\text { siemens }\end{array}$ & $\begin{array}{c}\text { al Dike } \\
\text { DEPTH* } \\
\text { m }\end{array}$ & $\begin{array}{c}\text { Mag. Corr } \\
\text { NT }\end{array}$ \\
\hline $\begin{array}{l}\text { LINE } \\
\text { C } \\
\text { D } \\
\text { E }\end{array}$ & $\begin{array}{r}50340 \\
1661.2 \\
1646.0 \\
1585.9\end{array}$ & $\begin{array}{r}\mathrm{S} ? \\
\mathrm{M} \\
\mathrm{M}\end{array}$ & $\begin{array}{l}656590 \\
656925 \\
658915\end{array}$ & $\begin{array}{l}6091885 \\
6091869 \\
6091968\end{array}$ & $\begin{array}{l}3.4 \\
0.3 \\
0.0\end{array}$ & $\begin{array}{l}4.5 \\
0.2 \\
1.3\end{array}$ & $\begin{array}{l}4.9 \\
0.3 \\
0.1\end{array}$ & $\begin{array}{l}5.1 \\
0.2 \\
1.2\end{array}$ & $\begin{array}{r}13.5 \\
0.2 \\
0.1\end{array}$ & $\begin{array}{r}16.1 \\
6.2 \\
5.8\end{array}$ & $\begin{array}{l}5.2 \\
--- \\
---\end{array}$ & $\begin{array}{r}23 \\
--- \\
---\end{array}$ & $\begin{array}{r}0 \\
373 \\
17\end{array}$ \\
\hline $\begin{array}{l}\text { LINE } \\
\text { A } \\
\text { B } \\
\text { C } \\
\text { D } \\
\text { E } \\
\text { F } \\
\text { G }\end{array}$ & $\begin{array}{l}50350 \\
1970.4 \\
1996.4 \\
2060.0 \\
2061.0 \\
2076.2 \\
2100.0 \\
2138.0\end{array}$ & $\begin{array}{r}\text { B? } \\
\text { B? } \\
\text { M } \\
\text { D? } \\
\text { S } \\
\text { M } \\
\text { M }\end{array}$ & $\begin{array}{l}656133 \\
656377 \\
657174 \\
657194 \\
657572 \\
658131 \\
658721\end{array}$ & $\begin{array}{l}6091481 \\
6091489 \\
6091553 \\
6091552 \\
6091560 \\
6091549 \\
6091554\end{array}$ & $\begin{array}{l}8.9 \\
4.7 \\
7.6 \\
8.7 \\
4.2 \\
0.0 \\
0.9\end{array}$ & $\begin{array}{l}7.5 \\
3.9 \\
7.9 \\
8.8 \\
9.9 \\
0.4 \\
0.9\end{array}$ & $\begin{array}{r}24.7 \\
15.9 \\
12.9 \\
12.9 \\
11.2 \\
8.8 \\
5.5\end{array}$ & $\begin{array}{r}17.7 \\
13.4 \\
11.7 \\
11.7 \\
29.3 \\
0.1 \\
0.5\end{array}$ & $\begin{array}{r}39.8 \\
30.1 \\
18.7 \\
18.7 \\
67.9 \\
6.6 \\
4.3\end{array}$ & $\begin{array}{r}16.3 \\
10.5 \\
12.8 \\
12.8 \\
66.7 \\
1.2 \\
1.6\end{array}$ & $\begin{array}{c}15.4 \\
--- \\
--- \\
9.7 \\
--- \\
--- \\
---\end{array}$ & $\begin{array}{r}15 \\
--- \\
--- \\
18 \\
--- \\
--- \\
---\end{array}$ & $\begin{array}{r}0 \\
50 \\
50 \\
50 \\
50 \\
0 \\
3\end{array}$ \\
\hline $\begin{array}{l}\text { LINE } \\
\text { A } \\
B\end{array}$ & $\begin{array}{l}50360 \\
2433.5 \\
2313.1\end{array}$ & $\begin{array}{r}\mathrm{M} \\
\mathrm{D} ?\end{array}$ & $\begin{array}{l}657196 \\
660655\end{array}$ & $\begin{array}{l}6091106 \\
6091248\end{array}$ & $\begin{array}{r}17.7 \\
3.5\end{array}$ & $\begin{array}{l}1.0 \\
2.4\end{array}$ & $\begin{array}{l}4.0 \\
2.3\end{array}$ & $\begin{array}{l}0.8 \\
0.9\end{array}$ & $\begin{array}{l}3.6 \\
4.9\end{array}$ & $\begin{array}{l}8.5 \\
5.2\end{array}$ & $\begin{array}{r}--- \\
12.7\end{array}$ & $\begin{array}{r}--- \\
32\end{array}$ & $\begin{array}{l}0 \\
0\end{array}$ \\
\hline $\begin{array}{l}\text { LINE } \\
\mathrm{A}\end{array}$ & $\begin{array}{l}50361 \\
2630.0\end{array}$ & S? & 655836 & 6091035 & 0.1 & 2.9 & 0.8 & 2.9 & 4.3 & 13.9 & 0.6 & 0 & 0 \\
\hline $\begin{array}{l}\text { LINE } \\
\text { A } \\
\text { B } \\
\text { C }\end{array}$ & $\begin{array}{r}50370 \\
2887.2 \\
2936.0 \\
2986.6\end{array}$ & $\begin{array}{l}\mathrm{M} \\
\mathrm{M} \\
\mathrm{M}\end{array}$ & $\begin{array}{l}656470 \\
657362 \\
658581\end{array}$ & $\begin{array}{l}6090695 \\
6090718 \\
6090732\end{array}$ & $\begin{array}{r}0.0 \\
10.1 \\
3.6\end{array}$ & $\begin{array}{l}1.3 \\
0.8 \\
0.2\end{array}$ & $\begin{array}{r}8.0 \\
36.4 \\
3.0\end{array}$ & $\begin{array}{l}1.5 \\
2.5 \\
0.0\end{array}$ & $\begin{array}{r}4.3 \\
30.8 \\
2.3\end{array}$ & $\begin{array}{r}7.9 \\
14.0 \\
1.3\end{array}$ & $\begin{array}{l}--- \\
--- \\
---\end{array}$ & $\begin{array}{l}--- \\
--- \\
---\end{array}$ & $\begin{array}{r}0 \\
189 \\
264\end{array}$ \\
\hline $\begin{array}{l}\text { LINE } \\
\text { A }\end{array}$ & $\begin{array}{l}50380 \\
3252.0\end{array}$ & M & 657160 & 6090277 & 1.1 & 0.1 & 0.7 & 0.1 & 0.2 & 3.2 & --- & --- & 0 \\
\hline $\begin{array}{l}\text { LINE } \\
\text { A } \\
\text { B } \\
\text { C }\end{array}$ & $\begin{array}{r}59010 \\
5520.8 \\
5293.4 \\
5281.2\end{array}$ & $\begin{array}{r}\mathrm{B} ? \\
\mathrm{~S} \\
\mathrm{~S}\end{array}$ & $\begin{array}{l}656144 \\
655951 \\
655948\end{array}$ & $\begin{array}{l}6091583 \\
6097731 \\
6098147\end{array}$ & $\begin{array}{r}17.6 \\
0.1 \\
1.0\end{array}$ & $\begin{array}{l}7.5 \\
1.1 \\
0.9\end{array}$ & $\begin{array}{r}10.1 \\
0.4 \\
0.7\end{array}$ & $\begin{array}{l}8.9 \\
1.6 \\
0.5\end{array}$ & $\begin{array}{r}23.9 \\
3.0 \\
0.7\end{array}$ & $\begin{array}{r}11.6 \\
7.6 \\
6.5\end{array}$ & $\begin{array}{r}27.3 \\
--- \\
---\end{array}$ & $\begin{array}{r}9 \\
--- \\
---\end{array}$ & $\begin{array}{r}0 \\
0 \\
23\end{array}$ \\
\hline $\begin{array}{l}\text { LINE } 5 \\
\text { A } \\
\text { B } \\
\text { C } \\
\text { D } \\
\text { E } \\
\text { F }\end{array}$ & $\begin{array}{r}59020 \\
4320.0 \\
4508.1 \\
4562.8 \\
4573.8 \\
4591.9 \\
4627.5\end{array}$ & $\begin{array}{r}\text { B? } \\
\text { S? } \\
\text { B } \\
\text { B } \\
\text { B } \\
\text { B }\end{array}$ & $\begin{array}{l}660935 \\
660733 \\
660661 \\
660637 \\
660679 \\
660652\end{array}$ & $\begin{array}{l}6093279 \\
6098980 \\
6100426 \\
6100781 \\
6101390 \\
6102197\end{array}$ & $\begin{array}{l}4.6 \\
1.2 \\
3.2 \\
6.9 \\
4.3 \\
3.2\end{array}$ & $\begin{array}{l}4.0 \\
2.5 \\
3.0 \\
8.8 \\
4.0 \\
5.0\end{array}$ & $\begin{array}{l}4.5 \\
1.4 \\
1.6 \\
4.6 \\
1.2 \\
0.3\end{array}$ & $\begin{array}{r}8.2 \\
3.3 \\
5.7 \\
11.7 \\
5.2 \\
5.8\end{array}$ & $\begin{array}{r}17.3 \\
6.5 \\
16.5 \\
37.4 \\
11.6 \\
10.5\end{array}$ & $\begin{array}{r}8.8 \\
22.7 \\
24.3 \\
34.1 \\
11.1 \\
33.8\end{array}$ & $\begin{array}{l}5.5 \\
--- \\
--- \\
3.9 \\
--- \\
2.5\end{array}$ & $\begin{array}{r}27 \\
---- \\
--- \\
20 \\
--- \\
24\end{array}$ & $\begin{array}{r}14 \\
24 \\
0 \\
0 \\
0 \\
13\end{array}$ \\
\hline $\begin{array}{l}C X=C C \\
C P=C C \\
\text { Ketchik }\end{array}$ & $\begin{array}{l}\text { COAXIAL } \\
\text { COPLANAR }\end{array}$ & \multicolumn{5}{|c|}{$\begin{array}{l}\text { Note: EM values shown above } \\
\text { are local amplitudes } \\
\text { f Keete Inlet }\end{array}$} & & $142-$ & \multicolumn{5}{|c|}{$\begin{array}{l}\text { *Estimated Depth may be unreliable because the } \\
\text { stronger part of the conductor may be deeper or } \\
\text { to one side of the flight line, or because of a } \\
\text { shallow dip or magnetite/overburden effects }\end{array}$} \\
\hline
\end{tabular}


APPENDIX D

TOTAL MAGNETIC FIELD INVERSE MODELS 


\section{APPENDIX D}

\section{TOTAL MAGNETIC FIELD INVERSE MODELS}

Appendix D contains the diagramatic results of inverse models for five magnetic anomalies in the Ketchikan area.

\section{Model 1}

Magnetic Unit, Salt Chuck

A large irregular-shaped magnetic body, possibly a volcanic intrusive, was modelled. Sharp, high amplitude, near-surface features were ignored. The contacts of the main body of the intrusive appear to dip to the south.

\section{Model 2, 3}

\section{Magnetic Pluton, Prince of Wales Island}

The southwestern and northeastern contacts of this broad magnetic unit were modelled separately. A step model was used to approximate the contact at the southwestern end. The model results indicate a susceptibility contrast of 0.0165 emu with a dip of 160 ?. The northeastern contact of the magnetic unit was fitted to a tabular-type model with finite depth extent. Both models suggest a shallow-dipping contact of approximately 20? in opposite directions.

\section{Model 4}

\section{Magnetic Unit, Prince of Wales Island}

The model results indicate a ribbon-like, north-dipping unit at a depth of 153 metres.

\section{Model 5}

SE Trending Fault, Gravina Island West

A step model was used to approximate a southeast-trending fault between two magnetic units. The model results indicate a susceptibility contrast of $0.00220 \mathrm{emu}$ with a dip of $90 ?$. 


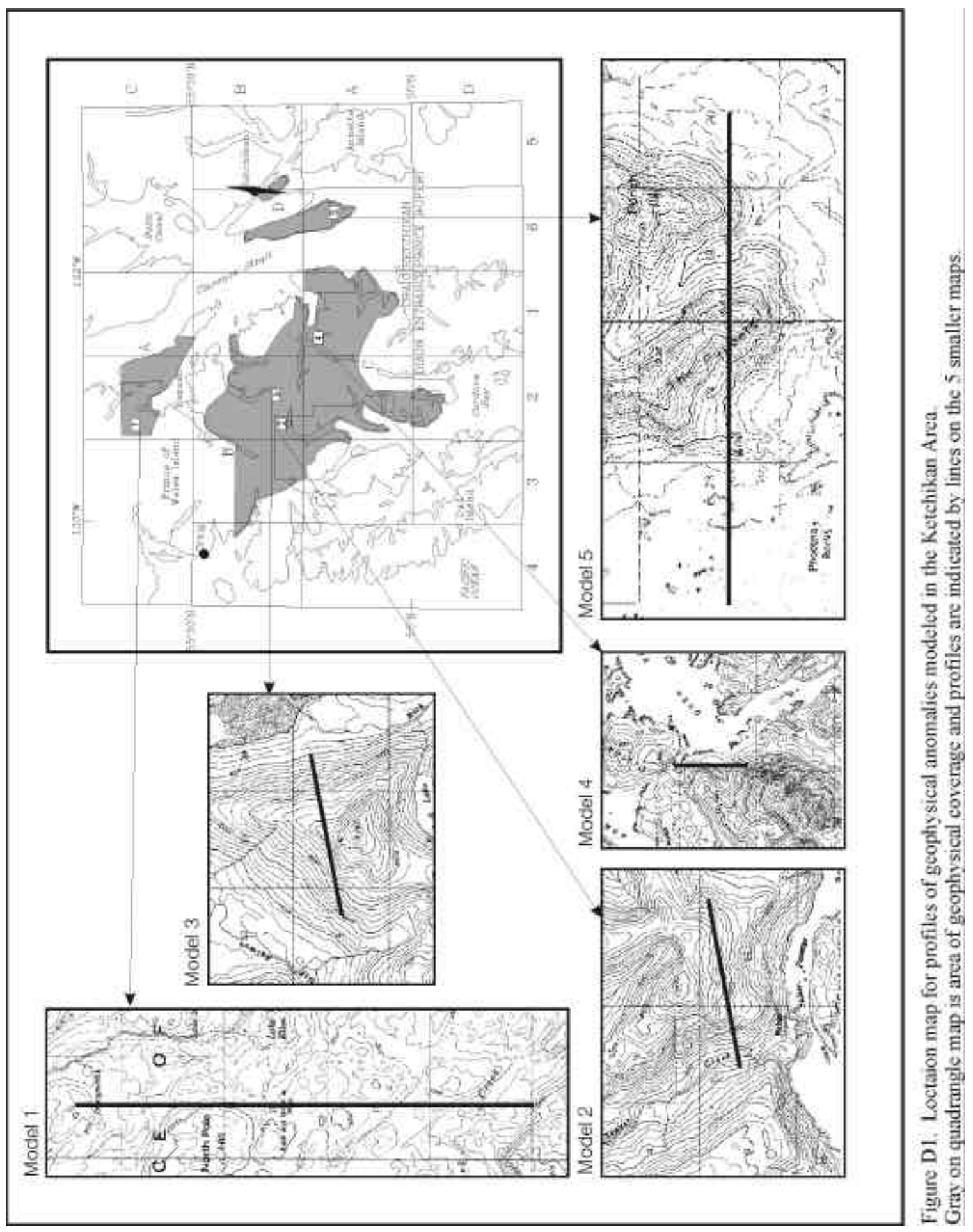




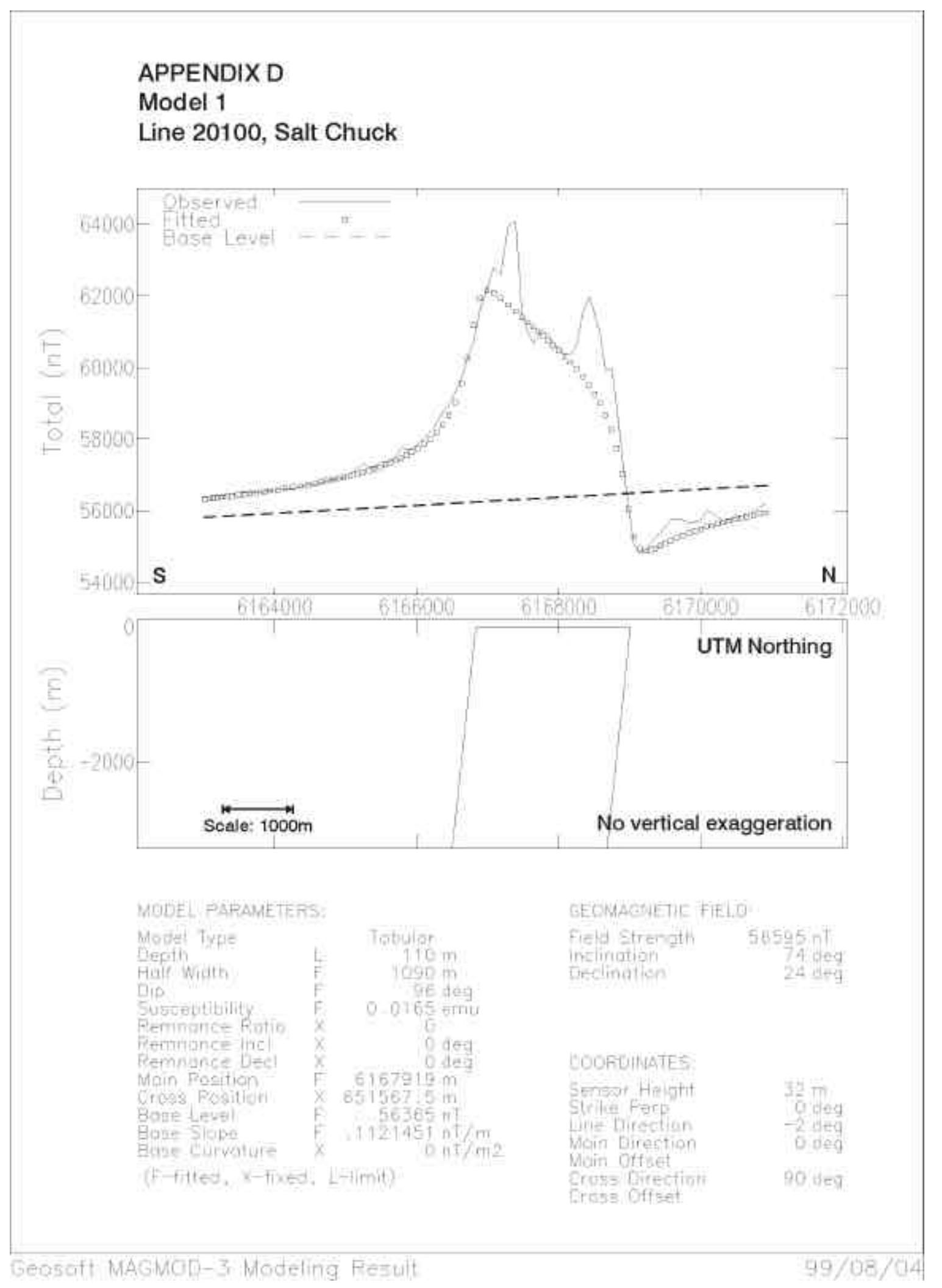




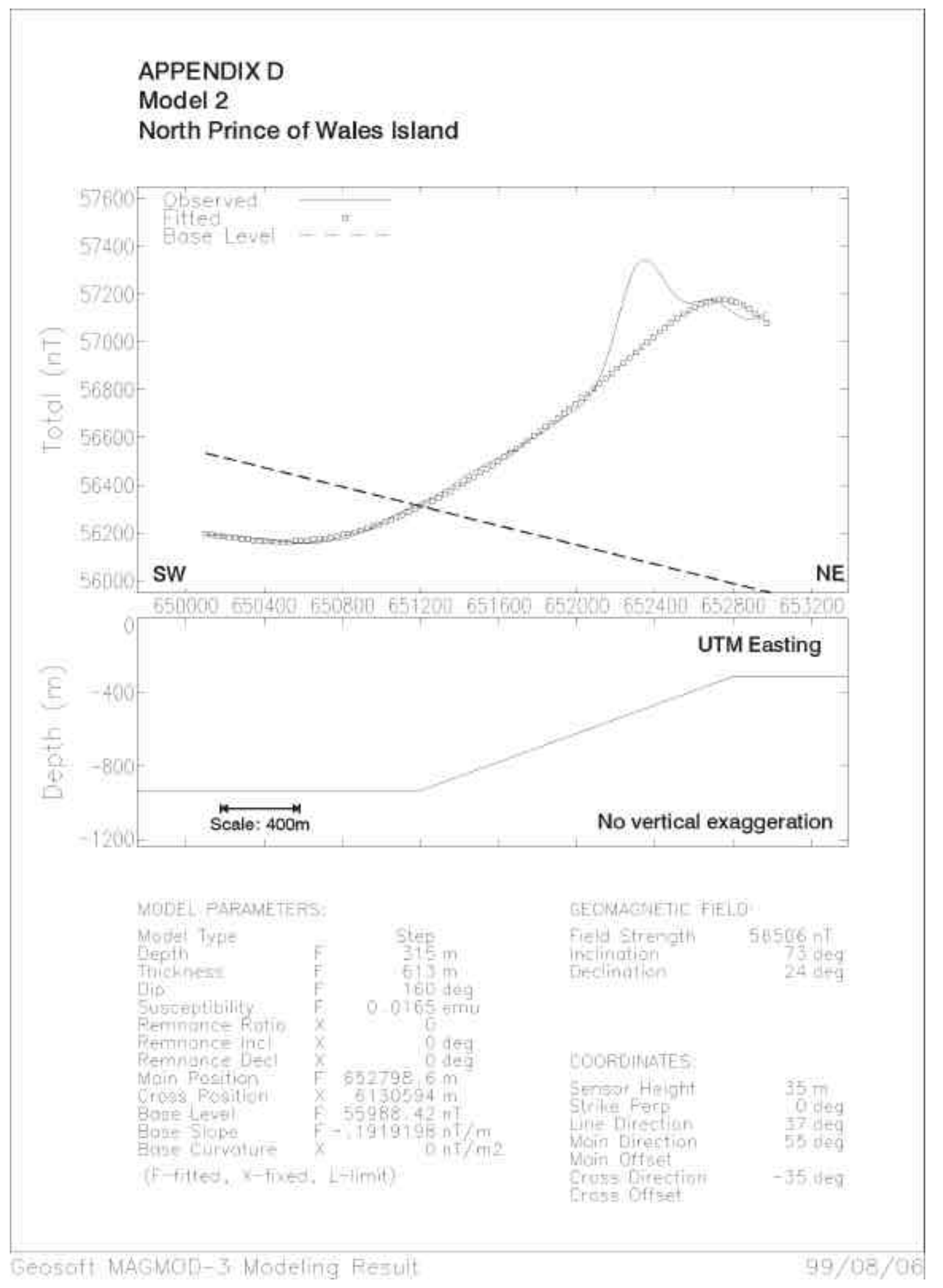




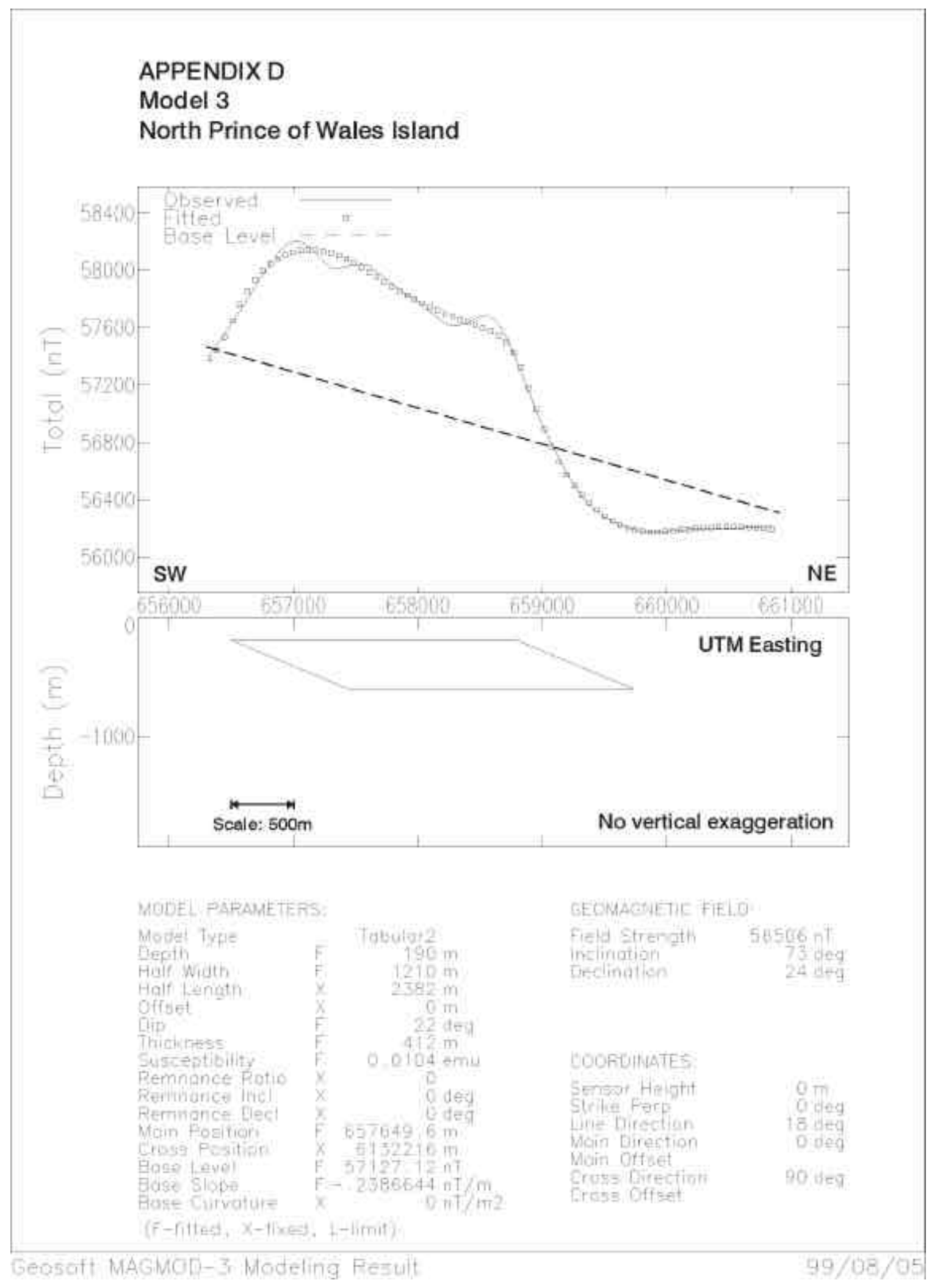




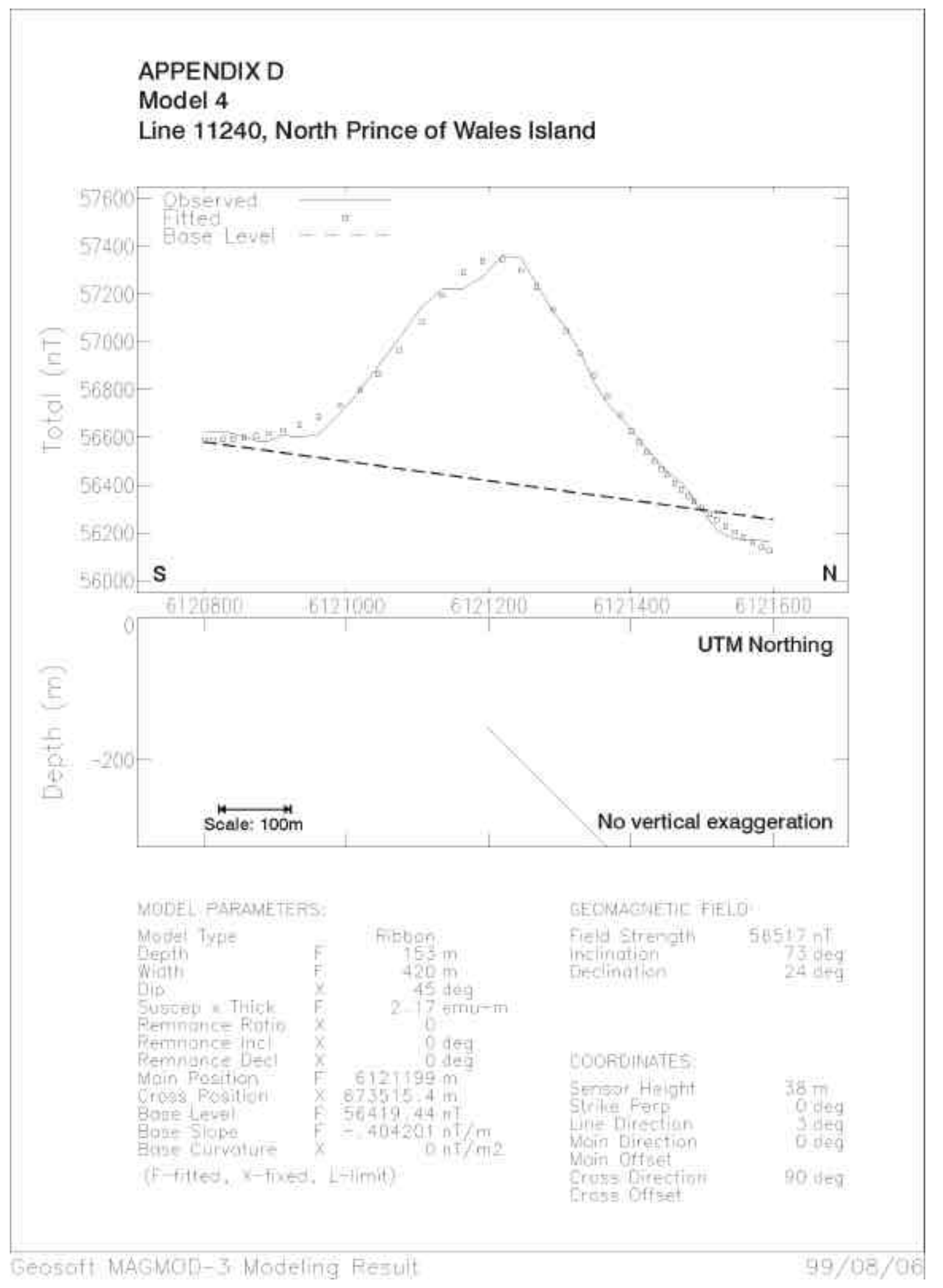




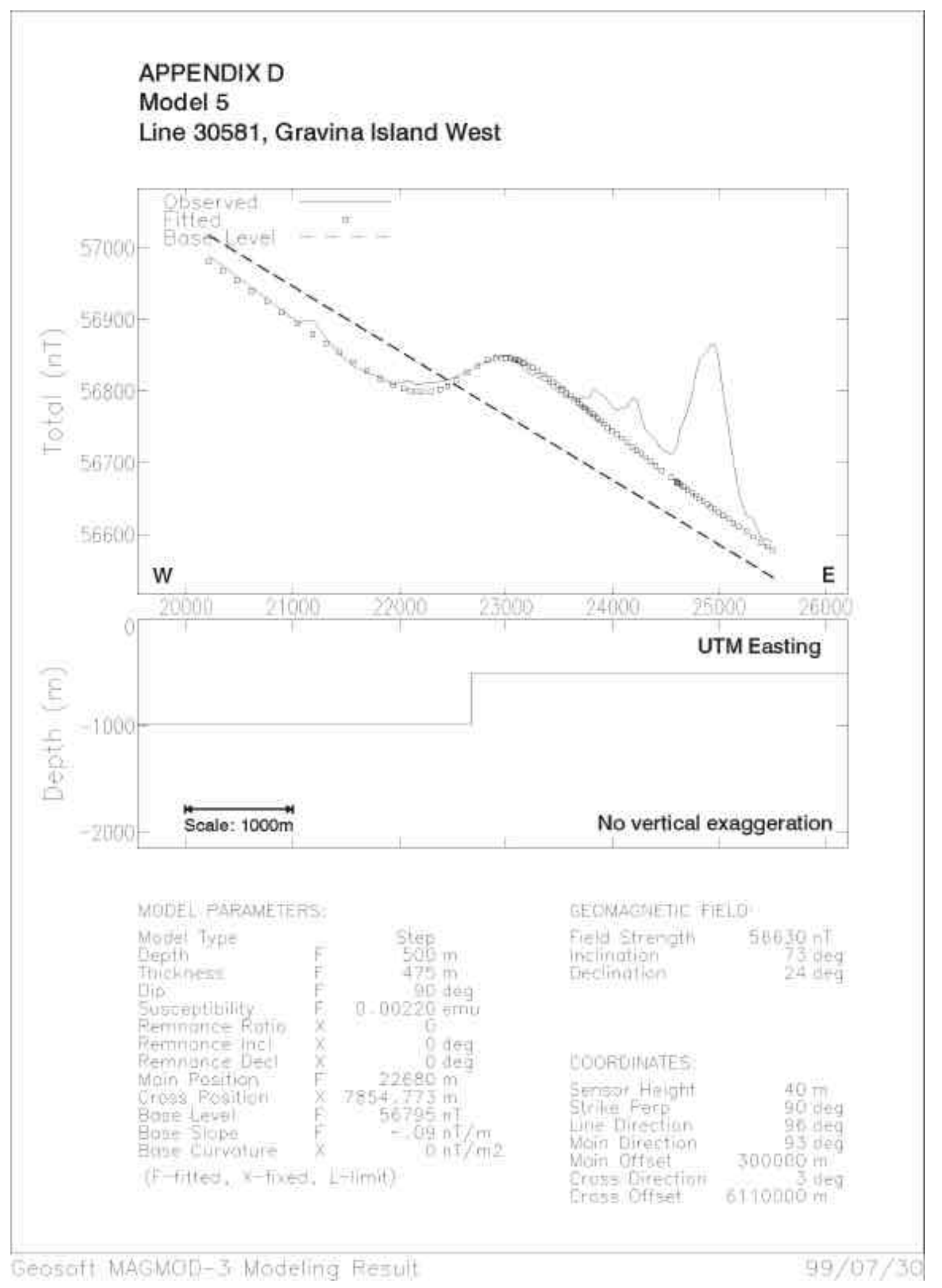


